\title{
Wilson-Leonard An 11,000-year Archeological Record of Hunter- Gatherers in Central Texas Volume II: Chipped Stone Artifacts
}

Michael B. Collins

Texas Archeological Research Laboratory

C. Britt Bousman

Department of Anthropology, Texas State University

Susan W. Dial

Texas Archeological Research Laboratory

Anne C. Kerr

Texas Archeological Research Laboratory

Keith Prillman

Texas Archeological Research Laboratory

Follow this and additional works at: https://scholarworks.sfasu.edu/ita

Part of the American Material Culture Commons, Archaeological Anthropology Commons, Environmental Studies Commons, Other American Studies Commons, Other Arts and Humanities Commons, Other History of Art, Architecture, and Archaeology Commons, and the United States History Commons

Tell us how this article helped you.

This Article is brought to you for free and open access by the Center for Regional Heritage Research at SFA ScholarWorks. It has been accepted for inclusion in Index of Texas Archaeology: Open Access Gray Literature from the Lone Star State by an authorized editor of SFA ScholarWorks. For more information, please contact cdsscholarworks@sfasu.edu. 


\section{Wilson-Leonard An 11,000-year Archeological Record of Hunter-Gatherers in Central Texas Volume II: Chipped Stone Artifacts}

\section{Creative Commons License}

\section{(c) (i) $\Theta($}

This work is licensed under a Creative Commons Attribution-NonCommercial-No Derivative Works 4.0 International License. 


\title{
WILSON-LEONARD
}

\section{An 11,000-year Archeological Record of Hunter-Gatherers in Central Texas}

\author{
Volume II: Chipped Stone Artifacts
}

\author{
Assembled and Edited by Michael B. Collins
}

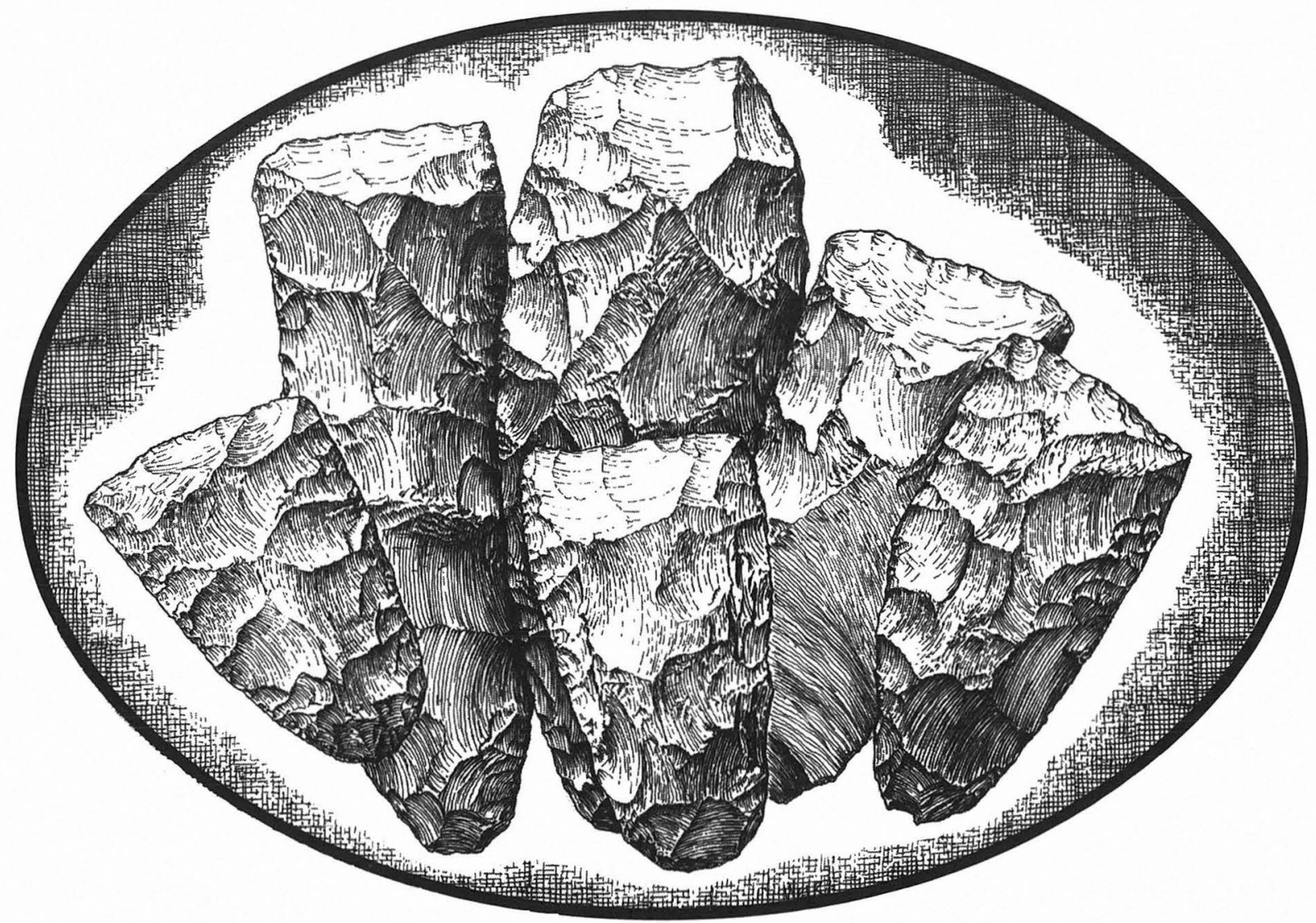

Studies in Archeology 31

Texas Archeological Research Laboratory The University of Texas at Austin
Archeology Studies Program, Report 10

Texas Department of Transportation Environmental Affairs Division 
"This page intentionally left blank" 


\title{
WILSON-LEONARD
}

\section{An 11,000-year Archeological Record of Hunter-Gatherers in Central Texas}

\section{Volume II: Chipped Stone Artifacts}

\author{
assembled and edited by \\ Michael B. Collins \\ with contributions by \\ C. Britt Bousman \\ Michael B. Collins \\ Susan W. Dial \\ Anne C. Kerr \\ Keith Prilliman
}

Principal Investigator: Nancy Kenmotsu

Frank Weir(1982-1998)

Texas Antiquities Committee Permit No. 300

Studies in Archeology 31

Texas Archeological Research Laboratory

The University of Texas at Austin

Archeology Studies Program, Report 10

Texas Department of Transportation

Environmental Affairs Division 
Cover drawing by Frank Weir

The University of Texas at Austin Texas Archeological Research Laboratory J. J. Pickle Research Campus 5 Austin, Texas 78712-1100

Printed 1998, on acid-free, 60-pound paper

ISBN 1-887072-26-8(Volume II)

ISBN 1-887072-24-1 (5-Volume Set) 


\section{TABLE OF CONTENTS \\ Volume II}

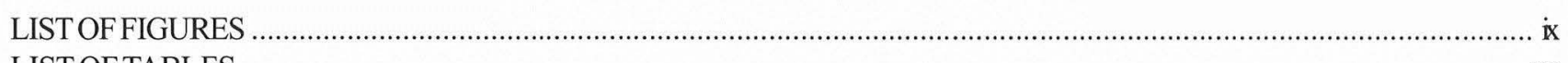

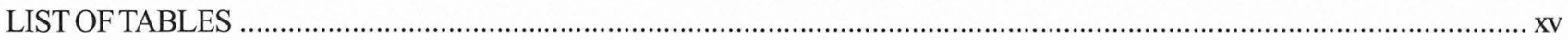

CHAPTER 12: INTRODUCTION TO THE CULTURALMATERIALS RECOVERED

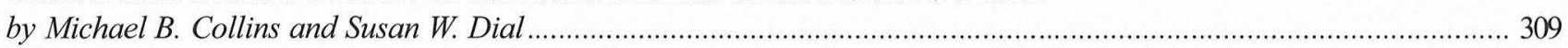

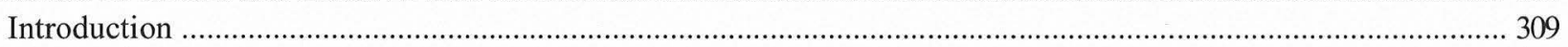

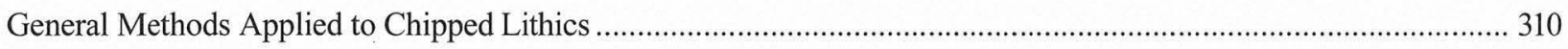

CHAPTER 13: PROJECTILEPOINTS

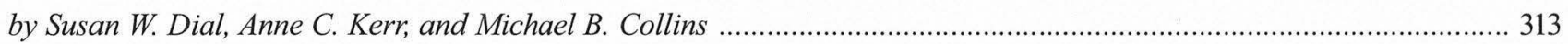

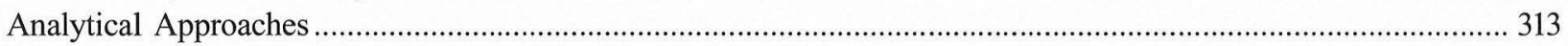

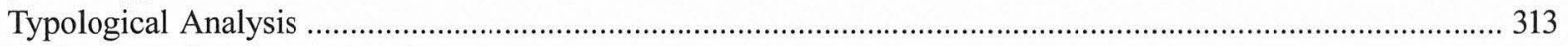

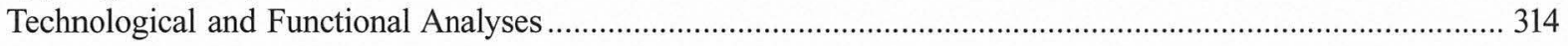

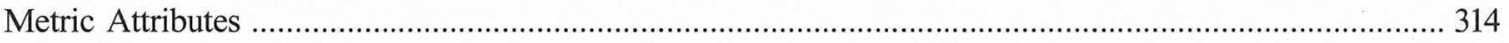

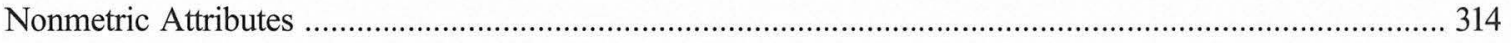

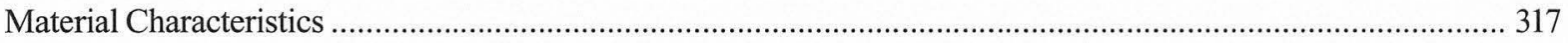

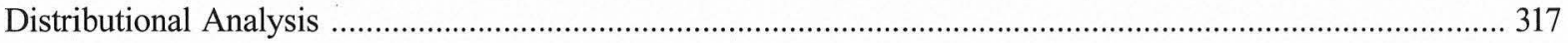

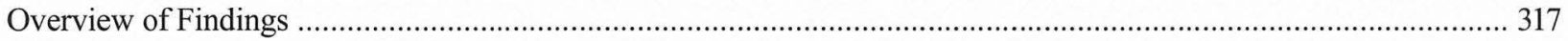

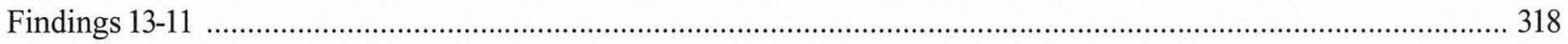

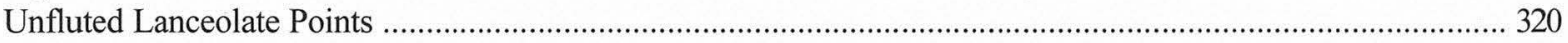

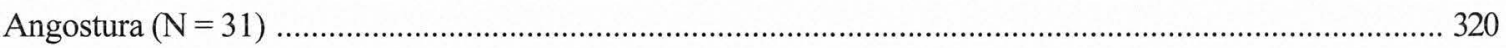

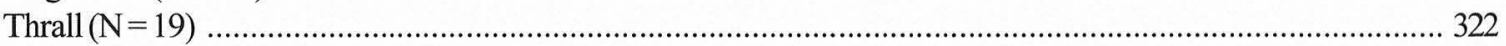

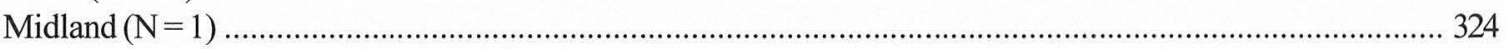

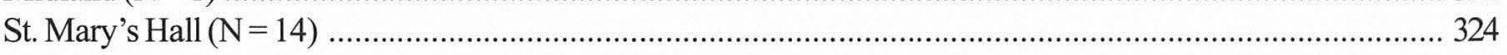

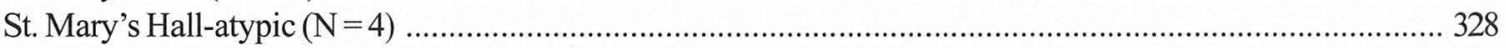

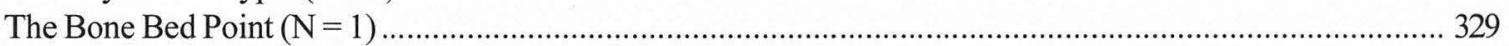

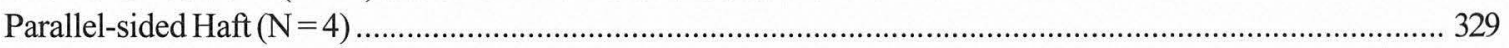

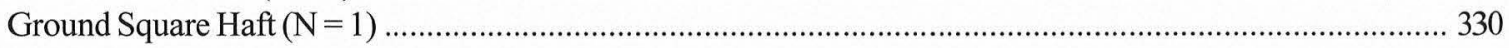

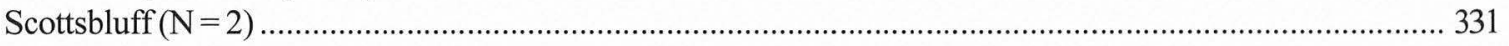

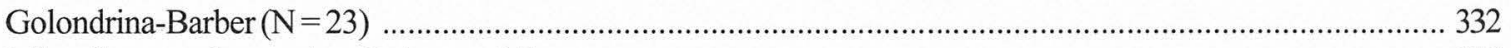

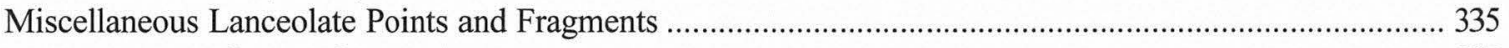

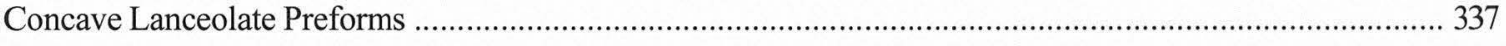

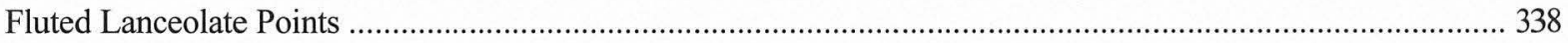

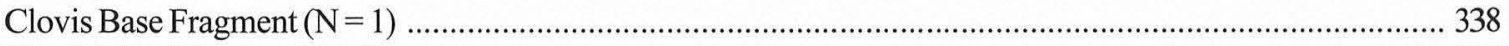

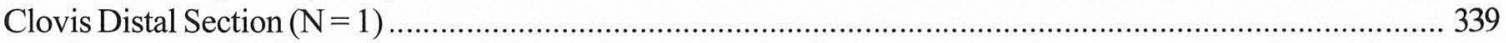

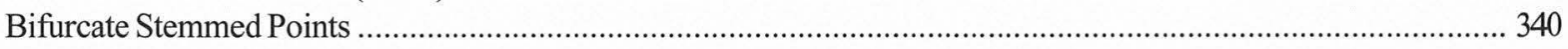

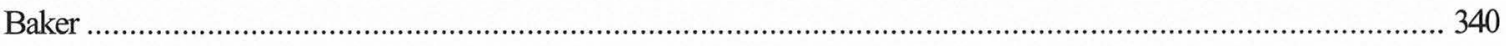

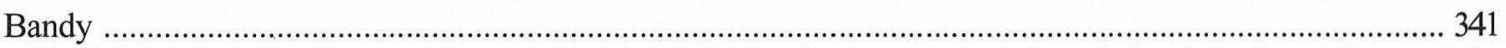

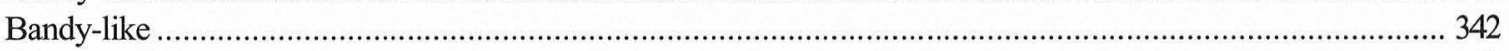

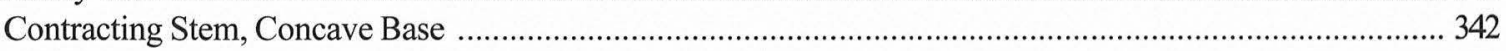

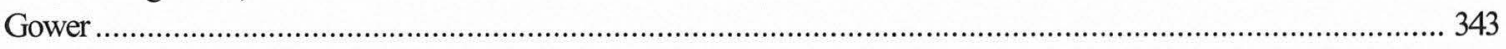

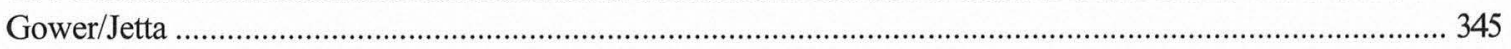

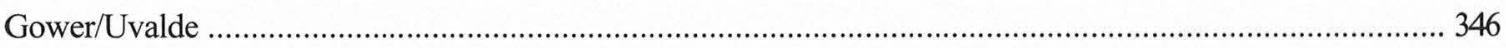

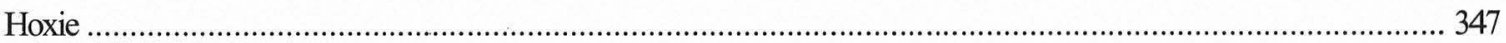

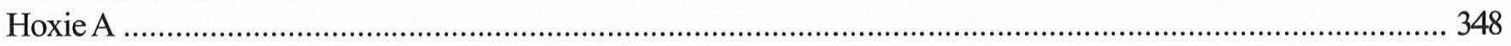

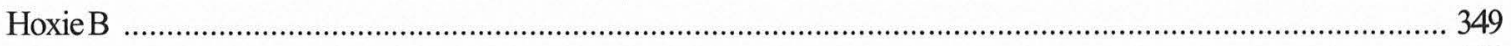

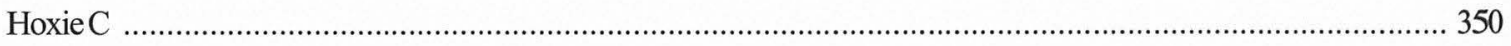

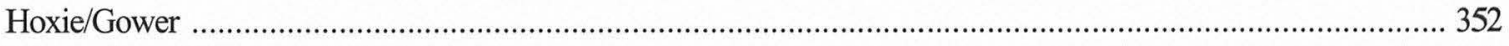

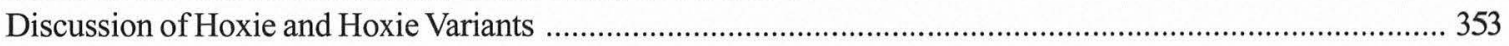

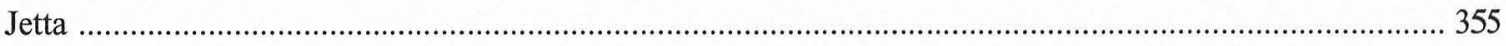

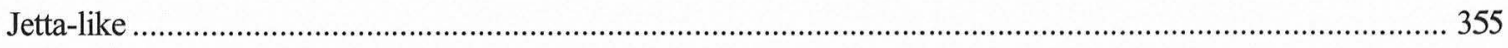




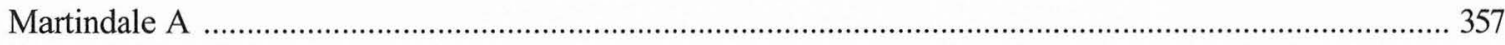

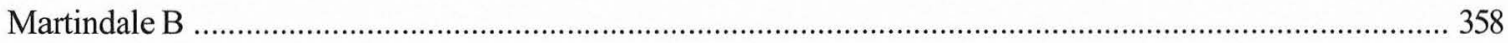

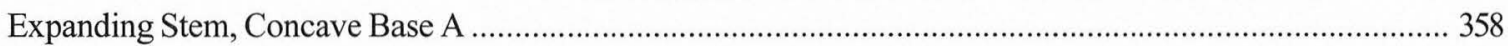

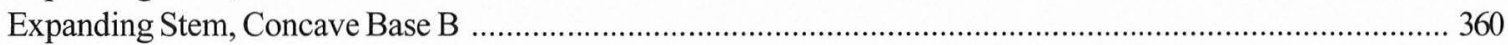

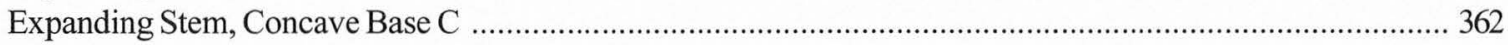

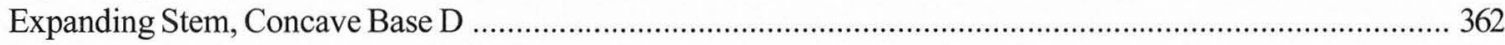

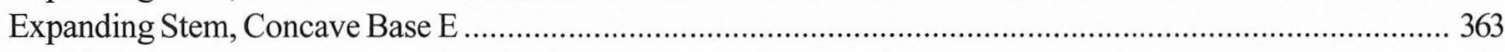

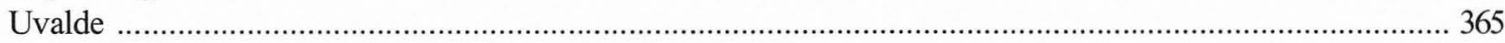

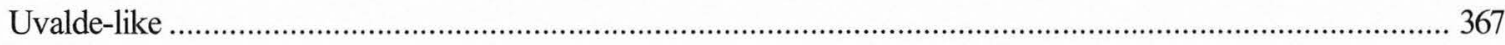

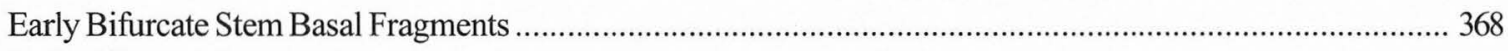

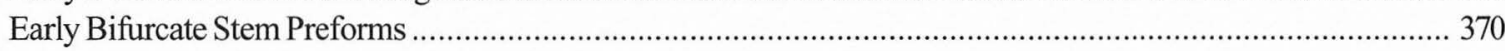

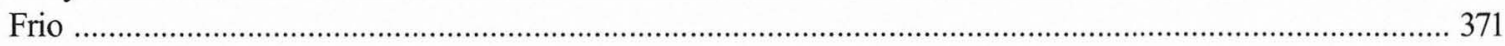

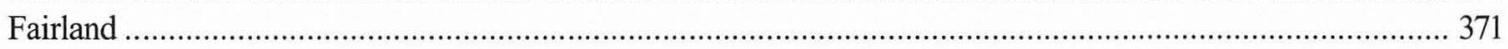

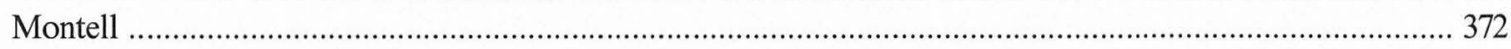

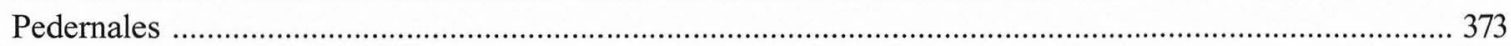

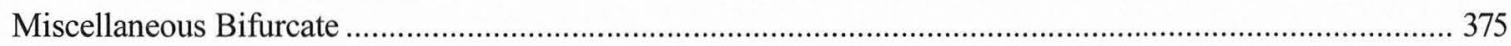

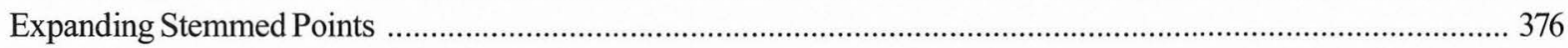

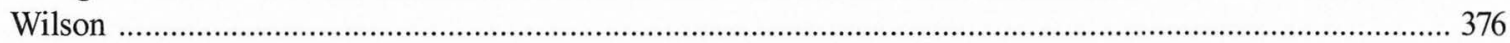

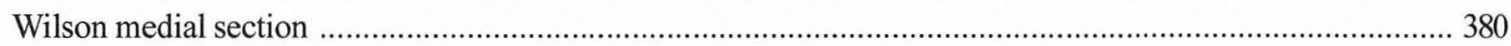

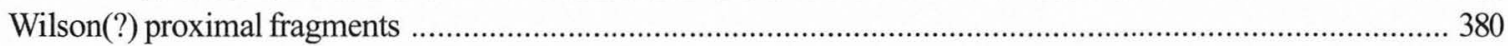

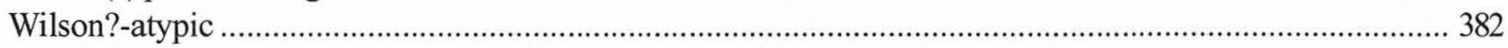

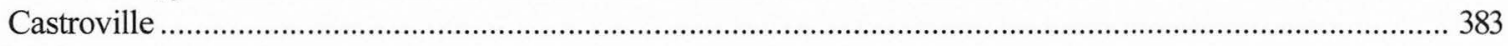

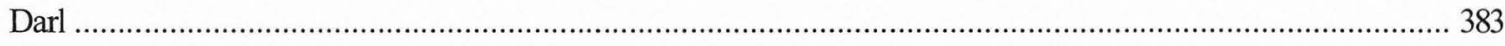

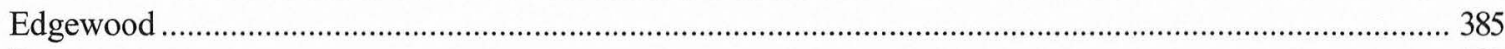

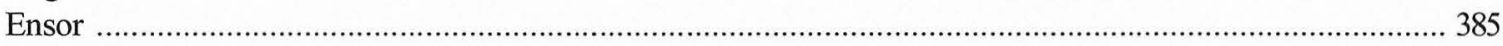

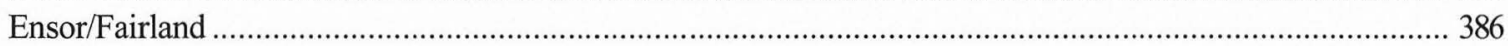

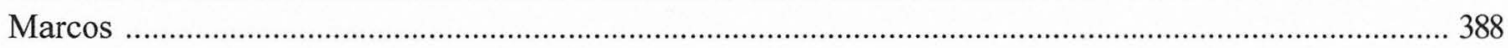

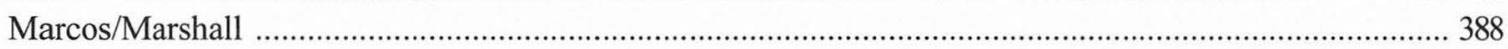

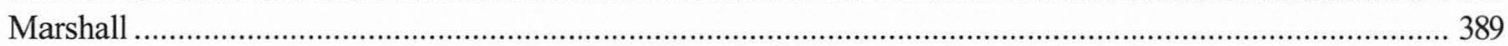

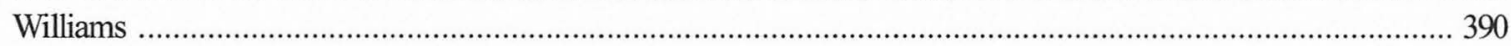

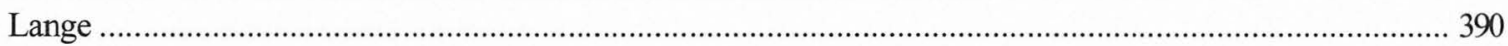

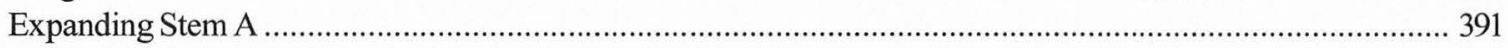

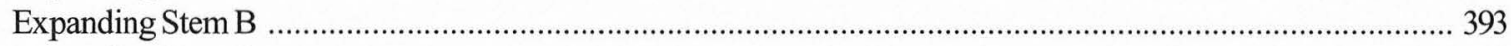

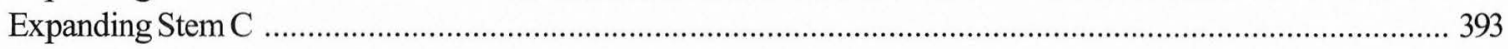

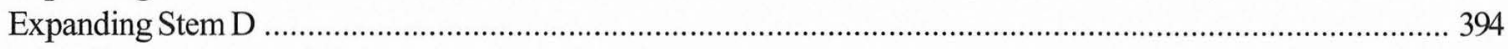

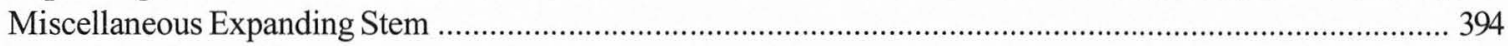

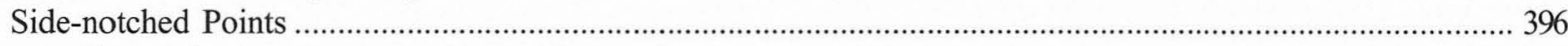

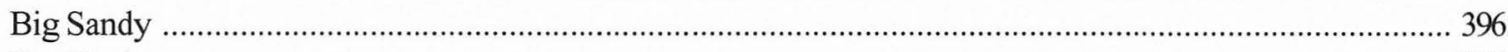

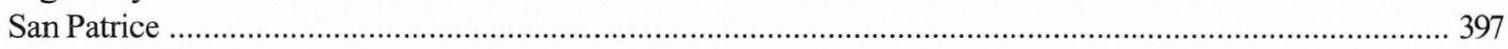

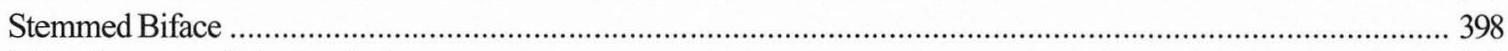

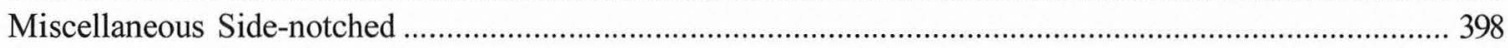

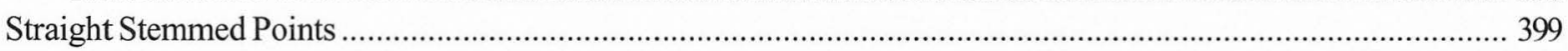

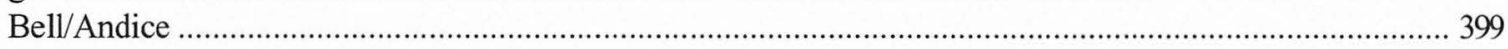

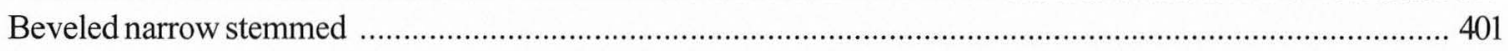

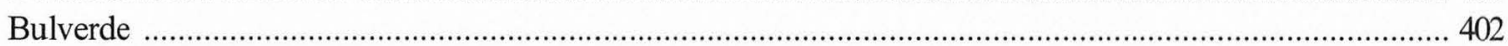

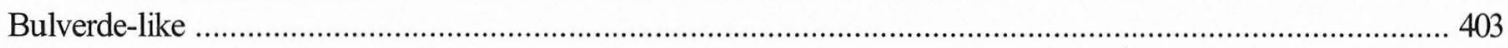

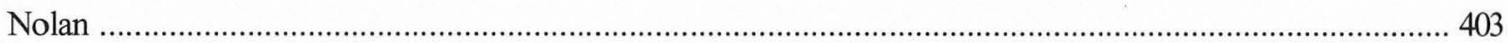

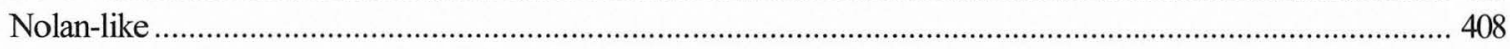

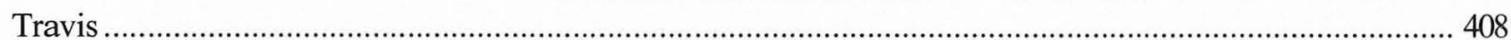

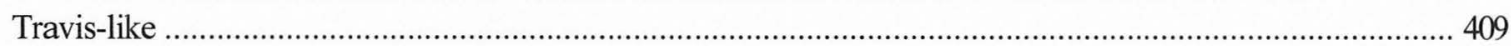

Wells

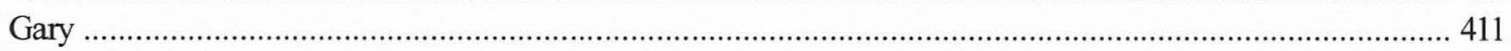

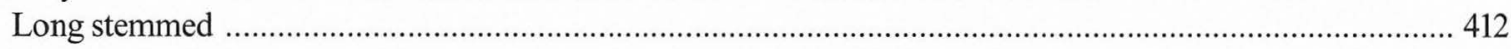

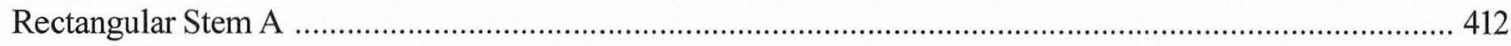

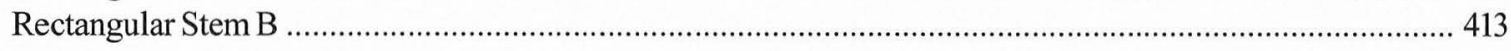




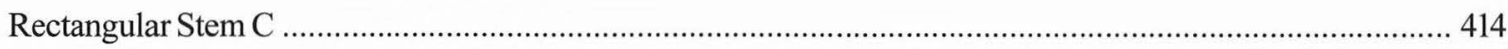

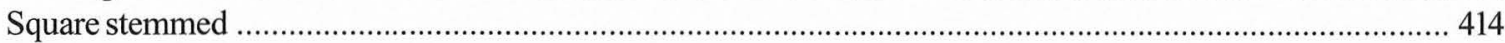

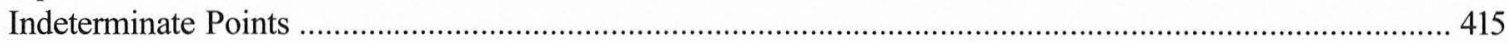

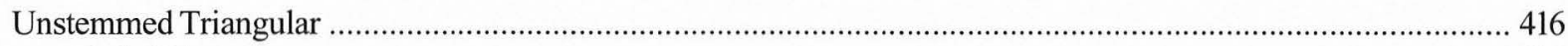

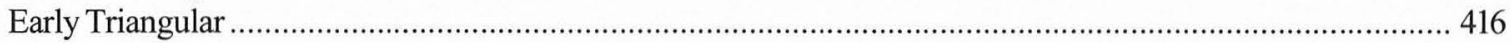

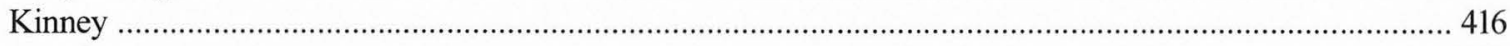

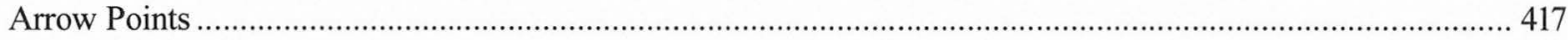

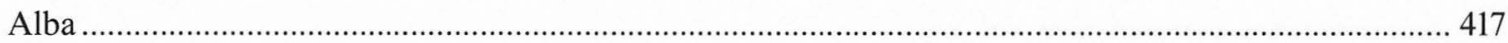

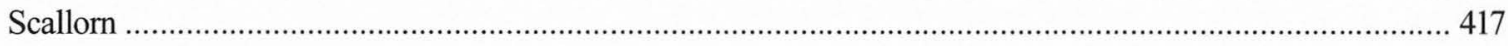

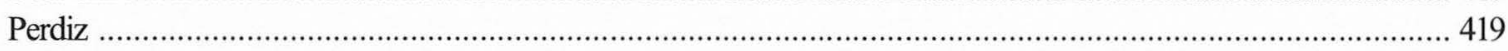

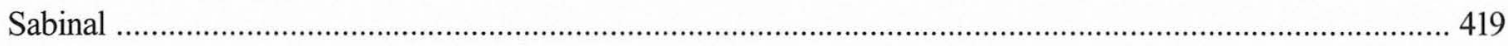

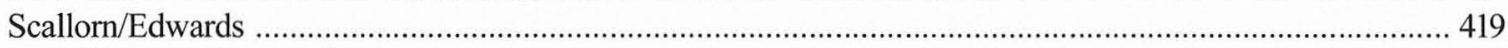

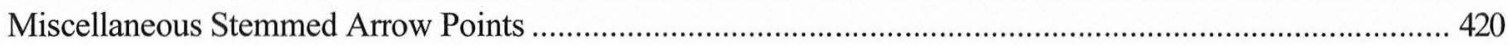

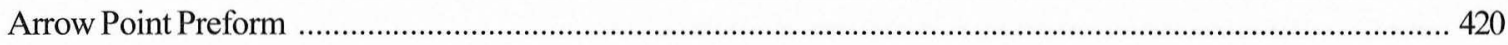

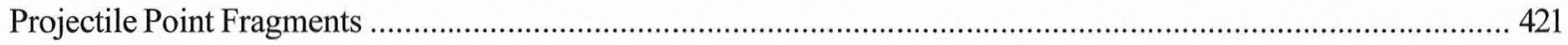

Summary and Discussion: The Wilson-Leonard Projectile Point Assemblage .......................................................... 424

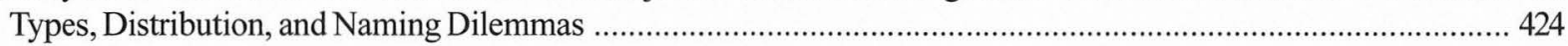

Tool State and Fracture Patterns ........................................................................................................... 425

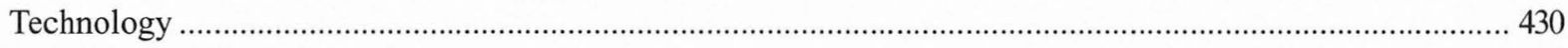

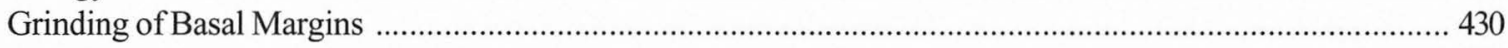

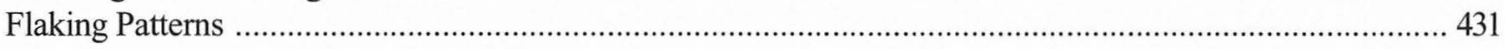

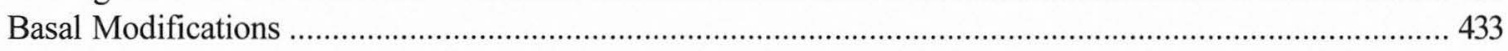

Material Selection and Effects of Natural and Cultural Processes ................................................................. 433

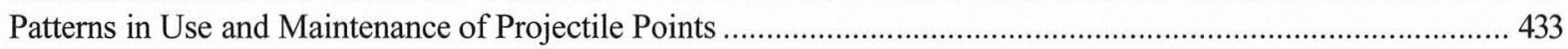

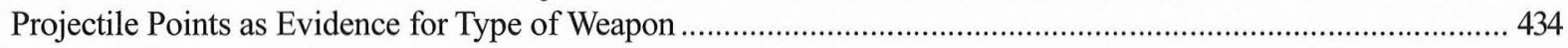

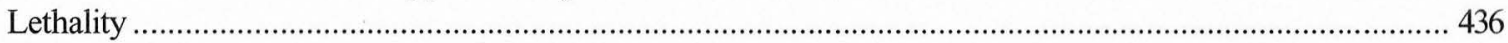

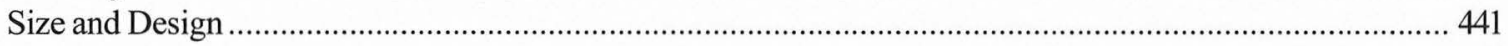

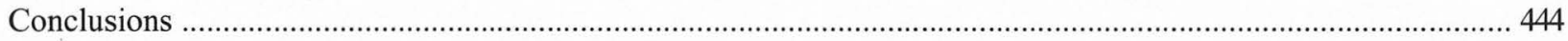

\section{CHAPTER 14: STATISTICALANALYSIS OF UNFLUTED LANCEOLATE AND}

EARLYBIFURCATESTEMPROJECTILEPOINTS

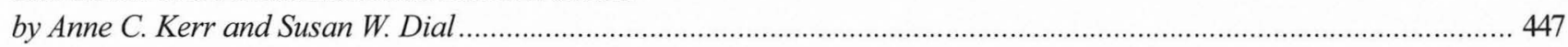

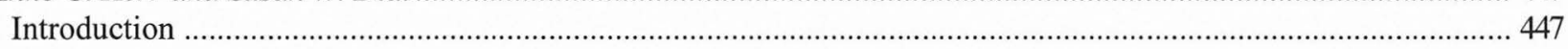

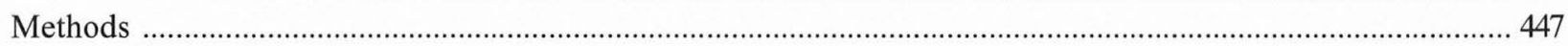

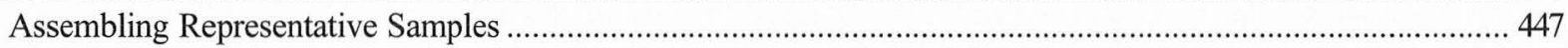

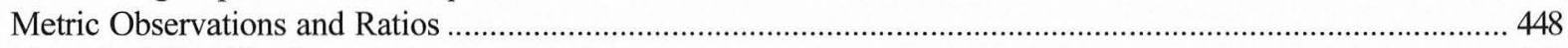

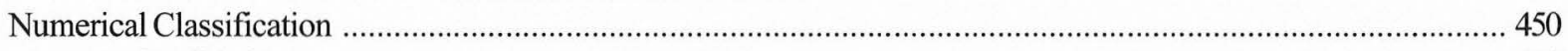

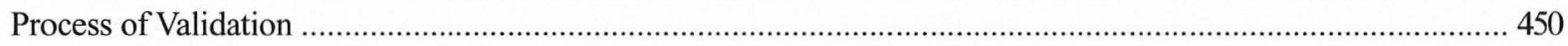

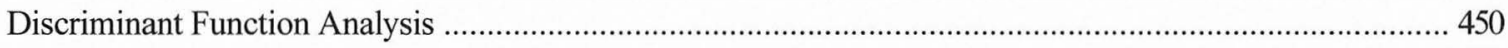

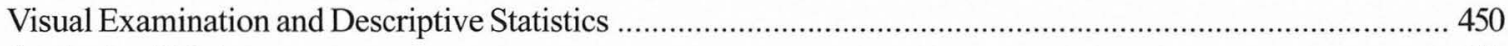

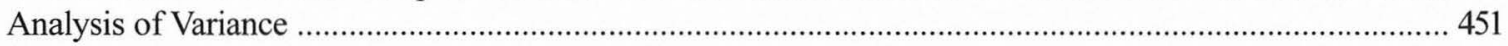

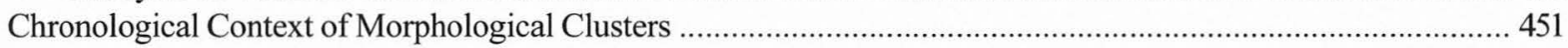

Systematic Analysis of Unfluted Lanceolate Projectile Points ............................................................................ 451

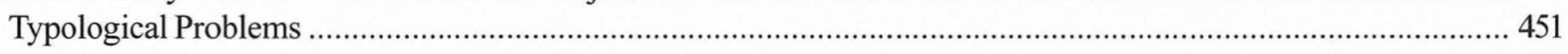

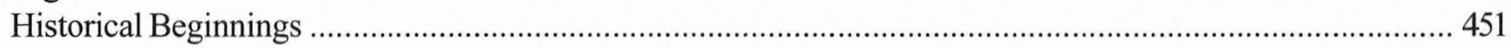

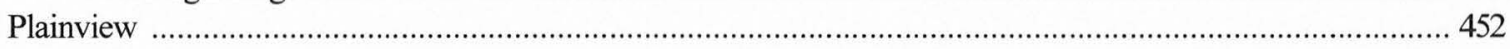

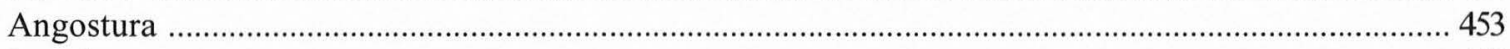

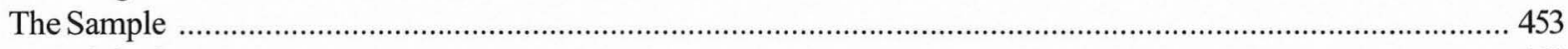

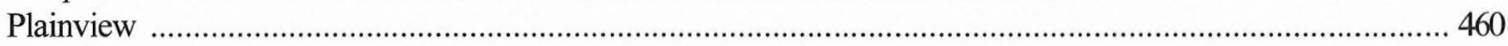

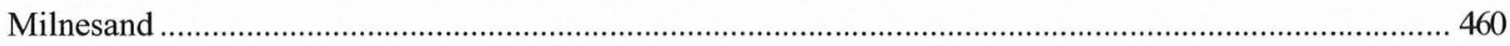

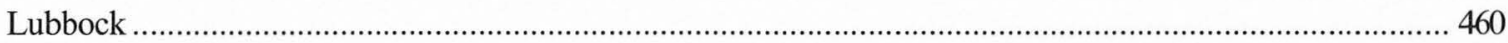

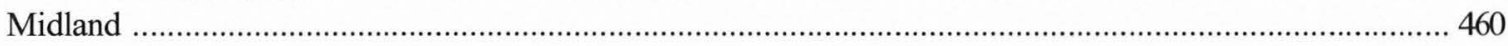

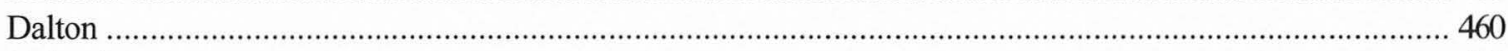

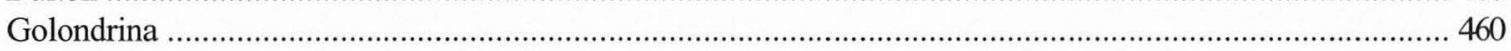

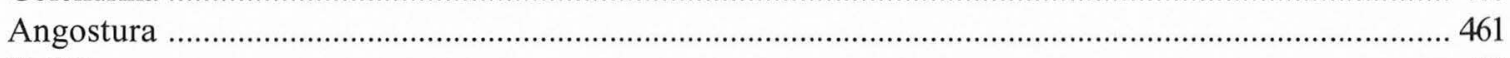

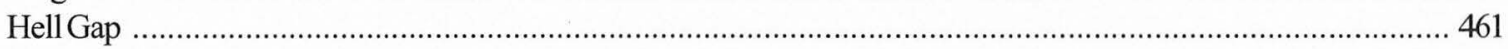




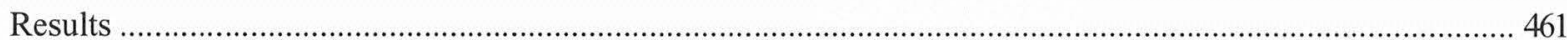

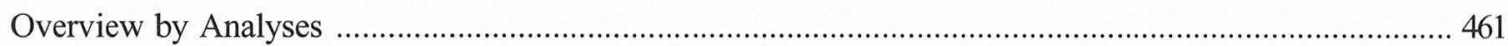

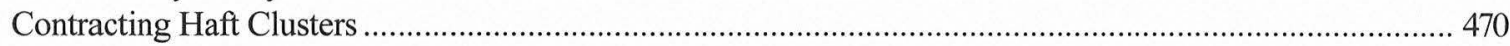

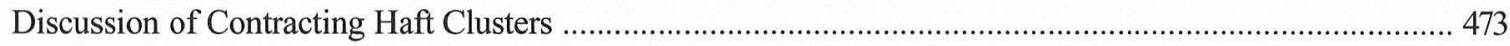

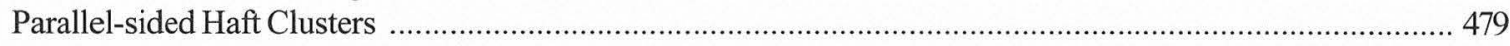

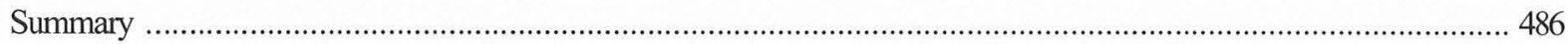

Statistical Analysis of Early Bifurcate Stem Projectile Points .................................................................................. 488

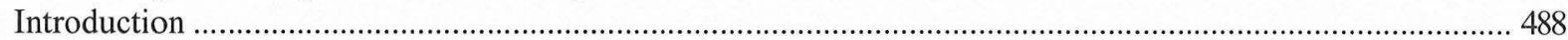

Background and Typological Problems among Early Bifurcate Stem Points .................................................. 488

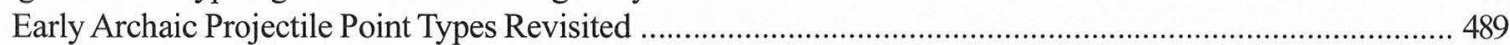

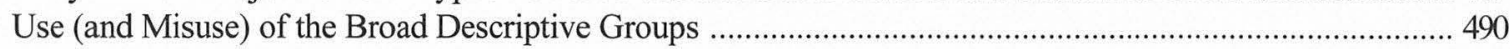

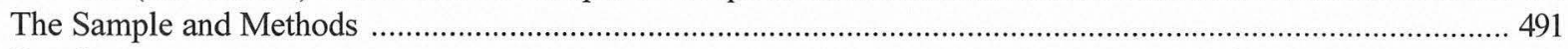

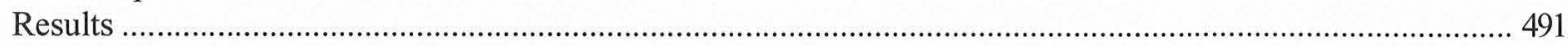

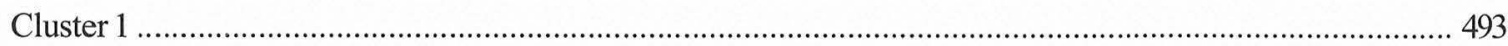

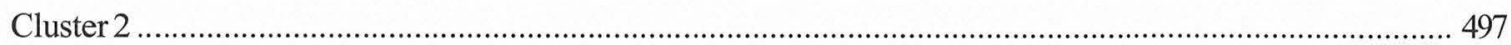

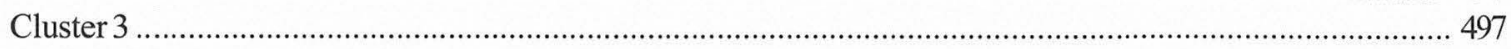

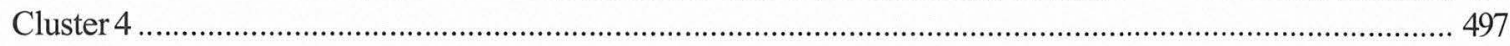

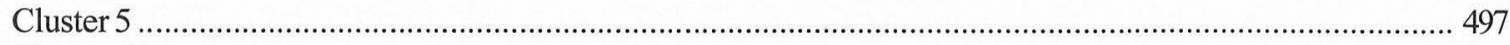

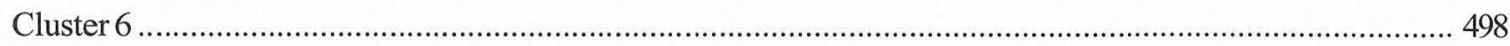

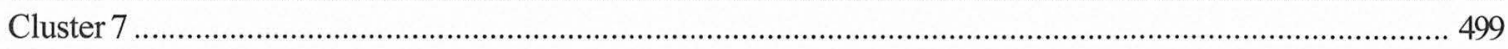

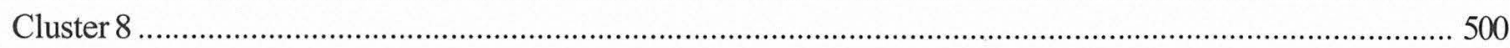

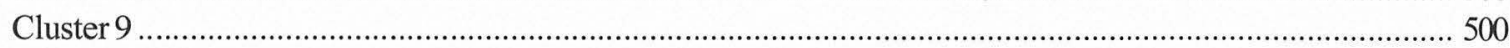

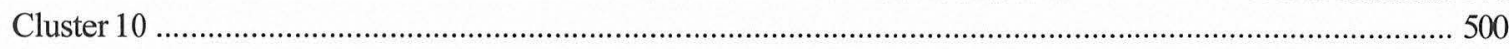

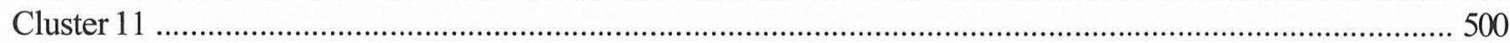

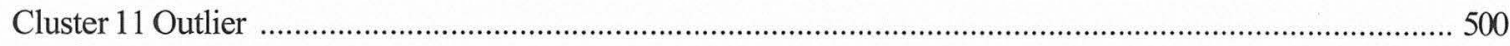

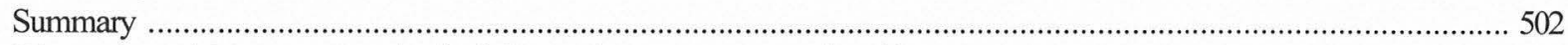

Discussion: A Note on Typological Conundrums among Early Bifurcate Stem Points ...................................... 503

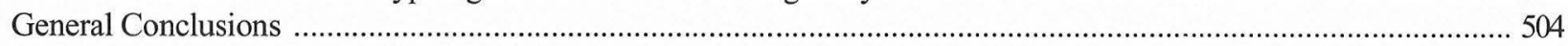

\section{CHAPTER 15: CLEARFORK TOOLS}

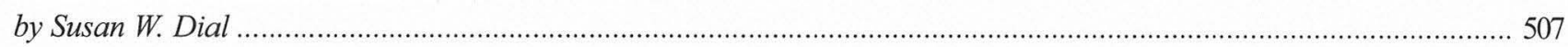

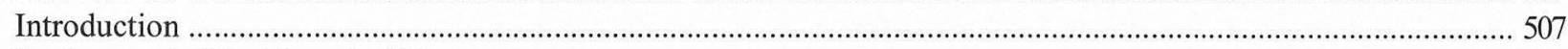

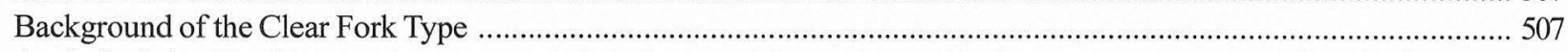

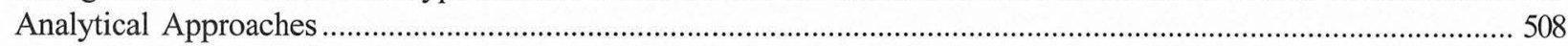

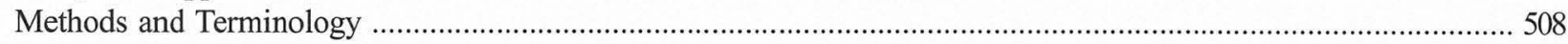

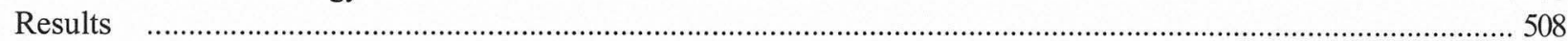

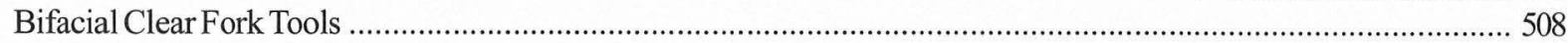

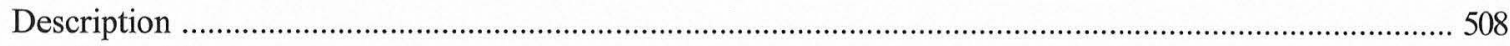

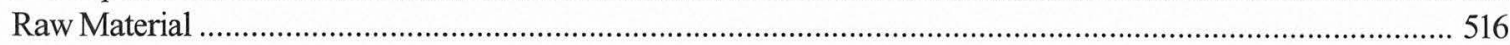

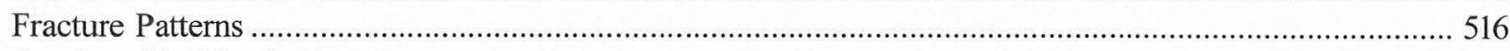

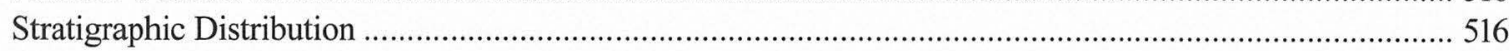

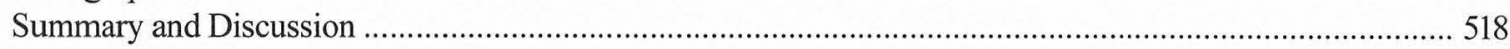

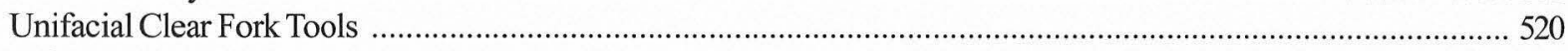

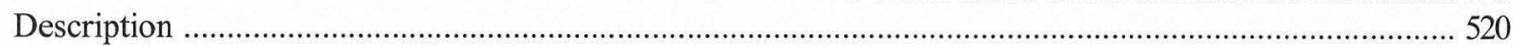

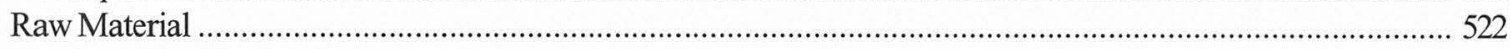

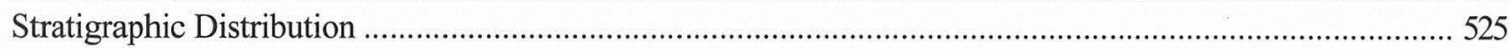

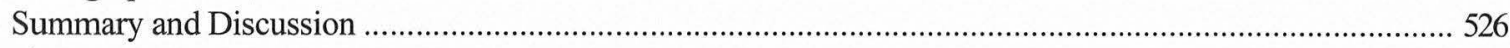

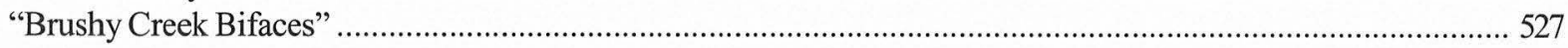

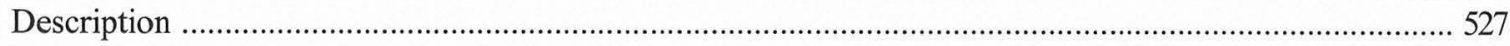

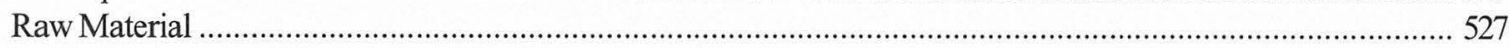

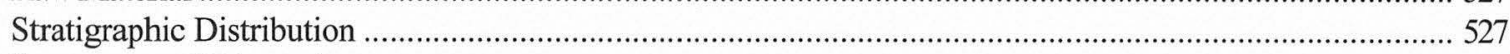

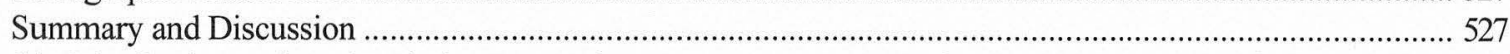

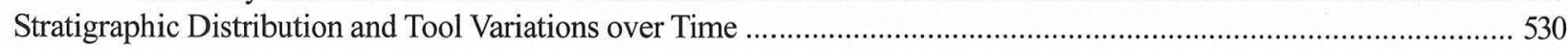

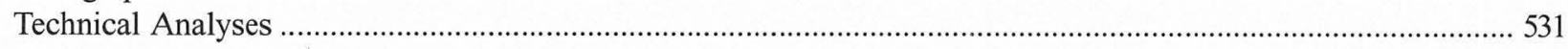

Variations in Clear Fork and other Distally Beveled Tools: A Use-resharpening Model ................................................... 533

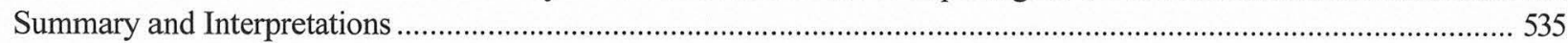


CHAPTER 16: BIFACES, BIFACIAL TOOLS, PERFORATORS, BURINS, AND SPALLS

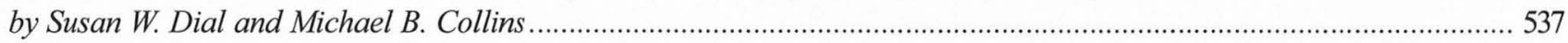

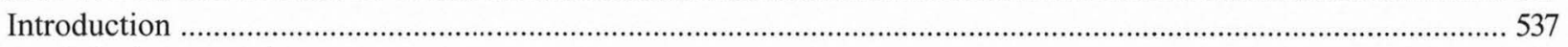

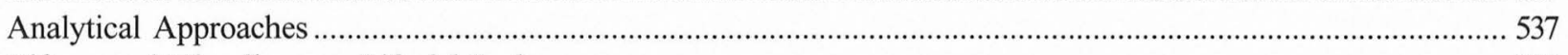

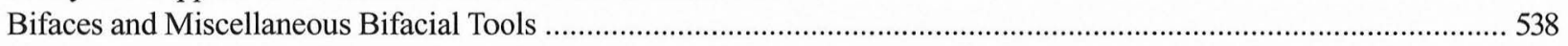

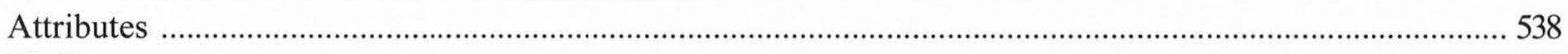

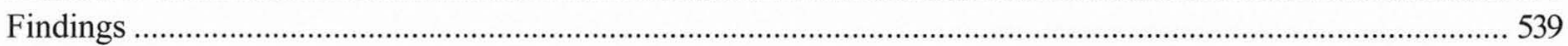

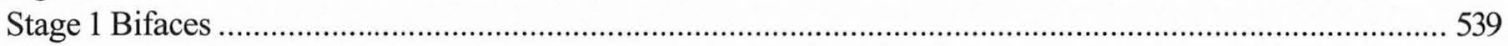

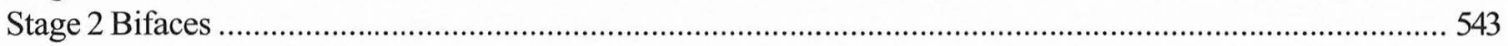

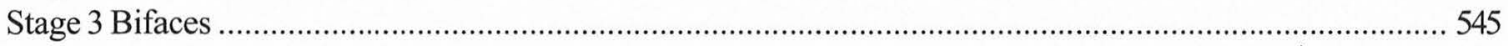

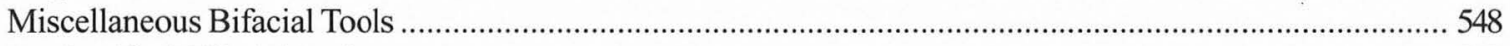

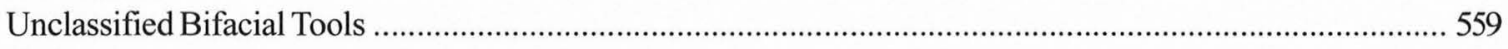

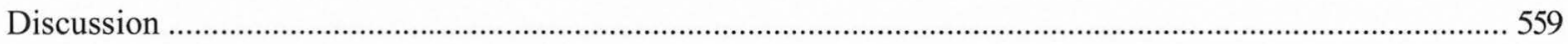

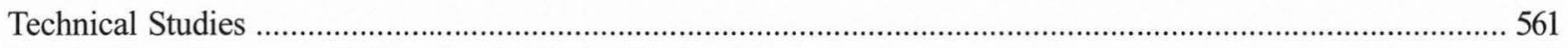

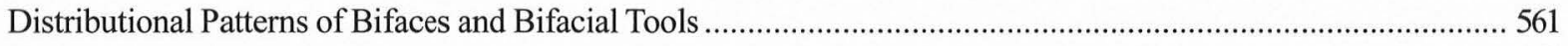

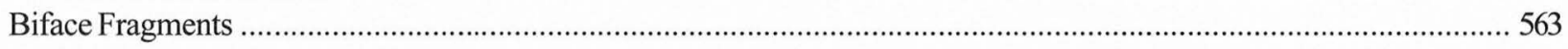

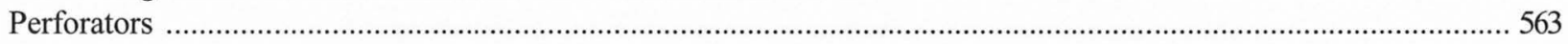

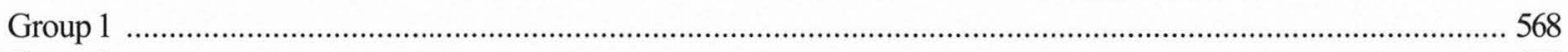

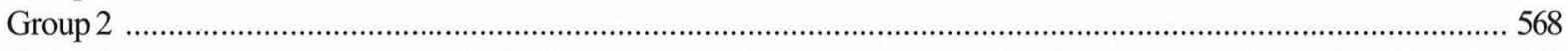

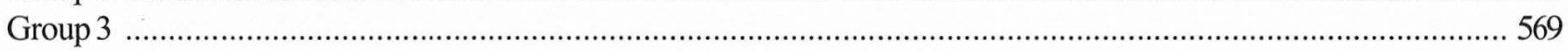

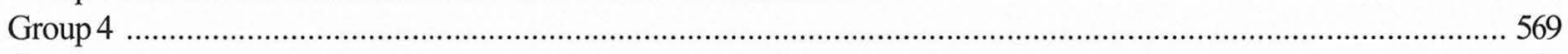

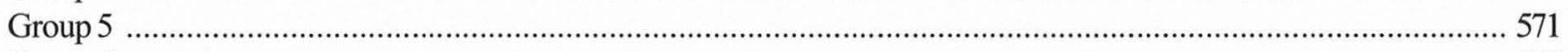

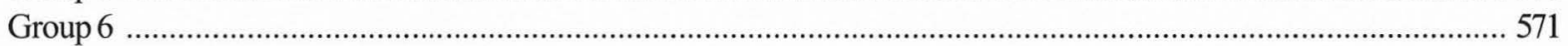

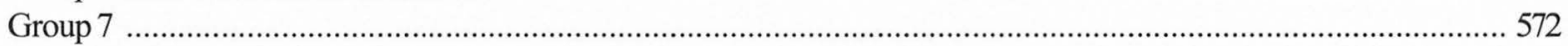

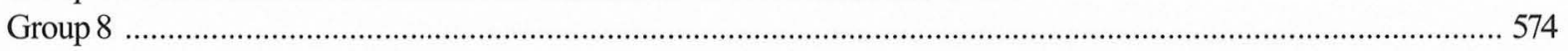

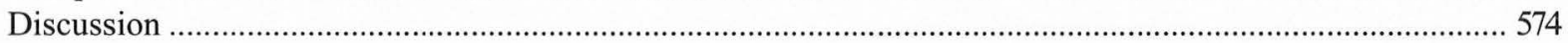

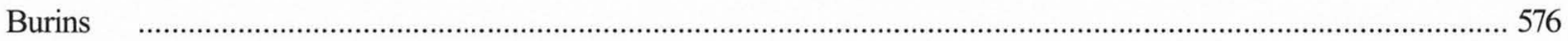

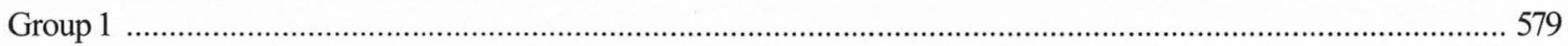

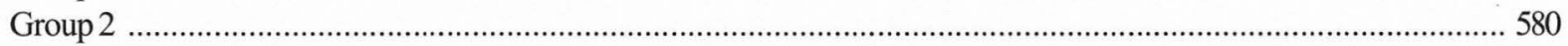

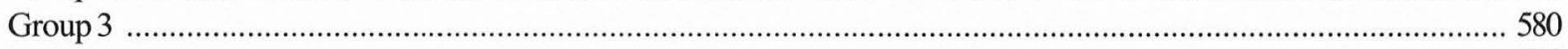

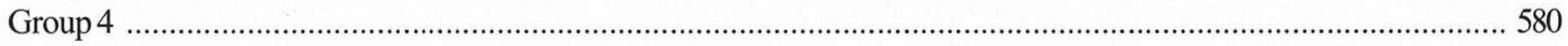

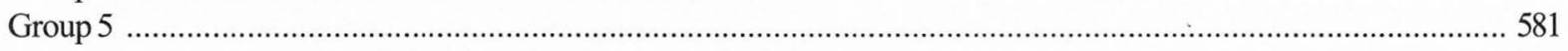

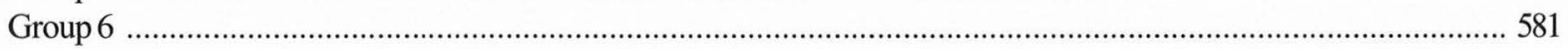

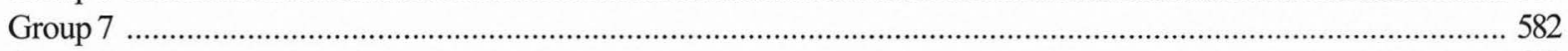

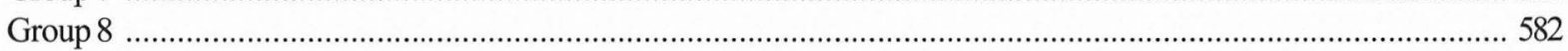

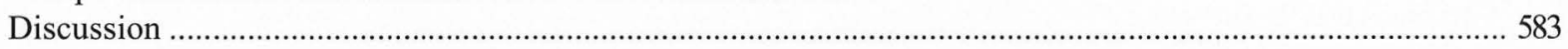

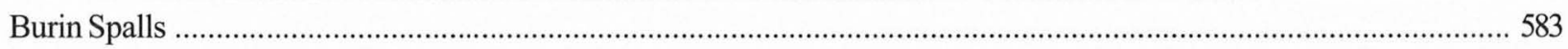

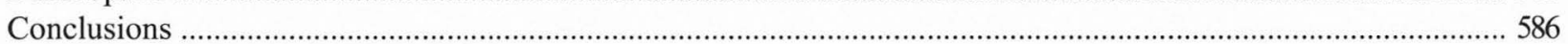

CHAPTER 17: UNIFACIAL TOOLS

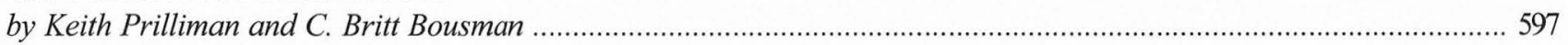

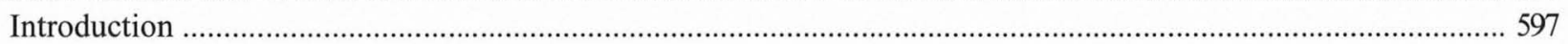

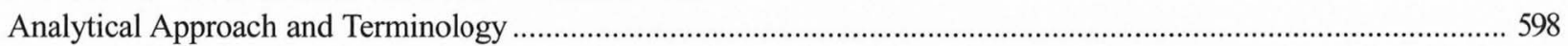

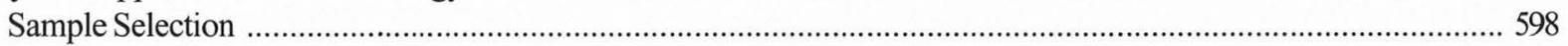

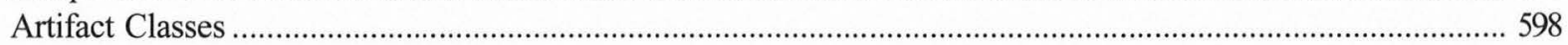

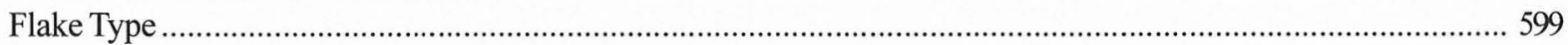

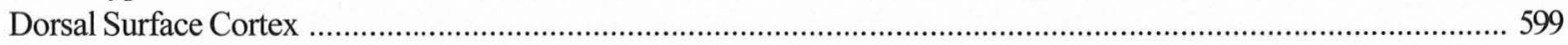

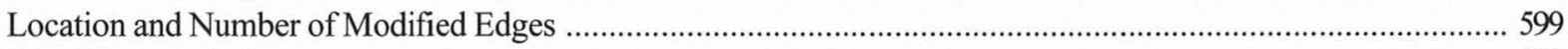

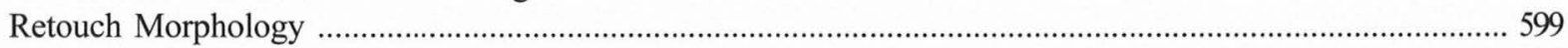

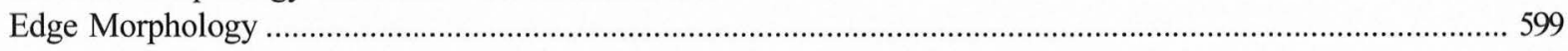

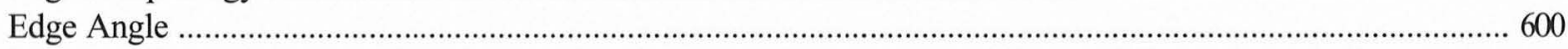

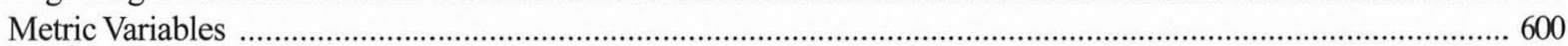

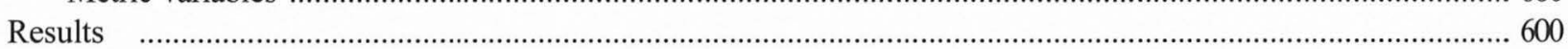

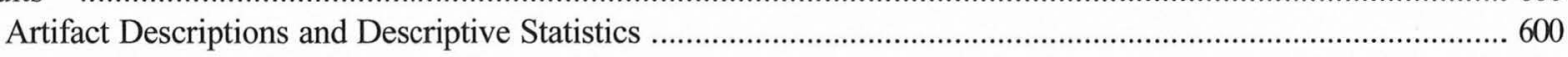

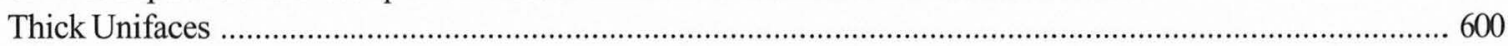




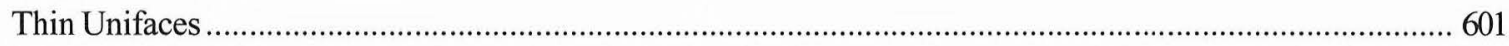

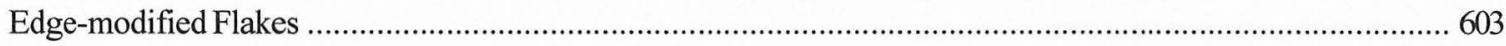

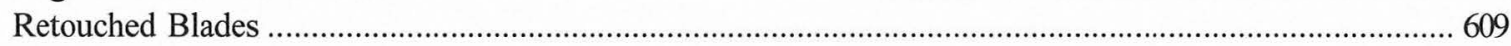

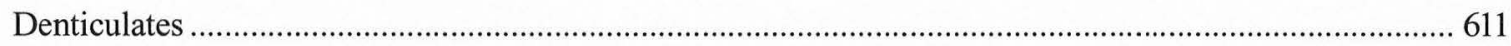

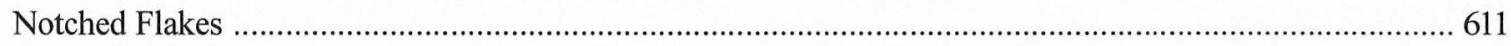

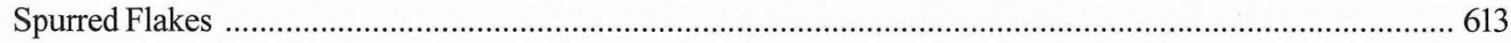

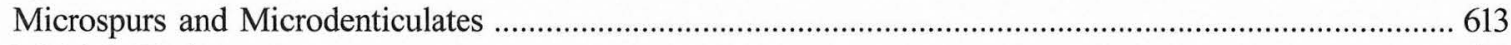

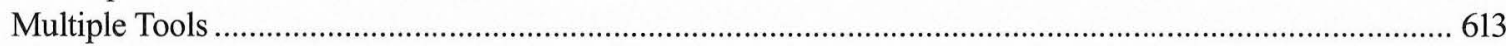

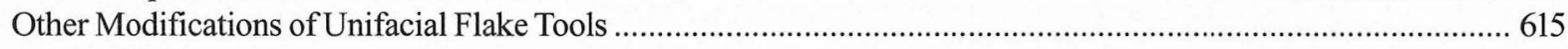

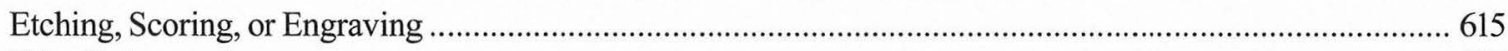

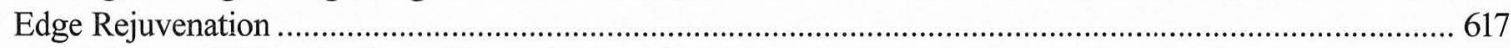

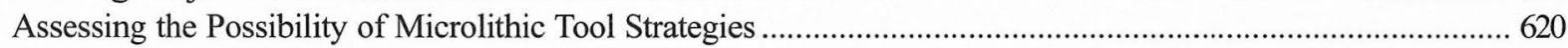

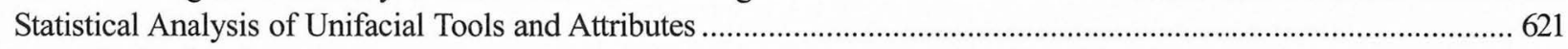

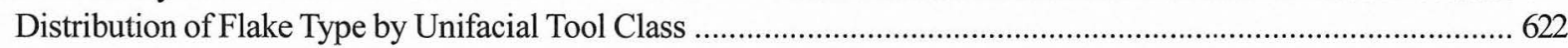

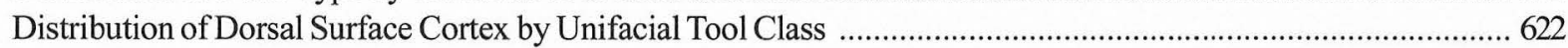

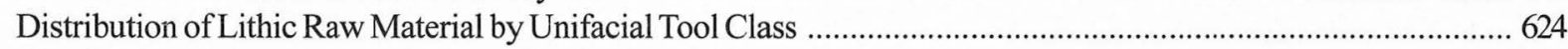

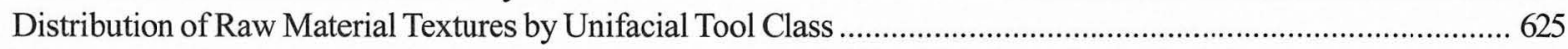

Distribution of Unifacial Tool Classes in the Valley Floor and Valley Margin Units

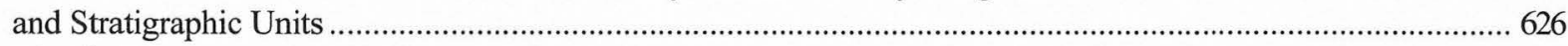

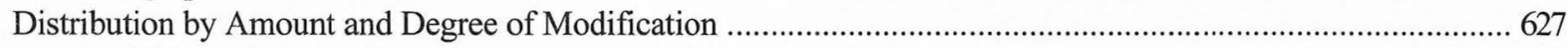

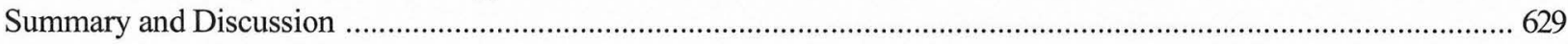

\section{CHAPTER 18: CORETOOLS, BATTERED STONES, CORES,ANDTESTED}

ANDUNMODIFIEDCHERT MATERIALS

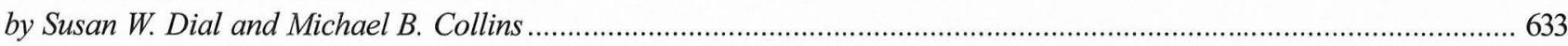

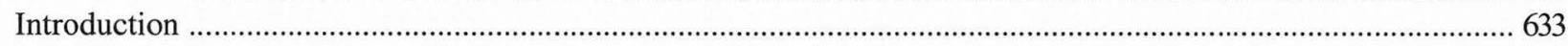

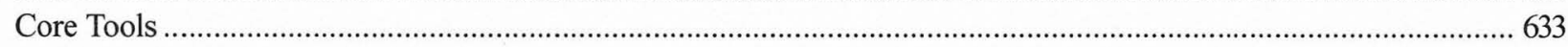

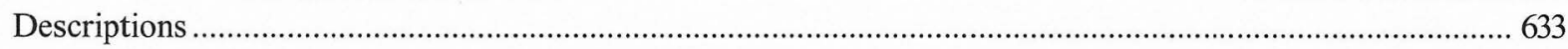

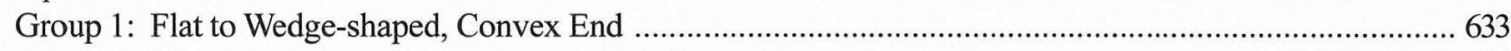

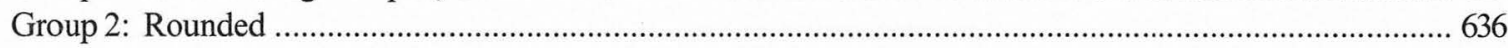

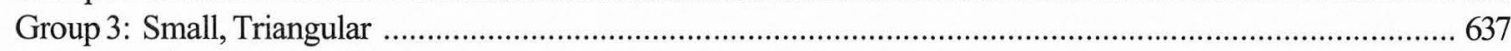

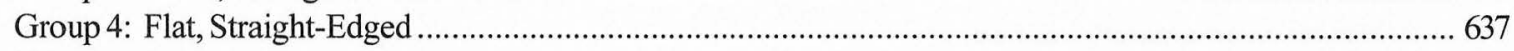

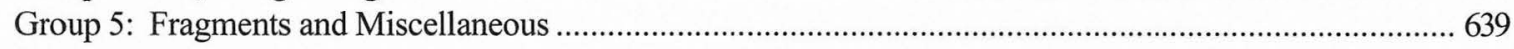

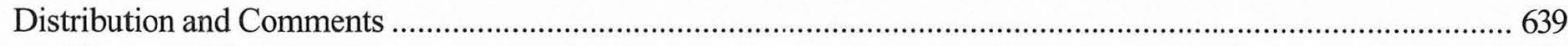

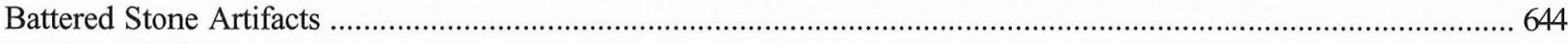

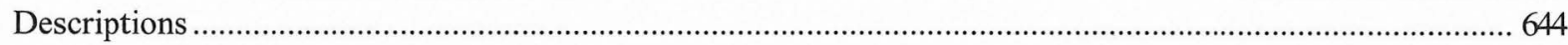

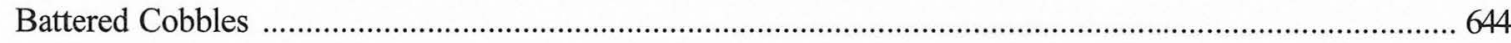

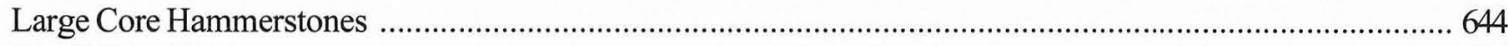

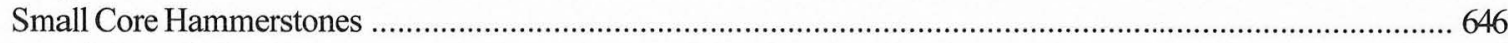

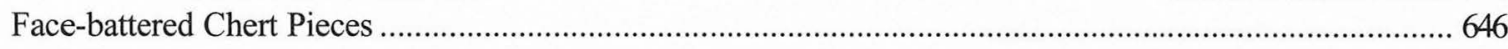

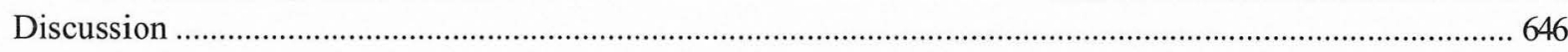

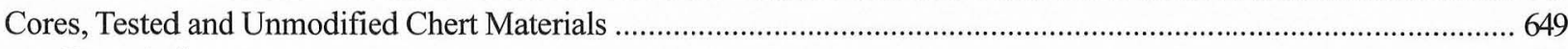

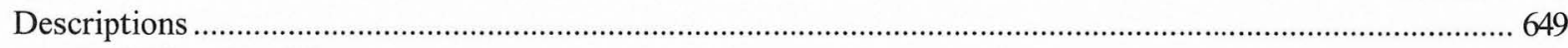

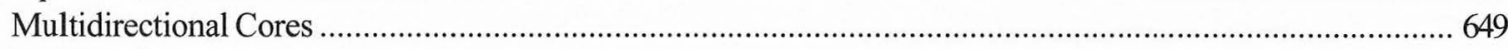

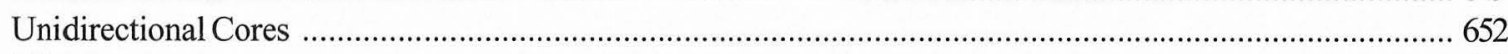

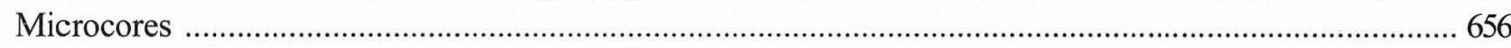

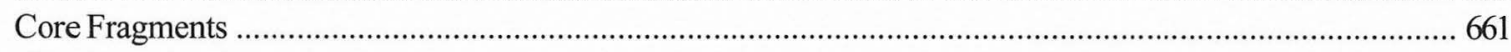

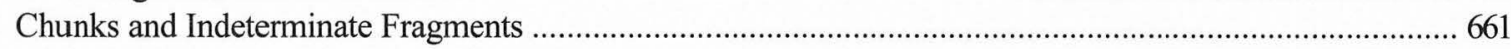

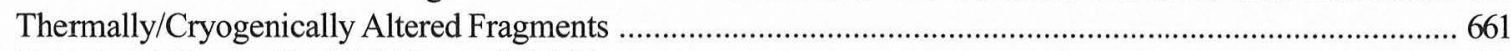

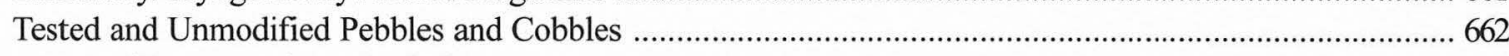

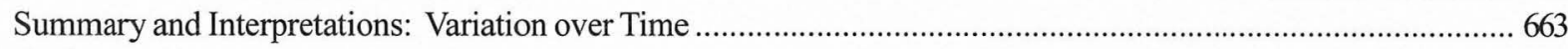

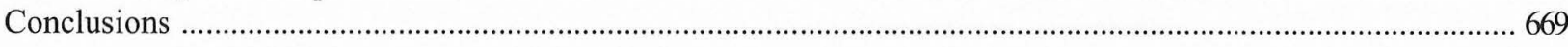

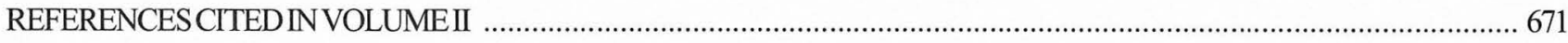




\section{LIST OF FIGURES}

\section{Volume II}

13-1. Measurement locations of metric attributes on various forms of projectile points ............................................... 315

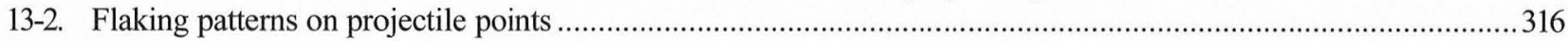

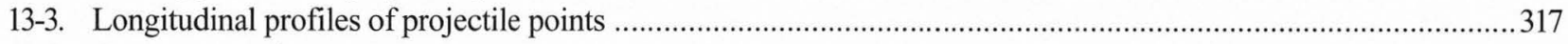

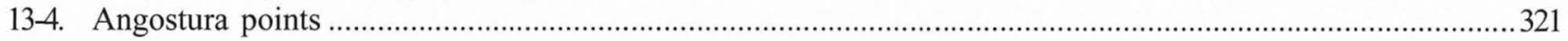

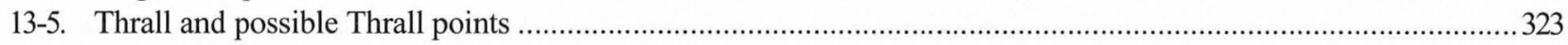

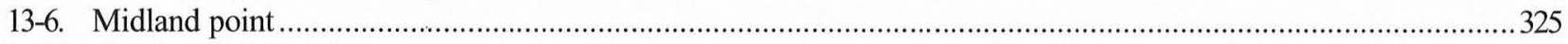

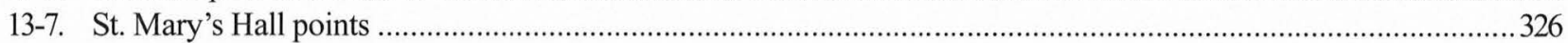

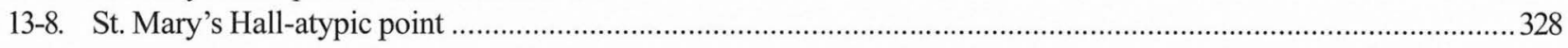

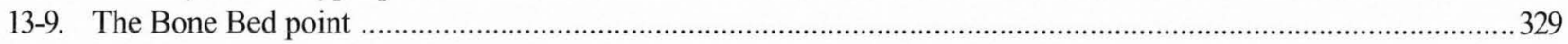

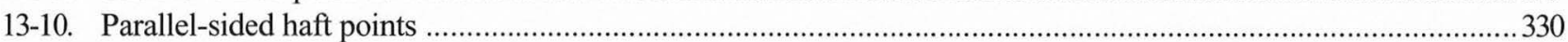

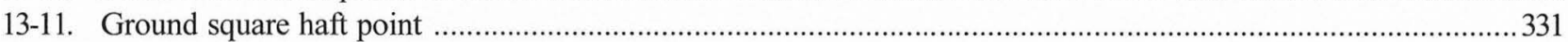

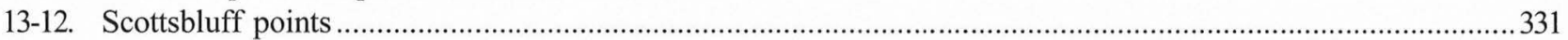

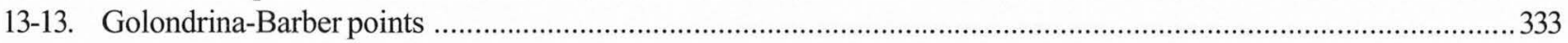

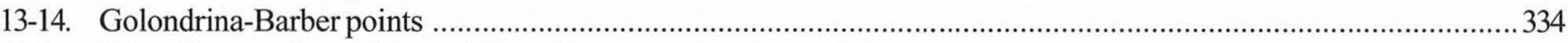

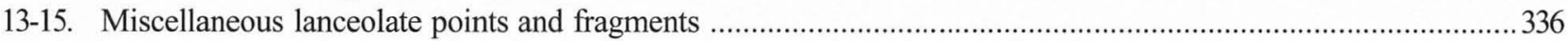

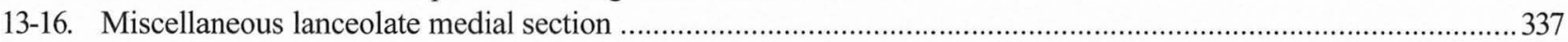

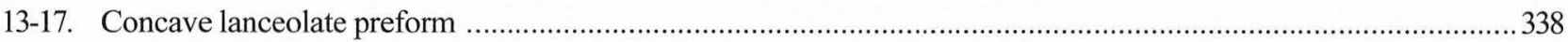

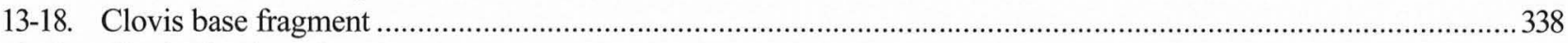

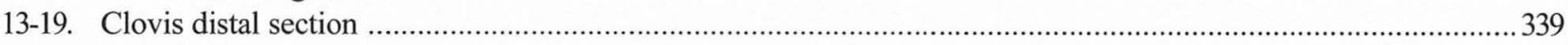

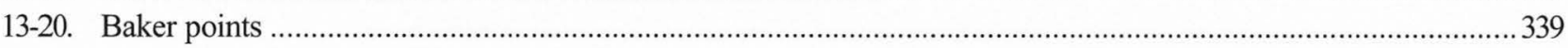

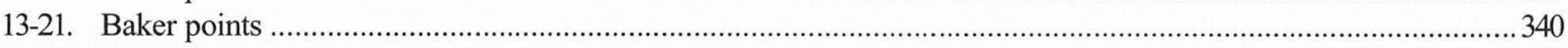

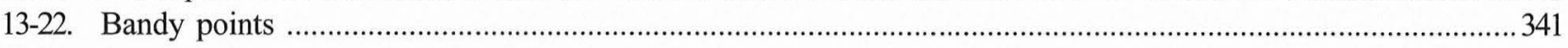

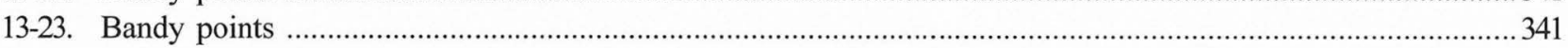

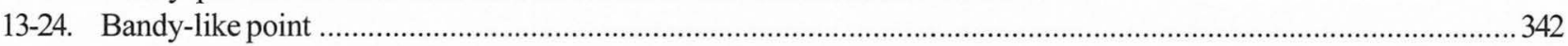

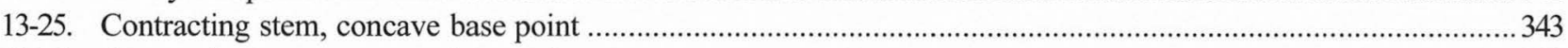

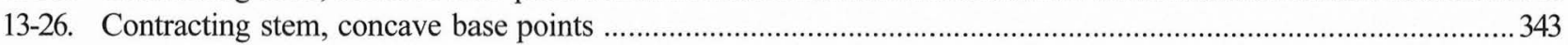

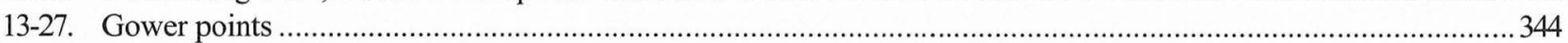

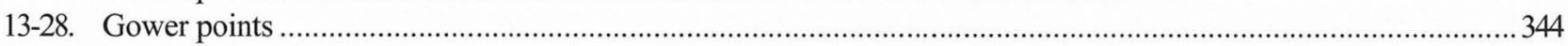

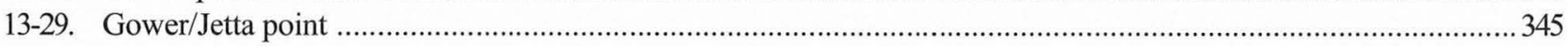

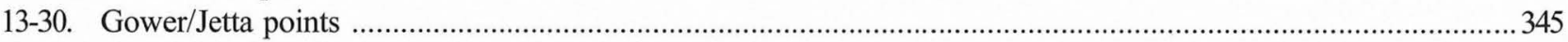

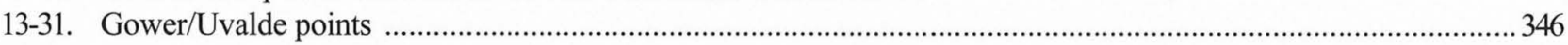

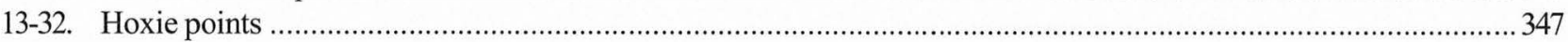

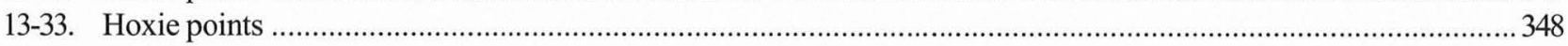

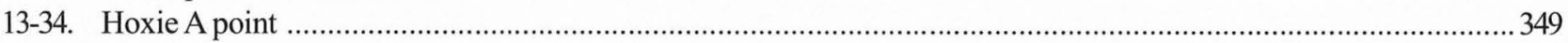

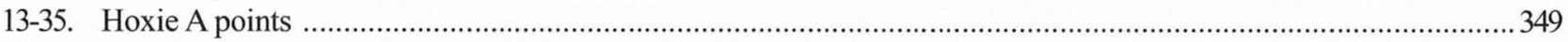

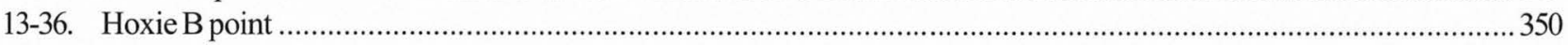

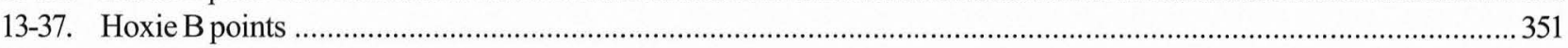

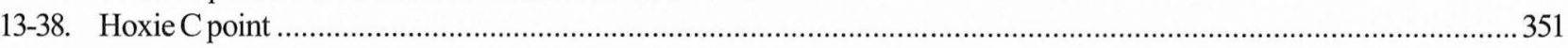

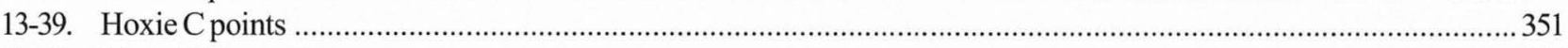

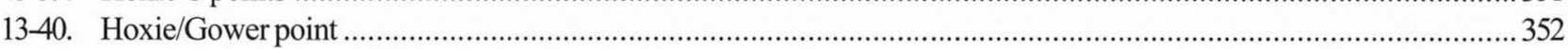

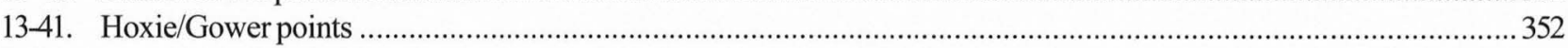

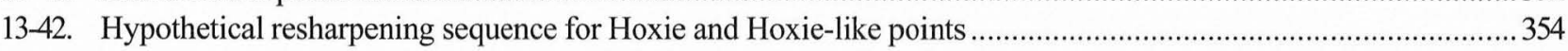

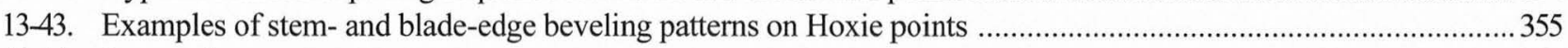

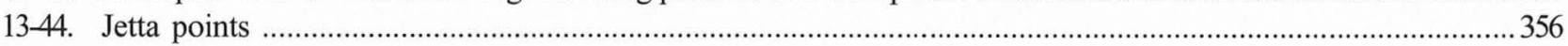

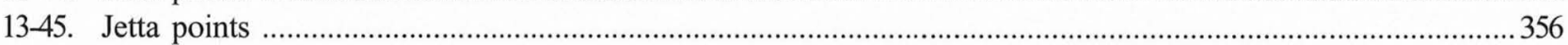

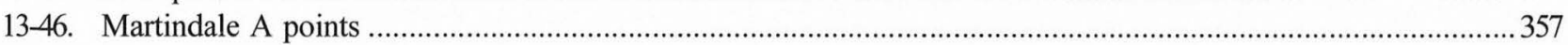

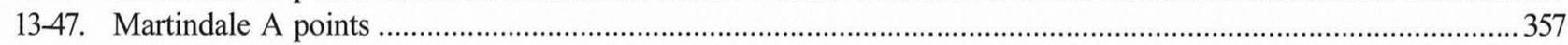

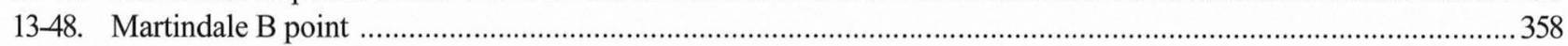

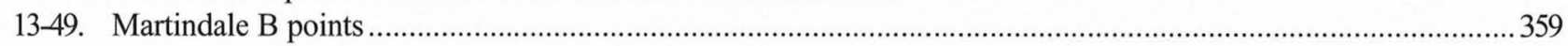

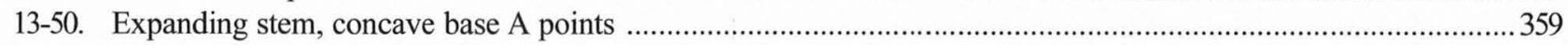

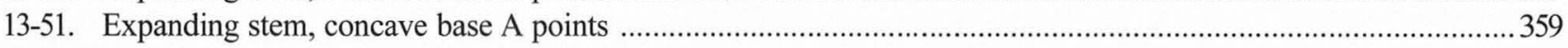

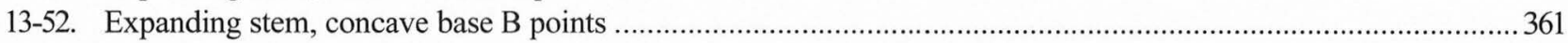




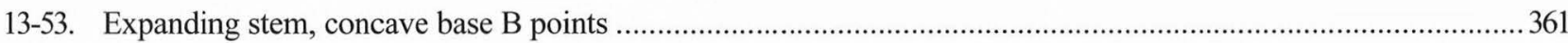

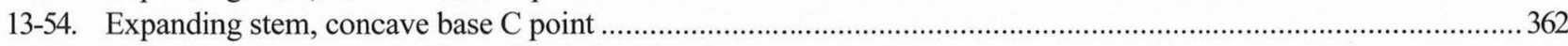

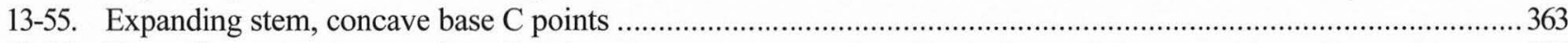

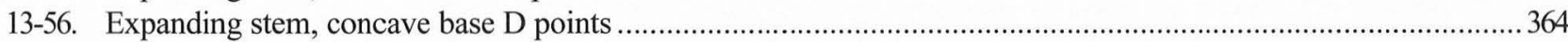

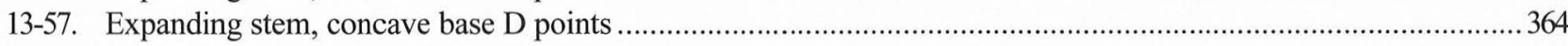

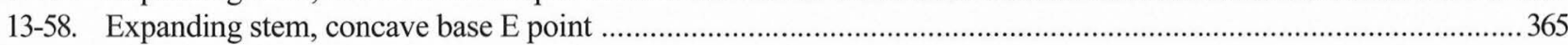

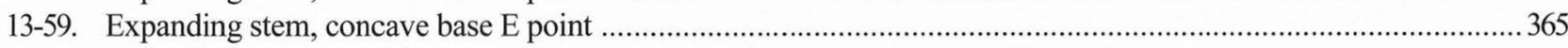

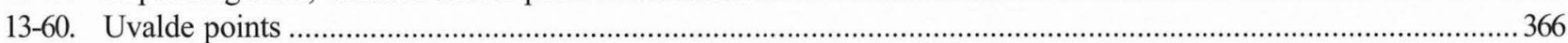

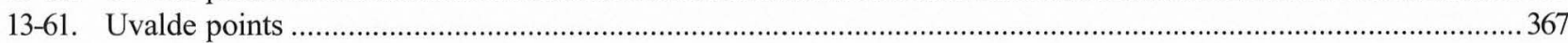

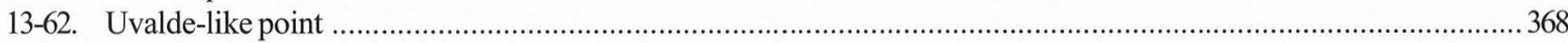

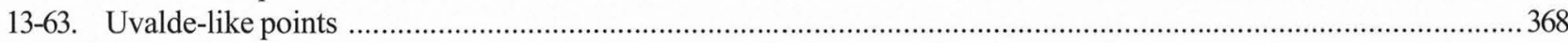

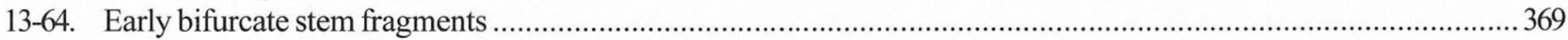

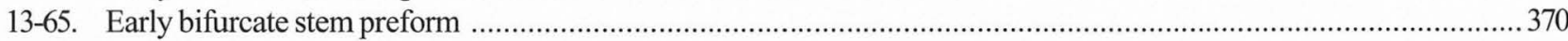

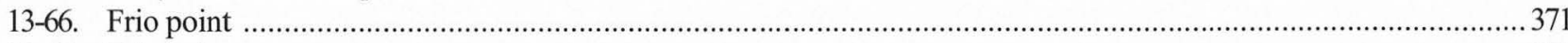

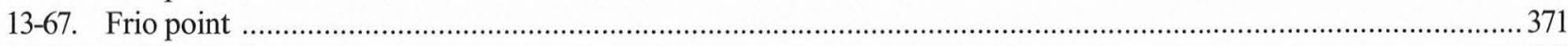

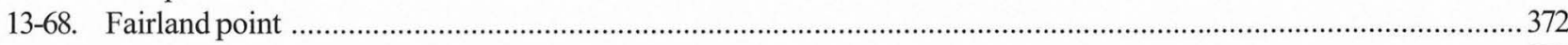

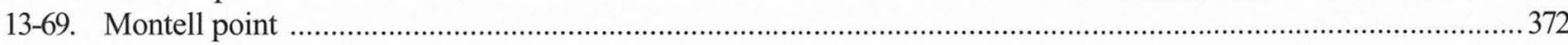

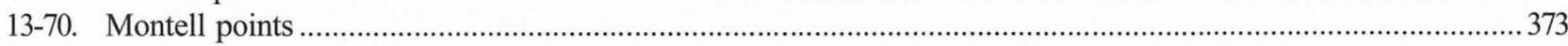

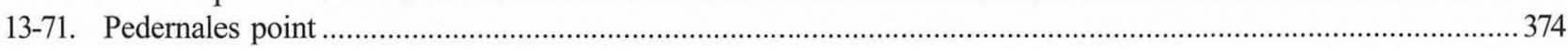

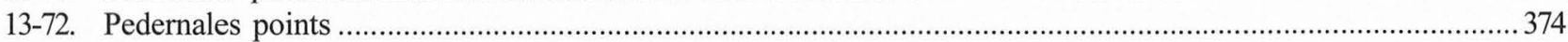

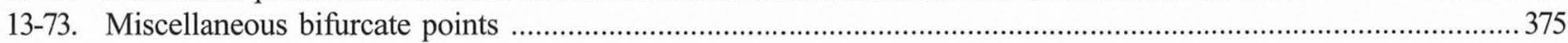

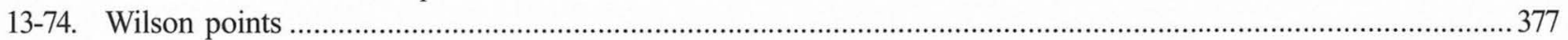

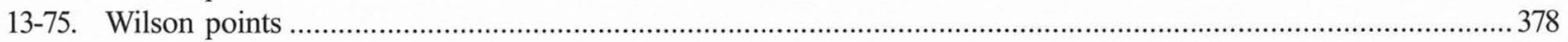

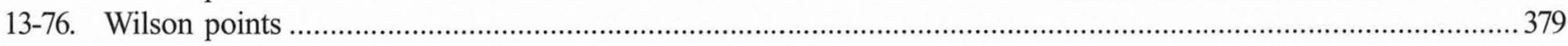

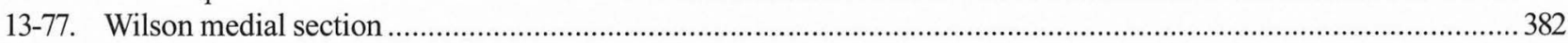

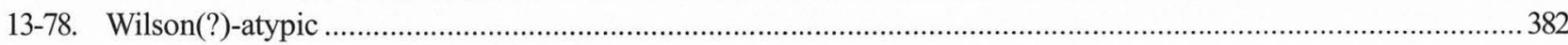

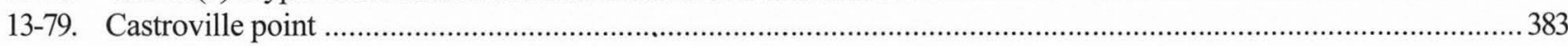

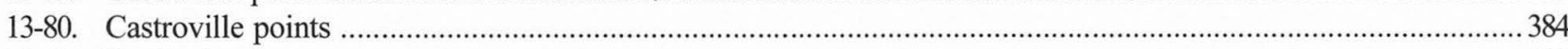

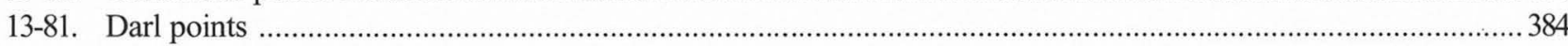

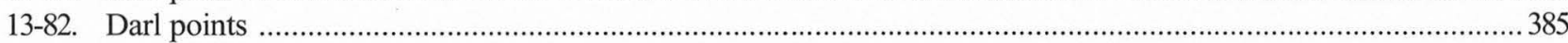

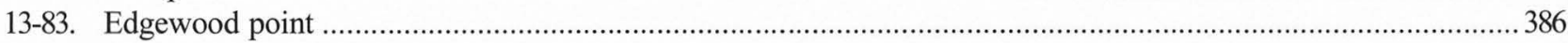

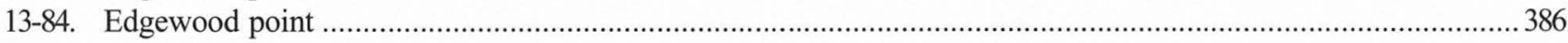

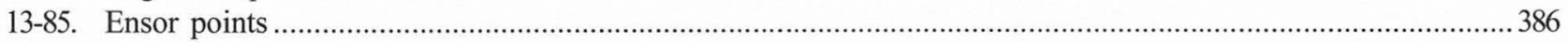

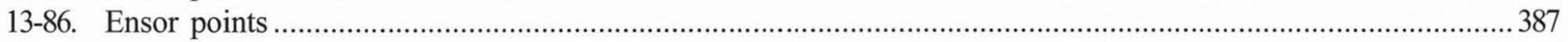

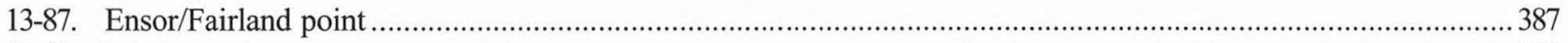

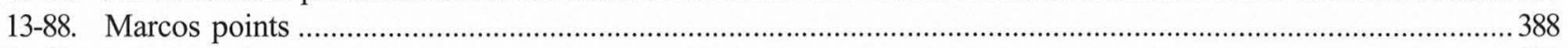

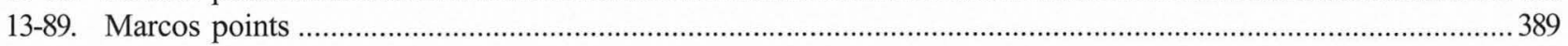

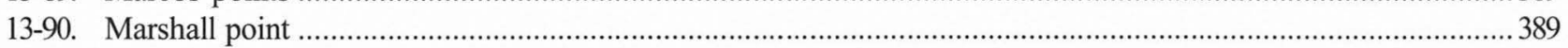

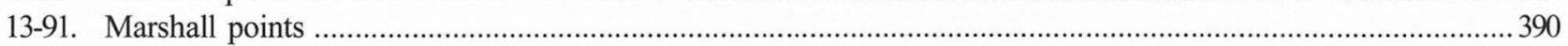

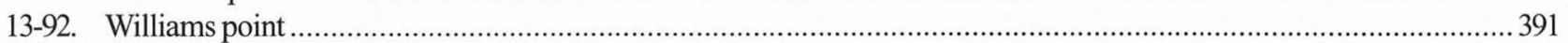

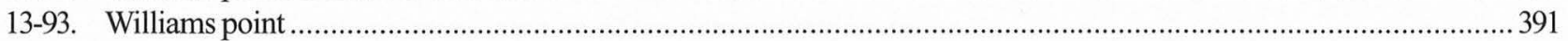

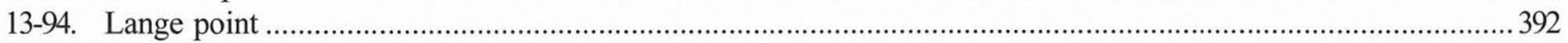

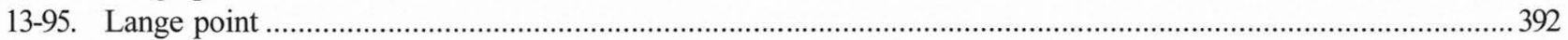

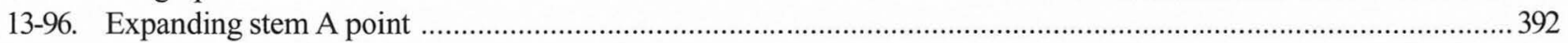

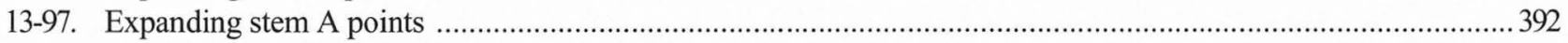

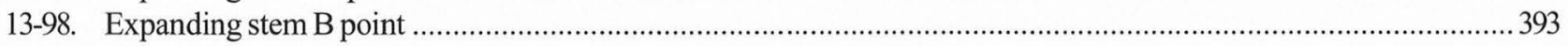

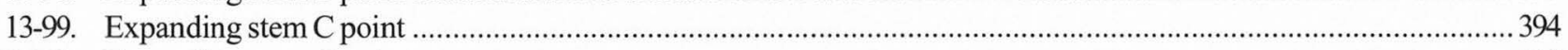

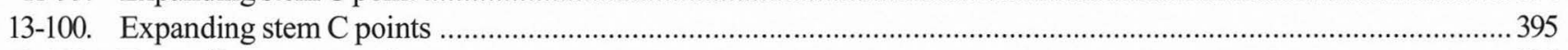

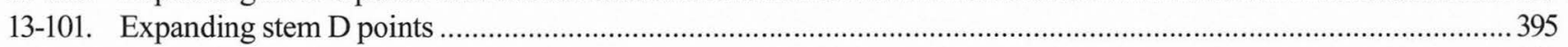

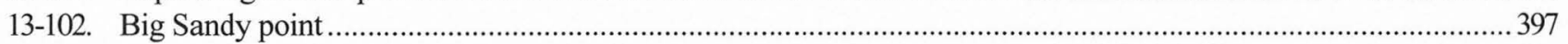

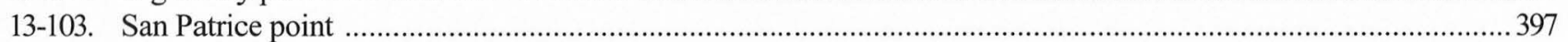

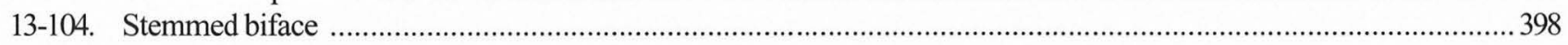

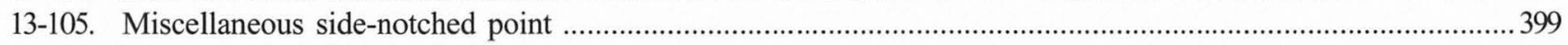

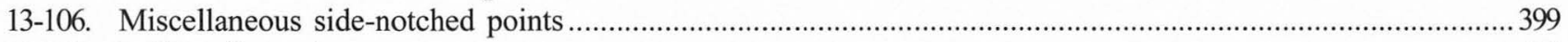

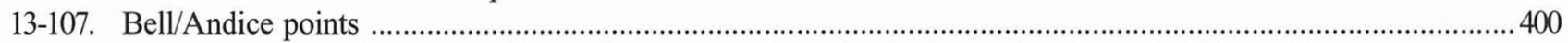

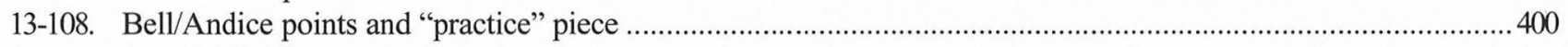




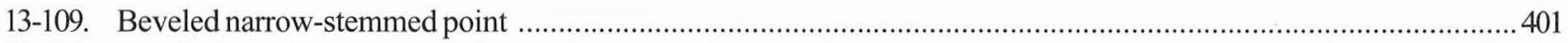

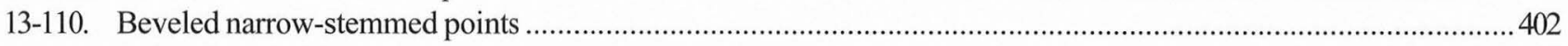

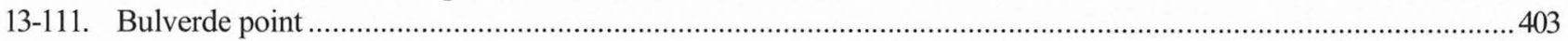

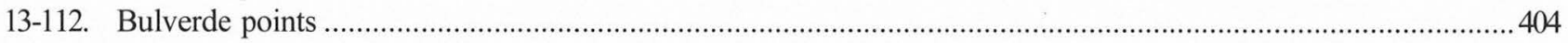

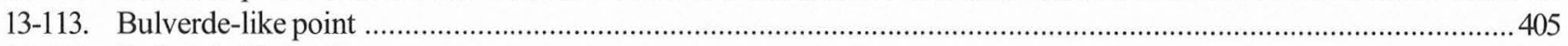

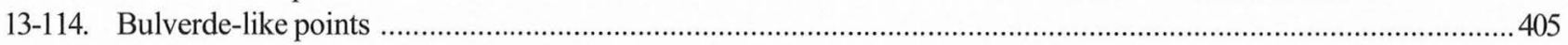

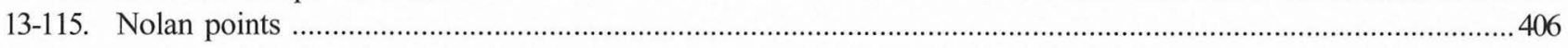

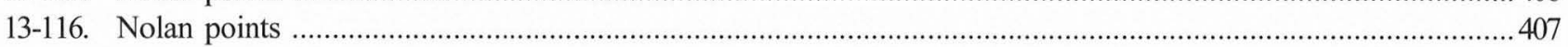

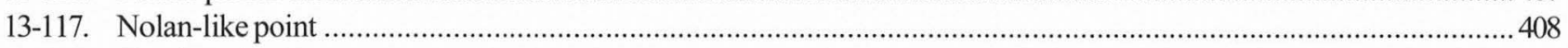

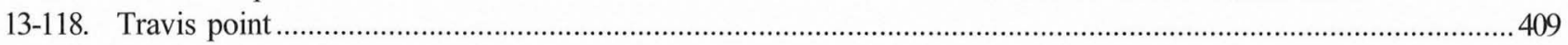

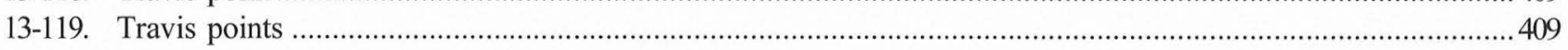

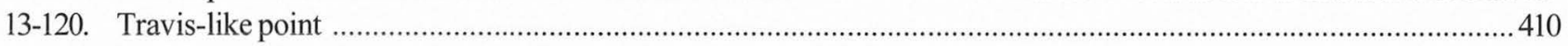

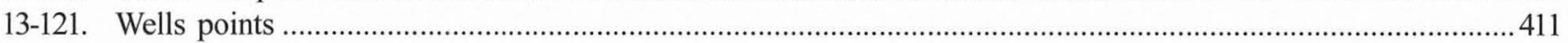

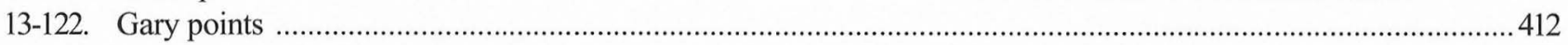

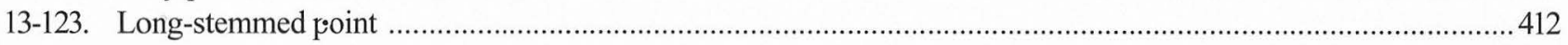

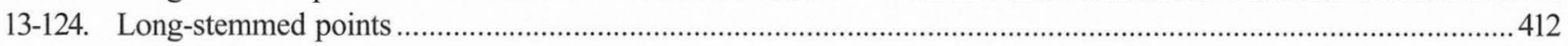

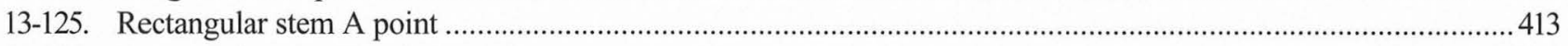

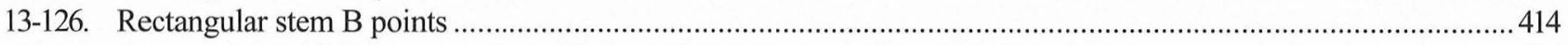

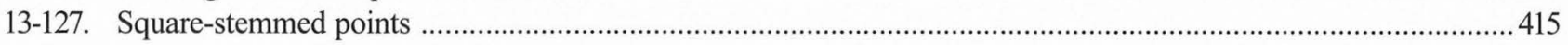

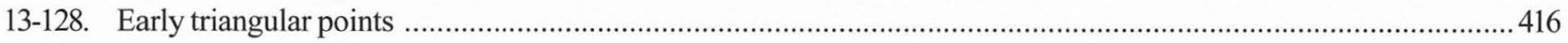

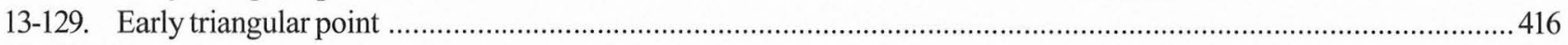

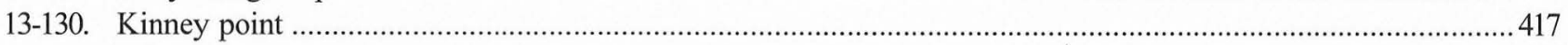

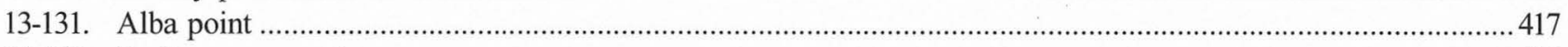

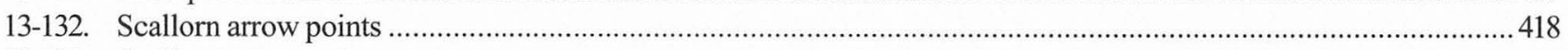

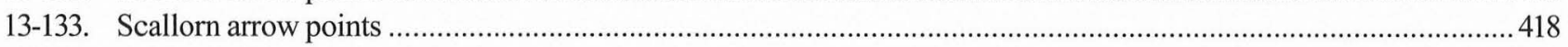

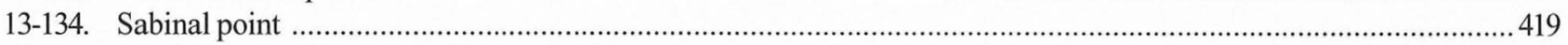

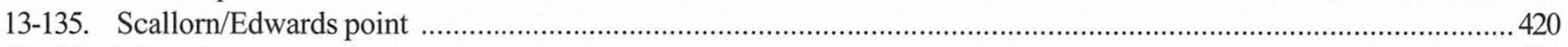

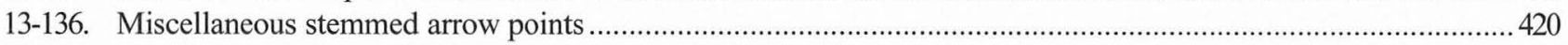

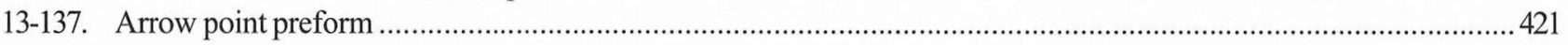

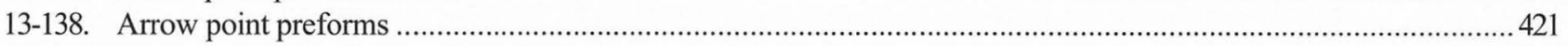

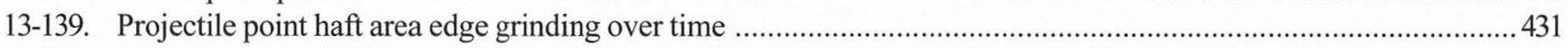

13-140. Regression of animal weights and lethal projectile point perimeters for seven Old

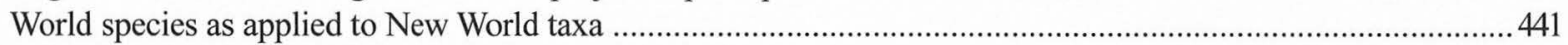

13-141. Wilson-Leonard projectile point widths and estimated shaft diameters by form over time .................................443

14-1. Measurements and ratios used in unfluted lanceolate point analysis .............................................................4 449

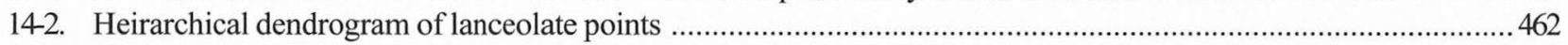

143. Mean shapes and extent of grinding for contracting haft clusters ................................................................ 472

144. Mean shapes and extent of grinding for parallel-sided haft clusters with shallow-to-moderate basal concavities and parallel-sided haft clusters with deep basal concavities ...................................................480

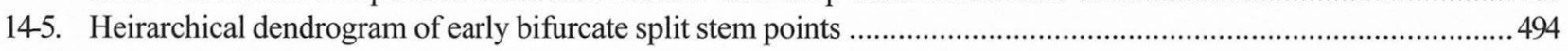

14-6. Representative specimens in early bifurcate stem morphological groups ..........................................................498

147. Representative specimens in early bifurcate morphological groups ................................................................499

14-8. Representative specimens in early bifurcate stem morphological groups .........................................................501

14-9. Representative specimens in early bifurcate stem morphological groups .......................................................502

15-1. Representative Clear Fork bifaces of varying size and morphology ................................................................512

15-2. Examples of varying bit shapes and profiles on Clear Fork bifaces ...................................................................513

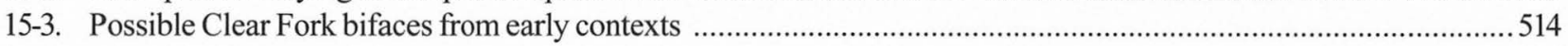

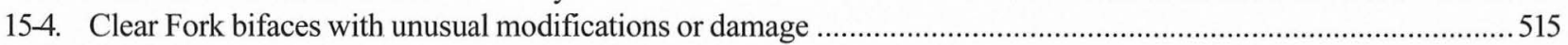

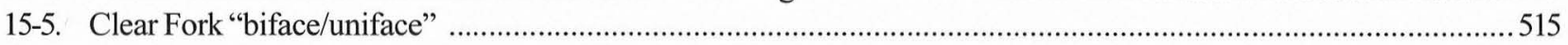

15-6. Examples of bit wear, edge damage, and breaks on Clear Fork bifaces .............................................................517

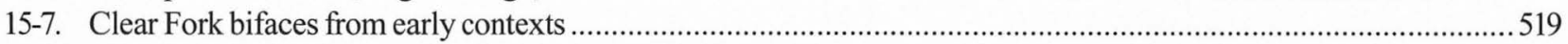

15-8. Clear Fork bifaces from Late Paleoindian and Early Archaic contexts ...............................................................520

15-9. Clear Fork bifaces from Early Archaic and later contexts .............................................................................521

15-10. Comparison of the metric attributes of Dalton adzes from the Sloan site cache with Wilson-Leonard 


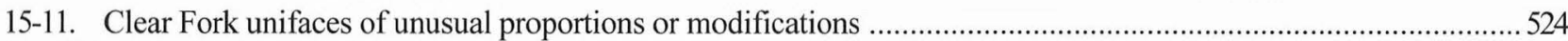

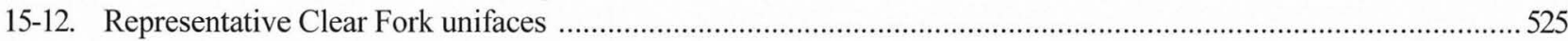

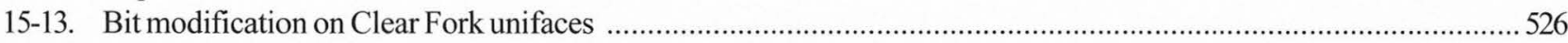

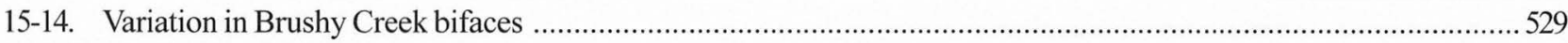

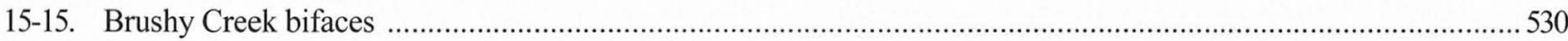

15-16. Patterns in form and distribution of Clear Fork bifaces, Clear Fork unifaces,

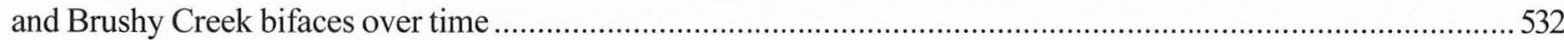

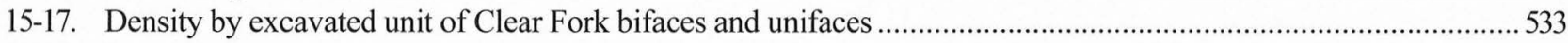

15-18. Hypothesized sequence of attrition and corresponding morphological changes with successive resharpening and rejuvenation episodes in Clear Fork tools ...........................................................534

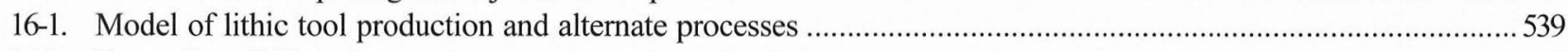

16-2. Examples of biface fracture types and manufacturing failures ..........................................................................5 540

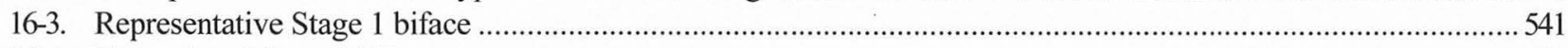

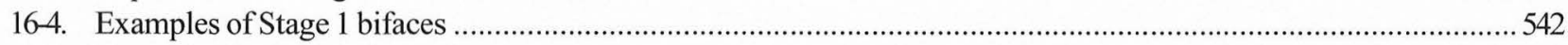

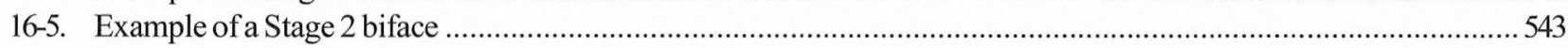

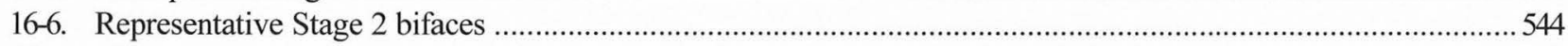

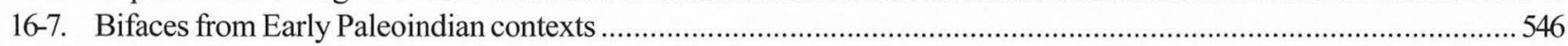

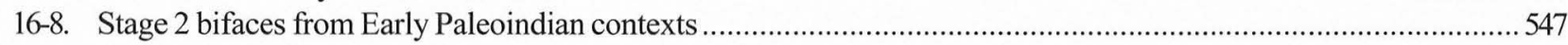

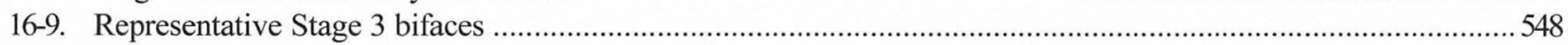

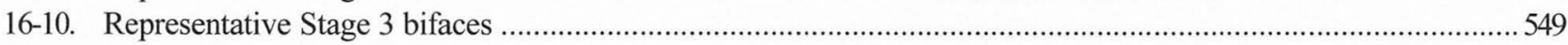

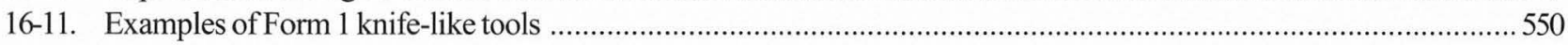

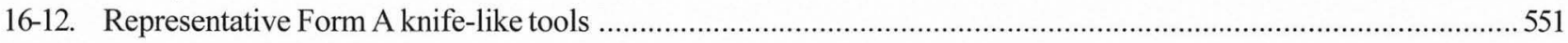

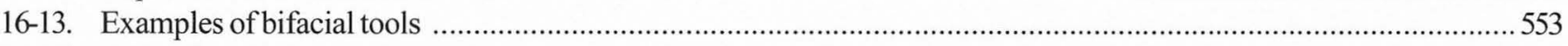

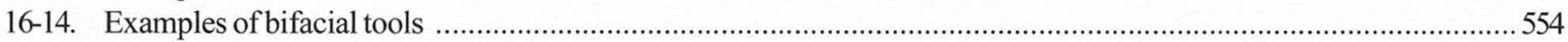

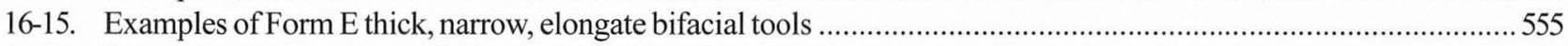

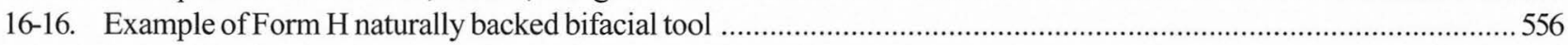

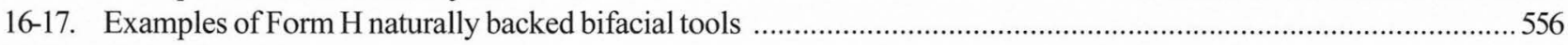

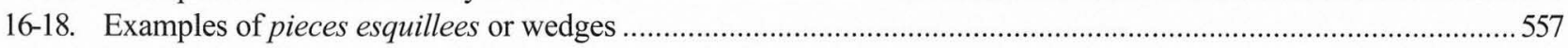

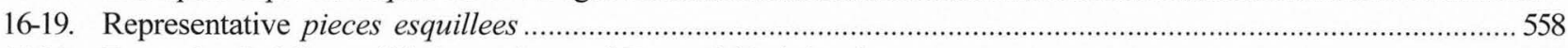

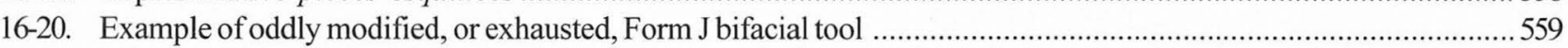

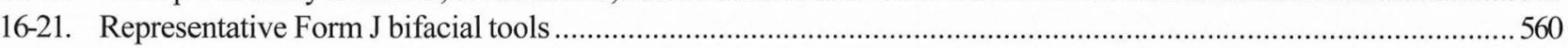

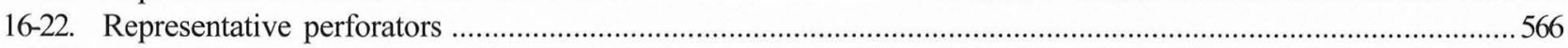

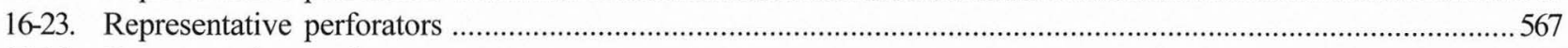

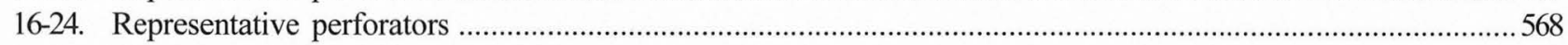

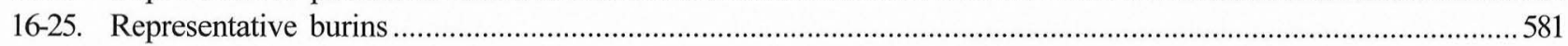

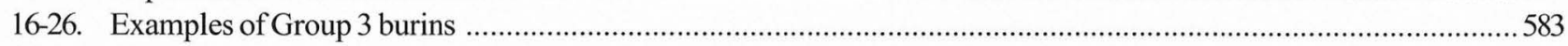

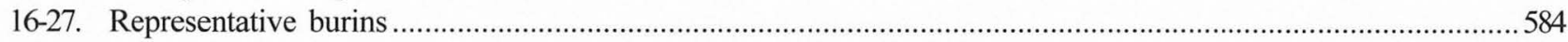

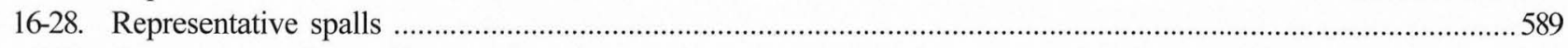

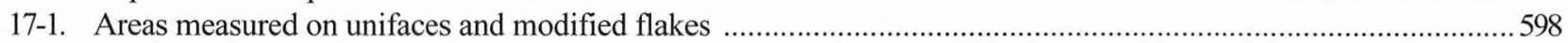

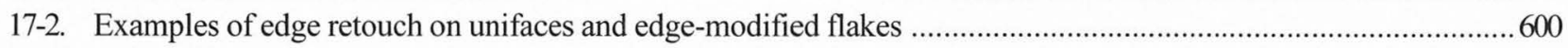

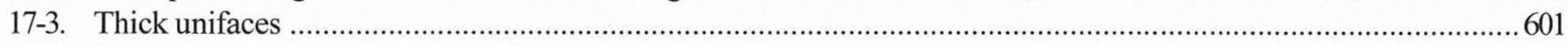

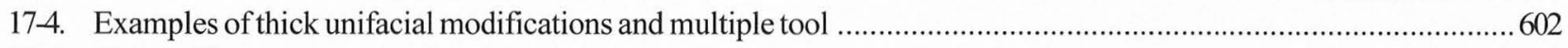

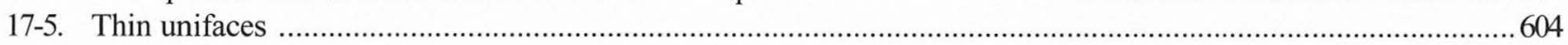

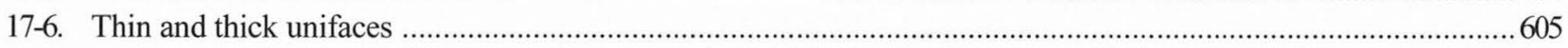

17-7. Distally convergent modification edge-modified flake, thin unifaces,

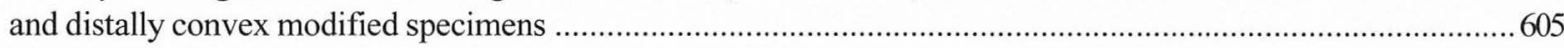

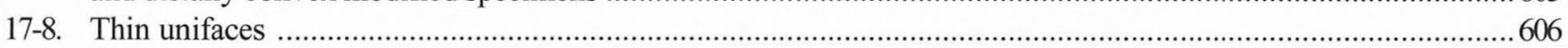

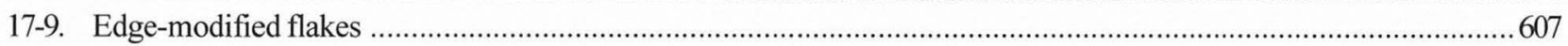

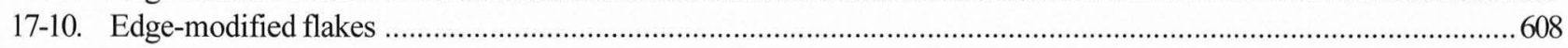

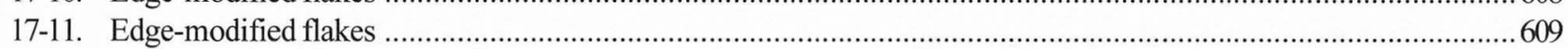

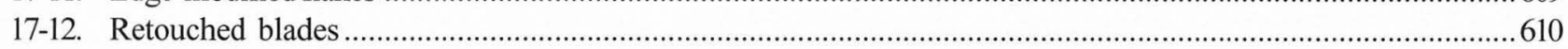

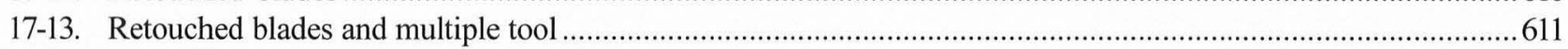

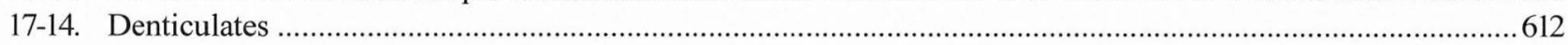

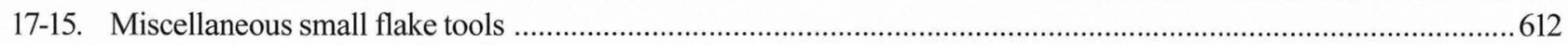

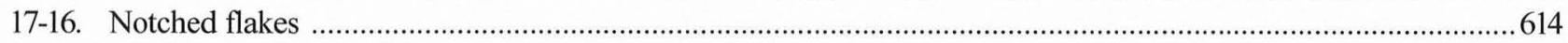




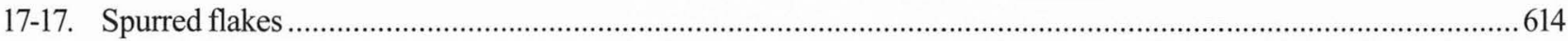

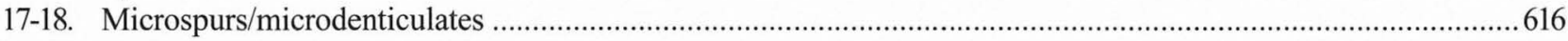

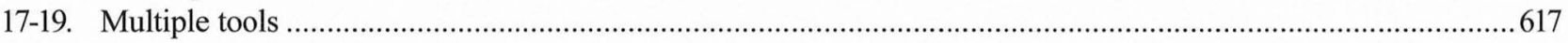

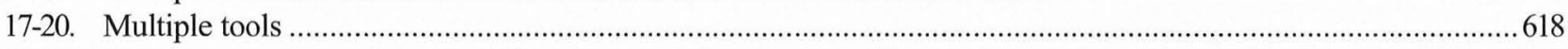

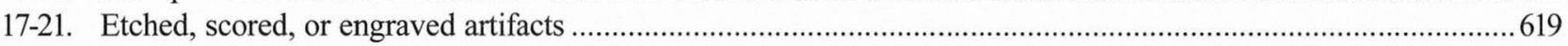

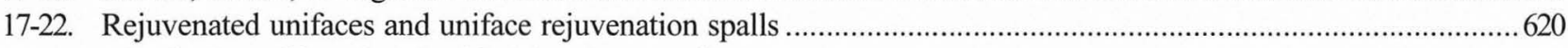

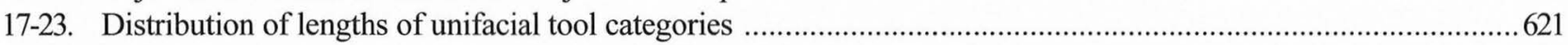

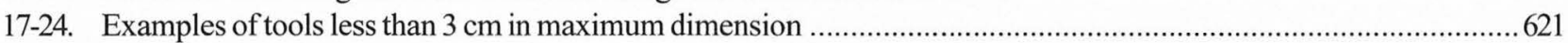

17-25. Distribution of mean potential edge length and mean total modification length by tool class .............................629

17-26. Distribution of unifacial tool class by mean percent of edge modification and630

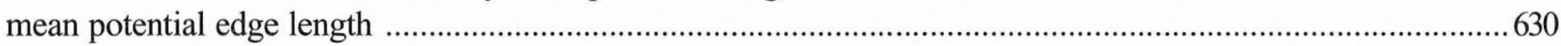

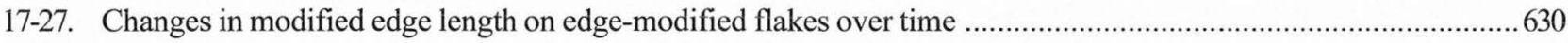

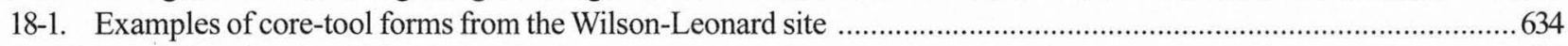

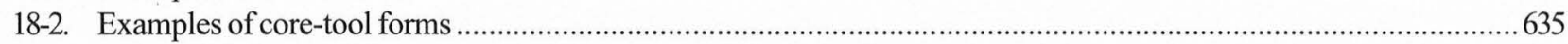

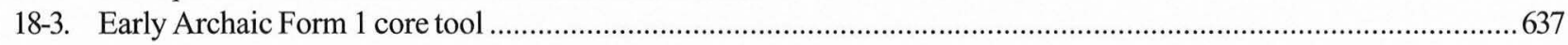

18-4. Group 4 tools from Early Paleoindian and Late Archaic contexts ................................................................641

18-5. Core tool fragment with both faunal and vegetal residues ..........................................................................642

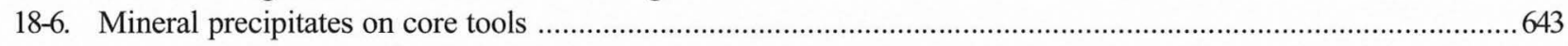

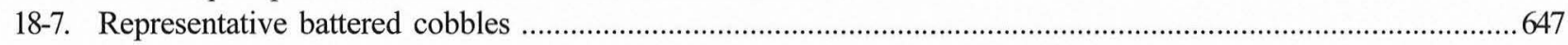

18-8. Large core hammerstone with concave edges from concentrated attrition of heavy battering ..............................649

18-9. Large core hammerstones and core hammerstones made on moderately thin biface fragments ............................650

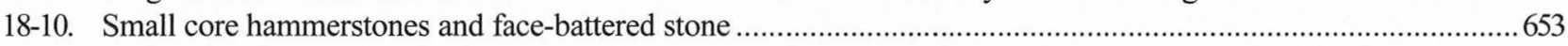

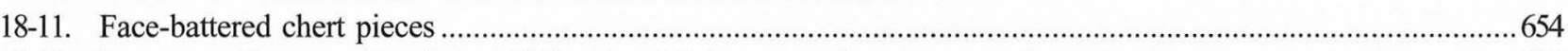

18-12. Representative core showing multidirectional flake removal ......................................................................... 655

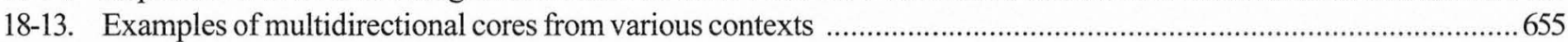

18-14. Quartz core from Late Archaic cache showing multidirectional and blade-like flake scars .................................656

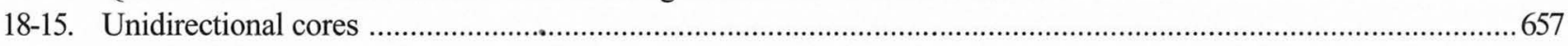

18-16. Representative microcores, showing varying flake scar sizes ...................................................................658

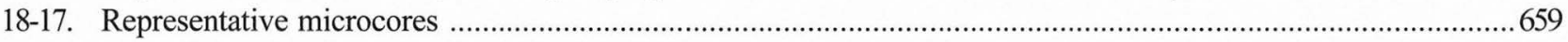

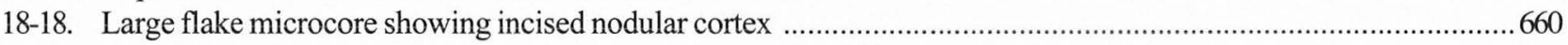

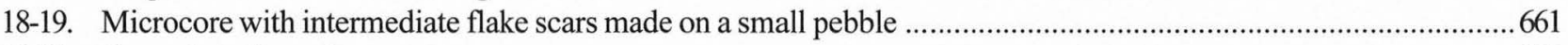

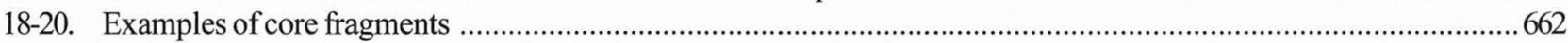

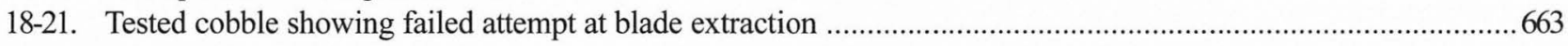


"This page intentionally left blank" 


\section{LIST OFTABLES}

\section{Volume II}

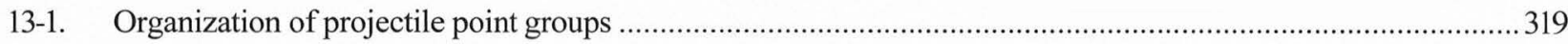

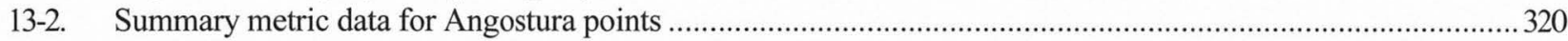

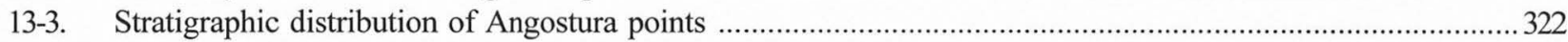

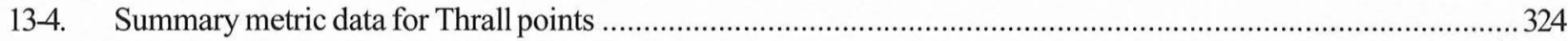

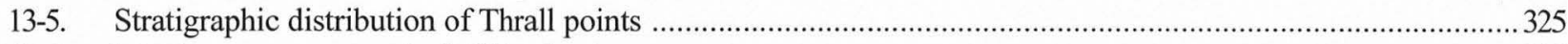

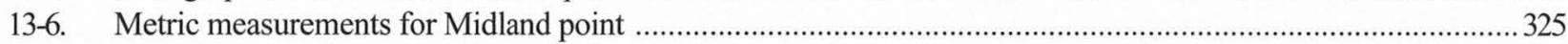

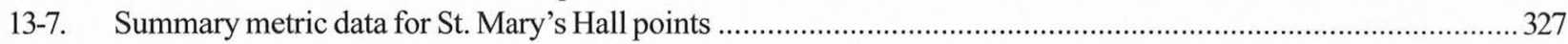

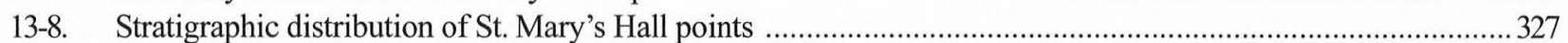

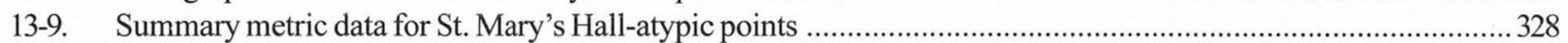

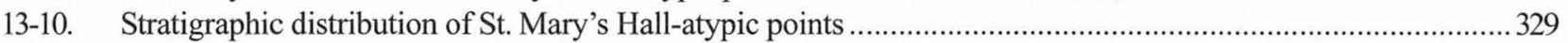

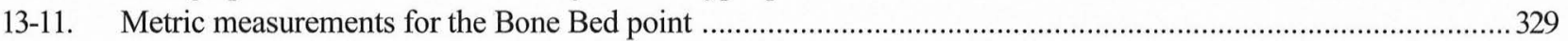

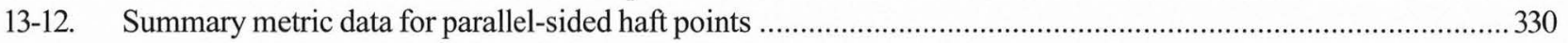

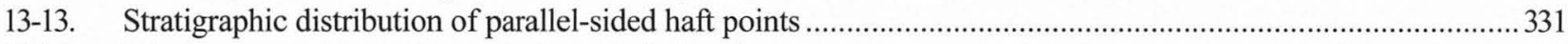

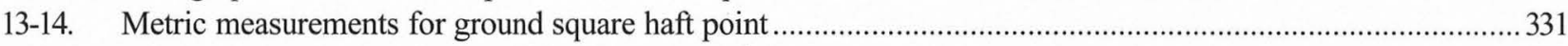

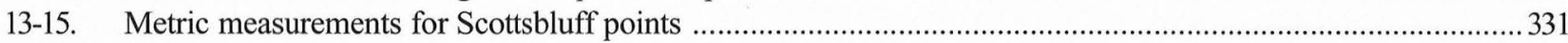

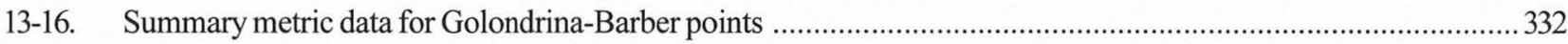

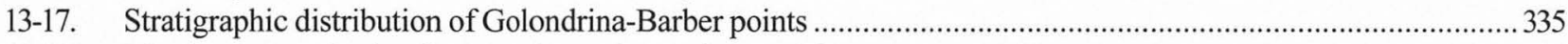

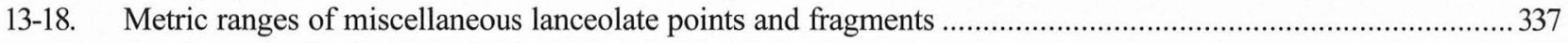

13-19. Stratigraphic distribution of miscellaneous lanceolate points and fragments .................................................. 338

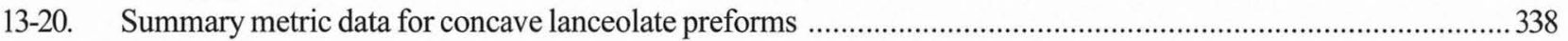

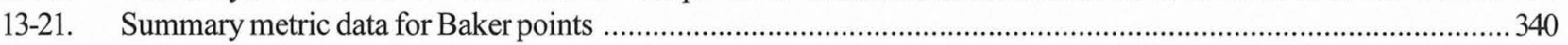

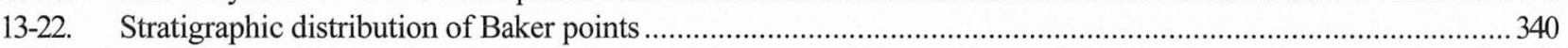

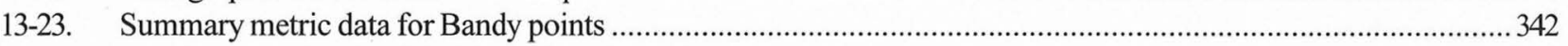

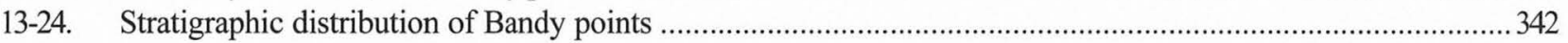

13-25. Summary metric data for contracting stem, concave base points ................................................................ 343

13-26. Stratigraphic distribution of points with constracting stems, concave bases ............................................... 343

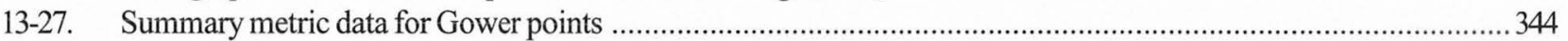

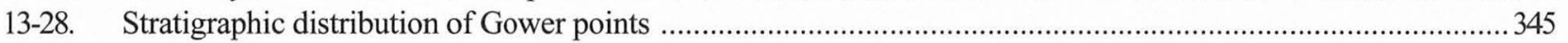

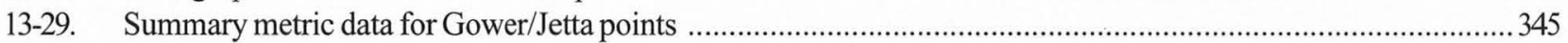

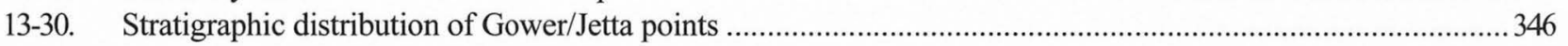

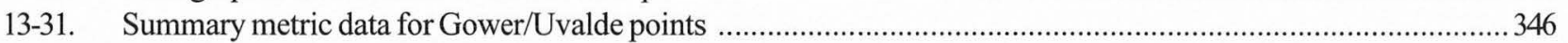

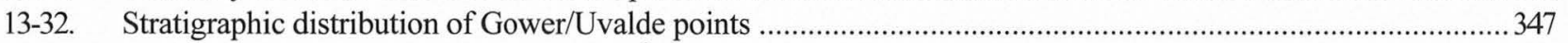

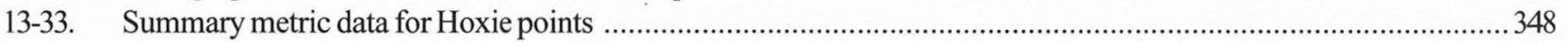

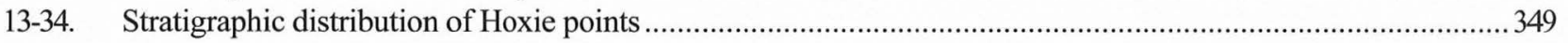

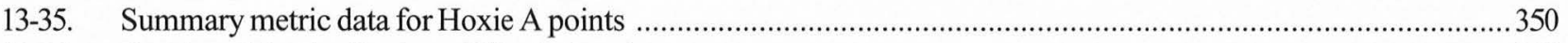

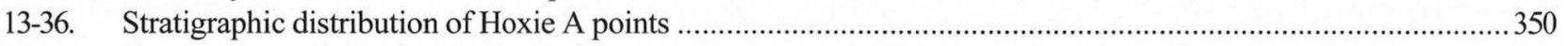

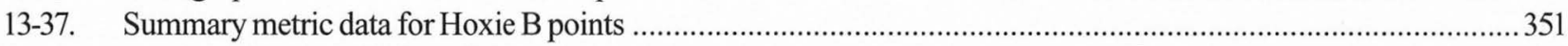

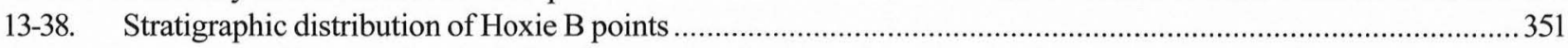

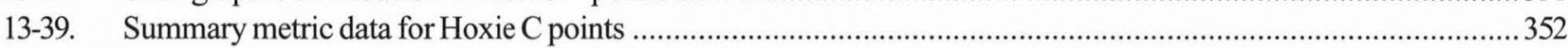

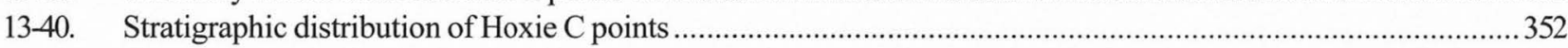

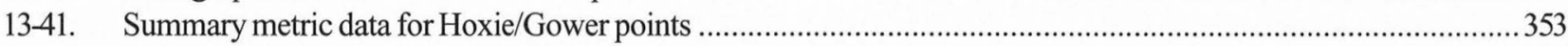

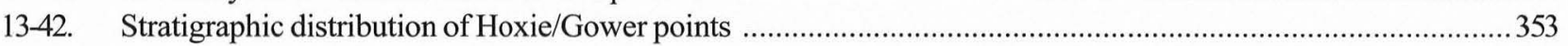

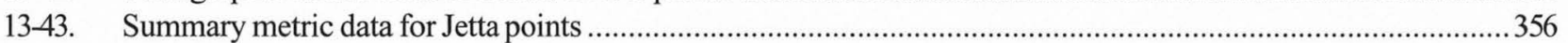

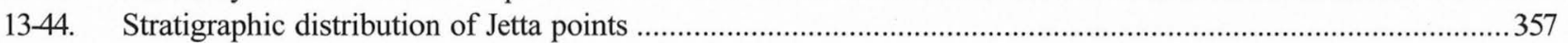

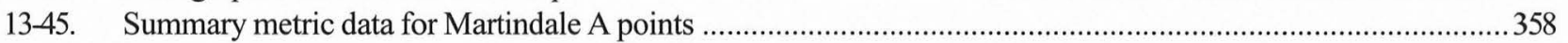

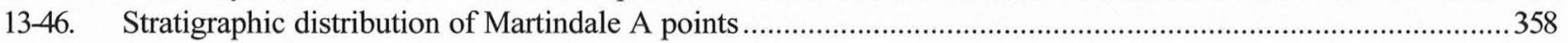

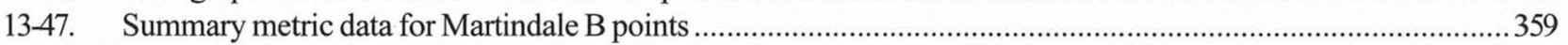

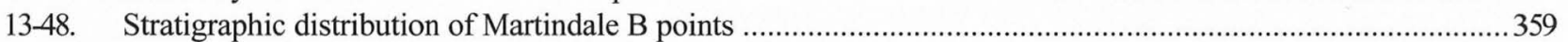

13-49. Summary metric data for expanding stem, concave, base A points ..................................................................360

13-50. Stratigraphic distribution of expanding stem, concave base A points ...........................................................360

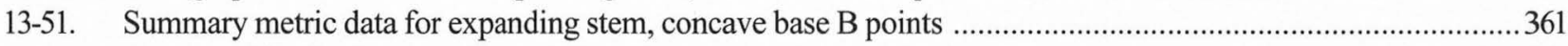

13-52. Stratigraphic distribution of expanding stem, concave base B points ............................................................362 


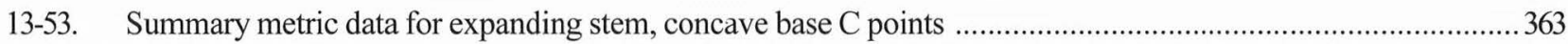

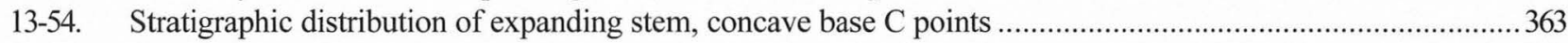

13-55. Summary metric data for expanding stem, concave base $D$ points ...............................................................365

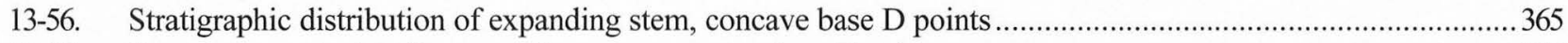

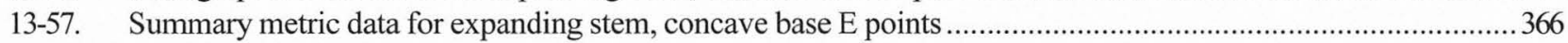

13-58. Stratigraphic distribution of expanding stem, concave base E points ……....................................................366

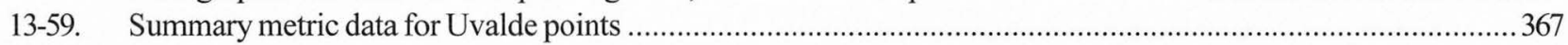

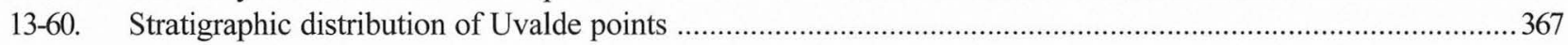

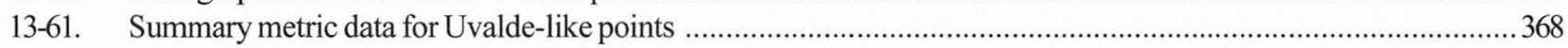

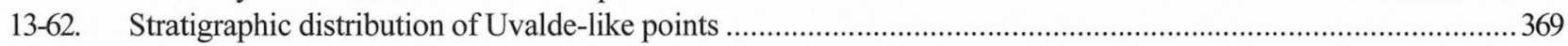

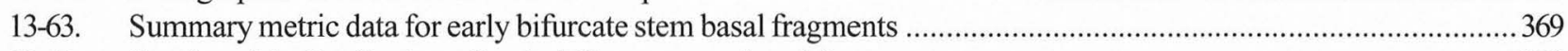

13-64. Stratigraphic distribution of early bifurcate stem basal fragments ..........................................................3 370

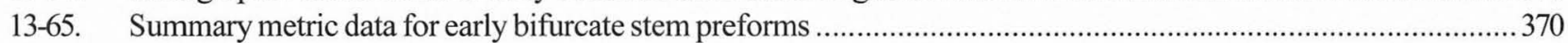

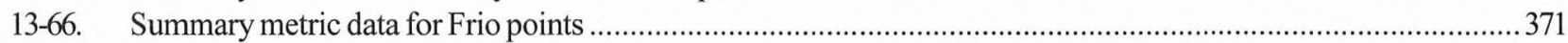

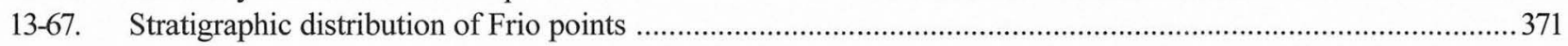

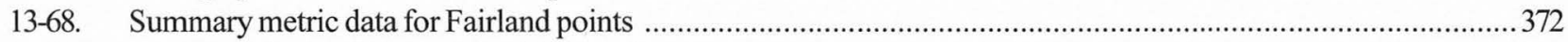

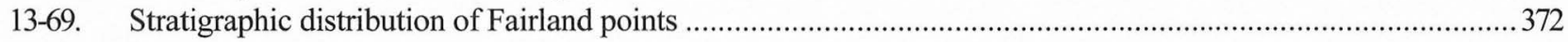

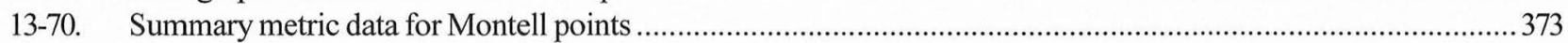

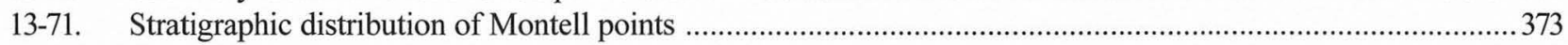

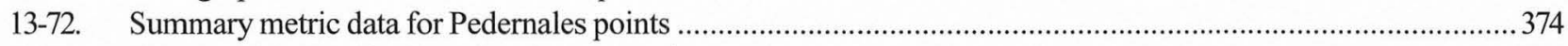

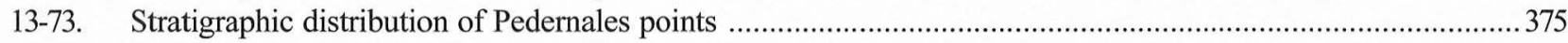

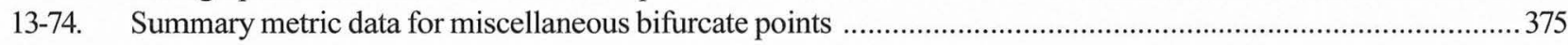

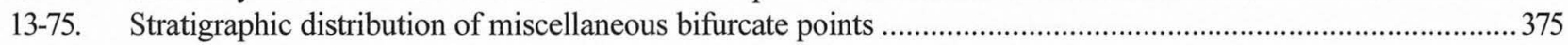

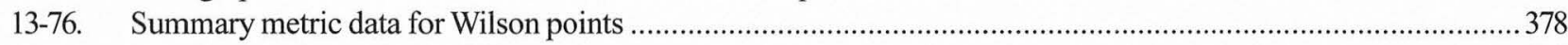

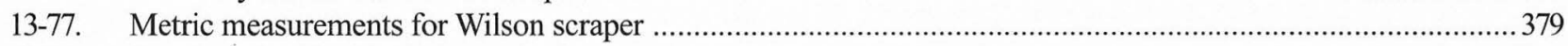

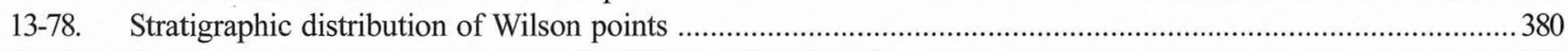

13-79. Comparative metric measurements for Wilson points from four sites ......................................................... 381

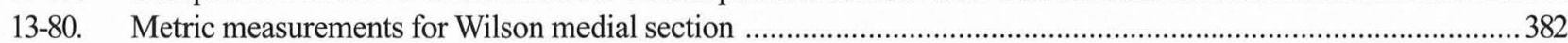

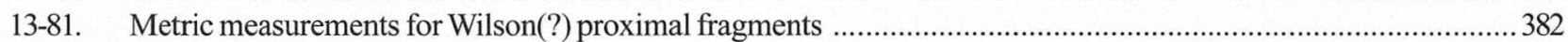

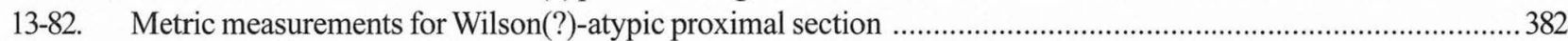

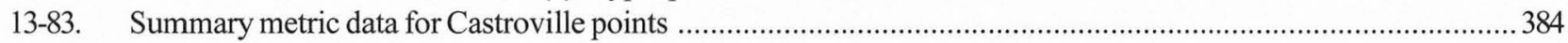

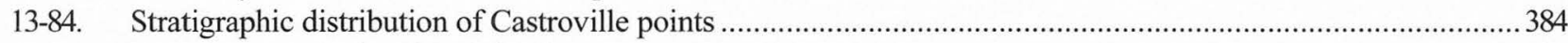

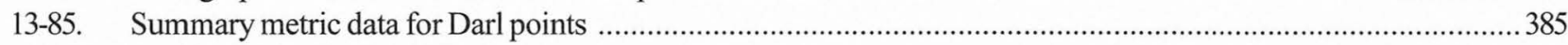

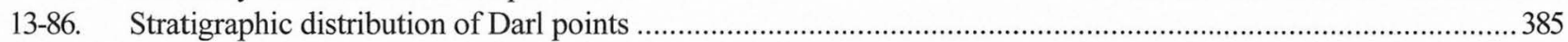

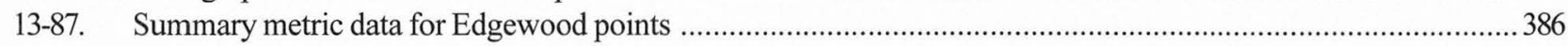

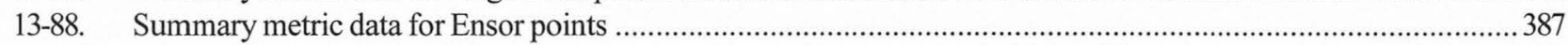

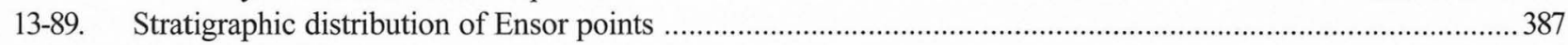

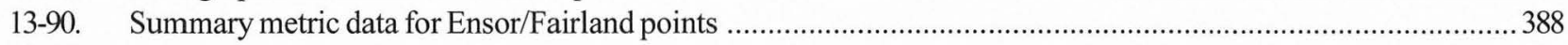

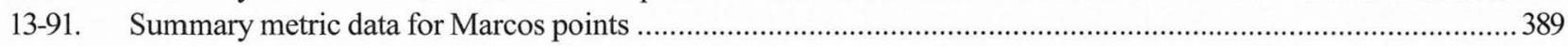

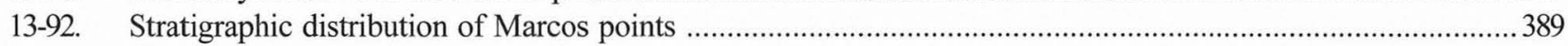

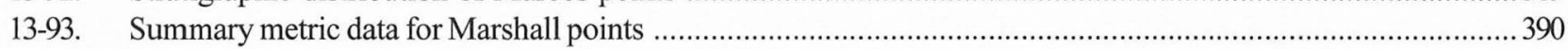

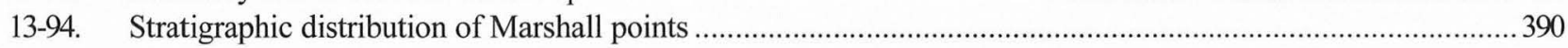

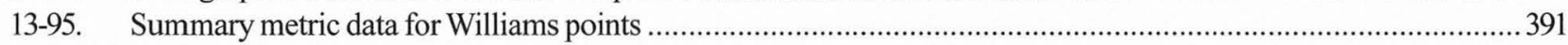

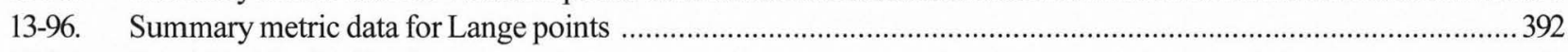

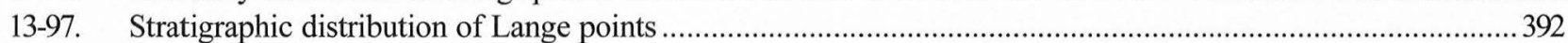

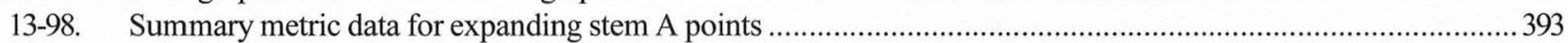

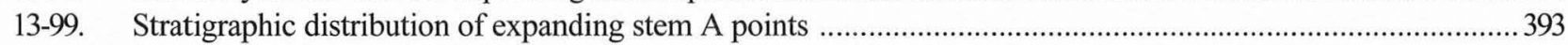

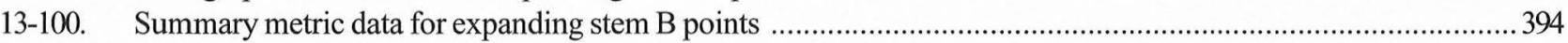

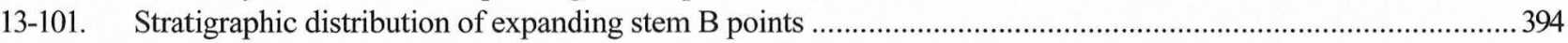

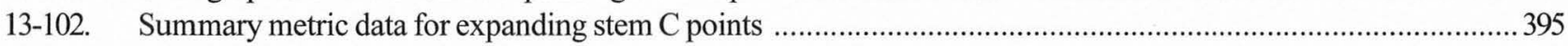

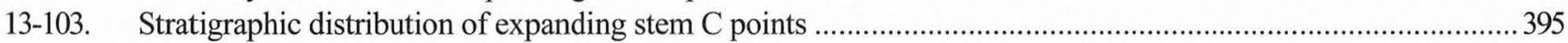

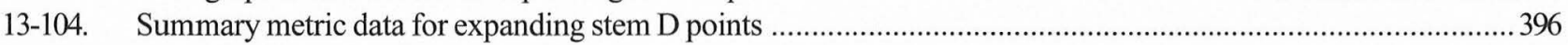

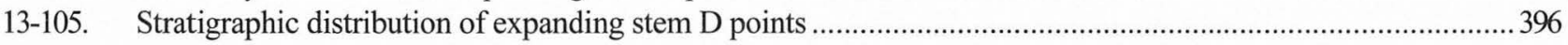

13-106. Summary metric data for miscellaneous expanding stem points …….............................................................396

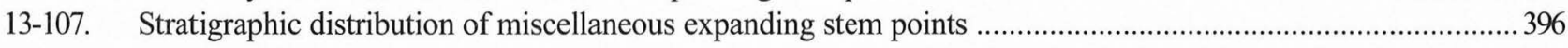




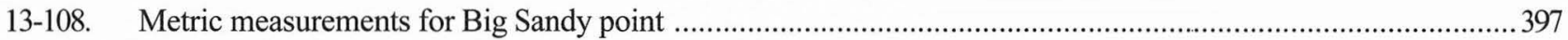

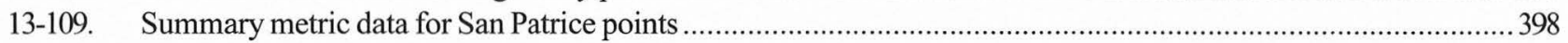

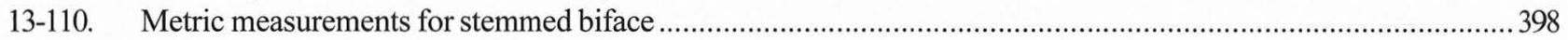

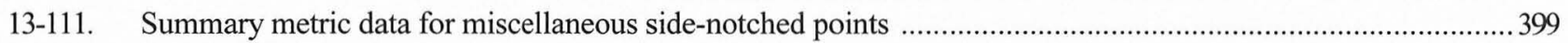

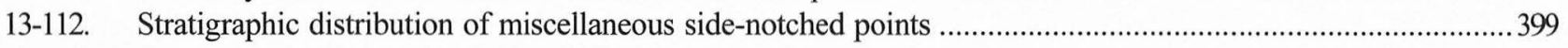

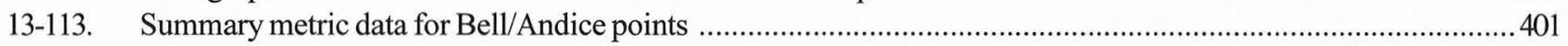

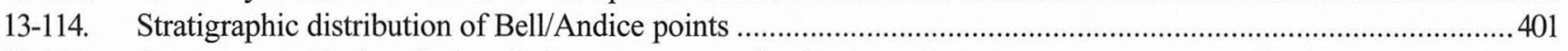

13-115. Summary metric data for beveled narrow stemmed points ............................................................................ 402

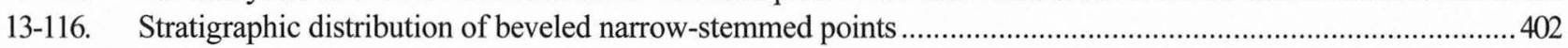

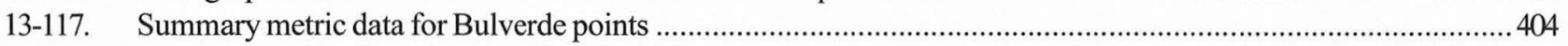

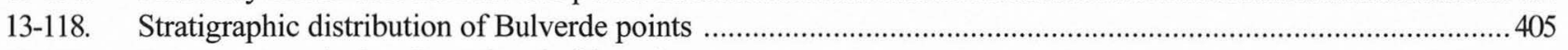

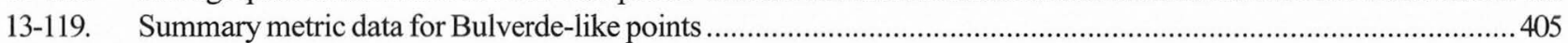

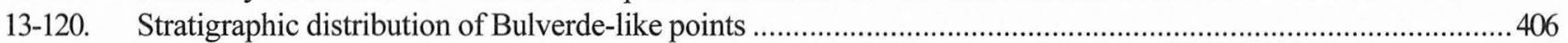

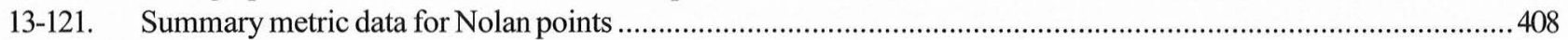

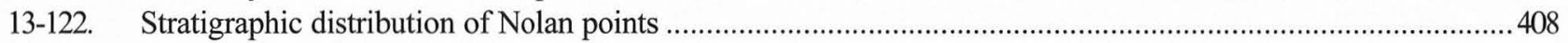

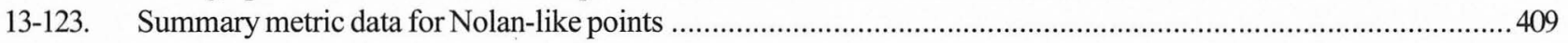

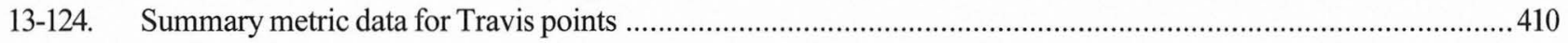

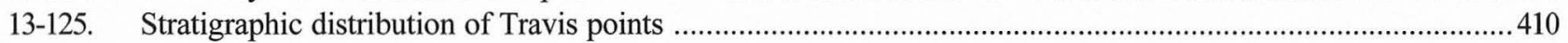

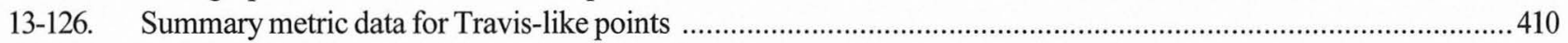

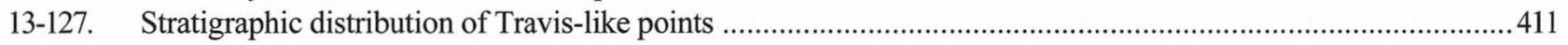

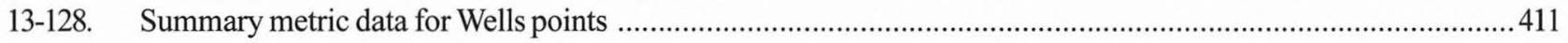

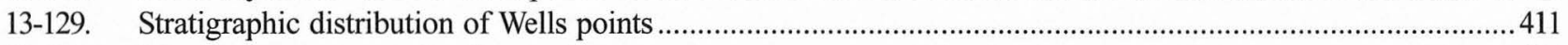

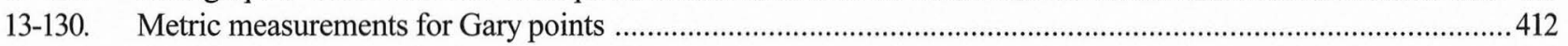

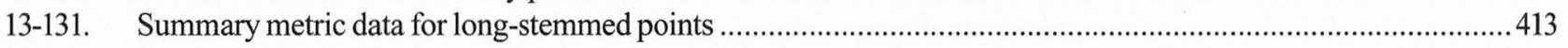

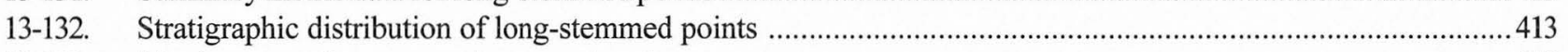

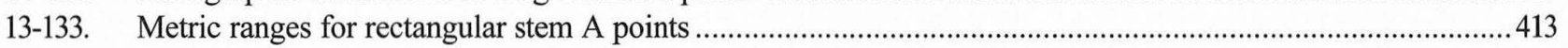

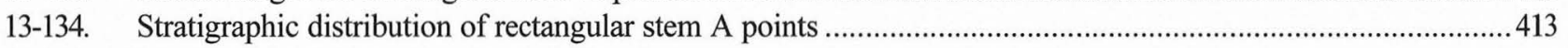

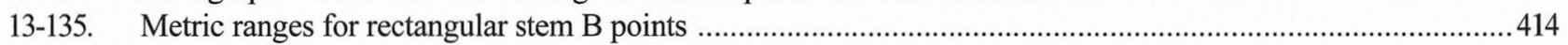

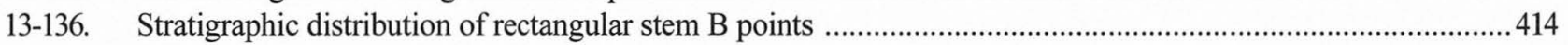

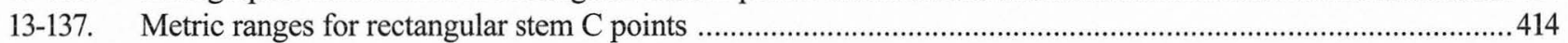

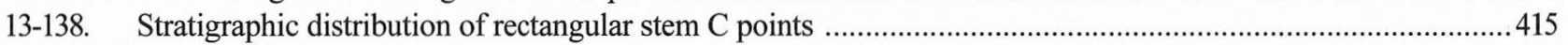

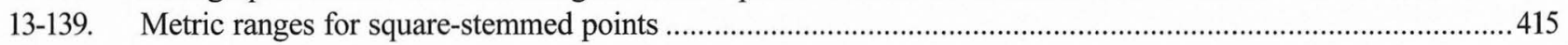

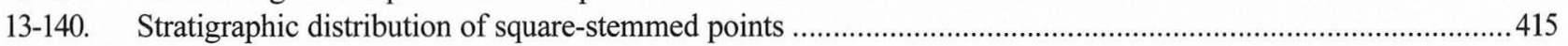

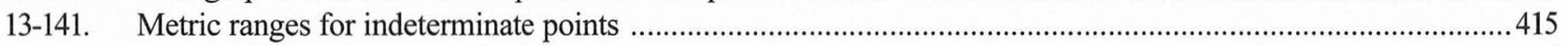

13-142. Stratigraphic distribution of indeterminate points ................................................................................... 416

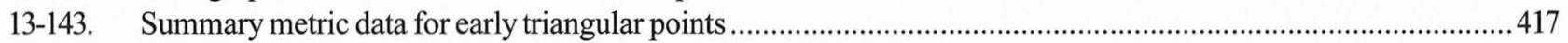

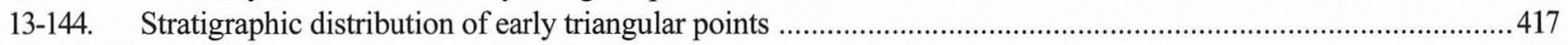

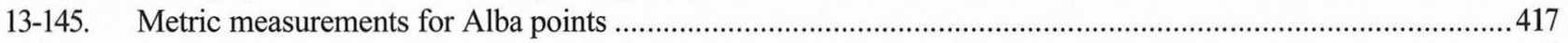

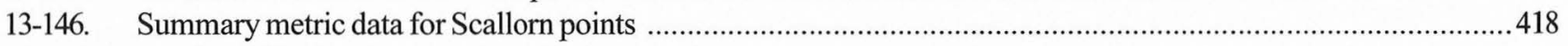

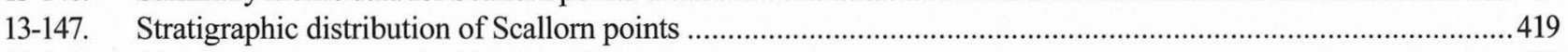

13-148. Metric measurements for Scallorn/Edwards points .............................................................................420

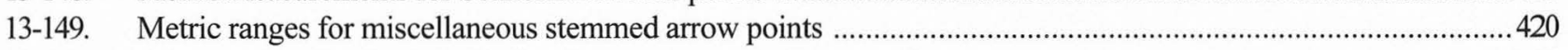

13-150. Stratigraphic distribution of miscellaneous stemmed arrow points ................................................................420

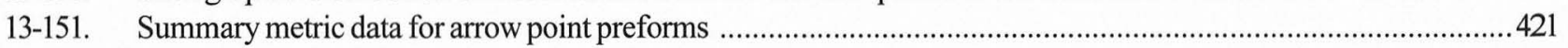

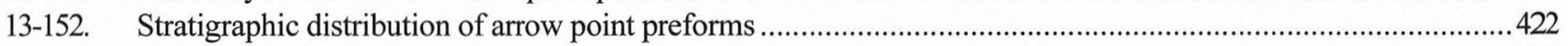

13-153. Stratigraphic distribution of projectile point fragments ........................................................................... 422

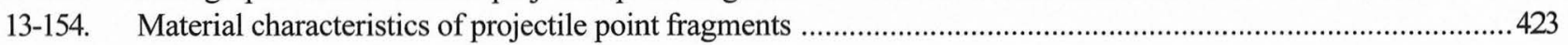

13-155. Distribution of technologically significant projectile point fragments ...............................................................424

13-156. Stratigraphic distribution of early split stem and early corner-notched points ..................................................426

13-157. Projectile point distribution by type and stratigraphic unit (Valley Floor) ......................................................427

13-158. Projectile point distribution by type and stratigraphic unit (Valley Margin) ....................................................429

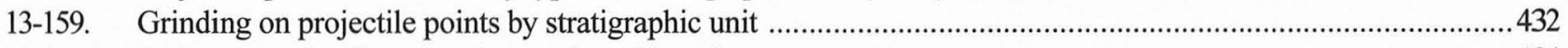

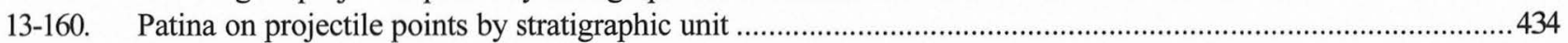

13-161. Mineral precipitates on projectile points by stratigraphic unit ..................................................................435

13-162. Heat alteration on projectile points by stratigraphic unit .........................................................................436 
13-163. Heat alteration on projectile point fragments by stratigraphic unit ............................................................ 437

13-164. Resharpening of projectile points by stratigraphic unit ............................................................................438

13-165. Anatomical factors determining the requirements for a fatal arrow wound .....................................................438

13-166. Projectile point width, shoulder form, estimated wound perimeter,

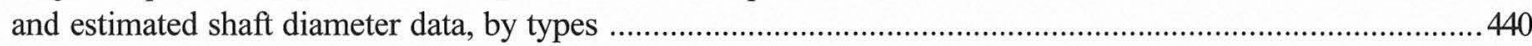

14-1. Sample list and specimen key for cluster analysis dendrogram ................................................................... 454

142. Radiocarbon ages associated with sample specimens from sites other than Wilson-Leonard ........................... 459

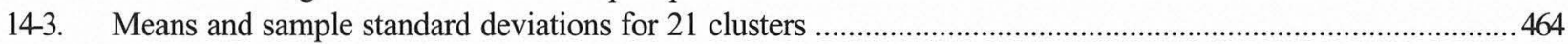

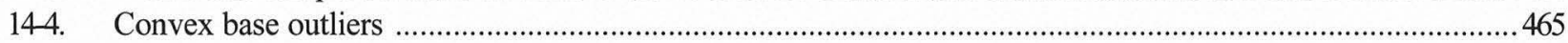

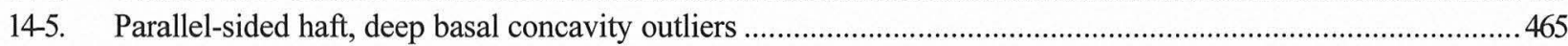

146. Geisser Classification Probabilities for unfluted lanceolate projectile points .................................................466

147. Analysis of Variance comparing nine contracting haft clusters by eight variables ......................................... 470

148. Analysis of Variance comparing six parallel-sided haft, shallow-to-moderate basal

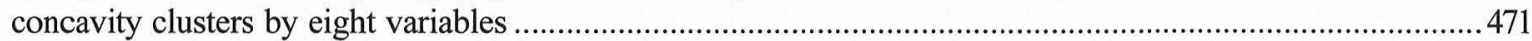

14-9. Analysis of Variance comparing six parallel-sided haft, deep basal concavity clusters

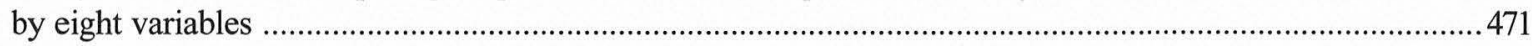

14-10. Analysis of Variance comparing Clusters 1-5 for Angostura Type ……....................................................474

14-11. Means and sample standard deviations for two sets of contracting haft clusters .............................................475

14-12. Analysis of Variance comparing Clusters 6 and 9 for Thrall type …...........................................................476

14-13. Analysis of Variance comparing Clusters 7 and 8 for Midland type ...........................................................47

1414. Analysis of Variance comparing five contracting haft point types ................................................................477

1415. Means and sample standard deviations for unfluted lanceolate types ..........................................................478

14-16. Analysis of Variance comparing Clusters 12-14 for Plainview type .............................................................. 481

14-17. Means and sample standard deviations for Clusters 12, 13, and 14 of Plainview type .....................................482

1418. Analysis of Variance comparing Clusters 10 and 11 for St. Mary's Hall type ................................................. 483

14-19. Analysis of Variance comparing parallel-sided haft, shallow-to-moderate basal concavity point types ......... 484

14-20. Distribution of types within parallel-sided haft, deep basal concavity clusters ...........................................485

1421. Analysis of Variance comparing three point types of parallel-sided haft,

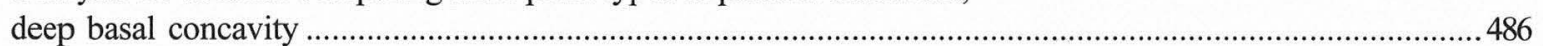

14-22. Geisser classification probabilities for early bifurcate stem projectile points ................................................... 492

1423. Means and standard deviations for early bifurcate stem clusters …….......................................................496

1424. Comparison of types of morphometric classifications of Bifurcate Stemmed projectile points ...........................504

1425. Early split stem morphological clusters by stratigraphic unit .........................................................................505

15-1. Measurements, attributes, and distribution of Clear Fork bifaces .................................................................509

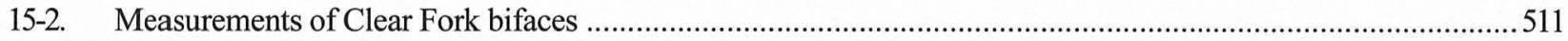

15-3. Measurements, attributes, and distribution of Clear Fork unifaces ...............................................................523

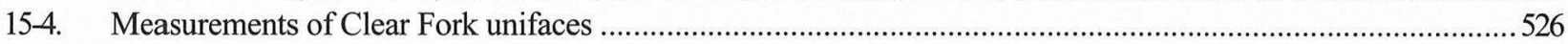

15-5. Measurements, attributes, and distribution of Brushy Creek bifaces ............................................................528

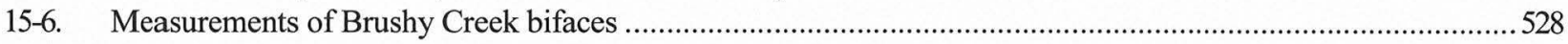

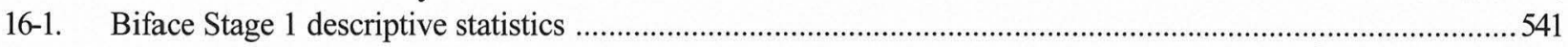

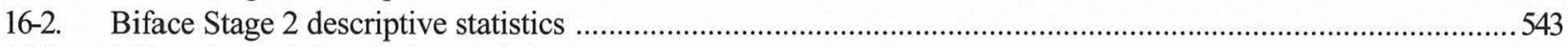

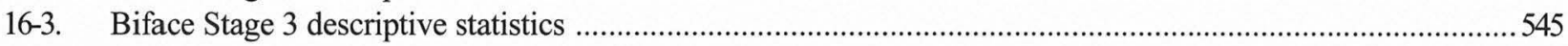

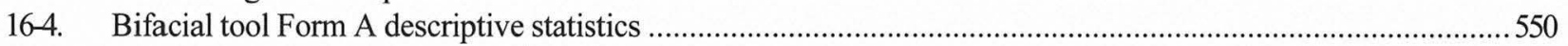

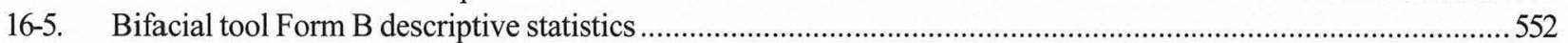

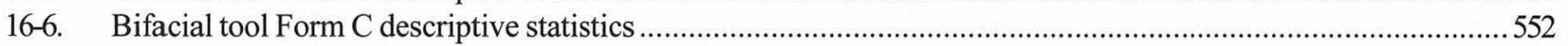

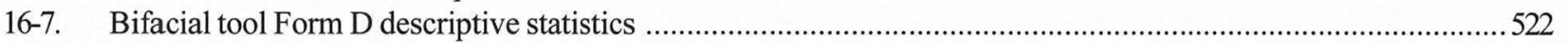

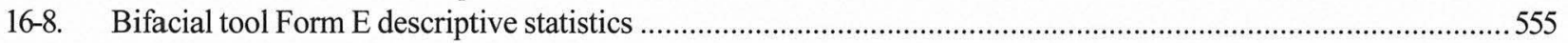

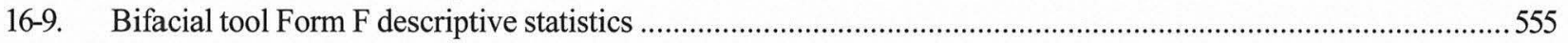

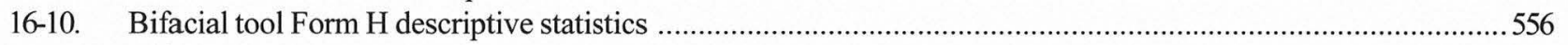

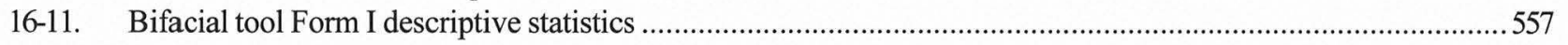

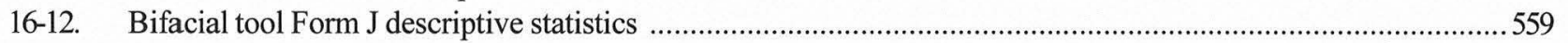

16-13. Stratigraphic distribution of Stage 1-3 bifaces in the Valley Floor ...............................................................56

16-14. Stratigraphic distribution of Stage 1-3 bifaces in the Valley Margin ..............................................................56

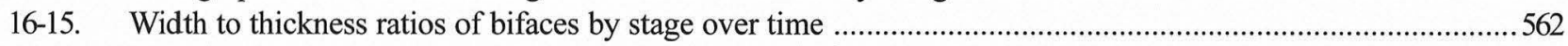

16-16. Distribution of bifacial tools in the Valley Floor ......................................................................................562 


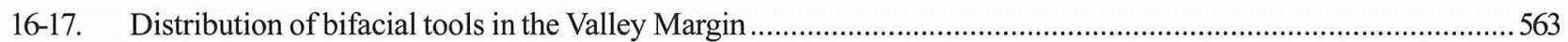

16-18. Raw material characteristics of bifaces and bifacial tools in selected Valley Floor units ..................................564

16-19. Cortex type on bifaces and bifacial tools in selected Valley Floor units ........................................................564

16-20. Heat alteration of bifaces and bifacial tools in selected Valley Floor units ......................................................564

16-21. Patina on bifaces and bifacial tools in selected Valley Floor units .................................................................564

16-22. Mineral precipitates on bifaces and bifacial tools in selected Valley Floor units ..............................................565

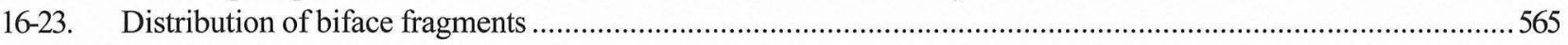

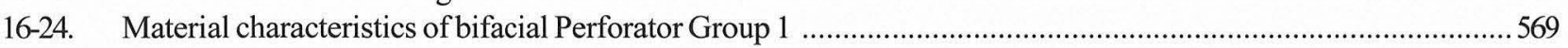

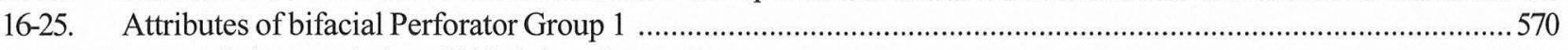

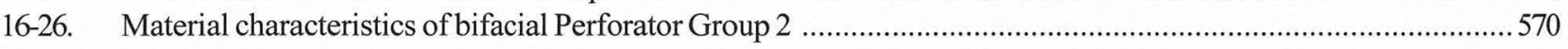

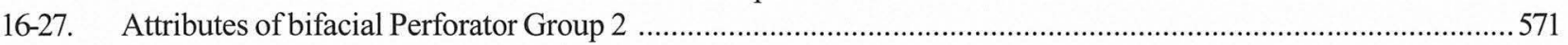

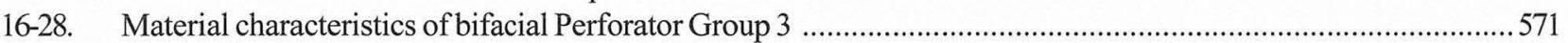

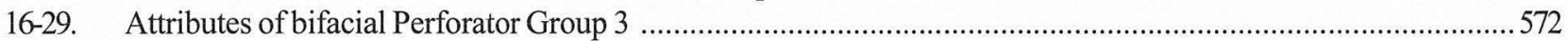

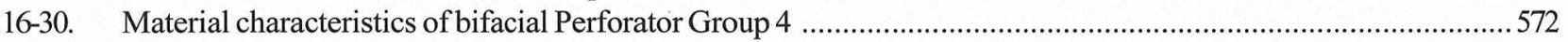

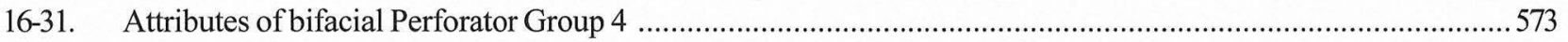

16-32. Material characteristics of bifacial Perforator Group 5 .................................................................................5

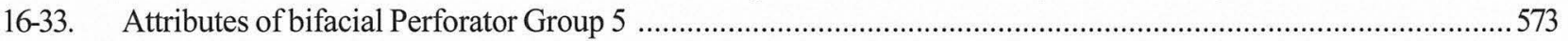

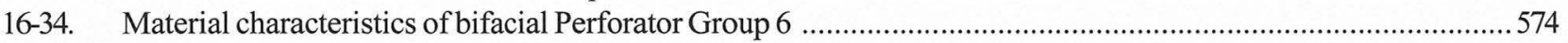

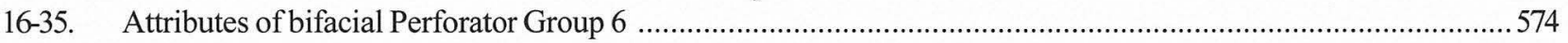

16-36. Material characteristics of bifacial Perforator Group 7 ..............................................................................5

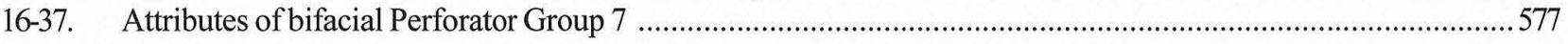

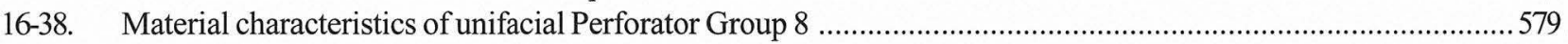

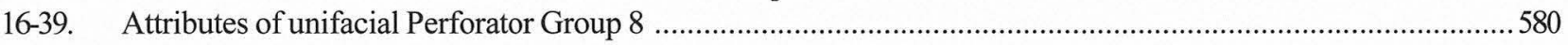

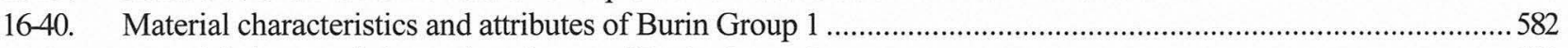

16-41. Material characteristics and attributes of Burin Group 2 ........................................................................582

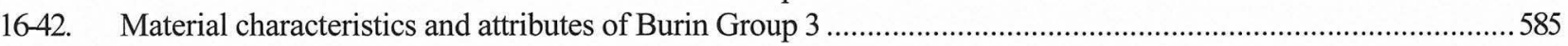

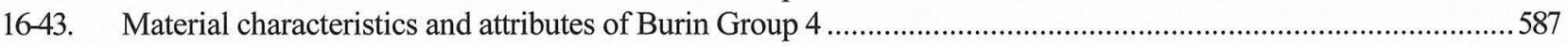

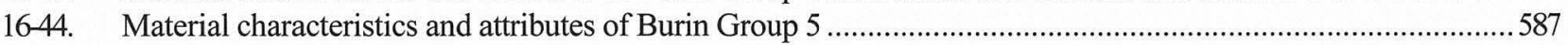

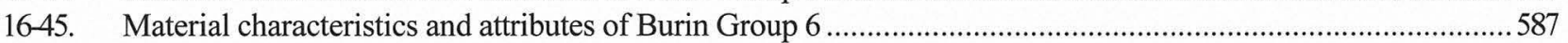

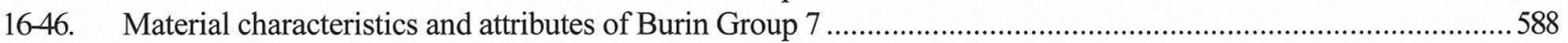

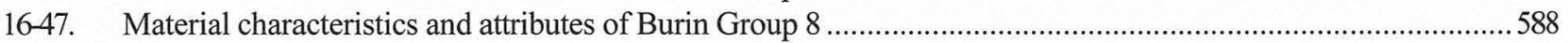

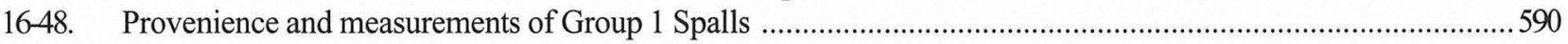

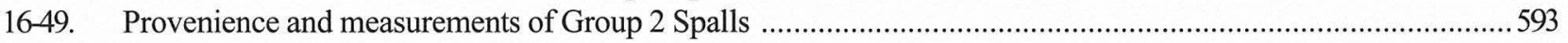

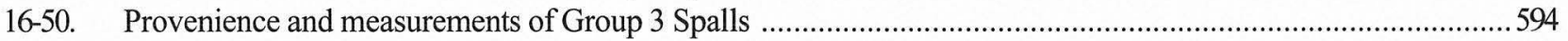

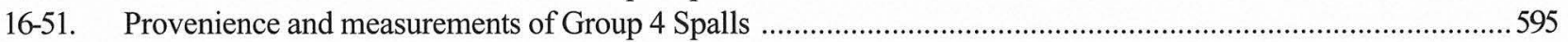

16-52. Provenience and measurements of Indeterminate type Spalls ....................................................................5 596

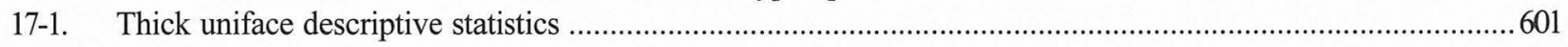

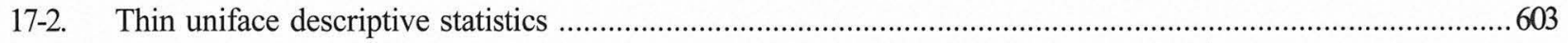

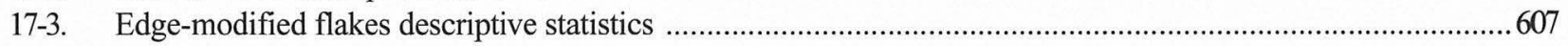

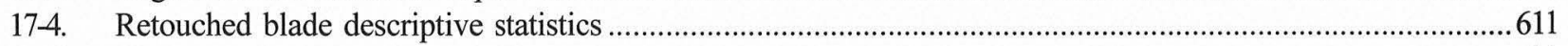

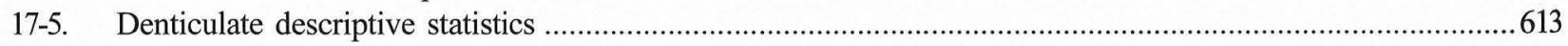

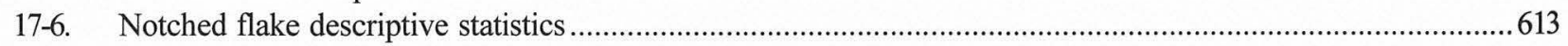

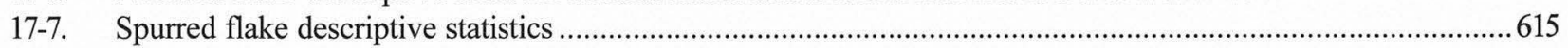

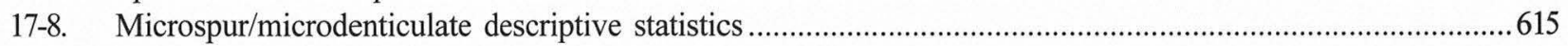

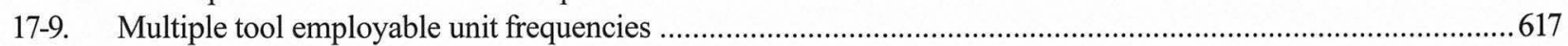

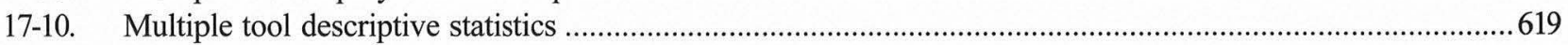

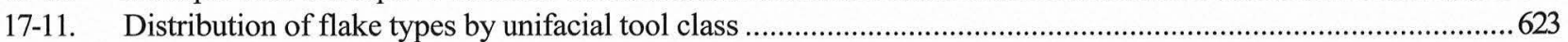

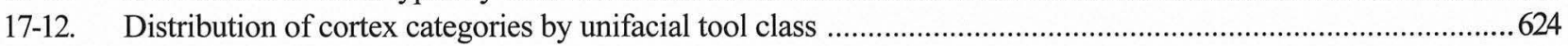

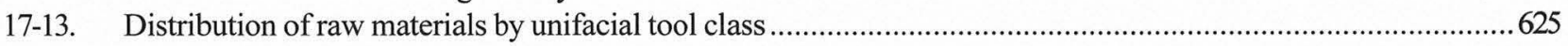

17-14. Distribution of raw material textures by unifacial tool class .............................................................................62

17-15. Distribution of unifacial tool classes in the Valley floor and Valley Margin ......................................................627

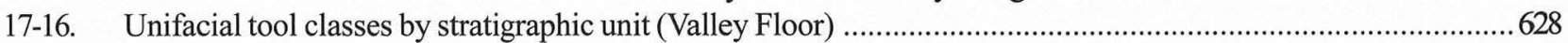

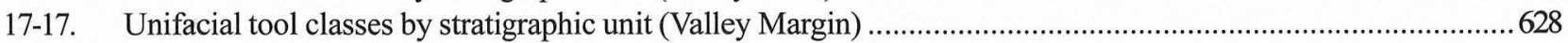

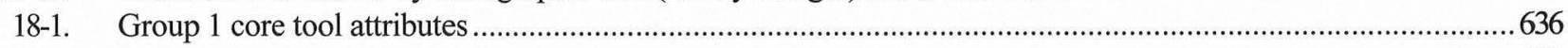

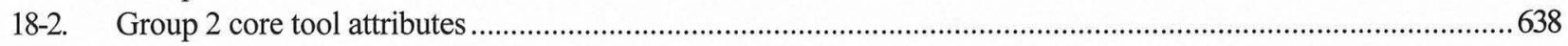




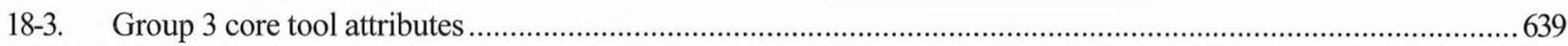

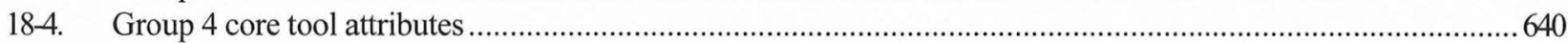

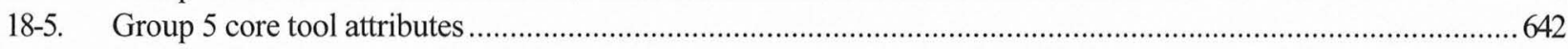

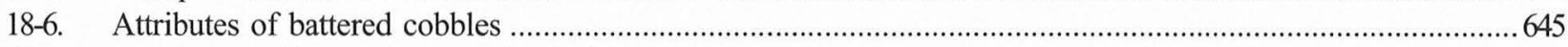

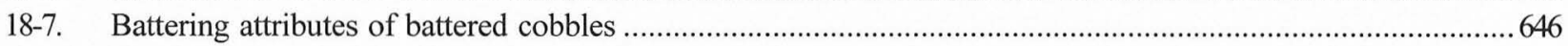

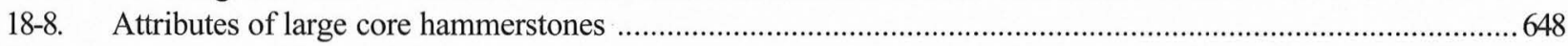

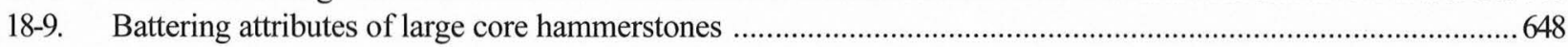

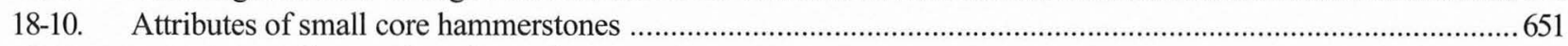

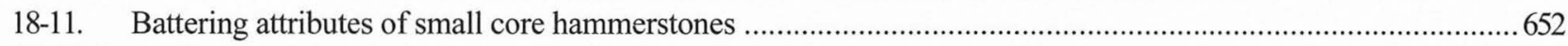

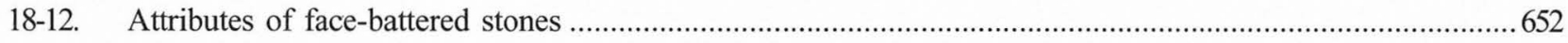

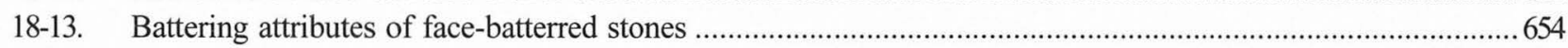

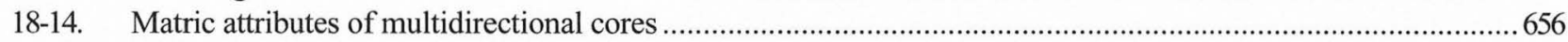

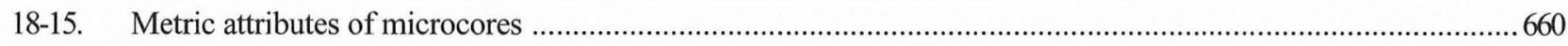

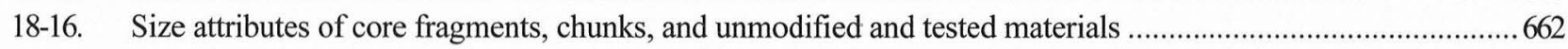

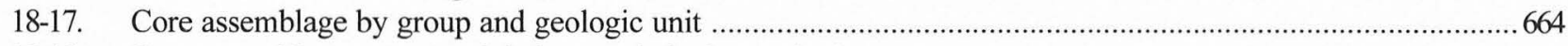

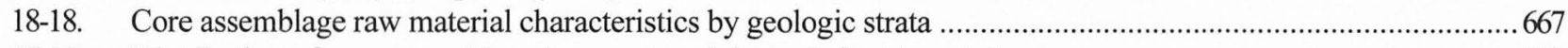

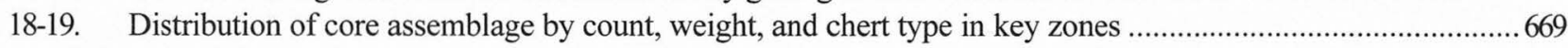




\section{Chapter 12}

\section{INTRODUCTION TO CULTURAL MATERIALS RECOVERED}

by Michael B. Collins and Susan W. Dial

\section{INTRODUCTION}

Prehistoric cultural materials recovered from WilsonLeonard are predominantly durable items of chipped and ground stone along with a few pieces of modified bone and shell. Also recovered were a number of fired clay objects (mostly mud dauber wasp nests) and a small number of historic metal and glass objects of twentieth- and possibly late nineteenth-century vintage. These are reported and tabulated in this and the next volume, Chapters 13 through 23 and Appendices 1 through 5. A single, small potsherd that was found in Late Prehistoric context is described in the interpretive discussion of Chapter 10 (Volume I).

Ethnohistoric and ethnographic accounts (especially photographs and films) graphically depict the vastly greater amounts of organic refuse and perishable material culture than of nonperishable refuse and material culture present at any given time at pre-industrial habitation sites. On rare occasions, ideal archeological conditions afford a similar perspective, as in wet sites such as Ozette (Daugherty 1988; Folsom and Folsom 1993:192) and Kalambo Falls (Clark 1969, 1974) or dry shelter sites such as Hogup (Aikens 1970), Humboldt (Heizer and Krieger 1956), Danger (Jennings 1957), the rockshelter in Bee Cave Canyon (Coffin 1932) and those of the "Ozark Bluff Dwellers" (Dellinger 1936; Early and Sabo 1988:15-17).

Closer to Wilson-Leonard, dry rockshelters in the lower Pecos Region probably give a reasonably analogous indication of what is missing from the more common open sites like Wilson-Leonard where the evanescent part of the record has vanished. The organic leavings such as wornout textiles, grass beds, broken gear of wood, food scrap, coprolites, and ash account for a far greater part of the record than do the durable materials left behind. Hinds Cave (Shafer and Bryant 1977), Baker Cave (Word and Douglas 1970), and the Perry Calk site (Collins 1969:4-24) with their yields of perishable artifacts, abundant organic food remains, and human coprolites illustrate the point well with some deposits containing considerably greater volume of normally perishable refuse than nonperishable.

One can imagine that repeatedly in its long history, Wilson-Leonard was probably the site of temporary shelters framed with wood and covered with brush and grass or with skins. Its occupants probably introduced quantities of grass or boughs as ground cover and produced substantial amounts of refuse from preparing plant foods along with scrap from manufacturing objects of wood, cane, and grass. Animal food scrap and the byproducts of working sinew and bone were undoubtedly present. Probably most perishable artifacts (such as clothing, mats, baskets, nets, animal traps and snares, and wooden tools) were carried away at each departure, but undoubtedly there was always some loss and discard from this inventory. There may at times have been bulkier organic objects, such as wooden mortars and pestles (Collins and Hester 1968; Prewitt 1981c), cached for the next time the site was used.

Even though it is certain that inhabitants introduced considerable organic material into their sites, it is equally certain that their presence disrupted natural vegetation which was probably trampled, burned, and removed. This would result in a mosaic with patches of bare, hard-packed ground alongside areas where organic residue was abundant.

It is necessary, and a bit humbling, to remind ourselves throughout the act of describing and interpreting the durable artifacts from open sites that in terms of volume, numbers, or ethnographic information yield, the absent organic record may exceed the nonperishable record, sometimes by orders of magnitude. Too often the daunt of orderly description and the guile of "artifactology" prevail over the objective of writing ethnographies of past peoples. Obviously, the necessary data do not survive for the writing of complete ethnographies, but when it is set as an ideal, ethnographic description forces the analyst to consider the 
behavioral implications of the surviving materials and to search for clues regarding the more transient materials.

The 11 chapters and 5 appendixes that follow present the obligatory orderly artifact descriptions, offer some "artifactology," report efforts to eke out a bit of information on the perished parts of the material cultures at WilsonLeonard, and discuss aspects of the behaviors that may be indicated by some classes of artifacts. Description prevails, first because the investigators are obliged to describe what was found and second because the greater costs of most approaches to investigating the ephemeral record limit the amount that can be attempted. The fuller ethnographic efforts of this project appear in the interpretive chapters of Volume I.

Of the eight topics guiding the research at WilsonLeonard (see Chapter 5), all but the bioarcheology of Burial 2 are addressed to greater or lesser degree by the study of the recovered materials. The site's chronology is an important aspect of the analysis of the diagnostic prehistoric artifacts (projectile points [Chapters 13 and 14] as well as Clear Fork tools and bifaces [Chapters 15 and 16, respectively]). The chronological dimension also relates to other artifact categories such as cores, debitage, ground stone objects, modified bone and shell, and the historic objects of metal and glass (Chapters 17 through 21 and Appendix 3).

Except for the preserved mud dauber wasp nests (Appendix 2), most of the materials reported in Volumes II and III have little direct bearing on the effort to interpret paleoenvironmental conditions. However, the broad perspective of human ecology guides a consideration of all classes of recovered materials as they relate to the environmental conditions inferred from other sources of information (see Volumes IV and especially V).

Densities of materials recovered by volume of natural strata and in relation to elapsed time for each stratum are useful indicators of how the site formed. Those artifact classes that are numerous, time diagnostic, or exhibit other indicators of site-formational conditions (such as the presence or absence of patination on chert objects) are considered to be particularly informative (see Chapters 13 through 19 and Appendix 1).

Virtually all of the recovered materials described in these two volumes inform on the topics of resource exploitation and of technological organization, and their analyses reflect this emphasis in the project. The synthetic chapters of Volume I rely heavily on the findings concerning sources of lithic raw materials, tool functions, patterns of tool breakage, wear and refurbishment, and indications of tool design criteria. For those archeological components sufficiently well defined, patterns in the horizontal distributions of most classes of recovered materials in relation to other data (such as the distribution of features) hint at the ways people used space in their sites.

The reader is reminded that at the outset of this project, a decision was made to conduct stratigraphic analyses according to geologic provenience to lessen the consequences of using the more inferential archeological components. Also, Wilson-Leonard is almost like two sites in that natural rates of deposition were much greater in the valley floor than on the valley margin, and in some of the artifact classes described below, stratigraphic analyses are reported separately for these two areas of the site.

Throughout the artifact analyses, three philosophical considerations prevail. First, the position is taken that since the collection is to be curated and that no single study can exhaust all of the analytical potential in any class of artifact, descriptions and analyses should emphasize those attributes considered most productive toward the research issues enumerated above. Second, there has been an effort to minimize use of functional terms to label artifact categories. Some terms, such as "projectile points," have become so ubiquitous that their use is continued here, but others, such as "scraper," are eschewed. Third, each analyst was encouraged to judge what was the best use of their analytical resources under the general guideline that it would be preferable to have more-comprehensive analysis of selected items or attributes than superficial consideration of most or all; in this vein, the preference would be to treat those items from more-secure contexts.

Ultimately, in an approach such as this and with a collection this large from diverse contexts of variable qualities, the level of analysis differs between artifact categories ("types") and between the higher-order classes of artifacts (as between chipped stone and ground stone). However, because the guiding philosophy was to maximize the recovery of data relevant to our eight research questions, these divergent results merge in the synthetic chapters of Volume I.

Chipped stone is by far the most abundant and diverse class of artifacts from Wilson-Leonard as reflected by the eight chapters below devoted to reporting their various subclasses and special analyses. Except for the chipped stone descriptive chapters, methods are discussed separately in each chapter below. Those methods common to the several chapters on chipped lithic artifact descriptions are presented here.

\section{GENERAL METHODS APPLIED TO CHIPPED LITHICS}

As a class, lithic artifacts are viewed from the perspective of the complete stone tool life cycle from selection of raw material to discard (cf. Driskell 1986). Sections are ordered somewhat in reverse of the production-use-discard sequence, to first consider end products of the full manufacturing cycle and the more temporally sensitive tool categories. Projectile points are considered first, and are addressed through two different approaches: results of a moretraditional analysis and classification are reported in Chapter 13 , followed by findings from a mathematical study of morphological variance in unfluted lanceolate and early 
bifurcate stem points in Chapter 14. A study of Clear Fork tools is presented in Chapter 15. In Chapter 16, bifaces and bifacial tools, including perforators and burins, are accounted for. An analysis of unifaces and modified flakes is detailed in Chapter 17 , followed by a report on early stage tool production, including analyses of cores, core tools, battered stone and tested and unmodified cherts in Chapter 18.

All lithic tools were fully analyzed with the exception of modified flakes, from which category a smaller sample was selected for analysis. Although analysis necessarily differed somewhat among tool categories depending on specific research issues, an overall objective was to collect a uniform data set for raw materials and material characteristics on all lithic tools examined. The methods used to record these and other general characteristics are described here. More specific attributes observed only for certain tool categories are discussed in detail in the pertinent chapters below.

Raw material observations were intended to elucidate general lithic sources as well as alterations to the material resulting from cultural or environmental processes (e.g., presence of patina, mineral precipitates, heat alteration). Material identification was keyed to categories generated through analysis of debitage (Chapter 19). In contrast to the more detailed, color framework used in the debitage analysis, however, raw material data for lithic tools was compressed and synthesized into a broader and more-abbreviated framework that recognizes general source (local and nonlocal Edwards chert and non-Edwards materials) and general texture (finegrained or medium/coarse-grained materials).

Presence/absence of mineral precipitates was noted and, in some tool categories, was further detailed according to density of accumulation and distribution. Precise identification of the deposits by chemical analysis (e.g., calcium carbonates or calcium phosphates) was not made on any specimens other than a selection of those submitted for residue analysis (Chapter 23).

Patina was observed according to relative coverage (e.g., slight overall, moderate partial); patina that was partially removed by flaking - a likely indication of reuse of a tool subsequent to patination-is termed "differential." In some analyses, only presence/absence of patina is reported.

Thermal alteration was recognized by the presence of potlid and/or crenated fractures, crazing, and, for certain raw materials, a pink, red, or dark gray coloration. Only in cases where differential glossiness of flake scars was noted was a specimen termed heat treated, with the potential implication that it may have been deliberately heated to enhance flaking properties.

An effort was made to evaluate all chert tools for fluorescent properties which might indicate chert source on a regional scale (i.e., Edwards or non-Edwards cherts). Tools were examined under long- and short-wave ultraviolet light using a high-intensity Mineralight ${ }^{\circledR}$ Lamp (see Hofman et al. 1991).

Linear measurements for lithic tools were taken with sliding calipers with a dial allowing for precision to $.01 \mathrm{~mm}$. Measurements representing the maximum for each dimension were rounded to the nearest millimeter for all lithic tool categories with the exception of projectile points. In that category, stem attributes of certain unfluted lanceolate and bifurcate stem projectile points were measured to a higher degree of precision for use in statistical analyses (Chapter 14).

Specimens collected for organic residue (OR) sampling posed a particular problem (Marchbanks and Collins 1993), as their analysis required the wearing of gloves and attempting to make observations on specimens that had only been lightly rinsed (Chapter 23). Each specimen was unwrapped but kept atop its foil packaging during analysis to prevent contamination. Artifacts were minimally handled during examination and measuring. Because of these restrictions, material characteristics and attributes such as grinding and mineral precipitates could not always be discerned and, in those cases, were coded as indeterminate. Attempts at evaluating fluorescent properties of OR specimens under ultraviolet light were abandoned after it was determined that adhering dirt on the specimens and reflection from the foil wrappings on which they were laid tended to skew the observations.

A voluminous body of information was collected during analysis of the Wilson-Leonard stone tool assemblage, and this information is available on computerized databases at TARL. Only a portion of those data are presented herein. For the very large data sets, in which quantities approached 1,000 specimens or more (e.g., bifaces, projectile points, unifaces, and flake tools), information is reported in summary tables rather than by individual specimens. The reader is referred to Appendix 5, Volume III, for a full listing of individual specimens by tool category and provenience. 
"This page intentionally left blank" 


\section{Chapter 13}

\section{PROJECTILE POINTS}

by Susan W. Dial, Anne C. Kerr, and Michael B. Collins

More than 1,350 projectile points and fragments were recovered from the Wilson-Leonard site, a sample representing technologies and traditions from earliest Paleoindian to Late Prehistoric times. The sheer size of the collection and stratified contexts from which it derives afford an opportunity to examine projectile point technology, style, and use over nearly 11 millennia in the same setting. With location held constant, changes in the projectile point assemblages over time can be examined alongside other factors, most particularly alterations in other aspects of culture, climate, and floral and faunal populations, issues addressed in the conclusions.

In this chapter, data collected on the full projectile point assemblage are presented. Variation is assessed first within traditional types and morphological groupings and then according to stratigraphic distribution within the site. Because of the large number of projectile points recovered, emphasis is placed on more fully describing and illustrating those groups for which critical typological questions might be addressed, specifically, Late Paleoindian unfluted lanceolate points and the bifurcate stemmed points from Early Archaic contexts.

\section{ANALYTICALAPPROACHES}

\section{Typological Analysis}

Toward the goal of organizing the sample as well as identifying archeological or chronological markers, points were sorted into broad categories based on morphology, metric attributes, and certain technological characteristics. Emphasis was placed on the basal and stem portions inasmuch as these sections were deemed less likely to be affected by resharpening and rejuvenation and thus more likely to preserve original attributes. In all cases, descriptions given refer to the Wilson-Leonard assemblage, although reference to other collections may be included in the comments sections. Morphological groups were further refined as possible into recognized types chiefly following Turner and Hester (1993) and Suhm and Jelks (1962) and based on comparisons with selected literature and type collections.

In numerous cases, type identifications could not be confidently made. Rather than amassing these points into a large and rather meaningless "miscellaneous" category, a number of variant and descriptive terms were devised. When points appear to conform at least in some aspects to type definitions as currently understood, such appellations as Gower-like, Bulverde-like, etc., are used. In cases of certain Early Archaic bifurcate points, group names such as GowerUvalde, and Gower-Jetta were designed to illuminate problems residing in these various types and to more explicitly convey the gradational nuances of typology in the Early Archaic. The larger heading, "Early Bifurcate Stem," is used herein to refer to that problematical series, as is further detailed in Chapter 14. For certain other points, a series of alphabetically lettered, descriptive group names is used. Points that could not be assigned to any of the groups remain in broad miscellaneous categories. The term "atypic" is used to denote specimens that are considered aberrant but appear to fall at the edge of the range of variance as presently understood for a particular type. Atypical points should not be used as type reference specimens. Other conventions include use of a "?" at the end of a type name to denote unresolvable doubt about application of the type name.

Although projectile point fragments are reported separately, specific small fragments that appear to be typologically significant are included instead in the larger projectile point category. Those fragments were assigned to pertinent typological or morphological groups as possible.

In Chapter 14, typological issues in unfluted lanceolate and Early Archaic bifurcate points are further discussed and results reported for systematic quantitative studies 
that focused specifically on those problems. Type/descriptors used herein for certain unfluted lanceolate points evolved chiefly from those analyses. However, categories used in this chapter for the bifurcate points are not linked to the statistical analysis; rather, they derive solely from traditional typological and morphological sortings completed prior to the statistical analysis. Considered together, results from the two studies reported in Chapters 13 and 14 serve to illuminate the complex problems residing in unfluted lanceolate and early bifurcate stem projectile point typological constructs.

\section{Technological and Functional Analyses}

Other analyses of projectile points focused on attributes related to manufacture, use, and discard. These include observations on size, haft modifications, flake patterns, fracture types, evidence of resharpening, and recycling. They are encompassed under the general headings of Metric Attributes, Nonmetric Attributes, Tool State and Fractures, and Rejuvenation. Specific terms used herein are defined below.

\section{Metric Attributes}

A variety of size attributes were recorded for projectile points, and locations of measurements are illustrated in Figure 13-1. In most cases, maximum length, width, thickness, and stem measurements were rounded to the nearest millimeter. Exceptions are measurements taken for all lanceolate points and for certain stemmed and bifurcate stemmed points from which a sample was chosen for statistical analyses (see Chapter 14). For lanceolate points, all measurements were taken to the nearest 1 of a millimeter; for those bifurcate points considered for statistical analysis, basal and stem attributes also were measured to the nearest 1 of a millimeter.

The attributes observed and terms used in measurements vary somewhat for certain early stemmed bifurcates and Paleoindian lanceolate and stemmed points. For lanceolate points, the term "haft grinding length" is used to describe the maximum extent of lateral edge grinding within the haft (i.e., nonblade) area. This usage of the term "haft" follows Justice (1987), Bradley (1993), and others who eschew use of the term "stem" for lanceolate points. Note that the same authors use "stem" for points of the Cody complex, such as Scottsbluff. Additional attributes observed as a basis for type definitions on this subset of points are included herein, as well as in Chapter 14. These include measurements of thickness and width taken $10 \mathrm{~mm}$ up from the base of the point (referred to respectively as $10-\mathrm{mm}$ haft thickness and 10-mm haft width). To obtain the 10-mm haft width, specimens were placed flat on a gridded block and measured with calipers at the fixed point; for the $10-\mathrm{mm}$ thickness measurement, the specimen was then turned on side, with fingertips marking the 10 -mm point. For the bifurcate points used in this study, the width and thickness of the distal stem as well as the width of the basal concavity were measured.

Metric data are summarized in tabular form for each type or group, with the exception of miscellaneous categories which are not intended as a group and for which metric averages have no meaning. For these, only minimum and maximum measurements for the group are presented. Because incomplete measurements are not included in summary statistics, sample totals within each group may vary from one attribute to the next; total number of specimens by variable are denoted under the "number" column in each table.

\section{Nonmetric Attributes}

Nonmetric attributes, described below, encompass a host of qualitative characteristics and by their nature are largely subjective. The intent in all cases, however, was to describe and encode a feature that might add stylistic or technological information.

Basal modification describes the type of flaking that serves to modify or thin the base. This includes unifacial or bifacial thinning and unifacial or bifacial fluting as well as less invasive basal treatments characterized as beveling, concave beveling, or simple secondary trimming. Although somewhat subjective, fluting is characterized herein as one or more long flakes struck from the base, creating a pronounced channel or hollow; by this measure, the length of the flute should be at least two times the width of the base. Basal thinning is distinguised from basal trimming in that the former constitutes flaking that creates a "dish-shaped" basal cross-section.

As illustrated in Figure 13-2a-i, nine flake patterns on blade areas were observed following Crabtree (1982:51). These patterns are intended to characterize flaking of the original piece rather than flaking due to subsequent resharpening. Those specimens on which flake patterns could not be discerned (including those extensively resharpened) were coded as indeterminate. Although the various patterns used are easily identified from the drawing, several need further explanation. Figure 13-2a illustrates a specimen on which a section of the parent flake blank remains. Where parallel and parallel oblique flaking was not highly regularized, the patterns were coded as "subparallel" or "oblique subparallel" (Figure 13-2d, e). Collateral flaking is distinguished by generally expanding lateral flake scars meeting to form an irregular longitudinal ridge in the center of the blade (Figure 13-2f). A flute or channel flake scar (Figure 13-2i) was recorded in this category when it obscured part or most of the lateral flaking. In numerous cases, specimens have different flake patterns on opposite faces; and these were coded accordingly.

For this report, flake patterns are summarized into three categories: organized (see Figure 13-2b-f, h, i), random (see 


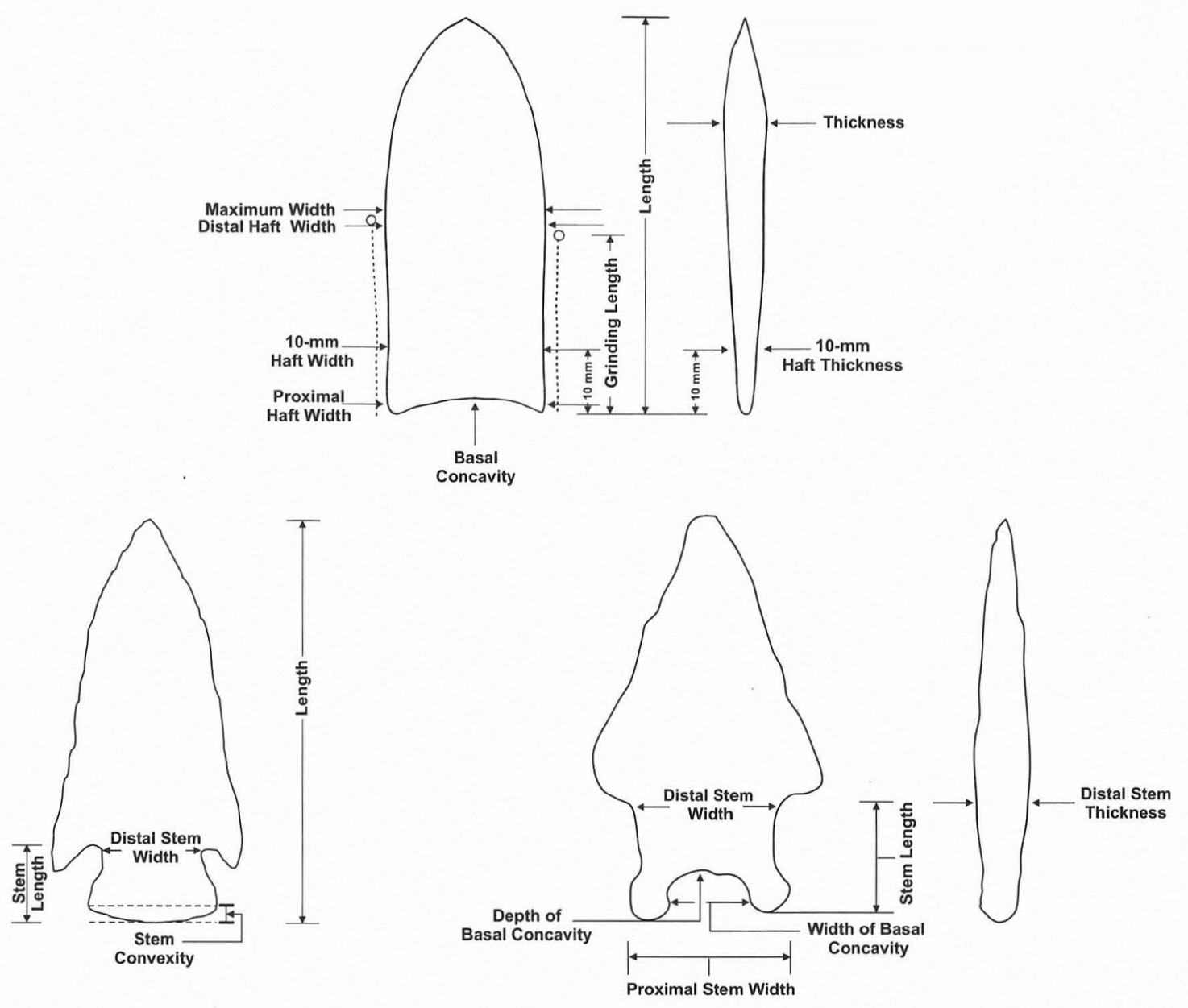

FIGURE 13-1. Measurement locations of metric attributes on various forms of projectile points.

Figure 13-2g), or indeterminate flaking. For purposes of this summary, a specimen is characterized as having organized flaking if it was observed on at least one face even if the other face was randomly flaked. Specific patterns consistently occurring within a type or group are noted.

Longitudinal profile was recorded on specimens deemed sufficiently complete to be representative. Typically, this required a specimen to be at least one-half to twothirds complete, although descriptive terms used in coding may suggest a complete point was observed (e.g, tapered base and tip; Figure 13-3a). Profile types range from the generally flat longitudinal section seen in Midland points (Figure 13-3d), to the somewhat thick-tipped profile associated with some Plainview points (Figure 13-3b), to the tapered base and tip that usually distinguish Bulverde (Figure 13-3a). The other profile types chiefly allow for gradations between these more distinctive categories.

Lateral stem or basal grinding was recorded within a range from absent to heavy. In most cases, "slight" grinding is considered equivalent to slight smoothing, with edges possibly exhibiting some minor protrusions. "Moderate" describes dulled to rounded edges, which may retain some coarseness. "Heavy" describes edges that have been ground to a rounded state, eliminating all coarseness and protrusions.

The tool state category serves to characterize the degree of completeness and/or specific section of a fragmentary point. Terms used include "base or stem," which refers to a basal or stem portion with none of the blade present and "proximal section," which describes a base or stem with part of the blade present above the grinding area or stem. Many points are described as complete or nearly so; in the latter case, small convergent portions (distal, barb, basal ear) may be lacking.

Six fracture types chiefly related to tool use or rejuvenation were identified and were recorded in correlation with break position and orientation (fracture types related to manufacturing are chiefly discussed in Chaper 16, although certain dart point and arrow point preforms thought to be related to specific types or groups are included in this section). Fracture terminology follows Crabtree (1982), Johnson (1979, 1981), and Whittaker (1994), and brief definitions of some of these terms, as used in the present analysis, are provided below. 


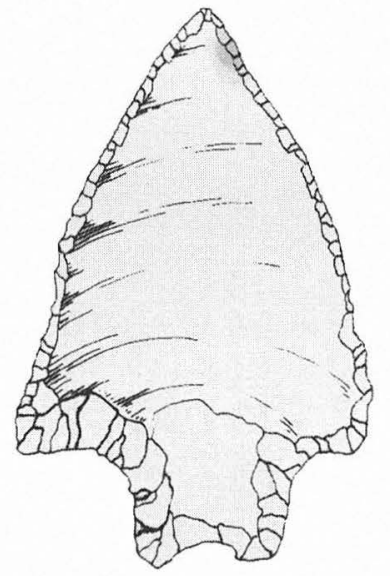

a

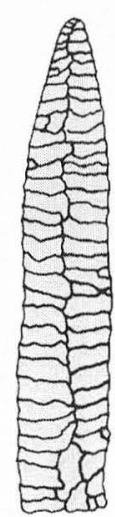

b

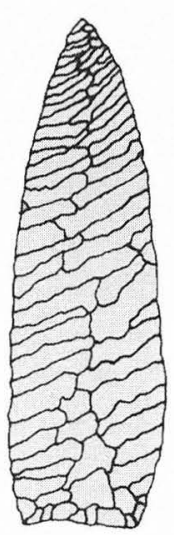

C

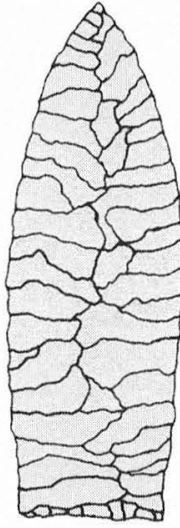

d

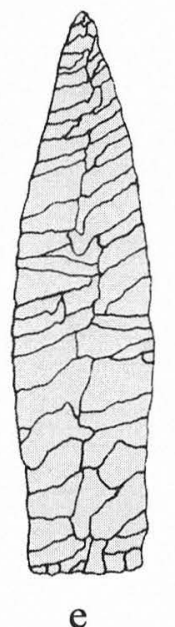

e

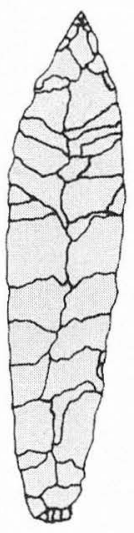

f

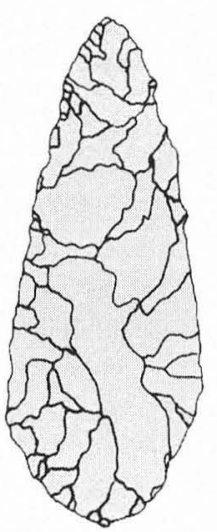

g

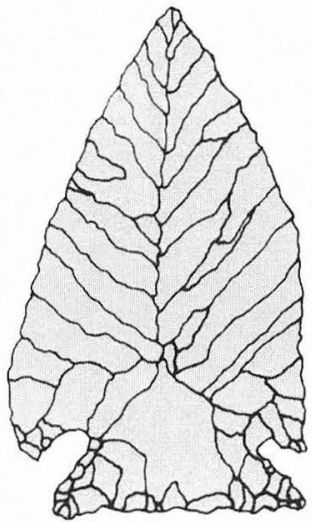

$\mathrm{h}$

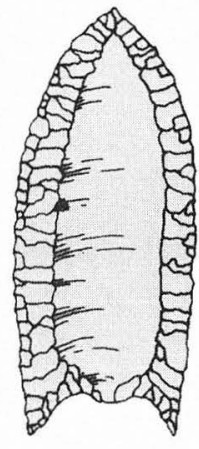

i

FIGURE 13-2. Flaking patterns on projectile points. (a) flake blank remnant; (b) parallel; (c) oblique parallel; (d) subparallel; (e) oblique subparallel; (f) collateral; (g) random or nonpatterned; (h) chevron; (i) flute scar.

Impact fractures commonly are distinguished by channel-like flake scars emanating from the distal tip and extending onto one or both faces of the point. Such breaks frequently are accompanied by a constellation of related fractures, including burin-like flake removals of lateral edges and oblique burin-like basal fractures that typically removed a basal corner or "ear." These complex fractures may have resulted from movement or reverberation of the point after impact or from attempts to remove the weapon from the animal or object in which it was embedded. Point fragments displaying what appear to be intentional burin blows (i.e., those struck with the apparent aim of recycling a broken point to another use) are examined within the burin tool section. 


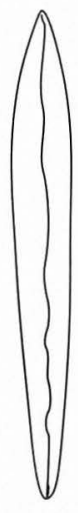

a

d

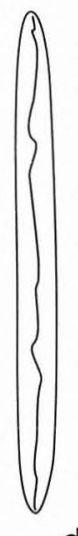

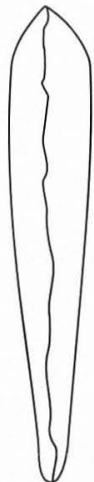

b

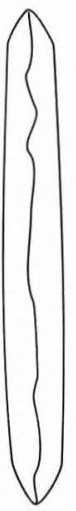

e
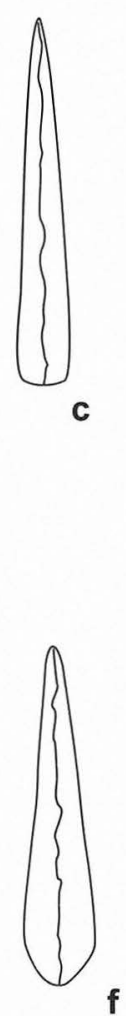

f
FIGURE 13-3. Longitudinal profiles of projectile points. (a) tapered base and tip (maximum thickness in medial section); (b) tapered base, increasing to maximum thickness at distal section; (c) thick base, flat or equal proportions; (d) generally flat overall; (e) wedgeshaped base and tip; (f) thick wedge-shaped base, generally thick body.

Bending or snap fractures are typically distinguished by a clean or lipped break resulting from force perpendicular to or at a right angle to the face of the object. These breaks commonly occur during both manufacturing and use.

Thermal or crenated fractures are recognized chiefly by sinuous or curved break facets often in combination with potlids, crazing, and/or color change. Johnson (1981:49) has observed that crenated fractures, when seen in plan view, often appear cut by a jigsaw. These fractures may occur during heat treatment of the chert or in a burning episode after discard.

An overshot may occur within the manufacturing or rejuvenation process and is characterized by a plunging flake which travels across the face, removing the opposite lateral edge of an object. Perverse fractures are recognized as twisting breaks emanating from the edge of the biface and plunging across and in a downward trajectory. Often these breaks, typically manufacturing errors, are marked by a flake scar leading into the break point. Edge damage is used somewhat loosely herein to describe extensive crushing or attrition of lateral margins that may have occurred during manufacture, use, or after discard.

Under the heading of rejuvenation, types and degree of tool resharpening, break compensation, and/or recycling to a different tool type are described. Resharpening was coded according to type and occurrence on one or both blade faces and includes unbeveled unifacial or bifacial resharpening, unifacial beveling, alternate beveling, and serration. Recycling to a different tool category is conjectured where modification to a specific form (e.g., scraper or burin) could be observed without microscopic examination of edges. Results of specialized microwear evaluation are briefly discussed as pertinent within the various point type categories, with complete results presented in Chapter 22.

\section{Material Characteristics}

Summaries of raw material type, presence/absence of mineral precipitates, and degree (if any) of patination and thermal alteration are presented by type or group. Specific attributes, terminology, and methods used for material observations are described in Chapter 12.

\section{Distributional Analysis}

Distribution of projectile points within the site was analyzed predominately according to occurrence within geological units as well as within the established horizontal sectors of the site (Valley Margin and Valley Floors A and B). The type and amount of data presented may vary somewhat due to different field recovery strategies employed for artifacts in certain contexts. Most artifacts in lower levels were point plotted, thus enabling a more precise depiction of their provenience and elevation. For all other groups, distributional summaries are organized by geological unit. Full provenience data and archeological chronology are reported in Appendix 5 to Volume III.

\section{OVERVIEW OF FINDINGS}

The Wilson-Leonard projectile point assemblage constitutes representatives of every central Texas archeological "style interval" (Collins 1995) including one of the largest Late Paleoindian and Early Archaic collections from any stratified Texas site. The assemblage enables a clearer understanding of the archeological sequence as well as a more complete picture of the degree of morphological variance contained within certain types and groups.

Three new point types are proposed: the large expanding-stemmed "Wilson," the unfluted lanceolate "St. Mary's Hall," and the thick lanceolate "Thrall" point. Identification of the Wilson point in contexts dated to ca. 950010,000 B.P.—a time previously associated with unfluted 
lanceolate points - significantly revises traditionally perceived trends in projectile point styles in this region. The St. Mary's Hall type, commonly identified at other sites as a variant of Plainview, is shown at Wilson-Leonard to be a distinctive type later than classic Plainview, occurring primarily in Late Paleoindian times. The Angostura point appears in many respects to herald the onset of the Early Archaic. The poorly understood Hoxie point also is seen to precede other bifurcate stem and expanding stem groups of the Early Archaic. Chronological placement of the Thrall point is more uncertain, but it too appears to derive chiefly from Early Archaic deposits.

As is discussed here and in the following chapter, the various Early Archaic bifurcate stem points cannot be well sorted and may be more appropriately considered as variants of a larger tradition displaying a continuum of morphological traits. On a very gross level, however, it is demonstrated that Early Archaic bifurcate-stem morphology appears to trend over time from roughly straight sided to expanding. In addition, certain technological attributes such as stem grinding and alternate beveling of the blade are shown in continuity with earlier or perhaps contemporaneous Late Paleoindian styles and may be sensitive temporal indicators within the larger bifurcate point group. For these reasons, use of such descriptive terms as "Early Split Stem" (Johnson 1991:111; Prikryl 1990:51) and "Early Corner Notched" (Hester 1971:71-73) is strongly advocated to help distinguish these enigmatic groups, although even these broad descriptors are problematical and difficult to assign. As is discussed further below, these terms need to be redefined to eliminate the ambiguity that has crept into their usage. Ideally, Early Split Stem should connote the earlier, typically ground and straighter-stemmed bifurcates and Early Corner Notched the later, more expanding, shallowly concave base forms.

Technological patterns traced over the millennia indicate a gradual change from finely crafted lanceolate points to less carefully prepared bifurcate stem points, coinciding to a large extent with the so-called Late Paleoindian/Archaic transition. Evident along with this shift is a heightened degree of efficiency, manifested in intensive resharpening and reuse of certain unfluted lanceolate and early bifurcate stem variants. A change in hafting technology also appears to be signalled at this juncture. These technological innovations, in tandem with certain stylistic changes, may indicate influences from other regions-particularly the southeastern United States-during this time period or alternatively, a shift in hunting organization and subsistence. Over the next several millennia in the site's history, patterns of less-intensive maintenance of points and different discard patterns are manifested, with virtually intact points being discarded in relatively large numbers at or around the time of the Middle to Late Archaic transition.

Use-wear data derived with high-power microscopy (see Chapter 22) suggest a complex history for certain points, involving cycles of use and reuse both as projectiles and as knives. These data not only call into question once again the use of the term "projectile point" but raise serious issues about traditional notions of point type and morphology. While the typological vagaries resulting from maintenance and resharpening have been noted (e.g., Flenniken and Raymond 1986), use-wear findings and observations recorded for rejuvenation underscore the possibility that certain point types-Hoxie and Wilson, for instance-may have been defined mainly on the basis of resharpened, exhausted points that bear little resemblance, except perhaps in their haft sections, to the specimens in their original form.

Archeologists working with projectile points in Texas have maintained an almost purely typological approach, an historical tradition strongly reflected in this study. However, the richness of this database warrants far greater effort toward deriving fuller information on human behavior from these interesting artifacts. As a tentative step toward that end, this chapter concludes with an attempt to interpret projectile points according to the weapon systems of which they were a part and to explore how projectile point design relates to the effective killing of game.

It is suggested that the evidence from the WilsonLeonard site, Texas and beyond in North America indicates use of the atlatl and dart, the hand-thrown spear, the thrusting lance, and the bow and arrow at different times and under varying conditions. It is further proposed that to accomplish the task of killing game, it is necessary to deliver a point of the correct form to a vital area of the prey. It is concluded that various ploys were needed to gain strategic advantage over such quarrry as deer, antelope, and bison to make these weapon systems effective.

\section{FINDINGS}

Of the 1,353 projectile points and fragments recovered, 910 were categorized as dart points, 93 as arrow points, and 350 as indeterminate fragments. A total of 90 types and groups was identified based on morphological similarities and established typologies. To facilitate presentation of data, these groups are ordered within 8 larger morphological categories: unfluted lanceolate, fluted lanceolate, bifurcate stemmed, straight stemmed, expanding stemmed, side notched, unstemmed triangular, and arrow points. These categories are neither clear-cut nor mutually exclusive due to morphological variations within groups. For ease of locating certain types within the chapter, a full listing of types and groups according to larger category is presented in Table 13-1.

Data collected on projectile point fragments are presented within relevant morphological categories, as possible. Information on other fragments that could not be placed within descriptive or typological categories is presented primarily in tabular form. 
TABLE 13-1

Organization of Projectile Point Groups

UNFLUTED LANCEOLATE
Angostura
Thrall
Midland
St. Mary's Hall
St. Mary's Hall atypic
Bone bed point
Parallel-sided haft
Ground square haft
Scottsbluff
Golondrina-Barber
Miscellaneous lanceolate
Concave lanceolate, preform

\section{FLUTED LANCEOLATE}

Clovis base fragment

Clovis distal section

\section{BIFURCATE STEMMED}

Baker

Bandy

Contracting stem, convex base

Gower

Gower/Jetta

Gower/Uvalde

Hoxie

Hoxie A

Hoxie B

Hoxie C

Hoxie-Gower

Jetta

Martindale A

Martindale B

Expanding stem concave base A

Expanding stem concave base B

Expanding stem concave base $\mathrm{C}$

Expanding stem concave base D

Expanding stem concave base $\mathrm{E}$

Uvalde

Uvalde-like

Early bifurcate stem fragments

Early bifurcate stem preform

Frio

Fairland

Montell

Pedernales

Miscellaneous bifurcate

EXPANDING STEMMED

Wilson

Wilson medial section

Wilson? proximal fragments

Wilson?-atypic

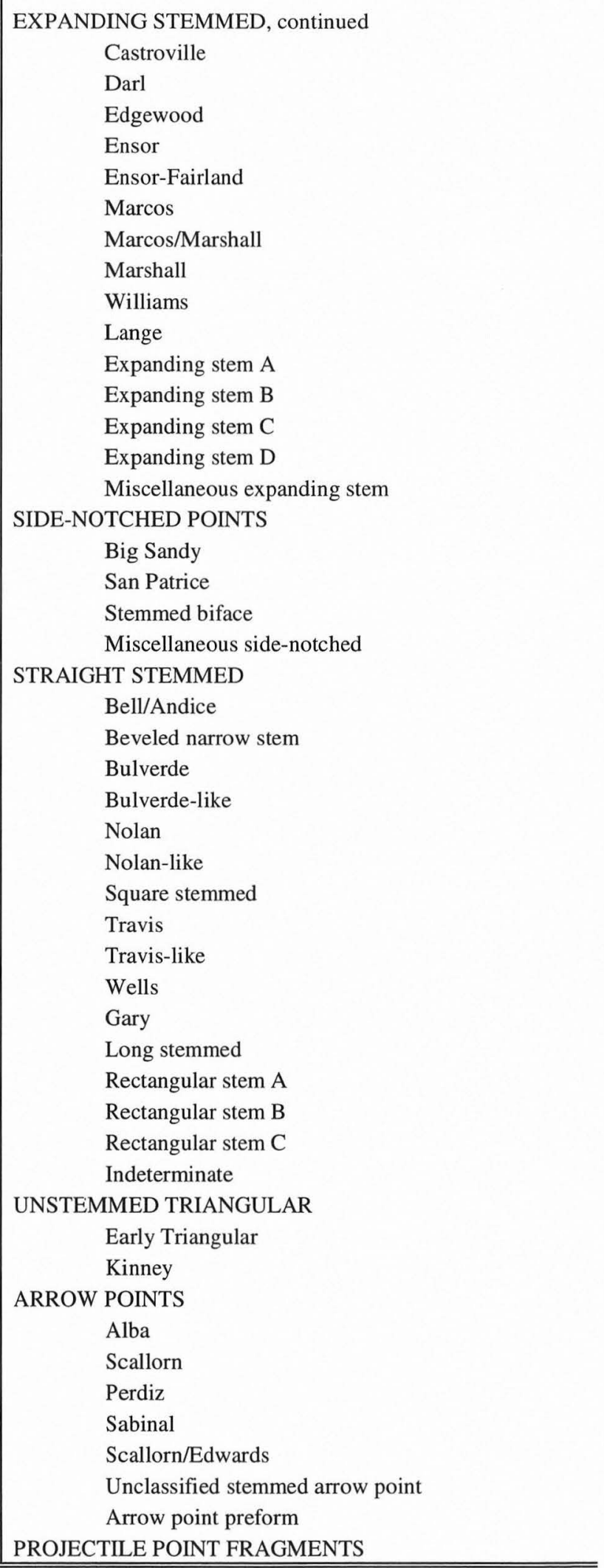




\section{LANCEOLATE POINTS}

The lanceolate points are divided into unfluted lanceolate and fluted lanceolate groups. Among the unfluted lanceolate points, Angostura, Thrall, Midland, St. Mary's Hall, the Bone Bed point, Parallel-Sided Stem, Ground Square Stem, Scottsbluff, St. Mary's Hall-atypic, and Golondrina-Barber were categorized in the systematic analysis of basal morphology discussed in detail in Chapter 14 and follow the same order here as in that chapter.

\section{Unfluted Lanceolate}

\section{Angostura $(N=31)$}

Morphology: These finely flaked points have narrow, moderately thick, extreme to slight contracting hafts usually with a slight basal concavity (Figure 13-4a-j); basal shape can, less frequently, vary from slight basal convexity to straight; bases are modified predominately by concave bevel, with secondary trimming and unifacial thinning occurring less frequently. Lateral haft edges are usually heavily ground or, less frequently, moderately ground; basal edges can have no grinding or slight to heavy grinding.

Metric Averages: Table 13-2

Nonmetric Attributes: Longitudinal profiles include 3 with generally equal proportions, 2 with tapered bases increasing to thick distal sections, 2 with short wedgeshaped bases with tapered tips, 23 indeterminate, and 1 not coded; flake patterns are organized on 19 (subparallel, oblique subparallel, oblique parallel, collateral, chevron), and 12 are indeterminate; basal modification includes 19 concave bevel, 5 secondary trimming, 4 unifacial thinning, 2 none, and 1 indeterminate; lateral grinding includes 24 heavy, 6 moderate, and 1 slight; and basal grinding includes 10 absent, 9 slight, 7 moderate/heavy, 4 indeterminate, and 1 not coded.

Material Attributes: Raw material includes local Edwards chert (13 fine grained, 5 coarse grained), nonlocal
Edwards chert ( 7 fine grained, 3 coarse grained), 1 indeterminate heat altered, and 2 indeterminate; patina is absent on 28 , slight overall on 1 , moderate overall on 1 , and heavy overall on 1 ; mineral precipitates are present on only 4 points; heat damage is slight on 5 points, extensive on 2 , absent on 23, and not coded on 1 .

Tool State and Fractures: One point is complete and 3 are nearly complete in length; of these, a single facial impact fracture occurs on 1, a single facial bend fracture on 1 , and a crushed edge on 1; slight serration of blade edges is evident on 2 . There are 2 proximal sections with a single impact break on 1 and bend and hinge breaks on 1; there are 25 base sections with a single impact break on 1 , a single bend fracture on 6 , a single thermal spall on 1 , multiple impact/bend breaks on 4 , multiple bend/burin-like breaks on 3, multiple bend breaks on 1, bend/thermal spalls on 2, a bend break and crushed edge on 1, impact/bend/ burin-like breaks on 2 , bend/burin-like/edge crushing breaks on 1, multiple bend/burin-like/thermal breaks on 1, multiple burin-like/impact breaks on 1, and multiple thermal breaks on 1 .

Rejuvenation: One point is not resharpened; resharpened points include 3 alternately beveled, 1 each unbeveled unifacial and unbeveled bifacial, and 25 indeterminate. Specimens 11Q-1, 16S2-1, 25R1-1, and 29T1C-1 were used as both a projectile point and a knife as determined by usewear analysis (see Chapter 22).

Stratigraphic Distribution: As shown in Table 13-3, Angostura points occurred in highest frequency $(n=12)$ in stratigraphic Unit IIIa, deposits attributed to the Early Archaic. Eight others were found in mixed deposits of Late Paleoindian and Early Archaic origins (the Unit II/IIIa transition zone in the Valley Floor and Unit X/II/IIIa in the Valley Margin). Three found in Unit II are the lowest occurring at the site. Although some Angostura points were recovered from basin-shaped hearth features, the large majority were found in Unit IIIa contexts well removed from the disturbed areas.

Comments: A recent review of the Angostura type

TABLE 13-2

Summary Metric Data for Angostura Points (in mm)

\begin{tabular}{|c|c|c|c|c|c|}
\hline Variable & Number & Minimum & Maximum & Mean & $1-\sigma$ \\
\hline Length & 3.0 & 64.0 & 79.1 & 73.0 & 8.0 \\
\hline Width & 26.0 & 15.0 & 28.0 & 21.0 & 3.0 \\
\hline Thickness & 31.0 & 5.0 & 9.0 & 6.6 & 0.9 \\
\hline Haft/grinding length 1 & 28.0 & 10.2 & 46.9 & 24.5 & 9.4 \\
\hline Haft/grinding length 2 & 26.0 & 8.7 & 34.9 & 19.4 & 6.7 \\
\hline Proximal haft width & 29.0 & 6.1 & 17.4 & 11.4 & 2.8 \\
\hline 10-mm haft width & 28.0 & 13.5 & 19.0 & 16.2 & 1.4 \\
\hline Distal haft width & 15.0 & 16.5 & 24.8 & 20.3 & 2.3 \\
\hline 10-mm haft thickness & 30.0 & 4.5 & 7.7 & 5.8 & 0.7 \\
\hline Basal concavity & 28.0 & 0.0 & 2.6 & 1.2 & 0.7 \\
\hline Basal convexity & 2.0 & 0.8 & 1.0 & & \\
\hline
\end{tabular}




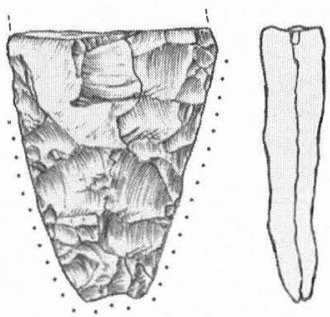

a
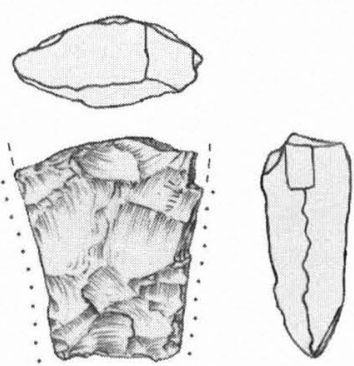

d

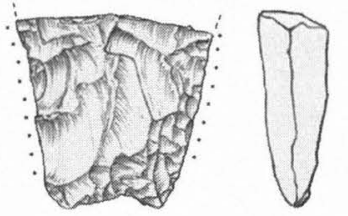

g

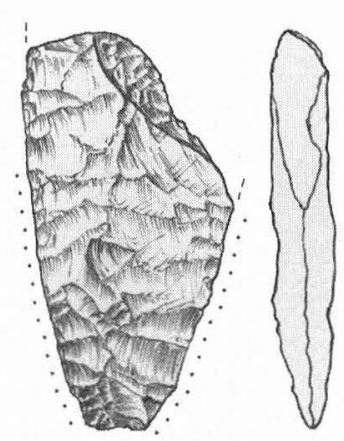

$\mathrm{b}$
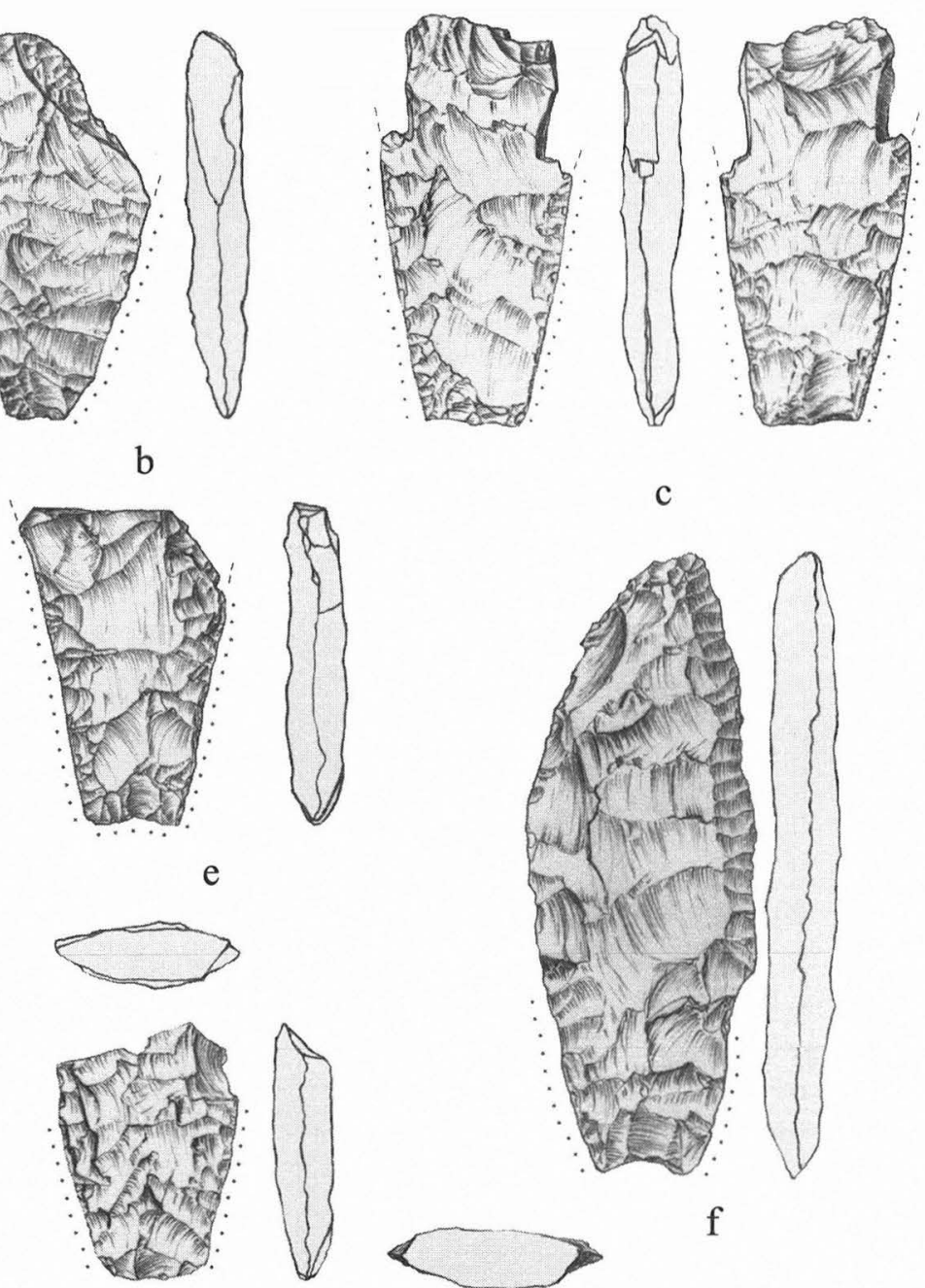

C

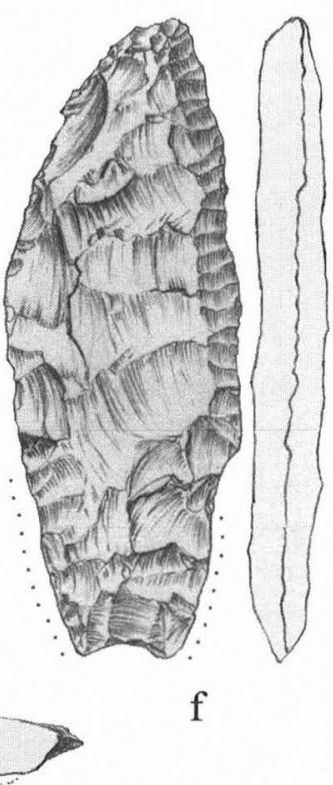

h

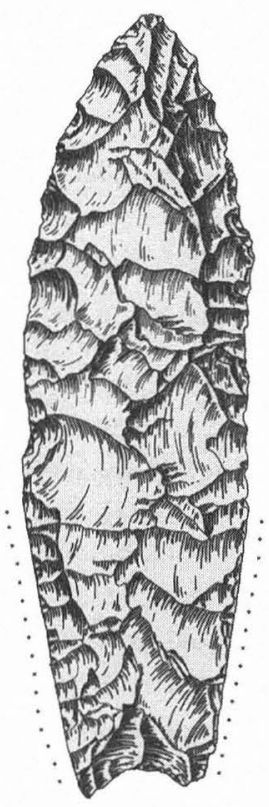

i
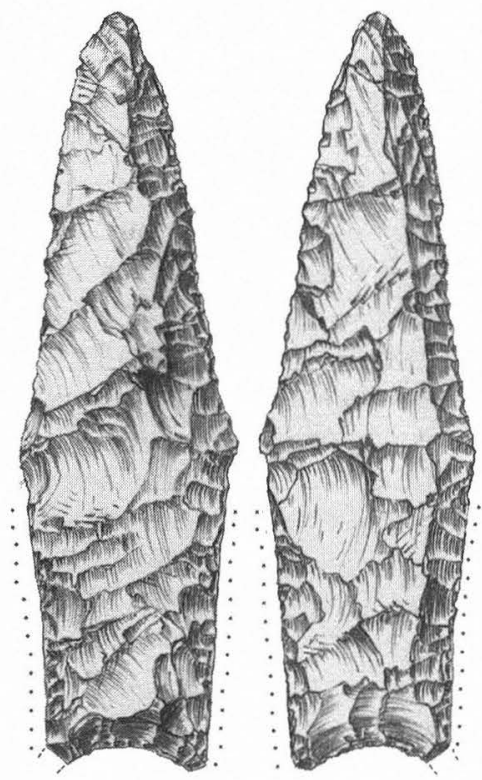

$\mathrm{j}$

FIGURE 13-4. Angostura points. Specimen numbers: (a) 7O-1; (b) 3M-1; (c) 16S2-1; (d) 23P-1; (e) 11O-1; (f) 29T1C-1; (g) 895-1; (h) 25R13; (i) 25R1-1; (j) 11Q-1. 
TABLE 13-3

Stratigraphic Distribution of Angostura Points

\begin{tabular}{|c|c|c|c|c|c|c|c|c|c|c|c|c|c|}
\hline \multirow{2}{*}{ Elevation } & & \multicolumn{7}{|c|}{ Valley Margin } & \multicolumn{4}{|c|}{ Valley Floor A } & \multirow[b]{2}{*}{ Surface } \\
\hline & & II & $\mathrm{X}$ & $\mathrm{X} / \mathrm{II} / \mathrm{IIII}$ & IIIa & $\mathrm{III} / \mathrm{b}$ & IIIb & IIIc & II & II/IIIa & IIIa & III $a / b$ & \\
\hline Surface & & & & & & & & & & & & & 1 \\
\hline 97.61 & - 97.70 & & 1 & & & & & & & & & & \\
\hline 97.51 & - 97.60 & & & & & & & & & & & & \\
\hline 97.41 & - 97.50 & & & & & & & & & & & & \\
\hline 97.31 & - 97.40 & & & & & & & & & & & & \\
\hline 97.21 & - 97.30 & & & & & & & 1 & & & & & \\
\hline 97.11 & - 97.20 & & & & & & & & & & & & \\
\hline 97.01 & - 97.10 & & & & & & & & & & & & \\
\hline 96.91 & - 97.00 & & & & & & & & & & & & \\
\hline 96.81 & - 96.90 & & 1 & & & & & & & & & & \\
\hline 96.71 & - 96.80 & & & & & & & & & & & & \\
\hline 96.61 & - 96.70 & & & & & & & & & & & & \\
\hline 96.51 & - 96.60 & & & & & & 1 & & & & & & \\
\hline 96.41 & - 96.50 & & & & & 1 & & & & & & & \\
\hline 96.31 & - 96.40 & & & 1 & & & & & & & & 1 & \\
\hline 96.21 & - 96.30 & & & 1 & 1 & & & & & & & & \\
\hline 96.11 & - 96.20 & & & & & & & & & & 1 & & \\
\hline 96.01 & - 96.10 & 1 & & & & & & & & 1 & 1 & 1 & \\
\hline 95.91 & - 96.00 & & & & & & & & & & 3 & & \\
\hline 95.81 & - 95.90 & & & & & & & & & 1 & 3 & & \\
\hline 95.71 & - 95.80 & & & & & & & & & 1 & 2 & & \\
\hline 95.61 & - 95.70 & & & & & & & & & 2 & 1 & & \\
\hline 95.51 & - 95.60 & & & & & & & & 1 & & & & \\
\hline 95.41 & - 95.50 & & & & & & & & 1 & 1 & & & \\
\hline Totals: & & 1 & 2 & 2 & 1 & 1 & 1 & 1 & 2 & 6 & 11 & 2 & 1 \\
\hline
\end{tabular}

concluded that there was little morphological consistency in the many competing descriptions (Thoms 1993:24). Suhm et al. (1954:402) emphasize shape, size, and grinding characteristics. While Wheeler (1995:415-420) describes shape, size, grinding, and flaking characteristics, his discussion of the distribution of Angostura makes clear that although a point may have the defined shape and proportions of the type, it is not a "true Angostura" unless the parallel diagonal ripple flake scars running from upper left to lower right are present. At the Wilson-Leonard site, Angostura is defined on the size and shape of the base and on chronological position. The analysis in Chapter 14 indicates that Angostura is a contracting haft point with a wide range of variability in haft width and shape but a narrow range in haft thickness. The chronological position of Angostura, based on dates from the Wilson-Leonard and Richard Beene (Thoms 1993:23) sites and new dates from the Ray Long site (Banks et al. 1995), is 9000 B.P. to 8000 B.P. Use-wear analysis of four Angostura points proved intriguing (see Kay, Chapter 22). Apparently three began as knives, were recycled to projectile points, and then back to knives.

\section{Thrall $(N=19)$}

Morphology: Thrall points have moderately wide, very thick, moderate to slight contracting hafts with slight basal concavity (Figure 13-5a-g); bases are modified predominately by concave bevel and rarely by secondary trimming or unifacial or bifacial thinning; lateral haft edges are usually heavily ground; basal edges are usually slightly ground or, less frequently, moderately to heavily ground.

Metric Averages: Table 13-4

Nonmetric Attributes: Longitudinal profiles include 2 with thick bases decreasing to thin distal sections, 2 with short wedge-shaped bases with tapered tips, 1 with generally equal proportions, and 14 indeterminate; flake patterns are organized on 11 (subparallel, oblique subparallel, and collateral), random on 5, and indeterminate on 3; basal modification includes 13 concave bevel, 2 secondary trimming, 2 unifacial thinning, 1 bifacial thinning, and 1 indeterminate; lateral grinding includes 13 heavy, 3 moderate, and 3 slight; and basal grinding includes 9 slight, 5 moderate/heavy, and 2 indeterminate. 


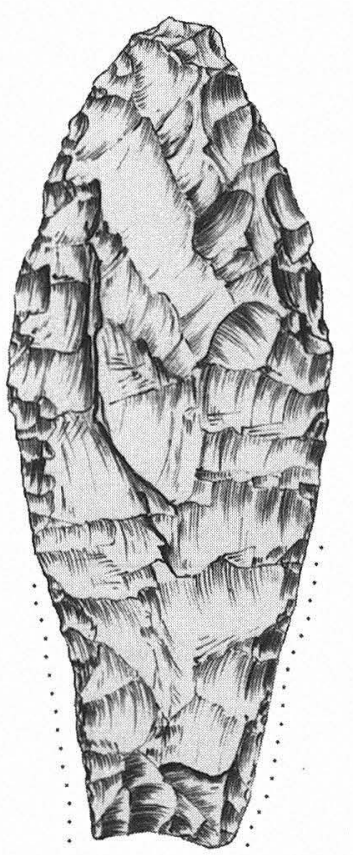

a
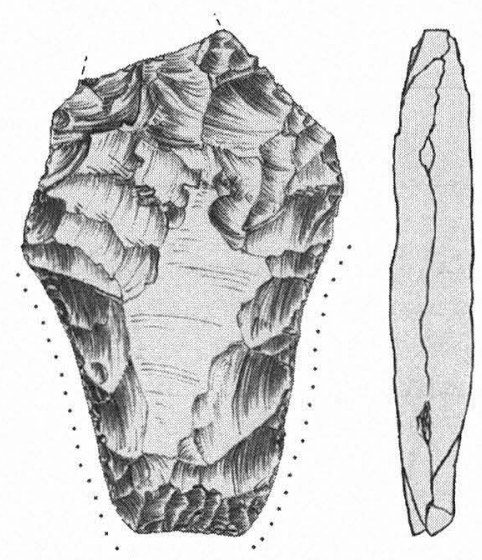

c

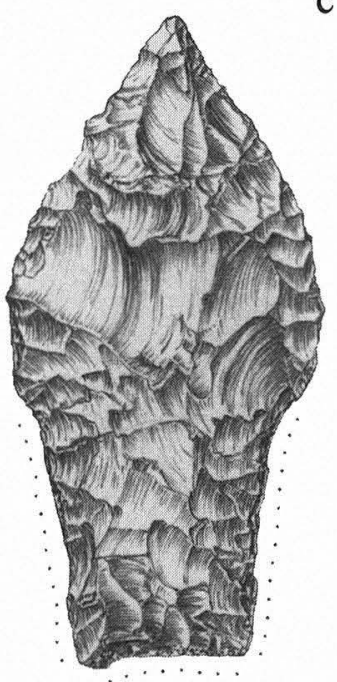

e
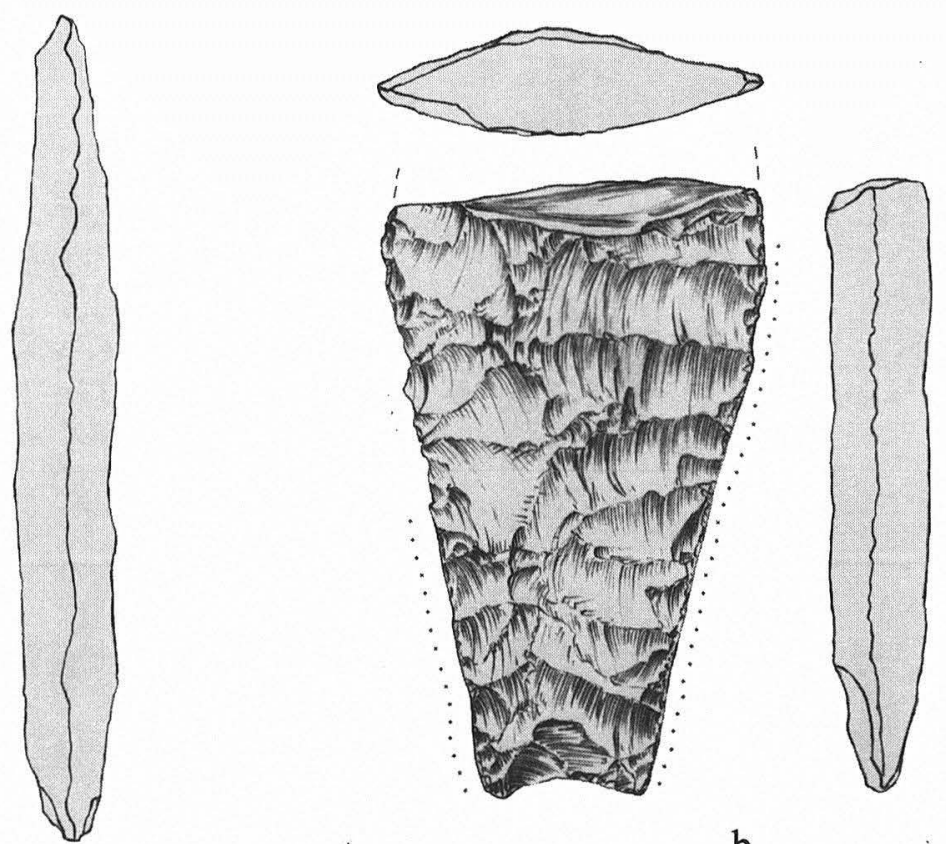

b
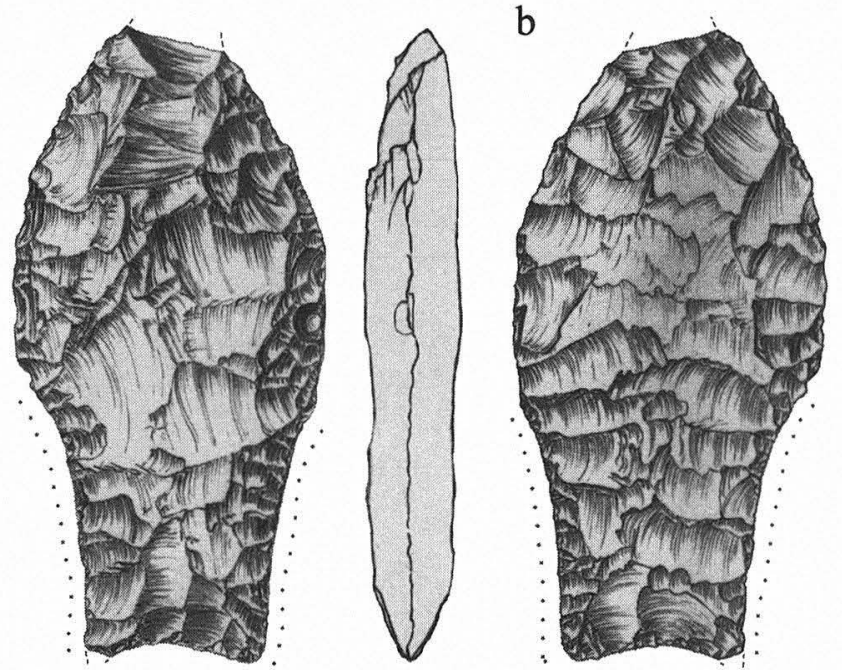

d
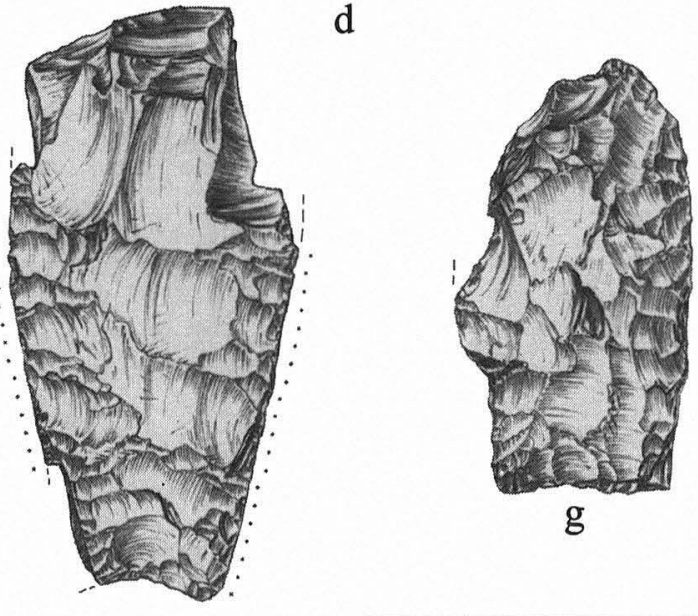

g
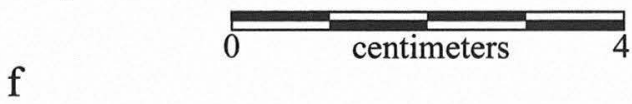

FIGURE 13-5. Thrall and possible Thrall points. Specimen numbers: (a) 19N-1; (b) 8K-1; (c) 7N-1; (d) 13N-2; (e) 31R1-1; (f) 25R1-2; (g) $5 J-4$. 
TABLE 13-4

Summary Metric Data for Thrall Points (in mm)

\begin{tabular}{|c|c|c|c|c|c|}
\hline Variable & Number & Minimum & Maximum & Mean & $1-\sigma$ \\
\hline Length & 3 & 52.0 & 84.0 & 67.0 & 16.1 \\
\hline Width & 17 & 19.5 & 39.1 & 26.7 & 5.5 \\
\hline Thickness & 19 & 6.9 & 10.5 & 8.5 & 1.1 \\
\hline Haft/grinding length 1 & 17 & 11.8 & 51.2 & 29.0 & 10.1 \\
\hline Haft/grinding length 2 & 15 & 10.7 & 35.2 & 23.3 & 6.7 \\
\hline Proximal haft width & 15 & 14.3 & 19.2 & 16.4 & 1.6 \\
\hline $10 \mathrm{~mm}$ haft width & 16 & 17.1 & 20.5 & 19.0 & 1.1 \\
\hline Distal haft width & 12 & 20.4 & 31.5 & 24.7 & 3.8 \\
\hline $10 \mathrm{~mm}$ haft thickness & 19 & 6.1 & 8.8 & 7.4 & 0.9 \\
\hline Basal concavity & 16 & 0.6 & 2.5 & 1.3 & 0.5 \\
\hline Basal convexity & 1 & & 1.0 & & \\
\hline
\end{tabular}

Material Attributes: Raw material includes local Edwards chert (11 fine grained, 2 coarse grained), nonlocal Edwards chert (3 fine grained, 1 coarse grained), 1 indeterminate heat altered, and 1 indeterminate; patina is not present; mineral precipitates are present on only 3 points; heat damage is slight on 1 point, moderate on 2 , extensive on 1 , and absent on 15 .

Tool State and Fractures: Two points are complete and 3 are nearly complete in length except for impact fractures near the tip; there are 3 proximal sections with a single bend break on 2 and impact and bend breaks on 1; there are 11 base sections with a single bend break on 2, a thermal spall on 1, multiple bend/burin-like breaks on 3, impact and bend breaks on 1 ; a bend break and crushed edge on 1 ; multiple bend breaks on 2 , and multiple thermal/bend fractures on 1.

Rejuvenation: One point is not resharpened; resharpened points include 6 unbeveled bifacial, 2 alternately beveled, 1 unbeveled unifacial, and 9 indeterminate. Specimens $13 \mathrm{~N}-2$, 19N-1, 31P1-1, and 31R1-1 were used as projectile points and knives as determined by Kay (see Chapter 22).

Stratigraphic Distribution: Twelve Thrall points occur in mixed Early Archaic strata of the Valley Margin (Table 13-5) and 4 in Unit IIIa of Valley Floor A. Chronologically, Thrall appears younger than Angostura. The mixed condition of Unit IIIa at the Wilson-Leonard site makes further chronological distinction from other Early Archaic points difficult.

Comments: Previously, Thrall points have been called Angostura, Fat Angostura, Victoria, and Hell Gap. Krieger (1947b:22) may have been referring to such specimens when he described an unnamed type with a "fat" stem present in small numbers in burned rock midden sites in Central Texas. The sample from the Wilson-Leonard site is the largest available from a single site and allowed definition of this distinct morphology and an indication of a chronological position between 8700 B.P. and 7000 B.P. (see Chapter 14 for further analysis). One Thrall point is recognized in the Wiley Williams site collection (TARL Records and Collections). The proposed type name, Thrall, comes from a small Williamson County community on a minor tributary of Brushy Creek about $45 \mathrm{~km}$ east of the Wilson-Leonard site.

\section{Midland $(N=1)$}

Morphology: This Midland specimen is a narrow, very thin, very slightly contracting base section; it has the broad facial flake pattern and very fine edge retouch typically described for the Midland type (Figure 13-6).

Metric Values: Table 13-6

Nonmetric Attributes: Longitudinal profile is of generally equal proportions; flake pattern is organized (subparallel); basal modification is unifacial thinning; lateral grinding is moderate; and basal grinding is absent.

Material Attributes: Raw material is local fine-grained Edwards chert; patina is slight, partial; mineral precipitates are absent; heat damage is absent.

Tool State and Fractures: The specimen is a base section with 2 bend fractures.

Rejuvenation: There is no apparent resharpening.

Stratigraphic Distribution: This Midland base is from Unit Id/II and is considered out of context.

Comments: This specimen compares well with those from Winkler-1 (Blaine 1968). See Chapter 14 for a comparative analysis.

\section{St. Mary's Hall $(N=14)$}

Morphology: St. Mary's Hall points have moderately wide, thick, parallel-sided hafts with moderately deep basal concavities (Figure 13-7a-h). Bases are modified predominately by bifacial thinning and rarely by unifacial thinning or secondary trimming. Lateral haft edges are usually heavily ground and basal edges are usually moderately to heavily ground. 
TABLE $13-5$

Stratigraphic Distribution of Thrall Points

\begin{tabular}{|c|c|c|c|c|c|c|c|c|c|c|c|c|c|c|c|}
\hline \multirow{2}{*}{ Elevation } & & \multicolumn{11}{|c|}{ Valley Margin } & \multicolumn{3}{|c|}{ Valley Floor A } \\
\hline & & $\mathrm{X}$ & $\mathrm{X} / \mathrm{II} / \mathrm{IIIa}$ & II/IIIa & $\mathrm{X} / \mathrm{Y}$ & $\mathrm{Y}$ & IIIa & $\mathrm{IIIa} / \mathrm{b}$ & $\mathrm{X} / \mathrm{IIIb}$ & $\mathrm{X} / \mathrm{Y} / \mathrm{IIIb}$ & Y/IIIb & $\mathrm{IIIb} / \mathrm{c}$ & IIIa & $\mathrm{IIIa} / \mathrm{b}$ & IIIC \\
\hline 97.71 & - 97.80 & & & & & 1 & & & & & & & & & \\
\hline 97.61 & - 97.70 & & & & & & & & & & & & & & \\
\hline 97.51 & - 97.60 & & & & & & & & & & & & & & \\
\hline 97.41 & $\begin{array}{l}-97.50 \\
-9\end{array}$ & & & & & & & & & & & & & & \\
\hline 97.31 & - 97.40 & & & & & & & & & & & & & & \\
\hline 97.21 & - 97.30 & & & & & & & & & & & & & & \\
\hline 97.11 & - 97.20 & & & & & 1 & & & & & & 1 & & & \\
\hline 97.01 & - 97.10 & & & & & & & & & & & & & & \\
\hline 96.91 & - 97.00 & & & & 1 & & & & & & 1 & & & & 1 \\
\hline 96.81 & - 96.90 & & & & & & & & & 1 & & & & & \\
\hline 96.71 & - 96.80 & & & & & & & & 1 & & & & & & \\
\hline 96.61 & - 96.70 & 2 & & & & & & & & & & & & & \\
\hline 96.51 & - 96.60 & & & & & & & & & & & & & & \\
\hline 96.41 & - 96.50 & & & & & & & 1 & & & & & & & \\
\hline 96.31 & - 96.40 & & & & & & & & & & & & & 1 & \\
\hline 96.21 & - 96.30 & & 1 & & & & 1 & & & & & & & & \\
\hline 96.11 & - 96.20 & & & 1 & & & & & & & & & & & \\
\hline 96.01 & - 96.10 & & & & & & & & & & & & 1 & & \\
\hline 95.91 & - 96.00 & & & & & & & & & & & & & & \\
\hline 95.81 & - 95.90 & & & & & & & & & & & & 1 & & \\
\hline 95.71 & - 95.80 & & & & & & & & & & & & 1 & & \\
\hline 95.61 & - 95.70 & & & & & & & & & & & & 1 & & \\
\hline Totals: & & 2 & 1 & 1 & 1 & 2 & 1 & 1 & 1 & 1 & 1 & 1 & 4 & 1 & 1 \\
\hline
\end{tabular}
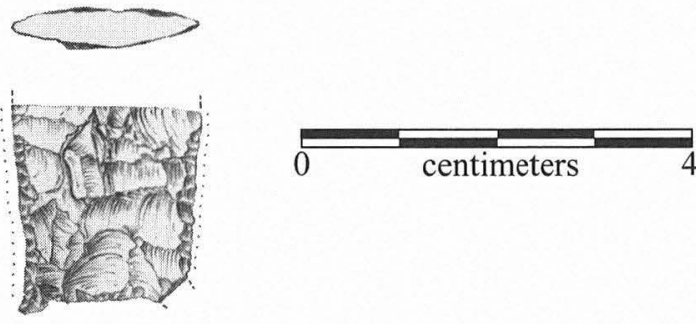

Figure 13-6. Midland point, Specimen 26Y2C-3.

TABLE 13-6

Metric Measurements for Midland Point (in mm)

\begin{tabular}{l|r}
\hline Variable & Maximum \\
\hline Width & 18.9 \\
Thickness & 3.9 \\
Haft/grinding length 1 & 20.9 \\
Haft/grinding length 2 & 16.7 \\
Proximal haft width & 18.0 \\
10-mm haft width & 17.4 \\
10-mm haft thickness & 3.2 \\
Basal concavity & 2.3 \\
\hline \hline
\end{tabular}

Metric Averages: Table 13-7

Nonmetric Attributes: Longitudinal profiles include 4 with tapered bases increasing to thick distal sections, 2 with tapered bases and maximum thicknesses at medial sections, 2 with generally equal proportions, and 6 indeterminate; flake patterns are organized on 7 (subparallel, oblique subparallel), random on 2, and 5 are indeterminate. Basal modification includes 9 bifacial thinning, 2 secondary trimming, 2 unifacial thinning, and 1 thinning and fluting; lateral grinding includes 11 heavy, 1 moderate, and 2 slight; and basal grinding includes 2 absent, 3 slight, and 8 moderate/heavy, and 1 indeterminate.

Material Attributes: Raw material includes local (10 fine grained) and nonlocal (3 fine grained) Edwards chert, and 1 indeterminate; patina is absent on 12 , heavy overall on 1 , and 1 is indeterminate; mineral precipitates are present on 4 ; heat damage is absent on 9 , moderate on 3 , extensive on 1 , and indeterminate on 1.

Tool State and Fractures: One point is complete and 3 are nearly complete in length. Of these, a single bend fracture occurs near the tip on 1, multiple facial impact/burinlike breaks occur on 1, and combination facial impact/bend/ burin-like breaks and edge crushing occur on 1; there are 6 proximal sections with a single bend break on 1 , multiple 

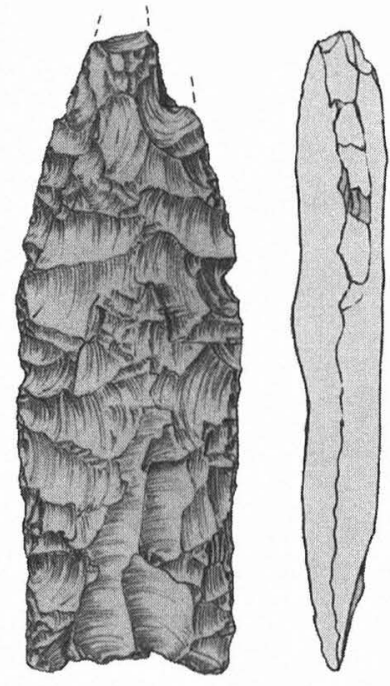

a

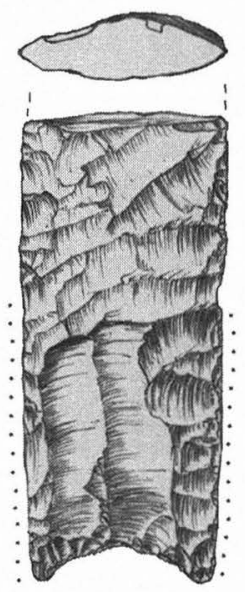

d
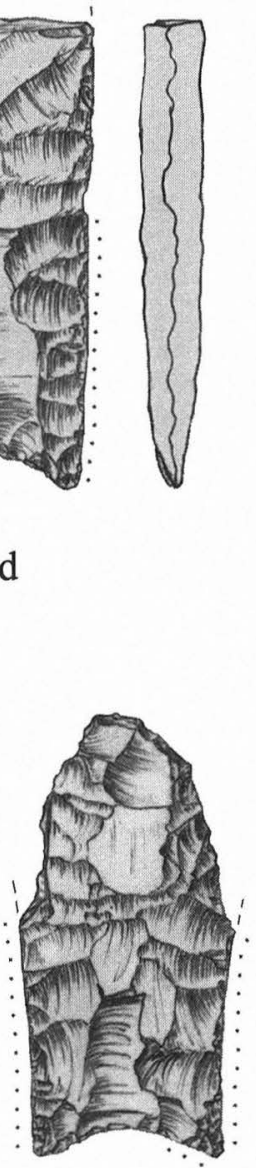

$\mathrm{g}$

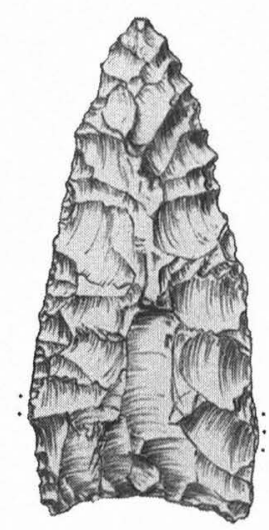

b

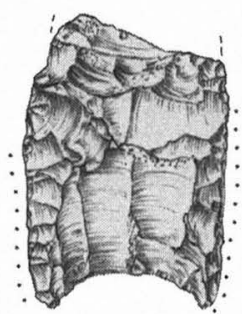

$\mathrm{e}$

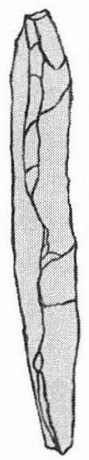

centimeters

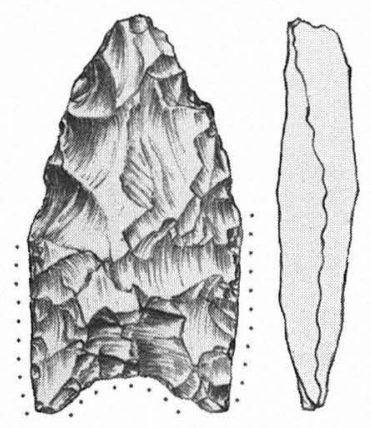

c

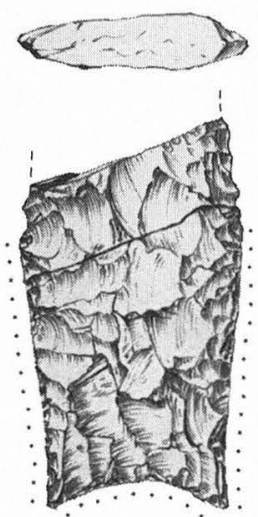

f

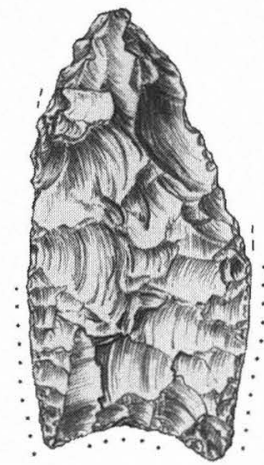

$\mathrm{h}$

FIGURE 13-7. St. Mary's Hall points. Specimen numbers: (a) 34U1B-1; (b) 28W2C-1; (c) 7Q-1; (d) 20W-1; (e) 50XD-1; (f) 38S1; (g) 25U21 ; (h) 26P1-2. 
bend/impact breaks on 2, impact and burin-like breaks on 1 , impact/bend/burin-like breaks on 1, and multiple thermal spalls on 1; there are 4 base sections with a single thermal spall on 1, impact and burin-like breaks on 1, multiple edge crushing/bend breaks on 1 , and bend/burin-like/thermal breaks on 1 .

Rejuvenation: One point is not resharpened; resharpened points include 7 alternately beveled, 2 unbeveled bifacial, and 4 indeterminate. One specimen, 34U1B-1, may have been recycled as a burin. According to Kay's microwear analysis (see Chapter 22), specimens 20W-1, 25U2-1, and $50 \mathrm{XD}-1$ were used as both a projectile point and a knife, and specimen 7Q-1 was a reworked, repointed projectile point.

Stratigraphic Distribution: Little patterning could be discerned in vertical distribution of St. Mary's Hall points, although four occur between elevations $95.30 \mathrm{~m}$ to 95.50 $m$ in the upper part of Units II and Isi-c/II in Valley Floor A (Table 13-8).

Comments: Note that Specimen 28W2C-1 (see Figure $13-7 b$ ) is a rebased tip with a basal configuration conforming to St. Mary's Hall.

Previously, St. Mary's Hall points have been subsumed within a very broadly defined Plainview type. The analysis

TABLE 13-7

Summary Metric Data for St. Mary's Hall Points (in mm)

\begin{tabular}{|c|c|c|c|c|c|}
\hline Variable & Number & Minimum & Maximum & Mean & $1-\sigma$ \\
\hline Length & 1 & & $51.0 *$ & & \\
\hline Width & 12 & 20.7 & 23.9 & 22.2 & 0.8 \\
\hline Thickness & 14 & 5.2 & 9.4 & 6.7 & 1.1 \\
\hline Haft/grinding length 1 & 13 & 12.3 & 34.9 & 22.5 & 5.4 \\
\hline Haft/grinding length 2 & 11 & 9.1 & 34.8 & 19.5 & 7.5 \\
\hline Proximal haft width & 13 & 18.3 & 21.5 & 19.9 & 1.2 \\
\hline 10 -mm haft width & 13 & 19.2 & 23.0 & 20.9 & 1.3 \\
\hline Distal haft width & 9 & 20.7 & 22.7 & 22.0 & 0.7 \\
\hline 10-mm haft thickness & 14 & 4.8 & 6.9 & 5.7 & 0.7 \\
\hline Basal concavity & 13 & 1.4 & 3.6 & 2.6 & 0.8 \\
\hline
\end{tabular}

* The only specimen with a complete length measurement is a rebased tip which is not representative of the type.

TABLE $13-8$

Stratigraphic Distribution of St. Mary's Hall Points

\begin{tabular}{|c|c|c|c|c|c|c|c|c|}
\hline \multirow[b]{2}{*}{ Elevation } & \multicolumn{2}{|c|}{ Valley Margin } & \multicolumn{4}{|c|}{ Valley Floor A } & \multicolumn{2}{|c|}{ Valley Floor B } \\
\hline & $\mathrm{X}$ & II/IIIa & Isi-c/II & II & II/IIIa & IIII & II & Surface \\
\hline Surface & & & & & & & & 1 \\
\hline $96.61-96.70$ & 1 & & & & & & & \\
\hline $96.51-96.60$ & & & & & & & & \\
\hline $96.41-96.50$ & & & & & & & & \\
\hline $96.31-96.40$ & & & & & & & & \\
\hline $96.21-96.30$ & & & & & & & & \\
\hline $96.11-96.20$ & & 1 & & & & & & \\
\hline $96.01-96.10$ & & & & & & & & \\
\hline $95.91-96.00$ & & & & & & 1 & & \\
\hline $95.81-95.90$ & 1 & & & & & & & \\
\hline $95.71-95.80$ & & & & & & 1 & & \\
\hline $95.61-95.70$ & & & & & & 1 & & \\
\hline $95.51-95.60$ & & & & & & & & \\
\hline $95.41-95.50$ & & & & 1 & 1 & & & \\
\hline $95.31-95.40$ & & & 1 & 1 & & & & \\
\hline $95.21-95.30$ & & & & 1 & & & & \\
\hline $95.11-95.20$ & & & & & 1 & & & \\
\hline $95.01-95.10$ & & & & & & & 1 & \\
\hline Totals: & 2 & 1 & 1 & 3 & 2 & 3 & 1 & 1 \\
\hline
\end{tabular}


in Chapter 14 indicates that St. Mary's Hall points are narrower and thicker in haft dimensions and have deeper basal concavities and shorter grinding lengths than Plainview points. Flake patterns are less regular on St. Mary's Hall specimens than on Plainviews. Radiocarbon data suggest that St. Mary's Hall dates from 9990 B.P. to 8700 B.P., while Plainview dates between 11,000 B.P. and 10,100 B.P. (see Chapter 14 for full discussion). St. Mary's Hall points have been identified at the St. Mary's Hall (Hester 1977, 1991), Lubbock Lake (Johnson and Holliday 1980), and Levi Rockshelter (Alexander 1963) sites. It is likely that the Miniature Plainviews identified by Kelly (1983b) are St. Mary's Hall. The type name was chosen from the St. Mary's Hall site where the component is stratigraphically isolated, although not radiocarbon dated. Observations of the St. Mary's Hall site collection indicate that thicker hafts might distinguish the type from Plainview.

\section{St. Mary's Hall-atypic $(N=4)$}

Morphology: St. Mary's Hall-atypic points have moderately wide, thick, parallel-sided hafts with moderately deep basal concavities; bases are modified by bifacial thinning, lateral haft edges are slightly to heavily ground, and basal edges are usually slightly ground (Figure 13-8).

Metric Averages: Table 13-9

Nonmetric Attributes: Longitudinal profiles include 1 with a tapered base increasing to a thick distal section, 1 with a tapered base with maximum thickness at medial section, and 2 indeterminate; flake patterns are organized on 2 (parallel and oblique subparallel), random on 1, and indeterminate on 1; basal modification includes 3 bifacial thinning and 1 unifacial thinning; lateral grinding includes 2 heavy, 1 moderate, and 1 slight; and basal grinding includes 1 absent, 2 slight, and 1 moderate/heavy.

Material Attributes: Raw material includes local (3 fine grained) and nonlocal (1 fine grained) Edwards chert; patina is absent on 3 points and slight, partial on 1; mineral precipitates are present on 2 points; heat damage is absent.

Tool State and Fractures: One point is complete; 2 are proximal sections with a single bend break on 1 and bend and burin-like breaks on 1; and 1 is a base section with a bend break.

Rejuvenation: Two are resharpened by alternate beveling and 2 are indeterminate.

Stratigraphic Distribution: Two points are from upper Unit II in Valley Floor A between the elevations of $95.11 \mathrm{~m}$ and $95.40 \mathrm{~m}$, which is consistent for St. Mary's Hall (Table 13-10).

Comments: These points are atypical of the St. Mary's Hall category because of a deeper basal concavity and a slightly wider haft. However, they have the same haft thickness and grinding length, and two of the four atypical points have proveniences similar to the more typical St. Mary's Hall points. Atypical specimens also occur at the St. Mary's Hall (Hester 1991:Figure 1d) and Devil's Mouth (Sorrow 1968:Figure 17f) sites.

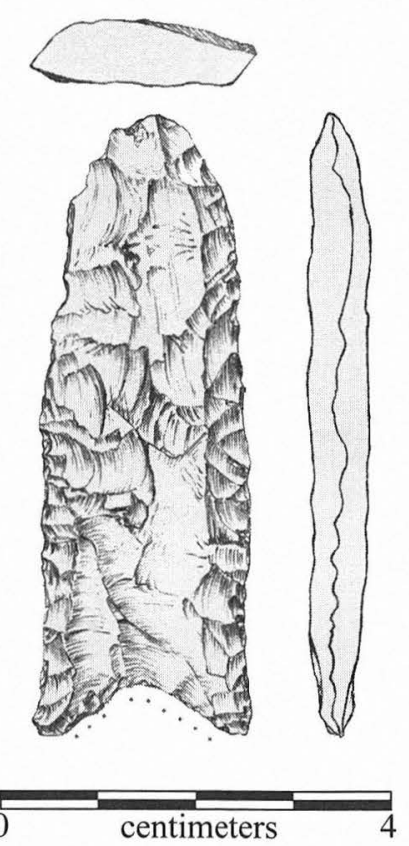

FIGURE 13-8. St. Mary's Hall-atypic point, Specimen 18L-1.

TABLE 13-9

Summary Metric Data for St. Mary's Hall-atypic Points (in mm)

\begin{tabular}{|c|c|c|c|c|c|}
\hline Variable & Number & Minimum & Maximum & Mean & $1-\sigma$ \\
\hline Length & 1 & & 63.0 & & \\
\hline Width & 4 & 22.0 & 28.1 & 24.5 & 2.6 \\
\hline Thickness & 4 & 5.8 & 7.2 & 6.6 & 0.8 \\
\hline Haft/grinding length 1 & 4 & 13.7 & 33.6 & 25.0 & 8.4 \\
\hline Haft/grinding length 2 & 4 & 13.4 & 23.0 & 18.6 & 4.8 \\
\hline Proximal haft width & 4 & 21.7 & 24.8 & 23.2 & 1.3 \\
\hline 10-mm haft width & 4 & 20.6 & 26.1 & 23.2 & 2.3 \\
\hline Distal haft width & 3 & 20.6 & 28.1 & 24.3 & 3.8 \\
\hline 10-mm haft thickness & 4 & 5.4 & 6.8 & 5.9 & 0.6 \\
\hline Basal concavity & 4 & 2.8 & 4.7 & 3.8 & 0.8 \\
\hline
\end{tabular}


TABLE $13-10$

Stratigraphic Distribution of St. Mary's Hall-atypic Points

\begin{tabular}{rr|c|cc}
\hline \multicolumn{2}{|c|}{} & Valley Margin & \multicolumn{2}{|c}{ Valley Floor A } \\
Elevation & & X/Y & II & IIIa \\
\hline 97.01 & -97.10 & 1 & & \\
95.71 & -95.80 & & & 1 \\
95.61 & -95.70 & & & \\
$95.51-95.60$ & & & \\
95.41 & -95.50 & & & \\
$95.31-95.40$ & & 1 & \\
$95.21-95.30$ & & & \\
$95.11-95.20$ & & 1 & \\
\hline Totals: & & 1 & 2 & 1 \\
\hline
\end{tabular}

\section{The Bone Bed Point $(N=1)$}

Morphology: The Bone Bed point has a narrow, thin, very slightly expanded parallel-sided haft with moderately shallow basal concavity; the base is modified by secondary trimming; lateral haft edges are heavily ground and the basal edge is slightly ground (Figure 13-9).

Metric Values: Table 13-11

Nonmetric Attributes: Longitudinal profile is of generally equal proportions; flake pattern is organized (subparallel); basal modification is secondary trimming; lateral grinding is heavy; and basal grinding is slight.

Material Attributes: Raw material is nonlocal mediumcoarse-grained Edwards chert; patina is moderate overall; mineral precipitates are absent; heat damage is absent.

Tool State and Fractures: This point is nearly complete in length, although a longitudinal impact fracture has severed a lateral edge of the blade.

Rejuvenation: There is no apparent resharpening. According to Kay's microwear analysis (see Chapter 22), this point was used a projectile point, a knife or butchering tool,

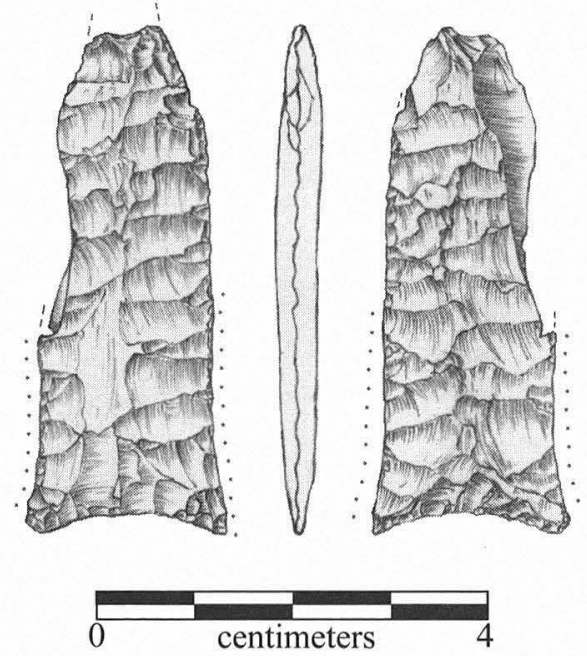

FIgURE 13-9. The Bone Bed point, Specimen 17JJ2/KK1-3. and finally as a burin for cutting hard material such as bone, antler, or wood.

Stratigraphic Distribution: The Bone Bed point is from Valley Floor A, Unit Isi/Icl at $93.92 \mathrm{~m}$ in elevation.

Comments: The Bone Bed point, so named because it was found with the numerous bison bones of Unit Isi, was first considered to be a Midland point, but the analysis in Chapter 14 suggests Plainview-atypic as an alternative type. The Bone Bed point did not conform well with the Midland sample in shape, size, and flake pattern. In contrast, it is within the Plainview range in proximal haft width, haft thickness, basal concavity, grinding length, haft thickness to haft width ratio, and basal concavity to grinding length ratio. Comparison of this specimen with Beidleman Ranch specimen 1054-1 (Suhm 1961:Figure 2B,B') shows close similarities in flake pattern and shape.

\section{Parallel-sided Haft $(\mathrm{N}=4)$}

Morphology: Parallel-sided haft points have wide, thick, parallel-sided haft areas with shallow to no basal concavities; bases are modified predominately by bifacial thinning; lateral haft edges are likely to be heavily ground, and basal edges are likely not to be ground (Figure 13-10).

Metric Averages: Table 13-12

Nonmetric Attributes: Longitudinal profiles include 1 with generally equal proportions, 1 with a tapered base increasing to a thick distal section, 1 with a short wedge-shaped base with tapered tip, and 1 indeterminate; flake patterns are organized on 3 (subparallel, collateral), and 1 is indeterminate; basal modification includes 3 bifacial thinning and 1 unifacial thinning; lateral grinding includes 2 heavy, 1 moderate, and 1 slight; and basal grinding includes 2 absent, 1 slight, and 1 moderate/heavy.

Material Attributes: Raw material includes local (1 fine grained and 2 medium coarse grained) and nonlocal (1 fine grained) Edwards chert; patina is absent; mineral precipitates are present on 1 point; heat damage is absent on 3 points and moderate on 1 .

Tool State and Fractures: There are 3 proximal sections

TABLE 13-11

Metric Measurements for the Bone Bed Point (in mm)

\begin{tabular}{l|r}
\hline Variable & Maximum \\
\hline Length* & 51.0 \\
Width & 20.1 \\
Thickness & 4.4 \\
Haft/grinding length 1 & 23.4 \\
Proximal haft width & 20.1 \\
10-mm haft width & 17.4 \\
10-mm haft thickness & 3.8 \\
Basal concavity & 2.1 \\
\hline * Maximum measurement & \\
\hline \hline
\end{tabular}


with multiple bend breaks on 1, impact/bend/edge crushing breaks on 1, and multiple bend/burin-like/thermal breaks on 1 (which refits with the potlid); and there is 1 base section with a bend break and crushed edge.

Rejuvenation: One point is not resharpened and 3 are indeterminate. According to Kay's microwear analysis (see Chapter 22), Specimen 26X2A-1 was used as a projectile point and knife, and the use of Specimen 25V1C-1/28X1C4 was uncertain, and it had been subjected to postdepositional movement.

Stratigraphic Distribution: Parallel-sided haft points appear in Unit Id/II and lower Unit II (Table 13-13); conjoined Specimens 25V1C-1 and 28X1C-4 (a potlid) were found at elevations $95.26 \mathrm{~m}$ and $95.40 \mathrm{~m}$ respectively, which runs counter to the dip of the deposits. This relationship suggests the possibility of postdepositional disturbance at the Unit I/II contact.

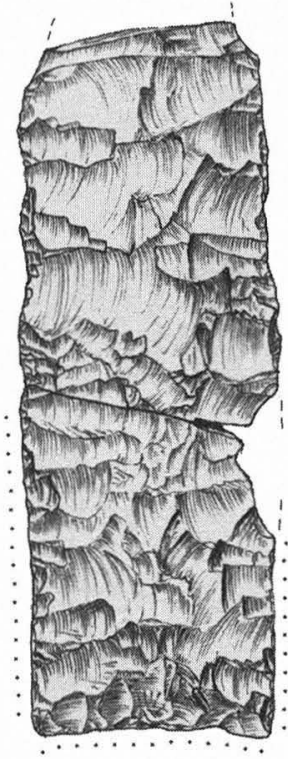

a

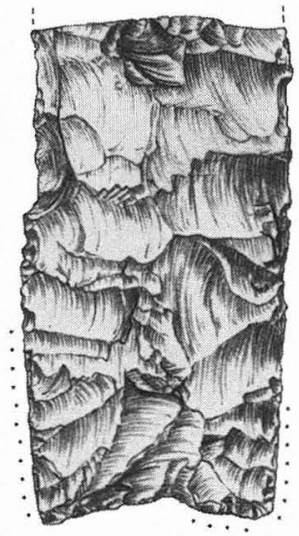

b
Comments: This morphological set was not firmly typed due to its small sample size and lack of an adequate comparative sample. Initially, these points were referred to as Firstview, but comparison with available measurements for the type-site Firstview points (Wheat 1972:Tables 21 and 22) indicate these Wilson-Leonard points are too large and do not really fit the Firstview description. Given the wide range of morphological variability accepted for the Firstview type, it is in need of careful systematic analysis with a larger sample size (see discussion in Hofman 1989:42-43).

\section{Ground Square Haft (N=1)}

Morphology: This specimen is a wide, thick, parallelsided base section with no basal concavity; the base is modified by bifacial thinning; lateral haft edges are slightly ground and the basal edge is not ground (Figure 13-11).

FIGURE 13-10. Parallel-sided haft points. Specimen numbers: (a) 22T-1; (b) 26X2A-1; (c) 25V1C-1 (conjoined with potlid, 28X1C-4).

TABLE 13-12

Summary Metric Data for Parallel-sided Haft Points (in mm)

\begin{tabular}{|c|c|c|c|c|c|}
\hline Variable & Number & Minimum & Maximum & Mean & $1-\sigma$ \\
\hline Width & 4 & 24.4 & 30.2 & 27.1 & 2.4 \\
\hline Thickness & 4 & 5.5 & 8.2 & 7.0 & 1.2 \\
\hline Haft/grinding length 1 & 4 & 16.1 & 34.0 & 24.3 & 7.8 \\
\hline Haft/grinding length 2 & 3 & 15.3 & 25.4 & 20.5 & 5.1 \\
\hline Proximal haft width & 4 & 23.6 & 24.7 & 24.1 & 0.6 \\
\hline $10-\mathrm{mm}$ haft width & 4 & 24.4 & 26.4 & 25.2 & 1.0 \\
\hline Distal haft width & 3 & 24.5 & 29.0 & 26.8 & 2.3 \\
\hline 10-mm haft thickness & 4 & 4.6 & 5.8 & 5.3 & 0.5 \\
\hline Basal concavity & 4 & 0.0 & 2.0 & 1.1 & 0.8 \\
\hline
\end{tabular}


TABLE 13-13

Stratigraphic Distribution of Parallel-sided Haft Points

\begin{tabular}{c|c|cc}
\hline & \multicolumn{2}{|c|}{ Valley Margin } & \multicolumn{2}{|c}{ Valley Floor A } \\
Elevation & II & Id/II & II \\
\hline $95.71-95.80$ & 1 & & \\
$95.61-95.70$ & & & \\
$95.51-95.60$ & & & \\
$95.41-95.50$ & & & $1 *$ \\
$95.31-95.40$ & & & \\
$95.21-95.30$ & & $1 *$ & 1 \\
$95.11-95.20$ & & & \\
$95.01-95.10$ & & 1 & \\
\hline Totals: & 1 & 2 & 2 \\
\hline
\end{tabular}

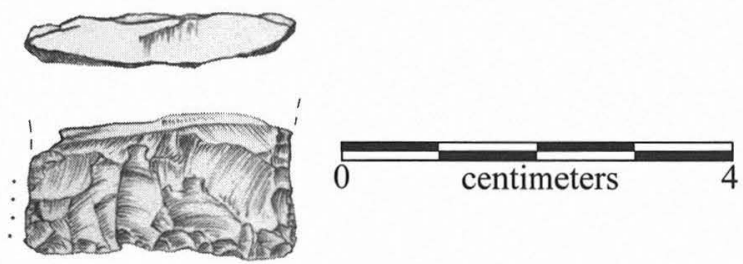

FIGURE 13-11. Ground square haft point, Specimen 15X1-3.

Metric Values: Table 13-14

Nonmetric Attributes: Longitudinal profile is indeterminate; flake pattern is indeterminate; basal modification is bifacial thinning; lateral grinding is slight; and basal grinding is absent.

Material Attributes: Raw material is local fine-grained Edwards; patina is absent; mineral precipitates are absent; heat damage is absent.

Tool State and Fractures: The specimen is a base section with a transverse bend fracture.

Rejuvenation: There is no apparent resharpening. This point base was recycled as a radial break tool according to Kay's microwear analysis (see Chapter 22).

Stratigraphic Distribution: Valley Floor A, Unit Id/II.

Comments: This specimen was too fragmented to type securely but is possibly Scottsbluff. It lacks the characteristic slight shoulder of Scottsbluff because it was fractured within the haft, but it clustered with the Scottsbluff points in the systematic analysis in Chapter 14.

TABLE 13-14

Metric Measurements for Ground Square Haft Point (in mm)

\begin{tabular}{l|c}
\hline Variable & Maximum \\
\hline Width & 27.7 \\
Thickness & 5.3 \\
Proximal haft width & 27.8 \\
10-mm haft width & 26.1 \\
10-mm haft thickness & 5.2 \\
Basal concavity & 0.0 \\
\hline \hline
\end{tabular}

\section{Scottsbluff $(N=2)$}

Morphology: Scottsbluff points have very slight shoulders and wide, very thick, parallel-sided stems with shallow to no basal concavities. Bases are modified by unifacial thinning; lateral stem edges are slight to heavily ground, and basal edges are slightly ground (Figure 13-12).

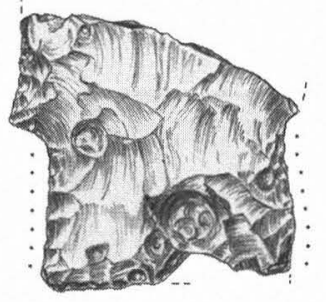

a

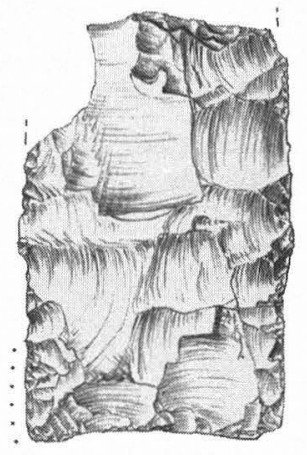

b

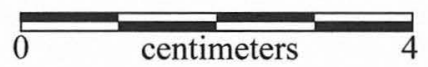

FIGURE 13-12. Scottsbluff points. Specimen numbers: (a) 21M-3; (b) 26V2B-2.

Metric Values: Table 13-15

Nonmetric Attributes: Longitudinal profiles are indeterminate on both; flake pattern is organized (subparallel) on 1 and indeterminate on 1 ; basal modification is unifacial thinning on both; lateral grinding is slight on 1 and heavy on 1 ; and basal grinding is slight on 1 and indeterminate on 1.

Material Attributes: Raw material includes 1 local medium-coarse-grained Edwards and 1 indeterminate heat altered; patina is absent; mineral precipitates are absent; heat damage is moderate on 1 point and absent on 1 .

Tool State and Fractures: Both are proximal sections with multiple impact/bend/burin breaks on 1 and multiple thermal spalls on 1 .

TABLE $13-15$

Metric Measurements for Scottsbluff Points (in mm)

\begin{tabular}{l|c|c}
\hline Variable & $\begin{array}{c}\text { Specimen } \\
21 \mathrm{M}-3 \\
\text { Maximum }\end{array}$ & $\begin{array}{c}\text { Specimen } \\
\text { 26V2B-2 } \\
\text { Maximum }\end{array}$ \\
\hline Width & 29.9 & 27.8 \\
Thickness & 8.8 & 7.1 \\
Stem/grinding length 1 & 14.3 & 17.3 \\
Stem/grinding length 2 & 13.8 & 16.5 \\
Proximal stem width & & 26.0 \\
10-mm stem width & 24.1 & 26.7 \\
Distal stem width & 24.3 & 26.9 \\
10-mm stem thickness & 6.6 & 6.6 \\
Basal concavity & 0.0 & 1.1 \\
\hline \hline
\end{tabular}


Rejuvenation: One point is not resharpened; resharpening is indeterminate on 1. Specimen 26V2B-2 was recycled as a burin according to Kay's microwear analysis (see Chapter 22).

Stratigraphic Distribution: One point was found in the Valley Margin, Unit IIIb and is considered out of context; the other point was found in Valley Floor A, lower Unit II at elevation $95.28 \mathrm{~m}$.

\section{Golondrina-Barber $(N=23)$}

Morphology: Golondrina-Barber points have moderate to very wide, thick, parallel-sided hafts with moderate to very deep basal concavities (Figures 13-13 and 13-14); bases are modified by bifacial or unifacial thinning; lateral haft edges are slightly to heavily ground and basal grinding is absent to slight.

Metric Averages: Table 13-16

Nonmetric Attributes: Longitudinal profiles include 5 with tapered bases with maximum thicknesses at their medial sections; 2 with generally equal proportions, 2 with tapered bases increasing to thick distal sections, 1 with a short wedge-shaped base with a tapered tip, 11 indeterminate, and 2 that was not coded; flake patterns are organized (subparallel, oblique subparallel, collateral), random on 1 , indeterminate on 5 , and 1 has a channel flake scar; basal modification includes 12 bifacial thinning, 7 unifacial thinning, 1 concave bevel, 1 secondary trimming, 1 unifacial fluting, and 1 thinning and fluting; lateral grinding includes 13 heavy, 3 moderate, and 7 slight; and basal grinding includes 11 absent, 8 slight, and 4 moderate/ heavy.

Material Attributes: Raw material includes local (14 fine grained and 2 medium coarse grained) and nonlocal (4 fine grained and 1 medium coarse grained) Edwards chert; 1 quartz, and 1 indeterminate; patina is absent; mineral precipitates are present on 8 points; heat damage is extensive on 1 point.

Tool State and Fractures: Two points are complete, and 2 are nearly complete in length; of these, a single facial impact break occurs near the tip on 1, and impact/bend/ burin-like breaks occur near the tip or basal edge on 1 ; there are 12 proximal sections with a single bend break on 3 , multiple bend breaks on 3 , impact and bend breaks on 1 , a hinge break and crushed edge on 1, impact/bend/edge crushing breaks on 1, multiple burin-like/bend breaks on 2, and multiple thermal spalls on 1 ; there are 7 base sections with a single bend break on 2 , multiple bend breaks on 1 , multiple bend/burin-like breaks on 3 , and multiple impact/burin-like breaks on 1 .

Rejuvenation: Eleven points are resharpened, including 6 alternately beveled, 3 unbeveled bifacial, and 2 unbeveled unifacial; 9 are indeterminate. According to Kay's microwear analysis (Chapter 22), Specimens 15X1-1, 15Y11 , and 50YA-1 were used as both projectile points and knives, Specimen 51Y1B-1 was used as both a knife and a radial break tool, and Specimen 13Q-2 was used as a projectile point, knife, and burin.

Stratigraphic Distribution: Seven Golondrina-Barber points thought to be in most secure context are distributed through Units Isi-c/Id, Isi-c/II, Id/II, lower Unit II between elevations $94.90 \mathrm{~m}$ and $95.20 \mathrm{~m}$ in Valley Floor A, and at the Unit I/II contact in Valley Floor B (Table 13-17).

Comments: One point is a rebased tip (Figure 13-14b) with basal morphology that fits within the range of Golondrina-Barber.

Golondrina points were first defined as a variety of Plainview at the Devil's Mouth site (41VV188) (Johnson 1964:46-49). Kelly (1982:3-9) tried to narrowly define Golondrina as an expanding stem unfluted lanceolate point and eventually rejected several of the original typesite points. Kelly (1983a:10-25) also attempted to define the contracting haft Barber points using four points from Wilson-Leonard site and five points from surface collections in Gillespie County. The analysis in Chapter 14 suggests that Golondrina and Barber encompass a wide range of morphological variability in haft shape from slightly contracted to slightly expanded; there is no detectable chronological significance. Golondrina-Barber points have a deeper basal concavity and a wider haft than other unfluted

TABLE $13-16$

Summary Metric Data for Golondrina-Barber Points (in $\mathrm{mm}$ )

\begin{tabular}{|c|c|c|c|c|c|}
\hline Variable & Number & Minimum & Maximum & Mean & $1-\sigma$ \\
\hline Length & 2 & 53.0 & 55.0 & & \\
\hline Width & 22 & 21.4 & 33.2 & 27.8 & 3.1 \\
\hline Thickness & 23 & 5.5 & 8.3 & 7.0 & 0.8 \\
\hline Haft/grinding length 1 & 22 & 12.4 & 36.0 & 25.0 & 6.9 \\
\hline Haft/grinding ength 2 & 16 & 10.8 & 35.5 & 21.9 & 6.7 \\
\hline Proximal haft width & 20 & 17.2 & 30.8 & 25.1 & 3.6 \\
\hline 10-mm haft width & 22 & 19.9 & 30.8 & 26.0 & 3.0 \\
\hline Distal haft width & 18 & 19.4 & 32.8 & 27.0 & 3.2 \\
\hline 10-mm haft thickness & 22 & 4.4 & 6.7 & 5.6 & 0.7 \\
\hline Basal concavity & 21 & 2.8 & 8.5 & 6.2 & 1.6 \\
\hline
\end{tabular}



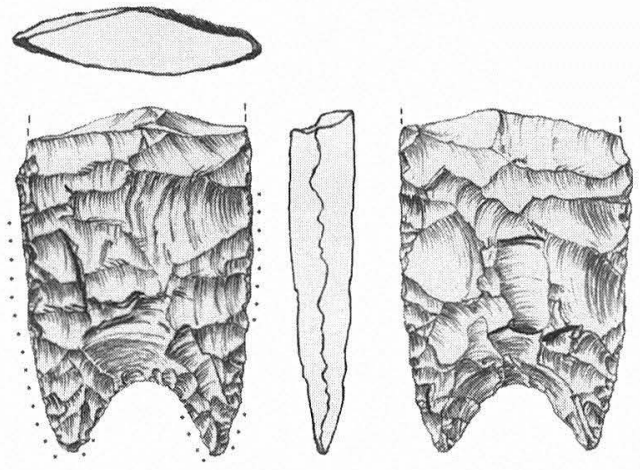

a

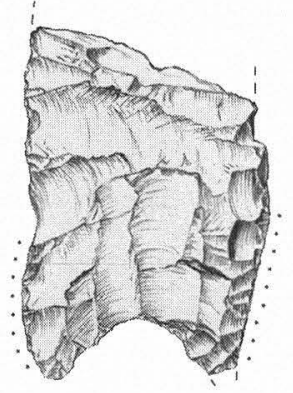

d

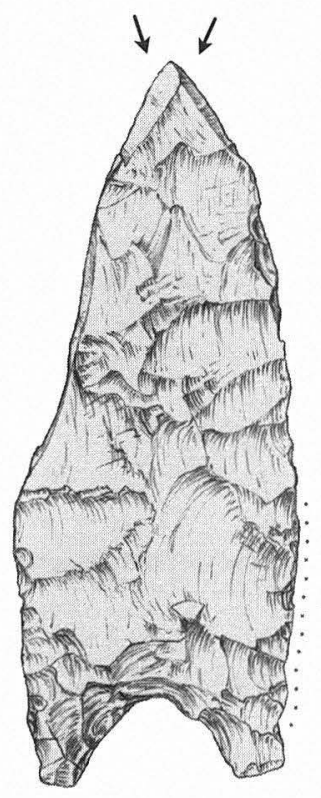

h
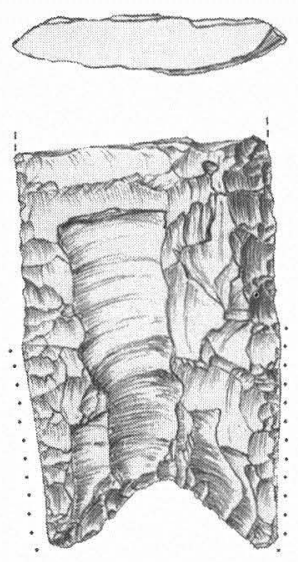

b
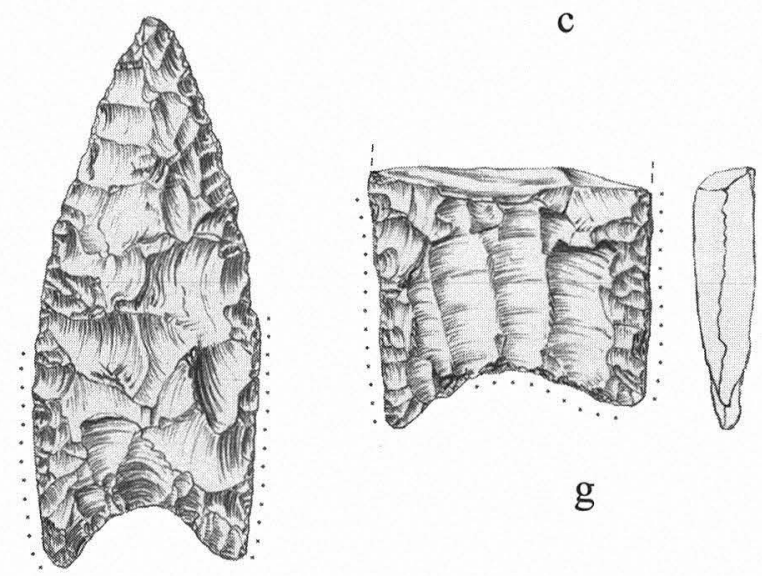

g

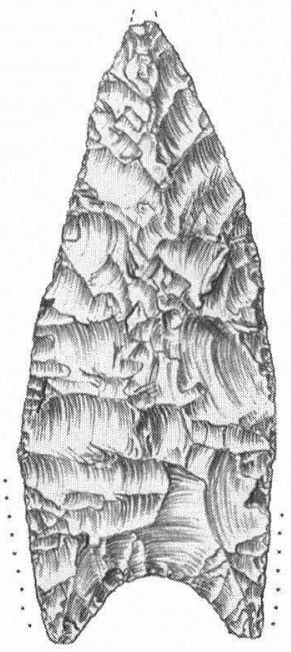

c
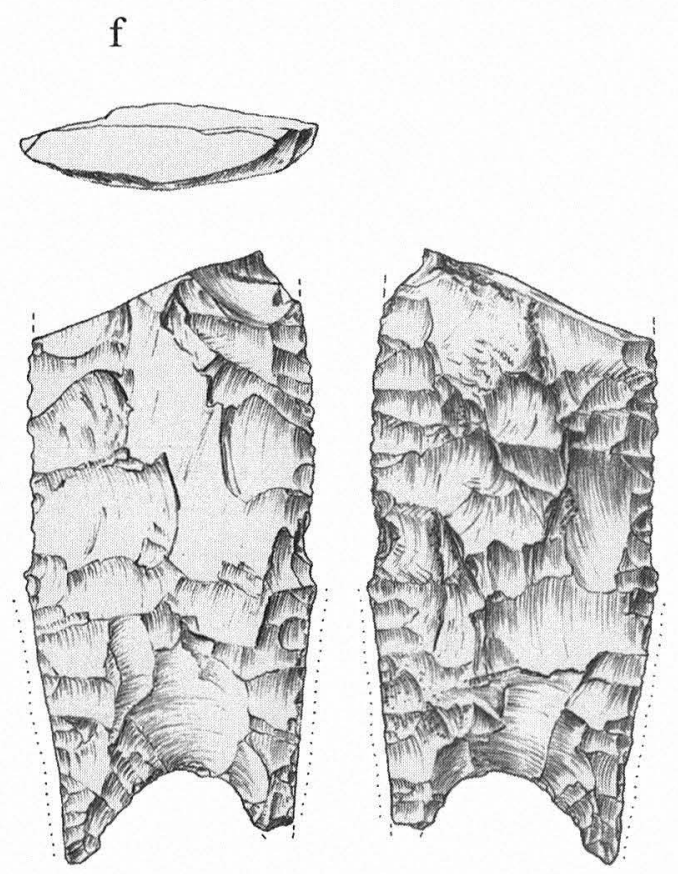

FIGURE 13-13. Golondrina-Barber points. Specimen numbers: (a) 33X2C-1; (b) 28T2B-1; (c) 15X1-1; (d) 23Q-1; (e) 15Y1-1; (f) 50YA-1; (g) $21 \mathrm{~L}-1$; (h) 13Q-2; (i) 18SB-2. 


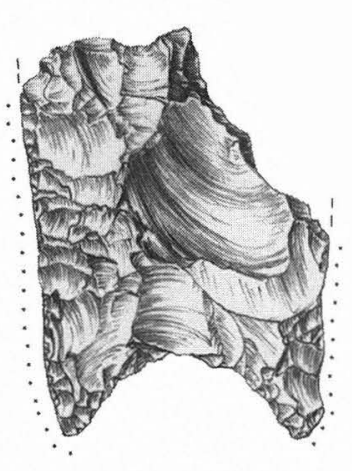

a
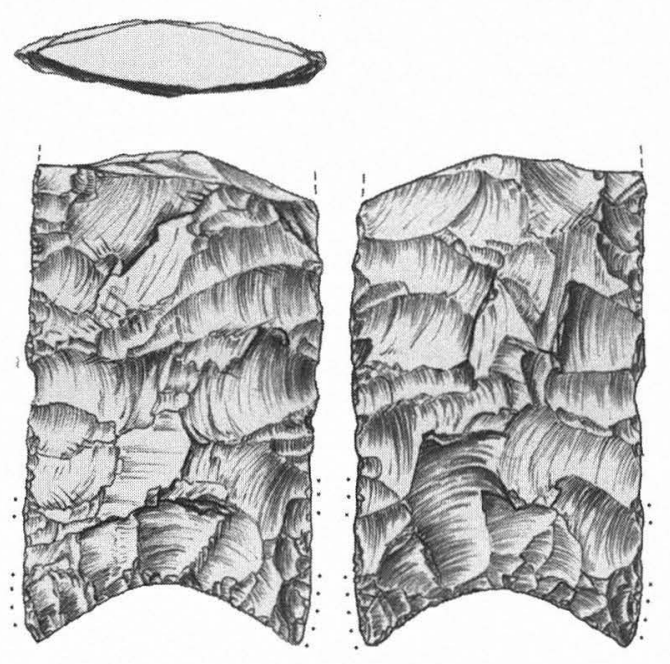

d
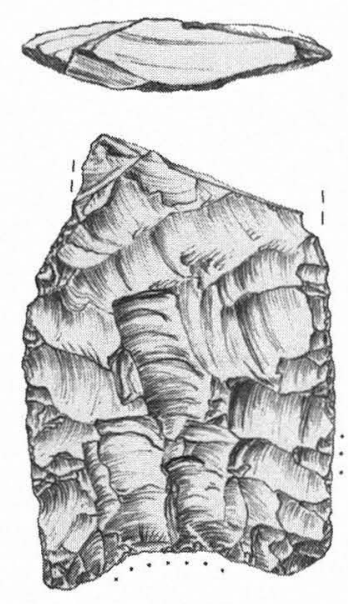

$\mathrm{f}$

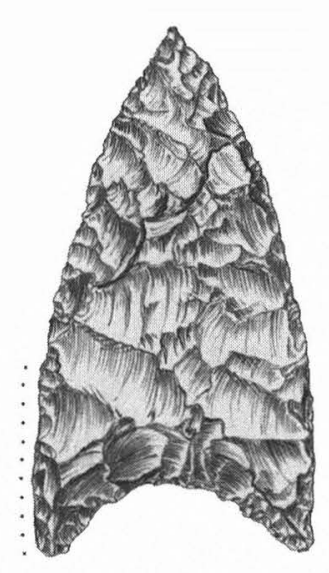

$\mathrm{b}$

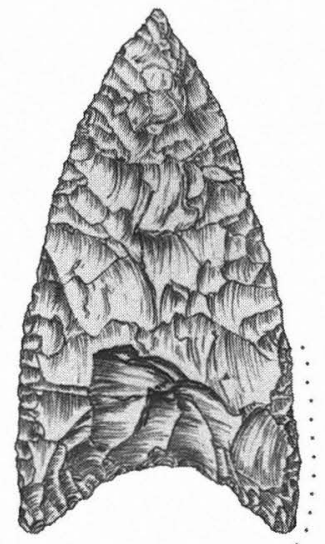

西
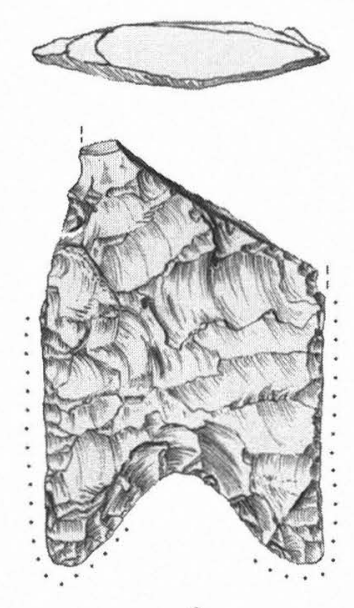

c

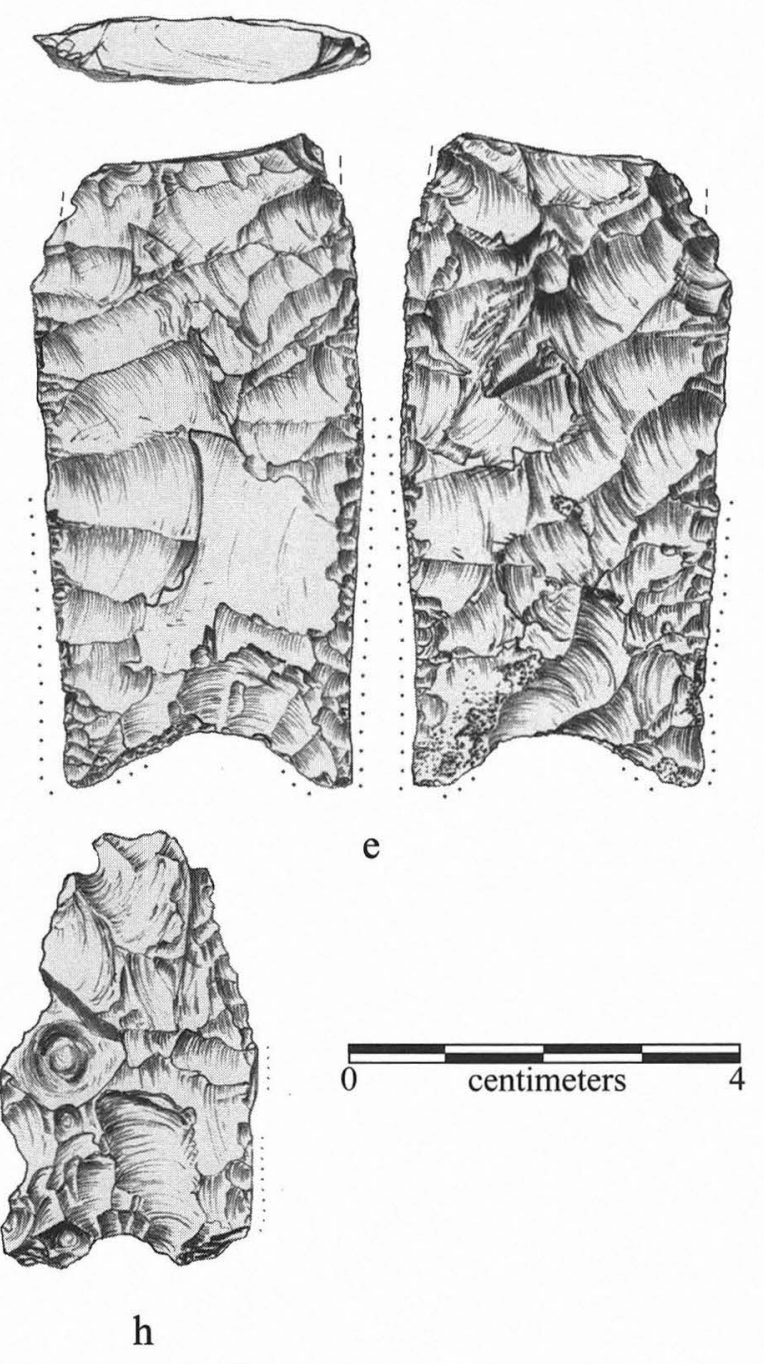

FIGURE 13-14. Golondrina-Barber points. Specimen numbers: (a) 26Y2C-1; (b) 10S-1; (c) 26R1-1; (d) 12U-1; (e) 51Y1B-1; (f) 25Z1C-1; (g) 37T2B-3; (h) 9O-3. 
TABLE 13-17

Stratigraphic Distribution of Golondrina-Barber Points

\begin{tabular}{|c|c|c|c|c|c|c|c|c|c|c|c|c|}
\hline \multirow[b]{2}{*}{ Elevation } & & \multicolumn{3}{|c|}{ Valley Margin } & \multicolumn{7}{|c|}{ Valley Floor A } & \multirow{2}{*}{\begin{tabular}{|c|} 
Valley Floor B \\
I/II
\end{tabular}} \\
\hline & & $\mathrm{X}$ & $\mathrm{X} / \mathrm{II}$ & IIIb & Isi-c & Isi-c/Id & Isi-c/II & $\mathrm{Id} / \mathrm{II}$ & II & II/IIIa & IIIa & \\
\hline 96.61 & -96.70 & & & 1 & & & & & & & & \\
\hline 96.51 & - 96.60 & 1 & & & & & & & & & & \\
\hline 96.41 & - 96.50 & & & & & & & & & & & \\
\hline 96.31 & - 96.40 & & & & & & & & & & & \\
\hline 96.21 & -96.30 & & & & & & & & & & & \\
\hline 96.11 & - 96.20 & 1 & & & & & & & & & & \\
\hline 96.01 & - 96.10 & & 1 & & & & & & & 1 & & \\
\hline 95.91 & -96.00 & & & & & & & & & 1 & & \\
\hline 95.81 & -95.90 & & & & & & & & & & & \\
\hline 95.71 & -95.80 & & & & & & & & 1 & 1 & 1 & \\
\hline 95.61 & - 95.70 & & & & & & & & & & & \\
\hline 95.51 & - 95.60 & & & & & & & & 2 & & & \\
\hline 95.41 & -95.50 & & & & & & & & 1 & 1 & & \\
\hline 95.31 & - 95.40 & & & & & & & & & & & \\
\hline 95.21 & -95.30 & & & & & & & & & & & \\
\hline 95.11 & - 95.20 & & & & & & 1 & 1 & 2 & & & \\
\hline 95.01 & - 95.10 & & & & & 1 & & 1 & & & & 1 \\
\hline 94.91 & -95.00 & & & & & & & 1 & & & & \\
\hline 94.81 & - 94.90 & & & & & & & & & & & 1 \\
\hline 94.71 & - 94.80 & & & & & & & & & & & \\
\hline 94.61 & -94.70 & & & & & & & & & & & \\
\hline 94.51 & $-\quad 94.60$ & & & & 1 & & & & & & & \\
\hline Totals: & & 2 & 1 & 1 & 1 & 1 & 1 & 3 & 6 & 4 & 1 & 2 \\
\hline
\end{tabular}

lanceolate points. Also, it should be noted that this same range of variability seems to be present in the points found in a cache in South Texas (Hester 1993:Figure 8, 1995a:Figure 10).

Golondrina-Barber points also share morphological characteristics with Dalton points but differ in flake patterning and chronological position (see Chapter 14 for more discussion.

\section{Miscellaneous Lanceolate Points and Fragments $(N=69)$}

Morphology: This group includes lanceolate specimens that do not fit type categories or are too fragmented to classify further. In haft shape, most are parallel-sided to contracting with straight to concave bases, and most are ground on lateral haft edges. Included in this group also are medial and distal sections with the typically organized flaking and blade outline common among lanceolate points (Figures 13-15 and 13-16).

Several specimens in this group are unusual or resemble other identified types and merit individual description. Specimen 30V2D-3 (see Figure 13-15a) is an odd point with a blunted, scraper-like tip and an indented, thick parallel-sided haft modified by basal thinning. The blade is ran- domly flaked, and blade edges, while resharpened bifacially, are not beveled.

Specimen 51PC-1 (see Figure 13-15b) is a thin (5.6 $\mathrm{mm}$ ), rather well-flaked point with a heavily ground parallel-sided haft and slightly ground basal concavity. A fine line of patina outlines the margins of this point in a pattern similar to that of the Clovis basal fragment (29S2D10; see below). In shape, this point most resembles St. Mary's Hall. Specimen 51PD-2 (see Figure 13-15c) is of similar thinness $(5.8 \mathrm{~mm})$; this specimen has been fractured longitudinally by burin-like impact fractures and bend breaks.

Specimen 33X1A-1 (see Figure 13-15d) appears to be a rebased tip, judging from its thick distal section and absence of lateral and basal edge grinding. The base has been modified by bifacial thinning. Specimen 25EE2B-1 was made on a thin flake and, as shown (see Figure 13-15g), retains a remnant scar of the original flake blank. In its exceptional thinness $(5.1 \mathrm{~mm})$, diminutive size, and haft shape, this point resembles the Midland type. Its early context (Unit I, in close proximity to the bison bone bed; see Chapter 7) would also support this contention. Specimen 15R1-1 (see Figure 13-15h) resembles Golondrina/Barber, as do three other lanceolate proximal sections in this 


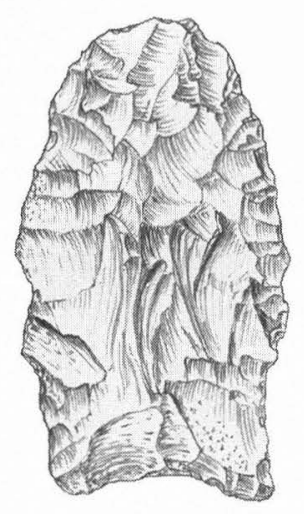

a

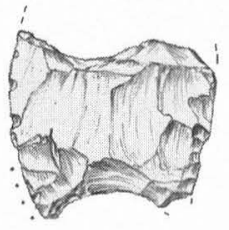

$\mathrm{e}$

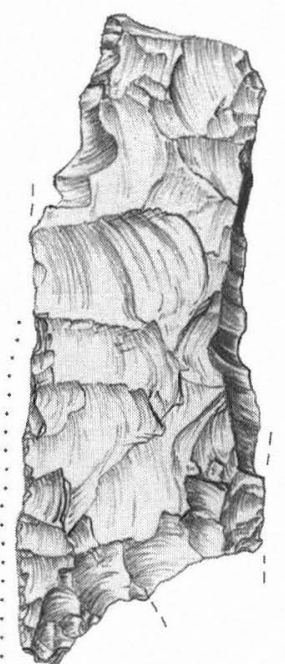

$\mathrm{h}$

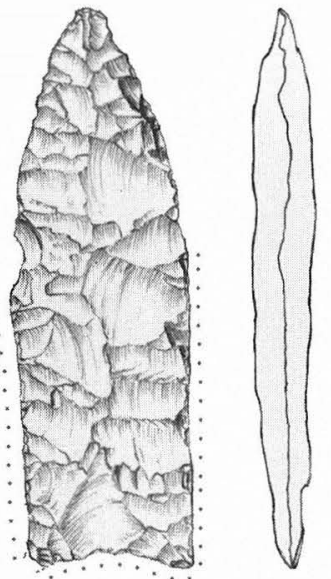

b
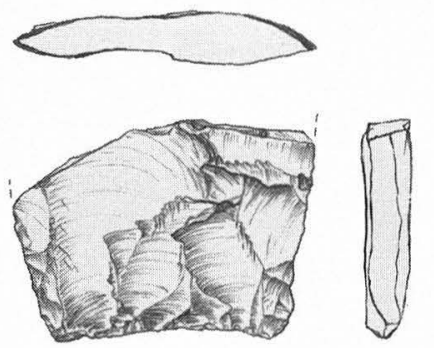

f
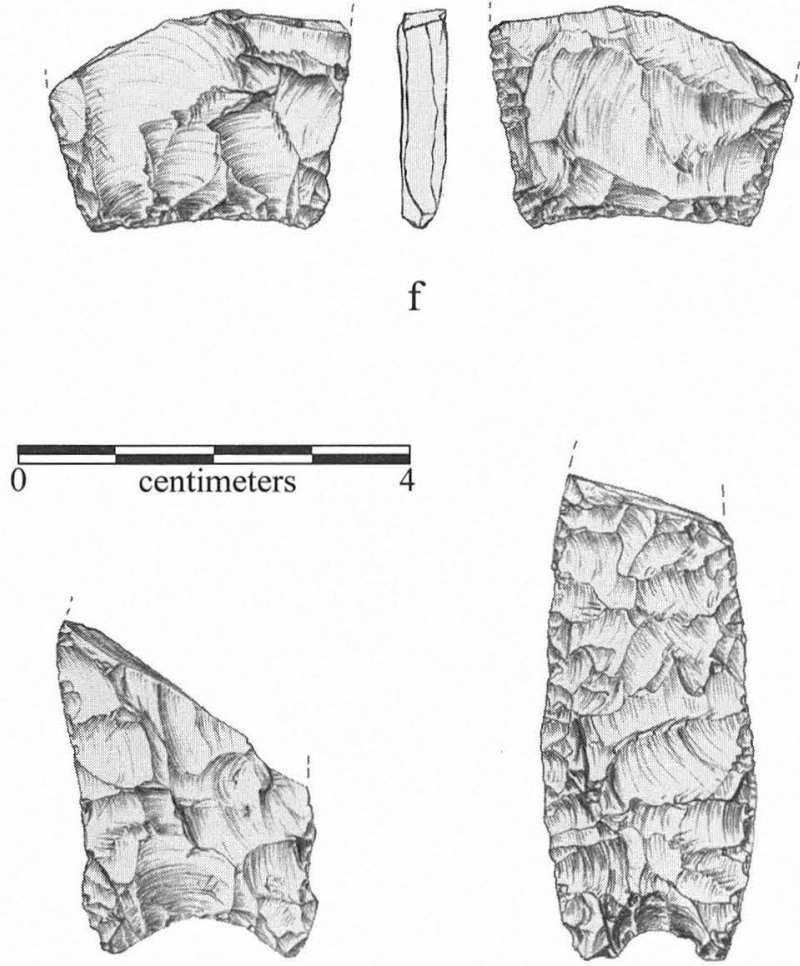

$\mathrm{j}$
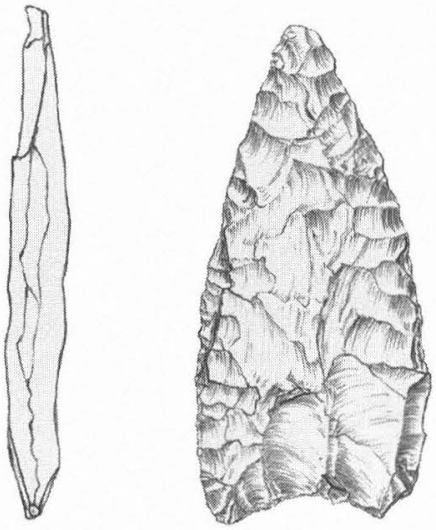

d

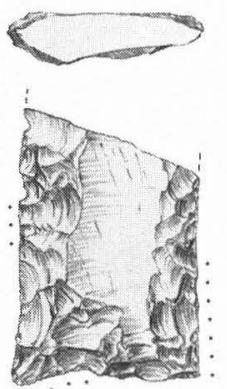

g

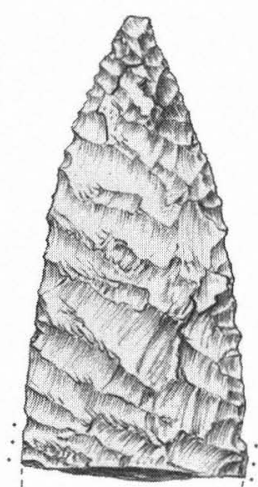

$\mathrm{k}$

FIGURE 13-15. Miscellaneous lanceolate points and fragments. Specimen numbers: (a) 30V2D-2; (b) 51PC-1; (c) 51PD-2; (d) 33X1A-4; (e) 50TC-3; (f) 25AA2C-4; (g) 25EE2B-1; (h) 15R1-1; (i) 10T-2; (j) 24R1-1; (k) 20\&21Q-19.

group. Figures 13-15k and 13-16 show distal and medial sections exhibiting very fine parallel-oblique flaking.

Metric Ranges: Table 13-18

Nonmetric Attributes: Longitudinal profiles are classed as tapered bases with thick medial sections on 2; tapered base with thick tip on 1 ; thick base with thin distal section on 1; generally equal proportions on 6 ; short wedge-shaped bases and tapered tips on 5; and wedge-shaped base, thick stem, and generally flat body on 1. Flake patterns are organized on 39 (oblique parallel, subparallel, oblique subparallel, chevron, collateral) random on 5 , and indeterminate on 25 . One specimen 


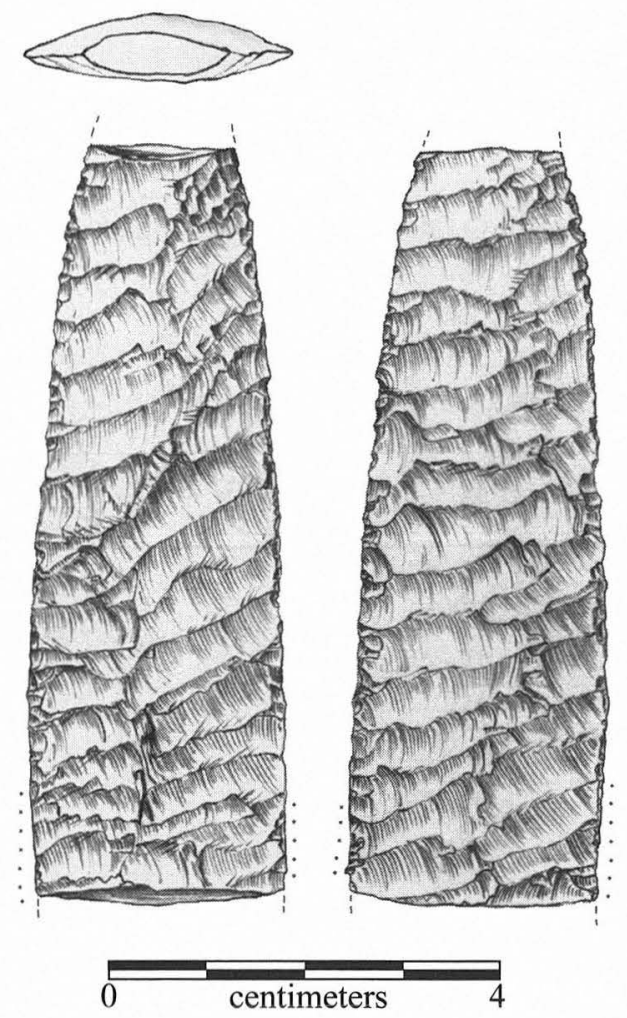

FIGURE 13-16. Miscellaneous lanceolate medial section 28T2B-2, with fine oblique-parallel flaking.

TABLE 13-18

Metric Ranges of Miscellaneous Lanceolate Points and Fragments (in mm)

\begin{tabular}{l|c|c|c}
\hline & Number & Minimum & Maximum \\
\hline Length & 5 & 48.0 & 81.0 \\
Width & 49 & 15.8 & 31.0 \\
Thickness & 67 & 3.7 & 12.0 \\
Haft grinding length & 20 & 4.8 & 34.4 \\
Proximal width & 11 & 12.9 & 23.6 \\
10-mm haft width * & 15 & 11.8 & 28.9 \\
10-mm haft thickness** & 17 & 3.7 & 8.4 \\
Basal concavity & 16 & 1.8 & 6.0 \\
\hline
\end{tabular}

*Not observed on 3 specimens.

**Not observed on 5 specimens.

showed a channel flake scar, and 2 also showed remnants of original flake blanks. Basal modification includes unifacial thinning on 10; bifacial thinning on 9; unifacial fluting on 1; bifacial fluting on 1 ; beveled base on 1 ; concave bevel on 10 ; secondary trimming on 4 ; and indeterminate on 33. Lateral haft grinding is slight on 13, moderate on 14 , heavy on 23 , and indeterminate on 11 . Basal grinding is slight on 8 , moderate/heavy on 9 , and indeterminate on 36 .
Material Attributes: Raw material is local Edwards chert (33 fine grained, 13 medium/coarse), nonlocal Edwards chert (13 fine grained, 6 medium/coarse), non-Edwards medium/ coarse chert on 1 , indeterminate on 1 , and indeterminate heat altered on 2. Patina is present on 14 (slight partial on 3 , slight overall on 2, moderate partial on 1 , moderate overall on 3, heavy partial on 3, and heavy overall on 2). Mineral precipitates are present on 9 . Heat alteration is slight on 6 , moderate on 9 , extensive on 5 , and indeterminate on 1 .

Tool State and Fractures: Four points are complete and 4 are nearly so; of these, 2 are damaged by impact and burinlike fractures, 1 by bend and burin-like fractures, and 1 by impact, bend, and burin-like fractures. Eleven others are proximal sections, 5 of which show multiple bend scars; 1 , impact and bend; 1 , multiple bend and thermal; 1 , multiple burin-like scars; 1 , multiple bend and burin-like; 1 , multiple burin-like and impact; and 1, burin and indeterminate fractures. Twentyseven are base sections, 2 of which were damaged by burin fractures, 5 by single bend fractures, 5 by multiple bend, 5 by thermal, 4 by bend and thermal, 3 by multiple bend and burin-like fractures, 1 by impact, bend, and thermal, 1 by impact, bend, and burin-like fractures, and 1 indeterminate. Six are distal sections, 1 of which shows a bend fracture; 2 , thermal; 1, multiple bend; 1, multiple bend and burin-like; and 1 bend and thermal. Sixteen are medial sections; fractures include bend on 1 , multiple bend on 6 , bend and thermal on 2 , thermal on 1 , impact and bend on 1 , multiple bend and burin-like on 4, and multiple bend and indeterminate on 1. The 1 remaining specimen is a lateral section displaying a longitudinal burin-like and bend break.

Rejuvenation: Twenty-four are resharpened (4 unifacially unbeveled, 12 alternately beveled, and 8 unifacially beveled) and 37 are indeterminate. Three specimens may have been rebased, including the concave lanceolate point shown in Figure 13-15d (33X1A-1).

Stratigraphic Distribution: As shown inTable 13-19, the indeterminate lanceolate specimens are widely distributed but are present in greatest numbers in stratigraphic units pertaining to the Early Archaic (Unit IIIa), Late Paleoinidian (Unit II), and the mixed deposits of the Unit II/ IIIa transition zone.

Comments: Assigning types to specimens in this category proved problematical for a variety of reasons, including the high degree of fragmentation and/or reworking of some. Other, more-complete specimens appear to fall outside the range of known variability for identified lanceolate types. Nonetheless, as mentioned above, it is likely that variants of St. Mary's Hall, Golondrina-Barber, possibly Midland, and such Archaic period lanceolates as Kinney are encompassed in this category.

\section{Concave Lanceolate, Preforms $(N=3)$}

Morphology: Relatively wide, medium thick to thick lanceolate bases with U-shaped to shallow arc-like basal con- 
TABLE 13-19

Stratigraphic Distribution of Miscellaneous Lanceolate Points and Fragments

\begin{tabular}{l|c|c|c}
\hline Unit & $\begin{array}{c}\text { Valley } \\
\text { Margin }\end{array}$ & $\begin{array}{c}\text { Valley Floor } \\
\text { A }\end{array}$ & $\begin{array}{c}\text { Valley Floor } \\
\text { B }\end{array}$ \\
\hline IIIc & 1 & 1 & \\
IIIb/c & 2 & 5 & \\
IIIb & 1 & & \\
Y & 1 & & \\
X/Y & 1 & 8 & \\
II/IIIa & 1 & & \\
X/II/IIIa & 1 & & \\
X & 2 & 12 & \\
X/II & 1 & 1 & \\
II & & & \\
IIIa/b & & & \\
IIIa & & & \\
III & & & \\
II/III & & & \\
Id/II & & & \\
Isi-c/II & & & \\
Isi-c & & & \\
Isi/Icl/Isi-c & & & \\
Other: & & & \\
Surface & & & \\
\hline \hline
\end{tabular}

cavities, although unfinished, are included herein (rather than in the "bifaces" section, below) to enable comparisons with the lanceolate projectile point categories (Figure 1317).

Metric Averages: Table 13-20

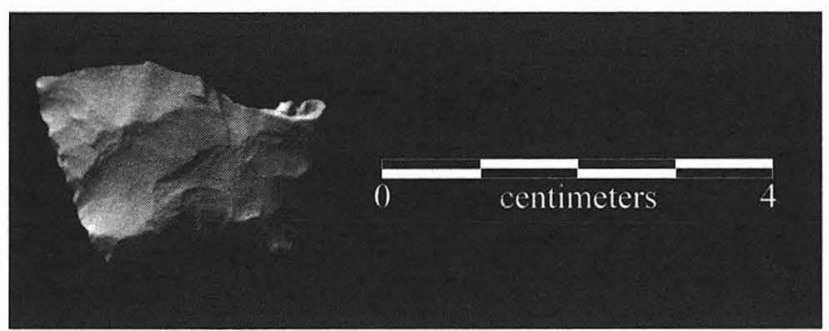

FIGURE 13-17. Concave lanceolate preform, Specimen 756-2.
Nonmetric Attributes: Longitudinal profiles are indeterminate. Flake patterns are random on 2 (with a flake blank scar on 1) and indeterminate on 1 . Basal modification is concave bevel on 1 and secondary trimming on 2 . Lateral haft grinding is absent.

Material Attributes: Raw material is local Edwards chert (2 fine grained, 1 medium/coarse). Heat damage is extensive on 1. No patina or mineral precipitates were observed.

Tool State and Fractures: All 3 are bases, 2 of which exhibit manufacturing breaks (combination overshot and bend), and the other, thermal fractures.

Stratigraphic Distribution: All 3 were recovered from Valley Floor A, 2 from Unit IIIa and 1 from transition Unit II/ IIII.

Comments: Although they may have been intended as bifacial knives, the shape and size of these specimens also suggests they may have been preforms for Angostura or certain Hoxie variants.

\section{Fluted Lanceolate}

\section{Clovis Base Fragment $(N=1)$}

Morphology: The Clovis base fragment has a thick, parallel-sided haft with a flute scar on each face (Figure 13-18); lateral haft edges are heavily ground and the basal edge is moderately to heavily ground.

Metric Values: Width is $22.7 \mathrm{~mm}$; thickness is $6 \mathrm{~mm}$; and $10-\mathrm{mm}$ haft thickness is $5.4 \mathrm{~mm}$.
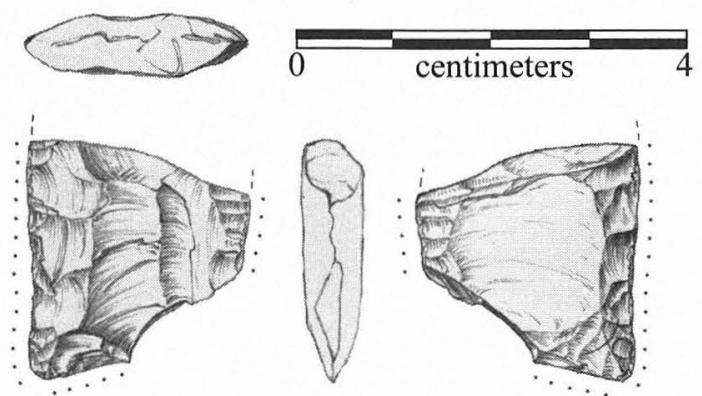

FIGURE 13-18. Clovis base fragment, Specimen 29S2D-10.

TABLE $13-20$

Summary Metric Data for Concave Lanceolate Preforms (in mm)

\begin{tabular}{l|c|c|c|c|c}
\hline & Number & Minimum & Maximum & Mean & $1-\sigma$ \\
\hline Width & 2 & 29.0 & 30.0 & 29.5 & 0.7 \\
Thickness & 3 & 5.0 & 8.0 & 6.3 & 1.5 \\
Proximal haft width & 2 & 20.0 & 22.0 & 21.0 & 1.4 \\
Basal concavity depth & 3 & 2.9 & 3.8 & 3.4 & 0.5 \\
10-mm thickness & 2 & 4.6 & 6.3 & 5.5 & 1.2 \\
Basal concavity width & 2 & 9.8 & 14.1 & 12.0 & 3.0 \\
\hline \hline
\end{tabular}


Nonmetric Attributes: Longitudinal profile is indeterminate; flake pattern is flute (or channel flake scar); basal modification is bifacial fluting; lateral grinding is heavy; and basal grinding is moderate/heavy.

Material Attributes: Raw material is local fine-grained Edwards cherts; patina is moderate, partial coverage; mineral precipitates are absent; heat damage is absent.

Tool State and Fractures: This base fragment exhibits 2 bend fractures.

Rejuvenation: Resharpening and recycling are indeterminate.

Stratigraphic Distribution: The Clovis base fragment is from Unit II/IIIa and is considered out of context.

Comments: This Clovis base fragment is not a clear indicator for Clovis occupation at the site since it might have been picked up off-site and carried in much later.

\section{Clovis Distal Section $(N=1)$}

Morphology: The Clovis distal section is thick with subparallel flaking (Figure 13-19).

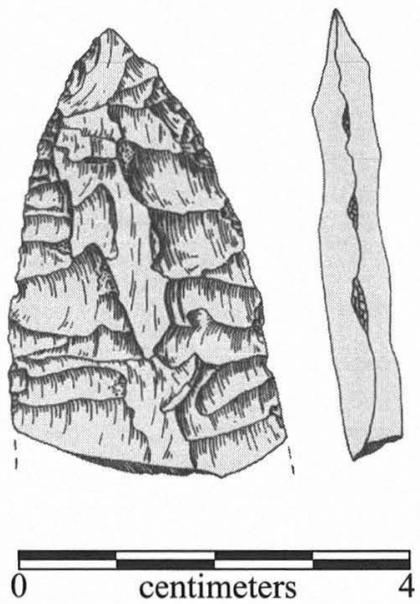

FIGURE 13-19. Clovis distal section, Specimen OR734.

Metric Values: Width is $28 \mathrm{~mm}$ and thickness is $7 \mathrm{~mm}$.

Nonmetric Attributes: Longitudinal profile is indeterminate; flake pattern is subparallel; basal modification is indeterminate; lateral and basal grinding are indeterminate.

Material Attributes: Raw material is local mediumcoarse-grained Edwards; patina, mineral precipitates, and heat damage are absent.

Tool State and Fractures: This distal section has a bend fracture.

Rejuvenation: Resharpening is indeterminate.

Stratigraphic Distribution: The Clovis distal section is from Unit $\mathrm{Igl} / \mathrm{Isi} / \mathrm{Icl}$ at elevation $93.09 \mathrm{~m}$.

Comments: A distal section is not ordinarily considered diagnostic but this specimen was found at considerable depth in the site and compares very favorably in terms of size, cross-section, longitudinal taper, convergence of the convex edges, and flake pattern with similar specimens from Clovis-age deposits at Blackwater Draw and elsewhere.

\section{Bifurcate Stemmed Points}

$$
\operatorname{Baker}(N=7)
$$

Morphology: These medium-sized points have strong shoulders and barbs and frequently evidence organized flake patterns. Stems are medium to long and slightly expanding with deep V-shaped or, less typically, U-shaped basal concavities. Basal ears are commonly pointed, and stem edges are generally straight (Figures 13-20 and 1321).

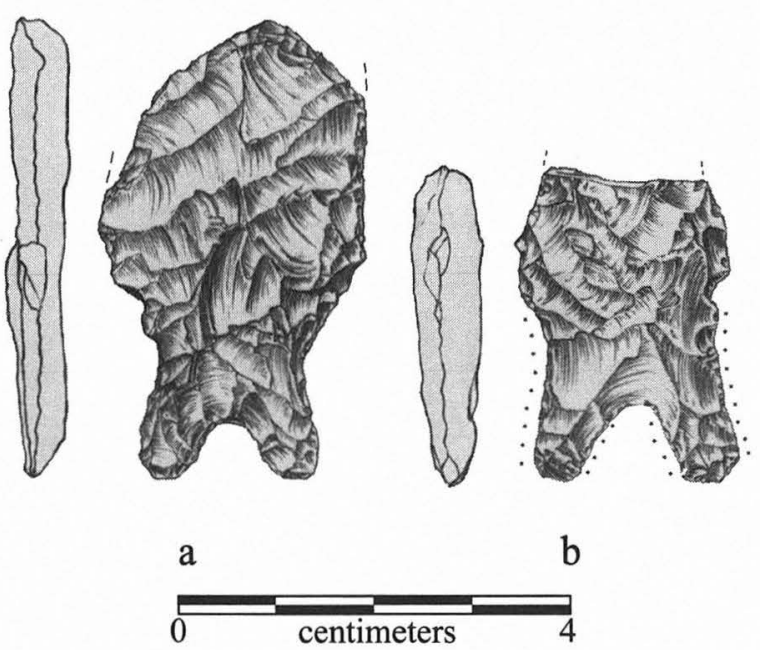

FIGURE 13-20. Baker points. Specimen numbers: (a) 20H-2; (b) 180-1.

Metric Averages: Table 13-21

Nonmetric Attributes: Longitudinal profiles include 2 tapered base and tip, 1 flat, and 4 indeterminate. Flake patterns are organized on 4 (all oblique subparallel), random on 1 , and indeterminate on 2 . Basal modification includes concave bevels on 4 ( 1 of which is in combination with flute-like flaking on the reverse face), unifacial thinning on 1, secondary trimming on 1 , and 1 indeterminate. Stem grinding is moderate to heavy on 2 , slight dulling on 3 .

Tool State/Fractures: All are proximal sections exhibiting a complex suite of impact fractures and/or burin-like breaks that removed distal and lateral blade sections on 4, including barbs on 3 of those. Two other specimens each lack barbs and a basal ear. Additional bend fractures were observed on 3 specimens, and another exhibits thermal damage.

Material Characteristics: Raw material is local finegrained Edwards chert. Heat damage is slight on 1. No patina or mineral precipitates are present. 


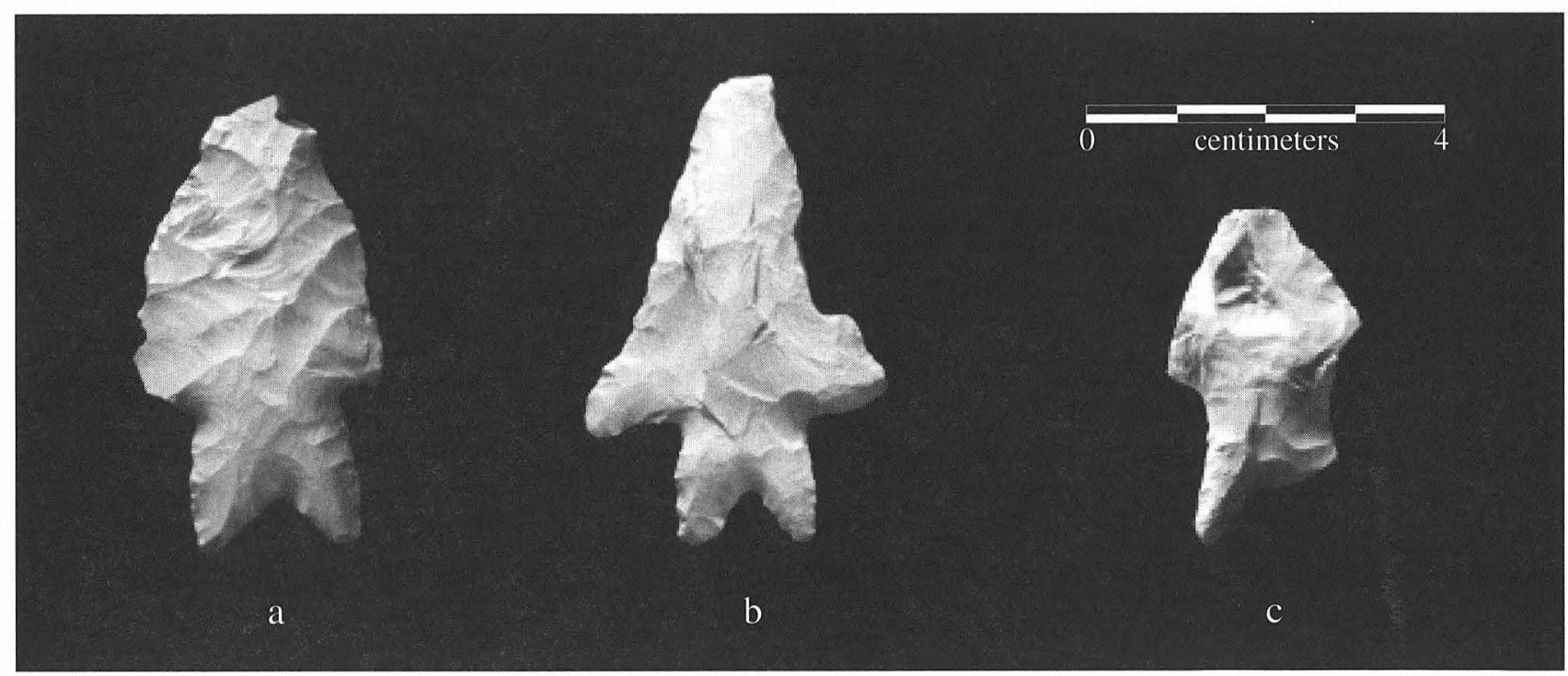

FIGURE 13-21. Baker points. Specimen numbers: (a) 12L-1; (b) 9O-1; (c) 36M-2.

TABLE 13-21

Summary Metric Data for Baker Points (in $\mathrm{mm}$ )

\begin{tabular}{|c|c|c|c|c|c|}
\hline & Number & Minimum & Maximum & Mean & $1-\sigma$ \\
\hline Width & 1 & & 28.0 & & \\
\hline Thickness & 7 & 5.0 & 9.0 & 7.00 & 1.41 \\
\hline Stem grinding length & 7 & 13.0 & 20.0 & 15.77 & 2.45 \\
\hline Proximal stem width & 4 & 15.7 & 21.0 & 18.30 & 2.23 \\
\hline Distal stem width & 6 & 13.0 & 18.0 & 15.50 & 1.98 \\
\hline Basal concavity depth & 6 & 5.6 & 13.0 & 7.40 & 2.88 \\
\hline Distal stem thickness & 3 & 5.9 & 6.7 & 6.40 & 0.44 \\
\hline Basal concavity width & 3 & 8.4 & 10.9 & 9.33 & 1.37 \\
\hline
\end{tabular}

Rejuvenation: One is resharpened unifacially, unbeveled; 3 are alternately beveled; and 1 is beveled unifacially.

\section{Stratigraphic Distribution: Table 13-22}

Comments: This type is more frequently reported in the Lower Pecos area (Turner and Hester 1993:77). Although it shares attributes with other early bifurcate stem varieties, it may be somewhat distinguished by a longer, generally straight-edged, expanding stem, as opposed to the more recurved, flared stem of Uvalde, and generally shorter, straighter and more heavily ground stem of Gower. Faulty classification may be a factor in the widespread distribution shown for this group (see Table 13-22).

\section{$\operatorname{Bandy}(N=10)$}

Morphology: These diminutive, thin triangular points have large barbs, sharply pointed distal tips, and typically fine flaking of the blades (Figures 13-22 and 13-23). The stems are expanding, formed by corner notching, and commonly are somewhat thicker than the body. The bases are
TABLE $13-22$

Stratigraphic Distribution of Baker Points

\begin{tabular}{l|c|c}
\hline Unit & Valley Floor A & Valley Margin \\
\hline IIIb/c & 1 & 1 \\
IIIb & 1 & 1 \\
IIIa & & 1 \\
X/Y & & 2 \\
X & & \\
\hline \hline
\end{tabular}

gently concave to slightly V-shaped and may be lightly smoothed on edge.

Metric Averages: Table 13-23

Nonmetric Attributes: Longitudinal profiles include 3 thick base; 1 tapered base and tip; 1 flat; 3 wedge-shaped base, moderately thick stem and flat body; and 2 indeterminate. Flake patterns are organized on 7 (oblique subparallel, subparallel, and 1 chevron), random on 2, and indeterminate on 1 ; basal modification consists of unifacial thinning on 4 , bifacial thinning on 3 , concave beveling on 1 , and secondary trimming on 2 . Lateral stem grinding is 


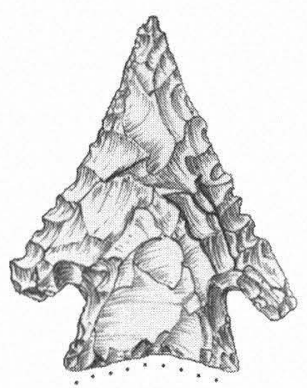

a

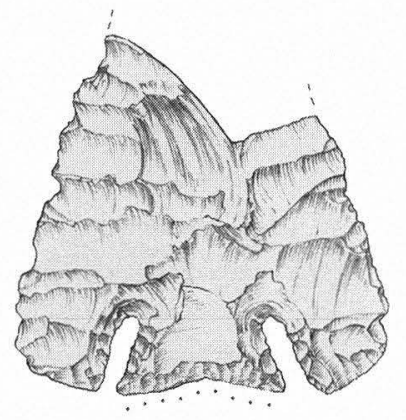

d

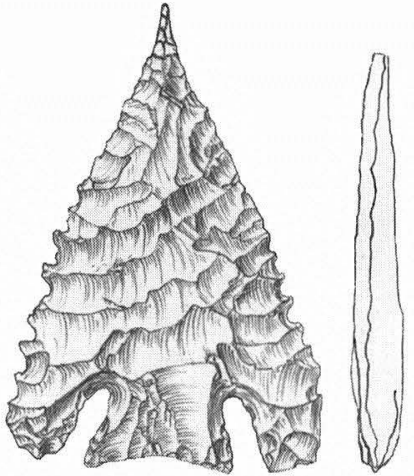

b
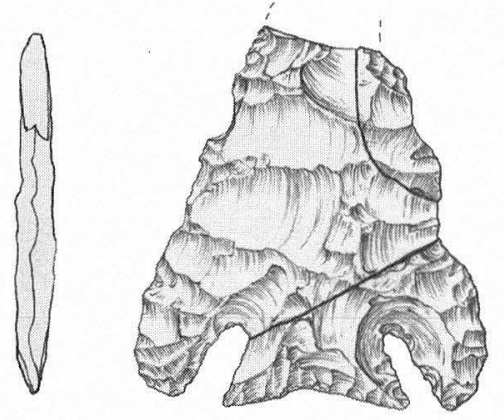
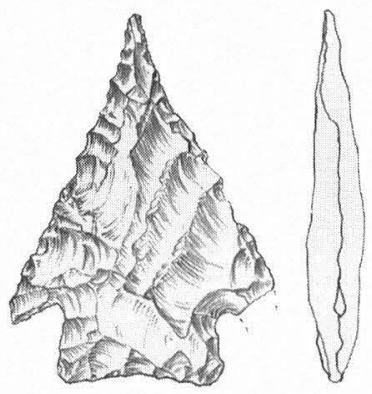

c

Figure 13-22. Bandy points. Specimen numbers: (a) 14K1-1; (b) 5H-1; (c) 21K-1; (d) 50LD-5; (e) 55M.

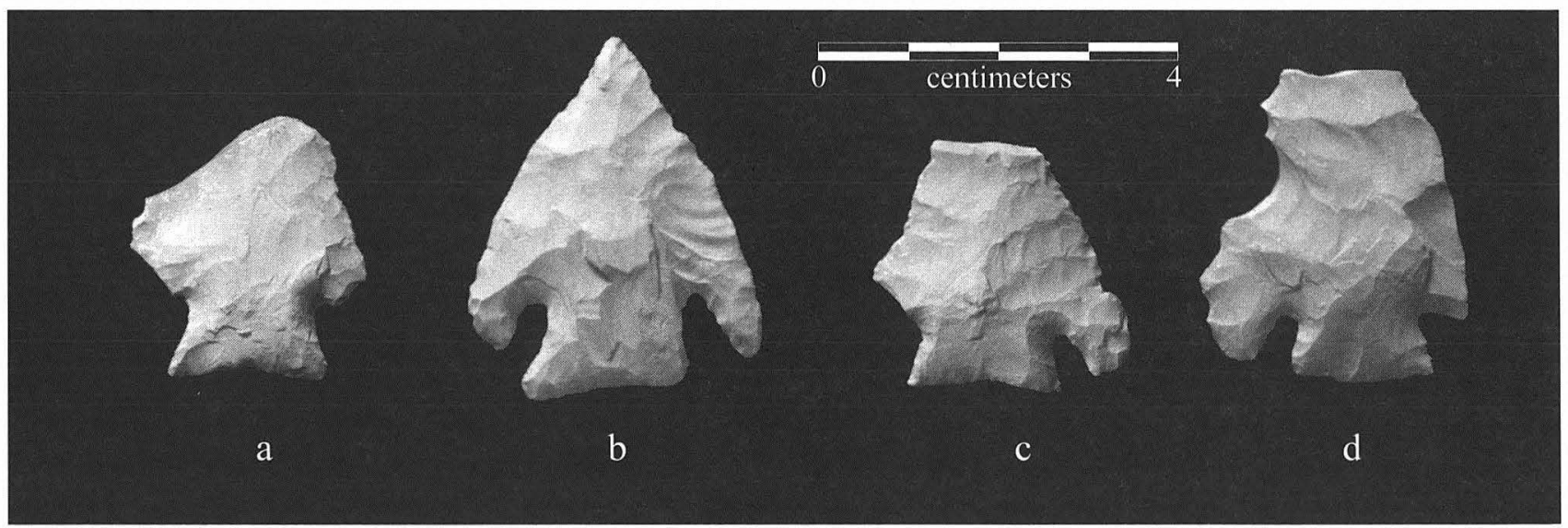

FIGURE 13-23. Bandy points. Specimen numbers: (a) 23M-1; (b) 21N-3; (c) 11M-1; (d) 698-3.

slight on 1, and the bases of 5 are smoothed.

Material Attributes: Raw material is local Edwards chert ( 8 fine grained, 1 medium/coarse), and nonlocal Edwards fine-grained chert $(n=1)$. Patina is moderate overall on 1 specimen; otherwise, no patina or mineral precipitates were observed.

Tool State and Fractures: Three are complete or nearly so, and 1 lacks only a barb tip. The other 6 are proximal sections exhibiting multiple bend fractures and, in 1 instance, heat damage.

Rejuvenation: One is resharpened unifacially, unbeveled; 2 bifacially, unbeveled; 1 alternately beveled; 1 unifacially beveled; 3 indeterminate; and 2 are not resharpened. 
TABLE 13-23

Summary Metric Data for Bandy Points (in mm)

\begin{tabular}{|c|c|c|c|c|c|}
\hline & Number & Minimum & Maximum & Mean & $1-\sigma$ \\
\hline Length & 3 & 37.0 & 41.0 & 38.3 & 2.3 \\
\hline Width & 8 & 28.0 & 38.0 & 31.9 & 3.7 \\
\hline Thickness & 10 & 4.0 & 7.0 & 5.1 & 0.9 \\
\hline Stem grinding length & 9 & 6.0 & 11.0 & 8.0 & 1.3 \\
\hline Proximal stem width & 10 & 15.0 & 19.0 & 17.3 & 1.3 \\
\hline Distal stem width & 9 & 12.0 & 16.0 & 14.3 & 1.3 \\
\hline Basal concavity depth & 9 & 1.1 & 2.1 & 1.6 & 0.3 \\
\hline Basal convexity length & 1 & 2.0 & 2.0 & & \\
\hline Distal stem thickness & 8 & 3.9 & 5.2 & 4.7 & 0.4 \\
\hline Basal concavity width & 8 & 12.4 & 15.3 & 13.9 & 1.0 \\
\hline
\end{tabular}

Stratigraphic Distribution: Bandy points at WilsonLeonard were confined chiefly to deposits pertaining to the latter part of the Early Archaic and Middle Archaic, with 2 in the Unit IIIa/b transition zone and 4 in Unit IIIb; dating can be estimated from ca. $6500 / 6000$ to 4000 в.P. (Table 13-24).

Comments: Like Baker, this point is traditionally recorded in the Lower Pecos and south Texas areas (McReynolds 1993; Turner and Hester 1993). Overall, it resembles Martindale - an apparent contemporary in Central Texasalthough it is generally thinner, more finely made, and typically lacks the characteristic "fishtail," recurved base. However, several Wilson-Leonard specimens classed as Bandy (see 50LD-5, 55M; Figure 13-22d, e) exhibit concave bases that show close affinities to the Martindale shape. Specimens from Wilson-Leonard and other collections frequently show needle-like distal tips (McReynolds 1993:Figure 1d, 2c, d, h).

\section{Bandy-like $(N=1)$}

Morphology: This is a thin, flat, broad-bladed point with strong shoulders and barbs; flaking is subparallel on one face. Unlike classic Bandy, the stem is widely expanding and slightly convex on the basal edge (Figure 13-24). Basal thinning is bifacial, and the point shows no resharpening. Possibly an unfinished point, it has been split obliquely by a bend or perverse fracture and exhibits scalloped edge damage.

TABLE 13-24

Stratigraphic Distribution of Bandy Points

\begin{tabular}{l|c|c|c}
\hline Unit & $\begin{array}{c}\text { Valley } \\
\text { Floor A }\end{array}$ & $\begin{array}{c}\text { Valley } \\
\text { Floor B }\end{array}$ & $\begin{array}{c}\text { Valley } \\
\text { Margin }\end{array}$ \\
\hline Y/IIIc & & & 1 \\
IIIb & 2 & & 3 \\
IIIa/b & 1 & 1 & 1 \\
III & & & \\
Other & & & \\
surface & 1 & & \\
\hline \hline
\end{tabular}

Metric Values: Dimensions are $47 \mathrm{~mm}$ in length, $29 \mathrm{~mm}$ in width (incomplete), and $4 \mathrm{~mm}$ in thickness. Stem dimensions are $10 \mathrm{~mm}$ in length, $16 \mathrm{~mm}$ in distal width, and $19 \mathrm{~mm}$ in proximal width, with a basal convexity of $2 \mathrm{~mm}$.

Material Attributes: Of fine-grained local chert, it shows no heat damage, patina, or mineral precipitates.

Stratigraphic Context: Unit IIIa/b, Valley Floor A.

\section{Contracting Stem, Convave Base $(N=4)$}

Morphology: These small to medium-sized triangular points have a medium length bifurcate stem with convex lateral edges contracting gently to a medium to narrow width base (Figures 13-25 and 13-26). In addition to a basal concavity of medium depth, several specimens display long, Vshaped basal thinning flake scars on their stem. Two retain small, splayed barbs on strong shoulders, and the others exhibit narrow blades resharpened in an alternate bevel opposing the slight beveling of the stems.

Metric Averages: Table 13-25

Nonmetric Attributes: Longitudinal profiles include 2 tapered base and tip and 2 wedge-shaped base and tip. Flake patterns are organized on 2 (oblique subparallel, chevron), random on 1, and indeterminate on 1; basal modifica-

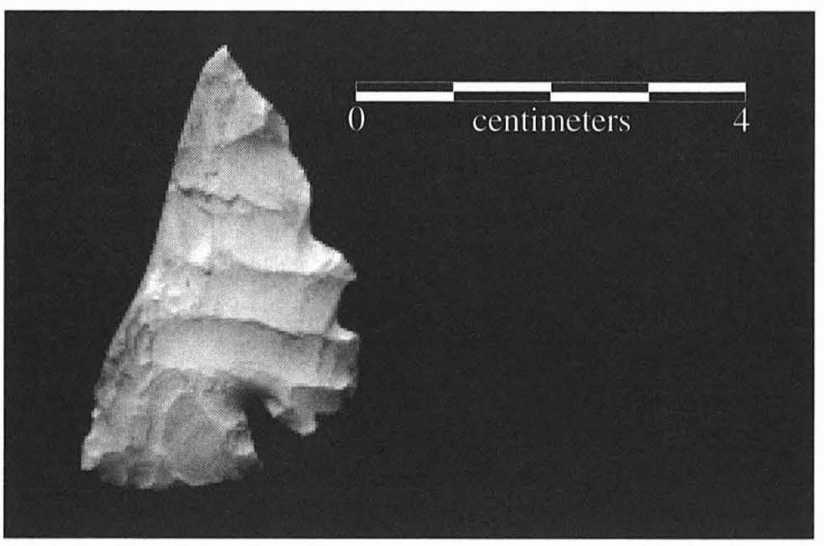

FIGURE 13-24. Bandy-like point, Specimen 692-1. 


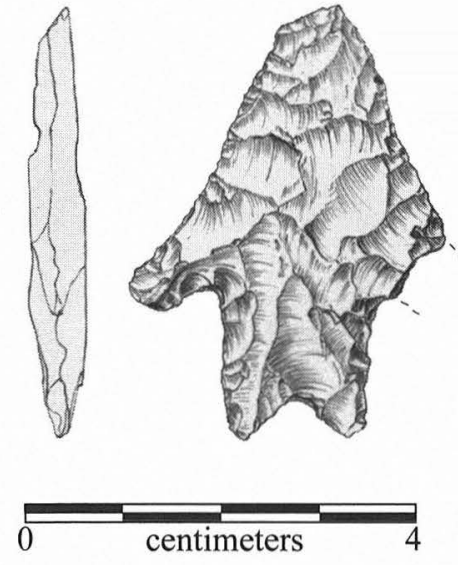

FIGURE 13-25. Contracting stem, concave base point, Specimen 21M2.

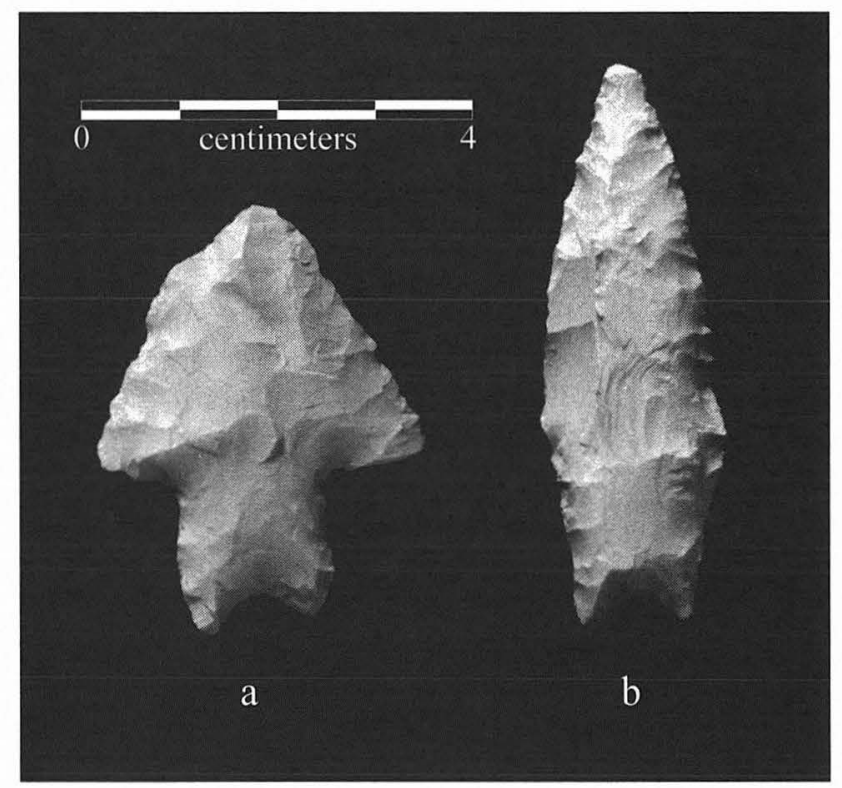

FIGURE 13-26. Contracting stem, concave base points. Specimen numbers: (a) $11 \mathrm{M}-2$; (b) $250-4$. tion consists of unifacial thinning on 1, bifacial thinning on 2 , and concave bevel on 1. Lateral stem grinding is slight on 2.

Material Attributes: Raw material is local Edwards finegrained chert on all. No patina, heat alteration, or mineral precipitates were observed.

Tool State and Fractures: Two are complete, 1 lacks only a barb, and 1 lacks a distal tip and barbs due to multiple impact and bend breaks.

Rejuvenation: All are resharpened ( 2 unifacially, unbeveled, 1 alternately beveled, and 1 unifacially beveled).

Stratigraphic Distribution: Table 13-26

Comments: Although these points resemble both Pedernales and Gower types, their stratigraphic placement suggests an Early Archaic derivation and affiliation with other early bifurcate stem groups.

TABLE 13-26

Stratigraphic Distribution of Points with

Contracting Stems, Concave Bases

\begin{tabular}{l|c|c}
\hline Unit & Valley Floor A & Valley Margin \\
\hline IIIb & 1 & 2 \\
IIIa/b & 1 & \\
IIIa & & \\
\hline \hline
\end{tabular}

\section{$\operatorname{Gower}(N=14)$}

Morphology: These are small triangular points with short to medium-length stems that are frequently dulled or ground on lateral margins (Figures 13-27 and 13-28). Stems are generally straight to slightly convex-edged and terminate in downward pointing, rounded basal "ears." Basal concavities are typically of medium depth, varying from a U- to Vshape and may exhibit a radially aligned retouch of the flake scar. Shoulders are "weak" on all specimens in this assemblage, and several display remnants of small barbs. Gower points may appear as thick or chunky in longitudinal profile, due in part to resharpening but also to poorly thinned bases.

TABLE 13-25

Summary Metric Data for Contracting Stem, Concave Base Points (in mm)

\begin{tabular}{l|c|c|c|c|c}
\hline & Numbers & Minimum & Maximum & Mean & $1-\sigma$ \\
\hline Length & 3 & 45.0 & 70.0 & 57.7 & 12.5 \\
Width & 2 & 19.0 & 21.0 & 20.0 & 1.4 \\
Thickness & 4 & 5.0 & 7.0 & 6.5 & 1.0 \\
Stem length & 4 & 13.0 & 16.0 & 15.0 & 1.4 \\
Proximal stem width & 4 & 12.0 & 14.0 & 13.5 & 1.0 \\
Distal stem width & 4 & 15.0 & 16.0 & 15.8 & 0.5 \\
Basal concavity depth & 4 & 2.7 & 3.8 & 3.4 & 0.5 \\
Distal stem thickness & 4 & 5.2 & 7.1 & 6.3 & 0.8 \\
Basal concavity width & 4 & 7.5 & 7.9 & 7.7 & 0.2 \\
\hline \hline
\end{tabular}




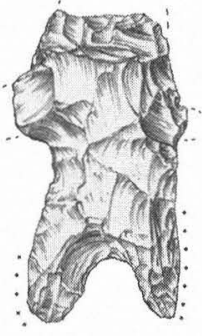

a
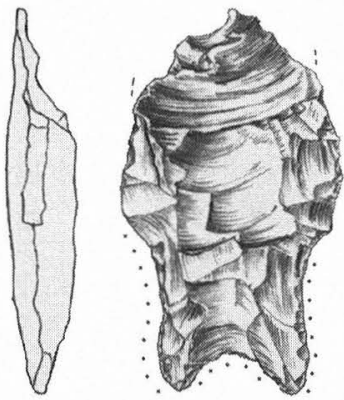

$\mathrm{b}$

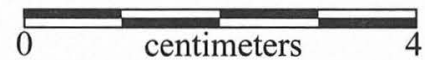

FIGURE 13-27. Gower points. Specimen numbers: (a) 280-3; (b) 50PB-1.

\section{Metric Averages: Table 13-27}

Nonmetric Attributes: Longitudinal profiles include 2 tapered base and tip; 3 thick tip; 2 wedge-shaped base and tapered tip; and 7 indeterminate. Flake patterns are organized on 3 (oblique subparallel), random on 3 (with original flake scar blank on 1), and 8 are indeterminate. Basal modification consists of unifacial thinning on 3 , concave bevel on 8 , secondary trimming on 2 , and 1 unmodified. Lateral stem grinding is slight on 1 , moderate on 4 , heavy on 7 , and absent on 2 .

Material Attributes: Raw material is local Edwards finegrained chert for 11, nonlocal Edwards fine-grained chert for 2 , and 1 indeterminate heat altered. Patina is slight overall on 1. Mineral precipitates were observed on 1 . Heat damage is slight on 2 and extensive on 1.

Tool State and Fractures: Four are largely complete, lacking only distal sections or, in 1 case, a basal ear; of these, 2 display bend fractures, and 2 , impact. The remaining 10 specimens are proximal sections, 5 of which exhibit multiple impact-related fractures; 2 , multiple bend fractures; 2 , thermal fractures; and 1, indeterminate breaks.

Rejuvenation: Seven are resharpened (1 bifacially unbeveled, 5 alternately beveled, and 1 beveled unifacially); 6 are indeterminate; and 1 is not resharpened.

Stratigraphic Distribution: The highest frequency of Gower is in the Unit IIIa/b transition zone $(n=5)$ and IIIb $(n=4)$, which may be pertinent to the later part of the Early Archaic (Table 13-28).

Comments: In his definition of Gower, Shafer (1963:65) identifies a distinctive basal concavity formed by the removal of one or two flakes, typically on a single face. Specimens further are described as rather crude. Lateral stem edge grinding was not characteristic of the type-site Gowers, although several identified by Shafer as Gower

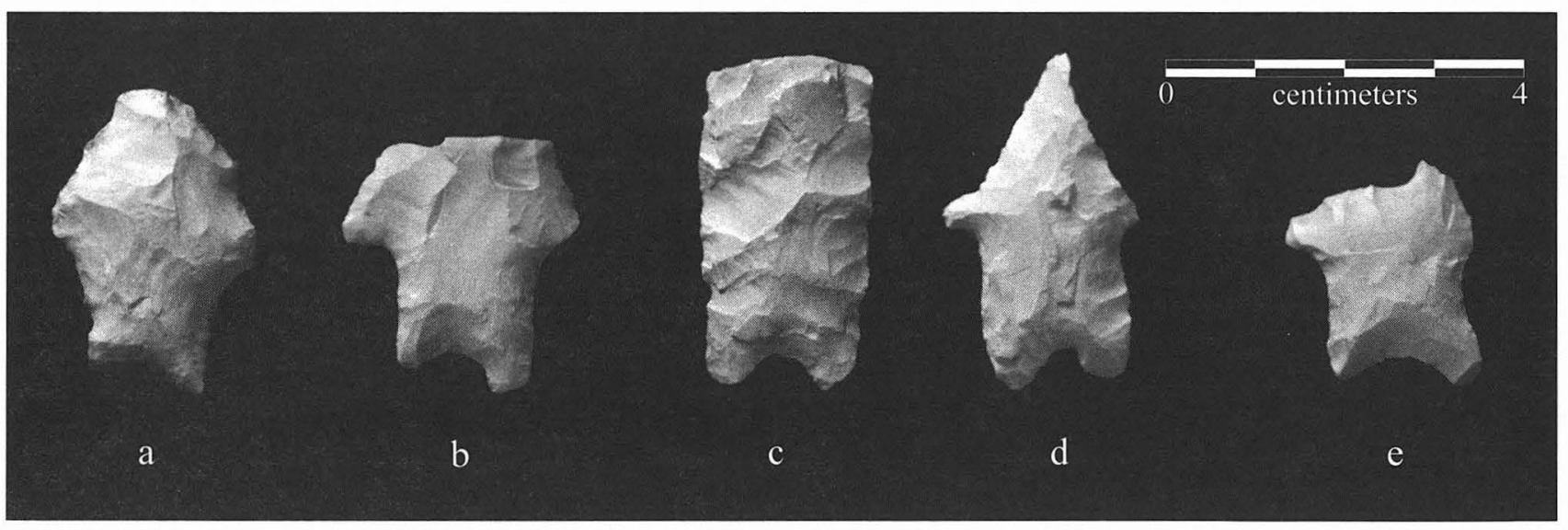

FigURE 13-28. Gower points. Specimen numbers: (a) 31R2-1; (b) 31O-2; (c) 24P2-3; (d) 21L-2; (e) 28O-2.

TABLE 13-27

Summary Metric Data for Gower Points (in mm)

\begin{tabular}{|c|c|c|c|c|c|}
\hline & Number & Minimum & Maximum & Mean & $1-\sigma$ \\
\hline$\overline{\text { Width }}$ & 8 & 17.0 & 26.0 & 20.8 & 2.9 \\
\hline Thickness & 14 & 5.0 & 8.0 & 6.4 & 1.1 \\
\hline Stem length & 10 & 11.0 & 18.0 & 15.1 & 2.2 \\
\hline Proximal stem width & 11 & 13.0 & 22.0 & 16.3 & 2.2 \\
\hline Distal stem width & 12 & 12.0 & 16.0 & 15.1 & 1.2 \\
\hline Basal concavity depth & 11 & 2.0 & 6.0 & 3.9 & 1.2 \\
\hline Distal stem thickness & 8 & 4.1 & 6.8 & 5.9 & 1.0 \\
\hline Basal concavity width & 8 & 5.2 & 10.8 & 8.8 & 1.7 \\
\hline
\end{tabular}


TABLE 13-28

Stratigraphic Distribution of Gower Points

\begin{tabular}{l|c|c|c}
\hline Unit & Valley Floor A & Valley Floor B & Valley Margin \\
\hline IIIc & 2 & & 1 \\
IIIb & 5 & & 2 \\
IIIa/b & 2 & & \\
IIIa & & 1 & \\
III & 1 & & \\
Other & & & \\
surface & 1 & & \\
\hline
\end{tabular}

variants displayed dulling (e.g., Shafer 1963:72, Figure 7I). This type, with its similarity in stem morphology to both Uvalde and Hoxie, is problematical (see Chapter 14 for further discussion).

\section{Gower/Jetta $(N=6)$}

Morphology: These specimens share attributes of both Gower and Jetta (e.g., U-shaped basal concavity, generally straight stems ground on lateral edges), although they are generally larger than most Gowers (Figures 13-29 and 13-30).

Metric Averages: Table 13-29

Nonmetric Attributes: Longitudinal profiles include 3 tapered base and tip and 3 indeterminate. Flake patterns are organized on 2 (oblique subparallel) and indeterminate on 4 . Basal modification consists of unifacial thinning on 2, concave bevel on 3, and absent on 1. Lateral stem grinding is slight on 2, moderate on 3, and heavy on 1 .

Material Attributes: Raw material is local Edwards chert (4 fine grained and 2 medium/coarse). Patina is slight partial on 1; otherwise, patina and mineral precipitates are absent.

Tool State and Fractures: Although 2 display full body length, all are heavily fractured by multiple bend and burinlike impact fractures, with the exception of a stem section which exhibits a single bend break.

Rejuvenation: Three are resharpened ( 2 unifacially, unbeveled and 1 bifacially, unbeveled), and 3 are indeterminate.

Stratigraphic Distribution: These points are widely distributed over several stratigraphic units pertaining to the Early and Middle Archaic (Table 13-30).

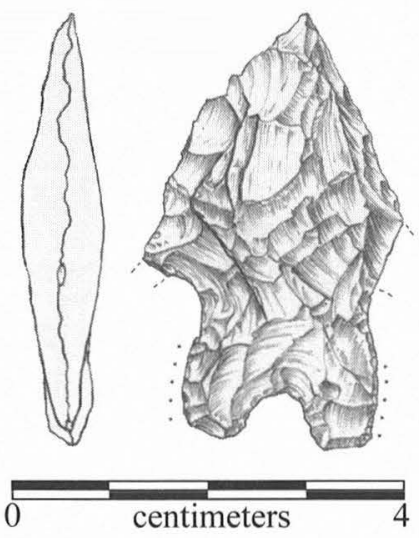

Figure 13-29. Gower/Jetta Specimen 15L2-1.

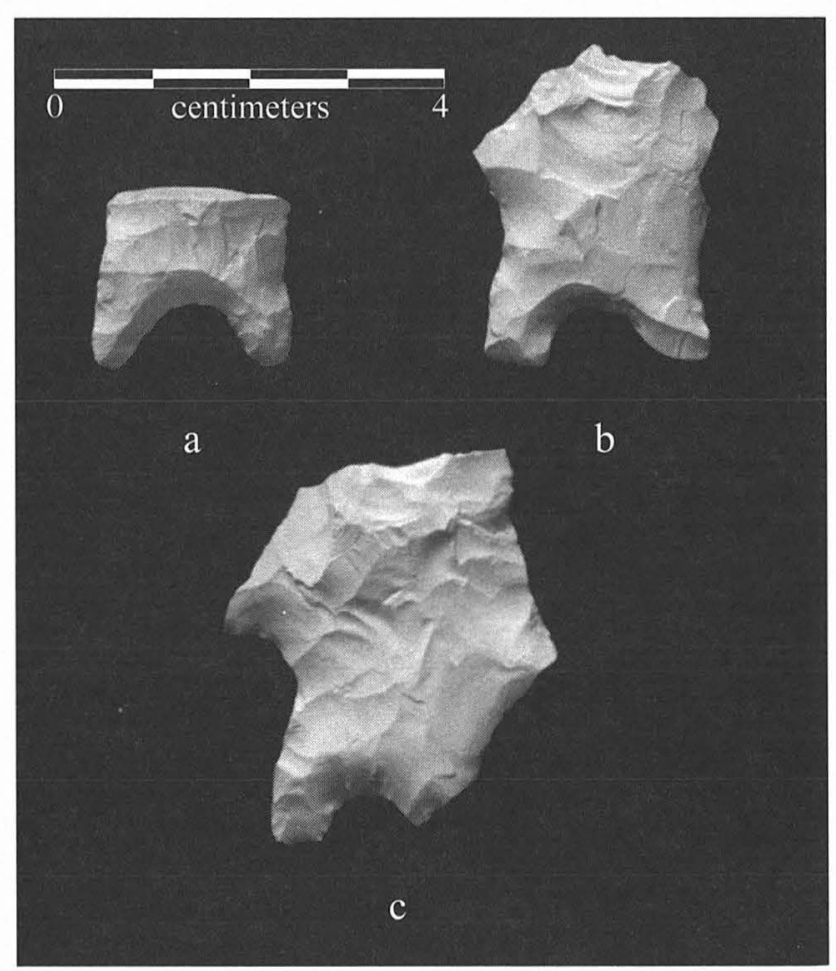

FIGURE 13-30. Gower/Jetta points. Specimen numbers: (a) 15I1-1; (b) $23 \mathrm{O}-9$; (c) $12 \mathrm{~N}-3$.

TABLE $13-29$

Summary Metric Data for Gower/Jetta Points (in mm)

\begin{tabular}{|c|c|c|c|c|c|}
\hline & Numbers & Minimum & Maximum & Mean & $1-\sigma$ \\
\hline Width & 4 & 26.0 & 35.0 & 30.8 & 4.4 \\
\hline Thickness & 5 & 5.0 & 8.0 & 6.6 & 1.1 \\
\hline Stem length & 5 & 15.0 & 20.0 & 17.4 & 2.5 \\
\hline Proximal stem width & 4 & 20.0 & 24.0 & 21.8 & 1.7 \\
\hline Distal stem width & 6 & 17.0 & 22.0 & 19.5 & 1.9 \\
\hline Basal concavity depth & 4 & 5.2 & 7.0 & 6.3 & 0.9 \\
\hline Distal stem thickness & 2 & 6.2 & 6.3 & 6.3 & 0.0 \\
\hline Basal concavity width & 2 & 8.4 & 11.6 & 10.0 & 2.3 \\
\hline
\end{tabular}


TABLE 13-30

Stratigraphic Distribution of Gower/Jetta Points

\begin{tabular}{l|c}
\hline Unit & Valley Floor A \\
\hline IIIb/c & 1 \\
IIIb & 2 \\
IIIa/b & 1 \\
IIIa & 2 \\
\hline \hline
\end{tabular}

\section{Gower/Uvalde $(N=8)$}

Morphology: This group combines attributes of Gower and Uvalde, including U-shaped basal concavities as well as flared basal ears more similar to Uvalde and stem grinding more typically associated with Gower on some (Figure 13-31). Stems are gently expanding and generally convex edged, with varying degrees of constriction at the neck. Small barbs and moderate shoulders are present on most.

Metric Averages: Table 13-31

Nonmetric Attributes: Longitudinal profiles include 1 tapered base and tip, 1 flat, and 6 indeterminate. Flake patterns are organized on 5 (subparallel and oblique subparallel), random on 2 , and 1 indeterminate. Basal modification constitutes concave beveling on 3 and secondary trimming on 5. Lateral stem grinding is slight on 1, heavy on 3 .

Material Attributes: Raw material is local Edwards fine-grained chert for 6 and nonlocal fine-grained Edwards chert for 2. Mineral precipitates are present on 2. Heat damage is extensive on 2. No patina was observed.

Tool State and Fractures: One is complete, and 1 lacks only distal and basal tips due to bend breaks. The remaining 6 are proximal sections, 3 of which display multiple bend and impact/burin-like fractures, and 3 others, multiple thermal, bend, and impact fractures.

Rejuvenation: One is resharpened unifacially, unbeveled; 5 are alternately beveled; and 2 are indeterminate.

Stratigraphic Distribution: Gower/Uvalde distribution appears to be chiefly in deposits attributed to the later part of the Early Archaic (Table 13-32).

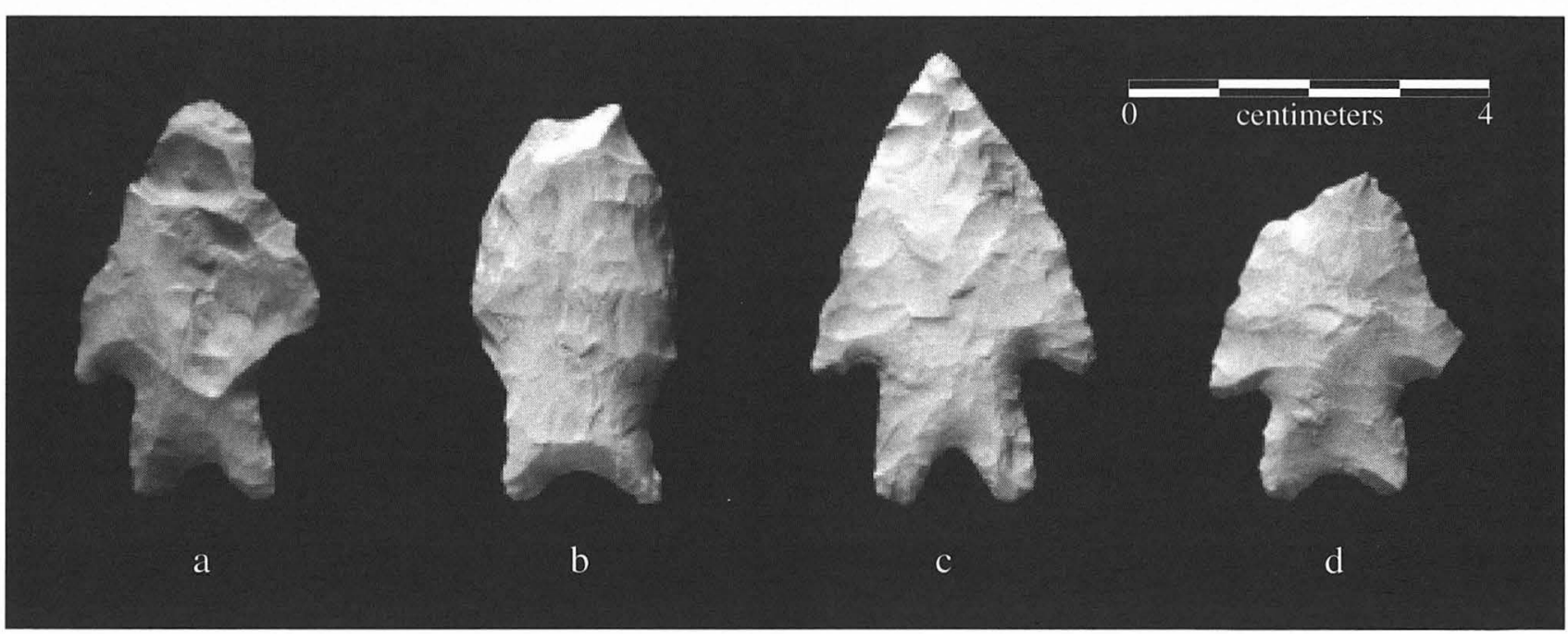

FIGURE 13-31. Gower/Uvalde points. Specimen numbers: (a) 20K-8; (b) 37LD-31; (c) 18N-1; (d) 8J-4.

TABLE 13-31

Summary Metric Data for Gower/Uvalde Points (in $\mathrm{mm}$ )

\begin{tabular}{|c|c|c|c|c|c|}
\hline & Numbers & Minimum & Maximum & Mean & $1-\sigma$ \\
\hline Length & 1 & 51.0 & 51.0 & 51.0 & \\
\hline Width & 2 & 23.0 & 32.0 & 27.5 & 6.4 \\
\hline Thickness & 8 & 6.0 & 9.0 & 7.0 & 1.1 \\
\hline Stem length & 8 & 12.0 & 16.0 & 13.8 & 1.4 \\
\hline Proximal stem width & 7 & 16.0 & 21.0 & 17.9 & 1.6 \\
\hline Distal stem width & 7 & 14.0 & 20.0 & 16.0 & 2.1 \\
\hline Basal concavity depth & 8 & 2.8 & 5.5 & 4.2 & 0.8 \\
\hline Distal stem thickness & 5 & 5.3 & 7.0 & 6.2 & 0.8 \\
\hline Basal concavity width & 5 & 7.0 & 11.2 & 8.7 & 1.5 \\
\hline
\end{tabular}


TABLE 13-32

Stratigraphic Distribution of Gower/Uvalde Points

\begin{tabular}{l|c|c}
\hline Unit & Valley Floor A & Valley Margin \\
\hline Y/IIIc & 2 & 2 \\
IIIb & & 1 \\
Y & & 1 \\
X & & 1 \\
Other & & \\
surface & 1 & \\
\hline \hline
\end{tabular}

\section{Hoxie $(N=26)$}

Morphology: The overall form of these points varies widely, in part due to characteristic resharpening of the blade which often takes the form of steep alternate beveling with fine parallel oblique flake scars (Figures 13-32 and 13-33). In most, shape is generally elongate with only slight angular protrusions and/or very weak shoulders at the stem/blade juncture. Somewhat greater congruity is seen when only stem portions are considered. Stems are straight to gently flaring and are generally heavily ground on lateral edges; some may exhibit beveled retouch on stem margins. Bases vary from a gentle arc to a moderately deep U-shape, generally formed by what is more a concave beveling of the stem rather than actual basal thinning. In longitudinal profile, bases appear rather thick and wedge-shaped.

Metric Averages: Table 13-33

Nonmetric Attributes: Longitudinal profiles include 1 tapered base and tip; 2 thick tip; 2 flat; 11 wedge-shaped base and tapered tip; and 10 indeterminate. Flake patterns are organized on 11 (subparallel and oblique subparallel), random on 7, and indeterminate on 8 . Six retain sections of the original flake blank. Basal modification encompasses unifacial thinning on 2 , beveling on 1 , concave beveling on 15 , secondary trimming on 3 , none on 3 , and indeterminate on 2. Lateral stem grinding is slight on 3 , moderate on 6 , and heavy on 17.

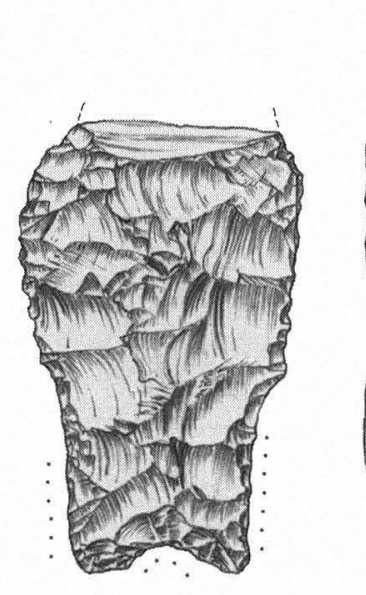

a

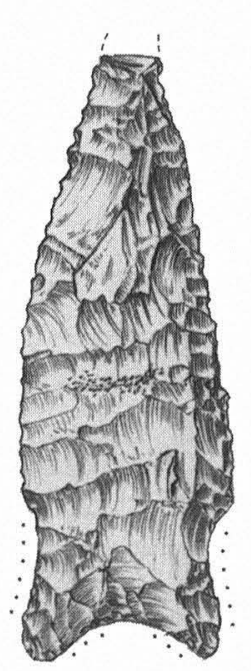

c
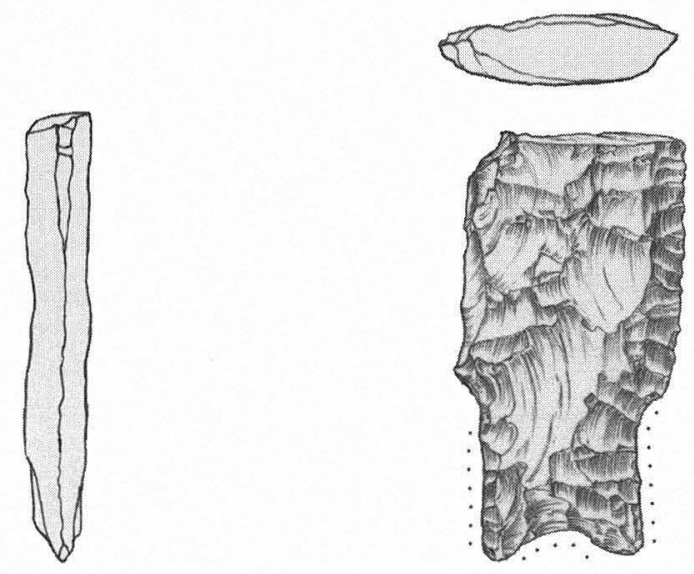

b
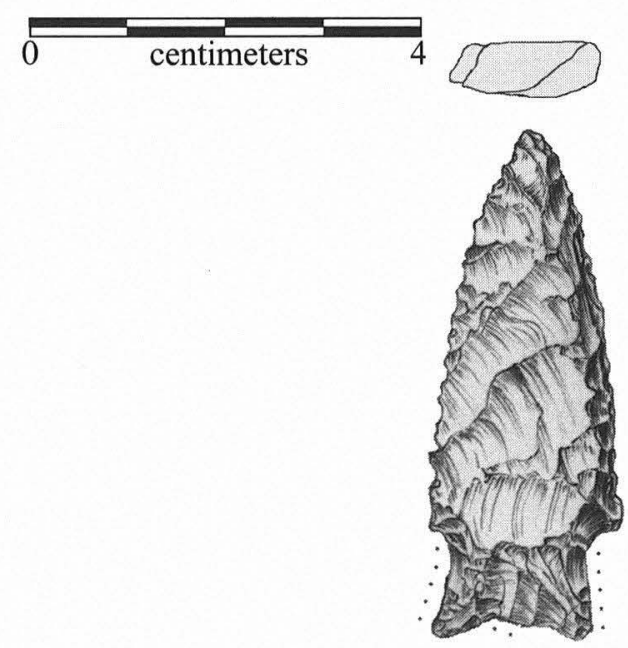

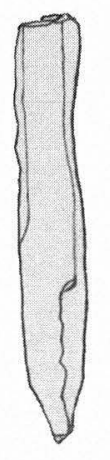

6

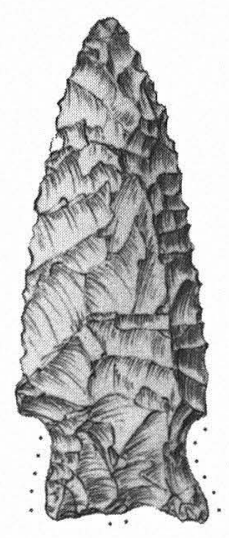

d

FIGURE 13-32. Hoxie points. Specimen numbers: (a) 37O2C-8; (b) 29P1-1; (c) 16R2-1; (d) 3K-1. 


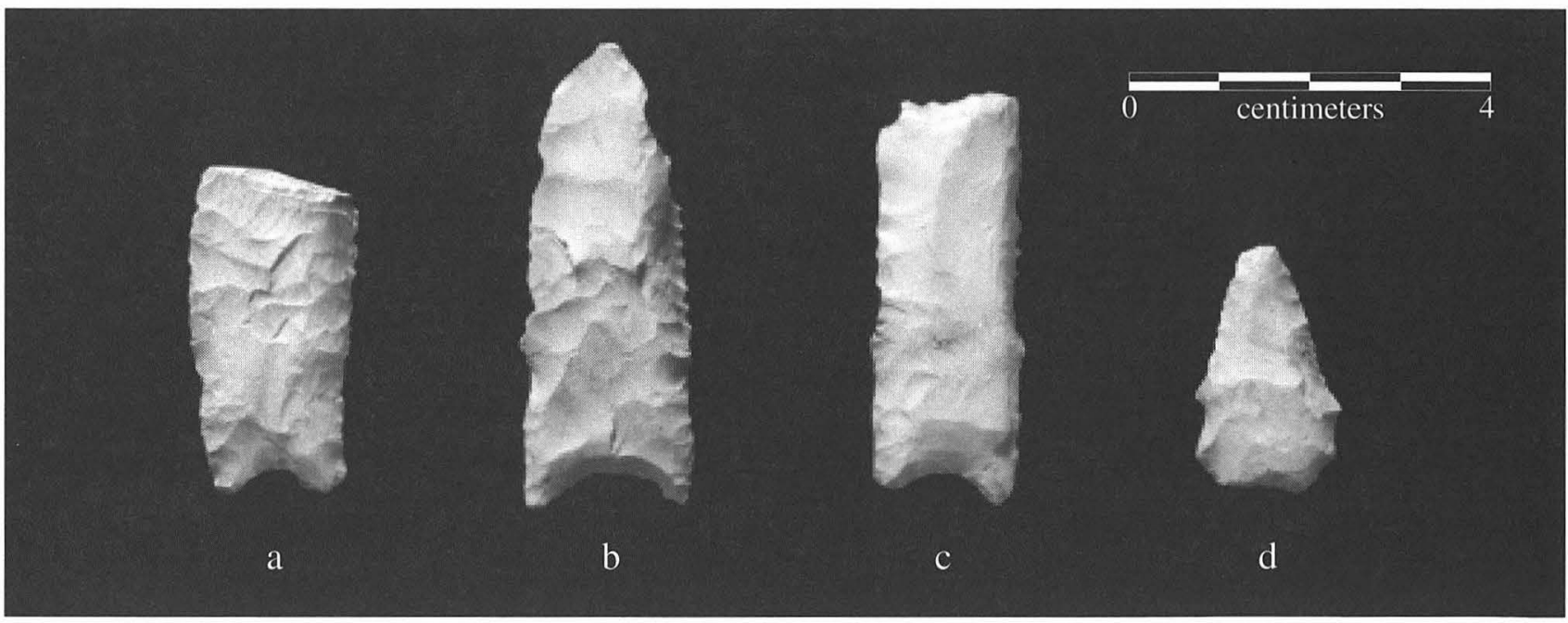

FIGURE 13-33. Hoxie points. Specimen numbers (a) 22Q-1; (b) 31S1-1; (c) 0-31; (d) 22O-2.

TABLE 13-33

Summary Metric Data for Hoxie Points (in mm)

\begin{tabular}{|c|c|c|c|c|c|}
\hline & Numbers & Minimum & Maximum & Mean & $1-\sigma$ \\
\hline Length & 4 & 27.0 & 69.0 & 44.0 & 19.9 \\
\hline Width & 21 & 16.0 & 31.0 & 21.7 & 3.7 \\
\hline Thickness & 26 & 5.0 & 9.0 & 6.5 & 0.9 \\
\hline Stem length & 25 & 8.0 & 19.0 & 14.9 & 2.9 \\
\hline Proximal stem width & 24 & 13.0 & 20.0 & 16.3 & 1.7 \\
\hline Distal stem width & 26 & 14.0 & 22.0 & 17.2 & 1.7 \\
\hline Basal concavity depth & 22 & 0.4 & 4.2 & 2.7 & 0.9 \\
\hline Distal stem thickness & 20 & 5.1 & 7.1 & 6.2 & 0.6 \\
\hline Basal concavity width & 20 & 6.3 & 13.0 & 9.4 & 1.9 \\
\hline
\end{tabular}

Material Attributes: Raw material is local Edwards chert for 15 (12 fine grained, 3 medium/coarse); nonlocal Edwards fine-grained chert for 8 ; and 3 indeterminate heat altered. Mineral precipitates are present on 2. No patina was observed. Heat damage is slight on 2, extensive on 4. Marked reddening of the basal ears of Figure 13-23d (3K-1) is likely due to heat alteration; the specimen, though complete, may have been discarded near a fire due to its highly resharpened, exhausted state.

Tool State and Fractures: Four are complete, and 6 lack only distal or lateral sections; of these, 1 exhibits a single bend break, 2 display impact fractures, and 3, multiple bend, impact, and/or hinge fractures. The remaining 16 are proximal sections; on these, a single impact break was recorded on 1 , single bend breaks on 3 , multiple bend and burin-like/impact fractures on 8 , thermal fractures on 3 (in addition to burin-like breaks on 1), and multiple bend breaks on 1.

Rejuvenation: One is resharpened unifacially, unbeveled; 2 bifacially, unbeveled; 19 alternately beveled, and 5 are indeterminate. Ten specimens display beveled or alternately beveled stem edges which, in some cases, were flaked in an opposite pattern to the beveling on the blade, producing a twisted longitudinal profile (e.g., Figure 13-32d). Specimen 220-2 (Figure 13-33d) has been reworked both vertically and laterally to the form of a steeply beveled nub.

Stratigraphic Distribution: Although the first occurrence of Hoxie is in Late Paleoindian deposits (Unit II), there is a pronounced expression of Hoxie in Early Archaic contexts; Unit IIIa, with a total of 10 Hoxie specimens, is estimated to date from 8700-6500/600 B.P. (Table 13-34).

Comments: Resharpening and maintenance, both of blade and stem elements, clearly plays a role in morphological variance in Hoxie groups. This is explored further in the discussion of Hoxie and Hoxie variants, below.

\section{Hoxie A $(N=9)$}

Morphology: Hoxie A bases have an overall rounded look due to thickness, width, and grinding (Figures 13-34 and 13-35). Stems are straight to slightly constricted, relatively thick, and medium to wide in width; basal concavities 
TABLE 13-34

Stratigraphic Distribution of Hoxie Points

\begin{tabular}{l|c|c|c}
\hline Unit & $\begin{array}{c}\text { Valley } \\
\text { Floor A }\end{array}$ & $\begin{array}{c}\text { Valley } \\
\text { Floor B }\end{array}$ & $\begin{array}{c}\text { Valley } \\
\text { Margin }\end{array}$ \\
\hline IIIc & 1 & & 1 \\
IIIb & 1 & & 1 \\
X/Y & & & 1 \\
IIIa/b & 2 & & 1 \\
X/IIIa/b & & 1 & 2 \\
IIIa & 8 & & \\
III & 2 & & 1 \\
II/IIIa & 1 & & \\
X & 1 & & \\
II & & & \\
Other & & & \\
\multicolumn{1}{c}{ surface } & & & \\
\hline \hline
\end{tabular}
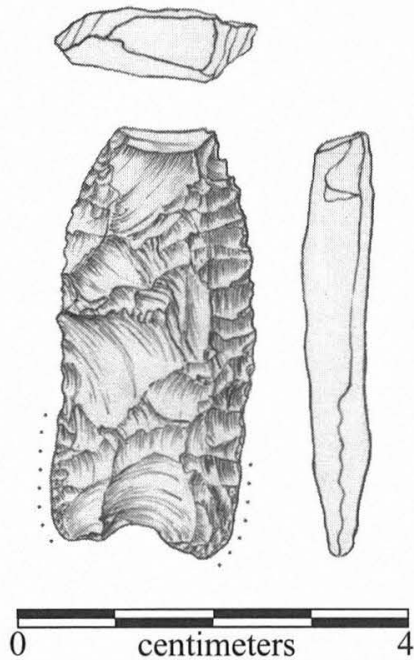

FIgURE 13-34. Hoxie A point, Specimen 10R-10. are shallow and U-shaped. Grinding is typically heavy, both in basal concavity and stem lateral edges. Most are damaged and thus difficult to characterize as to body form. However, the two nearly complete specimens, although heavily resharpened, exhibit small protrusions above the stem suggesting the one-time presence of shoulders, if not barbs.

Metric Averages: Table 13-35

Nonmetric Attributes: Longitudinal profiles include 1 tapered base and tip, 1 thick base, 1 wedge-shaped base, and 6 indeterminate. Flake patterns are organized on 3 (oblique subparallel and subparallel) and indeterminate on 6 . Two show original flake blank sections. Basal modification consists of 2 unifacially thinned, 1 bifacially thinned, 4 concave beveled, and 2 secondary trimming. Lateral stem grinding is moderate on 1 , heavy on 8 .

Material Attributes: Raw material is local Edwards chert (7 fine grained, 1 medium/coarse), and nonlocal Edwards medium/coarse chert for 1 . Patina is moderate overall on 1 and moderate partial on 2. Heat damage is slight on 1. No mineral precipitates were observed.

Tool State and Fractures: Two are complete, or nearly so, and another lacks a distal section. The 6 others are proximal and stem sections, 4 of which were fractured by single bend bend breaks, and the others by bend and burin-like breaks.

Rejuvenation: One is resharpened unifacially, unbeveled, 3 alternately beveled, and 5 are indeterminate. Stems on 2 are beveled, 1 of which appears to be in opposition to blade beveling.

Stratigraphic Distribution: These variants occur predominately in mixed Late Paleoindian/Early Archaic and Early Archaic deposits (Table 13-36).

Comments: Hoxie A bases have an overall thick, rounded character and resemble some Angostura variants.

\section{Hoxie $B(N=5)$}

Morphology: In this variant, the distal stem or "neck" portion is wider than the proximal section. The stem is generally

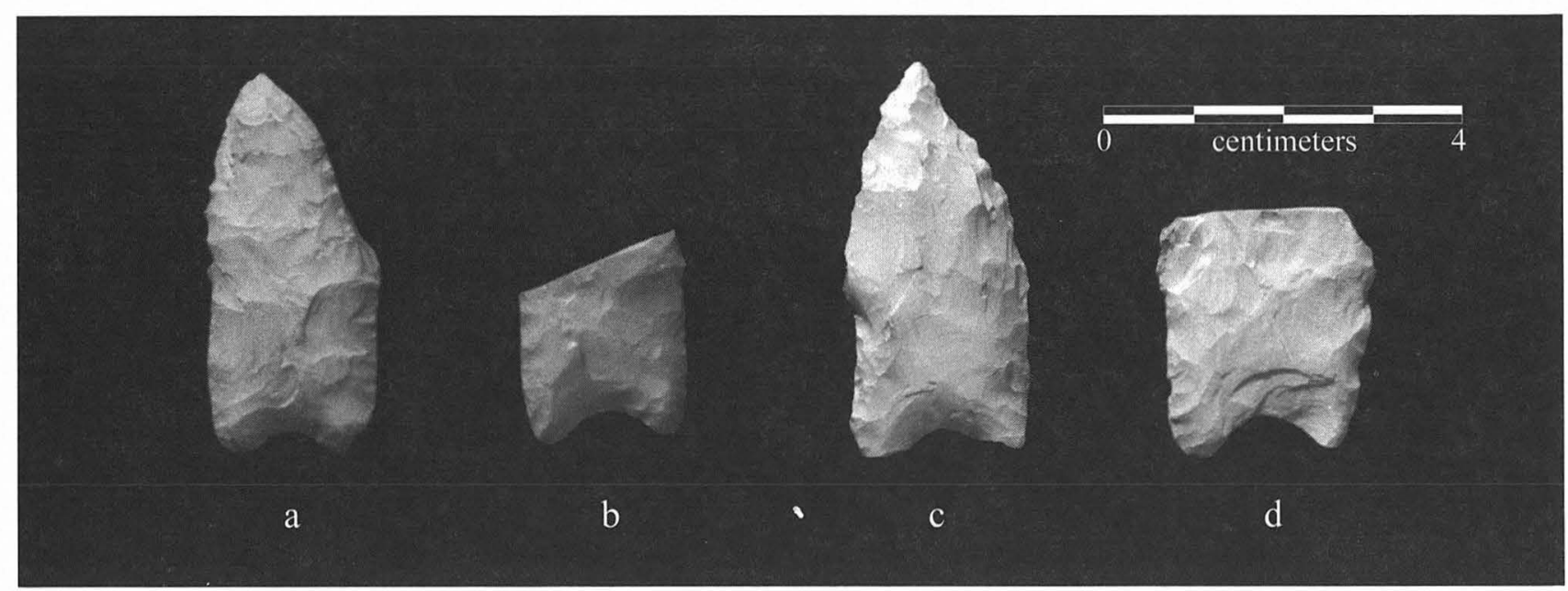

Figure 13-35. Hoxie A points. Specimen numbers: (a) 22P-3; (b) 12Q-1; (c) 26P1-1; (d) 27R1-1. 
TABLE 13-35

Summary Metric Data for Hoxie A Points (in mm)

\begin{tabular}{|c|c|c|c|c|c|}
\hline & Numbers & Minimum & Maximum & Mean & $1-\sigma$ \\
\hline Length & 2 & 40.0 & 44.0 & 42.0 & 2.8 \\
\hline Width & 5 & 18.0 & 21.0 & 19.6 & 1.1 \\
\hline Thickness & 9 & 6.0 & 8.0 & 6.6 & 0.7 \\
\hline Stem length & 7 & 15.0 & 20.0 & 17.1 & 1.7 \\
\hline Proximal stem width & 9 & 16.0 & 19.0 & 17.2 & 1.0 \\
\hline Distal stem width & 8 & 18.0 & 20.0 & 19.0 & 0.5 \\
\hline Basal concavity & 9 & 1.9 & 4.0 & 3.0 & 0.8 \\
\hline Distal stem thickness & 6 & 5.5 & 6.8 & 6.1 & 0.5 \\
\hline Basal concavity width & 6 & 6.6 & 9.8 & 8.4 & 1.3 \\
\hline
\end{tabular}

TABLE 13-36

Stratigraphic Distribution of Hoxie A Points

\begin{tabular}{l|c|c}
\hline Unit & Valley Floor A & Valley Margin \\
\hline Y & 1 & 1 \\
IIIa/b & 2 & \\
IIIa & 2 & 1 \\
II/IIIa & & 1 \\
X & & 1 \\
X/II & & \\
\hline \hline
\end{tabular}

contracting, or basal ears are downward pointing, thus giving the illusion of contraction at the base. Stem lateral edges are heavily ground, and basal concavity is shallow. On the more complete specimens, weak shoulders are evident in spite of resharpening by alternate beveling (Figures 13-36 and 13-37).

Metric Averages: Table 13-37

Nonmetric Attributes: Longitudinal profiles include 2 with wedge-shaped bases and 3 indeterminate. Flake patterns are organized on 4 (subparallel, collateral, oblique subparallel) and indeterminate on 1. A section of original flake blank is evident on 1. Basal modification constitutes 1 bifacially thinned, 1 beveled, and 3 concave beveled. Lateral stem grinding is heavy on all 5 .

Material Attributes: Raw material is local Edwards chert (3 fine grained and 1 medium/coarse), and 1 is nonlocal finegrained chert. Mineral precipitates are present on 1. Neither patina nor heat damage was observed.

Tool State and Fractures: Two are complete, with 1 displaying a reworked impact scar. Another is nearly complete, lacking only a distal tip and basal ear due to bend and burinlike fractures. The remaining 2 are proximal sections broken by multiple bend and impact fractures.

Rejuvenation: One is unifacially beveled, and 4 are alternately beveled. Stems on 3 are beveled, and Specimen $28 \mathrm{~S} 1-1$ is alternately beveled in a pattern opposite to blade beveling, which creates a torque-like longitudinal profile.
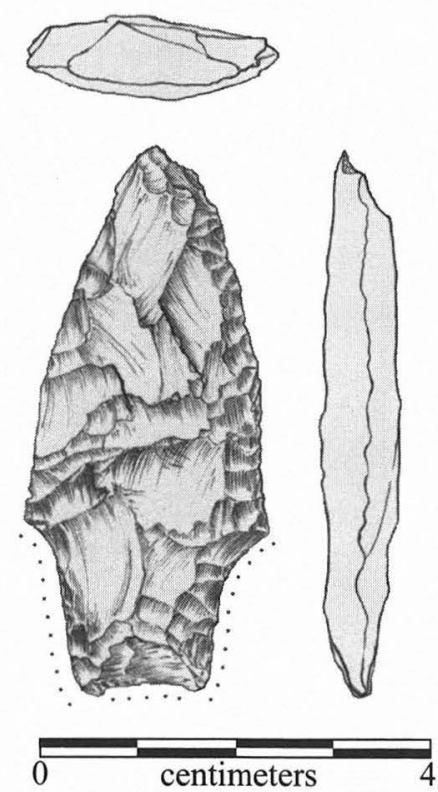

Figure 13-36. Hoxie B point, Specimen 29S1-5.

Stratigraphic Distribution: Hoxie B were recovered chiefly in lower Early Archaic deposits (Table 13-38).

\section{Hoxie $C(N=5)$}

Morphology: This group is distinguished by a very long, typically heavily ground stem of narrow to medium width with only a very slight, arc-shaped basal concavity. All specimens have been heavily reworked and display only slight shoulders (Figures 13-38 and 13-39).

Metric Averages: Table 13-39

Nonmetric Attributes: Longitudinal profiles include 3 wedge-shaped base and tip and 2 indeterminate. Flake patterns are organized on 2 (oblique subparallel), random on 2 , and indeterminate on 1. Basal modification consists of unifacial thinning on 1 and concave bevel on 4 . Lateral stem grinding is moderate on 2 and heavy on 3.

Material Attributes: Raw material is local medium/ coarse-grained Edwards chert for 2, and nonlocal Edwards 


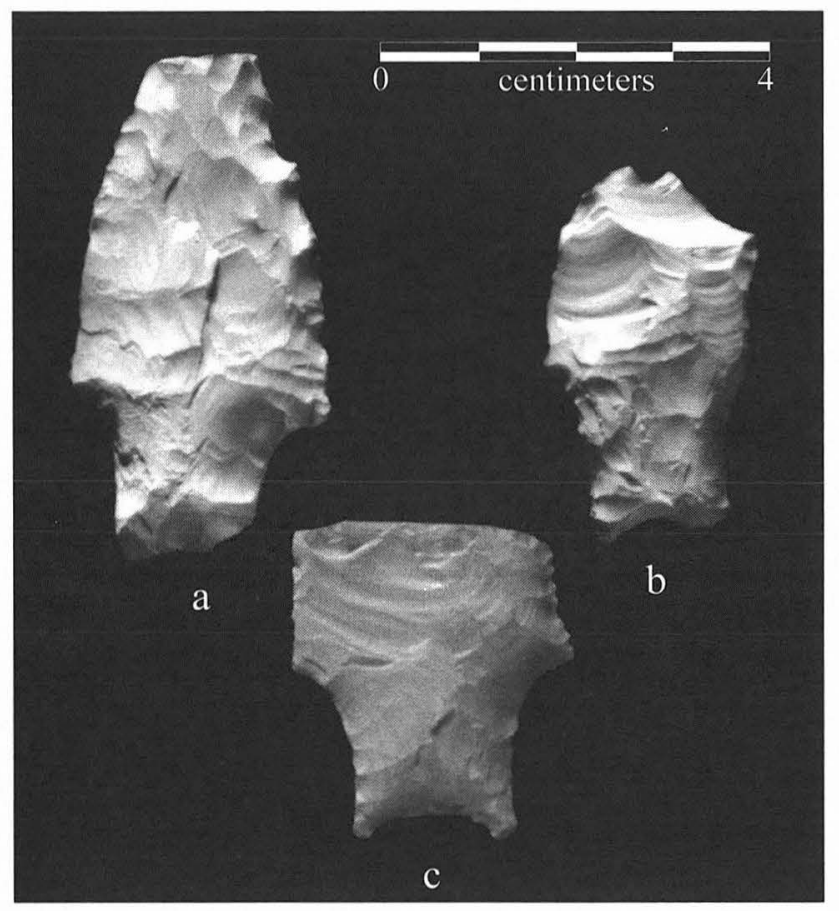

FIGURE 13-37. Hoxie B points. Specimen numbers: (a) 2I-1; (b) 310-1; (c) 360-1.

chert ( 2 fine grained, 1 medium/coarse). No mineral precipitates, heat damage, or patina was observed.

Tool State and Fractures: One is complete and 1 is nearly so, although marred by multiple impact fractures. The other 3 are proximal sections, 2 of which display single bend breaks and the other, multiple bend and impact fractures.

Rejuvenation: Three are alternately beveled and 2 beveled unifacially. The stem of 1 is beveled.

Stratigraphic Distribution: These long-stemmed variants occur somewhat higher in Early Archaic deposits than other Hoxie groups, with 4 in the Unit IIIa/b transition zone and 1 in Unit IIIa (Table 13-40)

Comments: This group is similar to Hoxie A, but their stems are generally longer, more narrow, and wedge-shaped in longitudinal profile.
TABLE $13-38$

Stratigraphic Distribution of Hoxie B Points

\begin{tabular}{l|c|c}
\hline Unit & Valley Floor A & Valley Margin \\
\hline Y & 1 & 1 \\
IIIa/b & 2 & \\
IIIa & 1 & \\
II/IIIa & & \\
\hline \hline
\end{tabular}
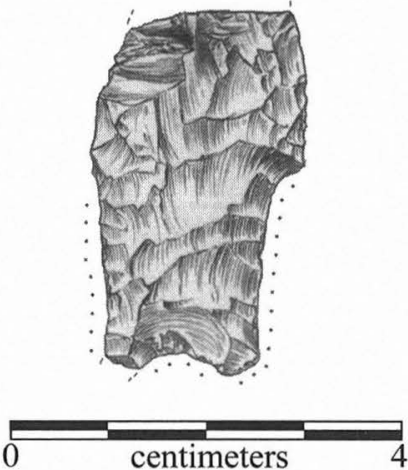

Figure 13-38. Hoxie C point, Specimen 32P2-12.

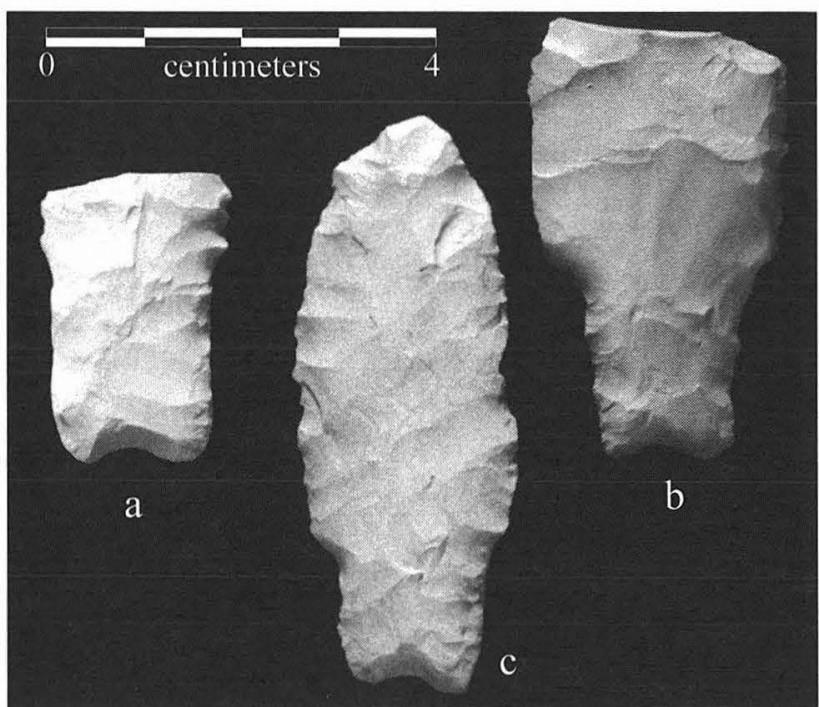

FIGURE 13-39. Hoxie C points. Specimen numbers: (a) $21 \mathrm{~N}-2$; (b) $22 \mathrm{M}-1$; (c) $13 \mathrm{~N}-1$.

TABLE 13-37

Summary Metric Data for Hoxie B Points (in mm)

\begin{tabular}{l|c|c|c|c|c}
\hline & Numbers & Minimum & Maximum & Mean & $1-\sigma$ \\
\hline Length & 3 & 33.0 & 56.0 & 47.3 & 12.5 \\
Width & 4 & 20.0 & 29.0 & 25.0 & 3.9 \\
Thickness & 5 & 6.0 & 8.0 & 6.8 & 0.8 \\
Stem length & 4 & 13.0 & 17.0 & 15.3 & 1.7 \\
Proximal stem width & 4 & 14.0 & 17.0 & 15.3 & 17.4 \\
Distal stem width & 5 & 16.0 & 20.0 & 1.8 & 1.7 \\
Basal concavity depth & 4 & 1.3 & 6.5 & 0.6 & 0.6 \\
Distal stem thickness & 4 & 5.5 & 11.9 & 9.0 & 2.6 \\
Basal concavity width & 4 & 6.8 & & \\
\hline \hline
\end{tabular}


TABLE 13-39

Summary Metric Data for Hoxie C Points (in mm)

\begin{tabular}{|c|c|c|c|c|c|}
\hline & Number & Minimum & Maximum & Mean & $1-\sigma$ \\
\hline Length & 1 & 61.0 & 61.0 & & \\
\hline Width & 3 & 20.0 & 28.0 & 23.7 & 4.0 \\
\hline Thickness & 5 & 7.0 & 8.0 & 7.4 & 0.5 \\
\hline Stem length & 4 & 15.0 & 21.0 & 19.5 & 3.0 \\
\hline Proximal stem width & 4 & 14.0 & 16.0 & 15.3 & 1.0 \\
\hline Distal stem width & 5 & 16.0 & 19.0 & 17.4 & 1.1 \\
\hline Basal concavity depth & 4 & 1.4 & 2.1 & 1.8 & 0.3 \\
\hline Distal stem thickness & 4 & 6.1 & 7.6 & 6.9 & 0.7 \\
\hline Basal concavity width & 3 & 5.6 & 7.7 & 6.9 & 1.1 \\
\hline
\end{tabular}

TABLE $13-40$

Stratigraphic Distribution of Hoxie C Points

\begin{tabular}{l|c|c}
\hline Unit & Valley Floor A & Valley Margin \\
\hline IIIa/b & 1 & 3 \\
IIIa & 1 & \\
\hline \hline
\end{tabular}

\section{Hoxie/Gower ( $N=9)$}

Morphology: Although many are damaged, these points appear to display attributes of both Gower and Hoxie (Figures 13-40 and 13-41). The stems are medium to long in length, and straight to slightly expanding, with well-ground, occasionally slightly beveled lateral edges. Basal ears are typically directed downward, framing a U-shaped basal concavity of medium depth. Resharpening clearly has altered the appearance of these points, although several evidence small barb remnants on very slight shoulders.

Metric Averages: Table 13-41

Nonmetric Attributes: Longitudinal profiles include 3 tapered base and tip; 1 thick tip; 3 wedge-shaped base and tapered tip; and 2 indeterminate. Flake patterns are organized on 4 (subparallel and oblique subparallel) random on 2 , and indeterminate on 3 . Basal modification consists of unifacial thinning on 3, concave bevel on 4 , and 2 indeterminate. Lateral stem grinding is moderate on 4 and heavy on 5 .

Material Attributes: Raw material is local Edwards chert (6 fine grained, 1 medium/coarse), and nonlocal Edwards fine-grained chert $(n=2)$. Mineral precipitates are present on 1. Heat damage is slight on 1. No patina was observed.

Tool State and Fractures: Three are complete except for distal tip and/or basal ears, which were severed by burin-like and bend fractures. The 6 others are proximal sections, 1 of which displays a single bend break, and the others, multiple bend and burin-like impact fractures.

Rejuvenation: All are alternately beveled.

Stratigraphic Distribution: Although several of this group appear in relatively early deposits, the majority are found in Unit IIIa and are attributed to the Early Archaic (Table 13-42).

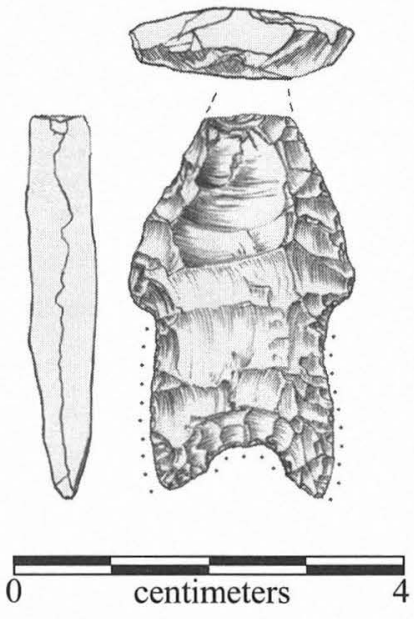

Figure 13-40. Hoxie/Gower, Specimen 18N-2.

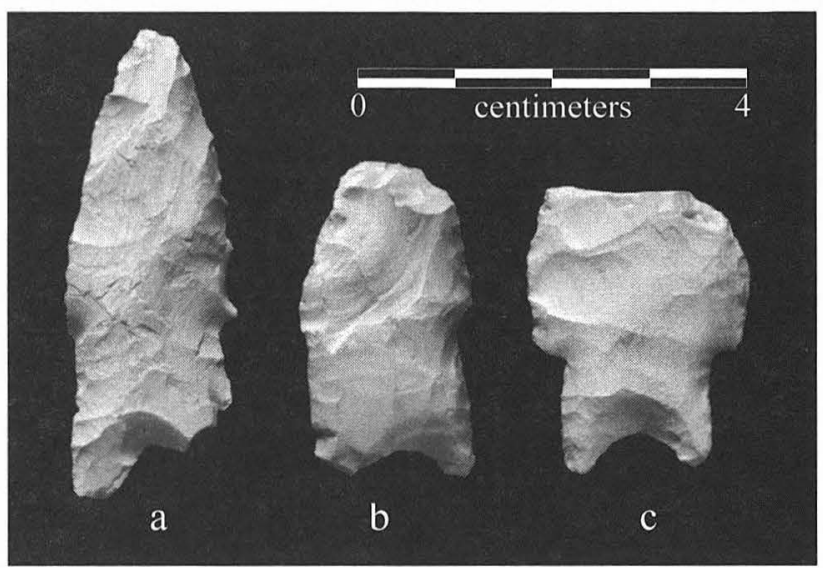

FIGURE 13-41. Hoxie/Gower points. Specimen numbers: (a) 12R-2; (b) $4 \mathrm{~J}-1$; (c) $6 \mathrm{G}-1$.

Comments: Most specimens have a stem somewhat longer and thicker than Gower (see Figure 13-41b, c) and, like some Hoxie, display beveled-stem edges. Specimen 12R-2 (see Figure 13-41a), although damaged, resembles a serrated-edged Hoxie but exhibits the more typical Gower flake removal forming a U-shaped basal concavity. 
TABLE 13-41

Summary Metric Data for Hoxie/Gower Points (in mm)

\begin{tabular}{|c|c|c|c|c|c|}
\hline & Number & Minimum & Maximum & Mean & $1-\sigma$ \\
\hline Length & 1 & & 37.8 & & \\
\hline Width & 9 & 17.0 & 25.0 & 20.78 & 2.77 \\
\hline Thickness & 9 & 6.0 & 7.0 & 6.33 & 0.50 \\
\hline Stem length & 9 & 7.0 & 18.2 & 14.70 & 3.66 \\
\hline Proximal stem width & 4 & 14.4 & 18.9 & 16.53 & 2.09 \\
\hline Distal stem width & 7 & 13.0 & 17.8 & 15.66 & 1.51 \\
\hline Basal concavity depth & 5 & 2.3 & 4.4 & 3.62 & 0.87 \\
\hline Distal stem thickness & 4 & 5.7 & 6.7 & 6.08 & 0.48 \\
\hline Basal concavity width & 4 & 8.7 & 11.2 & 10.05 & 1.12 \\
\hline
\end{tabular}

TABLE 13-42

Stratigraphic Distribution of Hoxie/Gower Points

\begin{tabular}{l|c|c}
\hline Unit & Valley Floor A & Valley Margin \\
\hline IIIc & & 1 \\
Y & 4 & 1 \\
IIIa & 1 & \\
II/IIIa & & 1 \\
X & 1 & \\
II & & \\
\hline \hline
\end{tabular}

Resharpening of these points and reworked stem areas may have blurred distinctions.

\section{Discussion of Hoxie and Hoxie Variants}

Hoxie points from the type site (Prewitt n.d.) and other sites encompass a fairly wide range of variation in stem morphology (see Peter 1982:Figure 14.1-2; Prewitt 1981a:Figure 82). Alternate beveling of blade, beveling of stems, and weakly defined shoulders are attributes that frequently have been associated with this type, although these might be more appropriately identified as results of rejuvenation rather than morphological type indicators. As discussed below, alternate beveling is a technique that saw maximum expression in the Late Paleoindian and Early Archaic contexts at the Wilson-Leonard site. Because of this, the original morphology of Hoxie and other points of this time period remains somewhat unclear. Within the large group of Hoxie and Hoxie variants from the Wilson-Leonard site, however, are points spanning the continuum from only slightly resharpened to fully rejuvenated and with these, morphological variance at successive junctures throughout the sequence can be roughly tracked.

In Figure 13-42a, a convex-bladed, stemmed point with expanding bifurcate stem is proposed as a Hoxie prior to intensive resharpening. Identified as "Hoxie?" from Zone $\mathrm{X}$ in the Valley Margin, this point has a bifurcate stem with heavily ground lateral edges. Its blade is slightly asymmetri- cal, and displays light bifacial beveling of the lateral edges. Within the proposed resharpening sequence-from slight bifacial reworking to extreme alternate beveling — blades are narrowed and blade edge angles become steeper, with concomitant alterations in cross section, from biconvex or lenticular to a sharp-angled parallelogram shape. As shown, some of the points in this group appear almost lanceolate in shape, with only a slight protrusion marking the shoulders and the vertical extent of stem grinding.

Although Hoxie points were not studied for microscopic use-wear, fracture and resharpening patterns suggest these points were used exhaustively both as cutting/scraping implements and as projectiles. Figure 13-33c (Specimen 0-31) exemplifies this in a somewhat remarkable constellation of fractures and recycling modifications, including two longitudinal burin-like impact fractures, one of which has severed a lateral blade margin, extreme alternate beveling and light serration of lateral edge, and apparent retouch of a transverse bend break at the distal tip, as if for use as a scraper.

Other notable characteristics observed in the Hoxie group are a relatively high incidence of original flake blank remnant scars on blade areas (present on $35 \%$ of all Hoxie and variants) as well as, somewhat paradoxically, a fairly high frequency of patterned flaking of blade (20 of 29 , or $69 \%$, of those large enough to observe). Bases are generally not well thinned, with predominate modification being concave beveling; this frequently produces a longitudinal profile that is wedge shaped at the base.

The beveling of stem edges in these groups is notable ( 26 of 45 Hoxie and variants, or 58\%), and implications of such are substantial in our understanding of this early bifurcate form. As shown in Figure 13-43, it appears that many of those with stem edges beveled in congruence with blade beveling were resharpened in the same episode and thus were unhafted at the time. In contrast, those with stems beveled in opposite patterns to the blade area likely were beveled prior to hafting. Blade areas on those specimens likely were resharpened while hafted.

It is clear, then, that much variance within these groups is introduced not only via blade resharpening but in 


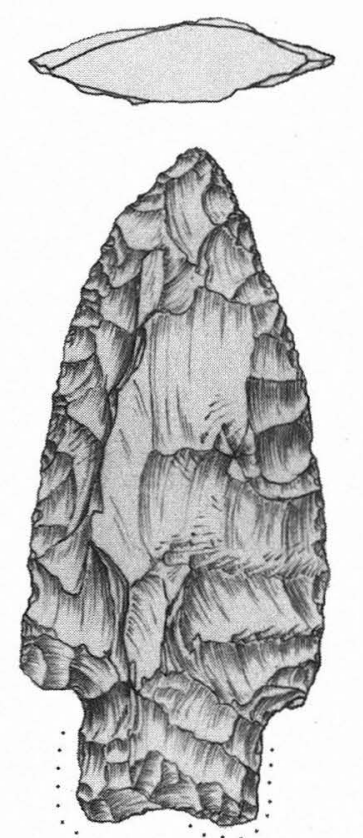

a
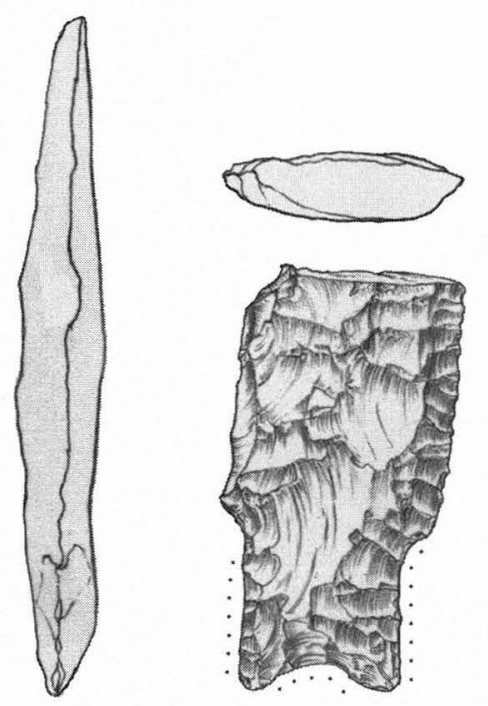

$\mathrm{b}$
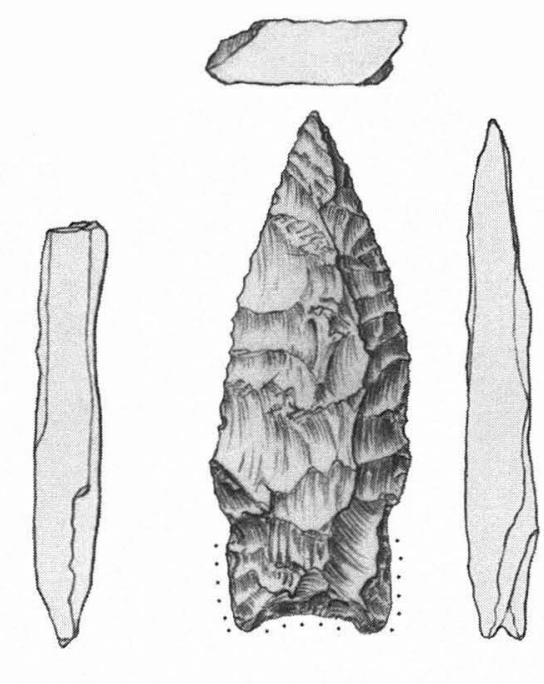

$\mathrm{c}$

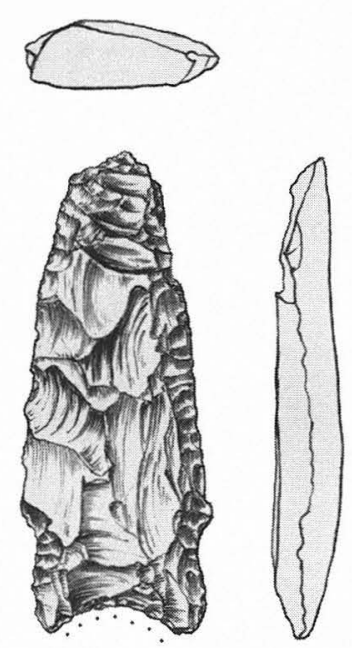

d

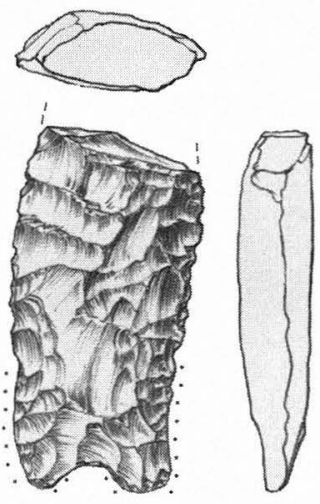

e

FIGURE 13-42. Hypothetical resharpening sequence for Hoxie and Hoxie-like points illustrating morphological changes as blade is increasingly beveled. Specimen "a" is proposed as a Hoxie point prior to resharpening. All points are shown are from Early Archaic contexts. Specimen numbers: (a) 19S-1; (b) 29P1-2; (c) 28S1-1; (d) 37O2B-7; (e) 22Q-1. 
modification of the haft area. Given the wide variety of bifurcate stem forms present in Early Archaic contexts, these technological data should be carefully considered in future analyses. Similarities between certain Hoxie variants and Angostura variants-not only in contracting stem form but in patterned flaking — raise the issue of possible rebasing and transformation from one "type" to another. This concept is explored further in the final discussion, below. Although the data were not directly compared, morphological similarities between Angostura and certain bifurcate stem points also were noted in a statistical analysis of selected projectile points (see Chapter 14).

Although restricted both spatially and temporally, Hoxie appears to be a somewhat problematical type. Distribution is chiefly in northeastern and north-central Texas (see Prewitt 1995) and, as shown in the Wilson-Leonard groups, temporal range is chiefly the early part of the Early Archaic or slightly earlier. As demonstrated in the WilsonLeonard sample and in a mathematical analysis of morphometric attributes, there is a significant degree of overlap with other early bifurcate types, particularly Gower (see Chapter 14). There are also similarities with certain east Texas points, including Yarbrough and Morrill, both of which also display stem grinding and alternate beveling of the blade. In some cases, there appear be affinities with the Early Stemmed Lanceolate group (Turner and Hester 1993:107); see also Goode's (1989:89, Figure 25f, g) comments along these lines.

\section{$\operatorname{Jetta}(N=12)$}

Morphology: This broad-bladed, typically well-flaked bifurcate-stem point is distinguished by the almost exaggerated size of its features, including strong shoulders, large barbs that often are splayed outward, and a long, wide stem that is generally straight to convex-sided (Figures 13-44 and 13-45). Stem lateral edges are typically ground, and basal concavity is deep and U-shaped, commonly in conjunction with simple unifacial thinning or concave beveling of the base. Although relatively thick overall, these specimens appear generally evenly proportioned in longitudinal profile.

Metric Averages: Table 13-43

Nonmetric Attributes: Longitudinal profiles include 5 tapered base and tip, 1 wedge-shaped base, and 6 indeterminate. Flake patterns are organized on 4 (oblique subparallel and oblique parallel), random on 1, and indeterminate on 7. Basal modification consists of unifacial thinning on 5, concave bevel on 4 , secondary trimming on 2 , and 1 indeterminate. Lateral stem grinding is absent on 1 , slight on 3 , moderate on 2 , and heavy on 6 .

Material Attributes: Raw material is local Edwards fine-grained chert for 8, nonlocal Edwards chert (2 fine grained, 1 medium/coarse), and chalcedony for 1 . Mineral precipitates are present on 2. Heat damage is slight on 1 . Patina is not present.

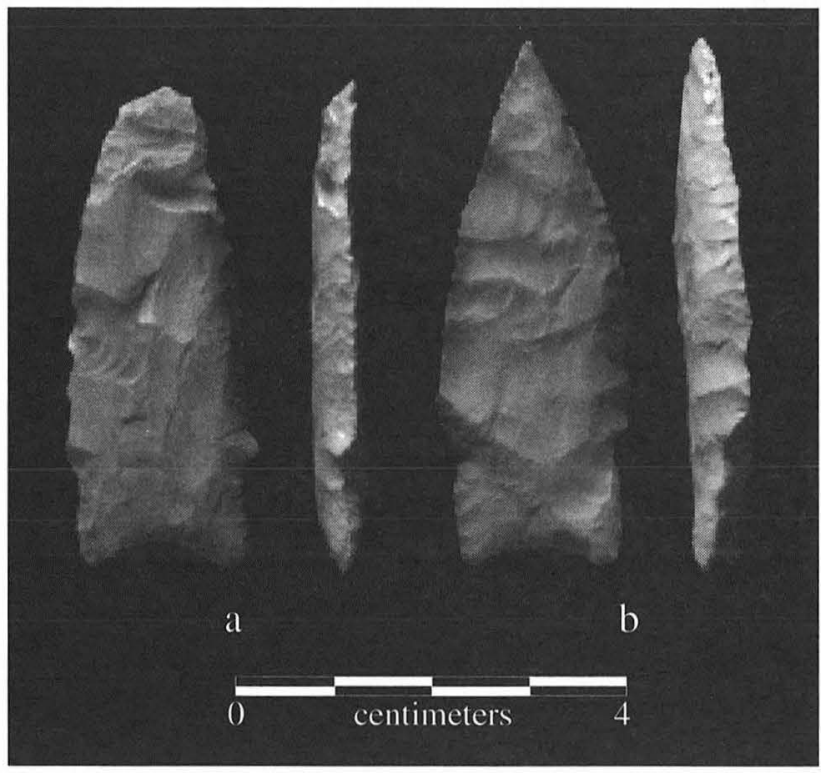

FIGURE 13-43. Examples of stem- and blade-edge beveling patterns on Hoxie points, shown face on and in longitudinal section. (a) alternate beveling of blade and stem in congruent pattern; (b) alternate beveling of stem opposing the alternate beveling of blade. Specimen numbers (a) 37O2B-7; (b) 28S1-1.

Tool State and Fractures: One is roughly complete and exhibits a reworked shoulder section; 10 are proximal sections, 6 of which have largely complete bases. One other is a stem section. Single bend fractures were recorded on 3 , multiple impact and burin-like fractures on 5, multiple bend on 1 , multiple bend and thermal on 1 , and bend and indeterminate on 2 .

Rejuvenation: One is resharpened unifacially, unbeveled, 5 are alternately beveled, and 6 are indeterminate.

Stratigraphic Distribution: As shown in Table 13-44, specimens typed as Jetta are widely distributed in chiefly Archaic deposits.

Comments: Jetta is a poorly understood point type (in part, perhaps, because most of the known examples appear to be heavily reworked), chiefly distinguished by attributes relative to other types (e.g., larger size, stem shape) and which may form a continuum with certain smaller early bifurcate types. Nonetheless, specimens typed as Jetta emerged as a discrete cluster in a statistical study of early bifurcate stemmed points (see Chapter 14).

\section{Jetta-like $(N=1)$}

Morphology: This is an oddly shaped triangular point with characteristic strong shoulders and barbs but with a short bifurcate stem, possibly due to rebasing.

Metric Values: Length is incomplete, width is $47 \mathrm{~mm}$, stem length is $10 \mathrm{~mm}$, proximal stem width is $20 \mathrm{~mm}$, distal stem width is $19 \mathrm{~mm}$, and basal concavity is $3 \mathrm{~mm}$. 


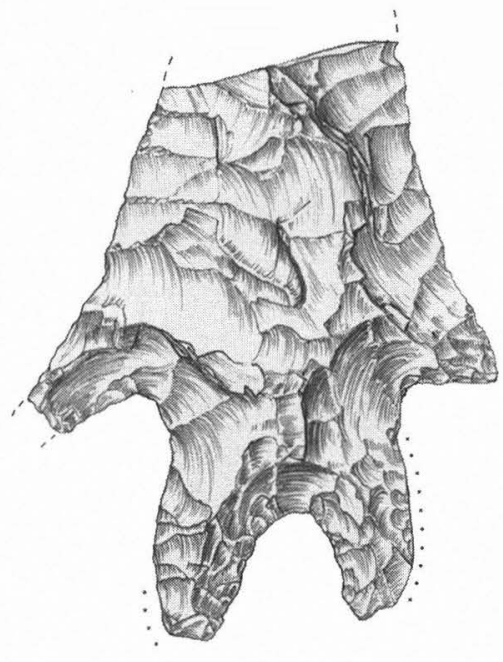

a

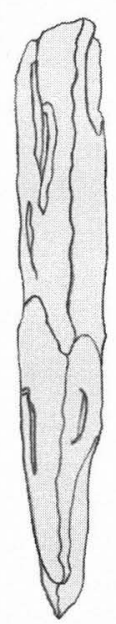

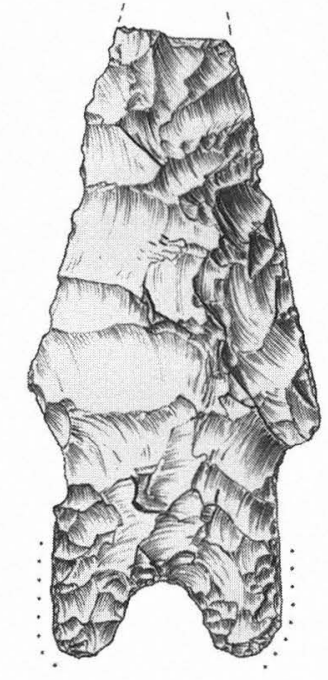

$\mathrm{b}$
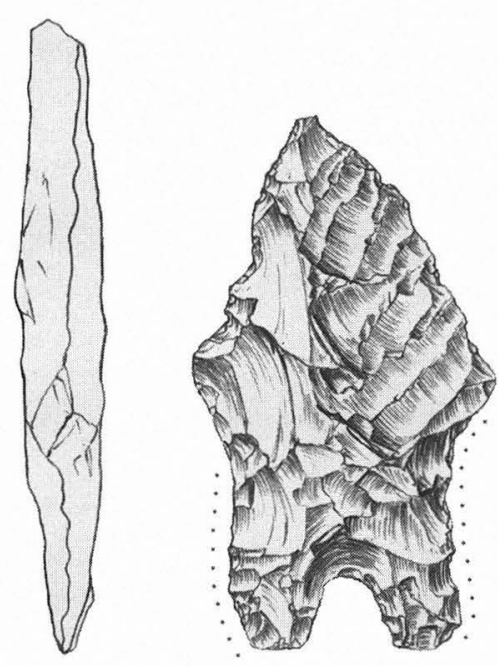

c

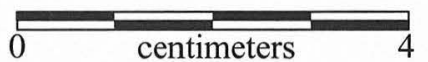

FIGURE 13-44. Jetta points. Specimen numbers: (a) 15N2-1; (b) 3M-2; (c) 3701D-1.

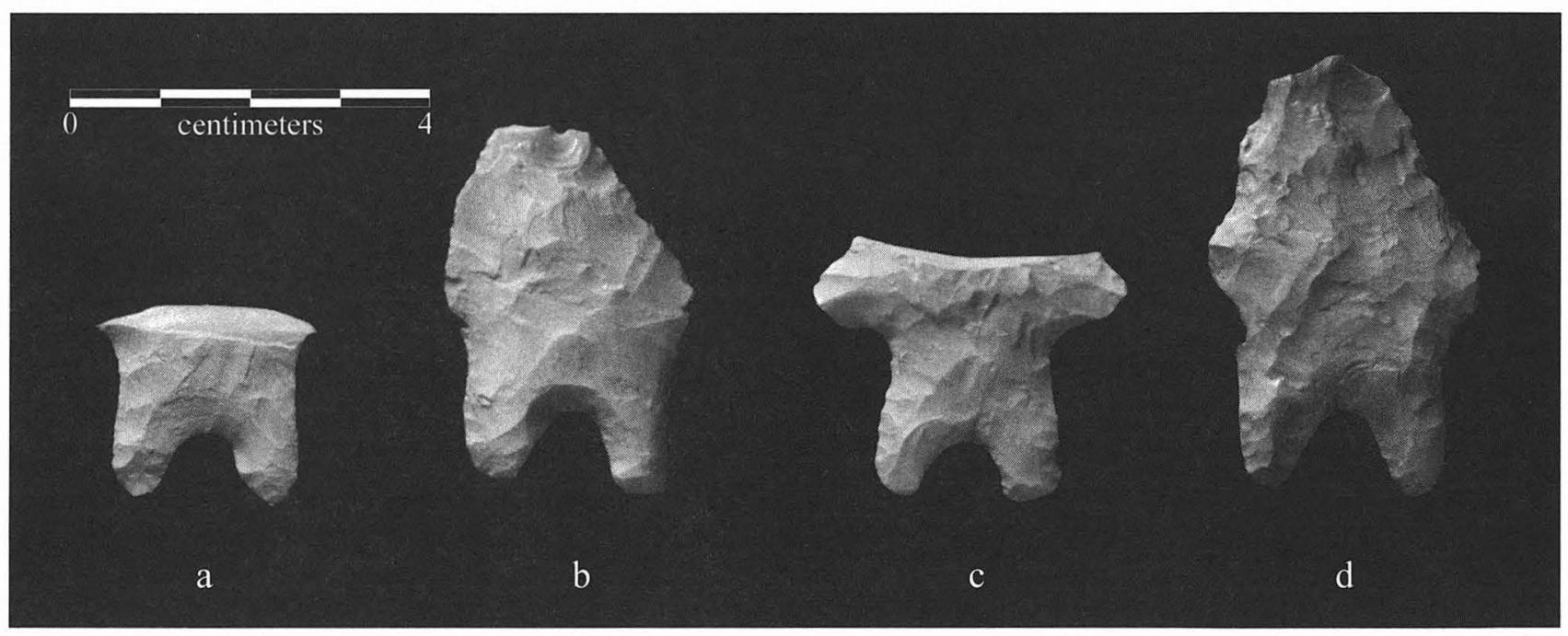

FIGURE 13-45. Jetta points. Specimen numbers: (a) 8O-1; (b) 8N-1; (c) 11R-1; (d) 13J-1.

TABLE $13-43$

Summary Metric Data for Jetta Points (in mm)

\begin{tabular}{|c|c|c|c|c|c|}
\hline & Number & Minimum & Maximum & Mean & $1-\sigma$ \\
\hline Width & 6 & 28.0 & 35.0 & 30.7 & 2.4 \\
\hline Thickness & 12 & 5.0 & 9.0 & 7.6 & 1.2 \\
\hline Stem length & 12 & 17.0 & 24.0 & 20.1 & 2.4 \\
\hline Proximal stem width & 8 & 20.0 & 26.0 & 22.3 & 1.8 \\
\hline Distal stem width & 10 & 18.0 & 26.0 & 21.9 & 2.5 \\
\hline Basal concavity depth & 11 & 6.1 & 12.6 & 8.8 & 2.0 \\
\hline Distal stem thickness & 8 & 4.9 & 8.4 & 6.5 & 1.2 \\
\hline Basal concavity width & 8 & 8.6 & 13.4 & 10.3 & 1.5 \\
\hline
\end{tabular}


TABLE 13-44

Stratigraphic Distribution of Jetta Points

\begin{tabular}{l|c|c}
\hline Unit & Valley Floor A & Valley Margin \\
\hline IIIc & 1 & 1 \\
IIIb & 1 & 1 \\
Y & 3 & \\
IIIa/b & 1 & 1 \\
IIIa & & 3 \\
II/IIIa & & \\
X & & \\
\hline \hline
\end{tabular}

Nonmetric Attributes: Longitudinal profile is wedgeshaped base and tip; flake patterns are random; basal modification is concave beveling; lateral stem grinding is absent.

Material Attributes: Raw material is nonlocal finegrained Edwards chert. No patina or mineral precipitates were observed. Differential luster suggests heat treatment prior to resharpening.

Tool State and Fractures: This is a relatively complete specimen with its distal tip severed by bending.

Rejuvenation: Resharpened bifacially, unbeveled, and possibly rebased.

Stratigraphic Distribution: Surface.

\section{Martindale $A(N=12)$}

Morphology: These are medium-sized, typically wellflaked triangular points with moderate, rounded shoulders and medium to wide expanding stems formed by corner notching. A distinguishing feature is an inwardly recurved or "fishtail" base which is typically well-thinned (Figures 13-46 and 13-47). Blade resharpening in this group typically takes the form of fine edge retouch or very slight beveling of blade edges. Dulling or grinding of stem lateral edges is not common.

Metric Averages: Table 13-45

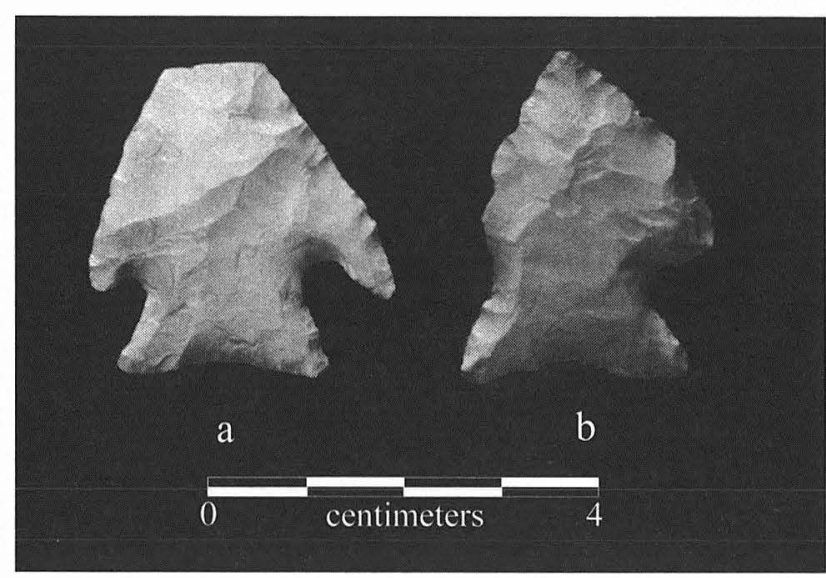

FiguRE 13-47. Martindale A points. Specimen numbers: (a) 13J-2; (b) $21 \mathrm{~J}-1$.

Nonmetric Attributes: Longitudinal profiles include 5 tapered base and tip; 1 thick tip; 2 wedge-shaped base and tapered tip; 1 wedge-shaped base, thick stem, and flat body; and 3 indeterminate. Flake patterns are organized on 6 (oblique subparallel, chevron), random on 2 , and indeterminate on 4. Basal modification consists of unifacial thinning on 8 , bifacial thinning on 2 , and secondary trimming on 2 . Lateral stem grinding is slight on 2, moderate on 1 .

Material Attributes: Raw material is local fine-grained Edwards chert $(n=7)$ and nonlocal Edwards chert (4 fine grained and 1 medium/coarse). Patina is heavy overall on 1 . Mineral precipitates are present on 1 . Heat damage is slight on 1 and extensive on 1.

Tool State and Fractures: Three are nearly complete, lacking only a distal tip, barb tip, or small basal ear section, due, in each case, to bend fracturing. Though near full size, 5 others are heavily damaged due to multiple bend and impact/burin-like fractures, thermal fractures, and indeterminate edge crushing. The remaining 4 are proximal sections displaying multiple bend and burin-like/impact fractures in 3 cases and thermal fractures in 1.

Rejuvenation: Ten are resharpened ( 2 unifacially,

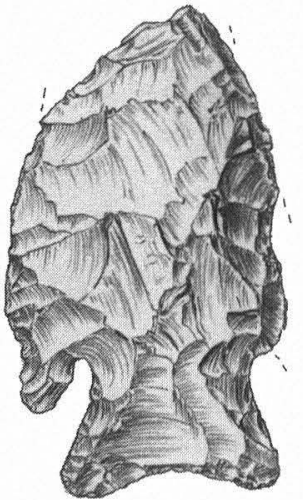

a

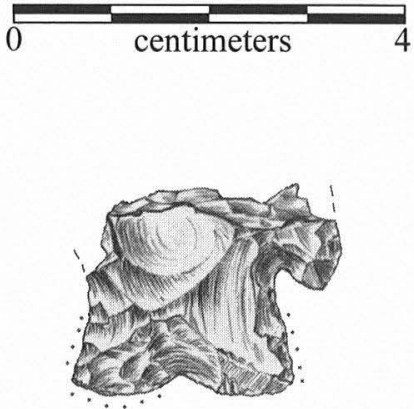

b

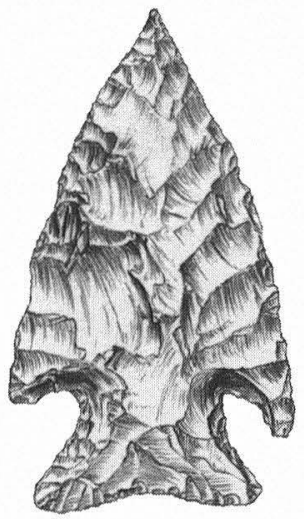

c

FIGURE 13-46. Martindale A points. Specimen numbers: (a) 10K-1; (b) 6F-1; (c) 37MA-4. 
TABLE $13-45$

Summary Metric Data for Martindale A Points (in mm)

\begin{tabular}{|c|c|c|c|c|c|}
\hline & Number & Minimum & Maximum & Mean & $1-\sigma$ \\
\hline Length & 3 & 34.0 & 50.0 & 39.7 & 9.0 \\
\hline Width & 6 & 27.0 & 37.0 & 29.7 & 3.9 \\
\hline Thickness & 12 & 6.0 & 8.0 & 7.3 & 0.8 \\
\hline Stem length & 12 & 11.0 & 15.0 & 12.0 & 1.1 \\
\hline Proximal stem width & 11 & 19.0 & 24.0 & 22.3 & 1.7 \\
\hline Distal stem width & 11 & 16.0 & 19.0 & 17.1 & 1.3 \\
\hline Basal concavity depth & 12 & 1.4 & 3.0 & 2.2 & 0.5 \\
\hline Distal stem thickness & 7 & 5.5 & 8.1 & 6.6 & 0.8 \\
\hline Basal concavity width & 7 & 12.2 & 17.0 & 14.8 & 1.8 \\
\hline
\end{tabular}

unbeveled; 4 bifacially, unbeveled; 3 alternately beveled; and 1 unifacially beveled).

Stratigraphic Distribution: Martindale A occurs in highest frequency in stratigraphic Unit IIIb, pertaining to Early Archaic and Middle Archaic times (Table 13-46).

\section{Martindale $B(N=6)$}

Morphology: This group overlaps with Martindale A but is set apart by a generally narrower stem and a somewhat asymmetrically recurved or divided base with rounded basal ears, rather than the characteristic Martindale "fishtail" (Figures 13-48 and 13-49).

Metric Averages: Table 13-47

Nonmetric Attributes: Longitudinal profiles include 3 tapered base and tip and 3 indeterminate. Flake patterns are or-ganized on 2 (oblique subparallel, subparallel), random on 1 , and indeterminate on 3 . Basal modification consists of uni-facial thinning on 2, bifacial thinning on 3 , and it is absent on 1. Lateral stem grinding is slight on 1 and moderate on 1 .

Material Attributes: Raw material is local Edwards finegrained chert for 4, nonlocal Edwards fine-grained chert for 1, and nonlocal Edwards medium/coarse chert for 1. No mineral precipitates, patina, or heat alteration was observed.

Tool State and Fractures: Three are complete or nearly so, lacking only distal or barb tips due to bend fractures; 2 others are stem sections, exhibiting multiple bend and burinlike fractures. The remaining specimen is a proximal section severed by a bend break.

Rejuvenation: Three are resharpened ( 1 bifacially unbeveled, 2 alternately beveled) and 3 are indeterminate.

Stratigraphic Distribution: Distribution of Martindale $\mathrm{B}$ mirrors that of Martindale $\mathrm{A}$, with highest frequency in Unit IIIb; based on this sample, there appears to be no clear temporal distinction between the two variants (Table $13-48)$.

Comments: Specimens in this group appear similar to Martindale Variety 2 from the Landslide site (Sorrow et al. 1967:Figure 121, m).
TABLE 13-46

Stratigraphic Distribution of Martindale A Points

\begin{tabular}{l|c|c}
\hline Unit & Valley Floor A & Valley Margin \\
\hline IIIc & 1 & 2 \\
IIIb/c & & 1 \\
Y/IIIc & 4 & 2 \\
IIIb & & 1 \\
Y & 1 & \\
IIIa/b & & \\
\hline \hline
\end{tabular}

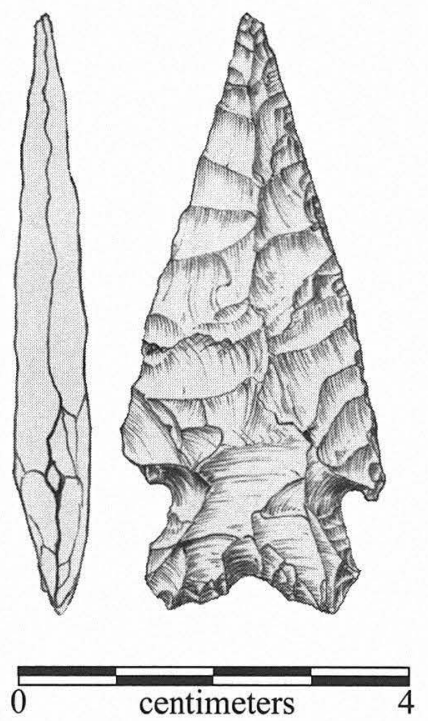

Figure 13-48. Martindale B point, Specimen 36J-1.

\section{Expanding Stem, Concave Base $A(N=6)$}

Morphology: Diminutive overall in size, these points have short, expanding stems with ground lateral edges and very shallow, arc-shaped basal concavities (Figures 13-50 and 13-51). Bases are not well thinned and appear wedgelike in cross section. The two specimens complete enough to characterize exhibit slight shoulders with slight barb remnants. Figure 13-50a (21R-8) exhibits more-patterned, ob- 


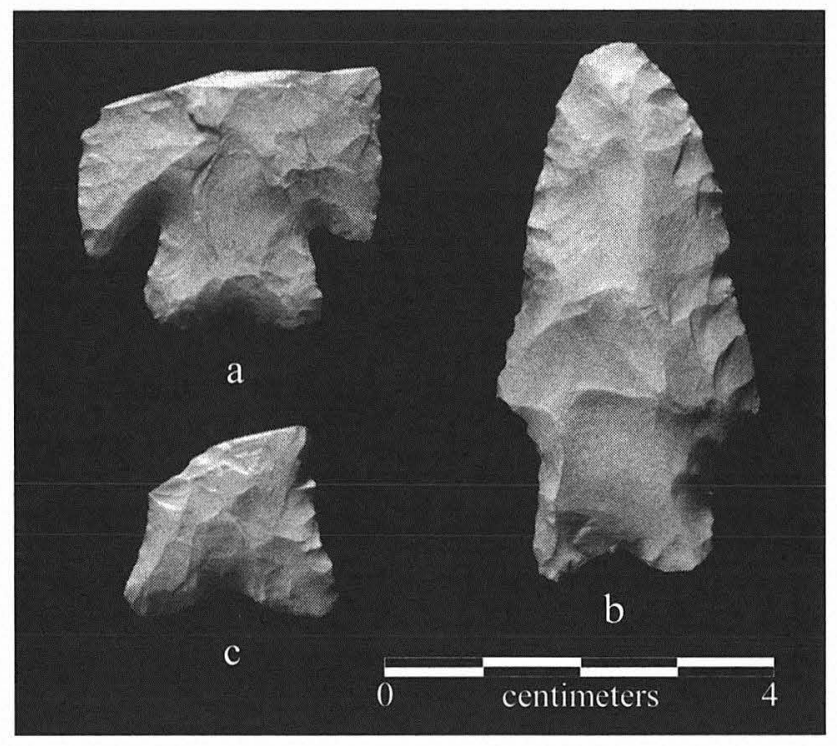

FiguRE 13-49. Martindale B points. Specimen numbers: (a) 21L-3; (b) $14 \mathrm{G}-2$; (c) 14J1-1.
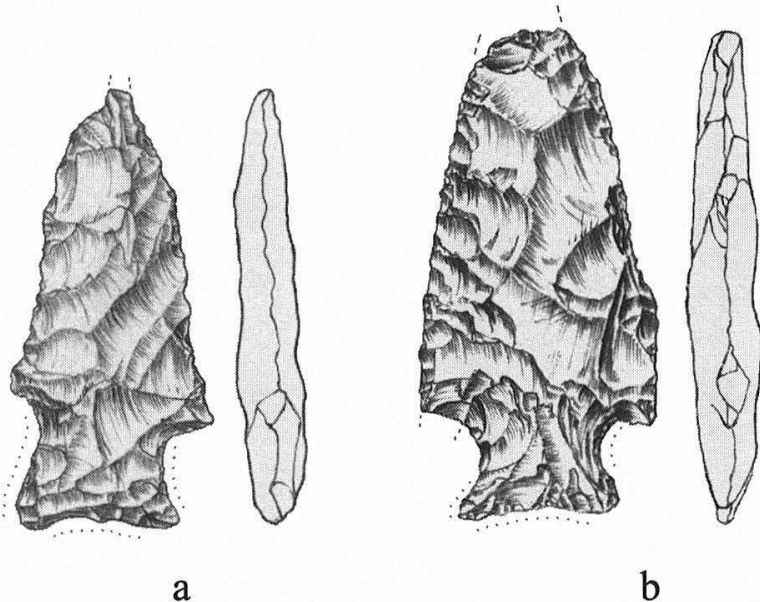

a

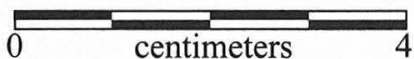

Figure 13-50. Expanding stem, concave base A points. Specimen numbers: (a) 21R-8; (b) 26W2A-1.

TABLE 13-47

Summary Metric Data for Martindale B Points (in mm)

\begin{tabular}{l|c|c|c|c|c}
\hline & Number & Minimum & Maximum & Mean & $1-\sigma$ \\
\hline Length & 3 & 31.0 & 61.0 & 48.7 & 15.7 \\
Width & 4 & 22.0 & 31.0 & 26.5 & 3.7 \\
Thickness & 6 & 5.0 & 8.0 & 6.3 & 1.2 \\
Stem length & 5 & 11.0 & 15.0 & 12.8 & 1.6 \\
Proximal stem width & 5 & 18.0 & 27.0 & 21.0 & 3.7 \\
Distal stem width & 4 & 15.0 & 18.0 & 16.5 & 1.3 \\
Basal concavity depth & 6 & 1.5 & 4.0 & 3.1 & 1.1 \\
Distal stem thickness & 2 & 5.3 & 5.6 & 5.5 & 0.2 \\
Basal concavity width & 2 & 8.0 & 9.7 & 8.9 & 1.2 \\
\hline \hline
\end{tabular}

TABLE 13-48

Stratigraphic Distribution of Martindale B Points

\begin{tabular}{l|c|c}
\hline Unit & Valley Floor A & Valley Margin \\
\hline IIIb/c & 1 & \\
IIIb & 4 & 1 \\
\hline \hline
\end{tabular}

lique subparallel flaking and shows slight alternate beveling of blade edges. The other (Figure 13-50b, 26W2A-1) is flaked more randomly and is reworked bifacially unbeveled with some dulling of blade lateral margins. Both show moderate to heavy stem and basal edge grinding.

Metric Averages: Table 13-49

Nonmetric Attributes: Longitudinal profiles include 1 flat; 3 wedge-shaped base and tip; and 2 indeterminate. Flake patterns are organized on 1 (oblique subparallel), random on 1 , and indeterminate on 4 . Basal modification includes 2 with concave beveling and 4 with secondary trimming. Lateral stem grinding is moderate on 1 and heavy on 2 .

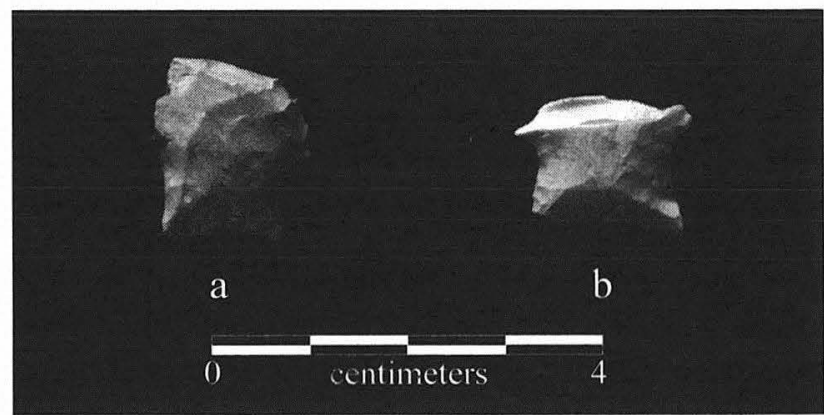

FIGURE 13-51. Expanding stem, concave base A points. Specimen numbers: (a) 16H-6; (b) 51SC-7.

Material Attributes: Raw material is local $(\mathrm{n}=5)$ and nonlocal $(n=1)$ Edwards fine-grained cherts. Patina is moderate partial on 1 . No mineral precipitates or heat alteration were observed.

Tool State and Fractures: Two are nearly complete, displaying only minor facial, burin-like, and bend breakage of 
TABLE 13-49

Summary Metric Data for Expanding Stem, Concave Base A Points (in mm)

\begin{tabular}{|c|c|c|c|c|c|}
\hline & Number & Minimum & Maximum & Mean & $1-\sigma$ \\
\hline Length & 1 & 45.0 & 45.0 & & \\
\hline Width & 3 & 18.0 & 25.0 & 21.3 & 3.5 \\
\hline Thickness & 6 & 5.0 & 8.0 & 6.0 & 1.3 \\
\hline Stem length & 4 & 8.0 & 11.0 & 9.8 & 1.3 \\
\hline Proximal stem width & 6 & 13.0 & 18.0 & 15.8 & 1.6 \\
\hline Distal stem width & 4 & 12.0 & 14.0 & 13.0 & 0.8 \\
\hline Basal concavity depth & 6 & 1.0 & 2.0 & 1.3 & 0.5 \\
\hline Distal stem thickness & 3 & 5.0 & 6.6 & 6.1 & 0.9 \\
\hline Basal concavity width & 3 & 7.3 & 10.5 & 9.3 & 1.8 \\
\hline
\end{tabular}

the distal tip. The remaining 4 are proximal sections with multiple bend and burin-like break scars.

Rejuvenation: Resharpening was observed on 3 (1 unbeveled bifacial, 1 alternately beveled, 1 beveled unifacial), and 3 are indeterminate.

Stratigraphic Distribution: As shown in Table 13-50, there is no clear pattern of distribution of this small group. The specimens shown in Figure 13-50 are both from Unit II, attributed to the Late Paleoindian.

Comments: With their small size and expanding stems, the two specimens recovered from Late Paleoindian contexts (see Figure 13-50) are particularly enigmatic. They may be related to Hoxie or the Zephyr variant. Prewitt's (n.d., 1981a:96) early discussions of the two, however, suggest that distinctions center somewhat on relative degree of blade beveling and stem grinding; the Zephyr variety was thought at the time to be somewhat younger than Hoxie, perhaps dating to the Middle Archaic. Farther afield, points of similar morphology and temporal context are seen among types Hardin Barbed and Kirk Corner Notched (Justice 1995).

\section{Expanding Stem, Concave Base B $(N=20)$}

Morphology: These are medium-sized, triangular, corner-notched points with fairly strong shoulders and barbs, and typically fine, organized flaking. Their stems expand from relatively narrow distal neck sections to medium to wide bases (Figures 13-52 and 13-53). Basal concavities are generally shallow and typically arc-shaped; stem lateral edges may be dulled or ground.

Metric Averages: Table 13-51

Nonmetric Attributes: Longitudinal profiles include 3 with tapered base and tip, 12 with wedge-shaped base and tip and uniform body, and 5 indeterminate; flake patterns are organized on 12 (oblique subparallel, subparallel), random on 4 , and indeterminate on 4 ; basal modification constitutes unifacial thinning on 6 , bifacial thinning on 1 , fluting unifacially on 1 , beveled base on 1 , concave bevel on 6 , and secondary trimming on 5; and lateral stem grinding is slight on 3, moderate on 2, and heavy on 2 .
TABLE 13-50

Stratigraphic Distribution of Expanding Stem, Concave Base A Points

\begin{tabular}{l|c|c|c}
\hline Unit & Valley Floor A & Valley Floor B & Valley Margin \\
\hline IIIb/c & 1 & & 1 \\
Y/IIIc & & & 1 \\
IIIb & & 1 & \\
III & & & 1 \\
II & 1 & & \\
\hline \hline
\end{tabular}

Material Attributes: Raw material includes local Edwards chert on 13 (11 fine grained, 2 medium/coarse), nonlocal Edwards on 5 (3 fine grained, 2 medium/coarse), and indeterminate heat altered on 2; patina is heavy overall on 2 , slight partial on 1 , and moderate partial on 1 ; mineral precipitates were observed on 4; and heat damage is moderate on 1 and extensive on 2 . An additional specimen exhibits differential gloss suggestive of heat treatment.

Tool State and Fractures: Twelve are complete except for distal and/or barb tips, due to single bend breaks (on 2), single impact fracture (on 1), multiple bend breaks (on 3), or multiple bend and burin-like or facial impact fractures (on 6); the remaining 8 are proximal sections exhibiting multiple bend, burin-like/impact, and/or thermal fractures.

Rejuvenation: All but 1 of those complete enough to observe were resharpened (1 unbeveled unifacial, 5 unbeveled bifacial, 6 alternately beveled).

Stratigraphic Distribution: This group is widely distributed over several stratigraphic units but shows highest frequency in the Unit IIIa/b transition zone and Unit IIIb (Table 13-52).

Comments: This group shares attributes with a number of the early expanding stem groups described herein. Varying chiefly in size; it appears to grade between Martindale and Uvalde, falling within the realm of the Early Corner Notched series (see Hester 1971; cf. Early Expanding Stem Form 1, Black and McGraw 1985: Figure 22a-f; also see Wesolowsky et al. 1976:Figure 15d:53). 


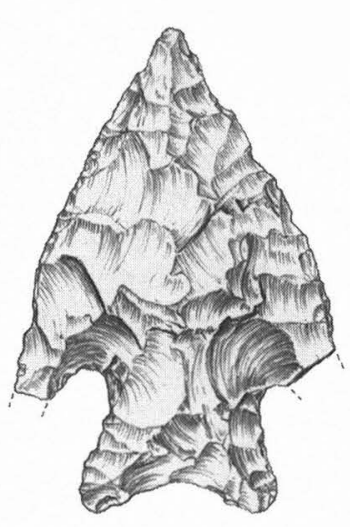

a
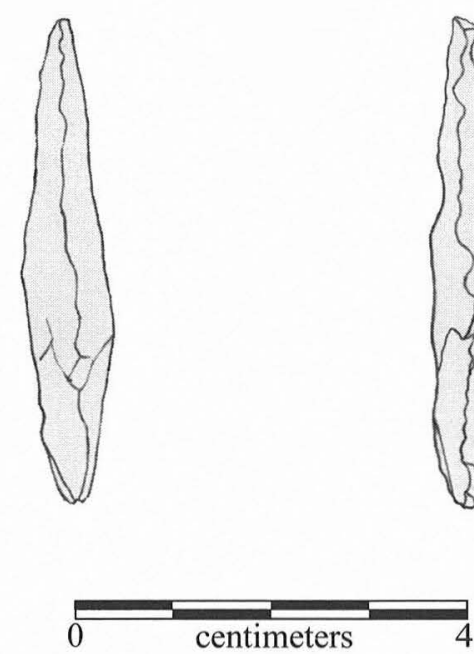

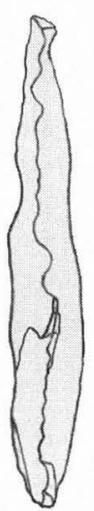

b

FIGURE 13-52. Expanding stem, concave base B points. Specimen numbers: (a) 7F-1; (b) 20K-9.

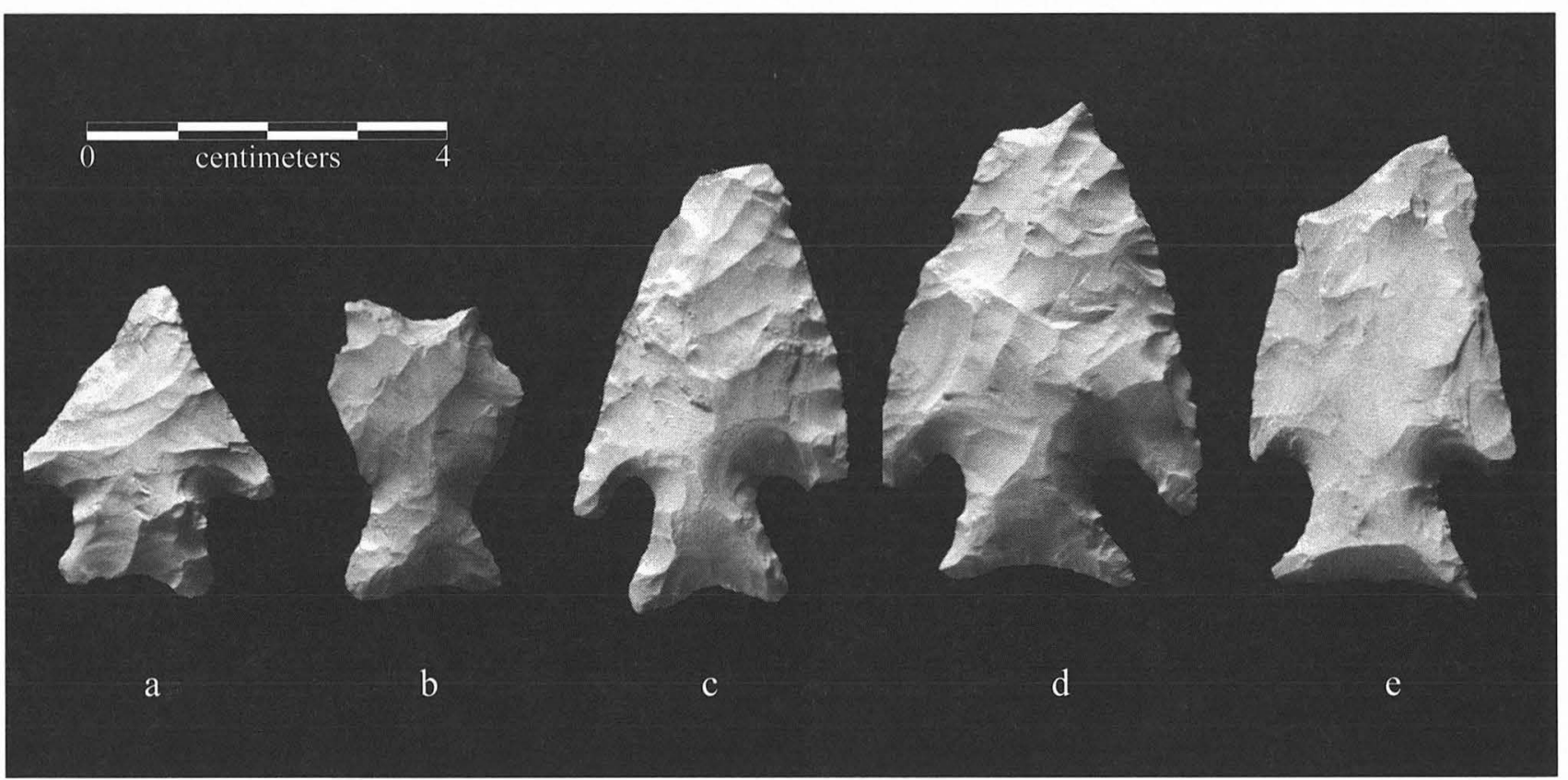

FIGURE 13-53. Expanding stem, concave base B points. Specimen numbers: (a) 14N1-1; (b) 5N-2; (c) 12G-1; (d) 17L-1; (e) $20 \mathrm{G}-9$.

TABLE 13-51

Summary Metric Data for Expanding Stem, Concave Base B Points (in mm)

\begin{tabular}{l|c|c|c|c|c}
\hline & Number & Minimum & Maximum & Mean & $1-\sigma$ \\
\hline Length & 3 & 36.0 & 61.0 & 48.7 & 12.5 \\
Width & 13 & 22.0 & 40.0 & 27.8 & 7.3 \\
Thickness & 20 & 5.0 & 8.6 & 12.9 & 1.0 \\
Stem length & 20 & 9.0 & 23.6 & 19.0 & 2.0 \\
Proximal stem width & 17 & 15.0 & 18.6 & 13.6 & 2.5 \\
Distal stem width & 20 & 10.0 & 4.0 & 2.2 & 0.9 \\
Basal concavity depth & 20 & 0.5 & 7.7 & 1.9 & 0.6 \\
Distal stem thickness & 10 & 5.5 & 18.9 & 12.4 & 3.7 \\
Basal concavity width & 9 & 6.6 &
\end{tabular}


TABLE 13-52

Stratigraphic Distribution of Expanding Stem, Concave Base B

\begin{tabular}{l|c|c}
\hline Unit & Valley Floor A & Valley Margin \\
\hline IIIc & 1 & 3 \\
IIIb/c & 2 & 2 \\
IIIb & 4 & 1 \\
IIIa/b & & 1 \\
Y/IIIb & & 1 \\
X/Y/IIIb & & 1 \\
X/IIIb & & 1 \\
Y & & 1 \\
X/Y & & \\
X & & \\
Other & 1 & \\
IIIc pot & & \\
IIIb/c fill & & \\
\hline \hline
\end{tabular}

\section{Expanding Stem, Concave Base $C(N=9)$}

Morphology: These are typically well-flaked, triangular, corner-notched points with small barbs and wide, expanding bases with shallow to moderately deep, arc-shaped basal concavities (Figures 13-54 and 13-55). Stems are usually well thinned from the base, and basal concavities may be lightly ground or dulled; stem lateral edges are generally not ground.

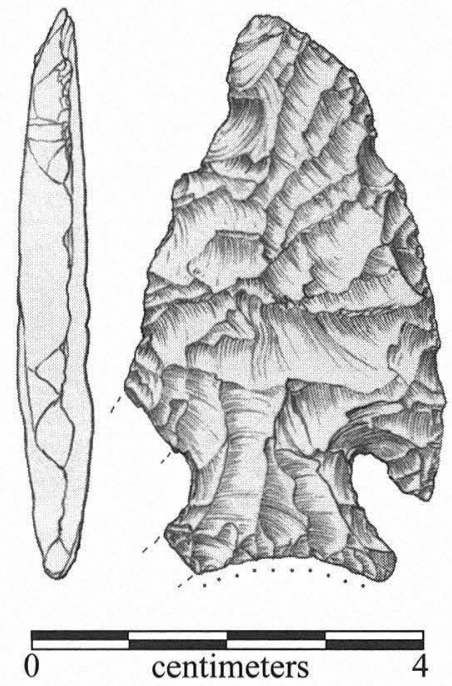

FIGURE 13-54. Expanding stem, concave base C point, Specimen $21 \mathrm{H}-2$.

Metric Averages: Table 13-53

Nonmetric Attributes: Longitudinal profiles inlude 4 with tapered base and tip, 1 with thick tip, 1 flat, and 3 with wedge-shaped base, thick stem, and flat body. Flake patterns are organized on 6 (oblique subparallel, subparallel, chevron), random on 2, and indeterminate on 1 . Basal modification consists of unifacial thinning on 6 and bifacial thinning on 3. Lateral stem grinding is slight on 3 .

Material Attributes: Raw material for 6 is local Edwards chert ( 5 fine grained, 1 medium/coarse), and for 3 is nonlocal Edwards fine-grained chert. Mineral precipitates are present on 1 . Heat damage is slight on 1 . No patina was observed.

Tool State and Fractures: One is complete. Eight lack distal and/or barb tips, and of these, 2 display multiple bend fractures, 1, multiple impact/burin-like fractures, 2 multiple bend and impact/burin-like fractures, 2 multiple impact and thermal fractures, and 1, multiple bend, burin-like, and thermal fractures.

Rejuvenation: Six are alternately beveled, 1 is resharpened unbeveled bifacially, 1 is indeterminate, and 1 is not resharpened.

Stratigraphic Distribution: This group is dispersed over contexts chiefly pertinent to the Early and Middle Archaic (Table 13-54).

Comments: With the widest base of the five expanding stem, concave base groups, Group C resembles Martindale but lacks the distinctive fishtail base. Stratigraphic contexts for the two groups are very similar (see Tables 13-46, 13-48, and 13-54).

\section{Expanding Stem, Concave Base D (N=15)}

Morphology: These are well-made, fairly broad-bladed points with large barbs; stems are narrow to medium with recurved or slightly convex edges and downward pointing basal ears, giving some of the stems a contracting appearance. With generally well-thinned bases, stems are tapered in longitudinal profile. Basal concavities vary from U- to Vshaped and are generally ground, as are stem lateral edges (Figures 13-56 and 13-57).

Metric Averages: Table 13-55

Nonmetric Attributes: Longitudinal profiles include tapered base and tip on 9, wedge-shaped base, thick stem, and flat body on 1, and 5 indeterminate. Flake patterns are organized on 7 (oblique subparallel, subparallel), random on 3 , and indeterminate on 5. Basal modification includes unifacial thinning on 4 , bifacial thinning on 8 , bifacial fluting on 1, concave beveling on 1 , and 1 indeterminate. Lateral stem grinding is slight on 4 , moderate on 5 , and heavy on 3 .

Material Attributes: Raw material for 11 is local Edwards chert (10 fine grained, 1 medium/coarse), 1 is nonlocal Edwards fine-grained chert, and 3 are indeterminate heat altered. Mineral precipitates are present on 3 . Heat damage is moderate on 1 and extensive on 2. No patina was observed.

Tool State and Fractures: Two are complete and 1 nearly so, lacking only distal and barb tips. Eight others are proximal sections, and of these, 1 exhibits a single bend fracture, 2 , multiple bend fractures, 2 , multiple bend and burin-like fractures, 2, multiple impact and burin-like fractures, and 1, multiple thermal fractures. Five are basal sections, 3 of which display single bend breaks, 1 , multiple bend breaks, and 1 multiple thermal breaks. 


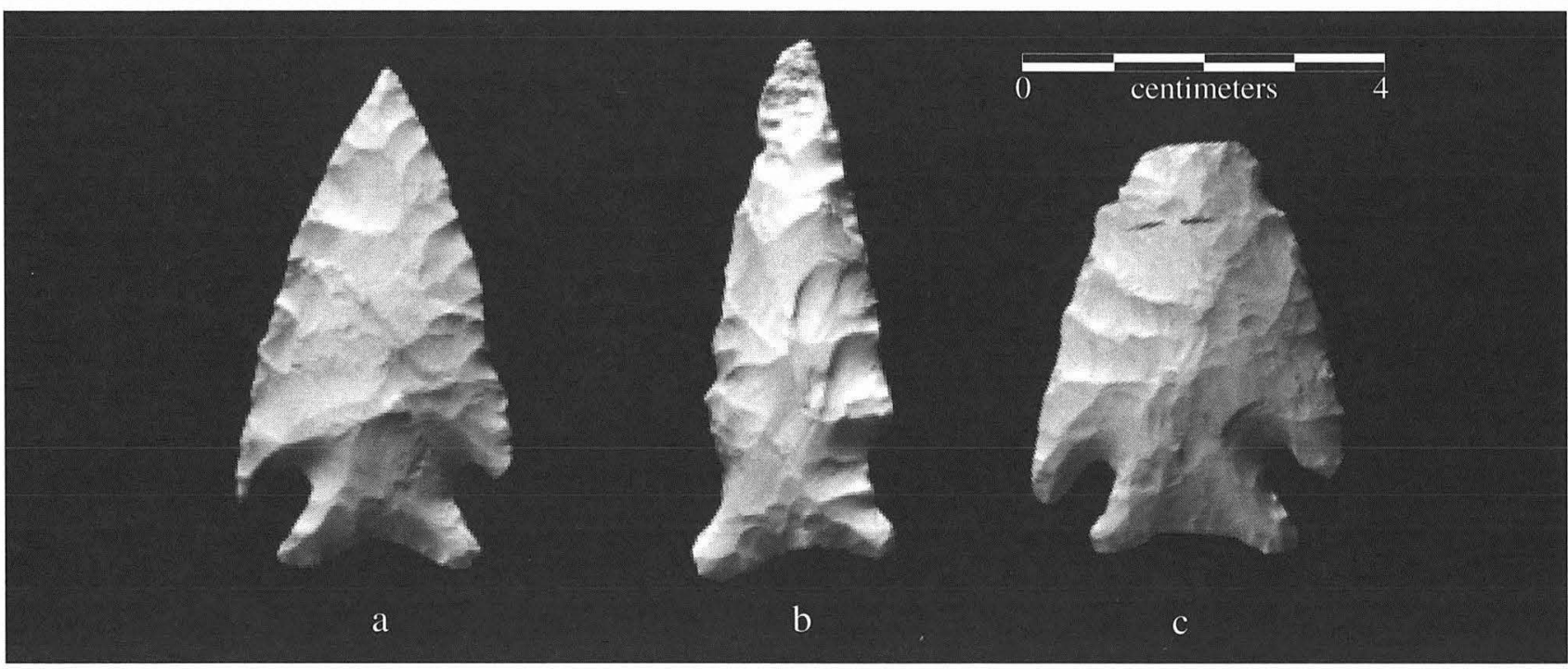

FIGURE 13-55. Expanding stem, concave base C points. Specimen numbers: (a) 9J-2; (b) 21H-3; (c) 7G-2.

TABLE 13-53

Summary Metric Data for Expanding Stem, Concave Base C Points (in mm)

\begin{tabular}{|c|c|c|c|c|c|}
\hline & Number & Minimum & Maximum & Mean & $1-\sigma$ \\
\hline Length & 3 & 51.0 & 58.0 & 54.3 & 3.5 \\
\hline Width & 7 & 25.0 & 34.0 & 29.9 & 3.3 \\
\hline Thickness & 9 & 6.0 & 9.0 & 7.0 & 0.0 \\
\hline Stem length & 8 & 10.7 & 15.8 & 12.5 & 1.8 \\
\hline Proximal stem width & 4 & 22.0 & 29.9 & 24.3 & 3.7 \\
\hline Distal stem width & 9 & 15.0 & 18.0 & 16.5 & 1.0 \\
\hline Distal stem thickness & 4 & 6.2 & 7.7 & 6.9 & 6.4 \\
\hline Basal concavity depth & 7 & 2.0 & 5.0 & 2.9 & 1.1 \\
\hline Basal concavity width & 4 & 11.0 & 24.0 & 15.9 & 5.6 \\
\hline
\end{tabular}

TABLE 13-54

Stratigraphic Distribution of Expanding Stem, Concave Base C

\begin{tabular}{l|c|c}
\hline Unit & Valley Floor A & Valley Margin \\
\hline IIIc & 1 & 1 \\
IIIb/c & & 2 \\
Y/IIIb/c & 1 & 1 \\
IIIb & 2 & 1 \\
IIIa/b & & \\
\hline \hline
\end{tabular}

Rejuvenation: Three are resharpened bifacially, unbeveled; 5 are alternately beveled; 1 is beveled unifacially, and 5 are indeterminate. One of the complete specimens (37NB-7; see Figure 13-56c) was recycled as a scraper after distal and barb sections were broken.

Stratigraphic Distribution: As shown in Table 13-56, almost half ( 6 of 14 with provenience, or $43 \%$ ) were recovered in stratigraphic Unit IIIb.

Comments: The large barbs, fine flaking, and well- thinned bases of this group are attributes shared with Bell/ Andice and the Calf Creek points (see Perino 1968). They are set apart, however, by distinct basal concavities that are not characteristic of Bell/Andice or Calf Creek. The present group can be more appropriately likened to both the Early Corner Notched group in south-central Texas (see Hester 1971) and the Early Barbed group of the Lower Pecos (Johnson 1964:3334) which resembles Bell. Two bifurcate stem points recovered from below an apparent Martindale zone at the Eckols site in Travis County (James Karbula, personal communication 1995) are similar to some specimens in this group, particularly Figure 13-56c, d.

\section{Expanding Stem, Concave Base $E(N=3)$}

Morphology: These broad triangular stemmed points have moderately strong shoulders and remnants of small barbs. Stems are wide and thick, with necks only slightly constricted; basal concavities are wide, shallow, and V- to arcshaped (Figures 13-58 and 13-59). Stem lateral edges are ground. 


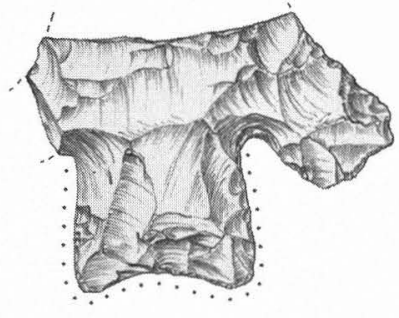

a

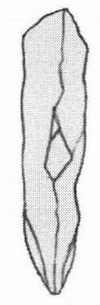

a
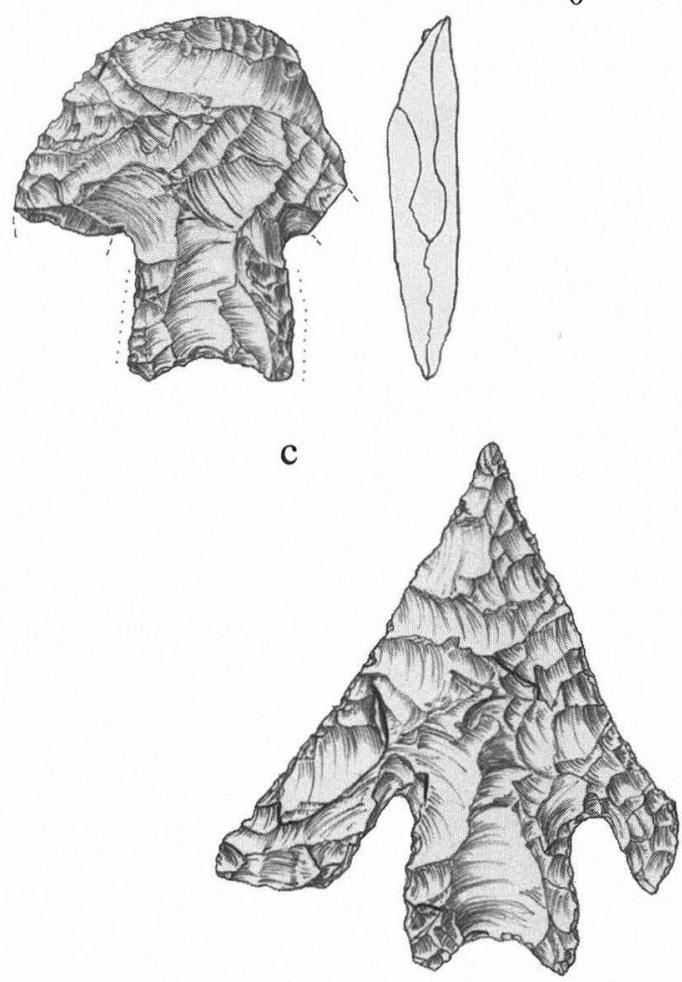

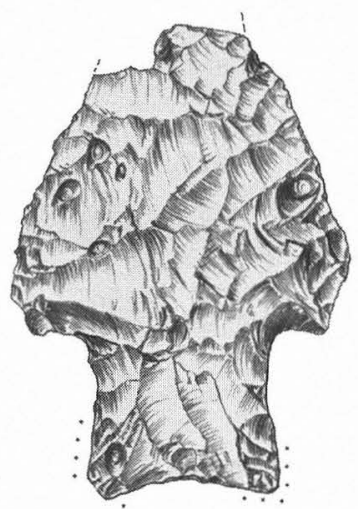

b
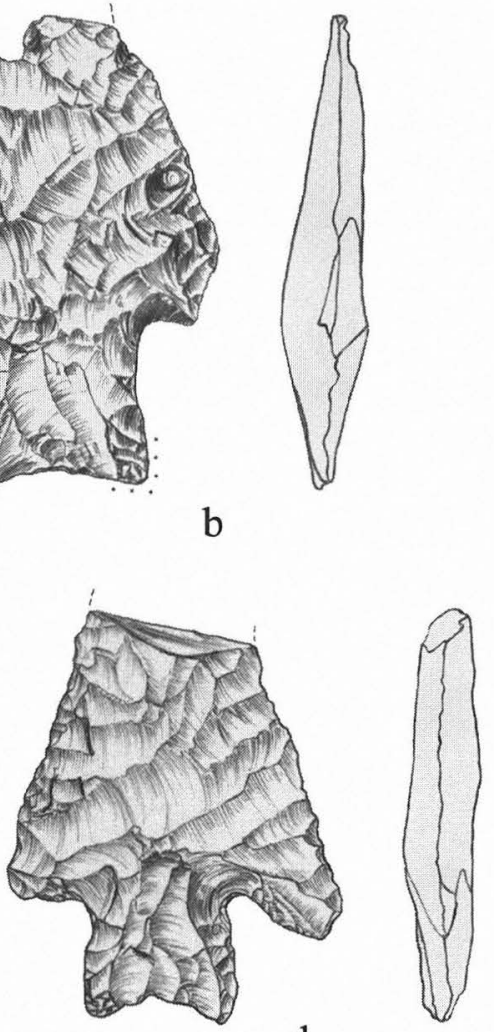

d
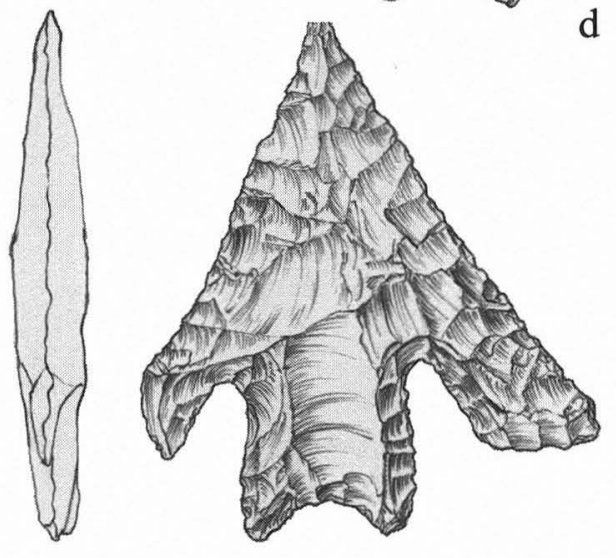

e

FiguRE 13-56. Expanding stem, concave base D points. Specimen numbers: (a) 19K-1; (b) 23K-1; (c) 37NB-7; (d) 37MC-16; (e) $20 \mathrm{~N}-1$.

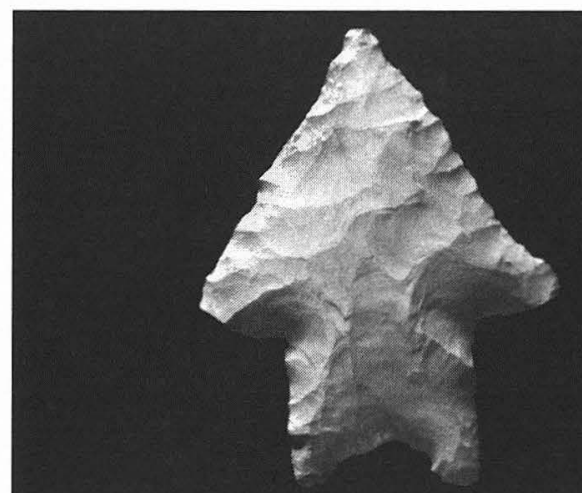

a

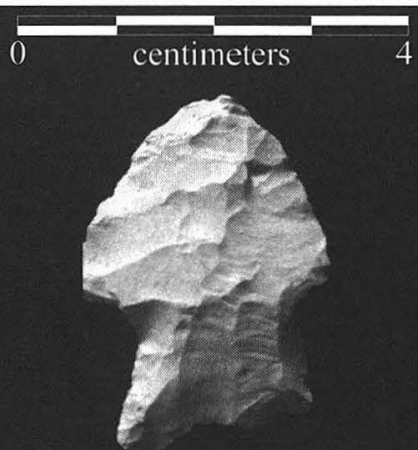

b

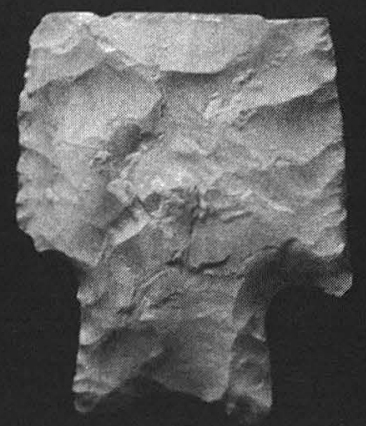

c

FIGURE 13-57. Expanding stem, concave base D points. Specimen numbers: (a) 17K1-1; (b) 17K2-2; (c) 8J-2. 
TABLE 13-55

Summary Metric Data for Expanding Stem, Concave Base D Points (in mm)

\begin{tabular}{|c|c|c|c|c|c|}
\hline & Number & Minimum & Maximum & Mean & $1-\sigma$ \\
\hline Length & 4 & 27.0 & 53.0 & 41.3 & 11.6 \\
\hline Width & 6 & 32.0 & 47.0 & 35.7 & 5.6 \\
\hline Thickness & 15 & 5.0 & 9.0 & 7.2 & 1.2 \\
\hline Stem length & 12 & 12.0 & 18.0 & 15.6 & 1.8 \\
\hline Proximal stem width & 13 & 16.0 & 20.9 & 18.2 & 1.6 \\
\hline Distal stem width & 12 & 14.6 & 20.4 & 17.5 & 1.9 \\
\hline Distal stem thickness & 9 & 5.5 & 8.8 & 7.1 & 1.1 \\
\hline Basal concavity depth & 13 & 1.0 & 4.1 & 2.3 & 1.8 \\
\hline Basal concavity width & 9 & 9.8 & 15.5 & 12.2 & 1.9 \\
\hline
\end{tabular}

TABLE 13-56

Stratigraphic Distribution of Expanding Stem, Concave Base D

\begin{tabular}{l|c|c|c}
\hline Unit & Valley Floor & Valley Floor B & Valley Margin \\
\hline IIIc & 3 & 1 & \\
IIIb/c & 6 & & \\
IIIb & & & 1 \\
Y/IIIc & & & 1 \\
Y/IIIb & & & 1 \\
X/IIIa/b & & & 1 \\
X & & & \\
Other & & & \\
Fill & 1 & & \\
\hline \hline
\end{tabular}

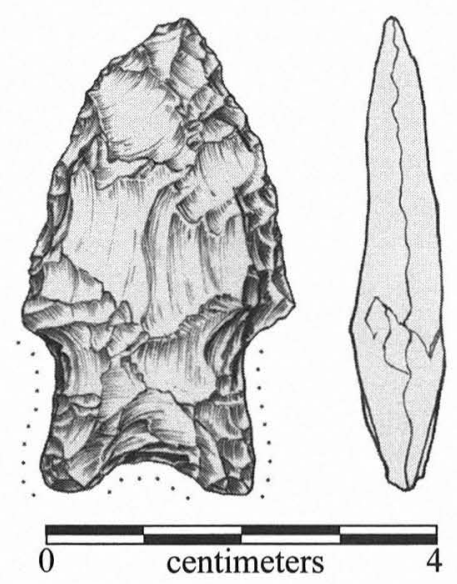

FIGURE 13-58. Expanding stem, concave base E point, Specimen $12 \mathrm{~N}-5$.

\section{Metric Averages: Table 13-57}

Nonmetric Attributes: Longitudinal profiles on all 3 are tapered base and tip. Flake patterns are organized on 1 (oblique subparallel), random on 1, and indeterminate on 1 due to resharpening. Basal modification consists of unifacial thinning on 2 and bifacial thinning on 1. Lateral stem grinding is moderate on 1 , heavy on 2 .

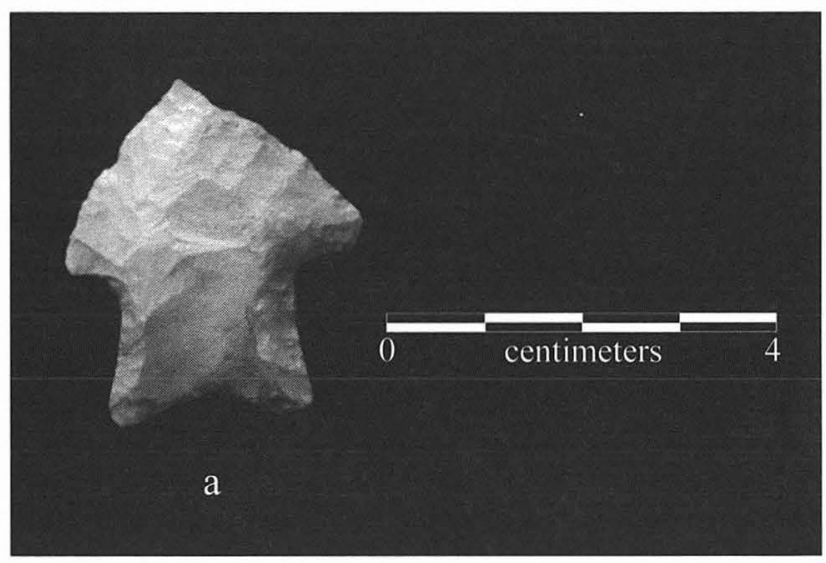

FIGURE 13-59. Expanding stem, concave base E point, Specimen $2 \mathrm{M}-1$.

Material Attributes: Raw material is local Edwards finegrained chert; heat damage is slight on 1 . No mineral precipitates or patina were observed.

Tool State and Fractures: One is complete and the other 2 nearly so, with 1 having lost a barb tip due to bend fracturing, and the other lacking distal section, due to impact fracturing.

Rejuvenation: Two are resharpened unifacially, unbeveled, and 1 is alternately beveled.

Stratigraphic Distribution: Stratigraphic placement suggests an Early Archaic derivation (Table 13-58).

\section{Uvalde $(N=14)$}

Morphology: These are small to medium-sized triangular points with small barbs and varied stem treatments. Stems are expanding and generally of short to medium length, but stem lateral edges may be recurved, with downward pointing, rounded basal ears or concave, flaring into outward-directed basal ears (Figures 13-60 amd 13-61). Commonly, stems on Uvalde points are distinguished by a relatively narrow distal "neck," and basal concavities are U-shaped. Lateral stem grinding may be present, but is not 
TABLE 13-57

Summary Metric Data for Expanding Stem, Concave Base E Points (in mm)

\begin{tabular}{l|c|c|c|c|c}
\hline & Number & Minimum & Maximum & Mean & $1-\sigma$ \\
\hline Length & 2 & 35 & 50 & 42.5 & 29 \\
Width & 2 & 27 & 31 & 7.333 & 2.828 \\
Thickness & 3 & 7 & 8 & 17 & 0.577 \\
Stem length & 3 & 16 & 18 & 21.333 & 0.577 \\
Proximal stem width & 3 & 21 & 22 & 19.667 & 0.577 \\
Distal stem width & 3 & 19 & 3.2 & 2.5 & 0.889 \\
Basal concavity depth & 3 & 1.5 & 8.8 & 7.7 & 0.954 \\
Distal stem thickness & 3 & 7.1 & 18.3 & 14.5 & 4.015 \\
Basal concavity width & 3 & 10.3 & & & 1 \\
\hline \hline
\end{tabular}

TABLE 13-58

Stratigraphic Distribution of Expanding Stem, Concave Base E Points

\begin{tabular}{l|c|c}
\hline Unit & Valley Floor A & Valley Margin \\
\hline IIIa/b & 1 & \\
IIIa & 1 & 1 \\
X & & \\
\hline \hline
\end{tabular}
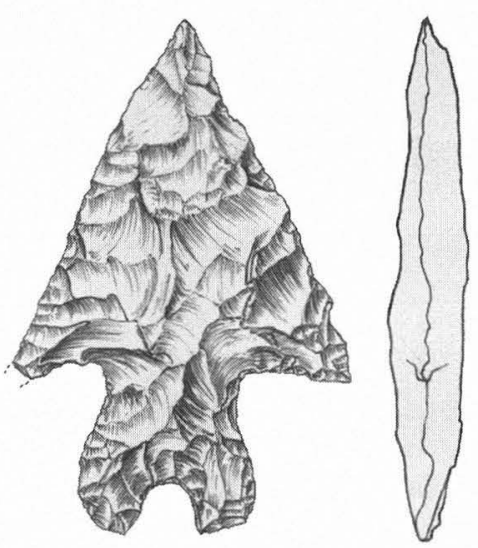

a

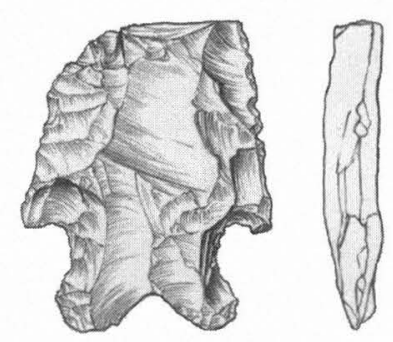

b

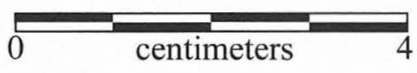

FiguRE 13-60. Uvalde points. Specimen numbers: (a) 6G-2; (b) $18 \mathrm{~J}-1$. characteristic, and blade flaking is more typically random than organized.

Metric Averages: Table 13-59

Nonmetric Attributes: Longitudinal profiles include 1 tapered base and tip; 2 flat; 4 wedge-shaped base and tip; and 7 indeterminate. Flake patterns are organized on 4 (oblique subparallel, subparallel), random on 3 , and indeterminate on 7. Basal modification constitutes unifacial thinning on 4; bifacial thinning on 1 ; unifacial fluting on 1 ; concave bevel on 2; and secondary trimming on 6 . Lateral stem grinding is moderate on 2, heavy on 2, indeterminate on 1 , and absent on 9 .

Material Attributes: Raw material is local Edwards chert (7 fine grained, 1 medium/coarse); nonlocal Edwards finegrained chert $(\mathrm{n}=4)$; and 2 indeterminate heat altered. Mineral precipitates are present on 4. Heat damage is extensive on 2. Patina was not observed.

Tool State and Fractures: Five are complete or nearly so, with 3 lacking distal tips due to bend or impact fractures, and 1 showing indeterminate edge damage. Six are proximal sections, 5 of which display multiple bend and burin-like/ impact damage, and 1 , a single bend break. The 3 remaining are largely stem fragments, 2 of which were severed by mulitiple bend and burin-like breaks and the other, thermal spalling.

Rejuvenation: One is resharpened unifacially, unbeveled; 5 are alternately beveled; 1 is beveled unifacially; and 5 are indeterminate.

Stratigraphic Distribution: As shown, distribution is widespread within middle and upper portions of Unit III (Table 13-60).

Comments: There is much variation in this group, with the most salient characteristics, other than U-shaped bifurcation of the stems, being the more-constricted stem necks and the generally more rounded and downward pointing basal ears. Uvalde is very similar to Baker, a more typically Lower Pecos type that has longer stems and often pointed basal ears (Turner and Hester 1993:77, 191), and it appears to form a continuum with several of the expanding stem concave base groups (above). See Chap- 


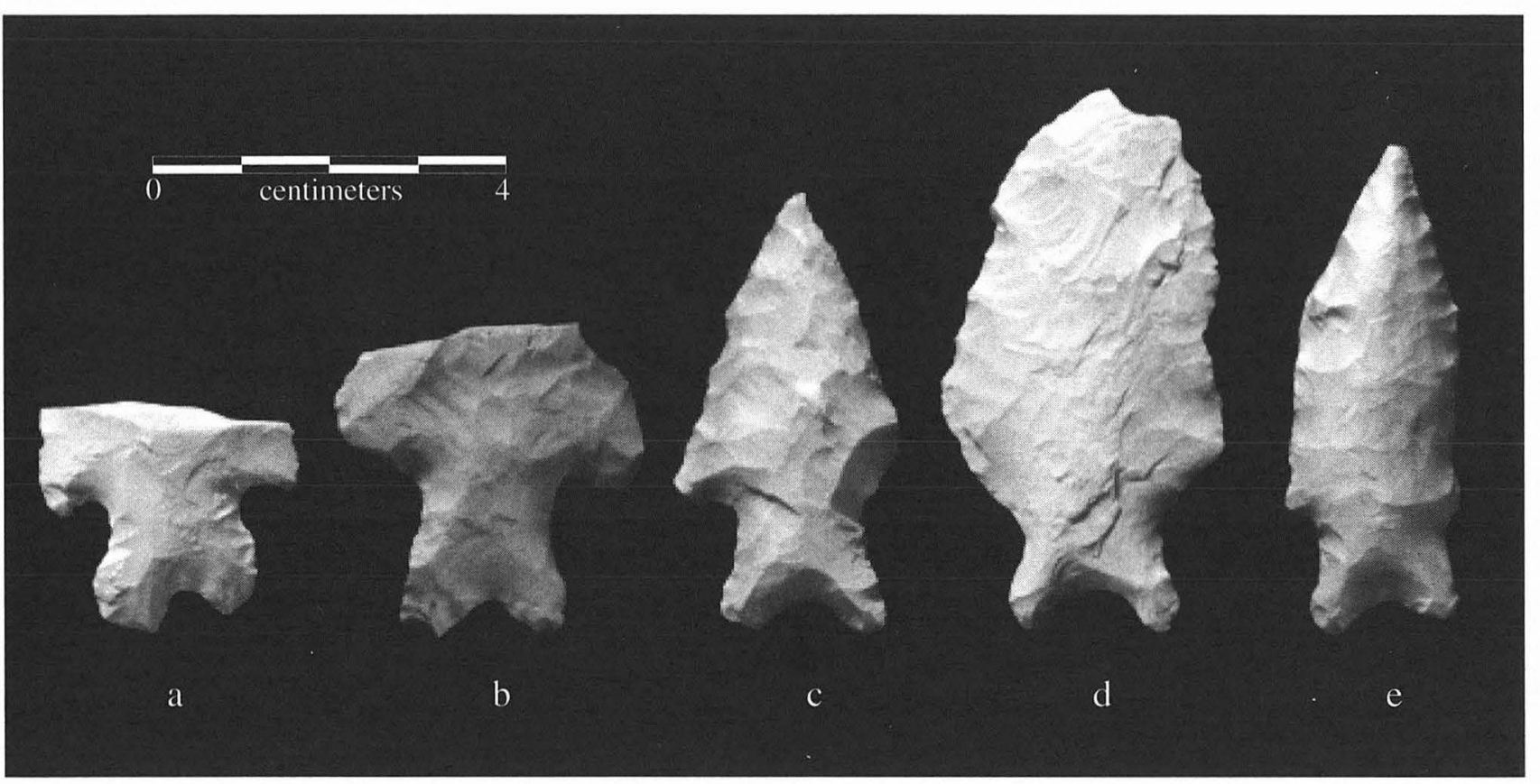

FIGURE 13-61. Uvalde points. Specimen numbers: (a) 5J-2; (b) 12G-3; (c) 19N-3; (d) 7J-2; (e) 12K-2.

TABLE 13-59

Summary Metric Data for Uvalde Points (in mm)

\begin{tabular}{l|c|c|c|c|c}
\hline & Number & Minimum & Maximum & Mean & $1-\sigma$ \\
\hline Length & 3 & 35.0 & 75.0 & 53.0 & 20.3 \\
Width & 9 & 20.0 & 34.0 & 27.1 & 4.9 \\
Thickness & 14 & 6.0 & 9.0 & 17.2 & 1.0 \\
Stem length & 11 & 11.0 & 20.0 & 17.7 & 1.9 \\
Proximal stem width & 10 & 14.0 & 17.0 & 14.7 & 1.9 \\
Distal stem width & 13 & 12.0 & 5.5 & 6.2 & 1.5 \\
Basal concavity depth & 14 & 2.7 & 9.9 & 8.0 & 1.5 \\
Distal stem thickness & 8 & 5.3 & 10.8 & & 1.9 \\
Basal concavity width & 8 & 5.6 & & \\
\hline \hline
\end{tabular}

TABLE $13-60$

Stratigraphic Distribution of Uvalde Points

\begin{tabular}{l|c|c}
\hline Unit & Valley Floor A & Valley Margin \\
\hline IIIc & 2 & 1 \\
IIIb/c & 2 & \\
IIIb & 1 & \\
IIIa/b & & 3 \\
Y/IIIc & & 3 \\
Y & & 1 \\
X/IIIb & & \\
Other & & \\
surface & & \\
\hline \hline
\end{tabular}

ter 14 for further discussion of issues surrounding the Uvalde type.

\section{Uvalde-like $(\mathrm{N}=9)$}

Morphology: Points in this category do not form a coherent group but may be odd variants of Uvalde. Most have slightly more straight-sided stems than the larger Uvalde group, and all have bifurcate bases with generally small basal concavities (Figures 13-62 and 13-63). Several are damaged, making further classification difficult.

Metric Averages: Table 13-61

Nonmetric Attributes: Longitudinal profiles include 3 tapered base and tip; 1 flat; 4 wedge-shaped base and tip; and 1 indeterminate. Flake patterns are organized on 4 (oblique subparallel), random on 4, and indeterminate on 1. Basal 


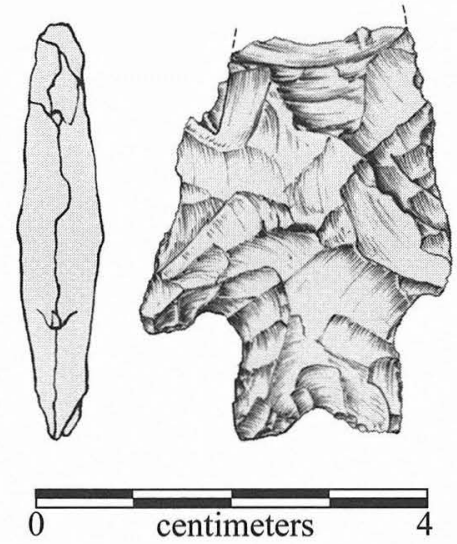

FIGURE 13-62. Uvalde-like point, Specimen 19I-2.

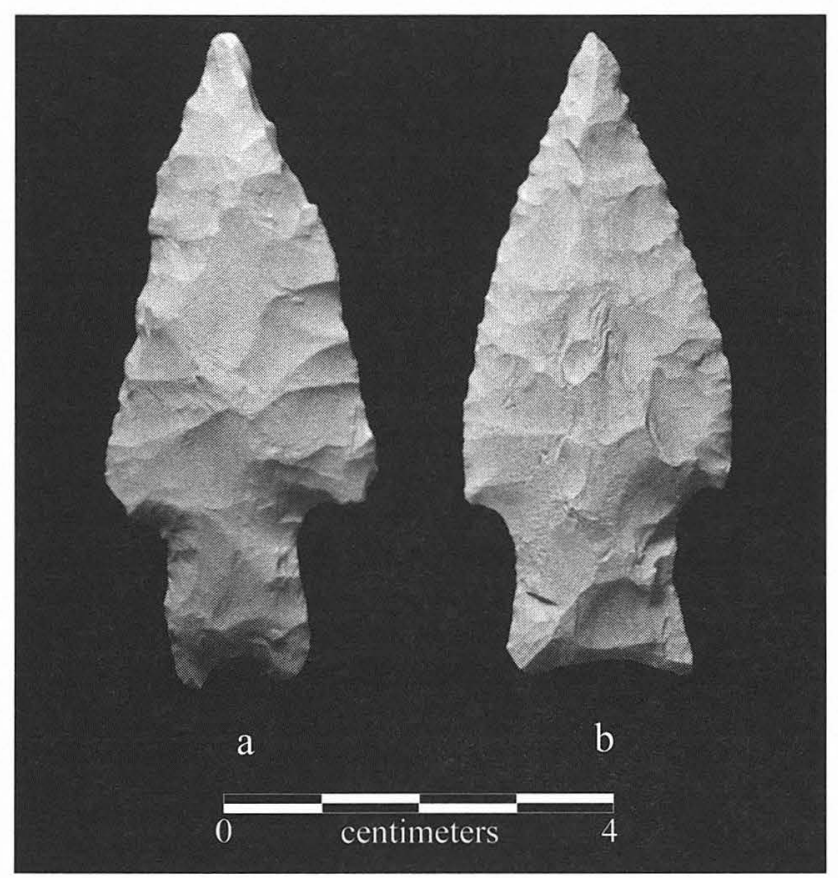

FIGURE 13-63. Uvalde-like points. Specimen numbers: (a) 9O-2; (b) 40 -I. modification consists of unifacial thinning on 4 , concave bevel on 2, secondary trimming on 2 , and 1 indeterminate. Lateral stem grinding is heavy on 1 .

Material Attributes: Raw material is local Edwards finegrained chert $(\mathrm{n}=6)$, nonlocal Edwards chert (1 fine grained, $1 \mathrm{medium} / \mathrm{coarse}$ ), and 1 indeterminate heat altered. Mineral precipitates are present on 3 . Heat damage is moderate on 1 and extensive on 1. Patina was not observed.

Tool State and Fractures: Three are largely complete except for distal sections, barb tips, or basal ear tips, due to bend or burin-like fractures. The other 6 are proximal sections, 2 of which display multiple bend breaks; 3 , multiple bend and burin-like impact fractures; and 1, thermal and bend damage.

Rejuvenation: Two are resharpened bifacially, unbeveled, 5 are alternately beveled, and 2 are indeterminate.

Stratigraphic Distribution: Distribution shown in Table 13-62 nearly mirrors that of the Uvalde group (see Table 1360 ) and suggests possible problems in classification of both groups.

\section{Early Bifurcate Stem Basal Fragments $(N=44)$}

Morphology: These small basal fragments display attributes characteristic of Early Archaic bifurcate stem groups (e.g., Hoxie, Gower, and Uvalde) but are too fragmented to classify further. Stems are concave based, varying from slight arc to U-shaped to V-shaped and are typically dulled or ground on lateral edges. Figure 13-64 illustrates the wide variety of sizes and shapes.

Metric Averages: Table 13-63

Nonmetric Attributes: Longitudinal profiles are indeterminate on all. Flake patterns are oblique subparallel on 2 and indeterminate on the remaining 42. Basal modification constitutes unifacial thinning on 7 , bifacial thinning on 4 , thinning and fluting on 1 , concave bevel on 9 , secondary trimming on 17, and is indeterminate on 4 , and absent on 2 . Lateral stem grinding is slight on 4 , moderate on 7 , and heavy on 19.

TABLE $13-61$

Summary Metric Data for Uvalde-like Points (in mm)

\begin{tabular}{|c|c|c|c|c|c|}
\hline & Number & Minimum & Maximum & Mean & $1-\sigma$ \\
\hline Length & 1 & 68.0 & 68.0 & & \\
\hline Width & 4 & 24.0 & 32.0 & 28.5 & 3.4 \\
\hline Thickness & 9 & 6.0 & 8.0 & 7.1 & 0.8 \\
\hline Stem length & 9 & 11.0 & 18.0 & 14.2 & 2.5 \\
\hline Proximal stem width & 6 & 14.0 & 17.0 & 15.2 & 1.2 \\
\hline Distal stem width & 9 & 11.0 & 18.0 & 13.8 & 2.1 \\
\hline Basal concavity depth & 8 & 2.6 & 5.0 & 3.6 & 1.0 \\
\hline Distal stem thickness & 3 & 6.2 & 6.9 & 6.7 & 0.4 \\
\hline Basal concavity width & 2 & 5.2 & 6.5 & 5.9 & 0.9 \\
\hline
\end{tabular}


TABLE 13-62

Stratigraphic Distribution of Uvalde-like Points

\begin{tabular}{l|c|c}
\hline Unit & Valley Floor A & Valley Margin \\
\hline IIIc & 2 & 2 \\
IIIb/c & & 1 \\
Y/IIIc & & 1 \\
X/Y & & 1 \\
X/IIIa & & 1 \\
$X$ & & 1 \\
\hline \hline
\end{tabular}

Material Attributes: Raw material is local Edwards chert (23 fine grained, 4 medium/coarse), nonlocal Edwards chert ( 7 fine grained, 2 medium/coarse), indeterminate heat altered $(n=7)$, and indeterminate $(n=1)$. Patina is present on 2 ; mineral precipitates were observed on 6 ; and heat damage is moderate on 1 and extensive on 8 .

Tool State and Fractures: All are stems and proximal sections; the most common break pattern is multiple bend and burin-like fractures (on 20), followed by single bend (on 11), multiple bend breaks (on 4), bend and thermal fractures

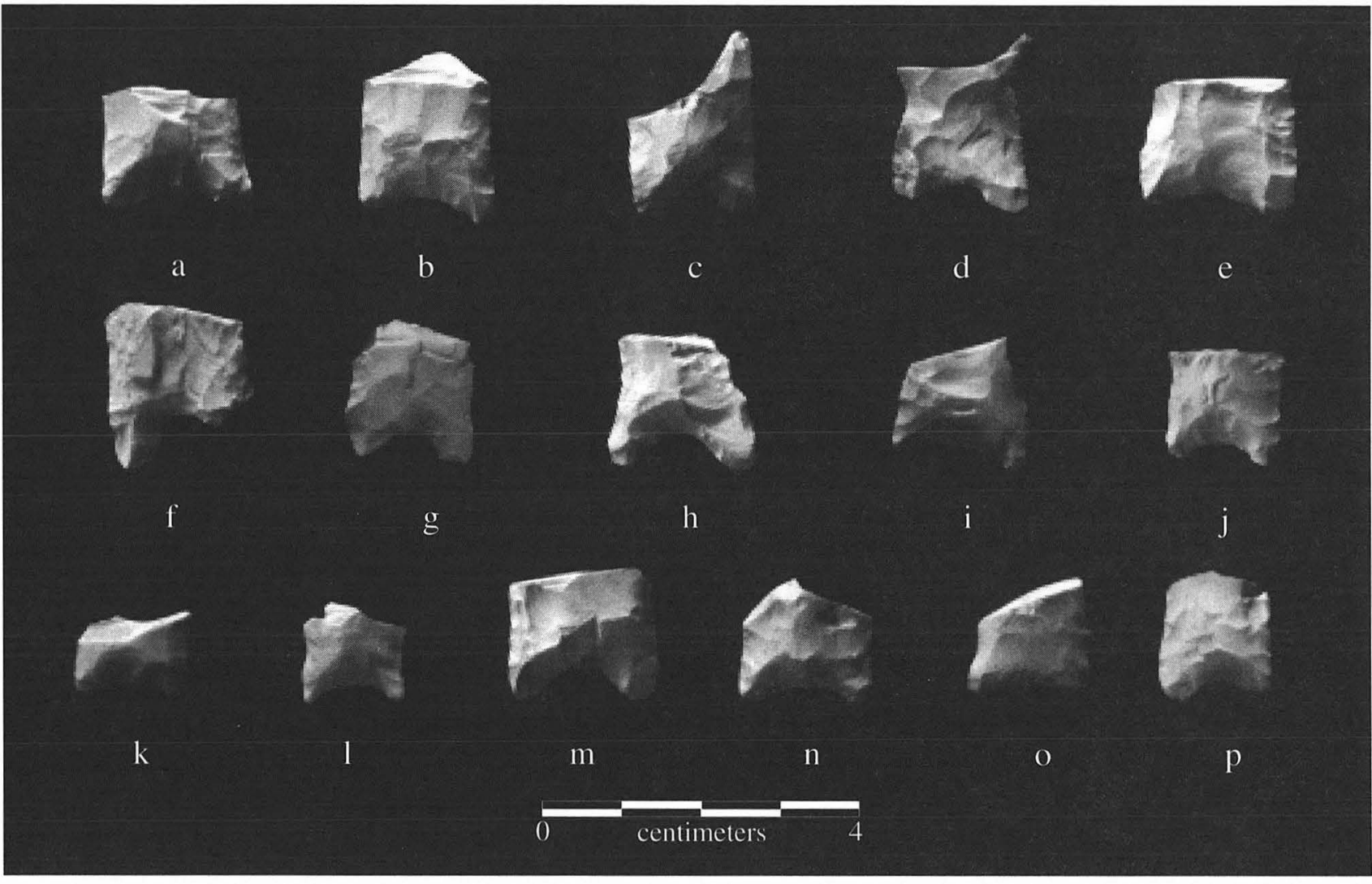

FIGURE 13-64. Early bifurcate stem fragments, from latest context (Unit IIIb/c), a-c, to earliest (X/II, II, IIIa), n-p, showing slight morphological changes from roughly straight-sided to more convex edged to expanding. Note consistent position of break point at or just above the haft. Specimens are: (a) 16J1-1; (b) 37IB-4; (c) 23G -3; (d) 22L-1; (e) 21M-7; (f) 13M-4; (g) 17K2-1; (h) 16Q2-1; (i) 27Q1-1; (j) 32Q1-1; (k) 32R1-2; (l) 33R2-12; (m) 10P-8; (n) 20R-2; (o) 20Q-2; (p) 35S2B-3.

TABLE 13-63

Summary Metric Data for Early Bifurcate Stem Basal Fragments (in mm)

\begin{tabular}{|c|c|c|c|c|c|}
\hline & Number & Minimum & Maximum & Mean & $1-\sigma$ \\
\hline Width & 12 & 15.0 & 20.0 & 17.6 & 1.7 \\
\hline Thickness & 39 & 4.0 & 9.0 & 5.8 & 1.0 \\
\hline Stem length & 11 & 10.0 & 18.0 & 13.5 & 2.2 \\
\hline Proximal stem width & 29 & 8.0 & 20.0 & 16.1 & 2.5 \\
\hline Distal stem width & 9 & 13.8 & 18.0 & 15.1 & 1.5 \\
\hline Basal concavity depth & 37 & 1.0 & 9.0 & 3.6 & 1.7 \\
\hline Distal stem thickness & 4 & 1.4 & 5.4 & 5.9 & 0.6 \\
\hline Basal concavity width & 4 & 6.5 & 11.7 & 8.4 & 2.4 \\
\hline
\end{tabular}


(on 3), burin-like and thermal fractures (on 4), and thermal fractures (on 2).

Stratigraphic Distribution: Table 13-64

Comments: These basal fragments, in their abbreviated state, rather dramatically highlight the morphological variation in bifurcate point stem treatments, particularly in Early Archaic and later Archaic contexts. As depicted in rough stratigraphic sequence (later specimens at the top) in Figure 13-64, these fragments trend toward slightly wider, more flared stems and shallower basal concavities over time.

The fragments also illuminate a fracture pattern that appears to have been quite common, that is, multiple bend and burin-like breaks occurring at or very near to the upper extent of the haft element. This may signify a use that caused a higher frequency of breaks at this point, and/or may indicate a weakness or flaw in the bifurcate stem hafting technology; whichever is the case, the broken stems were evidently extracted from the shafts at the site fairly frequently. This may also imply reliable recovery of the shaft or foreshaft. The nine (20\%) with heat damage could indicate extraction from meat after cooking.

TABLE 13-64

Stratigraphic Distribution of Early Bifurcate Stem Basal Fragments

\begin{tabular}{l|c|c|c}
\hline Unit & Valley Floor A & Valley Floor B & Valley Margin \\
\hline IIIc & 1 & & 2 \\
IIIb/c & 3 & & 4 \\
Y/IIIc & & & 1 \\
Y/IIIb/c & & & 3 \\
IIIb & 3 & & 4 \\
Y & & & 1 \\
X/IIIb & & & 1 \\
X/Y & & & 1 \\
IIIa/b & 2 & & \\
IIIa & 8 & & \\
III & 1 & & 4 \\
II/IIIa & 1 & & 1 \\
X/II/IIIa & & & \\
X & & & \\
X/II & & & \\
\hline \hline
\end{tabular}

Early Bifurcate Stem Preforms ( $N=2)$

Morphology: Although unfinished, these specimens exhibit stem characteristics of some of the early bifurcate stem groups. The more complete specimen (Figure 13-65) is a triangular bladed point with small barbs made on a flake blank; it evidently was broken during manufacture. Although the stem has been shaped and thinned, only one face of the blank has been flaked. The other point is mostly a proximal
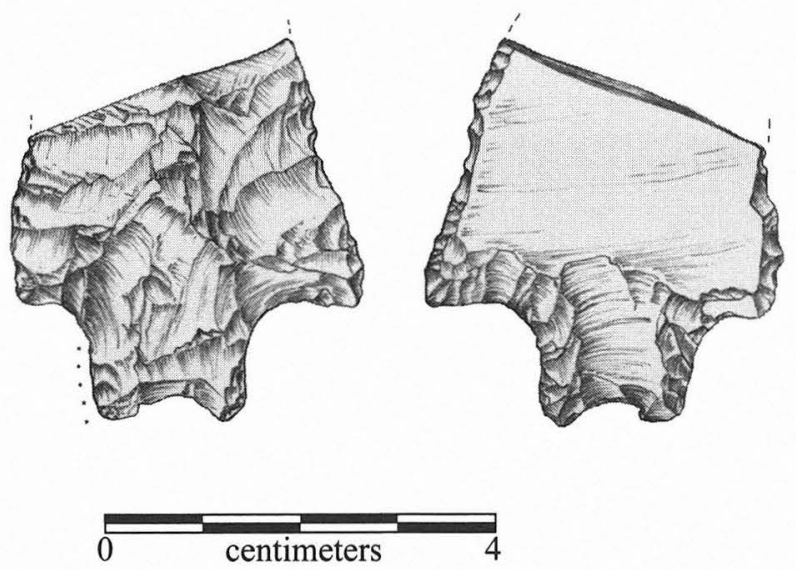

FIGURE 13-65. Early bifurcate stem preform, Specimen 9U-1.

section with a more arc-shaped concavity and narrow shoulders.

Metric Averages: Table 13-65

Nonmetric Attributes: Longitudinal profiles on both are indeterminate. Flake patterns include flake blank remnant on 1 and 1 indeterminate. Basal modification consists of unifacial thinning on 1 and secondary trimming on 1 . Lateral stem dulling appears slight on 1 .

Material Attributes: Raw material is local Edwards finegrained chert for 1 , and 1 is indeterminate. Mineral precipitates are present on 1 . Heat damage is moderate on 1 . No patina was observed.

Tool State and Fractures: Both are proximal sections. One, on a flake blank, was broken during manufacturing. The other shows thermal fracturing.

Stratigraphic Distribution: Both specimens were recovered from Unit X of the Valley Margin.

Comments: The specimen illustrated provides insights into early stage manufacturing of what may have been intended as a Gower or Hoxie point, considering stem shape and the early context. The wide blade and strong barbs are significant morphological information, inasmuch as many Gower and Hoxie points are recovered only after intensive resharpening.

TABLE 13-65

Summary Metric Data for Early Bifurcate Stem Preforms

\begin{tabular}{l|c|c}
\hline & $3 \mathrm{~N}-4$ & $9 \mathrm{U}-1$ \\
\hline Length & 26.0 & 35.0 \\
Width & 6.0 & 7.0 \\
Thickness & 11.5 & 11.3 \\
Stem length & 19.0 & 14.9 \\
Proximal stem width & 20.0 & 17.4 \\
Distal stem width & 3.0 & 2.5 \\
Basal concavity depth & & 5.6 \\
Distal stem thickness & & 7.3 \\
Basal concavity width & & \\
\hline \hline
\end{tabular}




\section{Frio $(N=5)$}

Morphology: These are small to medium-sized, cornernotched triangular points with expanding stems. The pronounced, U-shaped basal concavity commonly takes the form of a very regularized basal notch (Figures 13-66 and 13-67).

Metric Averages: Table 13-66

Nonmetric Attributes: Longitudinal profiles include 2 flat, 1 wedge-shaped base and tip, and 2 indeterminate. Flake patterns are organized on 1 (oblique subparallel), random on 3 , and indeterminate on 1. Basal modification consists of unifacial thinning on 1, and it is absent on 4.

Material Attributes: Raw material is local Edwards chert (3 fine grained, 1 medium/coarse) and 1 indeterminate heat altered. Patina is slight overall on 1 . Heat damage is slight on 1 , extensive on another. No mineral precipitates were observed.

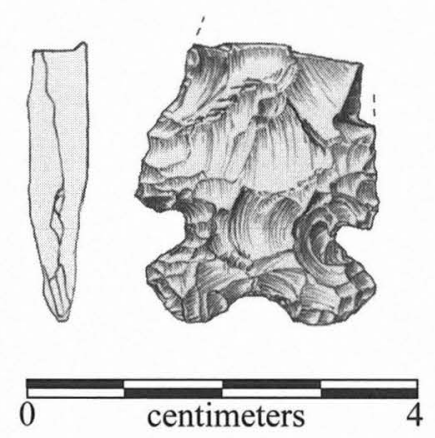

Figure 13-66. Frio point, Specimen 11D-2.

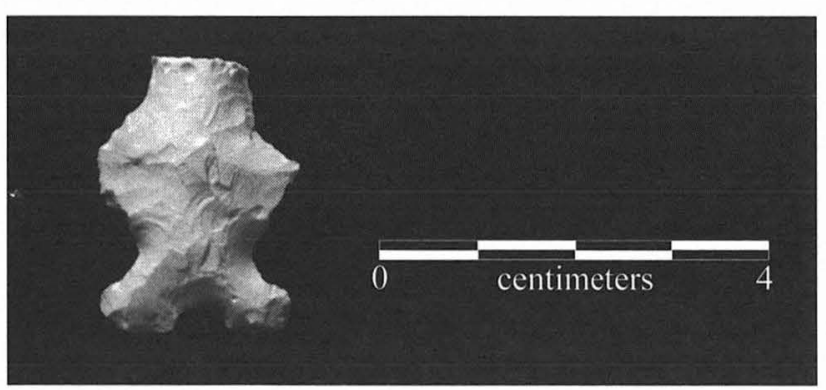

FIGURE 13-67. Frio point, Specimen 9A-8.
Tool State and Fractures: Three are proximal sections. Of these, 1 displays thermal fracturing; 1 , multiple bend breaks; and 1, multiple bend and burin-like fractures. The 2 remaining each lack a distal tip and basal ear due to thermal fractures in 1 case, and bending in the other.

Rejuvenation: One is resharpened bifacially, unbeveled, 1 is alternately beveled, and 3 are indeterminate. Although the distal section is missing, specimen 9A-8 (see Figure 1367) appears to have been reworked to a perforator.

Stratigraphic Distribution: All were recovered in Unit IIIc (Table 13-67).

TABLE 13-67

Stratigraphic Distribution of Frio Points

\begin{tabular}{l|c|c}
\hline Unit & Valley Floor A & Valley Margin \\
\hline IIIc & 1 & 3 \\
Other & & 1 \\
IIIc/pot & & \\
\hline \hline
\end{tabular}

Fairland $(N=5)$

Morphology: This group consists of triangular points with small shoulders and barbs. Their stems are wide and expanding, formed by corner or side notching; basal concavities are characteristically shallow and wide, framed by downward directed basal ears (Figure 13-68).

Metric Averages: Table 13-68

Nonmetric Attributes: Longitudinal profiles include 3 tapered base and tip, 1 wedge-shaped base and tip, and 1 indeterminate. Flake patterns are organized on 3 (oblique subparallel and subparallel), random on 1, and indeterminate on 1. Basal modification consists of unifacial thinning on 1 , bifacial thinning on 3 , and 1 indeterminate. Lateral stem grinding is absent.

Material Attributes: Raw material is local Edwards chert (1 fine grained, 1 medium/coarse), nonlocal medium/coarse Edwards chert $(\mathrm{n}=1)$, indeterminate $(\mathrm{n}=1)$, and indeterminate heat altered $(\mathrm{n}=1)$. Heat damage is extensive on 1. Mineral precipitates and patina are not present.

Tool State and Fractures: One is complete; 3 others are proximal sections manifesting combination bend and

TABLE 13-66

Summary Metric Data for Frio Points (in mm)

\begin{tabular}{l|c|c|c|c|c}
\hline & Number & Minimum & Maximum & Mean & $1-\sigma$ \\
\hline Length & 1 & 35.0 & 35.0 & 35.0 & \\
Width & 5 & 22.0 & 29.0 & 25.2 & 2.6 \\
Thickness & 5 & 5.0 & 8.0 & 6.4 & 1.1 \\
Stem length & 4 & 10.0 & 14.0 & 11.5 & 1.7 \\
Proximal stem width & 2 & 20.0 & 23.0 & 21.5 & 2.1 \\
Distal stem width & 4 & 12.0 & 18.0 & 15.5 & 2.7 \\
Basal concavity depth & 3 & 3.0 & 3.0 & 3.0 & 0.0 \\
\hline \hline
\end{tabular}




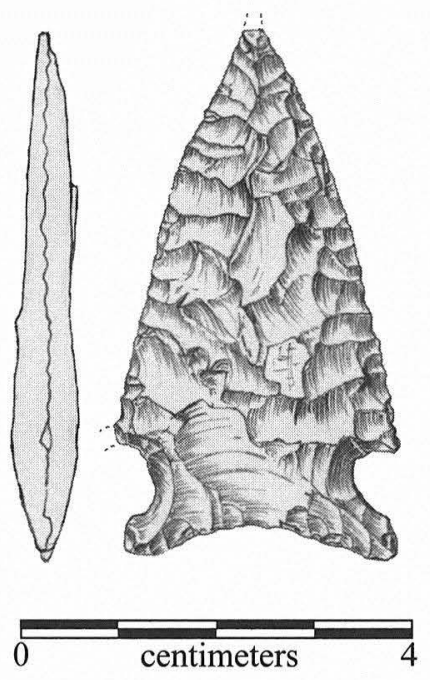

Figure 13-68. Fairland point, Specimen 2E-2.

burin-like damage. The other is a proximal fragment with thermal and bend damage.

Rejuvenation: One is resharpened bifacially, unbeveled, 2 are alternately beveled, and 2 are indeterminate.

Stratigraphic Distribution: Table 13-69

\section{Montell $(N=24)$}

Morphology: These are broad, typically thin and flat triangular points with pronounced shoulders and barbs and wide, straight to slightly expanding, short- to medium-length stems which usually are well thinned at the base. Characteristic attributes are a deep, narrow, U- to V-shaped basal notch and squared-off basal corners. Flake patterns across blade faces most commonly are random (Figures 13-69 and 13-70).

Metric Averages: Table 13-70

Nonmetric Attributes: Longitudinal profiles include 5 tapered base and tip, 1 thick base, 11 flat, and 7 indeterminate. Flake patterns are organized on 5 (subparallel, collateral), random on 12 , and indeterminate on 7 . Basal modification consists of unifacial thinning on 11, bifacial thinning on 5 , concave bevel on 1, 1 indeterminate, and 6 absent. Lateral stem grinding is absent.
TABLE 13-69

Stratrigraphic Distribution of Fairland Points

\begin{tabular}{l|c|c}
\hline Unit & Valley Floor A & Valley Margin \\
\hline IIIc & 1 & 1 \\
$\begin{array}{l}\text { Other } \\
\text { IIIc/pot } \\
\text { unknown }\end{array}$ & & 1 \\
\hline \hline
\end{tabular}

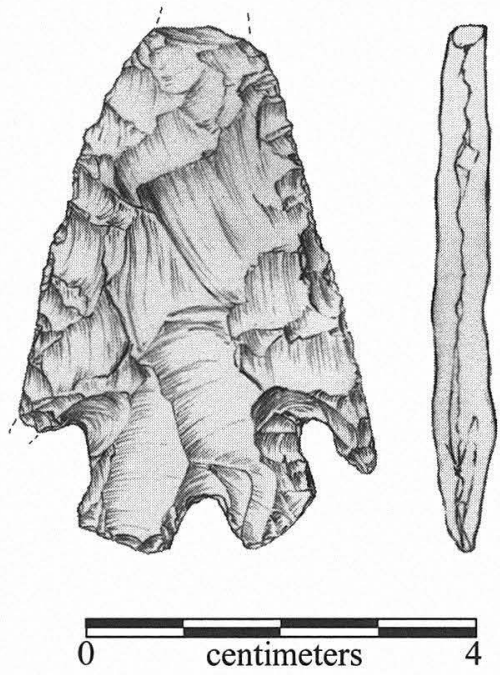

FIGURE 13-69. Montell point, Specimen 17C-4.

Material Attributes: Raw material is local Edwards chert (13 fine grained, 2 medium/coarse), nonlocal Edwards finegrained chert $(\mathrm{n}=6)$, and indeterminate and/or heat altered $(\mathrm{n}=3)$. Mineral precipitates are present on 1 . Heat damage is slight on 1, moderate on 2. Patina is not present.

Tool State and Fractures: Nine are complete or nearly so, lacking only small distal sections or small barbs or basal ear tips due to bend or combination bend and burin-like/ impact fractures. Seven others are proximal sections with largely intact bases. Of these, 3 display multiple bend and impact fractures; 1 , a single bend; 2 , multiple bend; and 1, bend and overshot fractures. Three other proximal sections

TABLE 13-68

Summary Metric Data for Fairland Points (in mm)

\begin{tabular}{l|c|c|c|c|c}
\hline & Number & Minimum & Maximum & Mean & $1-\sigma$ \\
\hline Width & 4 & 23 & 30 & 26.75 & 3.304 \\
Thickness & 4 & 5 & 8 & 6.25 & 1.258 \\
Stem length & 4 & 10 & 12 & 11.25 & 0.957 \\
Proximal stem width & 3 & 19 & 28 & 25 & 5.196 \\
Distal stem width & 4 & 14 & 20 & 2.65 & 0.943 \\
Basal concavity depth & 4 & 2 & & & \\
Distal stem thickness & 0 & & & & \\
Basal concavity width & 0 & & & & \\
\hline \hline
\end{tabular}




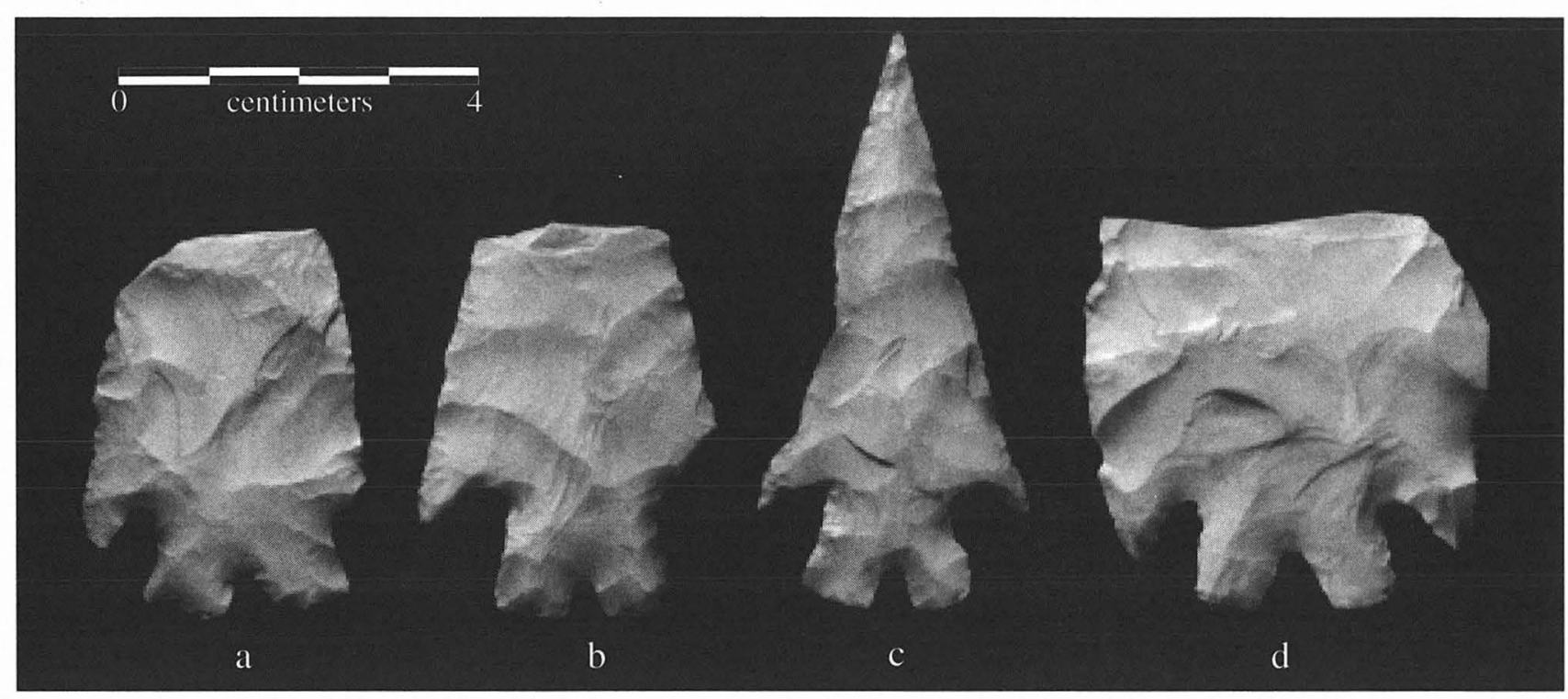

FIGURE 13-70. Montell points. (a) 24B; (b) 11D-3; (c) 399-1; (d) 37BD-25.

TABLE 13-70

Summary Metric Data for Montell Points (in mm)

\begin{tabular}{l|c|c|c|c|c}
\hline & Number & Minimum & Maximum & Mean & $1-\sigma$ \\
\hline Length & 4 & 38 & 65 & 55.0 & 11.8 \\
Width & 20 & 23 & 45 & 32.3 & 5.2 \\
Thickness & 24 & 4 & 8 & 6.1 & 1.1 \\
Stem length & 20 & 11 & 16 & 12.6 & 1.5 \\
Proximal stem width & 18 & 10 & 26 & 1.6 & 3.4 \\
Distal stem width & 19 & 14 & 6.5 & 2.6 \\
Basal concavity depth & 18 & 3 & 6.6 & 1.0 \\
\hline \hline
\end{tabular}

each have only a single basal tang. Of these, 2 display multiple bend fractures, and 1, bend and thermal fractures. The remaining 5 specimens are stem fragments, 4 of which were bend fractured, and 1 , thermally fractured.

Rejuvenation: One is resharpened unifacially, unbeveled; 2 bifacially, unbeveled; 6 alternately beveled; 3 beveled unifacially; and 8 indeterminate.

Stratigraphic Distribution: Table 13-71

\section{Pedernales $(N=18)$}

Morphology: Generally elongate triangular points with typically strong shoulders and barbs of varying sizes form this group. Stems are characteristically long and straight with strongly bifurcate bases formed by the removal of long, often flute-like thinning flakes. Flaking of the blades varies widely from crude and random to well patterned (Figures 1371 and 13-72).

Metric Averages: Table 13-72

Nonmetric Attributes: Longitudinal profiles include 9 tapered base and tip, 2 thick base, 1 flat, 3 wedge-shaped base and tip, and 3 indeterminate. Flake patterns are orga-
TABLE 13-71

Stratigraphic Distribution of Montell Points

\begin{tabular}{l|c|c}
\hline Unit & Valley Floor A & Valley Margin \\
\hline IIIc & 12 & 5 \\
IIIb/c & 2 & 3 \\
Other & & \\
surface & 1 & \\
unknown & 1 & \\
\hline \hline
\end{tabular}

nized on 8 (subparallel, oblique subparallel, collateral, chevron), random on 5, original flank blank on 1 , and indeterminate on 4. Basal modification consists of unifacial thinning on 5 , bifacial thinning on 8 , fluting unifacially on 1 , fluting bifacially on 2 , concave bevel on 1 , and secondary trimming on 1.

Lateral stem grinding is slight on 3.

Material Attributes: Raw material is local Edwards chert (15 fine grained, 2 medium/coarse) and 1 nonlocal finegrained Edwards chert. Patina is heavy overall on 1. No mineral precipitates or heat damage was observed. 


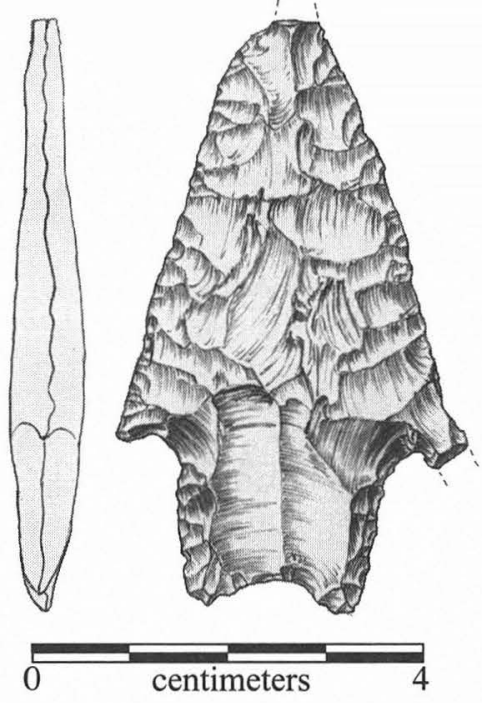

FIGURE 13-71. Pedernales point, Specimen 37EC-39.
Tool State and Fractures: Three are complete or largely so. Six others exhibit minor damage of distal, basal, or barb tips or lateral sections due to multiple bend breaks on 2, burin-like/impact breaks on 2, multiple bend and impact on 1 , and bend and edge crushing on 1, the latter likely a failure during blade resharpening. Eight are proximal sections, 1 of which shows a single bend fracture; 1 , multiple bend fractures; and 6, multiple bend and burin-like/impact fractures. The remaining specimen is a stem with a single bend fracture.

Rejuvenation: Two are resharpened unifacially, unbeveled; 4 bifacially, unbeveled; 2 alternately beveled; 1 beveled unifacially, 7 indeterminate; 2 are not resharpened.

Stratigraphic Distribution: Pedernales are concentrated in the upper portions of the site chiefly pertaining to Middle and Late Archaic occupations (Table 13-73).

Comments: Although longer overall and rarely ground on lateral stem edge, Pedernales stems are similar in overall shape to those of several early bifurcate variants.

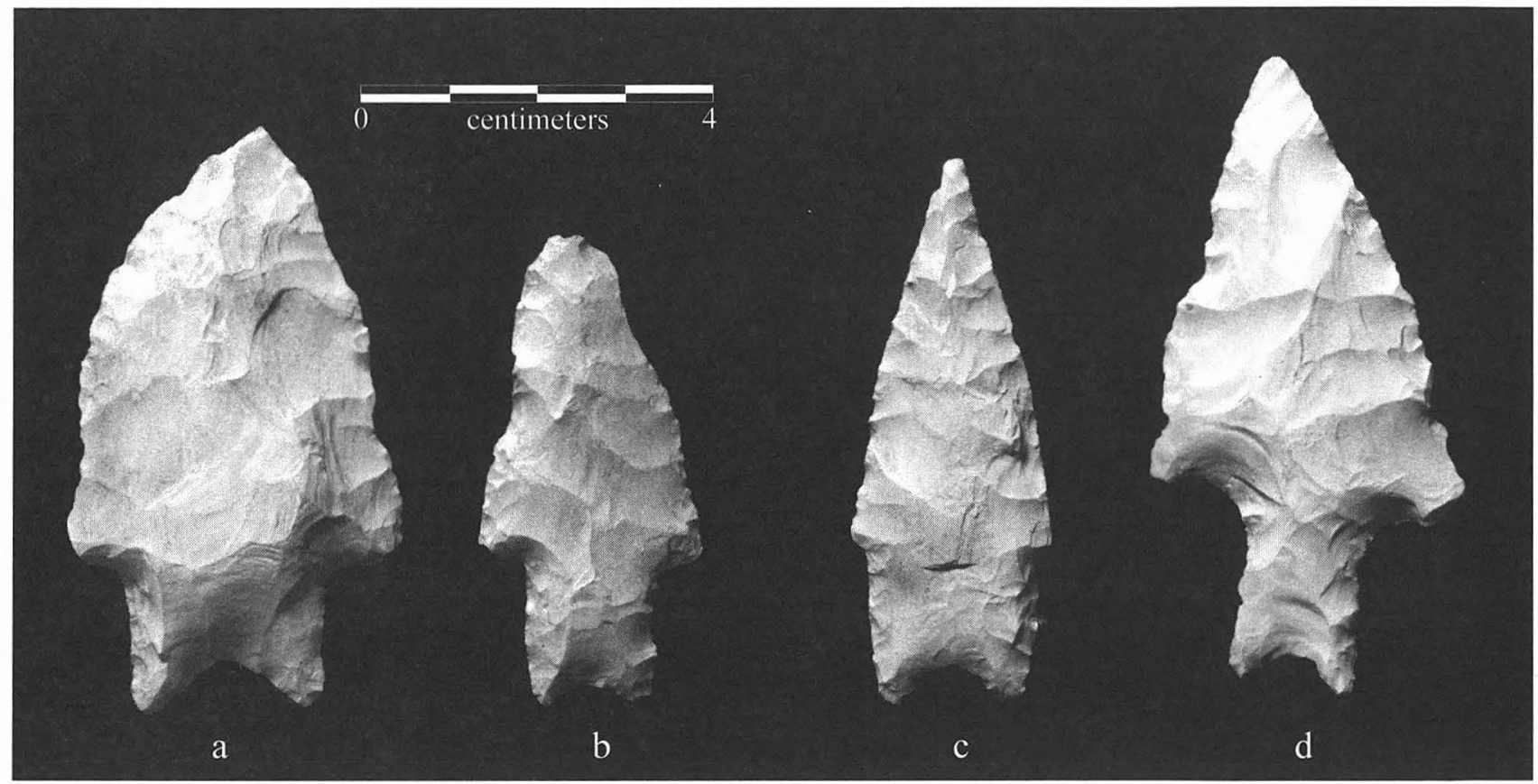

Figure 13-72. Pedernales points. Specimen numbers: (a) 100-1; (b) 36F-4; (c) 21G -8; (d) 332-4.

TABLE 13-72

Summary Metric Data for Pedernales Points (in mm)

\begin{tabular}{l|c|c|c|c|c}
\hline & Number & Minimum & Maximum & Mean & $1-\sigma$ \\
\hline Length & 5 & 49.0 & 75.0 & 65.2 & 11.0 \\
Width & 11 & 24.0 & 47.0 & 32.9 & 7.4 \\
Thickness & 18 & 6.0 & 10.0 & 8.1 & 1.3 \\
Stem length & 16 & 13.0 & 21.0 & 18.3 & 2.2 \\
Proximal stem width & 17 & 13.0 & 23.0 & 18.2 & 2.8 \\
Distal stem width & 17 & 15.0 & 26.0 & 19.4 & 3.1 \\
Basal concavity depth & 18 & 3.0 & 7.0 & 4.9 & 1.1 \\
\hline \hline
\end{tabular}


TABLE 13-73

Stratigraphic Distribution of Pedernales Points

\begin{tabular}{l|c|c}
\hline Unit & Valley Floor A & Valley Margin \\
\hline IIIc & 8 & 2 \\
IIIb/c & 2 & 1 \\
Other & & \\
surface & 3 & \\
unknown & 2 & \\
\hline \hline
\end{tabular}

\section{Miscellaneous Bifurcate $(N=18)$}

Morphology: Not intended as a morphological category, this group, includes bifurcate points or basal sections of bifurcate points that do not conform to other groups or types or are too damaged to classify further. Those complete enough to characterize are triangular with generally weak shoulders and, where present, indistinct to small barbs, as well as a variety of stem and blade modifications. Examples shown include Figure 13-73a, which exhibits a steeply beveled basal concavity as well as slight bifacial beveling of blade edges; stem lateral edges are slightly dulled. Flaking is oblique subparallel on one blade face. Figure 13-73b (12G-2) displays only retouch of the basal concavity and lateral stem edges are unground.

Metric Averages: Table 13-74

Nonmetric Attributes: Longitudinal profiles include 5 with tapered base and tip, 1 thick tip, 5 wedge-shaped base and tip, 1 wedge-shaped base, thick stem, flat body, and 6 indeterminate. Flake patterns are organized on 6 (oblique subparallel, subparallel, collateral), random on 5, and indeterminate on 7. Basal modification constitutes unifacial thinning on 2, concave bevel on 5, secondary thinning on 5, 3

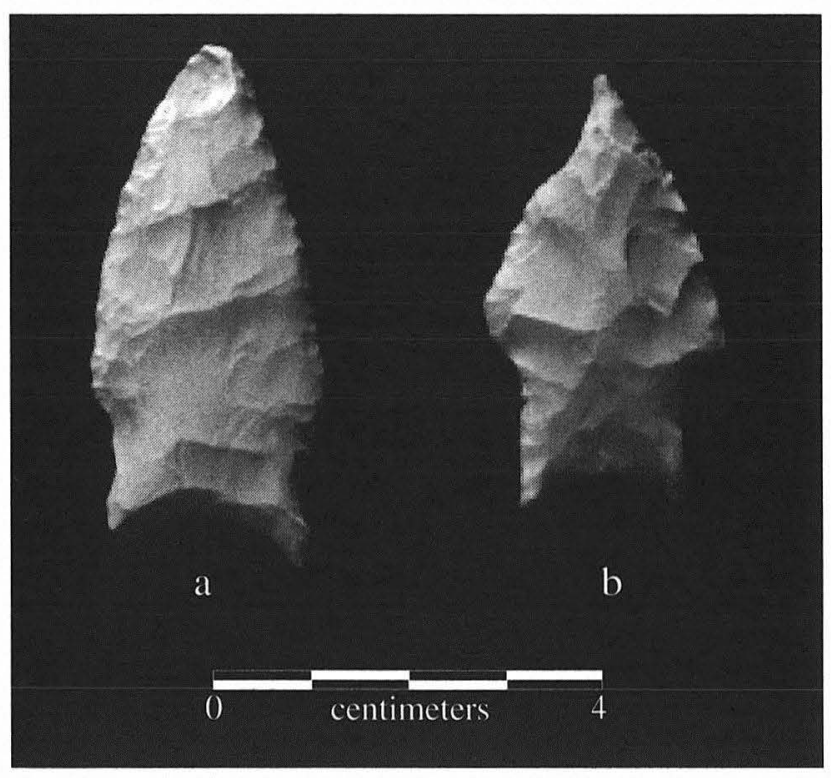

FIGURE 13-73. Miscellaneous bifurcate points. Specimen numbers: (a) $7 \mathrm{I}-1$; (b) $12 \mathrm{G}-2$.
TABLE 13-74

Summary Metric Data for Miscellaneous Bifurcate Points (in $\mathrm{mm}$ )

\begin{tabular}{l|c|c|c}
\hline & Number & Minimum & Maximum \\
\hline Length & 8 & 38.0 & 65.0 \\
Width & 11 & 19.0 & 35.0 \\
Thickness & 15 & 6.0 & 9.0 \\
Stem length & 10 & 10.7 & 25.0 \\
Proximal stem width & 7 & 15.9 & 20.0 \\
Distal stem width & 13 & 14.0 & 26.0 \\
Basal concavity depth & 9 & 1.8 & 5.0 \\
Distal stem thickness & 3 & 6.2 & 8.6 \\
Basal concavity width & 3 & 7.5 & 13.9 \\
\hline \hline
\end{tabular}

indeterminate, and 3 absent. Lateral stem grinding is slight on 4 , moderate on 1 , and indeterminate on 2 .

Material Attributes: Raw material is local Edwards finegrained chert $(n=10)$, nonlocal Edwards chert ( 2 fine grained, 2 medium/coarse), and 4 indeterminate and/or heat altered. Patina is heavy overall on 2. Mineral precipitates are present on 1. Heat damage is slight on 2 and extensive on 6 .

Tool State and Fractures: Eight are complete or nearly so, with 3 lacking distal tips due to bend breaks and 2 to thermal spalling. Two others lack basal ears due to apparent bend fractures. Eight are proximal sections, 4 of which display bend and burin-like/impact fractures, and 4 others, multiple bend, thermal, and burin-like fractures. The 2 remaining are largely stem fragments with extensive thermal damage.

Rejuvenation: Six are resharpened bifacially, unbeveled, 3 alternately beveled; 1 beveled unifacially; and 8 are indeterminate.

\section{Stratigraphic Distribution: Table 13-75}

Comments: Based on distribution, it is likely that some specimens included in the miscellaneous bifurcate group are related to the early bifurcate stem series while others likely are associated with Pedernales.

TABLE 13-75

Stratigraphic Distribution of Miscellaneous Bifurcate Points

\begin{tabular}{l|c|c}
\hline Unit & Valley Floor A & Valley Margin \\
\hline IIIc & 5 & 3 \\
IIIb/c & 1 & \\
IIIb & 1 & \\
IIIa/b & 1 & 2 \\
Y/IIIc & & 1 \\
Y & & 2 \\
X/IIIb & & 1 \\
Other & & \\
IIIc pot & & \\
surface & & \\
\hline \hline
\end{tabular}




\section{Expanding Stemmed Points}

\section{Wilson $(N=18)$}

The 18 Wilson points include a Wilson scraper (Specimen 17AA2-2), formed on a characteristic Wilson point base, a Wilson point (Specimen 23Z-2) which is missing from the collection but was identified from an outline drawing in the level records, and counts the Wilson distal section (Specimen 34EE1B-1) with the conjoined Wilson proximal section (Specimen 35EE1A-1) as one point.

Morphology: Wilson points have very thick, expanding stems with straight bases (Figures 13-74 through 13-76); bases are modified by secondary trimming, unifacial thinning, or bifacial thinning; lateral stem edges are moderately to heavily ground, and basal edges are usually moderately to heavily ground. Flake patterns tend to be random.

Metric Averages: Tables 13-76 and 13-77

Nonmetric Attributes: Longitudinal profiles include 6 with tapered base with maximum thickness at medial section, 2 with tapered base increasing to thick distal section, 1 with thick base decreasing to thinner distal section, and 9 indeterminate; flake patterns are organized on 7 (oblique subparallel, subparallel) random on 5 , and indeterminate on 6; basal modification includes 8 secondary trimming, 5 unifacial thinning, 4 bifacial thinning, and 1 indeterminate; lateral grinding includes 8 heavy, 6 moderate, 3 slight, and 1 indeterminate; and basal grinding includes 1 absent, 2 slight, 13 moderate/heavy, and 2 indeterminate.

Material Attributes: Raw material includes 12 local and 3 nonlocal fine-grained Edwards chert, 1 local and 1 nonlocal medium-coarse-grained Edwards chert, and 1 indeterminate. Patina is absent on 16 and indeterminate on 2. Mineral precipitates are present on 8 points, absent on 9 , and indeterminate on 1 . Heat damage is absent on 12 points, slight on 1 , extensive on 3 , and indeterminate on 2 .

Tool State and Fractures: Ten points are nearly complete in length; of these, a single bend break occurs on 2, a single impact fracture on 1, a single thermal spall on 1, multiple bend breaks on 1 , multiple thermal spalls on 1 , multiple burin-like breaks on 2, multiple burin/impact breaks on 1 , and 1 is indeterminate because it is identified only from an outline sketch in the level records. There are 4 proximal sections ( 1 of which conjoins with the distal section) with a single or multiple thermal spalls on 2, multiple impact/bend breaks on 1, and multiple bend breaks on 1 . There are 2 base sections (the Wilson scraper is included here) with multiple burin-like/bend breaks on 1 and an indeterminate break on 1; and there are 2 fragmented specimens with multiple bend/ impact breaks on 1 and multiple burin-like/bend breaks on 1 .

Rejuvenation: Resharpened points include 6 alternately beveled, 4 unbeveled bifacial, 1 unifacial bevel, 6 indeterminate, and 1 indeterminate. One specimen, $32 \mathrm{CC} 2 \mathrm{C}-2$, may have been recycled as a burin. Specimen 17AA2-2 was recycled as a scraper, which was verified by Kay's microwear analysis (see Chapter 22). Also according the Kay's analysis, Specimens 20X-1, 28Y2D-2, 30CC2A-1, 32DD1B-1, and $9 \mathrm{~V}-1$ were used both as a projectile points and knives, and Specimen 33EE1C-1 was used as a projectile point, knife, and burin. Specimens 32CC2B-1 and 35EE1A-1/34EE1B-1 were examined for microscopic use-wear evidence by Driskell (see Chapter 22) who found only a generic polish ("X-polish," likely related to soil abrasion).

Stratigraphic Distribution: Seven Wilson points occur in Unit Isi-c of Valley Floor A between elevations $94.30 \mathrm{~m}$ and $94.70 \mathrm{~m}$ (Table 13-78); these points include the conjoined Specimens 34EE1B-1 (distal section) and 35EE1A-1 (proximal section) found in contiguous squares within Unit Isi-c of Valley Floor A at elevations $94.37 \mathrm{~m}$ and $94.35 \mathrm{~m}$ respectively (and counted as 1 point with 2 proveniences for Table 13-78). Segregated from points of other types, this distribution may suggest a Wilson "zone." Another group of 4 Wilson specimens occurs in Valley Floor A, Units Id/II and II between $95.10 \mathrm{~m}$ and $95.15 \mathrm{~m}$. The position of these specimens overlaps with Golondrina-Barber and parallelsided stem specimens between $94.90 \mathrm{~m}$ and $95.20 \mathrm{~m}$.

Comments: The Wilson points were provisionally named by Weir (1985) upon recognizing their association with the Late Paleoindian Burial 2. Other expanding stem points thought to be Wilsons include unnamed expandingstem specimens (Johnson 1964:55-57) and Group 1 specimens (Sorrow 1968:19-21) from Devil's Mouth, 41VV188, an untyped point from Merrell, 41WM2 (Campbell 1948:Plate 2A,1), a misidentified Lange from Mauermann I, 41TV25 (Nichols 1959:Figure 2H), misidentified Castrovilles from La Perdida, 41SR25 (Weir 1956), Specimen L from Landslide, 41BL85 (Sorrow et al. 1967), a stem fragment from Strohacker, 41KR29 (Sollberger and Hester 1972:Figure 3a), Untyped 1 from 41SP99 (Holliday and Grombacher 1974:Figure 3c), Group 12 from 41WM57 and Cervenka, 41WM267 (Hays 1982), Untyped 2 from 41 TV753 (Prewitt 1984), a side-notched point from 41WH19 (Patterson and Hudgins 1985), an untyped point from Horn Shelter, 41BQ46 (Forrester 1985:Figure 2F), an untyped point from Jack Dies Ranch, 41TV102 (TARL collections and records), and a point from 41VV1654 (Tennis et al.1996). Turner and Hester (1985:87) referred to these broadly expanding stemmed points with ground edges as "Early Stemmed."

A comparative sample of 24 sufficiently complete specimens was assembled from the Devil's Mouth, Jack Dies Ranch, 41VV1654, and Wilson-Leonard sites. Fifteen specimens are from the Wilson-Leonard collection, 7 are from Devil's Mouth, and 1 each from Jack Dies Ranch and 41VV1654. The metric data indicate that the Devil's Mouth and Wilson-Leonard points are virtually the same in the three stem width measurements, basal concavity, and stem shape as indicated by stem shape ratio (Table 13-79; see also Chapter 14). Differences occur in thickness, 10-mm stem thickness, stem length, and stem thickness to stem width ratio, with the Devil's Mouth points averaging 

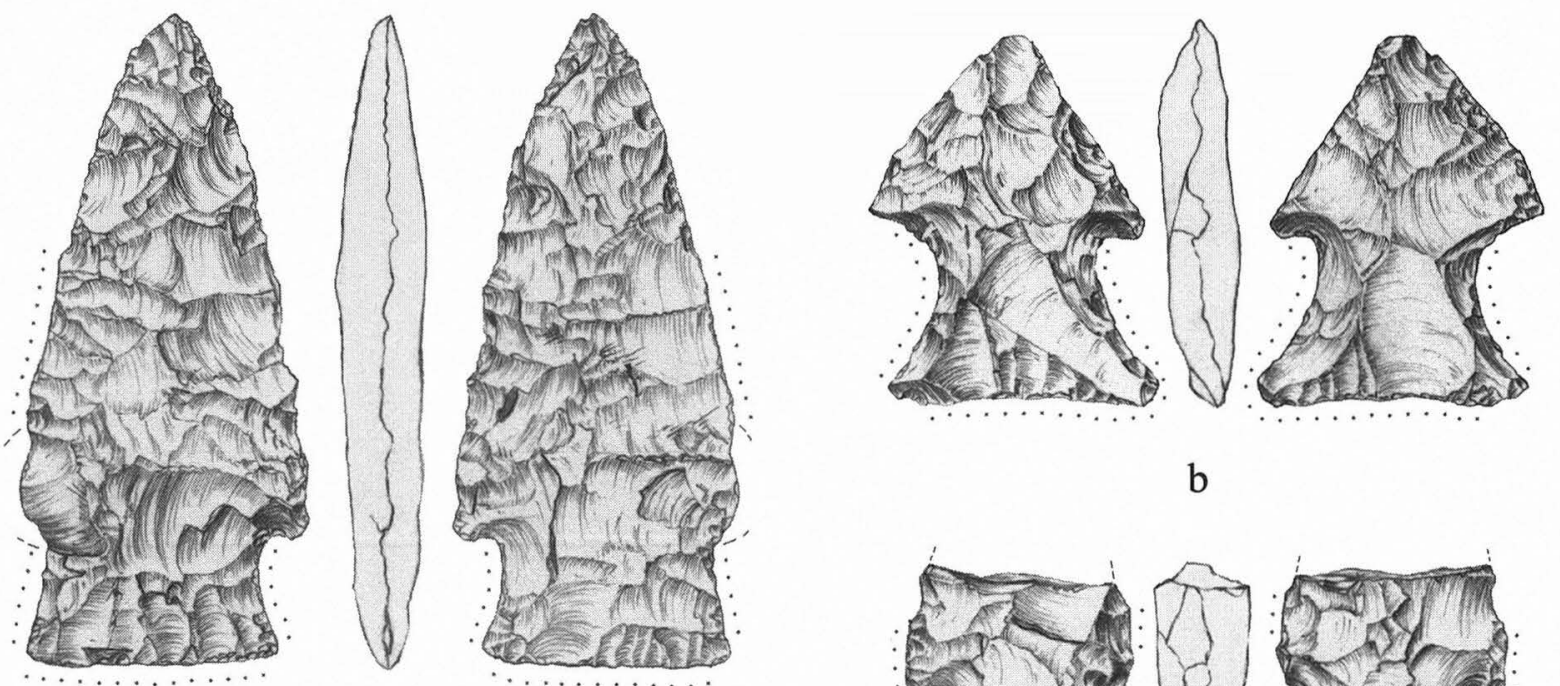

b

a
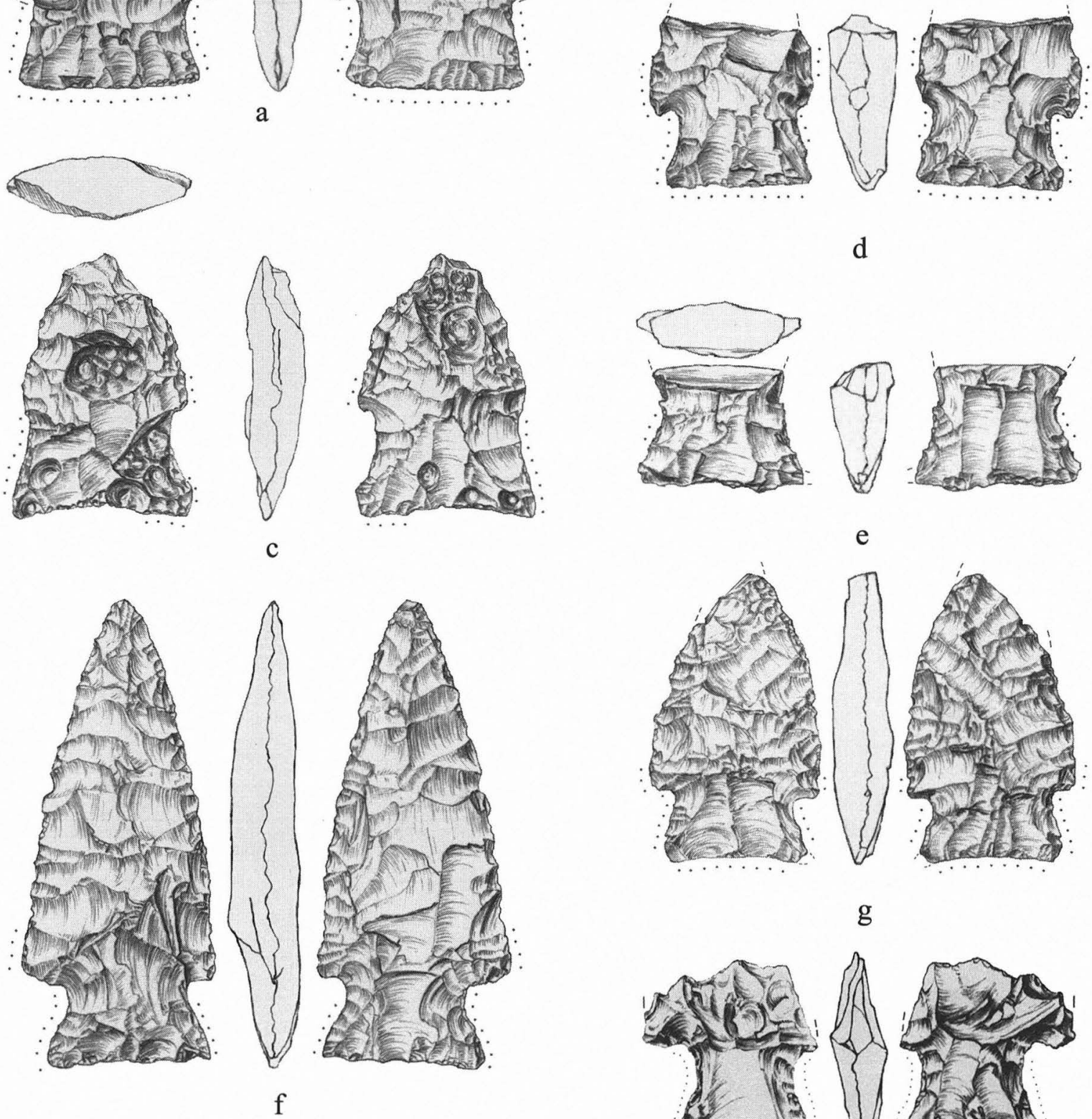

g

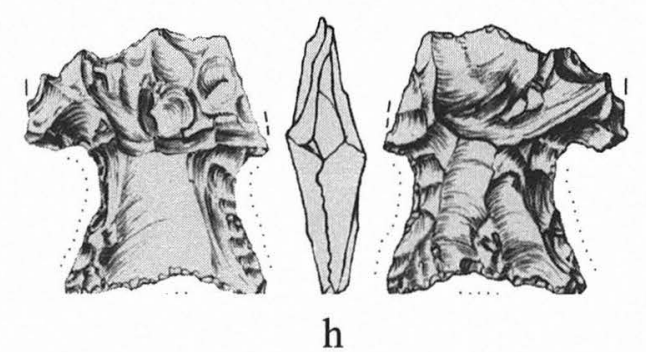

$0 \quad$ centimeters

FiGURE 13-74. Wilson points. Specimen numbers: (a) 9V-1; (b) 20X-1; (c) 15V1-1; (d) 13U-1; (e) 29X1-1; (f) 28Y2C-1; (g) 28Y2D-2; (h) 32Y2C-1. 

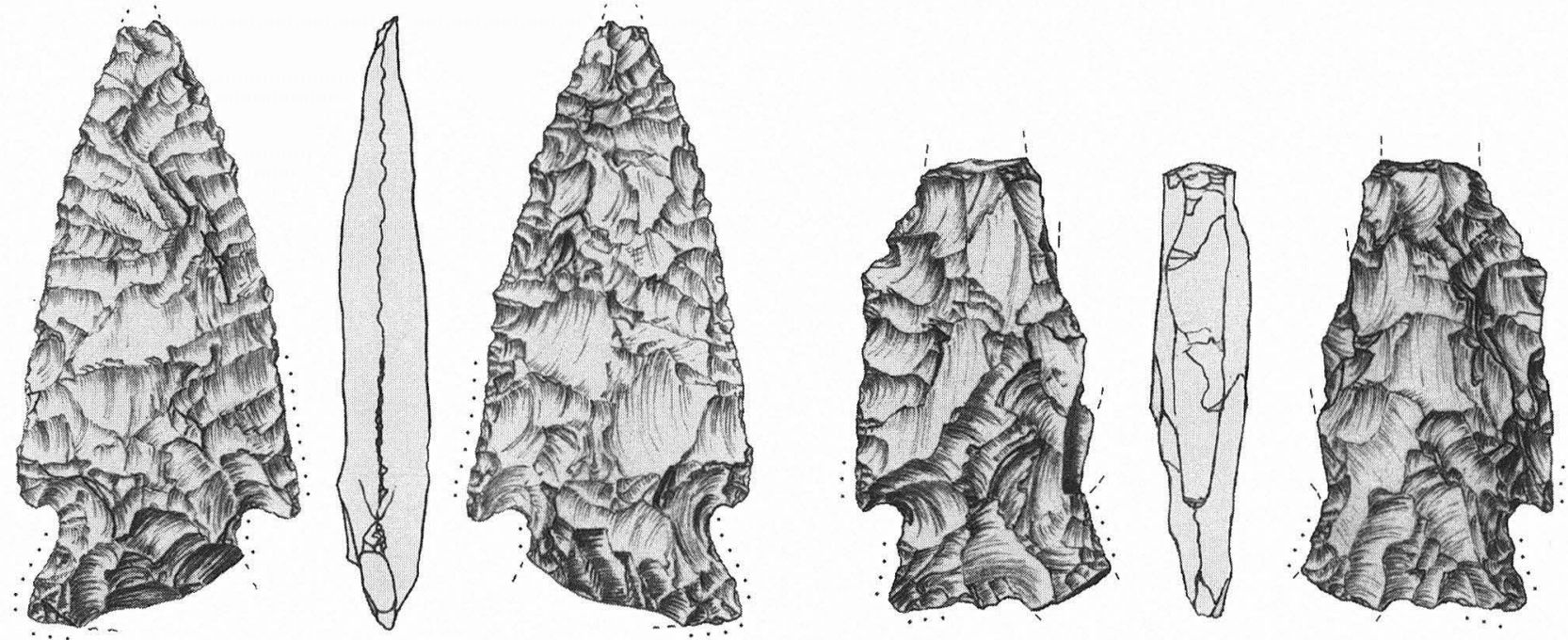

a
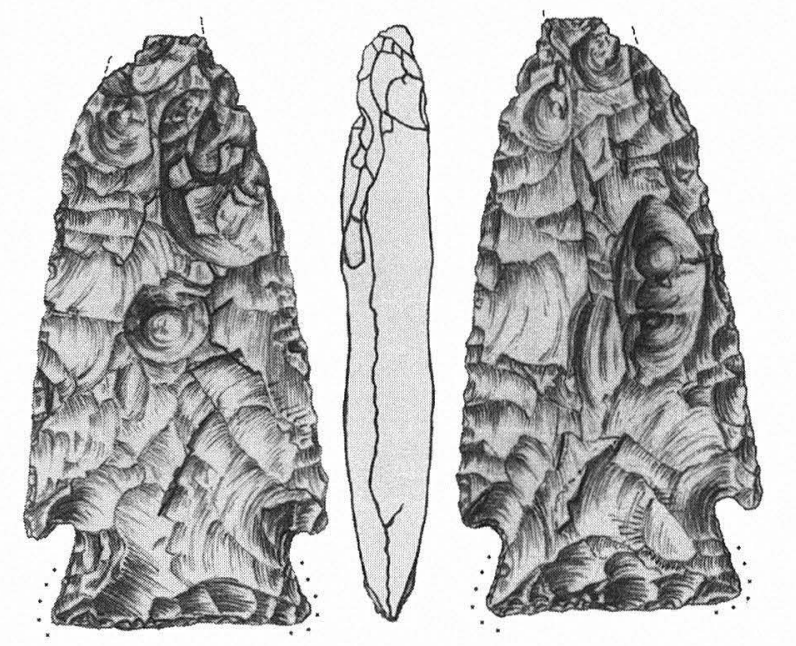

c
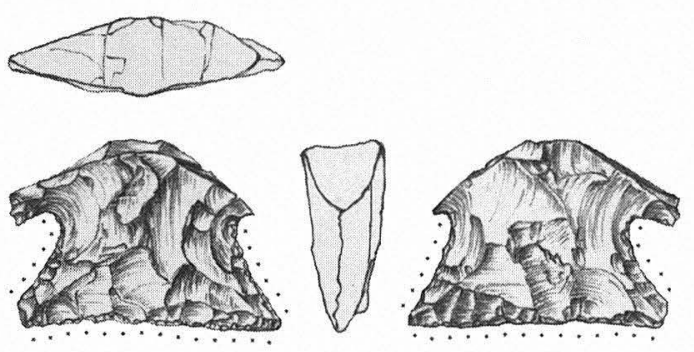

d

Figure 13-75. Wilson points. Specimen numbers: (a) 32CC2B-1; (b) 32CC2C-2; (c) 32DD1B-1; (d) 32DD2C-1.

TABLE $13-76$

Summary Metric Data for Wilson Points (in mm)

\begin{tabular}{|c|c|c|c|c|c|}
\hline Variable & Number & Minimum & Maximum & Mean & $1-\sigma$ \\
\hline Length & 5 & 37.5 & 73.0 & 63.1 & 14.7 \\
\hline Width & 12 & 22.9 & 31.3 & 27.2 & 2.5 \\
\hline Thickness & 16 & 7.1 & 10.4 & 8.8 & 1.0 \\
\hline Stem/grinding length 1 & 16 & 9.2 & 15.8 & 12.5 & 1.8 \\
\hline Stem/grinding length 2 & 12 & 8.8 & 15.8 & 11.8 & 2.0 \\
\hline Proximal stem width & 15 & 20.3 & 27.2 & 23.5 & 2.5 \\
\hline 10 -mm stem width & 15 & 15.5 & 21.6 & 18.3 & 1.6 \\
\hline Distal stem width & 14 & 15.9 & 22.7 & 18.8 & 2.1 \\
\hline 10-mm stem thickness & 16 & 6.4 & 8.5 & 7.2 & 0.5 \\
\hline Minimum stem width & 2 & 17.4 & 19.0 & & \\
\hline Basal concavity & 11 & 0.0 & 1.7 & 0.7 & 0.6 \\
\hline Basal convexity & 3 & 0.5 & 1.1 & 0.9 & 0.3 \\
\hline
\end{tabular}



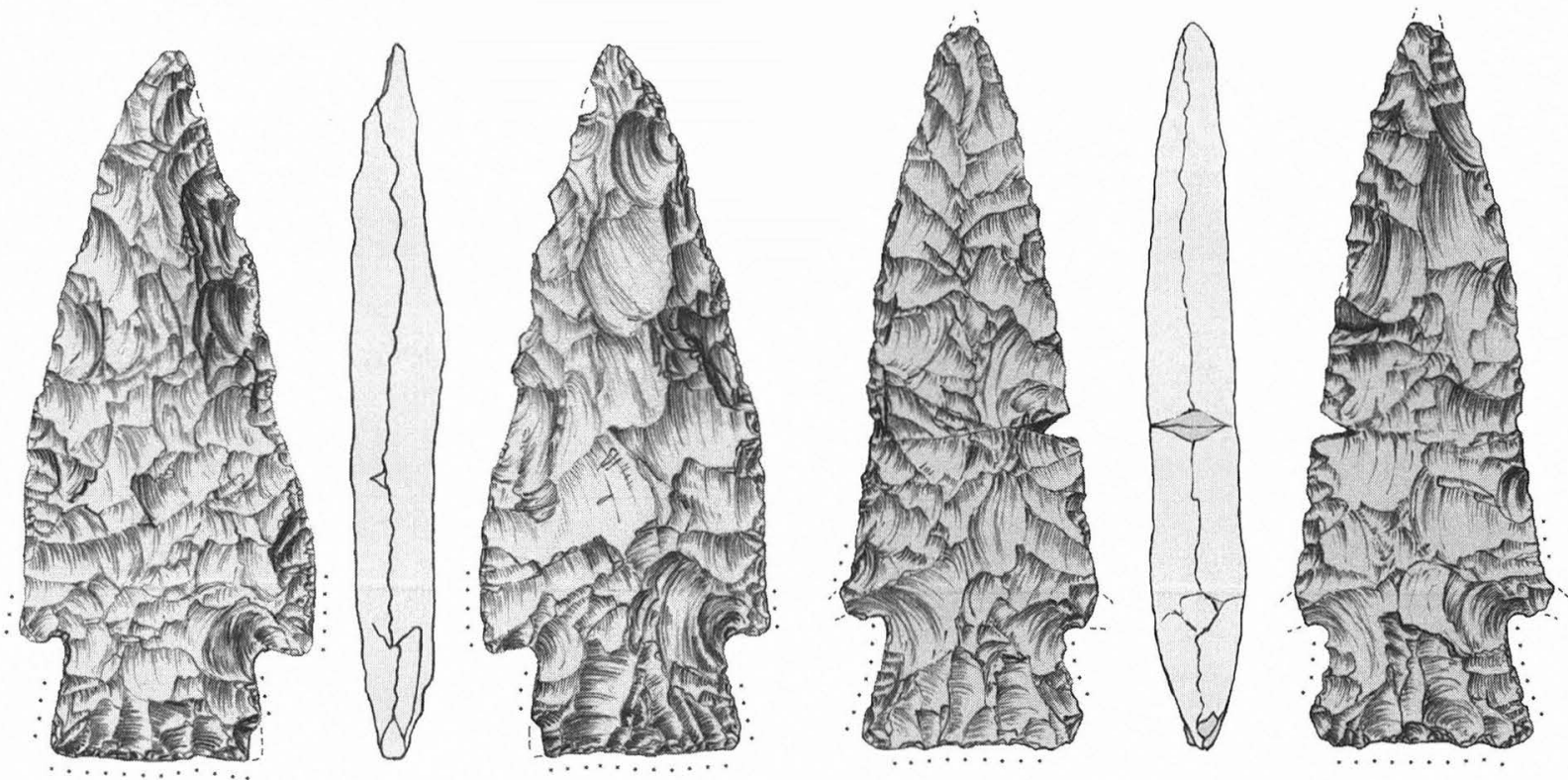

a
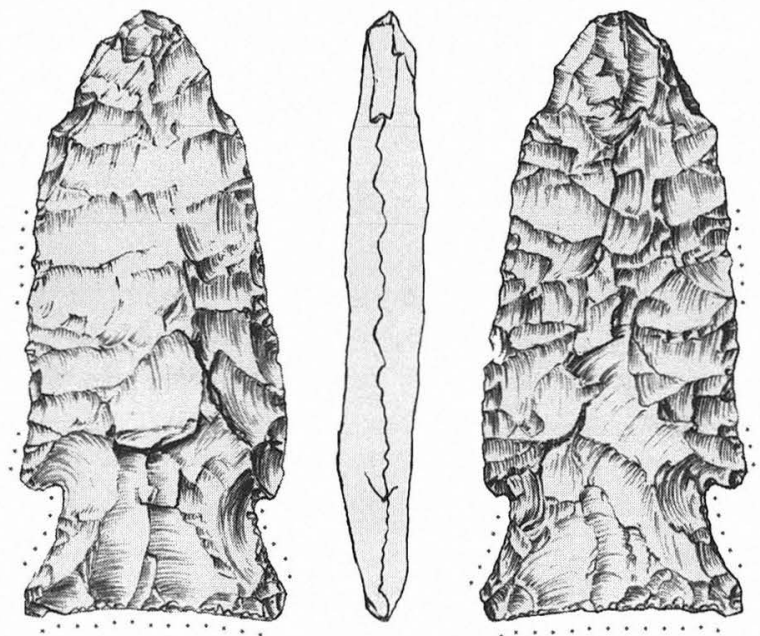

c

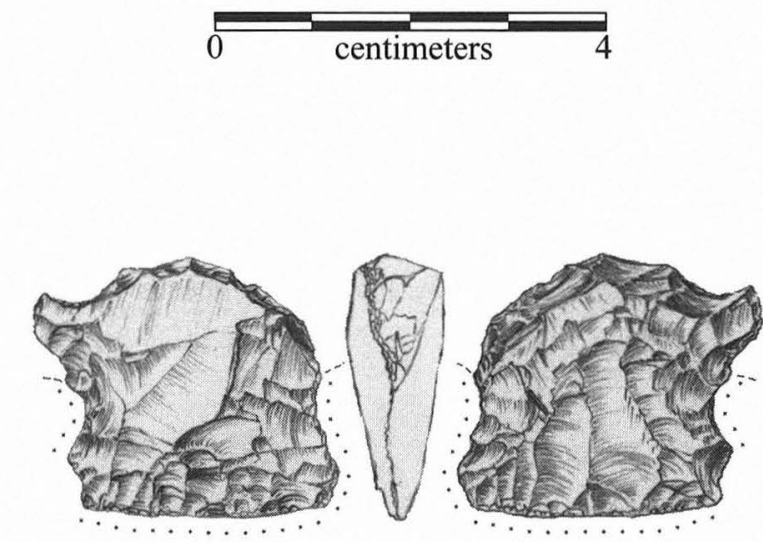

d

FIGURE 13-76. Wilson points. Specimen numbers: (a) 30CC2A-1; (b) 34EE1B-1 (distal), 35EE1A-1 (proximal); (c) 33EE1C-1; (d) 17AA2-2.

TABLE 13-77

Metric Measurements for Wilson Scraper

\begin{tabular}{l|c}
\hline Variable & Value \\
\hline Length* & 26.0 \\
Width & 28.8 \\
Thickness & 8.9 \\
Stem/grinding ength 1 & 11.7 \\
Proximal stem width & 27.0 \\
10 mm stem width & 23.3 \\
10 mm stem thickness & 6.8 \\
Minimum stem width & 22.9 \\
Basal convexity & 2.1 \\
\hline * Maximum measurement & \\
\hline \hline
\end{tabular}

slightly thinner in both thickness and stem thickness, and slightly longer in stem length than the Wilson-Leonard points. This result suggests some regional variation between the Lower Pecos and Central Texas. The individual specimens from Jack Dies Ranch, a Central Texas site, and 41VV1654, a Lower Pecos site, appear to bear out this dichotomy.

The chronological position of Wilson at the WilsonLeonard site is 10,000 B.P. to 9500 B.P. Devil's Mouth site has the only other radiocarbon date for Wilson, $8780 \pm 310$ B.P. (TX-526) from charcoal (Sorrow 1968:47).

Kay (see Chapter 22) examined eight Wilson points for evidence of microscopic use-wear. Four showed use first as projectile points and then as knives. The remaining five had more complex use histories, with some recycled to burins. 
TABLE $13-78$

Stratigraphic Distribution of Wilson Points

\begin{tabular}{|c|c|c|c|c|c|c|}
\hline \multirow[b]{2}{*}{ Elevation } & \multirow{2}{*}{$\begin{array}{l}\text { Valley Margin } \\
X\end{array}$} & \multicolumn{5}{|c|}{ Valley Floor A } \\
\hline & & Isi/Isi-c & Isi-c & Isi-c/Id/II & $\mathrm{Id} / \mathrm{II}$ & II \\
\hline $95.81-95.90$ & 1 & \multirow{16}{*}{1} & & \multirow{16}{*}{$1+$} & \multirow{16}{*}{3} & \\
\hline $95.71-95.80$ & 1 & & & & & \\
\hline $95.61-95.70$ & & & & & & 1 \\
\hline $95.51-95.60$ & & & & & & \\
\hline $95.41-95.50$ & & & & & & \\
\hline $95.31-95.40$ & & & & & & 1 \\
\hline $95.21-95.30$ & & & 1 & & & \\
\hline $95.11-95.20$ & & & & & & 1 \\
\hline $95.01-95.10$ & & & & & & \\
\hline $94.91-95.00$ & & & & & & \\
\hline $94.81-94.90$ & & & & & & \\
\hline $94.71-94.80$ & & & & & & \\
\hline $94.61-94.70$ & & & 2 & & & \\
\hline $94.51-94.60$ & & & 3 & & & \\
\hline $94.41-94.50$ & & & 1 & & & \\
\hline $94.31-94.40$ & & & $2 *$ & & & \\
\hline Totals: & 2 & 1 & 9 & 1 & 3 & 3 \\
\hline
\end{tabular}

Only one showed use first as a knife, then as a projectile. In Kay's estimation, the Wilson point evinces innovative design elements not fully developed until much later; as a knife, it must have functioned well, but as a projectile point, perhaps less than satisfactory.

\section{Wilson Medial Section ( $N=1)$}

Morphology: This specimen is a very thick medial section of a stemmed point (Figure 13-77).

Metric Values: Table 13-80

Nonmetric Attributes: Longitudinal profile is indeterminate; flake patterns of oblique parallel/random co-occur; basal modification is indeterminate; lateral and basal grinding are indeterminate.

Material Attributes: Raw material is local mediumcoarse-grained Edwards chert; patina is indeterminate; mineral precipitates and heat damage are absent.

Tool State and Fractures: This specimen has 1 transverse impact fracture on the stem and 1 oblique indeterminate fracture on the blade.

Rejuvenation: Resharpening is indeterminate.

Stratigraphic Distribution: Valley Floor A, Unit II.

Comments: This specimen, although missing the basal morphology used to assign type, has characteristics that suggest the Wilson type. The distal stem width is within the range for Wilson. Kay (see Chapter 22) indicates that the blades of the Wilson points he examined are heavily resharpened, and it is likely a pristine Wilson blade would be much wider. This specimen has a blade width well outside the range of the heavily resharpened Wilsons, and thus, suggests the size of an unresharpened Wilson. Also, this specimen is stratigraphically associated with Wilson Specimens 28Y2C-1, 28Y2D-2, 29X1-1, and 32Y2C-1.

\section{Wilson(?) Proximal Fragments $(\mathrm{N}=2)$}

Morphology: These points have very thick, expanding stems with straight bases; lateral stem edges are moderately ground and basal edges are moderately to heavily ground.

Metric Values: Table 13-81

Nonmetric Attributes: Longitudinal profiles are indeterminate on 2; flake patterns are random on 1 and 1 is indeterminate; basal modification is bifacial thinning on 1 and indeterminate on 1; lateral grinding is moderate on 2 ; and basal grinding is moderate/heavy on 2 .

Material Attributes: Raw material includes 1 local finegrained and 1 local medium-coarse-grained Edwards chert; patina is absent; mineral precipitates are absent; heat damage is absent on 1 and extensive on 1.

Tool State and Fractures: One specimen has an oblique bend fracture on the blade and a crushed edge on the stem; the other specimen has multiple thermal spalls.

Rejuvenation: Resharpening includes 1 unbeveled bifacial and 1 indeterminate.

Stratigraphic Distribution: With Specimen 280-1 in 
TABLE 13-79

Comparative Metric Data for Wilson Points from Four Sites

\begin{tabular}{|c|c|c|c|c|c|c|c|c|c|c|c|c|c|c|c|c|c|}
\hline \multirow[b]{2}{*}{ Site } & \multicolumn{5}{|c|}{$41 \mathrm{VV} 188$} & \multicolumn{5}{|c|}{ 41WM235 } & \multirow{2}{*}{ 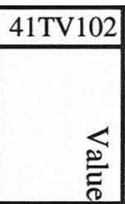 } & \multirow{2}{*}{ 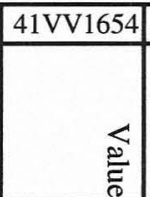 } & \multicolumn{5}{|c|}{ Total Sample } \\
\hline & $\begin{array}{l}Z \\
\text { Z } \\
\text { 总 } \\
\end{array}$ & 客 & 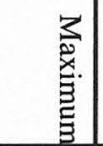 & 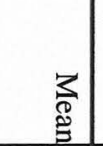 & 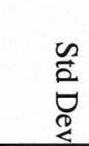 & $\begin{array}{l}\text { Zz } \\
\text { 空 }\end{array}$ & 客: & 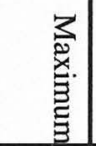 & 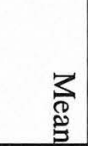 & 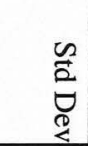 & & & $\begin{array}{l}Z \\
\text { Z } \\
\stackrel{\Xi}{G}\end{array}$ & 客 & 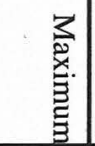 & 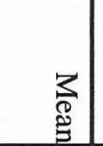 & 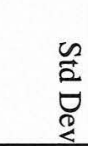 \\
\hline Length & 4 & 41.2 & 60.1 & 48.9 & 8.9 & 9 & 37.0 & 73.0 & 56.3 & 12.8 & 78.6 & 47.4 & 15 & 37.0 & 78.6 & 55.2 & 12.9 \\
\hline Width & 7 & 27.9 & 28.7 & 28.4 & 0.3 & 15 & 22.9 & 31.3 & 27.0 & 2.4 & 32.4 & 25.5 & 24 & 22.9 & 32.4 & 27.6 & 2.3 \\
\hline Thickness & 7 & 5.9 & 7.6 & 7.0 & 0.6 & 15 & 7.1 & 10.4 & 8.6 & 0.9 & 9.9 & 7.8 & 24 & 5.9 & 10.4 & 8.2 & 1.1 \\
\hline Stem Length & 7 & 12.3 & 16.9 & 14.9 & 1.9 & 15 & 9.2 & 15.8 & 12.4 & 1.9 & 14.8 & 16.0 & 24 & 9.2 & 16.9 & 13.4 & 2.2 \\
\hline Proximal Stem Width & 7 & 21.6 & 26.1 & 24.0 & 1.5 & 15 & 20.3 & 27.2 & 23.8 & 2.7 & 28.6 & 25.3 & 24 & 20.3 & 28.6 & 24.1 & 2.5 \\
\hline 10-mm Ster & 7 & 16.8 & 20.0 & 18.5 & 1.2 & 15 & 15.5 & 23.3 & 18.8 & 2.2 & 22.5 & 19.3 & 24 & 15.5 & 23.3 & 18.9 & 2.0 \\
\hline Distal S & 7 & 16.3 & 20.6 & 18.4 & 1.7 & 13 & 15.9 & 22.7 & 18.7 & 2.2 & 22.5 & 19.2 & 22 & 15.9 & 22.7 & 18.8 & 2.1 \\
\hline $10-\mathrm{mm} \mathrm{S}$ & 7 & 4.6 & 6.7 & 5.8 & 0.8 & 15 & 6.4 & 7.9 & 7.1 & 0.4 & 7.9 & 6.9 & 24 & 4.6 & 7.9 & 6.7 & 0.8 \\
\hline Basal Concavity & 7 & 0.0 & 1.5 & 0.7 & 0.7 & 15 & -2.1 & 1.7 & 0.2 & 1.0 & -1.1 & 0.0 & 24 & -2.1 & 1.7 & 0.3 & 1.0 \\
\hline Stem Shape Ratio & 7 & 0.690 & 0.853 & 0.770 & 0.050 & 15 & 0.673 & 0.919 & 0.795 & 0.077 & 0.787 & 0.763 & 24 & 0.673 & 0.919 & 0.786 & 0.067 \\
\hline Stem Thickness to Stem Width Ratio & 7 & 0.274 & 0.354 & 0.311 & 0.031 & 15 & 0.292 & 0.477 & 0.382 & 0.054 & 0.351 & 0.358 & 24 & 0.274 & 0.477 & 0.359 & 0.055 \\
\hline
\end{tabular}



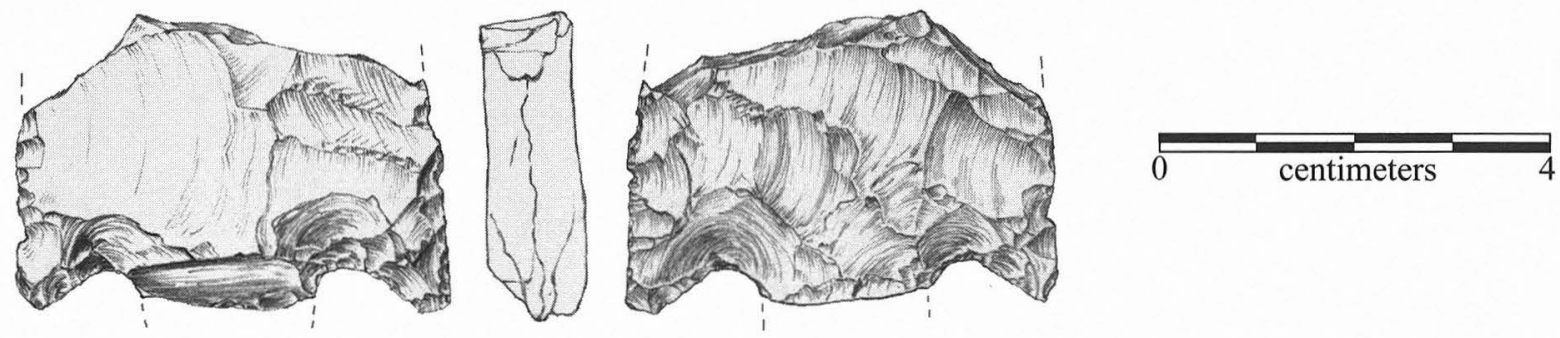

FIGURE 13-77. Wilson medial section, Specimen 34Y1D-1.

TABLE $13-80$

Metric Measurements for

Wilson Medial Section

\begin{tabular}{l|c}
\hline Variable & Value \\
\hline Length* & 28.0 \\
Width & 43.0 \\
Thickness & 9.5 \\
Distal Stem Width & 20.1 \\
10-mm Stem Thickness & 8.2 \\
\hline
\end{tabular}

* Maximum measurement of fragment

TABLE 13-81

Metric Measurements for Wilson(?)

Proximal Fragment (in mm)

\begin{tabular}{l|c|c}
\hline Variable & $\begin{array}{c}\text { Specimen } \\
280-1\end{array}$ & $\begin{array}{c}\text { Specimen } \\
\text { 37LD-30 }\end{array}$ \\
\hline Length* & 42.0 & 35.0 \\
Width & 33.0 & \\
Thickness & 8.0 & 7.0 \\
Stem/grinding length 1 & 15.5 & 12.2 \\
Proximal stem width & 22.4 & 19.4 \\
10-mm stem width & 17.7 & 16.9 \\
Distal stem width & 18.7 & \\
10-mm stem thickness & 8.1 & 6.1 \\
Minimum stem width & 17.4 & \\
Basal concavity & 0.0 & 0.6 \\
* Maximum measurement of fragment \\
\hline \hline
\end{tabular}

Unit IIIa/b and Specimen 37LD-30 in Unit IIIb, both are well outside the context for Wilson.

Comments: The fragmented condition and poor stratigraphic context prohibit firm type classification.

\section{Wilson(?)-atypic ( $N=1)$}

Morphology: This specimen has an extremely expanded stem with a shallow basal concavity (Figure 13-78); the base is modified by secondary trimming; lateral stem edges are slightly ground and the basal edge is not ground.

Metric Values: Table 13-82

Nonmetric Attributes: Longitudinal profile is short

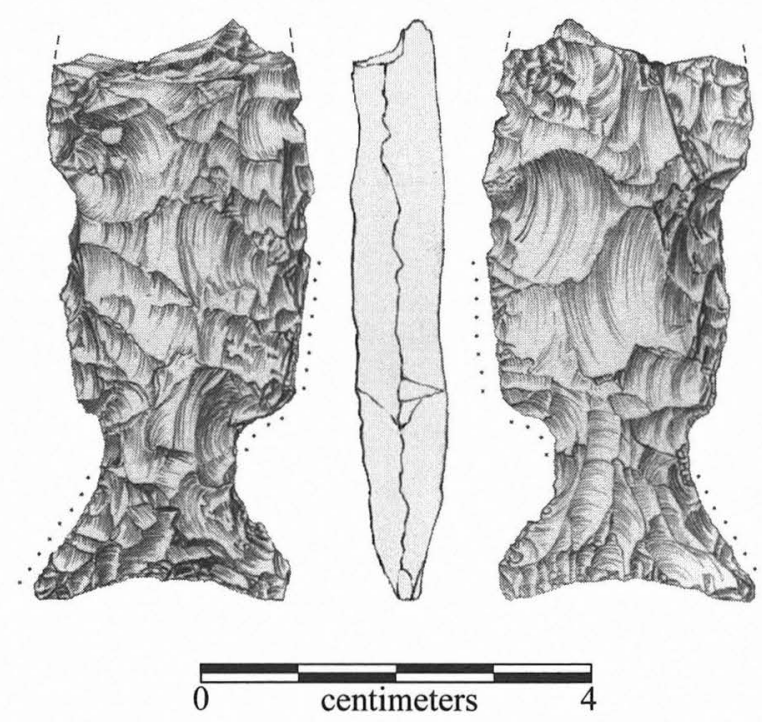

FiguRE 13-78. Wilson(?)-atypic, Specimen 28W1-1.

TABLE $13-82$

Metric Measurements for Wilson(?)-atypic Proximal Section (in mm)

\begin{tabular}{l|c}
\hline Variable & Value \\
\hline Length* & 58.0 \\
Width & 26.0 \\
Thickness & 9.0 \\
Stem/grinding length 1 & 17.9 \\
Proximal stem width & 26.9 \\
Distal stem width & 14.1 \\
Basal concavity & 1.7 \\
\hline
\end{tabular}

* Maximum measurement

wedge-shaped base with tapered tip; flake patterns of oblique subparallel/indeterminate co-occur; basal modification is secondary trimming; lateral grinding is slight; and basal grinding is absent.

Material Attributes: Raw material is local fine-grained Edwards chert; patina is absent; mineral precipitates are absent; heat damage is moderate.

Tool State and Fractures: This proximal section has multiple thermal spalls on the blade.

Rejuvenation: Resharpening is indeterminate. 
Stratigraphic Distribution: Valley Floor A, Unit II

Comments: Classification of this specimen is questionable. The stem length and distal stem width fall outside the range of the Wilson sample; the shape and flaking of the blade also do not conform to Wilson; and the stratigraphic context is not within good Wilson context. However, the remaining size, grinding, and basal modification data do fall within the Wilson range. Alternative interpretations suggest this specimen may be heavily reworked, losing part of the Wilson characteristics, or may be just a very atypical example of Wilson.

\section{Castroville $(N=17)$}

Morphology: These medium to large points are distinguished by broad blades, very wide, expanding stems with typically straight, although sometimes convex-edged bases, and large barbs formed by basal notching (Figures 13-79 and 13-80). Bases usually are well thinned, and basal edges may be lightly smoothed. Blade flaking is typically unpatterned.

\section{Metric Averages: Table 13-83}

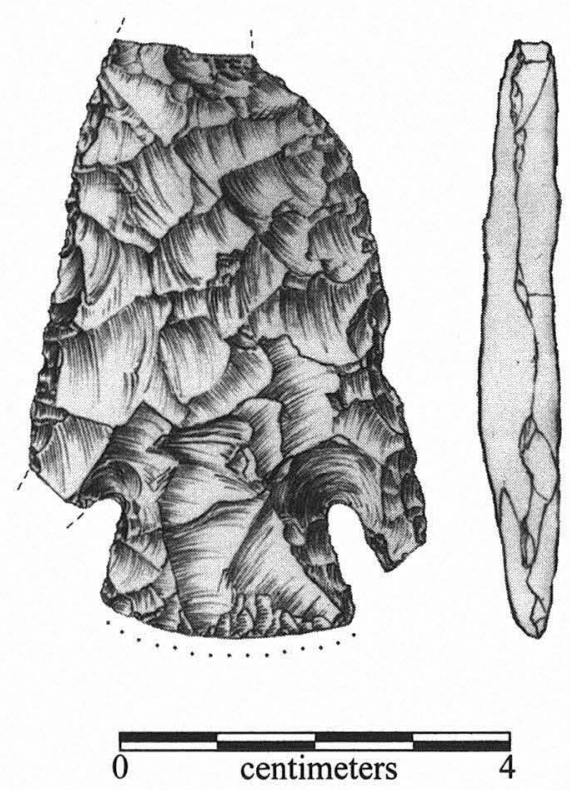

FIGURE 13-79. Castroville point, Specimen 108-14.

Nonmetric Attributes: Longitudinal profiles include 6 with tapered base and tip, 3 flat, 2 with wedge-like base and tip and generally uniform body, and 6 indeterminate. Flake patterns are organized on 1 (oblique subparallel), random on 14 , and indeterminate on 2 . Basal modification consists of unifacial thinning on 7 , bifacial thinning on 6 , secondary trimming on 3 , and absent on 1. Lateral stem grinding is slight on 1 .

Material Attributes: Raw material is local Edwards chert (11 fine grained, 2 medium/coarse) and nonlocal or indeterminate Edwards chert ( 3 fine grained, 1 medium/coarse). Heat damage is slight on 3 and extensive on 1 . No patina or mineral precipitates were observed.

Tool State and Fractures: One is complete, having been reworked to compensate for a broken barb; 6 lack distal tips and/or barb tips and evidence multiple impact and bend fractures; the 10 remaining are proximal sections with multiple impact and bend fractures ( 2 of these also exhibit thermal breaks).

Rejuvenation: Eight of the 10 complete enough to observe exhibit resharpening ( 3 bifacially, unbeveled; 4 alternately beveled, and 1 beveled unifacially); 2 of the above specimens display lateral edge modification to compensate for broken barbs.

Stratigraphic Distribution: Castroville points were recovered in highest frequency in Unit IIIc (Table 13-84).

Comments: Problems in distinguishing Castroville from Marcos have been previously noted; the former often may be set apart by their relatively larger overall size and straighter, less-expanding stems typically formed by basal notching as opposed to corner notching (Turner and Hester 1993:147). Differences are often slight, however, and in the Wilson-Leonard assemblage, there appears to be a morphological gradation between the two. As noted, 2 specimens were modified to compensate for broken barbs, indicating that the large barbs did not play a significant role either in hafting or for all uses of the tool.

\section{$\operatorname{Darl}(N=27)$}

Morphology: Generally well-flaked, small, narrow elongate points with slight, typically barbless shoulders comprise this group. Stems are straight to expanding with straight to slightly concave bases; stem lateral edges are not commonly ground. Blade sections and stem edges may be highly beveled, and cross sections range in shape from flat to lenticular to rhomboid (Figures 13-81 and 13-82).

Metric Averages: Table 13-85

Nonmetric Attributes: Longitudinal profiles include 8 with tapered base and tip, 1 with thick tip, 1 with thick base, 2 flat, 4 with wedge-like base and tip and uniform body proportions, and 11 indeterminate. Flake patterns are organized on 7 (subparallel, collateral, oblique subparallel), random on 5 , and indeterminate on 15 . Basal modification constitutes unifacial thinning on 6 , bifacial thinning on 7 , concave bevel on 3 , and 11 unmodified. Lateral stem grinding is slight on 2.

Material Attributes: Raw material is chiefly local Edwards chert (15 fine grained, 3 medium/coarse), nonlocal Edwards fine grained $(n=2)$, non-Edwards fine grained $(n=1)$, and indeterminate and/or heat altered $(\mathrm{n}=6)$. Mineral precipitates were identified on 1 . Heat damage is slight on 2 , moderate on 1 , and extensive on 4 . No patina was observed.

Tool State and Fractures: Ten are complete or nearly so, with 3 exhibiting impact damage to the distal section, and 2 have thermal damage. Seventeen others are proximal sec- 


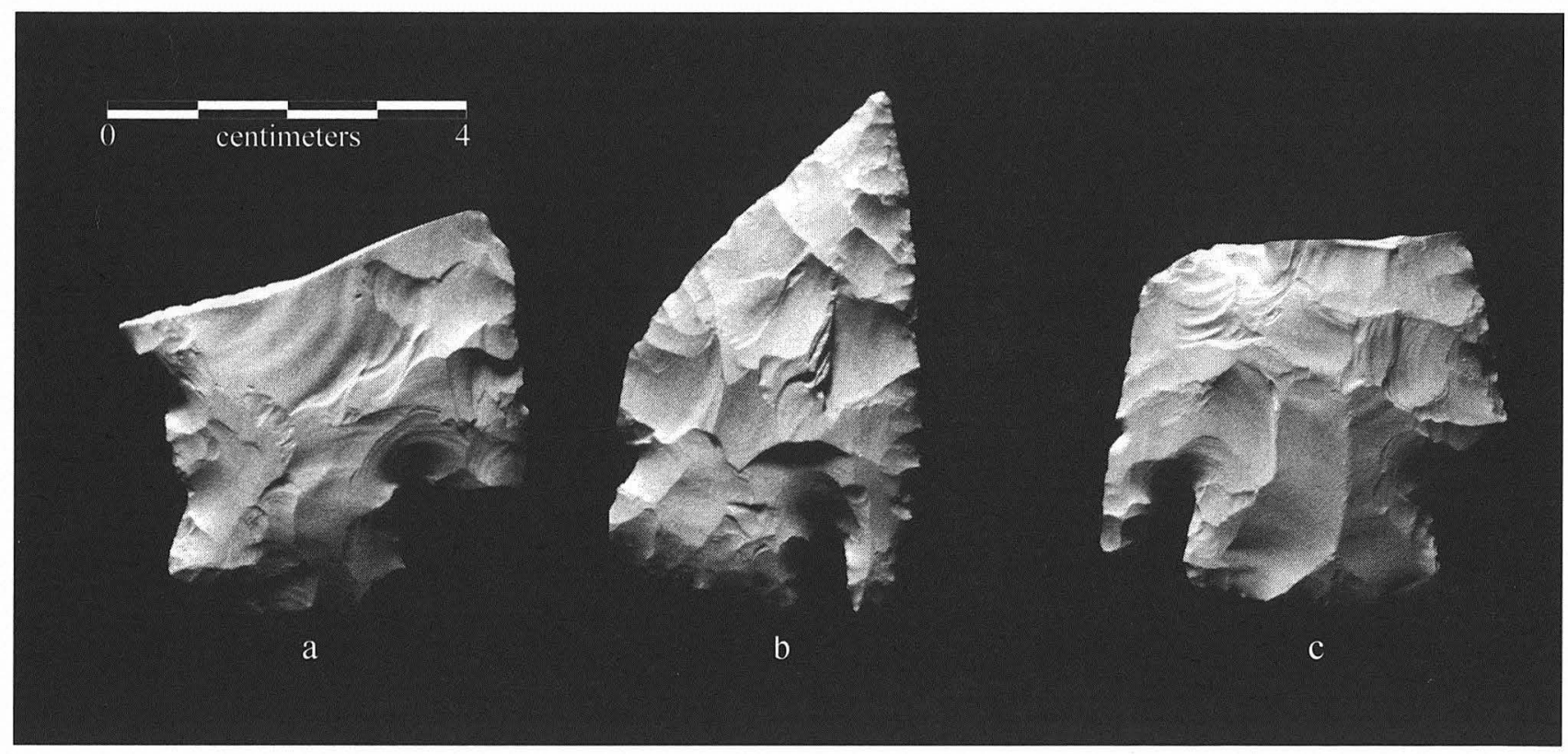

FIGURE 13-80. Castroville points. Specimen numbers: (a) 2730-1; (b) 36D-19; (c) 406-1.

TABLE 13-83

Summary Metric Data for Castroville Points (in mm)

\begin{tabular}{|c|c|c|c|c|c|}
\hline & Number & Minimum & Maximum & Mean & $1-\sigma$ \\
\hline Length & 4 & 50.0 & 59.0 & 56.5 & 4.4 \\
\hline Width & 8 & 30.0 & 39.0 & 33.8 & 2.9 \\
\hline Thickness & 17 & 6.0 & 10.0 & 7.5 & 1.1 \\
\hline Stem length & 17 & 11.0 & 16.0 & 14.0 & 1.4 \\
\hline Proximal stem width & 14 & 20.0 & 30.0 & 25.3 & 2.6 \\
\hline Distal stem width & 17 & 17.0 & 26.0 & 21.8 & 2.4 \\
\hline Basal concavity depth & 1 & 2.0 & 2.0 & & \\
\hline Basal convexity length & 14 & 1.0 & 5.0 & 2.9 & 1.3 \\
\hline
\end{tabular}

TABLE $13-84$

Stratigraphic Distribution of Castroville Points

\begin{tabular}{l|c|c}
\hline Unit & Valley Floor A & Valley Margin \\
\hline IIIc & 7 & 1 \\
IIIb/c & 3 & \\
Other & & \\
surface & 3 & \\
IIIb/c fill & 1 & \\
provenience unknown & 2 & \\
\hline
\end{tabular}

tions; of these, 7 exhibit one or more bend/hinge fractures, and 10 show multiple impact, bend, and/or thermal fractures.

Rejuvenation: Of the 15 complete enough to observe, all are resharpened ( 6 unbeveled bifacially, 6 alternately beveled, and 3 beveled unifacially).

Stratigraphic Distribution: Table 13-86

Comments: As Prewitt (n.d.; 1981a) recognized, there is
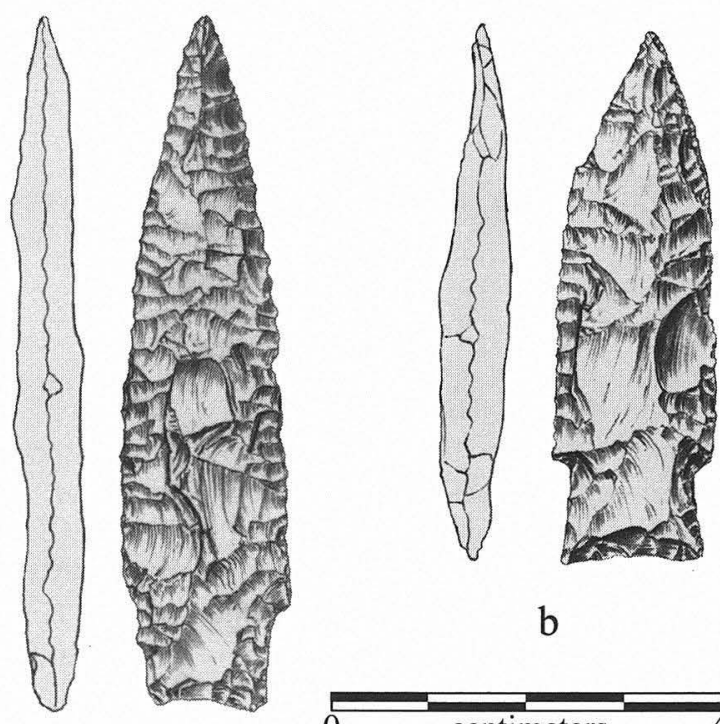

b

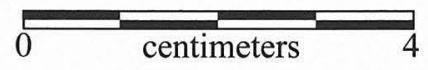

a

FIGURE 13-81. Darl points. Specimen numbers: (a) 8B-7; (b) 0-118. 


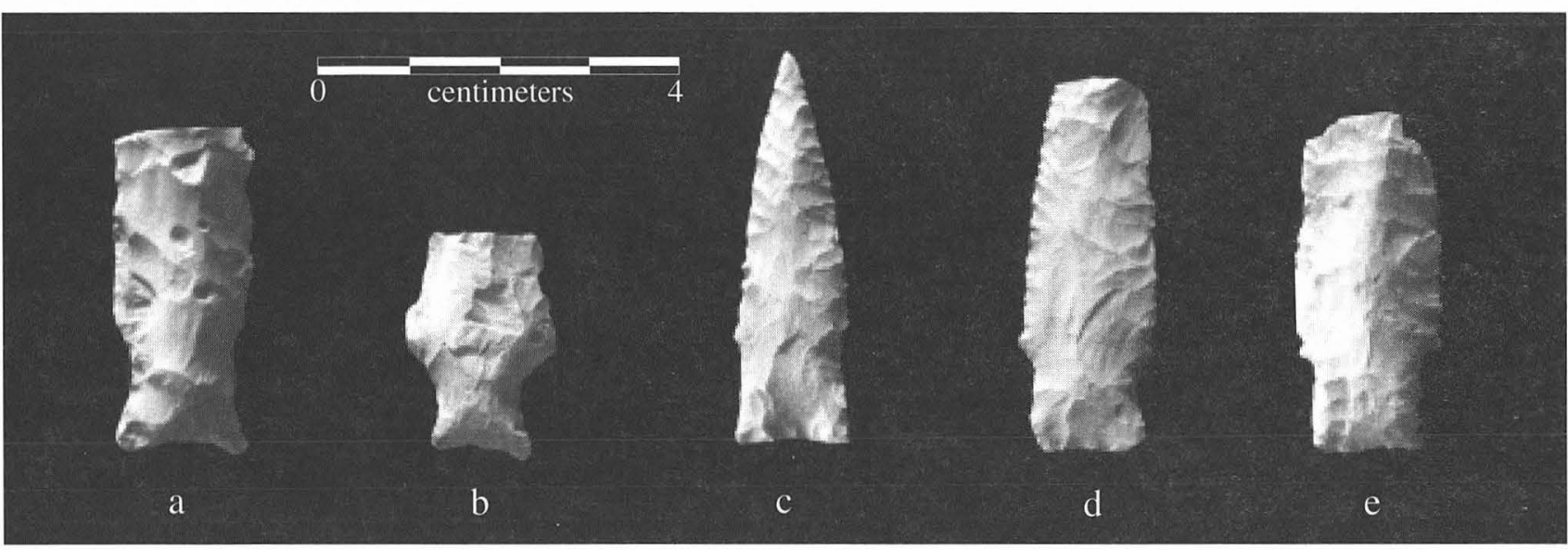

Figure 13-82. Darl points. Specimen numbers: (a) 3E-2; (b) 5B-1; (c) 36E-1; (d) 6A-13; (e) 9B-3.

TABLE 13-85

Summary Metric Data for Darl Points (in $\mathrm{mm}$ )

\begin{tabular}{l|c|c|c|c|c}
\hline & Number & Minimum & Maximum & Mean & $1-\sigma$ \\
\hline Length & 7 & 45.0 & 70.0 & 57.0 & 9.4 \\
Width & 23 & 14.0 & 21.0 & 17.3 & 1.7 \\
Thickness & 26 & 5.0 & 10.0 & 6.5 & 1.0 \\
Stem length & 23 & 11.0 & 18.0 & 13.1 & 1.7 \\
Proximal stem width & 22 & 12.0 & 16.0 & 13.3 & 1.2 \\
Distal stem width & 23 & 11.0 & 15.0 & 1.7 & 0.6 \\
Basal concavity depth & 25 & 1.0 & 3.0 & & \\
\hline \hline
\end{tabular}

TABLE 13-86

Stratigraphic Distribution of Darl Points

\begin{tabular}{l|c|c}
\hline Unit & Valley Floor A & Valley Margin \\
\hline IIIc & 4 & 16 \\
Other & & 3 \\
IIIc pot & \\
surface & 4 & \\
\hline \hline
\end{tabular}

a remarkable similarity in Darl and Hoxie, although the two types are separated in time by some 6,000-8,000 years. Both share similar stem morphologies, and both frequently display beveled resharpening and serration of blade edges. A key distinguishing attribute, however, is lateral stem grinding, which is almost always heavy on Hoxie but rarely present on Darl. In addition, Darl bases are more frequently wellthinned, whereas Hoxies more commonly display a beveled basal concavity. At Wilson-Leonard, morphological similarities are also seen with the diminutive Expanding Stem Concave A (see above).

\section{Edgewood $(N=4)$}

Morphology: These relatively small, triangular points have medium to wide expanding stems created by corner notching. Basal edges vary from slightly concave to straight, to slightly convex (Figures 13-83 and 13-84).

Metric Averages: Table 13-87

Nonmetric Attributes: Longitudinal profiles include wedge-shaped base and tip on 1, thick stem and flat body on 1 , and 2 indeterminate. Flake patterns are organized on 1 (chevron), random on 2 , and 1 indeterminate. Basal modification takes the form of unifacial thinning on 3 and concave bevel on 1. Lateral stem grinding is absent.

Material Attributes: Raw material is local $(n=2)$ and nonlocal $(n=1)$ Edwards fine-grained chert, and 1 is indeterminate. Heat damage is slight on 1 and extensive on 1 . No patina or mineral precipitates are present.

Tool State and Fractures: All lack either distal sections and/or barb tips, with 1 evidencing facial impact damage, 1 , thermal damage, and 2, bend fracture scars.

Rejuvenation: One exhibits alternate beveling, 1 unifacial beveling, 1 is indeterminate, and 1 is not resharpened.

Stratigraphic Distribution: All are from Valley Floor A, 3 from Unit IIIc and 1 from Unit IIIb/c.

$$
\operatorname{Ensor}(N=37)
$$

Morphology: These are generally well-made triangular points of varying sizes with typically small shoulders and barbs. Salient characteristics are shallow to moderately deep side notching and a typically wide distal neck. Bases most 


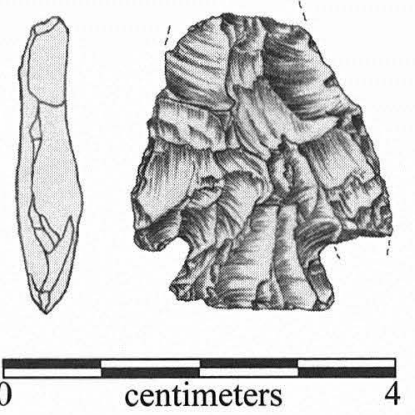

FigURE 13-83. Edgewood point, Specimen 13A-1.

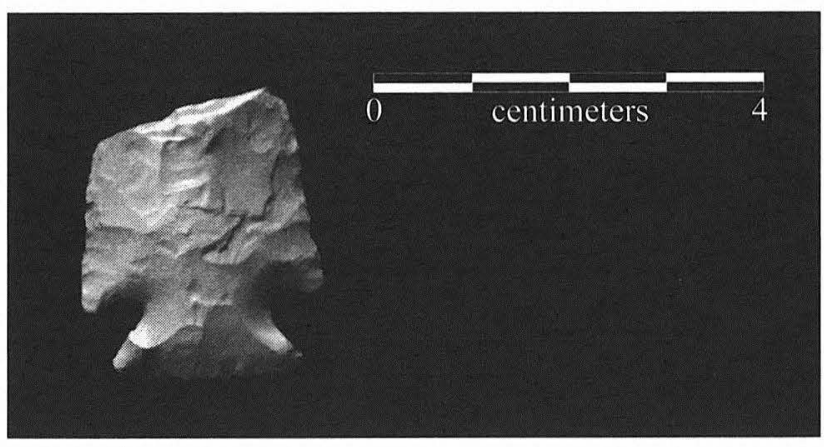

FIGURE 13-84. Edgewood point, Specimen 36D-18. coded as heavy partial on 1; heat damage is slight on 2 and extensive on 4 . No mineral precipitates are present.

Tool State and Fractures: Four are complete and 15 others nearly so, lacking only distal tips, barb tips, and/or basal ears; on these, single bend fractures were recorded on 2 , multiple bend fractures on 6 , multiple bend and burin-like fractures on 6 , and thermal spalling on 1 ; the remaining 18 are proximal and stem sections, evidencing single bend breaks $(n=6)$, multiple bend break and burin-like or facial impact breaks $(n=3)$, multiple bend and thermal breaks $(n=4)$, multiple bend and hinge breaks $(n=3)$, and indeterminate breaks $(n=2)$.

Rejuvenation: One is resharpened unifacially, beveled, 13 alternately beveled, 9 bifacially unbeveled, 10 are indeterminate, and 4 are not resharpened.

Stratigraphic Distribution: Ensor points are restricted to Unit IIIc (Table 13-89).

\section{Ensor/Fairland $(N=2)$}

Morphology: These points share attributes of both Ensor and Fairland points, namely, side notching and wide expanding bases; the shallow basal concavities, however, are unlike the more straight-edged Ensor bases, and somewhat

TABLE $13-87$

Summary Metric Data for Edgewood Points (in $\mathrm{mm}$ )

\begin{tabular}{|c|c|c|c|c|c|}
\hline & Number & Minimum & Maximum & Mean & $1-\sigma$ \\
\hline Length & 0 & & & & \\
\hline Width & 4 & 19.0 & 25.0 & 22.8 & 2.9 \\
\hline Thickness & 4 & 5.0 & 7.0 & 5.8 & 1.0 \\
\hline Stem length & 4 & 7.0 & 10.0 & 8.5 & 1.3 \\
\hline Proximal stem width & 4 & 14.0 & 20.0 & 17.5 & 2.6 \\
\hline Distal stem width & 4 & 12.0 & 13.0 & 12.8 & 0.5 \\
\hline Basal concavity depth & 2 & 1.0 & 2.0 & 1.5 & 0.7 \\
\hline Basal convexity length & 2 & 2.0 & 2.0 & 2.0 & 0.0 \\
\hline
\end{tabular}

commonly are straight and wide, often mirroring the width of the shoulders (Figures 13-85 and 13-86). Flaking is finely executed on some.

Metric Averages: Table 13-88

Nonmetric Attributes: Longitudinal profiles include 7 with a tapered base and tip, 9 flat, 12 with wedge-shaped base and tip, and 9 indeterminate. Flake patterns are organized on 17 (subparallel, oblique subparallel, chevron), random on 11, and indeterminate on 9. Basal modification constitutes unifacial thinning on 11 , bifacial thinning on 17 , thinning and fluting on 1 , secondary trimming on 6 , and 2 unmodified. Lateral stem grinding is absent. Basal edges of 8 are smoothed.

Material Attributes: Raw material is local (25 fine grained, 3 medium/coarse) and nonlocal $(n=3)$ Edwards fine-grained chert, and indeterminate and/or heat altered $(n=6)$; patina was
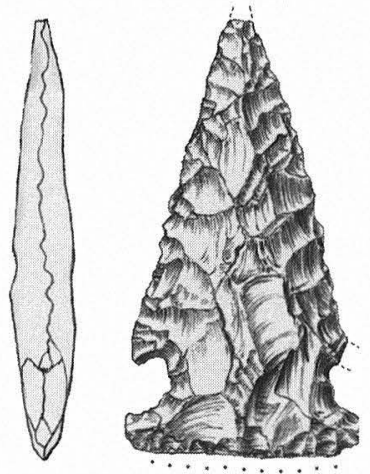

a

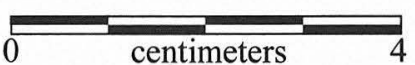

FIGURE 13-85. Ensor points. Specimen numbers: (a) 10B-7; (b) 12E-2. 


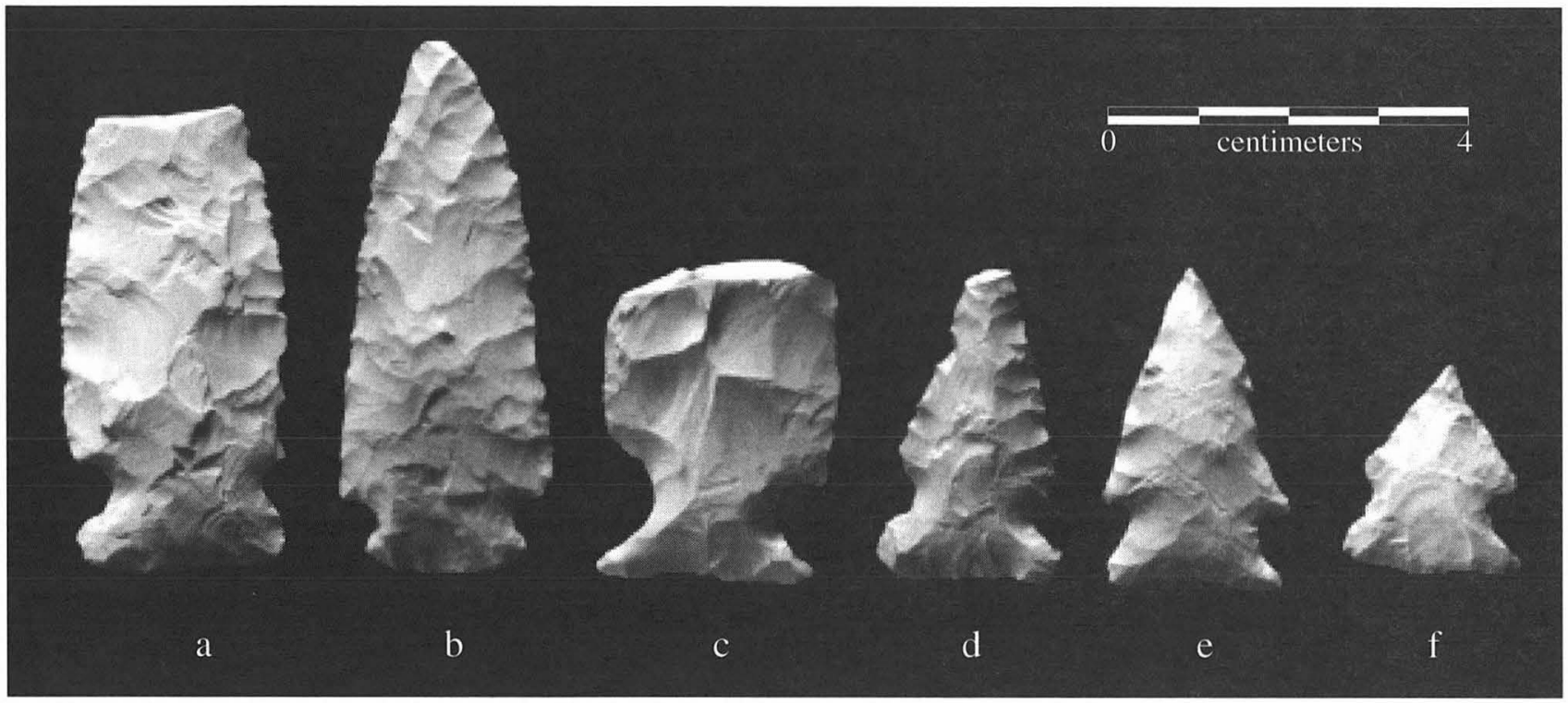

FIGURE 13-86. Ensor points. Specimen numbers: (a) 9C-3; (b) 2A-2; (c) 9D-1; (d) 202-3; (e) 11B-3; (f) $36 \mathrm{D}-2$.

TABLE $13-88$

Summary Metric Data for Ensor Points (in mm)

\begin{tabular}{|c|c|c|c|c|c|}
\hline & Number & Minimum & Maximum & Mean & $1-\sigma$ \\
\hline Length & 10 & 23.0 & 58.0 & 42.2 & 9.1 \\
\hline Width & 33 & 18.0 & 28.0 & 23.5 & 2.4 \\
\hline Thickness & 37 & 5.0 & 10.0 & 6.3 & 1.2 \\
\hline Stem length & 35 & 8.0 & 15.0 & 10.0 & 1.5 \\
\hline Proximal stem width & 20 & 18.0 & 25.0 & 21.2 & 2.0 \\
\hline Distal stem width & 37 & 11.0 & 20.0 & 14.9 & 2.1 \\
\hline Basal concavity depth & 19 & 1.0 & 3.0 & 1.4 & 0.7 \\
\hline Basal convexity length & 7 & 1.0 & 3.0 & 1.7 & 0.8 \\
\hline
\end{tabular}

TABLE 13-89

Stratigraphic Distribution of Ensor Points

\begin{tabular}{l|c|c}
\hline Unit & Valley Floor A & Valley Margin \\
\hline IIIc & 17 & 13 \\
Other & 1 & \\
surface & & \\
IIIc/pot \\
provenience unknown
\end{tabular}

unlike the "classic" Fairland base with downward pointing basal corners (Figure 13-87).

Metric Averages: Table 13-90

Nonmetric Attributes: Longitudinal profile on 1 is wedge-shaped base and tip, the other is indeterminate. Flake patterns are organized on 1 (oblique subparallel) and indeterminate on 1. Basal modification constitutes bifacial thinning on 1 and is indeterminate on 1. Lateral stem grinding is absent.

Material Attributes: Raw material is local Edwards fine

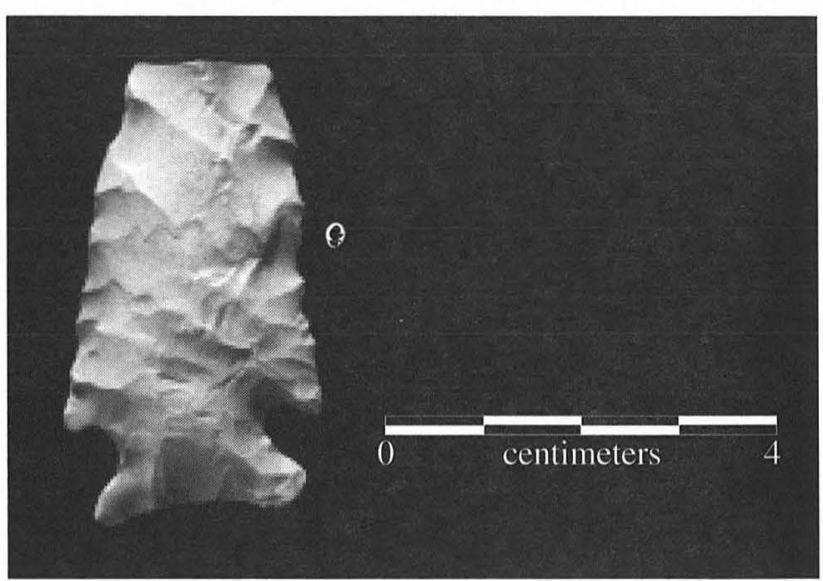

FIGURE 13-87. Ensor/Fairland point, Specimen 7A-10.

grained for 1 and indeterminate heat altered on 1 . Heat damage is extensive on 1 . No mineral precipitates or patina are present.

Tool State and Fractures: One lacks a distal tip due to a 
TABLE 13-90

Metric Measurements for Ensor/Fairland Points (in mm)

\begin{tabular}{l|c|c}
\hline Variable & $\begin{array}{c}\text { Specimen } \\
7 \mathrm{~A}-10\end{array}$ & $\begin{array}{c}\text { Specimen } \\
\text { TP3C-8 }\end{array}$ \\
\hline Width & 26.0 & \\
Thickness & 7.0 & 6.0 \\
Stem length & 10.0 & \\
Proximal stem width & 22.0 & \\
Distal stem width & 16.0 & 14.0 \\
Basal concavity depth & 2.0 & \\
\hline \hline
\end{tabular}

bend break; the other is a proximal section exhibiting bend and thermal fracture scars.

Rejuvenation: One displays unbeveled bifacial resharpening.

Stratigraphic Distribution: One of these specimens is from disturbed context (Unit IIIc/pot) in the Valley Margin, and the other is from an unknown provenience in Valley Floor A.

\section{$\operatorname{Marcos}(N=23)$}

Morphology: This group is composed of medium- to large-sized, barbed triangular points with wide expanding stems formed by deep corner notching (Figures 13-88 and 13-89). Bases are typically straight to gently convex.

Metric Averages: Table 13-91
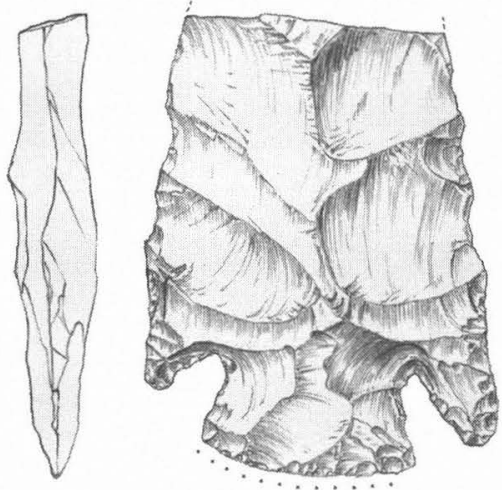

a
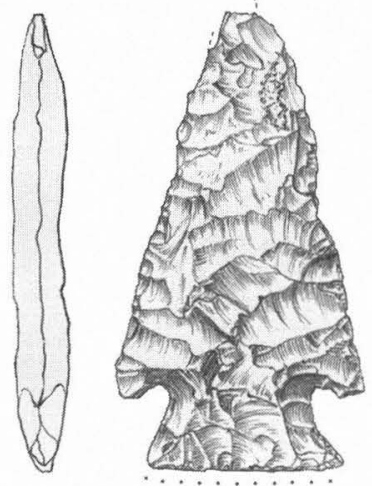

$\mathrm{b}$

FIGURE 13-88. Marcos points. Specimen numbers: (a) 36E-2; (b) 23F-1.
Nonmetric Attributes: Longitudinal profiles include 2 with tapered base and tip; 1 thick tip; 1 thick base; 4 flat; 4 wedge-shaped base and tip; and 11 indeterminate. Flake patterns are organized on 8 (oblique subparallel, subparallel, collateral), random on 7 , and indeterminate on 8 . Basal modification includes unifacial thinning on 10, bifacial thinning on 4 , secondary trimming on 7 , absent on 1 , and 1 indeterminate. Lateral stem grinding is absent. Basal edges of 6 are smoothed.

Material Attributes: Raw material is local Edwards chert (14 fine grained, 1 medium/coarse); nonlocal Edwards chert (4 fine grained, 3 medium/coarse), and 1 indeterminate heat altered. Patina is heavy overall on 1 . Mineral precipitates are present on 2. Heat damage is slight on 2, moderate on 1 , and extensive on 1.

Tool State and Fractures: Nine are nearly complete, lacking only a distal tip in 2 cases, barb(s) in 2 cases, barb(s) and distal tip on 3, and basal corner(s) and barb on 2. An additional 11 are proximal sections, with one or both barbs missing. The remaining 3 are largely stems. Single bend breaks were recorded on 4 , impact on 2 , multiple bend on 5 , multiple bend and burin-like impact on 9 , and burin-like or bend and thermal on 3 .

Rejuvenation: One is resharpened unifacially, unbeveled; 3 bifacially, unbeveled; 5 alternately beveled; 1 absent; and 13 indeterminate.

Stratigraphic Distribution: Highest frequency of Marcos is in Unit IIIc (Table 13-92).

Comments: As has been noted elsewhere, there is some typological confusion between Marcos and Castroville. The basal notching of Castroville - as opposed to the corner notching of Marcos - is typically held to be a distinguishing characteristic between the two; the difficulty remains, however, when the notching is not clearly one or the other (Turner and Hester 1993:147).

\section{Marcos/Marshall $(N=1)$}

Morphology: This small, triangular-bladed point with an expanding stem from which basal ears have been severed could belong to either group.

Metric Values: Length is $49 \mathrm{~mm}$; width, incomplete; thickness, $5 \mathrm{~mm}$; stem length, $8 \mathrm{~mm}$; proximal stem width, 17 $\mathrm{mm}$; and distal stem width, $14 \mathrm{~mm}$.

Nonmetric Attributes: Longitudinal profile is flat; flake pattern is random; basal modification consists of secondary trimming.

Material Attributes: Raw material is local Edwards finegrained chert; heat damage is indeterminate. No patina or mineral precipitates were present.

Tool State and Fractures: This point is complete except for barb and basal ears, broken by multiple bend and possible thermal fractures.

Rejuvenation: Point is resharpened bifacially, unbeveled. 


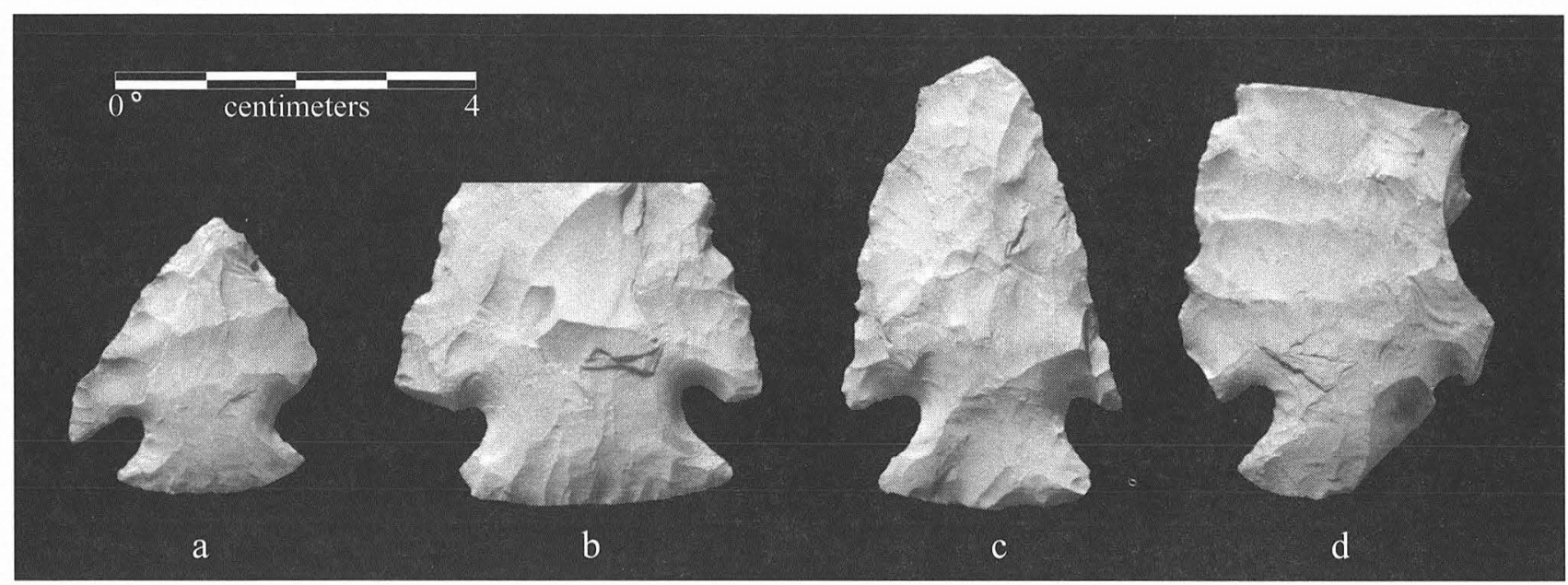

FIGURE 13-89. Marcos points. Specimen numbers: (a) 2350; (b) 11C-1; (c) 9G-1; (d) 15I2-1.

TABLE 13-91

Summary Metric Data for Marcos Points (in mm)

\begin{tabular}{l|c|c|c|c|c}
\hline & Number & Minimum & Maximum & Mean & $1-\sigma$ \\
\hline Length & 6 & 31.0 & 88.0 & 52.7 & 21.7 \\
Width & 12 & 25.0 & 39.0 & 31.3 & 6.3 \\
Thickness & 23 & 5.0 & 10.0 & 6.7 & 1.2 \\
Stem length & 23 & 9.0 & 15.0 & 31.3 & 1.6 \\
Proximal stem width & 18 & 18.0 & 22.0 & 17.6 & 3.6 \\
Distal stem width & 22 & 13.0 & 4.0 & 1.8 & 1.5 \\
Basal concavity depth & 4 & 1.0 & 4.0 & 2.4 & 0.9 \\
Basal convexity length & 14 & 1.0 & & \\
\hline \hline
\end{tabular}

TABLE 13-92

Stratigraphic Distribution of Marcos Points

\begin{tabular}{l|c|c}
\hline Unit & Valley Floor A & Valley Margin \\
\hline IIIc & 7 & 5 \\
IIIb/c & 2 & 1 \\
Y/IIIc & 1 & 2 \\
IIIb & & \\
Other & 1 & \\
surface & & \\
provenience unknown & 4 & \\
\hline \hline
\end{tabular}

Stratigraphic Distribution: Unit IIIc, Valley Floor A.

\section{Marshall $(\mathrm{N}=8)$}

Morphology: These are small- to medium-sized triangular points, commonly exhibiting long barbs. Stems are short and gently expanding with straight to slightly concave base (Figures 13-90 and 13-91).

Metric Averages: Table 13-93

Nonmetric Attributes: Longitudinal profiles include 2

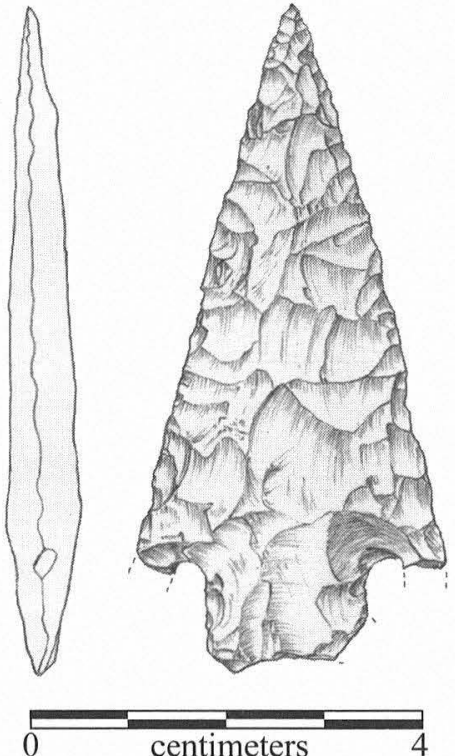

Figure 13-90. Marshall point, Specimen 36F-3.

with tapered base and tip, 1 wedge-shaped base and tip, and 5 indeterminate. Flake patterns are organized on 2 (subparallel, oblique subparallel), random on 3 , and indeterminate on 3 . 


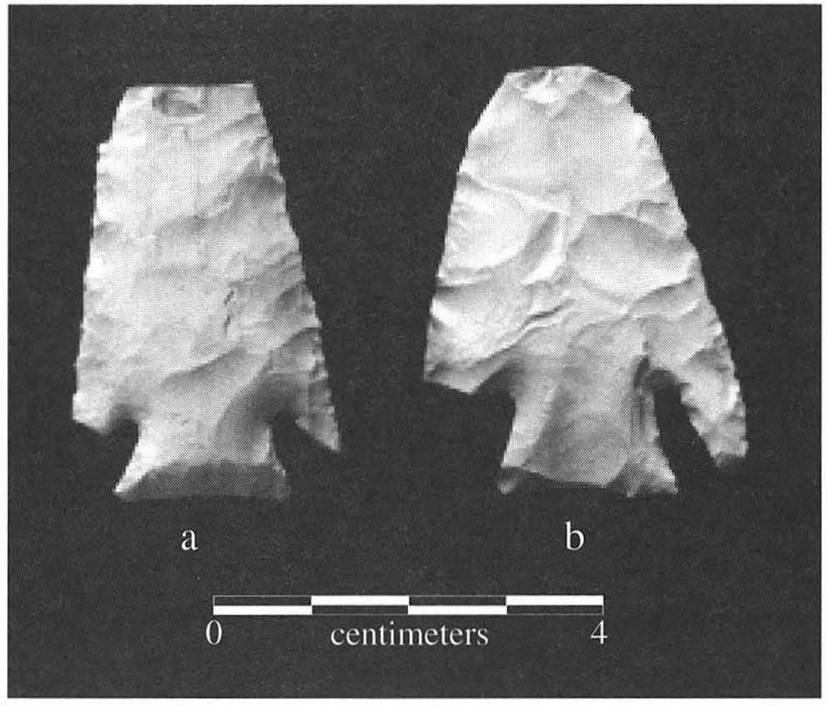

FIGURE 13-91. Marshall points. Specimen numbers: (a) 480-2; (b) 463-1.

\section{Williams $(N=4)$}

Morphology: These rather crude, broad-bladed points have small barbs and slightly expanding, often bulbous stems with convex bases (Figures 13-92 and 13-93).

Metric Averages: Table 13-95

Nonmetric Attributes: Longitudinal profiles include 1 tapered base and tip; 2 flat; and 1 indeterminate. Flake patterns are organized on 2 (subparallel, collateral), random on 1 , and indeterminate on 1. Basal modification consists of unifacial thinning on 2, and secondary trimming on 1 , and it is absent on 1. Lateral stem margins are not ground.

Material Attributes: Raw material is local Edwards chert (1 fine grained, 1 medium/coarse) and nonlocal Edwards chert (1 fine grained, 1 medium/coarse). No patina, mineral precipitates, or heat damage are present.

Tool State and Fractures: Three are largely complete except for distal, barb, and/or basal ear tips, due to multiple bend and burin-like/impact fractures. The other is a

TABLE 13-93

Summary Metric Data for Marshall Points (in mm)

\begin{tabular}{|c|c|c|c|c|c|}
\hline & Number & Minimum & Maximum & Mean & $1-\sigma$ \\
\hline Length & 1 & 66.0 & 66.0 & & \\
\hline Width & 6 & 26.0 & 31.0 & 28.0 & 1.8 \\
\hline Thickness & 7 & 5.0 & 8.0 & 6.4 & 1.1 \\
\hline Stem length & 8 & 8.0 & 12.0 & 10.0 & 1.5 \\
\hline Proximal stem width & 5 & 15.0 & 21.0 & 17.6 & 2.8 \\
\hline Distal stem width & 8 & 11.0 & 17.0 & 14.4 & 2.2 \\
\hline Basal concavity depth & 5 & 1.0 & 3.0 & 1.4 & 0.9 \\
\hline Basal convexity length & 1 & 2.0 & 2.0 & & \\
\hline
\end{tabular}

Basal modification consists of unifacial thinning on 1, bifacial thinning on 4 , beveling on 1 , and secondary trimming on 2. Lateral stem grinding is absent.

Material Attributes: Raw material is local Edwards chert (6 fine grained, 2 medium/coarse). Patina is moderate overall on 1. Mineral precipitates are present on 1 . Heat damage is moderate on 1 .

Tool State and Fractures: Two are nearly complete, lacking only distal tip, in one case, and basal ear and barb tips in the other, due to bend breaks. The other 6 are proximal sections, 5 of which have at least 1 barb intact. Of these, 1 exhibits a single bend fracture; 2 , multiple bend and burin-like/impact fractures; 2, multiple bend fractures; and 1, multiple bend and thermal fractures.

Rejuvenation: Two are resharpened bifacially, unbeveled; 1 is alternately beveled; 1 is beveled unifacially; 1 is not resharpened, and 3 are indeterminate.

Stratigraphic Distribution: Table 13-94.
TABLE 13-94

Stratigraphic Distribution of Marshall Points

\begin{tabular}{l|c|c}
\hline Unit & Valley Floor A & Valley Margin \\
\hline IIIc & 3 & 1 \\
IIIb/c & 2 & 1 \\
Y/IIIb/c & & \\
Other & & 1 \\
IIIc/pot & & \\
\hline \hline
\end{tabular}

proximal section, broken by bend and burin-like fractures.

Rejuvenation: Two are alternately beveled, 1 is indeterminate, and 1 is not resharpened.

Stratigraphic Distribution: Williams points are present in later contexts, the Unit IIIb/c transition zone $(n=3)$, and Unit IIIC ( $\mathrm{n}=1)$.

\section{Lange $(N=4)$}

Morphology: These are crude, somewhat nondescript, triangular points with strong shoulders, small barbs, and a 


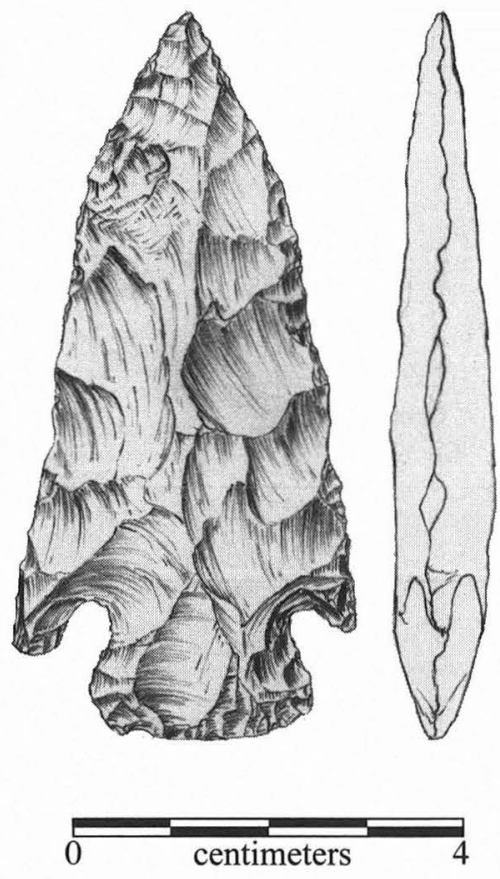

Figure 13-92. Williams point, Specimen 14D-1.

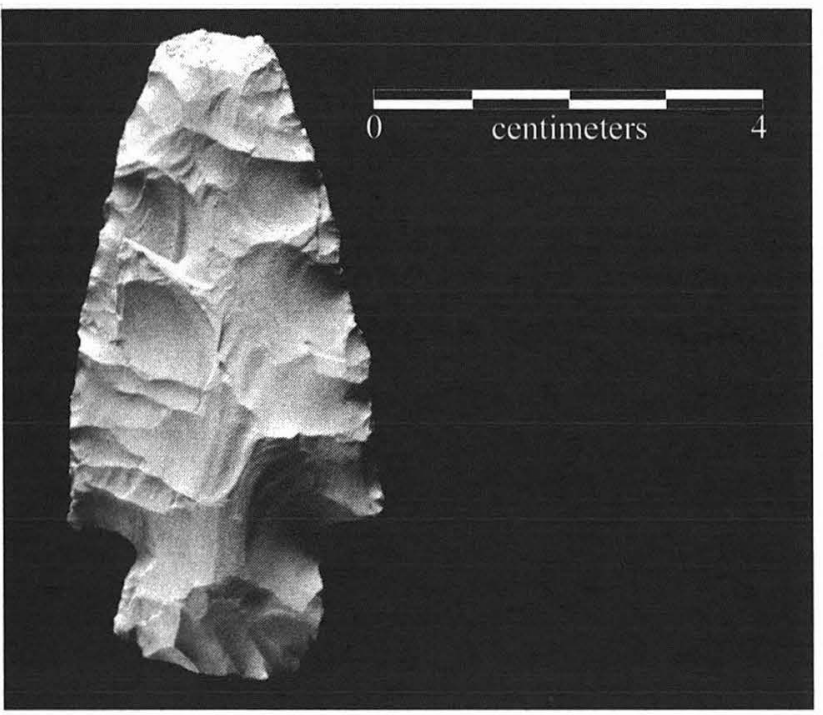

FIGURE 13-93. Williams point, Specimen 480-1. gently expanding stems with straight bases (Figures 13-94 and 13-95).

Metric Averages: Table 13-96

Nonmetric Attributes: Longitudinal profiles include 1 tapered base and tip; 2 wedge-shaped base and tip; and 1 indeterminate. Flake patterns on all are random. Basal modification consists of bifacial thinning on 1, secondary trimming on 2, and beveled base on 1. Lateral stem grinding is slight on 2 .

Material Attributes: Raw material is local Edwards finegrained chert for 3 and nonlocal Edwards fine-grained chert for 1 . Mineral precipitates are present on 2 . No heat damage or patina is present.

Tool State and Fractures: One is nearly complete, lacking only a distal tip due to impact fracture. The other 3 are proximal/ medial sections, each broken by bending fractures.

Rejuvenation: Three are resharpened bifacially, unbeveled, and 1 is alternately beveled.

Stratigraphic Distribution: Table 13-97.

\section{Expanding Stem $A(N=6)$}

Morphology: This is a somewhat miscellaneous group comprising chiefly stem sections, which are short and expanding with small- to medium-width, straight bases; stems are frequently ground on lateral edges. The two specimens complete enough to characterize have been heavily resharpened and one is ground on all basal margins (Figures 13-96 and 13-97a). The latter exhibits a thick, beveled basal edge.

Metric Averages: Table 13-98

Nonmetric Attributes: Longitudinal profiles include 1 tapered base and tip and 5 indeterminate. Flake patterns are organized on 1 (subparallel) and indeterminate on 5. Basal modification is 2 unifacial thinning, 1 beveled, and 4 secondary trimming. Lateral stem grinding is slight on 2 , moderate on 2 , and heavy on 1 .

Material Attributes: Raw material is local Edwards chert for 5 ( 3 fine grained, and 2 medium/coarse) and 1 indeterminate. Heat damage is slight on 1 . No mineral precipitates or patina are present.

Tool State and Fractures: One is complete. The others are small proximal and stem sections, 3 of which display a

TABLE 13-95

Summary Metric Data for Williams Points (in mm)

\begin{tabular}{l|c|c|c|c|c}
\hline & Number & Minimum & Maximum & Mean & $1-\sigma$ \\
\hline Length & 3 & 50.0 & 74.0 & 63.0 & 12.1 \\
Width & 2 & 32.0 & 34.0 & 33.0 & 1.4 \\
Thickness & 4 & 6.0 & 10.0 & 8.0 & 1.8 \\
Stem length & 4 & 13.0 & 13.0 & 13.0 & 0.0 \\
Proximal stem width & 4 & 21.0 & 23.0 & 21.8 & 1.0 \\
Distal stem width & 4 & 18.0 & 19.0 & 18.5 & 0.6 \\
Basal convexity length & 4 & 3.0 & 4.0 & 3.5 & 0.6 \\
\hline \hline
\end{tabular}




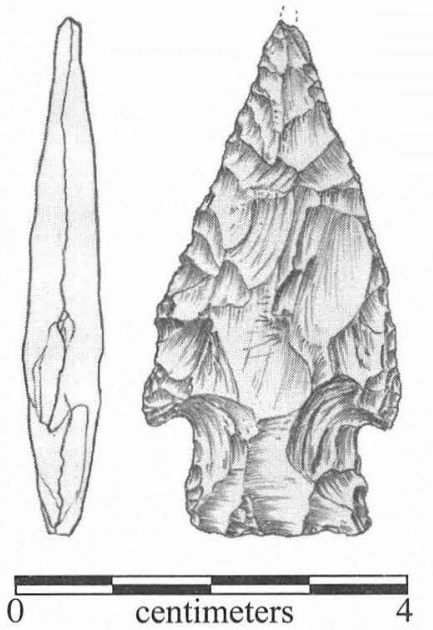

FIGURE 13-94. Lange point, Specimen 0-88.

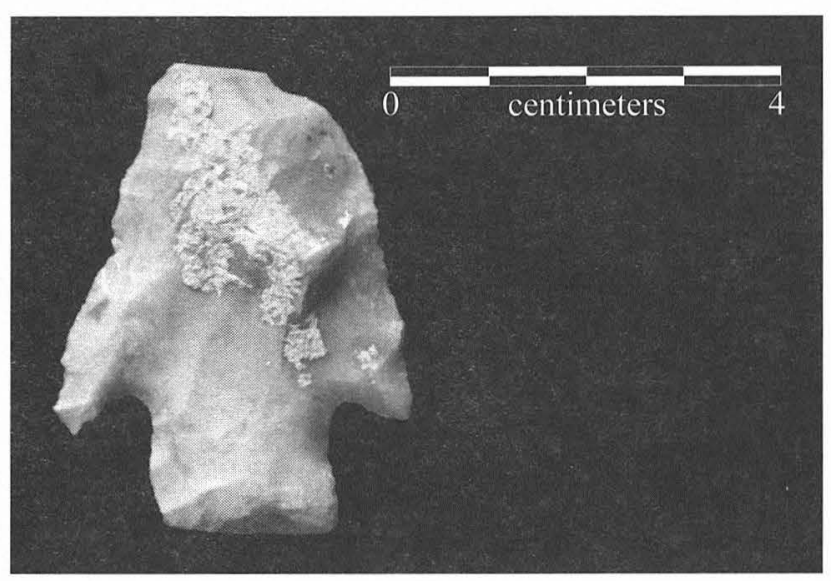

FIGURE 13-95. Lange point, Specimen 5G-1.
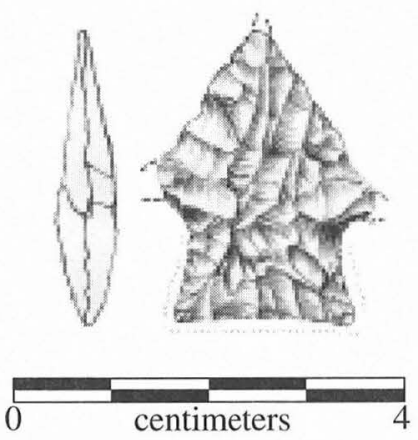

FIGURE 13-96. Expanding stem A point, Specimen 0-59.

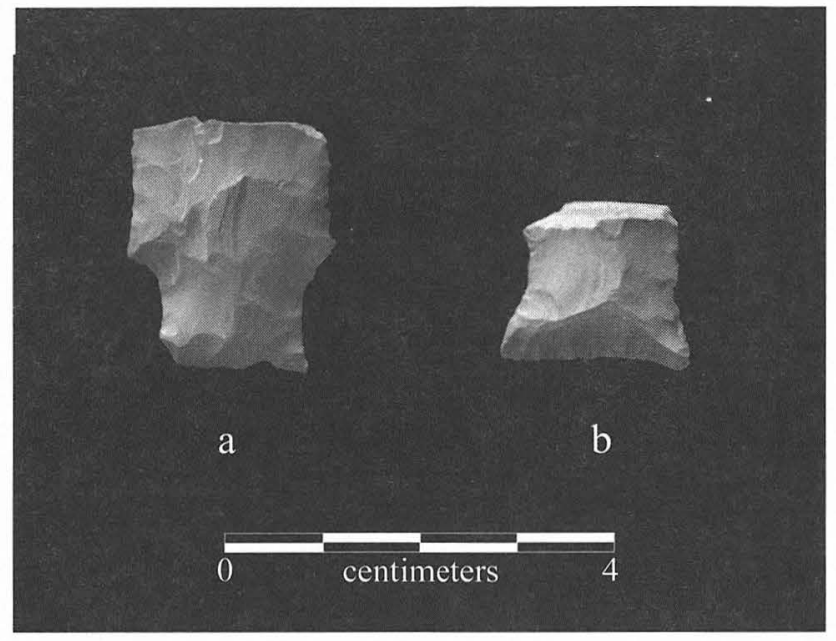

FIGURE 13-97. Expanding stem A points. Specimen numbers: (a) 596-1; (b) 652-3.

TABLE 13-96

Summary Metric Data for Lange Points (in mm)

\begin{tabular}{|c|c|c|c|c|c|}
\hline & Number & Minimum & Maximum & Mean & $1-\sigma$ \\
\hline Length & 1 & 54.0 & 54.0 & & \\
\hline Width & 4 & 27.0 & 35.0 & 31.8 & 3.4 \\
\hline Thickness & 4 & 7.0 & 15.0 & 9.3 & 3.9 \\
\hline Stem length & 4 & 11.0 & 17.0 & 13.0 & 2.8 \\
\hline Proximal stem width & 4 & 17.0 & 20.0 & 18.3 & 1.5 \\
\hline Distal stem width & 4 & 17.0 & 18.0 & 17.8 & 0.5 \\
\hline Basal convexity length & 1 & 1.0 & 1.0 & & \\
\hline
\end{tabular}

TABLE 13-97

Stratigraphic Distribution of Lange Points

\begin{tabular}{l|c|c|c}
\hline Unit & Valley Floor A & Valley Floor B & Valley Margin \\
\hline IIIc & 1 & 1 & 1 \\
IIIb & 1 & & \\
Other & & & \\
surface & 1 & & \\
\hline \hline
\end{tabular}

single bend break, and 2, multiple bend and burin-like fractures.

Rejuvenation: One is resharpened bifacially, unbeveled; 4 are indeterminate.

Stratigraphic Distribution: Table 13-99.

Comments: The specimen drawn (see Figure 13-96) is from the surface; its thinness $(5 \mathrm{~mm})$ and rather fine flaking (although heavily resharpened) suggest an affiliation with Bandy. 
TABLE 13-98

Summary Metric Data for Expanding Stem A (in mm)

\begin{tabular}{l|c|c|c|c|c}
\hline & Number & Minimum & Maximum & Mean & $1-\sigma$ \\
\hline Length & 1 & 30.0 & 30.0 & & \\
Width & 3 & 19.4 & 22.0 & 20.8 & 6.8 \\
Thickness & 5 & 5.0 & 9.0 & 1.3 & 1.5 \\
Stem length & 2 & 11.0 & 13.0 & 2.0 & 1.4 \\
Proximal stem width & 5 & 19.0 & 16.0 & 15.7 & 0.4 \\
Distal stem width & 3 & 15.0 & 1.1 & 1.0 & 0.6 \\
Basal concavity depth & 4 & 1.0 & & 0.0 \\
\hline \hline
\end{tabular}

TABLE 13-99

Stratigraphic Distribution of Expanding Stem A Points

\begin{tabular}{l|c}
\hline Unit & Valley Floor A \\
\hline IIIb/c & 3 \\
IIIb & 1 \\
IIIa/b & 1 \\
Other & \\
surface & 1 \\
\hline \hline
\end{tabular}

Expanding Stem B $(N=4)$

Morphology: Not intended as a coherent group, these points are of varying sizes with medium to wide, often thick expanding stems with straight basal edges. The specimen shown in Figure 13-98 exhibits strong shoulders with angular barb remnants and is alternately beveled along blade edges. Sections of the original flake blank are retained on both faces. The stem is wide, expanding, and only retouched rather than thinned on the basal edge; lateral stem margins are not ground.

Metric Averages: Table 13-100.

Nonmetric Attributes: Longitudinal profiles include tapered base and tip on 1, wedge-shaped base and tip on 1, and 2 indeterminate. Flake patterns are organized on 3 (oblique subparallel, subparallel, chevron) and random on 1. Basal modification consists of unifacial thinning on 1, bifacial thinning on 2, and secondary trimming on 1 . Lateral stem grinding is slight on 1, moderate on 1 .

Material Attributes: Raw material is local fine-grained Edwards chert. Mineral precipitates are present on 1. No patina or heat damage was observed.

Tool State and Fractures: Two are largely complete; 1 lacks a distal tip due to impact fracturing, the other, a shoulder and barb due to bend and burin-like impact damage. The 2 others are proximal sections displaying a single bend fracture in one case, and multiple bend and burin-like/impact damage in the others.

Rejuvenation: One is resharpened bifacially, unbeveled; 2 are alternately beveled; and 1 is indeterminate.

Stratigraphic Distribution: Table 13-101

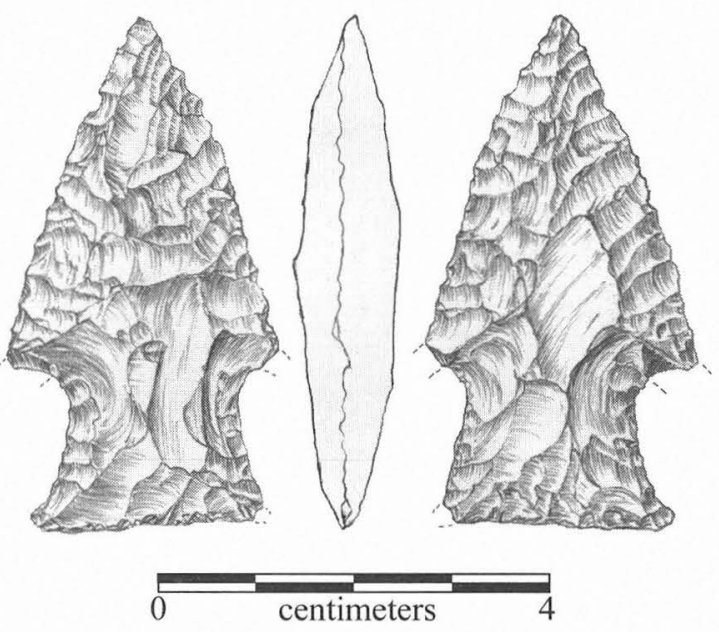

FIGURE 13-98. Expanding stem B point, Specimen 50MA-1.

Comments: Specimens in this group are problematic; some resemblance is seen to Marcos or Lange, which would be congruent with the contexts for some in this group. The point shown (see Figure 13-98), however, derives from relatively deep, although poorly defined geologic contexts (Level 13, Valley Floor B, undifferentiated Unit III). Although it lacks the characteristic stem grinding, it may belong to the Wilson type. Kay's analysis of this specimen for use-wear evidence suggests it was recycled from projectile point to knife (see Chapter 22).

\section{Expanding Stem $C(N=7)$}

Morphology: These small to medium-sized points have elongate triangular blades and short, expanding, rather bulbous stems with straight to convex basal edges (Figures 1399 and 13-100).

Metric Averages: Table 13-102

Nonmetric Attributes: Longitudinal profiles include wedge-shaped base and tip on 4 and 3 indeterminate; flake patterns are organized on 4 (oblique subparallel, subparallel), random on 2, and indeterminate on 1; basal modification constitutes unifacial thinning on 2, beveling on 3, and secondary trimming on 1 , and it is absent on 1; lateral stem grinding is slight on 1 . 
TABLE 13-100

Summary Metric Data for Expanding Stem B Points

\begin{tabular}{l|c|c|c|c|c}
\hline & Number & Minimum & Maximum & Mean & $1-\sigma$ \\
\hline Length & 2 & 36.0 & 52.0 & 44.0 & 11.3 \\
Width & 4 & 22.0 & 31.0 & 26.3 & 4.0 \\
Thickness & 4 & 8.0 & 10.0 & 16.0 & 1.2 \\
Stem length & 4 & 10.0 & 16.0 & 20.8 & 2.9 \\
Proximal stem width & 4 & 18.0 & 22.0 & 18.3 & 1.9 \\
Distal stem width & 4 & 16.0 & 21.0 & 1.0 & 2.1 \\
Basal concavity depth & 2 & 1.0 & 1.0 & & 0.0 \\
\hline \hline
\end{tabular}

TABLE 13-101

Stratigraphic Distribution of Expanding Stem B Points

\begin{tabular}{l|c|c|c}
\hline Unit & Valley Floor A & Valley Floor B & Valley Margin \\
\hline IIIc & 1 & & 1 \\
Y/IIIc & & 1 & \\
III & & & 1 \\
Other & & & \\
IIIc/pot & & & \\
\hline
\end{tabular}

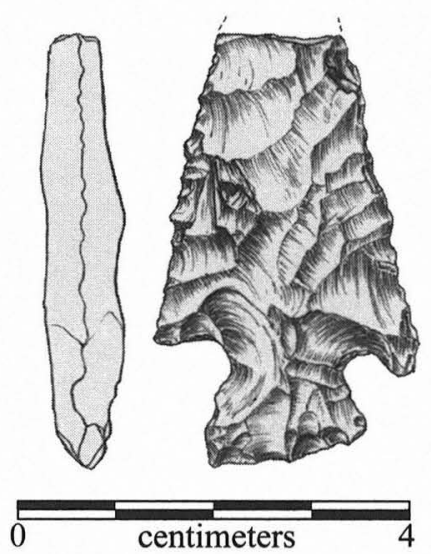

FIgURE 13-99. Expanding stem C point, Specimen 7J-1.

Material Attributes: Raw material is local Edwards chert (4 fine grained, 1 medium/coarse), nonlocal Edwards medium/coarse-grained chert for 1 , and 1 indeterminate finegrained chert; mineral precipitates are present on 3; heat damage is slight on 1 .

Tool State and Fractures: One is complete, and the 6 others are proximal or stem sections, 1 of which exhibits a single bend scar, 4 , multiple bend, burin-like, and/or hinge fractures, and 1, multiple bend and thermal fractures.

Rejuvenation: One is resharpened unifacially, unbeveled; 2 , bifacially, unbeveled; 1 , alternately beveled; 1 indeterminate; and 1 absent.

Stratigraphic Distribution: Table 13-103.

Comments: This group resembles Godley, typically distributed in east-central Texas (see Kyle site; Jelks 1962:Figure $16 \mathrm{~b}-\mathrm{e})$ as well as the widely distributed but poorly defined Palmillas type of the Middle to Late Archaic (Turner and Hester
1993:167). At Wilson-Leonard, there appears to be a stratigraphic clustering of the group in mid Unit III which may pertain roughly to the Early/Middle Archaic.

$$
\text { Expanding Stem } D(N=6)
$$

Morphology: This group of medium- to large-sized points have moderately strong, generally barbless shoulders and convex blade edges. Stems are medium to long and slightly expanding, with straight to slightly concave basal edges, some of which are slightly canted on end (Figure 13-101). Stem lateral edges may be ground.

Metric Averages: Table 13-104

Nonmetric Attributes: Longitudinal profiles include 1 tapered base and tip, 1 thick tip, 1 thick base, 2 wedge-shaped base and tip, and 1 indeterminate. Flake patterns are organized on 1 (collateral/subparallel) and random on 5 . Basal modification consists of unifacial thinning on 2 , bifacial thinning on 1 , beveled end on 1 , and concave bevel on 2. Lateral stem grinding is slight on 1 , and heavy on 3 .

Material Attributes: Raw material is local Edwards finegrained chert for 3; nonlocal Edwards chert (1 fine grained, 1 medium/coarse), and 1 indeterminate. Mineral precipitates are present on 1. Heat damage is slight on 1. No patina is present.

Tool State and Fractures: Four are complete or largely so; of these, 1 exhibits a reworked facial impact burin scar. The 2 others each lack distal sections and basal ears due to multiple impact fractures.

Rejuvenation: One is resharpened bifacially, unbeveled, and 4 are alternately beveled.

Stratigraphic Distribution: This group is widely scattered over Unit III (Table 13-105).

Comments: The long, slightly expanding stems and highly uniform, pointed tip blades of points in this group broadly suggest Nolan and Travis, found in similar stratigraphic contexts at Wilson-Leonard.

\section{Miscellaneous Expanding Stem ( $N=11)$}

Morphology: These are points with damaged, expanding stems which cannot be further classified. 


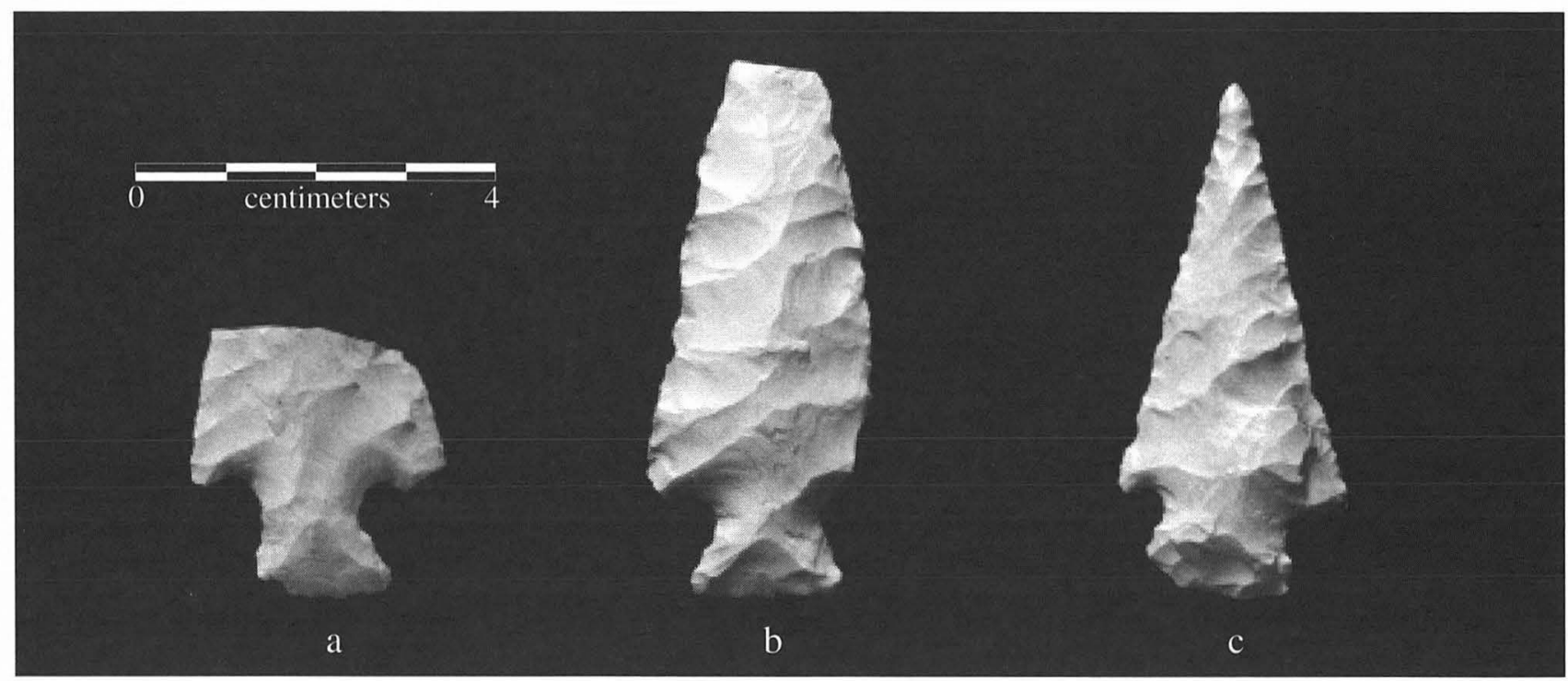

FIGURE 13-100. Expanding stem C points. Specimen numbers: (a) 12N-2; (b) 16M1-1; (c) 23I-1.

TABLE 13-102

Summary Metric Data for Expanding Stem C Points (in mm)

\begin{tabular}{|c|c|c|c|c|c|}
\hline & Number & Minimum & Maximum & Mean & $1-\sigma$ \\
\hline Length & 1 & 57.0 & 57.0 & & \\
\hline Width & 3 & 25.0 & 27.0 & 25.7 & 1.2 \\
\hline Thickness & 7 & 6.0 & 8.0 & 7.3 & 0.8 \\
\hline Stem grinding length & 6 & 11.0 & 13.0 & 11.8 & 1.0 \\
\hline Proximal stem width & 4 & 16.0 & 17.0 & 16.5 & 0.6 \\
\hline Distal stem width & 6 & 11.0 & 14.0 & 12.5 & 1.0 \\
\hline Basal convexity length & 5 & 1.0 & 3.0 & 1.6 & 0.9 \\
\hline Basal concavity width & 0 & & & & \\
\hline
\end{tabular}

TABLE 13-103

Stratigraphic Distribution of Expanding Stem C Points

\begin{tabular}{l|c|c}
\hline Unit & Valley Floor A & Valley Margin \\
\hline Y/IIIc & 3 & 1 \\
IIIb & 1 & 1 \\
X/Y & & \\
IIIa/b & & \\
Other & & \\
surface & 1 & \\
\hline \hline
\end{tabular}

Metric Averages: Table 13-106.

Nonmetric Attributes: Longitudinal profiles include tapered base and tip on 3, wedge-shaped base and tip on 4 , and 4 indeterminate. Flake patterns are organized on 3 (subparallel, oblique subparallel), random on 5, and indeterminate on 3. Basal modification is unifacial thinning on 2 , bifacial thinning on 1 , beveled stem on 2 , concave bevel on 1 , secondary trimming on 1, 3 indeterminate, and $1 \mathrm{ab}$ sent. Lateral stem grinding is slight on 1.

Material Attributes: Raw material is local Edwards

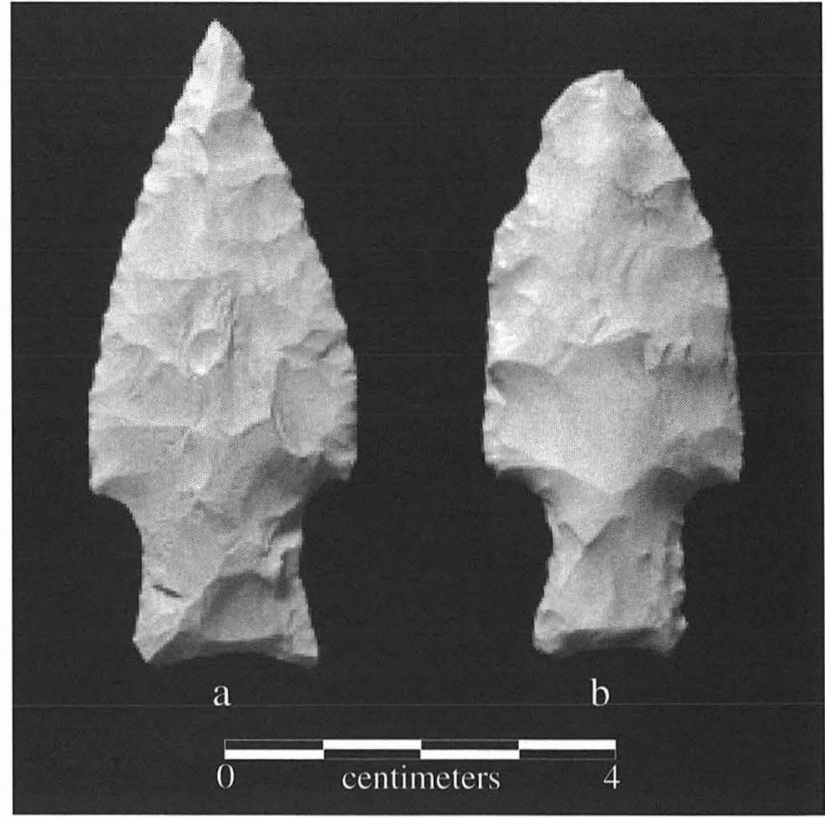

FIGURE 13-101. Expanding stem D points. Specimen numbers: (a) 4D-1; (b) $51 \mathrm{KD}-18$ 
TABLE 13-104

Summary Metric Data for Expanding Stem D Points

\begin{tabular}{|c|c|c|c|c|c|}
\hline & Number & Minimum & Maximum & Mean & $1-\sigma$ \\
\hline Length & 4 & 40.0 & 65.0 & 53.8 & 10.4 \\
\hline Width & 6 & 22.0 & 27.0 & 24.8 & 1.9 \\
\hline Thickness & 6 & 7.0 & 12.0 & 8.2 & 1.9 \\
\hline Stem length & 6 & 12.0 & 19.0 & 15.0 & 2.6 \\
\hline Proximal stem width & 6 & 16.0 & 19.0 & 17.0 & 1.3 \\
\hline Distal stem width & 6 & 14.0 & 17.0 & 15.7 & 1.2 \\
\hline Basal concavity depth & 5 & 1.0 & 2.0 & 1.3 & 0.4 \\
\hline Distal stem thickness & 1 & 7.0 & 7.0 & & \\
\hline Basal concavity width & 1 & 8.6 & 8.6 & & \\
\hline
\end{tabular}

TABLE 13-105

Stratigraphic Distribution of Expanding Stem D Points

\begin{tabular}{l|c|c|c}
\hline Unit & $\begin{array}{c}\text { Valley } \\
\text { Floor A }\end{array}$ & $\begin{array}{c}\text { Valley } \\
\text { Floor B }\end{array}$ & $\begin{array}{c}\text { Valley } \\
\text { Margin }\end{array}$ \\
\hline $\mathrm{IIIc}$ & 1 & 1 & 1 \\
$\mathrm{IIIb} / \mathrm{c}$ & 1 & & \\
$\mathrm{IIIb}$ & 1 & & \\
$\mathrm{III} / \mathrm{b}$ & & & \\
Other & 1 & & \\
provenience unknown & & & \\
\hline \hline
\end{tabular}

TABLE 13-106

Summary Metric Data for Miscellaneous

Expanding Stem Points (in mm)

\begin{tabular}{l|c|c|c}
\hline & Number & Minimum & Maximum \\
\hline Length & 2 & 36 & 97 \\
Width & 9 & 21 & 32 \\
Thickness & 11 & 6 & 9 \\
Stem length & 8 & 11 & 20 \\
Proximal stem width & 3 & 20 & 32 \\
Distal stem width & 7 & 11 & 20 \\
Basal concavity depth & 3 & 2 & 3 \\
\hline \hline
\end{tabular}

fine-grained chert for 10 and nonlocal Edwards medium/ coarse chert for 1 ; mineral precipitates are present on 2 . No heat damage or patina was observed.

Tool State and Fractures: Two are complete except for fractured stem sections due to multiple impact burination on 1 and bend break on the other. Five others lack basal, lateral, and/or distal sections due to multiple impact fractures (1 specimen), multiple bend and burin-like fractures ( 2 specimens), and bend and indeterminate breaks ( 2 specimens). Three are proximal sections displaying multiple bend and burin-like/indeterminate fractures. The remaining specimen is a very wide expanding base severed by a bend break.
Rejuvenation: Two are resharpened bifacially, unbeveled, 2 are alternately beveled, 1 is beveled unifacially, 1 is not resharpened, and 5 are indeterminate.

Stratigraphic Distribution: Table 13-107.

$$
\begin{gathered}
\text { Side-Notched Points } \\
\text { Big Sandy }(N=1)
\end{gathered}
$$

Morphology: Although it is smaller than average, this specimen conforms in all respects to the type. It has a moderately thick, side-notched stem with moderate basal concavity (Figure 13-102); the base is modified by secondary trimming; lateral stem edges and the basal edge are not ground.

Metric Values: Table 13-108

Nonmetric Attributes: Longitudinal profile is indeterminate; flake pattern is indeterminate; basal modification is secondary trimming; lateral grinding and basal grinding are absent.

Material Attributes: Raw material is indeterminate; patina is absent; mineral precipitates are absent; heat damage is absent.

Tool State and Fractures: This specimen has a transverse bend fracture on the blade.

Rejuvenation: Resharpening is indeterminate.

Stratigraphic Distribution: Valley Floor A, Unit II, elevation $95.53 \mathrm{~m}$.

TABLE 13-107

Stratigraphic Distribution of Miscellaneous Expanding Stem Points

\begin{tabular}{l|c|c}
\hline Unit & Valley Floor A & Valley Margin \\
\hline IIIc & 2 & 1 \\
Y/IIIc & 2 & 2 \\
IIIb & 1 & 1 \\
IIIa & & 1 \\
II/IIIa & & \\
Other & & 1 \\
IIIc/pot & & \\
\hline \hline
\end{tabular}



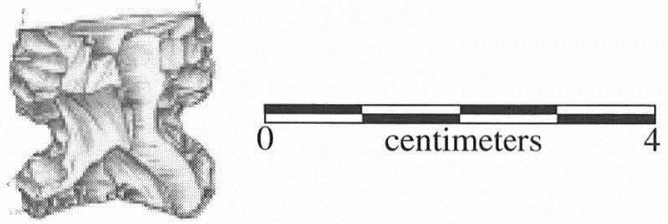

FIGURE 13-102. Big Sandy point, Specimen 24U1-1.

TABLE 13-108

Metric Measurements for Big Sandy Point (in mm)

\begin{tabular}{l|c}
\hline Variable & Value \\
\hline Thickness & 5.9 \\
Stem/grinding length 1 & 8.1 \\
10-mm stem width & 13.5 \\
Distal stem width & 13.5 \\
10-mm stem thickness & 5.5 \\
Basal concavity & 2.2 \\
\hline \hline
\end{tabular}

Comments: Points closely resembling the WilsonLeonard specimen at Dust Cave, Alabama, are dated to ca. 10,000 B.P. and referred to as Early Side-notched because of conflicting opinions of the status of dating on the Big Sandy Type (Driskell 1994).

\section{San Patrice $(N=4)$}

Morphology: These thin, well-flaked points have sidenotched stems with small, outward directed basal ears (Figure 13-103); bases are well-modified by bifacial thinning. Lateral stem edges have little or no grinding on this group, although grinding is typical. Full characterization of these points is difficult due to damage to basal area of each.

Metric Data: Table 13-109

Nonmetric Attributes: Longitudinal profiles include 2 with generally equal proportions, 1 with tapered base and maximum thickness at medial section, and 1 indeterminate;

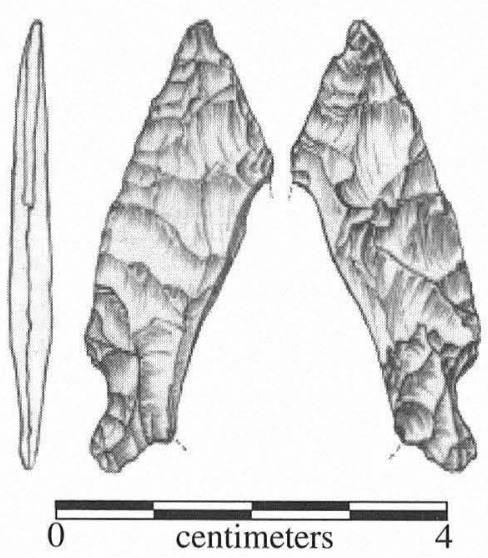

FigURE 13-103. San Patrice point, Specimen 1070-2. flake patterns are organized on 2 (subparallel, oblique subparallel), and 2 are indeterminate; basal modification is 3 bifacial thinning and 1 indeterminate; lateral grinding is absent on 2, slight on 1, and indeterminate on 1; basal grinding is absent on 1 and indeterminate on 3.

Material Attributes: Raw material includes 2 local and 1 nonlocal fine-grained Edwards chert, 1 local medium-coarsegrained Edwards chert; patina is absent on 3 specimens and slight partial on 1 ; mineral precipitates are present on only 1 specimen; heat damage is absent on 2 specimens and moderate on 2.

Tool State and Fractures: These 4 specimens are fragments with multiple bend breaks on 2, multiple burin-like/edge crushing breaks on 1, and multiple thermal spalls on 1 .

Rejuvenation: Resharpening includes 2 alternately beveled and 2 indeterminate. Specimen 1070-2, examined by Driskell, for microscopic evidence of use-wear, showed only a generic polish likely related to soil abrasion (see Chapter 22).

Stratigraphic Distribution: Valley Floor A, Units Isi-c/ II, Id/II, II, IIIa/b.

Comments: These specimens do not completely match descriptions of the two San Patrice variants described by Webb et al. (1971). Side-notched, Variety A (Webb et al. 1971:Figure 5i) seems to have a similar shape to the WilsonLeonard specimens, and Webb et al. (1971:41) considered this group part of the "San Patrice complex."

Typology and context of the San Patrice varieties have not been questioned (e.g., Johnson 1989; Story 1990). However, adequate geomorphological and formation process assessment has not been carried out at the John Pearce site (Webb et al. 1971), which is one of two acknowledged sites with an "isolated" San Patrice component. The second, the Whatley site (Thomas and Campbell 1978:40-49), had a moreintensive standardized examination of the sedimentary context, but the variety of cultural material was still accepted as an "assemblage." The question of whether the San Patrice "complex" or "assemblage" is mixed has not been examined.

Further, generalized comparisons can also be made between these Wilson-Leonard specimens and those referred to as "Brazos Fishtail" from Horn Shelter (Redder 1985), those called "Rodgers Hollow Stemmed" from Rodgers site (Willey et al. 1978), and several unnamed specimens from Kincaid Rockshelter (TARL Collections and Records, 41UV2); all of these are from contexts indicating archeological placement near the early part of the Late Paleoindian (Collins 1995).

Note similarities to Late Paleoindian Hardaway Side Notched (ca. $8000-7000$ B.c. or $9940-8940$ B.P.) of the southeastern United States, a type considered intermediate between Dalton and later side-notched types (Justice 1995:4344, Figure 8m-r); compare also with Hi-Lo type of the Great Lakes region, thought to correlate with the age of the Dalton horizon, $8500-8000$ в.C. (ca. 10,440-9940 в.P.) (Justice 1995:4446). 
TABLE 13-109

Summary Metric Data for San Patrice Points (in mm)

\begin{tabular}{l|c|c|c|c|c}
\hline Variable & Number & Minimum & Maximum & Mean & $1-\sigma$ \\
\hline Width & 1 & & 21.0 & & \\
Thickness & 4 & 4.0 & 5.0 & 4.5 & 0.6 \\
Stem/grinding length 1 & 2 & 6.6 & 7.0 & & \\
10 mm stem thickness & 1 & & 3.9 & & \\
\hline \hline
\end{tabular}

\section{Stemmed Biface $(n=1)$}

Morphology: This specimen is an asymmetrical, thinned, stemmed biface with a wide, deep notch on the blade edge. The stem is broken and was evidently broken while the piece was in use, as the notch appears to be a failed attempt to create a replacement haft. The former haft appears to have been formed by either basal or corner notches whereas the subsequent notch - if matched by one on the opposite edgewould likely have produced a side-notched form (Figure 13-104).

Metric Values: Table 13-110

Nonmetric Attributes: Longitudinal profile is indeterminate; flake pattern is oblique subparallel; basal modification is indeterminate; lateral grinding is indeterminate; and basal grinding is indeterminate.

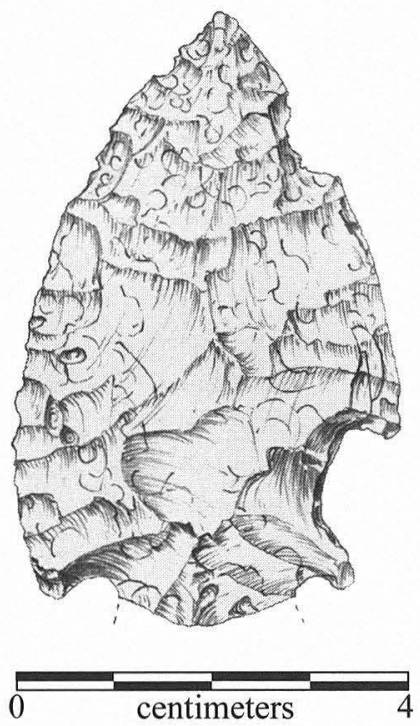

Figure 13-104. Stemmed biface, Specimen OR680.

TABLE 13-110

Metric Measurements for Stemmed Biface (in mm)

\begin{tabular}{l|c}
\hline Variable & Value \\
\hline Length* & 62.0 \\
Width & 40.0 \\
Thickness & 6.0 \\
Distal Stem Width & 18.0 \\
\hline
\end{tabular}

* Maximum measurement
Material Attributes: Raw material is indeterminate heat altered; patina is absent; mineral precipitates are absent; heat damage is extensive.

Tool State and Fractures: This specimen has a transverse bend fracture on the stem.

Rejuvenation: Resharpening is unbeveled bifacial.

Stratigraphic Distribution: Valley Floor A, Unit Isi-c, elevation $94.30 \mathrm{~m}$.

Comments: This specimen shares the oblique subparallel flake pattern and the same thickness with Specimen 26U2-2, a lanceolate distal section, but these two specimens come from widely separated stratigraphic positions. The stemmed biface was examined by Kay (see Chapter 22) who found wear traces on the blade indicating use as a knife, possibly for butchering.

\section{Miscellaneous Side-notched $(N=10)$}

Morphology: This miscellaneous category encompasses unclassifiable side-notched points. Most have short to medium length expanding stems with moderate to shallow side notching; stem bases vary from straight to slightly convex; basal ears vary from rounded to square (Figures 13-105 and 13-106).

Metric Averages: Table 13-111.

Nonmetric Attributes: Longitudinal profiles include 2 tapered base and tip; 1 flat; 2 wedge-shaped base and tip; and 5 indeterminate. Flake patterns are organized on 3 (collateral, oblique subparallel), random on 4 , and indeterminate on 3 .

Basal modification consists of unifacial thinning on 3 , bifacial thinning on 1 , secondary trimming on 2 , absent on 1 , and 3 indeterminate. Lateral stem grinding is absent.

Material Attributes: Raw material is local Edwards chert ( 6 fine grained, 2 medium/coarse); nonlocal Edwards finegrained chert for 1 ; and indeterminate heat altered for 1 . Heat damage is slight on 1, extensive on 1 . No mineral precipitates or patina were observed.

Tool State and Fractures: Three are complete except for small distal and basal ear tips severed by bend breaks. The remaining 7 are proximal sections showing multiple bend and thermal fractures (on 2); multiple bend and indeterminate fractures (on 1); multiple bend, hinge, and burin-like fractures (on 2); and burin-like impact fractures (on 2). 


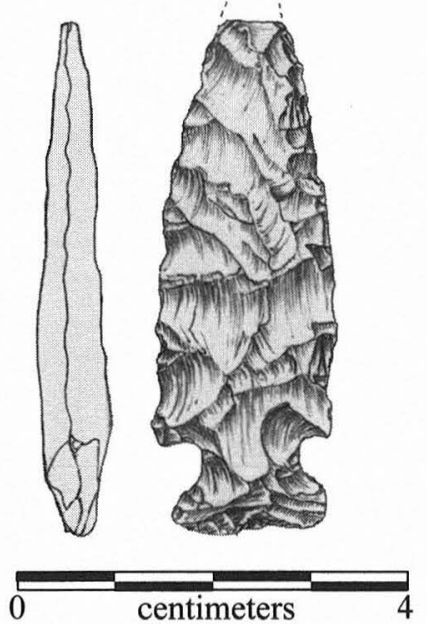

FIGURE 13-105. Miscellaneous side-notched point, Specimen 332-1.

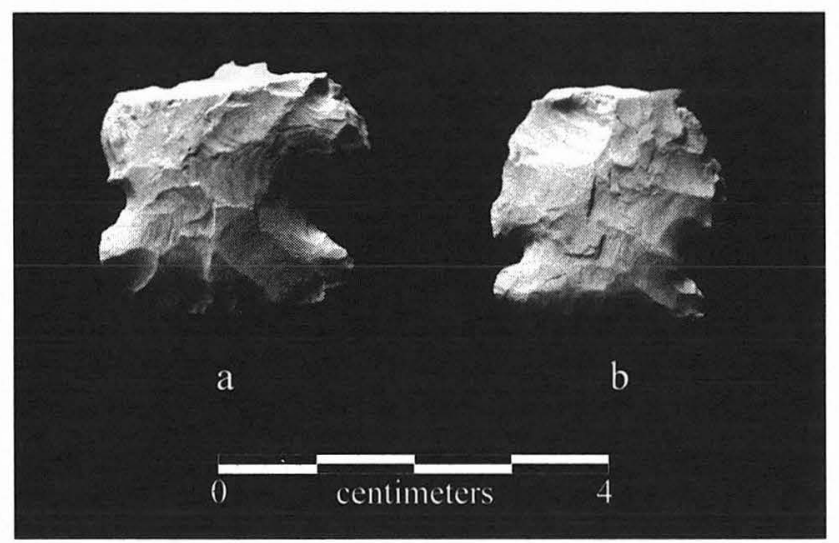

FIGURE 13-106. Miscellaneous side-notched points. Specimen numbers: (a) 12B-2; (b) 19L-3.

TABLE 13-111

Summary Metric Data for Miscellaneous Side-notched Points (in mm)

\begin{tabular}{l|c|c|c}
\hline & Number & Minimum & Maximum \\
\hline Length & 2 & 32.0 & 35.0 \\
Width & 7 & 18.3 & 29.0 \\
Thickness & 10 & 5.0 & 8.0 \\
Stem length & 8 & 8.0 & 14.0 \\
Proximal stem width & 4 & 16.0 & 21.0 \\
Distal stem width & 9 & 11.0 & 19.0 \\
Basal concavity depth & 2 & 1.0 & 2.0 \\
Basal convexity length & 3 & 1.0 & 5.0 \\
\hline \hline
\end{tabular}

Rejuvenation: One is resharpened unifacially, unbeveled, and 1 bifacially, unbeveled; 2 are alternately beveled; 1 is beveled unifacially; and 5 are indeterminate.

Stratigraphic Distribution: Table 13-112.

Comments: This category may include variants of Big
TABLE 13-112

Stratigraphic Distribution of Miscellaneous Side-notched Points

\begin{tabular}{l|c|c}
\hline Unit & Valley Floor A & Valley Margin \\
\hline IIIc & 4 & \\
IIIb/c & 1 & \\
IIIb & 1 & 1 \\
X/Y/IIIb & & \\
Other & & \\
surface & 3 & \\
\hline \hline
\end{tabular}

Sandy and Ensor, but most are not characteristic of either. Specimen 108-13 displays the more-squared basal ears of Big Sandy; basal edge is slightly dulled on this specimen. However, stratigraphic context of this and others in the group is generally high in the site, suggesting closer affiliation with Ensor for some.

\section{Straight Stemmed Points}

\section{Bell/Andice (N=18)}

Morphology: These large, thin, broad-bladed points are distinguished by deep basal notches and massive barbs; stems are straight to gently expanding, with straight to gently concave, well-thinned bases (Figures 13-107 and 13-108). Stems typically bear a series of remnant sequent flake scars aligned vertically along their lateral edges, which mark successive stages of the notching process (see Weber 1994). Two specimens in this group are wide, squared-off barb fragments typical of the type.

Metric Averages: Table 13-113.

Nonmetric Attributes: Longitudinal profiles on those 11 specimens complete enough to observe are all tapered base and tip. Flake patterns are organized on 7 (subparallel, oblique subparallel, collateral, chevron), random on 2, and indeterminate on 9. Basal modification consists of bifacial basal thinning on 12 , unifacial thinning on 1 , unifacial fluting on 1 , secondary trimming on 1 , and 3 indeterminate. Lateral stem grinding is slight on 1 , moderate on 4 .

Material Attributes: Raw material is local Edwards chert (10 fine grained, 4 medium/coarse), 3 nonlocal Edwards finegrained chert, and 1 indeterminate. Patina is moderate partial on 1 and heavy partial on another. Mineral precipitates are present on 2. Heat damage is slight on 2. No patina is present.

Tool State and Fractures: Seven are complete except for barb(s) or small distal or basal sections. Eight are proximal sections lacking barbs, and 3 are barb or barb/medial fragments. Ten exhibit single or multiple bend breaks; 6 show evidence of impact damage, 1 displays thermal fractures, and breaks on 1 are indeterminate.

Rejuvenation: Two are resharpened unifacially, unbeveled; 4 bifacially, unbeveled; 1 is alternately beveled; and 11 are indeterminate. 


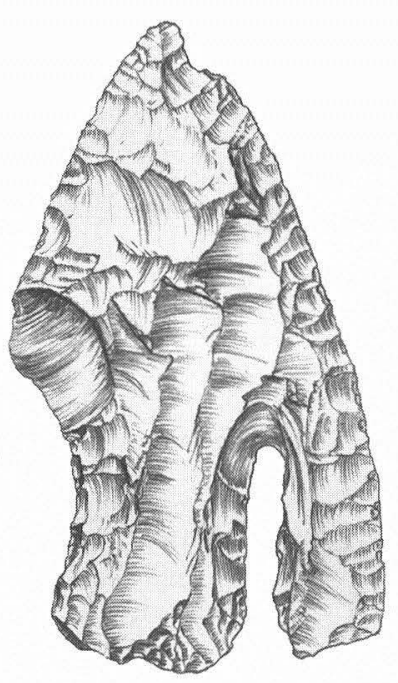

a

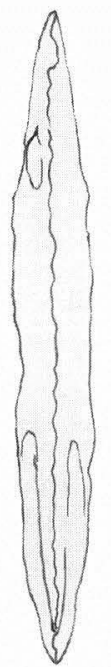

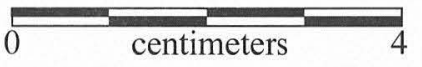

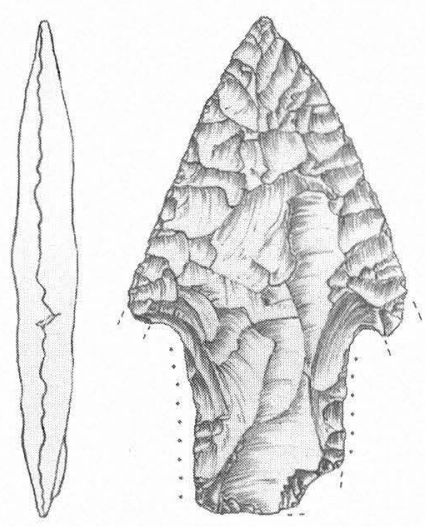

b

Figure 13-107. Bell/Andice points. Specimen numbers: (a) 12J-1; (b) 721-1.

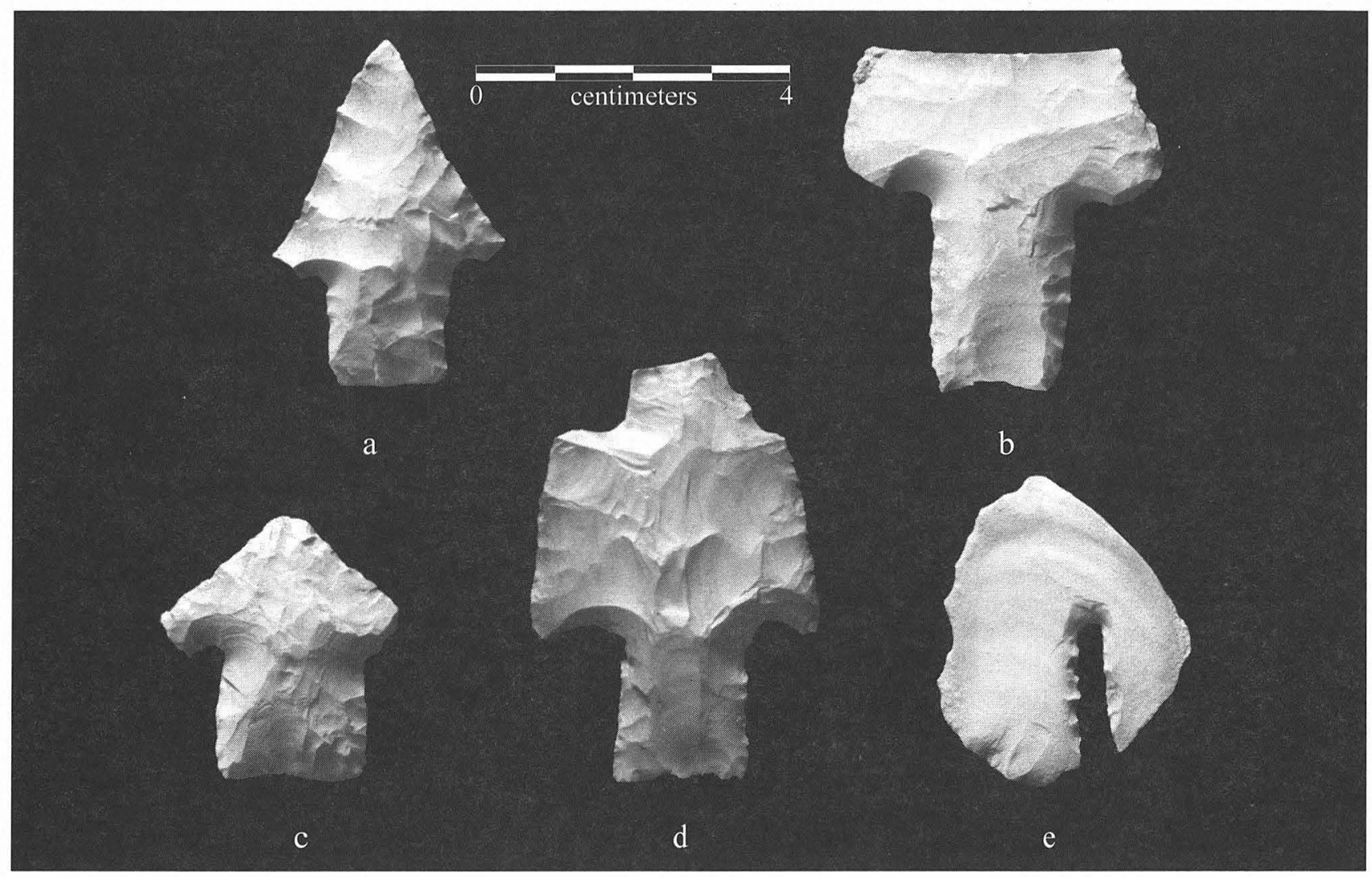

FIGURE 13-108. Bell/Andice points and "practice" piece. Points, a-d, practice piece, e. Specimen numbers: (a) 15J2-2; (b) 51MA-1; (c) $13 \mathrm{~K}-1$; (d) $16 \mathrm{E}-1$; (e) $556-4$.

Stratigraphic Distribution: As shown in Table 13-114, Bell/Andice are fairly well isolated in Unit IIIb and the Unit $\mathrm{IIIb} / \mathrm{c}$ transition zone.

Comments: To some Texas typologists, stem lengths have been considered sufficiently distinctive to require two types, with Andice recognized as longer stemmed, and Bell the shorter (e.g., Prewitt 1983:1-6; Turner and Hester 1993). Increasingly, these points are being considered as a continuum of stylistic expression, with more attention being placed on possible ties to the Calf Creek horizon described for similar time periods of south-central Oklahoma and Arkansas (e.g., Wyckoff 1994). Clearly, there are also resem- 
TABLE 13-113

Summary Metric Data for Bell/Andice Points (in mm)

\begin{tabular}{|c|c|c|c|c|c|}
\hline & Number & Minimum & Maximum & Mean & $1-\sigma$ \\
\hline Length & 5 & 33.0 & 66.0 & 46.2 & 12.7 \\
\hline Width & 2 & 29.0 & 36.0 & 32.5 & 5.0 \\
\hline Thickness & 16 & 4.0 & 9.0 & 6.7 & 1.1 \\
\hline Stem length & 13 & 15.0 & 25.0 & 19.0 & 3.5 \\
\hline Proximal stem width & 11 & 15.0 & 20.0 & 17.3 & 1.7 \\
\hline Distal stem width & 14 & 16.0 & 19.0 & 17.6 & 0.9 \\
\hline Basal concavity depth & 5 & 1.0 & 1.0 & 1.0 & 0.0 \\
\hline Basal convexity length & 3 & 2.0 & 2.0 & 2.0 & 0.0 \\
\hline
\end{tabular}

TABLE 13-114

Stratigraphic Distribution of Bell/Andice points

\begin{tabular}{l|c|c|c}
\hline Unit & $\begin{array}{c}\text { Valley } \\
\text { Floor A }\end{array}$ & $\begin{array}{c}\text { Valley } \\
\text { Floor B }\end{array}$ & $\begin{array}{c}\text { Valley } \\
\text { Margin }\end{array}$ \\
\hline IIIc & 2 & & 1 \\
IIIb/c & 3 & & 1 \\
Y/IIIc & 8 & & \\
IIIb & & 2 & \\
III & & & \\
Other & 1 & & \\
provenience unknown & 1 & \\
\hline \hline
\end{tabular}

blances to the group termed Early Barbed in the Lower Pecos (Johnson 1964).

It is notable that lateral stem edge grinding was observed on four of the Wilson-Leonard specimens. Similar modification has been observed on Bell/Andice points from South Texas (Robert Ricklis, personal communication 1995) and Calf Creek points (Perino 1968). This attribute is puzzling, in that accessing the stem for grinding, within the long, narrow basal notches, would seem logistically somewhat difficult as well as risky in terms of potential barb breakage. However, grinding with a flat flake would have been effective. At the present site, stem edge grinding occurred on specimens with at least partially broken off barbs; it is possible, although unlikely, that stem grinding was done after the breakage episode.

Figure 13-108e is a cortical flake shown here as a possible Bell/Andice notching "practice piece." Depth of notch is $22 \mathrm{~mm}$, well within the mean stem length (or notch depth) for this group. Context is Unit IIIb.

\section{Beveled Narrow Stemmed $(N=5)$}

Morphology: These points combine aspects of both Nolan and Travis. Stems are long, very narrow, thick, and generally rectangular in shape; some display unilateral beveling of lateral edges, although not the bilateral bevel of Nolan. Shoulders are weak and unbarbed. Two of the morecomplete specimens display a highly uniform, convex-sided blade outline, which is generally elongate and spatulate in shape (Figures 13-109 and 13-110).

Metric Averages: Table 13-115

Nonmetric Attributes: Longitudinal profiles include 1 flat, 3 wedge-shaped base and tip, and 1 indeterminate. Flake patterns are random on all. Basal modification constitutes unifacial thinning on 1,1 beveled base, 1 secondary trimming only, and 2 absent. Lateral stem grinding is not present.

Material Attributes: Raw material is local Edwards chert (1 fine grained, 3 medium/coarse) and nonlocal Edwards finegrained chert $(\mathrm{n}=1)$. Mineral precipitates, heat damage, and patina are not present.

Tool State and Fractures: Three are complete and 2 are proximal sections, 1 of which exhibits a bend fracture, the other, bend and burin-like breaks.

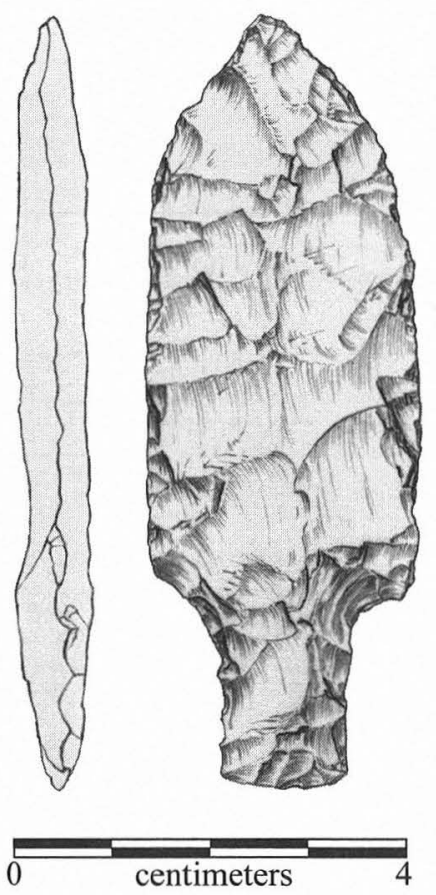

FIGURE 13-109. Beveled narrow-stemmed point, Specimen TR262. 


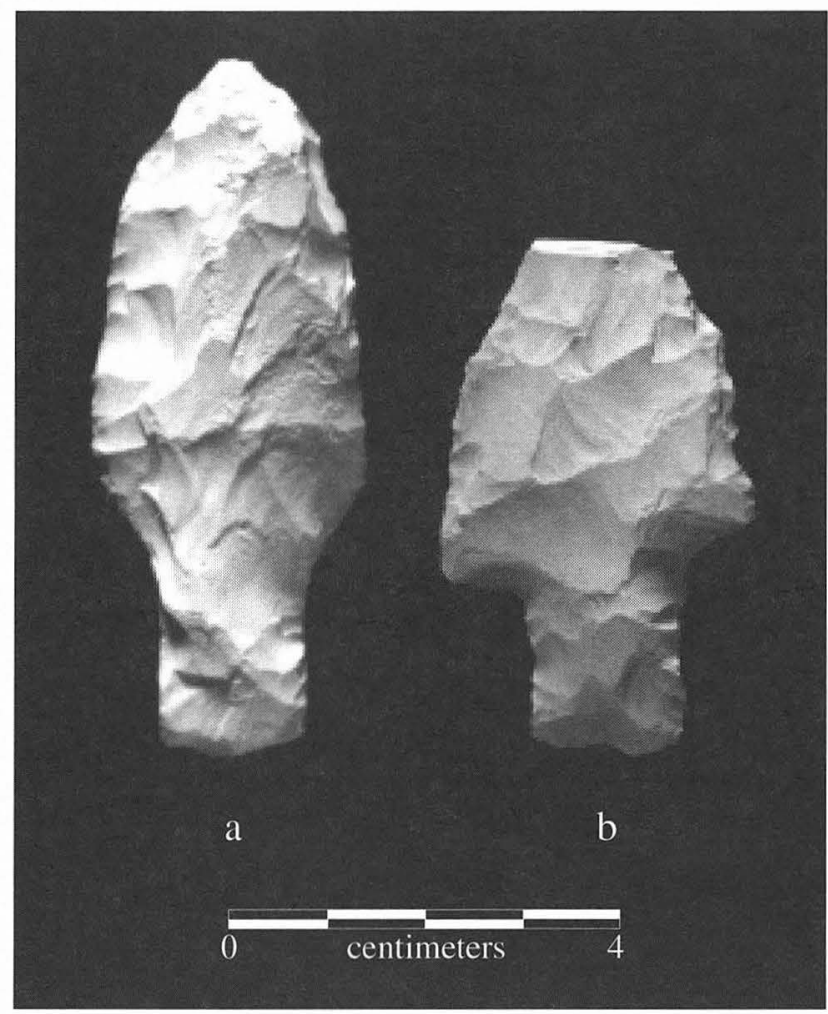

FIGURE 13-110. Beveled narrow-stemmed points. Specimen numbers: (a) 13E-1; (b) 2D-1.

Rejuvenation: Two are resharpened unifacially, unbeveled, 2 alternately beveled, and 1 beveled unifacially.

Stratigraphic Distribution: Stratigraphic placement suggests a Middle or Late Archaic time frame (Table 13-116).

Comments: These specimens are similar to some dubbed "Trolan" at the Crumley site (Kelly 1962:Figure 8h, i) because of their similarities to both Travis and Nolan. The stems on beveled narrow and Trolan are generally narrower than either Nolan or Travis.

\section{Bulverde $(N=29)$}

Morphology: These triangular points have strong shoulders and generally small barbs. Flake patterns commonly are nonregularized. Stems are typically rectangular to slightly
TABLE 13-116

Stratigraphic Distribution of Beveled Narrow-stemmed Points

\begin{tabular}{l|c|c}
\hline Unit & Valley Floor A & Valley Margin \\
\hline IIIc & 1 & 2 \\
Other & 1 & \\
surface & 1 & \\
provenience unknown & & \\
\hline \hline
\end{tabular}

contracting and vary in size as well as basal-edge form, which can be straight or slightly concave. Stem lateral edges may be dulled. Their characteristic attribute is a well-thinned base that may appear tapered or wedge-like in longitudinal profile (Figures 13-111 and 13-112).

Metric Averages: Table 13-117.

Nonmetric Attributes: Longitudinal profiles include 19 with tapered bases and tips, 1 with thick base form, 1 flat, 7 more-uniform overall with less-pronounced wedge-shaped base and tip, and 1 indeterminate. Flake patterns are organized on 8 (collateral, oblique subparallel, subparallel), random on 19, and indeterminate on 2. Basal modification was observed as unifacial thinning on 12 , bifacial thinning on 12 , and secondary trimming on 5; lateral stem grinding was slight on 8 , and moderate on 2 .

Material Attributes: Raw material is local Edwards chert for 23 (22 fine grained and 1 medium/coarse), nonlocal/indeterminate Edwards chert for 5 (1 fine grained, 4 medium/ coarse), and 1 indeterminate. Mineral precipitates are present on 3. Patina (moderate partial) was noted on 1. Heat damage is moderate on 1 .

Tool State and Fractures: Eight are complete; 14 are largely complete, lacking only distal and/or basal ear and/or barb tips; of these, 5 exhibit facial impact or burin-like impact fractures, 1 thermal damage, and 8 bend or indeterminate breaks. The remaining 7 are proximal/medial sections, 6 of which display single bend breaks, and 1 of which shows multiple bend breaks.

Rejuvenation: Of the 23 complete enough to observe, 16 exhibit bifacial unbeveled resharpening, 1 is resharpened unifacially, unbeveled, and 6 alternately beveled. One specimen, 50JB-5, may have been used, after fracturing, as a burin.

TABLE 13-115

Summary Metric Data for Beveled Narrow Stemmed Points (in mm)

\begin{tabular}{|c|c|c|c|c|c|}
\hline & Number & Minimum & Maximum & Mean & $1-\sigma$ \\
\hline Length & 3 & 52.0 & 78.0 & 67.0 & 13.5 \\
\hline Width & 5 & 23.0 & 34.0 & 29.0 & 4.2 \\
\hline Thickness & 5 & 6.0 & 9.0 & 7.6 & 1.1 \\
\hline Stem length & 5 & 17.0 & 21.0 & 19.2 & 1.5 \\
\hline Proximal stem width & 5 & 13.0 & 16.0 & 15.0 & 1.2 \\
\hline Distal stem width & 5 & 14.0 & 17.0 & 15.2 & 1.3 \\
\hline Basal concavity depth & 2 & 2.0 & 2.0 & 2.0 & 0.0 \\
\hline Basal convexity length & 2 & 2.0 & 2.0 & 2.0 & 0.0 \\
\hline
\end{tabular}




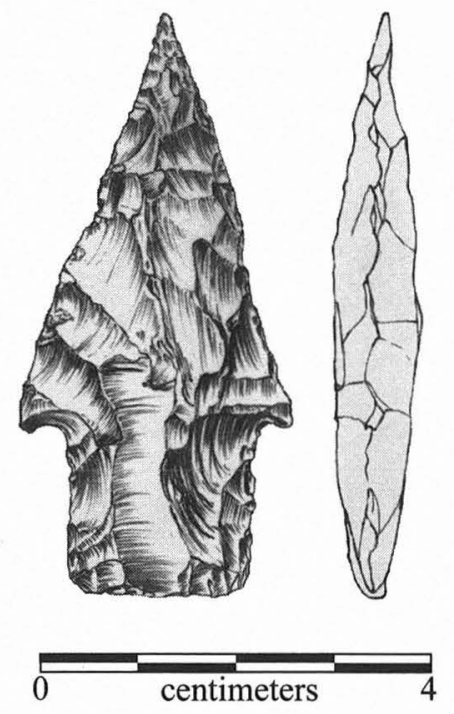

FIGURE 13-111. Bulverde point, Specimen 2E-1.

Stratigraphic Distribution: Highest frequency is in Unit IIIc (Table 13-118).

Comments: This group forms a continuum with Bulverde-like (see below). Based on stem morphology alone (e.g., long, straight to constricted stems with tapered bases), Bulverde can be confused with some reworked specimens of Bell/Andice where barbs have been removed.

\section{Bulverde-like $(N=19)$}

Morphology: Morphologically similar to Bulverde, this group lacks the distinctive basal thinning, exhibiting instead a generally thicker wedge-shaped base (Figures 13113 and 13-114). Dulling or grinding of stem lateral edges is common.

Metric Averages: Table 13-119.

Nonmetric Attributes: Longitudinal profiles include 9 with tapered base and stem, 1 flat, 5 with wedge-shaped base and tip, and 4 indeterminate; flake patterns are organized on 6 (subparallel, oblique subparallel, collateral), random on 9 , and indeterminate on 4 . Basal modification took the form of unifacial thinning on 9, bifacial thinning on 3 , beveled edge on 1 , secondary trimming on 4 , and absent on 2 ; lateral stem grinding was observed on 8 ( 4 slight, 3 moderate, 1 heavy).

Material Attributes: Raw material is local Edwards chert ( 7 fine grained and 5 medium/coarse), 4 nonlocal or indeterminate Edwards medium/coarse-grained chert, and 3 indeterminate/heat altered; patina was coded as moderate partial on 1 ; mineral precipitates were observed on 4 ; heat damage is moderate on 1, extensive on 3 .

Tool State and Fractures: Eleven are complete or nearly so, with 5 lacking distal or barb tips. The remaining 8 are proximal or stem sections, 4 of which exhibit thermal damage, 3 bend breaks, and 1 multiple bend and burin-like breaks.

Rejuvenation: All but 1 of those complete enough to observe showed resharpening ( 8 bifacial unbeveled and 4 alternately beveled).

Stratigraphic Distribution: As shown in Table 13-120, Bulverde-like points are present in greatest numbers in Unit IIIc.

Comments: This group appears temporally and morphologically related to Bulverde, but lacks the well-thinned, wedge-like base and more frequently displays stem grinding than does Bulverde (see also Collins 1972; Sorrow et al. 1967). It also is of possible significance that in the WilsonLeonard samples, Bulverde-like points are 7 times more frequently heat damaged than are Bulverde points (4 of 19 compared to 1 of 29).

\section{$\operatorname{Nolan}(N=45)$}

Morphology: These are distinctive, medium to large elongate points with leaf-shaped blades (Figures 13-115 and 13116). Their identifying characteristic is a straight to slightly expanding, alternately beveled stem of widely varying size. Basal edges vary from straight to concave and are generally wedge-shaped in profile. Stem lateral edges appear dulled or ground on some, typically in correlation with the degree of stem beveling. Blade edges are typically convex, and shoulders typically small and often rounded. Figure $13-115 b$ shows an unusual specimen with sinuous blade edges formed by three pairs of broad notches which appear to be dulled within their concavities; the distal section has been reworked to an elongate point.

Metric Averages: Table 13-121

Nonmetric Attributes: Longitudinal profiles include13 tapered base and tip, 1 thick tip, 1 thick base, 3 flat, 15 wedgeshaped base and tip, 1 wedge-shaped base, thick stem, and flat body, and 11 indeterminate. Flake patterns are organized on 19 (subparallel, collateral, oblique subparallel, chevron), random on 15, and indeterminate on 11. Basal modification consists of unifacial thinning on 16, bifacial thinning on 9, beveling on base on 3, secondary trimming on 11, absent on 4 , and indeterminate on 2 . Lateral stem grinding is slight on 17 , moderate on 13 , heavy on 4 , and indeterminate on 2 .

Material Attributes: Raw material is local Edwards chert (29 local fine grained, 6 medium/coarse), nonlocal Edwards chert ( 4 fine grained, 2 medium coarse), non-Edwards medium/coarse-grained chert $(\mathrm{n}=1)$, indeterminate heat altered $(\mathrm{n}=2)$, and indeterminate $(\mathrm{n}=1)$. Patina is slight partial on 2 . Mineral precipitates are present on 8 . Heat damage is slight on 2 and extensive on 2.

Tool State and Fractures: Eleven are complete, and 9 others are nearly so, displaying minor bend and impact fractures at the distal tip section and, on 2, at the shoulders as well; 1 lacks a distal tip and base section due to bending and indeterminate fractures. Eighteen are proximal sections. Of these, 10 display single bend fractures; 5 , multiple bend and burin-like/impact fractures; 2 , thermal breaks; and 1, bend and edge crushing. Two specimens are chiefly blade sec- 


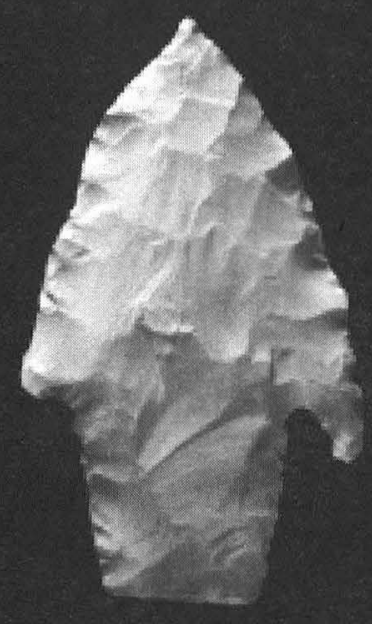

a

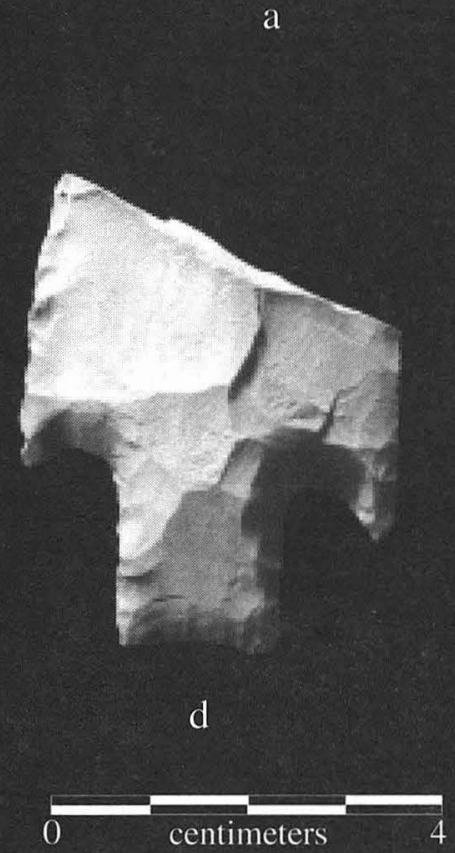

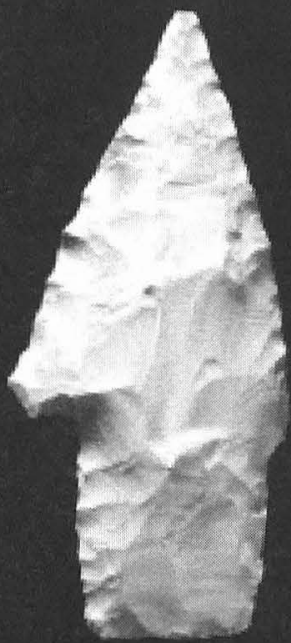

b

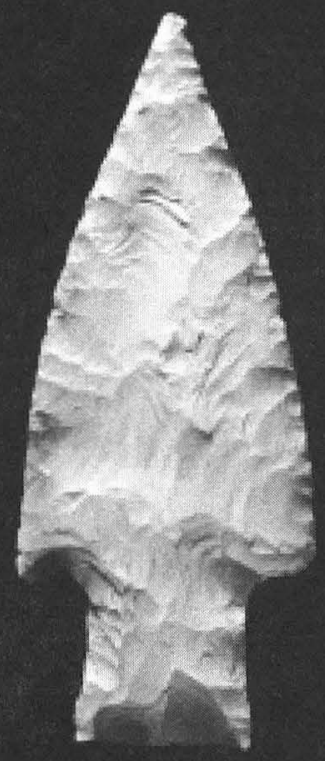

e

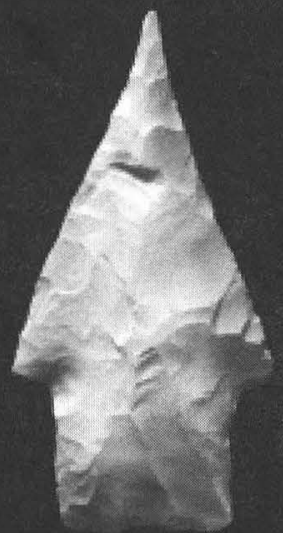

c

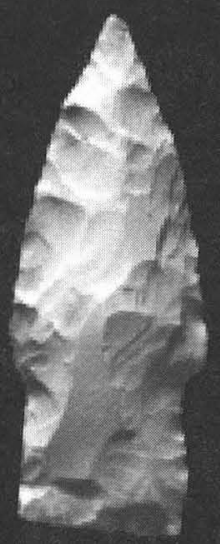

f

FIGURE 13-112. Bulverde points. Specimen numbers: (a) 0-117; (b) 15G-3; (c) 1434; (d) 0-99; (e) 50EB-7; (f) 13 G-1.

TABLE 13-117

Summary Metric Data for Bulverde Points (in mm)

\begin{tabular}{|c|c|c|c|c|c|}
\hline & Number & Minimum & Maximum & Mean & $1-\sigma$ \\
\hline Length & 10 & 36.0 & 74.0 & 53.4 & 10.7 \\
\hline Width & 24 & 19.0 & 39.0 & 29.1 & 5.4 \\
\hline Thickness & 28 & 5.0 & 9.0 & 7.6 & 1.0 \\
\hline Stem length & 29 & 15.0 & 22.0 & 17.5 & 1.9 \\
\hline Proximal stem width & 29 & 15.0 & 19.0 & 17.1 & 1.1 \\
\hline Distal stem width & 28 & 16.0 & 22.0 & 18.7 & 1.5 \\
\hline Basal concavity depth & 18 & 1.0 & 3.0 & 1.4 & 0.6 \\
\hline Basal convexity length & 2 & 1.0 & 1.0 & 1.0 & 0.0 \\
\hline
\end{tabular}




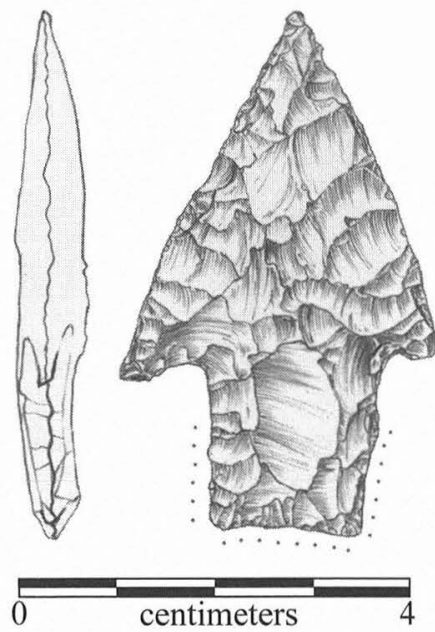

TABLE 13-118

Stratigraphic Distribution of Bulverde Points

\begin{tabular}{l|c|c|c}
\hline Unit & $\begin{array}{c}\text { Valley } \\
\text { Floor A }\end{array}$ & $\begin{array}{c}\text { Valley } \\
\text { Floor B }\end{array}$ & $\begin{array}{c}\text { Valley } \\
\text { Margin }\end{array}$ \\
\hline IIIc & 11 & 3 & 2 \\
IIIb/c & 2 & & \\
IIIb & 2 & & \\
Other & 8 & & \\
surface & 1 & & \\
provenience unknown & & & \\
\hline \hline
\end{tabular}

FIGURE 13-113. Bulverde-like point, Specimen 13F-1.

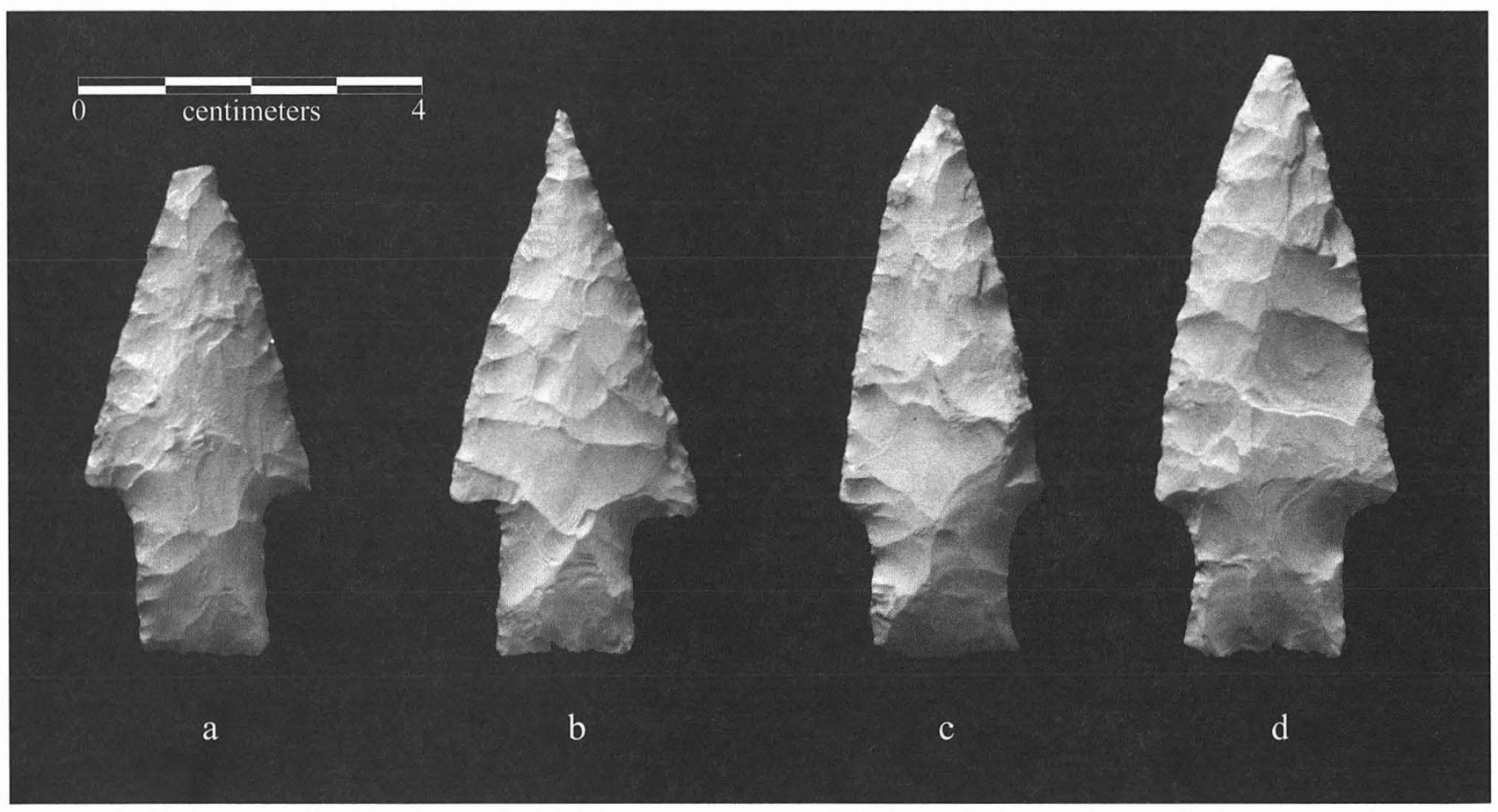

FIGURE 13-114. Bulverde-like points. Specimen numbers: (a) 51FB-4; (b) 20H-1; (c) 16E-3; (d) 37GB-3.

TABLE 13-119

Summary Metric Data for Bulverde-like Points (in mm)

\begin{tabular}{|c|c|c|c|c|c|}
\hline & Number & Minimum & Maximum & Mean & $1-\sigma$ \\
\hline Length & 8 & 46.0 & 74.0 & 57.3 & 10.2 \\
\hline Width & 15 & 19.0 & 35.0 & 26.1 & 4.0 \\
\hline Thickness & 19 & 6.0 & 9.0 & 7.6 & 1.0 \\
\hline Stem length & 19 & 13.0 & 21.0 & 18.1 & 1.8 \\
\hline Proximal stem width & 19 & 14.0 & 20.0 & 17.1 & 1.9 \\
\hline Distal stem width & 18 & 16.0 & 21.0 & 17.8 & 1.6 \\
\hline Basal concavity depth & 12 & 1.0 & 2.0 & 1.3 & 0.5 \\
\hline Basal convexity length & 3 & 1.0 & 2.0 & 1.7 & 0.6 \\
\hline
\end{tabular}


TABLE $13-120$

Stratigraphic Distribution of Bulverde-like Points

\begin{tabular}{l|c|c|c}
\hline Unit & Valley Floor A & Valley Floor B & Valley Margin \\
\hline IIIc & 4 & 3 & 2 \\
IIIb/c & 2 & & 2 \\
IIIb & 1 & & 1 \\
Y/IIIc & & & 1 \\
Other & 1 & & \\
surface & 1 & & \\
fill & 1 & & \\
IIIc fill & & & \\
\hline \hline
\end{tabular}

tions, retaining only the upper portion of alternately beveled stems. On these, stems were severed by a burin-like blow on 1, and bend on the other. Four are stems only, 1 of which exhibits multiple bend breaks, and the others bend, thermal, and indeterminate breaks. The remaining specimen is a medial section with a small, alternately beveled stem "neck" portion; it evidences multiple bend breaks.

Rejuvenation: Four are resharpened unifacially, unbeveled; 8 bifacially, unbeveled; 12 alternately beveled; 1 beveled unifacially; 14 indeterminate, and 6 absent. On 2 specimens, blade edges are alternately beveled in a pattern opposing that of the stem.

Stratigraphic Distribution: As shown in Table 13-122,
Nolan points are distributed fairly discretely, appearing most prominently in what likely are Middle Archaic and Late Archaic contexts (Units IIIb, IIIb/c, and IIIc). In the Valley Floor B area, Nolan appear to be present at or in the base of a burned rock midden (Burned Rock Midden 2; see Chapter 26). A similar pattern for Nolan has been observed at other Central Texas sites (e.g., 41GL160 [Kelly 1987]).

Comments: Several morphological and material attributes are unusual in the Wilson-Leonard Nolan assemblage. Many display a highly uniform, almost "chiseled" quality on both stem and blade edges (see Figure 13-116). This is due in part to the steeply angled beveling and dulling on the stem edges on some. The typically sharply pointed distal tip and uniform blade is a trait seen also in the Travis, Bulverde, and beveled narrow-stemmed groups, which share similar temporal contexts with Nolan.

It is notable that a relatively high percentage ( 20 of 45 or $44 \%$ ) of Nolan points were discarded (or lost) in a complete or virtually unbroken state. Fine-quality black cherts also are unusually prevalent in this group; 7 specimens $(16 \%)$ were made from these materials.

Of interest, too, is a proximal specimen $(0-101)$ that may be a preform. Although it is exceptionally wide stemmed, it displays the characteristic alternate beveling. It has not been resharpened, and may have been broken during manufacturing. Its dimensions (not included in the metric averages above) are $40 \mathrm{~mm}$ in width, $8 \mathrm{~mm}$ in thickness, $19 \mathrm{~mm}$ in stem length, $26 \mathrm{~mm}$ in proximal stem width, and $29 \mathrm{~mm}$ in distal

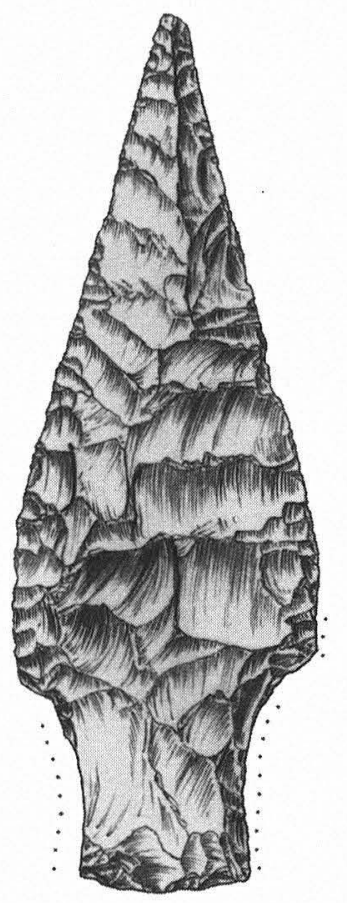

a
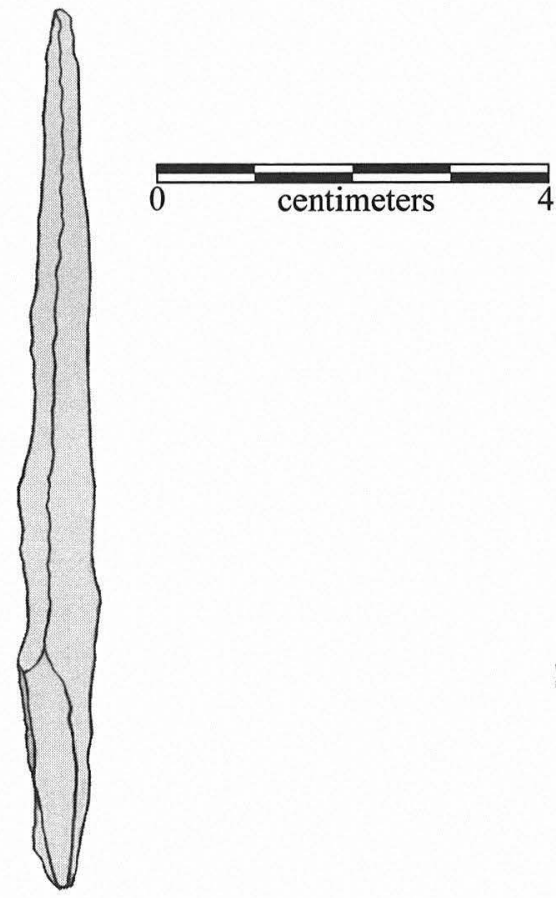

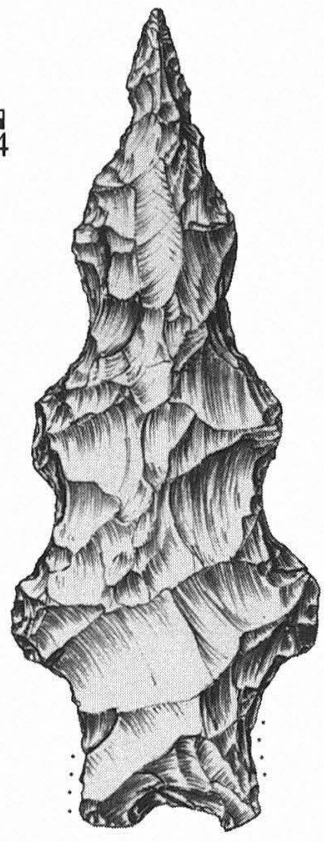

b

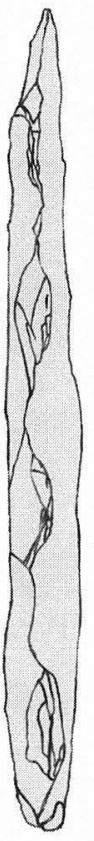

b

FIGURE 13-115. Nolan points. Specimen numbers: (a) 17H-1; (b) 37GD-18. The latter, "eccentric" specimen has been notched successively on the blade. 


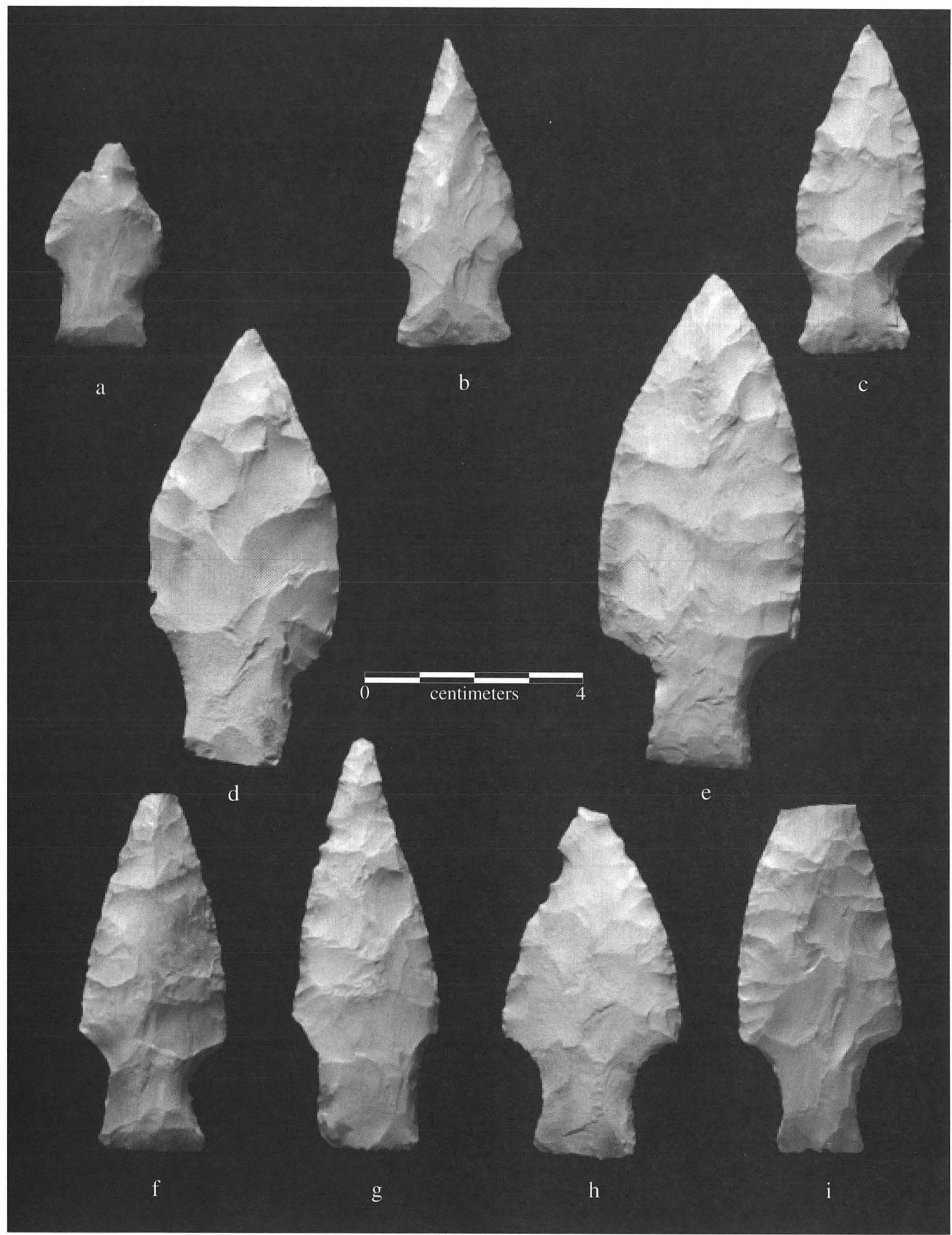

FiguRE 13-116. Nolan points. Specimen numbers: (a) 15J2-4; (b) 12F-1; (c) 14G-3; (d) 9F-2; (e) 22H-2; (f) 50FA-4; (g) 1246-1; (h) 3A15 ; (i) $22 \mathrm{H}-3$. 
TABLE 13-121

Summary Metric Data for Nolan Points (in mm)

\begin{tabular}{|c|c|c|c|c|c|}
\hline & Number & Minimum & Maximum & Mean & $1-\sigma$ \\
\hline Length & 12 & 32.0 & 89.0 & 64.2 & 18.4 \\
\hline Width & 38 & 21.0 & 37.0 & 29.7 & 4.7 \\
\hline Thickness & 43 & 5.0 & 10.0 & 7.8 & 1.2 \\
\hline Stem grinding length & 36 & 14.0 & 25.0 & 19.9 & 3.0 \\
\hline Proximal stem width & 38 & 13.0 & 22.0 & 18.1 & 2.0 \\
\hline Distal stem width & 18 & 12.0 & 23.0 & 17.9 & 2.2 \\
\hline Basal concavity depth & 4 & 1.0 & 3.0 & 1.5 & 1.0 \\
\hline Basal convexity length & 28 & 1.0 & 3.0 & 1.5 & 0.6 \\
\hline
\end{tabular}

Note: Nolan "eccentric" not included.

TABLE 13-122

Stratigraphic Distribution of Nolan Points

\begin{tabular}{l|c|c|c}
\hline Unit & $\begin{array}{c}\text { Valley } \\
\text { Floor A }\end{array}$ & $\begin{array}{c}\text { Valley } \\
\text { Floor B }\end{array}$ & $\begin{array}{c}\text { Valley } \\
\text { Margin }\end{array}$ \\
\hline IIIc & 5 & 6 & 6 \\
IIIc pot & 10 & & 2 \\
IIIb/c & 6 & & 3 \\
IIIb & 1 & & \\
IIIa/b & 1 & & \\
III & 3 & & \\
Other & 2 & & \\
surface & & \\
provenience unknown & 2 & \\
\hline \hline
\end{tabular}

stem width. The notched-blade specimen (Figure 13-115b) is particularly unusual; most "eccentric" lithic artifacts reported in Texas are related to Bell/Andice (see Hester 1990). The present specimen was recovered at the Unit IIIb/c transition zone, estimated to date ca. 4,000 B.P.

\section{Nolan-like $(N=3)$}

Morphology: These specimens display slightly different beveling of the stem, which makes them somewhat aberrant in the Nolan group. One exhibits a gentle beveling of the stem, opposing the steeply alternately beveled blade, resulting in a torque-like longitudinal profile not unlike Pandale (Figure 13-117). The other two show only slight beveling of the stems.

Metric Averages: Table 13-123

Nonmetric Attributes: Longitudinal profiles include 2 wedge-shaped base and tip and 1 indeterminate. Flake patterns are organized (collateral) on 1 and random on 2. Basal modification consists of unifacial thinning on 1 , beveled base on 1, and 1 absent. Lateral stem grinding is slight on 1 , heavy on another.

Material Attributes: Raw material is local Edwards finegrained chert on all. Mineral precipitates, heat alteration,

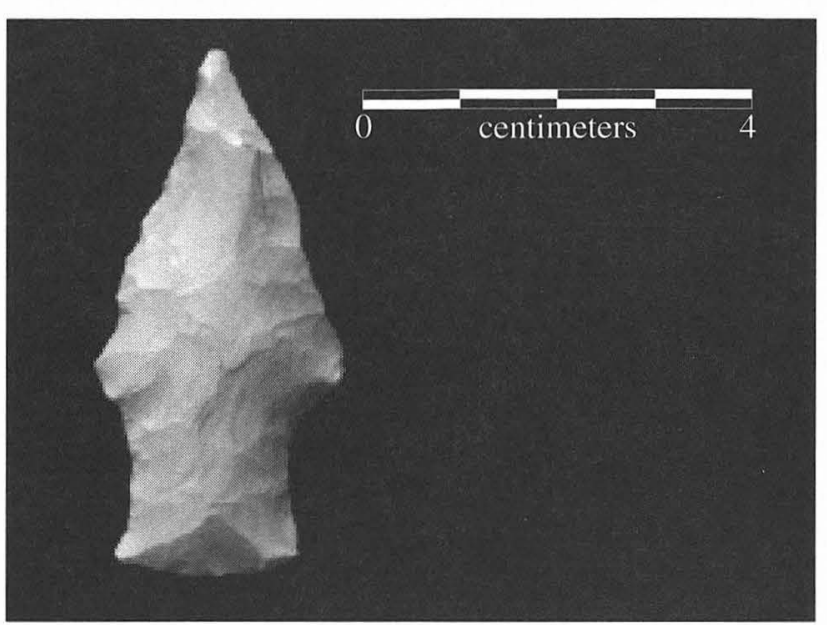

FIGURE 13-117. Nolan-like point, Specimen 11I-1.

and patina are absent.

Tool State and Fractures: One is complete, 1 exhibits a small bend break which severed the distal tip, and 1 is a proximal section exhibiting multiple bend and apparent impact damage.

Rejuvenation: One is resharpened unifacially, unbeveled, 1 is alternately beveled, and 1 is indeterminate.

Stratigraphic Distribution: All were recovered from the Unit IIIb/c transition zone, 2 in Valley Floor A and 1 in Valley Margin.

Comments: Based on stratigraphic context and overall morphology, these points are likely akin to Nolan but are not characteristic.

\section{Travis $(N=10)$}

Morphology: These rather elongate points characteristically exhibit convex-edged blades that tend to converge into the stem area with little or no demarcation of shoulders (Figures 13-118 and 13-119). Stems are generally square to gently expanding, and basal edges are typically straight to convex; lateral stem edges may be dulled or lightly ground. 
TABLE 13-123

Summary Metric Data for Nolan-like Points (in $\mathrm{mm}$ )

\begin{tabular}{l|c|c|c|c|c}
\hline & Number & Minimum & Maximum & Mean & $1-\sigma$ \\
\hline Length & 1 & 90.0 & 90.0 & & \\
Width & 3 & 26.0 & 32.0 & 29.0 & 3.0 \\
Thickness & 3 & 8.0 & 10.0 & 9.0 & 1.0 \\
Stem length & 3 & 18.0 & 19.0 & 18.3 & 0.6 \\
Proximal stem width & 3 & 16.0 & 19.0 & 17.0 & 1.7 \\
Distal stem width & 2 & 19.0 & 22.0 & 20.5 & 2.1 \\
Basal convexity length & 1 & 2.0 & 2.0 & 2.0 & \\
\hline \hline
\end{tabular}

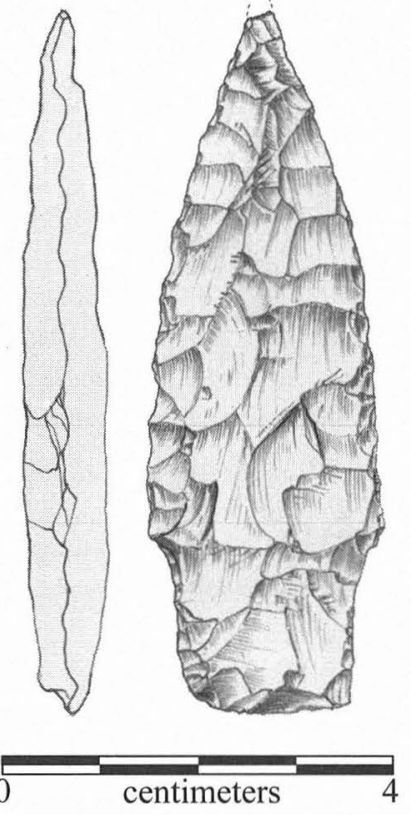

FIGURE 13-118. Travis point, Specimen 21G-2.

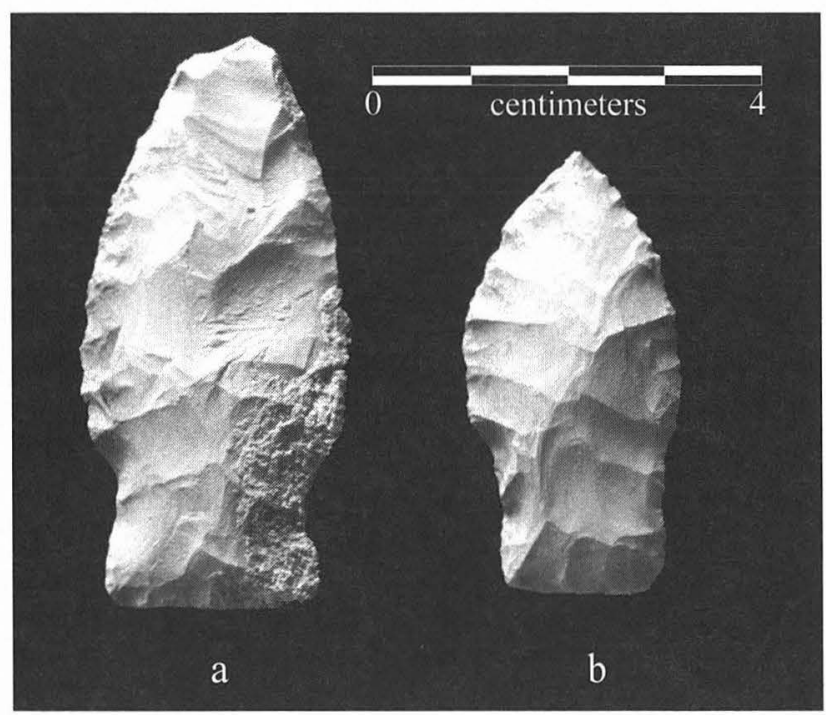

FigURE 13-119. Travis points. Specimen numbers: (a) 51JB-8; (b) $36 \mathrm{H}-1$.
Metric Averages: Table 13-124

Nonmetric Attributes: Longitudinal profiles include 1 tapered base and tip; 1 thick tip; 5 wedge-shaped base and tip; 1 wedge-shaped base, thick stem, and flat body; and 2 indeterminate. Flake patterns are organized on 5 (subparallel, collateral, chevron), random on 4 , and 1 indeterminate. Basal modification consists of unifacial thinning on 1 , bifacial thinning on 1 , beveled base on 4 , secondary trimming on 3 , and 1 not modified. Lateral stem grinding is slight on 3 , heavy on 1.

Material Attributes: Raw material is local Edwards chert (5 fine grained, 1 medium/coarse), nonlocal fine-grained Edwards chert $(\mathrm{n}=2)$, chalcedony $(\mathrm{n}=1)$, and 1 indeterminate heat altered. Mineral precipitates are present on 3 . Heat damage is slight on 1, extensive on 1. No patina was observed.

Tool State and Fractures: Eight are complete or nearly so. Of these, 2 exhibit minor bend damage to the distal sections; 1 , burin-like damage to shoulders; 2 , multiple burinlike and bend damage to distal tip and shoulder areas; 1 , impact damage to blade; and 1, edge crushing. Two others are near full length, although 1 was split vertically by a burinlike fracture, and the other, damaged extensively by thermal spalling.

Rejuvenation: Seven are resharpened unifacially, unbeveled; 2 are alternately beveled; and 1 is indeterminate.

Stratigraphic Distribution: Highest frequency in seen in the Unit IIIb/c transition zone (Table 13-125).

Comments: Travis points from this site tend to have heavily resharpened blades and sharp distal tips, giving these sections a very uniform, oval appearance, a pattern also seen in many Nolan points.

\section{Travis-like $(\mathrm{N}=3)$}

Morphology: These points have expanding stems, straight bases, and convex-edged blades which resemble Travis but are somewhat aberrant from the main group. The specimen shown in Figure 13-120 displays the elongate character and weak shoulders of Travis but is unusually narrow overall. Resharpening may be a factor but is difficult to discern due to burin-like impact fracturing of the blade.

Metric Averages: Table 13-126 
TABLE 13-124

Summary Metric Data for Travis Points (in mm)

\begin{tabular}{l|c|c|c|c|c}
\hline & Number & Minimum & Maximum & Mean & $1-\sigma$ \\
\hline Length & 3 & 45.0 & 54.0 & 50.3 & 4.7 \\
Width & 9 & 19.0 & 27.0 & 23.6 & 2.5 \\
Thickness & 10 & 7.0 & 10.0 & 8.2 & 0.9 \\
Stem length & 10 & 15.0 & 28.0 & 18.9 & 3.7 \\
Proximal stem width & 10 & 17.0 & 22.0 & 18.9 & 1.6 \\
Distal stem width & 4 & 17.0 & 20.0 & 18.5 & 1.3 \\
Basal concavity depth & 1 & 1.0 & 1.0 & & \\
Basal convexity length & 6 & 1.0 & 3.0 & & 0.7 \\
\hline \hline
\end{tabular}

TABLE 13-125

Stratigraphic Distribution of Travis Points

\begin{tabular}{l|c|c|c}
\hline Unit & Valley Floor A & Valley Floor B & Valley Margin \\
\hline IIIc & 5 & 2 & 1 \\
IIIb/c & 1 & & 1 \\
IIIb & & & \\
\hline \hline
\end{tabular}

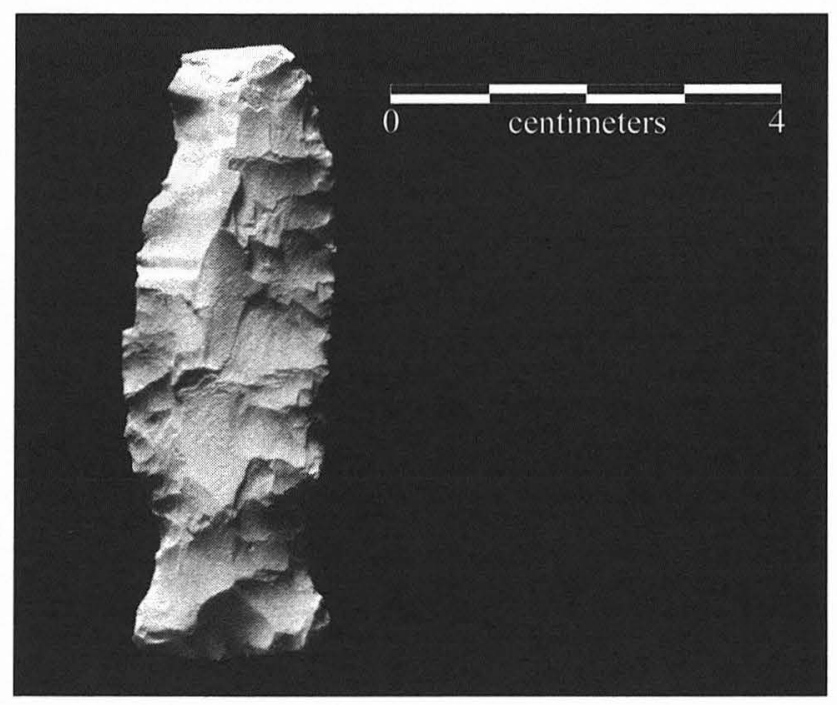

FIGURE 13-120. Travis-like point, Specimen 12E-1.
Nonmetric Attributes: Longitudinal profiles include 1 thick tip and 2 wedge-shaped base and tip. Flake patterns are organized on 2 (collateral, subparallel) and random on 1. Basal modification consists of unifacial thinning on 1 , beveled edge on 1, and secondary trimming on 1. Lateral stem grinding is slight on 1 .

Material Attributes: Raw material is local Edwards chert (1 fine grained, 2 medium/coarse). Mineral precipitates are present on 1. No patina or heat damage was observed.

Tool State and Fractures: Two are complete; the other lacks a distal section due to impact fracturing.

Rejuvenation: One is resharpened bifacially, unbeveled, 1 is alternately beveled, and 1 is indeterminate.

Stratigraphic Distribution: Table 13-127.

$$
\text { Wells }(N=5)
$$

Morphology: These are generally elongate points distinctive for their long, narrow, rectangular to slightly contracting stems, often with dulled or ground lateral edges (Figure 13-121). Blades are triangular in outline and may have small barbs.

Metric Averages: Table 13-128.

Nonmetric Attributes: Longitudinal profiles include 1 tapered base and tip; 1 wedge-shaped base and tip; 1 wedgeshaped base, thick stem, and flat body; and 2 indeterminate. Flake patterns are organized on 2 (collateral, oblique

TABLE 13-126

Summary Metric Data for Travis-like Points (in mm)

\begin{tabular}{l|c|c|c|c|c}
\hline & Number & Minimum & Maximum & Mean & $1-\sigma$ \\
\hline Length & 2 & 42.0 & 51.0 & 46.5 & 6.4 \\
Width & 3 & 21.0 & 26.0 & 23.0 & 2.6 \\
Thickness & 3 & 7.0 & 7.0 & 1.0 & 0.0 \\
Stem length & 3 & 15.0 & 18.0 & 19.7 & 1.5 \\
Proximal stem width & 3 & 19.0 & 20.0 & & \\
Distal stem width & 1 & 19.0 & 19.0 & & \\
Basal concavity depth & 2 & 1.0 & 1.0 & & \\
Basal convexity length & 1 & 2.0 & 2.0 & & \\
\hline \hline
\end{tabular}


TABLE 13-127

Stratigraphic Distribution of Travis-like Points

\begin{tabular}{l|c|c}
\hline Unit & Valley Floor A & Valley Floor B \\
\hline IIIc & 1 & 1 \\
$\begin{array}{l}\text { Other } \\
\text { surface }\end{array}$ & 1 & \\
\hline \hline
\end{tabular}

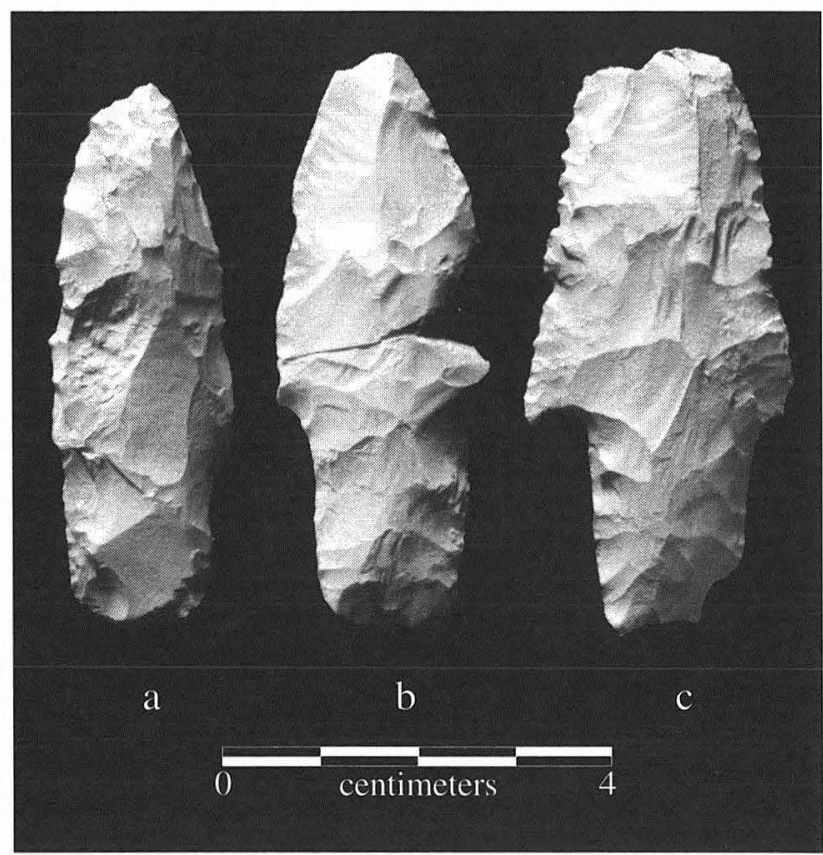

FIGURE 13-121. Wells points. Specimen numbers: (a) 19L-6; (b) 50LA-1; (c) 5D-1.

subparallel), random on 2 , and indeterminate on 1 . Two exhibit an original flake blank section. Basal modification consists of unifacial thinning on 2, concave bevel on 2 , and it is absent on 1. Lateral stem grinding is slight on 2, moderate on 1 , and heavy on 1 .

Material Attributes: Raw material is local Edwards finegrained chert on 4 and indeterminate heat altered on 1. Mineral precipitates are present on 2 . Heat damage is extensive on 2. Patina is not present.
Tool State and Fractures: Two are largely complete, and 1 lacks barb and basal ear tips due to bend and thermal fractures. The 2 others are large proximal sections, 1 of which displays impact damage to the distal section and edge crushing at base, and the other multiple impact, bend, and edge damage.

Rejuvenation: One is resharpened unifacially, unbeveled; 1 is alternately beveled; 1 is beveled unifacially, and 2 are indeterminate.

Stratigraphic Distribution: As shown in Table 13-129, Wells points are found chiefly in upper sectors of Unit III.

Comments: Although more common in East Texas, Wells points have been recovered throughout central Texas and into the Trans-Pecos (Prewitt 1995:135). Temporal range and cultural association is somewhat unclear. Prewitt (1981b) places Wells in the Early Archaic San Geronimo phase with Gower and Hoxie. At the Youngsport site (Shafer 1963), Wells points were found with a Morrill point in a stratum above Gower and below Nolan, Travis, and Bulverde. Distribution of the Wilson-Leonard specimens, although widespread, tends to suggest Middle to Late Archaic affiliations.

TABLE 13-129

Stratigraphic Distribution of Wells Points

\begin{tabular}{l|c|c|c}
\hline Unit & Valley Floor A & Valley Floor B & Valley Margin \\
\hline IIIc & 1 & & \\
IIIb/c & 1 & 1 & \\
III & & & 1 \\
X/Y/IIIb & & & \\
Other & & & \\
IIIc/pot & 1 & & \\
\hline \hline
\end{tabular}

\section{$\operatorname{Gary}(N=2)$}

Morphology: These are rather crude, wide-bladed points with strong shoulders and contracting stems with convex basal edges (Figure 13-122).

Metric Averages: Table 13-130

Nonmetric Attributes: Longitudinal profiles are indeterminate. Flake patterns on both are random. Basal

TABLE 13-128

Summary Metric Data for Wells Points (in mm)

\begin{tabular}{l|c|c|c|c|c}
\hline & Number & Minimum & Maximum & Mean & $1-\sigma$ \\
\hline Length & 2 & 55.0 & 71.0 & 63.0 & 11.3 \\
Width & 3 & 20.0 & 34.0 & 27.3 & 7.0 \\
Thickness & 5 & 7.0 & 9.0 & 24.0 & 1.0 \\
Stem length & 4 & 20.0 & 15.0 & 1.0 & 14.5 \\
Proximal stem width & 4 & 14.0 & 19.0 & 17.2 & 0.6 \\
Distal stem width & 5 & 16.0 & 2.0 & 1.5 & 0.7 \\
Basal concavity depth & 2 & 1.0 & 3.0 & & \\
Basal convexity length & 1 & 3.0 & & \\
\hline \hline
\end{tabular}




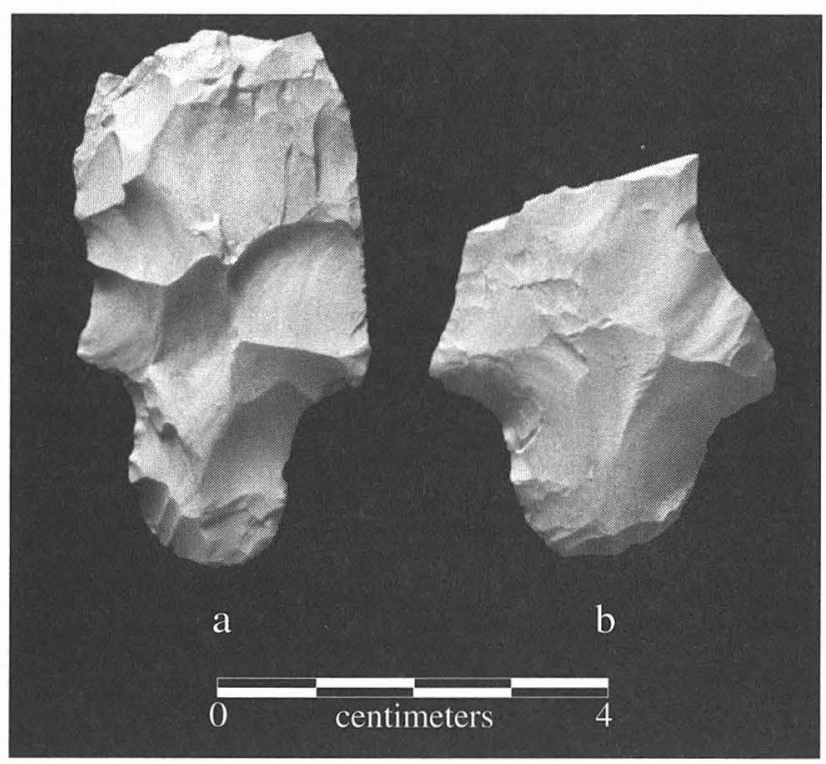

FIGURE 13-122. Gary points. Specimen numbers (a) 10F-1; (b) 11J-9.

TABLE $13-130$

Metric Measurements for Gary Points (in mm)

\begin{tabular}{l|c|c}
\hline & $\begin{array}{c}\text { Specimen } \\
10 \mathrm{~F}-1\end{array}$ & $\begin{array}{c}\text { Specimen } \\
11 \mathrm{~J}-9\end{array}$ \\
\hline Width & 30 & 36 \\
Thickness & 12 & 10 \\
Stem length & 19 & 15 \\
Proximal stem width & 15 & 16 \\
Distal stem width & 17 & 21 \\
Basal convexity length & 3 & 4 \\
\hline \hline
\end{tabular}

modification consists of unifacial thinning on 1 , and it is absent on the other. Lateral stem grinding is slight on 1.

Material Attributes: Raw material is local Edwards finegrained chert for both. Heat damage is moderate on 1. No mineral precipiates or patina were observed.

Tool State and Fractures: Both lack distal blade sections due to thermal fracturing in 1 case and bend breaks, in the other.

Rejuvenation: One is indeterminate and 1 is not resharpened.

Stratigraphic Distribution: Both are from the Valley Margin, 1 from Unit IIIc and the other from Unit IIIb/c.

Comments: A point type more commonly found in East Texas (Turner and Hester 1993:123), some of those found in Central Texas are made of non-Edwards chert.

\section{Long Stemmed ( $N=3)$}

Morphology: This group is comprised of mostly stem sections that are long and very narrow relative to their thickness; stem basal edges vary from a slight bevel to a very small concavity, and lateral edges are ground (Figures 13123 and 13-124).

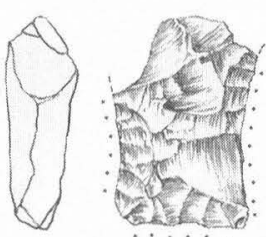

FigURE 13-123. Long-stemmed point, Specimen 50PD-6.

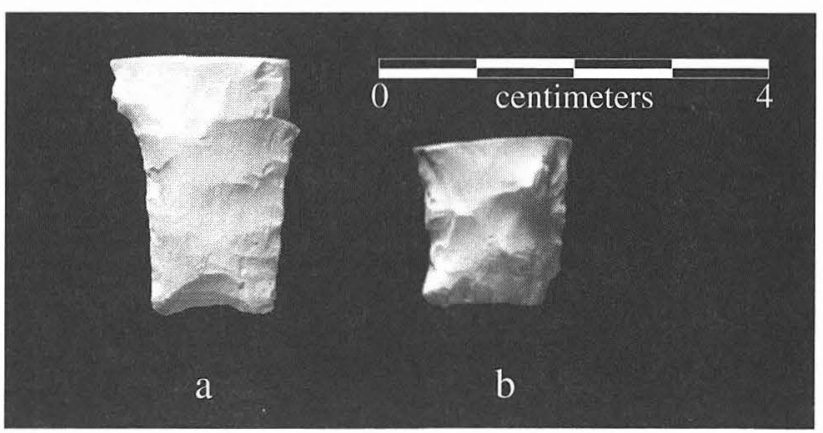

FIGURE 13-124. Long-stemmed points. Specimen numbers: (a) 5921 ; (b) 746-2.

Metric Averages: Table 13-131

Nonmetric Attributes: Longitudinal profiles are indeterminate. Flake patterns are organized on 1 (subparallel) and indeterminate on 2. Basal modification is concave beveling on all 3. Lateral stem grinding is slight on 1, moderate on 1 , and heavy on 1 .

Material Attributes: Raw material is local Edwards chert (2 fine grained, 1 medium/coarse). Mineral precipitates are present on 1 . No heat damage or patina was observed.

Tool State and Fractures: All are stem sections only, displaying multiple bend and burin-like fractures as well as edge crushing on base.

Rejuvenation: Indeterminate.

Stratigraphic Distribution: Table 13-132

Comments: These are unusual stems both in size and shape, as well as stratigraphic distribution; their contexts likely pertain to Early or Middle Archaic periods. They may be fragments of very narrow, ground-stemmed points such as the so-called Victoria point, often classed as Angostura (Kelly 1983b:21; see also Dial 1993:Figure 19d). Bases of similar morphology also are suggested in the Hoxie $\mathrm{C}$ group and early bifurcate stem fragments group (see above). At least 1 of the long-stemmed fragments (592-1) appears to have come from a shouldered point.

\section{Rectangular Stem $A(N=7)$}

Morphology: Not intended as a group, this miscellaneous category encompasses a variety of rectangularstemmed points and stem fragments with varying basal treatments and for which no further classification was possible (Figure 13-125). 
TABLE 13-131

Summary Metric Data for Long-stemmed Points (in mm)

\begin{tabular}{l|c|c|c|c|c}
\hline & Number & Minimum & Maximum & Mean & $1-\sigma$ \\
\hline Thickness & 3 & 7 & 7.3 & 7.1 & 20.3 \\
Stem length & 1 & & & 13.4 & 0.2 \\
Proximal stem width & 2 & 12.7 & 14 & 17.5 & 0.9 \\
Distal stem width & 1 & & & 1.1 & 0.2 \\
Distal stem thickness & 1 & & 1.3 & 7.9 & \\
Basal concavity depth & 3 & 1 & & & \\
Basal concavity width & 1 & & & & \\
\hline \hline
\end{tabular}

TABLE 13-132

Stratigraphic Distribution of Long-stemmed Points

\begin{tabular}{l|c|c}
\hline Unit & Valley Floor A & Valley Floor B \\
\hline IIIb & 1 & \\
IIIa/b & 1 & 1 \\
III & & \\
\hline \hline
\end{tabular}

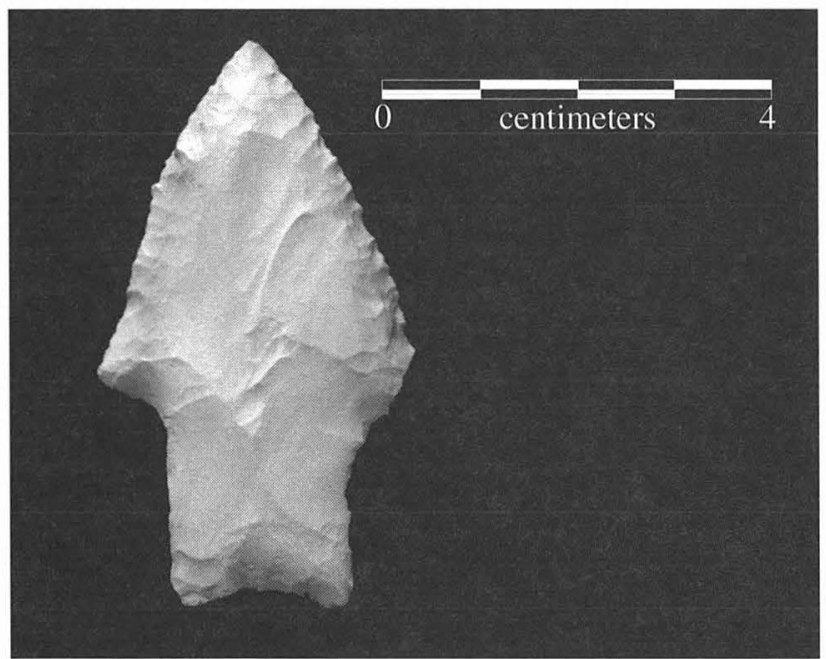

Figure 13-125. Rectangular stem A point, Specimen 22H-4.

\section{Metric Ranges: Table 13-133}

Nonmetric Attributes: Longitudinal profiles include 1 flat; 1 wedge-shaped base, thick stem, flat body; and 5 indeterminate. Flake patterns are random on 2 and indeterminate on 5. Basal modification consists of unifacial thinning on 2, and concave bevel on 5. Lateral stem grinding is moderate on 1 , heavy on 3 .

Material Attributes: Raw material is local Edwards chert (3 fine grained, 2 medium/coarse), 1 nonlocal Edwards finegrained chert, and 1 indeterminate heat altered. Mineral precipitates are present on 1 . Heat damage is slight on 2, moderate on 1 , and extensive on 2.

Tool State and Fractures: One is complete except for a barb severed by thermal spalling. Two are proximal sections, broken by bending and burin-like fractures. The remaining 4 are stems only, all of which display thermal damage.
TABLE 13-133

Metric Ranges for Rectangular Stem A Points (in mm)

\begin{tabular}{l|c|c|c}
\hline & Number & Minimum & Maximum \\
\hline Length & 1 & 59 & 59 \\
Width & 2 & 19 & 37 \\
Thickness & 7 & 6 & 8 \\
Stem length & 5 & 15 & 20 \\
Proximal stem width & 6 & 15 & 19 \\
Distal stem width & 7 & 16 & 20 \\
Basal concavity depth & 7 & 1 & 3 \\
Distal stem thickness & 2 & 7.5 & 7.9 \\
Basal concavity width & 2 & 11.7 & 11.9 \\
\hline \hline
\end{tabular}

Rejuvenation: One is resharpened bifacially, unbeveled, and 6 are indeterminate.

Stratigraphic Distribution: Table 13-134

Comments: Some specimens in this category may be related to Thrall, Bell/Andice, or Bulverde-like points. Damage due to heating likely has skewed classification for these specimens.

\section{Rectangular Stem B $(N=6)$}

Morphology: Not intended as a group, most are fragmented, medium to wide, straight stems with convex basal edges (Figure 13-126). The two more-complete specimens display strong shoulders and barbs.

Metric Ranges: Table 13-135

Nonmetric Attributes: Longitudinal profile and flake patterns could only be discerned on a single specimen that is randomly flaked and displays a tapered base and tip.

TABLE 13-134

Stratigraphic Distribution of Rectangular Stem A Points

\begin{tabular}{l|c|c|c}
\hline Unit & Valley Floor A & Valley Floor B & Valley Margin \\
\hline IIIc & 1 & 1 & 1 \\
IIIb/c & 1 & & 1 \\
IIIb & 1 & & \\
IIIa & 1 & & \\
\hline \hline
\end{tabular}




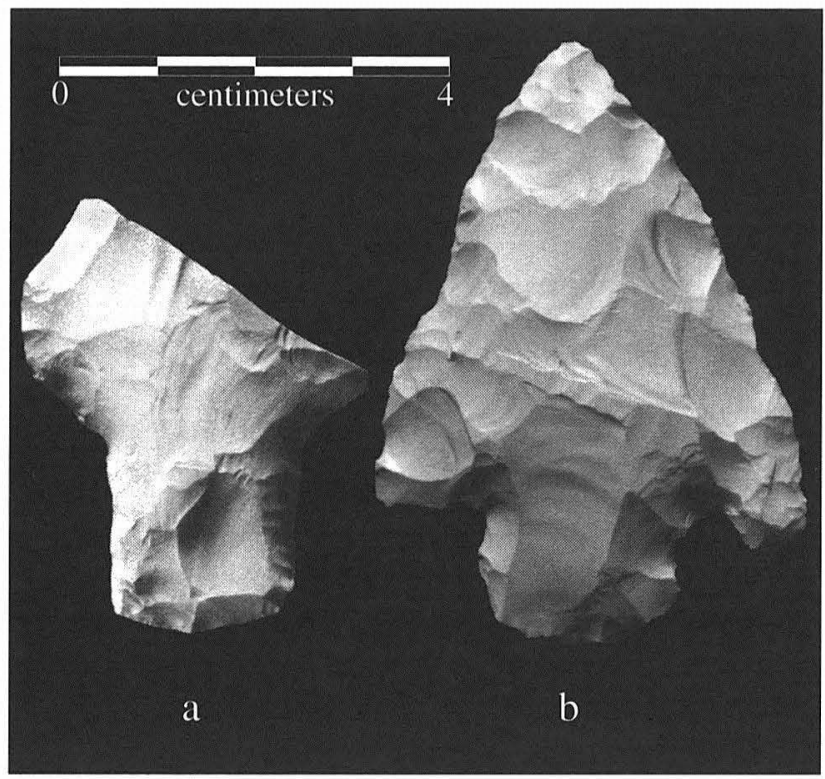

FiguRE 13-126. Rectangular stem B points. Specimen numbers: (a) 50FB-2; (b) 23G-2.

TABLE 13-135

Metric Ranges for Rectangular Stem B Points (in mm)

\begin{tabular}{l|c|c|c}
\hline & Number & Minimum & Maximum \\
\hline Length & 1 & & \\
Width & 2 & 35 & 45 \\
Thickness & 6 & 6 & 12 \\
Stem length & 3 & 14 & 20 \\
Proximal stem width & 5 & 15 & 21 \\
Distal stem width & 3 & 17 & 20 \\
Basal concavity width & 5 & 2 & 3 \\
\hline \hline
\end{tabular}

Basal modification includes 1 bifacially thinned, 1 beveled base, 2 with secondary trimming only, and 2 unmodified. Lateral stem grinding is slight on 1 and heavy on 1 .

Material Attributes: Raw material is local Edwards chert for 5 and indeterminate heat altered for 1 ; mineral precipitates were observed on 1; heat damage is slight on 1 , extensive on another.

Tool State and Fractures: One is largely complete and 1 lacks only distal tip and barb due to bend and thermal fractures; the other 4 are proximal or stem sections, 3 of which exhibit bend breaks, and the other, burin-like impact and thermal damage.

Rejuvenation: Indeterminate on all but 1 , which is unifacially resharpened.

Stratigraphic Distribution: Table 13-136

Comment: Some in this group resemble Bulverde-like points.

\section{Rectangular Stem $C(N=6)$}

Morphology: Not intended as a group, this category includes rectangular stemmed points, most of which have
TABLE 13-136

Stratigraphic Distribution of Rectangular Stem B Points

\begin{tabular}{l|c|c|c}
\hline Unit & Valley Floor A & Valley Floor B & Valley Margin \\
\hline IIIc & 2 & 2 & 1 \\
IIIb/c \\
$\begin{array}{l}\text { Other } \\
\text { surface }\end{array}$ & 1 & & \\
\hline \hline
\end{tabular}

been damaged and cannot be assigned more definitively.

Metric Ranges: Table 13-137

Nonmetric Attributes: Longitudinal profiles include 1 tapered base and tip, 2 wedge-shaped base and tip, and 3 indeterminate. Flake patterns are organized on 2 (oblique subparallel, collateral) and random on 4 . Basal modification consists of unifacial thinning on 2 , beveled base on 2 , and secondary trimming on 2. Lateral stem grinding is slight on 1 , moderate on 1 , and heavy on 1 .

Material Attributes: Raw material is local Edwards chert (3 fine grained, 1 medium/coarse), nonlocal Edwards finegrained chert $(n=1)$, and indeterminate heat altered $(n=1)$; mineral precipitates are present on 1 . Heat damage is slight on 1, extensive on 1. No patina was observed.

Tool State and Fractures: One is largely complete, lacking only the distal tip due to thermal spalling. Two others lack small distal and basal edge sections due to multiple bend and burin-like breaks. Three are proximal sections, 2 of which show bend and edge damage, and the third, bend and thermal damage.

Rejuvenation: Two are alternately beveled, 1 is beveled unifacially, 2 are indeterminate, and 1 is not resharpened.

Stratigraphic Distribution: Table 13-138

TABLE 13-137

Metric Ranges for Rectangular Stem C Points (in mm)

\begin{tabular}{l|c|c|c}
\hline & Number & Minimum & Maximum \\
\hline Length & 2 & 58 & 83 \\
Width & 5 & 25 & 38 \\
Thickness & 5 & 7 & 9 \\
Stem length & 5 & 15 & 17 \\
Proximal stem width & 2 & 14 & 16 \\
Distal stem width & 5 & 15 & 19 \\
Basal concavity depth & 2 & 1 & 2 \\
\hline \hline
\end{tabular}

\section{Square Stemmed $(N=11)$}

Morphology: This miscellaneous collection of square stems and square-stemmed points of varying sizes cannot be assigned further due to their fragmentary condition (Figure 13-127).

Metric Ranges: Table 13-139

Nonmetric Attributes: Longitudinal profiles include 1 
TABLE 13-138

Stratigraphic Distribution of Rectangular Stem C Points

\begin{tabular}{l|c|c|c}
\hline Unit & Valley Floor A & Valley Floor B & Valley Margin \\
\hline IIIc & 1 & 2 & 1 \\
IIIb/c & 1 & & \\
IIIb & 1 & & \\
\hline \hline
\end{tabular}

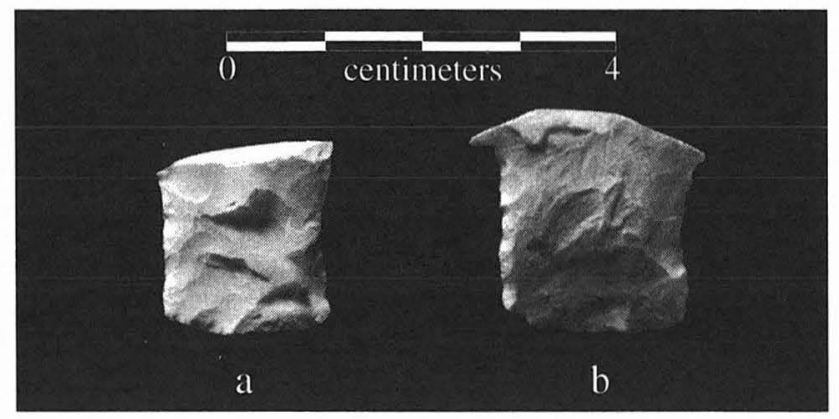

FIGURE 13-127. Square-stemmed points. Specimen numbers: (a) 51HD-11; (b) 15I-16.

TABLE 13-139

Metric Ranges for Square-stemmed Points (in mm)

\begin{tabular}{l|c|c|c}
\hline & Number & Minimum & Maximum \\
\hline Width & 3 & 19 & 38 \\
Thickness & 10 & 4 & 8 \\
Stem length & 4 & 11 & 15 \\
Proximal stem width & 9 & 13 & 19 \\
Distal stem width & 5 & 14 & 17 \\
Basal concavity length & 4 & 1 & 2 \\
Basal concavity depth & 5 & 1 & 2 \\
\hline \hline
\end{tabular}

tapered base and tip, 1 wedge-shaped base, thick stem, flat body; and 9 indeterminate. Flake patterns are organized on 1 (oblique subparallel), random on 1, and indeterminate on 9 . Basal modification consists of unifacial thinning on 2, bifacial thinning on 3 , beveled base on 1 , secondary trimming on 3, 1 indeterminate, and 1 absent. Lateral stem grinding is moderate on 2, heavy on 1 .

Material Attributes: Raw material is local Edwards finegrained chert $(n=7)$, nonlocal Edwards fine-grained chert $(\mathrm{n}=3)$, and indeterminate heat altered $(\mathrm{n}=1)$. Heat damage is extensive on 1. Mineral precipitates and patina are not present.

Tool State and Fractures: The large majority $(\mathrm{n}=8)$ are stems only, 5 of which exhibit single bend breaks; 2 , multiple bend and burin-like breaks; and 1, thermal damage. Two others are proximal sections that exhibit bend, thermal, and burinlike fractures. The remaining point lacks only a distal section, which was severed by a bend fracture.

Rejuvenation: One is resharpened bifacially, unbeveled; 1 is alternately beveled; and 9 are indeterminate.

Stratigraphic Distribution: Table 13-140
TABLE 13-140

Stratigraphic Distribution of Square-stemmed Points

\begin{tabular}{l|c|c|c}
\hline Unit & Valley Floor A & Valley Floor B & Valley Margin \\
\hline IIIc & 3 & 1 & \\
IIIb/c & 2 & & \\
IIIb & 1 & & \\
IIIa/b & 1 & & \\
IIIa & 1 & 1 & \\
III & & & 1 \\
X/II & & & \\
\hline \hline
\end{tabular}

\section{Indeterminate Points $(N=20)$}

Morphology: These are miscellaneous points that were not further classified due to damaged stems on most.

\section{Metric Ranges: Table 13-141}

Nonmetric Attributes: Longitudinal profiles include 5 with tapered base and tip, 1 flat, 7 wedge-shaped base and tip, and 7 indeterminate. Flake patterns are organized on 6 (oblique subparallel, subparallel), random on 7, and indeterminate on 7. Basal modification consists of unifacial thinning on 9, bifacial thinning on 3 , beveling on 1 , absent on 2 , and 5 indeterminate. Lateral stem grinding is slight on 1, moderate on 1 , and indeterminate on 3 .

Material Attributes: Raw material is local Edwards chert (10 fine grained, 2 medium/coarse); nonlocal Edwards chert (4 fine grained, 1 medium coarse); 2 indeterminate heat altered, and 1 indeterminate. Patina is present on 1 . Heat damage is moderate on 2 , extensive on 3 . No mineral precipitates were observed.

Tool State and Fractures: Three are nearly complete, lacking only small basal and distal sections due to thermal and indeterminate fractures. One is a proximal section, broken by bending. The remaining specimens exhibit a combination of bend, thermal, or burin-like/impact fractures that have damaged or removed distal sections on most, basal sections on all but 1 , and barb/shoulder areas on 10 .

Rejuvenation: One is resharpened unifacially, unbeveled, and 2, bifacially, unbeveled; 4 are alternately beveled; 2 , beveled unifacially; and 11 indeterminate.

Stratigraphic Distribution: Table 13-142

TABLE 13-141

Metric Ranges for Indeterminate Points (in mm)

\begin{tabular}{l|c|c|c}
\hline & Number & Minimum & Maximum \\
\hline Length & 6 & 24 & 56 \\
Width & 12 & 16 & 37 \\
Thickness & 20 & 5 & 12 \\
Stem length & 13 & 8 & 20 \\
Proximal stem width & 7 & 8 & 22 \\
Distal stem width & 12 & 12 & 22 \\
Basal concavity depth & 3 & 1 & 2 \\
Distal stem thickness & 1 & 7.5 & 7.5 \\
\hline \hline
\end{tabular}


TABLE 13-142

Stratigraphic Distribution of Indeterminate Points

\begin{tabular}{l|c|c|c}
\hline Unit & $\begin{array}{c}\text { Valley } \\
\text { Floor A }\end{array}$ & $\begin{array}{c}\text { Valley } \\
\text { Floor B }\end{array}$ & $\begin{array}{c}\text { Valley } \\
\text { Margin }\end{array}$ \\
\hline IIIc & 2 & 1 & 5 \\
IIIb/c & 2 & & \\
IIIb & 1 & & 1 \\
IIIa/b & 2 & & 1 \\
Y/IIIc & & 1 & 1 \\
III \\
$\begin{array}{l}\text { Other } \\
\text { provenience unknown (1) } \\
\text { surface (2) }\end{array}$ & & & \\
\hline \hline
\end{tabular}

\section{Unstemmed Triangular}

\section{Early Triangular $(N=5)$}

Morphology: Thin, generally finely flaked triangular points with typically well-thinned bases make up this group. Outlines range in shape from an equilateral to isosceles triangle; edges are sharp, alternately beveled, and some are serrated (Figures 13-128 and 13-129).

Metric Averages: Table 13-143

Nonmetric Attributes: Longitudinal profiles are tapered base and tip on 2, thick tip on 1, 1 flat, and 1 indeterminate. Flake patterns are random on 3 and indeterminate on 2. Basal modification consists of thinning bifacially on 4 and thinning and fluting on 1 .

Material Attributes: Raw material is local $(n=4)$ and nonlocal $(n=1)$ Edwards fine-grained chert; heat damage is slight on 1. Patina and mineral precipitates were not observed.

Tool State and Fractures: Three are complete or nearly so, although 1 displays ragged damage to the base; the 2 others both lack a basal corner and distal tip and exhibit multiple bend, thermal, and indeterminate fractures.

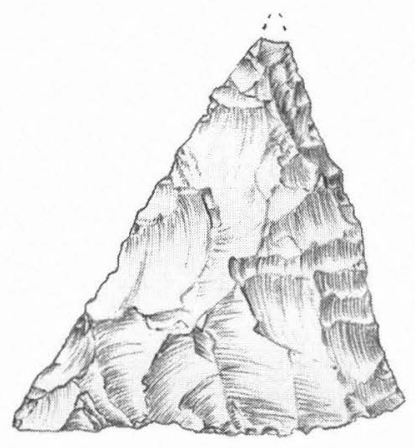

a

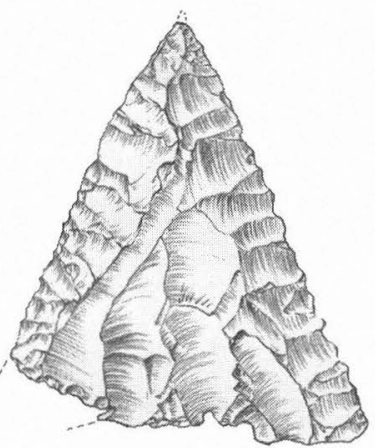

$\mathrm{b}$

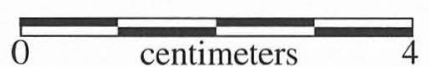

FIGURE 13-128. Early triangular points. Specimen numbers: (a) $16 \mathrm{H}-4$; (b) $14 \mathrm{H}-6$.

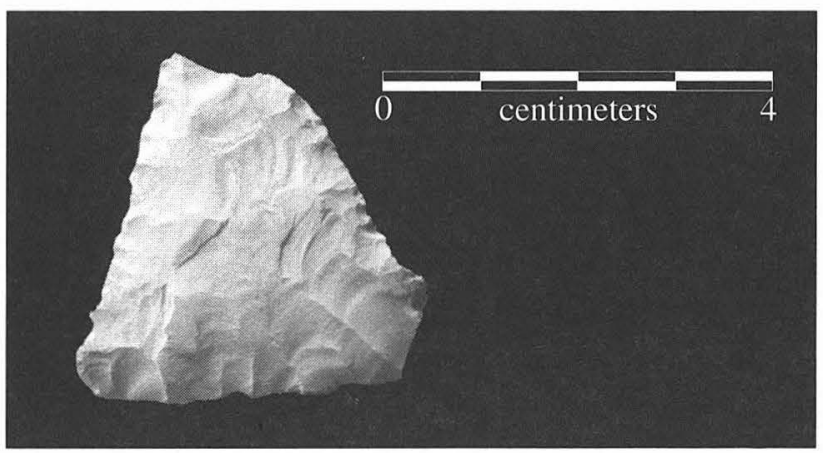

FIGURE 13-129. Early triangular point, Specimen 11K-3.

Rejuvenation: Four exhibit resharpening ( 1 unbeveled unifacially and 3 alternately beveled), and 1 is indeterminate.

Stratigraphic Distribution: The 5 specimens are chiefly confined to Unit IIIb and IIIb/c transition zone (Table 13144).

Comments: In their conjectured manufacturing and uselife sequence, Black and McGraw (1985:Figure 24) model the reduction of Early Triangular points; they hold that the wide variety of sizes and shapes is chiefly a result of use and edge rejuvenation. The likelihood of this scenario tends to mitigate against earlier typological distinctions based on morphology (e.g., the Taylor and Baird forms; see Kelley 1947 and Sorrow 1969). That Early Triangular points may have been preforms for Bell/Andice and/or Bandy, but used (and resharpened) in an unnotched state as knives, also has been suggested (Hester 1995b; McKinney 1981:111); however, dating of Early Triangular points is somewhat uncertain, as is their contemporaneity with those types.

$$
\text { Kinney }(N=1)
$$

Morphology: This elongate, triangular point has convex blade edges and a moderately deep basal concavity (Figure 13-130).

Metric Values: Length is $57 \mathrm{~mm}$; width, $26 \mathrm{~mm}$; thickness, $7 \mathrm{~mm}$; haft grinding length, $17 \mathrm{~mm}$; proximal width, 24 $\mathrm{mm}$; and basal concavity depth, $4 \mathrm{~mm}$.

Nonmetric Attributes: Longitudinal profile is wedgeshaped base and tip. Flake pattern is oblique subparallel. Basal modification is secondary trimming. Lateral stem grinding is slight.

Material Attributes: Raw material is local Edwards finegrained chert.

Tool State and Fractures: This specimen is complete except for a basal ear, removed by overshot, and lateral edge damage due to burin-like fracturing.

Rejuvenation: Resharpened by alternate beveling.

Stratigraphic Distribution: Unit IIII/Fill.

Comments: In size and shape, Kinney resembles many of the early lanceolate points but lacks the basal thinning, characteristic fine flaking, and heavy lateral edge grinding of the haft. 
TABLE 13-143

Summary Metric Data for Early Triangular Points (in mm)

\begin{tabular}{l|c|c|c|c|c}
\hline & Number & Minimum & Maximum & Mean & $1-\sigma$ \\
\hline Length & 3 & 41 & 43 & 42.0 & 1.0 \\
Width & 3 & 31 & 40 & 35.3 & 4.5 \\
Thickness & 4 & 5 & 6 & 5.8 & 0.5 \\
Basal convexity length & 1 & 1 & 1 & & \\
\hline \hline
\end{tabular}

TABLE 13-144

Stratigraphic Distribution of Early Triangular Points

\begin{tabular}{l|c|c|c}
\hline Unit & Valley Floor A & Valley Floor B & Valley Margin \\
\hline IIIc & 2 & 1 & \\
IIIb/c & 1 & & 1 \\
IIIb & & \\
\hline \hline
\end{tabular}

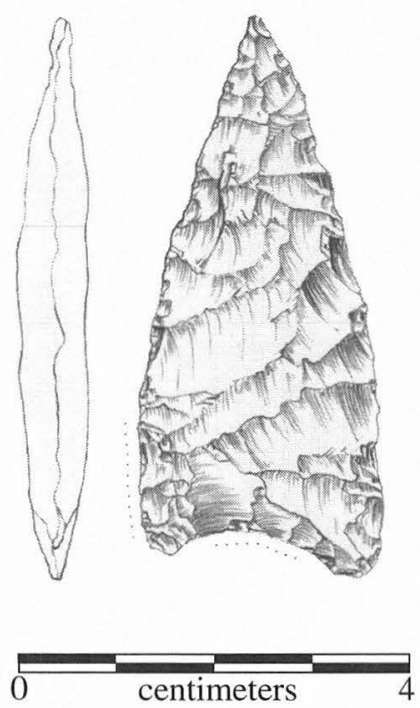

FIGURE 13-130. Kinney point, Specimen 153.

\section{Arrow Points}

$$
\operatorname{Alba}(N=2)
$$

Morphology: Parallel-stemmed points with straight bases, wide, barbed shoulders, and lightly serrated convex blade edges compose this group (Figure 13-131).

Metric Measurements: Table 13-145

Nonmetric Attributes: Longitudinal profiles are both wedge-shaped base and tip. Flake patterns are random on 1, and the other retains sections of the original flake blank on both faces. Basal modification on 1 is beveling; the other is unmodified.

Material Attributes: Raw material is local Edwards chert (1 fine grained, 1 medium/coarse). No mineral precipitates, heat alteration, or patina was observed.

Tool State and Fractures: One is complete, and 1 lacks a distal tip and barb, due to bend fractures.
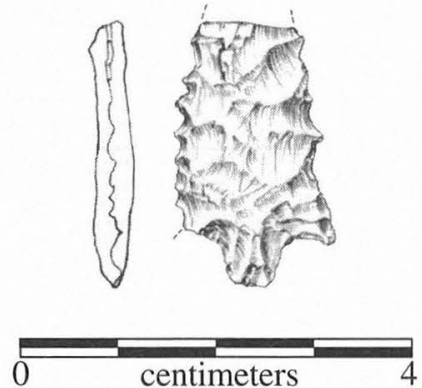

FIGURE 13-131. Alba point, Specimen 0-32.

TABLE 13-145

Metric Measurements for Alba Points (in mm)

\begin{tabular}{l|c|c}
\hline & $\begin{array}{c}\text { Specimen } \\
0-32\end{array}$ & $\begin{array}{c}\text { Specimen } \\
2 \mathrm{~A}-1\end{array}$ \\
\hline Length & & 21 \\
Width & 4 & 10 \\
Thickness & 5 & 2 \\
Stem length & 5 & 4 \\
Proximal stem width & 6 & 6 \\
Distal stem width & & 5 \\
Basal concavity depth & & 1 \\
\hline \hline
\end{tabular}

Rejuvenation: Not present, although both display serration.

Stratigraphic Distribution: One was recovered from Unit IIIc in the Valley Margin, while the other was a surface find.

$$
\text { Scallorn }(N=48)
$$

Morphology: These are small, often finely flaked triangular-bladed points with deep corner or side notches and relatively short- to medium-length expanding stems. Bases are typically straight to slightly concave or convex. There is much variation in size and shape (Figures 13-132 and 13133).

Metric Averages: Table 13-146

Nonmetric Attributes: Longitudinal profiles include 26 wedge-shaped base and tip; 1 thick tip; 1 thick base; 8 flat; 2 tapered base and tip, and 10 indeterminate. Flake patterns are organized on 19 (subparallel, oblique subparallel), random on 12 , indeterminate on 15 , and 2 retain sections of original flake blank. Basal modification consists of unifacial 


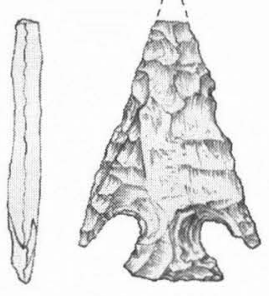

a

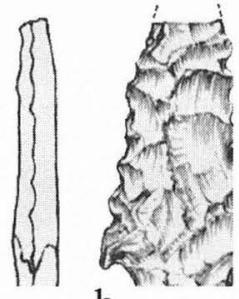

b

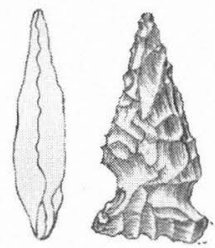

c

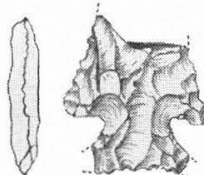

d

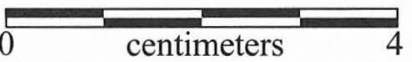

FIGURE 13-132. Scallorn arrow points. Specimen numbers: (a) 3A-2; (b) 9B-8; (c) 8A-3; (d) 0-33.

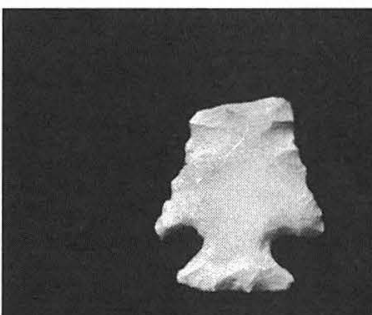

a

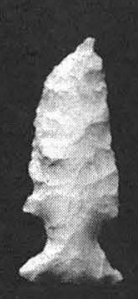

b

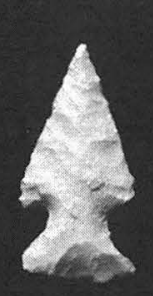

C

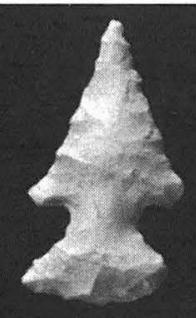

d

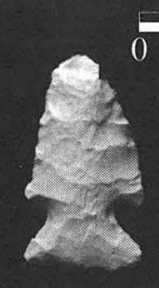

e

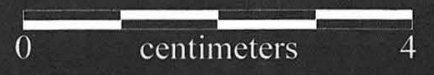

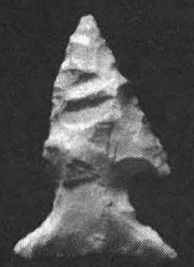

g

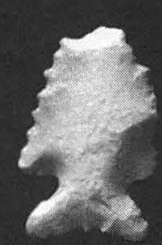

h

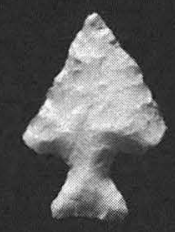

i

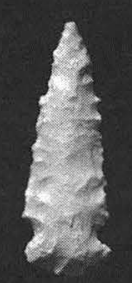

j

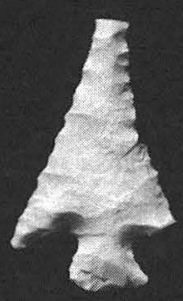

$\mathrm{k}$

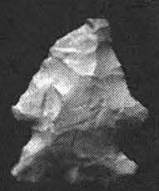

f

FIGURE 13-133. Scallorn arrow points. Specimen numbers: (a) 9B-7; (b) 6A-11; (c) 8A-2; (d) 9A-5; (e) 9A-3; (f) 146-2; (g) 4A-1; (h) 10B1 ; (i) 1838; (j) 9B-9; (k) 0-125; (l) 37AD-22; (m) 168-1.

TABLE 13-146

Summary Metric Data for Scallorn Points (in mm)

\begin{tabular}{l|c|c|c|c|c}
\hline & Number & Minimum & Maximum & Mean & $1-\sigma$ \\
\hline Length & 12 & 20 & 28 & 23.8 & 13.5 \\
Width & 37 & 9 & 21 & 3.3 & 2.8 \\
Thickness & 44 & 2 & 5 & 6.6 & 0.6 \\
Stem length & 44 & 4 & 12 & 10.6 & 1.2 \\
Proximal stem width & 33 & 7 & 14 & 6.3 & 2.0 \\
Distal stem width & 43 & 4 & 9 & 1.0 & 1.1 \\
Basal concavity depth & 15 & 1 & 1 & 1.3 & 0.0 \\
Basal convexity length & 19 & 1 & 3 & & 0.6 \\
\hline \hline
\end{tabular}

thinning on 22 , bifacial thinning on 7 , secondary trimming on 2, absent on 15, and indeterminate on 2. Lateral stem grinding is absent.

Material Attributes: Raw material is local Edwards chert (23 fine grained, 5 medium/coarse); nonlocal Edwards chert ( 8 fine grained, 1 medium/coarse), indeterminate heat altered $(\mathrm{n}=7)$, and indeterminate $(\mathrm{n}=4)$. Mineral precipitates are present on 1. Heat damage is slight on 1, extensive on 5. No patina was observed.

Tool State and Fractures: Five are complete. Eleven others lack only small distal, barb, or basal ear tips. Twentynine are proximal sections, and 3 are stems. Among the 
incomplete, 18 were fractured by single bend breaks, 7 by multiple bend breaks, 2 by impact fractures, 11 by multiple bend and burin-like/impact fractures, 2 by thermal damage, and 3 by multiple bend/hinge, impact, and thermal fractures.

Rejuvenation: Three are resharpened unifacially, unbeveled; 11 bifacially, unbeveled; 4 alternately beveled; 6 beveled unifacially; 14 are indeterminate; and 10 are not resharpened.

Stratigraphic Distribution: As shown in Table 13-147, all Scallorn points derive from Unit IIIc or disturbed contexts near the surface. Of particular note is the markedly patterned spatial distribution: 35 (79\% of those that can be sufficiently provenienced) were recovered from the Valley Margin, and most of these were from contexts that suggest postmidden use of the area.

Comments: Much variability is evinced in the Scallorn assemblage and - as in the dart points - this may be due in large measure to resharpening and maintenance. Serration of edges also is frequent in Scallorn points; a total of 17 (38\% of those sufficiently intact to observe) exhibit serrated blade edges, and at least 4 also display serrated basal edges (e.g., see Figure 13-1321, m).

TABLE 13-147

Stratigraphic Distribution of Scallorn Points

\begin{tabular}{l|c|c}
\hline Unit & Valley Floor A & Valley Margin \\
\hline IIIc fill & 1 & \\
IIIc & 7 & 26 \\
IIIc/pot & & 9 \\
Other & 3 & \\
surface & 2 & \\
provenience unknown & & \\
\hline \hline
\end{tabular}

\section{$\operatorname{Perdiz}(\mathrm{N}=1)$}

Morphology: This triangular point has an unusually short (possibly reworked) contracting stem.

Metric Values: Length is incomplete; width is $33 \mathrm{~mm}$; thickness, $5 \mathrm{~mm}$; stem length, $6 \mathrm{~mm}$; and distal stem width, $7 \mathrm{~mm}$.

Nonmetric Attributes: Longitudinal profile is beveled base and tip. Flake patterns are random. Basal modification is indeterminate.

Material Attributes: Raw material is nonlocal Edwards fine-grained chert, and no patina, mineral precipitates, or heat damage are present.

Tool State and Fractures: This point is complete except for distal and basal ear tips severed by bending fractures.

Rejuvenation: Blade is beveled unifacially.

Stratigraphic Distribution: Unit IIIc, Valley Floor A.

Comments: The single Perdiz was collected as an organic residue sample (OR80) and thus was unwashed; whether the short stem was fractured and/or reworked was difficult to discern. This and a single potsherd are the main evidence for Toyah-interval activity at Wilson-Leonard.

$$
\text { Sabinal }(N=1)
$$

Morphology: This specimen is narrow, elongate, triangular-bladed point with concave blade edges, large barbs, and a relatively long and narrow, slightly expanding stem (Figure 13-134).

Metric Values: Length is incomplete; width is $19 \mathrm{~mm}$; thickness is $4 \mathrm{~mm}$; stem length is $8 \mathrm{~mm}$; proximal stem width is $6 \mathrm{~mm}$; and distal stem width is $5 \mathrm{~mm}$.

Nonmetric Attributes: Longitudinal profile is flat. Flake patterns are subparallel. Basal modification is not present.

Material Attributes: Raw material is local Edwards finegrained chert; no patina, mineral precipitates, or heat damage are present.

Tool State and Fractures: This point lacks a distal tip due to a bend fracture.

Rejuvenation: The blade is resharpened bifacially, unbeveled.

Stratigraphic Distribution: Provenience unknown.

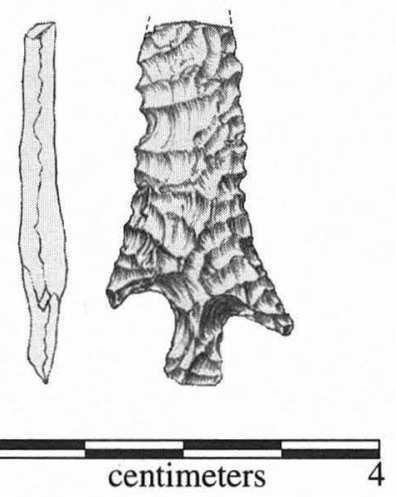

FIGURE 13-134. Sabinal point, Specimen 135.

\section{Scallorn/Edwards $(N=2)$}

Morphology: Although basal sections display the expanding stem and recurved, divided base more typical of Edwards, their small size is atypical of this type (Figure 13-135). Because of their fragmentary nature, classification is uncertain.

Metric Measurements: Table 13-148.

Nonmetric Attributes: Longitudinal profile is wedgeshaped base and tip on 1, indeterminate on the other. Flake patterns are oblique subparallel on 1 , indeterminate on the other. Basal modification consists of unifacial thinning on both.

Material Attributes: Raw material is local Edwards finegrained chert for both; no patina, mineral precipitates, or heat damage were observed.

Tool State and Fractures: Both are proximal sections, 1 damaged by a bend break, the other by bend and impact fractures. 


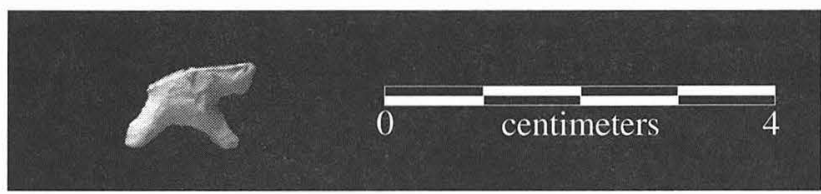

FIGURE 13-135. Scallorn/Edwards point, Specimen 16A-9.

TABLE $13-148$

Metric Measurements of Scallorn/Edwards Points (in mm)

\begin{tabular}{l|c|c}
\hline & $\begin{array}{c}\text { Specimen } \\
14 \mathrm{~B}-1\end{array}$ & $\begin{array}{c}\text { Specimen } \\
16 \mathrm{~A}-9\end{array}$ \\
\hline Width & 12 & \\
Thickness & 3 & \\
Stem grinding length & 4 & 6 \\
Proximal stem width & & 12 \\
Distal stem width & 7 & 7 \\
Basal concavity depth & 2 & 3 \\
\hline \hline
\end{tabular}

Rejuvenation: One is resharpened bifacially, unbeveled; the other is indeterminate.

Stratigraphic Distribution: Both were found in Unit IIIc, Valley Floor A.

\section{Miscellaneous Stemmed Arrow Points $(N=20)$}

Morphology: This is a miscellaneous group of arrow points and arrow point blade fragments that retain enough of the proximal section to indicate that the point was originally stemmed; however, no further classification was possible (Figure 13-136).

Metric Averages: Table 13-149

Nonmetric Attributes: Longitudinal profiles are indeterminate. Flake patterns are organized on 12 (subparallel, chevron, oblique subparallel), random on 4 , and indeterminate on 4. Basal modification is indeterminate.

Material Attributes: Raw material is local Edwards chert (7 fine grained, 7 medium/coarse); nonlocal fine-grained

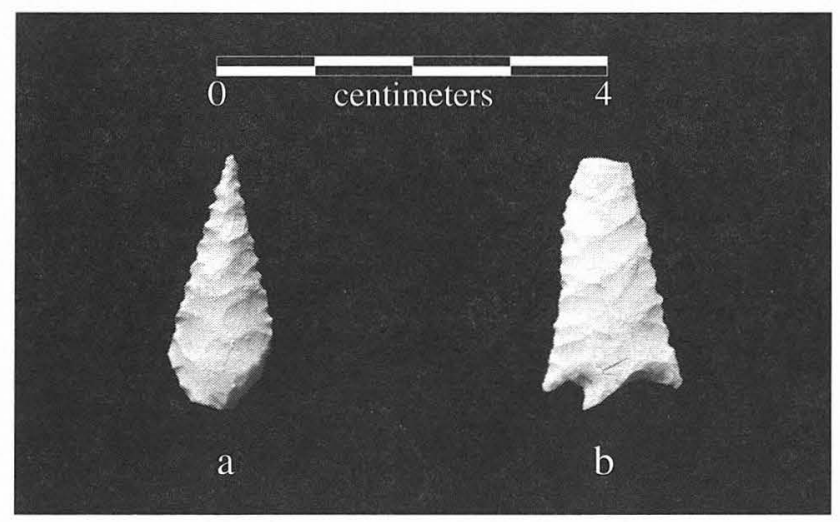

FIGURE 13-136. Miscellaneous stemmed arrow points. Specimen numbers: (a) $8 \mathrm{~B}-4$; (b) $5 \mathrm{~A}-2$.
TABLE 13-149

Metric Ranges for Miscellaneous

Stemmed Arrow Points (in mm)

\begin{tabular}{l|c|c|c}
\hline & Number & Minimum & Maximum \\
\hline Width & 11 & 11 & 23 \\
Thickness & 17 & 2 & 5 \\
Stem length & 5 & 4 & 9 \\
Proximal stem width & 4 & 6 & 9 \\
Distal stem width & 10 & 5 & 9 \\
\hline \hline
\end{tabular}

Edwards chert $(n=3)$; indeterminate heat altered $(n=2)$, and indeterminate $(n=1)$. Patina is slight overall on 1 and moderate partial on 1. Mineral precipitates are present on 1. Heat damage is slight on 2, extensive on 3 .

Tool State and Fractures: All — by virtue of definitionare blade sections, 15 of which exhibit bend fracturing; 2 , thermal fractures; 1, burin-like/impact break; and 2, multiple bend, thermal, and impact breaks.

Rejuvenation: One is unifacially beveled, 3 are alternately beveled, 13 are indeterminate, and 3 are not resharpened.

Stratigraphic Distribution: As in Scallorn points, the highest frequency (12 of 20, or 60\%) for the stemmed miscellaneous group is seen in Unit IIIc in the Valley Margin (Table 13-150).

Comments: The presence of patina on 2 arrow points is somewhat unusual. Interestingly, one was patinated after burning.

TABLE $13-150$

Stratigraphic Distribution of Miscellaneous Stemmed Arrow Points

\begin{tabular}{l|c|c}
\hline Unit & Valley Floor A & Valley Margin \\
\hline IIIc & 6 & 7 \\
Other & 1 & \\
IIIc fill & & 5 \\
IIIc/pot & 1 & \\
surface & & \\
\hline \hline
\end{tabular}

\section{Arrow Point, Preform (N=9)}

Morphology: These are small, thin, generally triangular bifaces with convex to straight lateral edges, some of which have been retouched (Figures 13-137 and 13-138). Basal shapes vary from straight to rounded. One specimen has been notched on one basal corner.

Metric Averages: Table 13-151

Nonmetric Attributes: Longitudinal profiles include 5 flat; 2 wedge-shaped base and tip; 1 tapered base and tip; and 1 indeterminate. Flake patterns are organized on 3 (subparallel, oblique subparallel, collateral), random on 5, and indeterminate on 1; 1 displays a section of original flake 


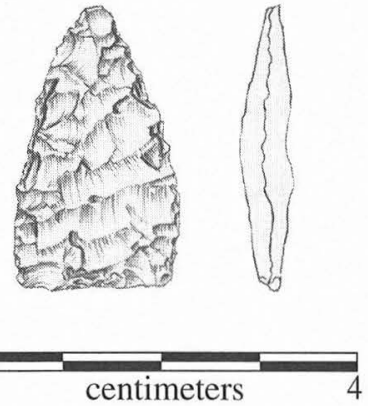

FIGURE 13-137. Arrow point preform, Specimen 9B-5.

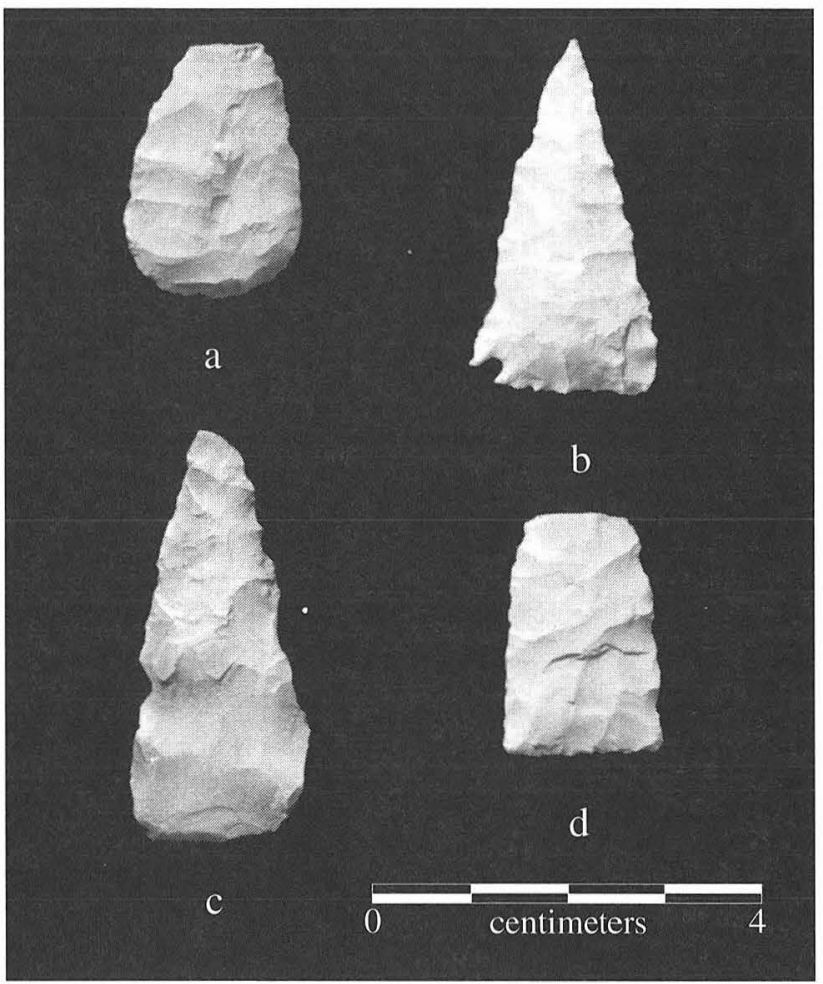

FIGURE 13-138. Arrow point preforms. Specimen numbers: (a) 1A3 ; (b) 4A-3; (c) 6A-2; (d) 16A-5.

blank. Basal modification consists of unifacial thinning on 2, bifacial thinning on 1 , secondary trimming on 4 , and is absent on 2 .

Material Attributes: Raw material is local Edwards chert (1 fine, 3 medium/coarse); nonlocal Edwards chert (2 fine, 1 medium/coarse); indeterminate heat altered $(\mathrm{n}=1)$, and inde- terminate $(n=1)$. Patina is differential on 1 . Heat damage is extensive on 1. No mineral precipitates were observed.

Tool State and Fractures: Five are complete or nearly so and another has been thermally damaged on the lateral edge. Two are proximal sections that lost distal tips to bend fractures. The remaining specimen is a blade section, broken by bend fracturing.

Rejuvenation: None displays resharpening.

Stratigraphic Distribution: As in the arrow point groups described above, arrow point preforms were recovered in highest frequency in the Valley Margin ( 7 of 9 or $78 \%$; Table 13-152).

Comments: The diminutive size and lack of blade resharpening suggests these bifaces were likely preforms for arrow points but were either broken or abandoned during final manufacturing stages. The differential patina on one suggests that a flake from an earlier context may have been recycled.

\section{Projectile Point Fragments}

This category includes projectile point fragments $(\mathrm{N}=350)$ that could not be placed into descriptive or typological projectile point categories. Although chiefly small sections, this group includes some larger points with badly damaged bases.

Several factors should be considered in evaluating the data from projectile point fragments. Relative counts among the six fragment categories (distal, medial, base, barb, blade, and indeterminate) are biased somewhat in that the large majority of basal fragments is reported in the main projectile point section. This was done so that as many specimens as possible could be evaluated typologically and chronologically. On the other hand, some very small fragments might well have been assigned to the biface fragments rather than the projectile point fragments category. As a general rule, the two categories were distinguished chiefly according to the degree of finishing or resharpening, with less-finished specimens assigned to the biface fragment group (presented in Chapter 16).

Distribution of projectile point fragments by stratigraphic unit and within the six fragment categories is presented in Table 13-153. As is shown, the highest counts of fragments are present in Unit IIIc (73 in the Valley Floor and 52 in the Valley Margin). Relatively few occur in contexts

TABLE 13-151

Summary Metric Data for Arrow Point Preforms (in mm)

\begin{tabular}{l|c|c|c|c|c}
\hline & Number & Minimum & Maximum & Mean & $1-\sigma$ \\
\hline Length & 6 & 20 & 41 & 32.0 & 7.1 \\
Width & 8 & 13 & 20 & 17.5 & 2.2 \\
Thickness & 8 & 3 & 5 & 4.4 & 0.7 \\
Proximal stem width & 2 & 10 & 16 & 13.0 & 4.2 \\
Basal concavity depth & 5 & 16 & 20 & 17.4 & 1.7 \\
\hline \hline
\end{tabular}


TABLE 13-152

Stratigraphic Distribution of Arrow Point Preforms

\begin{tabular}{l|c|c}
\hline Unit & Valley Floor A & Valley Margin \\
\hline IIIc & 2 & 5 \\
Other & & 2 \\
IIIc/pot & & \\
\hline \hline
\end{tabular}

lower than those pertaining to the Archaic. A total of 18 were recovered from Unit II, and only 1 was found in Unit Isi-c.

When distribution is further evaluated according to fragment section (and discounting the base sections), it appears that fragments were discarded in relatively consistant proportions throughout the stratigraphic units and across the defined spatial parameters. Distal sections consistently are most frequent, followed by medial and barb fragments.

As a group, projectile point fragments exhibit a pattern of material characteristics similar to that of the larger projectile point assemblage (Table 13-154). Raw materials identified are predominately local Edwards cherts (49\% fine grained, $5 \%$ coarse), with a smaller proportion of nonlocal Edwards cherts ( $7 \%$ fine grained, $3 \%$ coarse). One fragment from the Unit IIIa/b transition zone appears to be of chalcedony. An additional $15 \%$ of total specimens are indeterminate due to heat alteration or are unidentified (21\%).

TABLE 13-153

Stratigraphic Distribution of Projectile Point Fragments

\begin{tabular}{|c|c|c|c|c|c|c|c|}
\hline Stratigraphic Unit & Distal & Medial & Base & Barb & Blade & Indeterminate & Totals \\
\hline Valley Floor A: & & & & & & & \\
\hline IIIc/Fill & 2 & 1 & & 1 & & & 4 \\
\hline IIIb/c/Fill & 1 & & & & & & 1 \\
\hline IIIc & 29 & 18 & 8 & 13 & 1 & 4 & 73 \\
\hline $\mathrm{IIIb} / \mathrm{c}$ & 11 & 4 & & 6 & & 3 & 24 \\
\hline IIIb & 10 & 7 & 7 & 7 & & 3 & 34 \\
\hline $\mathrm{IIII} / \mathrm{b}$ & 9 & 5 & 3 & 2 & 2 & & 21 \\
\hline IIIa & 11 & 8 & 1 & 6 & 1 & 4 & 31 \\
\hline II/IIIa & 1 & 3 & & & & 4 & 8 \\
\hline II & 6 & 3 & 2 & 3 & & 1 & 15 \\
\hline $\mathrm{Id} / \mathrm{II}$ & 3 & 1 & 1 & & & & 5 \\
\hline Isi-c & & & & 1 & & & 1 \\
\hline Unknown & 2 & 2 & & 1 & & & 5 \\
\hline Valley Floor B: & & & & & & & \\
\hline IIIc & 3 & 4 & 2 & & & 2 & 11 \\
\hline III & & & & 1 & & & 1 \\
\hline II/III & 1 & & & & & & 1 \\
\hline Valley Margin: & & & & & & & \\
\hline IIIc & 20 & 13 & 3 & 10 & 1 & 5 & 52 \\
\hline IIIc/Pot & 4 & 4 & & 1 & & 1 & 10 \\
\hline $\mathrm{IIIb} / \mathrm{c}$ & 3 & 2 & 2 & & & & 7 \\
\hline Y/IIIc & & & 1 & & 1 & & 2 \\
\hline IIIb & 3 & & 1 & 3 & & 1 & 8 \\
\hline Y & 1 & 2 & & & & & 3 \\
\hline X/Y/IIIb & & 1 & & & & & 1 \\
\hline X/IIIb & 1 & 2 & & & & & 3 \\
\hline $\mathrm{X} / \mathrm{Y}$ & & & & 1 & & & 1 \\
\hline IIIa & & & & 2 & & & 2 \\
\hline II/IIIa & & & & 1 & & & 1 \\
\hline X/II/IIIa & 1 & 1 & & & 1 & & 3 \\
\hline$X$ & & 1 & 1 & 2 & & & 4 \\
\hline II & & 1 & & 1 & & 1 & 3 \\
\hline Totals: & 122 & 83 & 32 & 62 & 7 & 29 & 335 \\
\hline
\end{tabular}

Note: This table does not include 15 fragments of unknown Valley Floor/Margin proveniences. 
TABLE 13-154

Material Characteristics of Projectile Point Fragments

\begin{tabular}{|c|c|c|c|c|c|c|c|c|c|c|c|c|c|c|c|c|}
\hline \multirow[b]{3}{*}{ Unit } & \multicolumn{7}{|c|}{ Raw Material } & \multicolumn{2}{|c|}{ Patina } & \multicolumn{2}{|c|}{ Mineral Precipitates } & \multicolumn{5}{|c|}{ Heat Alteration } \\
\hline & \multicolumn{4}{|c|}{ Edwards Chert } & \multirow[b]{2}{*}{ 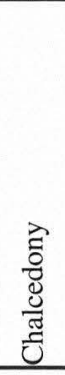 } & \multirow[b]{2}{*}{ 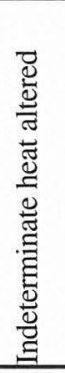 } & \multirow[b]{2}{*}{ 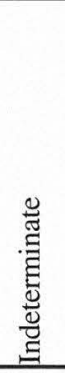 } & \multirow[b]{2}{*}{ 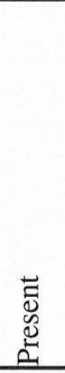 } & \multirow[b]{2}{*}{$\begin{array}{l}\overrightarrow{0} \\
\overline{0} \\
\stackrel{0}{4}\end{array}$} & \multirow[b]{2}{*}{ 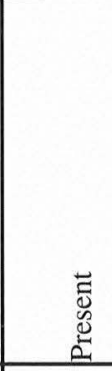 } & \multirow[b]{2}{*}{ 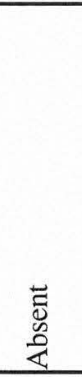 } & \multirow[b]{2}{*}{ 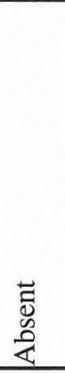 } & \multirow[b]{2}{*}{$\begin{array}{l}\frac{ \pm}{60} \\
\frac{-1}{05}\end{array}$} & \multirow[b]{2}{*}{$\begin{array}{l}\frac{\pi}{\pi} \\
\frac{\overrightarrow{0}}{0} \\
\sum\end{array}$} & \multirow[b]{2}{*}{ 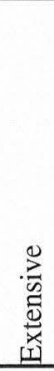 } & \multirow[b]{2}{*}{ 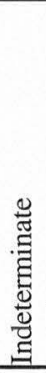 } \\
\hline & 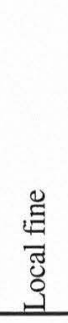 & 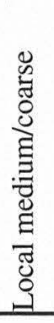 & 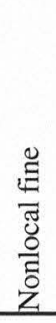 & 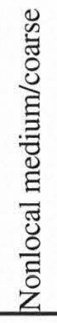 & & & & & & & & & & & & \\
\hline Surface & 8 & & 1 & 1 & & 3 & 1 & 4 & 10 & & 14 & 12 & & 1 & 1 & \\
\hline IIIc/Fill & & & & & & & 4 & & 4 & 1 & 3 & 4 & & & & \\
\hline IIIc/pot & 5 & & 2 & & & 3 & & & 10 & 1 & 9 & 5 & 1 & & 4 & \\
\hline IIIc & 70 & 7 & 8 & 3 & & 17 & 31 & 6 & 130 & 9 & 127 & 93 & 7 & 3 & 32 & 1 \\
\hline IIIb/c/Fill & & & & & & & 1 & 1 & & & 1 & & & & 1 & \\
\hline $\mathrm{IIIb} / \mathrm{c}$ & 17 & 1 & 2 & 1 & & 3 & 7 & & 31 & 1 & 30 & 21 & 2 & 1 & 7 & \\
\hline IIIb & 21 & 2 & 3 & & & 5 & 11 & & 42 & 1 & 41 & 27 & 2 & & 13 & \\
\hline $\mathrm{IIIa} / \mathrm{b}$ & 10 & & 2 & 1 & 1 & 3 & 4 & 1 & 20 & & 21 & 12 & & 2 & 7 & \\
\hline IIIa & 14 & 2 & & 1 & & 7 & 9 & 1 & 32 & 1 & 32 & 16 & 4 & 1 & 11 & 1 \\
\hline III & 1 & & & & & & & & 1 & & 1 & & 1 & & & \\
\hline II/IIIa & 5 & 2 & & & & 2 & & 2 & 7 & & 9 & 3 & 2 & & 4 & \\
\hline II/III & 1 & & & & & & & & 1 & & 1 & & & & 1 & \\
\hline II & 7 & 4 & 1 & & & 3 & 3 & 1 & 17 & 3 & 15 & 12 & 1 & & 5 & \\
\hline $\mathrm{Id} / \mathrm{II}$ & 2 & & 1 & & & 1 & 1 & & 5 & 1 & 4 & 3 & & 1 & 1 & \\
\hline Isi-c & 1 & & & & & & & & 1 & & 1 & 1 & & & & \\
\hline $\mathrm{X} / \mathrm{Y} / \mathrm{IIIb}$ & 1 & & & & & & & & 1 & 1 & & 1 & & & & \\
\hline X/IIIb & 3 & & & & & & & & 3 & & 3 & 1 & 1 & & 1 & \\
\hline X/II/IIIa & 1 & & & 2 & & & & & 3 & & 3 & 3 & & & & \\
\hline Y/IIIc & 1 & & & & & 1 & & & 2 & 2 & & 1 & & & 1 & \\
\hline $\mathrm{Y}$ & 2 & & & & & & 1 & & 3 & 1 & 2 & 3 & & & & \\
\hline $\mathrm{X} / \mathrm{Y}$ & 1 & & & & & & & & 1 & & 1 & 1 & & & & \\
\hline $\mathrm{X}$ & 2 & & 1 & 1 & & & & 1 & 3 & 2 & 2 & 4 & & & & \\
\hline Unknown & & & 2 & & & 3 & 1 & 1 & 5 & & 6 & 2 & & 1 & 3 & \\
\hline Totals: & 173 & 18 & 23 & 10 & 1 & 51 & 74 & 18 & 332 & 24 & 326 & 225 & 21 & 10 & 92 & 2 \\
\hline
\end{tabular}

Note: Specimens from surface, disturbed contexts, or unknown provenience not shown.

Patina and mineral precipitates were observed on a small number of fragments. Most were from contexts with such small sample sizes as to be of inconclusive significance. Patina was present on only 18 specimens, with one of the highest frequencies in the Unit II/IIIa transition zone ( 2 of 9 or $22 \%$ ). Mineral precipitates were noted on 24 specimens from almost all stratigraphic units, and most occurred in deposits with small sample sizes. As in the projectile points, a high frequency again was seen in Unit II specimens ( 3 of 15 or $20 \%$ ).

Heat alteration was observed on a total of 123 specimens. Highest frequencies (among samples large enough to consider) are seen in middle stratigraphic units, largely pertaining to Late Paleoindian and Early Archaic occupations.
In Unit II/IIIa, 6 of the 9 (67\%) fragments recovered show heat alteration, and 4 of those extensively so. The Unit IIIa group is similar with 16 of 33 or $48 \%$ of the fragments recovered showing heat alteration, with $11(69 \%)$ severely damaged. Somewhat lower frequencies were observed in samples from higher strata. Fifteen of $42(36 \%)$ are heat altered from Unit IIIb, and of those, 13 (87\%) are extensively damaged. In Unit IIIc, 42 of 136 fragments or $31 \%$ are heat altered, and of those, $32(76 \%)$ are severely heat damaged. The pattern in Unit IIIb/c is similar with $32 \%$ heat altered (10 of 31 ) and 7 (70\%) severely damaged.

An additional 20 fragments that may pertain to specific morphological projectile point categories are presented separately (Table 13-155). Included are 7 beveled edge sections 
TABLE 13-155

Distribution of Technologically Significant Projectile Point Fragments

\begin{tabular}{l|c|c|c|c|c|c}
\hline Unit & $\begin{array}{c}\text { Arrow Point } \\
\text { Medial Fragment }\end{array}$ & $\begin{array}{c}\text { Arrow Point } \\
\text { Tip Fragment }\end{array}$ & $\begin{array}{c}\text { Beveled Medial } \\
\text { Fragment }\end{array}$ & $\begin{array}{c}\text { Beveled Tip } \\
\text { Fragment }\end{array}$ & $\begin{array}{c}\text { Rectangular } \\
\text { Eccentric }\end{array}$ & $\begin{array}{c}\text { Notched } \\
\text { Flake }\end{array}$ \\
\hline IIIc & 3 & 8 & 1 & & 1 & 1 \\
IIIb & & & 1 & 1 & & \\
IIIa & & & 1 & 1 & & \\
II/IIIa & & & 1 & & & \\
II & & & 1 & & & \\
\hline \hline
\end{tabular}

(medial and tip) that likely relate to the heavily resharpened early bifurcate types (e.g., Hoxie) and 11 diminutive medial and tip sections that likely are arrow point fragments. While not a projectile point fragment, a primary flake with deep notch (see Figure 13-108e) is reported here. It was found in Unit IIIb and is of interest here inasmuch as it may have been a practice piece for executing the deep Bell/Andice basal notches. Points of that type were found chiefly in Unit $\mathrm{IIIb}$ and IIIb/c. A fragment of an eccentric point with multiple notches also was recovered in Unit IIII.

\section{SUMMARY AND DISCUSSION: THE WILSON- LEONARD PROJECTILE POINT ASSEMIBLAGE}

Projectile points recovered from the Wilson-Leonard site include representatives from every identified interval in the current Central Texas archeological sequence (Collins 1995; Johnson and Goode 1994; Prewitt 1985), spanning technologies from earliest Paleoindian times to the latter part of the Late Prehistoric. Given this extensive time depth, broad patterns and trends in projectile point manufacturing and use can be observed. In the following discussion, some of these behavioral patterns are briefly highlighted, problems in projectile point typology and classification are noted, and finally, the types of weapons upon which these projectile tips may have been deployed are considered.

\section{Types, Distribution, and Naming Dilemmas}

Of the total 1,003 projectile points, 591 were identified to 1 of 38 previously defined types or possible type variants, 60 were assigned to 1 of 3 proposed new types, and 352 were placed in descriptive or miscellaneous morphological groups. An additional 350 specimens were classified as projectile point fragments.

Among identified types, Scallorn arrow points were most numerous, totaling 48 , followed by Nolan with 45 specimens; 3 other specimens were identified as Nolan-like. Other high counts include Ensor with 37; Angostura with 31; Bulverde with 29, with an additional 19 classified as Bulverde-like; and Hoxie with 26, with 19 others classified as Hoxie variants
(A, B, C) for a total of 45 . It is noteworthy that, with the exception of Angostura and Hoxie, most of the above-mentioned specimens were recovered from upper portions of the site that were fully excavated only in Blocks 1-5.

Notwithstanding potentially important cultural information, these counts chiefly may serve to underscore the integrity of some of the type definitions and the relative ease with which many specimens can be assigned. The large Nolan count, for instance, is perhaps not surprising given that the type is one of the most distinctive and least controversial.

In contrast, some 135 early bifurcate-stem points reside in descriptive or mixed-type categories (signified by slash marks, question marks, or the suffix "like"). These classifications, encompassing many variants of the so-called early split stem and early corner-notched types, reflect some of the problems that thwart Early Archaic typology, specifically, types that overlap in many of their attributes.

When these points are plotted stratigraphically, however, the strength of broader morphological groupings is illuminated. As shown in Table 13-156, the more-strongly bifurcate varieties that tend to have ground, more-parallel-sided stems chiefly occur earlier in the site's history, whereas the more-expanding stem groups emerge somewhat later. Among these groups, Hoxie and Hoxie variants $\mathrm{A}$ and $\mathrm{B}$ are shown to generally predate other bifurcate types such as Gower, occurring predominately in Units II/IIIa and IIIa. Gower, Jetta, and Hoxie C occur in greatest numbers in slightly higher deposits, at the juncture of Unit IIIa and Unit IIIb and above. As shown in the center of Table 13-156, several unclassified expandingstem and contracting-stem groups mark a gradual transition into the early corner-notched categories. These latter generally are expanding-stem points exhibiting shallower and broader basal concavities and typically unground stems (e.g., Martindale, Uvalde, Bandy); they are most numerous in Unit IIIb.

Given the morphological continuum evinced in the early bifurcate groups, use of the terms Early Split Stem and Early Corner Notched is clearly appropriate in many cases, as Johnson (1991), Prikryl (1990), Hester (1971), and others have advocated. Unfortunately, these collective terms have acquired a variety of differing connotations, creating yet 
another layer of obfuscation in Early Archaic projectile point classifications (see Chapter 14 for further discussion). What is clearly needed is a consensus on what these collective terms imply. Based on the Wilson-Leonard assemblage, it is suggested that the term Early Split Stem be reserved for the earlier bifurcate forms that typically display ground, straighter-edged stems with concave beveling of base. The term Early Corner Notched would apply to the expandingstemmed forms with less-pronounced basal concavities and typically unground lateral stem edges. In cases where formal types can be identified, use of the broad category along with the form (e.g., Early Split Stem, Gower form) has been suggested (Johnson 1991). Clearly, this system will not solve all problems but may serve to highlight the more salient, time-sensitive attributes among the bifurcate groups.

The statistical analysis of metric attributes of early bifurcate stemmed and unfluted lanceolate points presented in Chapter 14 further considers typological issues. While the study of unfluted lanceolate points resulted in groupings which, in many cases, have both morphological and some temporal integrity, results of the early bifurcate-stem analysis served to emphasize the variability that has caused confusion in Early Archaic typology.

As shown in the provenience tables for projectile points (Tables 13-157 and 13-158), Angostura points also occur in highest frequencies in Early Archaic deposits along with the early bifurcate points. Judging from specimens at this site, there appears to be a certain degree of morphological and technological continuity between some early bifurcate forms (e.g., certain Hoxie variants) and Angostura (see Figure 13-4f and j; 29T1C-1, 11Q-1), namely lateral stem edge grinding; shallow, ground basal concavity in an arc-shaped configuration; and long narrow stems or bases. Both types may also exhibit alternate beveling of blade and oblique parallel flaking. As noted in Chapter 14, Angostura is a widely distibuted but poorly understood type encompassing a great degree of variability. Hoxie is poorly known, appearing in a much more localized area chiefly confined to the prairies and timbers east of the Edwards Plateau (see Prewitt 1995:111).

Given their similarities, it is unfortunate that time constraints prevented the merging and comparison of the bifurcate stem data with that of Angostura in the statistical analysis of metric attributes (see Chapter 14). What is clear from the present study is the large range of morphological variability encompassed in the intuitive Angostura "type." This degree of variability, from a technological standpoint, is more akin to that demonstrated in the early bifurcate sample than that of the more-standardized unfluted lanceolate points.

From a broad perspective, projectile point morphology can be seen to trend over time at the Wilson-Leonard site from early fluted and unfluted lanceolate, to ground expanding stem (Wilson) and late unfluted lanceolate, to ground stem bifurcate (early split stem), expanding stem bifurcate (early corner notched), rectangular and bifurcate stemmed, large expanding stem, small expanding stem, and finally into the diminutive arrow point varieties (see Tables 13-157 and 13-158). Although much of this pattern has been previously identified (with the notable exception of the Late Paleoindian expanding-stem Wilson), the Wilson-Leonard sequence provides a nearly continuous chronology and substantiation from a single site.

\section{Tool State and Fracture Patterns}

Among the full projectile point assemblage, certain categories exhibit markedly higher frequencies of complete or nearly complete points (i.e., those lacking only distal, barb, or basal ear tips, or combinations thereof). While admittedly somewhat arbitrary, the "nearly complete" category is nonetheless included, with the goal of deriving an "index" of relative completeness for point categories throughout the site's history. This in turn may provide a very conditional means of conceptualizing the likely correlated factors of intensity of tool use, efficiency of various haft technologies, the relative strength of certain material types, or simply what activities in relation to the lifecycle of projectile points transpired at Wilson-Leonard.

Highest percentage of completeness is seen among Travis points ( 8 of 10, or $80 \%$ ) followed by Bulverde with 22 of $29(76 \%)$, expanding stem D ( 4 of 6 , or $67 \%$ ), Early Triangular (3 of 5 , or $60 \%$ ), beveled narrow stem ( 3 of 5 , or $60 \%$ ), Bulverde-like (11 of 19, or 58\%), arrow point "preforms" (5 of 9 , or $56 \%$ ), Ensor ( 19 of 37 , or $51 \%$ ), and Nolan (20 of 45, or $44 \%)$. Slightly lower completeness frequencies are seen among Bandy ( 4 of 10, or 40\%), Scallorn arrow points ( 19 of 48 , or $40 \%$ ), Marcos (9 of 23, or $39 \%$ ), Montell ( 9 of 24 , or $38 \%$ ), Darl (10 of 27 , or $37 \%)$, and expanding stem concave base $\mathrm{A}$ ( 2 of 6 , or $33 \%)$.

Some of the lowest completeness frequencies are exhibited among lanceolate points. Among formal types, 5 of $18(28 \%)$ St. Mary's Hall are complete or nearly so, as are 5 of $19(26 \%)$ Thrall, 4 of 23 (17\%) Golondrina-Barber, and 4 of 31 (13\%) Angostura. Fracture locations among lanceolate points differ: among Angostura, $71 \%$ of the specimens are short basal sections, suggesting breaks occurred within or just above the inferred haft area; in contrast, St. Mary's Hall and Golondrina-Barber points most frequently display breaks within the blade section.

Lowest completeness frequencies among stemmed points are seen among Castroville; only 1 of the $17(6 \%)$ is complete. None of the 7 Baker points is complete. All of the bifurcate stem fragments, by virtue of their category definition as basal fragments, are highly incomplete, with most of the fractures occurring within or just above the inferred haft area.

Viewing the distribution of these groups, certain very broad patterns emerge. The more-complete points appear to cluster in stratigraphic units relevant to Middle Archaic, Late Archaic, and Late Prehistoric contexts. Morphologically, these points tend to fall within the rectangular-stemmed 
TABLE 13-156

Stratigraphic Distribution of Early Split Stem and Early Corner-notched Points

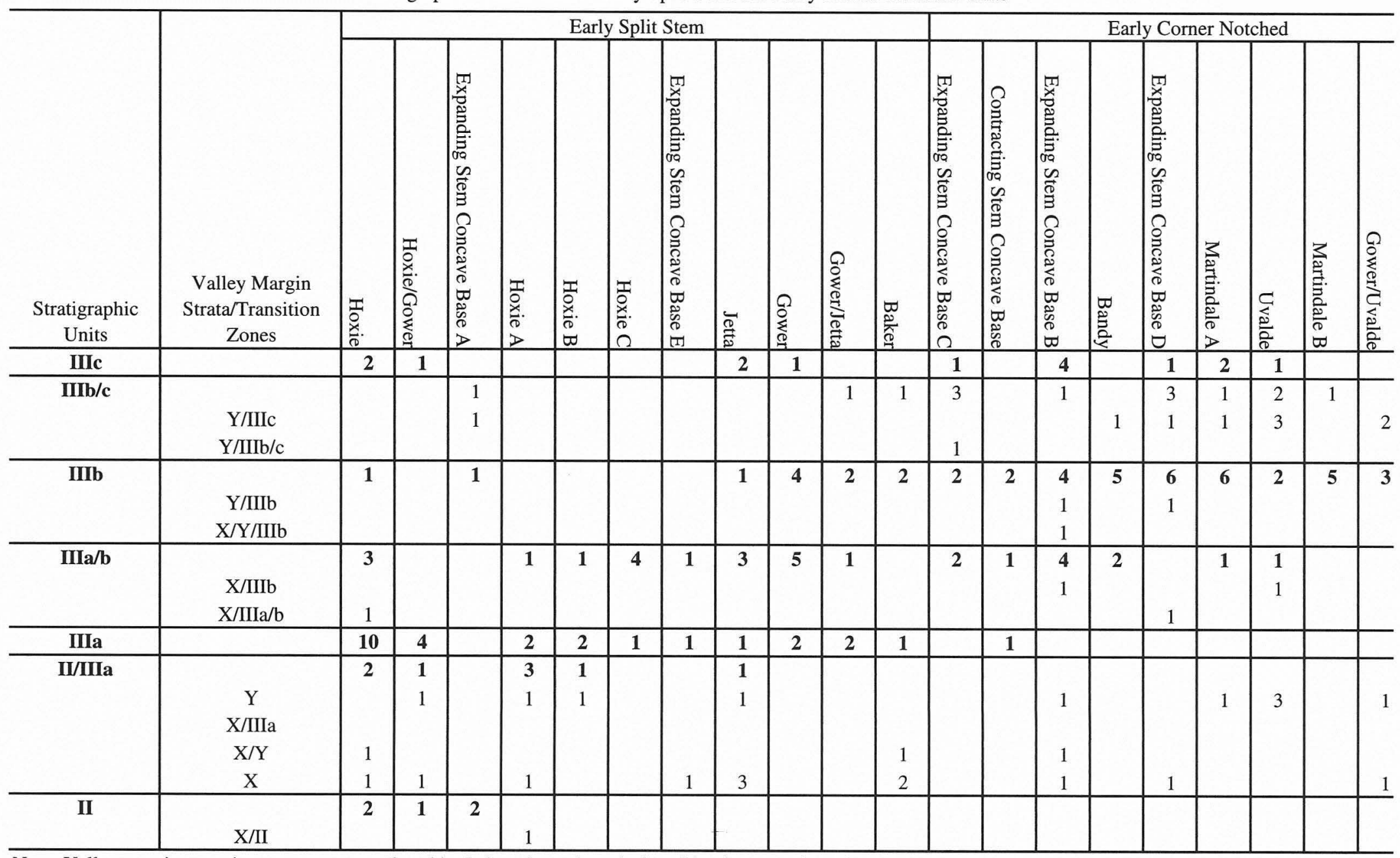

Note: Valley margin strata/contact zones are placed in their estimated vertical position between the other stratigraphic units. Positioning is not secure, so artifact counts from these units should be considered corollary information Valley Floor zones and counts are in bold. 
TABLE 13-157

Projectile Point Distribution by Type and Stratigraphic Unit (Valley Floor)

\begin{tabular}{|c|c|c|c|c|c|c|c|c|c|c|c|c|c|c|c|c|}
\hline & \multicolumn{16}{|c|}{ Stratigraphic Unit } \\
\hline Type & 表 & $\begin{array}{l}\text { 量 } \\
\text { 自 }\end{array}$ & 兰 & $\begin{array}{l}\text { 戛 } \\
\text { 首 }\end{array}$ & 首 & 自 & 學 & 覓 & 目 & 息 & 貝 & 曰 & $\xi$ & 岕 & 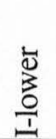 & 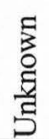 \\
\hline Arrow point, Scallorn/Edwards & & & 2 & & & & & & & & & & & & & \\
\hline Arrow point, stemmed & & 1 & 6 & & & & & & & & & & & & & \\
\hline Arrow point, Scallorn & & 1 & 7 & & & & & & & & & & & & & 1 \\
\hline Arrow point, preform & & & 2 & & & & & & & & & & & & & \\
\hline Arrow point, Perdiz & & & 1 & & & & & & & & & & & & & \\
\hline Kinney & 1 & & & & & & & & & & & & & & & \\
\hline Darl & & & 4 & & & & & & & & & & & & & \\
\hline Ensor & & & 17 & & & & & & & & & & & & & 2 \\
\hline Ensor/Fairland & & & & & & & & & & & & & & & & 1 \\
\hline Fairland & & & 1 & & & & & & & & & & & & & 2 \\
\hline Frio & & & 1 & & & & & & & & & & & & & \\
\hline Marcos/Marshall & & & 1 & & & & & & & & & & & & & \\
\hline Bevelled narrow stem & & & 1 & & & & & & & & & & & & & 1 \\
\hline Marshall & & & 3 & & 2 & & & & & & & & & & & \\
\hline Edgewood & & & 3 & & 1 & & & & & & & & & & & \\
\hline Montell & & & 12 & & 2 & & & & & & & & & & & 1 \\
\hline Pedernales & & & 8 & & 2 & & & & & & & & & & & 1 \\
\hline Castroville & & & 7 & 1 & 3 & & & & & & & & & & & 2 \\
\hline Marcos & & & 7 & & 2 & 1 & & & & & & & & & & 2 \\
\hline Side notched & & & 4 & & 1 & 1 & & & & & & & & & & \\
\hline Miscellaneous bifurcate stem & & & 5 & & 1 & 1 & 1 & & & & & & & & & \\
\hline Williams & & & 1 & & 3 & & & & & & & & & & & \\
\hline Rectangular stem B & & & 2 & & 2 & & & & & & & & & & & \\
\hline Bulverde & & & 14 & & 2 & 2 & & & & & & & & & & 1 \\
\hline Bulverde-like & 1 & 1 & 7 & & 2 & 1 & & & & & & & & & & \\
\hline Expanding stem B & & & 1 & & & & & & 1 & & & & & & & \\
\hline Nolan & & & 10 & & 9 & 6 & 1 & & 1 & & & & & & & 2 \\
\hline Bell/Andice & & & 2 & & 3 & 8 & & & 2 & & & & & & & 1 \\
\hline Square stem & & & 4 & & 2 & 1 & 1 & 1 & 1 & & & & & & & \\
\hline Rectangular stem A & & & 2 & & 1 & 1 & & 1 & & & & & & & & \\
\hline Rectangular stem C & & & 3 & & 1 & 1 & & & & & & & & & & \\
\hline Wells & & & 1 & & 1 & & & & 1 & & & & & & & \\
\hline Travis & & & 2 & & 5 & 1 & & & & & & & & & & \\
\hline Travis-like & & & 2 & & & & & & & & & & & & & \\
\hline Lange & & & 1 & & & & & & & & & & & & & \\
\hline Early Triangular & & & 1 & & 2 & 1 & & & & & & & & & & \\
\hline Expanding concave D & 1 & & 1 & & 3 & 6 & & & & & & & & & & \\
\hline Miscellaneous expanding stem & & & 2 & & & 2 & & 1 & & & & & & & & \\
\hline Nolan-like & & & & & 2 & & & & & & & & & & & \\
\hline Lange-like & & & & & & 1 & & & & & & & & & & \\
\hline Expanding stem A & & & & & 3 & 1 & 1 & & & & & & & & & \\
\hline Expanding stem D & & & 1 & & 1 & 1 & 1 & & & & & & & & & 1 \\
\hline Expanding concave B & & & & 1 & 1 & 2 & 4 & & & & & & & & & \\
\hline Expanding concave $\mathrm{C}$ & & & & & 1 & 1 & 2 & & & & & & & & & \\
\hline Uvalde & & & & & 2 & 2 & 1 & & & & & & & & & \\
\hline
\end{tabular}




\begin{tabular}{|c|c|c|c|c|c|c|c|c|c|c|c|c|c|c|c|c|}
\hline & \multicolumn{16}{|c|}{ Stratigraphic Unit } \\
\hline Type & 寻 & 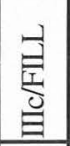 & 兰 & 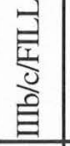 & 慁 & 自 & 丞 & $\stackrel{\Xi}{\equiv}$ & 三 & $\stackrel{\Xi}{\Xi}$ & 目 & $=$ & $\Xi$ & 营 & $\begin{array}{l}\overline{0} \\
\frac{0}{1} \\
\frac{0}{1} \\
\end{array}$ & $\begin{array}{l}\text { F } \\
\text { O } \\
\text { 至 } \\
\text { J } \\
\end{array}$ \\
\hline Uvalde-like & & & & & 2 & & & & & & & & & & & \\
\hline Martindale A & & & & & 1 & 4 & 1 & & & & & & & & & \\
\hline Martindale B & & & & & 1 & 4 & & & & & & & & & & \\
\hline Jetta & & & 1 & & & 1 & 3 & 1 & & & & & & & & \\
\hline Gower/Jetta & & & & & 1 & 2 & 1 & 2 & & & & & & & & \\
\hline Expanding stem $\mathrm{C}$ & & & & & & 3 & 1 & & & & & & & & & \\
\hline Baker & & & & & & 1 & & 1 & & & & & & & & \\
\hline Bandy & & & & & & 3 & 1 & & 1 & & & & & & & \\
\hline Bandy-like & & & & & & & 1 & & & & & & & & & \\
\hline Gower/Uvalde & & & & & & 2 & & & & & & & & & & \\
\hline Thrall & & & 1 & & 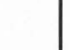 & & 1 & 4 & & & & & & & & \\
\hline Wilson? & & & & & . & 1 & 1 & & & & & & & & & \\
\hline Gower & & & & & 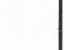 & 2 & 5 & 2 & 1 & & & & & & & \\
\hline Long stem & & & & & 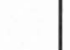 & 1 & 1 & & 1 & & & & & & & \\
\hline Bifurcate stem fragments & & & 1 & & 3 & 3 & 2 & 8 & 2 & 1 & & & & & & \\
\hline Hoxie & & & 1 & & 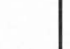 & 1 & 2 & 8 & 1 & 2 & & 1 & & & & \\
\hline Expanding concave $\mathrm{A}$ & & & & & 1 & & & & 1 & & & 1 & & & & \\
\hline Expanding concave $\mathrm{E}$ & & & & & & & 1 & 1 & & & & & & & & \\
\hline Contracting concave stem & & & & & & & 1 & 1 & & & & & & & & \\
\hline Hoxie C & & & & & & & 1 & 1 & & & & & & & & \\
\hline Hoxie B & & & & & & & 1 & 2 & & 1 & & & & & & \\
\hline Hoxie A & & & & & & & 1 & 2 & & 2 & & & & & & \\
\hline Angostura & & & & & . & . & 2 & 11 & & 6 & & 2 & & & & \\
\hline Hoxie/Gower & & & & & & & & 4 & & 1 & & 1 & & & & \\
\hline San Patrice & & & & & & & 1 & & & & & 1 & 2 & & & \\
\hline Concave lanceolate preform & & & & & & & & 2 & & 1 & & & & & & \\
\hline St. Mary's Hall & & & & & 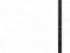 & & & 3 & & 2 & & 4 & 1 & & & \\
\hline Golondrina-Barber & & & & & . & & & 1 & & 4 & & 6 & 6 & 2 & & \\
\hline Miscellaneous Lanceolate & & & & & 1 & 5 & 1 & 17 & 3 & 8 & 1 & 12 & 5 & 4 & & \\
\hline St. Mary's Hall-atypic & & & & & & & & 1 & & & & 2 & & & & \\
\hline Scottsbluff & & & & & & & & & & & & 1 & & & & \\
\hline Parallel-sided haft & & & & & & & & & & & & 2 & 2 & & & \\
\hline Big Sandy & & & & & & & & & & & & 1 & & & & \\
\hline Wilson? atypic & & & & & & & & & & & & 1 & & & & \\
\hline Wilson & & & & & & & & & & & & 4 & 3 & 10 & & \\
\hline Midland & & & & & & & & & & & & & 1 & & & \\
\hline Ground square stem & & & & & & & & & & & & & 1 & & & \\
\hline Wilson scraper & & & & & & & & & & & & & 1 & & & \\
\hline Clovis & & & & & & & & & & 1 & & & & & 1 & \\
\hline The Bone Bed point & & & & & & & & & & & & & & & 1 & \\
\hline Stemmed biface & & & & & & & & & & & & & & & & 1 \\
\hline Indeterminate & & & 3 & & 2 & 1 & 2 & & 1 & & & & & & & 1 \\
\hline Total & 3 & 3 & 156 & 2 & 72 & 75 & 43 & 75 & 17 & 29 & 1 & \begin{tabular}{l|l}
40 \\
\end{tabular} & 22 & 16 & 3 & 19 \\
\hline
\end{tabular}


TABLE 13-158

Projectile Point Distribution by Type and Stratigraphic Unit (Valley Margin)

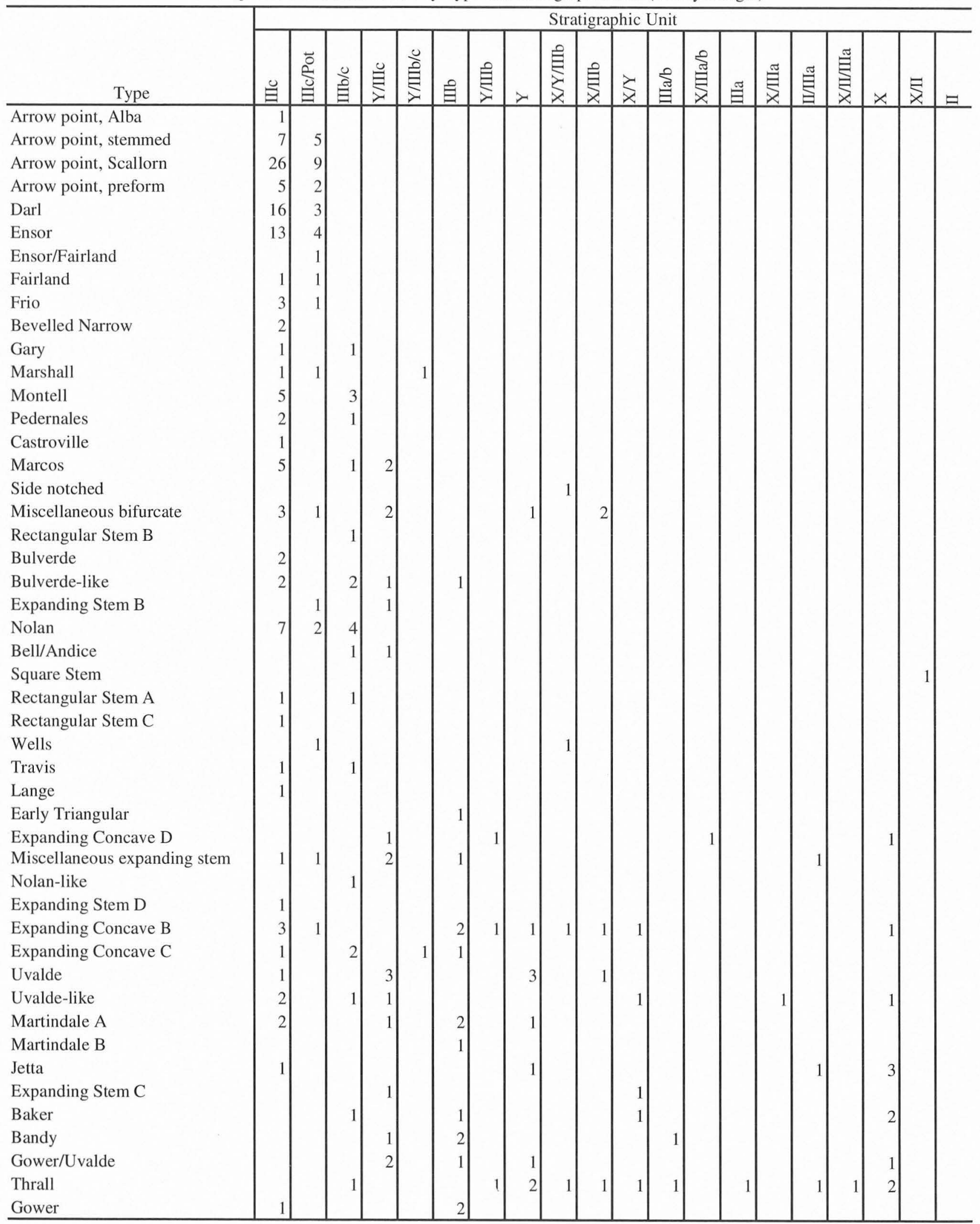




\begin{tabular}{|c|c|c|c|c|c|c|c|c|c|c|c|c|c|c|c|c|c|c|c|c|}
\hline \multirow[b]{2}{*}{ Type } & \multicolumn{20}{|c|}{ Stratigraphic Unit } \\
\hline & 首 & $\begin{array}{l}\overrightarrow{0} \\
\stackrel{0}{0} \\
\Leftrightarrow\end{array}$ & 首 & 塄 & 頁 & 目 & 貝 & $x$ & 貝 & 自 & 3 & 恖 & 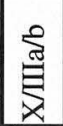 & $\cong$ & 罗 & 罗 & 買 & $x$ & 貝 & $=$ \\
\hline Bifurcate stem fragments & 2 & & & 4 & 1 & 3 & & 4 & & 1 & 1 & 1 & & & & & 2 & 4 & 1 & \\
\hline Early bifurcate preform & & & & & & & & & & & & & & & & & & 2 & & \\
\hline Hoxie & 1 & & & & & & & & & & 1 & 1 & 1 & 2 & & & & 1 & & 1 \\
\hline Hoxie? & & & & & & & & & & & & & & & & & & 1 & & \\
\hline Expanding Concave A & & & & 1 & & 1 & & & & & & & & & & & & & & 1 \\
\hline Expanding Concave E & & & & & & & & & & & & & & & & & & 1 & & \\
\hline Contracting concave stem & & & & & & 2 & & & & & & & & & & & & & & \\
\hline Hoxie C & & & & & & & & & & & & 3 & & & & & & & & \\
\hline Hoxie B & & & & & & & & 1 & & & & & & & & & & & & \\
\hline Hoxie A & & & & & & & & 1 & & & & & & & & 1 & & 1 & 1 & \\
\hline Angostura & 1 & & & & & 1 & & & & & & 1 & & 1 & & & 2 & 2 & & 1 \\
\hline Hoxie/Gower & 1 & & & & & & & 1 & & & & & & & & & & 1 & & \\
\hline Saint Mary's Hall & & & & & & & & & & & & & & & & 1 & & 2 & & \\
\hline Golondrina-Barber & & & & & & 1 & & & & & & & & & & & & 2 & 1 & \\
\hline Saint Mary's Hall-atypic & & & & & & & & & & & 1 & & & & & & & & & \\
\hline Scottsbluff & & & & & & 1 & & & & & & & & & & & & & & \\
\hline Parallel-sided haft & & & & & & & & & & & & & & & & & & & & 1 \\
\hline Wilson & & & & & & & & & & & & & & & & & & 2 & & \\
\hline Indeterminate & 5 & & & 1 & & 1 & & & & & & 1 & & & & & & & & \\
\hline Totals: & 130 & 34 & 22 & 25 & 3 & 25 & 3 & 17 & 4 & $\overline{6}$ & 8 & $\overline{9}$ & 2 & 4 & 1 & 5 & 5 & 30 & 4 & 4 \\
\hline
\end{tabular}

groups (Travis, Nolan, and Bulverde), expanding stem groups (Ensor, expanding stem D, Marcos), or the arrow points. In contrast, ground-stemmed bifurcates and lanceolates tend to be the least complete. These points occur in contexts relevant to early usage of the site (Paleoindian through Early Archaic). Exceptions, as noted, are Castroville and Scallorn in later contexts.

While these findings are somewhat impressionistic, they nevertheless may carry significant implications in the realm of use and technology. As has been noted previously, many of the points in Late Paleoindian and Early Archaic contexts saw extreme reworking and resharpening, many to the point of exhaustion; indeed, some Hoxie specimens bear evidence of reworking of both the blade and haft areas. That some lanceolate points saw a variety of uses is suggested by microscopic use-wear findings (see Kay, Chapter 22).

The fractured and reworked state of many of the unfluted lanceolates and early split stem points may suggest that they were products of an efficient system of intensive use and maintenance. The condition of these points may also attest to the effectiveness of the haft mechanisms employed for unfluted lanceolate (particularly Angostura) and early bifurcate stem points. These haft systems may be viewed as highly effective, inasmuch as they withstood apparent great use. On the other hand, they may be viewed as poorly designed, inasmuch as so many of the early bifurcate and unfluted lanceolate points appear as only basal fragments, having been broken in many cases just above the haft.

Changing site function over time also could explain differences in projectile point condition, or the state in which points were discarded or left behind. For instance, if the locality were used as a base camp, more retooling and multiple use of points would be expected. If it were a campsite used only briefly by mobile extractive parties, a much more limited set of activities might be reflected. These issues can be more fully addressed in conjunction with the full artifact assemblage and other contextual data (see Volume I).

\section{Technology}

Specific aspects of projectile point technology were evaluated within each point type and morphological group. Of particular interest are patterns related to lateral stem edge grinding, secondary flaking, and basal modification.

\section{Grinding of Basal Margins}

That the presence of lateral stem edge grinding is strongly patterned over time- being chiefly manifested in Paleoindian technologies-has long been noted. The degree to which this pattern continues into Early Archaic times, within the early split stem tradition, is clearly evinced in the Wilson-Leonard collection. In Figure 13-139, the percentage of ground stem projectile points recovered within specific stratigraphic units is plotted, as well as the percent- 


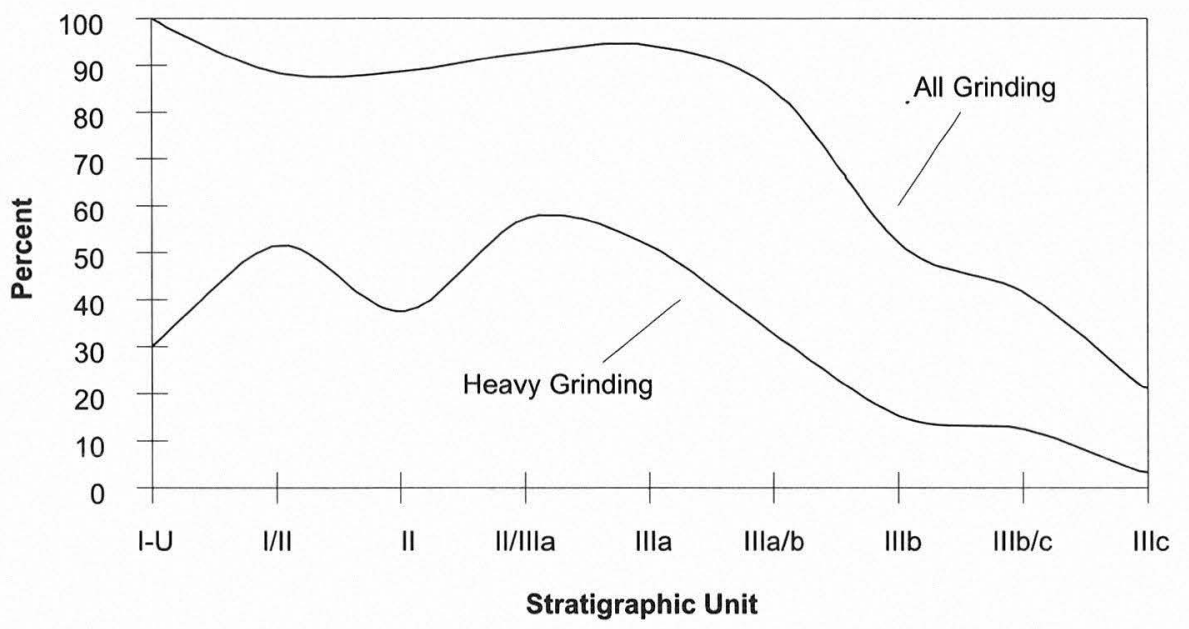

FIGURE 13-139. Projectile point haft area edge grinding over time.

age of those that are considered heavily ground. The sample plotted comprises 528 points drawn from more secure contexts in the Valley Floor; total counts by unit in all areas are given in Table 13-159. In plotting by unit, certain biases introduced by displaced points, particularly from earlier contexts, must be acknowledged; nonetheless, the pattern as shown appears to be significant. With a high of $100 \%$ in upper Unit I (based on a sample of 13 points from Units Isic, Isi/Isi-c, Isi-c/Id, and Isi/Icl/Isi-c) ), presence of grinding gently dips to $89 \%$ in slightly higher deposits, then rises to 93\% in the Unit II/IIIa transition zone, and 95\% in Unit IIIIa, deposits identified to the Late Paleoindian/Early Archaic and Early Archaic, respectively. The trajectory is downward in later deposits, with an intermediate plateau shown at Unit IIIb and the Unit IIIb/c transition zone.

The pattern of heavy stem grinding is somewhat less regular with peaks of $56 \%$ at Unit I/II (a zone of mixed Early Paleoindian and Late Paleoindian deposits) and 62\% at Unit II/IIIa (representing Late Paleoindian and Early Archaic deposits), with a marked interim dip to $41 \%$ in Unit II (Late Paleoindian). As shown, heavy stem grinding was observed much less frequently in specimens from deposits above Unit IIIa, although some degree of stem grinding or dulling continues on points from throughout the site's history.

Why haft areas were so commonly ground on certain projectile point forms is unclear, although a traditional notion has been that grinding would serve to protect haft wrappings from being severed by acute-edged margins of the point. The problem with this model, however, is that it fails to account for the many stemmed Archaic point forms that had no grinding and were likely hafted in split shafts with fiber or leather binding. A more plausible explanation is that suggested by Titmus and Woods (1991) in their study of fluted lanceolate points, namely, that grinding serves to strengthen basal portions of projectiles and prevent breakage within the shaft. Their work however, suggests that the identification of grinding is complex; while some points showed grinding with an abrasive agent, others had been further polished with a compound such as ocher, and it is the latter procedure which seems to have ensured more reliably against haft fracture.

Whether their findings have significance for the present assemblage is uncertain. What is suggested, however, is that certain hafting techniques or use activities during Paleoindian and Early Archaic times required the grinding of lateral stem edges in markedly higher frequencies than at other times. Further, in the Wilson-Leonard assemblage, stem grinding tends to correlate directly with certain lanceolate points such as Angostura and the more parallel-stemmed bifurcate points, both of which forms commonly display alternately beveled blade edges such as might be used for cutting or scraping. That grinding of basal edges tended to strengthen the haft area of the tool and improve its use for tasks requiring lateral motion is a possibility that should be further explored through microwear and replicative studies. Such studies would certainly further our understanding of the present assemblage and also help explain its significance within a much larger context, namely, the regional florescence of points bearing the compound attributes of bifurcated bases, stem grinding, and beveled and/or serrated edges during a ca. 10,000-8000 B.P. time period. To name but a few, these range from the Lecroy bifurcated stem form of the northeast (Justice 1995:91), to Dalton points and later variants of the southeast (Goodyear 1974; Justice 1995); Angostura on the Plains (Thoms 1993; Wormington 1957), to Pryor Stemmed (Frison 1978; Frison and Grey 1980) and possibly some of the Great Basin Stemmed series of the west (Beck and Jones 1993).

\section{Flaking Patterns}

Certain other technological attributes may point up periods when more skill or care was required in the production 
TABLE 13-159

Grinding on Projectile Points by Stratigraphic Unit

\begin{tabular}{|c|c|c|c|c|c|c|c|}
\hline Stratigraphic Unit & Absent & Slight & Moderate & Heavy & Totals & Heavy Grinding $\%$ & All Grinding \% \\
\hline \multicolumn{8}{|l|}{ Valley Floor: } \\
\hline IIIc & 117 & 21 & 10 & 5 & 153 & 3 & 24 \\
\hline $\mathrm{IIIb} / \mathrm{c}$ & 40 & 14 & 8 & 10 & 72 & 14 & 44 \\
\hline IIIb & 34 & 12 & 15 & 12 & 73 & 16 & 53 \\
\hline $\mathrm{III} / \mathrm{b}$ & 6 & 13 & 7 & 14 & 40 & 35 & 85 \\
\hline IIIa & 4 & 11 & 18 & 41 & 74 & 55 & 95 \\
\hline III & 8 & 1 & 2 & 6 & 17 & 35 & 53 \\
\hline II/IIIa & 2 & 4 & 5 & 18 & 29 & 62 & 93 \\
\hline II/III & 0 & 1 & 0 & 0 & 1 & 0 & 100 \\
\hline II & 4 & 9 & 9 & 15 & 37 & 41 & 89 \\
\hline $\mathrm{I} / \mathrm{II}$ & 2 & 4 & 2 & 10 & 18 & 56 & 89 \\
\hline I-upper & 0 & 3 & 6 & 4 & 13 & 31 & 100 \\
\hline I-lower & 0 & 0 & 0 & 1 & 1 & 100 & 100 \\
\hline \multicolumn{8}{|l|}{ Valley Margin: } \\
\hline IIIc & 111 & 9 & 5 & 8 & 133 & 6 & 17 \\
\hline $\mathrm{IIIb} / \mathrm{c}$ & 12 & 7 & 2 & 1 & 22 & 5 & 45 \\
\hline Y/IIIc & 19 & 1 & 1 & 3 & 24 & 13 & 21 \\
\hline $\mathrm{Y} / \mathrm{IIIb} / \mathrm{c}$ & 2 & 0 & 0 & 0 & 2 & 0 & 0 \\
\hline IIIb & 15 & 5 & 3 & 3 & 26 & 12 & 42 \\
\hline Y/IIIb & 1 & 0 & 0 & 2 & 3 & 67 & 67 \\
\hline $\mathrm{Y}$ & 8 & 2 & 3 & 5 & 18 & 28 & 56 \\
\hline $\mathrm{X} / \mathrm{Y} / \mathrm{IIIb}$ & 3 & 0 & 0 & 1 & 4 & 25 & 25 \\
\hline X/IIIb & 3 & 0 & 1 & 2 & 6 & 33 & 50 \\
\hline $\mathrm{X} / \mathrm{Y}$ & 4 & 1 & 2 & 2 & 9 & 22 & 56 \\
\hline $\mathrm{III} a / \mathrm{b}$ & 2 & 0 & 3 & 4 & 9 & 44 & 78 \\
\hline $\mathrm{X} / \mathrm{II} / \mathrm{b}$ & 1 & 0 & 1 & 0 & 2 & 0 & 50 \\
\hline IIIa & 0 & 0 & 2 & 2 & 4 & 50 & 100 \\
\hline X/IIIa & 1 & 0 & 0 & 1 & 2 & 50 & 50 \\
\hline II/IIIa & 0 & 1 & 1 & 4 & 6 & 67 & 100 \\
\hline X/II/IIIa & 0 & 0 & 1 & 4 & 5 & 80 & 100 \\
\hline$X$ & 7 & 3 & 3 & 17 & 30 & 57 & 77 \\
\hline $\mathrm{X} / \mathrm{II}$ & 2 & 0 & 1 & 3 & 6 & 50 & 67 \\
\hline II & 0 & 0 & 1 & 5 & 6 & 83 & 100 \\
\hline Totals: & 408 & 122 & 112 & 203 & 845 & 24 & 52 \\
\hline
\end{tabular}

Note: Points from surface finds, fill, and questionable stratigraphic proveniences are not included. Totals do not include points with indeterminate grinding.

of projectile points (e.g., organized flake patterns, extreme thinness, extraordinarily long barbs). Organized flake patterns (i.e., nonrandom) were shown in highest frequency in Unit Id/II (87\%) and Unit II (90\%), trending linearly toward less-organized, more-random patterning over time (e.g., Unit II/IIIa [61\% organized], IIIa [66\%], IIIa/b [53\%], IIIb [48\%], $\mathrm{IIIb} / \mathrm{c}[34 \%]$ and IIIc [34\%]). A notable exception to this pattern is seen Unit Isi-c, where Wilson points occurred. Their typically irregular flake patterns are among several attributes which betoken Archaic rather than Paleoindian technologies.
Again, there are other exceptions among certain point types (as well as vertically displaced points) that need to be acknowledged. Careful flaking as well as extraordinary thinness are distinguishing traits among Bandy and, to a lesser degree, among Martindale, Bell/Andice, Early Triangular, Montell, Castroville, and Scallorn points. Bell/Andice, in particular, are notable for their deep and intricate basal notches and long barbs, attributes that replicators have demonstrated as requiring no small amount of skill and patience (Weber 1994).

Why patterned flaking was favored in earlier times more 
so than later is unknown. What is seen in the present assemblage is the relative persistence of this trait, extending to more than two-thirds of the projectile points recovered in Early Archaic deposits.

\section{Basal Modification}

Modes of basal modification are varied, with certain point types-particularly Late Paleoindian unfluted lanceolates-displaying more-pronounced thinning than others. Among St. Mary's Hall points, 12 of $18(67 \%)$ are bifacially thinned, as are 12 of $23(52 \%)$ Golondrina-Barber. This pattern is also seen in Bell/Andice points, of which 12 of $18(67 \%)$ are bifacially thinned.

More frequently displaying the less-thinned, concave bevel basal modification are Angostura points (19 of 31 or $61 \%$ ); Thrall (13 of 19 or $68 \%$ ); and most of the early split stem forms including Gower ( 8 of 14 or $57 \%$ ), Hoxie ( 15 of 26 or $58 \%$ ), and Hoxie variants (11 of 19 or $58 \%$ ). Interestingly, later bifurcate types such as Montell and Pedernales rarely show concave-beveled bases, being more frequently fairly well thinned with either unifacial or bifacial thinning predominating. A trend toward simple unifacial thinning is seen among some of the early corner-notched groups including Martindale A and B ( 10 of 18 or $56 \%$ ) and expanding-stem concave base $C$ points ( 6 of 9 or $67 \%$ ). The least well-thinned among typed groups are the Late Paleoindian expanding-stem Wilson points, among which 8 of 18 or $44 \%$ showed only secondary trimming, 5 of $18(28 \%)$ unifacial thinning, and only 4 (22\%) bifacial thinning. This ill-defined basal modification is one of several attributes of this early type which makes it aberrant in comparison to the more typical, well-made lanceolate points of the Late Paleoindian period.

\section{Material Selection and Effects of Natural and Cultural Processes}

Raw materials used for projectile points were overwhelmingly local cherts. A total of $703(70 \%)$ was made of local Edwards chert (of these, 576 , or $82 \%$ are fine-grained, and 127 or $18 \%$ are medium to coarse). Among the local finegrained cherts is a distinctive dark gray/black variety likely derived from nearby sources in the present Round Rock and Georgetown areas (see Chapter 19). A total of 48 projectile points, including 7 Nolan points, are made of this fine material. It is somewhat surprising that highest frequencies of these distinctive cherts among projectile points are seen in Unit IIIc, inasmuch as cores and core fragments of the same black cherts occurred more prevalently in Unit I deposits (see Chapter 18).

A relatively small number (197 or 20\%) of projectile points were made of nonlocal Edwards cherts (150 or $76 \%$ finegrained; 49 or $25 \%$ medium to coarse). Unidentified nonEdwards cherts were used for only 3 points, and materials other than chert for 3 others; of these latter, 1 was identified as quartz and 2 as chalcedony. Raw materials for an additional 97 specimens were not classified, in most cases due to heat alteration of the material.

Only a small number of projectile points (42 or $5 \%$ ) display patination, and few of these were clustered in any one group. Exceptions were among the expanding stem concave base B group, with 4 of $19(21 \%)$ of its members showing patination; the Hoxie A group, in which 3 of the 9 $(30 \%)$ specimens are patinated, as are 3 of $18(17 \%)$ St. Mary's Hall points. When the assemblage is considered as a whole according to stratigraphic distribution, however, higher frequencies of patina are shown in certain early contexts (e.g., Valley Floor Unit II with 18\%; Table 13-160).

Deposits of mineral precipitates were observed on only 111 , or $13 \%$, of the projectile points and - as with patinaoccurrences were most frequent in lower stratigraphic units (Table 13-161). It is notable that similar distribution was seen in most other Wilson-Leonard tool categories with the exception of core tools, which showed markedly higher frequencies within all stratigraphic units. That pattern suggests the accumulation of precipitates may be use-related (see Chapter 18). Among morphological groups of projectile points, slightly higher percentages of mineral precipitates are seen among Uvalde, Uvalde-like, and several of the expanding stem concave base forms found in the later part of the Early Archaic.

Heat alteration was observed on $18 \%$ of the projectile points (157 of 875). As shown in Table 13-162, highest frequencies among samples large enough to be significant are in Unit I (upper, including Isi-c), Unit IIIa, and IIIc of the Valley Floor. Higher frequencies of extensive damage (i.e., burned) are in Valley Margin units, a pattern that may correlate with the large burned rock midden in that area during Archaic times and/or to disposal practices and occurrences of natural fires. Almost twice as many projectile point fragments were recorded with heat damage (108 of 311 or $35 \%$; Table 13-163), with Unit IIIa of the Valley Floor again showing a high frequency. Extensive damage on specimens in that sample showed a markedly different pattern with highest frequencies (among larger samples) within Valley Floor units.

\section{Patterns in Use and Maintenance of Projectile Points}

The large majority of points in the Wilson-Leonard collection appear to have been reworked, and a variety of resharpening techniques is manifested. Relative percentages of the four different resharpening techniques observed within stratigraphic units are shown in Table 13-164. Although resharpening clearly was an important technology throughout time, the technique of alternate beveling reached maximum expression in Unit IIIa and the Unit II/IIIa transition zone, pertaining chiefly to the Early Archaic. What is not codified in the present study is the degree of edge beveling; 
TABLE 13-160

Patina on Projectile Points by Stratigraphic Units

\begin{tabular}{|c|c|c|c|c|}
\hline Stratigraphic Unit & Absent & Present & Total & Percent Present \\
\hline \multicolumn{5}{|l|}{ Valley Floor: } \\
\hline IIIc & 154 & 3 & 157 & 2 \\
\hline $\mathrm{IIIb} / \mathrm{c}$ & 70 & 2 & 72 & 3 \\
\hline IIIb & 70 & 5 & 75 & 7 \\
\hline $\mathrm{IIIa} / \mathrm{b}$ & 40 & 4 & 44 & 9 \\
\hline IIIa & 74 & 3 & 77 & 4 \\
\hline III & 13 & 4 & 17 & 24 \\
\hline II/IIIa & 27 & 3 & 30 & 10 \\
\hline II/III & 0 & 1 & 1 & 100 \\
\hline II & 32 & 7 & 39 & 18 \\
\hline $\mathrm{I} / \mathrm{II}$ & 19 & 2 & 21 & 10 \\
\hline I-upper & 13 & 0 & 13 & 0 \\
\hline I-lower & 2 & 1 & 3 & 33 \\
\hline \multicolumn{5}{|l|}{ Valley Margin: } \\
\hline IIIC & 137 & 2 & 139 & 1 \\
\hline $\mathrm{IIIb} / \mathrm{c}$ & 22 & 0 & 22 & 0 \\
\hline Y/IIIc & 24 & 1 & 25 & 4 \\
\hline $\mathrm{Y} / \mathrm{IIIb} / \mathrm{c}$ & 3 & 0 & 3 & 0 \\
\hline IIIb & 27 & 0 & 27 & 0 \\
\hline Y/IIIb & 3 & 0 & 3 & 0 \\
\hline $\mathrm{Y}$ & 16 & 2 & 18 & 11 \\
\hline $\mathrm{X} / \mathrm{Y} / \mathrm{IIIb}$ & 4 & 0 & 4 & 0 \\
\hline X/IIIb & 6 & 0 & 6 & 0 \\
\hline $\mathrm{X} / \mathrm{Y}$ & 9 & 0 & 9 & 0 \\
\hline $\mathrm{IIIa} / \mathrm{b}$ & 9 & 0 & 9 & 0 \\
\hline $\mathrm{X} / \mathrm{III} / \mathrm{b}$ & 2 & 0 & 2 & 0 \\
\hline IIIa & 4 & 0 & 4 & 0 \\
\hline X/IIIa & 2 & 0 & 2 & 0 \\
\hline II/IIIa & 6 & 0 & 6 & 0 \\
\hline X/II/IIIa & 5 & 1 & 6 & 17 \\
\hline $\mathrm{X}$ & 30 & 1 & 31 & 3 \\
\hline $\mathrm{X} / \mathrm{II}$ & 6 & 0 & 6 & 0 \\
\hline II & 6 & 0 & 6 & 0 \\
\hline Totals: & 835 & 42 & 877 & 5 \\
\hline
\end{tabular}

Note: Points from surface finds, fill, and questionable stratigraphic proveniences are not included $(\mathrm{N}=15)$. Totals do not include points with indeterminate patina.

refinement of this observation would serve to further illuminate the steep-lateral edge beveling technique used for many of the early split stem points as well as the Late Archaic Darl points.

In his study of Plains beveled knives, Sollberger (1971:209-218) suggests that edge beveling is a technique of conservation to prolong the use-life of the tool, particularly in areas where chert or other good raw materials are scarce. As he explains, the technique of microflaking requires that only small amounts of material be removed to produce a sharp edge, as opposed to bifacial resharpening, which quickly attrites and concomitantly weakens lateral edges. For projectile points, which typically take the brunt of wear damage at or near the tip, extensive resharpening of lateral edges is evidence that they saw use as hafted knives or combination dart points and knives; beveled knives of the Plains may have been particularly useful and efficient in skinning bison hides (Sollberger 1971:209-211). Others have suggested, however, that the alternately beveled blade morphology is not ideal for cutting and have failed to observe use-wear evidence to support that use (Frison and Grey 1980). On Pryor Stemmed and Hoxie points, the beveling frequently extends well into hafted parts of the stem (see Figure 13-43; Frison and Grey 1980:30).

In the Wilson-Leonard assemblage, many of the extremely beveled specimens appear to be "exhausted" (Marvin Kay, personal communication 1995). Almost half of the points appear to have been broken by impact fracturing, suggesting a multiple use for these tools.

\section{Projectile Points as Evidence for Type of Weapon}

Projectile points in the local prehistory are divided into two groups based on size-larger "dart points" and smaller "arrow points," a distinction supported somewhat by finds elsewhere of bows and of atlatls in dry rockshelters. The identification of the smaller forms as tips of arrows propelled by 
TABLE 13-161

Mineral Precipitates on Projectile Points by Stratigraphic Unit

\begin{tabular}{|c|c|c|c|c|}
\hline Stratigraphic Unit & Absent & Present & Total & Percent Present \\
\hline $\begin{array}{l}\text { Valley Floor: } \\
\text { IIIc } \\
\text { IIIb/c } \\
\text { IIIb } \\
\text { IIIa/b } \\
\text { IIIa } \\
\text { III } \\
\text { II/IIIa } \\
\text { II/III } \\
\text { II } \\
\text { I/II } \\
\text { I-upper } \\
\text { I-lower } \\
\end{array}$ & $\begin{array}{c}138 \\
71 \\
73 \\
41 \\
75 \\
12 \\
28 \\
0 \\
29 \\
14 \\
8 \\
3 \\
\end{array}$ & $\begin{array}{c}19 \\
1 \\
1 \\
3 \\
3 \\
5 \\
2 \\
1 \\
11 \\
7 \\
6 \\
0 \\
\end{array}$ & $\begin{array}{c}157 \\
72 \\
74 \\
44 \\
78 \\
17 \\
30 \\
1 \\
40 \\
21 \\
14 \\
3 \\
\end{array}$ & $\begin{array}{c}12 \\
1 \\
1 \\
7 \\
4 \\
29 \\
7 \\
100 \\
28 \\
33 \\
43 \\
0\end{array}$ \\
\hline $\begin{array}{l}\text { Valley Margin: } \\
\text { IIIc } \\
\text { IIIb/c } \\
\text { Y/IIIc } \\
\text { Y/IIIb/c } \\
\text { IIIb } \\
\text { Y/IIIb } \\
\text { Y } \\
\text { X/Y/IIIb } \\
\text { X/IIIb } \\
\text { X/Y } \\
\text { IIIa/b } \\
\text { X/IIIa/b } \\
\text { IIIa } \\
\text { X/IIIa } \\
\text { II/IIIa } \\
\text { X/II/IIIa } \\
\text { X } \\
\text { X/II } \\
\text { II } \\
\end{array}$ & $\begin{array}{c}128 \\
22 \\
16 \\
2 \\
27 \\
1 \\
9 \\
4 \\
2 \\
7 \\
9 \\
2 \\
4 \\
2 \\
6 \\
5 \\
18 \\
6 \\
6 \\
\end{array}$ & $\begin{array}{c}11 \\
0 \\
9 \\
1 \\
0 \\
2 \\
9 \\
0 \\
4 \\
2 \\
0 \\
0 \\
0 \\
0 \\
0 \\
1 \\
13 \\
0 \\
0 \\
\end{array}$ & $\begin{array}{c}139 \\
22 \\
25 \\
3 \\
27 \\
3 \\
18 \\
4 \\
6 \\
9 \\
9 \\
2 \\
4 \\
2 \\
6 \\
6 \\
31 \\
6 \\
6 \\
\end{array}$ & $\begin{array}{c}8 \\
0 \\
36 \\
33 \\
0 \\
67 \\
50 \\
0 \\
67 \\
22 \\
0 \\
0 \\
0 \\
0 \\
0 \\
17 \\
42 \\
0 \\
0 \\
\end{array}$ \\
\hline Totals: & 768 & 111 & 879 & 13 \\
\hline
\end{tabular}

Note: Points from surface finds, fill, and questionable stratigraphic proveniences are not included. Totals do not include points with indeterminate mineral precipitation.

the bow is probably reasonably valid (Christen-son 1986; Fenenga 1953; Patterson 1985; Thomas 1978). What is less clear is what kinds of weapons were tipped with the larger projectile points. Among the likely possibilities are lances, javelins, and darts. Lances are thrusting weapons, javelins are hand-thrown spears, and darts are propelled by atlatls.

Hunting strategies call for different weapons depending upon the nature of the game, the composition of the hunting party, and what techniques are used to gain advantage over the quarry. Small animals are generally taken with snares, nets, traps, and other devices without the need for piercing weapons. Tipped piercing weapons are used primarily on the larger animals such as deer, antelope, bison, bear, or, in earlier times, mammoth or horse. Advantage can be gained over these larger animals by using decoys, disguises, blinds, drives, surrounds, pounds, natural traps, dogs, or other techniques that either allow the hunter longer time or shorter range for shots at unimpeded animals or impede the animals by injury, confinement, or the elimination of escape routes.

It would enhance our interpretations of prehistoric hunting strategies if the durable projectile points could be used to determine more about the weapons they tipped. In this study, two approaches to this problem were explored. In the first, the Wilson-Leonard projectile points were assessed using a model of projectile point lethality developed by Friis-Hansen (1990); in this effort, the result was that no matter what weapon system was in use, projectile points in the prehistory of Central Texas appear excessively large. In the second, the size and design of the WilsonLeonard points were surveyed for patterns that might indicate which of the weapons was in use. In this case, the results included definition of some intriguing patterns, but no conclusions regarding specific weapon systems. In spite of these limited results, we hope to stimulate Texas archeologists to move beyond typology in their consideration 
TABLE 13-162

Heat Alteration on Projectile Points by Stratigraphic Unit

\begin{tabular}{|c|c|c|c|c|c|c|c|}
\hline Stratigraphic Unit & Absent & Slight & Moderate & Extensive & Total & $\begin{array}{c}\text { Percent of } \\
\text { Extensive Damage }\end{array}$ & $\begin{array}{l}\text { Percent of All } \\
\text { Heat Damage }\end{array}$ \\
\hline $\begin{array}{l}\text { Valley Floor: } \\
\text { IIIc } \\
\text { IIIb/c } \\
\text { IIIb } \\
\text { IIIa/b } \\
\text { IIIa } \\
\text { III } \\
\text { II/IIIa } \\
\text { II/III } \\
\text { II } \\
\text { I/II } \\
\text { I-upper } \\
\text { I-lower } \\
\end{array}$ & $\begin{array}{c}128 \\
62 \\
66 \\
36 \\
60 \\
16 \\
25 \\
1 \\
31 \\
18 \\
8 \\
2 \\
\end{array}$ & $\begin{array}{c}11 \\
4 \\
5 \\
5 \\
4 \\
1 \\
2 \\
0 \\
2 \\
0 \\
1 \\
0 \\
\end{array}$ & $\begin{array}{l}2 \\
2 \\
1 \\
1 \\
7 \\
0 \\
1 \\
0 \\
2 \\
2 \\
3 \\
0 \\
\end{array}$ & $\begin{array}{c}15 \\
4 \\
4 \\
2 \\
7 \\
0 \\
2 \\
0 \\
3 \\
1 \\
1 \\
0 \\
\end{array}$ & $\begin{array}{c}156 \\
72 \\
76 \\
44 \\
78 \\
17 \\
30 \\
1 \\
38 \\
21 \\
13 \\
2 \\
\end{array}$ & $\begin{array}{c}10 \\
6 \\
5 \\
5 \\
9 \\
0 \\
7 \\
0 \\
8 \\
5 \\
8 \\
0 \\
\end{array}$ & $\begin{array}{c}18 \\
14 \\
13 \\
18 \\
23 \\
6 \\
17 \\
0 \\
18 \\
14 \\
38 \\
0 \\
\end{array}$ \\
\hline $\begin{array}{l}\text { Valley Margin: } \\
\text { IIIc } \\
\text { IIIb/c } \\
\text { Y/IIIc } \\
\text { Y/IIIb/c } \\
\text { IIIb } \\
\text { Y/IIIb } \\
\text { Y } \\
\text { X/Y/IIIb } \\
\text { X/IIIb } \\
\text { X/Y } \\
\text { IIIa/b } \\
\text { X/IIIa/b } \\
\text { IIIa } \\
\text { X/IIIa } \\
\text { II/IIIa } \\
\text { X/II/IIIa } \\
\text { X } \\
\text { X/II } \\
\text { II } \\
\end{array}$ & $\begin{array}{c}109 \\
20 \\
18 \\
3 \\
22 \\
2 \\
17 \\
2 \\
6 \\
8 \\
7 \\
1 \\
4 \\
2 \\
6 \\
4 \\
26 \\
4 \\
4 \\
\end{array}$ & $\begin{array}{l}9 \\
1 \\
2 \\
0 \\
0 \\
0 \\
0 \\
1 \\
0 \\
0 \\
0 \\
0 \\
0 \\
0 \\
0 \\
0 \\
1 \\
1 \\
1 \\
\end{array}$ & $\begin{array}{l}3 \\
1 \\
1 \\
0 \\
1 \\
1 \\
0 \\
0 \\
0 \\
0 \\
1 \\
0 \\
0 \\
0 \\
0 \\
0 \\
3 \\
0 \\
0 \\
\end{array}$ & $\begin{array}{l}18 \\
0 \\
4 \\
0 \\
4 \\
0 \\
1 \\
1 \\
0 \\
1 \\
1 \\
1 \\
0 \\
0 \\
0 \\
2 \\
1 \\
1 \\
0 \\
\end{array}$ & $\begin{array}{c}139 \\
22 \\
25 \\
3 \\
27 \\
3 \\
18 \\
4 \\
6 \\
9 \\
9 \\
2 \\
4 \\
2 \\
6 \\
6 \\
31 \\
6 \\
5 \\
\end{array}$ & $\begin{array}{c}13 \\
0 \\
16 \\
0 \\
15 \\
0 \\
6 \\
25 \\
0 \\
11 \\
11 \\
50 \\
0 \\
0 \\
0 \\
33 \\
3 \\
17 \\
0 \\
\end{array}$ & $\begin{array}{c}22 \\
9 \\
28 \\
0 \\
19 \\
33 \\
6 \\
50 \\
0 \\
11 \\
22 \\
50 \\
0 \\
0 \\
0 \\
33 \\
16 \\
33 \\
20 \\
\end{array}$ \\
\hline Totals: & 718 & 51 & 32 & 74 & 875 & 8 & 18 \\
\hline
\end{tabular}

Note: Points from surface finds, fill, and questionable stratigraphic proveniences are not included. Totals do not include points with indeterminate heat alteration.

of projectile points because we believe that behavioral interpretations should improve if archeologists continue to make greater effort toward determining what weapons were employed in what hunting strategies (cf. Christenson 1986; Shott 1993).

\section{Lethality}

Friis-Hansen (1990) combined archeological, ethnographic, and modern bow-hunting evidence to develop a model explaining the design and delivery requirements for a hunting arrow to be effective in the killing of large game. The Friis-Hansen model is explicitly intended for archeological interpretations, and, accordingly, it is built on easily measured attributes of projectile points. His sources mainly concerned Old World animal species, but the more important findings can be extrapolated to the pertinent New World game animals. Also, because the essence of his model is to be found in the size of the arrow point as it relates to location and depth of wound, it can be readily extrapolated to darts, javelins, and lances (Friis-Hansen 1990:499). Friis-Hansen notes (1990:499) that lance heads are ordinarily designed so that they can be easily withdrawn to be thrust again, meaning that, archeologically, large projectile points lacking barbs would be the expected form.

Basically, Friis-Hansen found that modern bow hunters strive to bring any large game animal down within $10 \mathrm{sec}-$ onds of being wounded in order to minimize tracking (thus, an animal that can run $6 \mathrm{~m} / \mathrm{second}$ should be found less than $60 \mathrm{~m}$ from where it was hit). Only rapid bleeding of the heart, lungs, and large thoracic veins or arteries will bring a large animal down in 10 seconds, so the objective is to deeply penetrate the chest cavity with a clean shot. In most cases, this means shots of less than $40 \mathrm{~m}$, and preferably less than 
TABLE $13-163$

Heat Alteration on Projectile Point Fragments by Stratigraphic Unit

\begin{tabular}{|c|c|c|c|c|c|c|c|}
\hline Stratigraphic Unit & Absent & Slight & Moderate & Extensive & Total & $\begin{array}{c}\text { Percent of Extensive } \\
\text { Alteration } \\
\end{array}$ & $\begin{array}{c}\text { Percent of All Heat } \\
\text { Alteration }\end{array}$ \\
\hline \multicolumn{8}{|l|}{ Valley Floor: } \\
\hline IIIc & 59 & 3 & 2 & 20 & 84 & 24 & 30 \\
\hline $\mathrm{IIIb} / \mathrm{c}$ & 16 & 2 & 0 & 6 & 24 & 25 & 33 \\
\hline IIIb & 22 & 0 & 2 & 10 & 34 & 29 & 35 \\
\hline $\mathrm{III} a / \mathrm{b}$ & 11 & 0 & 2 & 7 & 20 & 35 & 45 \\
\hline IIIa & 14 & 4 & 0 & 11 & 29 & 38 & 52 \\
\hline III & 1 & 0 & 0 & 0 & 1 & 0 & 0 \\
\hline II/IIIa & 3 & 2 & 0 & 3 & 8 & 38 & 63 \\
\hline II/III & 0 & 0 & 0 & 1 & 1 & 100 & 100 \\
\hline II & 10 & 1 & 0 & 4 & 15 & 27 & 33 \\
\hline $\mathrm{I} / \mathrm{II}$ & 3 & 0 & 1 & 1 & 5 & 20 & 40 \\
\hline I-upper & 1 & 0 & 0 & 0 & 1 & 0 & 0 \\
\hline \multicolumn{8}{|l|}{ Valley Margin: } \\
\hline IIIc & 35 & 4 & 1 & 12 & 52 & 23 & 33 \\
\hline $\mathrm{IIIb} / \mathrm{c}$ & 5 & 0 & 1 & 1 & 7 & 14 & 29 \\
\hline Y/IIIc & 1 & 0 & 0 & 1 & 2 & 50 & 50 \\
\hline $\mathrm{IIIb}$ & 5 & 0 & 0 & 3 & 8 & 38 & 38 \\
\hline $\mathrm{Y}$ & 3 & 0 & 0 & 0 & 3 & 0 & 0 \\
\hline X/Y/IIIb & 1 & 0 & 0 & 0 & 1 & 0 & 0 \\
\hline $\mathrm{X} / \mathrm{IIIb}$ & 1 & 1 & 0 & 1 & 3 & 33 & 67 \\
\hline $\mathrm{X} / \mathrm{Y}$ & 1 & 0 & 0 & 0 & 1 & 0 & 0 \\
\hline IIIa & 2 & 0 & 0 & 0 & 2 & 0 & 0 \\
\hline II/IIIa & 1 & 0 & 0 & 0 & 1 & 0 & 0 \\
\hline X/II/IIIa & 2 & 0 & 0 & 0 & 2 & 0 & 0 \\
\hline $\mathrm{X}$ & 4 & 0 & 0 & 0 & 4 & 0 & 0 \\
\hline II & 2 & 0 & 0 & 1 & 3 & 33 & 33 \\
\hline Totals: & 203 & 17 & 9 & 82 & 311 & 26 & 35 \\
\hline
\end{tabular}

Note: Points from surface finds, fill, and questionable stratigraphic proveniences are not included. Totals do not include fragments with indeterminate heat alteration.

$20 \mathrm{~m}$. It also means that the arrow point is most effective if it passes completely through the chest cavity, causing the longest possible cut. Therefore, the hunter attempts to avoid hitting bone, including ribs but especially shoulder elements.

Friis-Hansen found a linear relationship between body weight of a game animal and the size of the cut through the thorax necessary to bring about a 10-second drop. Size of cut, he determined, is best expressed as the surface area of the track of the projectile through the body, or length of wound (depth of penetration) times the perimeter of the track in cross section (Friis-Hansen 1990:495). Maximum depth of wound is the breadth of the chest cavity for a perpendicular shot, but this can be increased somewhat by directing the arrow diagonally from behind the rib cage toward the opposite shoulder. Perimeter of the track is calculated from the greatest width of the point and its thickness. Obviously, this relationship favors increased width of point either to shorten drop time or to take larger game animals, but a practical limitation is that with increased width of the point, there is increased probability of striking a rib. Openings between ribs vary significantly among game animals.

Friis-Hansen (1990:Table 1) summarizes these variables for the Old World animals (modern and fossil) he consid- ered to produce an estimate of the minimum perimeter of an arrow point required to achieve a 10 -second drop. The essential data from that table are presented here (Table 13165).

Contrary to a view that rough or serrated edges of points increase their lethality, Friis-Hansen (1990:497) reports that bow hunters have found that very sharp edges are actually the more effective for two reasons. First, a clean cut results in less production of fibrin, the clotting agent released when thrombocytes in the blood are broken. Second, there is less immediate pain caused by a sharp arrow point, and the hunter may have time to take a second shot before the quarry reacts.

An alternative to increasing the size of a single cut by increasing width of a point is to increase the number of wounds. Impeded animals can be dealt multiple wounds, each one of which adds to the bleeding surface area. This principle applies to arrows, darts, javelins, or lances.

Optimal lethality of weapon systems and hunting strategies, then, comes from a sharp, wide-bladed projectile delivered cleanly through the thorax or from multiple wounds that need not be as wide. Strategies that increase the hunter's advantage in delivering one or more such wounds improve the odds of success. 
TABLE 13-164

Resharpening on Projectile Points by Stratigraphic Unit

\begin{tabular}{|c|c|c|c|c|c|c|c|c|}
\hline Stratigraphic Unit & Absent & $\begin{array}{c}\text { Unbevelled } \\
\text { Unifacial }\end{array}$ & $\begin{array}{c}\text { Unbevelled } \\
\text { Bifacial }\end{array}$ & $\begin{array}{l}\text { Bevelled } \\
\text { Unifacial }\end{array}$ & $\begin{array}{l}\text { Alternate } \\
\text { Bevelling }\end{array}$ & Total & $\begin{array}{c}\text { Percent of } \\
\text { Alternate Bevelling }\end{array}$ & $\begin{array}{l}\text { Percent of All } \\
\text { Resharpening }\end{array}$ \\
\hline Valley Floor: & & & & & & & & \\
\hline IIIc & 12 & 2 & 34 & 10 & 25 & 83 & 30 & 86 \\
\hline $\mathrm{IIIb} / \mathrm{c}$ & 4 & 4 & 7 & 2 & 23 & 40 & 58 & 90 \\
\hline IIIb & 6 & 5 & 15 & 4 & 21 & 51 & 41 & 88 \\
\hline $\mathrm{III} \mathrm{a} / \mathrm{b}$ & 3 & 5 & 8 & 3 & 12 & 31 & 39 & 90 \\
\hline IIIa & 4 & 1 & 6 & 4 & 26 & 41 & 63 & 90 \\
\hline III & 2 & 0 & 1 & 0 & 4 & 7 & 57 & 71 \\
\hline II/IIIa & 1 & 1 & 1 & 1 & 7 & 11 & 64 & 91 \\
\hline II & 6 & 0 & 5 & 0 & 11 & 22 & 50 & 73 \\
\hline I/II & 2 & 0 & 2 & 1 & 5 & 10 & 50 & 80 \\
\hline I-upper & 2 & 1 & 2 & 1 & 3 & 9 & 33 & 78 \\
\hline I-lower & 1 & 0 & 0 & 0 & 1 & 2 & 50 & 50 \\
\hline Valley Margin: & & & & & & & & \\
\hline IIIC & 18 & 3 & 26 & 8 & 33 & 88 & 38 & 80 \\
\hline $\mathrm{IIIb} / \mathrm{c}$ & 2 & 2 & 7 & 3 & 3 & 17 & 18 & 88 \\
\hline Y/IIIc & 0 & 3 & 6 & 2 & 4 & 15 & 27 & 100 \\
\hline $\mathrm{Y} / \mathrm{IIIb} / \mathrm{c}$ & 0 & 0 & 0 & 0 & 2 & 2 & 100 & 100 \\
\hline IIIb & 2 & 2 & 4 & 0 & 6 & 14 & 43 & 86 \\
\hline Y/IIIb & 0 & 0 & 0 & 0 & 1 & 1 & 100 & 100 \\
\hline Y & 1 & 0 & 3 & 0 & 5 & 9 & 56 & 89 \\
\hline $\mathrm{X} / \mathrm{Y} / \mathrm{IIIb}$ & 0 & 1 & 0 & 0 & 0 & 1 & 0 & 100 \\
\hline X/IIIb & 0 & 0 & 1 & 1 & 3 & 5 & 60 & 100 \\
\hline $\mathrm{X} / \mathrm{Y}$ & 0 & 0 & 1 & 0 & 5 & 6 & 83 & 100 \\
\hline $\mathrm{III} \mathrm{a} / \mathrm{b}$ & 1 & 1 & 1 & 1 & 4 & 8 & 50 & 88 \\
\hline $\mathrm{X} / \mathrm{III} / \mathrm{b}$ & 0 & 0 & 0 & 0 & 2 & 2 & 100 & 100 \\
\hline IIIa & 0 & 0 & 0 & 0 & 3 & 3 & 100 & 100 \\
\hline Х/IIIa & 0 & 0 & 0 & 0 & 2 & 2 & 100 & 100 \\
\hline II/IIIa & 0 & 0 & 0 & 0 & 2 & 2 & 100 & 100 \\
\hline X/II/IIIa & 0 & 0 & 0 & 0 & 1 & 1 & 100 & 100 \\
\hline $\mathrm{X}$ & 1 & 2 & 4 & 1 & 9 & 17 & 53 & 94 \\
\hline X/II & 0 & 0 & 1 & 0 & 2 & 3 & 67 & 100 \\
\hline II & 1 & 0 & 0 & 0 & 2 & 3 & 67 & 67 \\
\hline Totals: & 69 & 33 & 135 & 42 & 227 & 506 & 45 & 86 \\
\hline
\end{tabular}

Note: Points from surface finds, fill, and questionable stratigraphic proveniences are not included. Totals do not include points with indetermindate resharpening.

TABLE 13-165

Anatomical Factors Determining the Requirements for a Fatal Arrow Wound (from Friis-Hansen 1990:Table 1)

\begin{tabular}{l|c|c|c|c|c}
\hline Game Animal & Weight $(\mathrm{kg})$ & Thorax Width & $\begin{array}{l}\text { Percent Free Space } \\
\text { Between Ribs }\end{array}$ & $\begin{array}{l}\text { Wound Surface } \\
\left(\mathrm{cm}^{2}\right)\end{array}$ & $\begin{array}{l}\text { Minimum Arrow Point } \\
\text { Perimeter }(\mathrm{cm})\end{array}$ \\
\hline Rangifer tarandus & 200 & 30 & 65 & 75 & 2.5 \\
Megaloceros giganteus & 600 & 40 & 50 & 100 & 2.5 \\
Alces alces & 300 & 35 & 60 & 80 & 2.3 \\
Bos primigenius & 800 & 45 & 45 & 115 & 2.6 \\
Bison bonasus & 500 & 40 & 70 & 95 & 2.4 \\
Bufalo caffer & 900 & 40 & 15 & 120 & 3.0 \\
Cervus elaphus & 250 & 35 & 60 & 75 & 2.2 \\
\hline \hline
\end{tabular}

That the essential principles ascertained by Friis-Hansen from modern European bow hunters were clearly understood by American Indian bison hunters is seen in the following accounts (assembled by Wheat 1972:92-95):
A buffalo's heart lies exceedingly low, so that to strike it the shot should enter not over one-fourth of the depth of the body above the lower edge of the breastbone [Gregg 1958:325]. 
... an arrow penetrates the bull's chest obliquely behind the last rib [McDermott 1940:192].

the hunter hopes to

...deliver his shot, taking aim a little below the center of the body, and about eight inches back of the shoulder...this will strike the vitals [Marcy 1963:302].

...a Teya was seen to shoot a bull right through both shoulders with an arrow [Winship 1896:507].

Turning to a consideration of projectile point data from Wilson-Leonard, a simplified characterization of form is employed that approximates the relationships noted by FriisHansen. Width times two (without factoring in thickness or estimated shaft diameters) affords a minimum estimate of the cross-section perimeter of the wound that any given point would make. The range and mean of those data show considerable variation through time at Wilson-Leonard (Table 13-166). In viewing the data in Table 13-166, the following information is helpful.

Projectile point forms are arrayed approximately in stratigraphic order from bottom to top at the left of the table. At least some members of each projectile point form exhibit impact fractures, an observation on which is based the inference that these are projectile points (possibly in addition to being used in other ways). In the second column is the number of specimens in each form for which the maximum width measurement is available. The third column subjectively divides the forms into two idealized groups - those with barbs or broad shoulders and those without barbs and having no or very slight shoulders. Barbed and broadly shouldered forms could only be withdrawn from a wound with difficulty whereas those lacking these elements could be withdrawn more easily. Next are tabulated the range and mean of the maximum width of each point form. These data represent minimum values because none of these specimens appears to be pristine, and many have been resharpened along the lateral edges reducing maximum width by an unknown amount. The wound perimeter, based on mean value of greatest width, of each form is tabulated next. Finally, the probable diameter of the shaft or foreshaft is estimated for each form using the distal stem width (which is the greater stem width for contracting and the lesser stem width for expanding stem forms).

The results of this tabulation are as follows. The 48 arrow points, all with shoulders or barbs, range in width from 9 to $23 \mathrm{~mm}$ (average $14.6 \mathrm{~mm}$ ) and were hafted on shafts estimated to range between 4 and $9 \mathrm{~mm}$ (average $6.25 \mathrm{~mm}$ ) in diameter. Two hundred forty-four dart points with barbs or broad shoulders range in width from 17 to $47 \mathrm{~mm}$ (average $28.4 \mathrm{~mm}$ ). Shafts for these are estimated to range from 10 to $26 \mathrm{~mm}$ (average about $18.6 \mathrm{~mm}$ ). And, 196 points without barbs or signficant shoulders range from 15 to $39 \mathrm{~mm}$ in width (average $23.8 \mathrm{~mm}$ ); shafts for these are estimated to range from 11 to $33 \mathrm{~mm}$ and to average $18.2 \mathrm{~mm}$.
Converting from centimeters to millimeters and plotting Friis-Hansen's minimum arrowhead perimeter against the body weights of the taxa he considered (see Table 13-165; see also Friis-Hansen 1990:Table 1) produces the graph shown in Figure 13-140. The data yield a calculated regression (Mosteller et al. 1983:315) as shown. The animals of concern in Central Texas are also shown on the vertical axis according to the weights of the larger individuals in each taxon (e.g., bison bulls, white-tailed bucks). From the regression, lethal projectile point perimeters can be predicted as follows: for a black bear, weighing $150 \mathrm{~kg}$, a point perimeter of about $21 \mathrm{~mm}$ should meet the Friis-Hansen requirement of a 10-second drop; for an antelope buck weighing $60 \mathrm{~kg}$, the perimeter estimate is less than $20 \mathrm{~mm}$; and, for a white-tailed buck weighing $100 \mathrm{~kg}$, the perimeter estimate is just over $20 \mathrm{~mm}$.

These figures mean that every single point form, even the arrow points, in Table 13-166 exceed the ca. 20 to $21 \mathrm{~mm}$ perimeter requirement of being lethal in 10 seconds or less by full penetration of the chest of these three kinds of animals.

A bison bull weighing $1,350 \mathrm{~kg}$ would require a cutting perimeter of $34.8 \mathrm{~mm}$, which is still exceeded by all but the arrow points in Table 13-166 (this perimeter is barely exceeded by the 35 -mm mean perimeter seen for Darl points). As calculated here, perimeter is simply maximum width doubled, meaning that points $17.4 \mathrm{~mm}$ in width should meet the lethality standard. The width ranges in Table 13-166 include only the following forms with a few specimens narrower than $17.4 \mathrm{~mm}$ : Darl, Gower, Hoxie, Angostura, and Hoxie/ Gower. In sum, the record is comprised virtually completely with what might theoretically be considered excessively wide points for all of the local game animals, except mammoths (see below). Why is this so?

In part, the answer probably lies in the fact that the hunting strategies and weapon systems could not reliably ensure wounds clean through the chest cavity of these game animals. Shock and as much bleeding as possible for wounds to less vital parts of the body is probably indicated, especially by the barbed and shouldered forms that could be expected to remain in a wound and cause pain and damage as the animal moved. Also, entire weapon systems may have been "over-designed" for increased reliability.

In regard to the design of points for the hunting of bison, it is apparent that wide points were commonly used to hunt bison (such as the Montell, Castroville, and Bell-Andice forms), but the data presented here do not indicate a particularly strong correlation between bison hunting and great projectile point widths. In part this is because Wilson-Leonard specimens of the types often associated with bison are not pristine and therefore do not reflect the greater widths that characterize such types. But there are also wide points (Jetta, for example) that are not known to be associated with bison hunting and narrow points (Midland, for example) that are associated with bison hunting. These are precisely the kinds of data that need to be more fully explored and understood. 
TABLE 13-166

Projectile Point Width, Shoulder Form, Estimated Wound Perimeter, and Estimated Shaft Diameter Data, by Types

\begin{tabular}{|c|c|c|c|c|c|c|c|c|}
\hline \multirow[b]{2}{*}{ Projectile Point Form } & \multirow[b]{2}{*}{ No. } & \multicolumn{2}{|c|}{ Barbs or Broad } & \multicolumn{2}{|c|}{ Width $(\mathrm{mm})$} & \multirow{2}{*}{$\begin{array}{r}\text { Perimeter } \\
\text { from Means }\end{array}$} & \multicolumn{2}{|c|}{ Estimated Shaft Diameter } \\
\hline & & Present & Absent & Range & Mean & & Range & Mean \\
\hline Scallorn & 37 & $\mathrm{X}$ & & $9-21$ & 14.5 & 29 & 4- 8 & 6.3 \\
\hline Other arrow points & 11 & $\mathrm{X}$ & & $11-23$ & 15.1 & 30 & 5- 9 & 6.2 \\
\hline Darl & 23 & & $\mathrm{X}$ & $14-21$ & 17.3 & 35 & $11-15$ & 13.3 \\
\hline Ensor & 35 & $X$ & & $18-28$ & 23.5 & 47 & $11-20$ & 14.9 \\
\hline Fairland & 4 & $\mathrm{X}$ & & $23-30$ & 26.8 & 54 & $14-20$ & 17.8 \\
\hline Frio & 5 & $\mathrm{X}$ & & $22-29$ & 25.2 & 50 & $12-18$ & 15.5 \\
\hline Marshall & 6 & $\mathrm{X}$ & & $26-31$ & 28.0 & 56 & $11-17$ & 14.3 \\
\hline Edgewood & 4 & $\mathrm{X}$ & & $19-25$ & 22.8 & 46 & $12-13$ & 12.8 \\
\hline Montell & 20 & $\mathrm{X}$ & & $24-45$ & 32.3 & 65 & $14-23$ & 19.5 \\
\hline Pedernales & 11 & $\mathrm{X}$ & & $24-47$ & 32.9 & 66 & $15-26$ & 19.4 \\
\hline Castroville & 8 & $\mathrm{X}$ & & $30-39$ & 33.8 & 68 & $17-26$ & 21.8 \\
\hline Marcos & 11 & $X$ & & $27-39$ & 31.3 & 63 & $13-22$ & 17.6 \\
\hline Williams & 2 & $\mathrm{X}$ & & $33-34$ & 33.5 & 67 & $18-19$ & 18.5 \\
\hline Bulverde & 24 & $\mathrm{X}$ & & $19-39$ & 29.1 & 58 & $16-22$ & 18.6 \\
\hline Bulverde-like & 15 & $\mathrm{X}$ & & $19-35$ & 28.1 & 56 & $16-21$ & 17.8 \\
\hline Nolan & 37 & & $\mathrm{X}$ & $21-37$ & 29.6 & 59 & $12-23$ & 17.9 \\
\hline Bell-Andice & 2 & $\mathrm{X}$ & & $29-36$ & 32.0 & 64 & $17-19$ & 17.6 \\
\hline Wells & 3 & & $\mathrm{X}$ & $20-34$ & 27.3 & 55 & $16-19$ & 17.2 \\
\hline Travis and Travis-like & 12 & & $\mathrm{X}$ & $19-27$ & 23.4 & 47 & $17-20$ & 18.6 \\
\hline Expanding Concave D & 6 & $\mathrm{X}$ & & $32-47$ & 35.6 & 71 & $15-20$ & 17.5 \\
\hline Expanding Stem D & 6 & $\mathrm{X}$ & & $22-27$ & 24.8 & 50 & $15-17$ & 15.7 \\
\hline Expanding Concave B & 13 & $\mathrm{X}$ & & $22-40$ & 27.8 & 56 & $10-19$ & 13.7 \\
\hline Expanding Concave $\mathrm{C}$ & 7 & $\mathrm{X}$ & & $25-34$ & 29.8 & 60 & $15-18$ & 16.6 \\
\hline Uvalde and Uvalde-like & 13 & $\mathrm{X}$ & & $20-34$ & 27.5 & 55 & $11-18$ & 14.3 \\
\hline Martindale A \& B & 10 & $\mathrm{X}$ & & $22-37$ & 25.5 & 51 & $15-19$ & 17.2 \\
\hline Jetta & 7 & $\mathrm{X}$ & & $28-47$ & 33.0 & 66 & $18-24$ & 22.0 \\
\hline Gower/Jetta & 4 & $\mathrm{X}$ & & $26-35$ & 30.7 & 61 & $17-22$ & 19.5 \\
\hline Baker, Bandy, Bandy-like & 9 & $\mathrm{X}$ & & $28-38$ & 31.4 & 63 & $12-19$ & 15.1 \\
\hline Thrall & 17 & & $\mathrm{X}$ & $20-39$ & 26.7 & 53 & $20-32$ & 24.8 \\
\hline Gower & 8 & $\mathrm{X}$ & & $17-26$ & 20.8 & 42 & $12-16$ & 15.1 \\
\hline Hoxie, Hoxie A, B, \& C & 32 & & $\mathrm{X}$ & $16-29$ & 21.6 & 43 & $14-21$ & 17.5 \\
\hline Angostura & 26 & & $\mathrm{X}$ & $15-28$ & 21.0 & 42 & $17-25$ & 20.7 \\
\hline Hoxie/Gower & 9 & & $\mathrm{X}$ & $17-25$ & 20.8 & 42 & $13-18$ & 15.7 \\
\hline Golondrina-Barber & 18 & & $\mathrm{X}$ & $21-33$ & 27.3 & 55 & $19-33$ & 26.5 \\
\hline St.Mary's Hall typic \& atypic & 15 & & $\mathrm{X}$ & $22-24$ & 23.0 & 46 & $21-28$ & 22.8 \\
\hline Wilson & 14 & $\mathrm{X}$ & & $23-43$ & 28.4 & 57 & $17-23$ & 19.1 \\
\hline Midland & 1 & & $\mathrm{X}$ & & & 38 & & \\
\hline Bone Bed point & 1 & & $\mathrm{X}$ & & & 40 & & \\
\hline Clovis & 2 & & $\mathrm{X}$ & $23-28$ & 25.5 & 51 & & \\
\hline
\end{tabular}

*Single specimen.

Further study of these issues must focus on site settings, projectile breakage patterns, and other indicators of hunting strategies and weapon systems design. For example, as is widely known and well reflected in Table 13-166, points without shoulders and barbs are more characteristic of the Paleoindian and earliest Archaic than they are of later time periods. One possible interpretation is that these points were attached to thrusting lances rather than to darts. If that were the case, it implies hunting strategies based on impeding or injuring animals to the extent that 


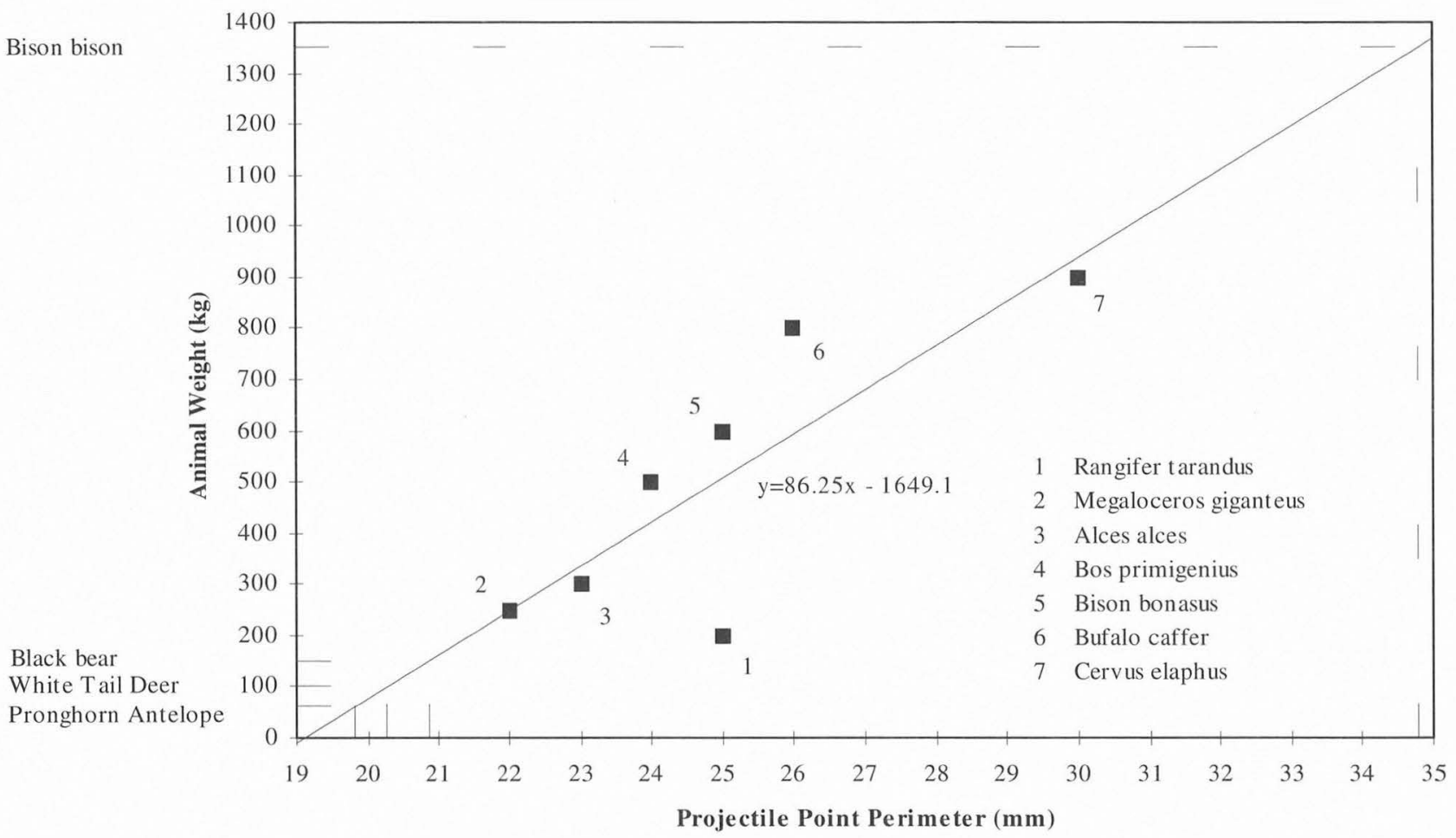

FIGURE 13-140. Regression of animal weights and lethal projectile point perimeters for seven Old World species as applied to New World taxa. (Old World data from Friis-Hansen 1990).

hunters could approach and lance them. This should be reflected arche-ologically in a consistent pattern of kill-site settings and lance-tip kinds of damage to points. Specific, testable hypotheses should emerge from continued inquiry along these lines.

As a final note, the relationship of body weight and lethal projectile point perimeter seems to apply with reasonable results to animals up to the size of a bison bull at ca. $1,350 \mathrm{~kg}$. Pushing it further to include mammoths at ca. $7,000 \mathrm{~kg}$ clearly does not yield reasonable results. The predicted perimeter of a projectile point needed to drop a mammoth would be ca. $106 \mathrm{~mm}$, or a point with a width of $53 \mathrm{~mm}$, close to twice that of typical Clovis points. This suggests several possibilities. Clovis "points" may be knives and Clovis "mammoth hunters" may have been scavengers more often than hunters. The extremely low frequency of impact damage on Clovis points would be consistent with that interpretation. It would also be consistent with Clovis points as tips of lances used in multiple stabbings of animals immobilized in some fashion. In lancing an animal mired in a bog, for example, a hunter could direct each thrust so as to minimize contact with bone and have the opportunity to inflict enough wounds to cause death.

\section{Size and Design}

Other variables needed to sort out weapon systems are elusive. The length, diameter, weight, and strength of the main shaft of arrows, darts, javelins, and lances should correlate with the mode of deployment and the nature of the target. In the absence of preserved shafts, these attributes can only be investigated indirectly, meaning, in this region, primarily through study of the stone points. Were it certain that these points were attached directly to main shafts, the haft modifications would be a clue to shaft size parameters, but nothing has been identified in projectile point design that indicates whether or not foreshafts were employed. Distal stem widths probably correlate well with the diameter of the shaft to which the point was hafted, but if that were a foreshaft, the diameter of the main shaft would probably be greater. Greater diameter does not necessarily mean greater weight or strength, however, because a hardwood shaft of identical proportions would be heavier and stronger than one of cane. This implies, therefore, that two point styles with similar estimated shaft diameter values could have been hafted in significantly different ways and been part of dissimilar weapon systems. Ensor and Uvalde/Uvalde-like forms, for example, have comparable estimated shaft diameter ranges and means (see Table 13-166), as follows: Ensor, range 11 to $20 \mathrm{~mm}$, mean 14.9; Uvalde/Uvalde-like, range 11 to $18 \mathrm{~mm}$, mean 14.3. Ensor points evidently were attached to foreshafts based on an example in the private collection of Jack Skiles, Langtry, Texas. The Skiles specimen consists of an Ensor point in a wooden foreshaft that was embedded in a human innominate bone recovered from a dry rockshelter near Langtry. Uvalde-style points may or may not have been 
employed with foreshafts, and further comparisons on the basis of estimated shaft diameters become entirely speculative.

Another attribute to consider is the configuration of the base of the projectile point forms. Three general categories are manifest in the forms under consideration herecontracting stem, bifurcate, and straight.

Tapered forms, such as those on Angostura points, are widest at the distal end of the hafting area and taper strongly toward the base. It is reasonable to hypothesize that these were inserted into a conical socket drilled into the end of a shaft or foreshaft and secured with adhesive and possibly wedges.

Forms with deeply concave or notched (bifurcate) bases (including some Darl, some Frio, Montell, Pedernales, the entire Early Archaic bifurcate series, and Golondrina-Barber types) range from straight to expanding stem varieties. These can reasonably be inferred to have been hafted into a slotted shaft or foreshaft that featured a central protrusion in the slot onto which the basal notch or concavity would be seated to reduce lateral movement of the stem in the haft. Interestingly, many of the specimens in this group are indicated as having been used as knives, an activity that produces lateral forces that would cause a loosely hafted blade to shift sideways out of alignment with its haft. The evidence is not exhaustive, but Golondrina-Barber points were found to have microscopic wear consistent with use as a knife (see Chapter 22), many of the Early Archaic bifurcate points are beveled in a manner suggestive of refurbishing a cutting edge, and a seriation of Pedernales points from Kincaid Shelter (Collins, notes on file, TARL) shows a pattern of lateral edge trimming more indicative of knife than of point refurbishing on most specimens.

The remainder of the forms have bases that range from shallow concavities, to straight, to convex. These are compatible with split-shaft hafting as well as with slotted-shaft hafting (without the central protrusion).

Mean estimated shaft diameters and the range of maximum widths of the Wilson-Leonard projectile points from Table 13-166 are presented graphically in Figure 13-141. In this graph, four categories are distinguished as withdrawable blades with bifurcate stems and without bifurcate stems along with barbed/shouldered blades with bifurcate stems and without bifurcate stems. These data are arrayed chronologically on the vertical axis and divided into the five major subperiods of the regional archeological chronology (from Collins 1995:Table 2).

Estimated mean shaft diameters show considerable variation in the Paleoindian and Early Archaic forms. Those of the Middle Archaic and earlier part of the Late Archaic are consistently close to $18 \mathrm{~mm}$. During the later part of the Late Archaic, shafts become smaller and more variable. Arrow shafts appear to be consisently close to $6 \mathrm{~mm}$ in diameter.

Withdrawable dart point forms are characteristic of the Paleoindian (except for Wilson points that are similar to a number of Archaic forms), some types of the early part of the Early Archaic, most of the Middle Archaic types, and only Darls in the Late Archaic. In contrast, barbed forms are particularly characteristic of the Late Prehistoric, Late Archaic, and the latter part of the Early Archaic.

Among Paleoindian points, only the Golondrina-Barber type has a deeply bifurcated base. Bifurcated stem points dominate the Early Archaic, are absent from the Middle Archaic, and are seen in only four types in the later part of the Late Archaic.

Among withdrawable Early Archaic and Paleoindian forms, there are six (Thrall, Hoxie, Angostura, GolondrinaBarber, St. Mary's Hall, and Clovis) where the mean of the estimated shaft diameters falls within, rather than below, the measured range of blade widths. This reflects considerable variation in blade width, possibly from resharpening.

Perhaps the data arrayed in Figure 13-141 depict patterns that are not evident at our present state of knowledge; the paradox is that, if it were known which types had been used with which kinds of weapons, correlative attributes might be immediately apparent. Some clue might be considered. Goodyear (1974:33) argues that beveled blades are more likely associated with foreshafts. If that is true and applies to these data, then the commonly beveled forms could represent the subpopulation of points used with foreshafts, and that subpopulation would almost certainly not include lances. Goodyear's argument was based on the logic that beveling results from resharpening of blades used as cutting tools and that cutting tools would be expected to have short handles (inferred to be dual-purpose handles and foreshafts). It is noteworthy that beveled blades in this data set are almost all on those types with bifurcate stems, already noted as a way to facilitate hafting to minimize lateral movement of points if they were used as a knife.

To summarize, all of the projectile point forms at Wilson-Leonard and common to the prehistory of most of Texas are much wider than expected from the perspective of being capable of producing an efficient kill. These were evidently hafted in at least three different ways - by being wedged into a conical socket; seated in a slot that engages a basal declevity in the point that, along with adhesives or bindings, decreases lateral movement; and in split or slotted shafts that depend entirely upon adhesives or binding to resist lateral movement. Data are extremely meager on the nature of shafts and foreshafts, and although there are some patterns to estimated shaft diameters in the Wilson-Leonard assemblage, it is not clear what they mean.

However, these and allied attributes warrant further scrutiny, especially using multivariate approaches that look in detail at correspondence between such attributes as use wear and haft form. Patterns of breakage, "burination" of barbs and shoulders, haft wear, blade and tip wear, and refurbishing need to be studied in relation to each other and to haft form. The modest number of hafted points from dry shelters and caves in western Texas should be studied in 

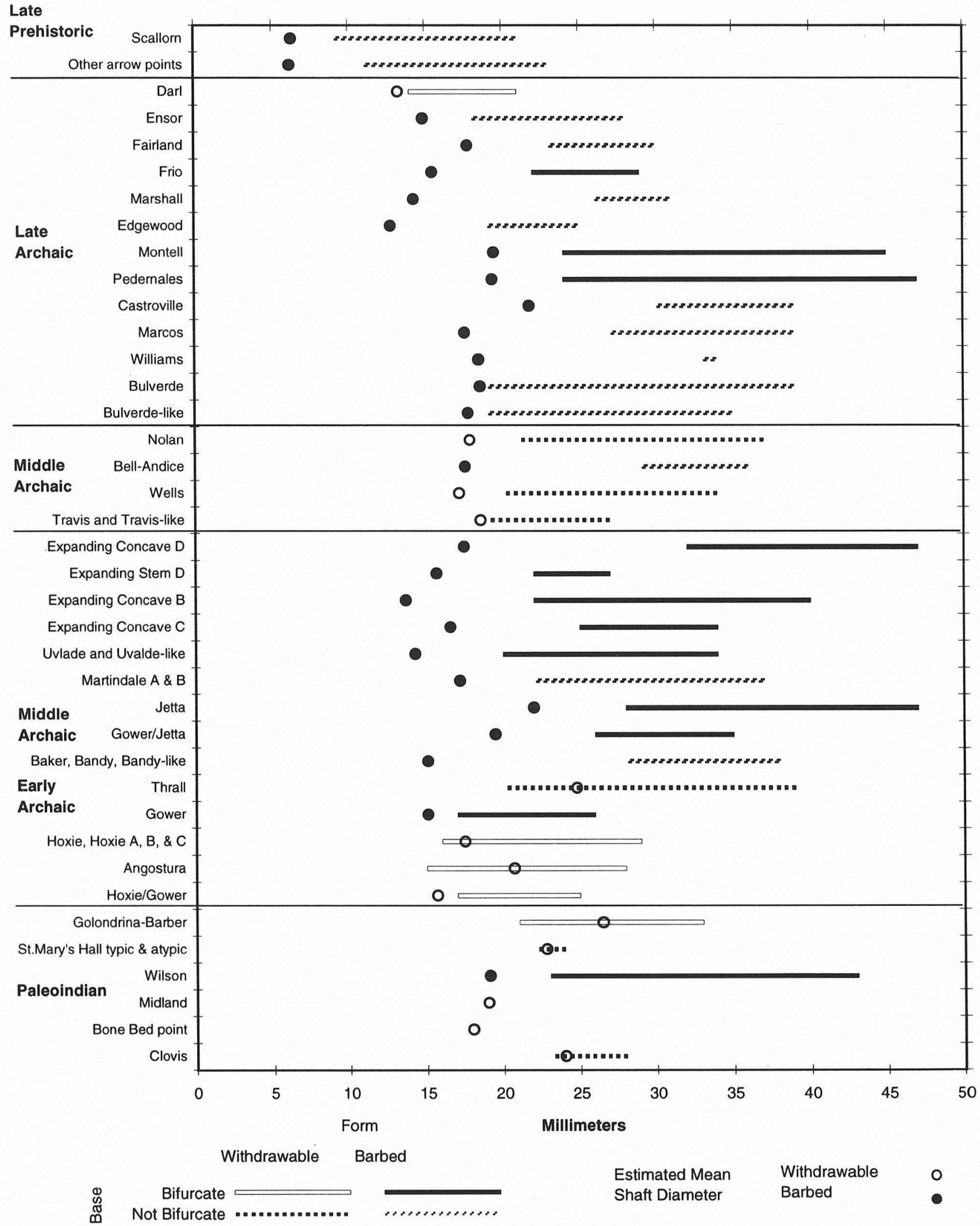

FIGURE 13-141. Wilson-Leonard projectile point widths and estimated shaft diameters by form over time. 
greater detail. Finally, greater use of contextual information is needed.

Among contextual information that warrants consideration are associations between point forms and specific kinds of fauna and whatever indicators we might recover on the kinds of hunting strategies that people were using. Occurrences are rare, but patterns among the few cases of points having been found in bone could afford clues as to the position of the hunter in relation to the anatomy of the prey animal. The Folsom point embedded in the vertebra of a bison found at the Lindenmeier site (Wilmsen and Roberts 1978:171, Figure 145) is such a case, indicating that the point entered from slightly above the animal (a standing animal speared from a high creek bank, or a disabled animal lying on the ground are among the indicated possibilities). If patterns could be discerned in the trajectories of weapons, more could be said about hunting strategies, and inferences should follow regarding the most appropriate weapon system for each indicated strategy. At present, research into weapons systems and hunting strategies for the prehistory of Texas has hardly begun, but there are relevant data, and the issue warrants greater effort.

\section{CONCLUSIONS}

In its quantity and great variety, the projectile point assemblage from Wilson-Leonard is a significant collection for typologists and archeological researchers. With few exceptions and three notable additions, the archeological sequence identified here well substantiates those frameworks laid forth by Suhm et al. (1954), Weir (1976), Prewitt (1981b, 1985), Johnson and Goode (1994), Collins (1995), and others. The addition of the Late Paleoindian expanding stem Wilson point to the sequence presents an intriguing link to stylistic traditions of the eastern United States, a connection which is further bolstered by other point types and tools from the site chiefly pertaining to Early Archaic occupations. Other influences are seen from the west, with the addition of the Early Archaic Bandy points to the Central Texas record.

The identification of the Late Paleoindian St. Mary's Hall point clears the way for greater understanding of unfluted lanceolate typology and dating. As is discussed in detail in the following chapter, these and other points are frequently classified as "Plainview," a distinctive type that appears much earlier in the archeological sequence and that was not recovered at Wilson-Leonard.

In a variety of technological traits in the projectile point sample, continuity with Paleoindian traditions is demonstrated well into Early Archaic times. These include lateral stem edge grinding and patterned flaking. To a large extent, edge resharpening in the form of alternate beveling also can be viewed as a trait that begins in Late Paleoindian times and reaches a peak of expression in the earlier part of the Early Archaic, particularly in Angostura and Hoxie points and other early bifurcate forms. An intriguing confluence of
Paleoindian and Archaic traits appears to be expressed within the early split stem and Angostura forms, both of which occur in high frequencies in the very Early Archaic deposits of Unit IIIa.

Our study has not focused exclusively on chronological markers and typological issues but also on possible human behavioral explanations and implications for the conformity and variability we see among certain projectile point forms. Specifically, here and in Chapter 14 we have sought to raise questions about changes in technology, hafting modes, and weaponry systems that might explain variation in projectile point styles seen over time. We have focused in somewhat greater measure on the point groups from Paleoindian and Early Archaic deposits, not only because of their more-secure contexts at this site but also in hopes of further elucidating the so-called Paleoindian/Archaic transition. With the Wilson-Leonard sample, questions can be focused on specific changes in point forms during this critical time period. What is the meaning of the standardization we see among certain Late Paleoindian lanceolate types, and which we, at present, fail to see among most early stemmed bifurcates? Is basal uniformity conditioned by style and tradition, or a need to conform to certain tightly drawn hafting restrictions, or aspects of both? If the answer is tightly drawn hafting restrictions, what is the purpose of those restrictions? Conversely, what does a relative lack of uniformity tell us about the makers of bifurcate stemmed points and the use of these points?

As Tomka and Prewitt (1993:49-58) have noted regarding the meaning of projectile point types, the role of stem and base shapes remains imperfectly understood. Based on the Wilson-Leonard sample, however, we believe that changes in projectile point morphology during these early periods in prehistory were not solely stylistically based. Alterations in the technoeconomic organization of populations over time appear to be reflected in some changes. These may have included moves toward greater efficiency by decreasing the amount of time and energy expended in projectile point manufacturing and maintenance (e.g., see Bleed 1986). Such choices over time likely included weighing the making of a finely flaked, more-standardized lanceolate point over a more-rapidly produced stemmed point with a hafting mode that ensured less breakage, longer use-life for varied tasks, and greater ease of resharpening. In our sample, the early bifurcate stemmed group may represent the inception of a more versatile hafting technology that could compensate for or tolerate greater variation in basal elements.

Perhaps more importantly, changes in projectile point form over time may carry larger implications reflecting alterations in weaponry systems, types of prey exploited and, concomitantly, the environment in which these early peoples subsisted. Whether a weapon was intended to be propelledor thrust and withdrawn - is a basic yet significant question which has received little attention in lithic technological research. Knowledge of the size range and habitat of various 
prey coupled with paleoenvironmental and faunal data through time will serve as valuable background information as these issues are considered. Of greater difficulty will be identifying the salient, distinguishing attributes on the stone points that tipped these various weapons. Nonetheless, it seems imperative that projectile point research move in these directions if we are to gain better understanding of the hunting practices and lifeways of early peoples. 
"This page intentionally left blank" 


\section{Chapter 14}

\section{STATISTICAL ANALYSIS OF UNFLUTED LANCEOLATE AND EARLY BIFURCATE STEM PROJECTILE POINTS}

by Anne C. Kerr and Susan W. Dial

\section{INTRODUCTION}

Morphological variation is considerable among unfluted lanceolate projectile points and early bifurcate stem projectile points. Prior to the analysis of the Wilson-Leonard sequence, this variation has not been rigorously scrutinized by typologists and sizable assemblages in good contexts have been lacking, rendering the typologies proposed for projectile points in these groups problematical (see Chapter 5). These problems affect our understanding and interpretation of Paleoindian and Archaic culture history and cultural behavior (Hester 1979b; Hofman 1989; Johnson 1991; Thoms 1993).

Two studies, one on unfluted lanceolate projectile points and the second on early bifurcate stem projectile points, were undertaken as a response to the quandaries over morphological variability. Every effort has been made to conduct these studies in a systematic and rigorous manner. Observations on the haft portions of projectile points were chosen for analysis inasmuch as the blade is subject to extensive modification through use and refurbishment. These observations evaluate the range of variation in size and shape. The goals were to delineate patterns in morphological variability and ascertain whether these patterns had relevant temporal and associational contexts (see discussions in Hoffman 1985; Krieger 1944; Thoms 1993; Tomka and Prewitt 1993).

Systematic analyses were separately carried out on unfluted lanceolate projectile points and early bifurcate stem projectile points in order to better address the morphological problems specific to each set. This being the case, the two analyses, with the exception of the methods, are presented in separate sections, each with specific discussions of background problems, results, and conclusions. The early bifurcate stem point analysis was initiated well after the unfluted lanceolate point study and due to time constraints, is more a work in progress, which does not quite reach the şame level of resolution as the unfluted lanceolate point study.

\section{METHODS}

These two analyses were first initiated by the assembly of representative samples and second, by the selection and recording of relevant observations of the sample specimens. Then a numerical classification technique was applied that enabled easier handling of large data sets and revealed morphological groupings in a consistent manner. This step was followed by evaluation of the results by use of another multivariate statistic and visual observation of the grouped specimens aided by reference to descriptive statistics. The unfluted lanceolate study also included an analysis of variance which clarified the significance of the morphological variability arrayed in the numerical classification. The final step was the comparison of the resulting morphological groups with available chronological data, as well as consideration of technological information and previous typological assignment.

\section{Assembling Representative Samples}

Two representative samples were required: one of unfluted lanceolate points and a second of early bifurcate stem points. The bifurcate-stem sample used in this study was drawn entirely from the Wilson-Leonard site whereas unfluted lanceolate points from Wilson-Leonard were studied along with comparable specimens from several sites. Ideally, each sample would include numerous specimens from well-stratified contexts with good radiocarbon age control, a condition not always found in the present archeological record. That being so, compromise on the side of representing as much variation as possible was necessary in some cases.

For the unfluted lanceolate point sample, specimens were included from original type-site collections when available, 
such as Plainview points from the Plainview site and Golondrina points from the Devil's Mouth site. Although neither of these two sites is particularly well dated and both have ambiguous stratigraphy as well, these Plainview and Golondrina specimens were considered the typological standards against which other unfluted lanceolate specimens were measured for the purpose of typology. The type-site specimens from poorly dated contexts were supplemented, when possible, by specimens from sites with better contextual or radiocarbon associations. For example, the Golondrina specimen from Baker Cave is described as coming from a hearth that produced a reliable radiocarbon date (Chadderdon 1983:22). Many of the unfluted lanceolate points from the Wilson-Leonard collection are from welldated deposits, although some specimens from poor context were included to maintain sample size and morphological variability.

As another important criterion, specimens had to be sufficiently complete for all relevant observations to be made. If a metric landmark was incomplete, then a judgment was made as to whether the fragmentation was too extensive for reasonable estimation, "reasonable estimation" being less than or equal to $2 \mathrm{~mm}$. Specimens were excluded if a single measurement could not be estimated or if it would cause too many of the observations to be based on estimation.

The unfluted lanceolate point sample of 182 specimens was drawn from the following named types: Angostura, Barber, Dalton, Golondrina, Midland, Milnesand, Plainview, and Scottsbluff. Some untyped or typologically ambiguous lanceolate specimens from the Wilson-Leonard site were also included. The minimum number of specimens from a named type, including possible but ambiguous specimens, was three. For example, from the Wilson-Leonard site there were two Scottsbluff specimens and one ground square stem specimen that might be Scottsbluff. These three specimens were included in the sample. A maximum number per named type was not set, but in order not to overwhelm the analysis with too many specimens of one type, a random sample was drawn of the single types that were represented by exceptionally large numbers of specimens. For example, a 19\% random sample was drawn from 75 Dalton points from the Sloan site. A more-detailed description of the unfluted lanceolate point sample is given below.

The 102 specimens for the early bifurcate stem point sample were drawn from the following named types: Uvalde, Martindale, Gower, Hoxie, Jetta, and Bandy. Untyped or typologically ambiguous early bifurcate stem specimens were also included. The problem of typological ambiguity is more apparent with this morphological set with some specimens referred to as Gower/Uvalde and Gower/Jetta.

The early bifurcate stem point sample was assembled from the Wilson-Leonard collection only and had sufficient numbers of specimens for each named type without including collections from other sites. However, a comparative sample, including type site specimens, would have been assembled if not for a time limit. Specimens from type sites were examined visually in order to guide selection of relevant observations on which to focus the analysis. A moredetailed description of the early bifurcate stem point sample is given below.

\section{Metric Observations and Ratios}

Variables for analysis were measurements and ratios that depict both size and shape of the artifacts. Measurements selected were taken from the basal portions of the points (Figure 14-1; see also Chapter 13). For the unfluted lanceolate point study, Kelly's (1982, 1983a, 1983b) work on a numerical classification scheme for lanceolate points suggests useful ways to look at a point base. Metric observations for this unfluted lanceolate point study included proximal haft width; 10 -mm haft width, measured at $10 \mathrm{~mm}$ above the base; depth of basal concavity; and haft/grinding length (haft/grinding length 1, the longer of the two grinding length measures, was chosen arbitrarily), all of which are similar to those used by Kelly (1982:Figure 1). A new measurement, 10-mm haft thickness (measured at $10 \mathrm{~mm}$ above the base), was added to include the third dimension of a point base. "Haft" rather than "stem" is used in this study to refer to that part of a lanceolate point that was secured in the haft. A ratio of 10-mm haft width to proximal haft width was calculated, rather than the haft index based on subtraction used by Kelly (1982:19). The haft width to proximal haft width ratio expresses stem shape. Other ratios include basal concavity depth to haft grinding length, indicating the relationship of void to solid in the shape of a point base, and 10-mm haft thickness to $10-\mathrm{mm}$ haft width, representing the haft thickness to haft width relationship. The measured observations indicate size, while the ratios represent shape irrespective of size.

Haft width was dropped as a direct variable from the cluster and discriminant function analyses due to redundancy and overemphasis on that variable, however, it is represented indirectly by the haft width to proximal haft width and haft thickness to haft width ratios. Haft grinding length was likewise not directly included because it had the highest variability as indicated by the standard deviation (8.372) for the whole sample and did not appear to have meaningful variation for this sample. Haft grinding length is indirectly represented in the basal concavity to haft grinding length ratio. All eight variables were used for the analysis of variance.

Six metric observations - stem length, distal stem width, distal stem thickness, proximal stem width, basal concavity depth, and basal concavity width - were taken on the early bifurcate stem points (see Figure 14-1). Of the ratios calculated, distal stem width divided by proximal stem width reflects stem shape, basal concavity depth divided by basal concavity width indicates a relative shallow-wide to narrow-deep basal concavity shape, and basal 


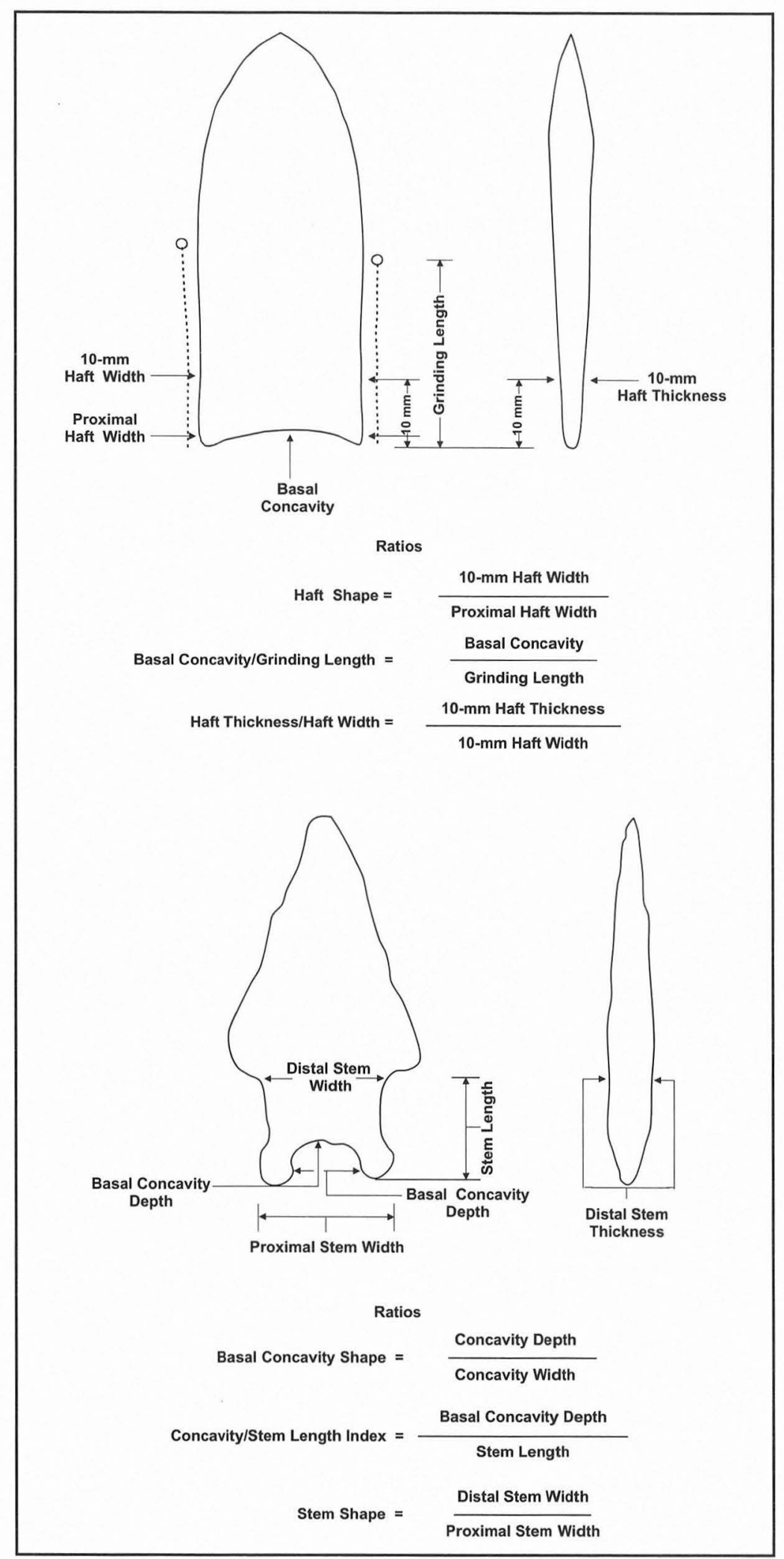

FIGURE 14-1. Measurements and ratios used in unfluted lanceolate point analysis.

concavity depth to stem length expresses the haft proportion of the stem length.

Both samples were tested through initial runs of statis- tical analyses to derive the optimum set of variables. The final set of measurements and ratios used was judged the best to describe the variation present in each sample. 


\section{Numerical Classification}

Numerical classification methods group items based on the values of variables that characterize them (Shennan 1988:193). The similarity to the traditional intuitive approach to doing artifact typology has made such methods easily applicable in archeology. The most compelling reasons to employ numerical classification are to make classification decisions explicit, to make handling of large data sets easier, to make the grouping process consistent, and to reveal patterning not easily apparent in large, complex data sets (Shennan 1988:195).

For both the unfluted lanceolate point and the early bifurcate stem point analyses, the multivariate statistics used

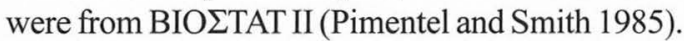

Cluster analysis was the numerical classification method chosen because it generates a pattern of groupings in a data set (Shennan 1988:196). This process is one of discovery. BIOETAT CLUST (Pimentel and Smith 1985: 117-123) was used to determine the groupings of specimens in both samples. An association matrix (a similarity measure of the relationships between individuals in a sample) is generated prior to running a cluster analysis. The association matrix was calculated by Percentage of Similarity, a symmetric matrix (Pimentel and Smith 1985:120; Shennan 1988:198). Although Euclidean Distance is a frequently used and easily understood measure of similarity (Pimentel and Smith 1985:117; Shennan 1988:198), it did not link similar specimens as effectively when tested against Percentage of Similarity for the data analyzed here. The clusters were calculated by group average, also known as the unweighted pair group method, which is a conventional agglomerative hierarchical method (Pimentel and Smith 1985:117; Shennan 1988:215-217). Such a method builds hierarchical groups beginning with the most similar items and then adds less similar items and groups until all items are linked in a large group that is displayed as a dendrogram (Shennan 1988:197).

\section{Process of Validation}

Cluster analysis tends to impose its own patterning to some degree on any data set (Shennan 1988:197). Since the resulting clusters may actually reflect real similarities or be somewhat arbitrary, it is important to apply some process of validation (Shennan 1988:197, 228-232). Both analyses employed discriminant function analysis and visual observation of clustered specimens with the assistance of descriptive statistics. The unfluted lanceolate point results were further evaluated by analysis of variance. It is important to remember that individual clusters do not necessarily represent types. To get from morphological clusters to types, it is necessary to look at the pattern of similarities and differences between pairs or groups of clusters. Thus, it is possible to see some morphological links between some clusters that may be indicative of types. Also, the consistent correlation of a previous type assignment with particular clusters may further suggest continuation of that type assignment. When more than one type assignment appears in a morphological cluster, consideration of technological (i.e., flake patterning) information may convey important type differences. Most importantly, the chronological or stratigraphic position of morphological clusters reflects the significance of type assignment.

\section{Discriminant Function Analysis}

Discriminant function analysis was used to check the results of the cluster analysis. Since this method presupposes existing groups and is concerned with checking the appropriate assignment of items to those groups (Shennan 1988:196), it helped to refine the groups and improve the initial cluster results.

Both analyses used BIOETAT MDA (Pimentel and Smith 1985:147-159). Particular attention was paid to the Geisser Classification Probabilities. The Geisser Classification Probability table shows the results of the evaluation of group membership for all individual items in the sample. This evaluation is reported in summary form by hits (correct assignments) and misses (incorrect assignments) (Pimentel and Smith 1985:157-158). In addition, by examining the full table of probabilities $(p)$ it is possible to discern how groups relate to one another, particularly those groups to which misses are reassigned from other groups. A criterion of $p=0.05$ is used to assess the strength of relationships between clusters. It is also possible to see how well individual specimens fit into their assigned group.

\section{Visual Examination and Descriptive Statistics}

The cluster analysis results were further checked by direct visual examination of the original artifacts or illustrated facsimiles for those specimens from distant collections. This allowed a further but very limited intuitive evaluation of other characteristics not measured directly in either analysis such as the quality and kind of flaking and general attributes of the blade including shape or breakage. The human eye was aided in this effort by reference to descriptive statistics such as mean, sample standard deviation, and range for each variable measured for each cluster. The examination of the descriptive statistics for each of the variables by cluster helped to illuminate the key morphological characteristics for each cluster.

A series of runs of the data through cluster analysis and discriminant function analysis, followed by visual examination of the clustered artifacts, was necessary in order to understand the behavior of the chosen variables and their ability to represent the morphology of the artifacts being analyzed. This allowed refinement of the data set and improved final interpretation of the results. In essence, this 
interplay of traditional visual sorting and statistical analysis of variables seeks to bring the best of both approaches to bear on the problem.

\section{Analysis of Variance}

An analysis of variance of the unfluted lanceolate point clusters further clarified the significance of the morphological variability arrayed by the cluster analysis. Analysis of variance (ANOVA) "partitions the total variation in a data set according to the sources of variation that are present" (Johnson and Bhattacharyya 1985:466). When comparing any number of means, the differences between means (or variation between groups) and inherent variation (or variation within groups), are the two sources of variation evaluated. Briefly, the Treatment Sum of Squares measures the variance between means; the Error Sum of Squares measures the inherent variation (Johnson and Bhattacharyya 1985:469-470). The $F$ ratio tests the significance of the difference between means and will be greater than the relevant $F$ distribution point as determined by the appropriate degrees of freedom when there is a significant difference between means and distributions (Johnson and Bhattacharyya 1985:476-477). The significant differences were determined at $\alpha=0.01$.

\section{Chronological Context of Morphological Clusters}

The final step for both analyses was to see if the morphological patterning of the defined and assessed clusters had any chronological patterning as well. Morphological groups were compared using their available stratigraphic or temporal context to determine chronological position. For clusters of specimens from a single site, the internal site stratigraphy was useful. However, when comparing clusters and specimens from different sites, radiocarbon age ranges were examined. A clear weakness in the reliance on the radiocarbon data for this purpose was apparent from the variety of materials dated that were not always directly comparable. For this reason, age ranges were considered rather than just midpoints or single dates. All previously published radiocarbon ages from the relevant cultural deposits were compiled and evaluated. Using age ranges instead of a single date occasionally suggested a reasonable alternative interpretation of previously published data.

For the Wilson-Leonard site, it was possible to use the stratigraphic and elevation data for the clustered unfluted lanceolate point specimens to examine the chronological patterning, and this accounted for about one-half of this sample. These data were then compared with the chronological information for specimens from other sites using radiocarbon age ranges when such data were available. Intuitive technological information and previous type assignment also had some influence at this stage.
The clustered early bifurcate stem point specimens were examined by stratigraphic unit and level for chronological patterning. Some cross dating from other sites also helped to determine chronological position. Previous type assignment was important in the assessment of the results for the early bifurcate stem point sample.

\section{SYSTEMATICANALYSIS OF UNFLUTED LANCEOLATE PROJECTILE POINTS}

This analysis is a response to the accumulating problems regarding morphological variability among unfluted lanceolate projectile points. Trying to document and understand this variability requires consistent comparison of morphology. The logical starting point was the basal portion of a representative sample of the unfluted lanceolate points.

\section{Typological Problems}

One way to understand the problems within the various named types of unfluted lanceolate points is to review the history of how these types were defined. Through this process, it is apparent that the recognition of a type is often cumulative in nature and includes the opinions of many different individual archeologists. Initial description and establishment of a type, based on a small sample, can be restricted and narrow, and then, as more evidence becomes available the morphological range of the type is expanded. In contrast, recognition of special characteristics or significant temporal context can bring about a restriction or a splitting-off of a type. An example of this kind can be seen with the Generalized Folsom and True Folsom of Howard (1935) and Cotter $(1937,1938)$ emerging as the Clovis and Folsom we recognize today (Hester 1972; Howard 1943; Krieger 1947b; Wormington 1957).

\section{Historical Beginnings}

In 1931 and 1932, Renaud published two papers that defined as "Yuma" a series of unfluted lanceolate projectile points. Renaud (1931) described Yuma varieties with observations of size, shape, and flaking (some of the basic morphological characteristics we still use today). However, use of surface specimens (collected mostly from Yuma County, Colorado) and primary reliance on morphology rather than context led to prematurely named types. At the time, Renaud (1932:10) thought that the Yuma types were about the same age as true Folsom because often they had been found together, albeit on the surface. Later Wormington (1957:104107) pointed out the Yuma types were flawed because of the morphological variation included among the types and subtypes and the reliance on morphology in the absence of chronological and contextual associations. The formal use of "Yuma" as a type was dropped in favor of the general morphological category of "parallel-flaked" points after the 
1941 Santa Fe conference (Howard 1943:227-228; Wormington 1957).

Basic morphological characteristics still remain as essential to the typological process of any artifact class, unfluted lanceolate points included. Documenting morphological variability and understanding its significance is an integral part of the typological process. Krieger's (1944:278) typological concept stressed the importance of considering spatial, temporal, and cultural distributions along with physical characteristics of specimens. The ideal criterion for determining types, that each type has essentially consistent, clearly recognizable patterns, is still a sensible approach to defining useful types. The question of how much variation to allow for any given type still plagues typologists, however. Also, the problem of maintaining consistency in the typological process is a continuous challenge.

The current confusion among all unfluted lanceolate point types can best be understood by review of the history of the Plainview and Angostura types. These are two of the most commonly identified Paleoindian projectile point types in southern Plains archeology, and they exemplify most of the problems typologists face or create as they deal with the unfluted lanceolate forms.

\section{Plainview}

The Plainview type was first described by Krieger (1947a:938-952) according to raw material, size, shape, smoothing or grinding of lateral edges, and flaking. The point type was described as being generally parallel sided, several perfectly so and others less so, with concave bases which rounded at the corners, grinding from base to midpoint or just beyond, and with two forms of flaking, irregular flaking over convex surfaces and collateral flaking of near equal size meeting at a medial ridge. The original definition of the type is extensive with specific specimens being compared and contrasted within all the categories of observation. Following this description is a lengthy and thoughtful discussion of the problems in typing of fluted and unfluted lanceolate points that is a good representative of its time within the history of archeology and typology in particular. Some specific questions and issues have changed (or been left behind like Yuma), but the general problem of recognizing significant attributes on lanceolate points which separate groups of points in cultural or temporal ways is still with us.

By 1954, the range of variation acceptable for the Plainview type had expanded beyond that indicated by the type-site specimens, and Krieger played an important role in altering the range for Plainview projectile points. This change is most evident in metric attributes. For example, the original maximum width of 22 to $26 \mathrm{~mm}$ (Krieger 1947a:939) was expanded to 18 to $28 \mathrm{~mm}$ (Suhm and Jelks 1962:239; Suhm et al. 1954:472). The most dramatic change was in basal concavity which went from 1 to $3.5 \mathrm{~mm}$, with a median of 2 mm (Krieger 1947a:942) to 1 to $8 \mathrm{~mm}$ (Suhm and Jelks
1962:239; Suhm et al. 1954:472). The specimens assembled to represent Plainview in Plate 116 of Suhm et al. (1954) also show considerable leeway in flaking pattern and shapes. Note particularly Specimen L, which would be typed as Golondrina today but was unrecognized at that time. As potential Plainview specimens were recognized at other sites, such as Red Smoke and Lime Creek (Davis 1962:84) and Bonfire Shelter (Dibble and Lorrain 1968:36), oblique flaking was added, and the shape of the base ranged from straight to concave.

The cumulative expansion of the Plainview type brought up questions about the application of the type name, however. Wheat (1972) observed that the Plainview type name was being applied indiscriminately. Upon the discovery of a Plainview-like component below a Folsom component at the Hell Gap site, Irwin-Williams et al. (1973), expressing doubts about the application of the Plainview type name to all generalized concave-based lanceolate points, selected a new type name, Goshen, for this component. Others (e.g., Johnson and Holliday 1980; Knudson 1973) have also noted the broad use of the Plainview type name for all unfluted concavebase lanceolate points.

Adding a slightly different twist to the Plainview type, Plainview golondrina was originally classified as a distinctive variety of Plainview (Johnson 1964:49) and described in contrast to Plainview. To distinguish the golondrina variety, the flared basal corners, deeper basal concavity, crescentshaped basal thinning flake scars, and generalized crude, random flake scars were emphasized. Temporal differences were unknown at the time of publication of the Devil's Mouth site.

During the 1970s, the Texas archeological community began referring to these points as Golondrina, a distinct type no longer considered a variety of Plainview (e.g., Hester 1977:175, 1978:2). Also, a temporal range for Golondrina of about 8700 to 9000 в.P. was established, and this was thought to be somewhat younger than Plainview.

Kelly (1982), interested in the application of numerical classification techniques to selected unfluted lanceolate point comparisons, attempted to make a consistent and objective study of the Plainview-Golondrina variability. In his comparisons, only specimens that fit an ideal type description for Golondrina were included (Kelly 1982:2-9). This selective approach to sampling, by which only 3 of the original 10 specimens from the Devil's Mouth site were included (Johnson 1964:46-49), made the resulting contrasts between Plainview and Golondrina impressive, but it did not address the full range of variability present in Golondrina specimens such as described at the type site of Devil's Mouth (Johnson 1964; Sorrow 1968).

In an expansion of the original numerical analysis, Kelly (1983b:28-32) recognized a Miniature Plainview as being similar to Plainview except for a shorter length and narrower width. Grinding length was also notably shorter for Miniature Plainviews (Kelly 1983b:Table 2). There was no chrono- 
logical information available to assist in interpreting the pattern.

Kelly (1983a) identified another variant within deeply concave-based unfluted lanceolate points which he proposed as an additional type, Barber. In contrast to Golondrina and Plainview, Barber points, with a deep basal concavity similar to Golondrina, have a contracting stem shape and lack the flared basal corners. The description was derived from only nine specimens: five from a surface collection in Gillespie County and four from the Wilson-Leonard site. Based on the stratigraphic positions at the Wilson-Leonard and St. Mary's Hall sites, the chronological position was interpreted as contemporary with and younger than Plainview but before Angostura (Kelly 1983a:21).

Despite efforts to split off some of the morphological variations, the Plainview type label has been the most frequently applied of any Paleoindian type. In a review of at least 460 known Paleoindian sites from Texas, $18 \%$ of the components were Plainview. Clovis, San Patrice, and Angostura were the next most frequent with $12 \%$ each. With such a wide ranging definition of the Plainview type, the distribution of Plainview sites is extensive in Texas (Collins and Kerr 1993:Figure 1h). It is time to question this cumulative and all-inclusive definition of Plainview. This type pattern may not be as meaningful or helpful for cultural interpretation as it could be.

\section{Angostura}

Angostura is another unfluted lanceolate type with a history of typological confusion. The first type description by Hughes (1949:270) consisted of one sentence, which vaguely indicated size, shape, and flaking for the proposed "Long" point, designated for the Ray Long site in South Dakota. Wheeler (1954:4), after further work at the site, changed the type name to Angostura and offered an extended verbal description emphasizing shape, flake pattern, cross section, and grinding characteristics. Meanwhile, Suhm et al. (1954:402), with the aid of Hughes, offered an alternative type description based on specimens from Texas, which stressed shape, size, and grinding attributes. Wheeler (1995:418-419) later rejected all but one of the specimens in Plate 80 of Suhm et al. (1954), and that specimen was not considered a "true Angostura" because the diagonal flaking runs from upper right to lower left. It has been a serious typological handicap that Wheeler's (1995) complete formal type description was not published for 40 years (Banks et al. 1995). The formal description of the Angostura point (Wheeler 1995:415) is of a large, slender, symmetrically convex-sided lanceolate point that tapers to a narrow base, with "parallel diagonal ripple flake scars running from upper left (tip) to lower right (base)." The published data on size unfortunately do not come from the original type-site specimens (see Wheeler 1995:416). It is clear from the discussion of the distributional data that Wheeler (1995:416-419) placed primary significance on the upper left to lower right parallel diagonal ripple flaking regardless of shape and size for designation of a specimen as Angostura. Wormington (1957:139) apparently concurred with the primary emphasis on upper left to lower right diagonal flaking, choosing to publish a photograph of an obliquely flaked lanceolate point from the University of Nebraska State Museum collection but none from the original type site. At the same time, Wormington (1957:138-141) criticized the extensive application of the Angostura type name in the absence of complete information about the original points from the type site. Later, Greiser (1985:84) pointed out the contrasts between the Nebraska specimen and those from the Ray Long site, suggesting the Nebraska specimen more closely resembles a Frederick point.

What is most apparent from this review of the Angostura type is the inconsistency in the choice of attributes among various typologists. The inconsistency led to very different conclusions about which specimens were or were not Angostura. Also, there was a general lack of contextual data used in formulating this type. In a review of the Angostura type, Thoms (1993) demonstrates how tenuous the evidence is for our cultural constructs. Kelly (1983b) attempted to circumvent the problems concerning the Angostura type by designating narrow, leaf-shaped unfluted lanceolate points as "Texas Angostura."

With the history of problems and confusion over unfluted lanceolate artifacts, the analysis of the WilsonLeonard site collection presented an obvious opportunity to analyze a wide range of morphological variability of unfluted lanceolate points from securely dated contexts and compare those to previously recognized types.

\section{The Sample}

The unfluted lanceolate projectile point sample consists of 182 specimens from 15 sites. The Wilson-Leonard site contributed 88 points $(48 \%)$ to the sample, which includes specimens that at various times have been referred to as Midland, Plainview, Golondrina, Barber, Dalton, Scottsbluff, Firstview, Angostura, Fat Angostura, and Hell Gap. As a result of this analysis, these preliminary type designations were reevaluated and accepted or modified as necessary. The original and revised type designations for the Wilson-Leonard site are listed in Table 14-1. Forty-four reliable radiocarbon ages referable to Early Paleoindian through Early Archaic contexts date or bracket the stratigraphic deposits that contained most of these specimens (see Chapter 25).

The comparative sample was assembled from 14 sites and consists of 94 specimens (52\%). The original type names include Plainview, Plainview-Angostura, Milnesand, Lubbock, Midland, Dalton, Plainview golondrina, Golondrina, Angostura, and Hell Gap (see Table 14-1). These original type designations were reevaluated by this analysis, and revised type names are suggested in some cases. 
TABLE 14-1

Sample List and Specimen Key for Cluster Analysis Dendrogram (see Figure 14-2)

\begin{tabular}{|c|c|c|c|c|c|}
\hline $\begin{array}{l}\text { Specimen } \\
\text { No. } \\
\end{array}$ & \begin{tabular}{|l|} 
Accession \\
No. \\
\end{tabular} & Site & Original Type & Revised Type & Reference \\
\hline 1 & $70-1$ & 41WM235, Wilson-Leonard & Angostura & Angostura & This report \\
\hline 2 & $3 \mathrm{M}-1$ & 41WM235, Wilson-Leonard & Angostura & Angostura & This report \\
\hline 3 & 29P1-4 & 41WM235, Wilson-Leonard & Unclassified & Angostura & This report \\
\hline 4 & 17Q1-1 & 41WM235, Wilson-Leonard & Angostura & Angostura & This report \\
\hline 5 & $738-1$ & 41WM235, Wilson-Leonard & Angostura & Angostura & This report \\
\hline 6 & $31 \mathrm{U} 1 \mathrm{C}-2$ & 41WM235, Wilson-Leonard & Unclassified & Angostura & This report \\
\hline 7 & 25Q1-1 & 41WM235, Wilson-Leonard & Angostura & Angostura & This report \\
\hline 8 & $16 \mathrm{~S} 2-1$ & 41WM235, Wilson-Leonard & Angostura & Angostura & This report \\
\hline 9 & 10Q-8 & 41WM235, Wilson-Leonard & Angostura & Angostura & This report \\
\hline 10 & $23 \mathrm{P}-1$ & 41WM235, Wilson-Leonard & Angostura & Angostura & This report \\
\hline 11 & $36 \mathrm{M}-1$ & 41WM235, Wilson-Leonard & Angostura & Angostura & This report \\
\hline 12 & $110-1$ & 41WM235, Wilson-Leonard & Angostura & Angostura & This report \\
\hline 13 & $14 \mathrm{M} 2-2$ & 41WM235, Wilson-Leonard & Unclassified & Angostura & This report \\
\hline 14 & TB-31 & 41WM165, Tombstone Bluff & $\begin{array}{l}\text { Angostura-like, Group } \\
3\end{array}$ & Angostura & Prewitt 1982:Fig. 81o \\
\hline 15 & $21 \mathrm{M}-1$ & 41WM235, Wilson-Leonard & Angostura & Angostura & This report \\
\hline 16 & $1345-1$ & 41WM235, Wilson-Leonard & Angostura & Angostura & This report \\
\hline 17 & 29T1C-1 & 41WM235, Wilson-Leonard & Angostura & Angostura & This report \\
\hline 18 & OR588 & 41WM235, Wilson-Leonard & Angostura & Angostura & This report \\
\hline 19 & OR337 & 41WM235, Wilson-Leonard & Angostura & Angostura & This report \\
\hline 20 & $0-17$ & 41WM235, Wilson-Leonard & Unclassified & Angostura & This report \\
\hline 21 & $895-1$ & 41WM235, Wilson-Leonard & Angostura & Angostura & This report \\
\hline 22 & 25R1-3 & 41WM235, Wilson-Leonard & Angostura & Angostura & This report \\
\hline 23 & $300-1$ & 41WM235, Wilson-Leonard & Angostura & Angostura & This report \\
\hline 24 & TB-8 & 41WM165, Tombstone Bluff & Angostura, Group 1 & Angostura & Prewitt 1982:276 \\
\hline 25 & $11 \mathrm{~S}-1$ & 41WM235, Wilson-Leonard & Angostura & Angostura & This report \\
\hline 26 & TB-17 & 41WM165, Tombstone Bluff & Angostura, Group 2 & Angostura & Prewitt 1982:276-277 \\
\hline 27 & TB-6 & 41WM165, Tombstone Bluff & Angostura, Group 1 & Angostura & Prewitt 1982:276 \\
\hline 28 & $\begin{array}{l}\text { TTU-A1- } \\
40469\end{array}$ & 41LU1, Lubbock Lake & Lubbock & Lubbock & $\begin{array}{l}\text { Holliday and Johnson } \\
\text { 1990:Fig. } 8\end{array}$ \\
\hline 29 & $25 \mathrm{R} 1-1$ & 41WM235, Wilson-Leonard & Angostura & Angostura & This report \\
\hline 30 & $\begin{array}{l}\text { TTU-A- } \\
39427\end{array}$ & 41LU1, Lubbock Lake & Lubbock & Lubbock & $\begin{array}{l}\text { Holliday and Johnson } \\
\text { 1990:Fig. } 8\end{array}$ \\
\hline 31 & TB-14 & 41WM165, Tombstone Bluff & Angostura, Group 1 & Angostura & Prewitt 1982:276 \\
\hline 32 & TB-13 & 41WM165, Tombstone Bluff & Angostura, Group 1 & Angostura & Prewitt 1982:276 \\
\hline 33 & TB-11 & 41WM165, Tombstone Bluff & Angostura, Group 1 & Angostura & Prewitt 1982:276 \\
\hline 34 & $121-2 / 6$ & 41TV49, Levi Rockshelter & Plainview-Angostura & Angostura & Alexander 1963:Fig. 3c \\
\hline 35 & TB-12 & 41WM165, Tombstone Bluff & Angostura, Group 1 & Angostura & Prewitt 1982:Fig. 81e \\
\hline 36 & $\begin{array}{l}\text { TTU-A- } \\
39340\end{array}$ & 41LU1, Lubbock Lake & Lubbock & Lubbock & $\begin{array}{l}\text { Holliday and Johnson } \\
\text { 1990:Fig. } 8\end{array}$ \\
\hline 37 & TB-21 & 41WM165, Tombstone Bluff & $\begin{array}{l}\text { Angostura-like, Group } \\
1\end{array}$ & Angostura & Prewitt 1982:Fig. $81 \mathrm{~h}$ \\
\hline 38 & 13Q-1 & 41WM235, Wilson-Leonard & Angostura & Angostura & This report \\
\hline 39 & TB-5 & 41WM165, Tombstone Bluff & Angostura, Group 1 & Angostura & Prewitt 1982:276 \\
\hline 40 & $\begin{array}{l}231-2 / 18, \\
231-2 / 12, \& \\
21-2 / 18\end{array} \mid$ & 41TV49, Levi Rockshelter & Plainview-Angostura & Angostura & Alexander 1963:Fig. 2h \\
\hline 41 & OR402 & 41WM235, Wilson-Leonard & Angostura & Angostura & This report \\
\hline 42 & 392 & 41VV218, Bonfire Shelter & Plainview & Lubbock & $\begin{array}{l}\text { Dibble and Lorrain 1968:Fig. } \\
\text { 14d }\end{array}$ \\
\hline 43 & TB-3 & 41WM165, Tombstone Bluff & Angostura, Group 1 & Angostura & Prewitt 1982:Fig. 81d \\
\hline 44 & TB-2 & 41WM165, Tombstone Bluff & Angostura, Group 1 & Angostura & Prewitt 1982:276 \\
\hline 45 & TB-7 & 41WM165, Tombstone Bluff & Angostura, Group 1 & Angostura & Prewitt 1982:Fig. 81b \\
\hline 46 & TB-9 & 41WM165, Tombstone Bluff & Angostura, Group 1 & Angostura & Prewitt 1982:276 \\
\hline 47 & $32 \mathrm{~T} 1 \mathrm{C}-3$ & 41WM235, Wilson-Leonard & Angostura & Angostura & This report \\
\hline
\end{tabular}


Table 14-1, continued

\begin{tabular}{|c|c|c|c|c|c|}
\hline $\begin{array}{c}\text { Specimen } \\
\text { No. }\end{array}$ & \begin{tabular}{|l} 
Accession \\
No. \\
\end{tabular} & Site & Original Type & Revised Type & Reference \\
\hline 48 & $11 \mathrm{Q}-1$ & 41WM235, Wilson-Leonard & Angostura? & Angostura & This report \\
\hline 49 & $\begin{array}{l}1-3,11-1, \& \\
02-1\end{array}$ & 41TV49, Levi Rockshelter & Plainview-Angostura & Angostura & Alexander 1963:Fig. 2h \\
\hline 50 & $19 \mathrm{~N}-1$ & 41WM235, Wilson-Leonard & Angostura & Thrall & This report \\
\hline 51 & $22 \mathrm{O}-10$ & 41WM235, Wilson-Leonard & Angostura & Thrall & This report \\
\hline 52 & 15G-1 & 41WM235, Wilson-Leonard & Angostura & Thrall & This report \\
\hline 53 & $8 \mathrm{~K}-1$ & 41WM235, Wilson-Leonard & Angostura & Thrall & This report \\
\hline 54 & 20Q-1 & 41WM235, Wilson-Leonard & Angostura & Thrall & This report \\
\hline 55 & $7 \mathrm{~N}-1$ & 41WM235, Wilson-Leonard & Angostura & Thrall & This report \\
\hline 56 & $22 \mathrm{P}-1$ & 41WM235, Wilson-Leonard & Angostura & Thrall & This report \\
\hline 57 & $21 \mathrm{~N}-1$ & 41WM235, Wilson-Leonard & Angostura & Thrall & This report \\
\hline 58 & $11 \mathrm{H}-1$ & 41WM235, Wilson-Leonard & Angostura? & Thrall & This report \\
\hline 59 & 2-61-1 & Winkler-1 & Midland & Midland & Blaine 1968 \\
\hline 60 & $2-38-5$ & Winkler-1 & Midland & Midland & Blaine 1968 \\
\hline 61 & 2-14-1 & Winkler-1 & Midland & Midland & Blaine 1968:Fig. $1 F$ \\
\hline 62 & $2-9-1$ & Winkler-1 & Midland & Midland & Blaine 1968:Fig. 1E \\
\hline 63 & $1-1-1$ & Winkler-1 & Midland & Midland & Blaine 1968:Fig. 1A \\
\hline 64 & $26 Y 2 C-3$ & 41WM235, Wilson-Leonard & Midland & Midland & This report \\
\hline 65 & $1-2-4$ & Winkler-1 & Midland & Midland & Blaine 1968:Fig. 1B \\
\hline 66 & $1-4-1$ & Winkler-1 & Midland & Midland & Blaine 1968:Fig. 1C \\
\hline 67 & $1146-16$ & 29RV1, Milnesand & Milnesand & Milnesand-1 & Sellards 1955:Fig. 100f,f' \\
\hline 68 & $1146-4$ & 29RV1, Milnesand & Milnesand & Milnesand-1 & Sellards 1955:Fig. 100i \\
\hline 69 & $1146-10$ & 29RV1, Milnesand & Milnesand & Milnesand-1 & Sellards 1955:Fig. 100e,e' \\
\hline 70 & $13 \mathrm{~N}-2$ & 41WM235, Wilson-Leonard & Angostura & Thrall & This report \\
\hline 71 & $\begin{array}{l}\text { CT- } \\
13 \mathrm{c} / 5836\end{array}$ & 41TV29, Wiley Williams & Hell Gap & Thrall & $\begin{array}{l}\text { TARL Records and } \\
\text { Collections }\end{array}$ \\
\hline 72 & 31P1-1 & 41WM235, Wilson-Leonard & Angostura & Thrall & This report \\
\hline 73 & 19L-1 & 41WM235, Wilson-Leonard & Angostura & Thrall & This report \\
\hline 74 & $18 \mathrm{P}-1$ & 41WM235, Wilson-Leonard & Angostura & Thrall & This report \\
\hline 75 & 31R1-1 & 41WM235, Wilson-Leonard & Angostura & Thrall & This report \\
\hline 76 & $16 \mathrm{~S} 2-2$ & 41WM235, Wilson-Leonard & Angostura & St. Mary's Hall & This report \\
\hline 77 & 21Q-1 & 41WM235, Wilson-Leonard & Plainview & St. Mary's Hall & This report \\
\hline 78 & $1291-1$ & 41WM235, Wilson-Leonard & Unclassified & St. Mary's Hall & This report \\
\hline 79 & 34U1B-1 & 41WM235, Wilson-Leonard & Plainview & St. Mary's Hall & This report \\
\hline $80+$ & 141 & 41VV218, Bonfire Shelter & Plainview & Plainview & $\begin{array}{l}\text { Dibble and Lorrain 1968:Fiq } \\
\text { 14b }\end{array}$ \\
\hline 81 & 28W2C-1 & 41WM235, Wilson-Leonard & Plainview & St. Mary's Hall & This report \\
\hline 82 & 7Q-1 & 41WM235, Wilson-Leonard & Plainview & St. Mary's Hall & This report \\
\hline 83 & $0-1$ & 41WM235, Wilson-Leonard & Plainview & St. Mary's Hall & This report \\
\hline 84 & $20 \mathrm{~W}-1$ & 41WM235, Wilson-Leonard & Plainview & St. Mary's Hall & This report \\
\hline 85 & 50XD-1 & 41WM235, Wilson-Leonard & Plainview & St. Mary's Hall & This report \\
\hline 86 & $38 \mathrm{~S} 1$ & 41WM235, Wilson-Leonard & Plainview & St. Mary's Hall & This report \\
\hline 87 & $\begin{array}{l}26 / 2 \mathrm{~W}- \\
10 \mathrm{H} 1\end{array}$ & 41BX229, St. Mary’s Hall & Plainview & St. Mary's Hall & Hester 1991:Fig. 1c \\
\hline 88 & $\begin{array}{l}\text { TTU- } \\
\text { A19941 }\end{array}$ & 41LU1, Lubbock Lake & Plainview & St. Mary's Hall & $\begin{array}{l}\text { Johnson and Holliday } \\
\text { 1980:Fig. } 4\end{array}$ \\
\hline 89 & $25 \mathrm{U} 2-1$ & 41WM235, Wilson-Leonard & Plainview & St. Mary's Hall & This report \\
\hline 90 & 26P1-2 & 41WM235, Wilson-Leonard & Plainview & St. Mary's Hall & This report \\
\hline 91 & OR498 & 41WM235, Wilson-Leonard & Plainview-like & St. Mary's Hall & This report \\
\hline 92 & $27-7$ & 41BX229, St. Mary's Hall & Plainview & St. Mary's Hall & Hester 1991:Fig. 1e \\
\hline 93 & $\begin{array}{l}31-2 / 24,1- \\
2 / 18, \& 31- \\
2 / 18\end{array}$ & 41TV49, Levi Rockshelter & Plainview-Angostura & St. Mary's Hall & Alexander 1963:Fig. 3d \\
\hline 94 & $725-29$ & 41HA1, Plainview & Plainview & Plainview-atypic & $\begin{array}{l}\text { Sellards et al. 1947:Fig. 5; } \\
\text { Knudson 1973:Fig. D.8c }\end{array}$ \\
\hline 95 & \begin{tabular}{|l} 
TTU-A1- \\
1196
\end{tabular} & 41LU72, Ryan's Site & Plainview & Plainview-atypic & Hartwell 1995:Fig. 10e,11e \\
\hline
\end{tabular}


Table 14-1, continued

\begin{tabular}{|c|c|c|c|c|c|}
\hline $\begin{array}{c}\text { Specimen } \\
\text { No. }\end{array}$ & $\begin{array}{l}\text { Accession } \\
\text { No. } \\
\end{array}$ & Site & Original Type & Revised Type & Reference \\
\hline 96 & $\begin{array}{l}\text { TTU-A1- } \\
14370\end{array}$ & 41LU72, Ryan's Site & Plainview & Plainview-atypic & Hartwell 1995:Fig. 10g,11g \\
\hline 97 & $\begin{array}{l}\text { TTU-A1- } \\
1185\end{array}$ & 41LU72, Ryan's Site & Plainview & Plainview-atypic & Hartwell 1995:Fig. 10d,11d \\
\hline 98 & $\begin{array}{l}\text { 17JJ2KK1- } \\
3\end{array}$ & 41WM235, Wilson-Leonard & Midland & Plainview-atypic & This report \\
\hline 99 & $725-\mathrm{X}$ & 41HA1, Plainview & Plainview & Plainview-atypic & Knudson 1973:Fig. D.8d \\
\hline 100 & $1146-12$ & 29RV1, Milnesand & Milnesand & Plainview-atypic & Sellards 1955:Fig. 98e \\
\hline 101 & $1054-2$ & 41SN2, Beidleman Ranch & Plainview & Plainview & Suhm 1961:Fig. 2B,B' \\
\hline 102 & $1146-39$ & 29RV1, Milnesand & Milnesand & Plainview & Sellards 1955:Fig. 99e,e' \\
\hline 103 & $\begin{array}{l}\text { TTU-A1- } \\
14348\end{array}$ & 41LU72, Ryan's Site & Plainview & Plainview & Hartwell 1995:Fig. 10h,11h \\
\hline 104 & $\begin{array}{l}\text { TTU-A1- } \\
1190\end{array}$ & 41LU72, Ryan's Site & Plainview & Plainview & Hartwell 1995:Fig. $10 \mathrm{j}, 11 \mathrm{j}$ \\
\hline 105 & $725-19$ & 41HA1, Plainview & Plainview & Plainview & $\begin{array}{l}\text { Sellards et al. 1947:Pl. 3,7; } \\
\text { Knudson 1973:Fig. D.5f }\end{array}$ \\
\hline $106+$ & 405 & 41VV218, Bonfire Shelter & Plainview & Plainview-atypic & $\begin{array}{l}\text { Dibble and Lorrain 1968:Fi६ } \\
\text { 14c }\end{array}$ \\
\hline 107 & $725-4$ & 41HA1, Plainview & Plainview & Plainview & $\begin{array}{l}\text { Sellards et al. 1947:Pl. } \\
\text { 3,3,3A; Knudson 1973:Fig. } \\
\text { D.7a }\end{array}$ \\
\hline 108 & $725-9$ & 41HA1, Plainview & Plainview & Plainview & $\begin{array}{l}\text { Sellards et al. 1947:Pl. } \\
\text { 4,2,2A; Knudson 1973:Fig. } \\
\text { D.4b }\end{array}$ \\
\hline 109 & $\begin{array}{l}\text { TTU-A1- } \\
14371\end{array}$ & 41LU72, Ryan's Site & Plainview & Plainview & Hartwell 1995:Fig. 10a,11a \\
\hline 110 & $725-8$ & 41HA1, Plainview & Plainview & Plainview & $\begin{array}{l}\text { Sellards et al. 1947:Pl. 4,3; } \\
\text { Knudson 1973:Fig. D.7d }\end{array}$ \\
\hline 111 & $725-13$ & 41HA1, Plainview & Plainview & Plainview & $\begin{array}{l}\text { Sellards et al. 1947:Pl. 3,6; } \\
\text { Knudson 1973:Fig. D.6c }\end{array}$ \\
\hline 112 & $\begin{array}{l}\text { TTU-A1- } \\
2007\end{array}$ & 41LU72, Ryan's Site & Plainview & Plainview & Hartwell 1995:Fig. 10f,11f \\
\hline 113 & $1146-13$ & 29RV1, Milnesand & Milnesand & Plainview & Sellards 1955:Fig. 98a,a' \\
\hline 114 & $\begin{array}{l}\text { TTU-A- } \\
14318\end{array}$ & 41LU72, Ryan's Site & Plainview & Plainview & Hartwell 1995:Fig. 101,111 \\
\hline 115 & $\begin{array}{l}\text { TTU-A1- } \\
14301\end{array}$ & 41LU72, Ryan's Site & Plainview & Plainview & Hartwell 1995:Fig. 10c,11c \\
\hline 116 & $725-10$ & 41HA1, Plainview & Plainview & Plainview & $\begin{array}{l}\text { Sellards et al. 1947:Pl. } \\
\text { 3,1,1A; Knudson 1973:Fig. } \\
\text { D.8a }\end{array}$ \\
\hline 117 & $\begin{array}{l}\text { TTU-A1- } \\
14347\end{array}$ & 41LU72, Ryan's Site & Plainview & Plainview & Hartwell 1995:Fig. 10b,11b \\
\hline 118 & $725-1$ & 41HA1, Plainview & Plainview & Plainview & $\begin{array}{l}\text { Sellards et al. 1947:Pl. } \\
\text { 5,3,3A; Knudson 1973:Fig. } \\
\text { D.4a }\end{array}$ \\
\hline 119 & $\begin{array}{l}\text { TTU-A1- } \\
14378\end{array}$ & 41LU72, Ryan's Site & Plainview & Plainview & Hartwell 1995:Fig. 10i,11i \\
\hline 120 & $725-5$ & 41HA1, Plainview & Plainview & Plainview-atypic & $\begin{array}{l}\text { Sellards et al. 1947:Pl. } \\
\text { 5,1,1A; Knudson 1973:Fig. } \\
\text { D.6d }\end{array}$ \\
\hline 121 & $725-2$ & 41HA1, Plainview & Plainview & Plainview-atypic & $\begin{array}{l}\text { Sellards et al. 1947:Pl. } \\
\text { 4,1,1A; Knudson 1973:Fig. } \\
\text { D.5d }\end{array}$ \\
\hline 122 & 16Y1-1 & 41WM235, Wilson-Leonard & Plainview & Parallel-sided Stem & This report \\
\hline 123 & $\begin{array}{l}\text { TTU-A1- } \\
14302\end{array}$ & 41LU72, Ryan's Site & Plainview & Plainview-atypic & Hartwell 1995:Fig. 10k,11k \\
\hline 124 & $1146-11$ & 29RV1, Milnesand & Milnesand & Plainview-atypic & Sellards 1955:Fig. 99b,b' \\
\hline
\end{tabular}


Table 14-1, continued

\begin{tabular}{|c|c|c|c|c|c|}
\hline $\begin{array}{l}\text { Specimen } \\
\text { No. }\end{array}$ & \begin{tabular}{|l|} 
Accession \\
No. \\
\end{tabular} & Site & Original Type & Revised Type & Reference \\
\hline 125 & $725-23$ & 41HA1, Plainview & Plainview & Plainview-atypic & $\begin{array}{l}\text { Sellards et al. 1947:Pl. 3,2; } \\
\text { Knudson 1973:Fig. D.5a }\end{array}$ \\
\hline 126 & $22 \mathrm{~T}-1$ & 41WM235, Wilson-Leonard & Plainview & Parallel-sided Stem & This report \\
\hline 127 & $26 \times 2 A-1$ & 41WM235, Wilson-Leonard & Plainview & Parallel-sided Stem & This report \\
\hline 128 & $\begin{array}{l}25 \mathrm{~V} 1 \mathrm{C}-1 \& \\
28 \times 1 \mathrm{C}-4\end{array}$ & 41WM235, Wilson-Leonard & Plainview & Parallel-sided Stem & This report \\
\hline 129 & $15 \times 1-3$ & 41WM235, Wilson-Leonard & Unclassified & Ground Square Stem & This report \\
\hline 130 & $21 \mathrm{M}-3$ & 41WM235, Wilson-Leonard & Scottsbluff & Scottsbluff & This report \\
\hline 131 & 26V2B-2 & 41WM235, Wilson-Leonard & Scottsbluff & Scottsbluff & This report \\
\hline 132 & 196 & 41VV218, Bonfire Shelter & Plainview & Plainview-Outlier & $\begin{array}{l}\text { Dibble and Lorrain 1968:Fiø } \\
14 \mathrm{a}\end{array}$ \\
\hline 133 & $33 \mathrm{X} 2 \mathrm{C}-1$ & 41WM235, Wilson-Leonard & Plainview & $\begin{array}{l}\text { Golondrina-Barber- } \\
\text { Outlier }\end{array}$ & This report \\
\hline 134 & 18L-1 & 41WM235, Wilson-Leonard & Plainview & $\begin{array}{l}\text { St. Mary's Hall- } \\
\text { atypic }\end{array}$ & This report \\
\hline 135 & $26 / 210 \mathrm{H} 1$ & 41BX229, St. Mary's Hall & Plainview & $\begin{array}{l}\text { St. Mary's Hall- } \\
\text { atypic }\end{array}$ & Hester 1991:Fig. 1d \\
\hline 136 & $741-2$ & 41VV188, Devil's Mouth & $\begin{array}{l}\text { Plainview, Golondrina } \\
\text { variety }\end{array}$ & $\begin{array}{l}\text { St. Mary's Hall- } \\
\text { atypic }\end{array}$ & Sorrow 1968:Fig. 17f \\
\hline 137 & 29R2-6 & 41WM235, Wilson-Leonard & Plainview & $\begin{array}{l}\text { St. Mary's Hall- } \\
\text { atypic }\end{array}$ & This report \\
\hline 138 & $17 \mathrm{~V} 1-4$ & 41WM235, Wilson-Leonard & Plainview & $\begin{array}{l}\text { St. Mary's Hall- } \\
\text { atypic }\end{array}$ & This report \\
\hline 139 & $29 \times 2 C-7$ & 41WM235, Wilson-Leonard & Barber & Golondrina-Barber & This report \\
\hline 140 & 89 & 3GE94, Sloan & Dalton & Dalton & $\begin{array}{l}\text { Morse and Morse 1983:72- } \\
75,89-95\end{array}$ \\
\hline 141 & OR561 & 41WM235, Wilson-Leonard & Plainview & Golondrina-Barber & This report \\
\hline 142 & 28T2B-1 & 41WM235, Wilson-Leonard & Plainview* & Golondrina-Barber* & This report \\
\hline 143 & $555-1$ & 41VV188, Devil's Mouth & Plainview Golondrina & Golondrina-Barber & $\begin{array}{l}\text { Johnson 1964:Fig. 15C; } \\
\text { Sorrow 1968:Fig. 3c }\end{array}$ \\
\hline 144 & 288 & 3GE94, Sloan & Dalton & Dalton & $\begin{array}{l}\text { Morse and Morse 1983:72- } \\
75,89-95\end{array}$ \\
\hline 145 & $15 \times 1-1$ & 41WM235, Wilson-Leonard & Plainview* & Golondrina-Barber* & This report \\
\hline 146 & 30 & 3GE94, Sloan & Dalton & Dalton & $\begin{array}{l}\text { Morse and Morse 1983:72- } \\
75,89-95\end{array}$ \\
\hline 147 & N2/E2 & 41VV213, Baker Cave & Golondrina & Golondrina-Barber & Chadderdon 1983:Fig. 22f \\
\hline 148 & 23Q-1 & 41WM235, Wilson-Leonard & Plainview & Golondrina-Barber & This report \\
\hline 149 & $15 Y 1-1$ & 41WM235, Wilson-Leonard & Plainview* & Golondrina-Barber* & This report \\
\hline 150 & 50YA-1 & 41WM235, Wilson-Leonard & Plainview & Golondrina-Barber & This report \\
\hline 151 & OR543 & 41WM235, Wilson-Leonard & Dalton or Plainview & $\begin{array}{l}\text { St. Mary's Hall- } \\
\text { atypic }\end{array}$ & This report \\
\hline 152 & 1313 & 41WM235, Wilson-Leonard & Golondrina? & Golondrina-Barber & This report \\
\hline 153 & $581-7$ & 41VV188, Devil's Mouth & Plainview Golondrina & Golondrina-Barber & Johnson 1964:Fig. 15H \\
\hline 154 & 581-18 & 41VV188, Devil's Mouth & Plainview Golondrina & Golondrina-Barber & Johnson 1964:Fig. 15J \\
\hline 155 & 308 & 3GE94, Sloan & Dalton & Dalton & $\begin{array}{l}\text { Morse and Morse 1983:72- } \\
75,89-95\end{array}$ \\
\hline 156 & $581-26$ & 41VV188, Devil's Mouth & Plainview Golondrina & Golondrina-Barber & Johnson 1964:Fig. 15G \\
\hline 157 & 184 & 3GE94, Sloan & Dalton & Dalton & $\begin{array}{l}\text { Morse and Morse 1983:72- } \\
75,89-95\end{array}$ \\
\hline 158 & $21 \mathrm{~L}-1$ & 41WM235, Wilson-Leonard & Plainview & Golondrina-Barber & This report \\
\hline 159 & 186 & 3GE94, Sloan & Dalton & Dalton & $\begin{array}{l}\text { Morse and Morse 1983:72- } \\
75,89-95\end{array}$ \\
\hline 160 & 74 & 3GE94, Sloan & Dalton & Dalton & $\begin{array}{l}\text { Morse and Morse 1983:72- } \\
75,89-95\end{array}$ \\
\hline 161 & 309 & 3GE94, Sloan & Dalton & Dalton & $\begin{array}{l}\text { Morse and Morse 1983:72- } \\
75,89-95\end{array}$ \\
\hline 162 & 13Q-2 & 41WM235, Wilson-Leonard & Plainview* & Golondrina-Barber* & This report \\
\hline 163 & 18SB-2 & 41WM235, Wilson-Leonard & Not Plainview [sic] & Golondrina-Barber & This report \\
\hline
\end{tabular}


Table 14-1, continued

\begin{tabular}{|c|c|c|c|c|c|}
\hline $\begin{array}{l}\text { Specimen } \\
\text { No. }\end{array}$ & $\begin{array}{l}\text { Accession } \\
\text { No. }\end{array}$ & Site & Original Type & Revised Type & Reference \\
\hline 164 & 408 & 3GE94, Sloan & Dalton & Dalton & $\begin{array}{l}\text { Morse and Morse 1983:72- } \\
75,89-95\end{array}$ \\
\hline 165 & 332 & 3GE94, Sloan & Dalton & Dalton & $\begin{array}{l}\text { Morse and Morse 1983:72- } \\
75,89-95\end{array}$ \\
\hline 166 & 60 & 3GE94, Sloan & Dalton & Dalton & $\begin{array}{l}\text { Morse and Morse 1983:72- } \\
75,89-95\end{array}$ \\
\hline 167 & 324 & 3GE94, Sloan & Dalton & Dalton & $\begin{array}{l}\text { Morse and Morse 1983:72- } \\
75,89-95\end{array}$ \\
\hline 168 & 452 & 3GE94, Sloan & Dalton & Dalton & $\begin{array}{l}\text { Morse and Morse 1983:72- } \\
75,89-95\end{array}$ \\
\hline 169 & 336 & 3GE94, Sloan & Dalton & Dalton & $\begin{array}{l}\text { Morse and Morse 1983:72- } \\
75,89-95\end{array}$ \\
\hline 170 & 26Y2C-1 & 41WM235, Wilson-Leonard & Plainview & Golondrina-Barber & This report \\
\hline 171 & $10 \mathrm{~S}-1$ & 41WM235, Wilson-Leonard & Plainview & Golondrina-Barber & This report \\
\hline 172 & 26R1-1 & 41WM235, Wilson-Leonard & Plainview & Golondrina-Barber & This report \\
\hline 173 & $581-9$ & 41VV188, Devil's Mouth & Plainview golondrina & Golondrina-Barber & $\begin{array}{l}\text { Johnson 1964:Fig. 15B; } \\
\text { Sorrow 1968:Fig. 3a }\end{array}$ \\
\hline 174 & $581-17$ & 41VV188, Devil's Mouth & Plainview golondrina & Golondrina-Barber & Johnson 1964:Fig. 15E \\
\hline 175 & $581-10$ & 41VV188, Devil's Mouth & Plainview golondrina & Golondrina-Barber & Johnson 1964:Fig. 15I \\
\hline 176 & $12 \mathrm{U}-1$ & 41WM235, Wilson-Leonard & Plainview & Golondrina-Barber & This report \\
\hline 177 & 51Y1B-1 & 41WM235, Wilson-Leonard & Golondrina & Golondrina-Barber & This report \\
\hline 178 & 25DD2A-1 & 41WM235, Wilson-Leonard & Plainview & Golondrina-Barber & This report \\
\hline 179 & $25 Z 1 C-1$ & 41WM235, Wilson-Leonard & Plainview & Golondrina-Barber & This report \\
\hline 180 & $1473-1$ & 41WM235, Wilson-Leonard & Plainview? & Golondrina-Barber & This report \\
\hline 181 & $\begin{array}{l}\text { TTU-A- } \\
40455\end{array}$ & 41LU1, Lubbock Lake & Lubbock & Lubbock-Outlier & $\begin{array}{l}\text { Holliday and Johnson } \\
\text { 1990:Fig. } 8\end{array}$ \\
\hline 182 & $1146-40$ & 29RV1, Milnesand & Milnesand & Milnesand-Outlier & Sellards 1955:Fig. $100 \mathrm{~g}$ \\
\hline
\end{tabular}

+ Reclassified after consideration of Discriminant Function Analysis results.

* Four of nine specimens Kelly (1983b) used to propose the Barber type.

\section{Plainview}

The Plainview specimens are drawn from seven sites. The first, the Plainview site (Holliday 1985, 1990; Knudson 1973; Sellards et al. 1947), has 12 specimens that are considered complete enough for analysis (see Table 14-1). There are seven radiocarbon ages from this site (Table 14-2), three of which suggest an age range of between 10,200 \pm 400 and $9800 \pm 500$ B.P. for the bone bed deposit. The materials that were dated, freshwater snail shell and bone apatite, indicate caution in interpretation of these dates, however. The three sediment sample ages, taken from about $400 \mathrm{~m}$ east of the original excavation, indicate a broad range of 11,000 to 8800 B.P. within which the Plainview site might have formed (Holliday 1990).

The second Plainview sample, Ryan's site (Hartwell 1995), has 12 specimens acceptable for analysis (see Table 14-1). The Ryan's site has three radiocarbon ages from playa sediments (see Table14-2). The radiocarbon samples were collected from positions lower than the point specimens within the playa. The uppermost age of $9220 \pm 220$ B.P. (SMU2448 ), collected about a meter below surface, is 1,430 years younger than the next sample down, $10,650 \pm 120$ B.P. (SMU2447) collected just $20 \mathrm{~cm}$ lower. The third sample,
$10,380 \pm 140$ в.P. (SMU-2446), collected $20 \mathrm{~cm}$ below the second, is 270 years younger than the second date. Conservatively such a pattern suggests the 9220 в.P. (SMU-2448) date might be too young due to unidentified contaminates introduced by soil illuviation processes. The remaining two ages suggest that the Plainview occupation at the Ryan's site probably took place after about 10,500 B.P.

Beidleman Ranch (41SN2) is the third Plainview site with one complete specimen that is acceptable for analysis. This specimen was recovered from a small poorly preserved bone bed of extinct bison, possibly Bison antiquus (Suhm 1961). The bone bed was within a pond deposit thought to be late Pleistocene in age. This site had only the one cultural deposit, and there are no radiocarbon dates.

The fourth Plainview site is Bonfire Shelter (Dibble and Lorrain 1968). It produced four unfluted lanceolate specimens, typed as Plainview, appropriate for analysis. The first thing that is notable in this collection is the morphological variability (Dibble and Lorrain 1968:Figure 14). As the remarks within the original descriptions make clear, these specimens tend to deviate from the Plainview type, particularly toward Milnesand or Midland in two of the cases (see Dibble and Lorrain 1968:36). Three of the four specimens (Specimens 80, 106, 132 in Table 14-1) are provenienced to the 
TABLE 14-2

Radiocarbon Ages Associated with Sample Specimens from Sites other than Wilson-Leonard

\begin{tabular}{|c|c|c|c|c|c|}
\hline Site Lab No. & \begin{tabular}{|l|} 
Uncalibrated \\
Years B.P. \\
\end{tabular} & Cultural Association* & Stratigraphy/Level/Feature & Technique-Material & Reference \\
\hline \multicolumn{6}{|c|}{ 41TV49, Levi Rockshelter } \\
\hline O-1128 & $7,350 \pm 150$ & Angostura & Zone IV-top & ${ }^{14} \mathrm{C}$-snail and mussel shell & Alexander 1963 \\
\hline O-1129 & $9,300 \pm 160$ & Angostura & Zone IV-middle & ${ }^{14} \mathrm{C}$-snail and mussel shell & Alexander 1963 \\
\hline O-1105 & $6,750 \pm 150$ & Angostura & Zone IV-bottom & ${ }^{14} \mathrm{C}$-snail and mussel shell & Alexander 1963 \\
\hline \multicolumn{6}{|c|}{ 41VV213, Baker Cave } \\
\hline RL-828 & $9,180 \pm 220$ & Golondrina & $\begin{array}{l}\text { Op. 2, Hearth Zones 17-23, } \\
\text { Level } 7\end{array}$ & ${ }^{14} \mathrm{C}$-charcoal & Chadderdon 1983:22 \\
\hline TX-2466 & $9,020 \pm 150$ & Golondrina & $\begin{array}{l}\text { Op. 2, Level 7, under point } \\
\text { sp. N2/E2 }\end{array}$ & ${ }^{14} \mathrm{C}$-charcoal & Chadderdon 1983:22 \\
\hline TX-128 & $8,910 \pm 140$ & Golondrina & Zone 1 & ${ }^{14} \mathrm{C}$-charcoal & Word and Douglas 1970 \\
\hline TX-129 & $9,030 \pm 230$ & Golondrina & Zone 1 & ${ }^{14} \mathrm{C}$-charcoal & Word and Douglas 1970 \\
\hline \multicolumn{6}{|c|}{ 41LU1, Lubbock Lake } \\
\hline SMU-759 & $9,330 \pm 70$ & $\begin{array}{l}\text { Plainview (St. Mary's } \\
\text { Hall) }\end{array}$ & $\begin{array}{l}\text { Area 6, Stratum 2B- } \\
\text { middle, top of FA6-11 }\end{array}$ & ${ }^{14} \mathrm{C}$-humic acid-marsh sediment & Holliday et al. 1983 \\
\hline SMU-828 & $9,870 \pm 140$ & $\begin{array}{l}\text { Plainview (St. Mary's } \\
\text { Hall) }\end{array}$ & $\begin{array}{l}\text { Area 6, Stratum 2B- } \\
\text { middle, top of FA6-11 }\end{array}$ & ${ }^{14} \mathrm{C}$-humic acid-marsh sediment & $\begin{array}{l}\text { Holliday et al. } 1985 \text {; Haas et al } \\
\text { 1986; Johnson } 1987\end{array}$ \\
\hline SMU-728 & $9,990 \pm 100$ & $\begin{array}{l}\text { Plainview (St. Mary's } \\
\text { Hall) }\end{array}$ & $\begin{array}{l}\text { Area 6, Stratum 2B- } \\
\text { middle, bottom of FA6-11 }\end{array}$ & ${ }^{14} \mathrm{C}$-humic acid-marsh sediment & $\begin{array}{l}\text { Holliday et al. 1983, 1985; } \\
\text { Haas et al. 1986; Johnson } 198 ;\end{array}$ \\
\hline SI-3198 & $11,770 \pm 140$ & $\begin{array}{l}\text { Plainview (St. Mary's } \\
\text { Hall) }\end{array}$ & $\begin{array}{l}\text { Area 6, Stratum 2B- } \\
\text { middle, bottom of FA6-11 }\end{array}$ & ${ }^{14} \mathrm{C}$-humin-marsh sediment & Holliday et al. 1985 \\
\hline SI-4591 & $7,245 \pm 90$ & $\begin{array}{l}\text { Plainview (St. Mary's } \\
\text { Hall) }\end{array}$ & $\begin{array}{l}\text { Area 6, Stratum 2B- } \\
\text { middle, bottom of FA6-11 }\end{array}$ & ${ }^{14} \mathrm{C}$-humin-marsh sediment & Holliday et al. 1985 \\
\hline SI-4974 & $9,605 \pm 195$ & $\begin{array}{l}\text { Plainview (St. Mary's } \\
\text { Hall) }\end{array}$ & $\begin{array}{l}\text { Area 6, Stratum 2B- } \\
\text { middle, bottom of FA6-11 }\end{array}$ & ${ }^{14} \mathrm{C}$-humin-marsh sediment & $\begin{array}{l}\text { Holliday et al. } 1985 \text {; Haas et al } \\
1986\end{array}$ \\
\hline SMU-1261f & $9,950 \pm 120$ & Lubbock & $\begin{array}{l}\text { Area 5, Stratum 2s LBb, } \\
\text { FA5-17 }\end{array}$ & ${ }^{14} \mathrm{C}$-humic acid-marsh sediment & $\begin{array}{l}\text { Holliday et al. 1985; Haas et al } \\
\text { 1986; Johnson } 1987\end{array}$ \\
\hline \multicolumn{6}{|c|}{ 41VV218, Bonfire Shelter } \\
\hline TX-657 & $9,920 \pm 150$ & Plainview (Lubbock) & $\begin{array}{l}\text { Bone Bed 2, Component C, } \\
\text { Hearth } 1\end{array}$ & ${ }^{14} \mathrm{C}$-charcoal & Dibble 1970 \\
\hline TX-658 & $10,100 \pm 300$ & Plainview (Lubbock) & $\begin{array}{l}\text { Bone Bed 2, Component C, } \\
\text { Hearth } 1\end{array}$ & ${ }^{14} \mathrm{C}$-charcoal & Dibble 1970 \\
\hline TX-153 & $10,230 \pm 160$ & Plainview (Lubbock) & $\begin{array}{l}\text { Bone Bed 2, Component C, } \\
\text { Hearth } 1\end{array}$ & ${ }^{14} \mathrm{C}$-charcoal & Dibble and Lorrain 1968 \\
\hline AA-346 & $10,280 \pm 430$ & Plainview (Lubbock) & Bone Bed 2, Stratum A & AMS ${ }^{14} \mathrm{C}$-charcoal & Bement 1986 \\
\hline \multicolumn{6}{|c|}{ 41HA1, Plainview } \\
\hline L-303 & $9,800 \pm 500$ & Plainview & $\begin{array}{l}\text { Bone bed, original } \\
\text { excavation }\end{array}$ & ${ }^{14} \mathrm{C}$-freshwater snail shell & $\begin{array}{l}\text { Broecker and Kulp 1957; } \\
\text { Campbell } 1959\end{array}$ \\
\hline O-171 & $7,100 \pm 160$ & Plainview & $\begin{array}{l}\text { bone on surface for } 10 \\
\text { years }\end{array}$ & ${ }^{14} \mathrm{C}$-bone & Campbell 1959 \\
\hline TX-3907 & $10,200 \pm 400$ & Plainview & bone from curated blocks & ${ }^{14} \mathrm{C}$-bone apatite & Speer 1990 \\
\hline TX-3908 & $9,860 \pm 180$ & Plainview & bone from curated blocks & ${ }^{14} \mathrm{C}$-bone apatite & Speer 1990 \\
\hline SMU-2341 & $8,860 \pm 110$ & above Plainview & $\begin{array}{l}\text { top of Stratum 2, from } \\
400 \text { m east of original } \\
\text { excavations }\end{array}$ & ${ }^{14} \mathrm{C}$-sediment $\mathrm{NaOH}$ soluble & Holliday 1990 \\
\hline SMU-1359 & $11,000 \pm 100$ & below Plainview & $\begin{array}{l}\text { near top of Stratum 1, from } \\
400 \text { m east of original } \\
\text { excavations }\end{array}$ & ${ }^{14} \mathrm{C}$-sediment $\mathrm{NaOH}$ soluble & Holliday 1990 \\
\hline SMU-1376 & $12,100 \pm 140$ & below Plainview & $\begin{array}{l}\text { bottom of Stratum 1, from } \\
400 \text { m east of original } \\
\text { excavations }\end{array}$ & ${ }^{14} \mathrm{C}$-sediment $\mathrm{NaOH}$ soluble & Holliday 1990 \\
\hline \multicolumn{6}{|c|}{ 41LU72, Ryan's Site } \\
\hline SMU-2448 & $9,220 \pm 220$ & below Plainview & $\begin{array}{l}\text { Playa, Stratum 2, } 100 \mathrm{~cm} \\
\text { below surface }\end{array}$ & ${ }^{14} \mathrm{C}-$-"soil humic fraction" & Hartwell 1995 \\
\hline SMU-2447 & $10,650 \pm 120$ & below Plainview & $\begin{array}{l}\text { Playa, Stratum 2, } 120 \mathrm{~cm} \\
\text { below surface }\end{array}$ & ${ }^{14} \mathrm{C}$-“soil humic fraction" & Hartwell 1995 \\
\hline SMU-2446 & $10,380 \pm 140$ & below Plainview & $\begin{array}{l}\text { Playa, Stratum } 1 / 2,140 \mathrm{~cm} \\
\text { below surface }\end{array}$ & ${ }^{14} \mathrm{C}$-"soil humic fraction" & Hartwell 1995 \\
\hline
\end{tabular}


unburned and undifferentiated areas of Bone Bed 2. The fourth specimen (Specimen 42 in Table 14-1) is provenienced to Component $\mathrm{C}$, the upper component of unburned bone in Bone Bed 2, within grid square N20/W60 (Dibble and Lorrain 1968:Table 2). A Folsom point is provenienced to Component $\mathrm{A}$, the lower unburned bone component in Bone Bed 2. Three radiocarbon ages were obtained on charcoal from Hearth 1, provenienced to the top of Component $\mathrm{C}$ of Bone Bed 2 in the southwestern quadrant of grid square N20/W60 (Dibble 1970; Dibble and Lorrain 1968:32-33). These three dates (see Table 14-2) range between $9920 \pm 150$ and 10,230 \pm 160 B.P. and average about 10,100 B.P. A fourth radiocarbon age of 10,280土430 (AA-346), obtained from loose charcoal fragments in the upper part of Bone Bed 2 during later research, tends to corroborate Dibble and Lorrain's conclusions (Bement 1986:9). Only one specimen, Specimen 42 (see Table 14-1) can be considered associated with the 10,100 в.P. age since it comes from the same grid square and bone bed component. The other three unfluted lanceolate specimens may be of similar or older age, but it is impossible to know with certainty since they are from undifferentiated portions of Bone Bed 2.

Two unfluted lanceolate point specimens, typed as Plainview, were found in bone concentration \#3 of Feature FA6-11 at the Lubbock Lake site (Johnson and Holliday 1980); only one is complete enough for analysis (Specimen 88 in Table 14-1). Six radiocarbon ages on marsh sediment date Feature FA6-11 (see Table 14-2). Two dates from above the feature are $9330 \pm 70$ в.P. and $9870 \pm 140$ в.P., and the two acceptable dates from the bottom of the feature are $9605 \pm 195$ B.P. and $9990 \pm 100$ B.P., suggesting an age range of about 9300 B.P. to 9990 B.P. for the feature and the artifacts found in association.

The St. Mary's Hall site (Hester 1978, 1991) had three specimens, previously typed as Plainview, that were complete enough for analysis. These specimens came from a stratigraphically isolated component below Golondrina and Angostura. No radiocarbon dates exist for this component.

From Levi Rockshelter (Alexander 1963), four specimens typed as Plainview-Angostura, were considered acceptable for analysis. It should be noted that three of the specimens appear to be nearly complete, but are actually made up of three refitted segments each (Alexander 1963:Figure 2d, h, and Figure 3d). Three radiocarbon ages, ranging from 7350 B.P. to 9300 B.P., on snail and mussel shell broadly date Zone IV which contained the Plainview-Angostura specimens (see Table 14-2).

\section{Milnesand}

Eight of the original Milnesand specimens from Sellards's (1955) excavation were available for study in the TARL collection and considered complete enough for analysis. Five of these specimens were excavated from the bone bed (or from blocks), and three specimens were found in or near an exposed part of the bone bed. There are no radiocarbon ages from the Milnesand site. The chronological position is estimated at 10,000 to 9000 B.P. (Hofman 1989:40).

\section{Lubbock}

From the Lubbock Lake site there are four Lubbock points (Holliday and Johnson 1990:Figure 8) complete enough for analysis. These specimens were associated with Feature FA5-17, a bison kill/butchering locale. There is one radiocarbon age of $9950 \pm 120$ (see Table 14-2) from marsh sediment associated with the feature.

\section{Midland}

Seven Midland specimens from Winkler-1 site (Blaine 1968) came from the P-1 and P-2 areas of the site. These two areas were considered to have had the best provenience and little or no mixing. These Midland specimens are somewhat unique in not having been found with Folsom points, as is usually the case for most Midland points (Amick 1995). This circumstance led Blaine (1968) to hypothesize that Midland may be a late, unfluted development of Folsom. There are no radiocarbon ages available from Winkler-1, but a chronological estimate, based on assumed contemporaneity with Folsom is about 11,000 to 10,000 B.P. (Hofman 1989).

\section{Dalton}

The Sloan site, a Dalton-age cemetery, is located in a sand dune near the Cache River floodplain in Arkansas (Morse and Morse 1983:89-94). Over 400 artifacts were recovered from this site. Measurements on 75 Dalton points were provided by $\mathrm{D}$. Morse, and 14 of these were randomly selected for this analysis. It should noted that the maximum thickness of stem measurement, provided by the Arkansas Archeological Survey database, had to be substituted for the 10-mm stem thickness measurement which was not made at the time the Sloan site Dalton points were observed. The minor inconsistency in the data is not significant for the morphological group of which Dalton is a part. There are no radiocarbon ages from the Sloan site, but Goodyear (1982) made a well-reasoned chronological estimate of 10,500 to 9900 B.P. for Dalton, based in part on radiocarbon dates from Rodgers Shelter (Wood and McMillan 1976).

\section{Golondrina}

The Devil's Mouth site (Johnson 1964; Sorrow 1968) is the type site for Golondrina. Eight specimens of Golondrina were complete enough for analysis. These specimens came from the upper gravels of Area $\mathrm{C}$, as did Angostura, Plainview- 
like, and Group 1 stemmed points (Sorrow 1968:19-21), later referred to as Early Stemmed (Turner and Hester 1985, 1993). There are no radiocarbon ages directly associated with the upper gravel deposits.

A temporal position for Golondrina was established with excavation at Baker Cave (Chadderdon 1983; Hester 1977, 1978; Word and Douglas 1970). One specimen (Specimen 147 in Table 14-1) was available for this analysis, and it was directly associated with a radiocarbon age of $9020 \pm 150$ B.P. (TX-2466) (Chadderdon 1983:22). Three more radiocarbon ages are also associated with the Golondrina levels, ranging from $8910 \pm 140$ B.P. to $9180 \pm 220$ B.P. (see Table 14-2).

\section{Angostura}

The Tombstone Bluff site (Prewitt 1981a) had 14 Angostura specimens that were complete enough for analysis. These specimens were recovered during surface collection.

\section{Hell Gap}

There was one specimen typed as Hell Gap in the records from the Wiley Williams site (TARL Records and Collections). There was no provenience or chronological information available. However, the variability offered by this specimen made it a desirable point for inclusion.

\section{Results}

The systematic analysis of unfluted lanceolate projectile points organizes the morphological variability by consistent characteristics of the point base as represented in the present sample. Descriptive data are presented within three large morphological groups, each of which is followed by a discussion of morphology, technology, chronology, and typology.

\section{Overview by Analyses}

\section{CLUSTERANALYSIS}

The cluster analysis produced 21 clusters and 4 outlier specimens from the 182 sample specimens (Figure 14-2). In general, this sample of unfluted lanceolate points is grouped by characteristics of haft width, haft shape, haft thickness, and basal concavity. The sample is composed of two large morphological groups of contracting haft and parallel-sided haft clusters. The parallel-sided haft clusters are further subdivided into those with shallow-to-moderate basal concavity and those with deep basal concavity. There are 9 contracting haft clusters (Clusters 1 through 9), 6 parallel-sided stem, shallow-to-moderate basal concavity clusters (Clusters 10 through 15 ), and 6 parallel-sided haft, deep basal concavity clusters (Clusters 16 through 21 ). The means and sample standard deviations of eight variables for each cluster are shown in Table 14-3.

By tracing the branches of the dendrogram (see Figure 14-2), it is apparent that Cluster 1, an extreme contracting haft cluster, is distinct from all the other clusters as it branches off first. Then Specimens 181 and 182, convex base outliers, separate from the rest of the sample at the second branch on the dendrogram. The moderate contracting haft Clusters 2 through 9 branch off from the parallel-sided haft clusters at the third branch on the dendrogram. The convex base outliers split into two outlier individuals at the fourth branch. This extreme basal convexity is not common in the sample and draws these specimens away from clustering with either contracting haft points, in the case of Specimen 181, or parallelsided haft points, in the case of Specimen 182 (Table 14-4).

In the moderate contracting haft groups, Cluster 2 separates from Clusters 3-9 at the fifth branch (see Figure 14-2). Cluster 9, with very thick hafts, branches off from Clusters 37 at 9 on the dendrogram. Clusters 7 and 8 separate from the remaining contracting haft clusters because both have very thin hafts and very low haft thickness/width ratios. Specimen 58 branches off at 15 on the dendrogram and could be treated as an outlier, but examination of the data on this specimen shows it shares significant characteristics with specimens from Cluster 6 , and it is different in only the insignificant characteristics. Therefore, it is placed with Cluster 6 . Clusters 5 and 6 , with wider hafts, branch off from Clusters 3 and 4 at 19 on the dendrogram. Cluster 6 , with much thicker hafts, differs from Cluster 5 and branches off at 23 .

The parallel-sided haft clusters separate into shallowto-moderate basal concavity and deep basal concavity clusters at branch 6 on the dendrogram (see Figure 14-2). Among the shallow-to-moderate basal concavity clusters, Cluster 15 , with nearly flat bases, is distinct from Clusters 10-14, separating at branch 7 . Clusters 13 and 14, having lower haft thickness/width ratios and very long grinding lengths, branch off from Clusters 10,11, and 12 at 17 on the dendrogram. Cluster 10 separates from Clusters 11 and 12 at branch 25. Cluster 11 branches off from Cluster 12 at 28 on the dendrogram. Cluster 13 separates from Cluster 14 at branch 27 .

Specimens 132 and 133 separate from the parallel-sided haft, deep basal concavity clusters at branch 8 on the dendrogram (see Figure 14-2). Then these two specimens split into deep basal concavity individuals at branch 12 . Specimens 132 and 133 share little in common except a very deep basal concavity (Table 14-5). They are so distinct in haft width, haft shape, and haft thickness from the rest of this morphological group that they have to be considered outliers. Among the deep basal concavity clusters, Clusters 20 and 21 branch off from Clusters 16-19 at 14 on the dendrogram. These two clusters have much wider hafts and lower haft thickness/width ratios than the other deep basal concavity clusters. Cluster 20, with deeper basal concavity, separates from Cluster 21 at branch 21. Cluster 19, having the deepest basal concavity, splits off from Clusters 16, 17, and 


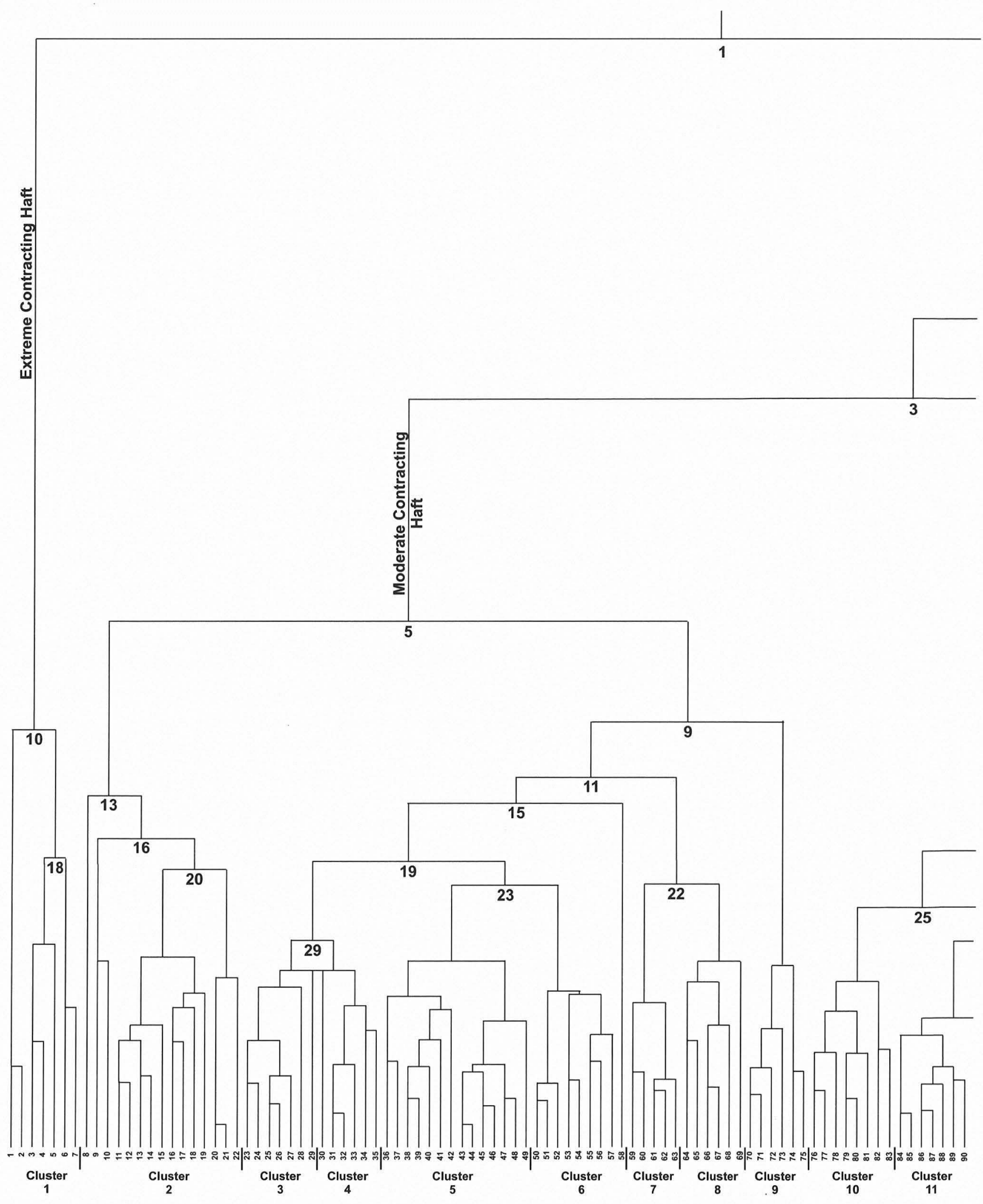

FIGURE 14-2. Hierarchical dendrogram of lanceolate points (see Table 14-1 for specimen key). 


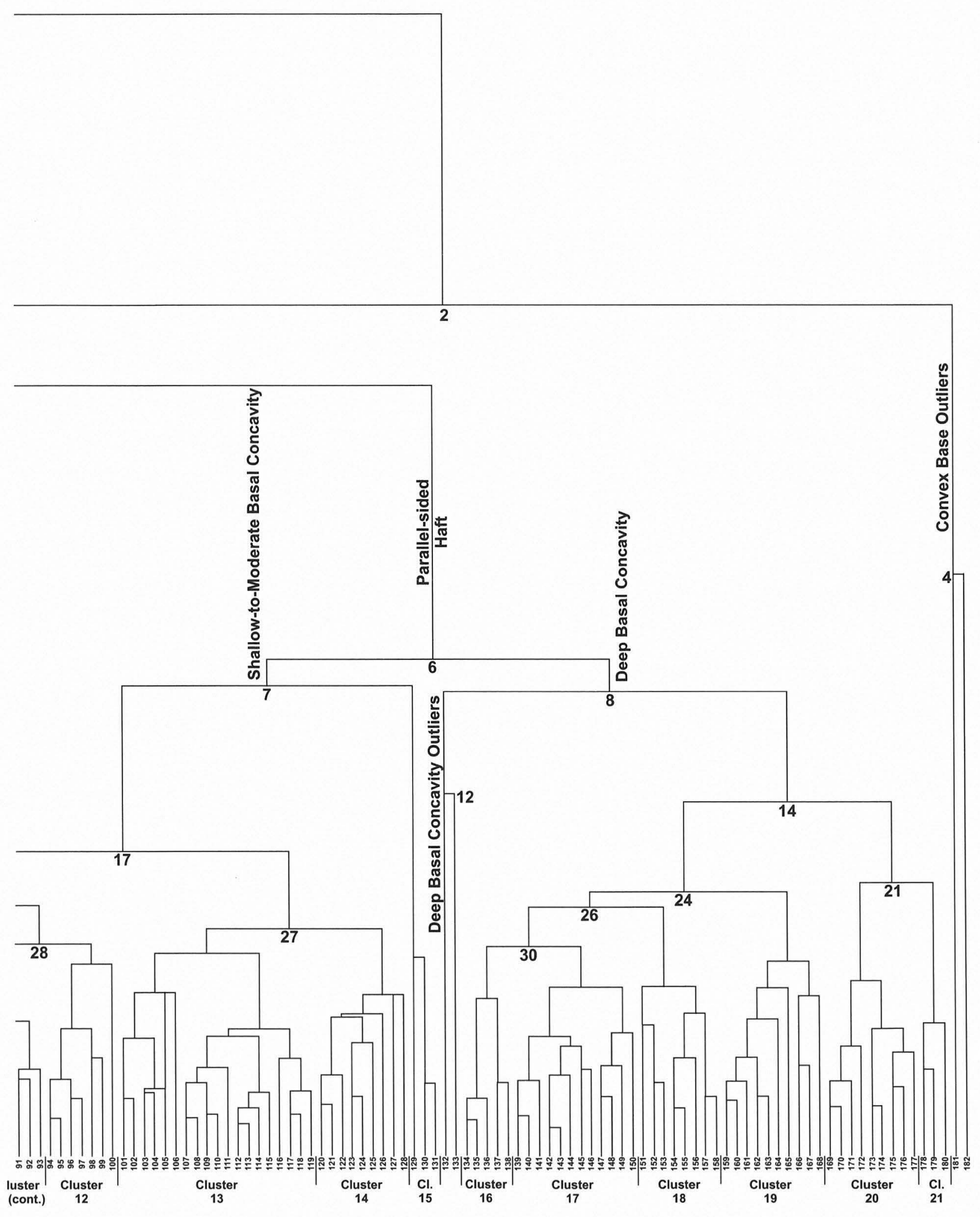

FIGURE 14-2 (CONTINUED). Hierarchical dendrogram of lanceolate points. 
TABLE 14-3

Means and Sample Standard Deviations for 21 Clusters

\begin{tabular}{|c|c|c|c|c|c|c|c|c|c|c|c|c|c|c|c|c|c|}
\hline \multirow[t]{2}{*}{ Cluster } & \multirow[t]{2}{*}{$\begin{array}{l}\text { Number of } \\
\text { Specimens }\end{array}$} & \multicolumn{2}{|c|}{$\begin{array}{l}\text { Proximal Haft } \\
\text { Width }\end{array}$} & \multicolumn{2}{|c|}{ 10-mm Haft Width } & \multicolumn{2}{|c|}{ Haft Shape Ratio } & \multicolumn{2}{|c|}{$\begin{array}{l}\text { 10-mm Haft } \\
\text { Thickness }\end{array}$} & \multicolumn{2}{|c|}{$\begin{array}{c}\text { Haft Thickness/ } \\
\text { Width Ratio }\end{array}$} & \multicolumn{2}{|c|}{ Basal Concavity } & \multicolumn{2}{|c|}{$\begin{array}{c}\text { Basal Concavity/ } \\
\text { Grinding Length } \\
\text { Ratio }\end{array}$} & \multicolumn{2}{|c|}{ Grinding Length } \\
\hline & & $\bar{x}$ & $\sigma$ & $\bar{x}$ & $\sigma$ & $\bar{x}$ & $\sigma$ & $\bar{x}$ & $\sigma$ & $\bar{x}$ & $\sigma$ & $\bar{x}$ & $\sigma$ & $\bar{x}$ & $\sigma$ & $\bar{x}$ & $\sigma$ \\
\hline 1 & 7 & 7.771 & 1.251 & 15.600 & 1.382 & 2.035 & 0.233 & 5.900 & 0.619 & 0.380 & 0.046 & 1.200 & 0.668 & 0.045 & 0.032 & 31.343 & 10.001 \\
\hline 2 & 15 & 11.313 & 0.800 & 15.793 & 1.031 & 1.401 & 0.112 & 5.707 & 0.748 & 0.362 & 0.045 & 0.900 & 0.844 & 0.042 & 0.044 & 24.067 & 10.335 \\
\hline 3 & 7 & 229 & 0.395 & 16.200 & 1.036 & 25 & 0.075 & .557 & 0.237 & 0.344 & 0.027 & .229 & 0.687 & 0.053 & 0.031 & 5.329 & 7.134 \\
\hline 4 & 6 & 14.150 & 0.432 & 15.817 & 1.560 & 1.117 & 0.096 & 4.783 & 0.325 & 0.305 & 0.039 & 0.833 & 0.516 & 0.038 & 0.022 & 24.183 & 6.574 \\
\hline 5 & 14 & 16.114 & 0.863 & 17.779 & 0.992 & 1.104 & 0.047 & .371 & 0.292 & 0.303 & 0.025 & 1.214 & 0.583 & 0.056 & 0.049 & 26.750 & 7.218 \\
\hline 6 & 9 & 15.233 & 0.740 & 18.711 & 0.805 & 1.230 & 0.050 & 7.133 & 0.592 & 0.382 & 0.040 & 1.144 & 0.853 & 0.040 & 0.026 & 32.678 & 10.511 \\
\hline 7 & 5 & 15.560 & 0.658 & 17.160 & 0.871 & 1.103 & 0.035 & 3.260 & 0.230 & 0.191 & 0.022 & 0.900 & 0.255 & 0.042 & 0.008 & 21.540 & 4.594 \\
\hline 8 & 6 & 17.583 & 0.397 & 18.200 & 1.310 & 1.035 & 0.069 & 3.833 & 0.476 & 0.212 & 0.032 & 1.250 & 0.817 & 0.074 & 0.066 & 20.000 & 5.726 \\
\hline 9 & 6 & 18.183 & 0.997 & 19.900 & 0.827 & 1.095 & 0.025 & 8.267 & 0.427 & 0.416 & 0.027 & 1.167 & 0.489 & 0.051 & 0.027 & 33 & 6.120 \\
\hline 10 & 7 & 20.829 & 0.571 & 21.714 & 0.807 & 3 & 0.041 & 6.186 & 0.463 & 0. & 0.025 & 2.443 & 0.872 & 0.129 & 0.049 & & 3.930 \\
\hline 11 & 10 & 18.710 & 0.238 & 19.910 & 0.980 & 1.064 & 0.055 & 5.410 & 0.606 & 0.272 & 0.028 & 2.660 & 0.640 & 0.106 & 0.028 & 00 & 5.820 \\
\hline 12 & 8 & 19.438 & 0.393 & 19.488 & 1.071 & 003 & 0.066 & 4.163 & 0.437 & 0.214 & 0.025 & 1.438 & 0.765 & 0.153 & 0.265 & 26.063 & 13.115 \\
\hline 13 & 19 & 21.653 & 0.716 & 22.284 & 1.239 & 1.029 & 0.037 & 4.458 & 0.494 & 0.201 & 0.029 & 1.647 & 0.737 & 0.058 & 0.027 & 9.274 & 6.416 \\
\hline 14 & 9 & 23.844 & 0.510 & 24.233 & 1.350 & 1.016 & 0.054 & 4.678 & 0.701 & 0.193 & 0.023 & 1.067 & 0.698 & 0.040 & 0.038 & 44 & 9.766 \\
\hline 15 & 3 & 26.567 & 1.069 & 25.633 & 1.361 & 0.966 & 0.053 & 6.133 & 0.808 & 0.240 & 0.038 & 0.367 & 0.635 & 0.021 & 0.037 & .767 & 2.335 \\
\hline 16 & 5 & 22.320 & 0.719 & 22.000 & 1.002 & 0.985 & 0.023 & 5.580 & 0.286 & 0.255 & 0.021 & 4.040 & 0.805 & 0.186 & 0.047 & 23.060 & 7.912 \\
\hline 17 & 12 & 22.308 & 1.107 & 22.975 & 1.673 & 1.032 & 0.087 & 5.592 & 0.652 & 0.244 & 0.029 & 6.383 & 0.395 & 0.311 & 0.108 & 22.858 & 7.752 \\
\hline 18 & 8 & 25.138 & 0.826 & 24.238 & 2.074 & 903 & 0.064 & 5.263 & 0.837 & 0.217 & 0.028 & 5.138 & 0.818 & 0.266 & 0.078 & 20.200 & 4.179 \\
\hline 19 & 10 & & 1.250 & 22.420 & 2.343 & & 0.086 & 50 & 0.700 & & 0.035 & 8.450 & 0.943 & 0.440 & 0.105 & 20.010 & 4.387 \\
\hline 20 & 9 & 21.861 & 0.735 & 27.667 & 1.826 & 0.992 & 0.051 & 5.433 & 0.903 & 0.198 & 0.038 & 6.922 & 1.095 & 0.270 & 0.090 & 27.322 & 6.799 \\
\hline 21 & 3 & 29.767 & 1.106 & 29.867 & 1.286 & 1.003 & 0.023 & 5.433 & 0.306 & 0.183 & 0.015 & 3.300 & 0.557 & 0.222 & 0.094 & 16.200 & 5.027 \\
\hline Totals: & 178 & 19.580 & 0.751 & 20.838 & 1.277 & 1.114 & 0.066 & 5.419 & 0.530 & 0.269 & 0.030 & 2.557 & 0.699 & 0.126 & 0.061 & 24.170 & 6.936 \\
\hline
\end{tabular}


TABLE 14-4

Convex Base Outliers

\begin{tabular}{l|c|c}
\hline Variable & $\begin{array}{c}181 \\
\text { TTU-A40455 }\end{array}$ & $\begin{array}{c}182 \\
1146-40\end{array}$ \\
\hline Proximal haft width & 14.600 & 20.800 \\
10-mm haft width & 17.100 & 23.000 \\
Stem shape ratio & 1.171 & 1.106 \\
10-mm haft thickness & 4.800 & 4.900 \\
Stem thickness/width ratio & 0.281 & 0.213 \\
Basal concavity & -3.000 & -4.000 \\
Basal concavity/grinding length ratio & -0.083 & -0.145 \\
Grinding length & 36.200 & 27.600 \\
\hline \hline
\end{tabular}

18 at branch 24 on the dendrogram. Cluster 18 separates from Clusters 16 and 17 at branch 26, and Cluster 16 branches off from Cluster 17 at 30 on the dendrogram.

\section{DISCRIMINANT FUNCTIONANALYSIS}

The groups defined by the cluster analysis just described were used as the predefined groups in a discriminant function analysis, and the individual projectile points were reclassified by the parameters of the group. The probability of accurate assignment of specimens to clusters was gauged through the Geisser Classification Probabilities (GCP) (Table 14-6). The discriminant function analysis was calculated with 23 clusters (the two pairs of outliers were treated as two groups with two specimens each, although the results are not meaningful except to indicate these four specimens could not be reassigned to other groups). The results indicate 179 hits and 3 misses, or $98 \%$ were correctly classified and $2 \%$ classified in contrast to the cluster analysis groupings.

All three misses and their reassignments are among the parallel-sided haft, shallow-to-moderate basal concavity clusters. This pattern suggests close morphological similarities between these clusters, with subtle differences not easily discerned.

The overall patterns in the probabilities indicate close similarities between adjacent clusters within the three large morphological groups (see Table 14-6). The patterns within the contracting haft clusters show very close similarities between Clusters 2,3,4, and 5 and between Clusters 7 and 8 . Cluster 8 also shows some similarity to Clusters 5 and 12 (of the parallel-sided haft, shallow-to-moderate basal concavity clusters). Points in Cluster 9 show some resemblance to specimens in Cluster 6; and Cluster 6 shows some similarity to Clusters 5 and 9. Points in Cluster 1 show only a slight resemblance with projectile points in Cluster 2, suggesting that Cluster 1 is at a morphological extreme within the contracting haft clusters.

The patterns of association within the parallel-sided haft, shallow-to-moderate basal concavity clusters are close for five of the six clusters. Close similarities are particularly the case for Clusters 10 and 13, which lost and received Specimen 80 (see Table 14-6) respectively; for Clusters 12
TABLE 14-5

Parallel-sided Haft, Deep Basal Concavity Outliers

\begin{tabular}{l|c|c}
\hline Variable & 132 & 133 \\
Proximal haft width & 196 & $33 \mathrm{X} 2 \mathrm{C}-1$ \\
10-mm haft width & 20.500 & 17.200 \\
Stem shape ratio & 21.400 & 21.800 \\
10-mm haft thickness & 1.044 & 1.267 \\
Stem thickness/width ratio & 3.000 & 4.600 \\
Basal concavity & 0.140 & 0.211 \\
Basal concavity/grinding length ratio & 5.400 & 6.600 \\
Grinding length & 0.216 & 0.230 \\
\hline \hline
\end{tabular}

and 13, which received and lost Specimen 106 (see Table 146) respectively; and for Clusters 13 and 14, which nearly share Specimen 108 (see Table 14-6). In addition, Clusters 10 and 11 show a close resemblance to each other, as do Clusters 12,13 , and 14 . Cluster 12 shows a close association with Cluster 8 of the contracting haft clusters. Cluster 15 appears unrelated to the other parallel-sided haft, shallow-to-moderate basal concavity clusters.

The patterns within the parallel-sided haft, deep basal concavity clusters show similarities between Clusters 17 and 18 and between Clusters 17 and 19. Cluster 18 shows a similarity with Cluster 20 . Cluster 16 has a similarity with Cluster 17 and slight associations with Clusters 10,13, and 14 of the parallel-sided haft, shallow-to-moderate basal concavity clusters.

\section{ANALYSIS OF VARIANCE}

Through analysis of variance (ANOVA), significance of the differences between the clusters within the three large morphological groups are delineated according to key characteristics. For the contracting haft clusters, significant differences between the cluster means are indicated for five out of eight variables (Table 14-7). These differences occur within proximal haft width, $10-\mathrm{mm}$ haft width, haft shape ratio, 10-mm haft thickness, and haft thickness/width ratio. The inherent variability in basal concavity/grinding length ratio and grinding length renders these variables meaningless for contracting haft clusters.

Significant differences between the cluster means are indicated for six of eight variables for parallel-sided haft, shallow-to-moderate basal concavity clusters (Table 14-8). These variables are proximal haft width, $10-\mathrm{mm}$ haft width, 10-mm haft thickness, haft thickness/width ratio, basal concavity, and grinding length. The greater inherent variability of the ratio of the haft shape ratio and the basal concavity/grinding length ratio indicates that these two variables are useless for describing these clusters.

For the parallel-sided haft, deep basal concavity clusters, significant differences between cluster means are indicated for five out of eight variables (Table 14-9). These differences occur within proximal haft width, 10-mm haft width, thickness/width ratio, basal concavity, and basal concavity/ 
TABLE 14-6

Geisser Classification Probabilities (GCP) for Unfluted Lanceolate Projectile Points (An * following an actual group number indicates a misclassification.)

\begin{tabular}{|c|c|c|c|c|c|c|c|c|c|c|c|c|c|c|c|c|c|c|c|c|c|c|c|c|}
\hline Specimen & Original & & & & & & & & & & & & isters & & & & & & & & & & & \\
\hline Number & Cluster & 1 & 2 & 3 & 4 & 5 & 6 & 7 & 8 & 9 & 10 & 11 & 12 & 13 & 14 & 15 & Ot.1 & 16 & 17 & 18 & 19 & 20 & 21 & Ot.2 \\
\hline$T$ & $T$ & 1.00 & 0.00 & 0.00 & 0.00 & 0.00 & 0.00 & 0.00 & 0.00 & 0.00 & 0.00 & 0.00 & 0.00 & 0.00 & 0.00 & 0.00 & 0.00 & 0.00 & 0.00 & 0.00 & 0.00 & 0.00 & 0.00 & 0.00 \\
\hline 2 & 1 & 1.00 & 0.00 & 0.00 & 0.00 & 0.00 & 0.00 & 0.00 & 0.00 & 0.00 & 0.00 & 0.00 & 0.00 & 0.00 & 0.00 & 0.00 & 0.00 & 0.00 & 0.00 & 0.00 & 0.00 & 0.00 & 0.00 & 0.00 \\
\hline 3 & 1 & 1.00 & 0.00 & 0.00 & 0.00 & 0.00 & 0.00 & 0.00 & 0.00 & 0.00 & 0.00 & 0.00 & 0.00 & 0.00 & 0.00 & 0.00 & 0.00 & 0.00 & 0.00 & 0.00 & 0.00 & 0.00 & 0.00 & 0.00 \\
\hline 4 & 1 & 1.00 & 0.00 & 0.00 & 0.00 & 0.00 & 0.00 & 0.00 & 0.00 & 0.00 & 0.00 & 0.00 & 0.00 & 0.00 & 0.00 & 0.00 & 0.00 & 0.00 & 0.00 & 0.00 & 0.00 & 0.00 & 0.00 & 0.00 \\
\hline 5 & 1 & 1.00 & 0.00 & 0.00 & 0.00 & 0.00 & 0.00 & 0.00 & 0.00 & 0.00 & 0.00 & 0.00 & 0.00 & 0.00 & 0.00 & 0.00 & 0.00 & 0.00 & 0.00 & 0.00 & 0.00 & 0.00 & 0.00 & 0.00 \\
\hline 6 & 1 & 1.00 & 0.00 & 0.00 & 0.00 & 0.00 & 0.00 & 0.00 & 0.00 & 0.00 & 0.00 & 0.00 & 0.00 & 0.00 & 0.00 & 0.00 & 0.00 & 0.00 & 0.00 & 0.00 & 0.00 & 0.00 & 0.00 & 0.00 \\
\hline 7 & 1 & 0.93 & 0.07 & 0.00 & 0.00 & 0.00 & 0.00 & 0.00 & 0.00 & 0.00 & 0.00 & 0.00 & 0.00 & 0.00 & 0.00 & 0.00 & 0.00 & 0.00 & 0.00 & 0.00 & 0.00 & 0.00 & 0.00 & 0.00 \\
\hline 8 & 2 & 0.00 & 1.00 & 0.00 & 0.00 & 0.00 & 0.00 & 0.00 & 0.00 & 0.00 & 0.00 & 0.00 & 0.00 & 0.00 & 0.00 & 0.00 & 0.00 & 0.00 & 0.00 & 0.00 & 0.00 & 0.00 & 0.00 & 0.00 \\
\hline 9 & 2 & 0.00 & 1.00 & 0.00 & 0.00 & 0.00 & 0.00 & 0.00 & 0.00 & 0.00 & 0.00 & 0.00 & 0.00 & 0.00 & 0.00 & 0.00 & 0.00 & 0.00 & 0.00 & 0.00 & 0.00 & 0.00 & 0.00 & 0.00 \\
\hline 10 & 2 & 0.00 & 0.76 & 0.20 & 0.00 & 0.00 & 0.04 & 0.00 & 0.00 & 0.00 & 0.00 & 0.00 & 0.00 & 0.00 & 0.00 & 0.00 & 0.00 & 0.00 & 0.00 & 0.00 & 0.00 & 0.00 & 0.00 & 0.00 \\
\hline 11 & 2 & 0.00 & 0.97 & 0.03 & 0.00 & 0.00 & 0.00 & 0.00 & 0.00 & 0.00 & 0.00 & 0.00 & 0.00 & 0.00 & 0.00 & 0.00 & 0.00 & 0.00 & 0.00 & 0.00 & 0.00 & 0.00 & 0.00 & 0.00 \\
\hline 12 & 2 & 0.00 & 0.99 & 0.01 & 0.00 & 0.00 & 0.00 & 0.00 & 0.00 & 0.00 & 0.00 & 0.00 & 0.00 & 0.00 & 0.00 & 0.00 & 0.00 & 0.00 & 0.00 & 0.00 & 0.00 & 0.00 & 0.00 & 0.00 \\
\hline 13 & 2 & 0.00 & 0.97 & 0.03 & 0.00 & 0.00 & 0.00 & 0.00 & 0.00 & 0.00 & 0.00 & 0.00 & 0.00 & 0.00 & 0.00 & 0.00 & 0.00 & 0.00 & 0.00 & 0.00 & 0.00 & 0.00 & 0.00 & 0.00 \\
\hline 14 & 2 & 0.00 & 1.00 & 0.00 & 0.00 & 0.00 & 0.00 & 0.00 & 0.00 & 0.00 & 0.00 & 0.00 & 0.00 & 0.00 & 0.00 & 0.00 & 0.00 & 0.00 & 0.00 & 0.00 & 0.00 & 0.00 & 0.00 & 0.00 \\
\hline 15 & 2 & 0.00 & 1.00 & 0.00 & 0.00 & 0.00 & 0.00 & 0.00 & 0.00 & 0.00 & 0.00 & 0.00 & 0.00 & 0.00 & 0.00 & 0.00 & 0.00 & 0.00 & 0.00 & 0.00 & 0.00 & 0.00 & 0.00 & 0.00 \\
\hline 16 & 2 & 0.00 & 0.99 & 0.01 & 0.00 & 0.00 & 0.00 & 0.00 & 0.00 & 0.00 & 0.00 & 0.00 & 0.00 & 0.00 & 0.00 & 0.00 & 0.00 & 0.00 & 0.00 & 0.00 & 0.00 & 0.00 & 0.00 & 0.00 \\
\hline 17 & 2 & 0.00 & 1.00 & 0.00 & 0.00 & 0.00 & 0.00 & 0.00 & 0.00 & 0.00 & 0.00 & 0.00 & 0.00 & 0.00 & 0.00 & 0.00 & 0.00 & 0.00 & 0.00 & 0.00 & 0.00 & 0.00 & 0.00 & 0.00 \\
\hline 18 & 2 & 0.00 & 0.97 & 0.03 & 0.00 & 0.00 & 0.00 & 0.00 & 0.00 & 0.00 & 0.00 & 0.00 & 0.00 & 0.00 & 0.00 & 0.00 & 0.00 & 0.00 & 0.00 & 0.00 & 0.00 & 0.00 & 0.00 & 0.00 \\
\hline 19 & 2 & 0.00 & 0.99 & 0.01 & 0.00 & 0.00 & 0.00 & 0.00 & 0.00 & 0.00 & 0.00 & 0.00 & 0.00 & 0.00 & 0.00 & 0.00 & 0.00 & 0.00 & 0.00 & 0.00 & 0.00 & 0.00 & 0.00 & 0.00 \\
\hline 20 & 2 & 0.00 & 0.93 & 0.07 & 0.00 & 0.00 & 0.00 & 0.00 & 0.00 & 0.00 & 0.00 & 0.00 & 0.00 & 0.00 & 0.00 & 0.00 & 0.00 & 0.00 & 0.00 & 0.00 & 0.00 & 0.00 & 0.00 & 0.00 \\
\hline 21 & 2 & 0.00 & 0.93 & 0.07 & 0.00 & 0.00 & 0.00 & 0.0 & 0.00 & 0.00 & 0.00 & 0.00 & 0.00 & 0.00 & 0.00 & 0.00 & 0.00 & 0.00 & 0.00 & 0.00 & 0.00 & 0.00 & 0.00 & 0.00 \\
\hline 22 & 2 & 0.00 & 0.75 & 0.23 & 0.02 & 0.00 & 0.00 & 0.00 & 0.00 & 0.00 & 0.00 & 0.00 & 0.00 & 0.00 & 0.00 & 0.00 & 0.00 & 0.00 & 0.00 & 0.00 & 0.00 & 0.00 & 0.00 & 0.00 \\
\hline 23 & 3 & 0.00 & 0.35 & 0.64 & 0.01 & 0.00 & 0.00 & 0.00 & 0.00 & 0.00 & 0.00 & 0.00 & 0.00 & 0.00 & 0.00 & 0.00 & 0.00 & 0.00 & 0.00 & 0.00 & 0.00 & 0.00 & 0.00 & 0.00 \\
\hline 24 & 3 & 0.00 & 0.05 & 0.89 & 0.04 & 0.00 & 0.01 & 0.00 & 0.00 & 0.00 & 0.00 & 0.00 & 0.00 & 0.00 & 0.00 & 0.00 & 0.00 & 0.00 & 0.00 & 0.00 & 0.00 & 0.00 & 0.00 & 0.00 \\
\hline 25 & 3 & 0.00 & 0.01 & 0.79 & 0.18 & 0.03 & 0.00 & 0.00 & 0.00 & 0.00 & 0.00 & 0.00 & 0.00 & 0.00 & 0.00 & 0.00 & 0.00 & 0.00 & 0.00 & 0.00 & 0.00 & 0.00 & 0.00 & 0.00 \\
\hline 26 & 3 & 0.00 & 0.01 & 0.85 & 0.14 & 0.01 & 0.00 & 0.00 & 0.00 & 0.00 & 0.00 & 0.00 & 0.00 & 0.00 & 0.00 & 0.00 & 0.00 & 0.00 & 0.00 & 0.00 & 0.00 & 0.00 & 0.00 & 0.00 \\
\hline 27 & 3 & 0.00 & 0.13 & 0.81 & 0.04 & 0.00 & 0.00 & 0.00 & 0.00 & 0.00 & 0.00 & 0.00 & 0.00 & 0.00 & 0.00 & 0.00 & 0.00 & 0.00 & 0.00 & 0.00 & 0.00 & 0.00 & 0.00 & 0.00 \\
\hline 28 & 3 & 0.00 & 0.01 & 0.64 & 0.33 & 0.02 & 0.00 & 0.00 & 0.0 & 0.00 & 0.00 & 0.00 & 0.00 & 0.00 & 0.00 & 0.00 & 0.00 & 0.00 & 0.00 & 0.00 & 0.00 & 0.00 & 0.00 & 0.00 \\
\hline 29 & 3 & 0.00 & 0.01 & 0.82 & 0.05 & 0.07 & 0.06 & 0.00 & 0.0 & 0.00 & 0.00 & 0.00 & 0.00 & 0.00 & 0.00 & 0.00 & 0.00 & 0.00 & 0.00 & 0.00 & 0.00 & 0.00 & 0.00 & 0.00 \\
\hline 30 & 4 & 0.00 & 0.00 & 0.02 & 0.67 & 0.30 & 0.00 & 0.00 & 0.00 & 0.00 & 0.00 & 0.00 & 0.00 & 0.00 & 0.00 & 0.00 & 0.00 & 0.00 & 0.00 & 0.00 & 0.00 & 0.00 & 0.00 & 0.00 \\
\hline 31 & 4 & 0.00 & 0.00 & 0.15 & 0.74 & 0.11 & 0.00 & 0.00 & 0.00 & 0.00 & 0.00 & 0.00 & 0.00 & 0.00 & 0.00 & 0.00 & 0.00 & 0.00 & 0.00 & 0.00 & 0.00 & 0.00 & 0.00 & 0.00 \\
\hline 32 & 4 & 0.00 & 0.00 & 0.23 & 0.70 & 0.07 & 0.00 & 0.00 & 0.00 & 0.00 & 0.00 & 0.00 & 0.00 & 0.00 & 0.00 & 0.00 & 0.00 & 0.00 & 0.00 & 0.00 & 0.00 & 0.00 & 0.00 & 0.00 \\
\hline 33 & 4 & 0.00 & 0.00 & 0.12 & 0.85 & 0.03 & 0.00 & 0.00 & 0.00 & 0.00 & 0.00 & 0.00 & 0.00 & 0.00 & 0.00 & 0.00 & 0.00 & 0.00 & 0.00 & 0.00 & 0.00 & 0.00 & 0.00 & 0.00 \\
\hline 34 & 4 & 0.00 & 0.00 & 0.08 & 0.42 & 0.41 & 0.00 & 0.0 & 0.0 & 0.0 & 0.00 & 0.00 & 0.00 & 0.00 & 0.00 & 0.00 & 0.00 & 0.00 & 0.00 & 0.00 & 0.00 & 0.00 & 0.00 & 0.00 \\
\hline 35 & 4 & 0.00 & 0.00 & 0.03 & 0.92 & 0.04 & 0.00 & 0.0 & 0.0 & 0.00 & 0.00 & 0.00 & 0.00 & 0.00 & 0.00 & 0.00 & 0.00 & 0.00 & 0.00 & 0.00 & 0.00 & 0.00 & 0.00 & 0.00 \\
\hline 36 & 5 & 0.00 & 0.00 & 0.00 & 0.02 & 0.97 & 0.01 & 0.0 & 0.0 & 0.0 & 0.00 & 0.00 & 0.00 & 0.00 & 0.00 & 0.00 & 0.00 & 0.00 & 0.00 & 0.00 & 0.00 & 0.00 & 0.00 & 0.00 \\
\hline 37 & 5 & 0.00 & 0.00 & 0.00 & 0.01 & 0.98 & 0.01 & 0.00 & 0.0 & 0.00 & 0.00 & 0.00 & 0.00 & 0.00 & 0.00 & 0.00 & 0.00 & 0.00 & 0.00 & 0.00 & 0.00 & 0.00 . & 0.00 & 0.00 \\
\hline 38 & 5 & 0.00 & 0.00 & 0.00 & 0.00 & 0.86 & 0.00 & 0.00 & 0.05 & 0.00 & 0.00 & 0.07 & 0.01 & 0.00 & 0.00 & 0.00 & 0.00 & 0.00 & 0.00 & 0.00 & 0.00 & 0.00 & 0.00 & 0.00 \\
\hline 39 & 5 & 0.00 & 0.00 & 0.00 & 0.00 & 0.86 & 0.00 & 0.00 & 0.02 & 0.00 & 0.00 & 0.12 & 0.00 & 0.00 & 0.00 & 0.00 & 0.00 & 0.00 & 0.00 & 0.00 & 0.00 & 0.00 & 0.00 & 0.00 \\
\hline 40 & 5 & 0.00 & 0.00 & 0.00 & 0.01 & 0.96 & 0.00 & 0.00 & 0.02 & 0.00 & 0.00 & 0.01 & 0.00 & 0.00 & 0.00 & 0.00 & 0.00 & 0.00 & 0.00 & 0.00 & 0.00 & 0.00 & 0.00 & 0.00 \\
\hline 41 & 5 & 0.00 & 0.00 & 0.00 & 0.00 & 0.74 & 0.00 & 0.00 & 0.02 & 0.00 & 0.00 & 0.21 & 0.02 & 0.00 & 0.00 & 0.00 & 0.00 & 0.00 & 0.00 & 0.00 & 0.00 & 0.00 & 0.00 & 0.00 \\
\hline 42 & 5 & 0.00 & 0.00 & 0.00 & 0.01 & 0.88 & 0.00 & 0.01 & 0.08 & 0.00 & 0.00 & 0.02 & 0.00 & 0.00 & 0.00 & 0.00 & 0.00 & 0.00 & 0.00 & 0.00 & 0.00 & 0.00 & 0.00 & 0.00 \\
\hline 43 & 5 & 0.00 & 0.00 & 0.02 & 0.14 & 0.84 & 0.00 & 0.00 & 0.00 & 0.00 & 0.00 & 0.00 & 0.00 & 0.00 & 0.00 & 0.00 & 0.00 & 0.00 & 0.00 & 0.00 & 0.00 & 0.00 & 0.00 & 0.00 \\
\hline 44 & 5 & 0.00 & 0.00 & 0.04 & 0.18 & 0.78 & 0.00 & 0.00 & 0.00 & 0.00 & 0.00 & 0.00 & 0.00 & 0.00 & 0.00 & 0.00 & 0.00 & 0.00 & 0.00 & 0.00 & 0.00 & 0.00 & 0.00 & 0.00 \\
\hline 45 & 5 & 0.00 & 0.00 & 0.02 & 0.14 & 0.84 & 0.00 & 0.00 & 0.00 & 0.00 & 0.00 & 0.00 & 0.00 & 0.00 & 0.00 & 0.00 & 0.00 & 0.00 & 0.00 & 0.00 & 0.00 & 0.00 & 0.00 & 0.00 \\
\hline
\end{tabular}




\begin{tabular}{|c|c|c|c|c|c|c|c|c|c|c|c|c|c|c|c|c|c|c|c|c|c|c|c|c|}
\hline \multicolumn{3}{|c|}{ Specimen Original } & \multicolumn{22}{|c|}{ Clusters } \\
\hline Number ${ }^{\top}$ & Cluster & 1 & 2 & 3 & 4 & 5 & 6 & 7 & 8 & 9 & 10 & 11 & 12 & 13 & 14 & 15 & Ot.1 & 16 & 17 & 18 & 19 & 20 & 21 & Ot.2 \\
\hline 46 & 5 & 0.00 & .00 & 0.06 & 0.12 & .81 & .01 & 0.00 & 0.00 & 0.00 & 0.00 & 0.00 & 0.00 & 0.00 & 0.00 & 0.00 & 0.00 & 0.00 & 0.00 & 0.00 & 0.00 & 0.00 & 0.00 & 0.00 \\
\hline 47 & 5 & 0.00 & 00 & .02 & 0.02 & .89 & 07 & 0.00 & 0.00 & 0.00 & 0.00 & 0.00 & 0.00 & 0.00 & 0.00 & 0.00 & 0.00 & 0.00 & 0.00 & 0.00 & 0.00 & 0.00 & 0.00 & 0.00 \\
\hline 48 & 5 & 0.00 & 00 & 03 & 0.06 & 90 & 0.01 & 0.00 & 0.00 & 0.00 & 0.00 & 0.00 & 0.00 & 0.00 & 0.00 & 0.00 & 0.00 & 0.00 & 0.00 & 0.00 & 0.00 & 0.00 & 0.00 & 0.00 \\
\hline 49 & 5 & 0.00 & .00 & 0.01 & 0.26 & 0.72 & 0.00 & 0.00 & 0.00 & 0.00 & 0.00 & 0.00 & 0.00 & 0.00 & 0.00 & 0.00 & 0.00 & 0.00 & 0.00 & 0.00 & 0.00 & 0.00 & 0.00 & 0.00 \\
\hline 30 & 6 & 0.00 & 0.00 & 0.00 & 0.00 & 0.00 & 1.00 & 0.00 & 0.00 & 0.00 & 0.00 & 0.00 & 0.00 & 0.00 & 0.00 & 0.00 & 0.00 & 0.00 & 0.00 & 0.00 & 0.00 & 0.00 & 0.00 & 0.00 \\
\hline 51 & 6 & 0.00 & 0.00 & 0.00 & 0.00 & 0.00 & 1.00 & 0.00 & 0.00 & 0.00 & 0.00 & 0.00 & 0.00 & 0.00 & 0.00 & 0.00 & 0.00 & 0.00 & 0.00 & 0.00 & 0.00 & 0.00 & 0.00 & 0.00 \\
\hline 52 & 6 & 0.00 & 0.00 & 0.00 & 0.00 & 0.00 & 0.99 & 0.00 & 0.00 & 0.01 & 0.00 & 0.00 & 0.00 & 0.00 & 0.00 & 0.00 & 0.00 & 0.00 & 0.00 & 0.00 & 0.00 & 0.00 & 0.00 & 0.00 \\
\hline 53 & 6 & 0.00 & 0.00 & 0.00 & 0.00 & 0.01 & 0.98 & 0.00 & 0.00 & 0.01 & 0.00 & 0.00 & 0.00 & 0.00 & 0.00 & 0.00 & 0.00 & 0.00 & 0.00 & 0.00 & 0.00 & 0.00 & 0.00 & 0.00 \\
\hline 54 & 6 & 0.00 & 0.00 & 0.00 & 0. & 0.01 & & 0.00 & 0.00 & 0.09 & 0.00 & 0.00 & 0.00 & 0.00 & 0.00 & 0.00 & 0.00 & 0.00 & 0.00 & 0.00 & 0.00 & 0.00 & 0.00 & 0.00 \\
\hline 55 & 6 & 0.00 & 0.00 & .00 & 0.00 & 0.10 & 5 & 0.00 & 0.00 & 0.00 & 0.00 & 0.00 & 0.00 & 0.00 & 0.00 & 0.00 & 0.00 & 0.00 & 0.00 & 0.00 & 0.00 & 0.00 & 0.00 & 0.00 \\
\hline 56 & 6 & 0 . & wo & 02 & 00 & 0.04 & 0.94 & 0.00 & 0.00 & 0.00 & 0.00 & 0.00 & 0.00 & 0.00 & 0.00 & 0.00 & 0.00 & 0.00 & 0.00 & 0.00 & 0.00 & 0.00 & 0.00 & 0.00 \\
\hline 57 & 6 & w & .00 & 18 & 0.01 & 0.02 & 0.79 & 0.00 & 0.00 & 0.00 & 0.00 & 0.00 & 0.00 & 0.00 & 0.00 & 0.00 & 0.00 & 0.00 & 0.00 & 0.00 & 0.00 & 0.00 & 0.00 & 0.00 \\
\hline 58 & 6 & 0.00 & 0.00 & 0.01 & 0.02 & 0.25 & 0.72 & 0.00 & 0.00 & 0.00 & 0.00 & 0.00 & 0.00 & 0.00 & 0.00 & 0.00 & 0.00 & 0.00 & 0.00 & 0.00 & 0.00 & .00 & 0.00 & 0.00 \\
\hline 39 & 7 & 0 & 0.00 & 0.00 & 0.00 & 0.00 & 0.00 & 0.84 & 0.16 & 0.00 & 0.00 & 0.00 & 0.00 & 0.00 & 0.00 & 0.00 & .00 & 0.00 & 0.00 & 0.00 & 0.00 & 0.00 & 0.00 & 0.00 \\
\hline 60 & 7 & 0.00 & 0.00 & 0.00 & 0.00 & 0.00 & 0.00 & 0. & 0.09 & 0.00 & 0.00 & 0.00 & 0.00 & 0.00 & 0.00 & 0.00 & 0.00 & 0.00 & 0.00 & 0.00 & 0.00 & 0.00 & 0.00 & 0.00 \\
\hline 61 & 7 & 0.00 & 0.00 & 0.00 & & & 0.00 & 0. & 0.1 & 0.0 & 0.00 & 0.00 & 0.00 & 0.00 & 0.00 & 0.00 & .00 & 0.00 & 0.00 & .00 & 0.00 & .00 & 0.00 & 0.00 \\
\hline 62 & 7 & 0.00 & 0.00 & & 0 & 0 & 0.00 & 0.9 & 0.02 & 0.00 & 0.00 & 0.00 & 0.00 & 0.00 & 0.00 & 0.00 & .00 & 0.00 & 0.00 & 0.00 & 0.00 & 0.00 & 0.00 & 0.00 \\
\hline 63 & 7 & 0.00 & 00 & 0. & 0.00 & 0.00 & 0.00 & 0.95 & 0.04 & 0.00 & 0.00 & 0.00 & 0.00 & 0.00 & 0.00 & 0.00 & 0.00 & 0.00 & 0.00 & 0.00 & 0.00 & 0.00 & 0.00 & 0.00 \\
\hline 64 & 8 & 0.06 & 00 & & & & 0.00 & 0.0 & 0.8 & 0.00 & 0.00 & 0.00 & 0.10 & 0.00 & 0.00 & 0.00 & 0.00 & 0.00 & 0.00 & 0.00 & 0.00 & 0.00 & .00 & 0.00 \\
\hline 65 & 8 & 0.00 & 0.00 & 0.0 & 0. & 0. & 0.00 & 0.0 & & & 0.00 & 0.03 & 0.32 & 0.00 & 0.00 & 0.00 & 0.0 & 0.00 & 0.00 & 0.00 & 0.00 & 0.00 & 0.00 & 0.00 \\
\hline 66 & 8 & & 0.00 & 0.00 & 0. & 0. & 0.00 & 0.12 & 0. & & 0.00 & 0.00 & 0.01 & 0.00 & 0.00 & 0.00 & 0.00 & 0.00 & 0.00 & 0.00 & 0.00 & 0.00 & 0.00 & 0.00 \\
\hline 67 & 8 & 0.00 & 0.00 & 0.00 & 0.00 & 0.0 & 0.00 & 0.05 & & & 0.00 & 0.00 & 0.05 & 0.00 & 0.00 & 0.00 & 0.00 & 0.00 & 0.00 & 0.00 & 0.00 & 0.00 & 0.00 & 0.00 \\
\hline 68 & 8 & 0.00 & 0.00 & & & & 0 & & & 0 & 0.00 & 0.00 & 0.01 & 0.00 & 0.00 & 0.00 & 0.00 & 0.00 & 0.00 & 0.00 & 0.00 & 0.00 & 0.00 & 0.00 \\
\hline 69 & 8 & 0. & 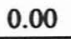 & & 0.00 & 0 & 0.00 & 0.0 & 0.51 & 0.00 & 0.00 & 0.04 & 0.07 & 0.00 & 0.00 & 0.00 & 0.00 & 0.00 & 0.00 & 0.00 & 0.00 & 0.00 & 0.00 & 0.00 \\
\hline 70 & 9 & $\pi$ & 0.00 & & 0 & & & & 0.00 & & 0.01 & 0.00 & 0.00 & 0.00 & 0.00 & 0.00 & 00 & 0.00 & 0.00 & 0.00 & 0.00 & 0.00 & 0.00 & 0.00 \\
\hline 71 & 9 & 00 & .00 & 0. & & & & & & & 0.00 & 0.00 & 0.00 & 0.00 & 0.00 & 0.00 & & 0.00 & 0.00 & .00 & 0 & 0.00 & 0.00 & 0.00 \\
\hline 72 & 9 & 0 & 0.00 & & & 0. & 0.00 & & & & 0.00 & 0.00 & 0.00 & 0.00 & 0.00 & 0.00 & ) & 0.00 & 0.00 & 0.00 & 0.00 & 0.00 & 0.00 & 0.00 \\
\hline 73 & 9 & & 0.00 & 0 . & 0.00 & 0. & 0.0 & & & & 0.00 & 0.00 & 0.00 & 0.00 & 0.00 & 0.00 & 0.00 & 0.00 & 0.00 & 0.00 & 0.00 & 0.00 & 0.00 & 0.00 \\
\hline 74 & 9 & 0.00 & 0.00 & 000 & $0 . C$ & & 0.2 & & & & 0.00 & 0.00 & 0.00 & 0.00 & 0.00 & 0.00 & 0.00 & 0.00 & 0.00 & 0.00 & 0.00 & 0.00 & 0.00 & 0.00 \\
\hline 75 & 9 & 0.00 & 0.00 & 0 & & & 0.03 & & 0. & 0.97 & 0.00 & 0.00 & 0.00 & 0.00 & 0.00 & 0.00 & 0.00 & 0.00 & 0.00 & 0.00 & 0.00 & 0.00 & 0.00 & 0.00 \\
\hline 76 & 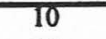 & & 0 & & 0 & 0. & 0 & 0. & 0.0 & 0.0 & 0.99 & 0.01 & 0.00 & 0.00 & 0.00 & 0.00 & 0.00 & 0.00 & 0.00 & 0.00 & 0.00 & 0.00 & 0.00 & 0.00 \\
\hline 77 & 10 & & 0 & 0. & 0.06 & 0 & & & & & 0.97 & 0.01 & 0.00 & 0.01 & 0.00 & 0.00 & 00 & 0.01 & 0.00 & 0.00 & 0.00 & 0.00 & 0.00 & 0.00 \\
\hline 7 & 10 & 0 & 0.00 & & & & & & & & 0.89 & 0.01 & 0.00 & 0.10 & 0.00 & 0.00 & 0 & 0.00 & 0.00 & 0.00 & & .00 & 0.00 & 0.00 \\
\hline 79 & 10 & 0. & 0.00 & 0.00 & & 0.0 & 0.00 & & & 0.00 & 0.74 & 0.13 & 0.01 & 0.11 & 0.00 & 0.00 & 0 & 0.00 & 0.00 & .00 & 0 & .00 & 0.00 & 0.00 \\
\hline 80 & & 0.00 & 0.00 & 0.00 & 0.0 & 0.0 & 0.0 & & & & $0.2 ;$ & 0.14 & 0.06 & 0.52 & 0.00 & 0.00 & 0 & 0.00 & 0.00 & .00 & 0.00 & 0.00 & 0.00 & 0.00 \\
\hline 81 & 10 & 0.00 & 0.00 & 0.00 & 0.0 & 0.0 & & & & & 0.83 & 0.10 & 0.01 & 0.05 & 0.00 & 0.00 & 0 & 0.00 & 0.00 & 0.00 & 0.00 & 0.00 & 0.00 & 0.00 \\
\hline 82 & 10 & 0.00 & 0.00 & & & & & & & & 0.75 & 0.22 & 0.00 & 0.00 & 0.00 & 0.00 & 0.0 & 0.02 & 0.00 & 0.00 & 0.00 & 0.00 & 0.00 & 0.00 \\
\hline 83 & 10 & 0.00 & 0.00 & & & & & & & 0 & 0.64 & 0.02 & 0.00 & 0.01 & 0.00 & 0.00 & 0.00 & 0.33 & 0.00 & 0.00 & 0.00 & 0.00 & 0.00 & 0.00 \\
\hline 84 & $\pi$ & & & & & & & & & & 0.01 & 0.98 & 0.01 & 0.00 & 0.00 & 0.00 & 0.00 & 0.00 & 0.00 & 0.00 & 0.00 & 0.00 & 0.00 & 0.00 \\
\hline 85 & & & & & & & & & 0 . & & 0.00 & 0.97 & 0.02 & 0.00 & 0.00 & 0.00 & 0.00 & 0.00 & 0.00 & 0.00 & 0.00 & 0.00 & 0.00 & 0.00 \\
\hline 86 & & & & & & & & & & 0.0 & 0.01 & 0.90 & 0.07 & 0.00 & 0.00 & 0.00 & 0.00 & 0.00 & 0.00 & 0.00 & 0.00 & 0.00 & 0.00 & 0.00 \\
\hline 8 & & & & & & & 0.0 & 0. & 0.0 & 0.00 & 0.02 & 0.86 & 0.08 & 0.00 & 0.00 & 0.00 & 0.00 & 0.00 & 0.00 & 0.00 & 0.00 & 0.00 & 0.00 & 0.00 \\
\hline 8 & & & & 0.0 & 0. & 0.0 & 0.0 & 0.0 & $0 . C$ & 0.00 & 0.05 & 0.79 & 0.12 & 0.01 & 0.00 & 0.00 & 0.00 & 0.00 & 0.00 & 0.00 & 0.00 & 0.00 & 0.00 & 0.00 \\
\hline 0 & & & & 0.00 & 0.0 & 0.0 & 0.0 & 0.0 & $0 . C$ & 0.00 & 0.05 & 0.90 & 0.03 & 0.01 & 0.00 & 0.00 & 0.00 & 0.00 & 0.00 & 0.00 & 0.00 & 0.00 & 0.00 & 0.00 \\
\hline 0 & & 0.00 & 0.00 & 0.00 & 0.0 & 0.00 & 0.0 & $0 . C$ & 0.0 & 0.0 & 0.04 & 0.94 & 0.01 & 0.00 & 0.00 & 0.00 & 0.00 & 0.00 & 0.00 & 0.00 & 0.00 & 0.00 & 0.00 & 0.00 \\
\hline 91 & 11 & 0.00 & 0.00 & 0.00 & 0.0 & 0.02 & & & & 0.0 & 0.20 & 0.77 & 0.00 & 0.00 & 0.00 & 0.00 & 0.00 & 0.00 & 0.00 & 0.00 & 0.00 & 0.00 & 0.00 & 0.00 \\
\hline 92 & 11 & 0.00 & 0.00 & 0.00 & & 0.0 & & & & 0. & 0.05 & 0.94 & 0.00 & 0.00 & 0.00 & 0.00 & 0. & 0.00 & 0.00 & 0.00 & 0.00 & 0.00 & 0.00 & 0.00 \\
\hline 93 & 11 & 0.00 & 0.00 & 0.00 & 0.00 & 0.00 & 0.00 & 0.00 & 0.00 & 0.00 & 0.17 & 0.82 & 0.00 & 0.00 & 0.00 & 0.00 & 0.00 & 0.00 & 0.00 & 0.00 & 0.00 & 0.00 & 0.00 & 0.00 \\
\hline
\end{tabular}




\begin{tabular}{|c|c|c|c|c|c|c|c|c|c|c|c|c|c|c|c|c|c|c|c|c|c|c|c|c|}
\hline \multirow{2}{*}{$\begin{array}{l}\text { Specimen } \\
\text { Number }\end{array}$} & \multirow{2}{*}{$\begin{array}{l}\text { Original } \\
\text { Cluster }\end{array}$} & \multicolumn{23}{|c|}{ Clusters } \\
\hline & & 1 & 2 & 3 & 4 & 5 & 6 & 7 & 8 & 9 & 10 & 11 & 12 & 13 & 14 & 15 & Ot.1 & 16 & 17 & 18 & 19 & 20 & 21 & Ot.2 \\
\hline 94 & 12 & 0.00 & 0.00 & 0.00 & 0.00 & 0.00 & 0.00 & 0.00 & 0.08 & 0.00 & 0.00 & 0.11 & 0.46 & 0.35 & 0.00 & 0.00 & 0.00 & 0.00 & 0.00 & 0.00 & 0.00 & 0.00 & 0.00 & 0.00 \\
\hline 95 & 12 & 0.00 & 0.00 & 0.00 & 0.00 & 0.00 & 0.00 & 0.00 & 0.15 & 0.00 & 0.00 & 0.09 & 0.58 & 0.17 & 0.00 & 0.00 & 0.00 & 0.00 & 0.00 & 0.00 & 0.00 & 0.00 & 0.00 & 0.00 \\
\hline 96 & 12 & 0.00 & 0.00 & 0.00 & 0.00 & 0.01 & 0.00 & 0.00 & 0.16 & 0.00 & 0.01 & 0.29 & .48 & 0.06 & 0.00 & 0.00 & 0.00 & 0.00 & 0.00 & 0.00 & 0.00 & 0.00 & 0.00 & 0.00 \\
\hline 97 & 12 & 0.00 & 0.00 & 0.00 & 0.00 & 0.00 & 0.00 & 0.00 & 0.40 & 0.00 & 0.00 & 0.04 & 0.53 & 0.03 & 0.00 & 0.00 & 0.00 & 0.00 & 0.00 & 0.00 & 0.00 & 0.00 & 0.00 & 0.00 \\
\hline 98 & 12 & 0.00 & 0.00 & 0.00 & 0.00 & 0.00 & .00 & 0.00 & 0.04 & 0.00 & 0.00 & 0.03 & 0.68 & 0.25 & 0.00 & 0.00 & 0.00 & 0.00 & 0.00 & 0.00 & 0.00 & 0.00 & 0.00 & .00 \\
\hline 99 & 12 & 0.00 & 0.00 & 0.00 & 0.00 & 0.00 & 0.00 & 0.00 & 0.01 & 0.00 & 0.03 & 0.33 & 0.50 & 0.13 & 0.00 & 0.00 & 0.00 & 0.00 & 0.00 & 0.00 & 0.00 & 0.00 & 0.00 & .00 \\
\hline 100 & 12 & 0.00 & 0.00 & 0.00 & 0.00 & 0.00 & 0.00 & 0.00 & 0.00 & 0.00 & 0.00 & 0.00 & 1.00 & 0.00 & 0.00 & 0.00 & 0.00 & 0.00 & 0.00 & 0.00 & 0.00 & 0.00 & 0.00 & .00 \\
\hline 101 & 13 & 0.00 & 0.00 & 0.00 & 0.00 & 0.00 & 0.00 & 0.00 & 0.00 & 0.00 & 0.07 & 0.01 & 0.03 & 0.88 & 0.00 & 0.00 & 0.00 & 0.00 & 0.00 & 0.00 & 0.00 & 0.00 & 0.00 & .00 \\
\hline 102 & 13 & 0.00 & 0.00 & 0.00 & 0.00 & 0.00 & 0.00 & 0.00 & 0.00 & 0.00 & 0.02 & 0.00 & 0.03 & 0.93 & 0.01 & 0.00 & 0.00 & 0.00 & 0.00 & 0.00 & 0.00 & 0.00 & 0.00 & 0.00 \\
\hline 103 & 13 & 0.00 & 0.00 & 0.00 & 0.00 & 0.00 & 0.00 & 0.00 & 0.00 & 0.00 & 0.01 & 0.00 & 0.01 & 0.98 & 0.00 & 0.00 & 0.00 & 0.00 & 0.00 & 0.00 & 0.00 & 0.00 & 0.00 & 0.00 \\
\hline 104 & 13 & 0.00 & 0.00 & 0.00 & 0.00 & 0.00 & 0.00 & 0.00 & 0.00 & 0.00 & 0.01 & 0.01 & 0.06 & 0.92 & 0.00 & 0.00 & 0.00 & 0.00 & 0.00 & 0.00 & 0.00 & 0.00 & 0.00 & 0.00 \\
\hline 105 & 13 & 0.00 & 0.00 & 0.00 & 0.00 & 0.00 & 0.00 & 0.00 & 0.00 & 0.00 & 0.00 & 0.00 & 0.03 & 0.97 & 0.00 & 0.00 & 0.00 & 0.00 & 0.00 & 0.00 & 0.00 & 0.00 & 0.00 & 0.00 \\
\hline 106 & $13 *$ & 0.00 & 0.00 & 0.00 & 0.00 & 0.01 & 0.00 & 0.00 & 0.14 & 0.00 & 0.01 & 0.05 & 0.53 & 0.27 & 0.00 & 0.00 & 0.00 & 0.00 & 0.00 & 0.00 & 0.00 & 0.00 & 0.00 & 0.00 \\
\hline 107 & 13 & 0.00 & 0.00 & 0.00 & 0.00 & 0.00 & 0.00 & 0.00 & 0.00 & 0.00 & 0.00 & 0.00 & 0.00 & 0.80 & 0.20 & 0.00 & 0.00 & 0.00 & 0.00 & 0.00 & 0.00 & 0.00 & 0.00 & 0.00 \\
\hline 108 & $13 *$ & 0.00 & 0.00 & 0.00 & 0.00 & 0.00 & 0.00 & 0.00 & 0.00 & 0.00 & 0.00 & 0.00 & 0.00 & 0.48 & 0.52 & 0.00 & 0.00 & 0.00 & 0.00 & 0.00 & 0.00 & 0.00 & 0.00 & 0.00 \\
\hline 109 & 13 & 0.00 & 0.00 & 0.00 & 0.00 & 0.00 & 0.00 & 0.00 & 0.00 & 0.00 & 0.00 & 0.00 & 0.00 & 0.94 & 0.05 & 0.00 & 0.00 & .00 & 0.00 & 0.00 & .00 & 0.00 & 0.00 & 00 \\
\hline 110 & 13 & 0.00 & 0.00 & 0.00 & 0.00 & 0.00 & 0.00 & 0.00 & 0.00 & 0.00 & 0.00 & 0.00 & 0.00 & 0.84 & 0.15 & 0.00 & 0.00 & 0.00 & 0.00 & 0.00 & 0.00 & 0.00 & 0.00 & 0.00 \\
\hline 111 & 13 & 0.00 & 0.00 & 0.00 & 0.00 & 0.00 & 0.00 & 0.00 & 0.00 & 0.00 & 0.00 & 0.00 & 0.00 & 0.98 & 0.01 & 0.00 & 0.00 & 0.00 & 0.00 & 0.00 & 0.00 & 0.00 & 0.00 & 0.00 \\
\hline 112 & 13 & 0.00 & 0.00 & 0.00 & 0.00 & 0.00 & 0.00 & 0.00 & 0.00 & 0.00 & 0.02 & 0.00 & 0.00 & 96 & 0.02 & 0.00 & 0.00 & .00 & 0.00 & 0.00 & 0.00 & 0.00 & 0.00 & 0.00 \\
\hline 113 & 13 & 0.00 & $0 . c$ & 0.1 & 0.00 & 0. & 0.00 & 0. & 0. & 0. & 0. & 0.00 & 0.00 & 0.95 & 0.04 & 0.00 & 0.00 & 0.00 & 0.00 & 0.00 & .00 & 0.00 & 0.00 & .00 \\
\hline 114 & 13 & 0.00 & 0.00 & 0.00 & 0.00 & 0.00 & 0.00 & 0.0 & 0.0 & 0.00 & 0.01 & 0.00 & 0.00 & 0.96 & 0.02 & 0.00 & 0.00 & 0.00 & 0.00 & 0.00 & 0.00 & 0.00 & 0.00 & .00 \\
\hline 115 & 13 & 0.00 & 0. & 0.00 & 0.00 & 0. & 0.00 & 0.0 & 0. & 0.00 & 0.06 & 0.00 & 0.00 & 0.86 & 0.08 & 0.00 & 0.00 & 0 & 0.00 & 0.00 & .00 & 0.00 & 0.00 & .00 \\
\hline 116 & 13 & 0.00 & 0.00 & 0.00 & 0.00 & 0.00 & 0.00 & 0.00 & 0.00 & 0.00 & 0.00 & 0.00 & 0.00 & 0.92 & 0.02 & 0.00 & 0.00 & 0.05 & 0.00 & 0.00 & 0.00 & 0.00 & 0.00 & 0.00 \\
\hline 117 & 13 & 0.00 & 0.00 & 0.00 & 0.00 & 0.00 & 0.00 & 0.00 & 0.00 & 0.00 & 0.00 & 0.00 & 0.01 & 0.98 & 0.00 & 0.00 & 0.00 & 0.00 & 0.00 & 0.00 & 0.00 & 0.00 & 0.00 & 0.00 \\
\hline 118 & 13 & 0.00 & 0.00 & 0.00 & 0.00 & 0.00 & 0.00 & 0.00 & 0.00 & 0.00 & 0.01 & 0.00 & 0.00 & 0.97 & 0.02 & 0.00 & 0.00 & 0.00 & 0.00 & 0.00 & .00 & 0.00 & & 0.00 \\
\hline 119 & 13 & 0.00 & 0. & 0.00 & 0.00 & 0.00 & 0.00 & 0. & 0. & 0.00 & 0.02 & 0.02 & 0.02 & 0.93 & 0.00 & 0.00 & $0 .($ & 0.01 & 0.00 & 0.00 & 0.00 & 0.00 & 0.00 & 0.00 \\
\hline 120 & 14 & 0.0 & 0. & 0.00 & 0.00 & 0.0 & 0.0 & 0. & 0. & 0.0 & 0.0 & 0.00 & 0.00 & 0.06 & 0.94 & 0.00 & 0.0 & 0.00 & 0.00 & 0.00 & 0.00 & 0.00 & 0.00 & 0.00 \\
\hline 121 & 14 & 0.00 & 0.00 & 0.00 & 0.00 & 0.0 & 0.0 & 0. & 0.0 & 0.00 & 0.0 & 0.00 & 0.00 & 0.05 & 0.95 & 0.00 & 0.00 & 0.00 & 0.00 & 0.00 & .00 & 0.00 & 0.00 & 0.00 \\
\hline 122 & 14 & 0.00 & 0.00 & 0.00 & 0.00 & 0.00 & 0.00 & 0. & 0.00 & 0.00 & 0.00 & 0.00 & 0.00 & 0.01 & 0.99 & 0.00 & 0.00 & .00 & 0.00 & 0.00 & 0.00 & 0.00 & 0.00 & 0.00 \\
\hline 123 & 14 & 0.00 & 0.00 & 0.00 & 0.00 & 0.00 & 0.00 & 0.00 & 0.00 & 0.00 & 0.00 & 0.00 & 0.00 & 0.30 & 0.70 & 0.00 & 0.00 & 0.00 & 0.00 & 0.00 & 0.00 & 0.00 & 0.00 & 0.00 \\
\hline 124 & 14 & 0.00 & 0.00 & 0.00 & 0.00 & 0.00 & 0.00 & 0.00 & 0.00 & 0.00 & 0.00 & 0.00 & 0.00 & 0.12 & 0.88 & 0.00 & 0.00 & 0.00 & 0.00 & 0.00 & 0.00 & 0.00 & 0.00 & 0.00 \\
\hline 125 & 14 & 0.00 & 0.00 & 0.00 & 0.00 & 0.00 & 0.00 & & 0.00 & 0.00 & 0.00 & 0.00 & 0.00 & 0.29 & 0.71 & 0.00 & 0.00 & 0.00 & 0.00 & 0.00 & 0.00 & 0.00 & 0.00 & 0.00 \\
\hline 126 & 14 & 0.00 & 0. & 0. & 0. & 0. & 0. & 0. & 0 & 0. & 0 & 0.00 & 0.00 & 0.09 & 0.90 & 0.00 & 0. & 0.00 & 0.00 & 0.00 & .00 & 0.00 & & 0.00 \\
\hline 127 & 14 & 0.00 & 0.00 & 0.00 & 0.00 & 0.00 & 0.00 & 0. & 0.0 & 0.00 & 0.0 & 0.00 & 0.00 & 0.06 & 0.92 & 0.01 & 0.00 & 0.00 & 0.00 & 0.00 & 0.00 & 0.00 & 0.00 & 0.00 \\
\hline 128 & 14 & 0.00 & 0.00 & 0.00 & 0.00 & 0.0 & 0.00 & 0.0 & 0. & 0.00 & 0.0 & 0.00 & 0.00 & 0.00 & 0.97 & 0.03 & 0.00 & 0.00 & 0.00 & 0.00 & 0.00 & 0.00 & 0.00 & 0.00 \\
\hline 129 & 15 & 0.00 & 0.0 & 0.00 & 0.00 & 0.0 & 0.0 & 0.0 & 0.0 & 0.0 & 0.0 & 0.00 & 0.00 & 0.00 & 0.00 & 1.00 & 0.00 & 0.00 & 0.00 & 0.00 & 0.00 & 0.00 & 0.00 & 0.00 \\
\hline 130 & 15 & 0.00 & 0.00 & 0.00 & 0.00 & 0.00 & 0.00 & 0.00 & 0.00 & 0.00 & 0.00 & 0.00 & 0.00 & 0.00 & 0.01 & 0.99 & 0.00 & 0.00 & 0.00 & 0.00 & 0.00 & 0.00 & 0.00 & 0.00 \\
\hline 131 & 15 & 0.00 & 0.00 & 0.00 & 0.00 & 0.00 & 0.00 & 0.00 & 0.00 & 0.00 & 0.00 & 0.00 & 0.00 & 0.00 & 0.01 & 0.99 & 0.00 & 0.00 & 0.00 & 0.00 & 0.00 & 0.00 & 0.00 & 0.00 \\
\hline 132 & Ot.1 & 0.00 & & 0.00 & 0.00 & 0.00 & 0.00 & 0.00 & & 0.00 & 0.00 & 0.00 & 0.00 & 0.00 & 0.00 & 0.00 & 1.00 & 0.00 & 0.00 & 0.00 & 0.00 & 0.00 & 0.00 & 0.00 \\
\hline 133 & Ot.1 & 0.00 & 0.00 & 0.00 & 0.00 & 0.00 & 0.00 & 0.00 & 0.0 & 0.00 & 0.0 & 0.00 & 0.00 & 0.00 & 0.00 & 0.00 & 1.00 & 0.00 & 0.00 & 0.00 & 0.00 & 0.00 & 0.00 & 0.00 \\
\hline 134 & 16 & 0.00 & 0.0 & 0.00 & 0.00 & 0.00 & 0.0 & 0.0 & 0.0 & 0.00 & 0.0 & 0.00 & 0.00 & 0.00 & 0.00 & $\overline{0 .}$ & 0.1 & 0.89 & 0.11 & 0.00 & 0.00 & 0.00 & 0.00 & 0.00 \\
\hline 135 & 16 & 0.00 & 0.0 & 0.00 & 0. & 0.0 & 0.0 & 0. & 0. & 0.00 & 0.0 & 0.00 & 0.00 & 0.00 & 0.00 & 0.00 & 0.00 & 0.89 & 0.07 & 0.00 & 0.00 & 0.00 & 0.00 & 0.00 \\
\hline 136 & 16 & 0.00 & 0.0 & 0.0 & 0. & 0.0 & 0.0 & 0. & & & 0. & 0.0 & 0.00 & 0.0 & 0.00 & 0. & 0.00 & 0.77 & 0.23 & 0.00 & 0.00 & 0.00 & 0.00 & 0.00 \\
\hline 137 & 16 & 0.00 & 0.00 & 0.00 & 0.00 & 0.00 & 0.00 & 0.00 & 0.00 & 0.00 & 0.09 & 0.00 & 0.00 & 0.06 & 0.09 & 0.00 & 0.00 & 0.75 & 0.00 & 0.00 & 0.00 & 0.00 & 0.00 & 0.00 \\
\hline 138 & 16 & 0.00 & 0.00 & 0.00 & 0.00 & 0.00 & 0.00 & 0.00 & 0.00 & 0.00 & 0.01 & 0.00 & 0.00 & 0.01 & 0.01 & 0.00 & 0.00 & 0.95 & 0.01 & 0.01 & 0.00 & 0.00 & 0.00 & 0.00 \\
\hline 139 & 17 & 0.00 & 0.00 & 0.00 & 0.00 & 0.00 & 0.00 & 0.00 & 0.00 & 0.00 & 0.00 & 0.00 & 0.00 & 0.00 & 0.00 & 0.00 & 0.00 & 0.01 & 0.97 & 0.02 & 0.00 & 0.00 & 0.00 & 0.00 \\
\hline 140 & 17 & 0.00 & 0.00 & 0.00 & 0.00 & 0.00 & 0.00 & 0.00 & 0.00 & 0.00 & 0.00 & 0.00 & 0.00 & 0.00 & 0.00 & 0.00 & 0.00 & 0.01 & 0.74 & 0.09 & 0.16 & 0.00 & 0.00 & 0.00 \\
\hline 141 & 17 & 0.00 & 0.00 & 0.00 & 0.00 & 0.00 & 0.00 & 0.00 & 0.00 & 0.00 & 0.00 & 0.00 & 0.00 & 0.00 & 0.00 & 0.00 & 0.00 & 0.01 & 0.60 & 0.39 & 0.01 & 0.00 & 0.00 & 0.00 \\
\hline
\end{tabular}




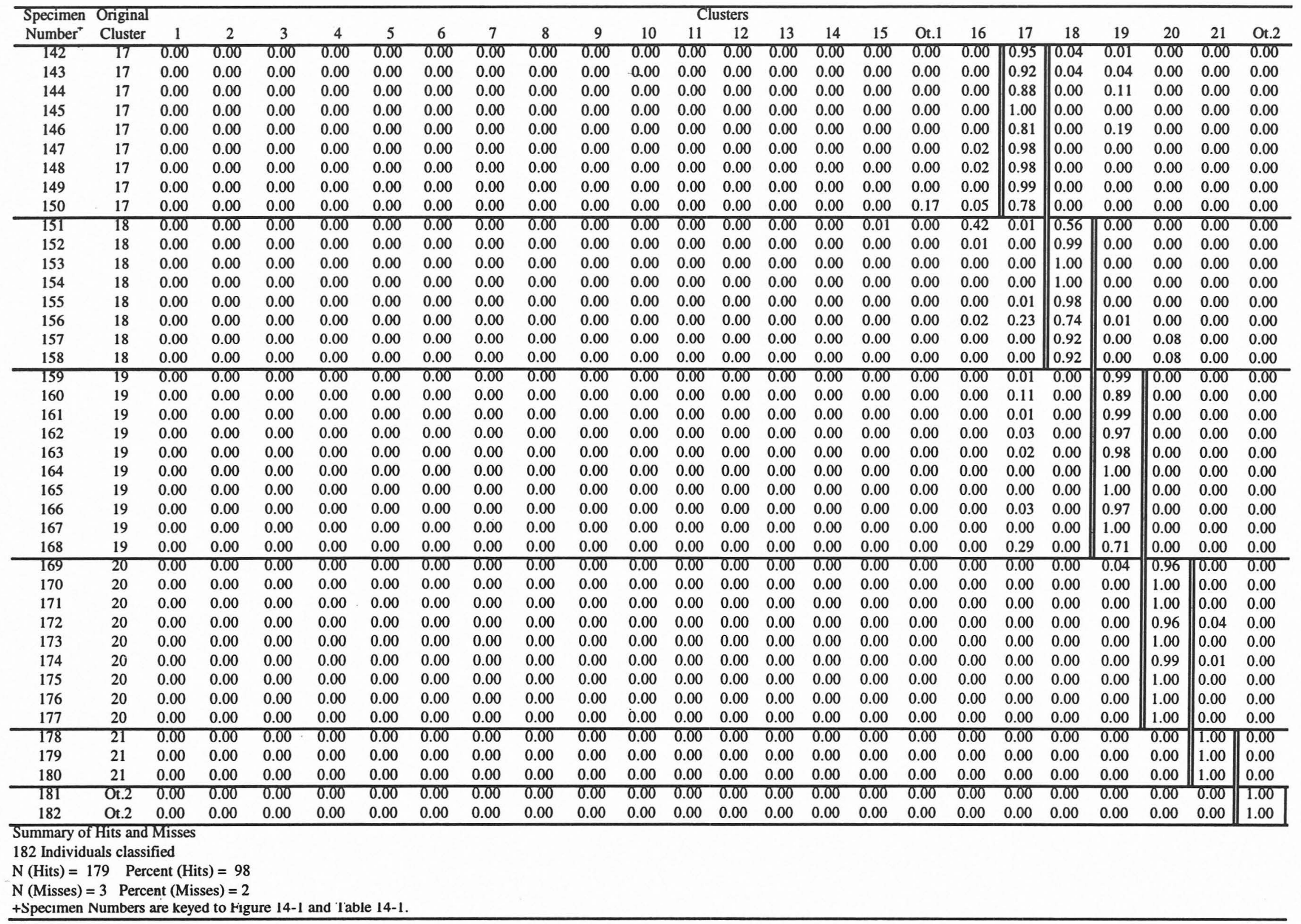

$N$ (Misses) $=3$ Percent (Misses) $=2$

+ Specimen Numbers are keyed to Higure 14-1 and I'able 14-1. 
grinding length ratio. The greater inherent variability within the haft shape, 10-mm haft thickness, and grinding length indicate these three variables are not useful for describing this set of clusters.

\section{Contracting Haft Clusters}

\section{CLUSTER 1 (SPECIMENS 1-7)}

Cluster 1 specimens have extremely contracted haft shapes, very narrow haft widths, moderately thick hafts, and thicker hafts relative to narrow haft widths (see Table 14-3 and Figure 14-3a). All seven specimens are correctly classified according to GCP (see Table 14-6), with $p=1.00$ in all but one case. This one specimen shows an accurate probability assignment of $p=0.93$ for Cluster 1 and a low probability $(p=0.07)$ for Cluster 2 indicating a slight affinity between Clusters 1 and 2.

\section{CLUSTER 2 (SPECIMENS 8-22)}

Cluster 2 is characterized by narrow haft width (see Table 14-3), a very contracted haft shape, and a moderately thick haft (see Figure 14-3b). These points also have a thicker haft compared to a narrow haft width. All 15 specimens are correctly classified according to GCP (see Table 14-6), with probabilities from 0.75 to 1.00 . Four specimens show probabilities greater than 0.05 for Cluster 3 indicating an affinity between Clusters 2 and 3.

\section{CLUSTER 3 (SPECIMENS 23-29)}

As Table 14-3 indicates, Cluster 3 specimens have, of the contracting haft clusters, moderately narrow haft widths, medium contracting haft shapes, and medium haft thicknesses (see Figure 14-3c). And they have medium haft thicknesses relative to moderately narrow haft widths. All seven specimens are correctly classified according to GCP (see Table 14-6), with probabilities from 0.64 to 0.89 . Four specimens show probabilities greater than or equal to 0.05 for Cluster 4. In addition, three specimens show probabilities greater than or equal to 0.05 for Cluster 2 , and one of these four shows $p=0.07$ for Cluster 5 and $p=0.06$ for Cluster 6 as well. Strong affinities are indicated between Cluster 3 and Clusters 2 and 4.

\section{CLUSTER 4(SPECIMENS 30-35)}

Of the contracting haft clusters (see Table 14-3), Cluster 4 points have moderately narrow hafts, slight contracting haft shapes, and moderately thin hafts (see Figure 14-3d). They have moderately thin hafts relative to moderately narrow haft widths. All six specimens are correctly classified according to GCP (see Table 14-6), with probabilities from 0.42 to 0.92 . Four specimens have probabilities greater than 0.05 for each of
TABLE 14-7

Analysis of Variance (ANOVA) Comparing Nine

Contracting Haft Clusters by Eight Variables

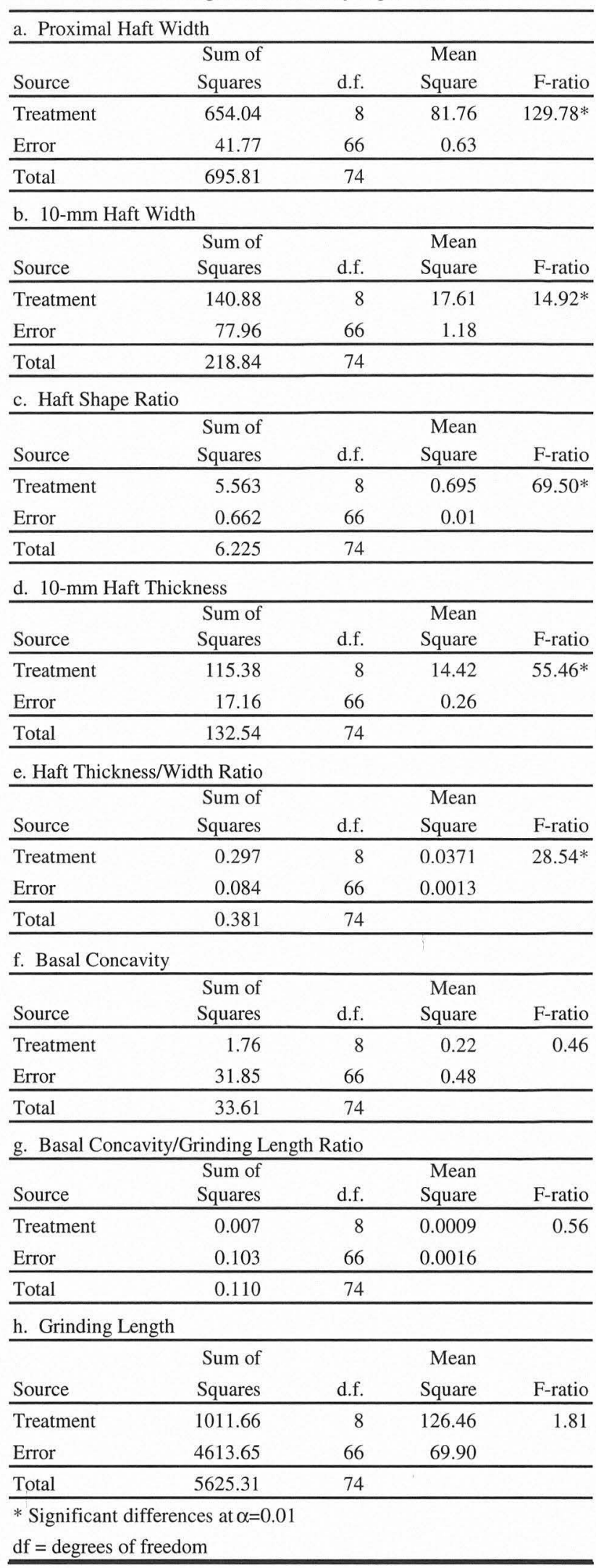


TABLE 14-8

Analysis of Variance (ANOVA) Comparing Six Parallel-Sided Haft, Shallow-to-Moderate Basal Concavity Clusters by Eight Variables

\begin{tabular}{|c|c|c|c|c|}
\hline \multirow[b]{2}{*}{ Source } & \multirow[b]{2}{*}{$\begin{array}{l}\text { Sum of } \\
\text { Squares }\end{array}$} & & & \\
\hline & & d.f. & $\begin{array}{r}\text { Mean } \\
\text { Square }\end{array}$ & F-ratio \\
\hline Treatment & 240.19 & 5 & 48.04 & $123.18^{*}$ \\
\hline Error & 19.36 & 50 & 0.39 & \\
\hline Total & 259.55 & 55 & & \\
\hline \multicolumn{5}{|c|}{ b. 10-mm Haft Width } \\
\hline Source & $\begin{array}{r}\text { Sum of } \\
\text { Squares } \\
\end{array}$ & d.f. & $\begin{array}{r}\text { Mean } \\
\text { Square } \\
\end{array}$ & F-ratio \\
\hline Treatment & 179.96 & 5 & 35.99 & $24.48^{*}$ \\
\hline Error & 73.48 & 50 & 1.47 & \\
\hline Total & 253.44 & 55 & & \\
\hline \multicolumn{5}{|c|}{ c. Haft Shape Ratio } \\
\hline Source & $\begin{array}{r}\text { Sum of } \\
\text { Squares }\end{array}$ & d.f. & $\begin{array}{r}\text { Mean } \\
\text { Square } \\
\end{array}$ & F-ratio \\
\hline Treatment & 0.032 & 5 & 0.00640 & 2.62 \\
\hline Error & 0.122 & 50 & 0.00244 & \\
\hline Total & 0.154 & 55 & & \\
\hline \multicolumn{5}{|c|}{ d. 10-mm Haft Thickness } \\
\hline Source & $\begin{array}{l}\text { Sum of } \\
\text { Squares }\end{array}$ & d.f. & $\begin{array}{r}\text { Mean } \\
\text { Square } \\
\end{array}$ & F-ratio \\
\hline Treatment & 27.20 & 5 & 5.44 & $18.13^{*}$ \\
\hline Error & 15.13 & 50 & 0.30 & \\
\hline Total & 42.33 & 55 & & \\
\hline \multicolumn{5}{|c|}{ e. Haft Thickness/Width Ratio } \\
\hline Source & $\begin{array}{l}\text { Sum of } \\
\text { Squares }\end{array}$ & d.f. & $\begin{array}{r}\text { Mean } \\
\text { Square } \\
\end{array}$ & F-ratio \\
\hline Treatment & 0.0689 & 5 & 0.0138 & $17.25^{*}$ \\
\hline Error & 0.0381 & 50 & 0.0008 & \\
\hline Total & 0.1070 & 55 & & \\
\hline \multicolumn{5}{|c|}{ f. Basal Concavity } \\
\hline Source & $\begin{array}{r}\text { Sum of } \\
\text { Squares }\end{array}$ & d.f. & $\begin{array}{r}\text { Mean } \\
\text { Square } \\
\end{array}$ & F-ratio \\
\hline Treatment & 22.55 & 5 & 4.51 & $7.64^{*}$ \\
\hline Error & 29.41 & 50 & 0.59 & \\
\hline Total & 51.96 & 55 & & \\
\hline \multicolumn{5}{|c|}{ g. Basal Concavity/Grinding Length Ratio } \\
\hline Source & $\begin{array}{r}\text { Sum of } \\
\text { Squares }\end{array}$ & d.f. & $\begin{array}{r}\text { Mean } \\
\text { Square } \\
\end{array}$ & F-ratio \\
\hline Treatment & 0.099 & 5 & 0.020 & 1.82 \\
\hline Error & 0.542 & 50 & 0.011 & \\
\hline Total & 0.641 & 55 & & \\
\hline \multicolumn{5}{|c|}{ h. Grinding Length } \\
\hline Source & $\begin{array}{r}\text { Sum of } \\
\text { Squares } \\
\end{array}$ & d.f. & $\begin{array}{r}\text { Mean } \\
\text { Square } \\
\end{array}$ & F-ratio \\
\hline Treatment & 1181.92 & 5 & 236.38 & $3.82 *$ \\
\hline Error & 3097.38 & 50 & 61.95 & \\
\hline Total & 4279.30 & 55 & & \\
\hline $\begin{array}{l}* \text { Significan } \\
\text { d.f. = degre }\end{array}$ & $\begin{array}{l}\text { nces at } \alpha=0 \\
\text { dom }\end{array}$ & & & \\
\hline
\end{tabular}

TABLE 14-9

Analysis of Variance (ANOVA) Comparing Six Parallel-Sided Haft, Deep Basal Concavity Clusters by Eight Variables a. Proximal Haft Width

\begin{tabular}{|c|c|c|c|c|}
\hline \multirow[b]{2}{*}{ Source } & \multirow{2}{*}{$\begin{array}{l}\text { Sum of } \\
\text { Squares }\end{array}$} & \multicolumn{3}{|c|}{ Mean } \\
\hline & & d.f. & Square & F-ratio \\
\hline Treatment & 277.30 & 5 & 55.46 & $55.46^{*}$ \\
\hline Error & 41.16 & 41 & 1.00 & \\
\hline Total & 318.46 & 46 & & \\
\hline \multicolumn{5}{|c|}{ b. 10 -mm Haft Width } \\
\hline Source & $\begin{array}{l}\text { Sum of } \\
\text { Squares }\end{array}$ & d.f. & $\begin{array}{r}\text { Mean } \\
\text { Square }\end{array}$ & F-ratio \\
\hline Treatment & 277.90 & 5 & 55.58 & $15.79 *$ \\
\hline Error & 144.31 & 41 & 3.52 & \\
\hline Total & 422.21 & 46 & & \\
\hline \multicolumn{5}{|c|}{ c. Haft Shape Ratio } \\
\hline Source & $\begin{array}{l}\text { Sum of } \\
\text { Squares }\end{array}$ & d.f. & $\begin{array}{r}\text { Mean } \\
\text { Square } \\
\end{array}$ & F-ratio \\
\hline Treatment & 0.044 & 5 & 0.0088 & 1.80 \\
\hline Error & 0.202 & 41 & 0.0049 & \\
\hline Total & 0.246 & 46 & & \\
\hline
\end{tabular}

d. 10-mm Haft Thickness

\begin{tabular}{lrrrr}
\hline Source & $\begin{array}{r}\text { Sum of } \\
\text { Squares }\end{array}$ & d.f. & $\begin{array}{r}\text { Mean } \\
\text { Square }\end{array}$ & F-ratio \\
\hline Treatment & 0.89 & 5 & 0.18 & 0.35 \\
Error & 21.01 & 41 & 0.51 & \\
\hline Total & 21.90 & 46 & & \\
\hline
\end{tabular}

e. Haft Thickness/Width Ratio

\begin{tabular}{lcrrr}
\hline Source & $\begin{array}{c}\text { Sum of } \\
\text { Squares }\end{array}$ & d.f. & Square & F-ratio \\
\hline Treatment & 0.0285 & 5 & 0.0057 & $5.70^{*}$ \\
Error & 0.0390 & 41 & 0.0010 & \\
\hline Total & 0.0675 & 46 & & \\
\hline
\end{tabular}

\section{f. Basal Concavity}

\begin{tabular}{lrrrr}
\hline & Sum of & \multicolumn{3}{c}{ Mean } \\
Source & Squares & d.f. & Square & F-ratio \\
\hline Treatment & 113.09 & 5 & 22.62 & $34.27^{*}$ \\
Error & 27.22 & 41 & 0.66 & \\
\hline Total & 140.31 & 46 & & \\
\hline
\end{tabular}

g. Basal Concavity/Grinding Length Ratio

\begin{tabular}{lrrrr}
\hline Source & $\begin{array}{r}\text { Sum of } \\
\text { Squares }\end{array}$ & d.f. & $\begin{array}{r}\text { Mean } \\
\text { Square }\end{array}$ & F-ratio \\
\hline Treatment & 0.297 & 5 & 0.059 & $6.56^{*}$ \\
Error & 0.361 & 41 & 0.009 & \\
\hline Total & 0.658 & 46 & & \\
\hline h. Grinding Length & & & & \\
\hline & Sum of & & Mean & \\
Source & Squares & d.f. & Square & F-ratio \\
\hline Treatment & 432.84 & 5 & 86.57 & 2.18 \\
Error & 1627.25 & 41 & 39.69 & \\
\hline Total & 2060.09 & 46 & & \\
\hline
\end{tabular}

* Significant differences at $\alpha=0.01$

d.f. $=$ degrees of freedom 


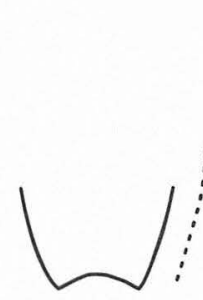

a

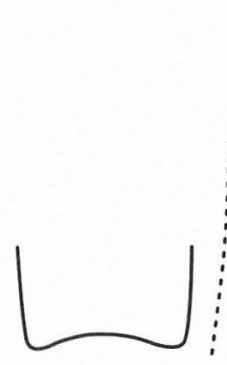

e

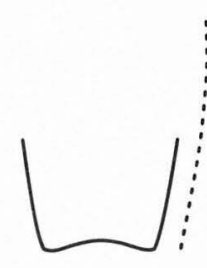

b

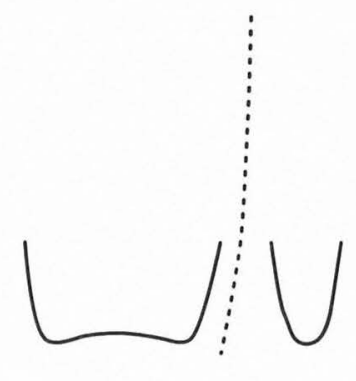

f

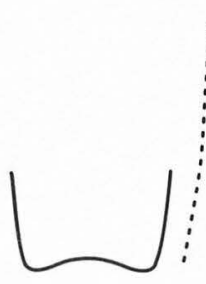

C

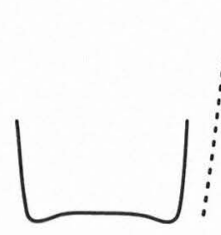

g

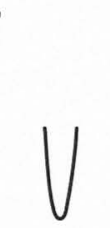

h

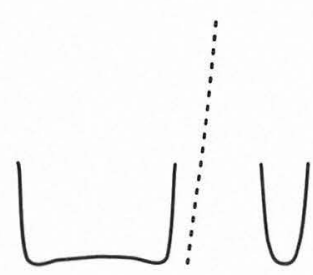

d

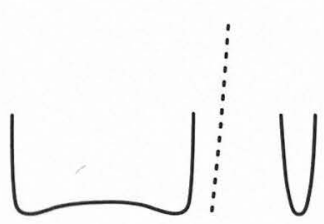

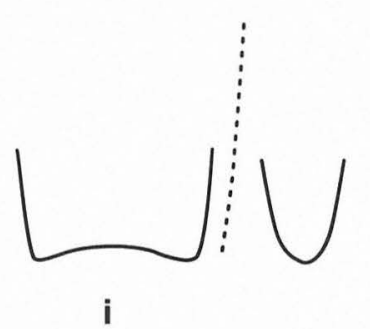

FIGURE 14-3. Mean shapes and extent of grinding (dotted line) for contracting haft clusters. (a) Cluster 1; (b) Cluster 2; (c) Cluster 3; (d) Cluster 4; (e) Cluster 5; (f) Cluster 6; (g) Cluster 7; (h) Cluster 8; (i) Cluster 9.

Clusters 3 and 5 , with Specimen 34 nearly split between Cluster $4(p=0.42)$ and Cluster $5(p=0.41)$, indicating a strong similarity between Clusters 3,4 , and 5 .

\section{CLUSTER 5 (SPECIMENS 36-49)}

Cluster 5 specimens have, of the contracting haft clusters, moderately wide hafts, slightly contracting haft shapes, moderate haft thicknesses, and moderately thick hafts relative to moderate haft widths (see Table 14-3 and Figure 143e). All 14 specimens are correctly classified according to GCP (see Table 14-6), with probabilities from 0.72 to 0.98 . Six specimens have probabilities greater than 0.05 for Cluster 4 , 3 specimens show greater than $p=0.05$ for Cluster 11, 2 specimens show probabilities greater than or equal to 0.05 for Cluster 8,1 specimen shows $p=0.06$ for Cluster 3 , and 1 specimen shows $p=0.07$ for Cluster 6 . The strongest affinity suggested is between Cluster 5 and Cluster 4 .

\section{CLUSTER 6(SPECIMENS 50-58)}

Of the contracting haft clusters, Cluster 6 points have moderate to wide haft widths, medium contracting haft shapes, very thick hafts, and very thick hafts compared to moderately wide hafts (see Table 14-3 and Figure 14-3f). All nine specimens are correctly classified according to GCP (see Table 14-6), with probabilities from 0.72 to 1.00 . There are two specimens with probabilities greater than 0.05 for Cluster 5 , one specimen shows $p=0.18$ for Cluster 3 , and another single specimen shows $p=0.09$ for Cluster 9. Slight similarities are indicated between Cluster 6 and Clusters 5,3, and 9.

\section{CLUSTER 7(SPECIMENS 59-63)}

Of the contracting haft clusters, Cluster 7 specimens have moderate haft widths, slight contracting haft shapes, and very thin hafts (see Table 14-3 and Figure 14-3g). They have very thin hafts relative to moderate haft widths. All five specimens are correctly classified according to GCP (see Table 14-6), with probabilities from 0.84 to 0.98 . Two specimens show probabilities greater than 0.05 for Cluster 8 indicating an association between the two clusters.

\section{CLUSTER 8(SPECIMENS 64-69)}

In relation to the other contracting haft clusters, Cluster 8 is defined by the following characteristics: wide hafts, very slight contracting haft shapes, very thin hafts (see Table 14-3 
and Figure 14-3h), and very thin hafts compared to haft widths. Also, it should be noted that Clusters 7 and 8 have the smallest mean haft thicknesses of all the clusters in the sample. According to GCP (see Table 14-6), all six specimens in Cluster 8 are correctly classified with probabilities from 0.51 to 0.88 . Four specimens show probabilities greater than or equal to 0.05 for Cluster 12, three specimens show probabilities greater than or equal to 0.05 for Cluster 7 , and one specimen shows $p=0.37$ for Cluster 5 . These suggest strong relationships between Cluster 8 and Clusters 12 and 7 .

\section{CLUSTER 9(SPECIMENS 70-75)}

Cluster 9 is unlike the other contracting haft clusters. Specimens in this cluster are characterized by very wide hafts, slight contracting haft shapes, very thick hafts (see Table 14-3 and Figure 14-3i), and very thick hafts relative to haft widths. All six specimens are correctly classified according to GCP (see Table 14-6), with probabilities from 0.76 to 1.00 . One specimen shows $p=0.24$ for Cluster 6 indicating a slight relationship between Clusters 6 and 9 .

\section{Discussion of Contracting Haft Clusters}

Clusters 1 through 9 represent the morphological variability of contracting haft points. It is clear from the dendrogram (see Figure 14-2) that first Cluster 1 and then Clusters 2 through 9 separate from the rest of the sample. All nine clusters share, to some extent, the primary characteristic of a contracting haft shape. But the degree of contraction ranges from extreme and obvious, as with Clusters 1 and 2, to slight, as with Clusters 4, 5, 7, 8, and 9 (see Figure 14-3). The secondary shared characteristic, which draws these five clusters away from the parallel-sided haft group, is the insignificance of basal concavity (see Table 14-7). In contrast to these general similarities, there still exist patterns of significant difference in haft width, haft shape, haft thickness, and haft thickness/width ratio. It is in the patterns of similarity and difference that clues are found to indicate the relationship of morphological clusters to types; individual clusters do not necessarily represent types.

Upon examination of contracting haft clusters, analysis of variance shows that significant differences exist between the cluster means, however, some clusters are more or less different than others. Among the contracting haft clusters, some important morphological similarities occur between Clusters 1, 2, 3, 4, and 5, Clusters 6 and 9, and Clusters 7 and 8 .

Clusters $1,2,3,4$, and 5 show some similarity in mean haft thickness (see Table 14-3). Clusters 4 and 5 have close similarities in haft shape ratio and haft thickness/width ratio. Clusters 1, 2, and 4 show near equivalence in $10-\mathrm{mm}$ haft width. However, the differences between means are what distinguish these five clusters. The differences are particularly apparent with proximal haft width and the haft shape ratio.
Type names previously applied to specimens in Clusters 1 through 5 are Angostura, Lubbock (in Clusters 3, 4, and 5), and one Plainview (in Cluster 5) from Bonfire Shelter (Specimen 42, see Table 14-1). The Lubbock and Plainview specimens show similarities in morphology and flake patterning suggesting that the Bonfire Plainview specimen can be considered a Lubbock point rather than a Plainview. It should be noted that this specimen from Bonfire Shelter is the only point from Bone Bed 2 that actually was found near the radiocarbon dated Hearth 1 as indicated by the similar grid coordinates and component assignment (Dibble and Lorrain 1968:32-36). When the radiocarbon age of $9950 \pm 120$ B.P. from humic acid (see Table 14-2) for the Lubbock points from Lubbock Lake site is compared with the three radiocarbon ages of $9920 \pm 150$ в.P., $10,100 \pm 300$ в.P., and 10,230 160 в.P. from charcoal in Hearth 1 (Dibble 1970; Dibble and Lorrain 1968 ) and a fourth radiocarbon age of $10,280 \pm 430$ B.P. from charcoal (Bement 1986) associated with Bone Bed 2 at Bonfire Shelter, there is some overlap, indicating a possible age range for Lubbock points between 9900 and 10,300 B.P., although some caution in interpretation remains due to the potential differences in dating humic acid and charcoal as well as the limited number of sampled sites.

Clusters 1 through 5, with Lubbock points excluded, still show significant differences between means for proximal haft width, 10-mm haft width, haft shape ratio, 10-mm haft thickness, and haft thickness/width ratio (Table 14-10). However, similarities do occur between Clusters 1, 2, and 3 and then between Clusters 4 and 5 in 10-mm haft thickness and haft thickness/width ratio (Table 14-11). Clusters 1,2,3, and 4 are similar in 10-mm haft width; Cluster 5 is much wider in 10-mm haft width. All five clusters are different in haft shape ratio, which is controlled by proximal haft width.

The Angostura points in Clusters 1 through 5 come from three sites: Wilson-Leonard, Levi Rockshelter, and Tombstone Bluff (see Table 14-1). The three specimens from Levi Rockshelter, previously published as Plainview-Angostura (Alexander 1963), can be considered Angostura.

Most of the Wilson-Leonard specimens are from Late Paleoindian and Early Archaic strata (Units II, II/IIIa, and IIIa) with a radiocarbon age range from 8800 B.P. to 8100 B.P. There is no indication of stratigraphic pattern between the clusters and therefore no apparent temporal significance to variability represented by the five clusters. The age range from Levi Rockshelter, about 6800 to 9300 B.P., is very wide probably due to problems in dating snail and mussel shell but does encompass the tighter range from Wilson-Leonard. The Tombstone Bluff specimens were surface collected and there can be no age determination.

There is no reason, based on this sample, to differentiate the variability represented by the five clusters of Angostura by five type or variant names, and so these specimens are considered to represent a single, variable type, Angostura. The Angostura type name is being retained because of its pervasive presence in practice and in the literature and, 
TABLE 14-10

Analysis of Variance (ANOVA) Comparing Clusters

1, 2, 3, 4, and 5 for Angostura Type (Lubbock Excluded)

\begin{tabular}{|c|c|c|c|c|}
\hline \multicolumn{5}{|c|}{ a. Proximal Haft Width } \\
\hline \multirow[b]{2}{*}{ Source } & \multirow{2}{*}{$\begin{array}{r}\text { Sum of } \\
\text { Squares }\end{array}$} & \multicolumn{3}{|c|}{ Mean } \\
\hline & & d.f. & Square & F-ratic \\
\hline Treatment & 344.55 & 4 & 86.14 & $118.00^{*}$ \\
\hline Error & 29.17 & 40 & 0.73 & \\
\hline Total & 373.72 & 44 & & \\
\hline \multicolumn{5}{|c|}{ b. 10-mm Haft Width } \\
\hline & Sum of & & Mean & \\
\hline Source & Squares & d.f. & Square & F-ratio \\
\hline Treatment & 30.32 & 4 & 7.58 & $5.57 *$ \\
\hline Error & 54.52 & 40 & 1.36 & \\
\hline Total & 84.84 & 44 & & \\
\hline \multicolumn{5}{|c|}{ c. Haft Shape Ratio } \\
\hline & Sum of & & Mean & \\
\hline Source & Squares & d.f. & Square & F-ratio \\
\hline Treatment & 4.419 & 4 & 1.10475 & $73.50 *$ \\
\hline Error & 0.601 & 40 & 0.01503 & \\
\hline Total & 5.02 & 44 & & \\
\hline
\end{tabular}

d. 10-mm Haft Thickness

\begin{tabular}{lrrrr}
\hline Source & $\begin{array}{c}\text { Sum of } \\
\text { Squares }\end{array}$ & d.f. & $\begin{array}{r}\text { Mean } \\
\text { Square }\end{array}$ & F-ratio \\
\hline Treatment & 4.63 & 4 & 1.16 & $4.00^{*}$ \\
Error & 11.62 & 40 & 0.29 & \\
\hline Total & 16.25 & 44 & & \\
\hline
\end{tabular}

e. Haft Thickness/Width Ratio

\begin{tabular}{lrrrr}
\hline Source & $\begin{array}{c}\text { Sum of } \\
\text { Squares }\end{array}$ & d.f. & $\begin{array}{r}\text { Mean } \\
\text { Square }\end{array}$ & F-ratio \\
\hline Treatment & 0.0383 & 4 & 0.0096 & $6.86^{*}$ \\
Error & 0.0575 & 40 & 0.0014 & \\
\hline
\end{tabular}

\begin{tabular}{lcrrr}
\hline Total & 0.0958 & 44 & & \\
\hline f. Basal Concavity & & & & \\
\hline & Sum of & & Mean \\
Source & Squares & d.f. & Square & F-ratio \\
\hline Treatment & 1.743 & 4 & 0.436 & 1.03 \\
Error & 16.970 & 40 & 0.424 & \\
\hline Total & 18.713 & 44 & & \\
\hline g. Basal Concavity/Grinding Length Ratio & & \\
\hline \multicolumn{5}{c}{ Sum of } \\
Source & Squares & d.f. & Square & F-ratio \\
\hline Treatment & 0.00333 & 4 & 0.00083 & 0.53 \\
Error & 0.06334 & 40 & 0.00158 & \\
\hline Total & 0.06667 & 44 & & \\
\hline h. Grinding Length & & & & \\
\hline & Sum of & & Mean & \\
Source & Squares & d.f. & Square & F-ratio \\
\hline Treatment & 338.52 & 4 & 84.63 & 1.07 \\
Error & 3149.03 & 40 & 78.73 & \\
\hline Total & 3487.55 & 44 & &
\end{tabular}

* Significant differences at $\alpha=0.01$

d.f. $=$ degrees of freedom through this analysis, its consistent characteristics of basal morphology supported by temporal placement. This is clearly preferable to reliance on a very specific flake pattern as Wheeler (1995:415-419) emphasized. Significantly, these samples came from a restricted region and this supports the proposition of Angostura representing a valid type in Texas. This analysis should help salvage the Angostura type and it should not be doomed to the scrap heap of typology like Yuma. As a final note, the possibility exists, of course, that future discoveries could produce evidence that some of the variability in this type has greater significance than is perceived at present and that the type will need to be refined.

Clusters 6 and 9 are alike among the contracting haft clusters by having points with very thick hafts, very high haft thickness/width ratio, and very wide $10-\mathrm{mm}$ haft widths. When the means of the two clusters are compared, significant differences are still apparent in proximal haft width, haft shape ratio, and 10-mm haft thickness (Table 14-12). Although the haft thickness remains significantly different for Clusters 6 and 9, when contrasted with the other contracting haft clusters, these two clusters are more like each other and very different from the others (see Table 143).

The Wilson-Leonard specimens give the best available chronological information. The majority of the specimens from the Wilson-Leonard site occur in the Valley Margin in Early Archaic contexts, along with Angostura and Hoxie variants. Stratigraphically, specimens from Clusters 6 and 9 show up slightly later than Angostura. In a more refined view, two specimens, Specimens 72 and 75 , are from Unit IIIa in Valley Floor A with an age range between 8700 B.P. and 7000 B.P. The stratigraphic patterning of the two clusters overlap so completely that there is no apparent chronological difference between Clusters 6 and 9 .

Some of the specimens from Clusters 6 and 9 have previously been referred to as "Hell Gap," "Fat Angostura," and "Victoria." And it may have been similar specimens that Krieger (1947b:22) was referring to when he described an asyet-unnamed type with a "fat" stem which had shown up in small numbers in burned rock midden sites in Central Texas. On the basis of morphological distinction and their chronological placement at the Wilson-Leonard site, these very thick, contracting-hafted points are being proposed as a new type, Thrall, named for a small Williamson County community on a tributary of Brushy Creek.

Clusters 7 and 8 are similar to each other in $10-\mathrm{mm}$ haft thickness (very narrow) and the haft thickness/width ratio (very low), while remaining significantly different in haft width and shape (Table 14-13). The type name previously applied to specimens of Cluster 7 is Midland, while specimens in Cluster 8 were typed Midland and Milnesand. It is apparent that the diminutive specimens from the original Milnesand type collection share morphological characteristics, such as similarities in haft width and haft shape, with specimens of the Midland type, more so than with other morphological 
TABLE 14-11

Means and Sample Standard Diviations for Two Sets of Contracting Haft Clusters

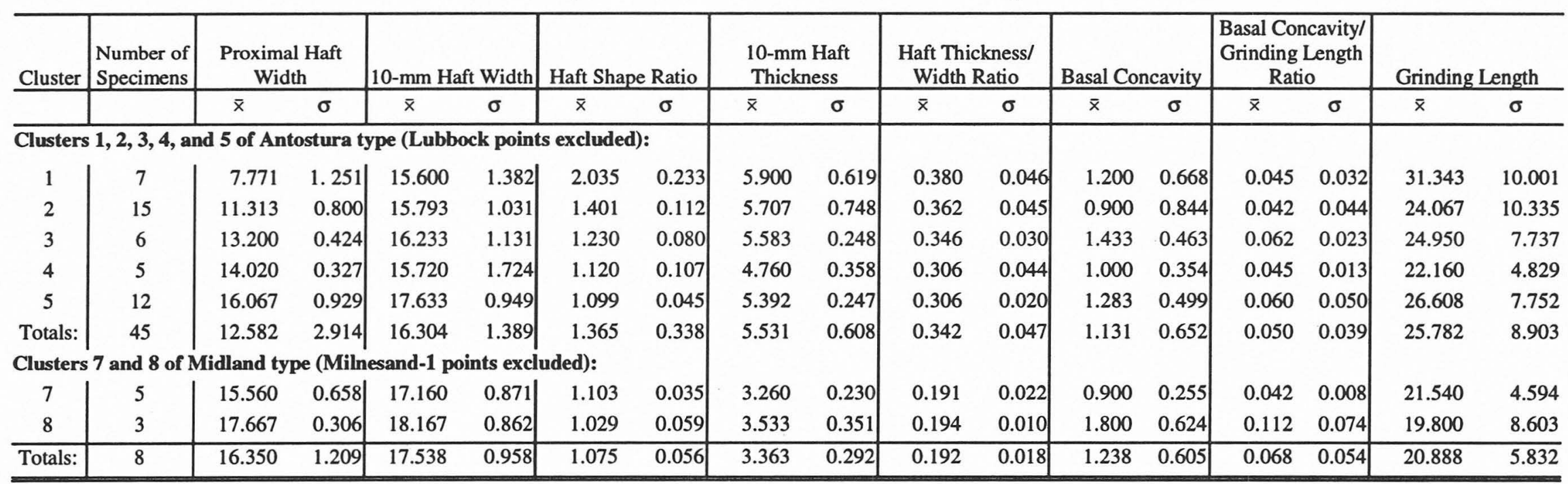


TABLE 14-12

Analysis of Variance (ANOVA) Comparing

Clusters 6 and 9 for Thrall Type

a. Proximal Haft Width

\begin{tabular}{lrrrr}
\hline Source & Sum of & & Mean & \\
Treatment & Squares & d.f. & Square & F-ratio \\
Error & 31.33 & 1 & 31.33 & $43.51^{*}$ \\
\hline Total & 9.35 & 13 & 0.72 & \\
\hline
\end{tabular}

\begin{tabular}{lll}
\hline b. 10-mm Haft Width & \\
\hline Sum of & Mean
\end{tabular}

\begin{tabular}{lrrrr} 
Source & Squares & d.f. & Square & F-ratio \\
\hline Treatment & 5.09 & 1 & 5.09 & 7.71 \\
Error & 8.61 & 13 & 0.66 & \\
\hline Total & 13.7 & 14 & & \\
\hline c. Haft Shape Ratio & & & &
\end{tabular}

c. Haft Shape Ratio

\begin{tabular}{lrrrr}
\hline Source & $\begin{array}{r}\text { Sum of } \\
\text { Squares }\end{array}$ & d.f. & $\begin{array}{r}\text { Mean } \\
\text { Square }\end{array}$ & F-ratio \\
\hline Treatment & 0.065 & 1 & 0.06500 & $36.72^{*}$ \\
Error & 0.023 & 13 & 0.00177 & \\
\hline Total & 0.088 & 14 & & \\
\hline
\end{tabular}

d. 10-mm Haft Thickness

\begin{tabular}{|c|c|c|c|c|}
\hline \multirow[b]{2}{*}{ Source } & \multirow{2}{*}{$\begin{array}{l}\text { Sum of } \\
\text { Squares }\end{array}$} & \multicolumn{3}{|c|}{ Mean } \\
\hline & & d.f. & Square & F-ratio \\
\hline Treatment & 4.63 & 1 & 4.63 & $15.97 *$ \\
\hline Error & 3.71 & 13 & 0.29 & \\
\hline Total & 8.34 & 14 & & \\
\hline \multicolumn{5}{|c|}{ e. Haft Thickness/Width Ratio } \\
\hline \multirow[b]{2}{*}{ Source } & \multirow{2}{*}{$\begin{array}{c}\text { Sum of } \\
\text { Squares }\end{array}$} & \multicolumn{3}{|c|}{ Mean } \\
\hline & & d.f. & Square & F-ratio \\
\hline Treatment & 0.0042 & 1 & 0.0042 & 3.23 \\
\hline Error & 0.0164 & 13 & 0.0013 & \\
\hline Total & 0.0206 & 14 & & \\
\hline \multicolumn{5}{|c|}{ f. Basal Concavity } \\
\hline \multirow[b]{2}{*}{ Source } & \multirow{2}{*}{$\begin{array}{l}\text { Sum of } \\
\text { Squares }\end{array}$} & \multicolumn{3}{|c|}{ Mean } \\
\hline & & d.f. & Square & F-ratio \\
\hline Treatment & 0.002 & 1 & 0.002 & 0.004 \\
\hline Error & 7.015 & 13 & 0.540 & \\
\hline Total & 7.017 & 14 & & \\
\hline \multicolumn{5}{|c|}{ g. Basal Concavity/Grinding Length Ratio } \\
\hline \multirow[b]{2}{*}{ Source } & \multirow{2}{*}{$\begin{array}{r}\text { Sum of } \\
\text { Squares } \\
\end{array}$} & \multicolumn{3}{|c|}{ Mean } \\
\hline & & d.f. & Square & F-ratio \\
\hline Treatment & 0.00043 & 1 & 0.00043 & 0.61 \\
\hline Error & 0.00922 & 13 & 0.00071 & \\
\hline Total & 0.00965 & 14 & & \\
\hline \multicolumn{5}{|c|}{ h. Grinding Length } \\
\hline \multirow[b]{2}{*}{ Source } & \multirow{2}{*}{$\begin{array}{r}\text { Sum of } \\
\text { Squares }\end{array}$} & \multicolumn{3}{|c|}{ Mean } \\
\hline & & d.f. & Square & F-ratio \\
\hline Treatment & 221.56 & 1 & 221.56 & 2.69 \\
\hline Error & 1071.17 & 13 & 82.40 & \\
\hline Total & 1292.73 & 14 & & \\
\hline \multicolumn{4}{|c|}{ * Significant differences at $\alpha=0.01$} & \\
\hline
\end{tabular}

clusters. However, the Milnesand specimens tend to have slightly thicker hafts than the Midland specimens, and the flake patterns of the two types appear different. These details suggest differences that should probably continue to be recognized at a typological level. Since the original Milnesand type specimens divide into at least two morphological types, the specimens morphologically similar to Midland are referred to as Milnesand-1 for the purpose of discussion here.

When the Milnesand- 1 specimens are excluded and only the Midland specimens are examined through ANOVA, the only significant difference existing between the two clusters is in proximal haft width (see Tables 14-11 and 14-13). Otherwise, these two clusters are morphologically the same, and therefore, are indicative of a morphological type.

There is only one Midland specimen in this sample from a dated context, Specimen 64 (see Table 14-1) from the Unit Id/II transition at the Wilson-Leonard site that dates about 9500 B.P. But this context is not considered reliable for this particular specimen. The specimens from the Winkler-1 and Milnesand sites do not have radiocarbon dates associated with them, nor do they have useful chronological information from their site context. Chronological estimate for Midland, which is thought to be contemporary with Folsom, is 11,000 to 10,000 B.P. and for Milnesand is 10,000 to 9000 B.P. Unfortunately, the absolute chronological placement of both types remains exceedingly weak.

From this analysis, Midland can be recognized as a morphological type, with a slight contracting haft shape, moderate haft width, and a very thin haft thickness. Without better temporal and contextual information, Midland cannot be considered a chronological type. Milnesand-1 can also be considered a morphological type, with a very slight contracting haft shape and with a haft thickness and haft thickness/width ratio more akin to Cluster 12 of the parallel-sided haft, shallow-to-moderate basal concavity clusters.

In summary, three types with good chronological data and two morphological types have been derived from the contracting haft clusters. The three chronological types are Angostura, Thrall, and Lubbock. The two morphological types are Midland and Milnesand-1. There are significant differences between the means of the five types (Tables 1414 and 14-15), and the pattern of significance parallels that of contracting haft clusters, with the same five variables remaining significant and the same three remaining insignificant. It is important to note, however, that the variance between means (Treatment Sum of Squares) relative to inherent variation (Error Sum of Squares) remains highest in 10-mm haft thickness and haft thickness/width ratio (see Table 1414) for the types, while proximal haft width, 10-mm haft width, and haft shape ratio (see Table 14-13) show considerable inherent variability while still remaining significant.

The Angostura type has considerable variation as seen in the spread across five clusters. The variation does not appear to have any temporal importance that can be determined 
TABLE 14-13

Analysis of Variance (ANOVA) Comparing Clusters

7 and 8 for Midland Type (Milnesand-1 Excluded)

a. Proximal Haft Width

\begin{tabular}{|c|c|c|c|c|}
\hline \multirow[b]{2}{*}{ Source } & \multirow{2}{*}{$\begin{array}{l}\text { Sum of } \\
\text { Squares }\end{array}$} & \multicolumn{3}{|c|}{ Mean } \\
\hline & & d.f. & Square & F-ratio \\
\hline Treatment & 8.32 & 1 & 8.32 & $26.00^{*}$ \\
\hline Error & 1.92 & 6 & 0.32 & \\
\hline Total & 10.24 & 7 & & \\
\hline \multicolumn{5}{|c|}{ b. 10-mm Haft Width } \\
\hline & Sum of & & Mean & \\
\hline Source & Squares & d.f. & Square & F-ratio \\
\hline Treatment & 1.90 & 1 & 1.90 & 2.53 \\
\hline Error & 4.52 & 6 & 0.75 & \\
\hline Total & 6.42 & 7 & & \\
\hline \multicolumn{5}{|c|}{ c. Haft Shape Ratio } \\
\hline & Sum of & & Mean & \\
\hline Source & Squares & d.f. & Square & F-ratio \\
\hline Treatment & 0.010 & 1 & 0.010 & 5.00 \\
\hline Error & 0.012 & 6 & 0.002 & \\
\hline Total & 0.022 & 7 & & \\
\hline \multicolumn{5}{|c|}{ d. 10-mm Haft Thickness } \\
\hline & Sum of & & Mean & \\
\hline Source & Squares & d.f. & Square & F-ratio \\
\hline Treatment & 0.14 & 1 & 0.14 & 1.75 \\
\hline Error & 0.46 & 6 & 0.08 & \\
\hline Total & 0.60 & 7 & & \\
\hline \multicolumn{5}{|c|}{ e. Haft Thickness/Width Ratio } \\
\hline & Sum of & & Mean & \\
\hline Source & Squares & d.f. & Square & F-ratio \\
\hline Treatment & 0.0000 & 1 & 0.0000 & 0.00 \\
\hline Error & 0.0021 & 6 & 0.0004 & \\
\hline Total & 0.0588 & 7 & & \\
\hline \multicolumn{5}{|c|}{ f. Basal Concavity } \\
\hline & Sum of & & Mean & \\
\hline Source & Squares & d.f. & Square & F-ratio \\
\hline Treatment & 1.519 & 1 & 1.519 & 8.78 \\
\hline Error & 1.040 & 6 & 0.173 & \\
\hline Total & 2.559 & 7 & & \\
\hline
\end{tabular}

g. Basal Concavity/Grinding Length Ratio

\begin{tabular}{lrrrr}
\hline Source & $\begin{array}{r}\text { Sum of } \\
\text { Squares }\end{array}$ & d.f. & $\begin{array}{r}\text { Mean } \\
\text { Square }\end{array}$ & F-ratio \\
\hline Treatment & 0.00919 & 1 & 0.00919 & 4.97 \\
Error & 0.01108 & 6 & 0.00185 & \\
\hline Total & 0.02027 & 7 & & \\
\hline h. Grinding Length & & & & \\
\hline & Sum of & & Mean & \\
Source & Squares & d.f. & Square & F-ratio \\
\hline Treatment & 5.68 & 1 & 5.68 & 0.15 \\
Error & 232.43 & 6 & 38.74 & \\
\hline Total & 238.11 & 7 & & \\
\hline
\end{tabular}

* Significant differences at $\alpha=0.01$

d.f. $=$ degrees of freedom
TABLE 14-14

Analysis of Variance (ANOVA) Comparing Five Contracting Haft Point Types

a. Proximal Haft Width

\begin{tabular}{|c|c|c|c|c|}
\hline \multirow[b]{2}{*}{ Source } & \multirow{2}{*}{$\begin{array}{l}\text { Sum of } \\
\text { Squares }\end{array}$} & \multicolumn{3}{|c|}{ Mean } \\
\hline & & d.f. & Square & F-ratio \\
\hline Treatment & 264.40 & 4 & 66.10 & $10.73 *$ \\
\hline Error & 431.40 & 70 & 6.16 & \\
\hline Total & 695.80 & 74 & & \\
\hline \multicolumn{5}{|c|}{ b. 10-mm Haft Width } \\
\hline & Sum of & & Mean & \\
\hline Source & Squares & d.f. & Square & F-ratio \\
\hline Treatment & 99.42 & 4 & 24.86 & $14.54^{*}$ \\
\hline Error & 119.47 & 70 & 1.71 & \\
\hline Total & 218.89 & 74 & & \\
\hline \multicolumn{5}{|c|}{ c. Haft Shape Ratio } \\
\hline & Sum of & & Mean & \\
\hline Source & Squares & d.f. & Square & F-ratio \\
\hline Treatment & 1.07 & 4 & 0.27 & $3.86^{*}$ \\
\hline Error & 5.16 & 70 & 0.07 & \\
\hline Total & 6.23 & 74 & & \\
\hline
\end{tabular}

d. 10-mm Haft Thickness

\begin{tabular}{|c|c|c|c|c|}
\hline \multirow[b]{2}{*}{ Source } & \multirow{2}{*}{$\begin{array}{l}\text { Sum of } \\
\text { Squares }\end{array}$} & \multicolumn{3}{|c|}{ Mean } \\
\hline & & d.f. & Square & F-ratio \\
\hline Treatment & 106.47 & 4 & 26.62 & $71.95^{*}$ \\
\hline Error & 26.09 & 70 & 0.37 & \\
\hline Total & 132.56 & 74 & & \\
\hline \multicolumn{5}{|c|}{ e. Haft Thickness/Width Ratio } \\
\hline & Sum of & & Mean & \\
\hline Source & Squares & d.f. & Square & F-ratio \\
\hline Treatment & 0.26 & 4 & 0.065 & $32.50 *$ \\
\hline Error & 0.13 & 70 & 0.002 & \\
\hline Total & 0.39 & 74 & & \\
\hline \multicolumn{5}{|c|}{ f. Basal Concavity } \\
\hline & Sum of & & Mean & \\
\hline Source & Squares & d.f. & Square & F-ratio \\
\hline Treatment & 2.67 & 4 & 0.67 & 1.52 \\
\hline Error & 30.96 & 70 & 0.44 & \\
\hline Total & 33.63 & 74 & & \\
\hline \multicolumn{5}{|c|}{ g. Basal Concavity/Grinding Length Ratio } \\
\hline & Sum of & & Mean & \\
\hline Source & Squares & d.f. & Square & F-ratio \\
\hline Treatment & 0.01 & 4 & 0.003 & 3.00 \\
\hline Error & 0.10 & 70 & 0.001 & \\
\hline Total & 0.11 & 74 & & \\
\hline \multicolumn{5}{|c|}{ h. Grinding Length } \\
\hline & Sum of & & Mean & \\
\hline Source & Squares & d.f. & Square & F-ratio \\
\hline Treatment & 542.95 & 4 & 135.74 & 1.87 \\
\hline Error & 5082.31 & 70 & 72.60 & \\
\hline Total & 5625.26 & 74 & & \\
\hline
\end{tabular}

* Significant differences at $\alpha=0.01$

d.f. $=$ degrees of freedom 
TABLE 14-15

Means and Sample Standard Deviations for Unfluted Lanceolate Types

\begin{tabular}{|c|c|c|c|c|c|c|c|c|c|c|c|c|c|c|c|c|c|}
\hline \multirow[b]{2}{*}{ Type } & \multirow{2}{*}{$\begin{array}{l}\text { Number of } \\
\text { Specimens }\end{array}$} & \multicolumn{2}{|c|}{$\begin{array}{c}\text { Proximal } \\
\text { Haft Width }\end{array}$} & \multicolumn{2}{|c|}{$\begin{array}{c}10-\mathrm{mm} \\
\text { Haft Width }\end{array}$} & \multicolumn{2}{|c|}{$\begin{array}{c}\text { Haft Shape } \\
\text { Radio }\end{array}$} & \multicolumn{2}{|c|}{$\begin{array}{c}10-\mathrm{mm} \\
\text { Haft Thickness }\end{array}$} & \multicolumn{2}{|c|}{$\begin{array}{l}\text { Haft Thickness/ } \\
\text { Width Ratio }\end{array}$} & \multicolumn{2}{|c|}{$\begin{array}{c}\text { Basal } \\
\text { Concavity }\end{array}$} & \multicolumn{2}{|c|}{$\begin{array}{l}\text { Basal Concavity/ } \\
\text { Grinding Length }\end{array}$} & \multicolumn{2}{|c|}{$\begin{array}{l}\text { Grinding } \\
\text { Length }\end{array}$} \\
\hline & & $\bar{x}$ & $\sigma$ & $\bar{x}$ & $\sigma$ & $\bar{x}$ & $\sigma$ & $\bar{x}$ & $\sigma$ & $\bar{x}$ & $\sigma$ & $\bar{x}$ & $\sigma$ & $\bar{x}$ & $\sigma$ & $\bar{x}$ & $\sigma$ \\
\hline \multicolumn{18}{|c|}{ Contracting Haft Types } \\
\hline Angostura & 45 & 12.582 & 2.914 & 16.304 & 1.389 & 1.365 & 0.338 & 5.531 & 0.608 & 0.342 & 0.047 & 1.131 & 0.652 & 0.050 & 0.039 & 25.782 & 8.903 \\
\hline Thrall & 15 & 16.413 & 1.705 & 19.187 & 0.989 & 1.176 & 0.079 & 7.587 & 0.772 & 0.396 & 0.038 & 1.153 & 0.708 & 0.044 & 0.026 & 29.540 & 9.609 \\
\hline Lubbock & 4 & 15.250 & 1.446 & 17.400 & 1.573 & 1.142 & 0.054 & 5.200 & 0.424 & 0.301 & 0.039 & 0.400 & 0.800 & 0.016 & 0.032 & 29.275 & 4.010 \\
\hline Midland & 8 & 16.350 & 1.209 & 17.538 & 0.958 & 1.075 & 0.056 & 3.363 & 0.292 & 0.192 & 0.018 & 1.238 & 0.605 & 0.068 & 0.054 & 20.888 & 5.832 \\
\hline Milnesand-1 & 3 & 17.500 & 0.529 & 18.233 & 1.882 & 1.041 & 0.091 & 4.133 & 0.416 & 0.229 & 0.038 & 0.700 & 0.608 & 0.035 & 0.032 & 20.200 & 2.800 \\
\hline Totals: & 75 & 14.089 & 3.066 & 17.148 & 1.720 & 1.271 & 0.290 & 5.637 & 1.338 & 0.330 & 0.072 & 1.091 & 0.674 & 0.048 & 0.039 & 25.975 & 8.719 \\
\hline \multicolumn{18}{|c|}{ Parallel-Sided Haft, Shallow-to-Moderate Basal Concavity Types } \\
\hline St. Mary's Hall & 17 & 19.582 & 1.144 & 20.653 & 1.274 & 1.055 & 0.049 & 5.729 & 0.665 & 0.277 & 0.027 & 2.571 & 0.726 & 0.115 & 0.038 & 23.124 & 5.909 \\
\hline Plainview & 32 & 21.409 & 1.481 & 21.769 & 1.810 & 1.017 & 0.048 & 4.344 & 0.470 & 0.201 & 0.028 & 1.500 & 0.746 & 0.077 & 0.136 & 29.847 & 9.092 \\
\hline Parallel-Sided Haft & 4 & 24.100 & 0.583 & 25.200 & 0.980 & 1.046 & 0.045 & 5.275 & 0.538 & 0.210 & 0.022 & 1.100 & 0.825 & 0.055 & 0.052 & 24.275 & 7.785 \\
\hline Scottsbluff & 3 & 26.567 & 1.069 & 25.633 & 1.361 & 0.966 & 0.053 & 6.133 & 0.808 & 0.240 & 0.038 & 0.367 & 0.635 & 0.021 & 0.037 & 14.767 & 2.335 \\
\hline Totals: & 56 & 21.323 & 2.163 & 21.882 & 2.117 & 1.028 & 0.053 & 4.927 & 0.882 & 0.227 & 0.044 & 1.736 & 0.948 & 0.084 & 0.108 & 26.600 & 8.840 \\
\hline \multicolumn{18}{|c|}{ Parallel-Sided Haft, Deep Basal Concavity Types } \\
\hline St. Mary's Hall-atypic & 6 & 22.733 & 1.199 & 22.683 & 1.899 & 0.997 & 0.034 & 5.783 & 0.560 & 0.256 & 0.019 & 4.000 & 0.727 & 0.181 & 0.044 & 23.250 & 7.092 \\
\hline Golondrina-Barber & 27 & 25.422 & 2.955 & 25.937 & 2.777 & 1.023 & 0.065 & 5.393 & 0.733 & 0.210 & 0.033 & 6.030 & 1.437 & 0.258 & 0.072 & 24.474 & 6.958 \\
\hline Dalton & 14 & 23.843 & 1.685 & 21.857 & 1.570 & 0.918 & 0.046 & 5.614 & 0.643 & 0.258 & 0.031 & 7.700 & 1.327 & 0.445 & 0.083 & 17.536 & 2.763 \\
\hline Totals: & 47 & 24.609 & 2.631 & 24.306 & 3.029 & 0.988 & 0.073 & 5.509 & 0.690 & 0.230 & 0.038 & 6.268 & 1.746 & 0.304 & 0.120 & 22.251 & 6.692 \\
\hline
\end{tabular}


by this sample. The wide range of variability within this type, then, is part of the overall characteristic of the type.

\section{Parallel-sided Haft Clusters (Specimens 76-182)}

The parallel-sided haft clusters further subdivide into shallow-to-moderate basal concavity and deep basal concavity clusters (see Figure 14-2).

\section{SHALLOW-TO-MODERATE BASAL CONCAVITY CLUSTERS (SPECIMENS 76-131)}

Cluster 10 (Specimens 76-79, 81-83)

Cluster 10 has, of the shallow-to-moderate basal concavity clusters, points with moderately wide, very thick hafts, moderately deep basal concavities, and short grinding lengths (Figure 14-4a). In addition, they have very thick hafts relative to moderate haft widths. Seven of eight specimens are correctly classified according to GCP (see Table 14-6), with probabilities from 0.64 to 0.99 . Specimen 80 is reclassified to Cluster 13 with $p=0.52$ and is not included in the description of Cluster 10. Of the remaining seven specimens, three show probabilities greater than 0.05 for Cluster 11, three specimens show probabilities greater than or equal to 0.05 or greater for Cluster 13, and one specimen shows $p=0.33$ for Cluster 16. Associations are suggested between Cluster 10 and Clusters 11 and 13 and a slight affinity between Cluster 10 and Cluster 16.

\section{Cluster 11 (Specimens 84-93)}

Of the shallow-to-moderate basal concavity clusters, Cluster 11 points have narrow, thick hafts, deep basal concavities, and moderately long grinding lengths as well as thick hafts relative to narrow haft widths (see Figure 14-4b). Compared to Cluster 12, Cluster 11 points have slightly narrower proximal haft widths, slightly wider $10-\mathrm{mm}$ haft widths, much thicker 10-mm haft thicknesses, much higher haft thickness/width ratios, deeper basal concavities, and slightly shorter grinding lengths (see Table 14-3). All 10 specimens are correctly classified according to GCP (see Table 14-6), with probabilities from 0.77 to 0.98 . Five specimens show probabilities greater than or equal to 0.05 for Cluster 10, and 3 specimens show probabilities greater than 0.05 for Cluster 12. Cluster 11 points have the strongest resemblance to those of Cluster 10.

\section{Cluster 12 (Specimens 94-100, 106)}

Cluster 12 points have, of the shallow-to-moderate basal concavity clusters, narrow, very thin hafts, moderately shallow basal concavities, and moderately long grinding lengths (see Figure 14-4c). They have very thin hafts relative to narrow haft widths. Seven specimens are correctly classi- fied according to GCP (see Table 14-6), with probabilities from 0.46 to 1.00 . Specimen 106 , with $p=0.53$, was received from Cluster 13 bringing the count to eight for Cluster 12. Six specimens show probabilities greater than 0.05 for Cluster 13 , five specimens show probabilities greater than or equal to 0.05 for Cluster 11, and five specimens show probabilities greater than 0.05 for Cluster 8 , suggesting strong relationships between these four clusters.

Cluster 13 (Specimens 80, 101-105, 107-119)

Of the shallow-to-moderate basal concavity clusters, Cluster 13 consists of points with moderately wide, thin hafts, moderately shallow basal concavities, and long grinding lengths along with thinner hafts relative to moderately wide haft widths (see Figure 14-4d). According to the GCP (see Table 14-6), 17 of 19 specimens are correctly classified, with probabilities ranging from 0.80 to 0.98 . Specimen 106, with $p=0.53$, was reclassified to Cluster 12 and is not included in the description of Cluster 13. Specimen 108 also has an inaccurate classification, with $p=0.48$ for Cluster 13 and $p=0.52$ for Cluster 14. After consideration of the raw data and the near equal probabilities between Clusters 13 and 14, this specimen has been left in Cluster 13 where it is at the wide end of the range for haft width but well within range for the other variables. Cluster 13 also received the reclassified Specimen 80 from Cluster 10, with $p=0.52$, and it is included in the description of Cluster 13. Five specimens show probabilities greater than or equal to 0.05 for Cluster 14, three specimens show probabilities greater than 0.05 for Cluster 10 , two specimens show $p=0.06$ for Cluster 12 , and one specimen shows $p=0.14$ for Cluster 11. Close similarities are suggested between Cluster 13 and Clusters 14, 10, and 12.

\section{Cluster 14 (Specimens 120-128)}

Of the shallow-to-moderate basal concavity clusters, Cluster 14 has points with wide, thin hafts, moderately shallow basal concavities, and very long grinding lengths (see Figure 14-4e). They have very thin hafts compared to wide haft widths. All nine specimens are correctly classified according to GCP (see Table 14-6) with probabilities from 0.70 to 0.99 . Seven specimens show probabilities greater than or equal to 0.05 for Cluster 13, indicating a strong relationship.

Cluster 15 (Specimens 129-131)

Of the shallow-to-moderate basal concavity clusters, Cluster 15 consists of specimens with very wide and very thick hafts, very shallow basal concavities, and very short grinding lengths. They also have very thick hafts relative to wide hafts (see Table 14-3 and Figure 14-4f). All three specimens are correctly classified according to GCP (see Table 14-6) with probabilities from 0.99 to 1.00 . No clear relationships are indicated with other clusters. 


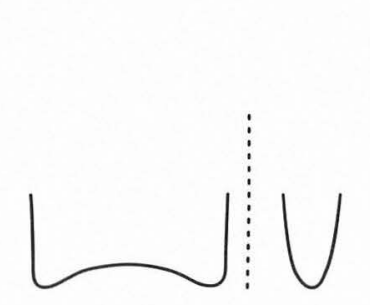

a

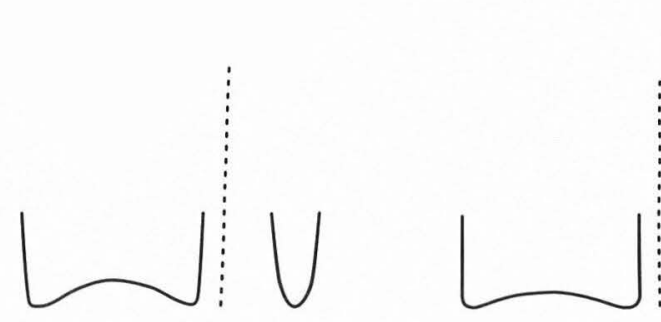

b

c
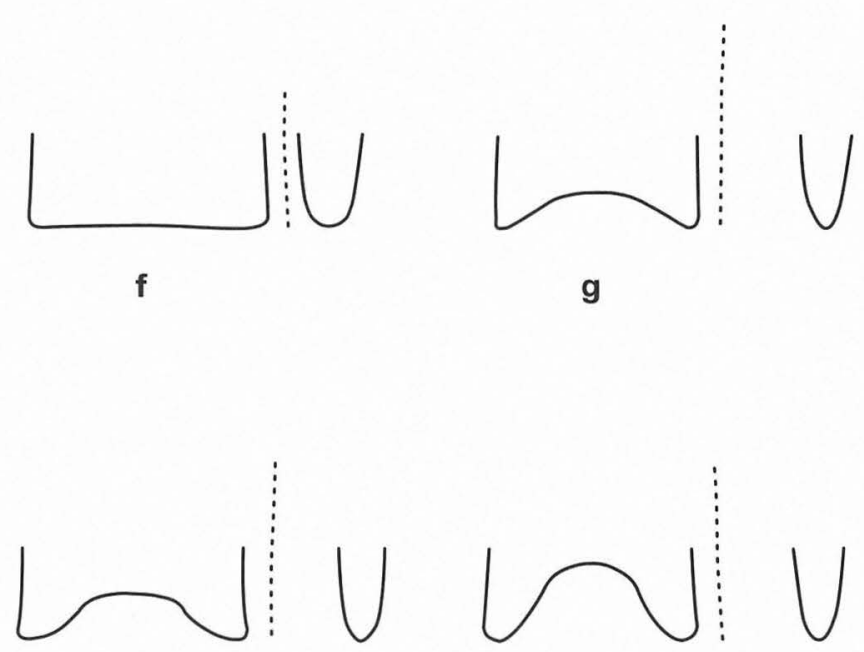

i

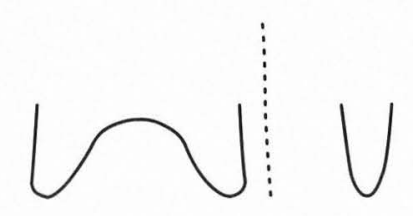

j

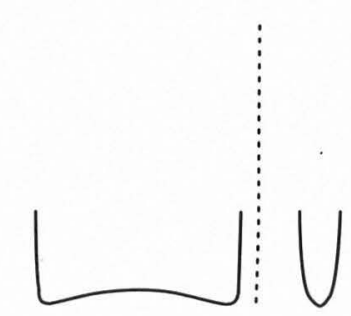

d

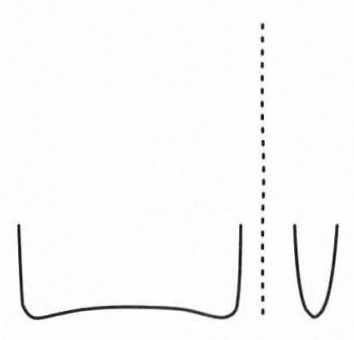

e

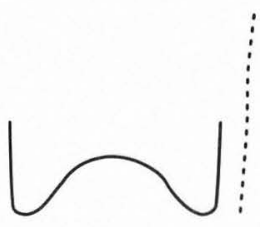

h

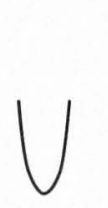

$\longrightarrow$

k

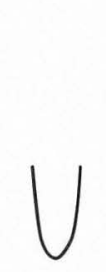

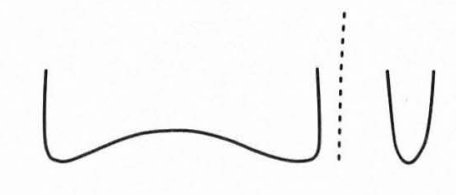

I

FIGURE 14-4. Mean shapes and extent of grinding (dotted line) for parallel-sided haft clusters with shallow-to-moderate basal concavities and parallel-sided haft clusters with deep basal concavaties. (a) Cluster 10; (b) Cluster 11; (c) Cluster 12; (d) Cluster 13; (e) Cluster 14; (f) Cluster 15; (g) Cluster 16; (h) Cluster 17; (i) Cluster 18; (j) Cluster 19; (k) Cluster 20; (1) Cluster 21.

\section{DISCUSSION OF SHALLOW-TO-MODERATE BASAL CONCAVITYCLUSTERS}

Clusters 10 through 15 represent the morphological variability of parallel-sided haft, shallow-to-moderate basal concavity points. The dendrogram (see Figure 14-2) shows that these six clusters separate from the remainder of the sample. In general, all six clusters have a parallel-sided haft shape and a shallow-to-moderate basal concavity (see Figure 14-4a-f). Any variability in the parallel-sided shape is not significant (see Table 14-8c). The significant differences occur in haft width, 10-mm haft thickness, haft thickness/width ratio, basal concavity, and grinding length (see Table 14-8). And it is these differences that suggest the relationship to types.

Following the same process as was done with the contracting haft clusters, these clusters were examined for appropriate typological assignment. Analysis of variance shows that significant differences exist between cluster means, but there are also patterns of morphological similarity between pairs or groups of clusters. Among the parallelsided haft, shallow-to-moderate basal concavity clusters, important similarities occur between Clusters 10 and 11 and between Clusters 12, 13, and 14, while Cluster 15 is unique within the general pattern. 
Cluster 12, 13, and 14 points share similarities in 10-mm haft thickness (thin), haft thickness/width ratio (low), and basal concavity (moderately shallow) but are still different in haft width (see Table 14-3). The type names previously applied to specimens of Clusters 12,13, and 14 are predominately Plainview and some Milnesand (referred to as Milnesand-2 in this discussion). The Milnesand-2 specimens are of similar size and shape to the Plainview specimens with which they cluster. The characteristics of flaking of the Milnesand-2 specimens, if not the exact patterns, are extremely similar to Plainview. Because of these similarities, it may not be worthwhile to continue recognition of Milnesand-2 specimens typologically, with the possible exception that good temporal information is lacking for Milnesand and the temporal significance cannot be assessed. However, the same can be said for Plainview.

Four specimens, all from the Wilson-Leonard site, in Cluster 14 (Specimens 122 and 126-128; see Table 14-1) appear to have a different flaking pattern and slightly wider and thicker hafts than the Plainview specimens with which they have been clustered. These four specimens represent an unnamed parallel-sided haft morphological type that needs further investigation with a larger sample, and therefore, they are excluded from the remaining discussion of the three clusters.

When Clusters 12, 13, and 14 are compared through analysis of variance, the only significant difference is in the haft width variables (Table 14-16). The three clusters are virtually the same in 10-mm haft thickness, haft thickness/ width ratio, and basal concavity (Table 14-17). These three clusters represent the morphological variability of the Plainview type. Cluster 13 is considered the most representative of the three and can be considered the "classic" Plainview. Clusters 12 and 14 indicate the narrower and wider range for Plainview and are somewhat atypical of the type.

Of importance for the Wilson-Leonard site, is the Bone Bed point (Specimen 98; see Table 14-1) which had previously been referred to as Midland, but this analysis indicates that it shares more similarities with Plainview. The GCP (see Table 14-6) show $p=0.68$ for Cluster 12 and $p=0.25$ for Cluster 13 , both predominately Plainview clusters, while showing only $p=0.04$ for Cluster 8 , a mixed MidlandMilnesand-1 cluster. The Bone Bed point has morphological characteristics of Plainview and shares flaking pattern and attributes with Plainview Specimen 101 (see Table 14-1) in Cluster 13, suggesting that the type designation of atypical Plainview may be most appropriate for this specimen.

Chronological information comes from four of the six sites with specimens in these three clusters (see Table 14-1). The Plainview site is dated at about 10,000 B.P., but the radiocarbon ages are on unreliable materials (see Table 14-2). Considering other new radiocarbon assays (Holliday 1990), perhaps an age estimate of younger than 11,000 B.P. is more appropriate. Ryan's site suggests a Plainview age younger than 10,500 B.P. Bonfire Shelter indicates Plainview at possibly
TABLE 14-16

Analysis of Variance (ANOVA) Comparing Clusters 12, 13, and 14 for Plainview type Parallel-Sided Haft Points Excluded)

a. Proximal Haft Width

\begin{tabular}{lrrrr}
\hline & Sum of & & Mean & \\
Source & Squares & d.f. & Square & F-ratio \\
\hline Treatment & 57.10 & 2 & 28.55 & $75.13^{*}$ \\
Error & 10.90 & 29 & 0.38 & \\
\hline Total & 68.00 & 31 & & \\
\hline b. $10-\mathrm{mm}$ Haft Width & & & &
\end{tabular}

b. 10-mm Haft Width

\begin{tabular}{lrrrr}
\hline & $\begin{array}{r}\text { Sum of } \\
\text { Source }\end{array}$ & \multicolumn{3}{c}{ Mean } \\
\hline Treatment & 60.96 & 2 & 30.48 & $21.77^{*}$ \\
Error & 40.63 & 29 & 1.40 & \\
\hline Total & 101.59 & 31 & & \\
\hline
\end{tabular}

c. Haft Shape Ratio

\begin{tabular}{lrrrr}
\hline Source & $\begin{array}{c}\text { Sum of } \\
\text { Squares }\end{array}$ & d.f. & Mean \\
Treatment & 0.008 & 2 & 0.00400 & 1.79 \\
Error & 0.065 & 29 & 0.00224 & \\
\hline Total & 0.073 & 31 & & \\
\hline
\end{tabular}

d. 10-mm Haft Thickness

\begin{tabular}{|c|c|c|c|c|}
\hline \multirow[b]{2}{*}{ Source } & \multirow{2}{*}{$\begin{array}{c}\text { Sum of } \\
\text { Squares }\end{array}$} & \multicolumn{3}{|c|}{ Mean } \\
\hline & & d.f. & Square & F-ratio \\
\hline Treatment & 0.61 & 2 & 0.31 & 1.48 \\
\hline Error & 6.23 & 29 & 0.21 & \\
\hline Total & 6.84 & 31 & & \\
\hline \multicolumn{5}{|c|}{ e. Haft Thickness/Width Ratio } \\
\hline & Sum of & & Mean & \\
\hline Source & Squares & d.f. & Square & F-ratio \\
\hline Treatment & 0.0038 & 2 & 0.0019 & 2.71 \\
\hline Error & 0.0207 & 29 & 0.0007 & \\
\hline Total & 0.0245 & 31 & & \\
\hline \multicolumn{5}{|c|}{ f. Basal Concavity } \\
\hline & Sum of & & Mean & \\
\hline Source & Squares & d.f. & Square & F-ratio \\
\hline Treatment & 1.5000 & 2 & 0.750 & 1.38 \\
\hline Error & 15.7380 & 29 & 0.543 & \\
\hline Total & 17.2380 & 31 & & \\
\hline \multicolumn{5}{|c|}{ g. Basal Concavity/Grinding Length Ratio } \\
\hline & Sum of & & Mean & \\
\hline Source & Squares & d.f. & Square & F-ratio \\
\hline Treatment & 0.06459 & 2 & 0.03230 & 1.85 \\
\hline Error & 0.50512 & 29 & 0.01742 & \\
\hline Total & 0.56971 & 31 & & \\
\hline \multicolumn{5}{|c|}{ h. Grinding Length } \\
\hline & Sum of & & Mean & \\
\hline Source & Squares & d.f. & Square & F-ratio \\
\hline Treatment & 459.70 & 2 & 229.85 & 3.17 \\
\hline Error & 2102.65 & 29 & 72.51 & \\
\hline Total & 2562.35 & 31 & & \\
\hline
\end{tabular}

* Significant differences at $\alpha=0.01$

d.f. $=$ degrees of freedom 
TABLE 14-17

Means and Sample Standard Diviations for Clusters 12, 13, and 14 of Plainview Type (Parallel-sided Haft Points Excluded)

\begin{tabular}{|c|c|c|c|c|c|c|c|c|c|c|c|c|c|c|c|c|c|}
\hline \multirow[t]{2}{*}{ Cluster } & \multirow[t]{2}{*}{$\begin{array}{l}\text { Number of } \\
\text { Specimens }\end{array}$} & \multicolumn{2}{|c|}{$\begin{array}{c}\text { Proximal Haft } \\
\text { Width }\end{array}$} & \multicolumn{2}{|c|}{ 10-mm Haft Width } & \multicolumn{2}{|c|}{ Haft Shape Ratio } & \multicolumn{2}{|c|}{$\begin{array}{l}\text { 10-mm Haft } \\
\text { Thickness }\end{array}$} & \multicolumn{2}{|c|}{$\begin{array}{c}\text { Haft Thickness/ } \\
\text { Width Ratio }\end{array}$} & \multicolumn{2}{|c|}{ Basal Concavity } & \multicolumn{2}{|c|}{\begin{tabular}{|c|} 
Basal Concavity/ \\
Grinding Length \\
Ratio \\
\end{tabular}} & \multicolumn{2}{|c|}{ Grinding Length } \\
\hline & & $\bar{x}$ & $\sigma$ & $\bar{x}$ & $\sigma$ & $\bar{x}$ & $\sigma$ & $\bar{x}$ & $\sigma$ & $\bar{x}$ & $\sigma$ & $\bar{x}$ & $\sigma$ & $\bar{x}$ & $\sigma$ & $\bar{x}$ & $\sigma$ \\
\hline 12 & 8 & 19.438 & 0.393 & 19.488 & 1.071 & 1.003 & 0.066 & 4.163 & 0.437 & 0.214 & 0.025 & 1.438 & 0.765 & 0.153 & 0.265 & 26.063 & 13.115 \\
\hline 13 & 19 & 21.653 & 0.716 & 22.284 & 1.239 & 1.029 & 0.037 & 4.458 & 0.494 & 0.201 & 0.029 & 1.647 & 0.737 & 0.058 & 0.027 & 29.274 & 6.416 \\
\hline 14 & 5 & 23.640 & 0.385 & 23.460 & 1.115 & 0.993 & 0.052 & 4.200 & 0.354 & 0.179 & 0.014 & 1.040 & 0.680 & 0.029 & 0.020 & 38.080 & 6.277 \\
\hline Totals: & 32 & 21.409 & 1.481 & 21.769 & 1.810 & 1.017 & 0.048 & 4.344 & 0.470 & 0.201 & 0.028 & 1.500 & 0.746 & 0.077 & 0.136 & 29.847 & 9.092 \\
\hline
\end{tabular}


about or before 10,100 в.P. The Wilson-Leonard site specimen dates to no younger than 10,800 B.P. and possibly older than 11,170 B.P. In general, these data suggest a time range for Plainview between 11,200 B.P. and 10,100 B.P.

Clusters 10 and 11 points (see Figure 14-4a, b) are similar to each other in 10-mm haft thickness (thick), thickness/ width ratio (high), and basal concavity (moderately deep) but are different in haft width (see Table 14-3). When the means of the two clusters are compared, the only significant differences are in proximal haft width and $10-\mathrm{mm}$ haft width (Table 14-18); otherwise, points in these two clusters are morphologically the same.

Some chronological information comes from all four sites with specimens in Clusters 10 and 11. St. Mary's Hall site, with two specimens in Cluster 11 (Specimens 87 and 92; see Table 14-1), indicates a stratigraphic position below Golondrina, which elsewhere dates about 9000 B.P. (Hester 1977, 1991). The Levi Rockshelter specimen (Specimen 93; see Table 14-1) comes from the lower portion of the zone also containing Angostura which dates between 6800 B.P. and 9300 в.P. The single specimen from Lubbock Lake site (Specimen 88, see Table 14-1) comes from bone concentration 3 in Feature FA6-11 (Johnson and Holliday 1980) and dates between 9990 B.P. and 9330 B.P. (see Table 14-2). The remaining 13 specimens are from Late Paleoindian contexts at the Wilson-Leonard site dating between 9400 B.P. and 8700 B.P. Some of these latter specimens occur slightly above GolondrinaBarber specimens in Unit II. In general, specimens of Clusters 10 and 11 occur between 9990 B.P. and 8700 B.P.

The type names previously assigned to specimens of Clusters 10 and 11 have been Plainview and occasionally Plainview-Angostura. However, with radiocarbon ages younger that 10,000 B.P., suggesting a Late Paleoindian chronological position and morphological characteristics that distinguish these two clusters from Plainview, a new name of St. Mary's Hall, is being proposed for the morphological pattern expressed by Clusters 10 and 11 . St. Mary's Hall was chosen because the thick haft, one of the characteristics which distinguishes these points from Plainview, was first noticed during observations of the collection from that site and because the component at the St. Mary's Hall site, although lacking radiocarbon assays, is stratigraphically better defined than at the Wilson-Leonard site. It is likely that Kelly's (1983b) Miniature Plainviews are St. Mary's Hall type.

Cluster 15 points, with their wide, thick hafts, and very shallow basal concavities, constitute a poorly represented morphological pattern. Two of the three specimens were classified as Scottsbluff (see Table 14-1), and the third specimen, with a broken haft that removed the distinctive shoulder of Scottsbluff, remains unclassified but it could be Scottsbluff. All three specimens are from the Wilson-Leonard site, and two come from Late Paleoindian contexts. Additional specimens are needed to further define and validate this morphological pattern.
TABLE 14-18

Analysis of Variance (ANOVA) Comparing

Clusters 10 and 11 for St. Mary's Hall Type

a. Proximal Haft Width

\begin{tabular}{|c|c|c|c|c|}
\hline \multirow[b]{2}{*}{ Source } & \multirow{2}{*}{$\begin{array}{l}\text { Sum of } \\
\text { Squares }\end{array}$} & \multicolumn{3}{|c|}{ Mean } \\
\hline & & d.f. & Square & F-ratio \\
\hline Treatment & 18.49 & 1 & 18.49 & $115.56^{*}$ \\
\hline Error & 2.46 & 15 & 0.16 & \\
\hline Total & 20.95 & 16 & & \\
\hline \multicolumn{5}{|c|}{ b. 10-mm Haft Width } \\
\hline & Sum of & & Mean & \\
\hline Source & Squares & d.f. & Square & F-ratio \\
\hline Treatment & 13.40 & 1 & 13.40 & $15.95^{*}$ \\
\hline Error & 12.56 & 15 & 0.84 & \\
\hline Total & 25.96 & 16 & & \\
\hline \multicolumn{5}{|c|}{ c. Haft Shape Ratio } \\
\hline & Sum of & & Mean & \\
\hline Source & Squares & d.f. & Square & F-ratio \\
\hline Treatment & 0.002 & 1 & 0.00200 & 0.81 \\
\hline Error & 0.037 & 15 & 0.00247 & \\
\hline Total & 0.039 & 16 & & \\
\hline
\end{tabular}

d. 10-mm Haft Thickness

\begin{tabular}{lrrrr}
\hline Source & $\begin{array}{r}\text { Sum of } \\
\text { Squares }\end{array}$ & d.f. & $\begin{array}{r}\text { Mean } \\
\text { Square }\end{array}$ & F-ratio \\
\hline Treatment & 2.48 & 1 & 2.48 & 8.00 \\
Error & 4.60 & 15 & 0.31 & \\
\hline Total & 7.08 & 16 & & \\
\hline
\end{tabular}

e. Haft Thickness/Width Ratio

\begin{tabular}{lrrrr}
\hline Source & $\begin{array}{c}\text { Sum of } \\
\text { Squares }\end{array}$ & d.f. & $\begin{array}{r}\text { Mean } \\
\text { Square }\end{array}$ & F-ratio \\
\hline Treatment & 0.0007 & 1 & 0.0007 & 1.00 \\
Error & 0.0108 & 15 & 0.0007 & \\
\hline Total & 0.0115 & 16 & & \\
\hline f. Basal Concavity & & & & \\
\hline & Sum of & & Mean & \\
Source & Squares & d.f. & Square & F-ratio \\
\hline Treatment & 0.194 & 1 & 0.194 & 0.35 \\
Error & 8.241 & 15 & 0.549 & \\
\hline Total & 8.435 & 16 & & \\
\hline
\end{tabular}

g. Basal Concavity/Grinding Length Ratio

\begin{tabular}{lrrrr}
\hline Source & $\begin{array}{c}\text { Sum of } \\
\text { Squares }\end{array}$ & d.f. & $\begin{array}{r}\text { Mean } \\
\text { Square }\end{array}$ & F-ratio \\
\hline Treatment & 0.00218 & 1 & 0.00218 & 1.54 \\
Error & 0.02129 & 15 & 0.00142 & \\
\hline Total & 0.02347 & 16 & & \\
\hline h. Grinding Length & & & & \\
\hline & Sum of & & Mean & \\
Source & Squares & d.f. & Square & F-ratio \\
\hline Treatment & 161.21 & 1 & 161.21 & 6.08 \\
Error & 397.52 & 15 & 26.50 & \\
\hline Total & 558.73 & 16 & & \\
\hline
\end{tabular}

* Significant differences at $\alpha=0.01$

d.f. $=$ degrees of freedom 
In summary, three named types and one unnamed morphological type are recognized in the parallel-sided haft, shallow-to-moderate basal concavity clusters. When grouped by type, there are significant differences in haft width, haft shape, haft thickness, haft thickness/width ratio, basal concavity, and grinding length (Table 14-19; see Table 14-15). Scottsbluff points in Cluster 15 and the unnamed parallel-sided haft points from Wilson-Leonard in Cluster 14 are not well represented in the sample and need further study.

The two named types, Plainview and St. Mary's Hall, had previously been grouped under Plainview. Plainview and St. Mary's Hall differ in haft width, 10-mm haft thickness, haft thickness/width ratio, basal concavity, basal concavity/grinding length ratio, and grinding length (see Table 14-15). Based on the data analyzed here, there appear to be two distinguishable morphological groups with Plainview points having wider, thinner stems with shallower basal concavities than St. Mary's Hall points. Temporally, these two types seem to represent earlier (Plainview) versus later (St. Mary's Hall) Paleoindian components. It is for these reasons that it seems justified to advocate a separation of these two morphological patterns under two type names in order to distinguish and study the cultural patterns that Plainview and St. Mary's Hall imply.

\section{DEEP BASAL CONCAVITY CLUSTERS (SPECIMENS 132- 180)}

\section{Cluster 16 (Specimens 134-138)}

Cluster 16 has, among deep basal concavity clusters, points with narrow haft widths and shallow basal concavities (see Figure 14-4g and Table 14-3). They have shallow basal concavities relative to moderate grinding lengths and thicker hafts relative to narrower haft widths. All five specimens are correctly classified according to GCP (see Table 14-6), with probabilities ranging from 0.75 to 0.95 . Three specimens show probabilities greater than 0.05 for Cluster 17, and one specimen shows $p=0.09$ for Cluster $10, p=0.06$ for Cluster 13, and $p=0.09$ for Cluster 14. The strongest similarity indicated is between Clusters 16 and 17.

\section{Cluster 17 (Specimens 139-150)}

Cluster 17 points have, of deep basal concavity clusters, narrow haft widths and moderately deep basal concavities (see Figure 14-4h and Table 14-3). And they have deeper basal concavities relative to moderately short grinding lengths and thicker hafts relative to narrow haft widths. All 12 specimens are correctly classified according to GCP (see Table 14-6), with probabilities ranging from 0.60 to 1.00 . Three specimens show probabilities greater than 0.05 for Cluster
TABLE 14-19

Analysis of Variance (ANOVA) Comparing

Parallel-sided Haft, Shallow-to-moderate Basal Concavity Point Types

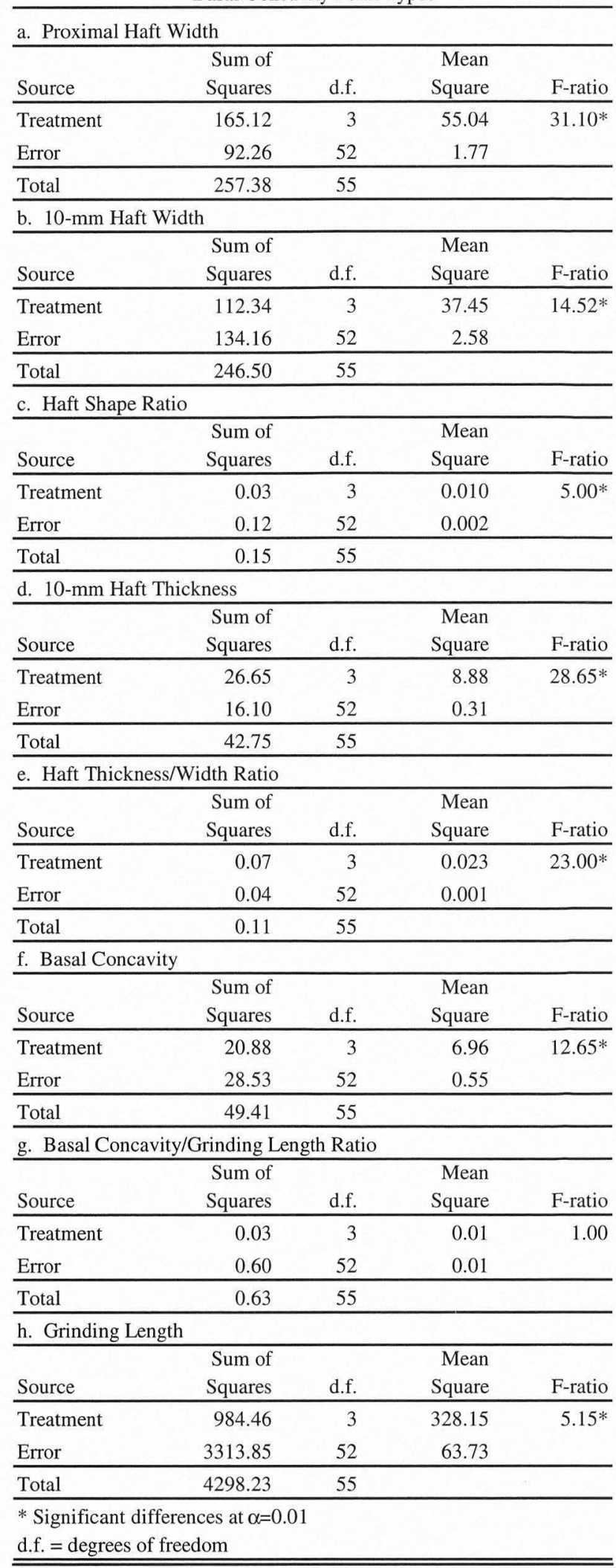


19, two specimens show probabilities greater than 0.05 for Cluster 18 , and one specimen has $p=0.05$ for Cluster 16 . Weak affinities are indicated between Cluster 17 and Clusters 16, 18 , and 19.

\section{Cluster 18 (Specimens 151-158)}

Cluster 18 has, among deep basal concavity clusters, points with moderate haft widths and moderately shallow basal concavities, as well as moderately shallow basal concavities relative to moderately short grinding lengths and moderately thin hafts relative to moderate haft widths (see Figure 14-4i; Table 14-3). All eight specimens are correctly classified according to GCP (see Table 14-6), with probabilities ranging from 0.56 to 1.00 . Specimen 151 has $p=0.42$ for Cluster 16 and $p=0.56$ for Cluster 18 , indicating this specimen is nearly split between the two clusters. Two specimens show probabilities greater than 0.05 for Cluster 20 , and one specimen shows $p=0.23$ for Cluster 17. Slight similarities between Cluster 18 and Clusters 20,16, and 17 are indicated.

\section{Cluster 19 (Specimens 159-168)}

Among deep basal concavity clusters, Cluster 19 has points with moderately narrow hafts and extremely deep basal concavities. They have moderately thick hafts relative to moderately narrow haft widths and extremely deep basal concavities relative to moderately short grinding lengths (see Figure 14-4j; Table 14-3). All 10 specimens are correctly classified according to GCP (see Table 14-6), with probabilities ranging from 0.71 to 1.00 . Two specimens have probabilities greater than 0.05 for Cluster 17, indicating similarities between Clusters 19 and 17 .

\section{Cluster 20 (Specimens 169-177)}

Among deep basal concavity clusters, Cluster 20 has points with wide hafts and very deep basal concavities. They have moderate haft thicknesses relative to wide haft widths and very deep basal concavities relative to long grinding lengths (see Figure 14-4k and Table 14-3). All nine specimens are correctly classified according to GCP (see Table 14-6), with probabilities ranging from 0.96 to 1.00 . There are no notable similarities with other clusters.

\section{Cluster 21 (Specimens 178-180)}

Cluster 21 has, of the deep basal concavity clusters, points with very wide hafts, much shallower basal concavities, moderately thick hafts relative to very wide haft widths, and shallow basal concavities relative to short grinding lengths (see Figure 14-41; Table 14-3). All three specimens are correctly classified according to GCP (see Table 14-6), with probabilities of 1.00 .

\section{DISCUSSION OF DEEPBASALCONCAVITY CLUSTERS}

Clusters 16 through 21 represent the morphological variability of parallel-sided haft, deep basal concavity specimens. These clusters separate from the rest of the sample as seen on the dendrogram (see Figure 14-2). The primary characteristics that distinguish these clusters from the rest of the sample are the much deeper basal concavity and wider hafts (see Table 14-3). The inherent variability in the haft shape ratio and $10-\mathrm{mm}$ haft thickness further separates these clusters from those of contracting haft and parallel-sided haft, shallow-to-moderate basal concavity.

In contrast to the results seen with contracting haft and parallel-sided haft, shallow-to-moderate basal concavity clusters, the parallel-sided haft, deep basal concavity clusters do not show clear patterns of similarities and differences that suggest types. When the assignment of previous types is examined by cluster, Dalton and Golondrina-Barber overlap throughout Clusters 17-20 (Table 14-20). This indicates that numerical analysis alone will not further distinguish these types.

TABLE $14-20$

Distribution of Types within Parallel-Sided Haft, Deep Basal Concavity Clusters

\begin{tabular}{|c|c|c|c|c|c|c|c|}
\hline \multirow[b]{2}{*}{ Type } & \multicolumn{6}{|c|}{ Cluster } & \multirow[b]{2}{*}{ Totals } \\
\hline & 16 & 17 & 18 & 19 & 20 & 21 & \\
\hline $\begin{array}{l}\text { St. Mary's Hall- } \\
\text { atypic }\end{array}$ & 5 & 0 & 1 & 0 & 0 & 0 & 6 \\
\hline Golondrina-Barber & 0 & 9 & 5 & 2 & 8 & 3 & 27 \\
\hline Dalton & 0 & 3 & 2 & 8 & 1 & 0 & 14 \\
\hline Totals: & 5 & 12 & 8 & 10 & 9 & 3 & 47 \\
\hline
\end{tabular}

An exception is seen for Cluster 16, which consists of points with narrower haft widths and moderately shallower basal concavities among the parallel-sided haft, deep basal concavity clusters (see Table 14-3). These specimens have been designated atypical St. Mary's Hall because this cluster includes one specimen (Specimen 135; see Table 14-1) from the St. Mary's Hall site and at least one specimen from the Wilson-Leonard site (Specimen 138; see Table 14-1) that is in a context consistent with other St. Mary's Hall specimens. The atypical label is applied because these specimens have wider hafts and deeper basal concavities than previously defined St. Mary's Hall specimens (cf., Table 14-3). St. Mary's Hall and atypical St. Mary's Hall points are almost the same in 10-mm haft thickness, haft thickness/width ratio, and grinding length.

Clusters 17-21 of the parallel-sided haft, deeper basal concavity clusters represent the morphological variability of Dalton and Golondrina-Barber types. This variability spans a wide range in haft width and basal concavity (see Table 14-3). Although not statistically significant, Cluster 17 points have slightly contracting haft shapes (see Figure 144h), an attribute that has been identified with the Barber 
type (Kelly 1983a). Cluster 18 points have the slightly expanding haft shapes (see Figure 14-4i) associated with Golondrina (Johnson 1964; Kelly 1982). Cluster 19 is dominated by Dalton points and reflects the deepest basal concavities (see Figure 14-4j). Points in Clusters 20 and 21 are noticeably wider in haft width (see Table 14-3) than those in the other clusters, and include Plainview golondrina specimens from Devil's Mouth site and Golondrina-Barber specimens from Wilson-Leonard site.

In contrast to the morphological overlap, flaking patterns for Dalton and Golondrina-Barber appear to be different. In addition, the chronological information for Dalton suggests an age range from 10,500 B.P. to 9900 в.P., while the temporal range for Golondrina-Barber is 9500 B.P. to 8800 B.P. These technological and chronological data separate Dalton and Golondrina-Barber into earlier and later types, respectively.

Concerning the matter of separating Golondrina and Barber, the stratigraphic distribution at the Wilson-Leonard site indicates no chronological difference; both are in a Late Paleoindian context. Instead, these two morphological patterns represent two ends of a continuum which extends from slight contracting haft shape, through parallel-sided haft shape, to slight expanding haft shape. These haft shape differences are not significant (see Table 14-9c), however, and so these specimens have been designated GolondrinaBarber in the Wilson-Leonard collection. That these two patterns are part of the same type is further supported by a cache from South Texas (Hester 1993:Figure 8, 1995a:Figure 10) in which the projectile points range from Golondrina to Barber. This cache presumably represents a single event and implies that this variation was not seen as significant by those responsible for caching the artifacts.

Three named types are recognized within the parallelsided haft, deep basal concavity clusters: atypical St. Mary's Hall, Golondrina-Barber, and Dalton. When compared by type, there are significant differences in 10-mm haft width, haft shape ratio, haft thickness/width ratio, basal concavity, basal concavity/grinding length ratio, and grinding length (Table 14-21; see Table 14-15). There is considerable variability in Golondrina-Barber, perhaps even more wide ranging than in Angostura. The variability as represented in the present sample does not appear to have temporal significance. Therefore, Golondrina-Barber includes a wide range of variability as a type characteristic.

\section{Summary}

This analysis has examined the basal morphology of unfluted lanceolate points irrespective of previously assigned types and then compared the resulting morphological patterns to those types. The results suggest some changes and improvements in some named chronological types and the recognition of two new chronological types. The systematic analysis of this sample of unfluted lanceolate
TABLE 14-21

Analysis of Variance (ANOVA) Comparing Three Point Types of Parallel-sided Haft, Deep Basal Concavity

a. Proximal Haft Width

\begin{tabular}{lrrrr}
\hline a. Proximal Haft Width & & & Mean & \\
Source & Sum of & & Square & F-ratio \\
\hline Treatment & Squares & d.f. & 23.59 & 3.83 \\
Error & 47.18 & 2 & 6.16 & \\
\hline Total & 271.21 & 44 & & \\
\hline
\end{tabular}

b. 10-mm Haft Width

\begin{tabular}{lrrrr}
\hline Source & $\begin{array}{r}\text { Sum of } \\
\text { Squares }\end{array}$ & d.f. & $\begin{array}{r}\text { Mean } \\
\text { Square }\end{array}$ & F-ratio \\
\hline Treatment & 171.59 & 2 & 85.80 & $15.05^{*}$ \\
Error & 250.58 & 44 & 5.70 & \\
\hline Total & 422.17 & 46 & & \\
\hline c. Haft Shape Ratio & & & & \\
\hline & Sum of & & Mean & \\
Source & Squares & d.f. & Square & F-ratio \\
\hline Treatment & 0.10 & 2 & 0.050 & $16.67^{*}$ \\
Error & 0.14 & 44 & 0.003 & \\
\hline Total & 0.24 & 46 & & \\
\hline
\end{tabular}

d. 10-mm Haft Thickness

\begin{tabular}{lrrrr}
\hline Source & $\begin{array}{r}\text { Sum of } \\
\text { Squares }\end{array}$ & d.f. & $\begin{array}{r}\text { Mean } \\
\text { Square }\end{array}$ & F-ratio \\
\hline Treatment & 0.96 & 2 & 0.48 & 1.00 \\
Error & 20.93 & 44 & 0.48 & \\
\hline Total & 21.89 & 46 & & \\
\hline
\end{tabular}

e. Haft Thickness/Width Ratio

\begin{tabular}{lrrrr}
\hline Source & $\begin{array}{r}\text { Sum of } \\
\text { Squares }\end{array}$ & d.f. & $\begin{array}{r}\text { Mean } \\
\text { Square }\end{array}$ & F-ratio \\
\hline Treatment & 0.03 & 2 & 0.015 & $15.00^{*}$ \\
Error & 0.04 & 44 & 0.001 & \\
\hline Total & 0.07 & 46 & & \\
\hline
\end{tabular}

f. Basal Concavity

\begin{tabular}{lrrrr}
\hline Source & Sum of & & Mean & \\
\hline Treatment & Squares & d.f. & Square & F-ratio \\
Error & 61.10 & 2 & 30.55 & $16.97^{*}$ \\
\hline Total & 79.20 & 44 & 1.80 & \\
\hline
\end{tabular}

g. Basal Concavity/Grinding Length Ratio

\begin{tabular}{|c|c|c|c|c|}
\hline \multirow[b]{2}{*}{ Source } & \multirow{2}{*}{$\begin{array}{l}\text { Sum of } \\
\text { Squares }\end{array}$} & \multicolumn{3}{|c|}{ Mean } \\
\hline & & d.f. & Square & F-ratio \\
\hline Treatment & 0.43 & 2 & 0.22 & $22.00 *$ \\
\hline Error & 0.23 & 44 & 0.01 & \\
\hline Total & 0.66 & 46 & & \\
\hline \multicolumn{5}{|c|}{ h. Grinding Length } \\
\hline & Sum of & & Mean & \\
\hline Source & Squares & d.f. & Square & F-ratio \\
\hline Treatment & 450.66 & 2 & 225.33 & $6.16^{*}$ \\
\hline Error & 1609.42 & 44 & 36.58 & \\
\hline Total & 2060.08 & 46 & & \\
\hline
\end{tabular}

* Significant differences at $\alpha=0.01$

d.f. $=$ degrees of freedom 
projectile points shows that there are two basic lanceolate shapes: contracting haft and parallel-sided haft. The significant characteristics within these two general groups are different.

Among contracting haft points, the morphological variability is parceled out by the amount of contraction in haft shape and the differences in haft width, 10-mm haft thickness, and the haft thickness/width ratio. Typologically, contracting haft points can be segregated primarily by differences in 10-mm haft thickness and haft thickness/width ratio, and then to a lesser extent by variations in haft shape and width. In this sample, there are three contracting haft morphological patterns: very thin hafted, moderately thick hafted, and very thick hafts. The points with very thin hafts correspond to two previously recognized types, Midland and Milnesand (here called Milnesand-1), which are further separated on apparent differences in flaking pattern. The moderately thick hafted points consist of Angostura and Lubbock types, which have different flaking patterns and chronological positions. The very thick hafted points represent a morphologically and chronologically distinct, newly named type, Thrall.

Within the contracting haft types, Angostura, dating from 8800 B.P. to 8100 B.P. at the Wilson-Leonard site, stretches across five morphological clusters with extreme to slightly contracting haft shapes. This wide span of variability does not show any temporal trend and so indicates a type that has considerable variation. Handling such a wide range of variation can be problematical for a typologist, but reference to contextual and chronological data supporting the morphological information is helpful. The age for Angostura at the Ray Long site, based on new research at the site, is about 8950 B.P. (Banks et al. 1995) and at the Richard Beene site is $8805 \pm 75$ B.P. (Beta- 47527 ; Thoms 1993:23). These data, together with that from the Wilson-Leonard site, suggest a range of 9000 B.P. to 8000 B.P. for Angostura.

Thrall, a new contracting haft type, dates between 8700 B.P. and 7000 B.P. at the Wilson-Leonard site. This slightly contracting, very thick-hafted point occurs in Early Archaic deposits which also contain Angostura, Hoxie, and other early bifurcate points. The level of resolution in these deposits at the Wilson-Leonard site indicates that Thrall is younger than Angostura.

Lubbock, a point type first recognized at the Lubbock Lake site, dates from about 10,300 B.P. to 9900 B.P. Only four specimens were identified in this analysis: three from the Lubbock Lake site and one from Bonfire Shelter. Lubbock is probably a valid chronological type, but it needs further investigation with a larger sample to fully understand its morphology, chronological position, and cultural pattern.

Parallel-sided haft points divide further on patterns in the depth of basal concavity. Within the shallow-to-moderate basal concavity points, the morphological variation divides primarily on the basis of haft width, $10-\mathrm{mm}$ haft thickness, haft thickness/width ratio, and basal concavity. Typologically, these same characteristics separate chronologically important types. Within the parallel-sided haft, shallow-to-moderate basal concavity points, there are three morphological patterns: very shallow basal concavity, moderately shallow basal concavity, and moderately deep basal concavity. The very shallow basal concavity specimens correspond to Scottsbluff, a form that is not well represented in this sample and should be studied further. The moderately shallow basal concavity points include Plainview and an unnamed parallel-sided haft form, which can be further differentiated by haft thickness, flaking pattern, and chronological position. The moderately deep basal concavity points denote a new type called St. Mary's Hall.

Among parallel-sided haft points with deep basal concavities there are considerable variations in haft width, haft thickness/width ratio, basal concavity, and basal concavity/ grinding length ratio, but when the clustered morphological patterns are compared with named types, it is clear that the patterns in morphological variability do not readily correspond to chronological types. At the typological level, these points are further identified by flaking pattern and chronological position in addition to morphology.

Within parallel-sided haft, shallow-to-moderate basal concavity points, true Plainview morphology was isolated as a relatively thin, moderately wide-hafted point with a moderately shallow basal concavity. A review of the radiocarbon ages associated with this Plainview sample suggests a range between 11,200 B.P. and 10,100 в.P. The results of this analysis suggest it might be time to adopt a more-restricted definition of Plainview, which probably represents an Early Paleoindian cultural pattern that needs to be investigated as such in future research.

In contrast to an Early Paleoindian Plainview, this analysis has identified a different morphological pattern represented by the proposed new type St. Mary's Hall, dating from 9990 B.P. to 8700 B.P. St. Mary's Hall appears to represent a Late Paleoindian cultural pattern that has been invisible because it was lumped into a large, morphologically unrestricted Plainview category.

Among the parallel-sided haft, deep basal concavity points, Golondrina-Barber, dating from 9500 B.P. to 8800 B.P. at the Wilson-Leonard site, spreads across five morphological clusters marked by insignificant variations in haft shape. These two types have previously been split, but this analysis indicates the temporal positions are the same, and so instead, represent a morphological continuum. GolondrinaBarber overlap morphologically with Dalton which dates between 10,500 B.P. to 9900 в.P. The differences in flaking pattern and chronology are necessary to further differentiate these two types.

Through this analysis, some types, such as Angostura versus Thrall, Plainview versus St. Mary's Hall, or Plainview versus Golondrina-Barber, have been recognized morphologically. However, the morphological overlap of Angostura 
and Lubbock, Midland and diminutive Milnesand (Milnesand-1), as well as Golondrina-Barber and Dalton demonstrate the next step in understanding significant attributes that delineate chronological types; that step is a systematic observation and analysis of flaking pattern/technology. Traditionally, observations of flake pattern and basal modification have tended to be exercises in verbal description using unstandardized terms. For example, Krieger (1947a:942) used the term "collateral flaking" defined as near equal size flakes removed from both sides of a medial ridge. In contrast, Crabtree (1982:28) defined collateral flaking as "expanding flakes removed from the lateral edges... at right angles to the longitudinal axis." From these descriptions, it is not clear whether they are describing the same type of pattern. The observations of lateral and vertical flaking need to be at the same level of detail as the morphological attributes in order to recognize the significant patterns that can be useful in typology.

Some suggestions for flake pattern analysis experiments include concentrating the observations of flaking on the base portion of a point in order to be directly comparable to the morphology under analysis, counting the number of lateral flake scars within the basal $10-\mathrm{mm}$ section, counting the number of vertical flake scars coming from the base, and measuring the length and width of lateral and vertical flake scars within the $10-\mathrm{mm}$ base section of the point which can be used in deriving flake size averages or length-width ratios. Recording the angle of flake scars, such as oblique, perpendicular, or horizontal to the long axis, may or may not be worthwhile. In spite of Wheeler's (1995) emphasis of upper left to lower right diagonal ripple flake scars to denote true Angosturas, the results of the analysis of WilsonLeonard site projectile points indicate oblique, subparallel flaking present on some specimens of Thrall, Angostura, St. Mary's Hall, atypical St. Mary's Hall, Golondrina-Barber, San Patrice, and Wilson. That oblique flaking crosscuts such morphological and temporal ranges suggests that angle of flake scars may not have much discriminating power for chronological types. If any of these flake pattern analysis experiments produce significant discriminating results, then they would become part of a process of systematic analysis of morphological and technological variability employed in understanding and interpreting Paleoindian culture history and cultural behavior.

\section{STATISTICALANALYSIS OF EARLY BIFURCATE STEM PROJECTILE POINTS}

\section{Introduction}

Among the most intractable projectile points to sort using traditional intuitive approaches are the Early Archaic bifurcate stem points. Preliminary results of statistical analyses of unfluted lanceolate points (above) prompted a decision to attempt a similar-if more-limited-study of the bifurcate stem points. In an effort to explore multivariate patterning among these, a sample was chosen for statistical analysis of morphometric attributes. The classes enumerated in this manner partially mirror the descriptive groups set forth in Chapter 13 and seem to reflect a chronologicalor at least stratigraphic - trend at this site.

\section{Background and Typological Problems among Early Bifurcate Stem Points}

An opportunity for evaluating projectile point typology in the Early Archaic period arose with the analysis of the large sample of bifurcate stem projectile points from the Wilson-Leonard site. More than 250 points bearing attributes of the "Early Split Stemmed" (Johnson 1991; Prikryl 1990) and "Early Corner Notched" (Hester 1971) series were recovered. Among these are forms that match established "types" as well as forms that do not. Pressing need for such an undertaking became more apparent as first attempts at morphological sorting and typological classification were undertaken for the Wilson-Leonard sample. Considered as a group, these points share a single common attribute, that is, a stem with a concave base. Beyond that, attributes vary widely, with some stems being generally straight, others slightly expanding, and others widely expanding. Some specimens could be assigned with relative ease to types Gower, Hoxie, Jetta, Uvalde, and Martindale, as originally defined. More typical, however, was the large number of specimens that seemed to share attributes of two or more of these types or grade between them.

Confusion among Early Archaic point types has long been noted, with several typologists questioning the usefulness and wisdom of certain types or contrived type variants (Black and McGraw 1985:121; Hester 1979b:5; Johnson 1991:111-112). One has only to review the lithic sections from reports on sites with Early Archaic remains (Black and McGraw 1985; Crawford 1965; Goode 1989; Hester 1971; Johnson 1991; Prewitt 1981a) to sense the difficulty inherent in adhering to the established types. Terms such as Martindalelike, Gower-Uvalde, and various untyped, numbered, and lettered forms and variants of specific types abound. Hester (1979b:5) noted that he "could not take a set of 'early corner notched points' and neatly sort them into Martindale, Uvalde, or other previously defined types."

Use of broad descriptive categories has been the solution for some typologists, although such terms are not without problems, as is discussed below. The term, "Early Barbed," was coined early on by Johnson (1964) to contend with variability among Early Archaic points at the Devil's Mouth site. Hester (1971) suggested the appellation "Early Corner Notched" to refer to an array of concave-based Early Archaic points from the La Jita site; he has also used the term "Early Basal Notched" to broadly describe specimens with morphological affinities to Bell, Andice, and Calf Creek types (Hester 1989:9). Prikryl (1990:51) advanced the classi- 
fication, "Early Split Stemmed," for a group of Early Archaic Gower-like, Martindale-like, and Uvalde-like points described from northeastern Texas sites. Terming Early Archaic point typology a "mess," Johnson (1991:111) called for use of the overarching rubric, "Early Split-Stem Series" to put to rest typological conundrums among Early Archaic types and suggested that "forms" within the series (e.g., Gower, Uvalde, Baker) be used for further definition, as possible.

Mindful of the confusing litany of classificatory devices already on hand, we are using herein yet another term, "early bifurcate stem," for descriptive purposes. It is intended purely as a organizational device to subsume the full panoply of early split-stemmed, early corner notched, and even some concave base specimens that seem to resemble early basal notched and early barbed.

As with any "lumping" strategy, the use of broad descriptive categories increases the risk of blurring or obscuring specific attributes that may be functionally or stylistically distinctive or that may have temporal significance. Yet, lacking an appropriate sample, evaluation of the finer distinctions within these larger groups and refining of existing typologies has not been practicable or particularly useful. Prior to this time, there has not been an excavated site reported in Texas with an Early Archaic projectile point sample of sufficient size to be statistically meaningful. Samples from more-discrete contexts (e.g., Youngsport [Shafer 1963]; Camp Pearl Wheat [Collins et al. 1990]; Sleeper [Johnson 1991]; Richard Beene [Thoms 1992]; and Eckols [Karbula 1993]) are quite small, numbering from only 2 to 19 specimens, and generally do not embrace the full range of evident variability. Larger samples are either from surface sites (e.g., Granite Beach [Crawford 1965]; High Bluff [Flinn and Flinn 1968]; and Tombstone Bluff [Prewitt 1981a]), or from apparently mixed depositional contexts bearing diagnostics of multiple archeological intervals (e.g., La Jita [Hester 1971]; and Panther Springs Creek [Black and McGraw 1985]). The WilsonLeonard assemblage afforded an opportunity to sort a reasonably large sample from better than average stratigraphic contexts not only by traditional methods (Chapter 13) but through morphometric and mathematical means.

Toward these ends, a variety of analytical approachessome overlapping in sample and scope - were employed, as described above for the lanceolate points. The early bifurcate stem analysis, however, differs from the lanceolate point study in that all specimens included in the sample are from the Wilson-Leonard site. Metric attributes also were tailored to the somewhat different set of questions posed for the bifurcate stem group.

Specimens from pertinent type sites were examined, as possible, in order to informally compare metric, morphological, and technological attributes to early bifurcate stemmed points in the Wilson-Leonard collection. These included collections from the following sites: Youngsport site for the Gower type (Shafer 1963); the San Gabriel Reservoir sites for Hoxie (Prewitt 1981a); and Jetta Court for the Jetta type
(Wesolowsky et al. 1976). Due to time constraints, however, data from these sites were not used in the statistical analysis; rather, the information contributed to the formulation of typological questions.

Indeed, what becomes apparent upon examining the type specimens is the degree of deviation from original specifications that has occurred since initial type definitions were made. This divergence is discussed in more detail below in conjunction with a reexamination of original type definitions.

\section{Early Archaic Projectile Point Types Revisited}

Present studies generally refer to six Early Archaic bifurcate stem point types in Central Texas. These are Uvalde, Martindale, Gower, Bandy, Hoxie, and Jetta. (To a lesser extent, the Baker type has significance, although it may be chiefly a Trans Pecos variant of other bifurcate types.)

These types are, for the most part, relative newcomers to Texas archeological chronologies. Only two-Uvalde and Martindale - appear in the type descriptions presented by Suhm et al. (1954) or the later revision by Suhm and Jelks (1962). Two significant changes in typological perspective have occurred since these two types were defined. First, most of what is now considered to be the "Early Archaic" was unknown 40 years ago, and, second, blade and shoulder/barb morphology is now known to be strongly modified by attrition and reworking.

In setting forth the Martindale type, Miller and Jelks (1952:171) credit Kelley with identification of the point type that he termed "Martindale Fishtail." The type was later refined by Suhm et al. (1954) and Suhm and Jelks (1962:213), who describe it as a well-barbed, triangular-bladed point with stems varying from parallel edged to strongly expanding. The distinctive base is "formed by two distinct concave curves meeting in a depression in the center." The two authors suggest that the base was a "result of a deliberate effort setting it apart from other bases." As defined and illustrated (Suhm and Jelks 1962:Plate 107), the Martindale type is tightly drawn.

Specimens of the Uvalde type are described by Suhm et al. (1954:486) as exhibiting triangular to leaf-shaped blades, prominent shoulders either rounded or barbed, and stems that expand strongly, sometimes to the width of the shoulders; bases have deep, U-shaped concavities, similar to those of Pedernales points (Suhm et al. 1954:486), although Uvalde stems are shorter and more outward flaring. The wide variability encompassed in this description is illustrated in Suhm et al. (1954:Plate 128); as noted by Suhm and Jelks (1962:255), similarities between Uvalde, Frio, and Martindale types can present difficulties in classification.

Shafer (1963:64) advanced the type, Gower, to describe a group of "crudely made, indented base points" with very short, parallel-edged stems, from the Youngsport site in Bell County. On the five points presented as type specimens, he notes basal concavities are formed "by the removal of a 
single (?) flake," the scar from which usually appears on only one side of the point and is sometimes lightly retouched. Lateral stem edge grinding was not observed on any of the five, although smoothing was observed on two of the nine "variants" recovered from the same stratum. Resemblances of Gower to Pedernales and Crumley points have been noted (Kelly 1962:247-249, 1979:13; Shafer 1963:64).

Based on findings at Baker Cave, the Bandy point was defined by Word and Douglas (1970) as well barbed, with corner notching producing a gently expanding stem. Distinctive attributes are thinness, overall fine quality of workmanship, and bases that "arc convexly" to the center, creating a fishtail appearance. Similarities to Martindale - particularly the fishtail base - suggest that Bandy may be its Lower Pecos equivalent (Turner and Hester 1993:78). However, in his review of Bandy points, McReynolds (1993:916, Figure 1 and 2) found the spatial distribution as well as range of morphological variability within the type to be more extensive than previously considered. The 26 specimens illustrated by McReynolds derive from Val Verde, Bexar, Atascosa, and Williamson counties. Basal modifications range from $\mathrm{V}$-shaped to almost straight edged, with basal ears or tangs varying from rounded to pointed. Like Word and Douglas, McReynolds found thinness to be a markedly consistent attribute, with stem thicknesses ranging from 3.0 $\mathrm{mm}$ to $4.1 \mathrm{~mm}$ (mean=3.6 $\mathrm{mm}$ ) (McReynolds 1993:12).

Hoxie is defined by Prewitt (n.d., 1981a:96) as a rectangular-stemmed point with a straight to mildly concave base. Stem lateral edges are usually alternately beveled and always smoothed or ground. Hoxies are similar to Darl but are cruder in flaking, and both types display narrow, elongate blades that are alternately beveled. An offshoot of Hoxie, the Zephyr type (Prewitt n.d., 1981a:96, 1995:138) apparently is distinguished by parallel flake scars on the blade resulting in serrated lateral edges; stems are moderately expanding, and bases are gently concave. As the Zephyr type is rather poorly understood, it is not used herein or in Chapter 13, which is consistent with Prewitt's (1995:138) recently published analysis placing Zephyrs primarily in the Rolling Plains and in North Central Texas rather than in Central Texas.

The type, Jetta, was defined by Hester (1971:6; Turner and Hester 1993:131) who recognized the distinctively large, bifurcate stem points in surface and museum collections and later correlated them with similar points from the lowest deposits of the Jetta Court site in Travis County. It is described as a carefully flaked point with widely flared barbs, a rectangular stem, and a deep basal notch.

As noted above, it is clear that substantial divergence from original definitions has occurred over the years. Somewhat impressionistically, it appears that Gower-perhaps more than any other Early Archaic type- - has been the subject of considerable expansion to include variant forms. Specifically, Shafer's original specification of a parallel-sided stem frequently is disregarded, with the result that some points now typed as Gower may more closely resemble ex- panding stem Uvaldes. Inasmuch as Wilson-Leonard findings strongly suggest that stem shape may be a time-sensitive characteristic, it is important that this attribute be carefully considered.

\section{Use (and Misuse) of the Broad Descriptive Groups}

In addition to the formal projectile point types, a plethora of descriptive classifications or series-each carrying the prefix, "early"- have been constructed by analysts in attempts to overcome typological problems among Late Paleoindian and Early Archaic points. While some are very broad in scope, apparently intended to encompass all Early Archaic bifurcate types and varieties, others are more limited, aimed at a more-discrete-although obviously problematical-morphological set. Unfortunately, misunderstanding and misapplication of some of these terms has served to add to the confusion. For example, the terms "Early Corner Notched" and "Early Barbed" currently are being used somewhat interchangeably (Johnson and Goode 1994:22), although they apparently were defined initially for two quite different morphological groups. In his original definition of the term, Johnson (1964:33) set forth Early Barbed to describe points with long barbs formed by basal notches, wide, thin triangular blades, and generally "rectanguloid" to slightly expanding stems with approximately straight basal edges (see also Johnson 1967:22-26 for description of Period Marker C "Early Barbed"). Based on illustrated examples in the Devil's Mouth site report (Johnson 1964: Plate 11J-S), these points appear to fall within the realm of the Bell/Andice/ CalfCreek series (Perino 1968:62; Turner and Hester 1993:71, 80; Wyckoff 1994). In contrast, the points included in the sets defined by Hester (1971:71-73) under the rubric Early Corner Notched, describe expanding, bifurcate stem varieties termed Uvalde, Bandy, and Martindale.

Some confusion also has occurred in the past in use of the terms "Early Corner Notched" and "Early Stemmed." While both point classifications refer to expanding-stem, corner-notched points, the latter describes a larger, Late Paleoindian point of quite different technology including, typically, ground lateral stem edges (Turner and Hester 1993:106). Formalization of the type name, Wilson, as proposed herein, may partially clarify these distinctions (see Chapter 13). Further contributing to typological confusion, points conforming to the "Early Stemmed Lanceolate" classification (Turner and Hester 1993:107) have been misidentified in the literature as "Early Stemmed" points, no doubt due in part to similarity in names.

The most recent broad descriptor for Early Archaic points, Early Split Stem, was introduced by Prikryl (1990:51) to describe points with "split" stems and basal grinding, including Gower-like, Martindale-like, and Uvalde-like. In his use of the term, however, Johnson (1991:111) defines Early Split-Stem Series to encompass Gower, Gower-like, Uvalde, Baker, and Jetta dart points and clearly excludes 
"Martindale or other points with fishtail tangs or only slightly concave bases." Points in this series have a "clearcut stem with a bifurcated base with a central notch or concavity; and usually exhibit very short barbs or barbless shoulders" (Johnson and Goode 1994:24).

In sum, early bifurcate stem types and descriptive groups can be seen as ambiguous and overlapping in their scope and application. These problems are an outgrowth of the apparent intergrading morphology of the early bifurcate stem points themselves but equally important are poor archeological contexts and less than rigorous analytical methods.

\section{The Sample and Methods}

As noted, focus of the study was exclusively on the stem/base section of projectile points, inasmuch as haft elements were deemed least likely to have been altered from their original state by resharpening. From the larger WilsonLeonard early bifurcate stem collection, a subset of projectile points was identified as being sufficiently complete for statistical examination, that is, having all or almost all of the stem section. By this criterion, points that may have been complete except for a single basal ear were eliminated. Conversely, numerous small basal/stem sections that were complete in all pertinent stem measurements but lacked most or all of the blade sections were retained in the study sample. In all, some 125 specimens were rejected as lacking complete stem measurements. "Marginal" specimens, which were missing only a small fraction of a basal element but for which a measurement could be "reasonably estimated," were generally included in the sample.

Selection of the sample was based on morphological and completeness criteria, as noted above, rather than on archeological context. As it developed, however, the 102 specimens comprising the final sample were affiliated chiefly with Early Archaic contexts ( $\mathrm{n}=67$ or $65 \%$ ). The remainder were from undifferentiated Unit III Archaic or surface contexts $(\mathrm{n}=13)$, and mixed deposits of Early Archaic/Late Paleoindian or Early Archaic/Middle Archaic affiliations $(\mathrm{n}=17)$. Three others were from Middle Archaic contexts, and 1 each from Late Archaic and mixed Late Archaic/Late Prehistoric contexts.

Although 11 different metric and qualitative attributes were recorded for stem sections, only 6 were selected for use in the final study (see Figure 14-1). An additional 3 attributes are derived ratios combining metric variables; these were added, as discussed above, to emphasize specific relationships in stem morphology. In all, this suite of 9 attributes was shown both statistically and observationally to be the strongest in the mathematical analyses undertaken.

Final classifications within groups, or degree of fit, were assessed via Geisser Classification Probabilities, or GCP (Table 14-22). In cases where individual specimens exhibited high probability for classification to another clus- ter, they were examined and assessed, both for metric and qualitative "fit" within the alternative cluster. In several of these cases, a final decision for regrouping was made intuitively. Visual observation also was employed to eliminate specimens that were clearly aberrant for reasons that could not be quantified or where particular attributes were weighted disproportionately. For example, in the case of Cluster 11, "Jetta-like" specimens, basal concavity depth did not emerge initially as a strong enough factor because the group was too small. For this reason, a ratio that more strongly emphasized attributes of the basal concavity was developed. In another example, presence or absence of basal grinding was factored in as attribute but eliminated after it became evident that it served to seriously distort morphological groupings.

Due to the differing kinds of results for the early bifurcate study and the unfluted lanceolate study, mode of presentation is somewhat different. For this section, findings are reported chiefly by individual clusters, with comparisons drawn between clusters. The range of morphological variation in each of the clusters is described with emphasis on the key attributes that served to segregate them and is illustrated in photographs of representative specimens constituting each cluster. Mean and standard deviation of metric variables for clusters identified in the analysis are shown in Table 14-23. An analysis of variance for bifurcate clusters was not undertaken.

To aid in description, type identifications or comparisons are offered for each cluster, when possible, as a means of correlating results of the present study to more traditional frameworks. These type identifications are based on classifications presented above in Chapter 13.

\section{Results}

A total of 11 major morphological groups within the final sample of 102 specimens was identified through cluster and discriminant function analyses. As shown in the hierarchical dendrogram (Figure 14-5), a wide range of subtle variability is present in the sample. The more-distinct clusters on the marginal extremes flank groups in the center displaying varying degrees of similarity.

The initial branching (Division I) of groups segregates thin, expanding-stemmed specimens (Cluster 1; Specimens 1-8) at a distance of approximately 147.865. Division II, at a distance of about 134.470 , isolates a cluster of long stemmed, deeply notched specimens (Cluster 11; Specimens 95-102). With several exceptions, discussed in more detail below, these two branchings proved to be the most significant.

Further divisions produced less singular, more morphologically similar groups. Although numerous subgroups within the larger clusters were examined, the final 11 clusters, drawn at distances of no less than 61.289 , appear to achieve the most meaningful balance between segregation and aggregation. 
TABLE 14-22

Geisser Classification Probabilities for Early Bifurcate Stem Projectile Points

\begin{tabular}{|c|c|c|c|c|c|c|c|c|c|c|c|c|}
\hline \multirow{3}{*}{$\begin{array}{l}\text { Key } \\
\text { No. } \\
\end{array}$} & \multirow{3}{*}{$\begin{array}{l}\text { Cluster } \\
\text { No. }\end{array}$} & \multicolumn{11}{|c|}{ Clusters } \\
\hline & & & & & & & & & & & & \\
\hline & & 1 & 2 & 3 & 4 & 5 & 6 & 7 & 8 & 9 & 10 & 11 \\
\hline 1 & 1 & 1.00 & 0.00 & 0.00 & 0.00 & 0.00 & 0.00 & 0.00 & 0.00 & 0.00 & 0.00 & 0.00 \\
\hline 2 & 1 & 1.00 & 0.00 & 0.00 & 0.00 & 0.00 & 0.00 & 0.00 & 0.00 & 0.00 & 0.00 & 0.00 \\
\hline 3 & 1 & 1.00 & 0.00 & 0.00 & 0.00 & 0.00 & 0.00 & 0.00 & 0.00 & 0.00 & 0.00 & 0.00 \\
\hline 4 & 1 & 1.00 & 0.00 & 0.00 & 0.00 & 0.00 & 0.00 & 0.00 & 0.00 & 0.00 & 0.00 & 0.00 \\
\hline 5 & 1 & 0.99 & 0.00 & 0.00 & 0.01 & 0.00 & 0.00 & 0.00 & 0.00 & 0.00 & 0.00 & 0.00 \\
\hline 6 & 1 & 0.99 & 0.00 & 0.00 & 0.01 & 0.00 & 0.00 & 0.00 & 0.00 & 0.00 & 0.00 & 0.00 \\
\hline 7 & 1 & 1.00 & 0.00 & 0.00 & 0.00 & 0.00 & 0.00 & 0.00 & 0.00 & 0.00 & 0.00 & 0.00 \\
\hline 8 & 1 & 1.00 & 0.00 & 0.00 & 0.00 & 0.00 & 0.00 & 0.00 & 0.00 & 0.00 & 0.00 & 0.00 \\
\hline 9 & 2 & 0.00 & 1.00 & 0.00 & 0.00 & 0.00 & 0.00 & 0.00 & 0.00 & 0.00 & 0.00 & 0.00 \\
\hline 10 & 2 & 0.00 & 1.00 & 0.00 & 0.00 & 0.00 & 0.00 & 0.00 & 0.00 & 0.00 & 0.00 & 0.00 \\
\hline 11 & 2 & 0.00 & 1.00 & 0.00 & 0.00 & 0.00 & 0.00 & 0.00 & 0.00 & 0.00 & 0.00 & 0.00 \\
\hline 12 & 2 & 0.00 & 1.00 & 0.00 & 0.00 & 0.00 & 0.00 & 0.00 & 0.00 & 0.00 & 0.00 & 0.00 \\
\hline 13 & 2 & 0.09 & 0.90 & 0.01 & 0.00 & 0.00 & 0.00 & 0.00 & 0.00 & 0.00 & 0.00 & 0.00 \\
\hline 14 & 2 & 0.00 & 1.00 & 0.00 & 0.00 & 0.00 & 0.00 & 0.00 & 0.00 & 0.00 & 0.00 & 0.00 \\
\hline 15 & 2 & 0.00 & 1.00 & 0.00 & 0.00 & 0.00 & 0.00 & 0.00 & 0.00 & 0.00 & 0.00 & 0.00 \\
\hline 16 & 2 & 0.00 & 1.00 & 0.00 & 0.00 & 0.00 & 0.00 & 0.00 & 0.00 & 0.00 & 0.00 & 0.00 \\
\hline 17 & 2 & 0.00 & 1.00 & 0.00 & 0.00 & 0.00 & 0.00 & 0.00 & 0.00 & 0.00 & 0.00 & 0.00 \\
\hline 18 & 3 & 0.00 & 0.00 & 1.00 & 0.00 & 0.00 & 0.00 & 0.00 & 0.00 & 0.00 & 0.00 & 0.00 \\
\hline 19 & 3 & 0.00 & 0.00 & 1.00 & 0.00 & 0.00 & 0.00 & 0.00 & 0.00 & 0.00 & 0.00 & 0.00 \\
\hline 20 & 3 & 0.00 & 0.00 & 0.90 & 0.01 & 0.00 & 0.00 & 0.00 & 0.09 & 0.00 & 0.00 & 0.00 \\
\hline 21 & 3 & 0.00 & 0.00 & 0.96 & 0.00 & 0.00 & 0.00 & 0.00 & 0.03 & 0.00 & 0.00 & 0.00 \\
\hline 22 & 3 & 0.00 & 0.00 & 1.00 & 0.00 & 0.00 & 0.00 & 0.00 & 0.00 & 0.00 & 0.00 & 0.00 \\
\hline 23 & 3 & 0.00 & 0.00 & 1.00 & 0.00 & 0.00 & 0.00 & 0.00 & 0.00 & 0.00 & 0.00 & 0.00 \\
\hline 24 & 3 & 0.00 & 0.00 & 1.00 & 0.00 & 0.00 & 0.00 & 0.00 & 0.00 & 0.00 & 0.00 & 0.00 \\
\hline 25 & 3 & 0.00 & 0.00 & 0.99 & 0.00 & 0.00 & 0.00 & 0.00 & 0.01 & 0.01 & 0.00 & 0.00 \\
\hline 26 & 4 & 0.00 & 0.00 & 0.00 & 0.89 & 0.03 & 0.00 & 0.02 & 0.05 & 0.01 & 0.00 & 0.00 \\
\hline 27 & 4 & 0.00 & 0.00 & 0.00 & 0.98 & 0.00 & 0.00 & 0.00 & 0.00 & 0.01 & 0.00 & 0.00 \\
\hline 28 & 4 & 0.00 & 0.00 & 0.00 & 0.99 & 0.01 & 0.00 & 0.00 & 0.00 & 0.00 & 0.00 & 0.00 \\
\hline 29 & 4 & 0.00 & 0.00 & 0.00 & 0.98 & 0.02 & 0.00 & 0.00 & 0.00 & 0.00 & 0.00 & 0.00 \\
\hline 30 & 4 & 0.00 & 0.00 & 0.00 & 0.97 & 0.02 & 0.00 & 0.01 & 0.00 & 0.00 & 0.00 & 0.00 \\
\hline 31 & 4 & 0.00 & 0.00 & 0.00 & 0.80 & 0.19 & 0.00 & 0.00 & 0.00 & 0.00 & 0.00 & 0.00 \\
\hline 32 & 4 & 0.00 & 0.00 & 0.00 & 0.63 & 0.36 & 0.00 & 0.00 & 0.01 & 0.00 & 0.00 & 0.00 \\
\hline 33 & 5 & 0.00 & 0.00 & 0.00 & 0.30 & 0.69 & 0.00 & 0.00 & 0.00 & 0.00 & 0.00 & 0.00 \\
\hline 34 & 5 & 0.00 & 0.00 & 0.00 & 0.07 & 0.92 & 0.00 & 0.00 & 0.01 & 0.00 & 0.00 & 0.00 \\
\hline 35 & 5 & 0.00 & 0.00 & 0.00 & 0.04 & 0.90 & 0.01 & 0.04 & 0.02 & 0.00 & 0.00 & 0.00 \\
\hline 36 & 5 & 0.00 & 0.00 & 0.00 & 0.19 & 0.76 & 0.00 & 0.03 & 0.01 & 0.00 & 0.00 & 0.00 \\
\hline 37 & 5 & 0.00 & 0.00 & 0.00 & 0.00 & 0.68 & 0.23 & 0.00 & 0.09 & 0.00 & 0.00 & 0.00 \\
\hline 38 & 5 & 0.00 & 0.00 & 0.00 & 0.05 & 0.87 & 0.00 & 0.00 & 0.07 & 0.00 & 0.00 & 0.00 \\
\hline 39 & 6 & 0.00 & 0.00 & 0.00 & 0.00 & 0.01 & 0.93 & 0.00 & 0.06 & 0.00 & 0.00 & 0.00 \\
\hline 40 & 6 & 0.00 & 0.00 & 0.00 & 0.00 & 0.01 & 0.95 & 0.00 & 0.04 & 0.00 & 0.00 & 0.00 \\
\hline 41 & 6 & 0.00 & 0.00 & 0.00 & 0.02 & 0.24 & 0.44 & 0.17 & 0.13 & 0.00 & 0.00 & 0.00 \\
\hline 42 & 6 & 0.00 & 0.00 & 0.00 & 0.00 & 0.02 & 0.82 & 0.00 & 0.16 & 0.00 & 0.00 & 0.00 \\
\hline 43 & 6 & 0.00 & 0.00 & 0.00 & 0.00 & 0.00 & 0.98 & 0.01 & 0.01 & 0.00 & 0.00 & 0.00 \\
\hline 44 & 6 & 0.00 & 0.00 & 0.00 & 0.00 & 0.00 & 0.99 & 0.00 & 0.01 & 0.00 & 0.00 & 0.00 \\
\hline 45 & 7 & 0.00 & 0.00 & 0.00 & 0.02 & 0.07 & 0.01 & 0.90 & 0.01 & 0.00 & 0.00 & 0.00 \\
\hline 46 & 7 & 0.00 & 0.00 & 0.00 & 0.01 & 0.01 & 0.01 & 0.41 & 0.49 & 0.08 & 0.00 & 0.00 \\
\hline 47 & 7 & 0.00 & 0.00 & 0.00 & 0.05 & 0.01 & 0.00 & 0.59 & 0.09 & 0.26 & 0.00 & 0.00 \\
\hline 48 & 7 & 0.00 & 0.00 & 0.00 & 0.00 & 0.00 & 0.00 & 0.86 & 0.01 & 0.13 & 0.00 & 0.00 \\
\hline 49 & 7 & 0.00 & 0.00 & 0.00 & 0.00 & 0.00 & 0.00 & 0.92 & 0.00 & 0.07 & 0.00 & 0.00 \\
\hline 50 & 7 & 0.00 & 0.00 & 0.00 & 0.00 & 0.00 & 0.00 & 0.99 & 0.00 & 0.01 & 0.00 & 0.00 \\
\hline 51 & 7 & 0.00 & 0.00 & 0.00 & 0.00 & 0.00 & 0.00 & 0.96 & 0.01 & 0.02 & 0.00 & 0.00 \\
\hline 52 & 7 & 0.00 & 0.00 & 0.00 & 0.00 & 0.00 & 0.00 & 0.99 & 0.00 & 0.01 & 0.00 & 0.00 \\
\hline 53 & 7 & 0.00 & 0.00 & 0.00 & 0.00 & 0.00 & 0.00 & 1.00 & 0.00 & 0.00 & 0.00 & 0.00 \\
\hline 54 & 7 & 0.00 & 0.00 & 0.00 & 0.00 & 0.00 & 0.00 & 0.97 & 0.00 & 0.03 & 0.00 & 0.00 \\
\hline
\end{tabular}


Table 14-22, continued

\begin{tabular}{|c|c|c|c|c|c|c|c|c|c|c|c|c|}
\hline \multirow{3}{*}{$\begin{array}{l}\text { Key } \\
\text { No. }\end{array}$} & \multirow{3}{*}{$\begin{array}{l}\text { Cluster } \\
\text { No. }\end{array}$} & \multicolumn{11}{|c|}{ Clusters } \\
\hline & & & & & & & & & & & & \\
\hline & & 1 & 2 & 3 & 4 & 5 & 6 & 7 & 8 & 9 & 10 & 11 \\
\hline 55 & 7 & 0.00 & 0.00 & 0.00 & 0.00 & 0.00 & 0.00 & 0.95 & 0.00 & 0.05 & 0.00 & 0.00 \\
\hline 56 & 7 & 0.00 & 0.00 & 0.00 & 0.00 & 0.00 & 0.00 & 0.99 & 0.00 & 0.01 & 0.00 & 0.00 \\
\hline 57 & 7 & 0.00 & 0.00 & 0.00 & 0.01 & 0.00 & 0.00 & 0.98 & 0.00 & 0.01 & 0.00 & 0.00 \\
\hline 58 & 7 & 0.00 & 0.00 & 0.00 & 0.00 & 0.00 & 0.00 & 1.00 & 0.00 & 0.00 & 0.00 & 0.00 \\
\hline 59 & 7 & 0.00 & 0.00 & 0.00 & 0.00 & 0.00 & 0.00 & 1.00 & 0.00 & 0.00 & 0.00 & 0.00 \\
\hline 60 & 7 & 0.00 & 0.00 & 0.00 & 0.00 & 0.00 & 0.00 & 0.99 & 0.00 & 0.01 & 0.00 & 0.00 \\
\hline 61 & 7 & 0.00 & 0.00 & 0.00 & 0.00 & 0.00 & 0.01 & 0.99 & 0.00 & 0.00 & 0.00 & 0.00 \\
\hline 62 & 7 & 0.00 & 0.00 & 0.00 & 0.00 & 0.00 & 0.00 & 1.00 & 0.00 & 0.00 & 0.00 & 0.00 \\
\hline 63 & 7 & 0.00 & 0.00 & 0.00 & 0.00 & 0.00 & 0.24 & 0.75 & 0.01 & 0.01 & 0.00 & 0.00 \\
\hline 64 & 7 & 0.00 & 0.00 & 0.00 & 0.00 & 0.00 & 0.00 & 0.99 & 0.00 & 0.00 & 0.00 & 0.00 \\
\hline 65 & 7 & 0.00 & 0.00 & 0.00 & 0.00 & 0.00 & 0.00 & 0.98 & 0.00 & 0.01 & 0.00 & 0.00 \\
\hline 66 & 8 & 0.00 & 0.00 & 0.00 & 0.00 & 0.00 & 0.00 & 0.04 & 0.63 & 0.33 & 0.00 & 0.00 \\
\hline 67 & 8 & 0.00 & 0.00 & 0.01 & 0.02 & 0.01 & 0.00 & 0.01 & 0.92 & 0.04 & 0.00 & 0.00 \\
\hline 68 & 8 & 0.00 & 0.00 & 0.02 & 0.02 & 0.02 & 0.00 & 0.00 & 0.92 & 0.02 & 0.00 & 0.00 \\
\hline 69 & 8 & 0.00 & 0.00 & 0.00 & 0.00 & 0.00 & 0.00 & 0.00 & 0.94 & 0.06 & 0.00 & 0.00 \\
\hline 70 & 8 & 0.00 & 0.00 & 0.00 & 0.00 & 0.00 & 0.02 & 0.00 & 0.98 & 0.00 & 0.00 & 0.00 \\
\hline 71 & 8 & 0.00 & 0.00 & 0.00 & 0.03 & 0.21 & 0.01 & 0.02 & 0.71 & 0.01 & 0.00 & 0.00 \\
\hline 72 & 8 & 0.00 & 0.00 & 0.00 & 0.01 & 0.07 & 0.04 & 0.02 & 0.86 & 0.00 & 0.00 & 0.00 \\
\hline 73 & 8 & 0.00 & 0.00 & 0.00 & 0.04 & 0.16 & 0.01 & 0.00 & 0.79 & 0.00 & 0.00 & 0.00 \\
\hline 74 & 8 & 0.00 & 0.00 & 0.01 & 0.00 & 0.07 & 0.01 & 0.00 & 0.92 & 0.00 & 0.00 & 0.00 \\
\hline 75 & 8 & 0.00 & 0.00 & 0.00 & 0.00 & 0.00 & 0.01 & 0.00 & 0.99 & 0.00 & 0.00 & 0.00 \\
\hline 76 & 8 & 0.00 & 0.00 & 0.00 & 0.00 & 0.00 & 0.85 & 0.00 & 0.15 & 0.00 & 0.00 & 0.00 \\
\hline 77 & 8 & 0.00 & 0.00 & 0.00 & 0.00 & 0.00 & 0.20 & 0.01 & 0.79 & 0.00 & 0.00 & 0.00 \\
\hline 78 & 9 & 0.00 & 0.00 & 0.00 & 0.01 & 0.01 & 0.00 & 0.60 & 0.02 & 0.37 & 0.00 & 0.00 \\
\hline 79 & 9 & 0.00 & 0.00 & 0.00 & 0.01 & 0.00 & 0.00 & 0.29 & 0.02 & 0.68 & 0.00 & 0.00 \\
\hline 80 & 9 & 0.00 & 0.00 & 0.00 & 0.01 & 0.00 & 0.00 & 0.02 & 0.20 & 0.77 & 0.00 & 0.00 \\
\hline 81 & 9 & 0.00 & 0.00 & 0.01 & 0.54 & 0.05 & 0.00 & 0.01 & 0.02 & 0.38 & 0.00 & 0.00 \\
\hline 82 & 9 & 0.00 & 0.00 & 0.00 & 0.00 & 0.00 & 0.00 & 0.01 & 0.00 & 0.99 & 0.00 & 0.00 \\
\hline 83 & 9 & 0.00 & 0.00 & 0.00 & 0.00 & 0.00 & 0.00 & 0.00 & 0.00 & 0.99 & 0.00 & 0.00 \\
\hline 84 & 9 & 0.00 & 0.00 & 0.00 & 0.00 & 0.00 & 0.00 & 0.00 & 0.00 & 0.94 & 0.06 & 0.00 \\
\hline 85 & 9 & 0.00 & 0.00 & 0.00 & 0.00 & 0.00 & 0.00 & 0.00 & 0.00 & 1.00 & 0.00 & 0.00 \\
\hline 86 & 9 & 0.00 & 0.00 & 0.00 & 0.00 & 0.00 & 0.00 & 0.10 & 0.00 & 0.90 & 0.00 & 0.00 \\
\hline 87 & 9 & 0.00 & 0.00 & 0.00 & 0.00 & 0.00 & 0.00 & 0.17 & 0.00 & 0.83 & 0.00 & 0.00 \\
\hline 88 & 9 & 0.00 & 0.00 & 0.00 & 0.00 & 0.00 & 0.00 & 0.00 & 0.00 & 0.99 & 0.00 & 0.00 \\
\hline 89 & 9 & 0.00 & 0.00 & 0.00 & 0.00 & 0.00 & 0.00 & 0.00 & 0.65 & 0.34 & 0.00 & 0.00 \\
\hline 90 & 9 & 0.00 & 0.00 & 0.00 & 0.00 & 0.00 & 0.00 & 0.01 & 0.01 & 0.98 & 0.00 & 0.00 \\
\hline 91 & 10 & 0.00 & 0.00 & 0.00 & 0.00 & 0.00 & 0.00 & 0.00 & 0.00 & 0.00 & 1.00 & 0.00 \\
\hline 92 & 10 & 0.00 & 0.00 & 0.00 & 0.00 & 0.00 & 0.00 & 0.00 & 0.00 & 0.00 & 1.00 & 0.00 \\
\hline 93 & 10 & 0.00 & 0.00 & 0.00 & 0.00 & 0.00 & 0.00 & 0.00 & 0.00 & 0.00 & 1.00 & 0.00 \\
\hline 94 & 10 & 0.00 & 0.00 & 0.00 & 0.00 & 0.00 & 0.00 & 0.00 & 0.00 & 0.00 & 1.00 & 0.00 \\
\hline 95 & 11 & 0.00 & 0.00 & 0.00 & 0.00 & 0.00 & 0.00 & 0.00 & 0.43 & 0.00 & 0.00 & 0.57 \\
\hline 96 & 11 & 0.00 & 0.00 & 0.00 & 0.00 & 0.00 & 0.00 & 0.00 & 0.01 & 0.00 & 0.00 & 0.99 \\
\hline 97 & 11 & 0.00 & 0.00 & 0.00 & 0.00 & 0.00 & 0.00 & 0.00 & 0.00 & 0.00 & 0.00 & 1.00 \\
\hline 98 & 11 & 0.00 & 0.00 & 0.00 & 0.00 & 0.00 & 0.00 & 0.00 & 0.00 & 0.00 & 0.00 & 1.00 \\
\hline 99 & 11 & 0.00 & 0.00 & 0.00 & 0.00 & 0.00 & 0.00 & 0.00 & 0.00 & 0.00 & 0.00 & 1.00 \\
\hline 100 & 11 & 0.00 & 0.00 & 0.00 & 0.00 & 0.00 & 0.00 & 0.00 & 0.00 & 0.00 & 0.00 & 1.00 \\
\hline 101 & 11 & 0.00 & 0.00 & 0.00 & 0.00 & 0.00 & 0.00 & 0.00 & 0.00 & 0.00 & 0.00 & 1.00 \\
\hline $102 *$ & 11 & 0.00 & 0.00 & 0.00 & 0.00 & 0.00 & 0.00 & 0.00 & 0.00 & 0.00 & 0.00 & 1.00 \\
\hline
\end{tabular}

*102 is an outlier of Cluster 11.

Summary of hits and misses: 102 individuals classified; hits $=97(95 \%)$; misses $=5(5 \%)$.

The 11 groups range in size from 4 to 21 members; each of these groups is described in some detail below.

\section{Cluster 1}

The eight specimens in Cluster 1 are distinctive in their thinness and exhibit short, slightly to moderately expanding stems (Figure 14-6a-c). Distal stem thickness ranges from 3.9 to $5.2 \mathrm{~mm}$, with mean of $4.7 \mathrm{~mm}$ (see Table 14-23). Stem shape is also a key determinant in this grouping, with mean ratio of .820 reflecting the narrowness of the neck in proportion to the width of the base. Basal concavities are shallow (mean=1.5 mm) and vary in form from a wide arc to "V" configuration. 


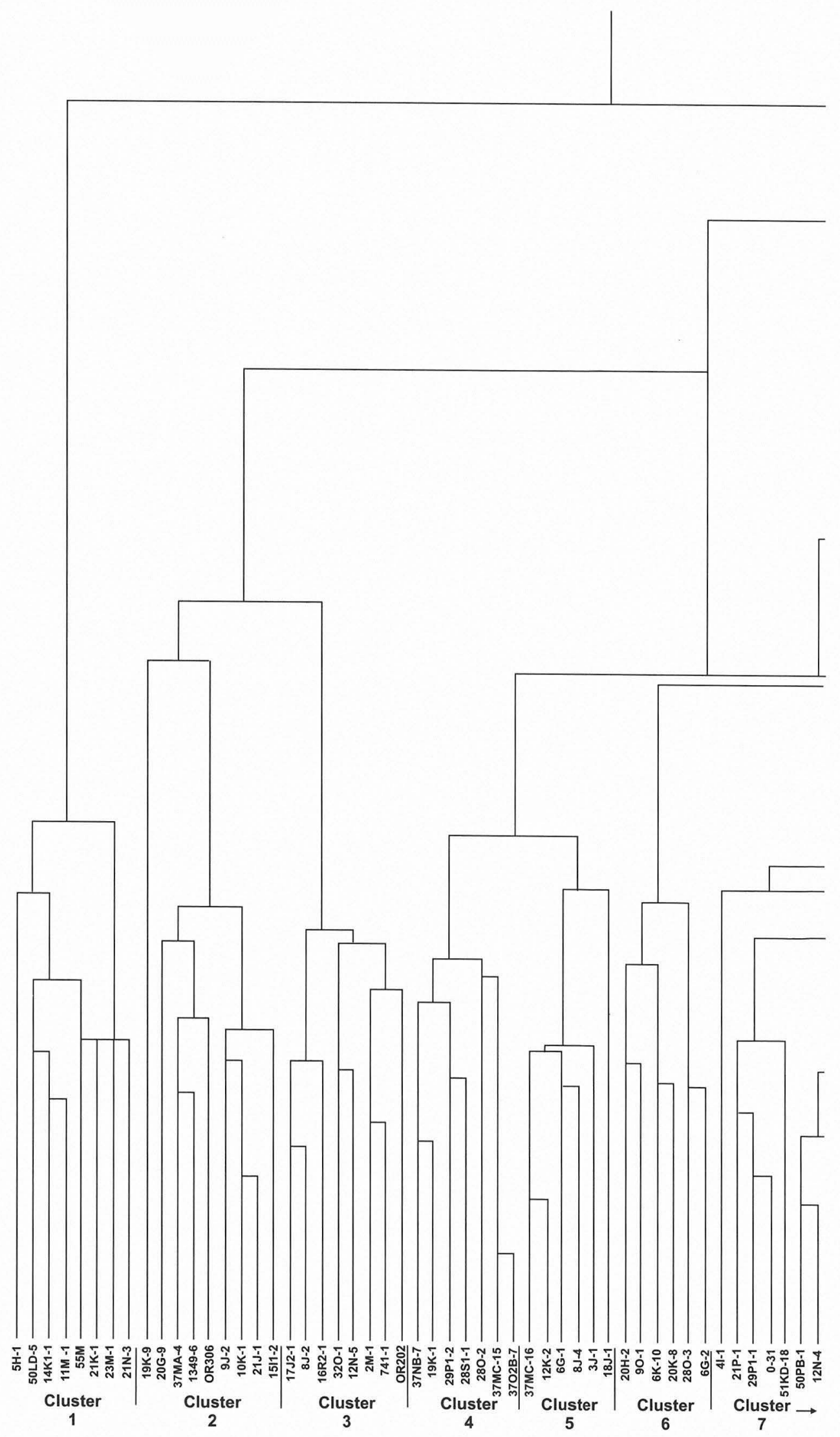

FIGURE 14-5. Hierarchical dendrogram of early bifurcate stem points. 


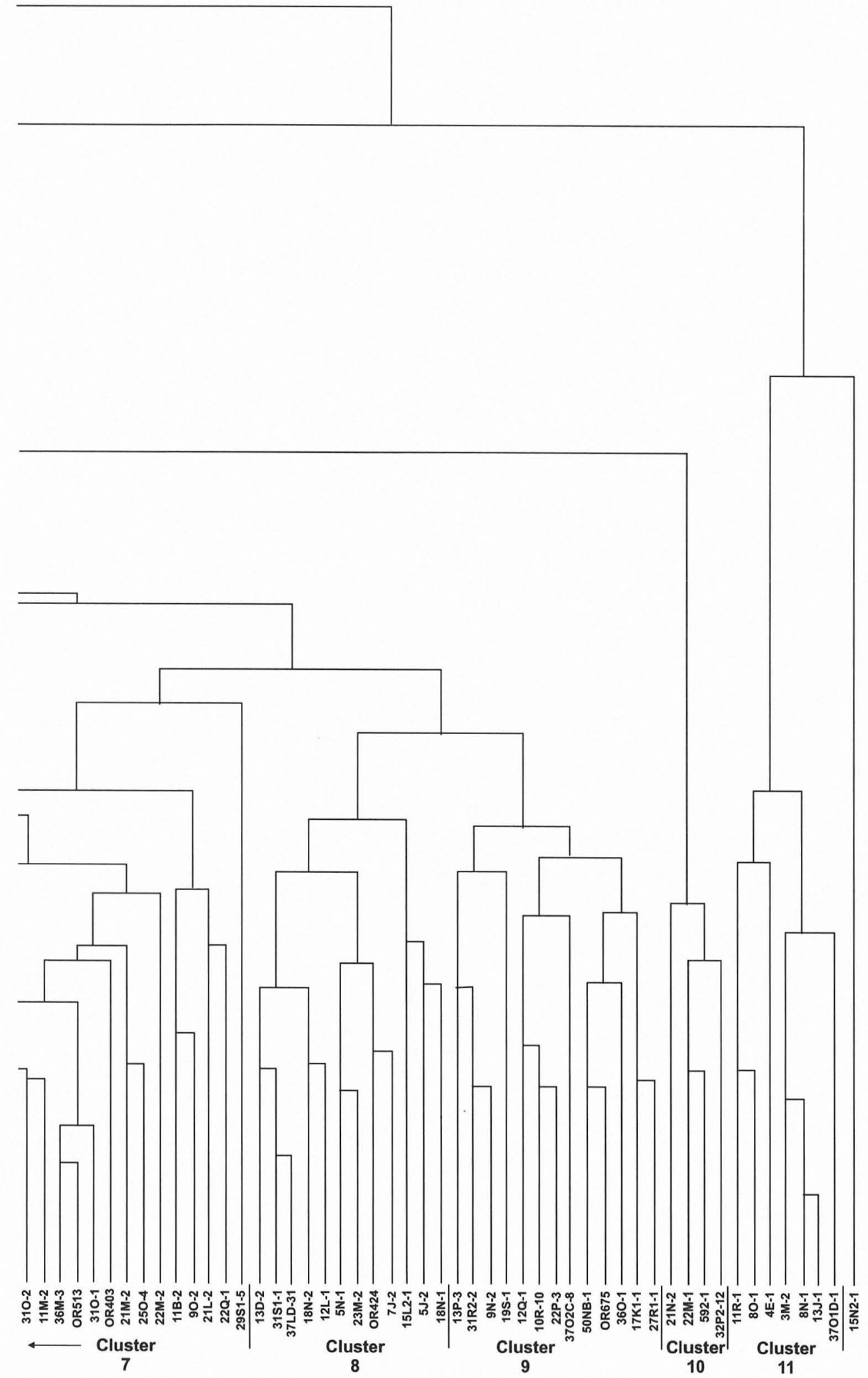

FigURE 14-5 (CONTINUED). Hierarchical dendrogram of early bifurcate stem points. 
TABLE 14-23

Means and Standard Deviations for Early Bifurcate Stem Clusters

\begin{tabular}{|c|c|c|c|c|c|c|c|c|c|c|c|c|c|c|c|c|c|c|c|}
\hline \multirow[b]{2}{*}{ Cluster } & \multirow{2}{*}{$\begin{array}{l}\text { Number of } \\
\text { Specimens }\end{array}$} & \multicolumn{2}{|c|}{$\begin{array}{l}\text { Distal Stem } \\
\text { Width }\end{array}$} & \multicolumn{2}{|c|}{ Stem Shape } & \multicolumn{2}{|c|}{\begin{tabular}{|c|} 
Basal Concavity/ \\
Stem Length \\
\end{tabular}} & \multicolumn{2}{|c|}{$\begin{array}{c}\text { Basal Concavity } \\
\text { Shape }\end{array}$} & \multicolumn{2}{|c|}{ Stem Length } & \multicolumn{2}{|c|}{$\begin{array}{c}\text { Proximal Stem } \\
\text { Width }\end{array}$} & \multicolumn{2}{|c|}{$\begin{array}{c}\text { Basal Concavity } \\
\text { Width }\end{array}$} & \multicolumn{2}{|c|}{$\begin{array}{c}\text { Distal Stem } \\
\text { Thickness }\end{array}$} & \multicolumn{2}{|c|}{$\begin{array}{c}\text { Basal Concavity } \\
\text { Depth }\end{array}$} \\
\hline & & $\overline{\bar{x}}$ & $\sigma$ & $\bar{x}$ & $\sigma$ & $\bar{x}$ & $\sigma$ & $\bar{x}$ & $\sigma$ & $\bar{x}$ & $\sigma$ & $\bar{x}$ & $\sigma$ & $\bar{x}$ & $\sigma$ & $\bar{x}$ & $\sigma$ & $\bar{x}$ & $\sigma$ \\
\hline 1 & 8 & 14.300 & 1.472 & 0.820 & 0.061 & 0.191 & 0.049 & 0.110 & 0.029 & 8.125 & 1.339 & 17.450 & 1.437 & 13.875 & 1.038 & 4.663 & 0.410 & 1.513 & 0.309 \\
\hline 2 & 9 & 16.056 & 2.011 & 0.709 & 0.107 & 0.177 & 0.044 & 0.144 & 0.042 & 12.033 & 1.079 & 22.733 & 1.054 & 15.000 & 2.349 & 6.400 & 0.585 & 2.110 & 0.494 \\
\hline 3 & 8 & 19.063 & 0.890 & 0.926 & 0.073 & 0.187 & 0.032 & 0.245 & 0.071 & 16.238 & 1.393 & 20.650 & 1.178 & 12.725 & 1.830 & 7.625 & 0.886 & 3.038 & 0.595 \\
\hline 4 & 7 & 16.343 & 0.624 & 0.959 & 0.025 & 0.172 & 0.063 & 0.197 & 0.052 & 12.929 & 1.802 & 17.057 & 0.842 & 10.914 & 0.620 & 5.514 & 0.706 & 2.143 & 0.535 \\
\hline 5 & 6 & 14.767 & 0.683 & 0.891 & 0.054 & 0.263 & 0.066 & 0.356 & 0.103 & 11.700 & 0.597 & 16.600 & 1.022 & 8.783 & 0.911 & 6.100 & 0.651 & 3.067 & 0.709 \\
\hline 6 & 6 & 14.233 & 0.480 & 0.854 & 0.063 & 0.352 & 0.075 & 0.681 & 0.089 & 14.817 & 1.817 & 16.717 & 0.950 & 7.583 & 0.958 & 6.400 & 0.632 & 5.167 & 0.909 \\
\hline 7 & 21 & 15.919 & 1.214 & 1.080 & 0.121 & 0.172 & 0.048 & 0.355 & 0.131 & 6.033 & 1.051 & 14.814 & 1.064 & 8.000 & 1.304 & 6.081 & 0.731 & 2.757 & 0.806 \\
\hline 8 & 12 & 16.792 & 0.672 & 0.922 & 0.040 & 0.281 & 0.056 & 0.467 & 0.164 & 15.783 & 1.752 & 18.233 & 0.838 & 9.917 & 1.783 & 6.525 & 0.550 & 4.400 & 0.807 \\
\hline 9 & 13 & 19.062 & 1.117 & 1.114 & 0.081 & 0.164 & 0.045 & 0.311 & 0.077 & 16.754 & 1.789 & 17.154 & 0.923 & 8.900 & 1.496 & 6.331 & 0.568 & 2.723 & 0.686 \\
\hline 10 & 4 & 17.850 & 0.985 & 1.247 & 0.122 & 0.083 & 0.015 & 0.246 & 0.060 & 20.775 & 0.457 & 14.400 & 1.383 & 7.150 & 1.054 & 6.875 & 0.695 & 1.725 & 0.330 \\
\hline 11 & 7 & 21.343 & 2.284 & 0.991 & 0.074 & 0.398 & 0.072 & 0.773 & 0.139 & 19.157 & 1.582 & 21.500 & 0.975 & 9.871 & 0.966 & 6.271 & 1.104 & 7.557 & 0.990 \\
\hline
\end{tabular}


Cluster 1 is a highly discrete group with only two of its members indicated at a very low probability for possible inclusion in another group, which in both cases is Cluster 4 . All of the specimens in Cluster 1 appear to conform to the Bandy type (McReynolds 1993:9-16; Turner and Hester 1993:78).

\section{Cluster 2}

Points $(n=9)$ in this cluster exhibit relatively thin, expanding stems; distal stem width is proportionately narrow in comparison to proximal width (Figure 14-6d-g). Basal concavities typically form a low, wide arc to "V" shape and are relatively shallow (mean $=2.1 \mathrm{~mm}$ ).

Although similar in outline shape, this group is set apart from Cluster 1 by generally larger stem proportions. Distal stem sections range from 5.5 to $7.2 \mathrm{~mm}$ in thickness (mean $=6.4 \mathrm{~mm}$ ). Proximal stem widths are among the largest in the sample, ranging from 20.4 to $24.0 \mathrm{~mm}$ (mean=22.7 mm) (see Table 14-19). Derived from those measurements, the mean stem shape ratio of .709 is lowest of all clusters and accentuates the extreme expanding stem common to this group. There is wide variability in overall stem morphology, as reflected in high standard deviations for stem length $(\sigma=1.1 \mathrm{~mm})$; proximal stem width $(\sigma=1.1 \mathrm{~mm})$; and basal concavity width $(\sigma=2.3 \mathrm{~mm})$. Nonetheless, as gauged by GCP, specimens are well grouped within this sample (see Table 14-22). Only one specimen was identified for alternate placement in different groups (Cluster 1: $p=.09$, and Cluster 3: $p=.01$ ).

In this group are found classic Martindale points (Turner and Hester 1993:151) and expanding stem, concave base variants outlined in Chapter 13.

\section{Cluster 3}

The eight specimens in Cluster 3 display relatively long, thick, slightly expanding stems with proportionately wide distal widths relative to proximal widths (Figure 14-6h-j). Distal stem thicknesses are among the highest in the sample, ranging from 6.7 to $8.8 \mathrm{~mm}$ (mean=7.6 mm). Basal concavity is shallow-to-moderately deep, ranging from 2.1 to $4.0 \mathrm{~mm}$, with a mean of $3 \mathrm{~mm}$. With stem distal widths ranging from 17.6 to $20.4 \mathrm{~mm}$ (mean=19.1 $\mathrm{mm}$ ), and stem lengths ranging from 14.4 to $18.0 \mathrm{~mm}$ (mean=16.2 $\mathrm{mm}$ ), these specimens generally display overall larger stem sections (with the exception of concavity width) than preceding and succeeding specimens in Clusters 1,2, 4, and 5 (see Table 14-23). Only Cluster 2 specimens exhibit wider proximal sections. In Cluster 3, however, there is high variability for this measurement: range is 18.3 to $21.4 \mathrm{~mm}$, with standard deviation of $1.2 \mathrm{~mm}$ from the mean of $20.7 \mathrm{~mm}$. Based on GCP, specimens are well placed within this sample, with only one showing a probability higher than .05 for grouping in another cluster (Cluster 8; see Table 14-22).
Specimens in Cluster 3 do not correspond well with any established types and are encompassed in various expanding stem groups in Chapter 13.

\section{Cluster 4}

Exhibiting slightly expanding stems of medium length, the seven specimens in Cluster 4 are distinctive for the relative thinness of their distal stem sections (Figure 14-7a-d). With a mean of $5.5 \mathrm{~mm}$, stems on these specimens are among the thinnest in the sample, differing from the mean of the thinnest group (Cluster 1) by only .9 mm (see Table 14-23). Unlike Cluster 1, however, specimens in Cluster 4 generally display a wider distal stem width (mean $=16.3 \mathrm{~mm}$ ) and less expanding stem shape. Basal concavities also are comparatively shallow, ranging from 1.4 to $2.8 \mathrm{~mm}$, with a mean of 2.1 $\mathrm{mm}$. Stem shape attributes (distal to proximal stem widths) are in good correlation for this set; standard deviation is only $.025 \mathrm{~mm}$ from a mean of .959 in this category.

Based on GCP, the cluster is unstable; three of the seven specimens showed high probability (greater than .10) for inclusion in other groups (see Table 14-22). The greatest correspondence is shown with Cluster 5 .

The points in Cluster 4 conform to traditional Hoxie (Turner and Hester 1993:130), and Gower (Turner and Hester 1993:128); others are expanding stem varieties, described in Chapter 13.

\section{Cluster 5}

In this rather diverse group of six specimens, relatively smaller stem size and relationship between stem length and basal concavity are significant (see Table 14-23). As shown in Figure 14-7e-h, some specimens exhibit slightly expanding, although somewhat recurved, short stems (mean $=11.7$ $\mathrm{mm}$ ). Compared to the other clusters with relatively short expanding stems (Clusters 1, 2, 4, and 6), Cluster 5 displays a much narrower basal concavity width (mean $=8.8 \mathrm{~mm}$ ). In stem shape, Cluster 5 resembles Cluster 6, but it is set apart by generally smaller dimensions. Basal concavity depths are moderate $($ mean $=3.1 \mathrm{~mm})$. Proximal stem width (mean $=16.6 \mathrm{~mm}$ ) is relatively narrow in proportion to distal stem width (mean=14.8 mm), accounting in some cases for the slightly recurved or almost contracting appearance of the stem.

Cluster 5 can be seen as a morphological transition between Clusters 4 (see Figure 14-7a-d) and 6 (Figure 14$7 \mathrm{i}-\mathrm{k}$ ). Based on GCP, none of the specimens in Cluster 5 is indicated at full probability for inclusion in the group (see Table 14-22). According to this measure, its members showed affinities with four other clusters, most strongly Cluster 4.

Most specimens in this group fall within the morphological parameters of the Uvalde (Turner and Hester 1993:191) and Gower types. 


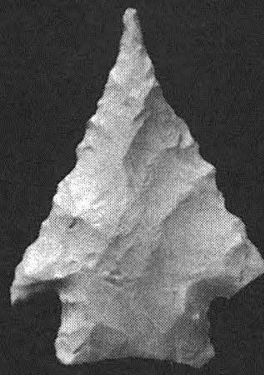

a

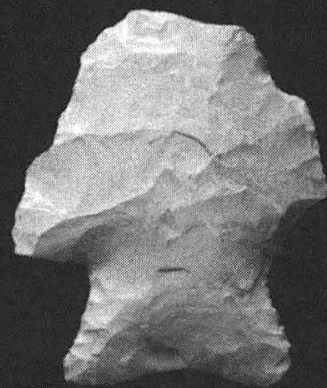

e

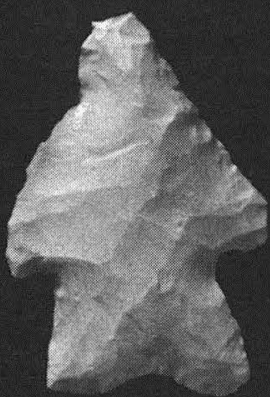

i

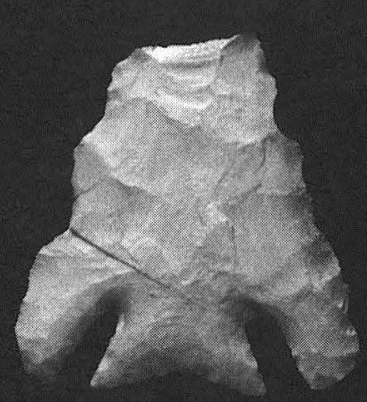

b

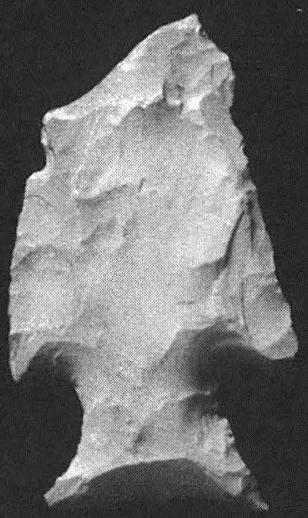

f

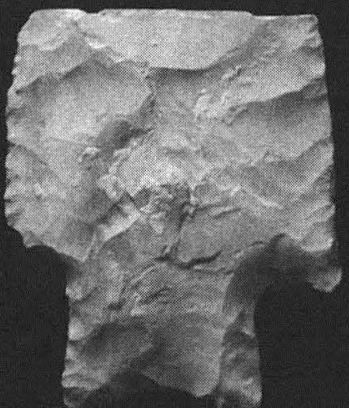

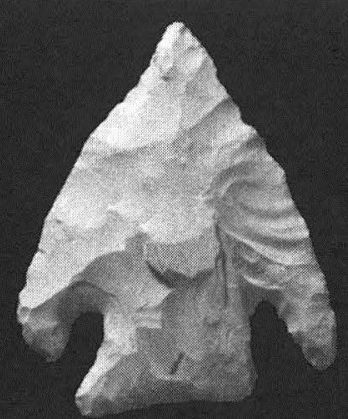

c

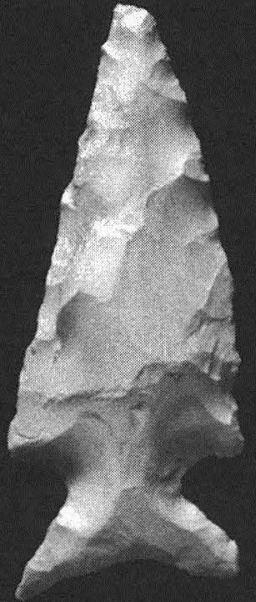

g

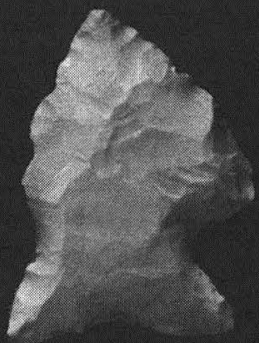

d

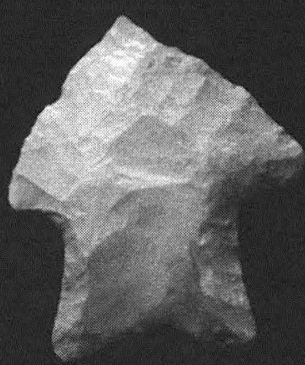

h

FIGURE 14-6. Representative specimens in early bifurcate stem morphological groups. (a-c) Cluster 1; (d-g) Cluster 2; (h-j) Cluster 3. Specimen numbers: (a) 21K-1; (b) 55M; (c) 21N-3; (d) 21J-1; (e) 1349-6; (f) 20G-9; (g) 19K-9; (h) 2M-1; (i) 17J2-1; (j) 8J-2.

\section{Cluster 6}

Members of Cluster $6(n=6)$ are similar in outline shape and dimensions to Cluster 5 but are set apart by slightly more expanding, generally longer stems and narrower, deeper basal concavities (see Figure 14-7i-k). Stem lengths range from 13.0 to $17.1 \mathrm{~mm}$ (mean=14.8 $\mathrm{mm}, \sigma=1.8 \mathrm{~mm}$ ) and basal concavity depths from 3.6 to $6.1 \mathrm{~mm}$ (mean=5.2 $\mathrm{mm}, \sigma=.9 \mathrm{~mm}$ ). As reflected in the index, this relationship of relatively long stem to deep basal concavity (ratio mean $=.352 \mathrm{~mm}$ ) is distinctive within the sample, second only to that of Cluster 11 (see Table 14-23).

According to GCP (see Table 14-22), Cluster 6 is a somewhat unstable group. Four of the members are candidates- 


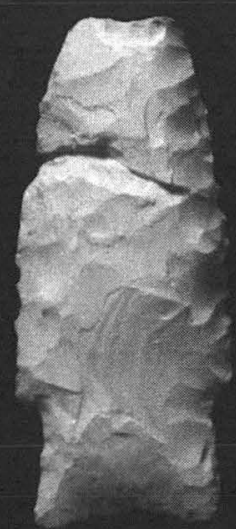

a

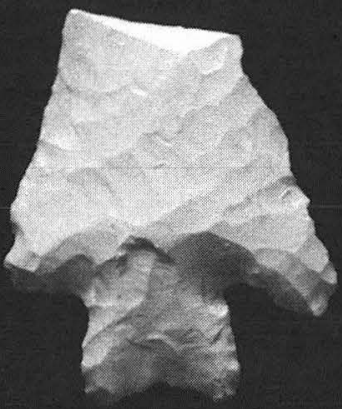

e

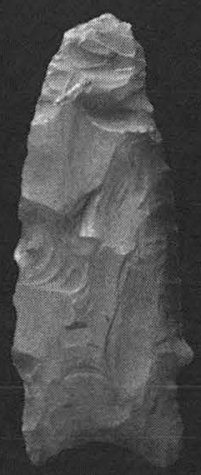

b

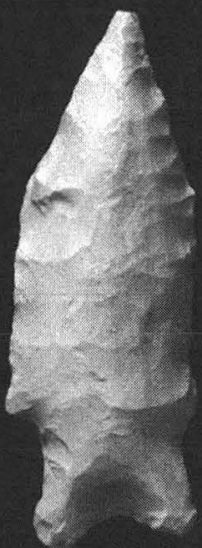

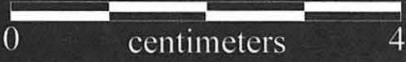

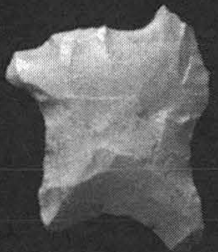

c

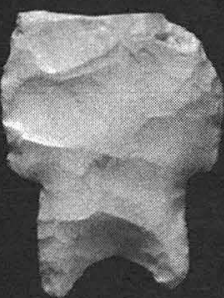

g

.

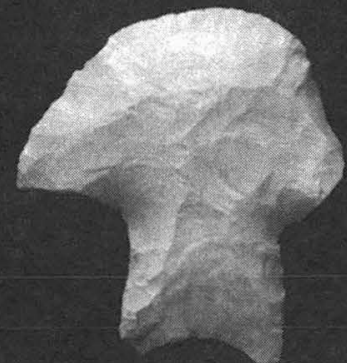

d

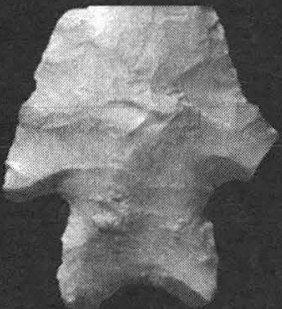

h

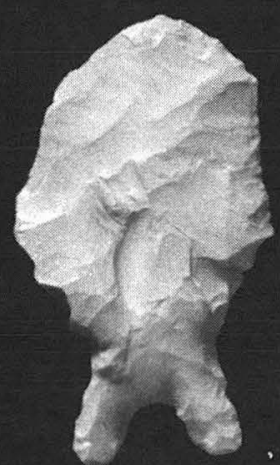

f

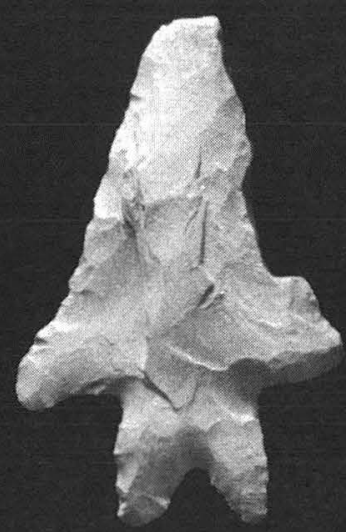

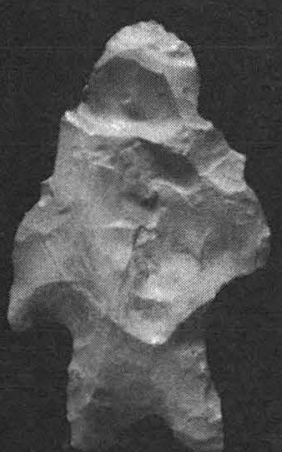

K

FIGURE 14-7. Representative specimens in early bifurcate morphological groups. (a-d) Cluster 4; (e-h) Cluster 5; (i-k) Cluster 6. Specimen numbers: (a) 29P1-2; (b) 37O2B-7; (c) 28O-2; (d) 37NB-7; (e) 37MC-16; (f) 12K-2; (g) 6G-1; (h) 8J-4; (i) 20H-2; (j) 9O-1; (k) 20K-8.

with probabilities greater than or equal to .05 - for inclusion in at least one other group; most consistent alternatives are Clusters 5 and 8 . One specimen was classified at only $p=.44$ for membership in Cluster 6 .

Specimens in this cluster share affinities with traditional Baker (Turner and Hester 1993:77), Uvalde, and Gower points.

\section{Cluster 7}

With 21 members, Cluster 7 is the largest group identified in the study and manifests an array of subtle variations (Figure 14-8a-f). Stems range from slightly constricted, to straightsided, to gently expanding. Stem sizes are generally in the 
moderate range in comparison to most other groups, with distal and proximal width in close congruity (mean of 15.9 and $14.8 \mathrm{~mm}$, respectively) producing an average stem shape ratio for the group of 1.080 (see Table 14-23). This latter figure reflects a more straight to recurved shape in contrast to the expanding stem Clusters 1,2,3, and 5, with mean stem shape ratios of $.820, .709, .926$, and .891 , respectively. Although more comparable in stem shape ratio to Clusters 9 and 10 (mean $=1.114$ and 1.247 , respectively), Cluster 7 is set apart from those clusters by smaller stem proportions in length and distal thickness (see Table 14-23).

By Geisser Classification, Cluster 7 is fairly stable, with six specimens showing probability greater than or equal to .05 for inclusion in other groups. One of these was classified as a "miss" in Cluster 7, with a probability of .49 for incorporation in Cluster 8. Another specimen, classified with $p=.59$ in the present cluster, shares affinities equal to or greater than $p=.05$ with three other clusters $(4,8$, and 9$)$.

Variation in this group is encompassed within types Hoxie, Gower, Uvalde, and a constricted stem group described in Chapter 13.

\section{Cluster 8}

Encompassing attributes of both the expanding stem and long, straighter stemmed groups, the 12 specimens in Cluster 8 form a very diverse group (Figure 14-8g-j). A key variable in composition, however, is proximal stem width, which, ranging from 17.1 to $20.2 \mathrm{~mm}$ (mean=18.2; $\sigma=.8 \mathrm{~mm}$ ), is among the largest for that dimension in all clusters (see Table 14-23). Basal concavity shape, reflected in concavity depth compared to width, is relatively distinctive (mean $=.467 \mathrm{~mm}$ ).

Cluster 8 is not a highly standardized group, according to GCP (see Table 14-22). All but two of its members showed probabilities greater than .05 for inclusion in other groups, and of these, five members showed a probability of .20 or higher. One of these was indicated as a "miss," demonstrating $p=.85$ for grouping in Cluster 6 .

Specimens in Cluster 8 share attributes with classic Hoxie, Uvalde, Gower, and Baker types.

\section{Cluster 9}

Stem shape ratio and basal concavity are two determinants in the formation of this group of 13 specimens (Figure $14-8 \mathrm{k}-\mathrm{n})$. Stems are generally parallel-sided to slightly convex (with one notable concave-sided exception; see Figure $14-8 \mathrm{n}$ ), and wide at distal sections (mean $=19.1 \mathrm{~mm}$ ) in proportion to proximal width (mean $=17.1 \mathrm{~mm}$ ), producing a comparatively high stem shape ratio $($ mean $=1.114 \mathrm{~mm})$. Basal concavities primarily are shallow, displaying a mean depth of $2.7 \mathrm{~mm}$.

According to GCP, Cluster 9 is somewhat weakly composed (see Table 14-22). Three specimens were identified as "misses," showing greater classification probability for Clus- ter $4(p=.54)$, Cluster $7(p=.60)$, and Cluster $8(p=.65)$. These specimens were reassessed through visual inspection, and a decision was made to retain them in Cluster 9 as originally assigned.

Cluster 9 constitutes specimens described as variants of Hoxie in Chapter 13.

\section{Cluster 10}

This small group of four specimens is exemplified by very long, narrow constricted stems with very shallow and narrow basal concavities (Figure 14-9a-c). Stem lengths vary from 20.3 to $21.3 \mathrm{~mm}$ (mean=20.8 $\mathrm{mm}$ ); stem proximal widths range from 12.7 to $15.8 \mathrm{~mm}$ (mean=14.4 $\mathrm{mm}$ ); and basal concavity depths range from 1.3 to $2.1 \mathrm{~mm}$ (mean=1.7 mm). Ratios derived from stem measurements express the distinctiveness of these specimens relative to the rest of the sample. As shown in Table 14-23, stem shape (mean=1.2) and basal concavity/stem length index (mean $=.083$ ) are at the extremes for those attributes among all clusters.

All specimens are classified with probability of 1.00 for inclusion in Cluster 10 (see Table 14-22). Specimens in this group chiefly conform to the Hoxie type.

\section{Cluster 11}

The seven Cluster 11 specimens are distinguished overall among the straighter stemmed groups by greater size in almost all dimensions (Figure 14-9d-g). As shown in Table 14-23, basal concavity depth is a key factor in segregation of this group. Ranging from 6.1 to $8.7 \mathrm{~mm}$, this measurement, on average, differs from that of nine other groups by at least $3 \mathrm{~mm}$. When this attribute is considered in tandem with stem length (mean $=19.2 \mathrm{~mm}$ ), the derived ratio is also higher than that for all clusters (mean=.398) The distinctiveness of this group is expressed in other ratios as well (see Table 14-23). Basal concavity shape, reflecting the ratio of basal concavity width to depth, is also significant; with a mean of .773, it differs by at least .306 from averages for nine of the other groups. As shown, the most similar group in these relational attributes is Cluster 6; however, it clearly differs in stem shape, displaying a markedly expanding stem, and in being proportionally smaller.

Based on GCP, one specimen (see Figure 14-9d) was identified with $p=.43$ for classification in Cluster 8. With the six other specimens each exhibiting a probability of 1.00 , this cluster can be considered well grouped within the present sample.

All of the specimens in Cluster 11 appear to fall within the established Jetta type (Turner and Hester 1993:131).

\section{Cluster 11 Outlier}

This specimen, because of it rather extraordinary size, was isolated from Cluster 11 for purposes of this statistical exercise, although it clearly follows the morphological pattern 


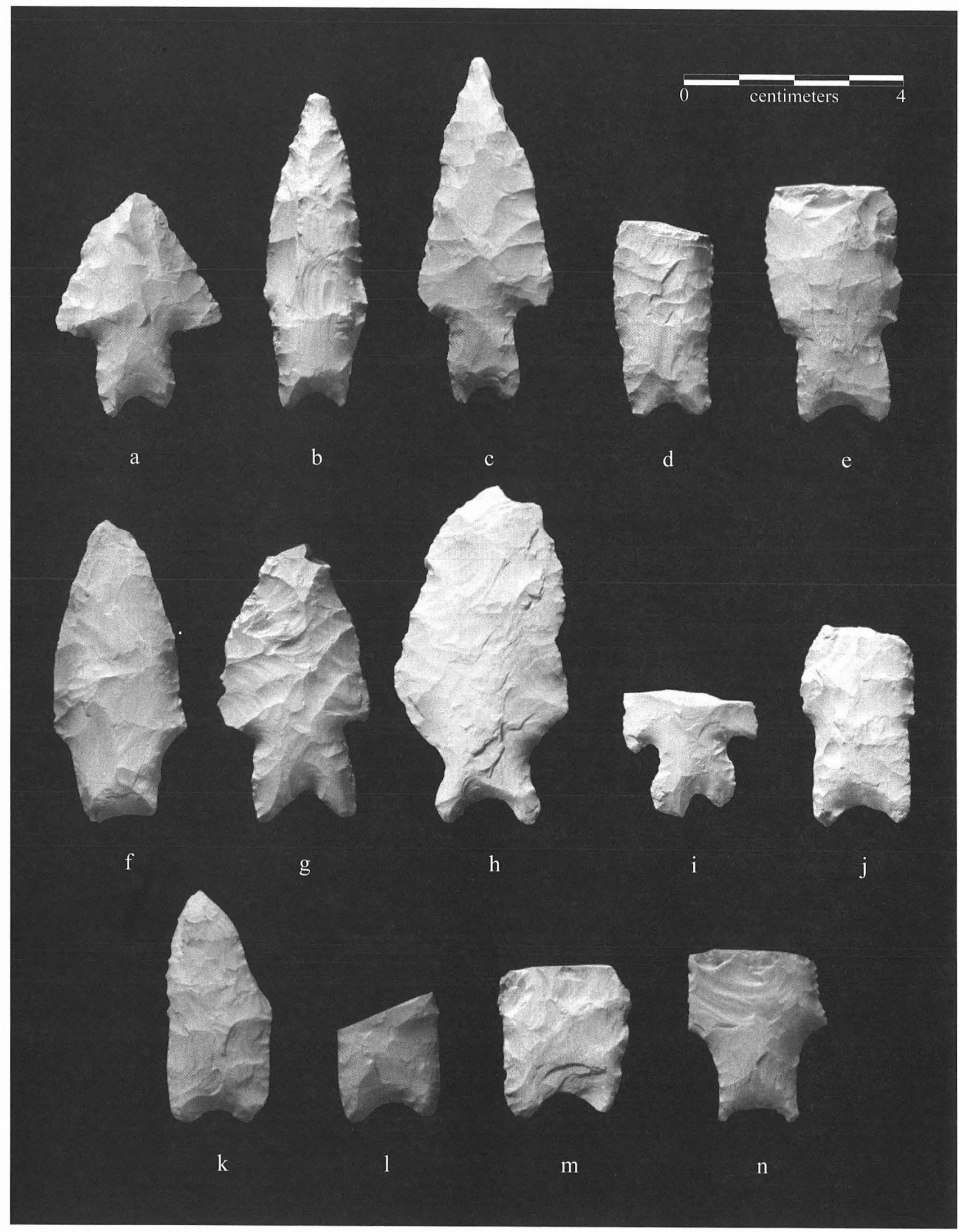

FIGURE 14-8. Representative specimens in early bifurcate stem morphological groups. (a-f) Cluster 7; (g-j) Cluster 8; (k-n) Cluster 9. Specimen numbers: (a) 11M-2; (b) 25O-4; (c) 9O-2; (d) 22Q-1; (e) 21P-1; (f) 29S1-5; (g) 12L-1; (h) 7J-2; (i) 5J-2; (j) 13D-2; (k) 22P-3; (l) $12 \mathrm{Q}-1$; (m) 27R1-1; (n) 36O-1. 


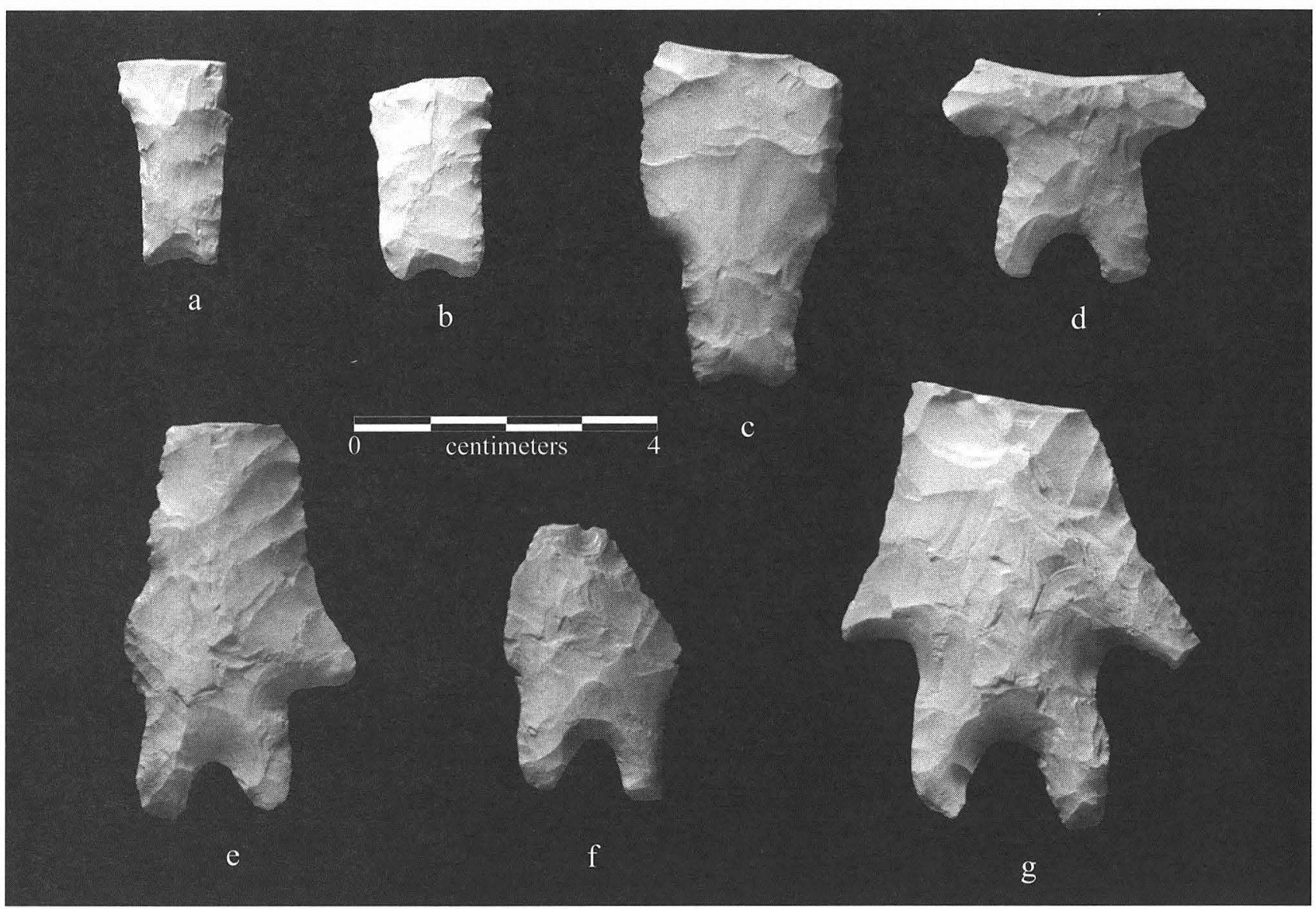

FIGURE 14-9. Representative specimens in early bifurcate stem morphological groups. (a-c) Cluster 10; (d-f) Cluster 11; (g) Cluster 11 outlier. Specimen numbers: (a) 592-1; (b) 21N-2; (c) 22M-1; (d) 11R-1; (e) 4E-1; (f) 8N-1; (g) 15N2-1.

of that group (see Figure 14-9g). Its stem measurements are at least $2 \mathrm{~mm}$ larger than any other specimen in Cluster 11 but is classed with a probability of 1.00 for inclusion in that group. Dimensions include a distal stem width of $25.7 \mathrm{~mm}$; stem shape ratio of $1.004 \mathrm{~mm}$; basal concavity/stem length index of $.461 \mathrm{~mm}$; basal concavity shape index of $.836 \mathrm{~mm}$; stem length of 24.3; proximal stem width of $25.6 \mathrm{~mm}$; basal concavity width of $13.4 \mathrm{~mm}$; distal stem thickness of $8.3 \mathrm{~mm}$; and basal concavity depth of $11.2 \mathrm{~mm}$. This specimen corresponds with the Jetta type.

\section{Summary}

Eleven clusters were identified in the Early Bifurcate Stem sample from the Wilson-Leonard site. While the central clusters proved to be the least stable in various sortings, groups on the margins were more fixed, consistently emerging in roughly the same three clusters. These comprise expanding stem forms found in Clusters 1 and 2 (cf. Bandy and Martindale-like, respectively) and the deeply concave, long-stemmed (Jetta-like) specimens in Cluster 11. Cluster 10 , encompassing four specimens with long, straightedged, heavily ground stems, also display relative stability.
Gauged by Geisser Classification Probabilities, the sample is generally well sorted. As classified within the final groupings, $95 \%$ of the specimens were judged as hits and $5 \%$ misses. Clusters 1,2 , and 10 showed highest probability as groups, followed by Clusters 3 and 11 . Cluster 9 exhibited the greatest number of "misses" ( $\mathrm{n}=3)$.

In almost all cases, key factors in segregating clusters proved to be derived ratios emphasizing relationships among metric attributes, rather than absolute measurements alone. Stem shape (ratio of stem distal width to proximal width) and index of basal concavity depth to stem length are particularly significant.

In cases where ratios did not come into play, certain metric attributes were sufficiently unusual to set apart groups of specimens. Examples are Cluster 1, which hinges strongly on thinness of distal stem, and Cluster 3, which keys, conversely, on thick distal stem measurements. Surprisingly, the same attribute proved to be the single-most unifying factor in the remainder of the sample. In the nine other clusters, mean distal thicknesses are all slightly below or above $6 \mathrm{~mm}$ with generally low standard deviations, a somewhat remarkable regularity in what is in many other ways a very diverse group of points. An exception is Cluster 11, which encompasses a fairly 
diverse range of values for this attribute, with a standard deviation of $1.104 \mathrm{~mm}$.

For other metric attributes, a fairly high range of variability is encompassed within most groups. Among the 55 means calculated for nonratio metric attributes for the 11 clusters, nearly half ( 27 of 55 , or $49.1 \%$ ) show standard deviations of more than $1 \mathrm{~mm}$ (see Table 14-23).

\section{Discussion: A Note on Typological Conundrums among Early Bifurcate Stem Points}

With several exceptions, the study essentially has borne out what many typologists have long suggested, namely, that the considerable variation within Early Archaic stemmed points can be demonstrated as a continuum of attributes and that several defined "types" appear to grade morphologically from one to another. Only in the case of two clusters (Bandy and Jetta) and, to some extent those containing the classic Martindale and Hoxie, could traditional types be statistically validated, and even in those cases, there will be typologists who will reach different interpretations.

Indeed, when the findings between computer-generated clusters are compared to groups identified by standard typological approaches (see Chapter 13), the results, for the most part, are graphic testimony to the torturous process of classifying early bifurcate stem points. Specimens in Chapter 13 were sorted chiefly on the basis of stem morphology as well as certain technological attributes, and according to established typologies in Turner and Hester (1993) and Suhm and Jelks (1962). Specimens that did not fit well into specific types were placed in broad, morphologically descriptive groups or in groups linking two type names (e.g., Gower/ Uvalde).

As shown in Table 14-24, the 8 members of Cluster 3 were classified in Chapter 13 among 5 different groups, the 7 members of Cluster 4 among 4 different groups, the 6 members of Cluster 5 among 4 groups, the 6 members of Cluster 6 among 5 groups, the 21 members of Cluster 7 among 8 groups, and the 12 members of Cluster 8 among 8 groups.

Exceptions to this pattern are Clusters 1 and 11, shown to be $100 \%$ in correlation with Chapter 13 type assignments (Bandy and Jetta, respectively). An intriguing degree of congruence also is seen in Cluster 9 and the Hoxie variants, as well as Cluster 10 and the Hoxie $\mathrm{C}$ group.

A certain degree of disparity between the sets of findings is to be expected, inasmuch as intuitive typology considers an array of qualitative as well as quantitative variables. As mentioned above, when the qualitative attribute of stem grinding was factored into the mathematical analysis, much less coherent clusters were the result. Although only a minor attempt, this particular experience brought to light some of the problems involved in attempting to statistically measure and integrate qualitative attributes. What the present statistical method served to highlight, however, were relationships among quantitative attributes that were difficult to recognize and integrate intuitively.

When bifurcate stem clusters are plotted stratigraphically, temporal patterning is evidenced in several cases and appears to hinge largely on stem shape (relative straightness of stem versus expansion), as shown in Table 14-25. Members of the expanding stem Bandy-like Cluster 1 and Martindale-like Cluster 2 occur later in the site's history, the former in the stratigraphic Unit IIIa/b transition zone, Unit IIIb, and associated transition zones in the Valley Margin, and the latter in the same units, as well as Unit IIIb/c transition zone and Unit IIIc. Another expanding stem group, the predominately Uvalde-like Cluster 5, is seen only in IIIb, IIIc, and related Valley Margin units. Occurring much earlier, the wide, relatively thick and straight-stemmed Hoxie-like specimens comprising Cluster 9 are confined chiefly to the Unit II/IIIa transition zone, Unit IIIa, and related Valley Margin zones. In contrast, members of the largest and least stable clusters are widely distributed; Cluster 7 specimens occur in virtually all stratigraphic units.

Although the individual clusters appear to have relatively slight temporal integrity, this study has verified certain previously recognized broad morphological or stylistic trends over time. That is, straighter-stemmed bifurcate points appear to occur earlier than the expanding stemmed bifurcates (see Chapter 13 for further discussion along these lines).

In assessing the findings and evaluating the implications of this study, several key issues should be addressed. One is the relatively large time depth represented in the deposits from which the sample was taken. Specimens were drawn from contexts spanning some 4,000 years and, in many cases, what were likely stable surfaces for long periods of time. In behavioral terms, those millennia saw the coming and going of many groups, with countless knappers leaving (resharpened) representatives of their work behind.

It would seem axiomatic, then, that points derived from more temporally discrete contexts-representing shorter time periods and fewer artisans - would be more likely to display less morphological variability. However, the variability displayed in points from perhaps more isolated components, such as that of the Sleeper site (Johnson 1991:105-111), tends to argue against this idea. The 13 points within the "Early Split Stem Series" include forms Johnson designates as both Gower and Uvalde.

With present data, it appears that considerable variability is the norm among early bifurcate stem points. Exceptions for the most part, are found at the morphological extremes, among clusters recognized as Bandy, Jetta, and, to some extent, Martindale. If there are other standardizations, we have not yet been able to identify them, either using traditional, intuitive typology or through mathematical analyses of metric variables.

This brings us to a second key point which is, if variability within a larger morphological class is the norm, what 
TABLE 14-24

Comparison of "Types" to Morphometric Classifications of Bifurcate-stemmed Projectile Points

\begin{tabular}{|c|c|c|c|c|c|c|c|c|c|c|c|c|c|c|c|c|c|c|c|c|c|c|}
\hline Cluster & 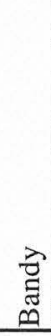 & $\begin{array}{l}\stackrel{\mathscr{X}}{x} \\
0 \\
\text { I }\end{array}$ & $\begin{array}{l}\varangle \\
.0 \\
\not \\
0 \\
\text { I } \\
\end{array}$ & 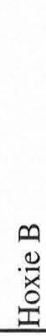 & $\begin{array}{l}u \\
0 \\
0 \\
0 \\
0 \\
\end{array}$ & $\begin{array}{l}\vec{D} \\
3 \\
0 \\
0\end{array}$ & 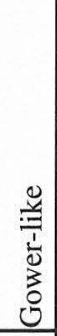 & $\begin{array}{l}\frac{0}{0} \\
\frac{\pi}{3} \\
2 \\
0 \\
0 \\
0 \\
0\end{array}$ & $\frac{\frac{\pi}{\pi}}{3}$ & 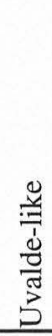 & 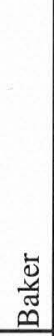 & 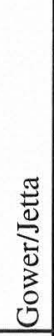 & $\underset{\Xi}{\tilde{E}}$ & 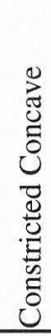 & 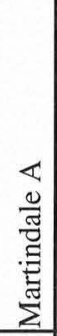 & 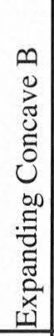 & 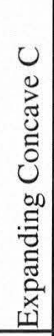 & 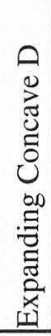 & 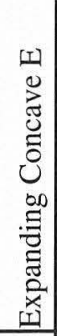 & 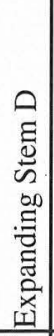 & $\begin{array}{l}E \\
0 \\
0 \\
0 \\
00 \\
0 \\
0 \\
\end{array}$ & 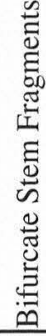 \\
\hline 1 & 8 & & & & & & & & & & & & & & & & & & & & & \\
\hline 2 & & & & & & & & & & & & & & & 5 & 2 & 2 & & & & & \\
\hline 3 & & 1 & & & & & & & & & & & & & 1 & 1 & & 3 & 2 & & & \\
\hline 4 & & 3 & & 1 & & 1 & & & & & & & & & & & & 2 & & & & \\
\hline 5 & & & & & & & 1 & 2 & 2 & & & & & & & & & 1 & & & & \\
\hline 6 & & & & & & 1 & & 1 & 1 & & 2 & & & & & & & & & & & 1 \\
\hline 7 & & 8 & & 2 & & 3 & 1 & & 1 & 2 & & & & 3 & & & & & & 1 & & \\
\hline 8 & & 2 & & & & 1 & 2 & 2 & 2 & & 1 & 1 & & & & & & & & & & 1 \\
\hline 9 & & 5 & 6 & 1 & & & & & & & & & & & & & & 1 & & & & \\
\hline 10 & & & & & 3 & & & & & & & & & & & & & & & & 1 & \\
\hline 11 & & & & & & & & & & & & & 8 & & & & & & & & & \\
\hline
\end{tabular}

does this variability imply, from functional, technological, and behavioral standpoints? Perhaps more so than searching for the cultural type(s) or template(s) for this period, focus should be directed toward the various types of activities and uses that allowed for diverse haft specifications corresponding to the early bifurcate stem points. As Keeley (1982:801) has noted, different hafting arrangements often require different shapes in tangs, but differences in hafts and stem shapes do not necessarily imply different uses, or for that matter, different individuals, groups, or cultures.

Finally, consideration should be given, in some instances, to stem variability as reflective of rehafting or reworking of the stem, an activity traditionally viewed as labor intensive and likely to occur infrequently. It should be recalled that, for too many years, typologists failed to recognize that certain differences in blade shape derived from use and refurbishing rather than stylistic considerations, as Collins (1975), Hoffman (1985), and others have noted. Such manifestations as asymmetrical, very narrow, or highly beveled blades have been included as diagnostic traits for certain types, rather than products of resharpening. In a similar vein, are we failing to weigh the possibilities of haft variability borne of rebasing, for instance, a projectile point broken in or just above the haft.

This study has focused exclusively on basal attributes that can be mathematically quantified and manipulated. As other analysts have noted, statistical analyses of this sort have not successfully integrated technological details that are often critical in separating certain types (Hester 1993:37). In future studies, focus may need to be directed toward incorporating qualitative variables, as well as toward understanding possible functional and technological significance of the subtle variabilities in early bifurcate stem points. Detailed microscopic examinations for haft and use wear in a large series of bifurcate stem points might shed light on some of these issues.

Finally, of course, the search for larger samples in more tightly constrained contexts should continue. Another parameter to consider more precisely is geographic ranges of variants in this series. Assemblages isolated either stratigraphically or spatially afford the opportunity to examine such technological evidence as the nature of preforms and knapping strategies that could provide additional clues toward understanding the variability among bifurcate stem points.

\section{GENERAL CONCLUSIONS}

The two analyses, of unfluted lanceolate and of early bifurcate stem projectile points, examined the morphological variability represented in these two groups. The results suggest the purpose that such carefully focused analyses fulfill. Such systematic analyses can help define patterns in morphology that can then be evaluated with chronological and technological information for the purpose of typology. The morphological variability is organized in a consistent manner which is reliable and not as vulnerable to intuitive distractions as can be the case with more traditional approaches.

Among the unfluted lanceolate point sample, some discrete morphological patterns with chronological integrity were identified, such as St. Mary's Hall and Plainview or Golondrina-Barber and Plainview. But, as can be seen with Golondrina-Barber and Dalton or Angostura and Lubbock, morphology alone did not define types that had consistent 
TABLE 14-25

Early Split-stem Morphological Clusters by Stratigraphic Unit

\begin{tabular}{|c|c|c|c|c|c|c|c|c|c|c|c|}
\hline \multirow[b]{2}{*}{ Unit } & \multicolumn{11}{|c|}{ Cluster } \\
\hline & 1 & 2 & 3 & 4 & 5 & 6 & 7 & 8 & 9 & 10 & 11 \\
\hline IIIc & & 2 & & & 1 & 1 & 2 & 1 & & & 1 \\
\hline $\mathrm{IIIb} / \mathrm{c}$ & & 1 & 1 & & & 1 & & & 1 & & \\
\hline Y/IIIc & 1 & & 1 & & 2 & & & 1 & & & \\
\hline $\mathrm{Y} / \mathrm{IIIb} / \mathrm{c}$ & & 1 & & & & & & & & & \\
\hline IIIb & 4 & 3 & 1 & 2 & 2 & 1 & 3 & 3 & & 1 & 1 \\
\hline $\mathrm{Y} / \mathrm{IIIb}$ & & 1 & & 1 & & & & & & & \\
\hline $\mathrm{Y}$ & & & & & 1 & 1 & 1 & 1 & & & \\
\hline $\mathrm{III} / \mathrm{b}$ & 2 & 1 & 2 & 2 & & 1 & 5 & 1 & & 2 & 1 \\
\hline IIIa & & & 1 & 1 & & & 6 & 2 & 5 & 1 & 1 \\
\hline II/IIIa & & & & 1 & & & 1 & & 3 & & 1 \\
\hline $\mathrm{X}$ & & & & & & 1 & 1 & 3 & 2 & & 3 \\
\hline $\mathrm{X} / \mathrm{II}$ & & & & & & & & & 1 & & \\
\hline II & & & & & & & 1 & & & & \\
\hline III & 1 & & & & & & 1 & & 1 & & \\
\hline Totals: & 8 & 9 & 6 & 7 & 6 & 6 & 21 & 12 & 13 & 4 & 8 \\
\hline
\end{tabular}

Note: Not shown are 5 specimens that had no provenience or were from undifferentiated Unit III.

temporal or geographic parameters. In future research, characteristics of flake patterns and basal modification, which might be chronologically sensitive, should be included.

For early bifurcate stem points, some discrete morphological patterns with some temporal estimate, such as Bandy and Jetta, and to a lesser extent Martindale, were recognized. In contrast, a morphological continuum including variations of Hoxie, Gower, and Uvalde overlap and may only be distinguished by characteristics other than basal morphology, and even then, such distinctions are questionable in many cases. Since the early bifurcate stem analysis did not receive the same amount of attention as the unfluted lanceolate analysis, it does not present the same level of resolution. The early bifurcate analysis could benefit from an analysis of variance among five of the clusters which cover the Hoxie-Gower-Uvalde continuum. At this stage in the analysis it is clear only that we still have not identified all the significant characteristics that would sort out the types in this continuum, Gower and Hoxie in particular. Further analysis on this part of the sample would be enhanced by including available specimens from the original type sites and from well-dated contexts at other sites. A better understanding of projectile point morphology in the Early Archaic could be achieved by comparing Gower and Hoxie morphology with that of Angostura and Thrall, all of which co-occur at the Wilson-Leonard site. A correlated study of the technology and manufacture of Gower, Hoxie, Thrall, and Angostura types also would be enlightening.
One of the broad implications for the typological process emerging from both of these analyses is the need for flexibility when setting the acceptable range of variability for a defined type. Setting a very rigid, narrow range, as Kelly $(1982,1983 a)$ did for Golondrina and Barber, can lead to a proliferation of types which all represent the same time period or cultural pattern. On the other hand, creating a very wide ranging type, as has been the case with Plainview or the broadly defined Early Split-Stem categories of Prikryl (1990) and Johnson (1991), may lead to a masking of cultural patterns which go unrecognized within a larger, possibly meaningless classification. It is important to look at as much of the available evidence on morphology, technology, chronology, and assemblage composition as possible in order to make informed interpretations about typology, a message first noted by Krieger a half century ago.

Finally, as the historical reviews suggest, named types are in a continuous process of refinement and reexamination as new evidence and larger samples become known. As defined types become meaningless, as with Yuma (Wormington 1957), or no longer meet current research needs, as with early bifurcates (Hester 1979b:5), changes should be made. These changes can be unsettling and require persistence on the part of analysts (see Krieger 1947b), but ultimately our understanding of the prehistoric past hinges on these definitions, not as ends but as means (Thoms 1993). 
"This page intentionally left blank" 


\section{Chapter 15}

\section{CLEAR FORK TOOLS}

by Susan W. Dial

\section{INTRODUCTION}

Clear Fork and other distally beveled tools from contexts spanning Paleoindian to Late Archaic times at the Wilson-Leonard site are reported in this chapter. Although considerable form and size variability is evinced in this sample, only two different groups of Clear Fork tools are recognized as significant, based on technological differences and temporal patterning. A third distally beveled tool form, provisionally termed the "Brushy Creek biface," is considered sufficiently distinct both morphologically and temporally to warrant consideration as a separate category. For the larger Clear Fork assemblage, it is suggested that much of the variation in size, outline, and bit shape is a product of the use-rejuvenation process. Use-wear studies suggest no functional correlates for morphological types as presently understood but indicate all forms were likely used on hard contact materials in tasks such as woodworking. Other uses, however, including working of bone and hide scraping, are also indicated.

\section{BACKGROUND OF THE CLEAR FORK TYPE}

Since its initial description by Cyrus Ray in 1929, the Clear Fork tool has been the subject of extensive morphological analyses (Brown et al. 1982; Goode 1989; Hall et al. 1982; Hall et al. 1986; Ray 1938, 1941, 1961; Taylor and Highley 1995) and, to a somewhat lesser extent, experimental replicative and use-wear studies (Chandler 1974; Hester et al. 1973; Howard 1975; Hudler 1997; Shiner 1975:179-188). The broadly defined classification encompasses a triangular to subtriangular biface or uniface, with a beveled or concave (hollowed or "scooped-out") distal bit, a rounded to pointed proximal or poll end, and a strongly planoconvex to biconvex profile. The tool is broadly distributed geographically, spanning much of Texas (Hall et al. 1982), northeastern Mexico (Epstein 1969), Oklahoma (Bell 1957; Hofman 1977), the central Plains (Holder and Wilke 1949; Hughes 1980; Mott Davis, personal communication 1995), and parts of Florida (Purdy 1981:29). Affinities also have been suggested with the Dalton adze which occurs over much of the Southeastern United States (Morse and Goodyear 1973).

Classification schemes have focused chiefly on elucidating differences in size, outline shape, and bit form. Relatively few have been tested within temporal frameworks, inasmuch as few assemblages derive from secure, excavated contexts and even fewer in conjunction with dated materials. Those that do appear to show little patterning based on outline or bit shape.

Technological differences, that is, bifacial versus unifacial flaking, appear to be strong temporal indicators, as Hester (1980:112, 1995a) and others have observed. Although a very early occurrence of a Clear Fork biface fragment was reported from possible Clovis-age levels at the Gault site (Hester et al. 1992:3), the preponderance of evidence points to bifacial Clear Fork tools first occurring in Late Paleoindian and very Early Archaic times, preceding unifacial varieties by several thousand years. Bifacial Clear Forks have been reported in Late Paleoindian contexts at the Devil's Mouth (Johnson 1964:63), St. Mary's Hall (Hester 1979a; Hester and Knepper 1991), and Baker Cave (Hester 1979a, 1983) sites and from surface contexts along with Golondrina and other Late Paleoindian points at the San Isidro site in northeastern Mexico (Epstein 1969:39). Johnson (1962) reported a bifacial Clear Fork in the same level as a Meserve point at the Yarbrough site; other bifacial specimens were found in later contexts there, with expanding and straight stem points.

At the Richard Beene site, a Clear Fork-like biface, a Plainview-like (St. Mary's Hall?) point, and an Angostura point were recovered from the lower part of deposits attributed to the Late Paleoindian period. In the slightly younger, overlying zone, numerous bifacial Clear Forks along with Angostura points, a Plainview-like (St. Mary's Hall?) point, and an early bifurcate stem point were found. The Late 
Paleoindian deposits in the Perez paleosol are estimated to range in age from 8700 to 9700 B.P., based on radiocarbon dated soil humates (Thoms 1995:10-17).

Smaller bifacial Clear Fork tools co-occur with unifacial Clear Fork tools during the Middle to Late Archaic periods in south Texas; these forms appear to coincide with use of unstemmed dart points (e.g., Early Triangular), beginning around 4450 в.P. (ca. 2500 в.с.; Hester 1995b:5). Data from Choke Canyon show these forms in prominence around $4300-2550$ B.P. (ca. 2360 B.c. to 600 B.C.; Hall et al. 1986:400). At Loma Sandia, short, broad, triangular Clear Fork tools predominately of unifacial form were found with Abasolo, Lange, Morhiss, and Tortugas points in contexts dated to around 2800 to 2500 в.P. (ca. 850 to 550 в.C.; Taylor and Highley 1995:467). A thick unifacial Clear Fork was recovered from what appears to be a discrete Martindale zone at the Eckols site in western Travis County (James Karbula, personal communication 1996).

Use-wear and replicative studies have elucidated possible hafting techniques and use for these tools but have been largely unsuccessful in correlating morphological variability with functional patterns. Howard (1975) found a " $T$ "-mount haft and a technique of short, vertically directed chopping strokes to be most efficient in felling small trees. Hudler (1997) identified polish on a large sample of unifacial and bifacial Clear Forks as being nearly identical to that created on replicated specimens used in woodworking experiments. Other studies have identified wear patterns indicating working of wood or other hard materials (Chandler 1974; Hester et al. 1973).

\section{ANALYTICAL APPROACHES}

Previous studies have defined numerous morphological variants and subgroups within the larger category of distally beveled or "Clear Fork" tools (e.g., Hall et al. 1982; Hall et al. 1986; Ray 1941). Following these precedents, 10 different groups based on size, overall shape, and bit modification initially were defined in the Wilson-Leonard collection. Since no significant temporal patterning could be detected among the groups, however, the 10-group morphological classification was dropped and a more basic approach adopted, that is, sorting broadly into bifacial and unifacial categories, following Hofman (1977) and Black and McGraw (1985:138-142). The reasoning for this approach is discussed briefly at this juncture and more fully in the interpretation section, below.

In departing from the more-established convention of using a multigroup classification system, it is suggested that, with some exceptions, morphological variations within Clear Fork tools exist as evidence of a dynamic process, that is, the manufacturing-use-resharpening cycle (Michael B. Collins, personal communication 1995). As such, many of the attributes hold no cultural or temporal significance, per se. Rather, these attributes may hold larger meaning within the technological and behavioral sphere, particularly as pertains to the economics of use, maintenance, and curation.

\section{METHODS AND TERMINOLOGY}

Clear Fork tools were evaluated as to technological group (bifacial or unifacial), size, shape, edge modifications, and raw material/surface characteristics. Linear measurements were taken using steel calipers, and edge angles were measured using a plastic goniometer. Length was measured as the maximum extent of distal to proximal end. Maximum width was measured perpendicular to the longitudinal axis, both at the maximum extreme for the tool in general as well as for the bit in particular. Typically, maximum width for the tool occurs at the bit. Thickness also was measured at the maximum for the tool overall, as well as at the bit end, at its juncture with the body plane. Bit "height" was taken at the same point as bit thickness, generally at the maximum extent of bit flake removal.

One of the most difficult measurements to take was bit angle or, as in this case, spine plane angle - the angle of the bit in relation to the body plane, as distinguished from the angle of merely the bit edge. Stated another way, spine plane angle more closely represents the production angle, as opposed to a working edge angle (Hayden and Kamminga 1979:7). Because these readings can be easily skewed by small protuberances and fracture scars, angle measurements were taken at three positions across the bit and then averaged.

Observations potentially pertinent to use and hafting were made and intermittently verified with the aid of a hand lens and low-power binocular microscope (10-20X). Chiefly, these observations include bit modification in the form of step and hinge flaking, battering, and, less frequently, polish. Dulling or grinding along lateral edges and along ridge lines also was recorded. As is discussed, a more-extensive use-wear study was performed by Hudler (see Chapter 22).

Raw materials and related characteristics were recorded following criteria described in Chapter 12. These include raw material type, evidence of patina, heat alteration, and mineral precipitates.

For this section, attributes are presented in tabular form, with specimens ordered according to stratigraphic occurrence. Examination of specimens reserved for organic residue analysis was necessarily somewhat limited; attributes which could not be observed are denoted with a question mark.

\section{RESULTS}

\section{Bifacial Clear Fork Tools}

\section{Description}

Of the 79 distally beveled tools recovered, the majority $(\mathrm{n}=60$ or $76 \%$ ) are of the bifacial, Clear Fork variety (Tables 15-1 and 15-2). Among these, 31 specimens are generally 
TABLE $15-1$

Measurements, Attributes, and Distribution of Clear Fork Bifaces ( $\mathrm{N}=60$ )

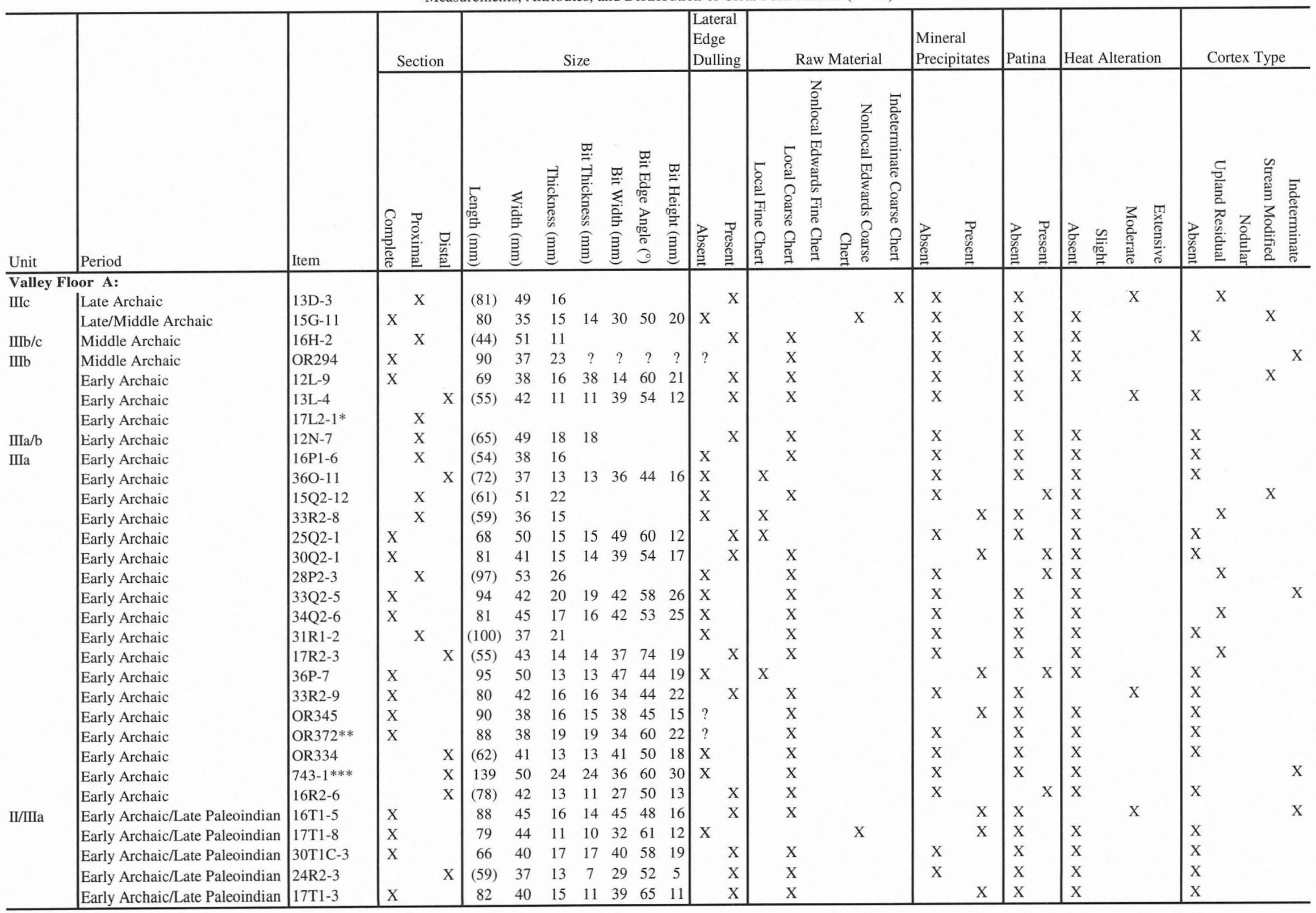




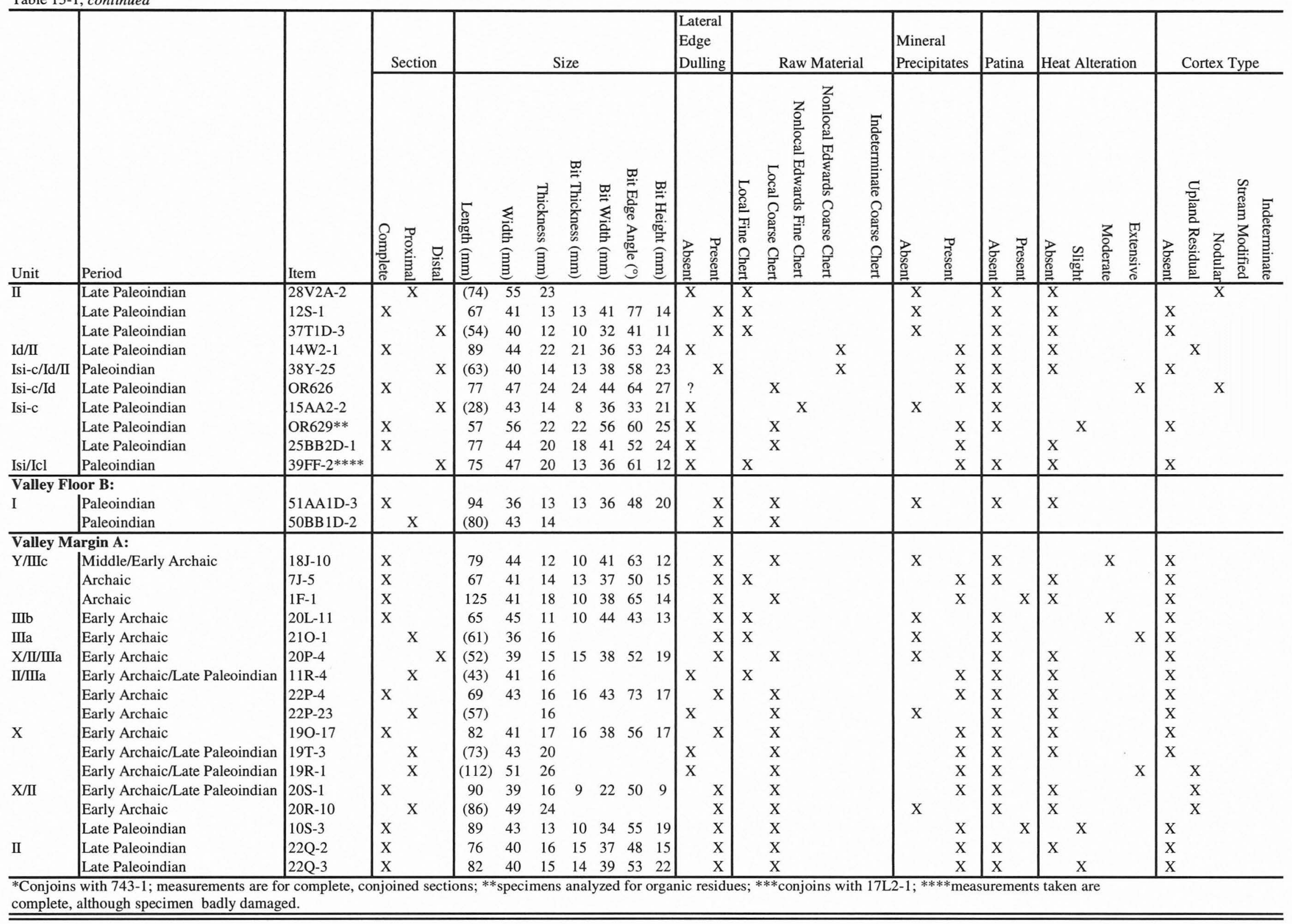


TABLE $15-2$

Measurements of Clear Fork Bifaces $(\mathrm{N}=60)$

\begin{tabular}{|c|c|c|c|c|c|c|c|}
\hline & Length* & Width* & Thickness* & $\begin{array}{l}\text { Bit } \\
\text { Thickness* }\end{array}$ & Bit Width* & $\begin{array}{l}\text { Bit Edge } \\
\text { Angle** }^{*}\end{array}$ & Bit Height* \\
\hline Mean & 82.85 & 42.98 & 16.63 & 14.49 & 37.30 & 54.56 & 17.67 \\
\hline Median & 81 & 42 & 16 & 14 & 38 & 53 & 17 \\
\hline Mode & 82 & 43 & 16 & 13 & 36 & 60 & 12 \\
\hline Standard deviation & 15.80 & 5.05 & 3.94 & 5.20 & 7.06 & 8.99 & 5.36 \\
\hline Coefficient of variation & 19.07 & 11.76 & 23.66 & 35.88 & 18.92 & 16.48 & 30.34 \\
\hline Range & 82 & 21 & 15 & 31 & 42 & 44 & 25 \\
\hline Minimum & 57 & 35 & 11 & 7 & 14 & 33 & 5 \\
\hline Maximum & 139 & 56 & 26 & 38 & 56 & 77 & 30 \\
\hline Count: & 33 & 58 & 59 & 42 & 42 & 42 & 42 \\
\hline
\end{tabular}

*Measurements in mm

**Measurements in degrees

complete. An additional 12 are distal ends with intact bits, and 17 others are proximal sections which conform in size and shape to the Clear Fork category but lack bit ends; among the fragmentary specimens, two are conjoinable to form a complete tool.

With several exceptions, these specimens generally are quite homogeneous in outline shape. The predominant form is narrow elongate with generally parallel lateral edges, varying to a roughly straight-edged triangle, and to a more convex-edged triangle commonly with assymmetric lateral edges. Proximal, or poll ends, on bifacial Clear Forks tools range from pointed, to rounded, to straight edged. In cross section, Clear Fork bifaces vary from plano-convex to biconvex.

Sizes are highly variable, particularly in the length category (see Tables 15-1 and 15-2; Figure 15-1). Lengths range from 57 to $139 \mathrm{~mm}$, with a mean of $82.9 \mathrm{~mm}$ and a standard deviation of $15.8 \mathrm{~mm}$ (see Table 15-2). Widths and thicknesses are somewhat more consistent. Widths range from 35 to $56 \mathrm{~mm}$, with standard deviation of $5.1 \mathrm{~mm}$ from the mean of $43 \mathrm{~mm}$. Thicknesses recorded are from 11 to $26 \mathrm{~mm}$, with standard deviation of $3.9 \mathrm{~mm}$ from the mean of $16.6 \mathrm{~mm}$.

When the standard deviations of these three body dimensions are considered proportionately in relation to their means (Coefficient of Variation, $V=(\mathrm{s} / \mathrm{m} .100$; see Table 15-2), a somewhat different perspective is afforded. Using this formulation, thickness is shown to be the most variable dimension ( $V=23.66)$, followed by length $(V=19.07)$. Maximum width ( $V=11.76)$ emerges as the most uniform of the body dimensions, a factor that may have significance in hafting considerations. Thickness, on the other hand, may be strongly influenced by raw material and its flakability.
Much of the length variation may be attributable to attrition during the use/rejuvenation process. Figure 15-1 illustrates a range of lengths, with the unusually large specimen (see Figure 15-1a), a possible Clear Fork preform, likely broken in its last stage of manufacturing prior to final shaping of the bit end. As such, its metric attributes may be representative of maximum dimensions of a tool in its original, nearly finished state: length is 139 $\mathrm{mm}$, width is $50 \mathrm{~mm}$, and thickness is $24 \mathrm{~mm}$. Microscopic examination of the bit end revealed no evidence of use wear (see Hudler, Chapter 22). Lateral edges are irregular and have not been dulled. A similar specimen, measuring $128 \mathrm{~mm}$ in length, is described by Brown (1985:21) as a Clear Fork "protoform" which manifests a rudimentary bit formed by heavy percussion scars on the distal end. Specimens of nearly comparable size have been described by Ray (1941:Plate 28, Specimens 1, 2, 3) and Goode (1989:Figure 33a). As shown in Table 15-1, the large majority of Wilson-Leonard Clear Fork bifaces exhibit dimensions well below those of the "preforms."

Bit modifications among bifacial specimens also are quite varied, likely depending on stage of use-rejuvenation. Bit shapes and sizes range from elongate "hollowed out" with a relatively acute edge angle (Figure 15-2a), to gently beveled with a moderate bit height (Figure 15-2b), to relatively short, steeply beveled, and markedly concave (Figure 15-2d). Bit thicknesses range from $7 \mathrm{~mm}$ to $38 \mathrm{~mm}$, with an average of $14.5 \mathrm{~mm}$; bit widths range from 14 to 56 $\mathrm{mm}$, with a mean of $37.3 \mathrm{~mm}$ (see Table 15-2). Edge angle on bit ends varies from a low of $33^{\circ}$, to a steeply beveled $77^{\circ}$; average among bifacial specimens is $54.6^{\circ}$. Bit heights are highly variable, ranging from 5 to $30 \mathrm{~mm}$, with a mean of $17.7 \mathrm{~mm}$ and a standard deviation of $5.4 \mathrm{~mm}$. 


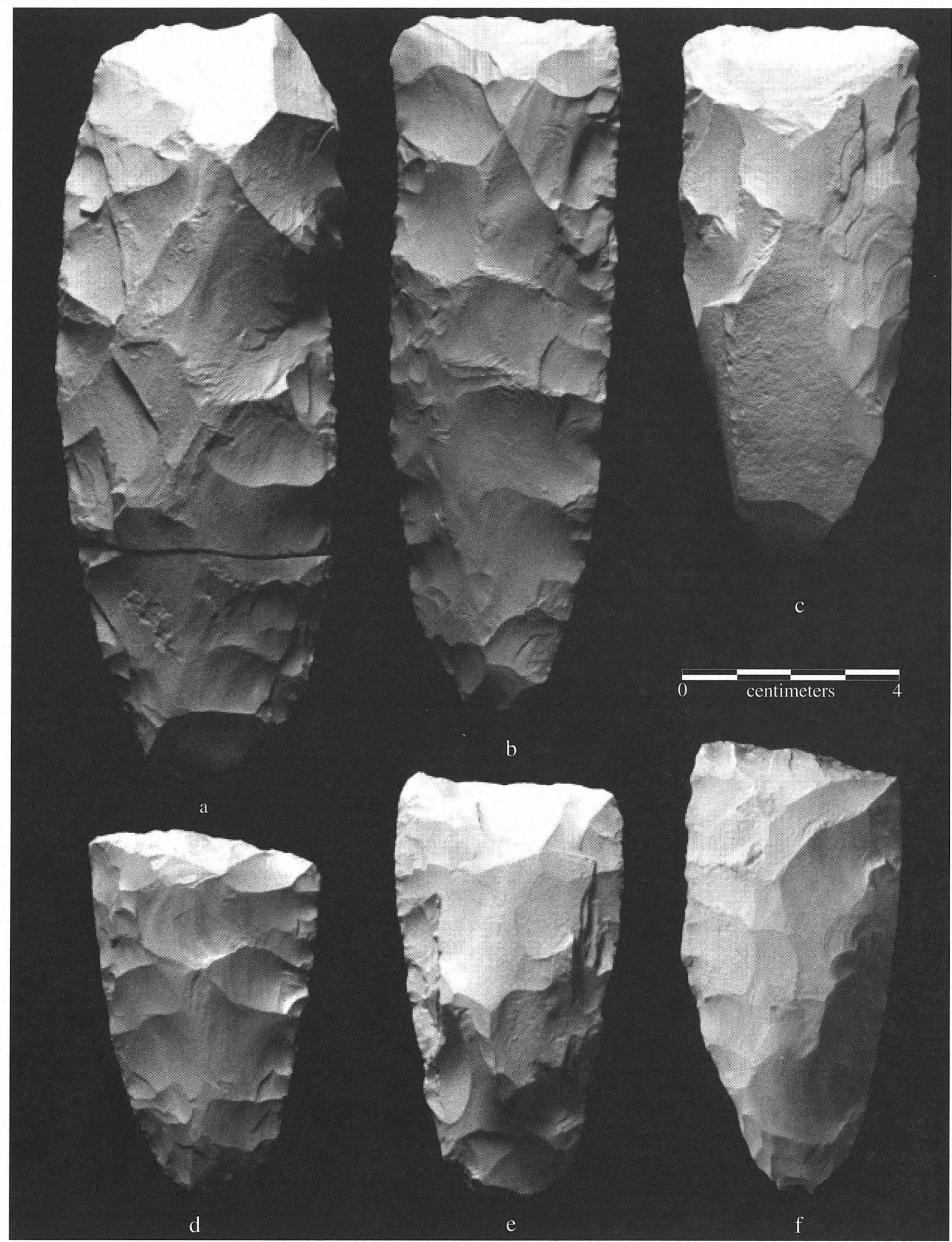

FIGURE 15-1. Representative Clear Fork bifaces of varying size and morphology. (a) possible preform, conjoined Specimens 743-11, 17L21; (b) Specimen 1F-1; (c) Specimen 33Q2-5; (d) Specimen 12S-1; (e) Specimen 22Q-2; (f) Specimen 17T1-3. 

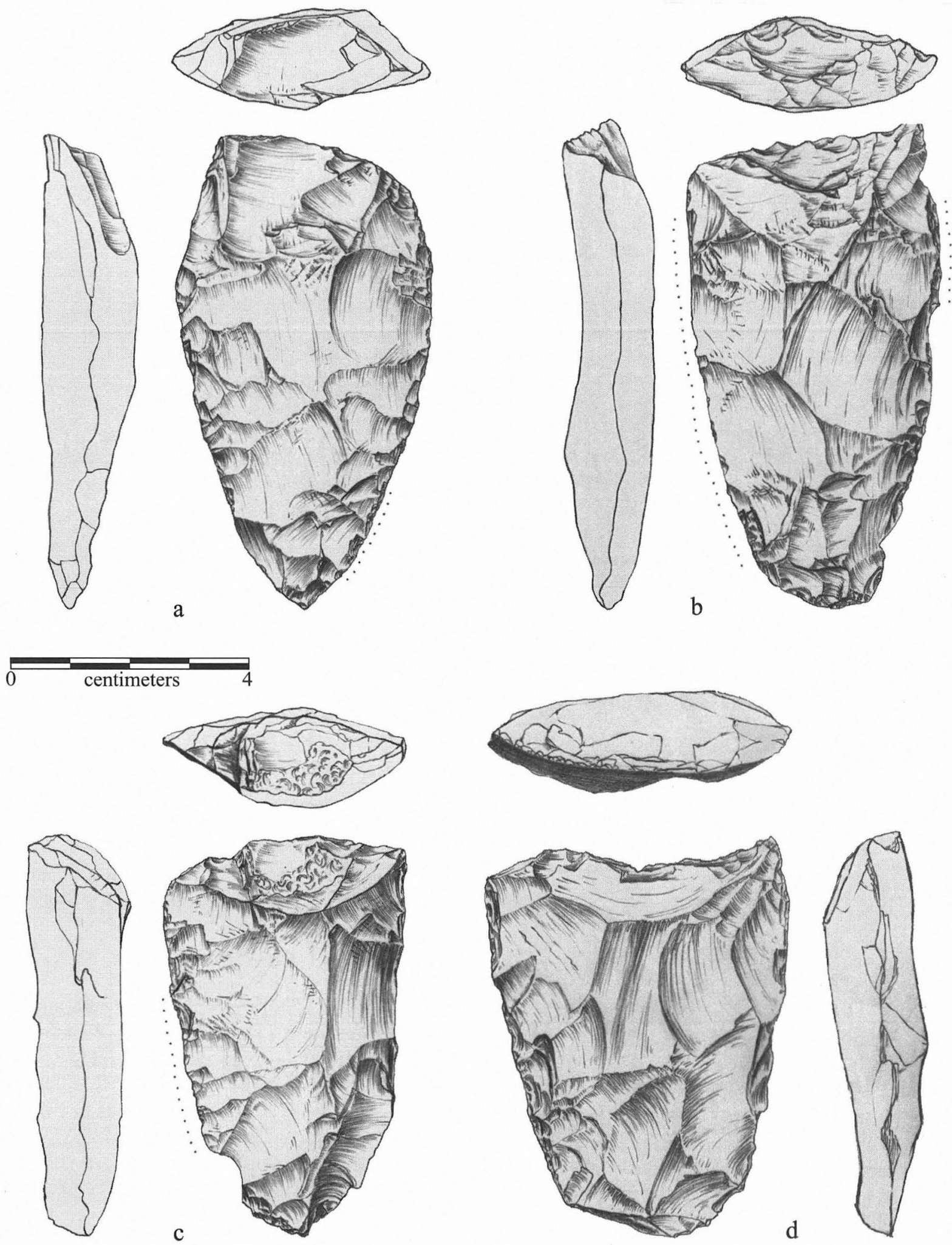

FIGURE 15-2. Examples of varying bit shapes and profiles on Clear Fork bifaces. (a) "hollowed-out," acute-edged bit (Specimen 33R2-9); (b) slightly beveled, less acute-edged bit (Specimen 30Q2-1); (c) shorter, rounded-bevel bit (Specimen 30T1C-3); (d) short, steeply beveled, concave-edged bit (Specimen 25Q2-1). 


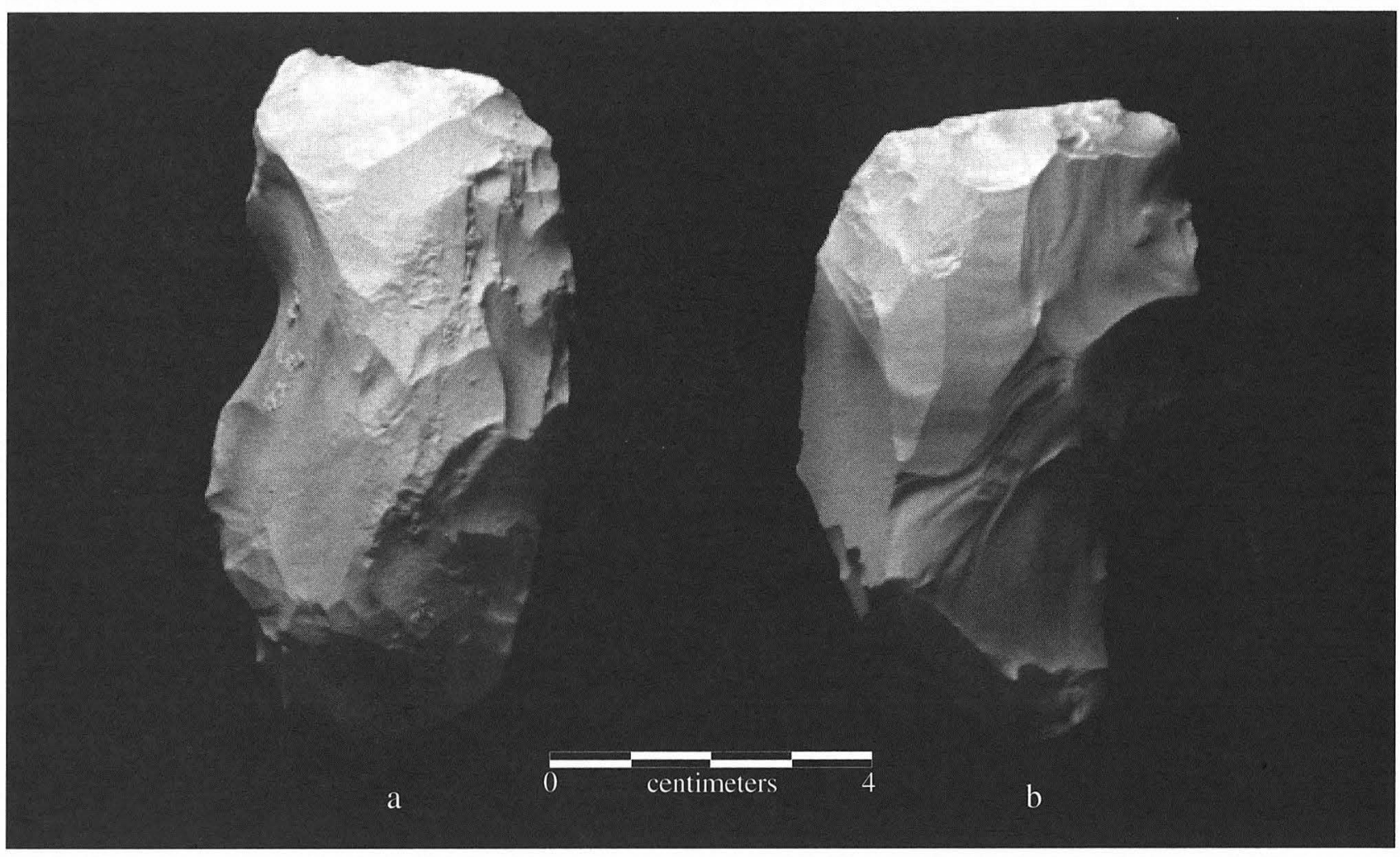

FIGURE 15-3. Possible Clear Fork bifaces from early contexts. (a) “double-bitted" Specimen 14W2-1; (b) Specimen 39 FF-2.

Modification of lateral edges is highly inconsistent. Edge dulling, recorded on 33 or 59\% of observed specimens, ranges from light smoothing on most to heavier grinding on 3. Although frequently noncontinuous and bilaterally asymmetrical (see, for example, Figure 15-2a-c) dulling occurs on both lateral edges on 22 specimens, and on 18 of these, dulling is present at or just below the bit area. On each of 30 specimens, at least one lateral edge was straightened or regularized by retouch. Dulling on ridge lines was observed on dorsal surfaces of 30 specimens and on ventral sides of 15. Macroscopic evidence of polish can be observed on bit areas of at least 5 specimens, including the "exhausted" tools shown in Figure 15-2c and 2d.

Several specimens that are somewhat aberrant in outline or bit modification merit further discussion. Three are from very early contexts. Figure 15-3a (Specimen 14W2-1) is a thick, convex-edged, double-bitted specimen from stratigraphic Unit Id/II. One end is beveled in a slight, scooped-out extension, and the opposing end is more rounded in profile, with a "bit" formed by three to four narrow longitudinal flakes. Both ends are about $50^{\circ}$ in edge angle and exhibit light dulling and step flakes along the dorsal and ventral surfaces of the bit edges; however, high-power microscopy failed to reveal distinctive polish on either end (see Chapter 22). A deep hinge flake has been removed from the left lateral margin of this specimen. Formed of coarse-grained chert, the specimen is encrusted on both faces with brownish mineral precipitates. Double-bitted specimens of various shapes have been reported by Ray (1941:157-158).

Figure 15-3b (Specimen 39FF-2), recovered from stratigraphic Unit Isi/Icl, is similarly convex edged but thinner than Specimen 14W2-1. Formed on dark gray, fine-grained local chert, it exhibits large percussion flake scars across both faces. The distal end of this specimen is only slightly canted at an angle of approximately $60^{\circ}$, forming a very short "bit" ca. $12 \mathrm{~mm}$ in length. Minor retouch in the form of "nibbling" is present along with step flaking on both bit faces. A deep hinge fracture has nearly halved the specimen vertically, removing much of the medial and proximal sections. Lacking a central ridge and a well-defined bit with clear evidence of use, this specimen could be categorized alternatively as a biface preform with a canted base. Use-wear analysis, however, revealed an unusual "fuzzy" polish possibly associated with hide working (see Hudler, Chapter 22); similar polish was noted on specimen 25BB2D-1 (see Figure 15-7a), a tool in the more "classic" Clear Fork shape.

An additional early specimen collected for organic residue analysis (OR629, not illustrated), is an apparent exhausted "nub" (length= $57 \mathrm{~mm}$; width [incomplete] $=56 \mathrm{~mm}$ ), the outline of which suggests lateral edges may have been similarly convex. Exhibiting a sharply planoconvex profile, the specimen is bifacially worked. The bit is steeply beveled 


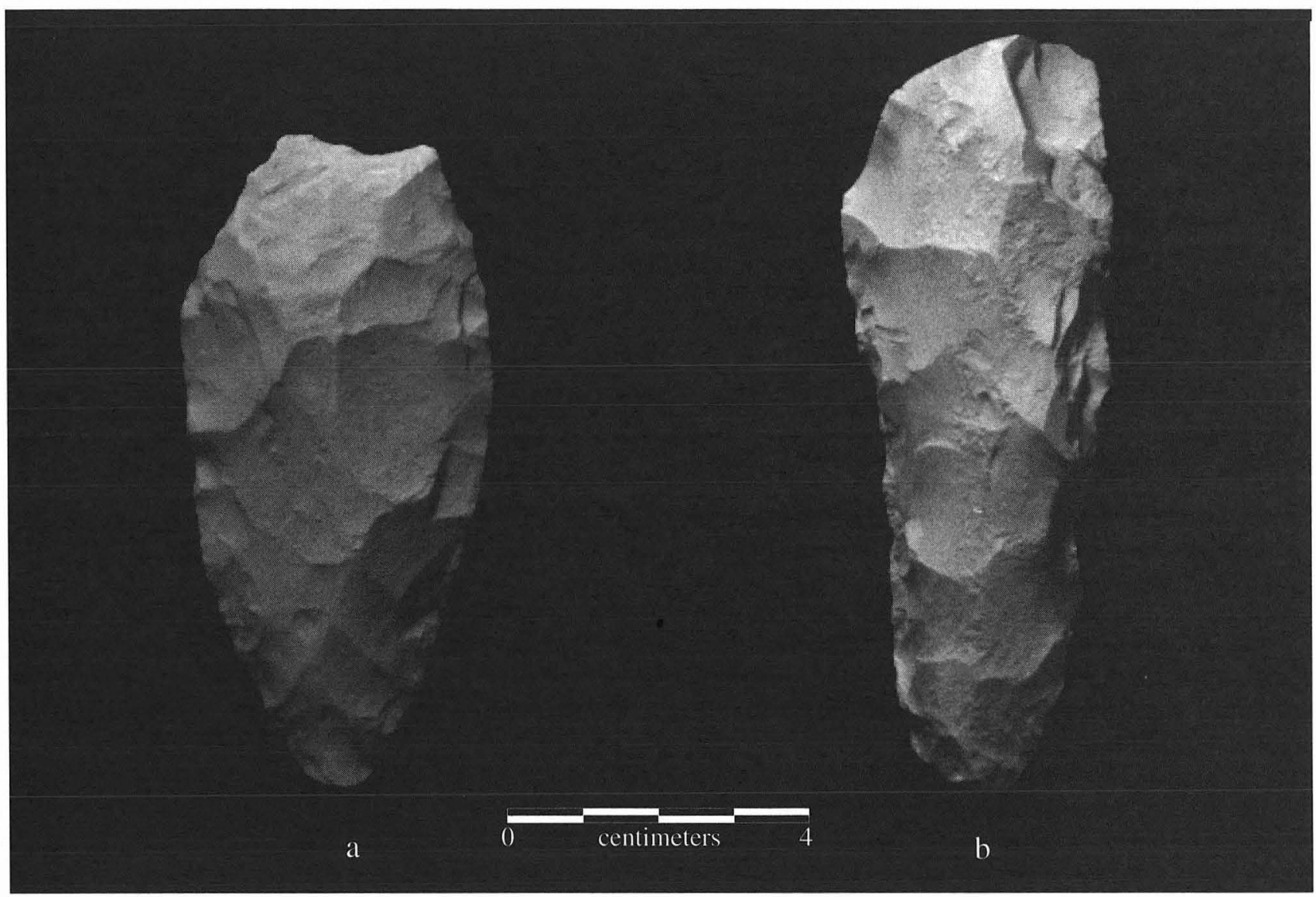

FIGURE 15-4. Clear Fork bifaces with unusual modifications or damage. (a) narrowed, bi-beveled bit (Specimen 20S-1); (b) failed manufacturing or rejuvenation attempt (Specimen 31R1-2).

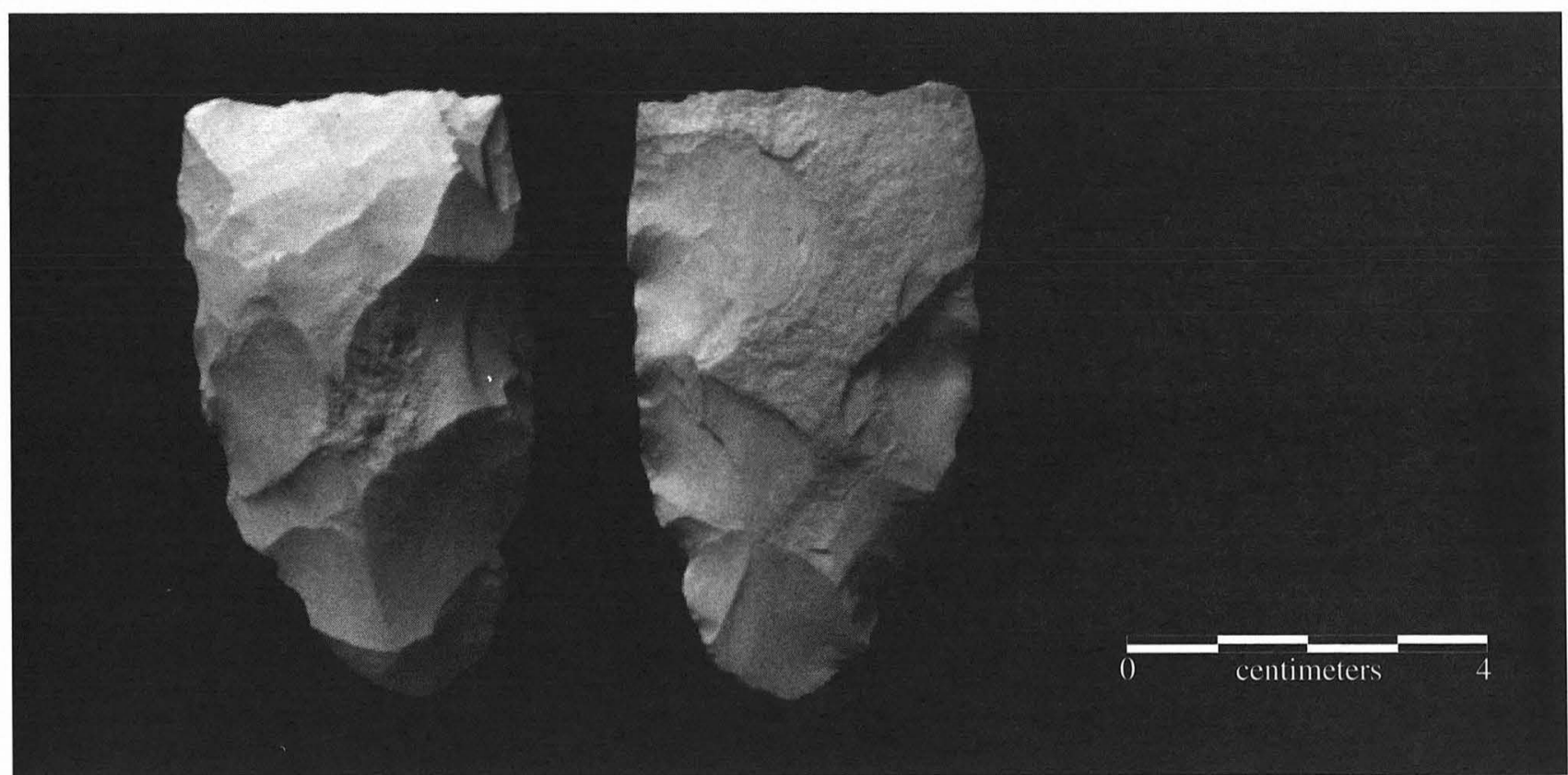

FIGURE 15-5. Clear Fork "biface/uniface" (Specimen 12L-9), two faces, showing partial cortex covering of ventral surface (right). 
to ca. $60^{\circ}$, but apparently it was damaged during excavation. Much of the tool is heavily encrusted with mineral precipitates, making observation of surfaces difficult.

Specimen 20S-1 (Figure 15-4a) has an unusually narrow bit $(22 \mathrm{~mm})$. In addition to the typical dorsal beveling of the distal end, the corner of the bit has been steeply beveled on the ventral surface, perhaps to overcome a prior fracture. Lacking a distal end, Specimen 31R1-2 (Figure 15-4b) is an example of failure during manufacturing or rejuvenation of the bit end, likely due to material flaw.

In Figure 15-5, both faces of Specimen 12L-9 are shown. With only partial removal of the ventral cortex covering, the specimen grades between the biface/uniface Clear Fork categories and, as such, is difficult to assign. Recovered from Unit IIIb, along with other bifacial and unifacial specimens, it may represent a somewhat intermediate type between the two technologies. Polish suggestive of woodworking was observed on this specimen (see Chapter 22).

A proximal fragment (OR294) from stratigraphic Unit IIIb is interesting in its similarity to the Guadalupe biface form, exhibiting the characteristic thick, elongate, planoconvex body form and steeply worked lateral edges. Lacking much of the distal bit, however, the specimen cannot be conclusively identified and, because it was collected for organic residue analysis, is not illustrated here. Guadalupe tools have been recovered from Early Archaic contexts, chiefly in south-central Texas and the coastal plain (Turner and Hester 1993:256).

\section{Raw Material}

Material attributes tend toward homogeneity in Clear Fork bifaces. A total of 48 or $80 \%$ of the bifacial tools was made on coarser cherts, while 13 or $21 \%$ were of fine-grained chert. Although most of the tools analyzed were fully decorticate ( 38 of 60 , or $63 \%$ ), a large percentage of those retaining cortex bear characteristics suggesting parent materials were weathered, upland residual nodules. The location of remnant cortex typically is the proximal area although a few (e.g., see Figure 15-1c) bear small patches on the dorsal or ventral surface. Evidence of heat alteration was observed on 15 specimens $(25 \%)$. Mineral precipitates are present on 25 specimens, or $41 \%$ of the sample. Only $7(11 \%)$ bear areas of patina, and none is fully patinated. As shown in Table 15-1, specimens evidencing patina and mineral precipates are chiefly concentrated in the lower stratigraphic units.

\section{Fracture Patterns}

Among the 29 broken specimens, the majority exhibit scars of bending fractures, with a smaller number attributable to impact, thermal damage, hinge fracture, or indeterminate causes (Figure 15-6a-c). Multiple breaks were observed on 6 of the tools, 2 of which are combination bend/snap and burin-like fractures. As shown in Figure 15-6b, Specimen
360-11 exhibits the scar of a bend break which has severed the proximal end, as well as a concentration of step flaking on the ventral bit surface. Two opposing, longitudinal burin-like fractures also are present, one emmanating from the bit end and the other from the broken proximal end. Multiple hinge flakes are located at the edge of the bend fracture. This constellation of damage suggests the tool may have been hammered wedge-like into an object or damaged in a haft while under impact stress. Possible recycling of broken adze-like tools as wedges has been suggested among Dalton assemblages (Goodyear 1974:42). The same tool is laterally asymmetrical and shows extensive retouch of the right lateral edge on both the ventral and dorsal surfaces. Impact and burin-like fractures, suggestive of use on a hard substance such as wood, were noted on a unifacial tool from the Kennedy Bluffs site (Goode 1989:120, Figure 37).

Two specimens (30T1C-3 and 7J-5) exhibit cones of percussion suggestive of battering on dorsal bit surfaces. Bit ends on both are steeply beveled at an angle of ca. $55^{\circ}$. The specimen shown in Figure 15-6c (Specimen 30T1C-3) also manifests polish adjacent to the battered area and a steeply beveled, heavily dulled bit edge. Rust-colored staining or residue is present in the battered area of Specimen 7J-5. Under high-power microscopy, both specimens exhibit polish suggestive of woodworking (see Hudler, Chapter 22).

Although the majority of breaks in the sample occurred on tools made of coarse-grained local cherts (21 of 29 or $72 \%$ ), there is a somewhat higher proportional incidence of breakage among fine-grained tools. More than half of the fine-grained tools are damaged or broken ( 7 of 12 , or $58 \%$ ), whereas a smaller percentage of the coarse-grained tools ( 21 of 48 , or $44 \%$ ) exhibit fractures.

\section{Stratigraphic Distribution}

Clear Fork bifaces are present within all major stratigraphic contexts at Wilson-Leonard but are most heavily represented in contexts pertaining to the Early Archaic, ca. 8700 to 6000 в.P. (see Table 15-1). Recovery was most sparse in very early and very late contexts.

In the Valley Floor area, four tools were recovered from deep levels characterized as undifferentiated Paleoindian. One of these, a thick biface tentatively identified as a Clear Fork, was recovered in Unit Isi/Icl, possibly pertaining to earlier Paleoindian times. As noted above, this specimen (Specimen 39FF-2; see Figure 15-3b) is damaged, and classification is somewhat questionable.

Eight specimens were recovered from Late Paleoindian contexts in the Valley Floor (Units Isi-c, II, and associated transition zones) and three others in the Valley Margin (Units $\mathrm{X} / \mathrm{II}, \mathrm{Id} / \mathrm{II}$ ) (Figures 15-7 and 15-8). Among these is seen perhaps the earliest occurrence of the more "classic" bifacial Clear Fork (e.g., Figure 15-7a, Specimen 25BB2D-1 from Unit Isi-c, possibly associated with the Wilson component). This form is seen in two other early specimens (Figures 15-7b 


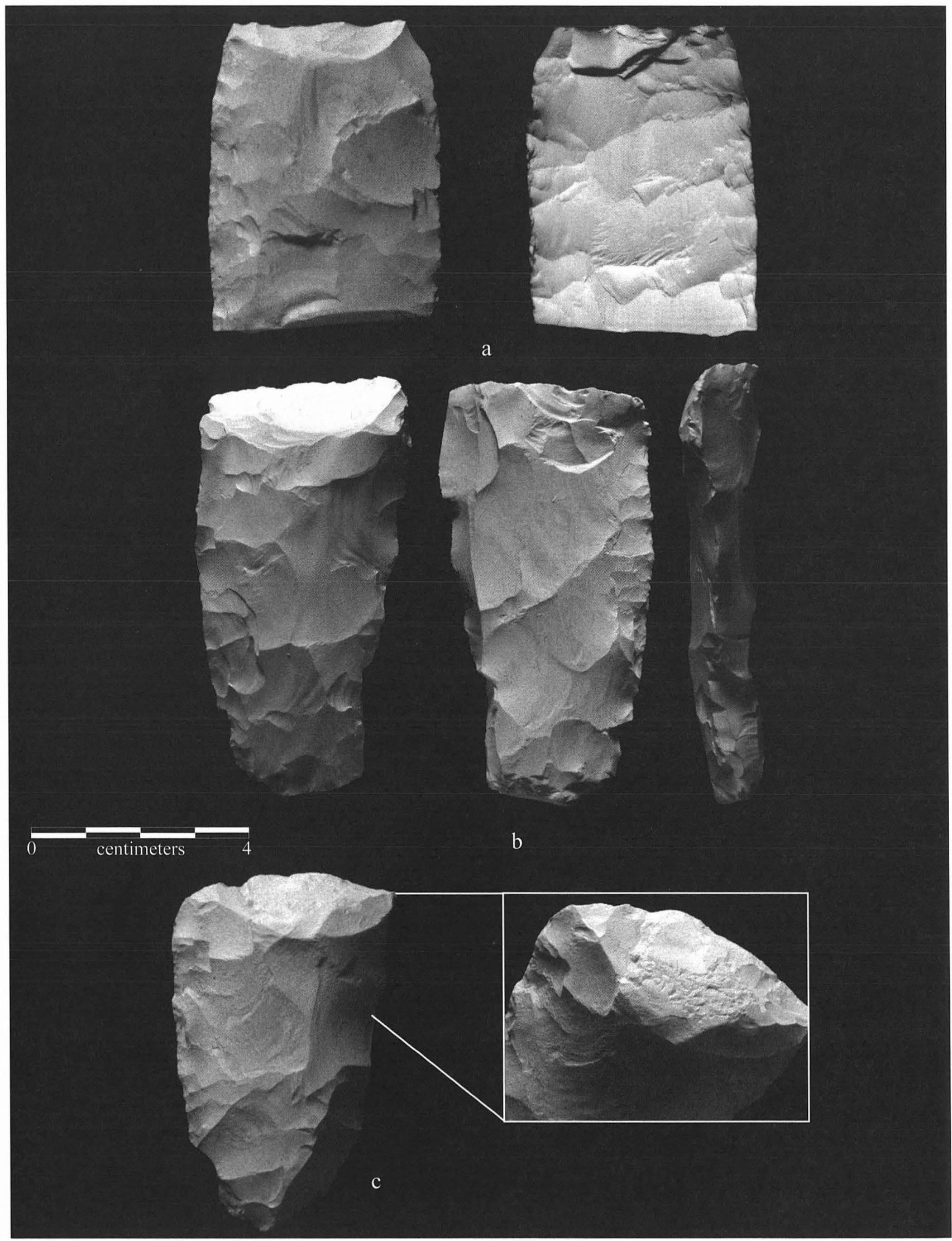

FIGURE 15-6. Examples of bit wear, edge damage, and breaks on Clear Fork bifaces. (a) extensive hinge and step flaking on bit area (Specimen 37T1D-3), dorsal (left) and ventral (right) faces; (b) burin-like damage to lateral edges and multihinge flaking of poll end (Specimen 36O11), both faces; (c) battering on dorsal bit surface (Specimen 30T1C-3), shown in closeup, bottom right. 
and $15-7 c$ ) - one of which is a characteristic poll end-although their contexts cannot be differentiated within the Unit I deposits of Valley Floor B.

A total of 22 specimens was recovered from Unit IIIa in the Valley Floor excavations, a context associated with Early Archaic occupations; an additional 5 were found in the underlying contact zone of Units II and IIIa (Figure 15-8c-e). At least 11 more specimens are from Valley Margin zones that can be loosely correlated to Early Archaic (Figure 15-8a and 15-8b) and mixed Late Paleoindian/Early Archaic deposits.

Only four bifacial specimens are from later contexts in the Valley Floor or mixed later deposits (Units IIIb, IIIb/c, IIIc, pertaining to the Middle and Late Archaic) (see Figures 15-2a and 15-5; Figure 15-9e). An additional one specimen is from related time periods in the Valley Margin (Figure 15-9f).

\section{Summary and Discussion}

Clear Fork bifaces at Wilson-Leonard occur in varied forms and sizes over several millennia. No clear temporal patterning of morphological attributes was observed. Rather, variation within the sample is suggested to derive chiefly from use, resharpening, constraints imposed by raw material, and individual knapping habits. Use-wear findings suggest several possible uses for these tools, including working on wood and possibly bone and hide (see Hudler, Chapter 22).

Almost half the sample of 60 tools (48\%) is broken. More than three-fourths of the tools were made on coarse cherts of apparent local origins, and-judging from corticate specimens-these were likely residual nodules from upland areas.

In many respects, bifacial Clear Fork tools are similar to the Dalton adzes of the Southeastern United States and Guadalupe tools of south-central Texas. Derived from Paleoindian and Early Archaic contexts, the Dalton and Guadalupe tools are apparently contemporaneous with early Clear Fork bifaces. Like the Clear Fork, Dalton adzes are percussion-chipped bifacial or unifacial tools made on elongate pebbles or small cobbles. Angled distal bits frequently evidence polish and dulling or grinding of lateral edges. As a rule, the outline of the Dalton adze is oval with lateral edges more convex, poll ends more rounded and typically heavily ground, and bit end distinguished by narrow, bladelike, vertical flake removals parallel to the long axis of the tool (see Morse and Goodyear 1973:Figure 1; Morse and Morse 1983:75-78). However, bit ends of Dalton adzes appear to become more steeply beveled and concave with use and in some cases, bear a close resemblance to the Clear Fork.

As shown in Figure 15-10, metric dimensions of bifacial Clear Fork tools from the Wilson-Leonard site are remarkably similar to Dalton adzes from the Sloan site in Arkansas, based on metric data provided by Dan F. Morse (personal communication to Michael B. Collins, 1995). These Sloan adzes, recovered as a "cache," have convex bit ends and appear, in some cases, to have been deposited in a freshly resharpened state (Michael B. Collins, personal communication 1995). What is described and illustrated as a "chisel" in the Hawkins cache in Arkansas (Morse 1971:17, Figure 4a) bears a striking resemblance in outline and bit to the typical elongate bifacial Clear Fork.

The few specimens classed as Dalton adzes from Texas sites show strong similarities to Clear Fork bifaces. The Gault site candidate (Specimen 59$)$ is relatively thin $(11.5 \mathrm{~mm})$ and of medium width $(46 \mathrm{~mm})$. Carefully bifaced, it has a gently biconvex profile and a pointed poll end. The bit is short (ca. $10 \mathrm{~mm}$ ) and has been relatively steeply beveled with the short retouch flaking found on many Clear Fork tools. No remnants of the long, narrow, vertically directed thinning flakes in the bit area, characteristic of the more classic Dalton adze, are evidenced. This specimen is not unlike several from Wilson-Leonard classed as Clear Fork bifaces. What was initially classified as a bifacial gouge from the "Plainview 1" substratum at Horn Shelter (Redder 1985:53) has been termed a Dalton adze (Johnson 1989:18; Story et al. 1990:196). Judging solely from its illustration (Johnson 1989:18, Figure 8), the Horn specimen appears very similar in outline shape and bit modification to many Clear Fork bifaces. Hester and Whatley (1992:5) report a possible Dalton adze from Dimmit County in southwestern Texas; both the bit treatment and raw material choice ( a glossy gray-brown chert) were quite different from that more typical of Clear Fork tools. Although few others are recorded, it is possible that other Dalton adzes reside in east and north-central Texas collections, unrecognized or confused with other tool forms (Dee Ann Story, personal communication 1996).

Although no tools from Wilson-Leonard could be conclusively assigned to the Guadalupe category, one proximal end specimen displays the characteristic thick, narrow, elongate body form with steeply worked lateral edges and triangular cross section. Lacking the distinctive truncated distal end, conclusive identification could not be made. These tools have been found in a rather localized area chiefly south of the Balcones Escarpment and are particularly concentrated along the Guadalupe and San Antonio river basins (Turner and Hester 1993:256-260). Nearer to Wilson-Leonard, a large collection was documented at the Kennedy Bluffs site in Bastrop County (Goode 1989:121-133).

These three tool forms - Clear Fork biface, Dalton adze, and Guadalupe tool — bear further examination and comparison for their possible similarities in function and technological traits. All three share a design predicated on a renewable distal bit, and their most frequently conjectured use is woodworking at varying degrees of force, based on microscopically observed polish, striations, and patterns of step fracturing on bit ends (see Brown 1985; Hudler 1997, Chapter 22, below). In form, the Guadalupe tool is distinctive. However, within the range of variability identified for the Clear Fork 


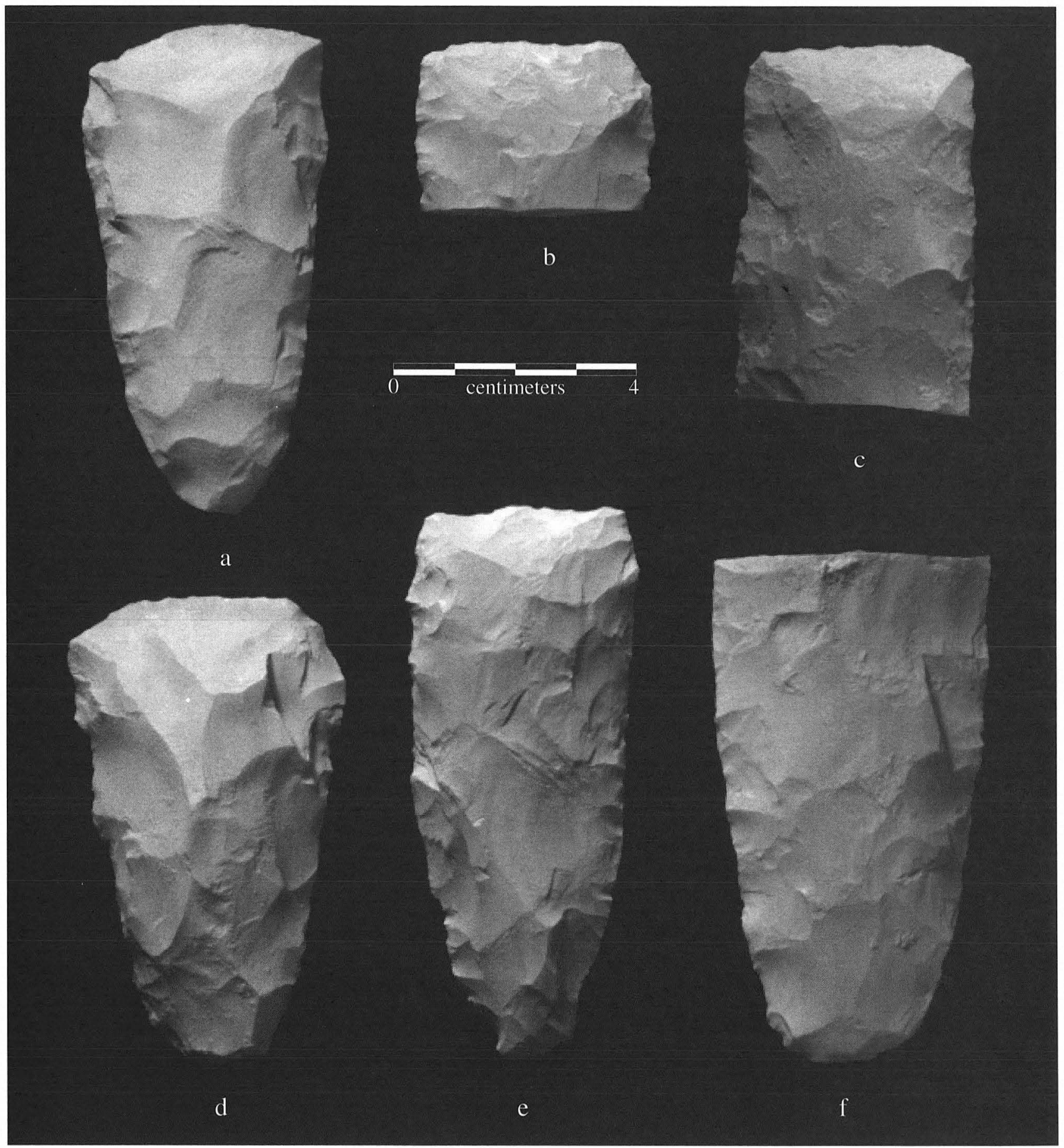

FIGURE 15-7. Clear Fork bifaces from early contexts. Specimen numbers: (a) 25BB2D-1; (b) 51AA1D-3; (c) 50BB1D-2; (d) 22Q-3; (e) 15AA2-2; (f) 38Y-25.

biface and Dalton adze, there appear to be substantial areas of overlap. Many of the thinner, more carefully made Clear Fork Tools with biconvex profiles are very similar to the body form of Dalton adze.

Certainly, there are striking similarities in cultural contexts of the three tool forms. Broadly, all derive mainly from assemblages in which beveled resharpening of lithic tools is fairly ubiquitous. This technique is particularly apparent in associated projectile point assemblages, specifically, the steeply beveled Dalton points, in the case of Dalton adzes, and the frequently beveled Angostura, Hoxie, and other early bifurcate forms that commonly are found with bifacial Clear Fork tools in Texas. And finally, all three appear to burgeon at peak frequency during Late Paleoindian and Early Archaic times across a wide swath of southeastern and central Plains states. 


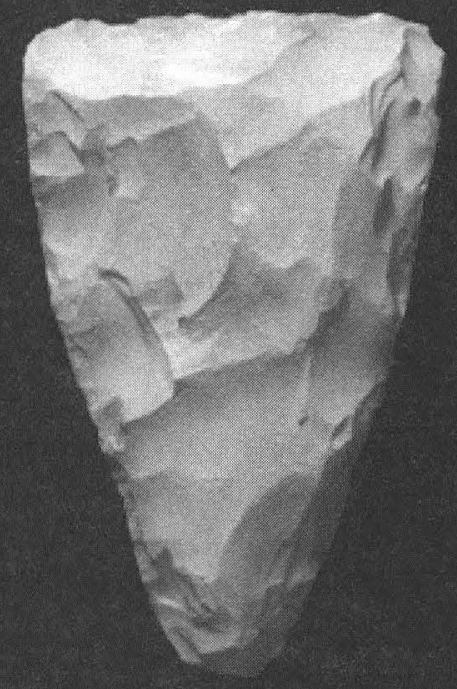

a

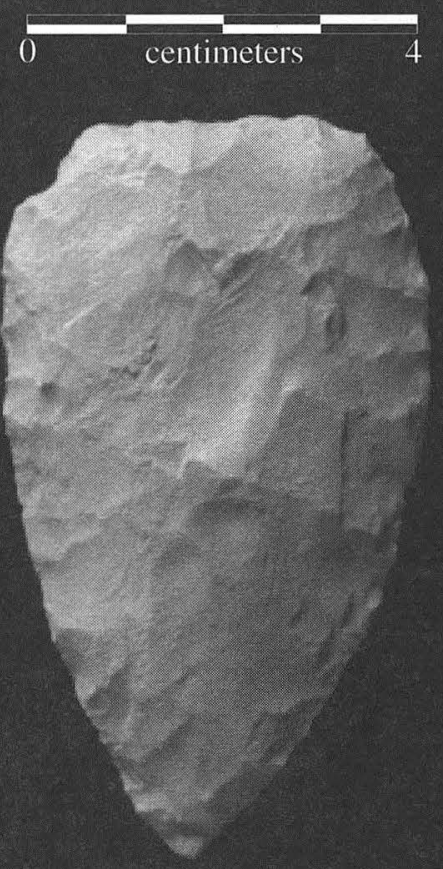

d

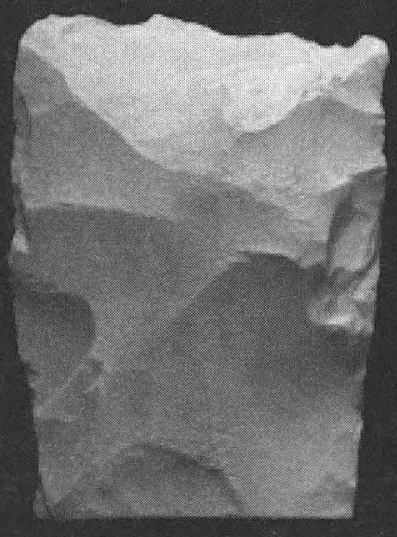

b

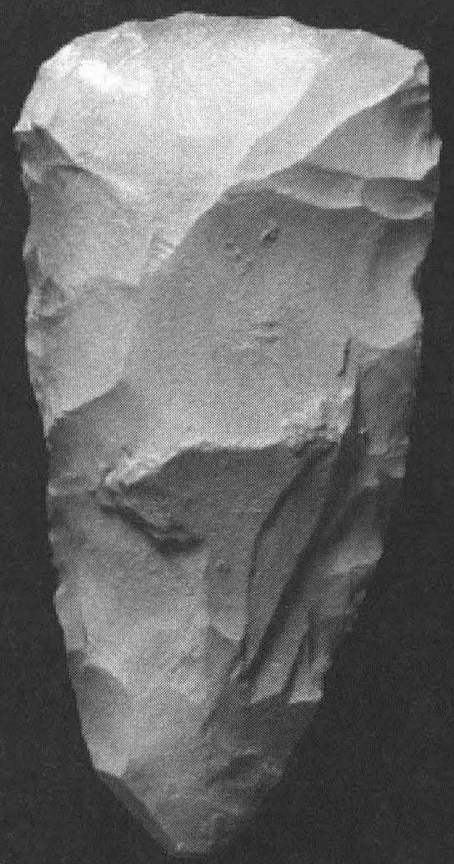

e

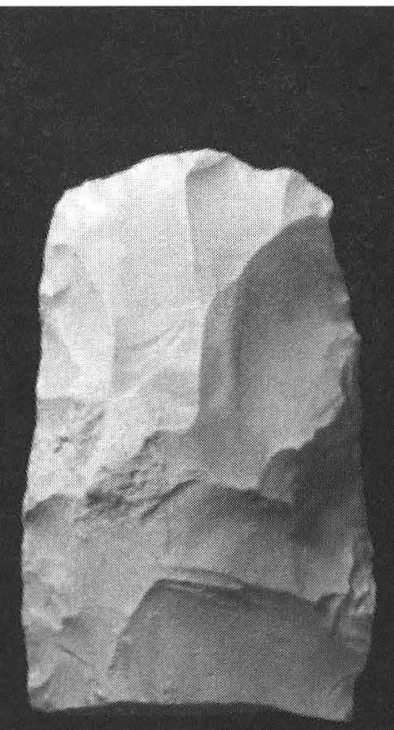

C

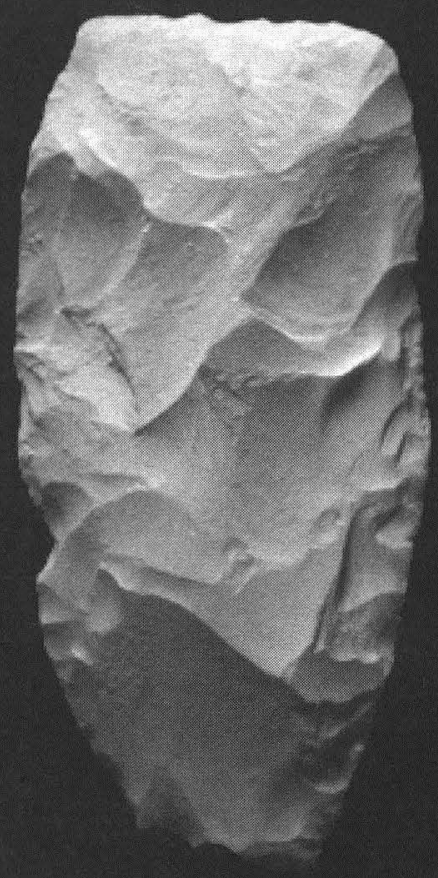

f

FIGURE 15-8. Clear Fork bifaces from Late Paleoindian and Early Archaic contexts. Specimen numbers: (a) 22P-4; (b) 20P-4; (c) 24R2-3; (d) $17 \mathrm{~T} 1-8$; (e) $16 \mathrm{~T} 1-5$; (f) $10 \mathrm{~S}-3$.

\section{Unifacial Clear Fork Tools}

\section{Description}

A total of 13 Clear Fork unifaces was recovered, chiefly from the upper portions of the site (Table 15-3; Figures 15-11 through 15-13). With two exceptions, all specimens were made on flakes, with distal beveling and flaking on dorsal surfaces. Flaking of ventral faces is minimal, typically along the lateral edges or to reduce bulbs of percussion. Within this group, outline shapes are predominately triangular, with straight to convex or asymmetric lateral margins and planoconvex to arc-like cross sections.

All specimens are complete or nearly so. With one notable exception, Clear Fork unifaces are generally smaller than most bifacial forms. Specimen 20I-4 (see Figure 15-11a) 


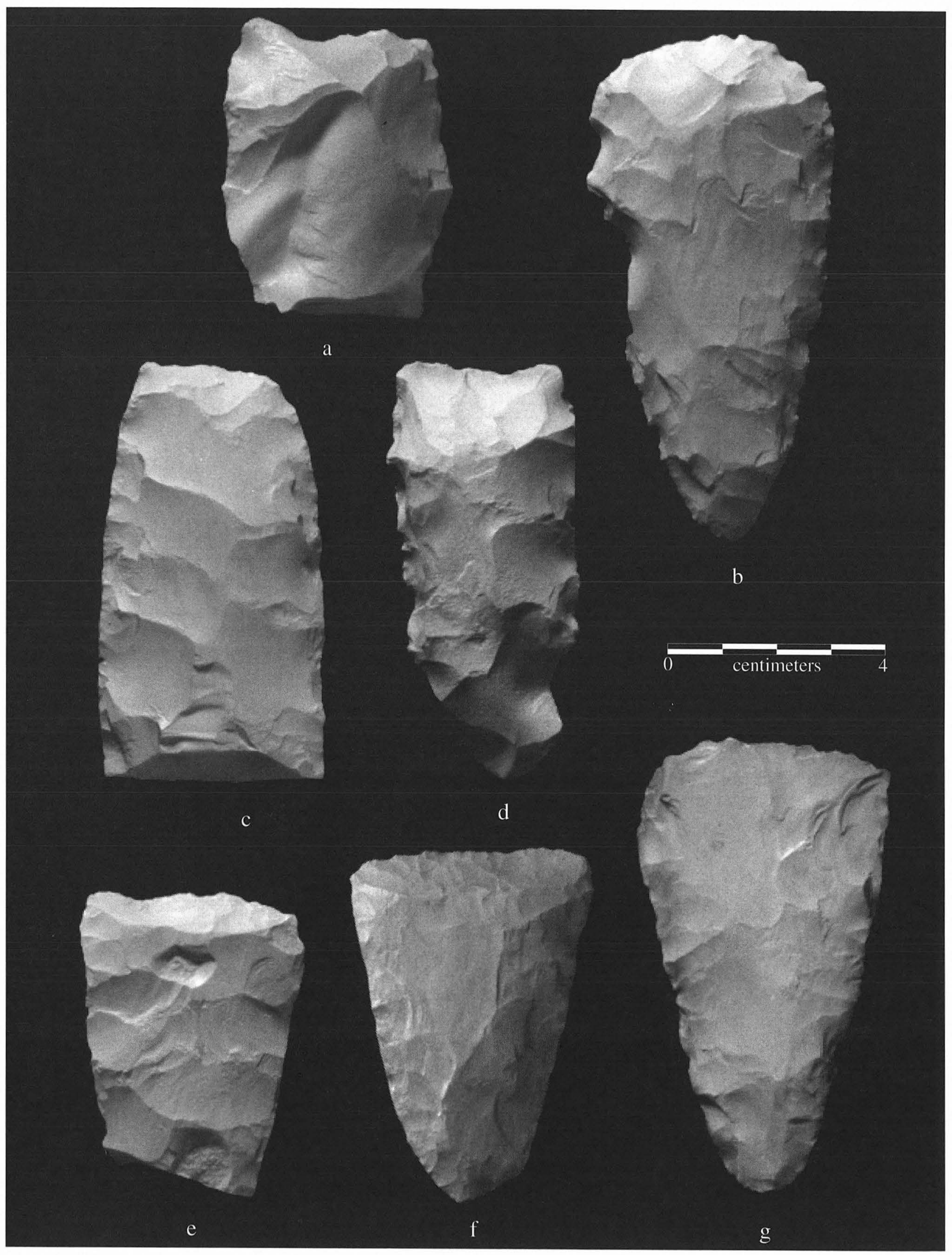

FIGURE 15-9. Clear Fork bifaces from Early Archaic and later contexts. Specimen numbers: (a) 17R2-3; (b) 36P-7; (c) 16R2-6; (d) 15G-11; (e) $13 \mathrm{~L}-4$; (f) $20 \mathrm{~L}-11$; (g) $18 \mathrm{~J}-10$. 


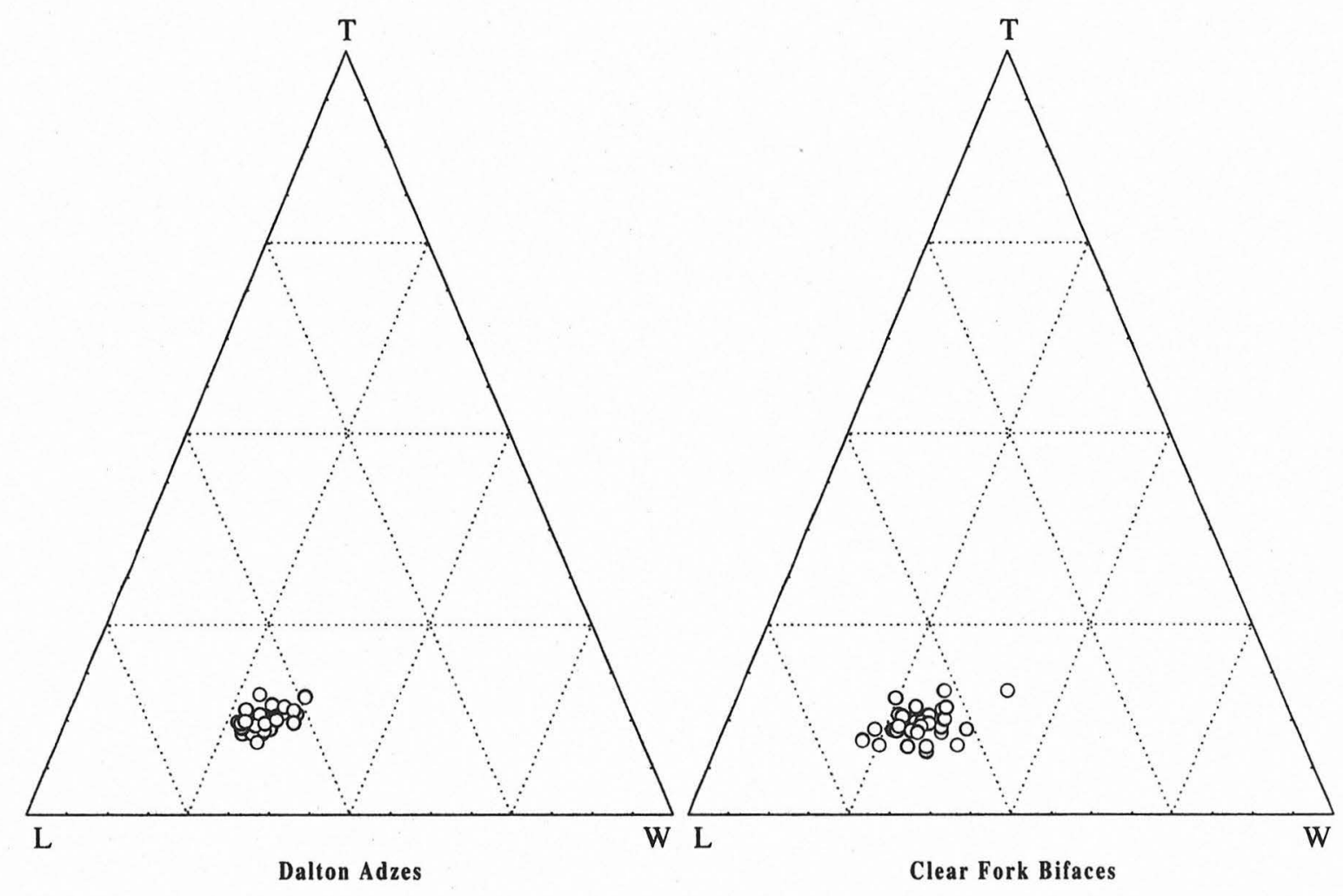

FIGURE 15-10. Comparison of the metric attributes of Dalton adzes from the Sloan site cache with Wilson-Leonard Clear Fork bifaces, based on metric attirbutes (length, width, and thickness).

is an aberrantly large, crudely chipped uniface recovered from Unit IIIb/c. Of coarse local chert, it measures $92 \mathrm{~mm}$ in length, $73 \mathrm{~mm}$ in width, and $21 \mathrm{~mm}$ in thickness. The bit is scooped out and acute $\left(40^{\circ}\right)$ in angle, and its edge is nibbled and dulled. The sizes of the other 12 specimens are somewhat more uniform (Figure 15-12a-f; Table 15-3 and 15-4). Overall lengths range from 49 to $85 \mathrm{~mm}$, with an average of $65 \mathrm{~mm}$; width varies from 34 to $58 \mathrm{~mm}$, with mean of $41 \mathrm{~mm}$. Thickness is the most consistent measurement, ranging from 10 to $15 \mathrm{~mm}$, with average of $12.5 \mathrm{~mm}$. As shown in Table 15-3, maximum thickness in most specimens occurs at the bit.

Bit modifications in Clear Fork unifaces are similar to those of their bifacial counterparts, ranging from acute edged and scooped out (Figure 15-13a) to short and steeply beveled (Figure 15-13b). Excluding the very large specimen, bit widths vary from 27 to $58 \mathrm{~mm}$ and bit heights from 10 to $29 \mathrm{~mm}$. In bit edge angle, the range is from 30 to $63^{\circ}$. Most specimens exhibit concentrations of step/hinge flaking along bit edges and, less frequently, light nibbling retouch.

A variety of other modifications are present on Clear Fork unifaces. The lateral edges on most are regularized and finely retouched; on three, however, they are sinuous with small protrusions (e.g., Figure 15-12b, d, e). One of these
(Figure 15-12d) shows polish suggestive of woodworking (see Hudler, Chapter 22). Two tools (e.g., Figure 15-11c) display beveled resharpening of one lateral edge. The specimen shown also exhibits a lightly serrated bit, which, according to Hudler (see Chapter 22) also evidenced polish suggesting use on soft contact material, such as animal hide. More than half the specimens have angular bit corners or beaked bit protrusions (e.g., Figure 15-12a, c, e, and f; Figure 15-11a and c).

The majority of unifacial specimens ( 7 of 12 observed, or 58\%) exhibit lateral edge dulling, including the large Specimen 20I-4. As in the biface sample, edge dulling is irregular and asymmetric, commonly occurring in disjointed patches (e.g., Figure 15-13a). Dulling of dorsal ridge scars is present on all specimens.

\section{Raw Material}

Raw material data for the unifacial group show that most were made on finer-grained cherts ( 7 or $54 \%)$. It is perhaps noteworthy that these specimens are generally smaller than those made on coarser materials. Only one exhibits mineral precipitates and patina (Specimen 9L-2). Three specimens (23\%) appear to have been heat altered. 
TABLE $15-3$

Measurements, Attributes, and Distribution of Clear Fork Unifaces $(\mathrm{N}=13)$

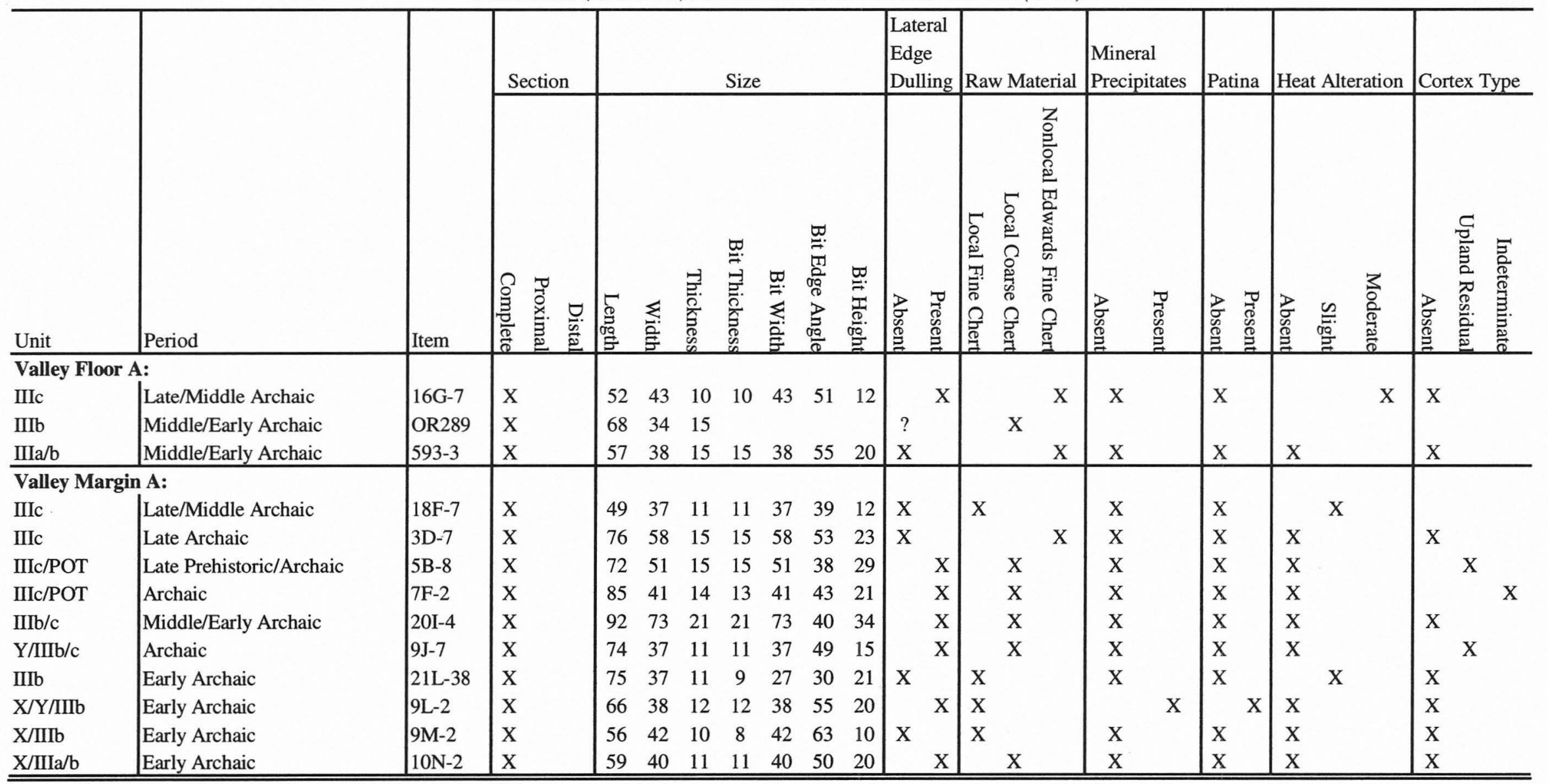




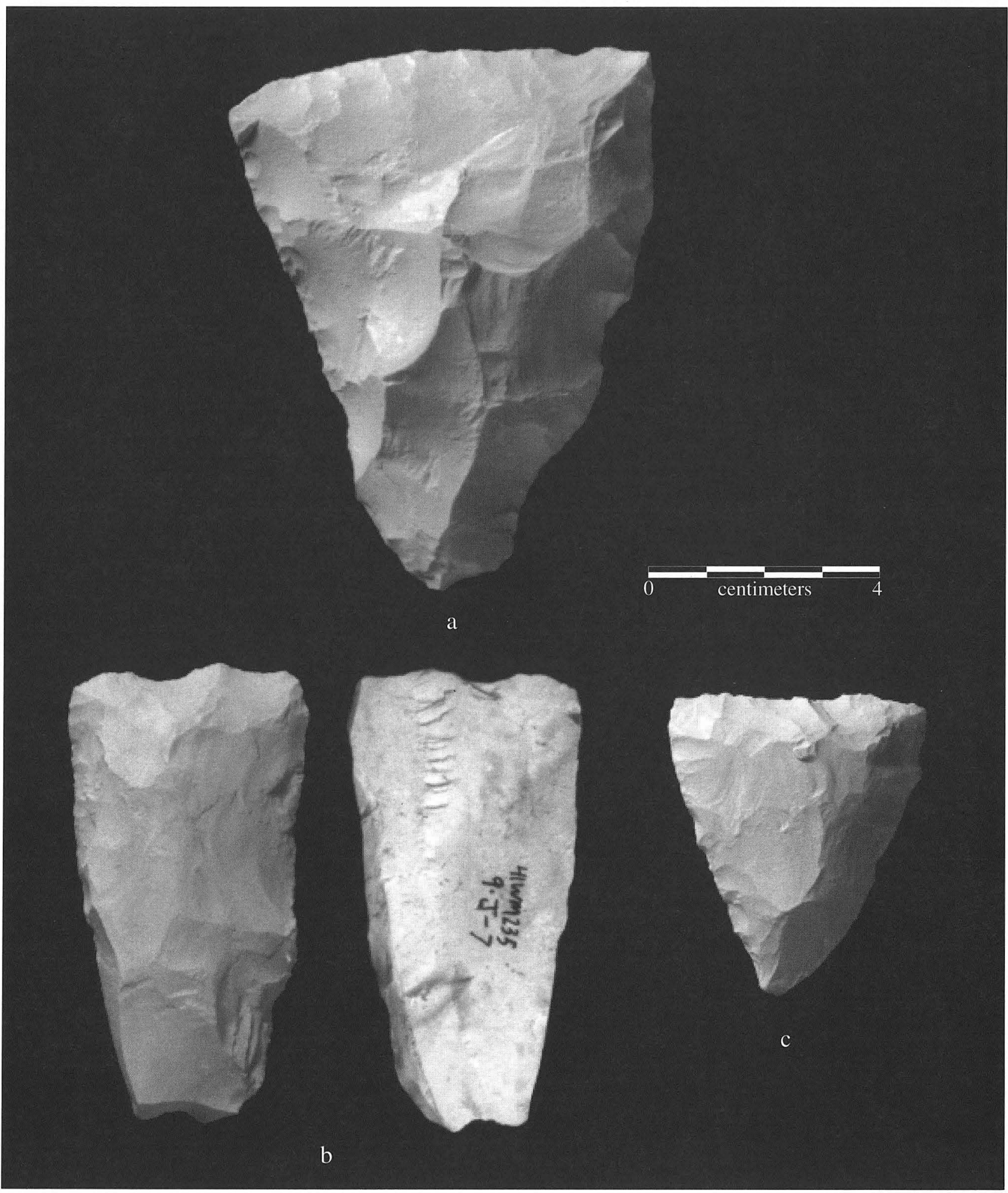

FIGURE 15-11. Clear Fork unifaces of unusual proportions or modifications. (a) Specimen 20I-4; (b) obverse and reverse of Specimen 9J7 , showing cortex covering of ventral surface; (c) Specimen 16G-7, with serrated bit.

Only three unifaces display cortex, and all are on coarse cherts. A small section of cortex suggestive of upland residual weathering remains on the poll end of one. Two other specimens are somewhat atypical in that they retain full cortex covering of their ventral surfaces. On Specimen 7F-2 (see Figure 15-12f), the smooth texture and odd coloration (pale gray) of the cortex suggests the parent material was a stream-rolled pebble rather than an upland or bedrock nodule. 


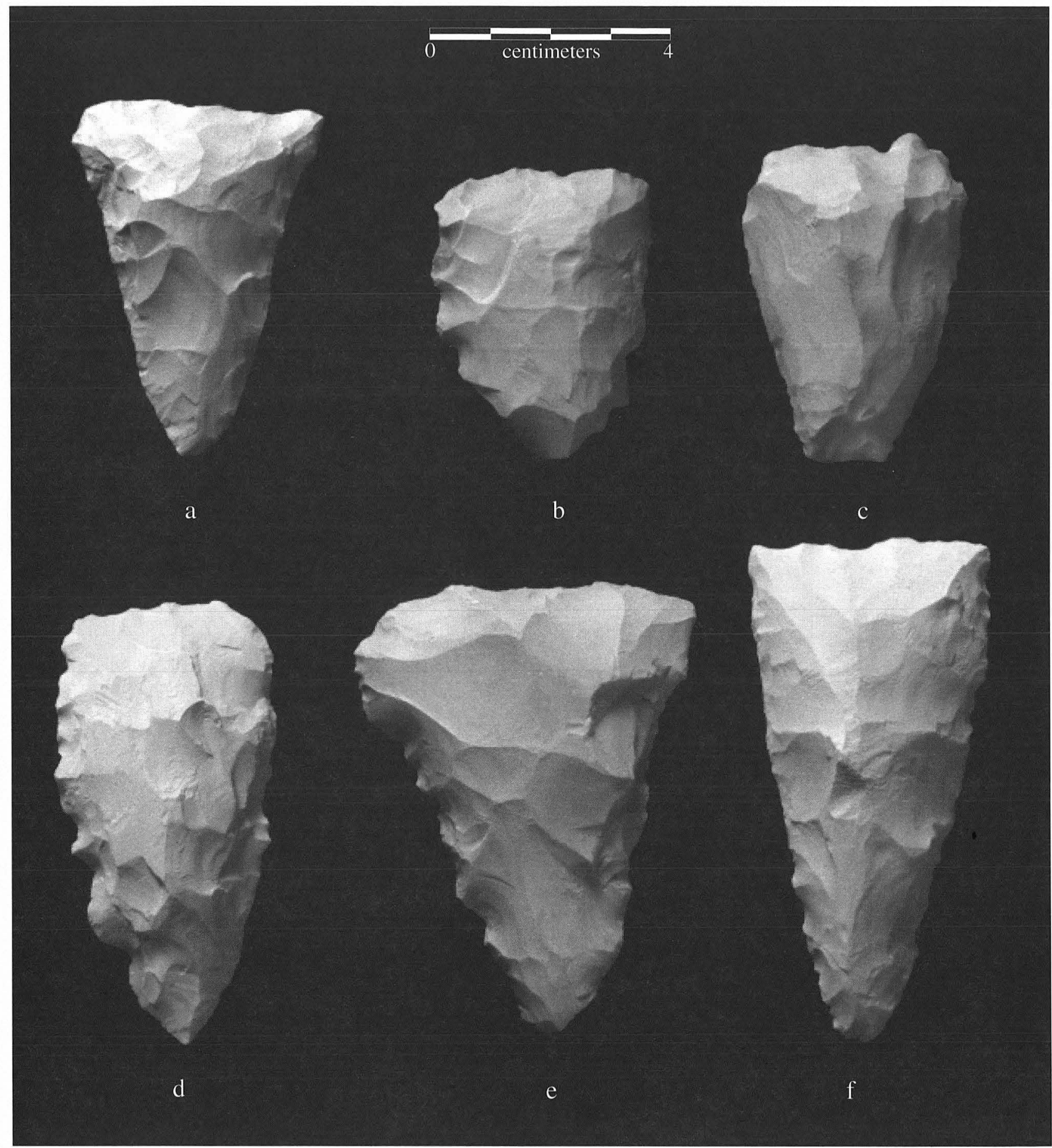

FiguRE 15-12. Representative Clear Fork unifaces. Specimen numbers: (a) 10N-2; (b) 18F-7; (c) 593-3; (d) 21L-38; (e) 3D-7; (f) 7F-2.

This specimen was either made on a thick primary flake and thinned on what became the dorsal face or made on a thin elongate pebble and modified only on the convex face. On Specimen 9J-7 (see Figure 15-11b), the ventral cortex covering suggests weathering on an upland surface. Both Chandler (1974) and Hofman (1977:107) have noted similar specimens and propose them as variants with some degree of regional significance within south Texas and Oklahoma.

\section{Stratigraphic Distribution}

As shown on Table 15-3, Clear Fork unifaces were restricted to Unit III (Archaic) deposits at the site. In the Valley Floor, the deepest recovery was from Units IIIb and IIIa/ b (Specimens 593-3, OR289; see Figure 15-12a and c, respectively), contexts likely pertaining to the later part of the Early Archaic and Early/Middle Archaic. 

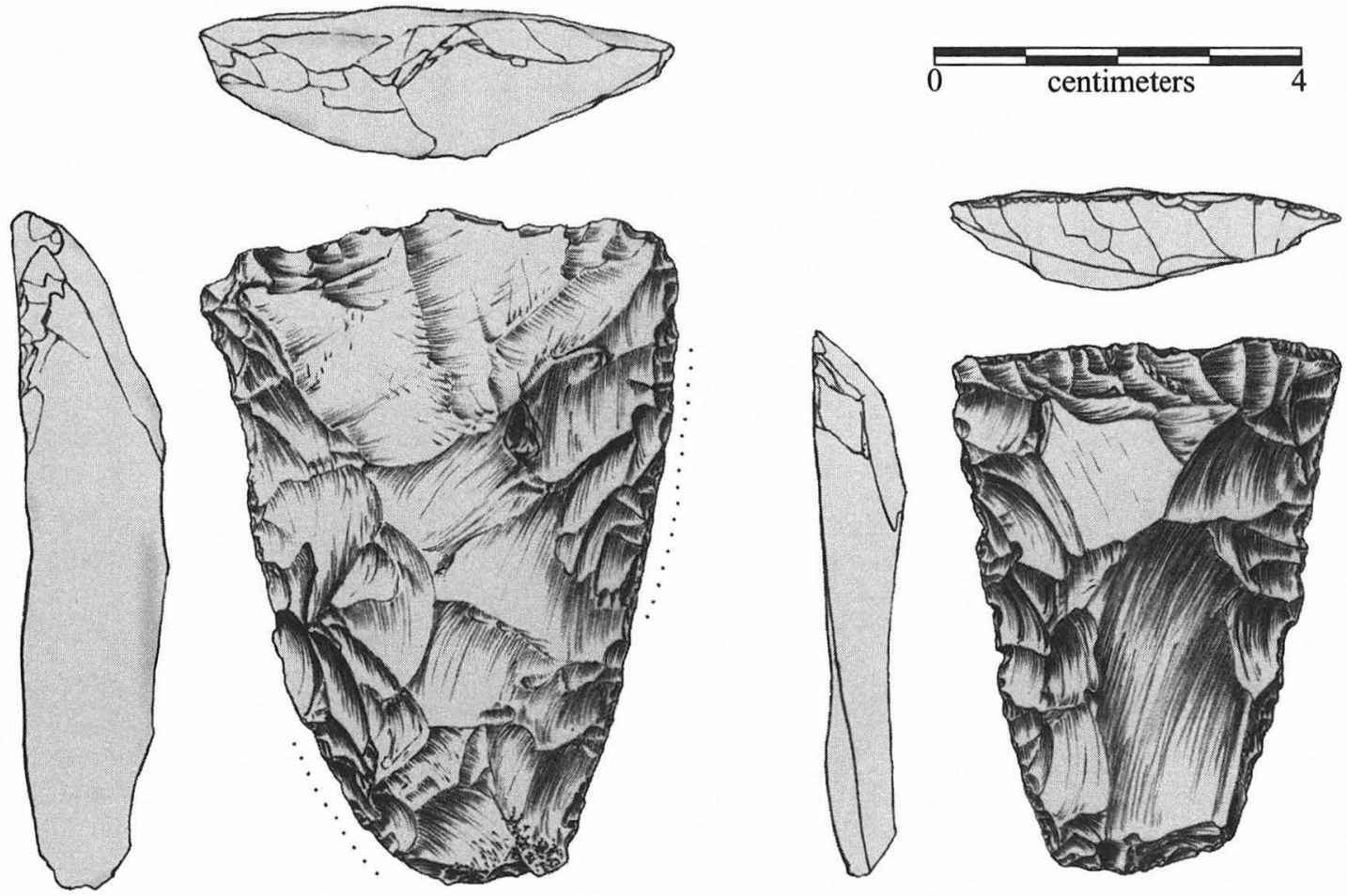

a

b

FIGURE 15-13. Bit modifications on Clear Fork unifaces. (a) "hollowed-out" acute-angled bit (Specimen 5B-8); (b) short and more steeply beveled bit (Specimen 9M-2).

TABLE $15-4$

Measurements of Clear Fork Unifaces $(\mathrm{N}=13)$

\begin{tabular}{l|c|c|c|c|c|c|c}
\hline & Length* & Width* & Thickness* & $\begin{array}{l}\text { Bit } \\
\text { Thickness* }\end{array}$ & Bit Width* & $\begin{array}{l}\text { Bit Edge } \\
\text { Angle** }\end{array}$ & $\begin{array}{l}\text { Bit Height* } \\
\text { Mean }\end{array}$ \\
\hline Median & 67.25 & 43.77 & 13.15 & 12.58 & 43.75 & 47.17 & 19.75 \\
Mode & - & 40.0 & 12.0 & 11.5 & 40.5 & 49.5 & 20.0 \\
Standard deviation & 13.33 & 10.93 & 3.11 & 3.53 & 11.96 & 55 & 20 \\
Coefficient of variation & 19.82 & 24.98 & 23.61 & 28.04 & 27.34 & 19.68 & 3.01 \\
Range & 43 & 39 & 11 & 13 & 46 & 33 & 24 \\
Minimum & 49 & 34 & 10 & 8 & 27 & 30 & 10 \\
Maximum & 92 & 73 & 21 & 21 & 73 & 63 & 34 \\
Count: & 12 & 13 & 13 & 12 & 12 & 12 & 12 \\
\hline
\end{tabular}

*Measurements in mm.

**Measurements in degrees.

Because a large percentage ( 10 of the 13 or $77 \%$ ) of the unifacial tools occurred in the Valley Margin, chronological placement for most is ambiguous. (It is not suggested that this spatial distribution is culturally patterned, rather that recovery may have been biased by machine excavation of Units IIIc and IIIb in Block 6 of the Valley Floor). Four specimens are from Unit III zones related to Early Archaic occupations, and one is from a zone roughly pertaining to the Late Archaic. The remainder are from mixed and undifferentiated contexts.

\section{Summary and Discussion}

Thirteen unifacial Clear Fork tools were recovered from Archaic contexts at Wilson-Leonard. Due to the preponderance of recovery in the Valley Margin, chronological placement for these tools is somewhat problematic, although their debut at the site can be correlated to Early Archaic times.

Generally smaller in size, unifacial Clear Forks show a continuum of bit forms and size gradations similar to their 
bifacial counterparts. As in that group, such variations likely are attributable in large part to the use-resharpening process. Several unifacial Clear Forks exhibit somewhat unusual edge modifications in the form of serration and small graver-like protuberances. The fact that all specimens are generally complete may suggest a less-intensive use for the unifacial forms.

\section{"Brushy Creek Bifaces"}

\section{Description}

A distinctive subgroup-provisionally termed the "Brushy Creek biface"-was identified in primarily Late Paleoindian contexts and merits attention as a possible type. On first inspection, several of these specimens appear to be thin bifaces with slightly canted "bases;" however, edge damage along the "base" in the form of step flaking is more indicative of the bit-like use seen in Clear Fork tools, and overall morphology suggests a diminutive form of that tool type.

Comprising four complete specimens and two distal sections, this group is distinguished by smaller dimensions in almost all areas measured (Tables 15-5 and 15-6; Figures 15-14 and 15-15). Typically narrow overall as well as narrow bitted, these tools are unusually thin and well made, in contrast to the larger, thicker, and generally cruder specimens that make up the two main groups (Clear Fork bifaces and unifaces). Lateral edges are more regularized and are generally lightly retouched.

As shown in Table 15-5, metric attributes are quite uniform and are key in the identification of the group. Widths range from 30 to $36 \mathrm{~mm}$, with a mean of $31.7 \mathrm{~mm}$; thicknesses 10 to $12 \mathrm{~mm}$, with a mean of $11.3 \mathrm{~mm}$; and lengths of the complete specimens from 59 to $79 \mathrm{~mm}$, with a mean of $68.3 \mathrm{~mm}$.

Bit attributes tend to be proportionately small as well. Bit thicknesses vary from 6 to $10 \mathrm{~mm}$ (mean $7.8 \mathrm{~mm}$ ), bit heights range from 7 to $23 \mathrm{~mm}$ (mean $12.2 \mathrm{~mm}$ ), and bit widths from 26 to $29 \mathrm{~mm}$ with a mean of $27 \mathrm{~mm}$. Bit edge angles are chiefly in the low or acute range $\left(31^{\circ}\right.$ to $50^{\circ}$; mean $\left.43^{\circ}\right)$. Three bit ends fall in the "scooped out" shape category (e.g., Figure 15-14a, b). Bit ends on the other three are formed in a more rounded bevel (e.g., Figure 15-14c).

One specimen, 37GB-9 (Figure 15-14a), recovered from Unit IIIc, is somewhat aberrant in form and context and may not be properly placed in the Brushy Creek category. Although more unifacial in form than the other specimens in the group, it exhibits extensive invasive bifacial retouch along the lateral margins of the ventral surface. Longitudinal profile is essentially planoconvex, although arc-like.

A variety of use/manufacturing evidence was recorded. Two specimens were broken above the proximal section by bend fractures. A third specimen (Figure 15-15c, 34X2A-1) is damaged by hinge flaking along one lateral margin and exhibits two long vertical flake scars on the ventral surface, a pattern of damage somewhat similar to that evidenced on Clear Fork biface 360-11 (see Figure 15-6b, above). On the Brushy Creek specimen, Hudler (see Chapter 22) found smooth linear polish suggestive of use on a high residue, hard material such as bone. Three complete specimens exhibit intermittent dulling along their lateral edges; four specimens show dulling on ridge scars. The ventral bit surfaces on two are lightly scarred by step and hinge flakes. Bit damage is more pronounced on two other specimens, 51WB-3 and 20W-2 (Figure 15-15a, b). The former specimen was found to have "invasive polish with a short linear look" and for which a possible use was not conjectured (see Hudler, Chapter 22). The latter specimen exhibits slight alternate beveling of the lateral margins as well as some crushing of the edges, including the bit end; no distinctive polish was noted in use-wear analysis, however.

\section{Raw Material}

Raw material selection is homogeneous throughout the group with all specimens made on fine-grained cherts (see Table 15-5). Three specimens appear heat altered. One of these, shown in Figure 15-14a (37GB-9), was resharpened after heating, as evidenced by flake scars that are markedly more lustrous in the distal section than on the tool proper. Three specimens bear small concentrations of mineral precipitates. Patina is present on another specimen (51WB-3), chiefly in fracture scars and along lateral margins and bit edges.

\section{Stratigraphic Distribution}

The six specimens described are homogeneous in form, metric attributes, and raw material. Four are derived from contexts attributable to Late Paleoindian occupations: Unit II $(n=3)$ and Valley Margin Unit $X(n=1)$. One is from the Unit II/III transitional zone deposits assigned to the Late Paleoindian/Early Archaic and an additional, slightly aberrant specimen was found in Unit IIIc.

\section{Summary and Discussion}

That all Brushy Creek bifaces are made on fine-grained cherts suggests these tools may have been made from failed bifaces. A generally finer flaking pattern on these tools, in comparaison to Clear Forks, would also tend to substantiate this contention.

A seemingly smaller version of the bifacial Clear Fork, this tool form has not been widely recognized, although a few similar specimens were observed by this author in a cursory search of literature and collections from diverse parts of the state. In size and shape, Brushy Creek bifaces appear to resemble Group 5 specimens recovered from the surface in the Choke Canyon investigations (Hall et al. 
TABLE 15-5

Measurements, Attributes, and Distribution of Brushy Creek Bifaces $(\mathrm{N}=6)$

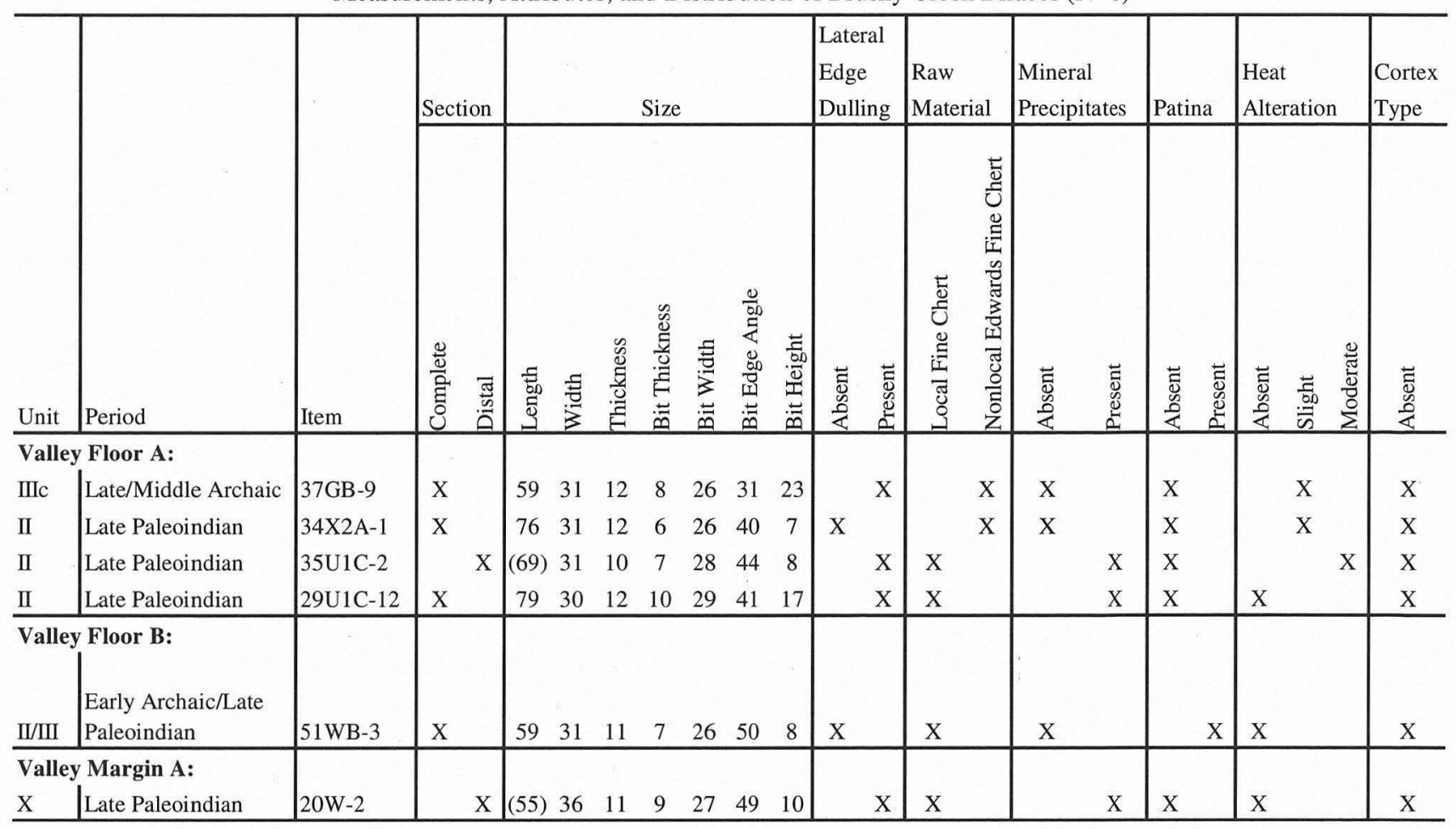

TABLE $15-6$

Measurements of Brushy Creek Bifaces

\begin{tabular}{l|c|c|c|c|c|c|c}
\hline & Length* & Width* & Thickness* & $\begin{array}{l}\text { Bit } \\
\text { Thickness* }\end{array}$ & $\begin{array}{l}\text { Bit Width* } \\
\text { Angle** }\end{array}$ & $\begin{array}{l}\text { Bit Height* } \\
\text { Bit }\end{array}$ \\
\hline Mean & 68.25 & 31.67 & 11.33 & 7.83 & 27.00 & 42.50 & 12.17 \\
Median & 67.5 & 31 & 11.5 & 7.5 & 26.5 & 42.5 & 9 \\
Mode & 59 & 31 & 12 & 7 & 26 & NA & 8 \\
Standard deviation & 10.75 & 2.16 & 0.82 & 1.47 & 1.26 & 6.95 & 6.43 \\
Coefficient of variation & 15.75 & 6.82 & 7.20 & 18.79 & 4.68 & 16.35 & 52.86 \\
Range & 20 & 6 & 2 & 4 & 3 & 19 & 16 \\
Minimum & 59 & 30 & 10 & 6 & 26 & 31 & 7 \\
Maximum & 79 & 36 & 12 & 10 & 29 & 50 & 23 \\
Count: & 4 & 6 & 6 & 6 & 6 & 6 & 6 \\
\hline
\end{tabular}

*Measurements in mm.

**Measurements in degrees.

1982:330; see also Hall et al. 1986:Figure 83:5-1, 5-2, 5-3). The three specimens examined are parallel-sided distal sections with fairly uniform bit dimensions (maximum width 22 to $28 \mathrm{~mm}$; thickness 8 to $9 \mathrm{~mm}$ ).

Specimens resembling the Wilson-Leonard tools were found on the surface in the north-central Texas Abilene area (Tull Collection, TARL). More-triangular and with very pointed proximal ends, they range from 25 to $35 \mathrm{~mm}$ in width and 8 to $11 \mathrm{~mm}$ in thickness. In the River Spur (41VT112) collection from the Victoria area are found a number of small, elongate distally beveled bifaces. Made of coarse material, these tools appear to be more crudely made and less uniform than the Brushy Creek bifaces from Wilson-Leonard. They range from 14 to $34 \mathrm{~mm}$ in maximum width and 8 to $15 \mathrm{~mm}$ in maximum thickness.

In east Texas and southern Oklahoma, Quince scrapers, described as small bifaces with beveled "bit" ends (Johnson as cited in Story et al. [1990:197]), have been found in Late Paleoindian contexts associated with Dalton points (see Perttula 1984), and these have been likened to Dalton adzes (Story et al. 1990:197). Two specimens from a surface collection at 41LR3 were examined for this study. With steep lateral edges beveled alternately in some sections, the specimens have a short, thick nubby appearance which 

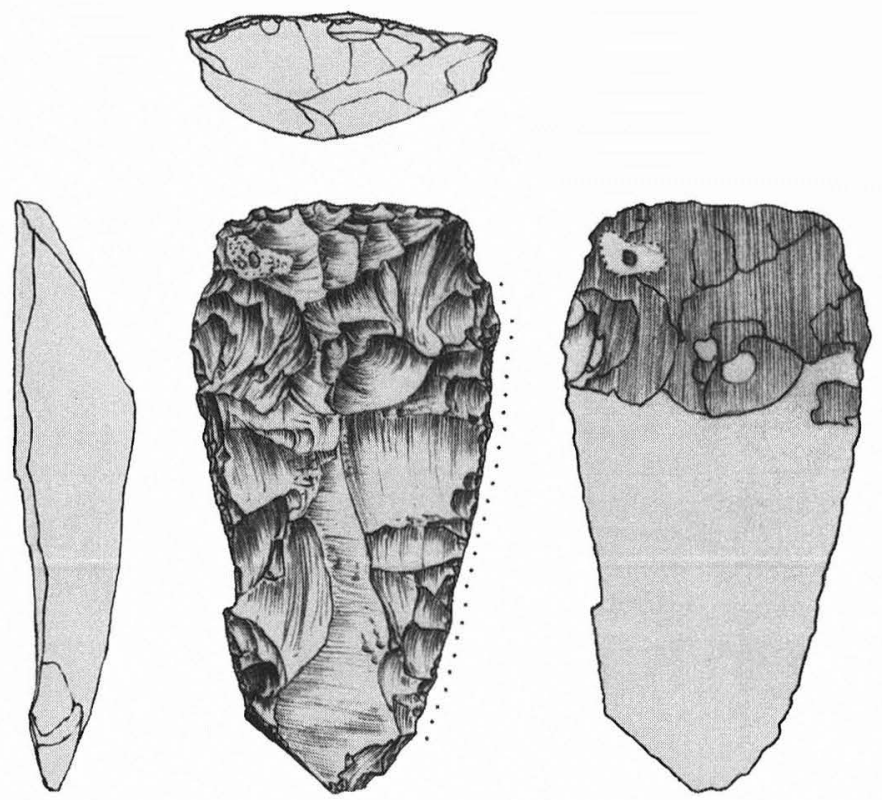

a
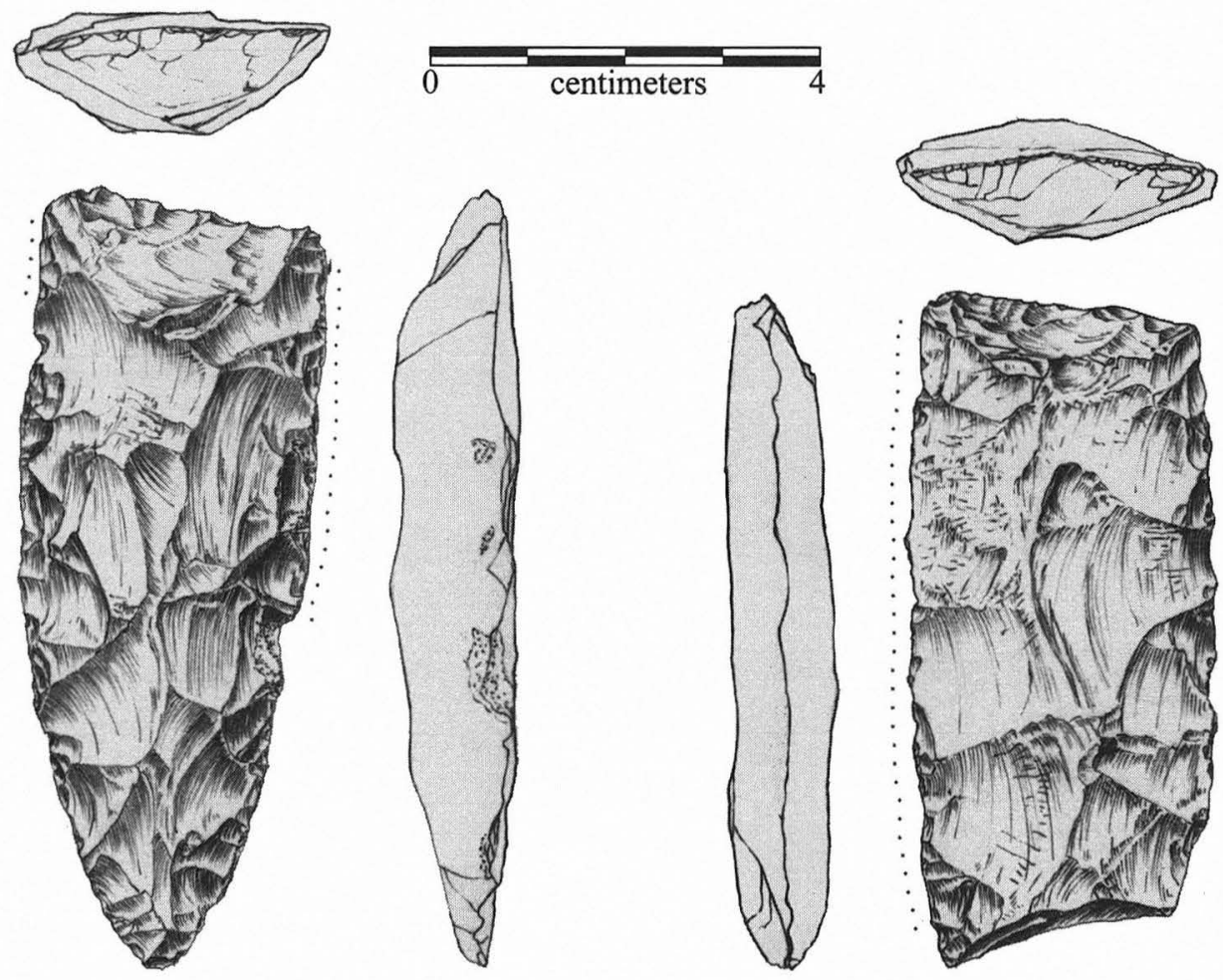

b
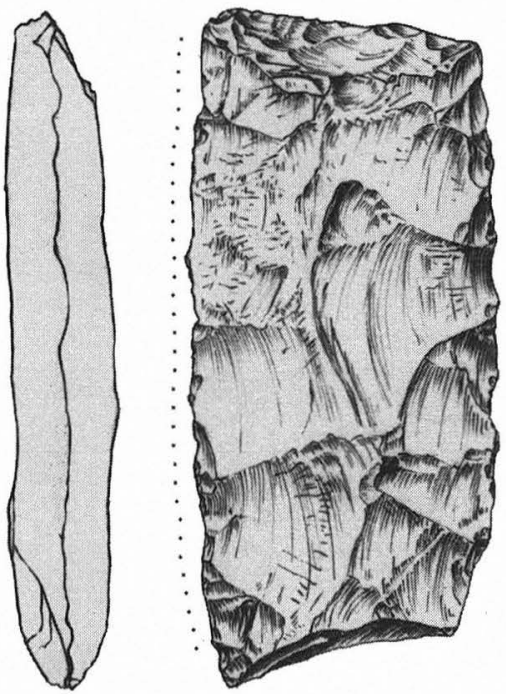

c

FIGURE 15-14. Variation in Brushy Creek bifaces. (a) differential luster in rejuvenated bit area on Specimen 37GB-9; (b) "hollowed-out" bit and elongate body form with intermittent lateral edge dulling, Specimen 29U1C-12; (c) short, beveled bit, Specimen 35U1C-2.

may be a function of heavy resharpening. Longitudinal profiles are more biconvex than any of the distally beveled bifaces from the Wilson-Leonard site, and the bit ends are more wedge-like than a beveled chisel. Dimensions of the two are length, $44 \mathrm{~mm}$ and $35 \mathrm{~mm}$; bit width, $32 \mathrm{~mm}$ and $21 \mathrm{~mm}$; bit thickness, $7.5 \mathrm{~mm}$ and $10 \mathrm{~mm}$; and bit height, $8 \mathrm{~mm}$ on both. In thickness dimensions, these specimens are comparable to Brushy Creek bifaces. According to Perttula's 


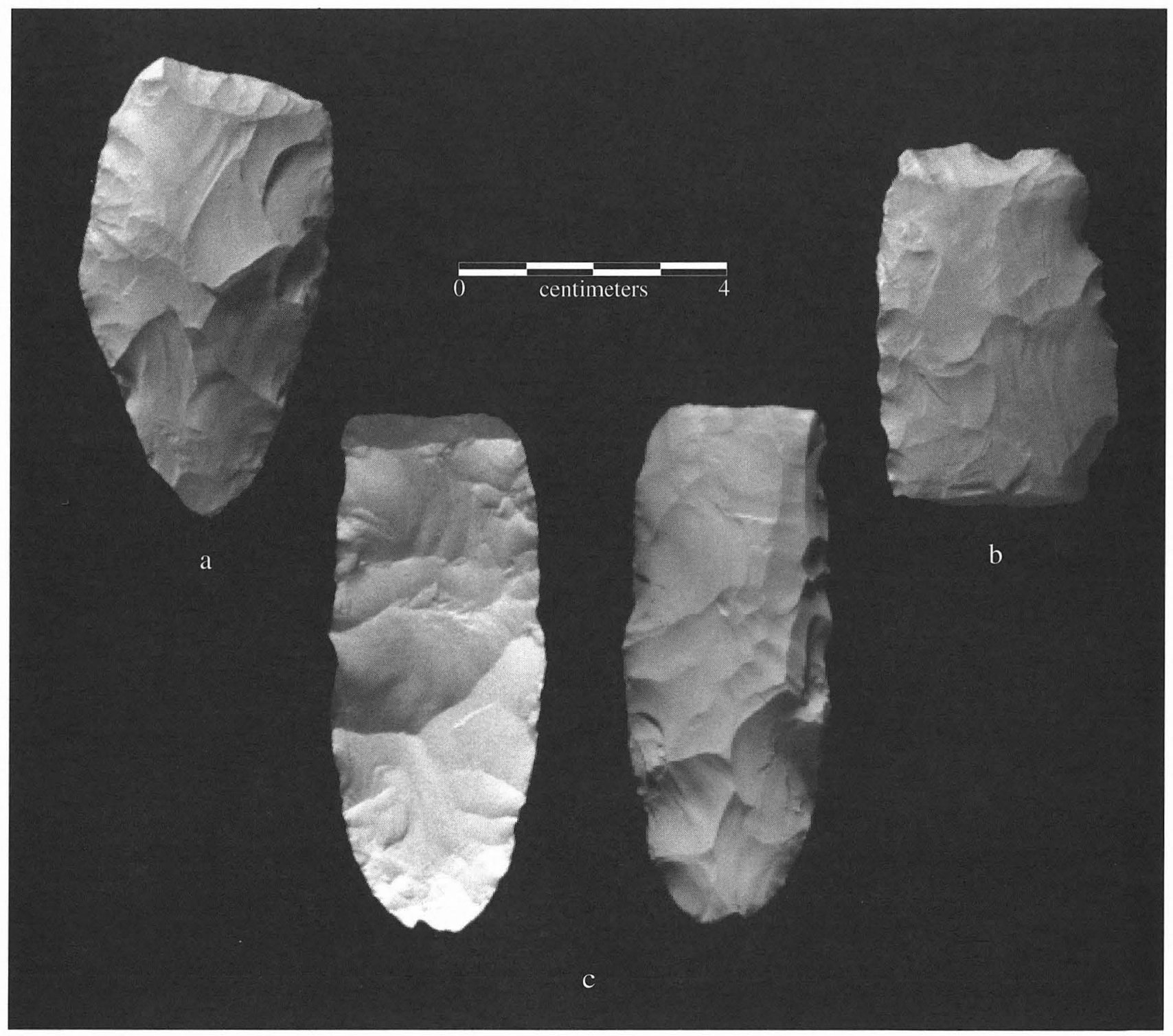

FIGURE 15-15. Brushy Creek bifaces. Specimen numbers: (a) 51WB-3; (b) 20W-2; (c) 34X2A-1, both faces, showing longitudinal flake removal in ventral bit area (right).

assessment of these tools in Arkansas sites, Quince scrapers are distinctive not only in their small size, which he does not attribute solely to resharpening, but in the consistent fine-grained raw material chosen for their manufacture in spite of the nearby sources of hard, coarse-grained materials that might have been more useful for heavy work (Timothy Perttula, personal communication 1996). As noted by Johnson (Story et al. 1990:197), these tools typically exhibit wear patterns and polish suggestive of use as scrapers.

Hester (1969) describes small, distally beveled bifaces from late Archaic contexts in Rio Grande Valley sites, which closely resemble Clear Fork tools in outline shape. Termed Olmos bifaces (Turner and Hester 1993:269), these tools are markedly smaller than any of the Wilson-Leonard Clear Fork bifaces and roughly half the size of Brushy Creek bifaces. With the exception of Olmos bifaces and Quince scrapers-neither of which are morphologically analogous to Brushy Creek bifaces-comparative specimens appear to be sparse, and none examined for this analysis is from a provenienced context.

\section{STRATIGRAPHICDISTRIBUTIONAND TOOL VARIATIONS OVER TIME}

Certain patterns in technology, temporal distribution, and use of Clear Fork tools can be observed in the data from Wilson-Leonard. As shown in Figure 15-16, Clear Fork bifaces were recovered in a continuous span over much of the site's history, from Paleoindian to Late Archaic times, whereas the unifacial variants were present only in deposits related to Archaic occupations (Unit III), ranging in age from 4000 в.P. 
to $6500 / 6000$ в.P. The six Brushy Creek bifaces were found chiefly in early contexts representing Late Paleoindian occupations, ca. 8700 to 9500 B.P.

This distribution and the peak of bifacial Clear Forks in Late Paleoindian and Early Archaic times is graphically depicted in a density chart, which compensates for the varying excavated volumes within stratigraphic units (Figure 15-17). In the Valley Floor, greatest density of bifacial Clear Fork tools is seen in Unit IIIa (ca. $6000 / 6500$ B.P. to 8700 B.P.), with almost .7 tools $/ \mathrm{m}^{3}$ of excavated volume $(\mathrm{n}=18)$, followed closely by Unit II/IIIa with .33 tools $/ \mathrm{m}^{3}(\mathrm{n}=8)$. In the more-compressed deposits of the Valley Margin area, densities are somewhat exaggerated by lower volumes. As shown, however, greatest densities occur in correlated units (IIIa, II/IIIa, and X/II/IIIa), mirroring in many ways the pattern in the Valley Floor.

The samples of 13 Clear Fork unifaces and 6 Brushy Creek bifaces are too small for evaluation of distributional patterns. The advent of Clear Fork unifaces in Early Archaic contexts and apparent co-occurrence with Clear Fork bifaces is illustrated in Figure 15-17; Brushy Creek biface densities are not shown.

Among the sample, the highest breakage rate by far is seen among the bifacial Clear Forks, which may suggest use for very heavy tasks and/or more-intensive use and rejuvenation until exhausted or broken beyond salvageability. In contrast, all of the unifacial Clear Forks and two-thirds of the Brushy Creek bifaces are complete, a somewhat paradoxical phenomenon, given the frequent use of the more-fragile, fine-grained cherts for these tool forms. This may indicate these tools saw either less-heavy or less-intensive use. Typical fracture types and wear observed among tools of coarse cherts include bend breaks, concentrated step and hinge flakes, and-in at least two cases-battering.

Manifesting a somewhat different pattern, several tools of the finer-grained cherts exhibit impact fractures, including burin-like spall removals along lateral edges. These fractures may be due to the less-durable nature of the chert or may indicate specialized tasks that produced different damage and wear.

Interestingly, raw material selection appears somewhat patterned in time for certain tools, as well. The majority of tools (8 of 15 or $53 \%$ ) recovered from Early and Late Paleoindian contexts in Unit II and lower in Valley Floor squares are made of fine-grained cherts. These include three Brushy Creek bifaces and five Clear Fork bifaces.

Clear Fork tools from other areas suggest a deliberate selection of coarser raw materials. Hester (1980:112) has observed that a very durable, hard, gray siliceous quartzite is common among Clear Fork tools recovered in parts of south Texas. At the Yarbrough site in East Texas, bifacial Clear Forks are made of ferruginous sandstone (Johnson 1962:191), and selection of gray quartzite for both unifacial and bifacial tools has been consistently noted in other East Texas sites (Crook and Harris 1952:21).
Heat alteration, recorded on nearly $25 \%$ of both Clear Fork bifaces and unifaces and on half of the Brushy Creek bifaces, may be a significant line of evidence in determining possible use. Purdy (1981:58) has tentatively speculated that Clear Fork tools may have been used in woodworking tasks including canoe building. Ethnographic accounts of this process detail the aboriginal method of incrementally charring a $\log$ and scraping out the burned interior with shells or "stone adzes" until an interior cavity of sufficient size was achieved (see, for example, Harriott 1893:Plate XII caption). Although it is not suggested that Clear Fork tools in this area were used for canoe building, it may be that similar tasks requiring use on hot, charred wood-such as the making of wooden mortars and other implements-may have resulted in patterns of heat alteration on some of the tools.

Observations related to hafting among the three tool groups does not point consistently to a singular hafting mode. It has been suggested that lateral edges of certain lithic tools and projectile points may have been dulled in preparation for hafting and to minimize potential breakage of sinew or fiber wrapping (Wormington 1957:23). In replicative studies on fluted points, however, Titmus and Woods (1991) found this explanation inadequate and have suggested alternatively that dulling was intended to strengthen lateral edges and diminish the possibility of tool fracture inside the haft element during use.

On the present three groups of tools under study, however, the asymmetrical and discontinuous distribution of dulling and grinding seems to suggest somewhat erratic edge modification effected in order to fit a tool to a particular haft. These patterns of dulling also may have occurred during use as a result of rubbing and friction in a hard, inflexible wooden socket. Among the three tool groups, dulling was more prevalent on Brushy Creek forms (66\%). Edge dulling was observed on $58 \%$ of both bifacial and unifacial Clear Forks. Dulling of ridge lines, another likely indicator of haft wear, was observed on specimens throughout all samples but was likely skewed by the different light reflective properties of particular raw materials.

\section{TECHNICALANALYSES}

Distally beveled tools in this study were examined by use-wear specialists Boyce Driskell and Dale Hudler, and an additional two were studied by Jeffrey Hurst for evidence of organic residues. Together, their findings provide intriguing insights into possible uses for these tools. Although results point most frequently to woodworking, other findings hint at use on bone and soft materials such as animal hide.

Among the 58 complete or distal sections of WilsonLeonard specimens examined by Hudler, distinctive polish was found on 23 (see Chapter 22). Of that group, 14 are Clear Fork bifaces, 5 are Clear Fork unifaces, and 3 Brushy Creek bifaces. The majority of specimens (13 of 23) display polishes suggestive of woodworking. It is interesting to note 

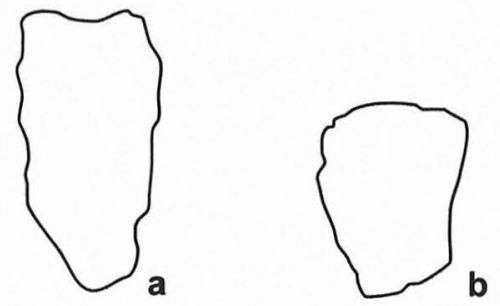

b

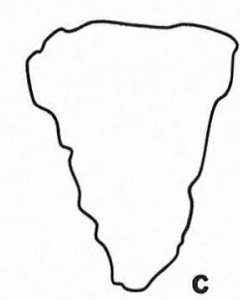

\{

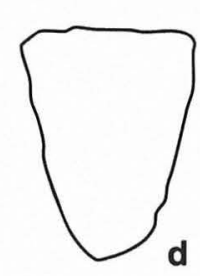

d
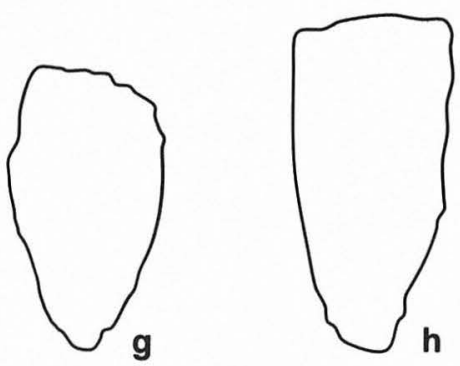

g

$$
\text { h }
$$
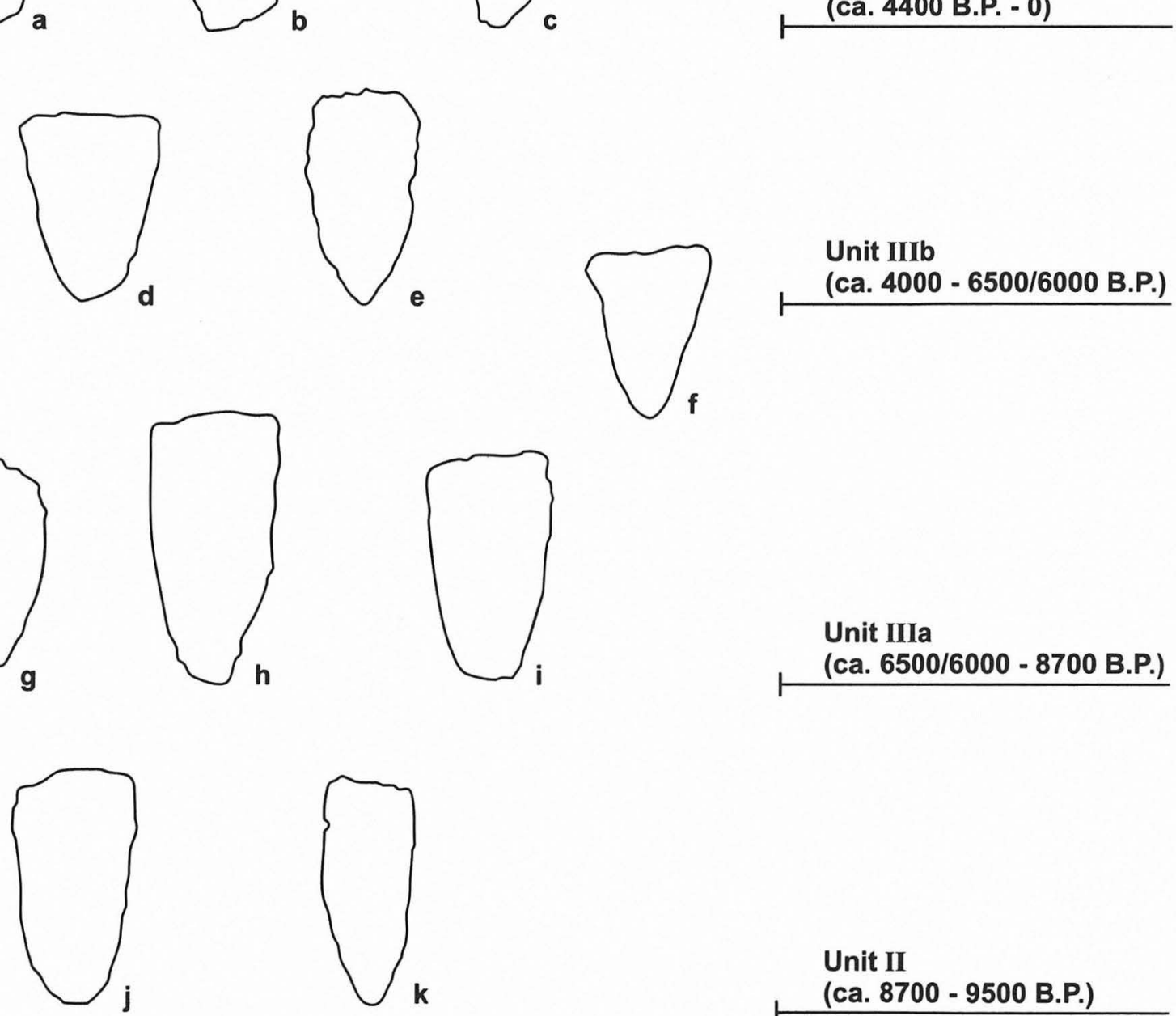

Unit IIIa

(ca. $6500 / 6000$ - 8700 B.P.)

(ca. 4400 B.P. - 0)

\section{Unit II}

(ca. 8700 - 9500 B.P.)

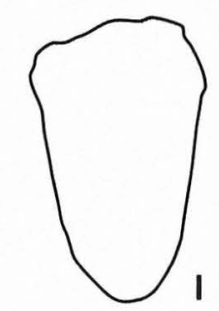

Unit Isi-c

(ca. 9500 - 10,000 B.P.)

Figure 15-16. Patterns in form and distribution of Clear Fork bifaces, Clear Fork unifaces, and Brushy Creek bifaces over time. Forms represented: Clear Fork bifaces (a, d, g-i, j, l); Brushy Creek biface (k); Clear Fork unifaces (b, c, e, f).

that in that group, the large majority are Clear Fork bifaces $(n=10)$, although Clear Fork unifaces $(n=2)$ as well as a single Brushy Creek biface are also represented.

Of particular interest are the eight tools that evidence use on a hard, high-residue material, such as bone. Of that group, four are from chiefly Late Paleoindian deposits (Units II, Isi-c, and II/III), and one is from a possible Early Paleoindian context. Two unifacial specimens, one from Early 


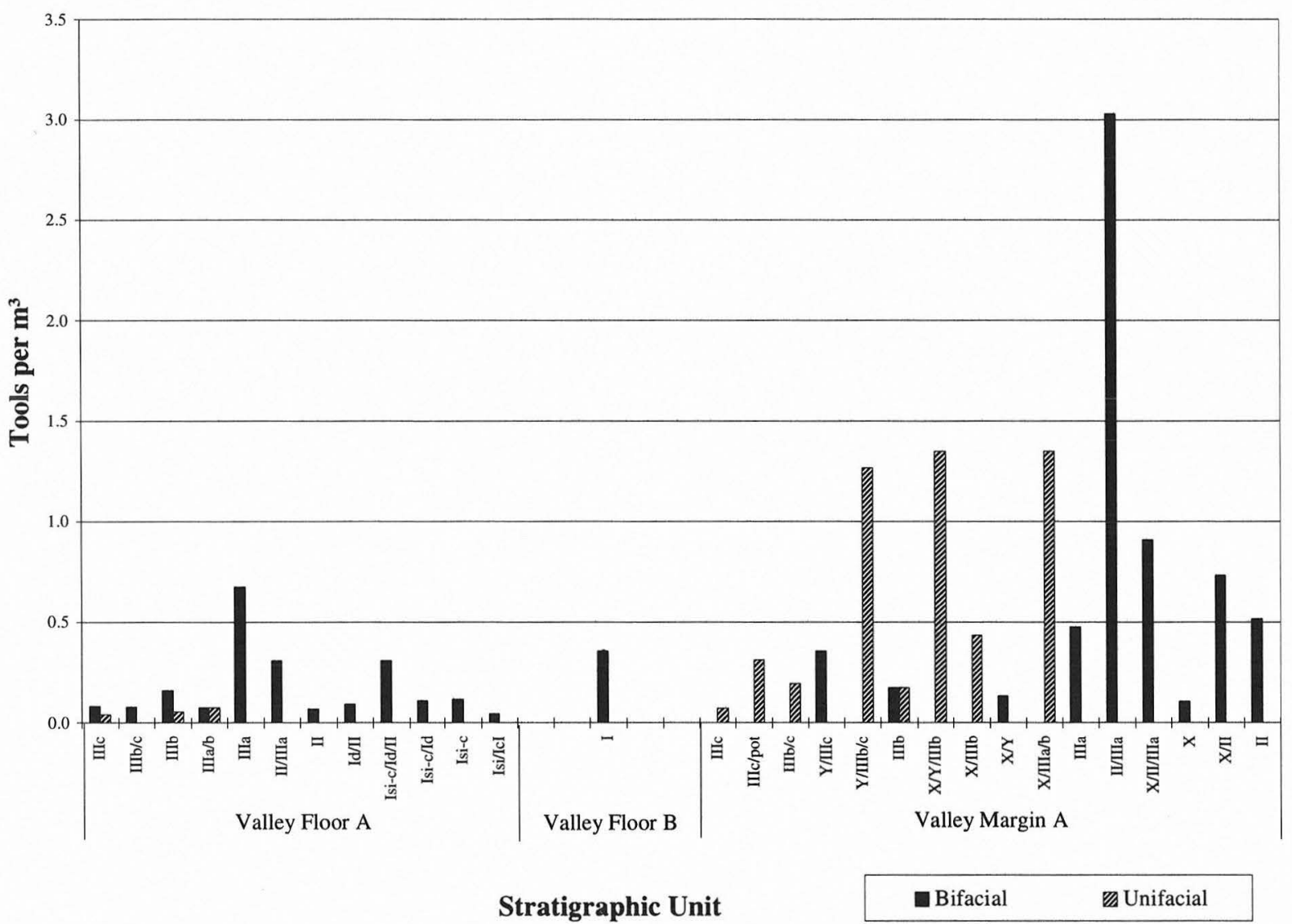

FIGURE 15-17. Density by excavated unit of Clear Fork bifaces and unifaces.

Archaic (9L-2) and one from Early/Middle Archaic (20I-4) contexts, are classed as multiuse tools, based on two different types of polish present on each.

Driskell (see Chapter 22) examined three distally beveled tools and one elongate proximal section but found no evidence of use. This was due largely, he believes, to the presence of a soil polish which may have obscured signs of use-wear at the level of magnification he employed.

In his examination of two Clear Fork tools for organic residues, Hurst found chloride, nitrate, and carbonate anions on each (see Chapter 23). The chloride and carbonate anions were interpreted as pedogenic in origin, but Hurst found the presence of nitrate intriguing. Although possibly deriving from agricultural runoff, it may have, alternatively, accumulated during prehistoric use of the artifacts.

\section{VARIATIONS INCLEAR FORKAND OTHER DISTALLY BEVELED TOOLS: A USE-RESHARPENINGMODEL}

Much variability in metric and morphological attributes has been measured, tabulated, and analyzed among Clear Fork assemblages from Wilson-Leonard and other sites. The significance and implications of this variability, from a technological, functional, and cultural standpoint, however, re- main to be more fully examined and addressed. What traditionally have been considered in classificatory schemes as typological attributes may be relics of the use-resharpening cycle. From this perspective, size and shape variations take on greater significance within the functional realm and carry less weight as typological indicators.

As suggested in graphic representations (Figure 15-18), variations in overall tool length and shape, bit shape, edge angle, and bit width and height will occur as the tool is used, resharpened, and the distal end truncated to rejuvenate a steeply beveled exhausted edge. Bit rejuvenation likely entailed one or a few hard-hammer blows directed from the ventral surface at the center of the bit, resulting in a more acute-edged, "scooped-out" profile (Brown et al. 1982:65; Taylor and Highley 1995:Figure 263) or bit removal by a tranchet-like blow to the bit lateral edge.

As a tool is used and resharpened, length diminishes, and the position of the bit moves progressively lower toward the proximal or butt end of the tool (see Figure 15-18a). This trajectory is suggested in specimens from the Wilson-Leonard sample, with the inferred preform (see Figure 15-1a) followed by a succession of shorter specimens. Length to width ratios for the preform (139:50 or 2.8) are nearly twice that of the likely exhausted specimens ( $67: 41$ or 1.6) illustrated in Figures 15-1d and 15-2d, and it may may be 


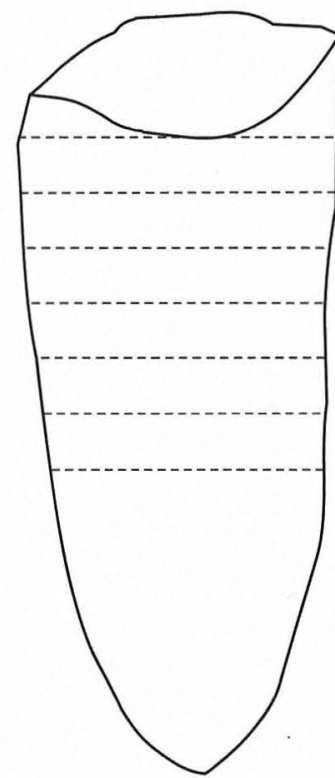

a

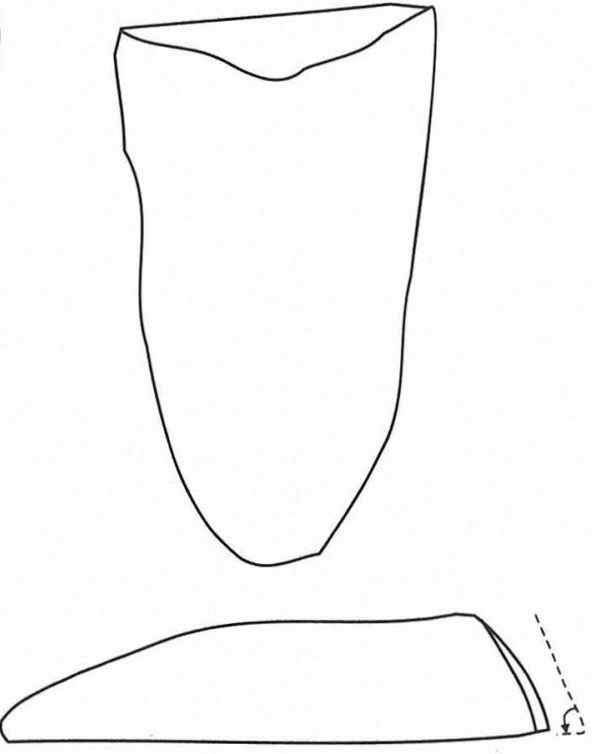

b

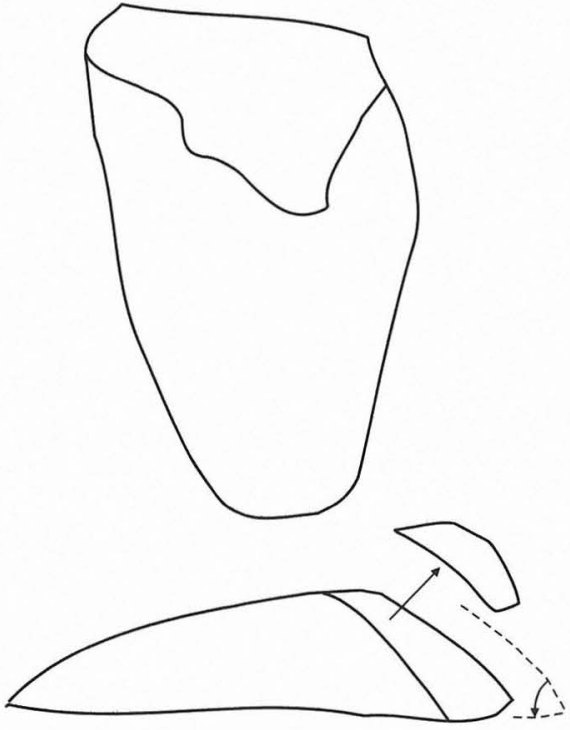

c

FIGURE 15-18. Hypothesized sequence of attrition and corresponding morphological changes with successive resharpening and rejuvenation episodes in Clear Fork tools (not at full size). (a) a tool marked with potential positions of bit removal for rejuvenation; (b) idealized tool with steeply beveled, "exhausted" bit; (c) idealized "rejuvenated" tool with low-angled bit and longer bit height.

that some formula along these lines may prove, with further studies, to be a rough indicator of "stage" of tool rejuvenation.

Along with diminished length, it is not inconceivable that changes in bit width and body shape might have occurred during the process. Specimens with markedly convex lateral edges might see an increase in bit width as the bit is repositioned, whereas triangular specimens might display diminished bit widths.

As shown in Figure 15-18b and c, certain bit dimensions tend to covary within the use cycle. With use and frequent resharpening, bit height diminishes as bit edge angle increases, causing the bit to become increasingly shorter and more steeply beveled. Rejuvenation produces a fresh, acute-angled bit of moderate to long length, as represented in a replicated tool shown before and after removal of the bit.

Other variables likely played a role in the form, size, and shape of Clear Fork tools. Individual knapping abilities or restraints imposed by raw material quality, size, and shape may have resulted in morphological differences among tool groups, as Hofman (1977) has noted.

Size and position of the haft would have served as limiting factors on all three body dimensions. Interestingly, based on calculations of Coefficient of Variation, width dimensions were shown to be the most standardized among Clear Fork and Brushy Creek bifaces, whereas length was the most regular dimension among Clear Fork unifaces. Thickness was proportionately most variable in relation to the mean among bifa- cial Clear Forks, width most variable among Clear Fork unifaces, and length most variable among Brushy Creek bifaces. What these factors imply in regard to the type(s) of haft employed is not clear, particularly when considered along with the highly irregular patterns of dulling and grinding of lateral edges.

From a behavioral standpoint, this use-resharpening model frames a critical perspective, that is, renewability and long use-life rather than stylistic or hafting considerations were key elements in the design and dimensions of many Clear Fork tools. This is strongly indicated by the unusual initial length of reported preforms and the experience of replicators whose hafting designs for Clear Forks are not predicated upon tools of this length (see Howard 1975; Hudler 1997).

Possible uses of Clear Forks and similar distally beveled tools have been further elucidated by recent studies, as noted above. In addition to intriguing hints of possible use on animal materials, in the case of several tools, Hudler (1997; see Chapter 22) has provided more solid support for the woodworking hypothesis proffered by many theorists. What remains to be further explored are the range of woodworking activities involving this tool form, and it is in this area that comparisons and replicative use studies with similar tools in other regions may prove most useful. Morse and Goodyear (1973) have suggested Dalton adzes may have been used for building structures and dugout canoes, and they note significantly higher distributional frequencies of usable adzes in larger "base" camp sites. Woodworking 
involving shaping, smoothing, and carving, and the manufacturing of wooden utensils and containers is also suggested (Morse and Morse 1983:78). These authors proffer the contemporary short-handled handaxe as an analog to the Dalton tool. In a similar vein, Purdy (1981:58) has suggested that the Clear Fork tool in Florida could have been used variously in planing, sawing, adzing, chopping, and wedging in the production of posts, houses, and canoes. Farther afield, replicated chert adzes were effectively used in hoeing and digging soil and limestone and in hollowing out charred logs, during a use-wear study of Maya stone tools at Cerros (Lewenstein 1987). While it is not suggested that Clear Fork tools are analogous to the tranchet bit adzes (Shafer 1983) of that region, it is perhaps useful to bring into consideration additional comparative data for future use-wear studies of Clear Fork tools. Finally, an alternative line of questioning needs to be deliberated, and that is, lacking adzes, axes, and other identifiable tree-felling and woodworking tools, by what means were aboriginal peoples in Texas and the Plains procuring and processing wood for posts, shelters, vessels, shafts, and tools? At this juncture, it would seem that the Clear Fork tool emerges as the most likely candidate for many of these tasks.

\section{SUMMARY AND INTERPRETATIONS}

A geographically widespread and apparently highly successful distally beveled tool form is shown in three different variations at the Wilson-Leonard site. Although somewhat temporally segregated, these forms - the bifacial Clear Fork, unifacial Clear Fork, and proposed Brushy Creek biface-appear to be, with several exceptions, functionally similar.

Bifacial Clear Fork tools appear first in Paleoindian contexts and reach a maximum population and density during Early Archaic times. Their strong presence at this time may indicate an intensification of woodworking or other activities requiring heavy tools. Similarities in certain metric attributes and resharpening design suggest this tool may be functionally analogous to the contemporaneous Dalton adze of the southeastern United States and the Guadalupe tool of south-central Texas. A much thinner and narrower distally beveled biface, tentatively termed the Brushy Creek biface, is restricted chiefly to Late Paleoindian times. The geographic distribution and use of this variant requires much more study.
The advent of the unifacial Clear Fork form marks a shift in a highly conservative tool manufacturing technology. At Wilson-Leonard, the first unifacial variants were detected in zones related to the later part of the Early Archaic, although bifacial tools still clearly predominate; this proportional pattern appears to continue through Middle Archaic zones. In Late Archaic contexts, the majority of Clear Fork tools are unifacial. These findings are in accord with data collected at Choke Canyon (Hall et al. 1982), which indicate unifacial Clear Fork tools occur later than their bifacial counterparts.

Although there are extremes represented, the bifacial Clear Fork tools overall evince substantial homogeneity, both in morphology and raw material. The greatest variation in bifacial Clear Forks occurs in the length dimension, and this likely reflects use and rejuvenation. The extraordinarily large Clear Fork preform provides maximum dimensions against which other bifacial Clear Forks may be gauged as to relative degree of resharpening and use.

In comparison to the other distally beveled tool forms from this sample, bifacial Clear Forks are generally larger in all dimensions and consistently are made of coarser, local cherts. Smaller specimens - unifacial Clear Forks and Brushy Creek bifaces - are more frequently formed on finer-grained cherts. That preferences for certain raw materials are keyed to tool type and use is not substantiated in use-wear analysis. Further, although six polish categories were defined by Hudler (see Chapter 22), none was exclusive to any one tool type, with the exception of a "fuzzy" polish suggestive of hideworking found on two very early Clear Fork bifaces. Hudler's findings strongly support use of the three tool types in woodworking activities and possibly on hard, high-residue substances such as bone.

The fact that complete as well as broken tools were recovered in all major stratigraphic units suggests they were variously being made, used, lost, or discarded, and possibly cached at the site throughout much of the site's history. The local occurrence of coarse, weathered cherts - the preferred raw material for the majority of the distally beveled tools, as well as for several other tool types (e.g., core tools, see Chapter 18) - may have been a critical factor in the selection of the site at different times for particular activities requiring heavy tools. This scenario seems particularly compelling in the Early Archaic period, when the density of Clear Fork tools reached a maximum. 
"This page intentionally left blank" 


\title{
Chapter 16
}

\section{BIFACES, BIFACIAL TOOLS, PERFORATORS, BURINS, AND SPALLS}

\author{
by Susan W. Dial and Michael B. Collins
}

\section{INTRODUCTION}

Perhaps more than any other traditional tool category, bifaces carry an illusory body of evidence emanating from both the manufacturing realm and the use context. The manufacturing/use history may at times be highly fluid and interwoven, and this aspect presents significant classification problems. This is due in large measure to what Kelly (1988) and others have termed the multifunctionality of bifaces.

In the present assemblage, the chief unifying attribute is, in simplest terms, bifacial technology, and it is within this framework that artifacts ranging from crude bifaces and expediently produced tools made on biface fragments to wellshaped preforms and finely crafted perforators are considered. Our construct attempts to recognize products of less-formalized technological pathways as well as the more traditionally described Stage 1-3 bifaces.

The manufacturing of bifaces requires a considerably higher investment of time, energy, and skills than that required for the extraction of flakes, and this would suggest that bifaces are not designed to be discarded quickly (Hayden et al. 1996; Kelly 1988). Rather, bifaces may serve in at least three different although not mutually exclusive roles: as cores, providing an efficient source of flakes with more useful edges; as tools designed for long use-life based on resharpenable bifacial edges; and as tools shaped for preexisting hafts (Kelly 1988:719). Fragments of bifaces also serve as blanks for such tools as burins and perforators. In this study, the term bifaces includes both flakes and core blanks that have been flaked on both faces. It excludes what we perceive to be finished projectile points, which are reported in Chapter 13, as well as certain formal tool forms such as Clear Fork bifaces (see Chapter 15).

Findings on the more than 2,000 specimens encompassed in this collection are reported under three larger categories: bifaces and miscellaneous bifacial tools; perfora- tors; and burins and burin spalls. Manufacturing attributes of shaped or "stage" bifaces from rudimentary stage to finished product are examined throughout the site's history. Of particular note are products of early Paleoindian technologies-specifically the large and finely crafted Clovis forms and the distinctive Folsom-age bifaces, some of which fall under the "ultrathin" rubric. Distinctions among biface reduction systems are more subtle during Archaic times, although changes in size and shape are explored. A large group of informal and likely expedient bifacial tools is examined, as well as pièces esquillées, perforators, burins, and acute-edged knife-like tools.

\section{ANALYTICAL APPROACHES}

Analytical approaches to these rather widely differing artifact forms have varied in other studies. In recent years, lithic technologists have used pathway models to graphically illuminate the often complex histories of stone tools (e.g., Collins 1974, 1975; Driskell 1986; Schiffer 1972). Use of such models, such as the example in Figure 16-1, allows for conceptualization of multistage, multidirectional production and the maintenance and recycling processes that likely flowed not only within technologically or culturally prescribed parameters but also within the realm of the circumstantial or expedient.

The analysis presented here is constructed to suggest technological, cultural, and temporal markers. Broad issues examined by stage or group and through time include patterning in selection of raw material, technology, maintenance, discard, and recycling. Inasmuch as these products assume a variety of somewhat disparate and unrelated forms, different attributes necessarily were developed to characterize each category. These are discusssed in the respective sections below. For each group, however, a uniform set of data pertaining to raw materials was collected. This data set includes raw material category, cortex presence/absence and 
type, heat alteration (absent or from slight to extensive), fluorescent properties, presence/absence of mineral precipitates, and presence/absence of patina. A more detailed discussion of methods and terminology used for these observations is outlined in Chapter 12.

As in many other lithic categories discussed previously, there is a certain amount of ambiguity and overlap not only among the present categories but also with those reported in other chapters. Some of the overlap is due to the variable aspect and multifunctionality of bifaces in general. In some cases, what are herein termed bifaces may have been used at times as cores for the extraction of flakes for the production of flake tools. Within the category identified as miscellaneous bifacial tools, there is likelihood that some were neither utilized nor intended as tools, although they display modifications suggestive of such. In like manner, artifacts characterized as bifaces within the Stage 1-3 manufacturing hierarchy may have been utilized as tools at some point in their history, but evidence for this was not recognized without high-power microscopy. Perforators and burins are presented in this chapter, although a small number are unifacially chipped and are somewhat similar to unifacial gravers and burinated flakes reported in Chapter 17.

\section{BIFACES AND MISCELLANEOUS BIFACIAL TOOLS}

Biface analysis followed the multistage manufacturing scheme advanced by Collins (1974) and Driskell (1986). Three stages were defined for this project, as explained below. An additional category, here termed miscellaneous bifacial tools, includes specimens that are aberrant within the stage continuum and/or have been modified for possible use as tools; these have been assigned to 11 groups based on morphology and edge modification. The final category, biface fragments, encompasses those specimens that are too incomplete to characterize further.

\section{Attributes}

A variety of attributes was recorded for bifaces. Those reported here include size, outline form, basal modification, raw material characteristics, state/condition, fracture type, section (for broken specimens), and inferred reason for discard. As is noted, somewhat different criteria necessarily were employed to describe complete and incomplete specimens.

Metric measurements recorded include maximum length, width, and thickness. A summary of only those measurements observed to be complete is reported; total specimen counts thus may vary for each size attribute presented. A mean ratio of width to thickness also was calculated for Stages 1-3 as an additional rather coarse indicator of degree of reduction. Typically, later-stage specimens evidence a higher value in this measure. As discussed in Chapter 12, somewhat different approaches were taken on specimens conserved for organic residue (OR) analysis. In the case of
OR bifaces, only complete specimens were measured, in order to minimize handling.

Eleven categories of shape or outline form for complete specimens were observed. These include ovate, bipointed, lanceolate, triangular, subtriangular with convex edges, discoidal, asymmetrical, constricted or "shoe-shaped," rectangular, stemmed, and miscellaneous.

Morphological characterization of broken specimens was more difficult, and thus only the complete end of the specimen was described, or when possible, the complete end and lateral margins. Shape categories for broken specimens include straight end, rounded end, concave end, pointed end, convex end, straight end with lanceolate sides, straight end with convex sides, concave end with lanceolate sides, and stemmed. On many unfinished Stage 1 and 2 bifaces, it was difficult to distinguish basal and distal ends; for this reason, assignment of section categories (distal, basal, and medial) for broken specimens was not comprehensive and is reported here only for Stage 3.

Basal modification was observed on complete specimens and on broken specimens that are classed as basal sections; this attribute is reported here for Stage 3 bifaces only. The eight categories of modification identified include thinning unifacial, thinning bifacial, beveling, concave bevel, simple retouch, multiple hinge/step flaking, dulling, and indeterminate.

Condition of Stage 1-3 bifaces was recorded as either complete or broken. Among broken specimens, 11 fracture types were observed. These include bend, perverse, overshot, burin-like, thermal, edge collapse, radial, multihinge, impact, edge bite, and longitudinal overshot. Other fracture scars that appeared to be recent or postdepositional in origin were coded according to that aspect rather than the nature of the break itself. These include excavation breaks or plow damage characterized by relatively fresh fracture scar surfaces.

As fracture types and their identification have been discussed fully in studies dedicated more specifically to that purpose (i.e., Crabtree 1982; Johnson 1979, 1981; Tomka n.d.; see also Chapter 13 herein), they are not discussed here in detail, except to note those types for which confusion in terminology may exist (Figure 16-2). Specifically, what is here termed a longitudinal overshot fracture has been called a "longitudinal reverse" (Johnson 1981:45). Figure 16-2a illustrates a biface severed at midpoint by a longitudinal overshot emmanating from the base. The more typical overshot, or outrepasse fracture (Crabtree 1982:46) is shown in Figure 16-2b, a bipointed biface with an overshot flake extending from one lateral edge across the biface and removing the opposite edge. A perverse fracture (Figure 16-2c) may appear as a twisting break emmanating from the lateral edge and plunging across in a downward trajectory (Crabtree 1982:46).

Specimens were evaluated as to reason for discard or abandonment, and five rather broad categories were developed to 


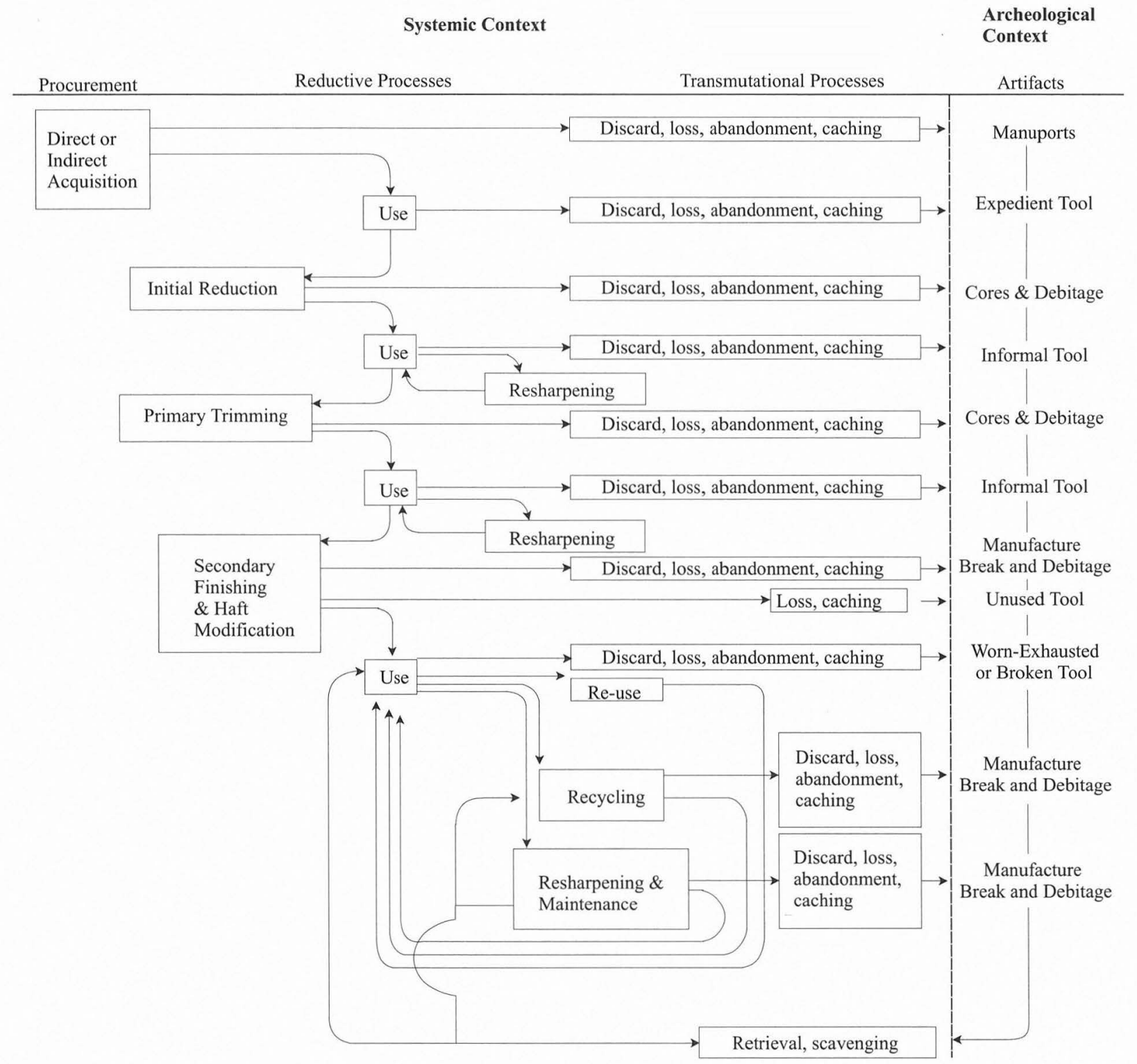

FIGURE 16-1. Model of lithic tool production and alternate processes, from acquisition of raw material to deposition into archeological context (following Bousman [1993] and Collins [1975]).

encompass myriad possibilities. These include breakage; failure to thin and/or presence of knot or stack; unsuccessful shaping; exhaustion (for tools); and indeterminate. Examples of some of these are shown in Figure 16-2, including failure to thin and edge collapse (Figure 16-2d) and unsuccessful shaping, likely due to failure to negotiate a tabular facet (see Figure 16-2e). Inasmuch as many Stage 4 bifacial tools were made on broken or aborted bifaces, it was not always possible to characterize state/condition, fracture type, or discard for that group.

\section{Findings}

Of the specimens recovered, 1,213 were classified as bifaces and 617 as unclassifiable biface fragments. In addi- tion, 209 miscellaneous bifacial tools are reported within an 11-group classification. Results of the analysis are presented first in a descriptive synopsis by stage of manufacture (1-3) and by tool category, then according to stratigraphic distribution. Biface fragments are reported last, chiefly in tabular form.

\section{Stage 1 Bifaces $(N=233)$}

Stage 1 bifaces generally are roughly chipped with irregular outlines and shape; most specimens retain large amounts of cortex (Figures 16-3 and 16-4). Sizes are widely varied. As shown in Table 16-1, Stage 1 bifaces range in length from 32 to $190 \mathrm{~mm}$, in width from 17 to $103 \mathrm{~mm}$, and in thickness from 5 to $38 \mathrm{~mm}$. Although most are relatively 


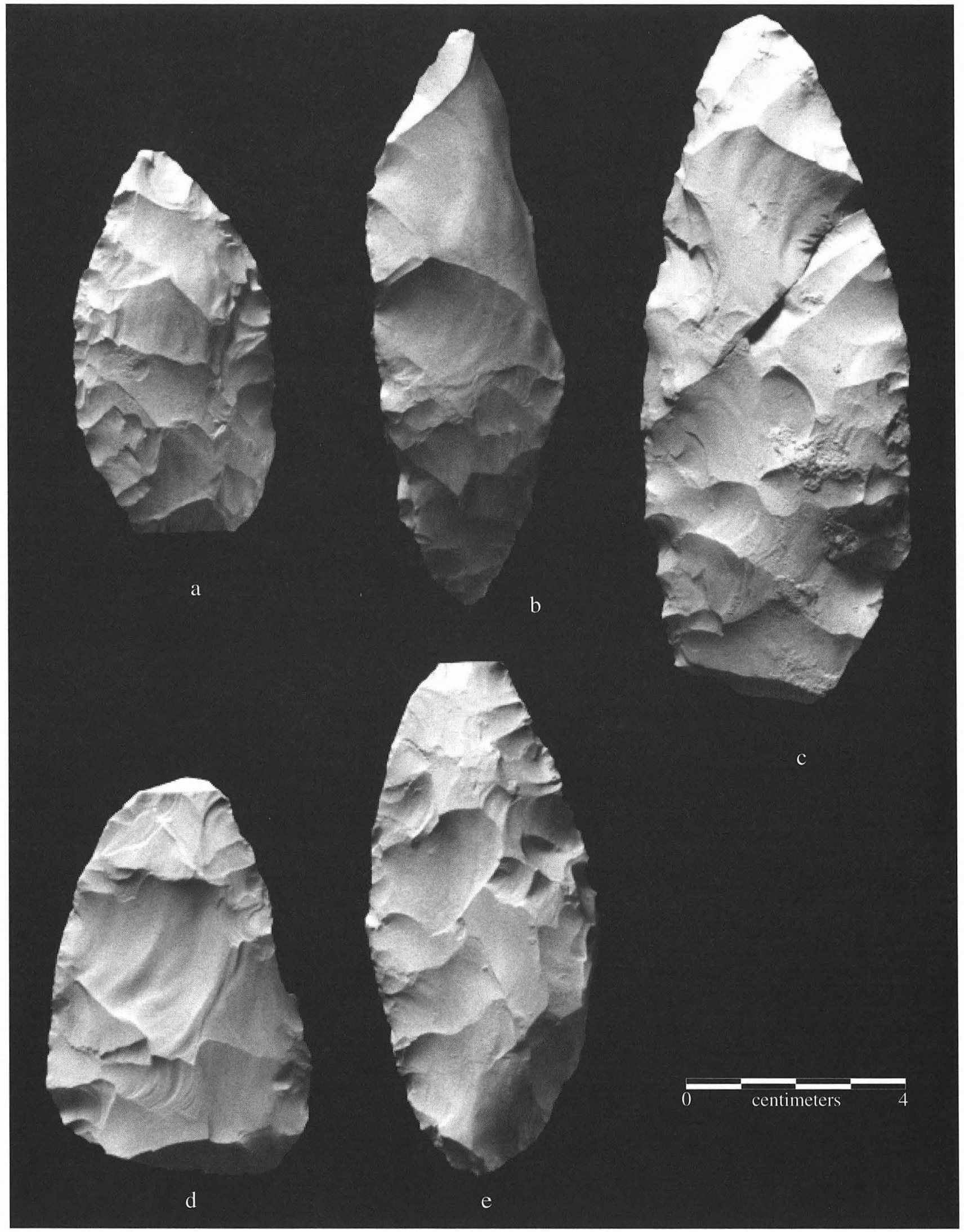

FIGURE 16-2. Examples of biface fracture types and manufacturing failures. (a) longitudinal overshot break; (b) overshot break; (c) perverse fracture; (d) edge collapse and failure to thin; (e) failure to negotiate a tabular facet. Specimen numbers: (a) 1095-1; (b) 50FD-8; (c) 27Y1A3 (proximal) and 31Y1B-2 (distal); (d) 37LD-34; (e) 37S1C-7. 

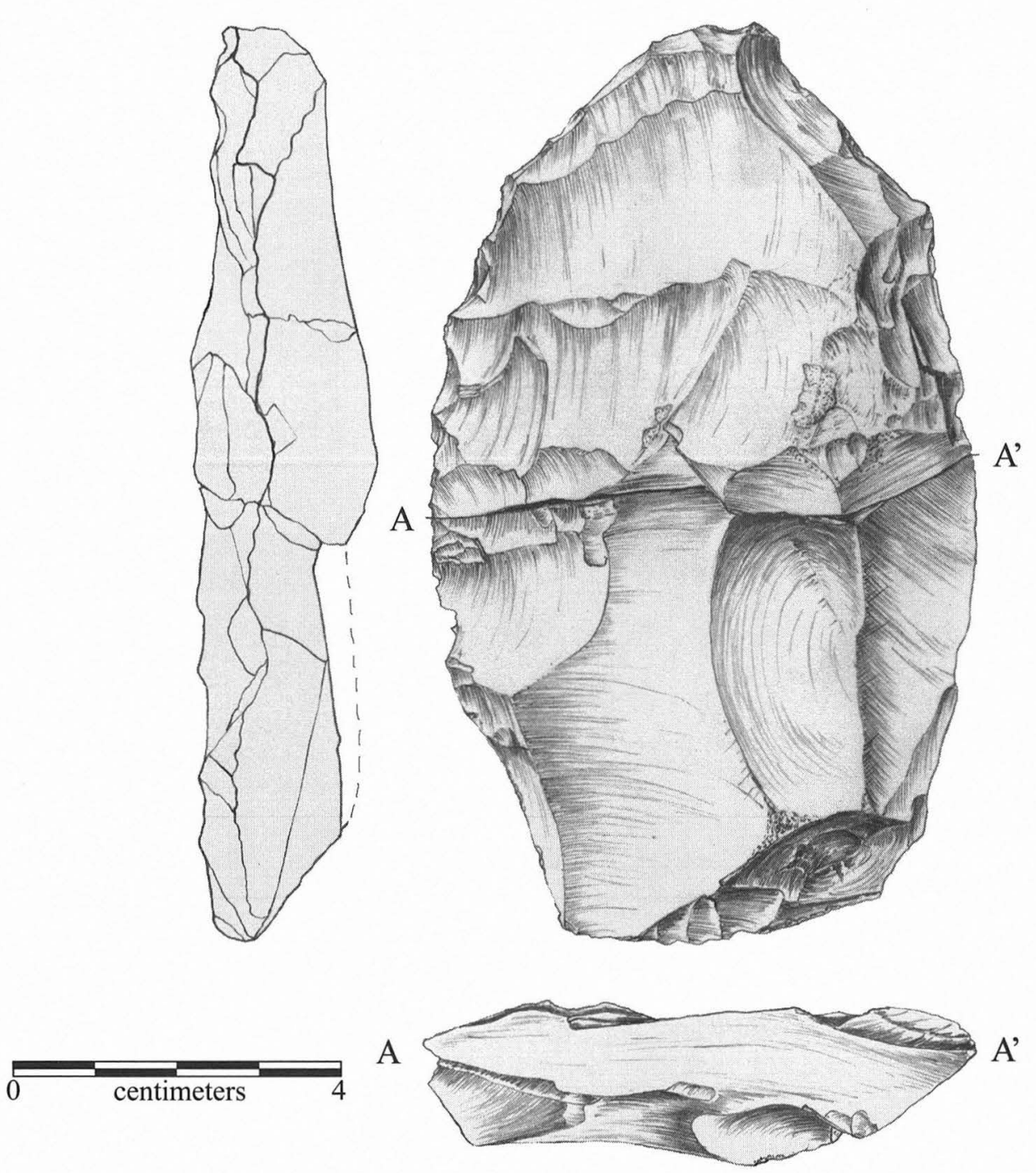

FIGURE 16-3. Representative Stage 1 biface: two conjoined specimens, 24T1D-5 (distal) and 14S1-4 (proximal); the latter section is heat damaged.

thick (averaging $17 \mathrm{~mm}$ ), a number of specimens - such as the one exhibiting the minimum thickness of $5 \mathrm{~mm}$ - are thinner cortex flakes with rudimentary bifacial flaking. Among Stage 1 bifaces, mean width to thickness ratio is very low at 2.78.

Of the total Stage 1 bifaces, $77(33 \%)$ are complete or nearly so. Among these, outline forms are varied, with the most numerous being subtriangular $(n=30 ; 39 \%)$ or ovate $(\mathrm{n}=21 ; 27 \%)$. Other forms include discoidal $(\mathrm{n}=6 ; 8 \%)$, rectangular $(\mathrm{n}=4 ; 5 \%)$, bipointed $(\mathrm{n}=3 ; 4 \%)$, and lanceolate $(\mathrm{n}=2$; $3 \%)$. Eleven (14\%) are miscellanous/indeterminate.

Raw material for Stage 1 specimens is predominately fine-grained Edwards chert (163 or $70 \%$ ), with the large majority of that being local $(97 \%)$. The remainder are of coarse Edwards cherts (45 [19\%] local and $9[4 \%]$ nonlocal), and $16(7 \%)$ are indeterminate. Cortex is present on 143 specimens, and on more than half $(\mathrm{n}=84 ; 59 \%)$ it is classi-
TABLE 16-1

Biface Stage 1 Descriptive Statistics

\begin{tabular}{l|c|c|c|c}
\hline & Length & Width & Thickness & $\begin{array}{c}\text { Width/ } \\
\text { Thickness }\end{array}$ \\
\hline Mean & 74.13 & 45.48 & 16.95 & 2.78 \\
Median & 68.5 & 43 & 16 & 2.75 \\
$1-\sigma$ & 26.68 & 13.01 & 6.11 & 1.08 \\
Range & 158 & 86 & 33 & 9 \\
Minimum & 32 & 17 & 5 & 0 \\
Maximum & 190 & 103 & 38 & 9 \\
Count & 76 & 201 & 209 & 209 \\
\hline
\end{tabular}

Note: Measurements are in $\mathrm{mm}$.

fied as upland residual, on $16(11 \%)$ as nodular, as stream altered on $9(6 \%)$, and as indeterminate on $34(24 \%)$. Patina is present on $46(20 \%)$ specimens; among those, distribution is heavy overall on 2 and differential on 10, the latter 


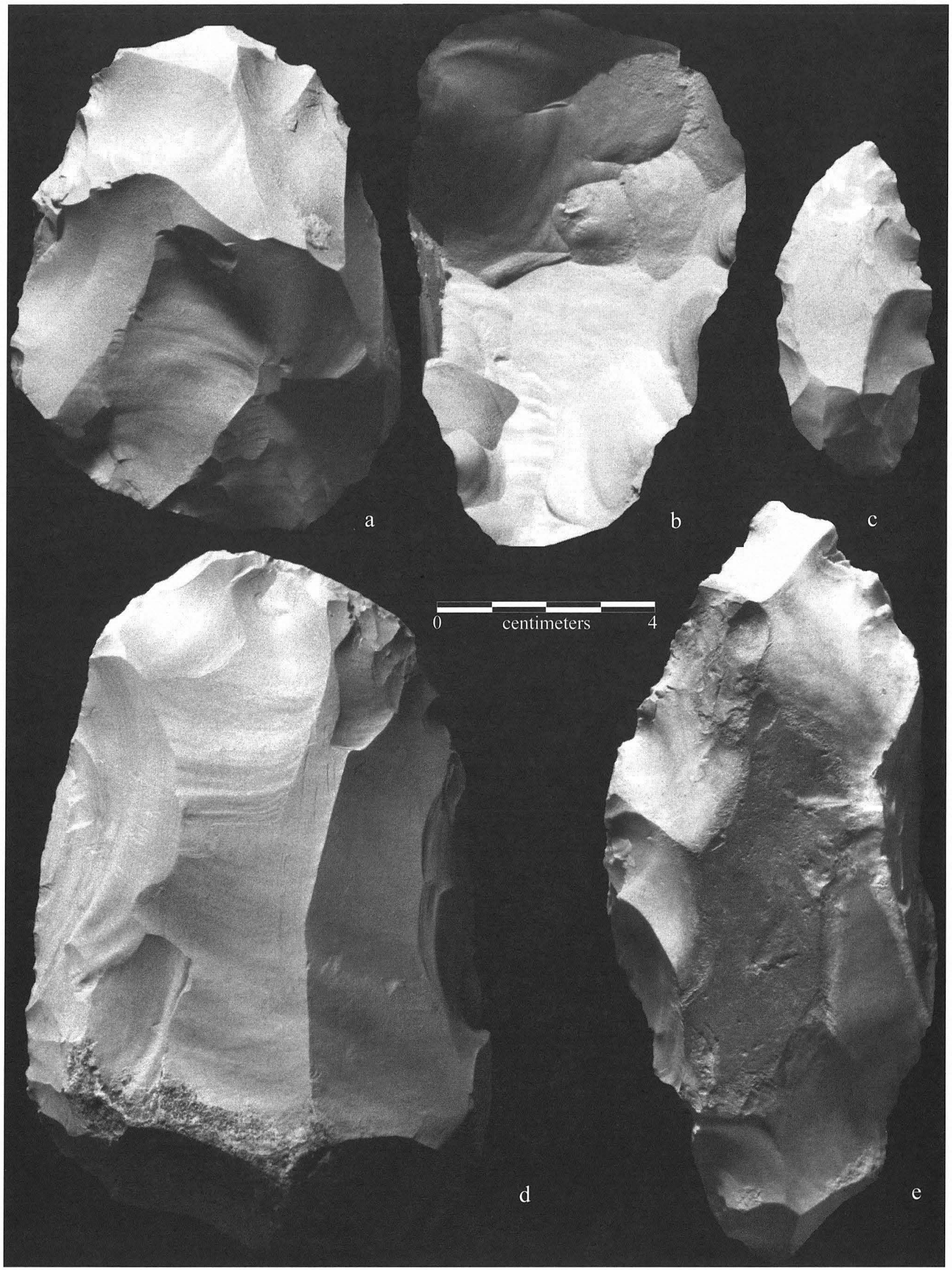

Figure 16-4. Examples of Stage 1 bifaces. Specimen numbers: (a) 20O-6; (b) 33R1-1; (c) 11A-10; (d) 10E-2; (e) $29 \mathrm{DD} 1 \mathrm{C}-3$. 
suggesting reduction of previously worked and patinated materials. A total of $31(13 \%)$ specimens is classified as heat altered ( 6 extensively so), and 60 (26\%) specimens showed mineral precipitates.

Among the 156 broken specimens, single bend fractures were observed on $59(38 \%)$ and thermal fractures on $24(15 \%)$. Present in smaller frequencies were overshot fractures $(n=5 ; 3 \%)$, longitudinal overshot $(n=2 ; 1 \%)$, and perverse fractures $(\mathrm{n}=1 ; 1 \%)$. Complex fractures were noted on 29 (19\%: 4 bend/burin-like; 3 multiple bend; 1 burin-like/ thermal; 1 perverse/longitudinal overshot; 5 bend/thermal; 1 bend/longitudinal overshot; 2 thermal/longitudinal overshot; 1 bend/edge bite; 1 burin-like/indeterminate; and 10 bend/indeterminate). Five specimens exhibit excavation breaks, and fractures on 32 are indeterminate.

Among the complete specimens, the most common manufacturing error or discard reason appears to be unsuccessful shaping coupled with failure to thin the biface. Fifteen complete specimens, however, were discarded or abandoned for no apparent reason.

\section{Stage 2 Bifaces $(N=788)$}

Bifaces in this stage generally have been thinned to the extent that no characteristics of the original core or flake morphology remain. They are characterized by a moreregularized outline, although some sections of cortex and knots or stacks of hinge flakes may remain (Figures 16-5 and 16-6). Sizes are generally intermediate between Stage 1 and Stage 3, as shown in Table 16-2. Mean width to thickness ratio for this group is 3.74 , markedly higher than that for Stage 1.

Of the 788 specimens, the great majority are broken. Only slightly over $13 \%(n=101)$ can be categorized as complete or nearly so. Outline shapes among the complete specimens generally are confined to two forms. Nearly half $(\mathrm{n}=45$; $45 \%)$ are subtriangular, while a smaller number $(n=23 ; 23 \%)$ are ovate. Other forms observed include asymmetrical $(\mathrm{n}=8$; $8 \%)$, lanceolate $(n=7 ; 7 \%)$, bipointed $(n=4 ; 4 \%)$, triangular $(n=3 ; 3 \%)$, discoidal $(n=2 ; 2 \%)$, and miscellaneous/indeterminate $(\mathrm{n}=14 ; 14 \%)$.

Raw materials for Stage 2 bifaces are predominately finegrained Edwards cherts $(\mathrm{n}=626 ; 79 \%)$. Of that number, 581 (93\%) are local and $45(7 \%)$ nonlocal. Only $106(13 \%)$ specimens are of coarse-grained Edwards cherts (87 [82\%] local and $19[18 \%]$ nonlocal). Additionally, $2(.3 \%)$ Stage 2 bifaces are made on chalcedony and $1(.1 \%)$ on quartz; $52(7 \%)$ others are indeterminate, in most cases due to heat alteration. Cortex, present on $200(25 \%)$ specimens, suggests an upland residuum derivation for almost half of those $(97 ; 49 \%)$ and stream alteration for $3(2 \%)$. Nodular cortex was observed on $27(14 \%)$, and cortex was indeterminate on 73 $(37 \%)$. Patina is present on $94(12 \%)$ specimens; of those, it is heavy overall on $10(11 \%)$ and differential on $8(9 \%)$. Mineral precipitates were noted on $128(16 \%)$ specimens. Heat

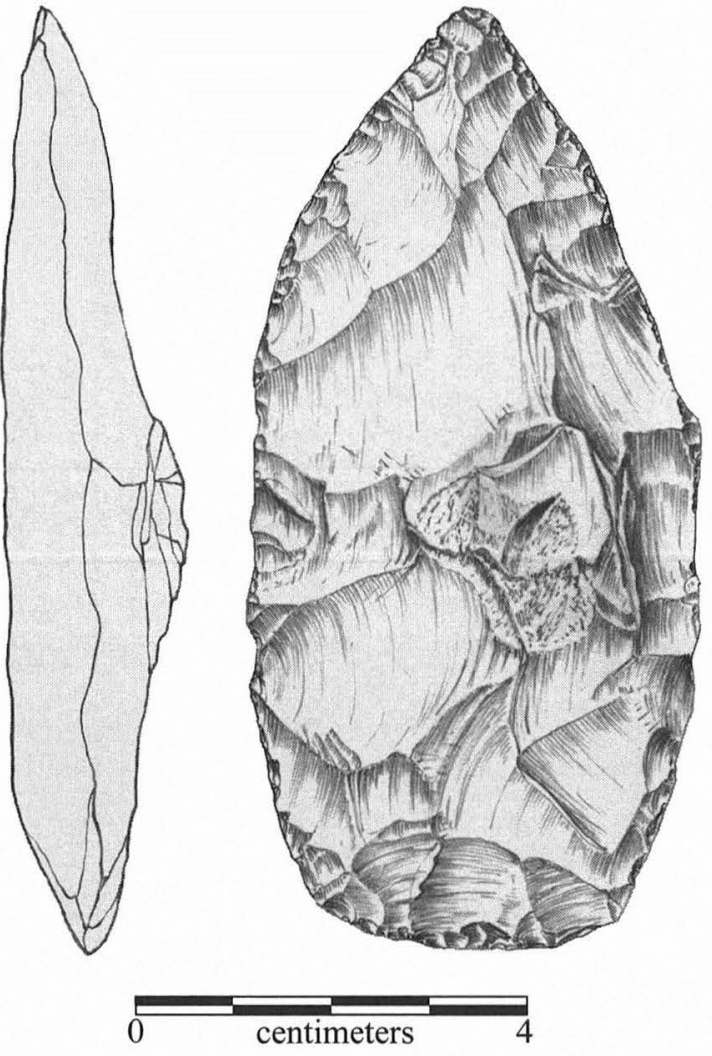

FIGURE 16-5. Example of Stage 2 biface, Specimen 37HC-13.

TABLE 16-2

Biface Stage 2 Descriptive Statistics

\begin{tabular}{l|c|c|c|c}
\hline & Length & Width & Thickness & $\begin{array}{c}\text { Width/ } \\
\text { Thickness }\end{array}$ \\
\hline Mean & 67.92 & 37.20 & 9.94 & 3.74 \\
Median & 67.00 & 36.00 & 9.00 & 4.00 \\
$\sigma$ & 18.42 & 10.16 & 3.69 & 2.76 \\
Range & 105.00 & 72.00 & 47.00 & 1.53 \\
Minimum & 28.00 & 8.00 & 3.00 & 2.67 \\
Maximum & 133.00 & 80.00 & 50.00 & 1.60 \\
Count & 100.00 & 689.00 & 720.00 & 0.96 \\
\hline
\end{tabular}

Note: Measurements are in $\mathrm{mm}$.

alteration was observed on $195(25 \%)$, of which $66(34 \%)$ were extensively damaged.

Among the 687 broken specimens, distal or pointed sections are present in greatest numbers $(n=246 ; 36 \%)$, followed by apparent bases $(194 ; 28 \%)$, medial sections ( $\mathrm{n}=117 ; 17 \%)$, and lateral edge fragments $(n=7 ; 1 \%)$. Others are classified as miscellaneous or indeterminate $(\mathrm{n}=123 ; 18 \%)$.

Although a wide variety of fracture patterns were displayed on Stage 2 bifaces, roughly half $(n=345 ; 50 \%)$ were broken by single bend breaks or end shock. Other break patterns observed include thermal fractures on $78(11 \%)$; perverse fracture on $55(8 \%)$; multiple bend breaks on 48 $(7 \%)$; bend and thermal on $35(5 \%)$; miscellaneous fracture 


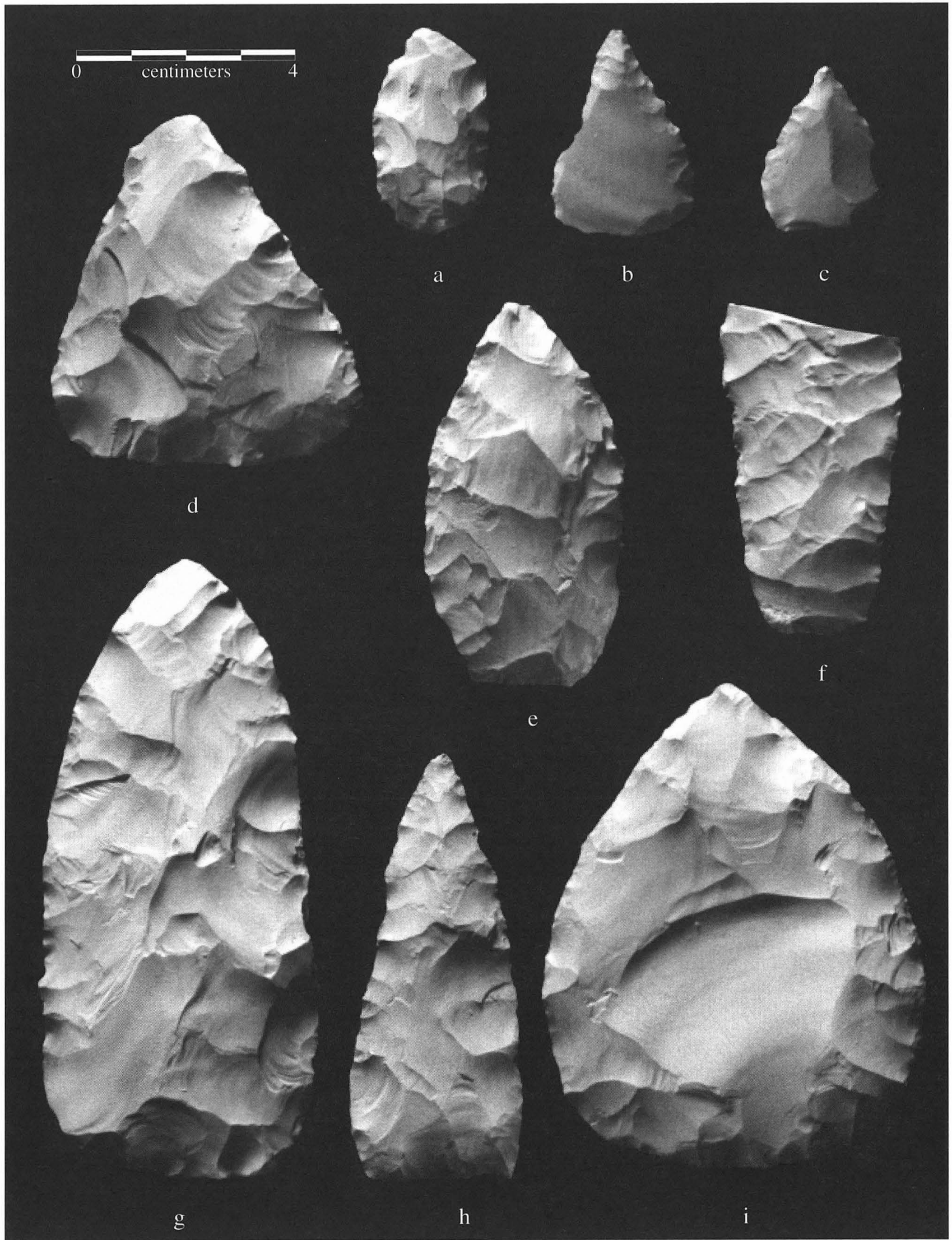

FiguRE 16-6. Representative Stage 2 bifaces, showing variation in size and shape. Specimen numbers: (a) 6A-4; (b) 5B-7; (c) 1A-5; (d) 7H3; (e) 22Q-27; (f) $31 \mathrm{~T} 1 \mathrm{~A}-2$; (g) 640-1 conjoined with 679-1; (h) 11Q-2; (i) $13 \mathrm{~K}-5$. 
combinations on $32(5 \%)$; bend and indeterminate on 23 (3\%); bend and excavation damage on $12(2 \%)$; bend and edge bite on $17(2 \%)$; bend and perverse on $10(1 \%)$; combination perverse and other fracture types on $8(1 \%)$; longitudinal overshot on $7(1 \%)$; overshot on $6(1 \%)$; bend and burin-like fractures on $5(1 \%)$; edge bite on $5(1 \%)$; single burin-like fractures on $4(1 \%)$; bend and overshot on $2(<1 \%)$; and indeterminate breaks on 21 (3\%).

Discard among complete or nearly complete specimens is attributed to failure to thin on 39 (39\%), unsuccessful shaping on $6(6 \%)$, or a combination of the two factors on 36 $(36 \%)$. An additional $6(6 \%)$ were discarded or abandoned for no clear reason, and $14(14 \%)$ were indeterminate.

Of particular note among this group are several specimens of Early Paleoindian derivation, exemplifying technologies distinctive to Folsom and Clovis (see also Chapter 7). Figures 16-7 and 16-8 are large Stage 2 bifaces of fine-grained Edwards chert; they are fully decorticate and exhibit the large, percussion flake scars characteristic of Early Paleoindian workmanship. Figure 16-7a shows a thick $(19 \mathrm{~mm})$ biface section severed at midpoint by a bend-like fracture; this break likely occurred in conjunction with failed attempts to thin a tabular facet on one lateral edge (shown in longitudinal profile). The opposite edge is acute.

The specimen shown in Figure 16-7b is a late Stage 2 proximal section severed by bending. The base has been thinned unifacially, and the concavity has been slightly smoothed. Lateral edges have been regularized, although a small knot remains in the margin area of one side. Workmanship on this specimen is reminiscent of that seen among Clovis bifaces from Kincaid rockshelter, the Vara Daniel site, and elsewhere (Collins n.d.).

Specimens in Figure 16-8 display dimensions and workmanship characteristic of Folsom technology including high width to thickness ratios and broad, hinged flake scars that intersect near the midline (Collins n.d.; Root 1994:40, as cited in Kay n.d.). Figure 16-8a shows a late stage distal biface section with flake scars approaching a collateral pattern on one face and regularized retouch along lateral margins. The maximum thickness of $7 \mathrm{~mm}$ is found toward the outer margins of the biface while the central, interior section is slightly thinner, creating the appearance of a dip or concavity. Width to thickness ratio exceeds 5.5. This specimen appears remarkably similar in size and form to a distal biface section of Knife River flint from the Bobtail Wolf site (see Root 1994:150-151) on which Kay (n.d.), in a microwear study, found striae suggestive of usage as a butchering knife. Although the Wilson-Leonard specimen has not been examined microscopically, edge modification suggests use as a knife.

The exceptionally flat, well-flaked, medial fragment shown in Figure 16-8b has been thermally fractured on all lateral edges. Maximum thickness is $6 \mathrm{~mm}$ and width is 46 $\mathrm{mm}$. Even with an incomplete width dimension, the width to thickness ratio for this specimen is unusually high at 7.7.

Kay (n.d.) has suggested the edges of ultrathin biface tools were intended to be straight and razor sharp for use in cutting and butchering, with initial extreme breadth designed to accommodate repeated resharpenings. Maximum thickness nearest the edges would tend to make the cutting wedge more efficient by reducing frictional drag on adjacent flatter and thinner tool surfaces near the center.

The Figure 16-8c specimen is a distal section of an ultrathin biface. Wide and generally flat in longitudinal profile, it shows a high width to thickness ratio of 8.13. At this stage, flaking is not fully regularized but generally collateral; lateral edges are somewhat sinuous.

\section{Stage 3 Bifaces $(N=192)$}

The specimens in this group show more-regularized lateral edges and overall outline; no cortex remains except in a very few cases where minute patches were left on bases or faces of otherwise finished pieces. Secondary thinning has been completed or nearly so, and final flaking and edge trimming may be underway. This stage represents a more final "preform" stage for either production of projectile points or certain bifacial tools. It is likely that some bifaces in this category in fact may have been utilized as tools but are unrecognized as such, lacking usewear analyses.

Representative examples of Stage 3 bifaces, shown in Figures 16-9 and 16-10, indicate the range of shapes and finishing encompassed in this group. A stemmed biface (Figure 16-9a), broken prior to finishing, has slightly sinuous lateral edges and an asymmetrical outline form. The specimen shown in Figure 16-9b is more representative of a finished Stage 3 biface with fully regularized edges, secondary flaking, and edge retouch. A small hinge flake stack remains near one lateral edge (reverse face, not shown).

Only six Stage 3 specimens are complete. Metric averages suggest the more-finished aspect of Stage 3 bifaces, with widths reduced on average to roughly $35 \mathrm{~mm}$ and thicknesses to a little over $8.5 \mathrm{~mm}$; mean width to thickness ratio is 4.56 (Table 16-3).

TABLE $16-3$

Biface Stage 3 Descriptive Statistics

\begin{tabular}{l|c|c|c|c}
\hline & Length & Width & Thickness & $\begin{array}{c}\text { Weight/ } \\
\text { Thickness }\end{array}$ \\
\hline Mean & 82.20 & 35.16 & 8.66 & 4.56 \\
Median & 86 & 32 & 7 & 4.31 \\
Standard & & & & \\
Deviation & 25.73 & 10.26 & 2.09 & \\
Range & 66 & 56 & 14 & \\
Minimum & 40 & 13 & 3 & 3.2 \\
Maximum & 106 & 60 & 15 & 5.45 \\
Count & 5 & 6 & 6 & 6 \\
\hline
\end{tabular}

Note: Measurements are in $\mathrm{mm}$. 

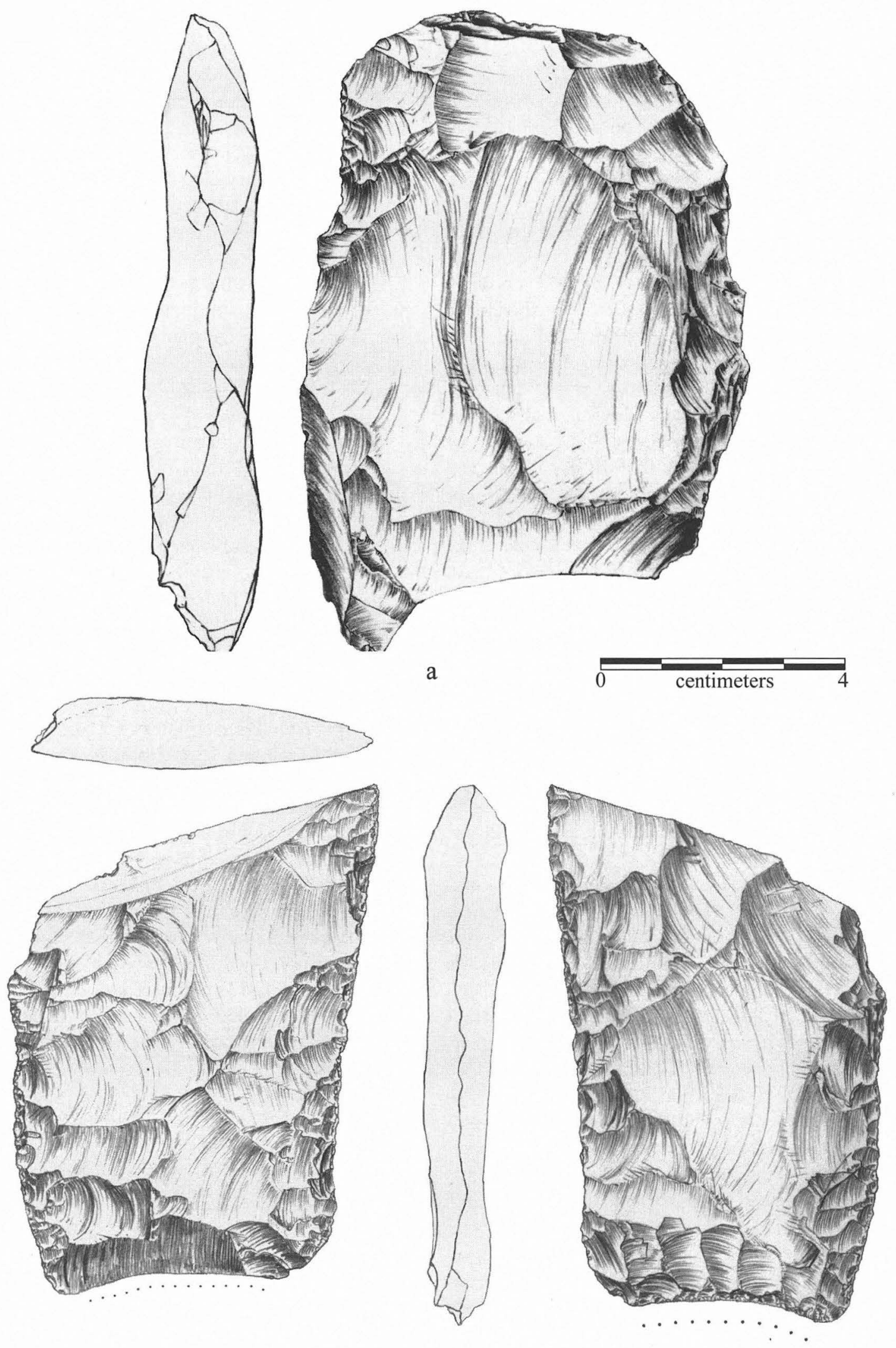

FigURE 16-7. Bifaces from Early Paleoindian contexts. Specimen numbers: (a) 35NN1\&2B-3; (b) 32KK2B-2. 


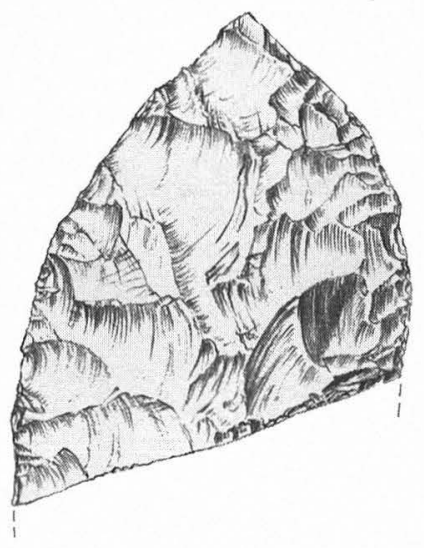

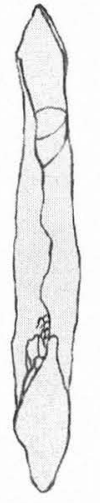

a

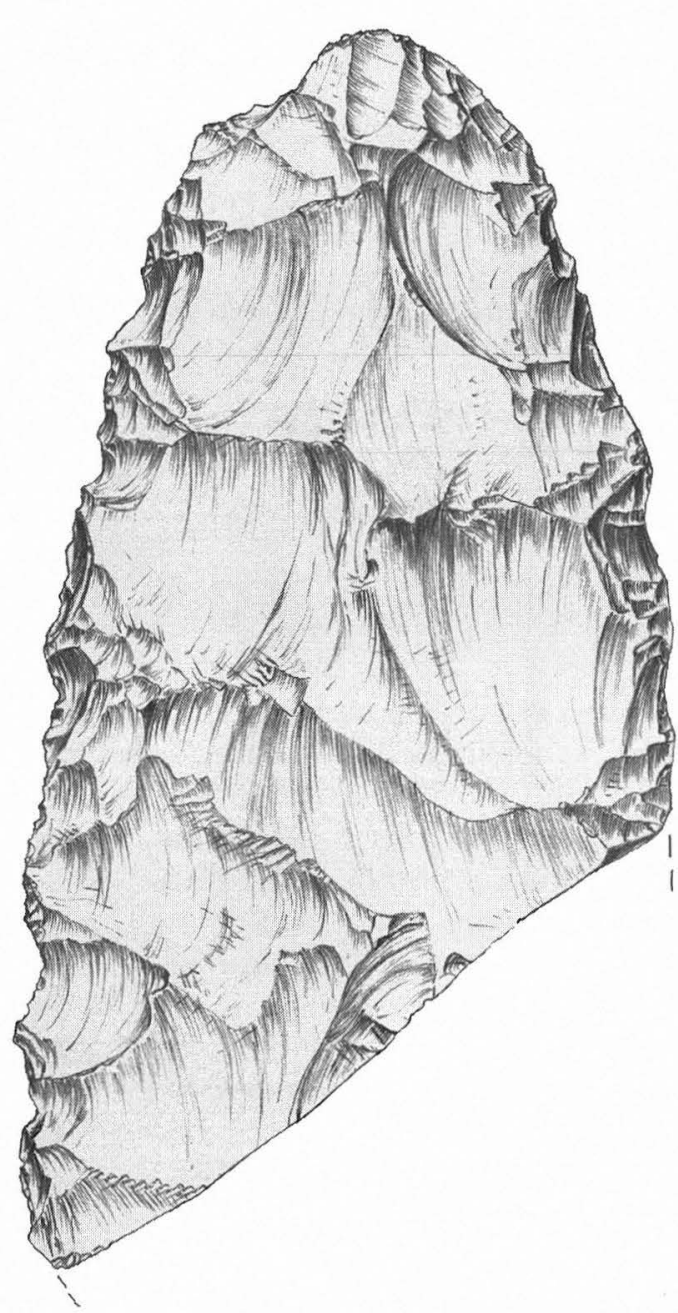

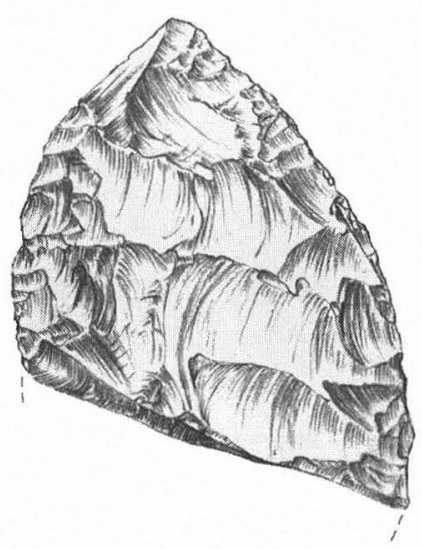

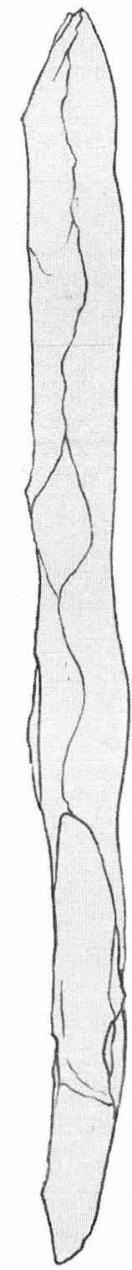

C
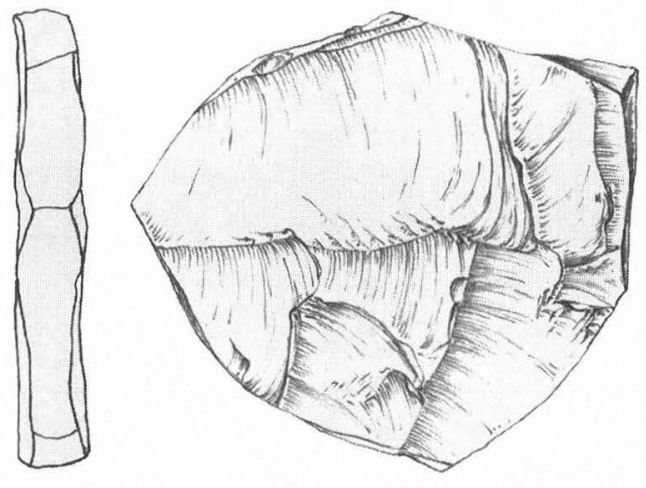

b

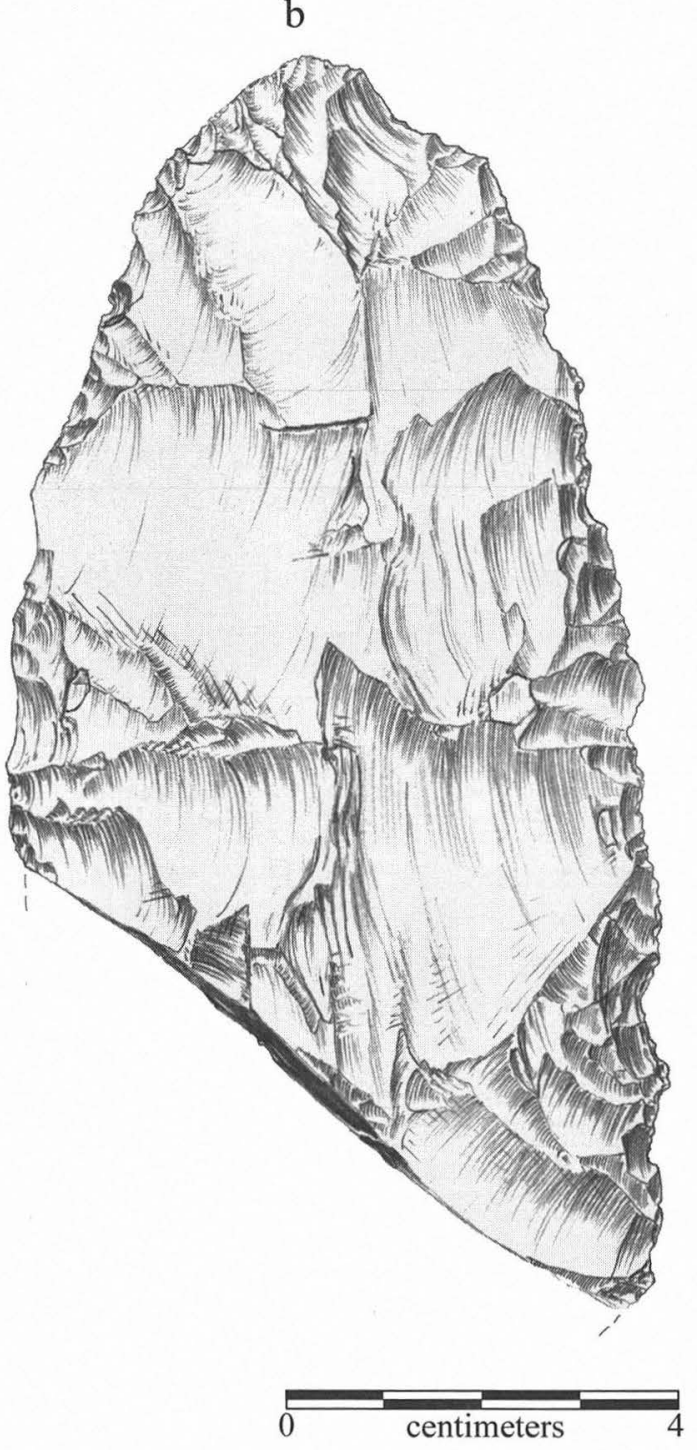

Figure 16-8. Stage 2 bifaces from Early Paleoindian contexts. Specimen numbers: (a) 2585-1; (b) 32MM1A-1; (c) 31MM2A-2.

Outline forms among complete specimens include one each of ovate, rectangular, bipointed, triangular, and two of subtriangular form. Among incomplete specimens, 71 (38\%) are distal sections, followed by $61(33 \%)$ basal, $32(17 \%)$ medial, $3(2 \%)$ lateral edge sections, and $19(10 \%)$ miscellaneous/indeterminate. Outline shapes on these sections vary from pointed on $75(40 \%)$, to straight end with lanceolate sides on $24(13 \%)$, rounded end on $21(11 \%)$, concave base 

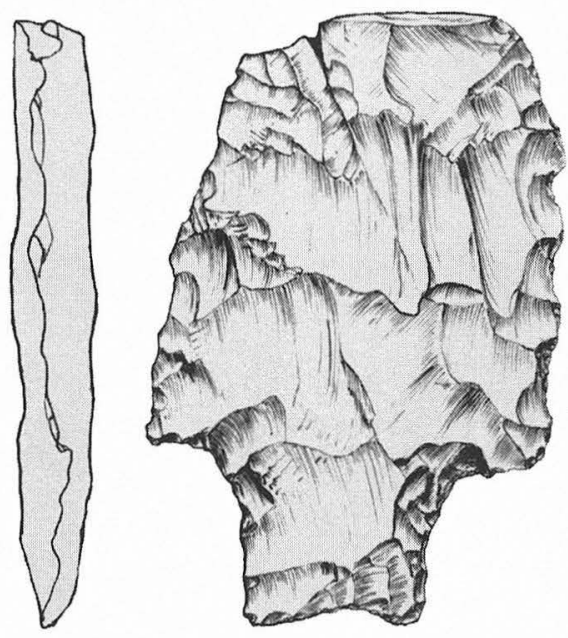

a

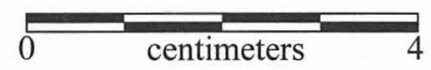

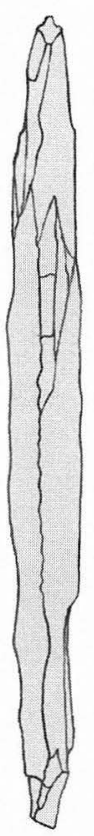

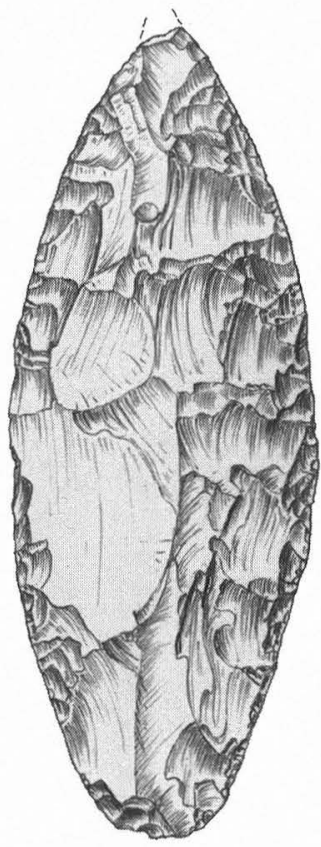

$\mathrm{b}$

FIGURE 16-9. Representative Stage 3 bifaces. Specimen numbers: (a) 17H-2; (b) 28R2-1.

on $7(4 \%)$, concave base lanceolate on $3(2 \%)$, straight end on $1(1 \%)$, straight end with convex sides on $4(2 \%)$, and 6 $(10 \%)$ each of convex-sided medial sections, convex end sections, and stemmed proximal sections. An additional 33 $(18 \%)$ are miscellaneous/indeterminate.

Raw materials for Stage 3 bifaces are chiefly fine-grained Edwards cherts ( $\mathrm{n}=147 ; 77 \%)$ with the majority of that local $(\mathrm{n}=131 ; 89 \%)$. An additional $24(13 \%)$ are coarse-grained Edwards cherts (17 [71\%] local and $7[29 \%]$ nonlocal). Twenty $(10 \%)$ specimens were classified as indeterminate/heat altered.

Of particular note is a Stage 3 biface of Tecovas jasper, a material found more than $700 \mathrm{~km}$ to the northwest of the site. A proximal section, the specimen was recovered from Unit II, a Late Paleoindian context.

Cortex was present on $16(8 \%)$ specimens, but generally in amounts too small to classifiy. One appears to be of upland origins, one nodular, and the remainder are indeterminate.

Mineral precipitates were observed on 14 (7\%) specimens, and patina on $22(11 \%)$; among the latter group, patina was differential on 1 and heavy overall on 5 . A total of $63(33 \%)$ specimens showed heat alteration, and of these, 16 $(25 \%)$ were extensively damaged.

Among broken specimens, one-half showed single bend fractures $(\mathrm{n}=93)$. Multiple bend fractures were noted on 23 $(12 \%)$, perverse fractures on $14(8 \%)$, thermal fractures on 16 $(9 \%)$, bend and thermal on $15(8 \%)$, and bend and burin-like fractures on $6(3 \%)$. The remainder showed miscellaneous breaks or combinations of breaks. Of the 6 complete specimens, 2 were likely discarded due to failure to thin or presence of knot, 1 due to unsuccessful shaping, and 3 for no apparent reason.

\section{Miscellaneous Bifacial Tools}

Eleven groups of miscellaneous bifaces/tools were identified based on overall morphology and identifiable potential working edge(s). Somewhat loosely formed, these groups are informal and may overlap in some cases due to shared characteristics.

Bifacial tools are a problematical category for several reasons. The large majority of specimens appear to be made on unfinished, broken, and/or discarded bifaces in reduction Stage 1, 2, or 3; in some cases, use as a tool is largely inferential, lacking microscopic use-wear analysis. A smaller number of specimens in this category may have been specifically crafted as tools and thus are derived from a somewhat different branch in the production process. To a large extent, there is no established convention for classifying and reporting bifacial tools. With several exceptions-notably knife-like or acute-edged bifaces and pièces esquillées or wedges - most bifacial tools are treated somewhat differently from one report to another.

Acknowledging these problems, our chief goal in attempting to identify groups of bifacial tools is to focus on what appears to be a large degree of reuse of broken and discarded bifaces and to highlight the many possible tool forms that may have come into play as a result. Although described by morphological characteristics, these tool groups may represent a variety of functions including cutting, scraping, and wedging. Several groups of tools are highly enigmatic. These are bifaces clearly aberrant from the biface reduction process and may be tools that have been recycled to the extent that they bear little resemblance to their original form. 


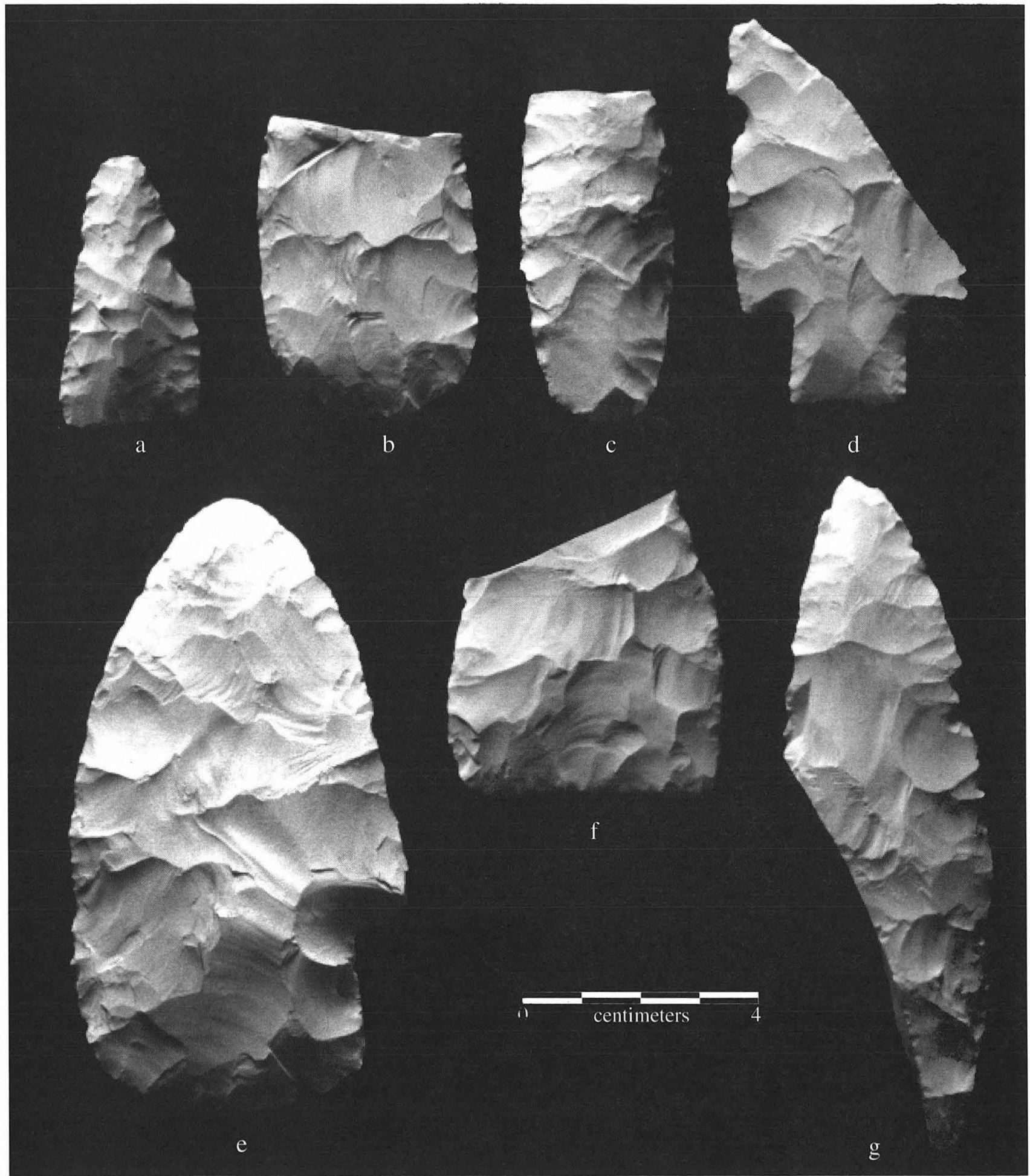

FIGURE 16-10. Representative Stage 3 bifaces, showing variation in size and shape. Specimen numbers: (a) 8C-1; (b) 13E-5; (c) 1F-3; (d) 15D-1; (e) 5J-5; (f) 21I-3; (g) 22G-9.

\section{FORM A: KNIFE-LIKE $(\mathrm{N}=63)$}

Although specimens in this group vary in outline shape from bipointed to lanceolate to triangular, all have in common acute lateral edges, some of which have been resharpened (Figures 16-11 and 16-12). Most are thin and well made and represent middle and late stages of manufacturing. Several, however, exhibit manufacturing or material flaws, including steep hinge stacks, which apparently did not impede the finishing of lateral edges for possible use as tools. Traditionally, most of the bifaces in this group would have been termed "knives" or thin "blades." 


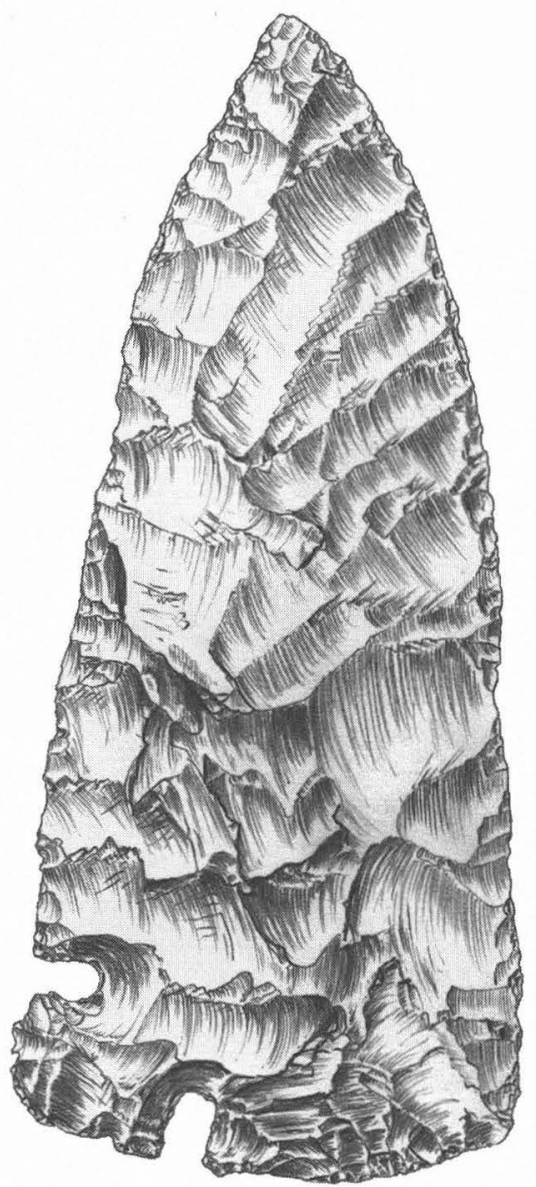

a

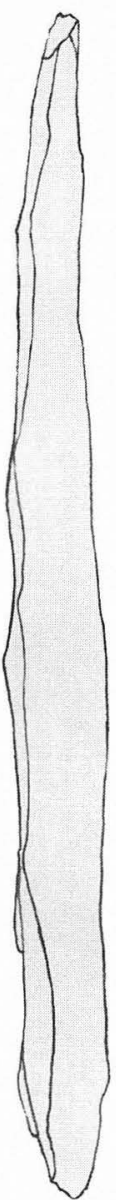

$\mathrm{b}$

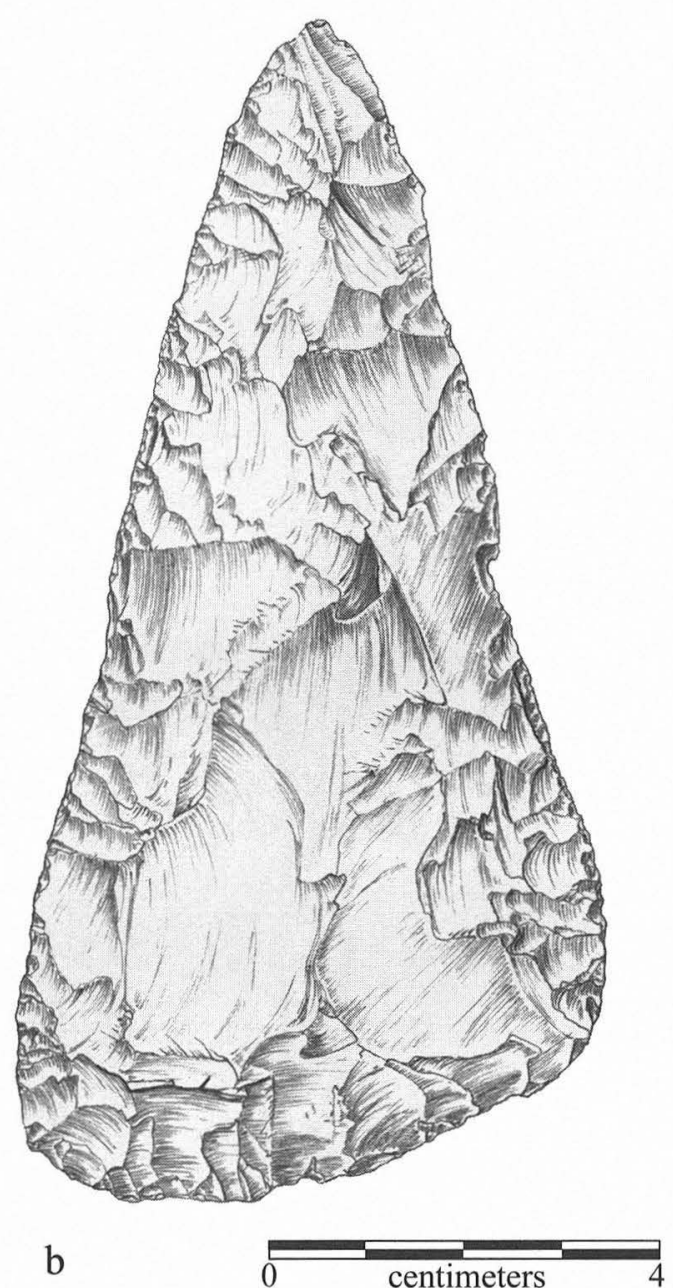

FIGURE 16-11. Examples of Form 1 knife-like tools. Specimen numbers: (a) 37DA-11; (b) 19I-5.

TABLE 16-4

Bifacial Tool Form A Descriptive Statistics

\begin{tabular}{l|c|c|c}
\hline & Length & Width & Thickness \\
\hline Mean & 75.92 & 34.48 & 10.25 \\
$1 \sigma$ & 22.71 & 12.44 & 4.50 \\
Range & 82.00 & 64.00 & 20.00 \\
Minimum & 41.00 & 13.00 & 5.00 \\
Maximum & 123.00 & 77.00 & 25.00 \\
Count & 36.00 & 62.00 & 63.00 \\
\hline
\end{tabular}

Note: Measurements are in mm.

Of the total, more than half $(\mathrm{n}=36 ; 57 \%)$ are complete or nearly so. Of these, sizes are highly varied, ranging from 41 to $123 \mathrm{~mm}$ in length, 13 to $77 \mathrm{~mm}$ in width, and 5 to $25 \mathrm{~mm}$ in thickness; mean width to thickness ratio for this group is 3.36 (Table 16-4). Among damaged specimens (including some termed nearly complete), the majority evidence single bend breaks $(n=16)$, followed by multiple bend breaks on five. Other fractures include perverse on four, bend and perverse fractures on four, thermal fractures on two, and burin-like fractures on two.
The remainder are combinations of miscellaneous break types.

Roughly one-third of these tools show marginal retouch, and most appear to have been resharpened and/or beveled on lateral edges. Serration is present on the edges of five specimens.

Form A tools are predominately of fine-grained Edwards chert $(\mathrm{n}=49 ; 78 \%)$ with 44 local and 5 nonlocal, and $10(16 \%)$ were made on local coarse cherts. An additional four are indeterminate. Patina was observed on $5(8 \%)$, mineral preciptates on $11(17 \%)$, and heat alteration on $9(14 \%)$. The large majority $(\mathrm{n}=47 ; 75 \%)$ are fully decorticate, while the others had small remnant patches of cortex chiefly on basal or facial sections.

Included in this group is a Corner Tang biface (see Figure 16-11a) which conforms to the more common form as described by Turner and Hester (1993:250-251) and to Patterson's (1936) diagonal form, Type 2 Corner Tang knife. Of fine-grained local chert, the specimen is thin and well flaked on one face in a subparallel pattern and more randomly flaked on the reverse. Dimensions are length $119 \mathrm{~mm}$, width $52 \mathrm{~mm}$, and thickness $14 \mathrm{~mm}$; basal tang is $14 \mathrm{~mm}$ in 

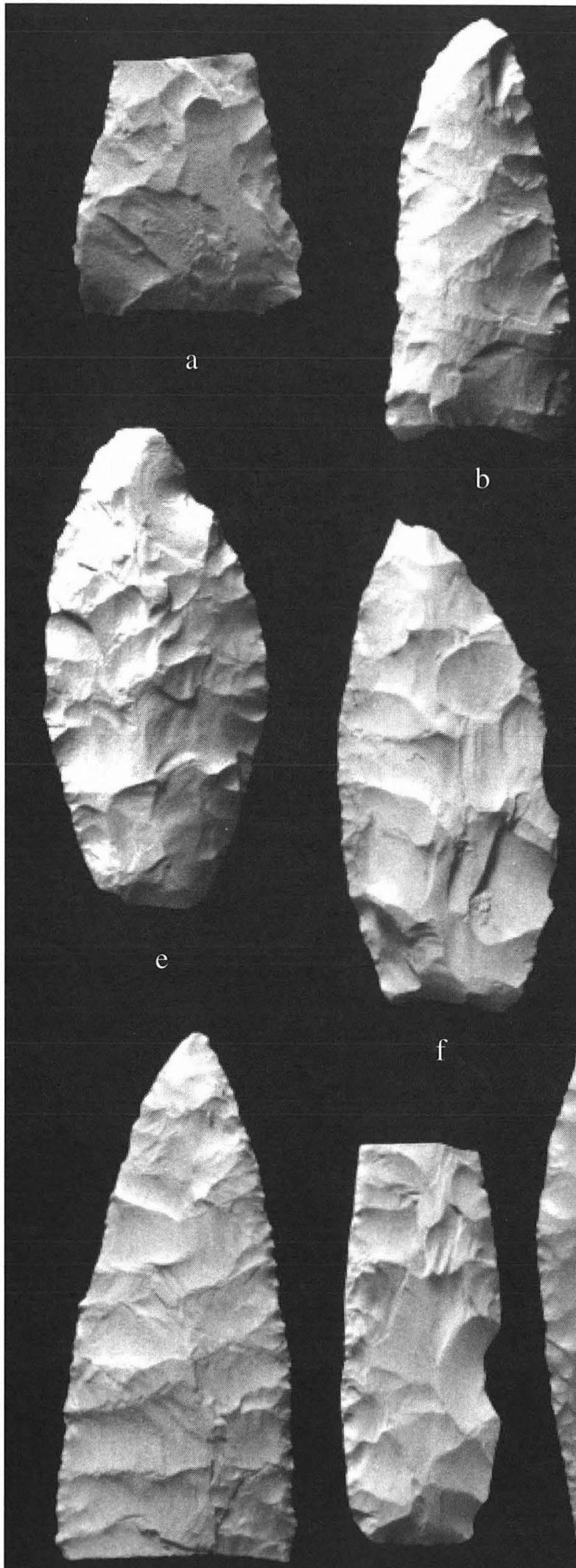

i b
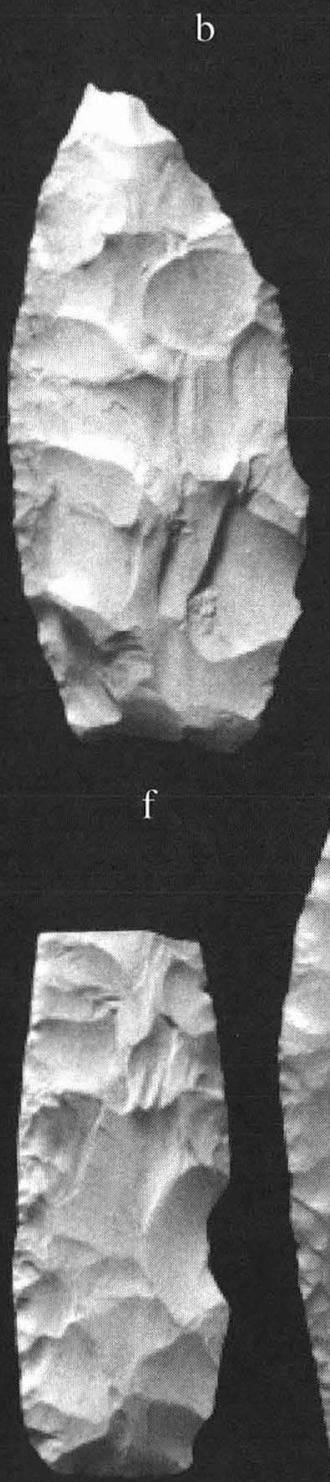

j

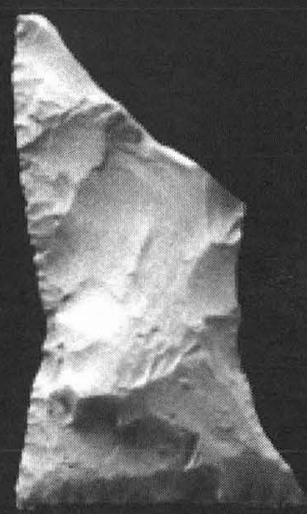

C

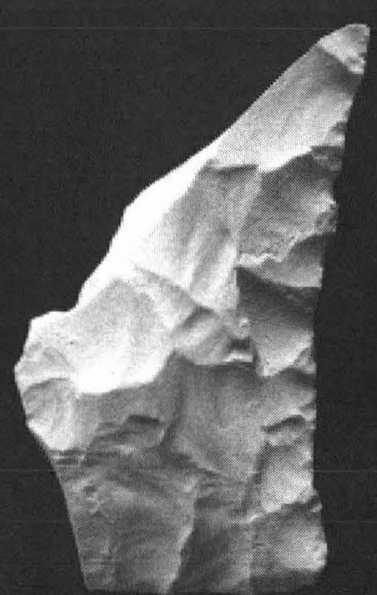

d

FIGURE 16-12. Representative Form A knife-like tools, showing variation in size and shape. Specimen numbers: (a) 202-1; (b) 6E-1; (c) 5A10; (d) 9B-11; (e) 37Q1A-1; (f) 3K-3; (g) 570-1; (h) 51ED-10; (i) 28R2-2; (j) 8B-25; (k) 10D-1. 
the present specimen, other studies have suggested use for cutting soft animal or plant tissues (see Chandler et al. 1983; McReynolds 1984). Hall (1981) has conjectured that a trade network for these specialized bifaces may have evolved during the Late Archaic. The Wilson-Leonard specimen from stratigraphic Unit IIIc is attributed to that period. Its recovery is of interest in that it was found oriented vertically as though it had been inserted into the ground or perhaps had slipped through a crack in the soil.

Other specimens, shown in Figure 16-12, illustrate the variety of shapes among these knife-like tools. Those in Figures $16-12 \mathrm{c}$ and d, although fragmentary, are suggestive of the characteristic basal morphology of the Late Prehistoric Gahagan biface (Turner and Hester 1993:255). Edgewear studies by Shafer (1973) suggest use of such tools for cutting vegetable products or meat.

\section{FORMB: STEEP-EDGED $(\mathrm{N}=28)$}

Most of the specimens in this group have steep and often beveled lateral or distal edges and a scraper-like appearance (Figures 16-13a-c, 16-14a-c). Others are of more aberrant shapes but exhibit one or more steep lateral edges. As shown in Table 16-5, a wide range of size variation is present in this group; lengths range from 30 to $90 \mathrm{~mm}$, widths from 23 to $68 \mathrm{~mm}$, and thicknesses from 3 to $28 \mathrm{~mm}$. Of the total, $14(50 \%)$ are complete, and these vary in outline form from ovate to "shoe-shaped." Among the broken specimens, single bend fractures were noted on 10 , thermal fractures on 2 , longitudinal overshot on 1 , and 1 is indeterminate.

Raw material is fine-grained Edwards chert for $23(82 \%)$

TABLE $16-5$

Bifacial Tool Form B Descriptive Statistics

\begin{tabular}{l|c|c|c}
\hline & Length & Width & Thickness \\
\hline Mean & 54.33 & 38.73 & 14.1 \\
$1 \sigma$ & 16.95 & 9.95 & 5.74 \\
Range & 60 & 45 & 25 \\
Minimum & 30 & 23 & 3 \\
Maximum & 90 & 68 & 28 \\
Count & 28 & 28 & 28 \\
\hline
\end{tabular}

Note: Measurements are in $\mathrm{mm}$.

with 22 of those local, and coarse-grained Edwards chert on 7 (25\%) with 6 local. Patina was noted on 3 and of that, was differential on 1. Mineral precipitates were observed on 4, and heat damage on 3 . Roughly two-thirds of the specimens $(\mathrm{n}=20 ; 71 \%)$ are decorticate.

\section{FORMC: $P L A N O C O N V E X(N=7)$}

These specimens have the appearance of traditional unifaces but are flaked bifacially (see Figures 16-13d and 1614d). Except for invasive retouch scars along the lateral edges, most are cortex-covered on one face; however, they are fully flaked on the ventral surface. Four specimens are complete, and among these outline forms vary from subtriangular to discoidal to shoe-shaped. Sizes range from 38 to $76 \mathrm{~mm}$ in length, 30 to $63 \mathrm{~mm}$ in width, and 7 to $25 \mathrm{~mm}$ in thickness (Table 16-6).

TABLE 16-6

Bifacial Tool Form C Descriptive Statistics

\begin{tabular}{l|c|c|c}
\hline & Length & Width & Thickness \\
\hline Mean & 54.67 & 40.33 & 14.67 \\
$1 \sigma$ & 14.33 & 11.94 & 6.15 \\
Range & 38 & 33 & 18 \\
Minimum & 38 & 30 & 7 \\
Maximum & 76 & 63 & 25 \\
Count & 6 & 6 & 6 \\
\hline
\end{tabular}

Note: Measurements are in $\mathrm{mm}$.

Raw materials among planoconvex specimens are all local cherts (five fine grained and two coarse grained). Only one specimen exhibits patina, and none shows mineral precipitates or heat alteration.

\section{FORMD: COMPOSITE EDGEMODIFICATION (N=4)}

Specimens in this group exhibit a range of edge modifications in combinations of two or more, including beveled edges, knife-like acute edges, gravers and spurs, retouched breaks, and/or notches (see Figure 16-13e). These tools are somewhat analogous to unifacial multiple tools (see Chapter 17).

Sizes are varied within this small group (Table 16-7). All are made of local fine-grained cherts. Patina was observed on two, mineral precipitates on one, and heat alteration on one.

TABLE 16-7

Bifacial Tool Form D Descriptive Statistics

\begin{tabular}{l|c|c|c}
\hline & Length & Width & Thickness \\
\hline Mean & 52.75 & 41.75 & 11.25 \\
$1 \sigma$ & 26.89 & 10.31 & 4.11 \\
Range & 58 & 24 & 9 \\
Minimum & 25 & 27 & 6 \\
Maximum & 83 & 51 & 15 \\
Count & 4 & 4 & 4 \\
\hline
\end{tabular}

Note: Measurements are in $\mathrm{mm}$.

\section{FORME: THICK, NARROW,ELONGATE (N=25)}

These are odd, chunky, elongate specimens with steep lateral edges (see Figure 16-13f; Figure 16-15). Width to thickness ratios for specimens in this group are very low, and longitudinal profiles on some are of an odd planoconvex, or "hump-backed" form. 

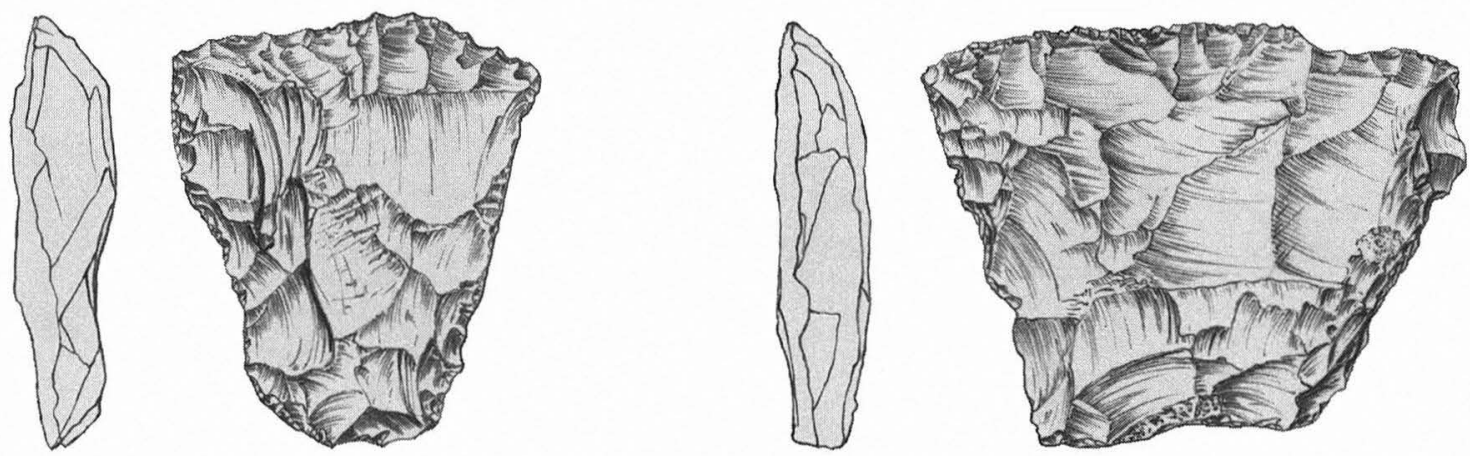

a
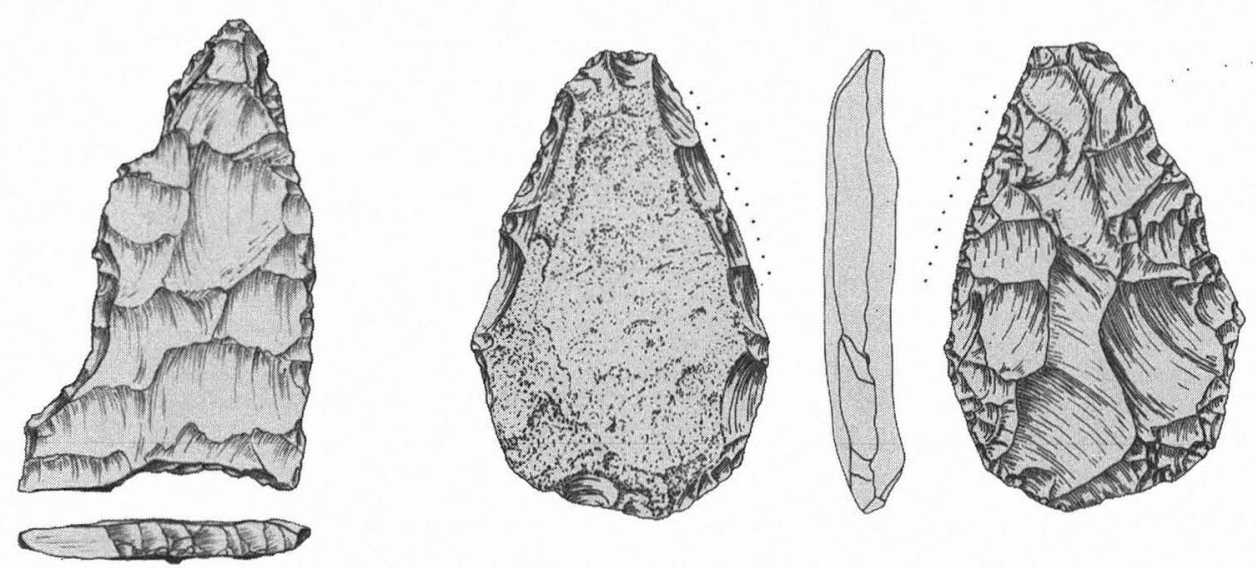

d

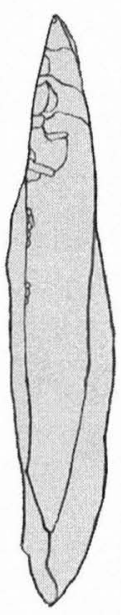

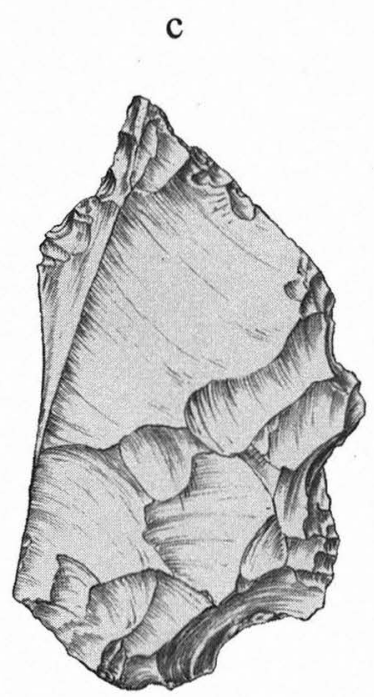

e

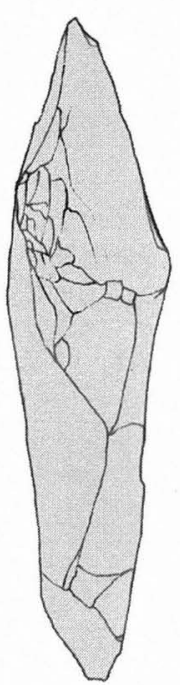

f

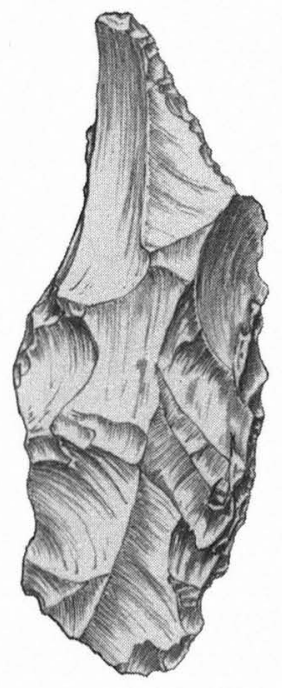

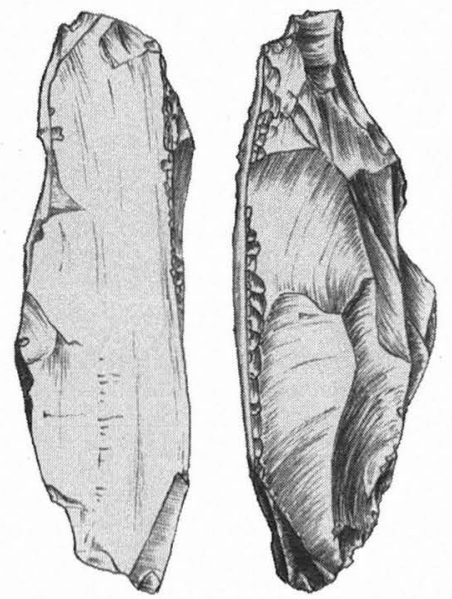

$\mathrm{g}$

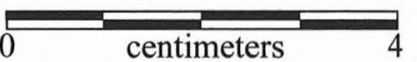

FIGURE 16-13. Examples of bifacial tools. (a-c) Form B, steep-edged; (d) Form C, planoconvex; (e) Form D, composite; (f) Form E, thick, narrow, elongate; (g) Form F, retouched break. Specimen numbers: (a) Specimen 10N-1; (b) Specimen 19I-4; (c) 5A-7; (d) 13N-3, (e) 13P1 ; (f) $12 \mathrm{M}-3$; (g) $51 \mathrm{AC}-3$. 


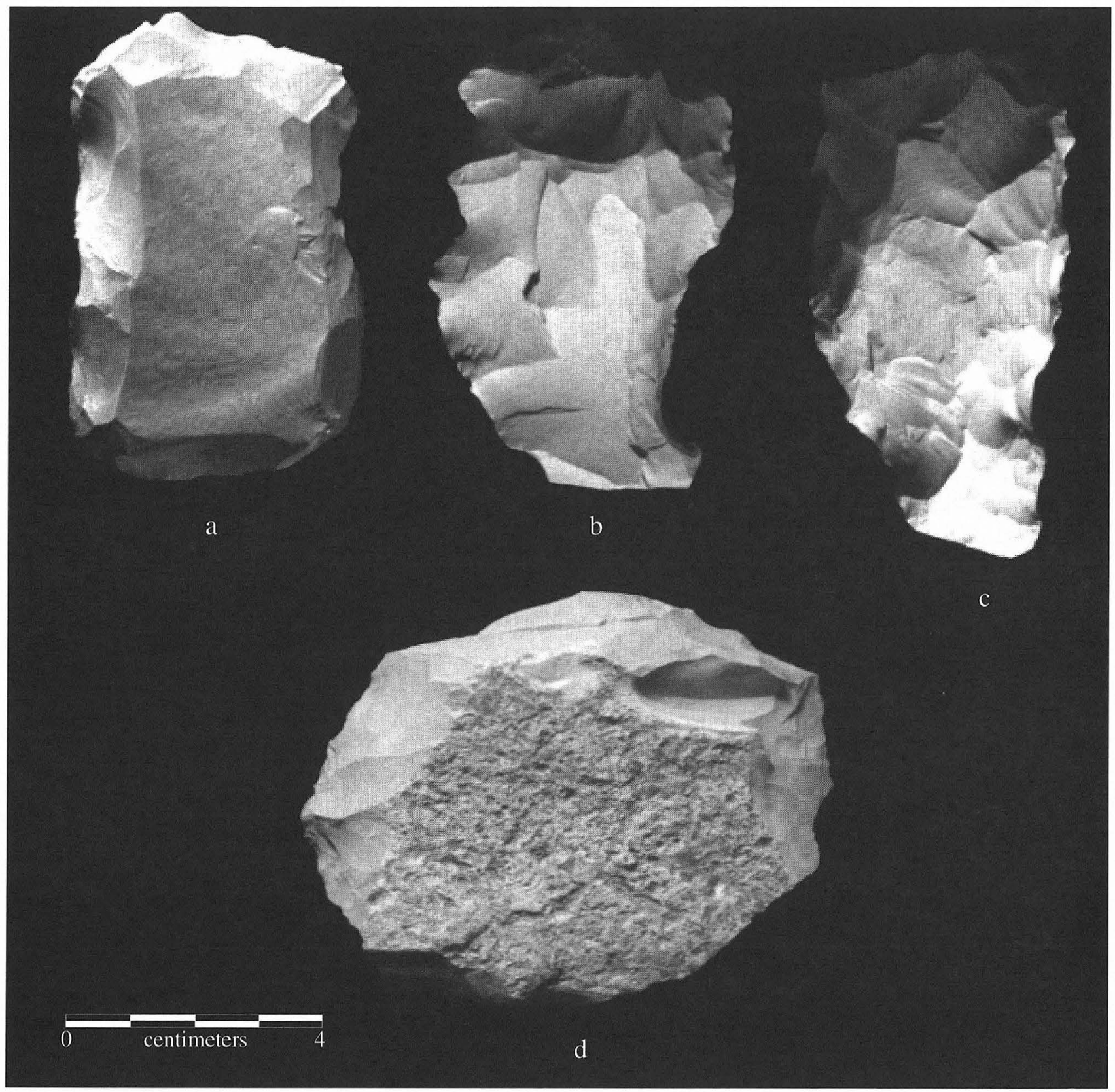

FIGURE 16-14. Examples of bifacial tools. (a-c) Form B, steep edged; (d) Form C, planoconvex. Specimen numbers: (a) 11S-6; (b) 9A-27; (c) $27 \mathrm{~T} 2 \mathrm{D}-7$; (d) 28P2-6.

Sizes range from 30 to $86 \mathrm{~mm}$ in length, 20 to $93 \mathrm{~mm}$ in width, and 8 to $16 \mathrm{~mm}$ in thickness; mean width to thickness ratio is 2.45 (Table 16-8). The specimen shown in Figure 16$13 \mathrm{f}$ exhibits an exceptionally low width to thickness ratio of 1.6 , exemplifying the aberrant nature of the tools in this group. Among the total, seven are complete; outline forms include bipointed, lanceolate, subtriangular, and "shoe-shaped." Raw material is local fine-grained chert on all but one specimen which is local coarse chert. Patina is present on three, mineral precipitates on one, and heat alteration on six.

Many of the specimens in this group may be tools that were reworked to the point of exhaustion. A possible ana- $\log$ is the exhausted adze tool form seen among lithic assemblages of past and present-day western desert aborigines of Australia (Gould 1980:128-129). Although those tools began their use-life as hafted discoidal unifacial scrapers predominately employed in woodworking tasks, they were resharpened repeatedly (an average of 20 times) to a thick, narrow form that could no longer be contained in a haft; at this exhausted stage, they are termed adze "slugs." While similar uses for these two tools-the Australian unifacial adze and Wilson-Leonard bifacial tool Form Eare not suggested, their maintenance histories may be similar and of interest in understanding the present assemblage. 


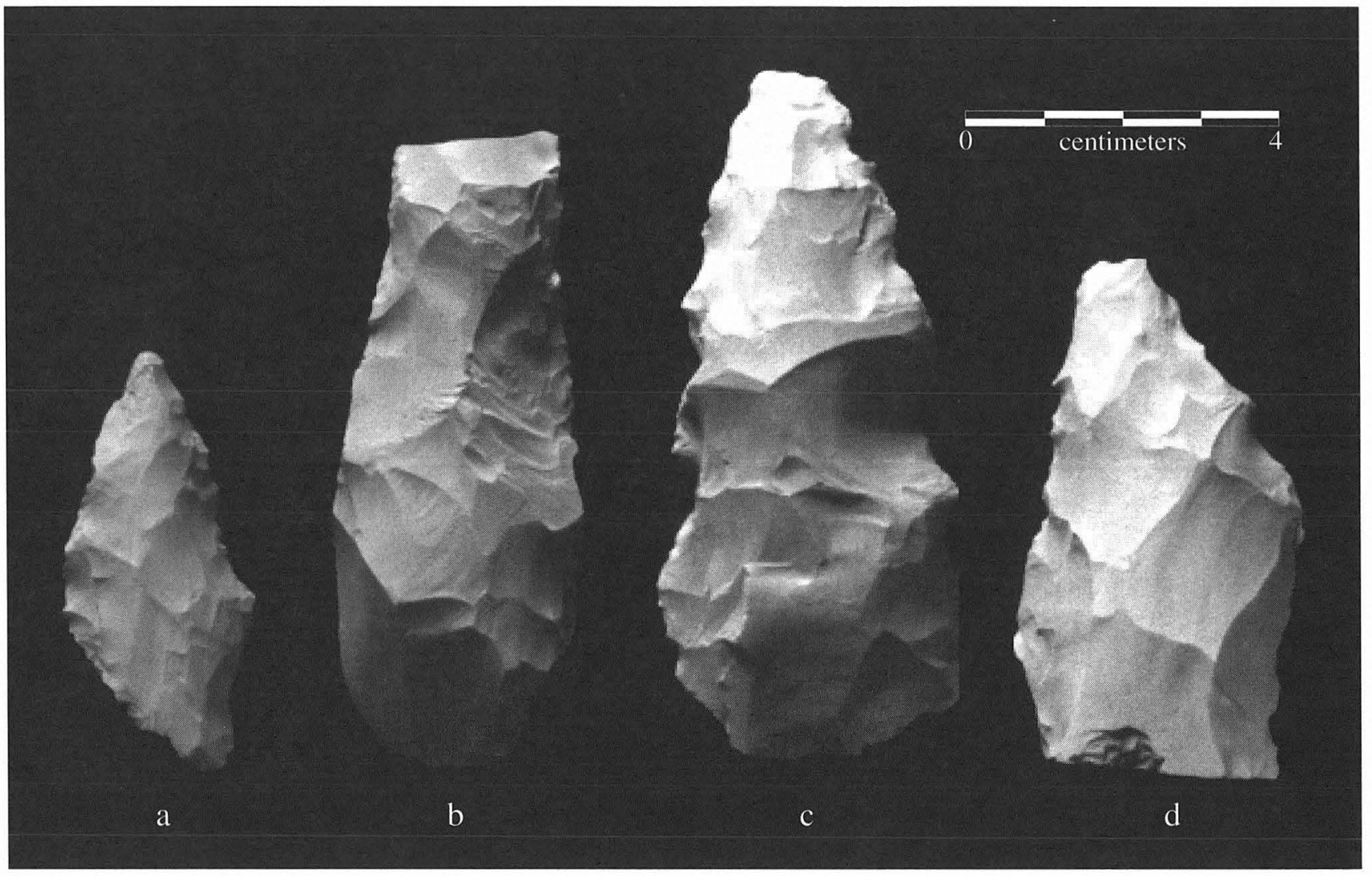

FIGURE 16-15. Examples of Form E thick, narrow, elongate bifacial tools. Specimen numbers: (a) 31S1-3; (b) 27O-4; (c) 0-69; (d) 532-3. The latter specimen is a broken section.

TABLE 16-8

Bifacial Tool Form E Descriptive Statistics

\begin{tabular}{|c|c|c|c|c|}
\hline & Length & Width & Thickness & $\begin{array}{c}\text { Width/ } \\
\text { Thickness }\end{array}$ \\
\hline Mean & 56.09 & 30.17 & 12.33 & 2.45 \\
\hline $1 \sigma$ & 15.36 & 14.47 & 2.65 & 5.46 \\
\hline Range & 56 & 73 & 8 & 9.13 \\
\hline Minimum & 30 & 20 & 8 & 2.50 \\
\hline Maximum & 86 & 93 & 16 & 5.81 \\
\hline Count & 23 & 23 & 24 & \\
\hline
\end{tabular}

Note: Measurements are in $\mathrm{mm}$.

\section{FORMF: RETOUCHED BREAKS ON BIFACE FRAGMENTS $(\mathrm{N}=5)$}

These are sections of broken bifaces on which the fracture has been retouched, likely in use either as a scraper or knife (see Figure 16-13g). Specimens range from 41 to $59 \mathrm{~mm}$ in length, 14 to $61 \mathrm{~mm}$ in width, and 9 to $20 \mathrm{~mm}$ in thickness (Table 16-9). Raw material on all is local fine-grained chert. Patina is evident on one and mineral precipitates on one. No heat damage was observed. Four of the specimens show simple retouch on the break facets, while the fifth, broken by perverse and bend fractures, has a notch formed on the fracture facet of the latter.
TABLE 16-9

Bifacial Tool Form F Descriptive Statistics

\begin{tabular}{l|c|c|c}
\hline & Length & Width & Thickness \\
\hline Mean & 49.4 & 34.6 & 14 \\
$1 \sigma$ & 8.96 & 17.56 & 4.06 \\
Range & 18 & 47 & 11 \\
Minimum & 41 & 14 & 9 \\
Maximum & 59 & 61 & 20 \\
Count & 5 & 5 & 5 \\
\hline
\end{tabular}

Note: Measurements are in $\mathrm{mm}$.

\section{FORM G: HAFTED BIFACE $(\mathrm{N}=1)$}

This specimen, OR445, is a stemmed biface with a rounded, beveled, scraper-like distal end. Its stem and blade edges are alternately beveled in an opposing pattern. Both blade faces are completely flaked, although randomly. Longitudinal and facial burin-like fractures to the stem suggest damage in the haft. Dimensions are length $51 \mathrm{~mm}$, width 33 $\mathrm{mm}$, thickness $6 \mathrm{~mm}$; stem length is $11 \mathrm{~mm}$ and distal stem width $11 \mathrm{~mm}$.

\section{FORM H: NATURALLY BACKED BIFACES (N=11)}

These are bifaces with a tabular facet or steep, cortexcovered edge opposing an edge that is either acute or slightly 
beveled (Figures 16-16 and 16-17). The natural backing on these tools likely would have facilitated prehension during use of the other lateral edge. A wide range of sizes is present in this group (Table 16-10). Five are unbroken and are classed variously as ovate, subtriangular, or miscellaneous in outline form.

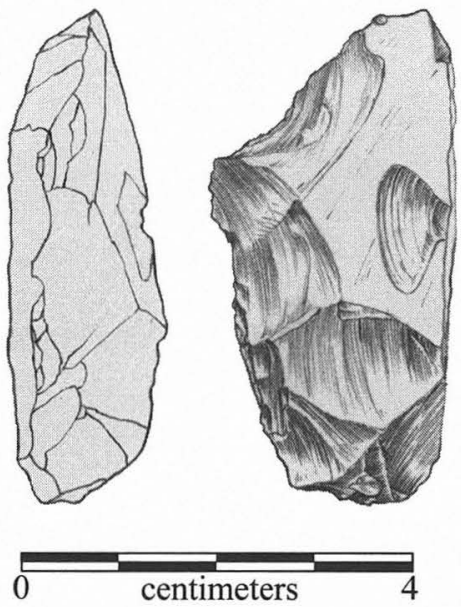

FIGURE 16-16. Example of Form H naturally backed bifacial tool (Specimen number 20X-4).
All specimens in this group are of local fine-grained chert, except for one which is indeterminate. Patina is present on two, and mineral precipitates and heat alteration are absent.

TABLE $16-10$

Bifacial Tool Form H Descriptive Statistics

\begin{tabular}{l|c|c|c}
\hline & Length & Width & Thickness \\
\hline Mean & 73 & 46.36 & 20.55 \\
$1 \sigma$ & 19.16 & 10.66 & 6.27 \\
Range & 66 & 43 & 19 \\
Minimum & 38 & 24 & 11 \\
Maximum & 104 & 67 & 30 \\
Count & 11 & 11 & 11 \\
\hline
\end{tabular}

Note: Measurements are in $\mathrm{mm}$.

\section{FORM I: PIÈCES ESQUILLÉES OR WEDGES (N=22)}

Although there is much morphological variation, this group typically comprises small- to medium-sized decorticate bifaces exhibiting bipolar flaking and often battering on one or more ends or lateral edges. Generally, the group is composed of two morphologies. The first form, comprising 18 specimens, is roughly flat to biconvex and varies in

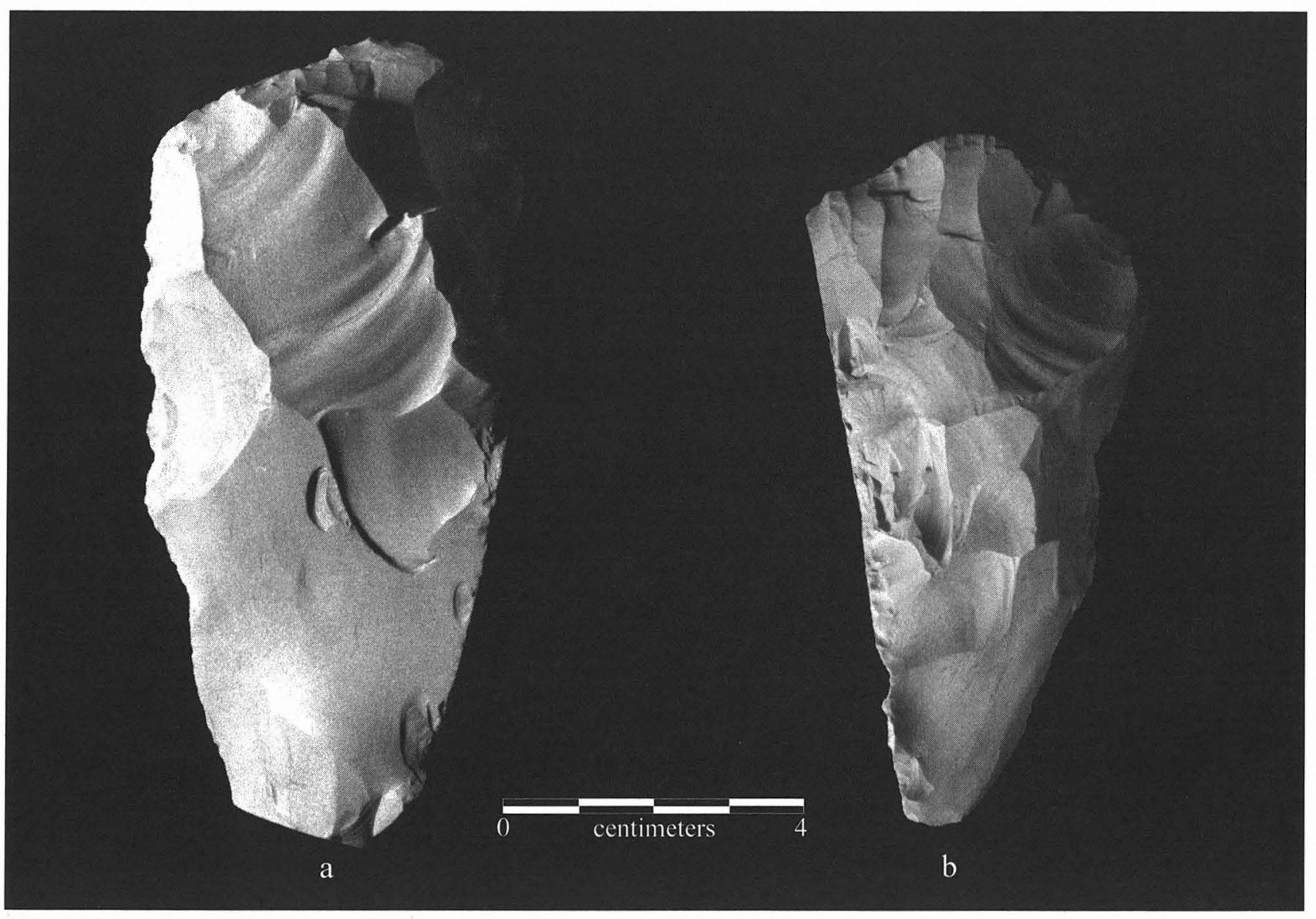

FIGURE 16-17. Examples of Form H naturally backed bifacial tools. Specimen numbers: (a) 20G-4; (b) 9H-1. 
outline from square or rectangular to discoidal. This form typically exhibits battering or concentrations of step flaking on one or more lateral edges, as well as vertical short to medium length flake scars emanating from both the battered edges and their opposing edges (Figures 16-18a, b, 16-19a-f). These specimens appear to have been made on broken or discarded bifaces.

Four specimens were classed into a second category (Figures 16-18c, 16-19g, h). More circular in outline than the first form specimens, these are typically thicker, rounded, and heavily battered on one end and have an opposing end that is somewhat thinner. Longitudinal profiles range from arced to planoconvex. These specimens appear to have been formed from cores-possibly microcores, as described in Chapter 18-or thick Stage 1 bifaces. Battering resembles that exhibited on hammerstones. One specimen (see Figure 16-18c) is somewhat similar to a small fist axe in configuration, with a thick (ca. 19 $\mathrm{mm}$ ) more clearly delineated "butt" end exhibiting a small cortex remnant as well as heavy battering. The opposing end is thin (ca. $7 \mathrm{~mm}$ ) and slightly convex.

A single specimen did not fall within either group, being larger, more elongate and flat, but evidencing vertical flake scars off one edge. This specimen is shown in Figure 16-19i. Within the full assemblage, 12 specimens are complete or largely so. Mean dimensions of pièces esquillées are roughly $45 \mathrm{~mm}$ in length, $38 \mathrm{~mm}$ in width, and $13 \mathrm{~mm}$ in thickness (Table 16-11). Battering was noted on $6(27 \%)$ specimens (e.g., see Figure 16-19g, h), and hinge and step flaking of lateral edges is prevalent. Fracture types are relatively homogeneous within this group: complex bend and burin-like fractures were identified on 6 and single bend breaks on an additional 6 . The only other break types noted are thermal and perverse fractures; an additional specimen is indeterminate.

TABLE $16-11$

Bifacial Tool Form I Descriptive Statistics

\begin{tabular}{l|c|c|c}
\hline & Length & Width & Thickness \\
\hline Mean & 44.81 & 37.59 & 12.95 \\
$1 \sigma$ & 8.36 & 6.82 & 4.2 \\
Range & 33 & 33 & 14 \\
Minimum & 31 & 28 & 6 \\
Maximum & 64 & 54 & 20 \\
Count & 16 & 19 & 21 \\
\hline
\end{tabular}

Note: Measurements are in $\mathrm{mm}$.

All pièces esquillées were made on local fine-grained cherts. Patina is present on three, and of these, is differential on one. Mineral precipitates were noted on one, and heat alteration on five.

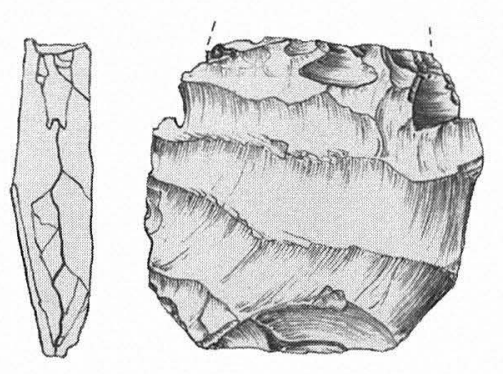

a

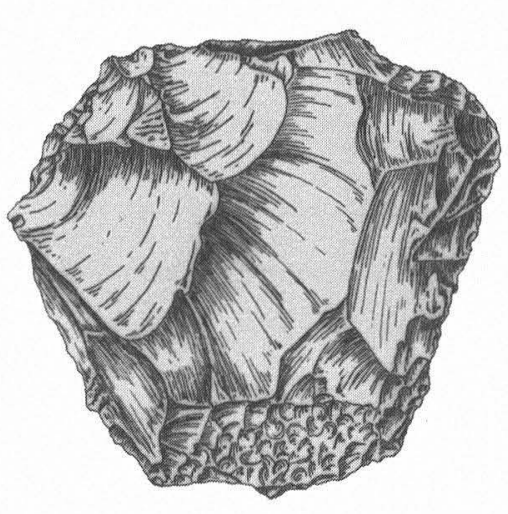

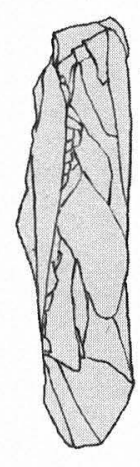

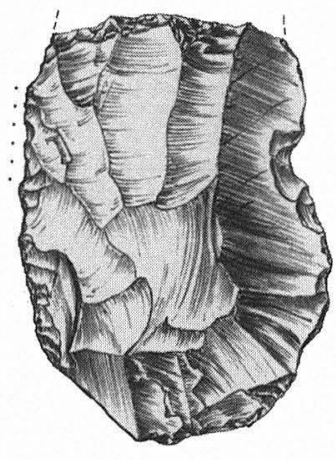

b
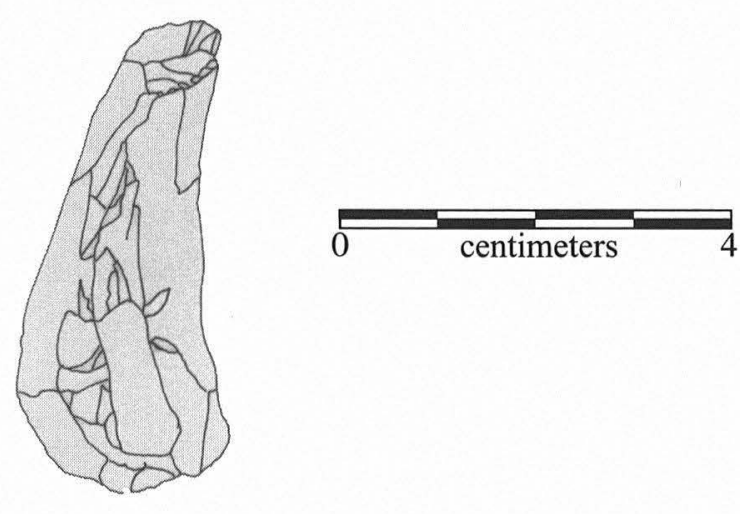

c

FIGURE 16-18. Examples of pièces esquillées, or wedges. (a, b) Form1; (c) Form 2. Specimen numbers: (a) 16B-2; (b) 1G-5; (c) 25S2-3. 


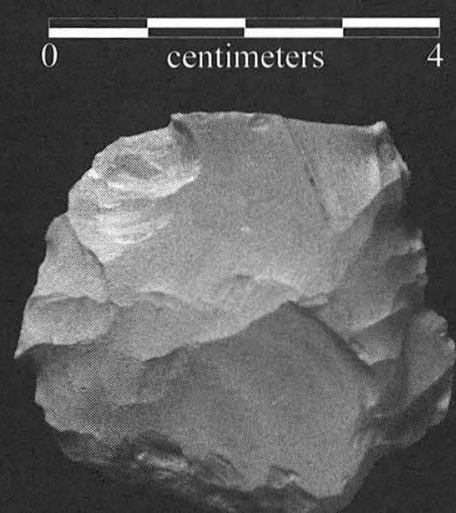

a

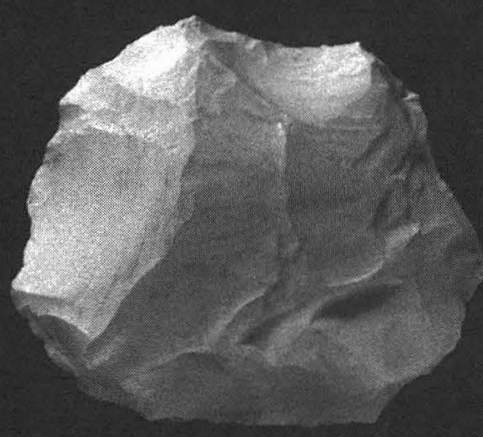

d

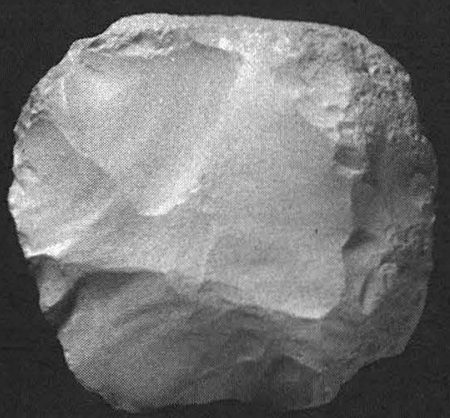

g
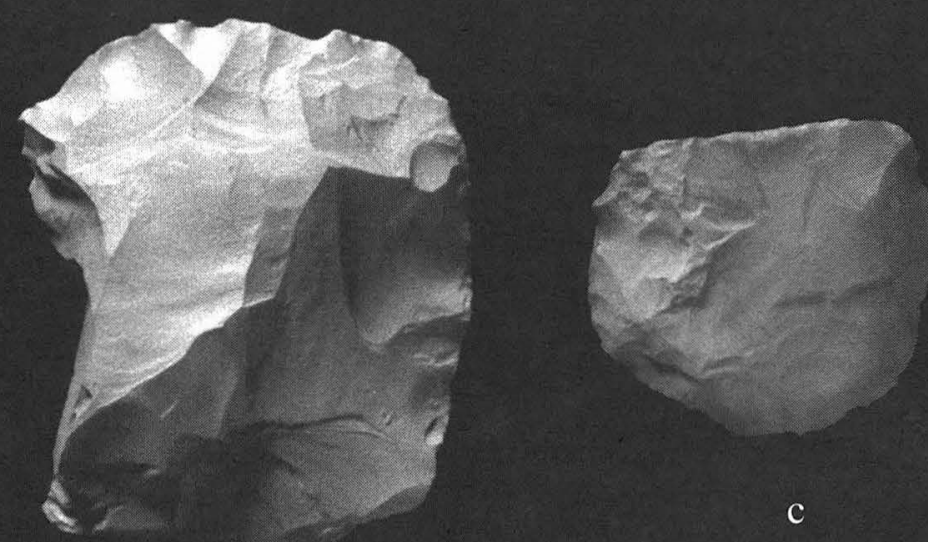

b

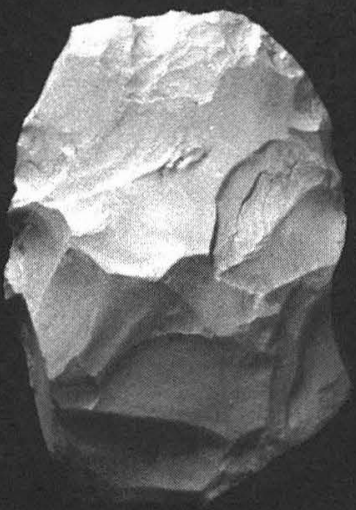

e
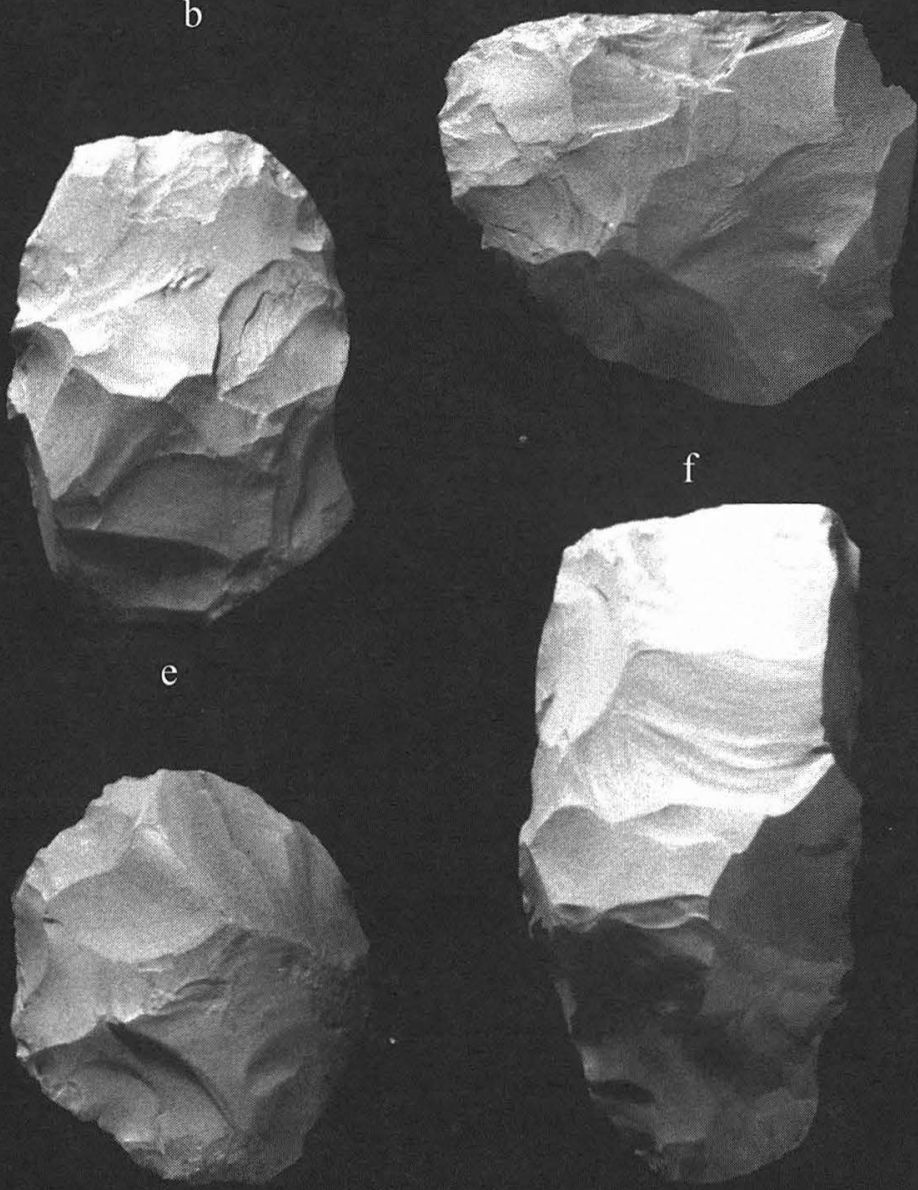

h

FIGURE 16-19. Representative pièces esquillées. (a-f) Form 1; (g, h) Form 2; (i) miscellaneous. Specimen numbers: (a) 37LB-10; (b) 21R6; (c) 36I-4; (d) 14K2-2; (e) 11K-5; (f) 15P2-1; (g) 21P-10; (h) 10P-15; (i) 10B-19.

Pièces esquillées typically have been likened to wedges or slotting tools for use in splitting bone, antler, or wood (Goodyear 1974; Le Blanc 1992; MacDonald 1968). If used as a wedge, these bifaces likely would have received applied force as one end was struck, as well as indirect pressure as the wedge end of the biface was forced into the wood or bone object being split. Battering and/or edge crushing, as well as vertical flake scars, would typically be produced on the directly struck end, whereas the bit end might exhibit edge crushing and vertical flake scars on a slightly smaller scale. 
Due to their varying shapes and sizes as well as morphological overlap with, if not recycling from, other tool groups, identification is often difficult. Many resemble small, bipolar cores. Runnels (1985) noted their similarity to tinder flints. A great deal more study - particularly microscopic use-wear analysis - is needed to help interpret this tool type.

\section{FORM J: INDETERMINATE BIFACIAL TOOLS ( $\mathrm{N}=11)$}

These enigmatic specimens were termed "mystery" bifaces during analysis, due to oddness in shape, size, and modifications (Figures 16-20 and 16-21). Likely they are not all tools, but are here grouped together because they have no logical place in the biface stage continuum. Many specimens evince what appear to have been insurmountable problems in shaping or thinning but were reduced further, regardless. Some specimens may be odd cores or crude bifaces later used as tools.

Sizes, shown in Table 16-12, range from 35 to $73 \mathrm{~mm}$ in length, 23 to $49 \mathrm{~mm}$ in width, and 7 to $23 \mathrm{~mm}$ in thickness. Eight of the total are complete, varying in shape from ovate, to subtriangular, to asymmetrical. Fractures on broken specimens were due to bending and hinge breaks. Raw material is local fine-grained chert on all but two, one of which is local coarse chert and the other, indeterminate. Patina was observed on three, one of which had differential distribution. Mineral precipitates are present on three and heat alteration on one.

\section{Unclassified Bifacial Tools $(N=32)$}

This category comprises possible tools that could not be further classified into an existing category. Included are bifaces with a denticulate or notched edge and chunks with bifacial edge modification.

Raw material in this group is fine-grained Edwards chert $(n=22 ; 69 \%)$ of which 19 are local and 3 nonlocal; $4(13 \%)$ are of coarse chert (3 local and 1 nonlocal), and $6(19 \%)$ are indeterminate. Patina is present on 7, 1 of which shows differential distribution. Mineral precipitates are present on 6 and heat alteration on 4 .

\section{Discussion}

Eleven categories of bifacial "tools" were identified. Without microscopic examination of wear characteristics, these specimens cannot be conclusively classed as tools or their uses determined. However, particular criteria argue in favor of their removal from classification within the Stage 13 biface reduction continuum and their examination within separate groups, at least on an exploratory basis. These criteria include unusual shape, low ratio of width to thickness, and/or edges exhibiting flaking or damage associated with use or preparation for use (e.g., step and hinge flaking, dulling). On some specimens, shape and edges are such that

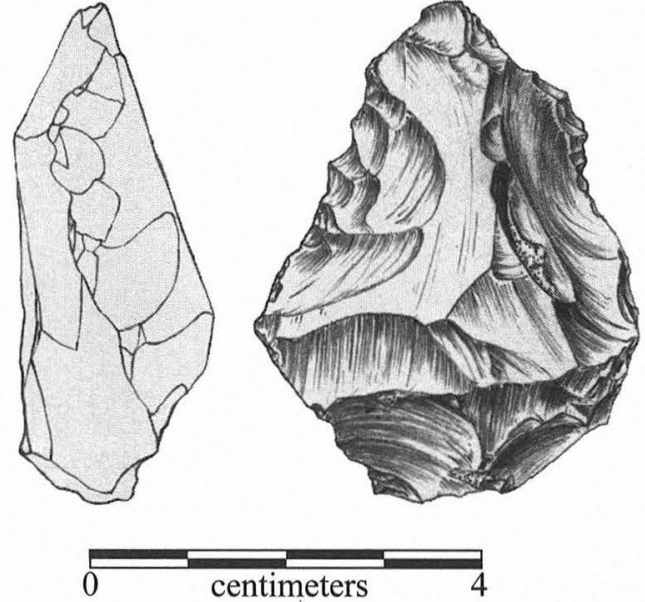

FIGURE 16-20. Example of oddly modified, or exhausted, Form J bifacial tools, Specimen 1F-8.

TABLE 16-12

Bifacial Tool Form J Descriptive Statistics

\begin{tabular}{l|c|c|c}
\hline & Length & Width & Thickness \\
\hline Mean & 54.82 & 36.73 & 15.82 \\
$1 \sigma$ & 11.05 & 6.6 & 5.44 \\
Range & 38 & 26 & 16 \\
Minimum & 35 & 23 & 7 \\
Maximum & 73 & 49 & 23 \\
Count & 11 & 11 & 11 \\
\hline
\end{tabular}

Note: Measurements are in mm.

further thinning and refinement toward the production of a thin biface seemed impossible and that use as a tool seemed the more plausible explanation. On others, such as those of the knife-like category, lateral edges appear resharpened. Form $\mathrm{F}$ comprises specimens with retouched, angular breaks. Such "tools of convenience" (Driskell 1986:47) suggest a separate mode of tool-use behavior, in which discarded or abandoned tools or site lithic detritus are utilized for their sharp edges or other serviceable features.

However, edge modification implicitly attributed to use as tools in the present categories may be, in some cases, either spontaneous retouch created during a break episode or postdepositional damage, as Mallouf (1981) demonstrated in his analysis of specimens from the Brokeen Cache. Conversely, it is certain that many specimens assigned to Stages 1-3 as unused manufacturing discards would show evidence of use wear if subjected to high power microscopy. Analysis by Kay (see Chapter 22) on a sample of what appeared to be unmodified flakes suggests a high rate of expedient use. Further, on so-called modified flakes submitted for analysis, Kay found use wear on what appeared to be the unmodified edge. He found little or no use-related wear on the clearly modified edges; rather, he suggested these were likely modified to provide a backed edge to aid in prehension. 


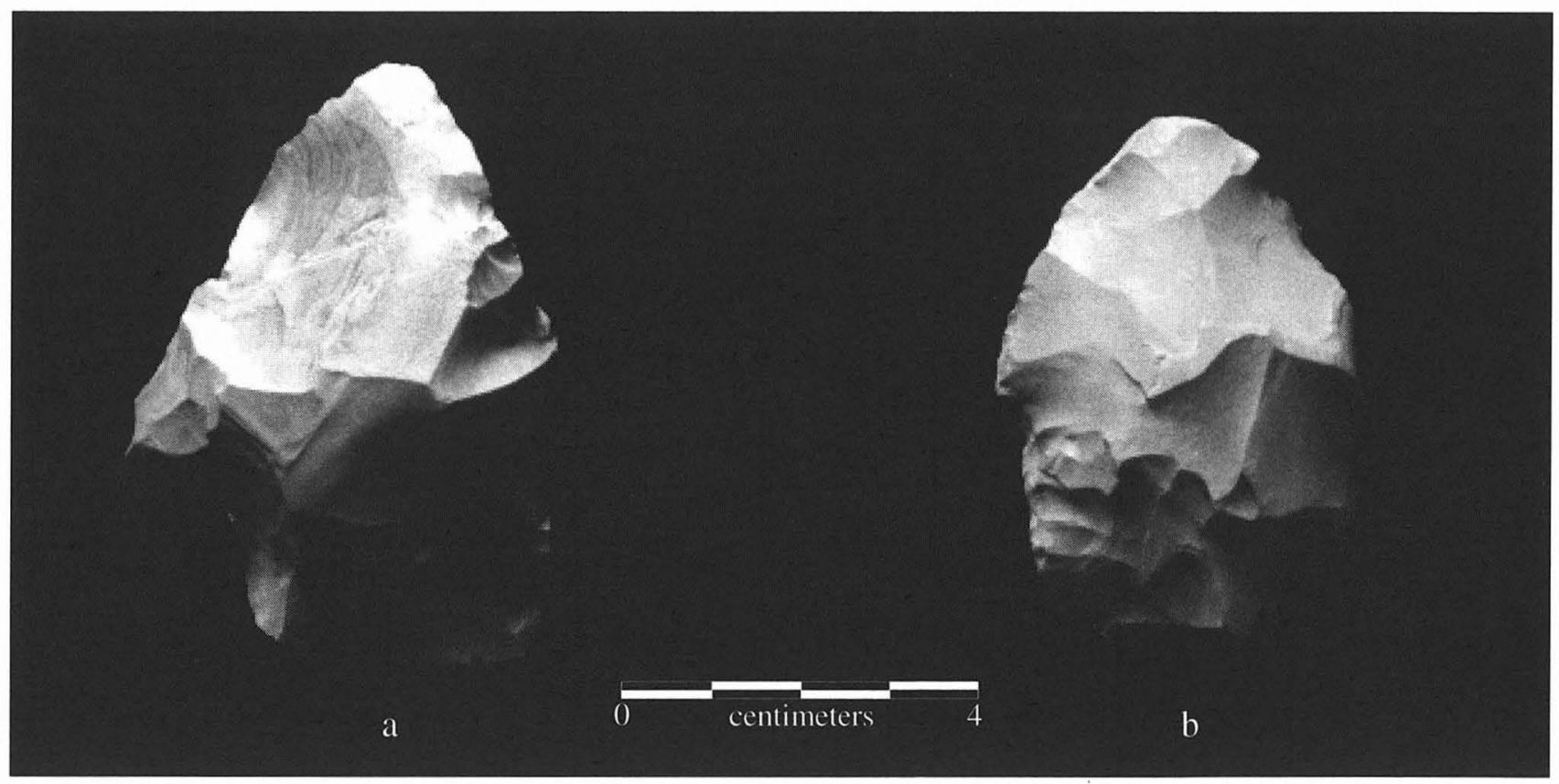

FIGURE 16-21. Representative Form J bifacial tools. Specimen numbers: (a) 14D-8; (b) 36D-23.

TABLE 16-13

Stratigraphic Distribution of Stage 1-3 Bifaces in the Valley Floor

\begin{tabular}{|c|c|c|c|c|c|c|c|}
\hline \multirow[b]{2}{*}{ Unit } & \multicolumn{2}{|c|}{ Stage 1} & \multicolumn{2}{|c|}{ Stage 2} & \multicolumn{2}{|c|}{ Stage 3} & \multirow[b]{2}{*}{ Total } \\
\hline & No. & $\%$ & No. & $\%$ & $\mathrm{No}$ & $\%$ & \\
\hline IIIc & 25 & 16 & 99 & 64 & 30 & 19 & 154 \\
\hline IIIc/fill & 3 & 38 & 4 & 50 & 1 & 13 & 8 \\
\hline $\mathrm{IIIb} / \mathrm{c}$ & 15 & 25 & 33 & 55 & 12 & 20 & 60 \\
\hline $\mathrm{IIIb} / \mathrm{c} /$ fill & & 0 & 1 & 100 & & 0 & 1 \\
\hline IIIb & 19 & 22 & 55 & 65 & 11 & 13 & 85 \\
\hline $\mathrm{III} / \mathrm{b}$ & 10 & 20 & 34 & 68 & 6 & 12 & 50 \\
\hline IIIa & 32 & 22 & 94 & 65 & 19 & 13 & 145 \\
\hline III & 2 & 22 & 7 & 78 & & 0 & 9 \\
\hline II/IIIa & 14 & 29 & 29 & 59 & 6 & 12 & 49 \\
\hline II/III & & 0 & 2 & 100 & & 0 & 2 \\
\hline II & 8 & 19 & 28 & 67 & 6 & 14 & 42 \\
\hline $\mathrm{Id} / \mathrm{II}$ & 1 & 8 & 8 & 67 & 3 & 25 & 12 \\
\hline $\mathrm{I} / \mathrm{II}$ & & 0 & 2 & 100 & & 0 & 2 \\
\hline Isi-c/II & & 0 & 3 & 100 & & 0 & 3 \\
\hline Isi-c/Id/II & & 0 & 4 & 100 & & 0 & 4 \\
\hline Id & & 0 & 1 & 100 & & 0 & 1 \\
\hline Isi-c/Id & 2 & 29 & 4 & 57 & 1 & 14 & 7 \\
\hline Isi-c & 2 & 29 & 5 & 71 & & 0 & 7 \\
\hline Isi/Isi-c & 1 & 17 & 4 & 67 & 1 & 17 & 6 \\
\hline Icl/Isi-c & 1 & 100 & & 0 & & 0 & 1 \\
\hline Isi/Icl/Isi-c & & 0 & 1 & 100 & & 0 & 1 \\
\hline I & 1 & 25 & 3 & 75 & & 0 & 4 \\
\hline Icl & & 0 & 3 & 100 & & 0 & 3 \\
\hline Isi/Icl & 4 & 27 & 11 & 73 & & 0 & 15 \\
\hline Isi/Icl/D & & 0 & 1 & 50 & 1 & 50 & 2 \\
\hline & 1 & 100 & & 0 & & 0 & 1 \\
\hline $\mathrm{Igl} / \mathrm{Isi}$ & & 0 & 2 & 100 & & 0 & 2 \\
\hline Totals: & 141 & 21 & 438 & 65 & 97 & 14 & 676 \\
\hline
\end{tabular}

Note: Includes Valley Floor A and B. Not shown are 61 specimens from unknown Valley Floor/Valley Margin proveniences and 15 specimens from unknown proveniences in the Valley Floor. 
TABLE 16-14

Stratigraphic Distribution of Stage 1-3 Bifaces in the Valley Margin

\begin{tabular}{|c|c|c|c|c|c|c|c|}
\hline \multirow[b]{2}{*}{ Unit } & \multicolumn{2}{|c|}{ Stage 1} & \multicolumn{2}{|c|}{ Stage 2} & \multicolumn{2}{|c|}{ Stage 3} & \multirow[b]{2}{*}{ Total } \\
\hline & No. & $\%$ & No. & $\%$ & No. & $\%$ & \\
\hline IIIc & 24 & 18 & 84 & 64 & 24 & 18 & 132 \\
\hline IIIc/pot & 1 & 3 & 29 & 74 & 9 & 23 & 39 \\
\hline $\mathrm{IIIb} / \mathrm{c}$ & 5 & 13 & 27 & 69 & 7 & 18 & 39 \\
\hline Y/IIIc & 5 & 15 & 24 & 73 & 4 & 12 & 33 \\
\hline $\mathrm{Y} / \mathrm{III} / \mathrm{c}$ & & 0 & 3 & 75 & 1 & 25 & 4 \\
\hline IIIb & 10 & 19 & 37 & 69 & 7 & 13 & 54 \\
\hline Y/IIIb & & 0 & 1 & 100 & & 0 & 1 \\
\hline Y & 6 & 25 & 16 & 67 & 2 & 8 & 24 \\
\hline X/Y/IIIb & 2 & 67 & 1 & 33 & & 0 & 3 \\
\hline X/IIIb & 4 & 22 & 11 & 61 & 3 & 17 & 18 \\
\hline $\mathrm{X} / \mathrm{Y}$ & 5 & 45 & 4 & 36 & 2 & 18 & 11 \\
\hline $\mathrm{IIIa} / \mathrm{b}$ & 1 & 20 & 4 & 80 & & 0 & 5 \\
\hline $\mathrm{X} / \mathrm{III} \mathrm{a} / \mathrm{b}$ & & 0 & 1 & 50 & 1 & 50 & 2 \\
\hline IIIa & 3 & 25 & 6 & 50 & 3 & 25 & 12 \\
\hline X/IIIa & 1 & 33 & 2 & 67 & & 0 & 3 \\
\hline II/IIIa & & 0 & 5 & 100 & & 0 & 5 \\
\hline X/II/IIIa & 2 & 18 & 7 & 64 & 2 & 18 & 11 \\
\hline $\mathrm{X}$ & 8 & 22 & 22 & 59 & 7 & 19 & 37 \\
\hline $\mathrm{X} / \mathrm{II}$ & 4 & 50 & 4 & 50 & & 0 & 8 \\
\hline II & 1 & 9 & 8 & 73 & 2 & 18 & 11 \\
\hline $\mathrm{Id} / \mathrm{II}$ & & 0 & 1 & 50 & 1 & 50 & 2 \\
\hline $\mathrm{X} / \mathrm{Isi}$-c/Id/II & & 0 & 1 & 100 & & 0 & 1 \\
\hline Isi-c/Id & & 0 & 3 & 100 & & 0 & 3 \\
\hline Totals: & 82 & 18 & 301 & 66 & 75 & 16 & 458 \\
\hline
\end{tabular}

Note: not shown are 61 specimens from unknown Valley Floor/Valley Margin proveniences. An additional specimen is from an unknown provenience in the Valley Margin.

\section{Technical Studies}

A small sample of Stage 2 and Stage 3 bifaces was submitted for microscopic examination of edge wear and for analysis of possible organic residues. Driskell examined four specimens from early Paleoindian contexts; he noted only a fuzzy polish ("X-polish"), which he attributed to movement in the soil rather than cultural processes (see Chapter 22).

On three Stage 2 bifaces conserved for residue analyses, Beck identified palmitic acid on two and stearic acid on one (see Chapter 23). As those acids are widely distributed in both animal and plant fats, the implications of their presence on the three bifaces is unclear. On an additional Stage 2 biface, Hurst found chloride, carbonate, and sulfate anions (see Chapter 23); while the chloride and carbonates likely were pedogenic in origin, the source of the sulfate is unknown.

\section{Distributional Patterns of Bifaces and Bifacial Tools}

Bifaces from the site were classified within a three-stage framework based on variations in shape and size or within 1 of 11 miscellaneous bifacial tool groups. Within the stage continuum, a mean reduction of nearly $10 \mathrm{~mm}$ in each of the three measurements was observed.

Of the 1,213 bifaces classed to one of three stages, almost two-thirds are in the intermediate Stage 2 category.
Fully thinned bifaces (Stage 3), requiring more skill and effort to complete, were present in lowest numbers; these may have been made into projectiles or other tools or carried from the site. As a tool group, bifaces have been considered an exceptional form for use by mobile groups, given their multifunctionality and economy of raw material use (Hayden et al. 1996). Thick, large specimens within the Stage 1 classification may have been used as cores for flake extraction, and there is likely some overlap between the Stage 1 biface category and the amorphous cores category (see Chapter 18). Thin cortex bifaces, found at the extreme minimum of the thickness dimension among Stage 1 specimens, are likely early stage arrow point preforms; these are found predominately in upper Unit IIIc, chiefly attributed to the Late Prehistoric.

Over time, staged bifaces are present in frequencies remarkably similar to those of the assemblage as a whole. As shown in Tables 16-13 and 16-14, Stage 2 bifaces constitute roughly $65 \%$ of the staged biface assemblage in almost all stratigraphic units and transition zones (in which total counts are greater than 10). An exception is seen in an early Paleoindian context-Unit Isi/Icl(Valley Floor) - where a total of 11 or $73 \%$ Stage 2 bifaces were recorded; no Stage 3 bifaces were identified in that context (see Table 16-13).

Highest counts of bifaces in all stages are seen in Unit IIIc, with totals of 154 and 132 in the Valley Floor and Valley 
TABLE $16-15$

Width to Thickness Ratios of Bifaces by Stage over Time

\begin{tabular}{|c|c|c|c|}
\hline Cultural Period & $\begin{array}{c}\text { Biface } \\
\text { Stage }\end{array}$ & $\begin{array}{c}\text { Mean Width/ } \\
\text { Thickness }\end{array}$ & Count \\
\hline \multirow[t]{3}{*}{ Late Prehistoric } & 1 & 3.27 & 7 \\
\hline & 2 & 3.73 & 22 \\
\hline & 3 & 5.21 & 4 \\
\hline \multirow{3}{*}{$\begin{array}{l}\text { Late Archaic/ } \\
\text { Late Prehistoric }\end{array}$} & 1 & 2.69 & 7 \\
\hline & 2 & 3.95 & 40 \\
\hline & 3 & 4.02 & 4 \\
\hline \multirow{3}{*}{$\begin{array}{l}\text { Archaic/Late Prehistoric } \\
\text { (undifferentiated) }\end{array}$} & 1 & 2.67 & 1 \\
\hline & 2 & 3.89 & 21 \\
\hline & 3 & 4.44 & 5 \\
\hline \multirow{3}{*}{ Late Archaic } & 1 & 2.89 & 22 \\
\hline & 2 & 4.11 & 58 \\
\hline & 3 & 4.76 & 13 \\
\hline \multirow[t]{3}{*}{ Middle/Late Archaic } & 1 & 2.80 & 10 \\
\hline & 2 & 3.76 & 37 \\
\hline & 3 & 4.93 & 4 \\
\hline \multirow[t]{3}{*}{ Middle Archaic } & 1 & 3.23 & 14 \\
\hline & 2 & 4.11 & 38 \\
\hline & 3 & 4.44 & 10 \\
\hline \multirow[t]{3}{*}{ Early/Middle Archaic } & 1 & 2.71 & 17 \\
\hline & 2 & 4.43 & 52 \\
\hline & 3 & 5.49 & 4 \\
\hline \multirow[t]{3}{*}{ Early Archaic } & 1 & 2.85 & 69 \\
\hline & 2 & 3.94 & 204 \\
\hline & 3 & 4.13 & 30 \\
\hline \multirow{3}{*}{$\begin{array}{l}\text { Late Paleoindian/ } \\
\text { Early Archaic }\end{array}$} & 1 & 2.87 & 15 \\
\hline & 2 & 3.80 & 43 \\
\hline & 3 & 4.28 & 7 \\
\hline \multirow[t]{3}{*}{ Late Paleoindian } & 1 & 2.72 & 17 \\
\hline & 2 & 3.70 & 64 \\
\hline & 3 & 4.32 & 10 \\
\hline \multirow[t]{3}{*}{ Early Paleoindian } & 1 & 2.27 & 4 \\
\hline & 2 & 4.82 & 14 \\
\hline & 3 & 4.22 & 1 \\
\hline Total: & & & 868 \\
\hline
\end{tabular}

Margin areas, respectively. Unit IIIa in the Valley Floor also showed a high count, with a total of 147 (see Table 16-13). Of the total staged biface assemblage, the great majority are broken sections; only slightly more than $15 \%$ can be characterized as complete. However, a relatively high frequency of complete bifaces is seen in Unit IIIb in the Valley Floor, with $24 \%$ complete. In other Valley Floor units, frequency of complete bifaces is much lower, including $6 \%$ in Unit Isi/Icl (total sample $=15$ ), $17 \%$ in Unit II (total sample $=42$ ), $13 \%$ in Unit IIIa (total sample=147), and 15\% in Unit IIIc (total sample=154).

Among complete bifaces, reasons for discard frequently appeared to derive from more than one manufacturing error, most typically failure to thin and unsuccessful shaping. Within the Valley Floor A sample, roughly $70 \%$ of complete bifaces recovered in Unit IIIa showed this combination. In contrast, 5 complete bifaces (28\%) in the Unit IIIb sample, evidenced no manufacturing errors and may have been lost or intentionally abandoned prior to completion.

Size variation over time within the biface assemblage can be traced somewhat impressionistically through ratios of width to thickness derived for bifaces in each of the three stages. In Table 16-15, mean width to thickness ratios according to stage are shown for all bifaces with both complete width and thickness measurements $(\mathrm{N}=868)$; these are further sorted by cultural period, based on stratigraphic context.

Of the 11 groups of miscellaneous bifacial tools identified, several appear to be strongly patterned in their distribution over time (Tables 16-16 and 16-17). Of particular interest is the clustering of narrow, elongate tool Form E in contexts pertinent chiefly to the Early Archaic. As shown, $61 \%$ (14 of 23) are present in Units IIIa, IIIa/b, and IIIb in the Valley Floor A and B; the remainder are distributed singly or in small counts in a variety of other units. Pièces esquillées (Form I) also show higher frequencies in similar contexts, with $64 \%$ of the Valley Floor total concentrated in Units IIIa, $\mathrm{III} / \mathrm{b}$, and IIIb, and $64 \%$ of the Valley Margin total in Units IIIb and Y.

TABLE 16-16

Distribution of Bifacial Tools in the Valley Floor (A and B)

\begin{tabular}{|c|c|c|c|c|c|c|c|c|c|c|c|c|}
\hline & Form A & Form B & Form C & Form D & Form E & Form F & Form $\mathrm{G}$ & Form $\mathrm{H}$ & Form I & Form $\mathrm{J}$ & Form K & Totals \\
\hline$\overline{\text { IIIc }}$ & 8 & 4 & & & 1 & 2 & & 4 & & 3 & & 22 \\
\hline IIIb/b/FILL & & & & & & & & & 2 & & 1 & 3 \\
\hline $\mathrm{IIIb} / \mathrm{c}$ & 5 & 2 & & & 1 & & & 1 & 1 & & 1 & 11 \\
\hline $\mathrm{IIIb}$ & 2 & 2 & 2 & & 4 & & & 1 & 3 & 2 & 3 & 19 \\
\hline $\mathrm{IIIa} / \mathrm{b}$ & 3 & 1 & 1 & & 6 & & & & 2 & & 3 & 16 \\
\hline IIIa & 9 & 4 & 2 & 1 & 4 & 1 & & 2 & 2 & 3 & 7 & 35 \\
\hline II/IIIa & 3 & 1 & & & & & & & 1 & & 5 & 10 \\
\hline II & 2 & & 1 & & 1 & & & & & & & 4 \\
\hline $\mathrm{I} / \mathrm{II}$ & & & & & & & & & & & 1 & 1 \\
\hline $\mathrm{Id} / \mathrm{II}$ & 1 & & & & & & & & & & & 1 \\
\hline Isi/Icl & & & & & & & & & & & 2 & 2 \\
\hline & 1 & & & & & & & & & & & 1 \\
\hline Totals: & 34 & 14 & 6 & 1 & 17 & 3 & & 8 & 11 & 8 & 23 & 125 \\
\hline
\end{tabular}


TABLE 16-17

Distribution of Bifacial Tools in the Valley Margin

\begin{tabular}{|c|c|c|c|c|c|c|c|c|c|c|c|c|}
\hline Unit & Form A & Form B & Form C & Form D & Form $\mathrm{E}$ & Form F & Form G & Form $\mathrm{H}$ & Form I & Form J & Form $\mathrm{K}$ & Totals \\
\hline IIIc/POT & 2 & 2 & & & & & & & & & & 4 \\
\hline IIIc & 11 & 5 & & 1 & & & & 2 & 1 & 1 & 3 & 24 \\
\hline $\mathrm{IIIJ} / \mathrm{c}$ & & 1 & & 1 & & & & & & & & 2 \\
\hline Y/IIIc & 5 & 1 & & & 1 & & & & & 2 & & 9 \\
\hline IIIb & & & & & & 1 & & 2 & 4 & & & 7 \\
\hline $\mathrm{Y}$ & & & & & & & & & 3 & & & 3 \\
\hline X/Y/IIIb & 1 & & & & & & & & & & & 1 \\
\hline X/IIIb & 1 & 1 & & 1 & & 1 & & & & & & 4 \\
\hline $\mathrm{X} / \mathrm{Y}$ & 1 & 1 & & & 1 & & & & & & & 3 \\
\hline $\mathrm{IIIa} / \mathrm{b}$ & 1 & & & & & & & & & & & 1 \\
\hline $\mathrm{X} / \mathrm{III} / \mathrm{b}$ & & 1 & & & & & & & & & & 1 \\
\hline IIIa & & & & & & & & & 1 & & & 1 \\
\hline X/IIIa & & & & 1 & & 1 & & & & & & 2 \\
\hline II/IIIa & & & & & 1 & & & & & & & 1 \\
\hline X/II/IIIa & 1 & & & & & & & & 1 & & 1 & 3 \\
\hline & 1 & 1 & & & 1 & & & 1 & & & 2 & 6 \\
\hline $\mathrm{X} / \mathrm{II}$ & & & & & 1 & & & & & & & 1 \\
\hline II & 1 & 2 & & & 1 & & & 1 & 1 & & 1 & 7 \\
\hline Totals: & 25 & 15 & & 4 & 6 & 3 & & 6 & 11 & 3 & 7 & 80 \\
\hline
\end{tabular}

Among the Form A knife-like tools, a quite different distribution is seen, with higher frequencies at Unit IIIc both in the Valley Floor ( 8 of 34 or 24\%) and in the Valley Margin (11 of 25 or 44\%) (see Tables 16-16 and 16-17). A high frequency (9 of 34 or 26\%) also occurs in Unit IIIa in the Valley Floor. Viewed another way, Form A tools comprise nearly half of the total bifacial tool assemblage in Unit IIIc, both in the Valley Floor and Valley Margin, and one-quarter of the total bifacial tool assemblage in Unit IIIa.

Within the full group of bifaces and bifacial tools, little variation is seen in raw material or other material characteristics over time, with several perhaps significant exceptions (Table 16-18). Based on the Valley Floor sample, a comparatively high use of nonlocal Edwards fine-grained chert (41\%) is seen in the Early Paleoindian Unit Isi/Icl. The small assemblage of bifaces from that zone includes the high-quality dark and greenish cherts, some of which may have been stained by groundwater and which resemble cherts from similarly early contexts at the nearby Gault site in Bell County (see Chapter 19 for further discussion of raw materials). The highest use of local fine-grained cherts (78\%) is in Unit IIIb, chiefly attributed to later Early Archaic and Middle Archaic occupations. A somewhat disproportionate use of local coarse cherts (21\%) is shown in Unit II and Unit Isi-c (29\%); both are Late Paleoindian contexts. Very low use of coarse cherts $(6 \%)$ is shown in Unit Isi/Icl.

Cortex was present on roughly $29 \%$ of the sample (Table 16-19). It appears that raw materials from upland residual sources were favored through time, although nodules and stream-altered cobbles also were procured. The highest proportions of upland cherts are in Units IIIa (62\%) and II (65\%), contexts attributed to the Early Archaic and Late Paleoindian, respectively. A slightly larger percentage of nodular cherts is shown in Unit IIIb (6 or $21 \%$ ) than in other stratigraphic units with reasonably large sample sizes.

Heat alteration was noted in only $28 \%$ of the biface and bifacial tool sample (Table 16-20). By stratigraphic unit, frequencies range from $24 \%$ to $38 \%$, with the latter frequency shown in Late Paleoindian contexts (Unit II). Among all units, highest frequency of extensively damaged specimens was seen in Unit IIIc (14 of 42 heat altered, or 33\%).

Patination is not prevalent among specimens in the sample, ranging generally around $10 \%$ within each unit, and roughly $11 \%$ overall (Table $16-21$ ). Of note is the unusually high frequency of patina (21\%) in Unit II, a pattern seen in other tool categories (see Chapter 13). A similar high frequency is seen in mineral precipitates on samples from Unit II (Table 16-22).

\section{BIFACE FRAGMENTS $(\mathrm{N}=617)$}

This category comprises portions of bifaces, including biface lateral edges, for which neither a complete length or width measurement could be taken, nor could they be oriented. For this reason, no metric attributes were recorded. Specimens in this category may derive from Stage 1, 2, or 3 bifaces or from miscellaneous bifacial tools, but generally, they are too small to characterize further. The stratigraphic distribution of the fragments, shown in Table 16-23, is in a pattern similar to Stage 1-3 bifaces, with highest counts in Units IIIa and IIIc.

\section{PERFORATORS}

Perforators in this study are defined as chipped stone implements with long tapering to cylindrical tips. It is generally 
TABLE 16-18

Raw Material Characteristics of Bifaces and Bifacial Tools In Selected Valley Floor Geologic Units

\begin{tabular}{|c|c|c|c|c|c|c|c|c|c|c|c|}
\hline \multirow[b]{2}{*}{ Unit } & \multicolumn{2}{|c|}{$\begin{array}{c}\text { Local Fine-grained } \\
\text { Chert }\end{array}$} & \multicolumn{2}{|c|}{ Local Coarse Chert } & \multicolumn{2}{|c|}{$\begin{array}{l}\text { Nonlocal Edwards } \\
\text { Fine-grained Chert }\end{array}$} & \multicolumn{2}{|c|}{$\begin{array}{c}\text { Nonlocal Edwards } \\
\text { Coarse Chert }\end{array}$} & \multicolumn{2}{|c|}{ Indeterminate } & \multirow{2}{*}{$\frac{\text { Totals }}{\text { Count }}$} \\
\hline & Count & $\%$ & Count & $\%$ & Count & $\%$ & Count & $\%$ & Count & $\%$ & \\
\hline IIIc & 101 & 72 & 19 & 14 & 6 & 4 & 5 & 4 & 9 & 6 & 140 \\
\hline IIIb & 82 & 78 & 16 & 15 & 2 & 2 & 1 & 1 & 4 & 4 & 105 \\
\hline IIIa & 133 & 73 & 24 & 13 & 10 & 5 & 5 & 3 & 10 & 5 & 182 \\
\hline II & 29 & 62 & 10 & 21 & 3 & 6 & 1 & 2 & 4 & 9 & 47 \\
\hline Isi-c & 3 & 43 & 2 & 29 & 2 & 29 & 0 & - & 0 & - & 7 \\
\hline Isi/Icl & 8 & 47 & 1 & 6 & 7 & 41 & 0 & - & 1 & 6 & 17 \\
\hline Totals: & 356 & & 72 & & 30 & & 12 & & 28 & & 498 \\
\hline
\end{tabular}

TABLE 16-19

Cortex Type on Bifaces and Bifacial Tools in Selected Valley Floor Geological Units

\begin{tabular}{l|c|c|c|c|c|c|c|c|c|c}
\hline \multirow{2}{*}{ Unit } & \multicolumn{2}{|c|}{ Upland Residual } & \multicolumn{2}{|c|}{ Nodular } & \multicolumn{2}{c|}{ Stream Altered } & \multicolumn{2}{c|}{ Indeterminate } & Total Cortex & Cortex Absent \\
\cline { 2 - 10 } & Count & $\%$ & Count & $\%$ & Count & $\%$ & Count & $\%$ & Count & Count \\
\hline IIIc & 18 & 47 & 5 & 13 & 1 & 3 & 14 & 37 & 38 & 102 \\
IIIb & 14 & 50 & 6 & 21 & 0 & - & 8 & 29 & 28 & 77 \\
IIIa & 32 & 62 & 9 & 17 & 2 & 4 & 9 & 17 & 52 & 130 \\
II & 11 & 65 & 1 & 6 & 1 & 6 & 4 & 24 & 17 & 30 \\
Isi-c & 1 & 50 & 1 & 50 & 0 & - & 0 & - & 2 & 5 \\
Isi/Icl & 3 & 60 & 0 & - & 0 & - & 2 & 40 & 5 & 12 \\
\hline Totals: & 79 & & 22 & & 4 & & 37 & & 142 & 355 \\
\hline \hline
\end{tabular}

TABLE 16-20

Heat Alteration of Bifaces and Bifacial Tools in Selected Valley Floor Geologic Units

\begin{tabular}{l|c|c|c|c|c|c|c|c|c|c|c}
\hline \multirow{2}{*}{ Unit } & \multicolumn{2}{|c|}{ Absent } & \multicolumn{2}{c|}{ Slight } & \multicolumn{2}{c|}{ Moderate } & \multicolumn{2}{c|}{ Extensive } & \multicolumn{2}{c}{ Indeterminate } & \multicolumn{2}{c}{ Total } \\
\cline { 2 - 11 } & No. & $\%$ & No. & $\%$ & No. & $\%$ & No. & $\%$ & No. & $\%$ & No. \\
\hline IIIc & 98 & 70 & 8 & 6 & 13 & 9 & 14 & 10 & 7 & 5 & 140 \\
IIIb & 79 & 75 & 8 & 8 & 5 & 5 & 4 & 4 & 9 & 9 & 105 \\
IIIa & 139 & 76 & 16 & 9 & 14 & 8 & 10 & 5 & 3 & 2 & 182 \\
II & 29 & 62 & 6 & 13 & 5 & 11 & 3 & 6 & 4 & 9 & 47 \\
Isi-c & 5 & 71 & 1 & 14 & 1 & 14 & 0 & - & 0 & - & 7 \\
Isi/Icl & 11 & 65 & 0 & - & 3 & 18 & 0 & - & 3 & 18 & 17 \\
\hline Totals: & 361 & & 39 & & 41 & & 31 & & 26 & & 498 \\
\hline \hline
\end{tabular}

TABLE 16-21

Patina on Bifaces and Bifacial Tools in Selected Valley Floor Geologic Units

\begin{tabular}{|c|c|c|c|c|c|c|c|}
\hline \multirow[b]{2}{*}{ Unit } & \multicolumn{2}{|c|}{ Absent } & \multicolumn{2}{|c|}{ Present } & \multicolumn{2}{|c|}{ Indeterminate } & \multirow[b]{2}{*}{ Totals } \\
\hline & Count & $\%$ & Count & $\%$ & Count & $\%$ & \\
\hline IIIC & 124 & 89 & 12 & 9 & 4 & 3 & 140 \\
\hline IIIb & 90 & 86 & 13 & 12 & 2 & 2 & 105 \\
\hline IIIa & 160 & 88 & 17 & 9 & 5 & 3 & 182 \\
\hline II & 35 & 74 & 10 & 21 & 2 & 4 & 47 \\
\hline Isi-c & 4 & 57 & 2 & 29 & 1 & 14 & 7 \\
\hline$\underline{\mathrm{Isi} / \mathrm{Icl}}$ & 14 & 82 & 1 & 6 & 2 & 12 & 17 \\
\hline Totals: & 427 & & .55 & & 16 & & 498 \\
\hline
\end{tabular}

presumed that such forms represent tools used to perforate objects either by drilling or by punching. It is likely that harder materials such as wood or bone would require drilling whereas softer materials such as hide or leather might be perforated by punching. It is also possible that some of these tools were awls used in weaving or sewing where the long slender tip was used to press an opening between weave elements for the insertion of another such element or to press a thong or cordage through a perforation in sewing.

Two basic kinds of perforators are recognized in this study, those made by bifacial (Groups 1-7) and those made by unifacial (Group 8) flaking technology. Generally the bifacially flaked variety have longer and more regular bits, and the unifacially flaked ones have shorter and less uniform 
TABLE $16-22$

Mineral Precipitates on Bifaces and Bifacial Tools in Selected Valley Floor Geologic Units

\begin{tabular}{|c|c|c|c|c|c|c|c|}
\hline \multirow[b]{2}{*}{ Unit } & \multicolumn{2}{|c|}{ Absent } & \multicolumn{2}{|c|}{ Present } & \multicolumn{2}{|c|}{ Indeterminate } & \multirow[b]{2}{*}{ Totals } \\
\hline & Count & $\%$ & Count & $\%$ & Count & $\%$ & \\
\hline IIIc & 135 & 96 & 1 & 1 & 4 & 3 & 140 \\
\hline IIIb & 102 & 97 & 0 & - & 3 & 3 & 105 \\
\hline IIIa & 168 & 92 & 10 & 5 & 4 & 2 & 182 \\
\hline II & 20 & 43 & 25 & 53 & 2 & 4 & 47 \\
\hline Isi-c & 4 & 57 & 3 & 43 & 0 & - & 7 \\
\hline Isi/Icl & 13 & 76 & 2 & 12 & 2 & 12 & 17 \\
\hline Totals: & 442 & & 41 & & 15 & & 498 \\
\hline
\end{tabular}

TABLE 16-23

Distribution of Biface Fragments

\begin{tabular}{|c|c|c|}
\hline & Unit & No. \\
\hline Valley Floor A & $\begin{array}{l}\text { IIIc/FILL } \\
\text { IIIc } \\
\text { IIIb/c/FILL } \\
\text { IIIb/c } \\
\text { IIIb } \\
\text { IIIa/b } \\
\text { IIIa } \\
\text { III } \\
\text { II/IIIa } \\
\text { II } \\
\text { Id/II } \\
\text { Id } \\
\text { Isi-c/II } \\
\text { Isi-c } \\
\text { Isi-c/Id } \\
\text { Isi/Isi-c } \\
\text { Icl/Isi-c } \\
\text { Isi/D } \\
\text { Isi/Icl } \\
\text { Igl/Isi/Icl } \\
\text { Isi } \\
\text { Igl }\end{array}$ & $\begin{array}{c}5 \\
75 \\
3 \\
25 \\
55 \\
32 \\
92 \\
1 \\
31 \\
43 \\
1 \\
2 \\
1 \\
3 \\
2 \\
3 \\
2 \\
1 \\
7 \\
1 \\
4 \\
3\end{array}$ \\
\hline Valley Floor B & $\begin{array}{l}\text { I } \\
\text { II } \\
\text { III } \\
\text { IIIc }\end{array}$ & $\begin{array}{l}1 \\
1 \\
8 \\
6\end{array}$ \\
\hline Valley Margin & 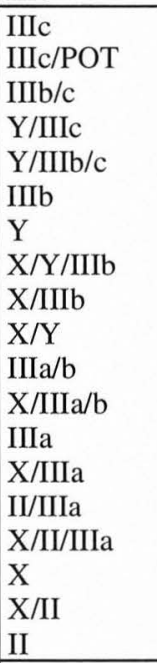 & $\begin{array}{c}55 \\
7 \\
9 \\
8 \\
4 \\
11 \\
13 \\
1 \\
5 \\
5 \\
5 \\
1 \\
11 \\
3 \\
4 \\
8 \\
14 \\
6 \\
7 \\
\end{array}$ \\
\hline Total: & & 585 \\
\hline
\end{tabular}

Note: An additional 32 fragments are from unknown proveniences. bits. Among the bifacial variety, seven morphological subgroups were identified. For all perforators, basic descriptive information was recorded in coded form as to each specimen's morphology, material, condition, and size. The specific attributes and their states are described here and reported in the accompanying tables.

Completeness/section is noted as complete, bit fragment, proximal fragment, medial fragment, lateral edge fragment, or medial bit fragment. A piece coded as complete may, in fact, have some small amount of breakage, but it is possible to confidently determine the complete form. Also, refitted fragments that together constitute a complete piece are coded as complete; in the case of refitted fragments from the same provenience for which the time and cause of breakage is unknown and could include excavation damage, these are reported in the tables as the single, reassembled piece. In the case of pieces definitely broken in antiquity and found in separate proveniences, the individual pieces are tabulated by their provenience with a notation that they refit; data on the conjoined specimen are reported only once in the tables.

Breakage is noted according to its nature, location, and orientation in the same manner as for other chipped stone artifacts except that breaks across the basal end of the perforators are ignored on the presumption that the bit was fashioned on a broken biface as the blank (see further discussion, below). Similarly, the presence or absence of adhering mineral precipitates and the degrees of patination or of heat alteration, if any, are noted as for other artifact classes.

Measurements (in mm) of overall length, maximum width, and maximum thickness are tabulated for those pieces without any breakage; incomplete dimensions are reported for broken pieces. No satisfactory way of measuring bit dimensions was identified in this study. Most bits taper, so width varies with location along the bit length, and the juncture of the bit with the base is usually gradational, making bit length a subjective dimension.

Fourteen of the bifacial perforators have been examined microscopically. Kay found use-related wear on all five of the specimens he viewed and Driskell found wear on one of the three he examined (see Chapter 22). Hudler conducted a brief and preliminary scan of six other specimens simply to 


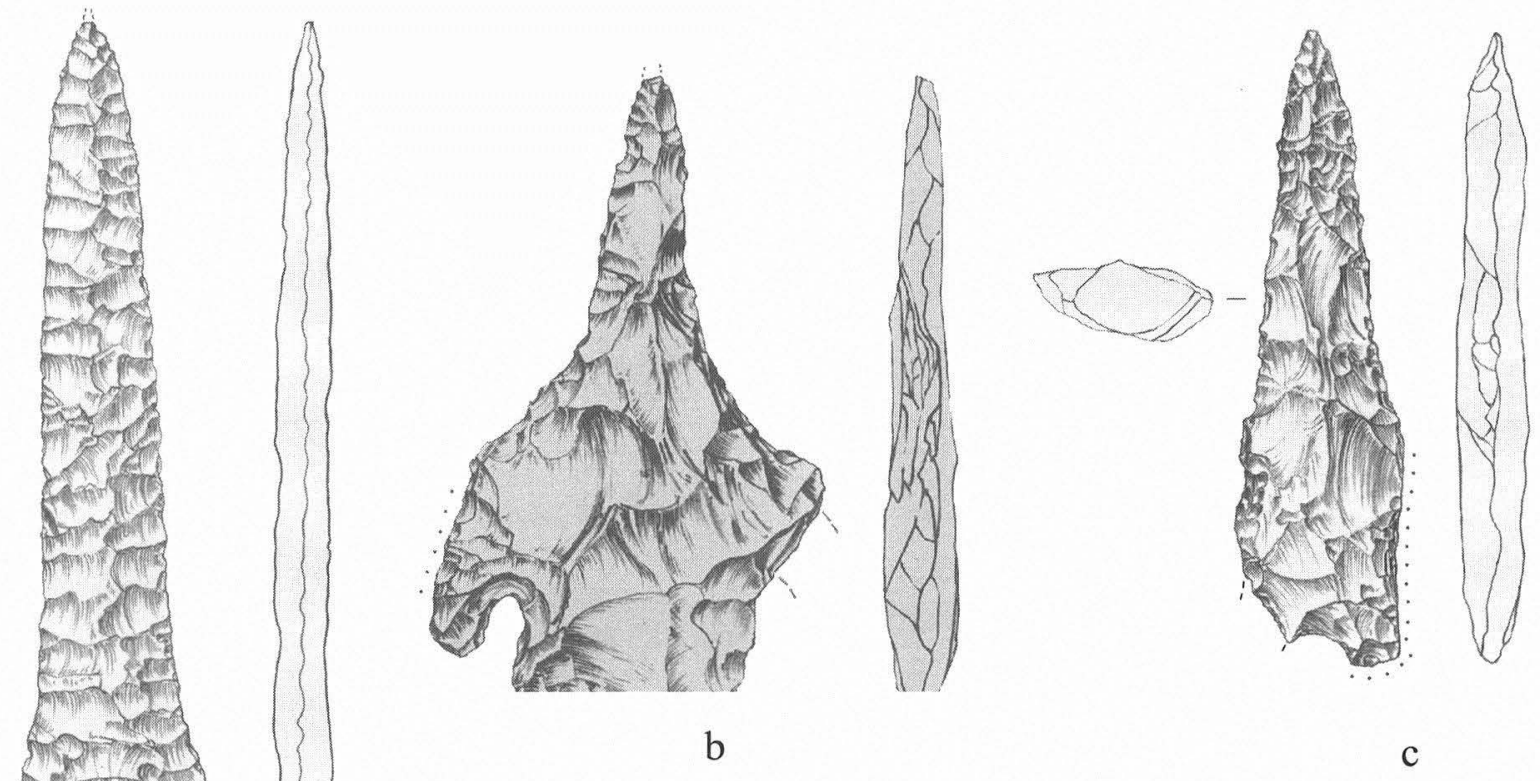

a
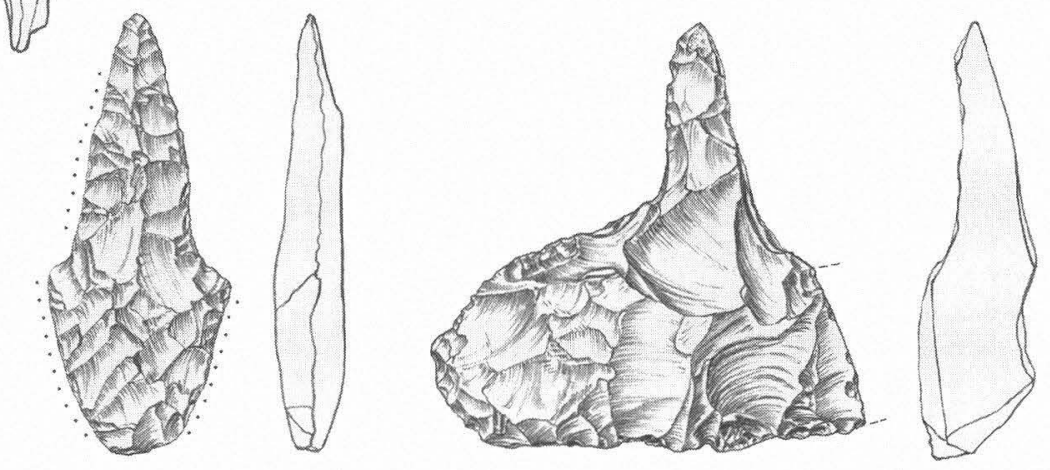

d

e

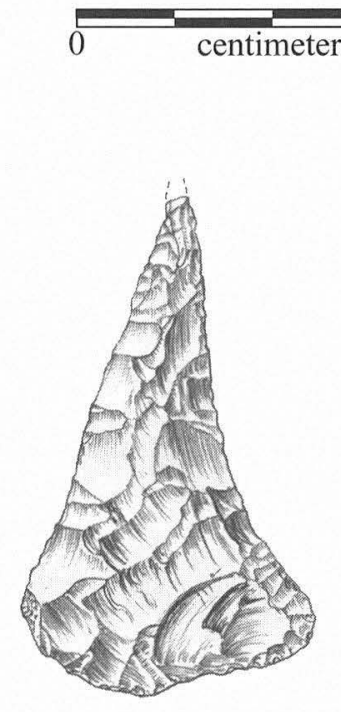

f

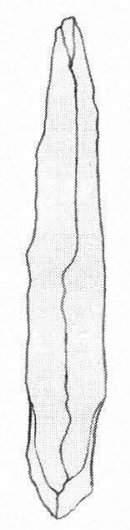

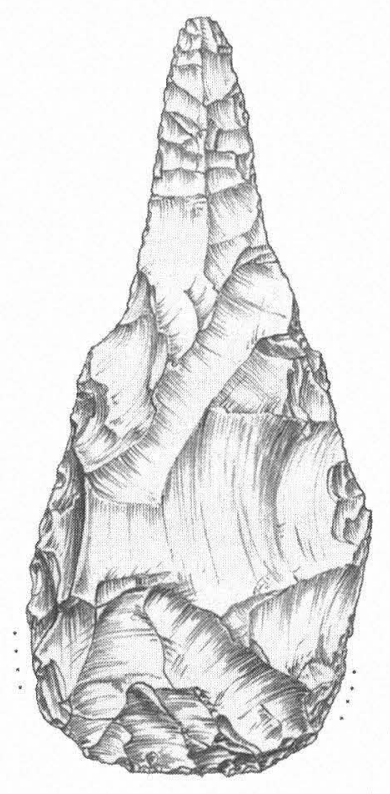

g
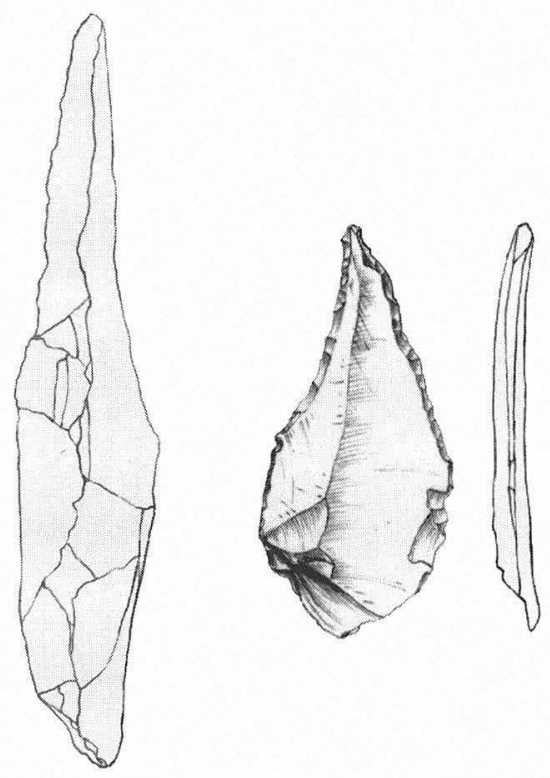

h

FIGURE 16-22. Representative perforators. (a) Group 2; (b-d) Group 3; (e, f) Group 5; (g) Group 6; (h) Group 8. Specimen numbers: (a) 32W1B-1; (b) 14D-2; (c) 25O-1; (d) 14P1-1; (e) 51VA-1; (f) 1H-4; (g) 6G-6; (h) 36O-13. 


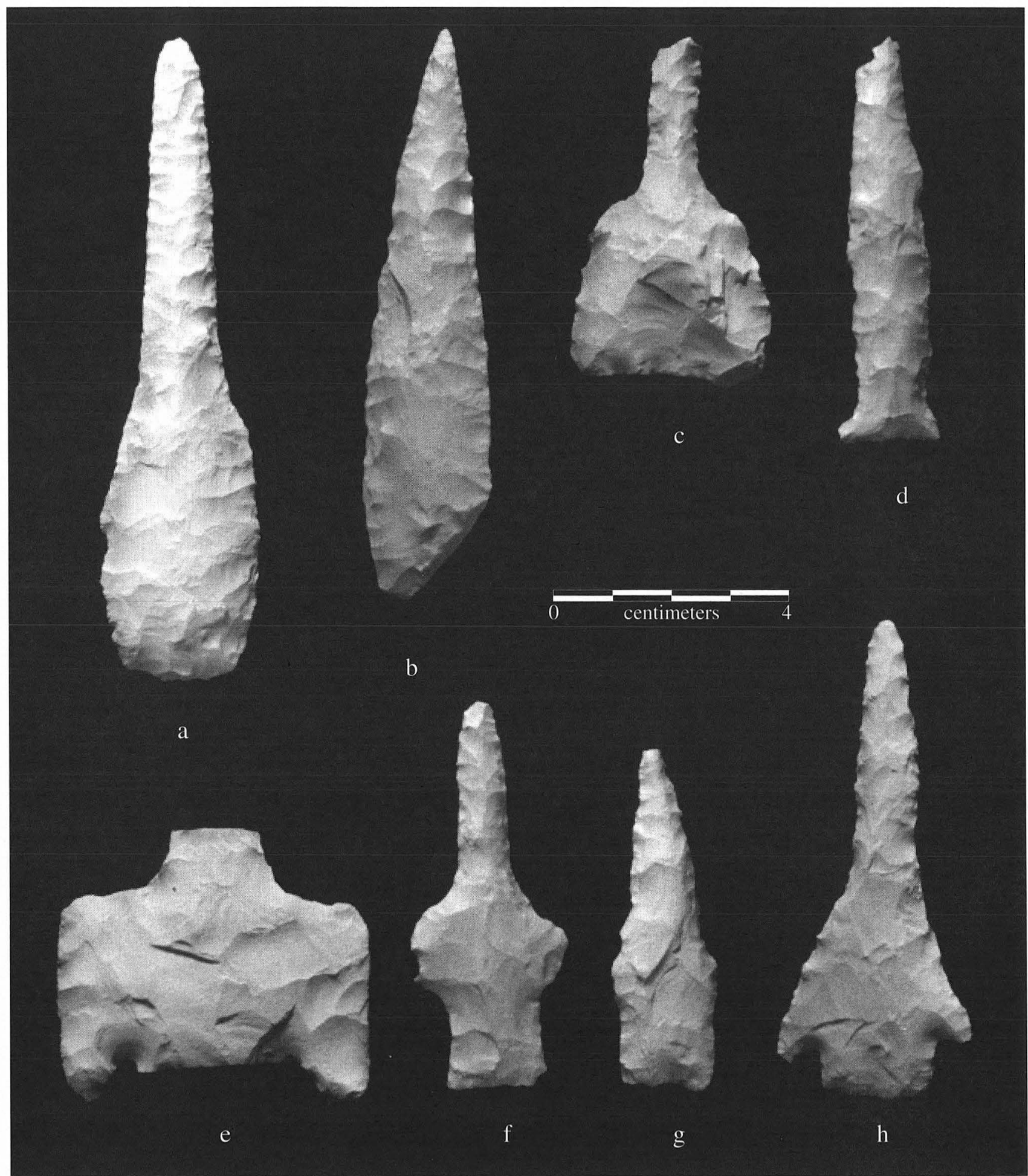

FIGURE 16-23. Representative perforators. (a-c) Group 1; (d) Group 2; (e-h) Group 3. Specimen numbers: (a) 25CC1A-1 conjoined with 26Y1C-2; (b) 3B-7; (c) 0-39; (d) 37LA-2; (e) 36D-8; (f) 8B-11; (g) 13J-29; (h) 21H-4.

determine whether striae were present and, if so, how they were oriented; his results are noted below. Kay also examined one unifacial perforator (see Chapter 22). Additionally, two perforators were examined by Beck for organic residues (see Chapter 23).
Each of the descriptive categories is discussed below followed by some overall observations on this artifact class. Metric, attribute, and provenience data are presented in the accompanying tables. Examples are illustrated in Figures 16$22,16-23$, and 16-24. 


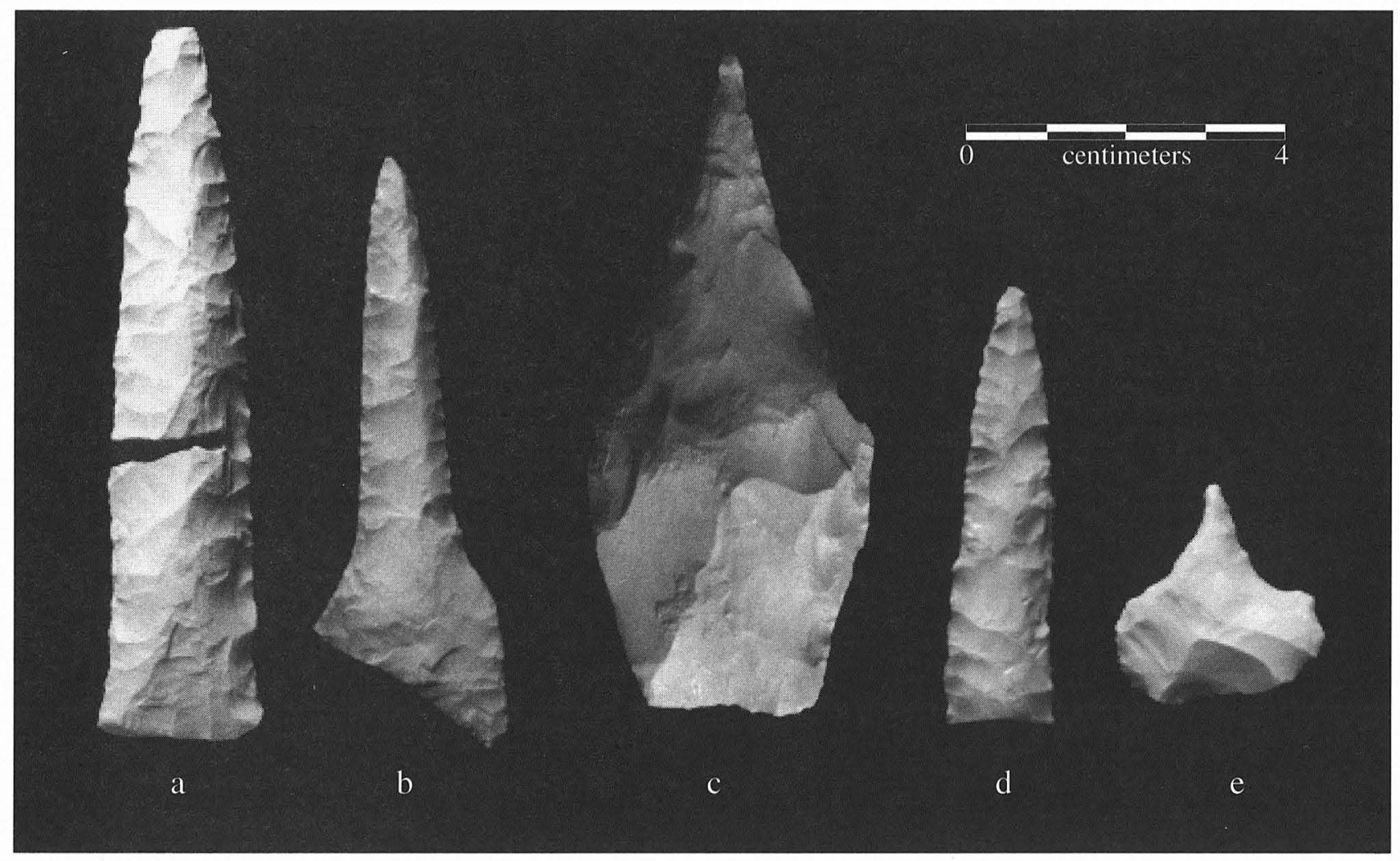

FIGURE 16-24. Representative perforators. (a, b) Group 4; (c) Group 6; (d) Group 7; (e) Group 8. Specimen numbers: (a) 31EE1D-1 conjoined with 16AA2-1; (b) 26U1B-3; (c) 7N-3; (d) 14U2-5; (e) 30T1C-1.

\section{Group $1(\mathbf{N}=18)$}

These are perforator bits fashioned on what appear to be various bifaces showing a range of shapes and workmanship (Figure 16-23a-c; Tables 16-24 and 16-25). Generally these perforators have round to oval to nearly square basal portions and comparatively long, well-made bits. Among the more-complete bits there are 2 that taper and 5 that are cylindrical (18 are too fragmentary for taper to be observed). The inference is that bits were fashioned on bifaces produced for other purposes and diverted to become perforator blanks during manufacture or recycled to that need after another use was over. In some cases, there are knapping errors present on the broad part of the biface suggesting abandoned preforms (a stack knot on one and hinge fractures on four others). One member of this group appears pristine. It (see Figure 16-23a) was found as two fragments in different proveniences but otherwise is in a very freshly flaked state both on its surfaces and along the edges.

Three specimens in this group were examined for use wear. Driskell confirmed that the large, pristine piece (conjoined fragments 26Y1C-2 and 25CC1A-1; see Figure 16-23a) has no evidence of use; Kay confirmed drilling of medium to hard material as the probable use of one (26R1-4); and Hudler noted one minor striation on the bit of one specimen (O-39) oriented perpendicular to the long axis of the bit (too little evidence to support any interpretation of its manner of use).
On one specimen in this group as well as a Group 6 specimen, Beck (see Chapter 23) found residues of multiple nondiagnostic fatty acids as well as traces of pentadecanoic acid. The latter is inferred to derive from the prickly ash.

\section{Group $2(\mathrm{~N}=12)$}

Awl-shaped perforators mostly with long tapering bits constitute this group (Figures 16-22a and 16-23d; Tables 1626 and 16-27); one specimen has a more cylindrical bit. Basal portions are small, the tapering bit extending all the way to the basal edge in some cases. Basal edges are bifacially flaked on all but one which is cortical. Bases range from concave to convex. Generally bits are lenticular rather than round in cross section. These may well represent refurbished pieces that once had larger, bulbous basal portions like those in Group 1. If that is true, reworking of the lateral edges has removed all but the most proximal part of the expanded basal portion. One indication of resharpening is the steep edges and stepped flaking along the bit edges on all but one of these for which the blade is reasonably intact.

Driskell found drill-type wear on one of these specimens (1397-1), and Kay infers that the one specimen (32W1B1) he examined was used as a knife. Hudler examined two and found linear features and striations perpendicular to the long axis on one (19G-4) and polish streaks perpendicular as 
TABLE 16-24

Material Characteristics of Bifacial Perforator Group $1(\mathrm{~N}=18)$

\begin{tabular}{|c|c|c|c|c|c|c|c|c|c|c|c|c|}
\hline \multirow[b]{2}{*}{ 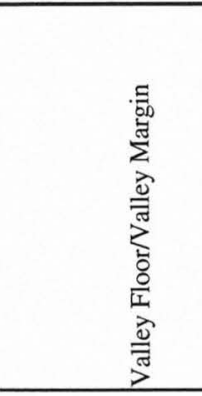 } & \multirow[b]{2}{*}{ 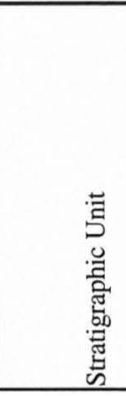 } & \multirow[b]{2}{*}{ 胥 } & \multicolumn{3}{|c|}{ Patination } & \multicolumn{2}{|c|}{$\begin{array}{c}\text { Mineral } \\
\text { Precipitates }\end{array}$} & \multicolumn{3}{|c|}{ Heat Alteration } & \multicolumn{2}{|c|}{ Raw Material } \\
\hline & & & 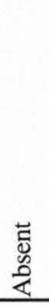 & 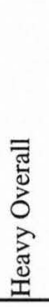 & 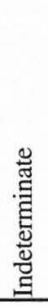 & $\begin{array}{l}\text { 䔍 } \\
\text { 号 } \\
\end{array}$ & 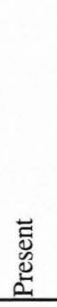 & $\begin{array}{l}\text { 芯 } \\
\text { 足 } \\
\end{array}$ & 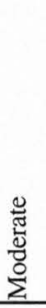 & 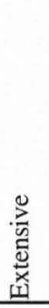 & 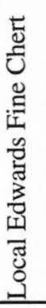 & 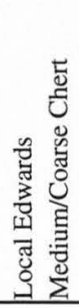 \\
\hline na & Surface & $0-145$ & $\mathrm{X}$ & & & $\mathrm{X}$ & & & & $\mathrm{X}$ & $\mathrm{X}$ & \\
\hline na & Surface & $0-39$ & & $\mathrm{X}$ & & $\mathrm{X}$ & & $\mathrm{X}$ & & & $\mathrm{X}$ & \\
\hline \multirow[t]{10}{*}{ Valley Floor A: } & IIIIc & $36 \mathrm{E}-11$ & $\mathrm{X}$ & & \multirow{10}{*}{$\mathrm{X}$} & $\mathrm{X}$ & \multirow{8}{*}{$\mathrm{X}$} & $\mathrm{X}$ & \multirow{10}{*}{$\mathrm{X}$} & & $\mathrm{X}$ & \\
\hline & $\mathrm{IIIb} / \mathrm{c}$ & OR55 & & & & & & $\mathrm{X}$ & & & $\mathrm{X}$ & \\
\hline & IIIa/b & $290-10$ & $\mathrm{X}$ & & & $\mathrm{X}$ & & $\mathrm{X}$ & & & $\mathrm{X}$ & \\
\hline & IIIa/b & $12 \mathrm{~N}-14$ & $\mathrm{X}$ & & & $\mathrm{X}$ & & $\mathrm{X}$ & & & $\mathrm{X}$ & \\
\hline & IIIa & $23 \mathrm{O}-3$ & $\mathrm{X}$ & & & $\mathrm{X}$ & & $\mathrm{X}$ & & & $\mathrm{X}$ & \\
\hline & IIIa & 26R1-4 & $\mathrm{X}$ & & & $\mathrm{X}$ & & $\mathrm{x}$ & & & $\mathrm{X}$ & \\
\hline & IIIa & $36 \mathrm{~N}-1$ & $\mathrm{X}$ & & & $\mathrm{X}$ & & & & & $\mathrm{X}$ & \\
\hline & II & 26Y1C-2 & $\mathrm{X}$ & & & $\mathrm{X}$ & & $\mathrm{X}$ & & & $\mathrm{X}$ & \\
\hline & II & OR575 & $\mathrm{X}$ & & & & \multirow[t]{2}{*}{$\mathrm{X}$} & $\mathrm{X}$ & & & $\mathrm{X}$ & \\
\hline & Isi-c & $25 \mathrm{CC} 1 \mathrm{~A}-1$ & $\mathrm{X}$ & & & $\mathrm{X}$ & & $\mathrm{X}$ & & & $\mathrm{X}$ & \\
\hline Valley Floor B: & III & $51 \mathrm{SA}-2$ & $\mathrm{X}$ & & & $\mathrm{X}$ & & $\mathrm{X}$ & & & & $\mathrm{X}$ \\
\hline \multirow[t]{5}{*}{ Valley Margin: } & IIIIc & $11 \mathrm{E}-5$ & $\mathrm{X}$ & & & $\mathrm{X}$ & & $\mathrm{x}$ & & \multirow[b]{5}{*}{$\mathrm{X}$} & $\mathrm{X}$ & \\
\hline & IIIIc & $3 \mathrm{~B}-7$ & $\mathrm{X}$ & & & $\mathrm{X}$ & & $\mathrm{X}$ & & & $\mathrm{X}$ & \\
\hline & IIIlb & $21 \mathrm{~L}-12$ & $\mathrm{X}$ & & & $\mathrm{X}$ & & $\mathrm{X}$ & & & $\mathrm{X}$ & \\
\hline & IIIa & $210-24$ & $\mathrm{X}$ & & & $\mathrm{X}$ & & $\mathrm{X}$ & & & $\mathrm{X}$ & \\
\hline & $\mathrm{X}$ & 19S-2 & $\mathrm{X}$ & & & $\mathrm{X}$ & & & & & $\mathrm{X}$ & \\
\hline
\end{tabular}

well as almost perpendicular but slightly oblique to the long axis on another (37LA-2).

\section{Group $3(\mathbf{N}=8)$}

Eight perforators are evidently made on recycled projectile points with the basal portion of the perforator corresponding to the proximal blade and haft area of the projectile point (Figures 16-22b-d and 16-23e-h; Tables 16-28 and 1629). In seven cases, the haft area of the projectile point is substantially complete (e.g., Figures 16-22b-d and 16-23f-h), and in one case, it is broken (see Figure 16-23e). Typologically, these basal portions can be called Hoxie-like (two specimens; see Figures 16-22c and 16-23g), Angostura (see Figure 16-22d), Marcos-like (see Figure 16-23h), Bulverde-like (see Figure 16-23f), and Castroville-like (see Figure 16-22b). In addition, one resembles Golondrina-Barber but lacks grinding, and another (see Figure 16-23e) is possibly a broken Castroville. Bits on all of these specimens are more nearly cylindrical than tapering. One of the perforators made on a
Hoxie-like projectile point (see Figure 16-23g) has an interesting pattern of heat alteration. The bit is reddened from the tip back about a centimeter. The question that arises is whether this is a result of drilling into hot material, such as charred wood.

Wear on the two specimens examined by Kay (13J-29 and 14D-2) indicates use histories consistent with the form of these artifacts in that both showed sequential uses as projectile points, knives, and drills. Hudler also examined the bit of one member of this group (8B-11) and found minor polish streaks obliquely across the distal bit.

\section{Group 4(N=10)}

This group of 10 perforators is similar to Group 1 except that the basal edge on all specimens is a fracture plane (Figure 16-24a, b; Tables 16-30 and 16-31). One way to interpret this form would be to suggest that the basal portion of the perforator was broken away, but instead, these are inferred to be perforators fashioned on broken bifaces. 
TABLE $16-25$

Attributes of Bifacial Perforator Group 1

\begin{tabular}{|c|c|c|c|c|c|c|c|c|c|c|c|c|c|c|c|c|c|c|c|c|c|c|c|c|c|c|c|}
\hline \multirow[b]{2}{*}{ 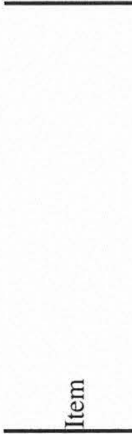 } & \multicolumn{3}{|c|}{ Section } & \multicolumn{4}{|c|}{ Break 1} & \multicolumn{4}{|c|}{$\begin{array}{c}\text { Break Position } \\
1\end{array}$} & \multicolumn{4}{|c|}{$\begin{array}{c}\text { Break } \\
\text { Orientation } 1 \\
\end{array}$} & \multicolumn{2}{|c|}{\begin{tabular}{|c} 
Break \\
2 \\
\end{tabular}} & \multicolumn{2}{|c|}{\begin{tabular}{|c|} 
Break \\
Position \\
2 \\
\end{tabular}} & \multicolumn{2}{|c|}{$\begin{array}{c}\text { Break } \\
\text { Orien- } \\
\text { tation } 2 \\
\end{array}$} & \multicolumn{6}{|c|}{ Metrics (mm) } \\
\hline & 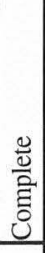 & 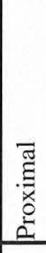 & 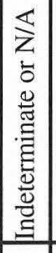 & 气̆ & 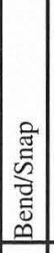 & 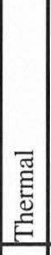 & 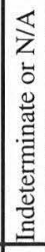 & $\begin{array}{l}\cong \\
z \\
\end{array}$ & 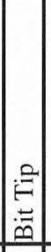 & : & 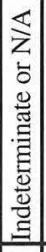 & ż & 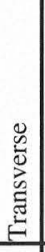 & 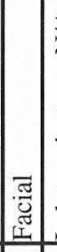 & 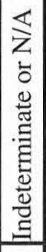 & 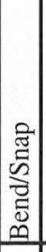 & 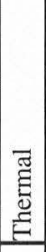 & 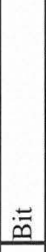 & 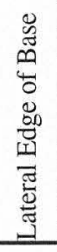 & 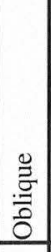 & 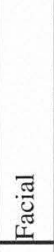 & 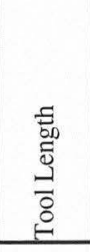 & 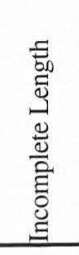 & 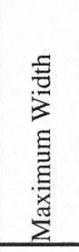 & 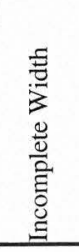 & 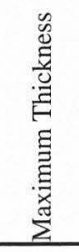 & 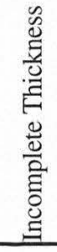 \\
\hline $0-145$ & & $\mathrm{X}$ & & & & $\mathrm{x}$ & & & $\mathrm{X}$ & & & & $\mathrm{x}$ & & & & $\mathrm{X}$ & & $\mathrm{X}$ & & $\mathrm{X}$ & & 46.22 & 31.6 & & & 5.74 \\
\hline $0-39$ & & $\mathrm{X}$ & & & $\mathrm{x}$ & & & & & $\mathrm{x}$ & & & $\mathrm{x}$ & & & & & & & & & & 59.08 & 34.03 & & 10.04 & \\
\hline $36 \mathrm{E}-11$ & & $\mathrm{X}$ & & & $\mathrm{X}$ & & & & & $x$ & & & & $x$ & & & & & & & & & 34.29 & 34.5 & & 9.34 & \\
\hline OR55 & $\mathrm{x}$ & & & & & & $\mathrm{X}$ & & & & $\mathrm{x}$ & & & & $\mathrm{x}$ & & & & & & & 95.16 & & 31.51 & & 8 & \\
\hline $290-10$ & & $\mathrm{X}$ & & & $\mathrm{X}$ & & & & & $\mathrm{X}$ & & & $\mathrm{x}$ & & & & & & & & & & 45.4 & 28.24 & & 9.88 & \\
\hline $12 \mathrm{~N}-14$ & & $\mathrm{X}$ & & & $\mathrm{x}$ & & & & & $x$ & & & $\mathrm{x}$ & & & & & & & & & & 64 & 29.4 & & 10.6 & \\
\hline $26 Y 1 C-2$ & & & $\mathrm{x}$ & & $\mathrm{x}$ & & & & & $x$ & & & $\mathrm{x}$ & & & & & & & & & 111.44 & & 27.39 & & 10.31 & \\
\hline OR575 & $\mathrm{x}$ & & & $\mathrm{x}$ & & & & $\mathrm{x}$ & & & & $\mathrm{x}$ & & & & & & & & & & 53.98 & & 30.9 & & 8.38 & \\
\hline $23 \mathrm{O}-3$ & & $\mathrm{X}$ & & & $\mathrm{x}$ & & & & & $x$ & & & $\mathrm{x}$ & & & & & & & & & & 40.63 & 28.82 & & 8.86 & \\
\hline $26 \mathrm{R} 1-4$ & & $\mathrm{X}$ & & & $\mathrm{x}$ & & & & & $\mathrm{X}$ & & & $\mathrm{X}$ & & & & & & & & & & 42.55 & 20.91 & & 5.72 & \\
\hline $36 \mathrm{~N}-1$ & & $\mathrm{X}$ & & & $\mathrm{x}$ & & & & & $\mathrm{X}$ & & & $\mathrm{x}$ & & & & & & & & & & 49 & 27.1 & & 6 & \\
\hline $25 \mathrm{CC} 1 \mathrm{~A}-1$ & $\mathrm{x}$ & & & & $\mathrm{x}$ & & & & & $x$ & & & & & $\mathrm{x}$ & & & & & & & 111.44 & & 27.39 & & 10.31 & \\
\hline 51SA-2 & & $\mathrm{X}$ & & & $\mathrm{x}$ & & & & & $\mathrm{x}$ & & & $\mathrm{x}$ & & & & & & & & & & 73.49 & 33.94 & & 9.67 & \\
\hline $11 \mathrm{E}-5$ & $\mathrm{X}$ & & & & $\mathrm{X}$ & & & & & $x$ & & & $\mathrm{x}$ & & & $\mathrm{x}$ & & $\mathrm{x}$ & & $\mathrm{x}$ & & 77.52 & & 43.35 & & 11.13 & \\
\hline $3 \mathrm{~B}-7$ & $x$ & & & $x$ & & & & $x$ & & & & $x$ & & & & & & & & & & 99.41 & & 22.03 & & 10.94 & \\
\hline $21 \mathrm{~L}-12$ & & $X$ & & & $\mathrm{X}$ & & & & & $x$ & & & $\mathrm{X}$ & & & & & & & & & & 60.86 & 30.17 & & 10.56 & \\
\hline $210-24$ & $x$ & & & $x$ & & & & $x$ & & & & $x$ & & & & & & & & & & 55.51 & 55.51 & 29.84 & & 12.54 & \\
\hline $19 \mathrm{~S}-2$ & & $X$ & & & $\mathrm{X}$ & & & & & $x$ & & & $\mathrm{X}$ & & & & $\mathrm{X}$ & & $\mathrm{X}$ & & $X$ & & 48.92 & & 32.45 & & 8.07 \\
\hline
\end{tabular}

TABLE $16-26$

Material Characteristics of Bifacial Perforator Group $2(\mathrm{~N}=12)$

\begin{tabular}{|c|c|c|c|c|c|c|c|c|}
\hline & \multirow[b]{2}{*}{ Unit } & \multirow[b]{2}{*}{ Item } & \multirow{2}{*}{\begin{tabular}{|c|} 
Patination \\
Absent \\
\end{tabular}} & \multicolumn{2}{|c|}{ Mineral Precipitates } & \multirow{2}{*}{$\begin{array}{c}\text { Heat Alteration } \\
\text { Absent }\end{array}$} & \multicolumn{2}{|l|}{ Raw Material } \\
\hline & & & & Absent & Present & & $\begin{array}{l}\text { Local Edwards } \\
\text { Fine Chert }\end{array}$ & $\begin{array}{c}\text { Local Edwards } \\
\text { Medium/Coarse Chert }\end{array}$ \\
\hline Valley Floor A: & \begin{tabular}{|l} 
FILL \\
IIIb/c \\
IIIb \\
IIIa \\
IIIa \\
IIIa \\
II/IIIa \\
II/IIIa \\
II \\
Isi-c/Id
\end{tabular} & \begin{tabular}{|l}
$110-1$ \\
$23 \mathrm{H}-12$ \\
$37 \mathrm{LA}-2$ \\
$34 \mathrm{R} 2-8$ \\
$35 \mathrm{Q} 1-2$ \\
$802-1$ \\
$17 \mathrm{~T} 1-7$ \\
$33 \mathrm{~S} 2-2$ \\
$32 \mathrm{~W} 1 \mathrm{~B}-1$ \\
$1397-1$ \\
\end{tabular} & $\begin{array}{l}X \\
X \\
X \\
X \\
X \\
X \\
X \\
X \\
X \\
X\end{array}$ & $\begin{array}{l}X \\
X \\
X \\
X \\
X \\
X \\
X \\
X \\
\\
X\end{array}$ & $\mathrm{X}$ & $\begin{array}{l}X \\
X \\
X \\
X \\
X \\
X \\
X \\
X \\
X \\
X \\
\end{array}$ & $\begin{array}{l}X \\
X \\
X \\
X \\
X \\
X \\
X \\
X \\
X \\
X \\
\end{array}$ & \\
\hline Valley Margin: & $\begin{array}{l}\text { IIIC } \\
\text { IIIC }\end{array}$ & $\begin{array}{l}19 \mathrm{G}-4 \\
11 \mathrm{G}-7\end{array}$ & $\begin{array}{l}X \\
X\end{array}$ & $\begin{array}{l}\mathrm{X} \\
\mathrm{X}\end{array}$ & & $\begin{array}{l}\mathrm{X} \\
\mathrm{X}\end{array}$ & $\mathrm{X}$ & $\mathrm{X}$ \\
\hline
\end{tabular}

Driskell examined one specimen (conjoined fragments 16AA2-1 and 31EEID-1; see Figure 16-24a) in this group and found that it had not been used. This drill was found in two pieces in different proveniences and overall appears to have been broken while otherwise in pristine condition. Another (26U1B-3), scanned by Hudler, has striations perpendicular to and almost parallel to the long axis of the bit, there being more development of those features perpendicular to the long axis. 
TABLE 16-27

Attributes of Bifacial Perforator Group 2

\begin{tabular}{|c|c|c|c|c|c|c|c|c|c|c|c|c|c|c|c|c|c|c|c|c|}
\hline \multirow[b]{2}{*}{ 昜 } & \multicolumn{3}{|c|}{ Section } & \multicolumn{3}{|c|}{ Break 1} & \multicolumn{4}{|c|}{ Break Position 1} & \multicolumn{3}{|c|}{$\begin{array}{c}\text { Break } \\
\text { Orientation } \\
1\end{array}$} & \multirow{2}{*}{ 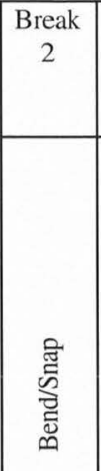 } & \multirow{2}{*}{$\begin{array}{c}\text { Break } \\
\text { Posi- } \\
\text { tion } 2 \\
\\
\\
\\
=\end{array}$} & \multirow{2}{*}{ 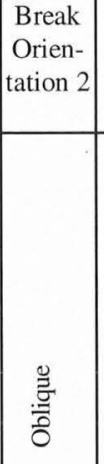 } & \multicolumn{4}{|c|}{ Metrics (mm) } \\
\hline & 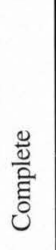 & 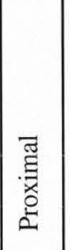 & 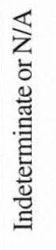 & 总 & 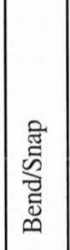 & 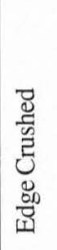 & 苋 & $\begin{array}{l}\because \\
\overline{0}\end{array}$ & 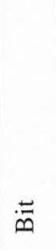 & 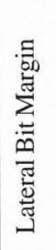 & $\begin{array}{l}\text { 足 } \\
\text { ż }\end{array}$ & 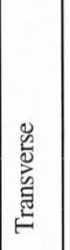 & 啴 & & & & 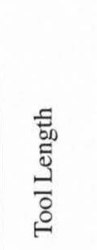 & 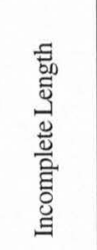 & 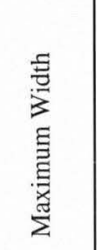 & 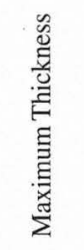 \\
\hline $110-1$ & & $\mathrm{X}$ & & & $\mathrm{X}$ & & & & $\mathrm{X}$ & & & $\mathrm{X}$ & & & & & & 36.22 & 21.37 & 6.95 \\
\hline $23 \mathrm{H}-12$ & $\mathrm{X}$ & & & $\mathrm{X}$ & & & $\mathrm{X}$ & & & & $\mathrm{X}$ & & & & & & 52.45 & & 16.25 & 6.96 \\
\hline 37LA-2 & & $\mathrm{X}$ & & & & $\mathrm{X}$ & & & & $\mathrm{X}$ & & & $\mathrm{X}$ & & & & & 70 & 18.15 & 7.85 \\
\hline $34 \mathrm{R} 2-8$ & & $\mathrm{X}$ & & & $\mathrm{X}$ & & & & $X$ & & & $\mathrm{X}$ & & & & & & 20.49 & 24.03 & 5.89 \\
\hline $35 \mathrm{Q} 1-2$ & & $\mathrm{X}$ & & & $\mathrm{X}$ & & & & $\mathrm{X}$ & & & & $\mathrm{X}$ & & & & & 40.04 & 23.03 & 7.63 \\
\hline $802-1$ & & $\mathrm{X}$ & & & $\mathrm{X}$ & & & $\mathrm{X}$ & & & & $\mathrm{X}$ & & & & & & 38.47 & 20.29 & 7.7 \\
\hline $17 \mathrm{~T} 1-7$ & & & $\mathrm{X}$ & & $\mathrm{X}$ & & & $\mathrm{X}$ & & & & $\mathrm{X}$ & & & & & & 40.23 & 16.76 & 6.01 \\
\hline $33 \mathrm{~S} 2-2$ & & $\mathrm{X}$ & & & $\mathrm{X}$ & & & $\mathrm{x}$ & & & & $\mathrm{X}$ & & & & & & 46.18 & 22.81 & 6.96 \\
\hline 32W1B-1 & $\mathrm{X}$ & & & $\mathrm{X}$ & & & $\mathrm{X}$ & & & & $\mathrm{X}$ & & & & & & 84.69 & & 20.21 & 5.28 \\
\hline $1397-1$ & $\mathrm{X}$ & & & & $\mathrm{X}$ & & & & $X$ & & & $\mathrm{X}$ & & $\mathrm{X}$ & $\mathrm{X}$ & $\mathrm{X}$ & 51.8 & & 24.57 & 6.33 \\
\hline $19 \mathrm{G}-4$ & $\mathrm{X}$ & & & & $\mathrm{X}$ & & & $x$ & & & & & $x$ & & & & & 49.46 & 20.14 & 7.16 \\
\hline $11 \mathrm{G}-7$ & $\mathrm{X}$ & & & & $\mathrm{X}$ & & & $\mathrm{x}$ & & & & $\mathrm{X}$ & & & & & & 65.35 & 23.35 & 9.01 \\
\hline
\end{tabular}

TABLE 16-28

Material Characteristics of Bifacial Perforator Group 3 (N=8)

\begin{tabular}{|c|c|c|c|c|c|c|c|c|c|}
\hline & \multirow[b]{2}{*}{ Unit } & \multirow[b]{2}{*}{ Item } & \multirow{2}{*}{$\begin{array}{l}\text { Patina } \\
\text { Absent }\end{array}$} & \multicolumn{2}{|c|}{ Mineral Precipitates } & \multicolumn{2}{|c|}{ Heat Alteration } & \multicolumn{2}{|c|}{ Raw Material } \\
\hline & & & & Absent & Present & Absent & Slight & $\begin{array}{l}\text { Local Edwards } \\
\text { Fine Chert }\end{array}$ & $\begin{array}{c}\text { Local Edwards } \\
\text { Medium/Coarse Chert }\end{array}$ \\
\hline Valley Floor A: & \begin{tabular}{|l|} 
IIIc \\
IIIc \\
IIIb \\
IIIa/b \\
IIIa \\
\end{tabular} & \begin{tabular}{|l|}
$14 \mathrm{D}-2$ \\
$36 \mathrm{D}-8$ \\
$13 \mathrm{~J}-29$ \\
$25 \mathrm{O}-1$ \\
$14 \mathrm{P} 1-1$ \\
\end{tabular} & $\begin{array}{l}\mathrm{X} \\
\mathrm{X} \\
\mathrm{X} \\
\mathrm{X} \\
\mathrm{X} \\
\end{array}$ & $\begin{array}{l}X \\
X \\
X \\
X \\
X \\
\end{array}$ & & $\mathrm{X}$ & $\begin{array}{l}X \\
X \\
X\end{array}$ & $\begin{array}{l}\mathrm{X} \\
\\
\mathrm{X} \\
\mathrm{X}\end{array}$ & $\begin{array}{l}X \\
X\end{array}$ \\
\hline Valley Margin: & \begin{tabular}{|l|} 
IIIc \\
IIIb \\
Y
\end{tabular} & $\begin{array}{l}8 \mathrm{~B}-11 \\
21 \mathrm{H}-4 \\
3 \mathrm{~J}-2\end{array}$ & $\begin{array}{l}X \\
X \\
X\end{array}$ & $\begin{array}{l}\mathrm{X} \\
\mathrm{X}\end{array}$ & $\mathrm{X}$ & $\begin{array}{l}X \\
X \\
X\end{array}$ & & $\begin{array}{l}X \\
x\end{array}$ & $\mathrm{X}$ \\
\hline
\end{tabular}

\section{Group 5 (N=5)}

Perforators made on irregular blanks or fashioned into an irregular form constitute this group (Figure 16-22e, f; Tables 16-32 and 16-33). Basal areas of these range from unmodified flakes to bifacially flaked pieces. Bits tend to be nearly cylindrical to moderately tapering. The salient characteristic of this category is that fairly regular bits have been fashioned onto irregular, asymmetrical bases of various forms. Macroscopically, one of these appears to have seen heavy use (Figure 16-22e), but none has been examined microscopically.

\section{Group 6(N=3)}

Three perforators are made on the basal section of gouges (Figures 16-22g and 16-24c; Tables 16-34 and 16-35). In each case, the gouge bit is intact and shows the characteristic nibbled edge damage seen on used gouges. The drill bits on the opposite end are tapered longitudinally and thick in cross section.

Kay found evidence that the one specimen he examined (6G-6) had been used in drilling medium to hard material (see Chapter 22). Hudler's scan of another (7N-3) found no directional indicators of its use (see Chapter 22). As noted above, 
TABLE 16-29

Attributes of Bifacial Perforator Group 3

\begin{tabular}{|c|c|c|c|c|c|c|c|c|c|c|c|c|c|c|c|c|}
\hline \multirow[b]{2}{*}{ 焉 } & \multicolumn{3}{|c|}{ Section } & \multicolumn{3}{|c|}{ Break 1} & \multicolumn{3}{|c|}{$\begin{array}{c}\text { Break } \\
\text { Position } 1\end{array}$} & \multicolumn{3}{|c|}{$\begin{array}{c}\text { Break } \\
\text { Orientation } 1\end{array}$} & \multicolumn{4}{|c|}{ Metrics (mm) } \\
\hline & $\frac{\frac{0}{2}}{\frac{0}{2}}$ & 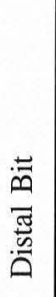 & 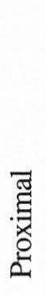 & $\begin{array}{l}\stackrel{\text { Z }}{Z} \\
\text { Z }\end{array}$ & 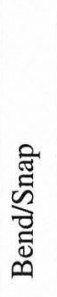 & 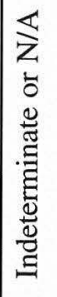 & $\begin{array}{l}0 \\
\text { D } \\
z\end{array}$ & $\mathscr{\oplus}$ & 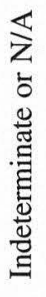 & $\begin{array}{l}\stackrel{0}{0} \\
\text { Z }\end{array}$ & 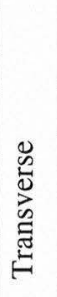 & 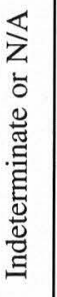 & $\begin{array}{l}\text { 䓃 } \\
\overline{0} \\
\stackrel{0}{\mid} \\
\overline{8} \\
0\end{array}$ & 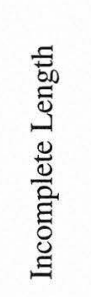 & 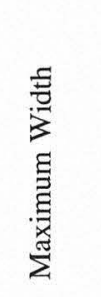 & 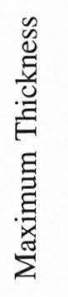 \\
\hline $14 \mathrm{D}-2$ & $\mathrm{X}$ & & & & & $\mathrm{X}$ & & & $\mathrm{X}$ & & & $\mathrm{X}$ & 63.03 & & 37.61 & 6.77 \\
\hline $36 \mathrm{D}-8$ & & & $\mathrm{X}$ & & $\mathrm{X}$ & & & $\mathrm{X}$ & & & $\mathrm{X}$ & & & 48.27 & 54.53 & 9.8 \\
\hline $250-1$ & $\mathrm{X}$ & & & $\mathrm{X}$ & & & $\mathrm{X}$ & & & $\mathrm{X}$ & & & 64.63 & & 16.97 & 6.77 \\
\hline $13 \mathrm{~J}-29$ & $\mathrm{X}$ & & & $\mathrm{X}$ & & & $\mathrm{X}$ & & & $\mathrm{X}$ & & & 59.29 & & 18.87 & 6.49 \\
\hline 14P1-1 & $\mathrm{X}$ & & & $\mathrm{X}$ & & & $\mathrm{X}$ & & & $\mathrm{X}$ & & & 44.83 & & 18.62 & 6.61 \\
\hline 8B-11 & & $\mathrm{X}$ & & $\mathrm{X}$ & & & $\mathrm{X}$ & & & $\mathrm{X}$ & & & 66.38 & & 27.24 & 8.1 \\
\hline $21 \mathrm{H}-4$ & $\mathrm{X}$ & & & $\mathrm{X}$ & & & $\mathrm{X}$ & & & $\mathrm{X}$ & & & 82.85 & & 33.93 & 7.37 \\
\hline $3 J-2$ & $\mathrm{X}$ & & & $\mathrm{X}$ & & & $\mathrm{X}$ & & & $\mathrm{X}$ & & & 59.35 & & 24.52 & 5.91 \\
\hline
\end{tabular}

TABLE $16-30$

Material Characteristics of Bifacial Perforator Group $4(\mathrm{~N}=10)$

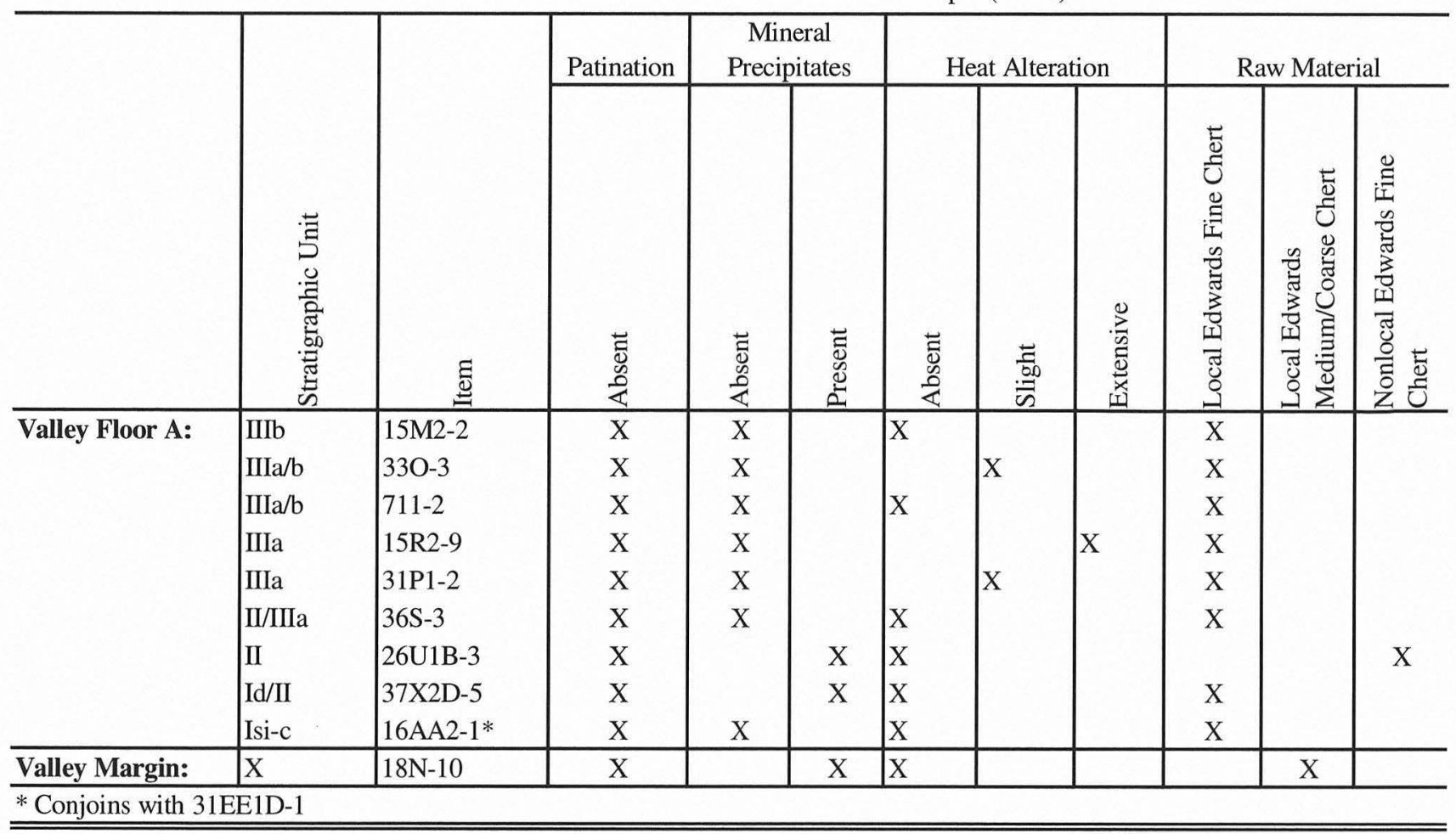

Beck found residues of multiple fatty acids and pentadecanoic acid on one of the specimens in this group (OR419) as well as a Group 1 specimen (see Chapter 23); pentadecanoic acid is inferred to derive from the prickly ash tree.

\section{Group $7(\mathrm{~N}=67)$}

Bifacial tip and bit fragments of perforators constitute this category (Figure 16-24d; Tables 16-36 and 16-37). Many 
TABLE 16-31

Attributes of Bifacial Perforator Group 4

\begin{tabular}{|c|c|c|c|c|c|c|c|c|c|c|c|c|c|c|c|c|}
\hline \multirow[b]{2}{*}{$\underset{\Xi}{\mathbb{E}}$} & \multicolumn{4}{|c|}{ Section } & \multicolumn{3}{|c|}{ Break } & \multicolumn{2}{|c|}{ Break Position } & \multicolumn{2}{|c|}{ Break Orientation } & \multicolumn{5}{|c|}{ Metrics (mm) } \\
\hline & 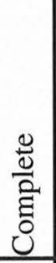 & $\begin{array}{l}\overline{\tilde{J}} \\
\stackrel{\tilde{n}}{0} \\
\end{array}$ & 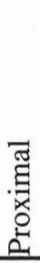 & 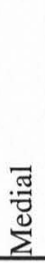 & $\begin{array}{l}\text { Z } \\
\text { Z }\end{array}$ & 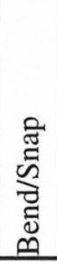 & $\begin{array}{l}\text { चี } \\
\text { है } \\
\text { है }\end{array}$ & 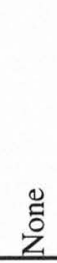 & 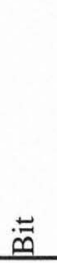 & $\begin{array}{l}\text { ஜ } \\
\text { Z }\end{array}$ & 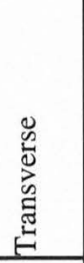 & 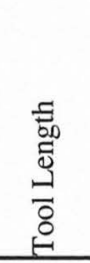 & 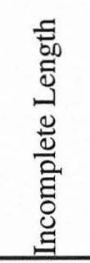 & 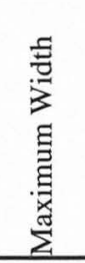 & 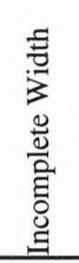 & 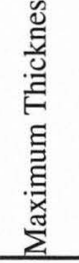 \\
\hline $15 \mathrm{M} 2-2$ & $\mathrm{X}$ & & & & $\mathrm{X}$ & & & $\mathrm{X}$ & & $\mathrm{X}$ & & 40.66 & & 25.75 & & 7.02 \\
\hline $33 \mathrm{O}-3$ & $\mathrm{X}$ & & & & $\mathrm{X}$ & & & $\mathrm{X}$ & & $\mathrm{X}$ & & 40 & & 15.01 & & 5.21 \\
\hline $711-2$ & $\mathrm{X}$ & & & & $X$ & & & $X$ & & $\mathrm{X}$ & & 18.19 & & 12.78 & & 2.82 \\
\hline $15 \mathrm{R} 2-9$ & & & & $\mathrm{X}$ & & & $\mathrm{X}$ & & $\mathrm{X}$ & & $\mathrm{X}$ & & 29.98 & & 26.13 & 7.37 \\
\hline $31 \mathrm{P} 1-2$ & & & $\mathrm{X}$ & & & & $\mathrm{X}$ & & $\mathrm{X}$ & & $\mathrm{X}$ & & 30.35 & 25.13 & & 8.12 \\
\hline $36 \mathrm{~S}-3$ & $\mathrm{X}$ & & & & $\mathrm{X}$ & & & $\mathrm{X}$ & & $\mathrm{X}$ & & 56.07 & & 23.36 & & 8.43 \\
\hline 26U1B-3 & $\mathrm{X}$ & & & & $\mathrm{X}$ & & & $X$ & & $X$ & & 76.72 & & 24.55 & & 7.51 \\
\hline $37 \times 2 D-5$ & & & $\mathrm{X}$ & & & $\mathrm{X}$ & & & $\mathrm{X}$ & & $X$ & & 56.04 & 27.14 & & 11.38 \\
\hline $16 \mathrm{AA} 2-1^{*}$ & $\mathrm{X}$ & & & & & $X$ & & & $\mathrm{X}$ & & $\mathrm{X}$ & 87.43 & & 21.36 & & 9.19 \\
\hline $18 \mathrm{~N}-10$ & & $\mathrm{X}$ & & & $\mathrm{X}$ & & & $\mathrm{X}$ & & $\mathrm{X}$ & & 76.22 & & 33.79 & & 13.44 \\
\hline
\end{tabular}

* Conjoins with 31EE1D-1

TABLE 16-32

Material Characteristics of Bifacial Perforator Group 5 ( $\mathrm{N}=5$ )

\begin{tabular}{l|c|c|c|c|c|c|c}
\hline & & & Patination & Mineral Precipitates & Heat Alteration & Raw Material \\
\cline { 4 - 7 } & Unit & Item & Absent & Absent & Present & Absent & Local Edwards Fine Chert \\
\hline Valley Floor A: & IIIb/c & $36 \mathrm{I}-10$ & $\mathrm{X}$ & $\mathrm{X}$ & & $\mathrm{X}$ & $\mathrm{X}$ \\
& $\mathrm{IIIb} / \mathrm{c}$ & $15 \mathrm{H}-6$ & $\mathrm{X}$ & $\mathrm{X}$ & & $\mathrm{X}$ & $\mathrm{X}$ \\
\hline Valley Floor B: & $\mathrm{III}$ & $51 \mathrm{VA}-1$ & $\mathrm{X}$ & & $\mathrm{X}$ & $\mathrm{X}$ & $\mathrm{X}$ \\
\hline Valley Margin: & $\mathrm{Y}$ & $1 \mathrm{H}-4$ & $\mathrm{X}$ & & $\mathrm{X}$ & $\mathrm{X}$ & $\mathrm{X}$ \\
& $\mathrm{X} / \mathrm{II}$ & $20 \mathrm{~S}-7$ & $\mathrm{X}$ & $\mathrm{X}$ & & $\mathrm{X}$ & $\mathrm{X}$ \\
\hline \hline
\end{tabular}

TABLE 16-33

Attributes of Bifacial Perforator Group 5

\begin{tabular}{|c|c|c|c|c|c|c|c|c|c|c|c|c|c|}
\hline \multirow[b]{2}{*}{ Item } & \multicolumn{2}{|c|}{ Section } & \multicolumn{2}{|c|}{ Break } & \multicolumn{2}{|c|}{ Break Position } & \multicolumn{2}{|c|}{$\begin{array}{c}\text { Break } \\
\text { Orientation }\end{array}$} & \multicolumn{5}{|c|}{ Metrics $(\mathrm{mm})$} \\
\hline & $\begin{array}{l}\frac{0}{0} \\
\frac{0}{\tilde{E}} \\
0 \\
0\end{array}$ & 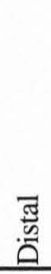 & $\begin{array}{l}\text { Z } \\
\text { z }\end{array}$ & 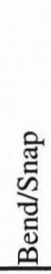 & $\begin{array}{l}\text { Z } \\
\text { Z }\end{array}$ & 常 & $\begin{array}{l}\text { : } \\
\text { z }\end{array}$ & 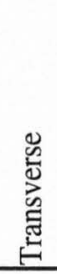 & 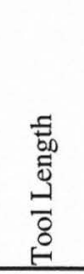 & 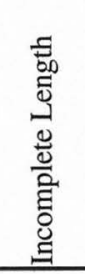 & 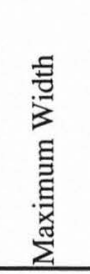 & 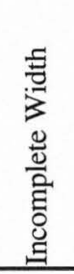 & 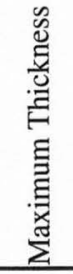 \\
\hline $36 \mathrm{I}-10$ & $\mathrm{X}$ & & $\mathrm{X}$ & & $\mathrm{X}$ & & $\mathrm{X}$ & & 39.91 & & 17.27 & & 4.77 \\
\hline $15 \mathrm{H}-6$ & $X$ & & $\mathrm{X}$ & & $\mathrm{X}$ & & $\mathrm{X}$ & & 54.29 & & 43.03 & & 13.06 \\
\hline 51VA-1 & $\mathrm{X}$ & & $\mathrm{X}$ & & $\mathrm{X}$ & & $\mathrm{X}$ & & 43.59 & & & 41.4 & 10.36 \\
\hline $1 \mathrm{H}-4$ & $\mathrm{X}$ & & $\mathrm{X}$ & & $\mathrm{X}$ & & $\mathrm{X}$ & & 51.5 & & 30 & & 8.1 \\
\hline 20S-7 & & $\mathrm{X}$ & & $\mathrm{X}$ & & $X$ & & $\mathrm{X}$ & & 54.56 & 35.28 & & 7.97 \\
\hline
\end{tabular}


TABLE 16-34

Material Characteristics of Bifacial Perforator Group 6 (N=3)

\begin{tabular}{|c|c|c|c|c|c|c|c|c|c|}
\hline & \multirow[b]{2}{*}{ Unit } & \multirow[b]{2}{*}{ Item } & \multicolumn{2}{|c|}{ Patination } & \multicolumn{2}{|c|}{$\begin{array}{c}\text { Mineral } \\
\text { Precipitates }\end{array}$} & \multirow{2}{*}{$\begin{array}{c}\text { Heat } \\
\text { Alteration } \\
\text { Absent }\end{array}$} & \multirow{2}{*}{$\begin{array}{c}\text { Raw Material } \\
\text { Local Edwards } \\
\text { Fine Chert }\end{array}$} & \multirow[b]{2}{*}{$\begin{array}{c}\text { Local Edwards } \\
\text { Medium/Coarse Chert }\end{array}$} \\
\hline & & & Absent & Present & Absent & Present & & & \\
\hline Valley Margin: & $\begin{array}{c}\text { IIIc } \\
\mathrm{X} / \mathrm{Y} \\
\mathrm{X}\end{array}$ & $\begin{array}{c}6 \mathrm{G}-6 \\
7 \mathrm{~N}-3 \\
18 \mathrm{QB}-2\end{array}$ & $\begin{array}{l}X \\
X\end{array}$ & $X$ & $\mathrm{X}$ & $\begin{array}{l}X \\
X\end{array}$ & $\begin{array}{l}\mathrm{X} \\
\mathrm{X} \\
\mathrm{X}\end{array}$ & $\begin{array}{l}X \\
X\end{array}$ & $X$ \\
\hline
\end{tabular}

TABLE 16-35

Attributes of Bifacial Perforator Group 6

\begin{tabular}{|c|c|c|c|c|c|c|c|c|c|c|c|c|}
\hline \multirow[b]{2}{*}{ Item } & \multicolumn{2}{|c|}{ Section } & \multicolumn{2}{|c|}{ Break } & \multicolumn{2}{|c|}{ Break Position } & \multicolumn{2}{|c|}{ Break Orientation } & \multicolumn{4}{|c|}{ Metrics (mm) } \\
\hline & 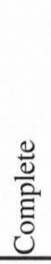 & 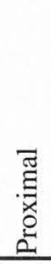 & $\begin{array}{l}\text { ठ̃ } \\
\text { ż }\end{array}$ & 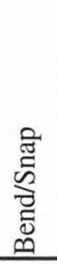 & $\begin{array}{l}0 \\
\tilde{Z} \\
\text { z }\end{array}$ & 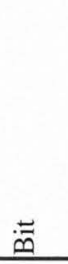 & $\begin{array}{l}0 \\
\text { हे } \\
\text { ż }\end{array}$ & $\begin{array}{l}\cong \\
. \\
0\end{array}$ & $\begin{array}{l}5 \\
5 \\
0 \\
0 \\
0 \\
0 \\
0 \\
0\end{array}$ & 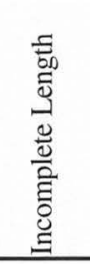 & 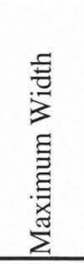 & 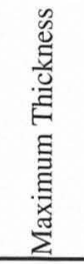 \\
\hline 6G-6 & $\mathrm{X}$ & & $\mathrm{X}$ & & $\mathrm{X}$ & & $\mathrm{X}$ & & 76.1 & & 34.24 & 14.07 \\
\hline $7 \mathrm{~N}-3$ & $\mathrm{X}$ & & $\mathrm{X}$ & & $\mathrm{X}$ & & $\mathrm{X}$ & & 86.3 & & 43.5 & 16.6 \\
\hline 18QB-2 & & $\mathrm{X}$ & & $\mathrm{X}$ & & $\mathrm{X}$ & & $\mathrm{X}$ & & 53.31 & 38.33 & 12.75 \\
\hline
\end{tabular}

of these are small fragments, but the narrow widths, nearly parallel edges, and thick cross sections are more consistent with perforator forms than with any other bifacial blade forms.

\section{Group $8(\mathrm{~N}=20)$}

Twenty tools, each with a prominent, unifacially flaked eminence on one edge, are referred to as unifacial perforators (Figures 16-22h and 16-24e; Tables 16-38 and 16-39). This class of tools is arbitrarily distinguished from gravers by the length and strength of the protruding bit, but in some cases, the distinction is minor and subjective.

Bits for the most part are subtriangular in cross section and taper toward a point; none has the fully cylindrical bit seen among some bifacial perforators. These are made on flakes ( $n=13)$, a uniface, and a crude biface. Five are tip fragments for which the parent piece cannot be identified. Kay's examination of one of these (30T1C-1) revealed evidence of drilling action on medium hard material.

\section{Discussion}

Perforators are not particularly numerous in the Wilson-Leonard sample except from Early Archaic contexts (Unit IIIa, primarily) where almost half of the total sample was found. Although several variants are noted as the groups described above, the form of the basal portion consistently seems to have been of little importance to the makers and users of these tools. This is reflected in the various blanks upon which perforators were made and the various sizes and shapes of the basal sections. It would seem that these are "handles" for simple prehended tools and that none of these was hafted. This further implies that drilling was not so demanding that bow- or hand-propelled shafts were required; no artifacts resembling the whorl of a bow drill occur in any frequency in the region, which may be another indication that compound drills were not in use.

The most significant variation in form, though not in chronological occurrence, is a distinction between cylindrical and tapering bits. The distinction is not perfect as some gradation is seen between the modal forms. In terms of form, the broader, thinner, tapering bits would seem more likely to have functioned as awls or punches whereas the more cylindrical bits would appear to be used in drilling. As punches, the cylindrical forms would produce round holes whereas the tapered bits would make elliptical holes; as drills, the cylindrical bits would produce cylindrical holes and the tapered ones would leave conical holes.

For any of these tools used as punches, wear should be in the form of striae roughly parallel to the long axis of the piece, whereas if they were used as drills, wear striae should be perpendicular to that axis. The observations of Driskell, Kay, and Hudler revealed that drilling was definitely or probably the manner of use on most of the specimens for which such determinations could be made; however, there is also ample evidence of complex directions of use on some of these tools, probably reflecting multiple functions. As noted above, Beck found residue inferred to derive from prickly ash on two specimens. If this is correct, perhaps these tools were used to drill of ash wood. This would be a reasonable interpretation given the high quality of this medium-hard white wood. 
TABLE 16-36

Material Characteristics of Bifacial Perforator Group 7 ( $N=67$ )

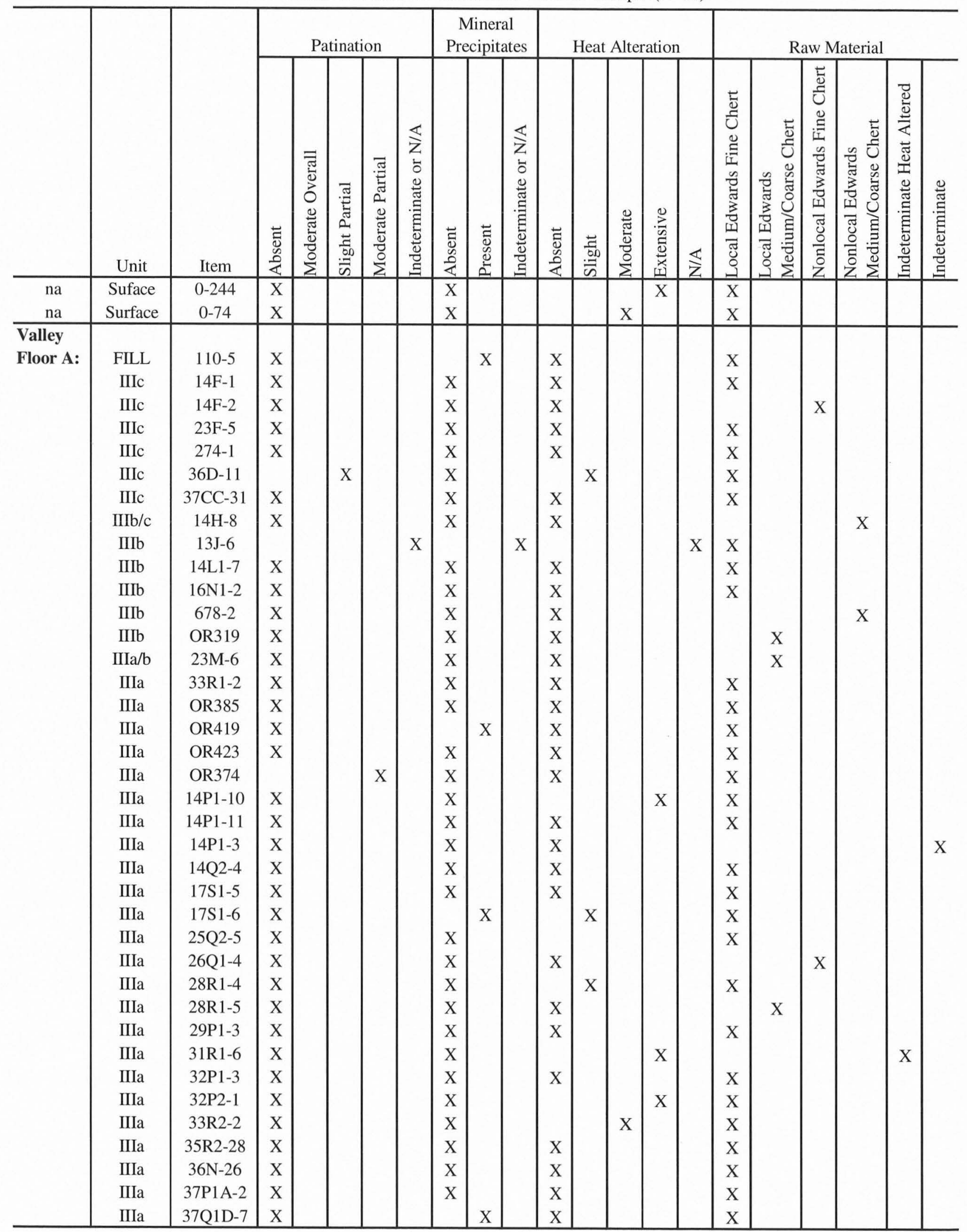


Table 16-36, continued

\begin{tabular}{|c|c|c|c|c|c|c|c|c|c|c|c|c|c|c|c|c|c|c|c|c|c|}
\hline & \multirow[b]{2}{*}{ Unit } & \multirow[b]{2}{*}{ Item } & \multicolumn{5}{|c|}{ Patination } & \multicolumn{3}{|c|}{$\begin{array}{c}\text { Mineral } \\
\text { Precipitates }\end{array}$} & \multicolumn{5}{|c|}{ Heat Alteration } & \multicolumn{6}{|c|}{ Raw Material } \\
\hline & & & $\begin{array}{l}\text { 若 } \\
\text { 足 } \\
\end{array}$ & 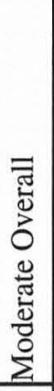 & 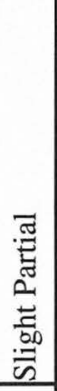 & 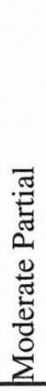 & 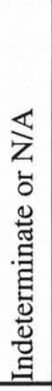 & 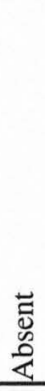 & 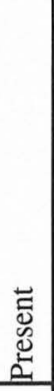 & 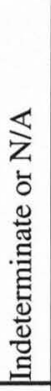 & $\begin{array}{l}\overrightarrow{0} \\
0 \\
\text { 足 } \\
\text { 安 }\end{array}$ & 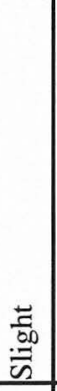 & $\begin{array}{l}0 \\
\frac{0}{\pi} \\
\frac{\pi}{0} \\
0 \\
0 \\
\end{array}$ & 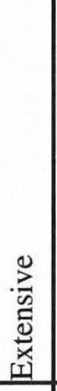 & 更 & 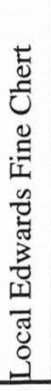 & 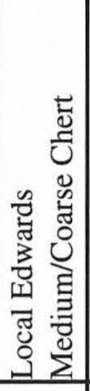 & 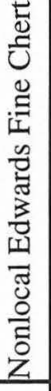 & 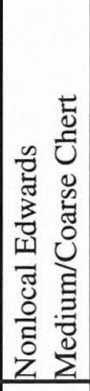 & 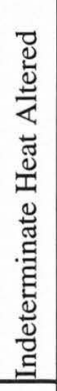 & 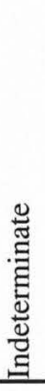 \\
\hline & $\begin{array}{c}\text { IIIa } \\
\text { IIIa } \\
\text { III } \\
\text { II/IIIa } \\
\text { II } \\
\text { II } \\
\text { Id/II } \\
\end{array}$ & $\begin{array}{c}710-5 \\
\text { OR339 } \\
52 \mathrm{PC}-11 \\
13 \mathrm{Q}-6 \\
28 \mathrm{~W} 1 \mathrm{~B}-3 \\
37 \mathrm{X} 2 \mathrm{C}-3 \\
14 \mathrm{U} 2-5 \\
\end{array}$ & $\begin{array}{l}X \\
X \\
X \\
X \\
X \\
X\end{array}$ & & $\mathrm{X}$ & & & $\begin{array}{l}X \\
X \\
X \\
X \\
X \\
X\end{array}$ & $\mathrm{X}$ & & $\begin{array}{l}\mathrm{X} \\
\mathrm{X} \\
\mathrm{X} \\
\mathrm{X} \\
\mathrm{X}\end{array}$ & $\begin{array}{c}\mathrm{X} \\
\mathrm{X}\end{array}$ & & & & $\begin{array}{l}\mathrm{X} \\
X \\
X \\
X \\
X \\
X \\
X\end{array}$ & & & & & \\
\hline $\begin{array}{l}\text { Valley } \\
\text { Floor B: }\end{array}$ & $\begin{array}{c}\text { IIIc } \\
\text { III } \\
\text { I } \\
\end{array}$ & $\begin{array}{c}50 \mathrm{~EB}-6 \\
51 \mathrm{PA}-3 \\
50 \mathrm{AA} 2 \mathrm{~B}-1 \\
\end{array}$ & $\begin{array}{l}X \\
X \\
2 \\
\end{array}$ & $\mathrm{X}$ & & & & $\begin{array}{l}X \\
X \\
X \\
\end{array}$ & & & $\begin{array}{l}X \\
X \\
X \\
\end{array}$ & & & & & $\begin{array}{l}X \\
X \\
\end{array}$ & $X$ & & & & \\
\hline $\begin{array}{l}\text { Valley } \\
\text { Margin: }\end{array}$ & $\begin{array}{c}\text { IIIc } \\
\text { IIIc } \\
\text { IIIc/POT } \\
\text { IIIc/POT } \\
\text { Y/IIIc } \\
\text { Y/IIIb } \\
\text { Y } \\
\text { X/IIIb } \\
\text { X/Y } \\
\text { IIIa } \\
\text { IIIa } \\
\text { X/IIIa/b } \\
\text { X/IIIa } \\
\text { X } \\
\text { X } \\
\text { X } \\
\text { X/II } \\
\end{array}$ & $\begin{array}{c}1349-8 \\
6 \mathrm{E}-4 \\
5 \mathrm{~A}-24 \\
6 \mathrm{~A}-19 \\
8 \mathrm{I}-16 \\
9 \mathrm{~K}-4 \\
1 \mathrm{I}-3 \\
19 \mathrm{M}-5 \\
5 \mathrm{~L}-2 \\
22 \mathrm{O}-11 \\
22 \mathrm{O}-12 \\
10 \mathrm{~N}-3 \\
10 \mathrm{O}-2 \\
18 \mathrm{~TB}-2 \\
19 \mathrm{Q}-2 \\
19 \mathrm{~S}-9 \\
20 \mathrm{~S}-8 \\
\end{array}$ & $\begin{array}{l}X \\
X \\
X \\
\\
X \\
X \\
X \\
X \\
X \\
X \\
X \\
X \\
X \\
X \\
X \\
X \\
X \\
\end{array}$ & & & & $\mathrm{X}$ & $\begin{array}{l}\text { X } \\
X \\
X \\
X \\
X \\
X \\
X \\
X \\
X \\
X \\
X\end{array}$ & $\mathrm{X}$ & $\mathrm{X}$ & $\begin{array}{l}X \\
X \\
X \\
X \\
X \\
X \\
X \\
X \\
X \\
X \\
X \\
X \\
X \\
\end{array}$ & \begin{tabular}{l|}
$\mathrm{X}$ \\
$\mathrm{X}$
\end{tabular} & $X$ & $X$ & & $\begin{array}{l}X \\
X \\
X \\
X \\
X \\
X \\
X \\
X \\
X \\
X \\
X \\
X \\
X \\
X\end{array}$ & $\begin{array}{l}X \\
X \\
\end{array}$ & & & & \\
\hline
\end{tabular}

BURINS

Burins are chisel-like tools formed by the intersection of two facets at a promontory on a piece of chert. The piece of chert in this study is referred to as the blank and is found in a variety of forms, as described below. The facets forming the burin include at least one specialized flake scar, known as a burin facet, detached from the edge of the blank in a direction away from the apex, or bit, of the burin. The intersecting facet may also be a burin facet or it may be a fracture plane or a truncation. Burins are well-known kinds of tools characteristic of the Old World Upper Paleolithic where they occur in large numbers and in a wide variety of distinctive types. Most Upper Paleolithic burins are made on blades (Knecht 1988). Burins are also common in some New World assemblages but generally are typologically far less formalized than in the Old World. New World burins are typically made on flakes or on recycled unifacially or bifacially flaked objects and fragments of such objects. Studies of burin function, both archeological and experimental, point to this as a versatile tool with the bit, each bit corner, and the edges of each burin facet all having functional utility in grooving, engraving, boring, planing, and scraping tasks on medium to hard materials (especially bone, antler, and wood). Knecht (1988) offers a useful study of burins as well as a review of past studies. 
TABLE 16-37

Attributes of Bifacial Perforator Group 7

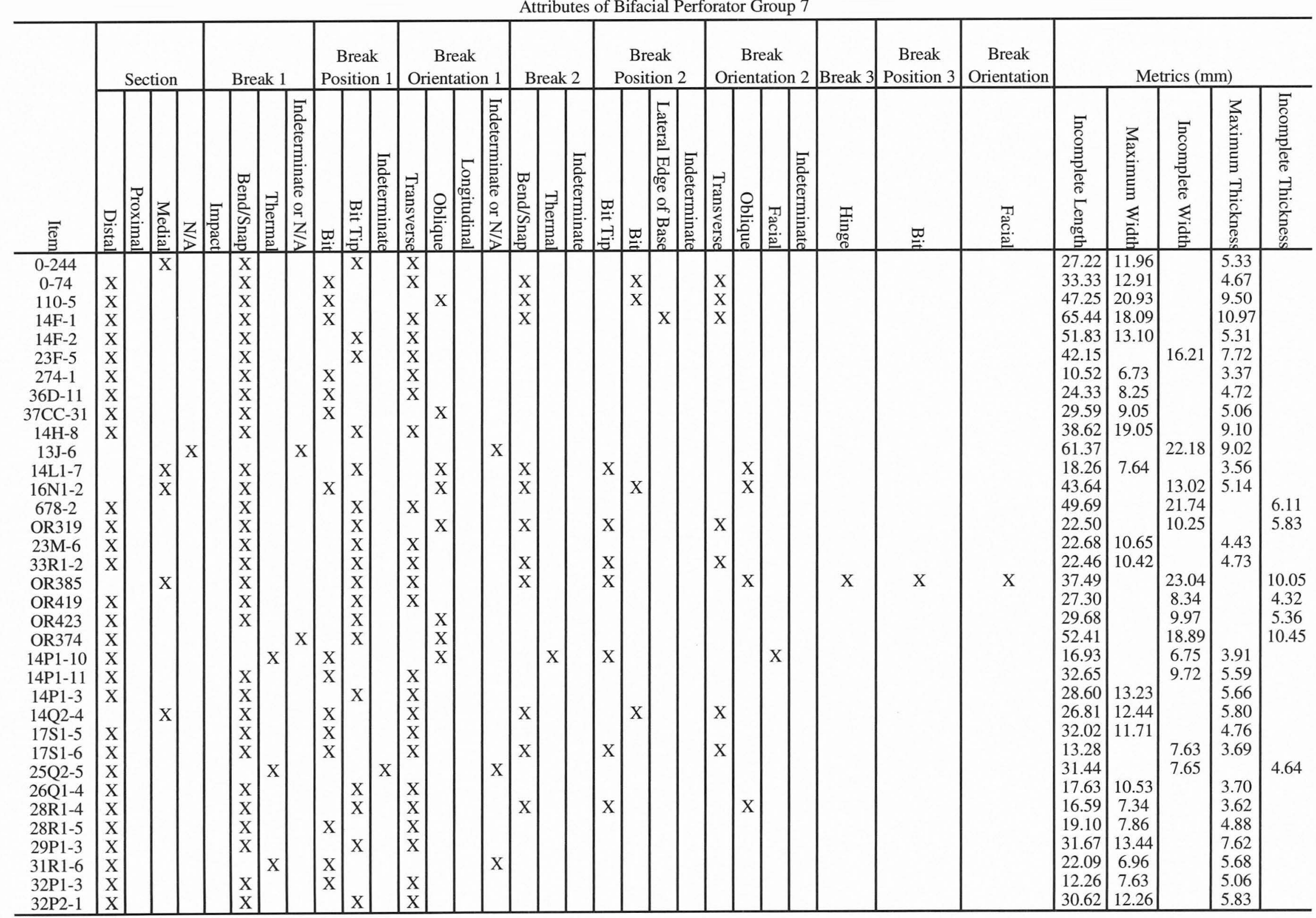




\begin{tabular}{|c|c|c|c|c|c|c|c|c|c|c|c|c|c|c|c|c|c|c|c|c|c|c|c|c|c|c|c|c|c|c|}
\hline \multirow[b]{2}{*}{$\overrightarrow{9}$} & \multicolumn{3}{|c|}{ Section } & \multicolumn{4}{|c|}{ Break 1} & \multicolumn{2}{|c|}{$\begin{array}{c}\text { Break } \\
\text { Position } 1\end{array}$} & \multicolumn{4}{|c|}{$\begin{array}{c}\text { Break } \\
\text { Orientation } 1\end{array}$} & \multicolumn{2}{|c|}{ Break 2} & \multicolumn{3}{|c|}{$\begin{array}{c}\text { Break } \\
\text { Position } 2 \\
\end{array}$} & \multicolumn{4}{|c|}{$\begin{array}{c}\text { Break } \\
\text { Orientation } 2 \\
\end{array}$} & \multirow[b]{2}{*}{ 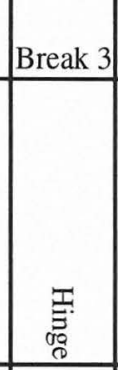 } & \multirow{2}{*}{$\begin{array}{c}\text { Break } \\
\text { Position } 3 \\
\\
\\
\stackrel{\sigma}{=}\end{array}$} & \multirow{2}{*}{\begin{tabular}{c|c}
$\begin{array}{c}\text { Break } \\
\text { Orientation }\end{array}$ \\
\\
\end{tabular}} & \multicolumn{5}{|c|}{ Metrics (mm) } \\
\hline & 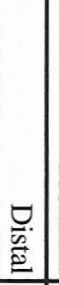 & 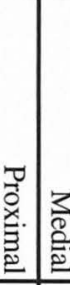 & & z) & 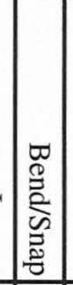 & 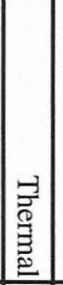 & 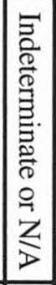 & & 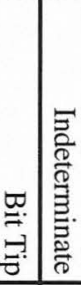 & 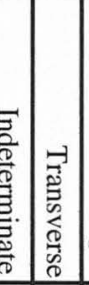 & 。 & 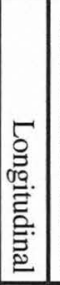 & 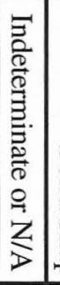 & 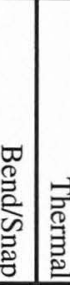 & 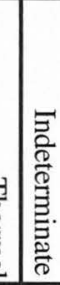 & 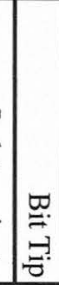 & 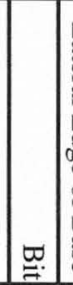 & 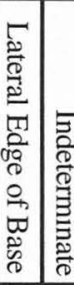 & 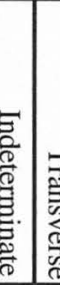 & 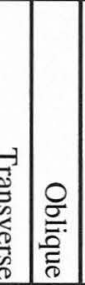 & 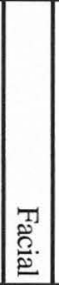 & 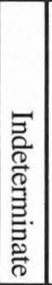 & & & & 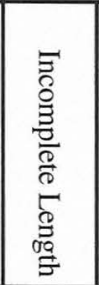 & 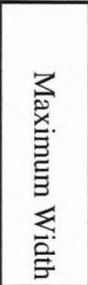 & 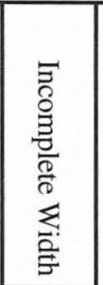 & 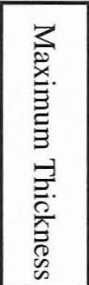 & 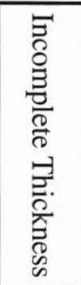 \\
\hline $33 R 2-2$ & $\mathrm{X}$ & & & & $\mathrm{X}$ & & & $\mathrm{X}$ & & $\mathrm{X}$ & & & & $\bar{x}$ & & & $x$ & & & \begin{tabular}{|l|}
$\mathrm{X}$ \\
\end{tabular} & & & & & & 41.40 & 14.40 & & 8.17 & \\
\hline $35 \mathrm{R} 2-28$ & $\mathrm{x}$ & & & & & & $x$ & $\mathrm{x}$ & & & & $\mathrm{x}$ & & & & & & & & & & & & & & 23.89 & 13.01 & & 5.91 & \\
\hline $\begin{array}{r}36 \mathrm{~N}-26 \\
37 \mathrm{P} 1 \mathrm{~A}-2\end{array}$ & $\mathrm{x}$ & $\mathrm{x}$ & & & $\begin{array}{l}\mathrm{X} \\
\mathrm{y}\end{array}$ & & & & $\mathrm{X}$ & $\begin{array}{l}\mathrm{X} \\
\mathrm{X}\end{array}$ & & & & & & & & & & & & & & & & 14.56 & & 9.61 & 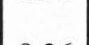 & 4.98 \\
\hline 37Q1D-7 & $\mathrm{x}$ & & & $X$ & & & & $\mathrm{X}$ & & $\hat{x}$ & & & & $\mathrm{X}$ & & & $x$ & & & & & & & & & $\left|\begin{array}{l}53.99 \\
47.80\end{array}\right|$ & 16.66 & 1300 & 9.36 & 720 \\
\hline $710-5$ & $\mathrm{x}$ & & & & & & $\mathrm{x}$ & $\mathrm{x}$ & & & & & $\mathrm{x}$ & & $\mathrm{X}$ & $\mathrm{x}$ & & & & & & $\mathrm{x}$ & & & & 14.29 & & $7.51 \mid$ & 3.17 & 1.20 \\
\hline OR339 & & $X$ & $x$ & & $\mathrm{x}$ & & & & $\mathrm{X}$ & $x$ & $\mathrm{x}$ & & & $x$ & & & & $\mathrm{x}$ & $\mathrm{x} \mid \mathrm{X}$ & $\mathrm{x}$ & & & & & & 41.07 & & 18.27 & & 9.44 \\
\hline 52PC-11 & $\mathrm{X}$ & X & $\mathrm{X}$ & & $\begin{array}{l}\mathrm{X} \\
\mathrm{x}\end{array}$ & & & & & \begin{tabular}{|l}
$\mathrm{x}$ \\
$\mathrm{y}$
\end{tabular} & & & & $\mathrm{X}$ & & & $x$ & & & & & & & & & 30.84 & 18.04 & & 7.15 & \\
\hline $\begin{array}{l}\text { 13Q-6 } \\
28 \text { W1B-3 }\end{array}$ & $\begin{array}{l}A \\
X\end{array}$ & & & & $\begin{array}{l}x \\
x\end{array}$ & & & & $\begin{array}{l}\mathrm{X} \\
\mathrm{X}\end{array}$ & & $x$ & & & & & & & & & & & & & & & \begin{tabular}{|l|}
28.38 \\
18.99
\end{tabular} & $\begin{array}{c}14.77 \\
776\end{array}$ & & \begin{tabular}{|l|}
6.93 \\
4.27
\end{tabular} & \\
\hline $37 \mathrm{X} 2 \mathrm{C}-3$ & $\mathrm{x}$ & & & & $\mathrm{x}$ & & & & $\mathrm{x}$ & $\mathrm{x}$ & & & & & & & & & & & & & & & & $\begin{array}{l}10.99 \\
22.42\end{array}$ & $\begin{array}{l}1.10 \\
10.58\end{array}$ & & \begin{tabular}{|l|}
4.22 \\
4.35
\end{tabular} & \\
\hline 14U2-5 & $\mathrm{X}$ & & & & $\mathrm{X}$ & & & & $\mathrm{X}$ & $\mathrm{X}$ & & & & & & & & & & & & & & & & 56.36 & 14.30 & & 4.36 & \\
\hline $50 \mathrm{~EB}-6$ & $\mathrm{x}$ & & & & $\mathrm{x}$ & & & & $\mathrm{X}$ & & $\mathrm{x}$ & & & & & & & & & & & & & & & 42.51 & & 17.07 & 6.73 & \\
\hline 51PA-3 & & $x$ & & & $\mathrm{X}$ & & & & $X$ & $\mathrm{x}$ & & & & $\mathrm{X}$ & & $X$ & & & & $\mathrm{x}$ & & & & & & 24.76 & 12.74 & & 4.80 & \\
\hline 50AA2B-1 & $\begin{array}{l}\mathrm{X} \\
\mathrm{x}\end{array}$ & & & & $\mathrm{x}$ & & & & $\mathrm{x}$ & & $\mathrm{x}$ & & & $\mathrm{X}$ & & $\mathrm{X}$ & & & & $\mathrm{x}$ & & & & & & 28.73 & 11.65 & & 4.80 & \\
\hline $\begin{array}{c}1349-8 \\
6 \mathrm{E}-4\end{array}$ & $\begin{array}{l}X \\
X\end{array}$ & & & & $\mathrm{x}$ & & $\mathrm{X}$ & $\mathrm{x}$ & $\mathrm{x}$ & & $\mathrm{x}$ & & $\mathrm{X}$ & & & & & & & & & & & & & $\begin{array}{l}20.76 \\
36.93\end{array}$ & 1012 & 8.57 & 663 & 3.91 \\
\hline $5 \mathrm{~A}-24$ & $\mathrm{x}$ & & & & $\mathrm{X}$ & & & & $\mathrm{X}$ & $\mathrm{x}$ & & & & & & & & & & & & & & & & 38.76 & $\begin{array}{l}10.12 \\
15.19\end{array}$ & & $\left|\begin{array}{l}0.03 \\
5.61\end{array}\right|$ & \\
\hline $6 \mathrm{~A}-19$ & $\mathrm{X}$ & & & & $\mathrm{X}$ & & & $\mathrm{x}$ & & & $\mathrm{X}$ & & & $\mathrm{X}$ & & & $\mathrm{X}$ & & & & & & & & & 15.92 & 9.48 & & 4.80 & \\
\hline $8 \mathrm{I}-16$ & $\mathrm{x}$ & & & & $\mathrm{x}$ & & & & $\mathrm{X}$ & & $\mathrm{X}$ & & & & & & & & & & & & & & & 36.88 & & 12.47 & & 16.97 \\
\hline $9 \mathrm{~K}-4$ & $\mathrm{X}$ & & & & $\mathrm{X}$ & & & & $\mathrm{X}$ & $\mathrm{X}$ & & & & & & & & & & & & & & & & 15.26 & & 11.55 & & 4.28 \\
\hline $1 \mathrm{I}-3$ & & $x$ & & & $\mathrm{X}$ & & & & $\mathrm{X}$ & $\mathrm{x}$ & & & & $\mathrm{X}$ & & $\mathrm{x}$ & & & & $\mathrm{x}$ & & & & & & 15.61 & 7.55 & & 5.38 & \\
\hline $\begin{array}{c}19 \mathrm{M}-5 \\
5 \mathrm{~L}-2\end{array}$ & $X$ & $x$ & $x$ & & $\begin{array}{l}\mathrm{X} \\
\mathrm{X}\end{array}$ & & & & $\begin{array}{l}x \\
x\end{array}$ & $x$ & \begin{tabular}{|l|}
$X$ \\
$X$
\end{tabular} & & & & $x$ & & & & $x$ & $x$ & & & & & & $\begin{array}{l}44.31 \\
3850\end{array}$ & 15.07 & 21,41 & 8.71 & \\
\hline $22 \mathrm{O}-11$ & & $\mathrm{x}$ & & & $\mathrm{x}$ & & & $\mathrm{X}$ & & $\mathrm{x}$ & & & & $\mathrm{X}$ & & & $\mathrm{X}$ & & $x$ & $\mathrm{x}$ & & & & & & 22.51 & 11.45 & & 5.33 & \\
\hline $22 \mathrm{O}-12$ & $\mathrm{x}$ & & & & & $\mathrm{x}$ & & $\mathrm{x}$ & & & $\mathrm{X}$ & & & & & & & & & & & & & & & & 8.67 & & 7.36 & \\
\hline $10 \mathrm{~N}-3$ & $\mathrm{X}$ & & & & $\mathrm{x}$ & & & & $\mathrm{X}$ & & $\mathrm{X}$ & & & & & & & & & & & & & & & & 18.10 & & 5.47 & \\
\hline $10 \mathrm{O}-2$ & $\underset{\mathrm{y}}{\mathrm{X}}$ & & & & $\underset{\mathrm{X}}{\mathrm{X}}$ & & & & $\mathrm{X}$ & $\mathrm{X}$ & & & & & & & & & & & & & & & & & 7.59 & & 4.79 & \\
\hline 18TB-2 & $\mathrm{X}$ & & & & $\mathrm{X}$ & & & $\mathrm{X}$ & & $\mathrm{X}$ & & & & & & & & & & & & & & & & 29.12 & 8.81 & & 4.94 & \\
\hline & $\mathrm{X}$ & & & & $\mathrm{X}$ & & & & $\mathrm{X}$ & $\mathrm{X}$ & $\mathrm{Yl}$ & & & & & & & & & & & & & & & 35.52 & 10.21 & & 4.82 & \\
\hline $\begin{array}{l}19 S-9 \\
20 S-8\end{array}$ & $\begin{array}{l}X \\
X\end{array}$ & & & & $\begin{array}{l}X \\
X\end{array}$ & & & & $\begin{array}{l}X \\
X\end{array}$ & $x$ & $X$ & & & & & & & & & & & & & & & $\begin{array}{l}49.30 \\
30.87\end{array}$ & $\begin{array}{l}17.75 \\
10.11\end{array}$ & & \begin{tabular}{|l|}
6.25 \\
5.52
\end{tabular} & \\
\hline
\end{tabular}


TABLE 16-38

Material Characteristics of Unifacial Perforator Group $8(\mathrm{~N}=20)$

\begin{tabular}{|c|c|c|c|c|c|c|c|c|c|c|c|}
\hline & \multirow[b]{2}{*}{ Unit } & \multirow[b]{2}{*}{ Item } & \multicolumn{2}{|c|}{ Patination } & \multicolumn{2}{|c|}{$\begin{array}{c}\text { Mineral } \\
\text { Precipitates } \\
\end{array}$} & \multicolumn{3}{|c|}{ Heat Alteration } & \multicolumn{2}{|c|}{ Raw Material } \\
\hline & & & $\begin{array}{l}\text { 苞 } \\
\text { है } \\
\text { 逢 }\end{array}$ & 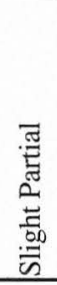 & 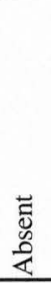 & 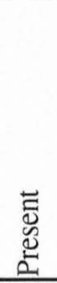 & 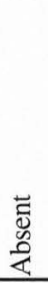 & 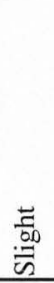 & 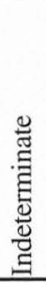 & 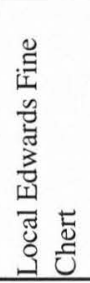 & 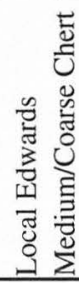 \\
\hline na & Surface & $0-225$ & $\mathrm{X}$ & & $\mathrm{X}$ & & $\mathrm{X}$ & & & $\mathrm{X}$ & \\
\hline Valley Floor A: & $\begin{array}{c}\text { IIIc/FILL } \\
\text { IIIc } \\
\text { IIIa/b } \\
\text { IIIa } \\
\text { IIIa } \\
\text { IIIa } \\
\text { IIIa } \\
\text { IIIa } \\
\text { IIIa } \\
\text { IIIa } \\
\text { II/IIIa } \\
\text { II/IIIa } \\
\text { II/IIIa } \\
\text { II/IIIa } \\
\text { II/IIIa } \\
\end{array}$ & \begin{tabular}{|l}
$185-11$ \\
$37 \mathrm{EC}-25$ \\
$36 \mathrm{~L}-4$ \\
$14 \mathrm{Q} 1-3$ \\
$36 \mathrm{O}-13$ \\
$31 \mathrm{R} 1-11$ \\
$15 \mathrm{R} 2-8$ \\
$28 \mathrm{P} 2-8$ \\
$28 \mathrm{Q} 2-10$ \\
$35 \mathrm{Q} 2-2$ \\
$12 \mathrm{R}-8$ \\
OR473 \\
$1830-2$ \\
$30 \mathrm{~T} 1 \mathrm{C}-1$ \\
$2008-1$ \\
\end{tabular} & $\begin{array}{l}X \\
X \\
X \\
X \\
X \\
X \\
X \\
X \\
X \\
X \\
X \\
X \\
X\end{array}$ & X & $\begin{array}{l}X \\
X \\
X \\
X \\
X \\
X \\
X \\
X \\
X \\
X \\
X \\
X \\
X\end{array}$ & $\mathrm{X}$ & $\begin{array}{l}X \\
X \\
X \\
X \\
X \\
X \\
X \\
X \\
X \\
X \\
X \\
X \\
X\end{array}$ & $\mathrm{X}$ & $\mathrm{X}$ & $\begin{array}{l}\mathrm{X} \\
X \\
X \\
X \\
X \\
X \\
X \\
X \\
X \\
X \\
X \\
X \\
X \\
X \\
X \\
\end{array}$ & \\
\hline Valley Margin: & $\begin{array}{l}\text { IIIc } \\
\text { Y/IIIc } \\
\text { IIIb/c } \\
\text { II/IIIa }\end{array}$ & $\begin{array}{l}4 \mathrm{~B}-10 \\
18 \mathrm{~J}-11 \\
21 \mathrm{I}-14 \\
22 \mathrm{P}-33\end{array}$ & $\begin{array}{l}X \\
X \\
X \\
X\end{array}$ & & $\begin{array}{l}X \\
X \\
X \\
X\end{array}$ & & $\begin{array}{l}X \\
X \\
X \\
X\end{array}$ & & & $\mathrm{X}$ & $\begin{array}{l}X \\
X\end{array}$ \\
\hline
\end{tabular}

Eight descriptive groups are recognized among the 126 burin specimens in the collections from Wilson-Leonard. These groups are based primarily on the number and form of burins per piece. The blanks upon which these various kinds of burins were made are described but not thought to be particularly important in either the archeological classification or in the prehistoric functions of these tools.

It is important to note that the count of 126 items for this category refers to the pieces upon which burins were made, some of which have one, two, three, or more burins on a single blank. Attributes noted in this study include provenience, kind of blank, bit thickness, raw material, patination, and heat alteration as reported in the accompanying tables as well as an estimate of bit angle and observations on the nature of the burin facets discussed in the text. In the conceptual ideal, each burin facet consists of a single plane formed by a single burin blow; in reality, many have been formed by more than one blow and the facet actually consists of two or more facets, a pattern referred to as carination in the Old World terminology (e.g., Demars and Laurent 1989). Also, refurbishing of a burin is often evidenced by stepped facet terminations where successive detachments were progressively shorter (these are denoted as primary, secondary, tertiary, etc). Bit angle is an estimate of the angle at which the two main burin facet planes intersect. This cannot be measured to great precision in many cases because of such vagaries as curvature of one or both of the facet planes or a cant to, or twist in, one or both planes. These characteristics are described below to the extent that they can be determined.

Each burin, whether or not other burins are present on the same blank, potentially affords a dihedral bit formed at the intersecion of the burin facets, two trihedral points at the corners of the bit, and dihedral edges along both margins of each facet. There is undoubtedly much potential to a comprehensive and systematic study of the evidence for use on these various aspects of burins from Wilson-Leonard, an effort beyond the capabilities of the present project. Eleven specimens have been subjected to microscopic examination. Only two were tested for chemical residues.

\section{Group $1(\mathbf{N}=20)$}

This group consists of 20 pieces, each with a single burin formed by the intersection of two burin facets, a type known as dihedral burins (Figure 16-25a-c; Table 16-40). There are 5 made on flakes, 9 on bifaces, 5 on unifaces, and 1 on a large burin spall. These flakes are mostly large and 
TABLE 16-39

Attributes of Unifacial Perforator Group 8

\begin{tabular}{|c|c|c|c|c|c|c|c|c|c|c|c|c|c|c|c|c|c|}
\hline \multirow[b]{2}{*}{ Item } & \multicolumn{3}{|c|}{ Section } & \multicolumn{3}{|c|}{ Break } & \multicolumn{3}{|c|}{ Break Position } & \multicolumn{3}{|c|}{ Break Orientation } & \multicolumn{5}{|c|}{ Metrics (mm) } \\
\hline & 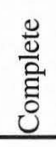 & 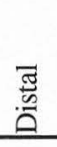 & 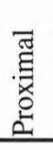 & 气̆ & $\begin{array}{l}\text { 迆 } \\
\text { 家 }\end{array}$ & 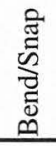 & ¿ัँ & $\vec{p}$ & $\begin{array}{l}\overrightarrow{2} \\
\vec{p} \\
\vec{p}\end{array}$ & 气̆ & 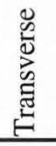 & 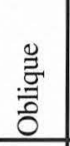 & 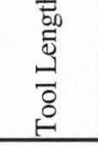 & 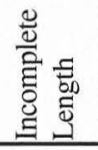 & 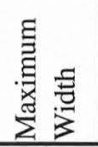 & 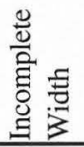 & 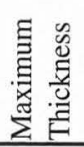 \\
\hline $0-225$ & & $\mathrm{X}$ & & & & $\mathrm{X}$ & & & $\mathrm{X}$ & & $\mathrm{X}$ & & & 26.63 & & 9.27 & 7.13 \\
\hline $185-11$ & $\mathrm{X}$ & & & $\mathrm{X}$ & & & $\mathrm{X}$ & & & $\mathrm{X}$ & & & 36.64 & & 32.82 & & 7.66 \\
\hline $37 \mathrm{EC}-25$ & $\mathrm{X}$ & & & $\mathrm{X}$ & & & $\mathrm{x}$ & & & $\mathrm{X}$ & & & 52.25 & & 23.56 & & 6.84 \\
\hline $36 \mathrm{~L}-4$ & $\mathrm{X}$ & & & $\mathrm{X}$ & & & $\mathrm{X}$ & & & $\mathrm{X}$ & & & 68.77 & & 38.44 & & 18.19 \\
\hline $14 \mathrm{Q} 1-3$ & $\mathrm{X}$ & & & $\mathrm{X}$ & & & $\mathrm{x}$ & & & $\mathrm{X}$ & & & 43.72 & & 23.2 & & 3.58 \\
\hline $360-13$ & $\mathrm{X}$ & & & $\mathrm{X}$ & & & $\mathrm{X}$ & & & $\mathrm{X}$ & & & 41.43 & & 18.74 & & 3.93 \\
\hline 31R1-11 & & & $\mathrm{x}$ & & $\mathrm{x}$ & & & & $\mathrm{x}$ & & $\mathrm{x}$ & & & 28.5 & 21 & & 3.8 \\
\hline $15 \mathrm{R} 2-8$ & & $\mathrm{X}$ & & & & $\mathrm{X}$ & & & $\mathrm{X}$ & & & $\mathrm{X}$ & & 28.87 & & 9.05 & 3.91 \\
\hline $28 \mathrm{P} 2-8$ & $\mathrm{X}$ & & & $\mathrm{X}$ & & & $\mathrm{X}$ & & & $\mathrm{x}$ & & & 48.76 & & 29.03 & & 7.22 \\
\hline 28Q2-10 & & $\mathrm{X}$ & & $\mathrm{X}$ & & & $\mathrm{X}$ & & & $\mathrm{X}$ & & & 28.05 & & 25.2 & & 3.64 \\
\hline $35 \mathrm{Q} 2-2$ & & $\mathrm{X}$ & & & & $\mathrm{X}$ & & & $\mathrm{X}$ & & $\mathrm{X}$ & & & 17.41 & 8.19 & & 4.01 \\
\hline $12 \mathrm{R}-8$ & $\mathrm{X}$ & & & $\mathrm{X}$ & & & $\mathrm{X}$ & & & $\mathrm{X}$ & & & 49.38 & & 36.49 & & 14.71 \\
\hline OR473 & $\mathrm{X}$ & & & $\mathrm{X}$ & & & $\mathrm{X}$ & & & $\mathrm{X}$ & & & 39.36 & & 35.25 & & 6.36 \\
\hline $1830-2$ & & $\mathrm{X}$ & & & & $\mathrm{X}$ & & $\mathrm{X}$ & & & $\mathrm{X}$ & & & 11.6 & & 5.7 & 2 \\
\hline $30 \mathrm{~T} 1 \mathrm{C}-1$ & $\mathrm{X}$ & & & $\mathrm{X}$ & & & $\mathrm{X}$ & & & $\mathrm{X}$ & & & 33.8 & & 29.35 & & 8.64 \\
\hline $2008-1$ & & $\mathrm{X}$ & & & & $\mathrm{X}$ & & & $\mathrm{X}$ & & $\mathrm{X}$ & & & 28.62 & 14.08 & & 9.81 \\
\hline $18 \mathrm{~J}-11$ & $\mathrm{X}$ & & & $\mathrm{X}$ & & & $\mathrm{x}$ & & & $\mathrm{X}$ & & & 37 & & 34.4 & & 8 \\
\hline $21 \mathrm{I}-14$ & $\mathrm{X}$ & & & $\mathrm{X}$ & & & $\mathrm{x}$ & & & $\mathrm{X}$ & & & 59.29 & & 26.62 & & 9.82 \\
\hline $4 \mathrm{~B}-10$ & $\mathrm{X}$ & & & & & $\mathrm{X}$ & & $\mathrm{X}$ & & & $\mathrm{X}$ & & & 36.12 & 26.72 & & 6.86 \\
\hline $22 \mathrm{P}-33$ & $\mathrm{X}$ & & & $\mathrm{X}$ & & & $\mathrm{X}$ & & & $X$ & & & 42.06 & & 23.15 & & 6.25 \\
\hline
\end{tabular}

thick, the unifaces are also thick and most have cortex, and the bifaces are fragments from early stages of biface manufacture. The burin facets on 3 pieces are both primary. On 6,1 facet is primary, and the companion facet has been refubished at least once. Ten specimens consist of a carinated facet intersecting a single facet, and 1 specimen is formed by the juncture of 2 carinated facets. Bit thicknesses range from 3.4 to $19.3 \mathrm{~mm}$, averaging over $8 \mathrm{~mm}$. Bit angles range from approximately $50^{\circ}$ to $90^{\circ}$, averaging close to $72^{\circ}$. Four Group 1 burins were examined by Kay for microscopic use wear; on 2 , he reports negative findings and on another 2, his findings were indeterminate (see Chapter 22).

\section{Group $2(\mathrm{~N}=6)$}

Six specimens, each with two dihedral burins on a single blank, form this category (Figure 16-25d; Table 1641). Three of these are made on flakes, two on bifaces, and one on a uniface. One biface blank is thin, the other is thick; two of the flake blanks are thin and one is thick; the unifacial blank is moderately thick. Both bits on four pieces are formed by single faceted burin blows, and two pieces each have one burin bit with one carinated facet. Bit thicknesses range from 3.1 to $11.5 \mathrm{~mm}$ and average 6.6 $\mathrm{mm}$. Burin angles range from near $55^{\circ}$ to near $100^{\circ}$ and average about $71^{\circ}$.

\section{Group 3(N=71)}

Specimens in this group each have a single burin formed by a single burin facet originating at a fracture plane on the parent piece (Figures 16-25e-f, 16-26a, b; 16-27a-c; Table 1642). Eighteen are on flakes, 41 on bifaces, and 10 on unifaces; 1 is made on a core fragment and another on an indeterminate blank. Blanks are highly variable, ranging from thin to thick and being with or without cortex. Bit angles range from $48^{\circ}$ to $96^{\circ}$, averaging $73^{\circ}$. Bit thicknesses vary between 2.2 and 15.6 $\mathrm{mm}$, averaging $6.0 \mathrm{~mm}$. Of the 71 burins, 23 show evidence of having been resharpened. Twelve are carinated. Hurst (see Chapter 23) found chloride and carbonate to be present on the one burin he examined from this group; he interprets these to derive from natural geologic or pedogenic sources. Kay examined five of these burins, finding no evidence for use on three and indications of use on medium to hard materials on two (see Chapter 22).

\section{Group 4(N=10)}

This small group consists of pieces, each with two burins formed by the juncture of a burin facet with a broken edge (Figure 16-27d; Table 16-43). Most are on fairly thick and often cortical blanks of which 4 are flakes, 3 are bifaces, 1 is a uniface, 1 is a core fragment, and 1 is indeterminate. Six of the 10 have 1 burin with evidence for resharpening, 2 have 


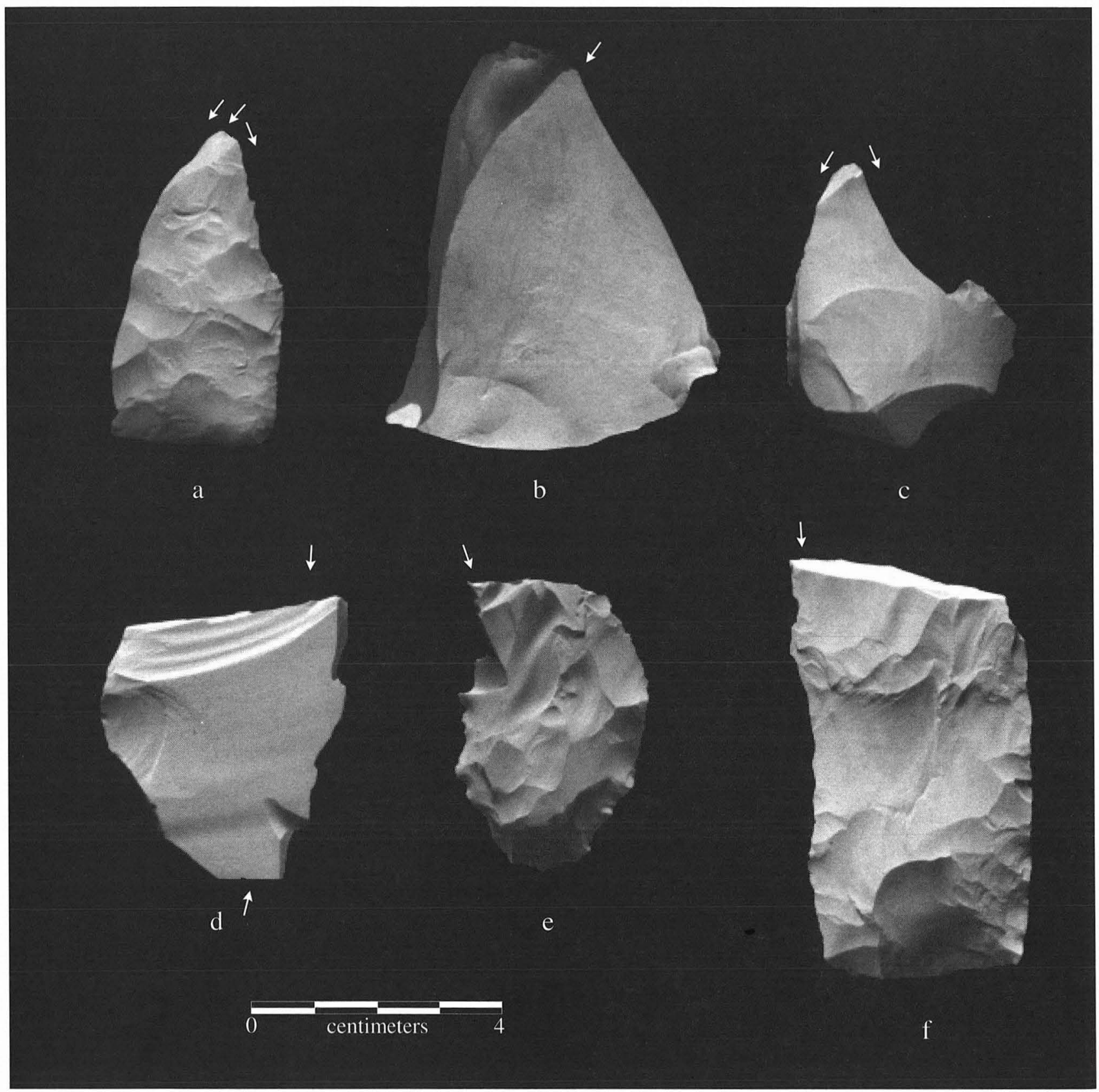

FIGURE 16-25. Representative burins. (a-c) Group 1; (d) Group 2; (e, f) Group 3. Specimen numbers: (a) 0-142; (b) 31R1-4; (c) 24V1D2 ; (d) 8D-4; (e) 701-4; (f) 11S-2.

similar evidence for both burins, and the burins on 2 show no evidence of resharpening. None is carinated. Bit thicknesses range from 2.8 to $15.7 \mathrm{~mm}$ and average $6.6 \mathrm{~mm}$. Bit angles range from $55^{\circ}$ to $110^{\circ}$ and average $76^{\circ}$. The single burin in this group examined by Kay yielded indeterminate evidence for use (see Chapter 22).

\section{$\operatorname{Group} 5(\mathrm{~N}=5)$}

Burins struck from steeply chipped edges, interpreted as truncations, comprise this group (Table 16-44). Each speci- men has a single burin. Blanks consist of two flakes, two bifaces, and a uniface. One flake is large and irregular and the other is long and blade-like. The bifaces are thin. The uniface is long and blake-like. Bit thicknesses range from 2.1 to $6.3 \mathrm{~mm}$, averaging $4.4 \mathrm{~mm}$. Bit angles range from $50^{\circ}$ to $96^{\circ}$ and average $75^{\circ}$.

\section{Group 6(N=1)}

The single specimen in this group consists of a large flake with a deeply concave truncation chipped into one end 
TABLE $16-40$

Material Characteristics and Attributes of Burin Group $1(\mathrm{~N}=20)$

\begin{tabular}{|c|c|c|c|c|c|c|c|c|c|c|c|}
\hline \multirow[b]{2}{*}{ Item } & \multirow[b]{2}{*}{ Unit } & \multirow[b]{2}{*}{ Cultural Period } & \multicolumn{4}{|c|}{ Blank } & \multirow{2}{*}{\begin{tabular}{|c} 
Thickness \\
Bit \\
\end{tabular}} & \multirow{2}{*}{$\begin{array}{c}\text { Raw } \\
\text { Material } \\
\text { Local } \\
\text { Edwards } \\
\text { Fine Chert } \\
\end{array}$} & \multicolumn{2}{|c|}{ Patina } & \multirow{2}{*}{$\begin{array}{c}\text { Heat } \\
\text { Alteration } \\
\text { Absent }\end{array}$} \\
\hline & & & Flake & Biface & Uniface & Other & & & Present & Absent & \\
\hline $\begin{array}{l}\text { Surface: } \\
0-142 \\
\end{array}$ & Surface & Unknown & & $\mathrm{X}$ & & & 7.8 & $\mathrm{X}$ & & $\mathrm{X}$ & $X$ \\
\hline \multicolumn{2}{|c|}{ Valley Floor A: } & & & & & & & & & & \\
\hline $208-1$ & IIIc/fill & Unassigned & & $\mathrm{X}$ & & & 8.5 & $\mathrm{X}$ & & $\mathrm{X}$ & $\mathrm{X}$ \\
\hline 37DA-7 & IIIc & Late Archaic & & & $\mathrm{X}$ & & 7.4 & $\mathrm{X}$ & & $\mathrm{X}$ & $\mathrm{X}$ \\
\hline 37DD-32 & IIIC & Late Archaic & & $\mathrm{X}$ & & & 7.6 & $\mathrm{X}$ & & $\mathrm{X}$ & \\
\hline $387-2$ & IIIc & Late Archaic & $\mathrm{X}$ & & & & 7.7 & $\mathrm{X}$ & & $\mathrm{X}$ & $\mathrm{X}$ \\
\hline $16 \mathrm{~N} 1-1$ & IIIb & Early Archaic & & $\mathrm{X}$ & & & 10.1 & $\mathrm{X}$ & $\mathrm{X}$ & & $\mathrm{X}$ \\
\hline $593-9$ & IIIa/b & Middle/Early Archaic & $\mathrm{X}$ & & & & 3.4 & $\mathrm{X}$ & & & $X$ \\
\hline 871-1 & IIIa/b & Early Archaic & & & & $\mathrm{X}$ & 9.4 & $\mathrm{X}$ & & $X$ & \\
\hline $31 \mathrm{R} 1-4$ & IIIa & Early Archaic & $\mathrm{X}$ & & & & 19.3 & $\mathrm{X}$ & & $\mathrm{X}$ & $\mathrm{X}$ \\
\hline $1199-1$ & II/IIIa & Early Archaic/Late Paleoindian & & & $\mathrm{X}$ & & 11.4 & $\mathrm{X}$ & & $\mathrm{X}$ & $\mathrm{X}$ \\
\hline $24 \mathrm{R} 2-5$ & II/IIIa & Early Archaic/Late Paleoindian & & $\mathrm{X}$ & & & 9.2 & $\mathrm{X}$ & & $\mathrm{X}$ & $\mathrm{X}$ \\
\hline 37S1C-4 & II/IIIa & Early Archaic/Late Paleoindian & & $\mathrm{X}$ & & & 5.9 & $\mathrm{X}$ & & $\mathrm{X}$ & $\mathrm{X}$ \\
\hline 24V1D-2 & II & Late Paleoindian & $\mathrm{X}$ & & & & 9.1 & $\mathrm{X}$ & & $\mathrm{X}$ & $\mathrm{X}$ \\
\hline \multicolumn{2}{|c|}{ Valley Margin: } & & & & & & & & & & \\
\hline $20 \mathrm{H}-5$ & $\mathrm{IIIb} / \mathrm{c}$ & Middle/Early Archaic & & $\mathrm{X}$ & & & 5.7 & $X$ & & $X$ & $\mathrm{X}$ \\
\hline $20 \mathrm{H}-9$ & IIIIb/c & Middle/Early Archaic & $\mathrm{X}$ & & & & 5.2 & $\mathrm{X}$ & & $\mathrm{X}$ & \\
\hline $11 \mathrm{~L}-13$ & $\mathrm{IIIb}$ & Early Archaic & & $\mathrm{X}$ & & & 4.4 & $\mathrm{X}$ & & $\mathrm{X}$ & \\
\hline $21 \mathrm{~J}-7$ & IIIb & Early Archaic & & & $\mathrm{X}$ & & 10.8 & $\mathrm{X}$ & & $\mathrm{X}$ & $\mathrm{X}$ \\
\hline $3 \mathrm{I}-1$ & $\mathrm{Y}$ & Early Archaic & & $\mathrm{X}$ & & & 10.1 & $\mathrm{X}$ & & $\mathrm{X}$ & $X$ \\
\hline $6 \mathrm{P}-1$ & $\mathrm{X}$ & Early Archaic & & & $\mathrm{X}$ & & 4.0 & $X$ & & $\mathrm{X}$ & $\mathrm{X}$ \\
\hline $18 \mathrm{~N}-8$ & $\mathrm{X}$ & Early Archaic & & & $\mathrm{X}$ & & 5.3 & $\mathrm{X}$ & & $\mathrm{X}$ & $\mathrm{X}$ \\
\hline
\end{tabular}

TABLE 16-41

Material Characteristics and Attributes of Burin Group $2(\mathrm{~N}=6)$

\begin{tabular}{|c|c|c|c|c|c|c|c|c|c|c|c|c|}
\hline \multirow[b]{2}{*}{ Item } & \multirow[b]{2}{*}{ Unit } & \multirow[b]{2}{*}{ Cultural Period } & \multicolumn{3}{|c|}{ Blank } & \multicolumn{2}{|c|}{ Thickness } & \multicolumn{2}{|c|}{ Raw Material } & \multicolumn{2}{|c|}{ Patina } & \multirow{2}{*}{$\begin{array}{c}\text { Heat } \\
\text { Alteration } \\
\\
\text { Absent } \\
\end{array}$} \\
\hline & & & Flake & Biface & Uniface & Bit 1 & Bit 2 & $\begin{array}{c}\text { Local } \\
\text { Edwards } \\
\text { Fine Chert }\end{array}$ & $\begin{array}{l}\text { Nonlocal } \\
\text { Edwards } \\
\text { Fine Chert }\end{array}$ & Present & Absent & \\
\hline \multicolumn{2}{|c|}{ Valley Floor A: } & & & & & & & & & & & \\
\hline $527-3$ & |IIIb & Middle Archaic & $\mathrm{X}$ & & & 3.1 & 4.0 & & $\mathrm{X}$ & $\mathrm{X}$ & & $\mathrm{X}$ \\
\hline 24P2-1 & IIIa & Early Archaic & & $\mathrm{X}$ & & 11.5 & 9.2 & $X$ & & & $\mathrm{X}$ & $X$ \\
\hline OR322 & IIIIa & Early Archaic & & & $X$ & 8.7 & 7.7 & $\mathrm{X}$ & & & $\mathrm{X}$ & $\mathrm{X}$ \\
\hline \multicolumn{2}{|c|}{ Valley Floor B: } & & & & & & & & & & & \\
\hline $51 \mathrm{SA}-1$ & III & Early Archaic & & $\mathrm{X}$ & & 5.5 & 4.8 & $\mathrm{X}$ & & & $\mathrm{X}$ & $\mathrm{X}$ \\
\hline \multicolumn{2}{|c|}{ Valley Margin: } & & & & & & & & & & & \\
\hline $8 \mathrm{D}-4$ & |IIIc & Late Archaic & $\mathrm{X}$ & & & 5.6 & 3.6 & $\mathrm{X}$ & & & $X$ & $\mathrm{X}$ \\
\hline $10 \mathrm{C}-16$ & IIIc & Late Archaic & $\mathrm{X}$ & & & 7.3 & 8.3 & $\mathrm{X}$ & & & $\mathrm{X}$ & $\mathrm{X}$ \\
\hline
\end{tabular}

(Figure 16-27e; Table 16-45). Two burins, one on each edge of the flake, have been struck from this truncation, but the burin facets converge at the opposite end of the flake to form another burin-like bit. The three bit angles are $55^{\circ}, 60^{\circ}$, and $88^{\circ}$; and bit thicknesses are 9.2, 9.0, and $11.6 \mathrm{~mm}$, which average $9.9 \mathrm{~mm}$.

\section{Group $7(\mathrm{~N}=5)$}

Specimens in this group are four fragmentary and indeterminate burin forms and one that was lost after being pre- liminarily classified (Table 16-46). Two of these are on bifaces, and two are on core fragments. Bit thicknesses range from $3.8 \mathrm{~mm}$ to $10.2 \mathrm{~mm}$, averaging about $6 \mathrm{~mm}$. Bit angles range from $80^{\circ}$ to $83^{\circ}$, averaging $82^{\circ}$.

\section{Group $8(\mathrm{~N}=8)$}

All of the items in this group have burin-like bits formed in ways other than by removal of a burin spall, but each one has macroscopic damage and nibbling that suggests the bits were used (Table 16-47). One is a thermal fragment, one 


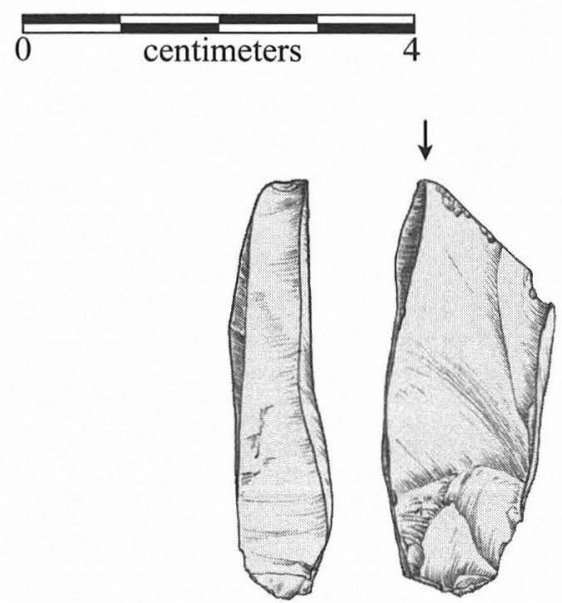

a

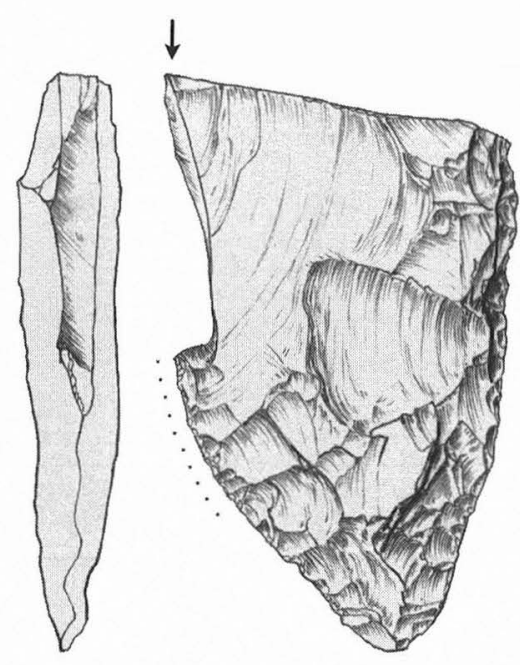

b

FIGURE 16-26. Examples of Group 3 burins. Specimen numbers: (a) 29R2-3; (b) 22J-7.

is formed by the intersection of a break and a thermal fracture, and five are formed by intersecting breaks. The final specimen is an irregular pyramidal-shaped core fragment with two promontories resembling burins, both of which show possible signs of use. The blanks are two thin flakes, four thin bifaces, a thin uniface, and a thick core fragment. "Bit" angles range from $62^{\circ}$ to $87^{\circ}$, averaging $75^{\circ}$; bit thicknesses range from 2.9 to $13.1 \mathrm{~mm}$, averaging $6.2 \mathrm{~mm}$. One of these pseudo burins was examined by Kay and found to have been used in the same fashion as bona fide burins in the working of medium to hard material (see Chapter 22).

\section{Discussion}

For the most part, burins seem to be somewhat expediently produced tools made on diverse pieces of chert. Resharpening occurs commonly in these tools, and in many cases, more than one burin is formed on a single blank. A few specimens show remnants of additional burins that have been removed almost completely by later burin spalls from another direction. All of this suggests that these were used heavily enough to require repeated maintenance. Kay's positive evidence for use-wear matches that seen by others (cf. Knecht 1988) in indicating use of the trihedral points, the dihedral bits, and the dihedral edges in various work modes (see Chapter 23). Kay's negative and inconclusive evidence raises two immediate questions. The first is whether or not all of the burins in this sample were produced intentionally, and the second is whether or not bona fide burins may have been used in light tasks (Kay's indeterminate use-wear category) or perhaps lost or discarded in a freshly made or refurbished state (Kay's negative results category). The former question derives from the ease by which burin-like fractures can occur inadvertently during knapping or during use of stone objects. Cultural affiliations of the WilsonLeonard burins range from late Paleoindian to Late Prehistoric but are most commonly with the Early Archaic.

\section{BURIN SPALLS}

The small prismatic, blade-like flakes produced in forming or resharpening a burin are distinctive and retain some indications of the status of the burin at the time of spall removal. In the Wilson-Leonard assemblage are 299 such pieces. These are tabulated as to spall form and the kind of blank from which they were detached and are accompanied by length and thickness measurements.

The comparatively simple morphology of burin spalls lends itself to a very straightforward scheme of classification and description. In this study, four categories have been recognized in regard to the evidence for prior spall removals on the exterior surface of the spall being classified. Primary spalls have no evidence for prior spall detachments, secondary spalls have evidence for a single prior removal, and so forth.

Group 1, primary spalls, number 128 and since their exterior surfaces represent the edge of the piece from which no previous spall was detached, the blank can often be fairly confidently identified (Figure 16-28a-e; Table 16-48). In this case 25 are on flakes, 40 are on bifaces, 55 are on unifaces, and 8 are indeterminate.

Seventy secondary spalls (Group 2) were recognized (Figure 16-28f-j; Table 16-49). In some of these, the entire exterior surface is the facet of a single previous spall removal whereas in others, the former spall ends short of the spall length and a portion of the original blank edge survives intact. It sometimes happens that removal of a long spall will erase evidence of earlier, shorter spalls such that 


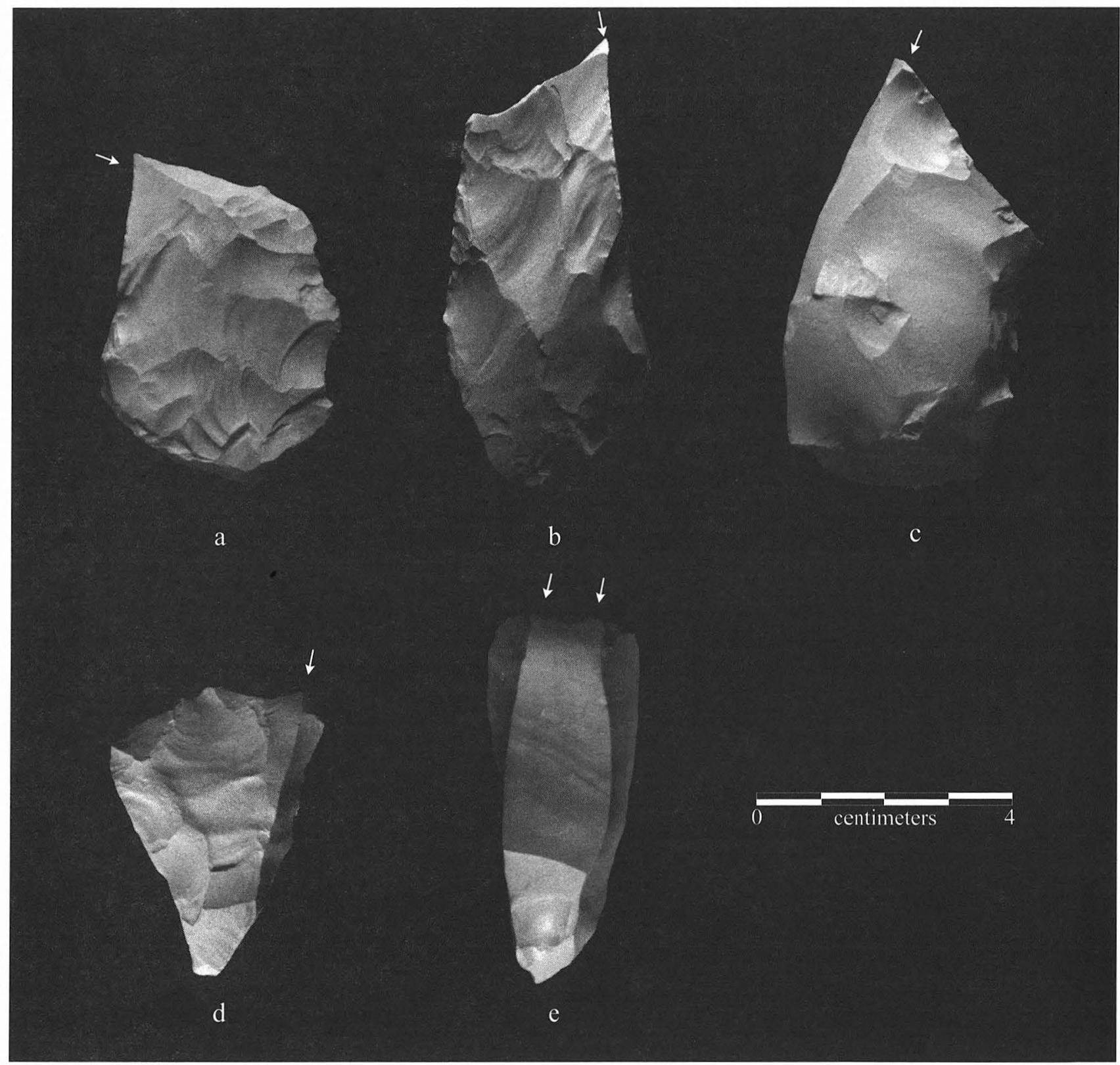

FIGURE 16-27. Representative burins. (a-c) Group 3; (d) Group 4; (e) Group 6. Specimen numbers: (a) 8H-1; (b) 11H-5; (c) 9U-2; (d) 1P3 ; (e) $34 \mathrm{~W} 1 \mathrm{C}-5$.

secondary spalls on which the exterior is completely faceted by a single previous scar could, in fact, be tertiary spalls. In this sample, 16 were produced from flakes, 11 from bifaces, 14 from unifaces, and 29 from indeterminate pieces. One secondary spall tested by Beck (see Chapter 23) retained residues of fatty acids.

There are 31 tertiary spalls (Group 3 ) in this collection (Figure 16-28k-n; Table 16-50). At least 2 previous spall facets are apparent on these; parent pieces were flakes $(n=11)$, bifaces $(n=9)$, unifaces $(n=1)$, and indeterminate $(n=10)$.

Fifty-two fragmentary burin spalls (Group 4) were identified (Figure 16-28o-q; Table 16-51). These were made on 8 flakes, 7 bifaces, 25 unifaces, and 12 indeterminate pieces. In addition, 18 specimens comprise a miscellaneous category of burins spalls for which no further classification was possible (Table 16-52).

Burin spalls, like burins, are presumed to have been intentionally produced, but there are undoubtedly some spalls in this grouping of artifacts that were produced inadvertently during the manufacture or use of diverse chert objects. Among the better lines of evidence supporting the inference that most of these are intentionally knapped pieces is the number (101) of secondary and tertiary spalls that are less likely to have been produced accidently. The number of spalls found in the excavated area of the site also suggests that burins were being made as well as refurbished at the 
TABLE $16-42$

Material Characteristics and Attributes of Burin Group $3(\mathrm{~N}=71)$

\begin{tabular}{|c|c|c|c|c|c|c|c|c|c|c|c|c|c|c|c|c|c|c|c|}
\hline \multirow[b]{2}{*}{ Item } & \multirow[b]{2}{*}{ Unit } & \multirow[b]{2}{*}{ Cultural Period } & \multicolumn{5}{|c|}{ Blank } & \multirow[b]{2}{*}{\begin{tabular}{|c|} 
Thickness \\
\\
\\
Bit \\
\end{tabular}} & \multicolumn{4}{|c|}{ Raw Material } & \multicolumn{2}{|c|}{ Patina } & \multicolumn{5}{|c|}{ Heat Alteration } \\
\hline & & & 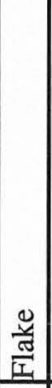 & 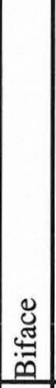 & 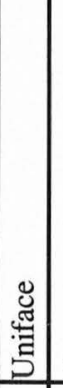 & نे & 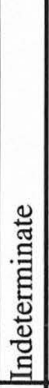 & & 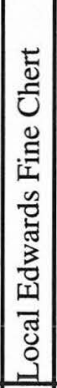 & 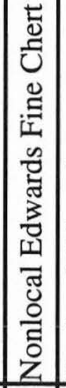 & 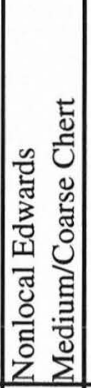 & 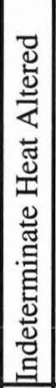 & 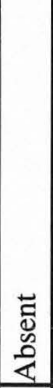 & 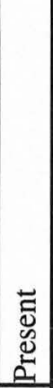 & 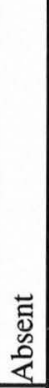 & 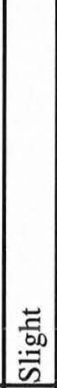 & 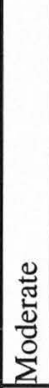 & 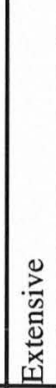 & 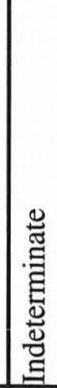 \\
\hline Surface: & & & & & & & & & & & & & & & & & & & \\
\hline $0-18$ & Surface & Cannot be determined & & $\mathrm{x}$ & & & & 4.6 & $\mathrm{x}$ & & & & $\mathrm{x}$ & & $\mathrm{x}$ & & & & \\
\hline $0-28$ & Surface & Cannot be determined & & $\mathrm{x}$ & & & & 5.9 & $\mathrm{x}$ & & & & $\mathrm{X}$ & & & $\mathrm{x}$ & & & \\
\hline $108-2$ & Surface & Unassigned & & $\mathrm{x}$ & & & & 4.5 & $\mathrm{x}$ & & & & $\mathrm{X}$ & & $\mathrm{x}$ & & & & \\
\hline \multicolumn{2}{|c|}{ Valley Floor A: } & & & & & & & & & & & & & & & & & & \\
\hline $208-5$ & IIIc/FILL & Unassigned & $\mathrm{x}$ & & & & & 2.2 & $x$ & & & & $\mathrm{x}$ & & & $\mathrm{x}$ & & & \\
\hline $13 \mathrm{C}-2$ & IIIIc & Late Archaic & & $\mathrm{x}$ & & & & 3.7 & $x$ & & & & $\mathrm{X}$ & & $\mathrm{x}$ & & & & \\
\hline $15 \mathrm{D}-7$ & IIIC & Late Archaic & $\mathrm{x}$ & & & & & 3.8 & $x$ & & & & $\mathrm{X}$ & & $\mathrm{x}$ & & & & \\
\hline $16 \mathrm{D}-1$ & IIIIc & Late Archaic & & & $\mathrm{x}$ & & & 3.4 & $\mathrm{x}$ & & & & $\mathrm{x}$ & & $\mathrm{x}$ & & & & \\
\hline $176-1$ & IIIIc & Late Prehistoric/Late Archaic & & $\mathrm{x}$ & & & & 3.2 & $\mathrm{x}$ & & & & $\mathrm{X}$ & & $\mathrm{x}$ & & & & \\
\hline $231-2$ & IIIIc & Late Archaic & $\mathrm{x}$ & & & & & 6.4 & $\mathrm{x}$ & & & & $\mathrm{x}$ & & $\mathrm{x}$ & & & & \\
\hline $36 \mathrm{D}-3$ & IIIIc & Late Archaic & $\mathrm{x}$ & & & & & 3.4 & $\mathrm{x}$ & & & & $\mathrm{X}$ & & & & & $\mathrm{x}$ & \\
\hline $36 \mathrm{E}-8$ & IIIIc & Late Archaic & & $\mathrm{x}$ & & & & 5.6 & $x$ & & & & $\mathrm{X}$ & & $\mathrm{x}$ & & & & \\
\hline $387-1$ & IIIIc & Late Archaic & & $\mathrm{x}$ & & & & 6 & $\mathrm{x}$ & & & & $\mathrm{x}$ & & $\mathrm{x}$ & & & & \\
\hline $387-3$ & IIIIC & Late Archaic & $\mathrm{x}$ & & & & & 7.8 & $\mathrm{x}$ & & & & $\mathrm{X}$ & & & & & & $\mathrm{x}$ \\
\hline $455-1$ & IIIIc & Late Archaic & & $\mathrm{x}$ & & & & 5.4 & $\mathrm{x}$ & & & & & $x$ & $\mathrm{x}$ & & & & \\
\hline OR99 & IIII & Late Archaic & & $\mathrm{x}$ & & & & 10.8 & $\mathrm{x}$ & & & & $\mathrm{x}$ & & $\mathrm{X}$ & & & & \\
\hline OR113 & IIII & Late Archaic & $\mathrm{x}$ & & & & & 6.9 & $\mathrm{x}$ & & & & & $\mathrm{x}$ & $\mathrm{x}$ & & & & \\
\hline $12 \mathrm{H}-1$ & $\mathrm{IIIb} / \mathrm{c}$ & Middle/Early Archaic & & $\mathrm{x}$ & & & & 3.5 & $\mathrm{x}$ & & & & $\mathrm{x}$ & & & & & $\mathrm{x}$ & \\
\hline $13 \mathrm{H}-2$ & $\mathrm{IIIb} / \mathrm{c}$ & Late/Middle Archaic & & $\mathrm{x}$ & & & & 4.9 & $\mathrm{X} \mid$ & & & & $\mathrm{X}$ & & $\mathrm{X}$ & & & & \\
\hline $15 \mathrm{~J} 1-7$ & $\mathrm{IIIb} / \mathrm{c}$ & Middle/Early Archaic & & & $\mathrm{X}$ & & & 4.7 & $|x|$ & & & & $\mathrm{x}$ & & $\mathrm{X}$ & & & & \\
\hline $16 \mathrm{~J} 2-2$ & $\mathrm{IIIb} / \mathrm{c}$ & Middle/Early Archaic & & $\mathrm{x}$ & & & & 7.5 & $\mathrm{x}$ & & & & $\mathrm{X}$ & & $\mathrm{x}$ & & & & \\
\hline $13 \mathrm{M}-13$ & IIIb & Early Archaic & & $\mathrm{x}$ & & & & 5.4 & & $\mathrm{x}$ & & & $\mathrm{x}$ & & $\mathrm{x}$ & & & & \\
\hline $14 \mathrm{~K} 1-3$ & IIIlb & Early Archaic & & $\mathrm{x}$ & & & & 8.7 & & $\mathrm{x}$ & & & & $\mathrm{x}$ & $\mathrm{X}$ & & & & \\
\hline $14 \mathrm{~L} 2-5$ & IIIb & Early Archaic & & $\mathrm{x}$ & & & & 4.7 & $\mathrm{x}$ & & & & $\mathrm{x}$ & & $\mathrm{X}$ & & & & \\
\hline $15 \mathrm{~J} 2-9$ & IIIlb & Middle/Early Archaic & $\mathrm{x}$ & & & & & 5.4 & $\mathrm{x}$ & & & & $\mathrm{X}$ & & & $x$ & & & \\
\hline 37LB-12 & IIIb & Middle/Early Archaic & & & & $\mathrm{x}$ & & 10.4 & $\mathrm{x}$ & & & & $x$ & & $\mathrm{x}$ & & & & \\
\hline 37LB-15 & IIIlb & Middle/Early Archaic & & & $\mathrm{x}$ & & & 6.8 & $\mathrm{x}$ & & & & $\mathrm{X}$ & & $\mathrm{X}$ & & & & \\
\hline $701-4$ & IIIb & Middle/Early Archaic & & $\mathrm{x}$ & & & & 10.2 & $\mathrm{x}$ & & & & $\mathrm{x}$ & & $\mathrm{x}$ & & & & \\
\hline $13 \mathrm{~N}-5$ & III $/ \mathrm{b}$ & Early Archaic & & $\mathrm{x}$ & & & & 5.8 & $\mathrm{x}$ & & & & $\mathrm{X}$ & & $\mathrm{X}$ & & & & \\
\hline $23 \mathrm{M}-13$ & IIIa/b & Early Archaic & & & $\mathrm{x}$ & & & 5 & $\mathrm{x}$ & & & & $\mathrm{X}$ & & $\mathrm{X}$ & & & & \\
\hline $32 \mathrm{O}-10$ & III $/ \mathrm{b}$ & Early Archaic & & $\mathrm{x}$ & & & & 4.2 & & $\mathrm{x}$ & & & $\mathrm{X}$ & & $\mathrm{x}$ & & & & \\
\hline 29R2-3 & IIIIa & Early Archaic & $\mathrm{x}$ & & & & & 4.4 & $\mathrm{x}$ & & & & $\mathrm{X}$ & & $\mathrm{x}$ & & & & \\
\hline OR318 & IIIIa & Early Archaic & & $\mathrm{x}$ & & & & 5.5 & $\mathrm{x}$ & & & & $\mathrm{X}$ & & $\mathrm{x}$ & & & & \\
\hline $52 \mathrm{PA}-2$ & III & Archaic & $\mathrm{x}$ & & & & & N/A & $\mathrm{x}$ & & & & $\mathrm{x}$ & & $\mathrm{X}$ & & & & \\
\hline $25 \mathrm{~S} 2-2$ & II/IIIa & Early Archaic/Late Paleoindian & & $\mathrm{x}$ & & & & 7.1 & $\mathrm{x}$ & & & & & & & & & & $\mathrm{x}$ \\
\hline 40R1-1 & II/IIIa & Early Archaic/Late Paleoindian & $\mathrm{x}$ & & & & & 15.6 & $x$ & & & & & & $\mathrm{x}$ & & & & \\
\hline 24U2-2 & II & Late Paleoindian & & $\mathrm{x}$ & & & & 2.4 & $\mathrm{x}$ & & & & $x$ & & $\mathrm{X}$ & & & & \\
\hline \multicolumn{2}{|c|}{ Valley Floor B: } & & & & & & & & & & & & & & & & & & \\
\hline $50 \mathrm{IB}-6$ & & Middle Archaic & & $\mathrm{x}$ & & & & 3.7 & $\mathrm{x}$ & & & & $\mathrm{x}$ & & $\mathrm{x}$ & & & & \\
\hline $50 \mathrm{KA}-6$ & IIIc & Middle/Early Archaic & & & & & $\mathrm{X}$ & 7.7 & $\mathrm{x}$ & & & & $\mathrm{x}$ & & $\mathrm{X}$ & & & & \\
\hline $51 *-1$ & Unknown & Cannot be determined & & $\mathrm{x}$ & & & & 3.6 & $\mathrm{x}$ & & & & $\mathrm{X}$ & & $\mathrm{X}$ & & & & \\
\hline
\end{tabular}




\begin{tabular}{|c|c|c|c|c|c|c|c|c|c|c|c|c|c|c|c|c|c|}
\hline \multirow[b]{2}{*}{ Item } & \multirow[b]{2}{*}{ Unit } & \multirow[b]{2}{*}{ Cultural Period } & \multicolumn{4}{|c|}{ Blank } & \multirow[b]{2}{*}{\begin{tabular}{|c|} 
Thickness \\
\\
\\
Bit \\
\end{tabular}} & \multicolumn{4}{|c|}{ Raw Material } & \multicolumn{2}{|c|}{ Patina } & \multicolumn{4}{|c|}{ Heat Alteration } \\
\hline & & & 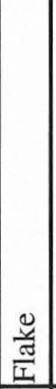 & $\begin{array}{l}\mathscr{\mathscr { \varpi }} \\
\stackrel{\mathscr{m}}{0}\end{array}$ & 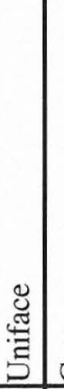 & 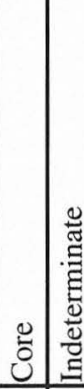 & & 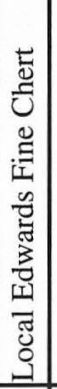 & 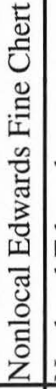 & 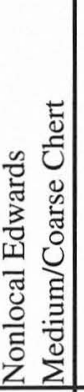 & 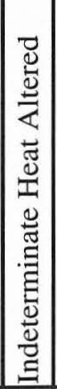 & 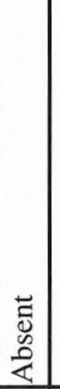 & 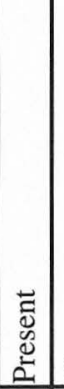 & $\begin{array}{l}\overrightarrow{0} \\
\overline{0} \\
\stackrel{0}{<}\end{array}$ & $\begin{array}{l}\frac{\overrightarrow{5}}{50} \\
\overline{0}\end{array}$ & $\begin{array}{l}\frac{0}{\pi} \\
\frac{\pi}{0} \\
\frac{\pi}{0} \\
\Sigma\end{array}$ & 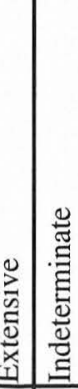 \\
\hline \multicolumn{2}{|c|}{ Valley Margin: } & & & & & & & & & & & & & & & & \\
\hline $10 \mathrm{~A}-5$ & IIIIc & Late Prehistoric & & $\mathrm{x}$ & & & 4.1 & $\mathrm{x}$ & & & & & & $\mathrm{x}$ & & & \\
\hline 10B-23 & IIIIc & Late Prehistoric/Late Archaic & & & $\mathrm{x}$ & & 5.5 & $\mathrm{x}$ & & & & $\mathrm{X}$ & & $\mathrm{x}$ & & & \\
\hline $1422-1$ & IIIIc & Archaic & & $\mathrm{x}$ & & & 3.8 & $\mathrm{x}$ & & & & $\mathrm{X}$ & & $\mathrm{x}$ & & & \\
\hline $5 \mathrm{G}-5$ & IIIIc & Archaic & $\mathrm{x}$ & & & & 4.9 & $\mathrm{x}$ & & & & $\mathrm{x}$ & & $\mathrm{x}$ & & & \\
\hline $6 \mathrm{~F}-3$ & IIIIc & Archaic & & & $\mathrm{x}$ & & 6.3 & $\mathrm{x}$ & & & & $\mathrm{x}$ & & $\mathrm{x}$ & & & \\
\hline $8 B-28$ & IIIIc & Late Prehistoric/Late Archaic & & $\mathrm{x}$ & & & 3.6 & & $\mathrm{X}$ & & & & $\mathrm{x}$ & $\mathrm{x}$ & & & \\
\hline $8 \mathrm{H}-1$ & IIIIc & Archaic & & $\mathrm{x}$ & & & 4.3 & $\mathrm{x}$ & & & & $\mathrm{x}$ & & $\mathrm{x}$ & & & \\
\hline $9 \mathrm{~A}-14$ & IIIIc & Late Prehistoric & & $\mathrm{x}$ & & & 4.8 & & & & $x$ & $\mathrm{x}$ & & & & $\mathrm{x}$ & \\
\hline $9 \mathrm{H}-3$ & IIIIc & Archaic & & $\mathrm{x}$ & & & 4.7 & & $\mathrm{X}$ & & & $\mathrm{x}$ & & $\mathrm{x}$ & & & \\
\hline $9 \mathrm{E}-17$ & IIIIc & Late Archaic & & & $\mathrm{x}$ & & 4.5 & $\mathrm{x}$ & & & & $\mathrm{x}$ & & $\mathrm{x}$ & & & \\
\hline $18 \mathrm{H}-6$ & Y/IIIc & Late/Middle Archaic & & $\mathrm{x}$ & & & 3.5 & $\mathrm{x}$ & & & & $\mathrm{x}$ & & $\mathrm{x}$ & & & \\
\hline 10I-11 & $\mathrm{IIIb} / \mathrm{c}$ & Middle/Early Archaic & $\mathrm{x}$ & & & & 11.5 & $\mathrm{x}$ & & & & $\mathrm{X}$ & & & & & $\mathrm{X}$ \\
\hline $11 \mathrm{H}-5$ & $\mathrm{IIIb} / \mathrm{c}$ & Late/Middle Archaic & & $\mathrm{x}$ & & & 7.4 & $\mathrm{x}$ & & & & & $\mathrm{x}$ & $\mathrm{x}$ & & & \\
\hline $20 \mathrm{~J}-5$ & $\mathrm{IIIb} / \mathrm{c}$ & Middle/Early Archaic & $\mathrm{x}$ & & & & 5.6 & $\mathrm{x}$ & & & & $\mathrm{x}$ & & $\mathrm{x}$ & & & \\
\hline $11 \mathrm{~K}-6$ & IIIIb & Middle/Early Archaic & & $\mathrm{x}$ & & & 5.3 & & $\mathrm{X}$ & & & $\mathrm{x}$ & & $\mathrm{x}$ & & & \\
\hline $20 \mathrm{~L}-6$ & IIIlb & Early Archaic & & $\mathrm{x}$ & & & 4.5 & $\mathrm{x}$ & & & & $\mathrm{x}$ & & $\mathrm{x}$ & & & \\
\hline $22 \mathrm{~J}-7$ & IIIlb & Middle/Early Archaic & & $\mathrm{x}$ & & & 4 & $\mathrm{x}$ & & & & $\mathrm{x}$ & & $\mathrm{x}$ & & & \\
\hline $19 \mathrm{~K}-5$ & Y/IIIb & Early Archaic & $\mathrm{x}$ & & & & 3.2 & $\mathrm{x}$ & & & & $\mathrm{x}$ & & $\mathrm{x}$ & & & \\
\hline $8 \mathrm{~K}-14$ & $\mathrm{Y}$ & Early Archaic & & & $\mathrm{X}$ & & 4.1 & & & $\mathrm{X}$ & & $\mathrm{x}$ & & $\mathrm{x}$ & & & \\
\hline OR648 & $\mathrm{Y}$ & Early Archaic & & $\mathrm{x}$ & & & 5.6 & $\mathrm{x}$ & & & & $\mathrm{X}$ & & $\mathrm{x}$ & & & \\
\hline 11P-10 & IIIIa & Early Archaic & $x$ & & & & 5 & $\mathrm{x}$ & & & & $\mathrm{X}$ & & $\mathrm{x}$ & & & \\
\hline $11 \mathrm{P}-5$ & III & Early Archaic & & $\mathrm{x}$ & & & 4.5 & $\mathrm{x}$ & & & & $\mathrm{X}$ & & $\mathrm{x}$ & & & \\
\hline 210-19 & IIIIa & Early Archaic & & $\mathrm{x}$ & & & 10.7 & & $\mathrm{X}$ & & & $\mathrm{x}$ & & $\mathrm{x}$ & & & \\
\hline 10P-16 & $\mathrm{X} / \mathrm{II} / \mathrm{III}$ & Early Archaic & $\mathrm{x}$ & & & & 9.1 & $\mathrm{x}$ & & & & $\mathrm{x}$ & & $\mathrm{x}$ & & & \\
\hline 20Q-9 & X/II/IIIa & Early Archaic & & $\mathrm{x}$ & & & 13.2 & $\mathrm{x}$ & & & & $\mathrm{x}$ & & $\mathrm{x}$ & & & \\
\hline $11 \mathrm{~S}-2$ & II & Late Paleoindian & & $\mathrm{x}$ & & & 4 & $\mathrm{x}$ & & & & & & $\mathrm{x}$ & & & \\
\hline 18RA-8 & $\mathrm{x}$ & Early Archaic/Late Paleoindian & $\mathrm{x}$ & & & & 14.5 & $\mathrm{x}$ & & & & $\mathrm{x}$ & & $\mathrm{x}$ & & & \\
\hline 19P-3 & $\mathrm{x}$ & Early Archaic/Late Paleoindian & & $\mathrm{x}$ & & & 11.6 & & $\mathrm{X}$ & & & & $\mathrm{x}$ & $\mathrm{x}$ & & & \\
\hline $19 \mathrm{~S}-8$ & $\mathrm{x}$ & Early Archaic/Late Paleoindian & $\mathrm{x}$ & & & & 4.1 & $\mathrm{x}$ & & & & $\mathrm{x}$ & & $\mathrm{x}$ & & & \\
\hline 5Q-1 & $\mathrm{x}$ & Early Archaic/Late Paleoindian & & & $\mathrm{X}$ & & 8.8 & $\mathrm{x}$ & & & & $\mathrm{x}$ & & $\mathrm{x}$ & & & \\
\hline $8 \mathrm{P}-2$ & $\mathrm{x}$ & Early Archaic & & $\mathrm{x}$ & & & 6.9 & $\mathrm{x}$ & & & & & $\mathrm{x}$ & $\mathrm{x}$ & & & \\
\hline $9 \mathrm{U}-2$ & $\mathrm{x}$ & Early Archaic/Late Paleoindian & & & $\mathrm{x}$ & & 14.2 & $\mathrm{x}$ & & & & $\mathrm{X}$ & & & & $\underline{X}$ & \\
\hline
\end{tabular}

site, the latter almost certainly reflecting their use at the site as well.

\section{CONCLUSIONS}

From a technological standpoint, there are several striking differences in the biface assemblage that have proved significant in distinguishing cultural assemblages in the lower units. Although small in number, products of the early Clovis culture are clearly manifested in several large bifaces, many of which evidence large percussion flake scars spanning the full breadth of the specimen.

Somewhat later in time, Folsom technology is signalled in remarkably thin, flat, and well-shaped bifaces typically displaying an unusually high width to thickness ratio. Two types of Folsom bifaces were recognized in the Wilson- 
TABLE 16-43

Material Characteristics and Attributes of Burin Group $4(\mathrm{~N}=10)$

\begin{tabular}{|c|c|c|c|c|c|c|c|c|c|c|c|c|c|c|}
\hline \multirow[b]{2}{*}{ Item } & \multirow[b]{2}{*}{ Unit } & \multirow[b]{2}{*}{ Cultural Period } & \multicolumn{5}{|c|}{ Blank } & \multicolumn{2}{|c|}{ Thickness } & \multirow{2}{*}{\begin{tabular}{|c|}
$\begin{array}{c}\text { Raw } \\
\text { Material }\end{array}$ \\
Local \\
Edwards \\
Fine Chert
\end{tabular}} & \multirow{2}{*}{$\begin{array}{l}\text { Patina } \\
\text { Absent }\end{array}$} & \multicolumn{3}{|c|}{ Heat Alteration } \\
\hline & & & Flake & Biface & Uniface & Core & Indeterminate & Bit 1 & Bit 2 & & & Absent & Slight & Moderat \\
\hline \multicolumn{2}{|c|}{ Valley Floor A: } & & & & & & & & & & & & & \\
\hline $120-5$ & |IIIc & $\begin{array}{l}\text { Late Prehistoric/ } \\
\text { Late Archaic }\end{array}$ & & & & & $\mathrm{X}$ & 11.5 & 15.7 & $\mathrm{X}$ & $\mathrm{X}$ & & $\mathrm{X}$ & \\
\hline $37 \mathrm{FB}-5$ & IIII & Late Archaic & $\mathrm{X}$ & & & & & 5.2 & 12.9 & $\mathrm{X}$ & $\mathrm{X}$ & $\mathrm{X}$ & & \\
\hline $37 \mathrm{CC}-34$ & IIII & Late Archaic & & & $\mathrm{X}$ & & & 5.2 & 4.7 & $\mathrm{X}$ & $\mathrm{X}$ & & & $\mathrm{X}$ \\
\hline $14 \mathrm{~L} 1-20$ & IIIb & Early Archaic & $\mathrm{X}$ & & & & & 6.2 & 7.3 & $\mathrm{X}$ & $\mathrm{X}$ & $\mathrm{X}$ & & \\
\hline $30 \mathrm{R} 2-3$ & IIIa & Early Archaic & $\mathrm{X}$ & & & & & 8.5 & 10.7 & $\mathrm{X}$ & $\mathrm{X}$ & $\mathrm{X}$ & & \\
\hline \multicolumn{2}{|c|}{ Valley Margin: } & & & & & & & & & & & & & \\
\hline $7 \mathrm{M}-2$ & Y & Early Archaic & & $\mathrm{X}$ & & & & 6.8 & 5.6 & $\mathrm{X}$ & & $\mathrm{X}$ & & \\
\hline $2 \mathrm{~K}-3$ & $\mathrm{X} / \mathrm{Y}$ & Early Archaic & & $\mathrm{X}$ & & & & 7.4 & 5.0 & $\mathrm{X}$ & $\mathrm{X}$ & $\mathrm{X}$ & & \\
\hline $11 \mathrm{P}-3$ & IIIa & Early Archaic & & & & $\mathrm{X}$ & & 2.8 & 3.6 & $\mathrm{X}$ & $\mathrm{X}$ & $\mathrm{X}$ & & \\
\hline $60-1$ & $\mathrm{X}$ & Early Archaic & & $\mathrm{X}$ & & & & 3.7 & 3.4 & $\mathrm{X}$ & $\mathrm{X}$ & $\mathrm{X}$ & & \\
\hline $190-15$ & $\mathrm{X}$ & Early Archaic & $\mathrm{X}$ & & & & & 4.3 & 5.3 & $\mathrm{X}$ & $\mathrm{X}$ & $\mathrm{X}$ & & \\
\hline
\end{tabular}

TABLE 16-44

Material Characteristics and Attributes of Burin Group $5(\mathrm{~N}=5)$

\begin{tabular}{|c|c|c|c|c|c|c|c|c|c|}
\hline \multirow[b]{2}{*}{ Item } & \multirow[b]{2}{*}{ Unit } & \multirow[b]{2}{*}{ Cultural Period } & \multicolumn{3}{|c|}{ Blank } & \multirow{2}{*}{$\begin{array}{c}\text { Thickness } \\
\text { Bit }\end{array}$} & \multirow{2}{*}{$\begin{array}{l}\text { Raw Material } \\
\text { Local Edwards } \\
\text { Fine Chert }\end{array}$} & \multirow{2}{*}{\begin{tabular}{|l|} 
Patina \\
Absent \\
\end{tabular}} & \multirow{2}{*}{$\begin{array}{c}\begin{array}{c}\text { Heat } \\
\text { Alteration }\end{array} \\
\text { Absent } \\
\end{array}$} \\
\hline & & & Flake & Biface & Uniface & & & & \\
\hline $\begin{array}{l}\text { Surface: } \\
0-243\end{array}$ & Surface & Unknown & & $\mathrm{X}$ & & 3.0 & $\mathrm{X}$ & $\mathrm{X}$ & $\mathrm{X}$ \\
\hline $\begin{array}{l}\text { Valley Fl } \\
934-1\end{array}$ & $\begin{array}{l}\text { or } \mathbf{A}: \\
\text { |II/IIIa }\end{array}$ & Early Archaic/Late Paleoindian & $\mathrm{X}$ & & & 5.8 & $\mathrm{X}$ & $\mathrm{X}$ & $\mathrm{X}$ \\
\hline $\begin{array}{l}\text { Valley M } \\
21 \mathrm{M}-10 \\
7 \mathrm{O}-2 \\
22 \mathrm{~T}-5\end{array}$ & \begin{tabular}{|l} 
rgin: \\
IIIb \\
$X$ \\
II
\end{tabular} & $\begin{array}{l}\text { Early Archaic } \\
\text { Early Archaic } \\
\text { Late Paleoindian }\end{array}$ & $\mathrm{X}$ & $\mathrm{X}$ & $\mathrm{X}$ & $\begin{array}{l}2.1 \\
6.3 \\
4.9\end{array}$ & $\begin{array}{l}X \\
X \\
X\end{array}$ & $\begin{array}{l}X \\
X \\
X\end{array}$ & $\begin{array}{l}X \\
X \\
X\end{array}$ \\
\hline
\end{tabular}

TABLE 16-45

Material Characteristics and Attributes of Burin Group $6(\mathrm{~N}=1)$

\begin{tabular}{|c|c|c|c|c|c|c|c|c|c|}
\hline \multirow[b]{2}{*}{ Item } & \multirow[b]{2}{*}{ Unit } & \multirow[b]{2}{*}{ Cultural Period } & \multirow{2}{*}{$\begin{array}{l}\text { Blank } \\
\text { Flake } \\
\end{array}$} & \multicolumn{3}{|c|}{ Thickness } & \multirow{2}{*}{$\begin{array}{c}\text { Raw Material } \\
\text { Local Edwards } \\
\text { Fine Chert }\end{array}$} & \multirow{2}{*}{$\begin{array}{l}\text { Patina } \\
\text { Absent }\end{array}$} & \multirow{2}{*}{$\begin{array}{c}\text { Heat Alteration } \\
\text { Absent } \\
\end{array}$} \\
\hline & & & & Bit 1 & Bit 2 & Bit 3 & & & \\
\hline $\begin{array}{l}\text { Valle } \\
34 \mathrm{~W} 1\end{array}$ & $\begin{array}{l}\text { or A: } \\
\text { |II }\end{array}$ & Late Paleoindian & $\mathrm{X}$ & 9.2 & 9.0 & 11.6 & $\mathrm{X}$ & $\mathrm{X}$ & $\mathrm{X}$ \\
\hline
\end{tabular}

Leonard collection: the ultrathin biface, likely intended as a cutting tool, and the slightly smaller biface, likely designed as a preform for the Folsom point.

With few exceptions, technological differentiation in biface production in succeeding periods is less apparent. The use of small flakes for production of arrow points in Late Prehistoric times is well evidenced at the site. Small preforms and points made on such flakes are often only minimally worked with bifacial retouch.

These patterns in technological variation were also detected, if only somewhat impressionistically, by comparing mean width to thickness ratios over time. Although high width to thickness ratios were expected in Early Paleoindian and Late Paleoindian contexts, high ratios also were evident in the Early/Middle Archaic and Middle/Late Archaic and Late Archaic assemblages. These would suggest that a large number of those bifaces were intended as preforms for the wide, thin projectile points characteristic of those periods, specifically Bandy and Bell/Andice in the former case and Castroville and Montell in the latter cases.

A variety of tool types were observed in contexts spanning the site's history, although certain groups showed more patterned distributions. Burins and spalls were found from Late Paleoindian to Late Prehistoric times, but were in somewhat higher frequencies during the Early Archaic. Perforators, pièces esquillées, and Form E narrow, elongate bifacial 
TABLE 16-46

Material Characteristics and Attributes of Burin Group 7 (N=5)

\begin{tabular}{|c|c|c|c|c|c|c|c|c|c|c|}
\hline \multirow[b]{2}{*}{ Item } & \multirow[b]{2}{*}{ Unit } & \multirow[b]{2}{*}{ Cultural Period } & \multicolumn{3}{|c|}{ Blank } & \multirow{2}{*}{$\begin{array}{c}\text { Thickness } \\
\text { Bit } \\
\end{array}$} & \multirow{2}{*}{$\begin{array}{c}\text { Raw Material } \\
\text { Local Edwards } \\
\text { Fine Chert }\end{array}$} & \multirow{2}{*}{$\begin{array}{l}\text { Patina } \\
\text { Absent }\end{array}$} & \multicolumn{2}{|c|}{ Heat Alteration } \\
\hline & & & Flake & Biface & Core & & & & Absent & Slight \\
\hline \multicolumn{11}{|c|}{ Valley Floor A: } \\
\hline $163-2$ & IIIIc/fill & Unassigned & & $\mathrm{X}$ & & 3.8 & $\mathrm{X}$ & $\mathrm{X}$ & $\mathrm{X}$ & \\
\hline OR61 & $\mathrm{IIIb} / \mathrm{c}$ & Late/Middle Archaic & & & $X$ & 4.3 & $\mathrm{X}$ & $x$ & $X$ & \\
\hline 31P1-6 & IIIIa & Early Archaic & & $X$ & & 6.2 & $\mathrm{X}$ & $\mathrm{X}$ & $X$ & \\
\hline 850-1 & IIIa & Early Archaic & & & $\mathrm{X}$ & 10.2 & $\mathrm{X}$ & $\mathrm{X}$ & & $\mathrm{X}$ \\
\hline \multicolumn{2}{|c|}{ Valley Margin: } & & & & & & & & & \\
\hline $6 \mathrm{~A}-20$ & IIIc/pot & Late Prehistoric/Archaic & - & - & - & - & - & - & - & - \\
\hline
\end{tabular}

TABLE 16-47

Material Characteristics and Attributes of Burin Group $8(\mathrm{~N}=8)$

\begin{tabular}{|c|c|c|c|c|c|c|c|c|c|c|c|c|}
\hline \multirow[b]{2}{*}{ Item } & \multirow[b]{2}{*}{ Unit } & \multirow[b]{2}{*}{ Cultural Period } & \multicolumn{4}{|c|}{ Blank } & \multirow{2}{*}{\begin{tabular}{|c|} 
Thickness \\
Bit \\
\end{tabular}} & \multirow{2}{*}{\begin{tabular}{|c|} 
Raw Material \\
Local Edwards \\
Fine Chert \\
\end{tabular}} & \multicolumn{2}{|c|}{ Patina } & \multicolumn{2}{|c|}{ Heat Alteration } \\
\hline & & & Flake & Biface & Uniface & Core & & & Present & Absent & Absent & Moderate \\
\hline \multicolumn{2}{|c|}{ Valley Floor A: } & & & & & & & & & & & \\
\hline $399-12$ & IIIb/c & Late Archaic & & & $\mathrm{X}$ & & 7.3 & $X$ & & $X$ & $X$ & \\
\hline $30 \mathrm{O}-2$ & $\mathrm{IIIa} / \mathrm{b}$ & Early Archaic & & $\mathrm{X}$ & & & 6.4 & $\mathrm{X}$ & & $\mathrm{X}$ & $\mathrm{X}$ & \\
\hline $25 \mathrm{P} 2-1$ & IIIIa & Early Archaic & & $\mathrm{X}$ & & & 5.3 & $X$ & & $X$ & & $\mathrm{X}$ \\
\hline 30R2-13 & IIIIa & Early Archaic & $\mathrm{X}$ & & & & 2.9 & $\mathrm{X}$ & & $\mathrm{X}$ & $X$ & \\
\hline \multicolumn{2}{|c|}{ Valley Floor B: } & & & & & & & & & & & \\
\hline $51 \mathrm{SA}-4$ & IIII & Early Archaic & & & & $\mathrm{X}$ & 4.8 & $\mathrm{X}$ & & $\mathrm{X}$ & $\mathrm{X}$ & \\
\hline \multicolumn{2}{|c|}{ Valley Margin: } & & & & & & & & & & & \\
\hline $3 G-2$ & |Y/IIIc & Archaic & & $\mathrm{X}$ & & & 5.9 & $\mathrm{X}$ & & $\mathrm{X}$ & $\mathrm{X}$ & \\
\hline $5 \mathrm{~K}-1$ & $\mathrm{Y}$ & Early Archaic & & $\mathrm{X}$ & & & 13.1 & $\mathrm{X}$ & & $\mathrm{X}$ & $\mathrm{X}$ & \\
\hline $180-17$ & $\mathrm{X}$ & Early Archaic & $\mathrm{X}$ & & & & 3.7 & $\mathrm{X}$ & $\mathrm{X}$ & & $\mathrm{X}$ & \\
\hline
\end{tabular}

tools also derive predominately from Early Archaic contexts, suggesting a wide range of activities during that period.

If we consider the odd shape and size of Form E tools as indicative of resharpening and heavy use, as discussed above, their high frequency in Early Archaic contexts assumes greater meaning, in light of data from other tool types. Projectile points and Clear Fork tools from those contexts show a very high rate of resharpening (see Chapters 13 and 15), and the presence of other exhausted tool forms at that juncture carries significant implications as to site use and behavior.

For the full assemblage considered here, raw material patterns are fairly consistent, with local fine-grained cherts preferred over time. Import of exotic materials for stone tools is not indicated with the exception of a single specimen of Tecovas Jasper found in Late Paleoindian contexts.

Certain material characteristics, indicative of either procurement patterns or site depositional processes, bear further discussion. Based on the biface and bifacial tool Valley Floor sample, it appears that upland cherts were favored during much of the site's occupation and that stream-rolled cobbles of chert were used only rarely. Heat damage to bifaces is seen in more than one-third of the Unit II subsample. The most extensive heat damage, however, appears among specimens in Unit IIIc; this occurrrence may be correlated to the large burned rock middens present in that zone. Patina among bifaces was unusually high among the Unit II (Late Paleoindian sample). Whether materials remained exposed on the surface for longer periods during the roughly 95008700 B.P. juncture and patinated with greater frequencies is not clear. Mineral precipitates also are seen in highest frequencies within the Unit II sample. Given the rather consistent co-occurrence of these two patterns (higher frequencies of patina and mineral precipitates) among other tool groups from Unit II contexts, it is evident that some alteration in site formation processes during that time period is indicated.

On a conceptual level, this study has sought to categorize bifaces within traditional manufacturing stages and tool categories while illuminating alternative, and in some cases, unusual morphologies which suggest use as tools. While this framework underlines the fluid interchange between the manufacturing and use realms suggested in many pathway models, it is complicated from a practical standpoint. Assignment of individual specimens to categories is problematical and often intuitive, due in some part to possible multifunctional roles of various biface types.

As discussed above, many bifaces in all stages in the collection were abandoned during the manufacturing process because of knapping error, material flaws, or for no apparent reason. Although these specimens did not exhibit 


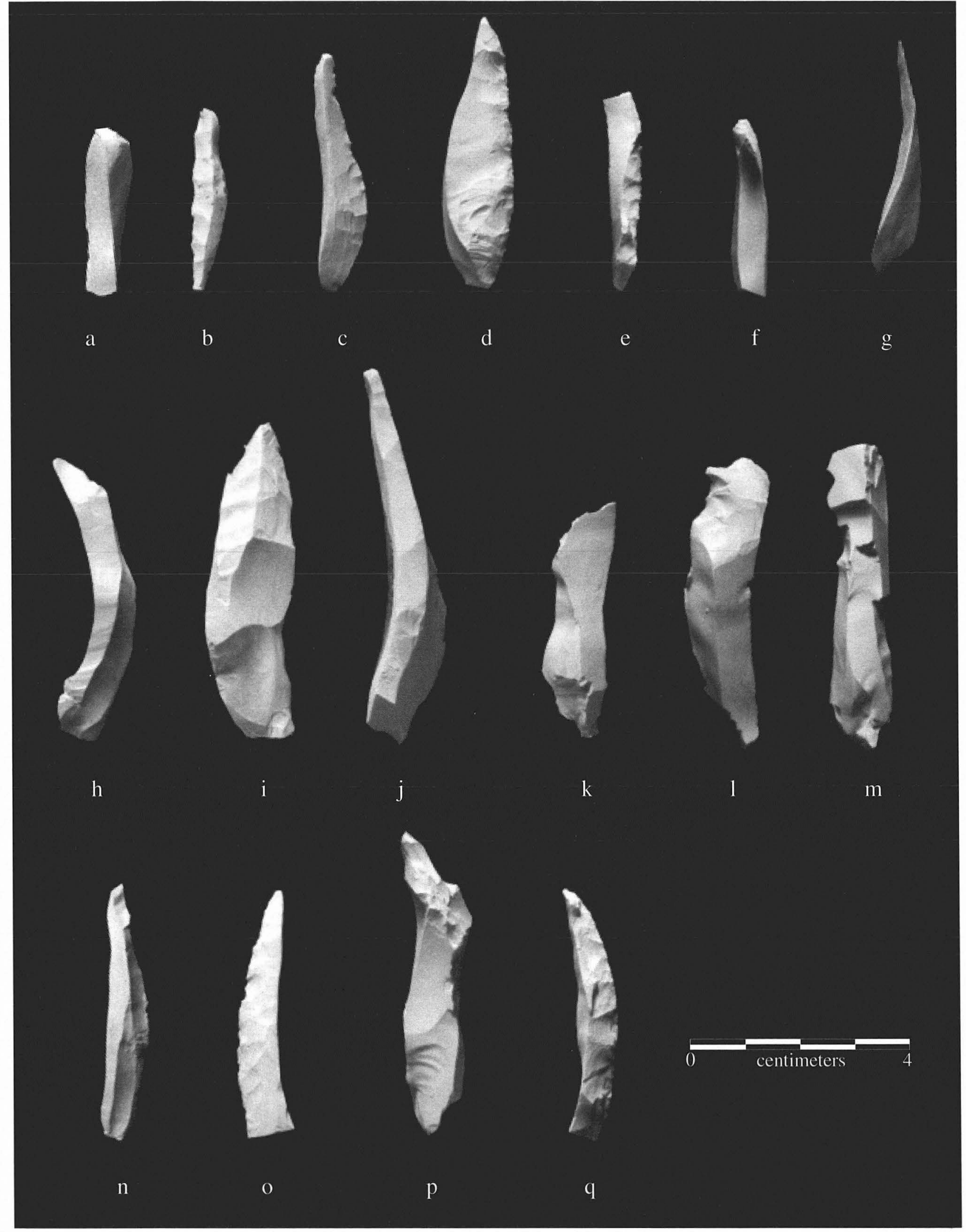

FIGURE 16-28. Representative spalls. (a-e) Group 1; (f-j) Group 2; (k-n) Group 3; (o-q) Group 4. Specimen numbers: (a) 1601-4; (b) 27X1A-1; (c) 1685-1; (d) 22L-8; (e) 18G-7; (f) 15Q2-1; (g) 29V1A-1; (h) 14L1-22; (i), 31Q2-11; (j) 27R1-6; (k) 16C-6; (l) 15R2-4; (m) $16 \mathrm{HH} 2 \mathrm{~B}-1$; (n) $29 \mathrm{X} 2 \mathrm{~A}-1$; (o) $11 \mathrm{~K}-7$; (p) $32 \mathrm{X} 2 \mathrm{~B}-1$; (q) $22 \mathrm{M}-6$. 
TABLE $16-48$

Provenience and Measurements of Group 1 Spalls $(\mathrm{N}=128)$

\begin{tabular}{|c|c|c|c|c|}
\hline Unit & Specimen No. & Blank & Length & Thickness \\
\hline $\begin{array}{l}\text { Unknown } \\
\text { Surface } \\
\text { Surface } \\
\text { Surface } \\
\end{array}$ & $\begin{array}{l}\text { Lot } 3 \\
0-176 \\
0-220 \\
0-29 \\
\end{array}$ & $\begin{array}{l}2 \\
4 \\
4 \\
2 \\
\end{array}$ & $\begin{array}{l}\text { N/A } \\
27.1 \\
27.4 \\
42.3 \\
\end{array}$ & $\begin{array}{c}\text { N/A } \\
6.0 \\
3.1 \\
10.0 \\
\end{array}$ \\
\hline Valley Floor A: & & & & \\
\hline Unknown & $36 *-11$ & 9 & 65.0 & 6.6 \\
\hline IIIC & $37 \mathrm{DB}-16$ & 1 & 29.2 & 5.4 \\
\hline IIIC & $452-1$ & 1 & 48.0 & 11.0 \\
\hline IIIc & $260-4$ & 1 & 34.0 & 2.3 \\
\hline IIIc & $391-1$ & 2 & 32.3 & 4.7 \\
\hline IIIc & $311-1$ & 2 & 36.4 & 3.3 \\
\hline IIIc & $36 \mathrm{E}-31$ & 2 & 35.3 & 7.2 \\
\hline IIIc & $16 \mathrm{C}-10$ & 4 & 20.5 & 5.7 \\
\hline IIIc & $14 D-6$ & 1 & 36.0 & 4.6 \\
\hline IIIc & $13 C-4$ & 2 & 61.3 & 10.2 \\
\hline IIIC & $12 \mathrm{~A}-8$ & 1 & 40.0 & 6.1 \\
\hline $\mathrm{IIIJ} / \mathrm{c}$ & $480-3$ & 2 & 52.8 & 5.5 \\
\hline IIIb & $580-1$ & 2 & 31.7 & 7.1 \\
\hline IIIb & $748-2$ & 4 & 18.3 & 3.8 \\
\hline IIIb & $538-8$ & 4 & 21.1 & 3.6 \\
\hline IIIb & $508-2$ & 4 & 51.0 & 10.5 \\
\hline IIIb & $17 \mathrm{M} 2-2$ & 4 & 28.0 & 5.0 \\
\hline IIIb & $16 \mathrm{~N} 1-3$ & 4 & 40.3 & 8.1 \\
\hline IIIb & $14 \mathrm{M} 1-2$ & 9 & 53.0 & 8.7 \\
\hline IIIb & $13 \mathrm{M}-6$ & 4 & 43.3 & 9.2 \\
\hline IIIb & $13 \mathrm{~J}-11$ & 4 & 64.8 & 6.2 \\
\hline $\mathrm{III} / \mathrm{b}$ & $611-6$ & 1 & 48.0 & 8.0 \\
\hline $\mathrm{IIIa} / \mathrm{b}$ & $729-9$ & 2 & 20.1 & 6.2 \\
\hline $\mathrm{III} / \mathrm{b}$ & $628-6$ & 1 & 44.0 & 5.9 \\
\hline $\mathrm{III} / \mathrm{b}$ & $611-5$ & 2 & 29.5 & 2.7 \\
\hline $\mathrm{III} a / \mathrm{b}$ & $340-7$ & 2 & 38.7 & 6.1 \\
\hline $\mathrm{III} a / \mathrm{b}$ & $340-5$ & 9 & 43.8 & 6.5 \\
\hline $\mathrm{III} / \mathrm{b}$ & $300-28$ & 4 & 56.2 & 7.7 \\
\hline $\mathrm{IIIa} / \mathrm{b}$ & $300-16$ & 4 & 36.5 & 5.5 \\
\hline $\mathrm{III} / \mathrm{b}$ & $290-7$ & 1 & 31.5 & 3.4 \\
\hline $\mathrm{III} / \mathrm{b}$ & $270-10$ & 4 & 46.0 & 10.0 \\
\hline IIIa & $25 \mathrm{Q} 2-12$ & 1 & 26.8 & 4.6 \\
\hline IIIa & $230-17$ & 4 & 26.8 & 5.3 \\
\hline IIIa & $974-1$ & 4 & 48.3 & 10.0 \\
\hline IIIa & $853-1$ & 1 & 13.8 & 3.1 \\
\hline IIIa & $744-2$ & 4 & 31.8 & 5.1 \\
\hline IIIa & $713-1$ & 2 & 23.4 & 4.3 \\
\hline IIIa & $37 \mathrm{Q} 2 \mathrm{~B}-5$ & 1 & 35.7 & 4.0 \\
\hline IIIa & $37 \mathrm{O} 2 \mathrm{C}-10$ & 1 & 32.5 & 1.0 \\
\hline IIIa & $36 \mathrm{P}-5$ & 1 & 23.9 & 1.0 \\
\hline IIIa & $360-6$ & 4 & 37.0 & 8.0 \\
\hline IIIa & $35 \mathrm{R} 2-10$ & 4 & 27.3 & 4.0 \\
\hline IIIa & $35 \mathrm{Q} 2-4$ & 4 & 33.6 & 4.0 \\
\hline IIIa & 28Q2-3 & 9 & 46.2 & 11.9 \\
\hline IIIa & 28Q2-2 & 4 & 55.1 & 6.0 \\
\hline IIIa & 26Q2-1 & 4 & 32.2 & 9.7 \\
\hline IIIa & $25 Q 1-6$ & 4 & 44.2 & 4.4 \\
\hline IIIa & 17Q2-1 & 4 & 34.3 & 5.8 \\
\hline IIIa & $16 \mathrm{P} 2-2$ & 4 & 50.3 & 4.5 \\
\hline IIIa & $15 \mathrm{Q} 2-4$ & 2 & 46.4 & 5.6 \\
\hline IIIa & $15 \mathrm{Q} 2-3$ & 2 & 37.1 & 10.6 \\
\hline IIIa & $15 \mathrm{P} 1-3$ & 2 & 47.2 & 12.3 \\
\hline III & $520 A-1$ & 2 & 43.1 & 6.0 \\
\hline II/IIIa & $1161-3$ & 4 & 40.2 & 6.8 \\
\hline II/IIIa & $949-1$ & 2 & 25.3 & 4.3 \\
\hline II/IIIa & $34 \mathrm{~T} 1 \mathrm{C}-4$ & 4 & 36.1 & 4.0 \\
\hline II/IIIa & $33 \mathrm{~S} 2 \mathrm{~A}-3$ & 4 & 31.2 & 7.9 \\
\hline
\end{tabular}


Table 16-48, continued

\begin{tabular}{|c|c|c|c|c|}
\hline Unit & Specimen No. & Blank & Length & Thickness \\
\hline$\overline{\mathrm{II} / \mathrm{IIIa}}$ & $31 \mathrm{~T} 2 \mathrm{C}-3$ & 2 & 35.5 & 5.2 \\
\hline II/IIIa & $17 \mathrm{~T} 2-2$ & 4 & 59.9 & 8.2 \\
\hline II/IIIa & $14 S 2-1$ & 4 & 46.2 & 5.2 \\
\hline II & $23 \mathrm{~S}-3$ & 2 & 60.0 & 30.0 \\
\hline II & $1215-1$ & 4 & 24.0 & 4.0 \\
\hline II & $1685-1$ & 2 & 44.0 & 5.1 \\
\hline II & $37 \mathrm{X} 2 \mathrm{~B}-2$ & 2 & 13.0 & 2.6 \\
\hline II & $37 \mathrm{X} 1 \mathrm{~A}-1$ & 4 & 27.5 & 3.0 \\
\hline II & 37T1D-4 & 4 & 30.7 & 8.1 \\
\hline II & $35 \mathrm{~W} 2 \mathrm{~A}-1$ & 4 & 41.2 & 10.9 \\
\hline II & $34 \mathrm{~V} 1 \mathrm{~B}-3$ & 9 & 35.6 & 6.0 \\
\hline II & $27 \mathrm{X} 1 \mathrm{~A}-1$ & 2 & 34.1 & 6.8 \\
\hline II & $27 \mathrm{~W} 2 \mathrm{~B}-1$ & 4 & 23.0 & 4.4 \\
\hline II & 17V2-4 & 4 & 26.0 & 4.6 \\
\hline $\mathrm{Id} / \mathrm{II}$ & $32 Y 2 B-2$ & 2 & 26.7 & 7.8 \\
\hline $\mathrm{Id} / \mathrm{II}$ & $14 \mathrm{~V} 1-4$ & 1 & 36.0 & 5.2 \\
\hline $\mathrm{Id} / \mathrm{II}$ & 14V1-1 & 1 & 41.6 & 3.8 \\
\hline $\mathrm{Id} / \mathrm{II}$ & 14U2-2 & 4 & 39.7 & 10.0 \\
\hline$\underline{\text { Isi }}$ & $1601-4$ & 1 & 26.8 & 6.4 \\
\hline Valley Floor B: & & & & \\
\hline Unknown & $50-1$ & 2 & 42.0 & 50.0 \\
\hline III & $51 \mathrm{VA}-3$ & 9 & 40.3 & 8.1 \\
\hline Valley Margin: & & & & \\
\hline IIIC & $3 \mathrm{~A}-31$ & 4 & 19.38 & 7.5 \\
\hline IIIc & $1265-13$ & 1 & 20.0 & 0.3 \\
\hline IIIc & $1265-12$ & 9 & 11.1 & 1.2 \\
\hline IIIc & $9 \mathrm{C}-17$ & 4 & 32.8 & 5.5 \\
\hline IIIc & $8 \mathrm{C}-4$ & 4 & 54.8 & 6.8 \\
\hline IIIC & $8 \mathrm{~A}-47$ & 2 & 36.2 & 7.1 \\
\hline IIIC & $8 \mathrm{~A}-32$ & 1 & 19.9 & 3.8 \\
\hline IIIC & $6 \mathrm{E}-2$ & 4 & 52.6 & 7.5 \\
\hline IIIC & $3 \mathrm{E}-10$ & 4 & 30.0 & 4.1 \\
\hline IIIC & $2 \mathrm{~B}-12$ & 2 & 27.2 & 6.0 \\
\hline IIIC & $2 \mathrm{~B}-10$ & 2 & 43.5 & 5.0 \\
\hline IIIC & $18 \mathrm{G}-7$ & 4 & 37.0 & 4.6 \\
\hline IIIc/pot & $5 \mathrm{~A}-12$ & 1 & 36.0 & 4.5 \\
\hline $\mathrm{IIIb} / \mathrm{c}$ & $20 \mathrm{I}-1$ & 2 & 49.6 & 12.0 \\
\hline Y/IIIc & $1502-4$ & 1 & 37.1 & 4.3 \\
\hline Y/IIIc & $1505-2$ & 2 & 31.7 & 10.0 \\
\hline Y/IIIc & $9 \mathrm{~K}-2$ & 4 & 30.6 & 7.0 \\
\hline IIIb & $22 \mathrm{~L}-8$ & 4 & 50.3 & 4.9 \\
\hline IIIb & $22 \mathrm{~L}-7$ & 2 & 60.0 & 9.5 \\
\hline IIIb & $22 \mathrm{~K}-4$ & 4 & 41.8 & 8.4 \\
\hline IIIb & $21 \mathrm{~J}-16$ & 2 & 41.2 & 4.7 \\
\hline $\mathrm{Y}$ & $1668-4$ & 4 & 45.0 & 5.0 \\
\hline $\mathrm{Y}$ & $8 K-9$ & 2 & 43.6 & 12.6 \\
\hline $\mathrm{Y}$ & $8 \mathrm{~K}-18$ & 4 & 30.6 & 10.2 \\
\hline $\mathrm{Y}$ & $7 \mathrm{M}-4$ & 2 & 40.0 & 8.4 \\
\hline $\mathrm{X} / \mathrm{Y} / \mathrm{IIIb}$ & $19 \mathrm{~L}-5$ & 2 & 43.2 & 6.8 \\
\hline $\mathrm{X} / \mathrm{IIIb}$ & $19 \mathrm{M}-4$ & 4 & 39.3 & 5.9 \\
\hline $\mathrm{X} / \mathrm{IIIb}$ & $10 \mathrm{M}-10$ & 4 & 46.0 & 6.7 \\
\hline $\mathrm{X} / \mathrm{Y}$ & $4 \mathrm{~L}-2$ & 2 & 35.2 & 6.3 \\
\hline $\mathrm{X} / \mathrm{III} / \mathrm{b}$ & $10 \mathrm{~N}-16$ & 1 & 51.2 & 8.1 \\
\hline IIIa & $11 \mathrm{P}-9$ & 2 & 52.6 & 9.5 \\
\hline IIIa & $11 \mathrm{P}-11$ & 1 & 43.6 & 6.2 \\
\hline X/IIIa & $200-20$ & 1 & 59.4 & 8.7 \\
\hline II/IIIa & $22 \mathrm{P}-18$ & 1 & 23.7 & 3.8 \\
\hline II/IIIa & $22 \mathrm{P}-17$ & 1 & 30.3 & 3.7 \\
\hline X/II/IIIa & 20Q-11 & 4 & 55.4 & 7.6 \\
\hline Х/I/IIа & $20 \mathrm{P}-5$ & 2 & 37.0 & 5.2 \\
\hline $\mathrm{X}$ & $9 R-4$ & 2 & 34.9 & 4.8 \\
\hline $\mathrm{X}$ & $8 R-4$ & 4 & 35.1 & 5.5 \\
\hline$\underline{X}$ & $6 \mathrm{P}-5$ & 4 & 25.8 & 7.1 \\
\hline
\end{tabular}


Table 16-48, continued

\begin{tabular}{l|l|c|c|c}
\hline Unit & Specimen No. & Blank & Length & Thickness \\
\hline $\mathrm{X}$ & $3 \mathrm{M}-4$ & 4 & 51.6 & 6.1 \\
$\mathrm{X}$ & $18 \mathrm{RC}-13$ & 4 & 35.0 & 6.5 \\
$\mathrm{X}$ & $18 \mathrm{P}-2$ & 2 & 40.0 & 5.3 \\
$\mathrm{X}$ & $18 \mathrm{~N}-7$ & 2 & 32.4 & 4.7 \\
$\mathrm{X} / \mathrm{II}$ & $21 \mathrm{~T}-4$ & 9 & 52.3 & 10.0 \\
X/II & 20R-11 & 4 & 50.0 & 7.0 \\
\hline \hline
\end{tabular}

any obvious signs of use, it is clearly possible that use-wear on many bifaces was not recognized, lacking microscopic study of the edges. Such was the case with a sample of ostensibly unmodified flakes from the site which, when examined under high-power microscopy by Kay, revealed evidence of use (see Chapter 22). What the present analysis has sought to elucidate is the likelihood that recycling and re-use is present in biface assemblages at a much higher degree than is commonly recognized. Although only hinted at in the miscellaneous bifacial tools, surely many specimens classified as broken or discarded bifaces were exploited for their sharp or broken edges. Certainly, greater focus needs to be directed to expedient tool use and recycling in all tool categories. Without benefit of such insights, the complete role of bifaces and bifacial technology in prehistoric economies cannot be understood. 
TABLE $16-49$

Provenience and Measurements of Group 2 Spalls $(\mathrm{N}=70)$

\begin{tabular}{|c|c|c|c|c|}
\hline Unit & Specimen No. & Blank & Length & Thickness \\
\hline Surface & $0-219$ & 9 & 29.1 & 7.6 \\
\hline Surface & $0-236$ & 1 & 39.7 & 5.0 \\
\hline Surface & $0-37$ & 4 & 41.6 & 4.5 \\
\hline \multicolumn{5}{|l|}{ Valley Floor A: } \\
\hline IIIc & OR45 & 4 & 27.3 & 7.6 \\
\hline IIIc & $310-5$ & 1 & 40.8 & 6.2 \\
\hline IIIb & $653-6$ & 4 & 58.0 & 7.3 \\
\hline IIIb & $572-1$ & 9 & 52.0 & 22.5 \\
\hline IIIb & $531-4$ & 9 & 30.0 & 4.1 \\
\hline IIIb & $15 \mathrm{M} 1-5$ & 1 & 51.3 & 8.2 \\
\hline IIIb & $15 \mathrm{~J} 2-11$ & 4 & 29.8 & 4.8 \\
\hline IIIb & $14 \mathrm{~L} 1-22$ & 9 & 52.2 & 7.8 \\
\hline IIIb & $12 \mathrm{~L}-4$ & 2 & 39.3 & 7.2 \\
\hline IIIa/b & $628-9$ & 9 & 40.1 & 6.0 \\
\hline $\mathrm{IIIa} / \mathrm{b}$ & $613-1$ & 9 & 32.4 & 6.2 \\
\hline $\mathrm{IIIa} / \mathrm{b}$ & $290-6$ & 1 & 39.6 & 5.5 \\
\hline IIIa/b & $270-11$ & 9 & 54.7 & 5.4 \\
\hline $\mathrm{IIIa} / \mathrm{b}$ & $26 \mathrm{O}-4$ & 9 & 60.2 & 8.2 \\
\hline $\mathrm{III} a / \mathrm{b}$ & $12 \mathrm{~N}-12$ & 4 & 61.3 & 8.0 \\
\hline IIIa & $710-7$ & 9 & 25.4 & 5.5 \\
\hline IIIa & 37Q2A-1 & 4 & 39.9 & 6.6 \\
\hline IIIa & 37Q1D-6 & 1 & 36.7 & 4.3 \\
\hline IIIa & 37P1D-8 & 9 & 39.9 & 6.8 \\
\hline IIIa & $36 \mathrm{~N}-17$ & 4 & 31.2 & 6.7 \\
\hline IIIa & $33 \mathrm{P} 1-1$ & 9 & 29.6 & 5.5 \\
\hline IIIa & $33 \& 32 \mathrm{P} 2-8$ & 1 & 50.2 & 11.1 \\
\hline IIIa & $32 \mathrm{R} 2-2$ & 1 & 37.0 & 6.3 \\
\hline IIIa & $32 \mathrm{P} 1-1$ & 4 & 36.0 & 6.2 \\
\hline IIIa & 31Q2-11 & 9 & 51.0 & 9.8 \\
\hline IIIa & $30 S 1-1$ & 1 & 50.7 & 13.3 \\
\hline IIIa & $30 Q 2-4$ & 5 & 32.8 & 7.0 \\
\hline IIIa & $28 \mathrm{P} 2-4$ & 5 & 35.6 & 6.4 \\
\hline IIIa & 27R1-6 & 1 & 70.2 & 10.2 \\
\hline IIIa & 27R1-2 & 9 & 36.8 & 6.9 \\
\hline IIIa & 25Q1-7 & 9 & 43.8 & 6.0 \\
\hline IIIa & $25 \mathrm{P} 2-5$ & 9 & 34.8 & 4.4 \\
\hline IIIa & 24Q2-2 & 9 & 33.9 & 5.7 \\
\hline IIIa & $17 S 1-1$ & 2 & 35.9 & 6.8 \\
\hline IIIa & 17Q1-2 & 2 & 35.5 & 13.0 \\
\hline IIIa & $16 \mathrm{R} 2-5$ & 9 & 50.7 & 10.4 \\
\hline IIIa & $15 \mathrm{Q} 2-2$ & 1 & 30.7 & 4.7 \\
\hline IIIa & $15 \mathrm{Q} 2-1$ & 9 & 33.3 & 4.2 \\
\hline II/IIIa & $995-2$ & 4 & 32.5 & 3.0 \\
\hline II/IIIa & 31T2A-1 & 9 & 48.9 & 7.1 \\
\hline II/IIIa & $25 \mathrm{~T} 1 \mathrm{C}-3$ & 1 & 46.1 & 10.0 \\
\hline II/IIIa & 12Q-6 & 4 & 55.4 & 7.4 \\
\hline II & OR585 & 4 & 60.4 & 10.3 \\
\hline II & 34 U2A-1 & 4 & 30.4 & 8.0 \\
\hline II & 29V1A-1 & 1 & 0.5 & 5.6 \\
\hline II & $1685-3$ & 1 & 45.0 & 7.5 \\
\hline $\mathrm{Id} / \mathrm{II}$ & $32 Y 1 C-2$ & 9 & 50.4 & 7.4 \\
\hline $\mathrm{Id} / \mathrm{II}$ & $28 \times 2 A-1$ & 2 & 33.7 & 3.3 \\
\hline $\mathrm{Id} / \mathrm{II}$ & $14 \mathrm{U} 2-4$ & 4 & 31.3 & 5.8 \\
\hline Isi-c/II & OR456 & 1 & 59.9 & 6.6 \\
\hline$\underline{\mathrm{Icl} / \mathrm{Isi}-\mathrm{c}}$ & 33GG1C-1 & 9 & 15.0 & 20.0 \\
\hline Valley Floor B: & & & & \\
\hline IIIC & $51 \mathrm{~KB}-4$ & 2 & 54.3 & 6.2 \\
\hline III & $51 \mathrm{TC}-3$ & 9 & 41.6 & 5.4 \\
\hline III & 51OD-2 & 9 & 30.85 & 2.6 \\
\hline$\underline{\text { II/III }}$ & 50WC-2 & 9 & 50.5 & 4.7 \\
\hline $\begin{array}{l}\text { Valley Margin: } \\
\text { IIIC }\end{array}$ & $1206-42$ & 9 & 197 & 40 \\
\hline
\end{tabular}


Table 16-49, continued

\begin{tabular}{l|l|c|c|c} 
Unit & Specimen No. & Blank & Length & Thickness \\
\hline IIIb/c & $11 \mathrm{H}-7$ & 9 & 29.8 & 3.1 \\
IIIb & $21 \mathrm{~L}-28$ & 2 & 36.0 & 12.5 \\
Y & $6 \mathrm{~K}-4$ & 4 & 26.2 & 5.5 \\
Y & $1 \mathrm{G}-8$ & 2 & 60.4 & 10.0 \\
X/IIIb & $20 \mathrm{M}-5$ & 9 & 29.1 & 4.1 \\
X/Y & $8 \mathrm{M}-3$ & 2 & 45.4 & 7.5 \\
X/Y & $18 \mathrm{M}-7$ & 1 & 38.0 & 4.7 \\
X/II/IIIa & $20 \mathrm{Q}-10$ & 9 & 47.0 & 4.6 \\
X & 6Q-2 & 2 & 41.1 & 4.3 \\
X & $19 \mathrm{Q}-1$ & 9 & 37.2 & 3.6 \\
II & $11 \mathrm{~S}-4$ & 1 & 37.0 & 4.9 \\
\hline \hline
\end{tabular}

TABLE 16-50

Provenience and Measurements of Group 3 Spalls $(\mathrm{N}=31)$

\begin{tabular}{|c|c|c|c|c|}
\hline Unit & Specimen No. & Blank & Length & Thickness \\
\hline $\begin{array}{l}\text { Surface } \\
\text { Surface } \\
\end{array}$ & $\begin{array}{l}0-237 \\
0-238 \\
\end{array}$ & $\begin{array}{l}2 \\
9 \\
\end{array}$ & $\begin{array}{c}0.4 \\
34.8 \\
\end{array}$ & $\begin{array}{l}4.1 \\
3.8 \\
\end{array}$ \\
\hline $\begin{array}{l}\text { Valley Floor A: } \\
\text { IIIc } \\
\text { IIIc } \\
\text { IIIb } \\
\text { IIIa/b } \\
\text { IIIa/b } \\
\text { IIIa/b } \\
\text { IIIa } \\
\text { IIIa } \\
\text { IIIa } \\
\text { IIIa } \\
\text { IIIa } \\
\text { IIIa } \\
\text { II/IIIa } \\
\text { II/IIIa } \\
\text { II/IIIa } \\
\text { II } \\
\text { II } \\
\text { II } \\
\text { Isi/Icl }\end{array}$ & $\begin{array}{l}176-3 \\
16 \mathrm{C}-6 \\
37 \mathrm{MA}-10 \\
26 \mathrm{O}-3 \\
37 \mathrm{NC}-10 \\
30 \mathrm{O}-20 \\
985-7 \\
37 \mathrm{O} 1 \mathrm{D}-8 \\
28 \mathrm{R} 2-5 \\
37 \mathrm{P} 2 \mathrm{C}-4 \\
23 \mathrm{P}-12 \\
15 \mathrm{R} 2-4 \\
28 \mathrm{~S} 1-3 \\
33 \mathrm{~S} 2 \mathrm{~B}-4 \\
23 \mathrm{Q}-13 \\
37 \mathrm{U} 1 \mathrm{~A}-1 \\
29 \mathrm{X} 2 \mathrm{~A}-1 \\
27 \mathrm{U} 2 \mathrm{~B}-1 \\
16 \mathrm{HH} 2 \mathrm{~B}-1\end{array}$ & $\begin{array}{l}2 \\
1 \\
1 \\
2 \\
1 \\
1 \\
9 \\
9 \\
2 \\
1 \\
9 \\
1 \\
9 \\
9 \\
1 \\
2 \\
1 \\
2 \\
9\end{array}$ & $\begin{array}{c}44.8 \\
44.2 \\
46.1 \\
35.2 \\
0.4 \\
43.7 \\
39.6 \\
42.7 \\
33.0 \\
34.4 \\
35.3 \\
53.6 \\
31.1 \\
40.0 \\
45.4 \\
55.1 \\
47.8 \\
50.4 \\
56.5\end{array}$ & $\begin{array}{c}6.3 \\
4.9 \\
10.6 \\
9.5 \\
4.7 \\
5.8 \\
12.9 \\
9.5 \\
10.0 \\
5.9 \\
4.5 \\
11.4 \\
6.8 \\
6.5 \\
6.7 \\
12.3 \\
5.1 \\
13.3 \\
8.9\end{array}$ \\
\hline $\begin{array}{l}\text { Valley Floor B: } \\
\text { III } \\
\end{array}$ & $50 \mathrm{MA}-2$ & 9 & 65.3 & 6.8 \\
\hline $\begin{array}{l}\text { Valley Margin: } \\
\text { IIIc } \\
\text { IIIc/pot } \\
\text { IIIb } \\
\text { X/Y/IIIb } \\
\text { X/IIIa } \\
\text { X } \\
\text { X } \\
\text { X } \\
\text { X/II } \\
\end{array}$ & $\begin{array}{l}1 \mathrm{~A}-10 \\
7 \mathrm{~F}-8 \\
20 \mathrm{~L}-7 \\
19 \mathrm{~L}-8 \\
20 \mathrm{O}-26 \\
8 \mathrm{Q}-3 \\
8 \mathrm{Q}-2 \\
19 \mathrm{~V}-1 \\
10 \mathrm{~T}-3\end{array}$ & $\begin{array}{l}4 \\
2 \\
2 \\
2 \\
1 \\
1 \\
9 \\
1 \\
9\end{array}$ & $\begin{array}{l}33.8 \\
33.6 \\
34.5 \\
47.1 \\
54.0 \\
42.0 \\
42.0 \\
70.0 \\
47.2 \\
\end{array}$ & $\begin{array}{c}7.3 \\
7.8 \\
5.4 \\
6.1 \\
10.0 \\
6.6 \\
6.0 \\
10.2 \\
12.2 \\
\end{array}$ \\
\hline
\end{tabular}


TABLE 16-51

Provenience and Measurements of Group 4 Spalls $(\mathrm{N}=52)$

\begin{tabular}{|c|c|c|c|c|}
\hline Unit & Specimen No. & Blank & Length & Thickness \\
\hline Surface & $0-235$ & 9 & 26.1 & 5.5 \\
\hline $\begin{array}{l}\text { Valley Floor A: } \\
\text { IIIc } \\
\text { IIIc } \\
\text { IIIc } \\
\text { IIIb/c } \\
\text { IIIb/c } \\
\text { IIIb } \\
\text { IIIb } \\
\text { IIIa/b } \\
\text { III/a/b } \\
\text { IIII/b } \\
\text { IIIa/b } \\
\text { IIIa } \\
\text { IIIa } \\
\text { IIIa } \\
\text { IIIa } \\
\text { IIIa } \\
\text { IIIa } \\
\text { IIIa } \\
\text { IIIa } \\
\text { IIIa } \\
\text { IIIa } \\
\text { II/IIIa } \\
\text { II/IIIa } \\
\text { II/IIIa } \\
\text { II/IIIa } \\
\text { II/IIIa } \\
\text { II } \\
\text { II } \\
\text { II } \\
\text { II } \\
\text { Isi/Icl }\end{array}$ & $\begin{array}{l}169-10 \\
37 \mathrm{DC}-21 \\
37 \mathrm{BD}-22 \\
37 \mathrm{IC}-8 \\
17 \mathrm{H}-4 \\
649-1 \\
14 \mathrm{~L} 2-6 \\
26 \mathrm{O}-5 \\
628-12 \\
14 \mathrm{M} 2-3 \\
12 \mathrm{~N}-11 \\
\text { OR395 } \\
34 \mathrm{P} 1-7 \\
599-3 \\
36 \mathrm{O}-7 \\
36 \mathrm{~N}-24 \\
32 \mathrm{P} 2-7 \\
30 \mathrm{~S} 2 \mathrm{D}-6 \\
25 \mathrm{P} 2-2 \\
16 \mathrm{R} 1-2 \\
13 \mathrm{P}-6 \\
\text { 29S2-11 } \\
35 \mathrm{~T} 2 \mathrm{~B}-2 \\
33 \mathrm{~T} 1 \mathrm{~B}-5 \\
\text { 24R2-4 } \\
\text { 24R2-11 } \\
\text { 32X2C-6 } \\
\text { 32X2B-1 } \\
\text { 15U1-1 } \\
14 \mathrm{~T} 1-4 \\
\text { 37OO2B-5 } \\
\end{array}$ & $\begin{array}{l}9 \\
4\end{array}$ & $\begin{array}{c}32.5 \\
30.7 \\
26.8 \\
36.0 \\
39.5 \\
29.7 \\
21.2 \\
26.2 \\
19.0 \\
25.6 \\
35.4 \\
0 \\
28.9 \\
22.5 \\
30.0 \\
23.1 \\
39.8 \\
32.4 \\
20.4 \\
31.7 \\
31.9 \\
38.4 \\
24.7 \\
36.0 \\
.34 .4 \\
29.7 \\
24.5 \\
56.1 \\
20.5 \\
20.3 \\
27.0 \\
\end{array}$ & $\begin{array}{c}10.0 \\
3.6 \\
3.7 \\
7.4 \\
4.7 \\
4.2 \\
5.5 \\
4.6 \\
2.4 \\
5.9 \\
6.0 \\
0 \\
4.8 \\
5.4 \\
4.7 \\
4.5 \\
5.4 \\
4.1 \\
4.3 \\
5.0 \\
4.3 \\
8.4 \\
3.5 \\
6.0 \\
6.5 \\
3.3 \\
5.0 \\
10.0 \\
5.1 \\
5.0 \\
16.0 \\
\end{array}$ \\
\hline $\begin{array}{l}\text { Valley Margin: } \\
\text { IIIc } \\
\text { IIIc } \\
\text { IIIc } \\
\text { IIIb/c } \\
\text { Y/IIIc } \\
\text { IIIb } \\
\text { IIIb } \\
\text { Y/IIIb } \\
\text { Y } \\
\text { Y } \\
\text { Y } \\
\text { X/IIIb } \\
\text { X/Y } \\
\text { X/Y } \\
\text { IIIa/b } \\
\text { IIIa/b } \\
\text { II/IIIIa } \\
\text { X } \\
\text { X } \\
\text { X } \\
\end{array}$ & $\begin{array}{l}9 \mathrm{~A}-28 \\
1265-15 \\
1265-14 \\
21 \mathrm{H}-10 \\
6 \mathrm{H}-15 \\
20 \mathrm{~L}-12 \\
11 \mathrm{~K}-7 \\
9 \mathrm{~K}-3 \\
1560-6 \\
1560-5 \\
6 \mathrm{~K}-3 \\
10 \mathrm{~L}-7 \\
2 \mathrm{~K}-4 \\
18 \mathrm{~L}-4 \\
22 \mathrm{M}-7 \\
22 \mathrm{M}-6 \\
22 \mathrm{P}-13 \\
6 \mathrm{Q}-1 \\
3 \mathrm{M}-3 \\
18 \mathrm{UC}-1 \\
\end{array}$ & $\begin{array}{l}9 \\
9 \\
9 \\
4 \\
4 \\
4 \\
4 \\
1 \\
9 \\
4 \\
4 \\
2 \\
2 \\
2 \\
4 \\
4 \\
1 \\
4 \\
2 \\
4 \\
\end{array}$ & $\begin{array}{l}23.6 \\
17.0 \\
13.3 \\
43.5 \\
21.8 \\
49.4 \\
45.1 \\
40.9 \\
14.2 \\
23.0 \\
31.8 \\
40.1 \\
26.3 \\
21.6 \\
50.8 \\
47.0 \\
40.7 \\
46.6 \\
39.6 \\
30.3 \\
\end{array}$ & $\begin{array}{l}4.5 \\
3.6 \\
1.6 \\
10.0 \\
3.6 \\
7.5 \\
5.1 \\
6.0 \\
3.2 \\
4.0 \\
6.1 \\
6.7 \\
6.0 \\
4.2 \\
3.8 \\
7.4 \\
4.4 \\
5.4 \\
7.7 \\
7.2 \\
\end{array}$ \\
\hline
\end{tabular}


TABLE $16-52$

Provenience and Measurements of Indeterminate Type Spalls $(\mathrm{N}=18)$

\begin{tabular}{l|l|c|c|c}
\hline Unit & Specimen No. & Blank & Length & Thickness \\
\hline Valley Floor A: & & & & \\
IIIb/c & $513-1$ & & 31.4 & 5.0 \\
IIIb & $592-6$ & & 17.0 & 5.5 \\
IIIb & $580-1$ & & 31.7 & 6.1 \\
IIIb & 12 L-12 & & 46.8 & 8.0 \\
IIIa/b & $613-2$ & & 27.5 & 3.7 \\
IIIa/b & 613 & & 32.4 & 6.0 \\
IIIa/b & $17 O 1-7$ & & 26.5 & 6.1 \\
IIIa & 853 & & 14.0 & 3.3 \\
IIIa & $599-1(4) ?$ & & 28.2 & 8.1 \\
IIIa & $582-1$ & & 15.0 & 3.7 \\
IIIa & 36P-6 & & 23.9 & 4.4 \\
IIIa & 36O-5 & 4 & 64.2 & 8.6 \\
IIIa & 28Q2-11 & & 37.4 & 5.2 \\
II & 35AA1D-1 & & 15.5 & 3.5 \\
II & 23S-5 & & 62.4 & 5.9 \\
Isi & 124-6 & & 5.3 \\
Valley Margin: & & & & \\
IIIc & 3A-8 & & 65.5 & 11.7 \\
X & 5Q-1 & & 42.0 & 4.6 \\
\hline \hline
\end{tabular}




\section{Chapter 17}

\section{UNIFACIAL TOOLS}

by Keith L. Prilliman and C. Britt Bousman

\section{INTRODUCTION}

A total of 4,247 formal unifaces and edge-modified flakes was recovered from the TARL and TxDOT excavations at the Wilson-Leonard site. Eighty-two percent $(n=$ 3,467 ) were analyzed, and the results are reported in this chapter (proveniences of all are presented in Appendixes 4 and 5). Two levels of analysis were undertaken. Fully analyzed specimens include 736 of the 747 unifacial tools excavated by TARL and 355 of the 3,500 unifacial tools excavated by TxDOT. A more-limited set of attributes was collected from 2,376 of the TxDOT collection (see discussion of the sample, below). This treatment of the collection was adopted in order to direct the effort toward fuller analysis of those parts of the assemblage with the tightest stratigraphic controls. For the TxDOT portion of the collection, the artifacts from stratigraphic Units I and II received the greater emphasis in this study.

Formal unifaces, as defined below, are sufficiently distinctive to be reliably sorted during laboratory processing, but edge-modified flake tools are less distinctive, and some with minimal edge modification probably escaped recognition during initial laboratory sorting. No serious effort was spent in this analysis toward identifying microscopic traces of wear such as polish or striations. Therefore, the total number of edge-modified flake tools is unknown for this large assemblage. Numerous researchers assume that the edge-modified flakes are expedient tools, but expediency is not a simple concept. For example, Nelson (1991) has argued that expediency as discussed by archeologists is actually two separate strategies: true expedient technological strategies and opportunistic technological behaviors. The latter is an unplanned response to an unanticipated need. Nelson (1991) suggests that expedient technology is a planned response to an expected task incorporating minimal preparation of tools, short period of use, and artifact discard at the activity locus.
Often, edge-modified flakes or so-called "expedient" tools are analyzed minimally or neglected in archeological reports in favor of more in-depth analyses of temporally and functionally diagnostic chipped stone artifacts such as projectile points, knives, adzes, and the like. Usually, edgemodified flakes in these assemblages are lumped into gross categories variously termed "trimmed," "utilized," or "retouched" flakes, and merely counted. Others, such as Forrester (1996) and Goodyear (1974), include these artifacts in techno-functional categories without microwear confirmation of their functional status. Judge (1973) separates some forms of edge-modified flakes into functional categories (e.g., flake knives, spokeshaves) while continuing to address others as either "modified" or "utilized" forms present in the debitage sample. More recent studies, such as those by Black (1986), Robinson and Turpin (1993), Fields (1984), and Tomka and Fields (1990), make a more systematic attempt at analyzing these artifacts while avoiding functional descriptors, though none has samples approaching the size of the Wilson-Leonard assemblage.

Theoretical models regarding these ad hoc tool forms have been advanced by Kuhn (1994) and Bousman (1993), among others. Kuhn's optimization model posits that the choices made regarding mobile toolkits are driven by economic decisions, specifically those of obtaining the greatest potential utility from a tool-kit while expending the least total weight cost. Bousman (1993) explores hunter-gatherer economic risk, and then models hunter-gatherer technological strategies designed to cope with various forms and degrees of economic risk. He also offers a general model of analysis geared toward identification of forager-collector technological strategies in terms of tool design, use-life, and production costs. In addition to these models, other analysts have attempted to address flake or unifacial tool forms by means of indexes (Kuhn 1990), or transformation of scraper "types" via resharpening and reduction (Dibble 1987), or by using statistical manipulations (Fish 1979; Wilmsen 1970). 
This study of the edge-modified flake and unifacial chipped stone tool assemblage recovered from the WilsonLeonard site makes no assumptions regarding the functions of these tools but instead presents a morphological description that permits a future investigation of function. There is considerable data potential in an assemblage this large. These artifacts are more than just nondiagnostic chipped stone tools. They reflect past human behavior and offer insight into changing small-tool-use patterns over a temporally significant duration of North American prehistory.

\section{ANALYTICAL APPROACHAND TERMINOLOGY}

In this study, definitions, descriptions, and preliminary interpretations of the unifacial and edge-modified flake tools are presented. Descriptive categories based on form are followed by an analysis of flake attributes and tool types. Summary provenience data are provided in this chapter, while specific artifact provenience is reported in Appendix 5.

A sample of tools was selected for analysis, and various categorical and metric observations were made. Due to the size and complexity of the artifact sample, not all observations were made on all artifacts (see Sample Selection below). The attributes defined below include artifact form, flake type, dorsal surface cortex, location and number of modified edge segments, retouch morphology, edge morphology, edge angle, and raw material. The metric attributes are maximum axial dimensions, thickness, total length of modified edge, edge length available for modification, and flake circumference. Figure 17-1 illustrates these measurements.

A number of these variables (e.g., location and number of modified edge segments, edge morphology) are drawn from Knudson's (1983:10) concept of "employable unit," defined as "that implement segment or portion (continuous edge or projection) deemed appropriate for use in performing a specific task." This term allows convenient discussion of relevant edge morphology and is especially useful when discussing complex morphologies such as those found on multiple tools.

\section{Sample Selection}

A total of 3,467 unifacial tools and edge-modified flakes was analyzed in this study. It was not feasible to conduct a detailed analysis on all of the unifacial tools recovered, therefore a nonrandom sampling strategy was adopted to collect metric and other data. Sixty-eight percent $(2,376)$ of the artifacts recovered from the general TxDOT excavations was subjected to a minimal level of classification consisting of tool type, flake type, raw material, and retouch morphology.

A total of 736 unifaces and edge-modified flake tools recovered from excavations conducted by TARL was analyzed using the attributes listed above. This amounts to

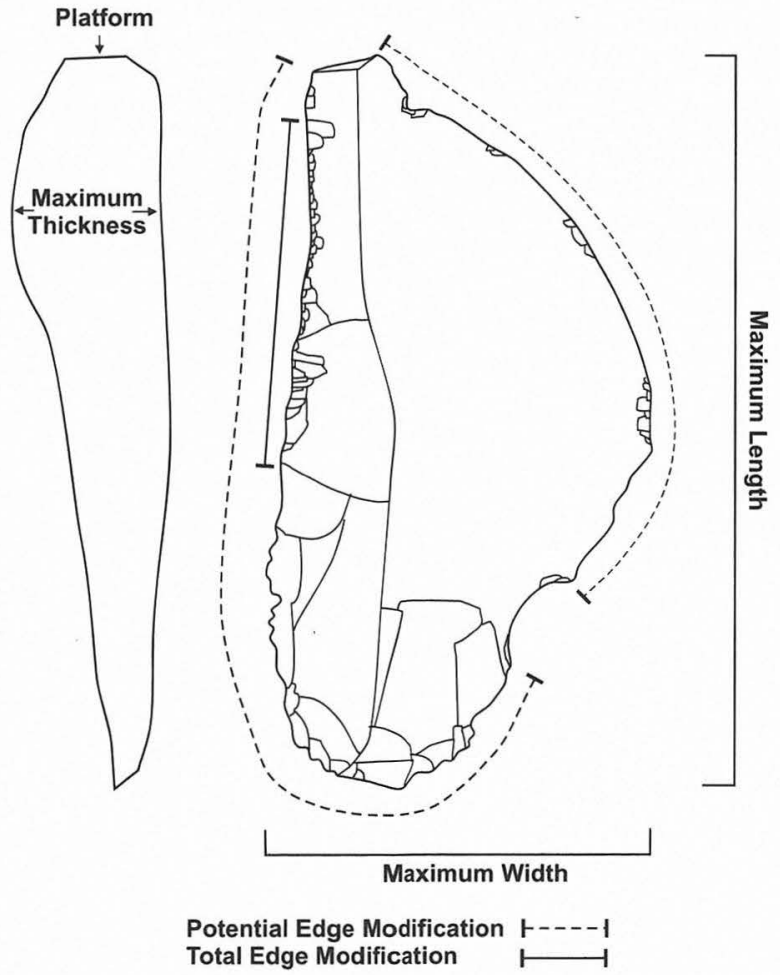

FIGURE 17-1. Areas measured on unifaces and modified flakes.

$21.2 \%$ of the analyzed sample. In addition to the TARL-collected artifacts, 355 artifacts recovered from the TxDOT fine screen unit (E28/S78) and other nonrandomly selected TxDOT artifacts, representing $10.2 \%$ of the analyzed sample, were analyzed using these same attributes. Artifact totals in summary tables vary due to missing data on individual specimens. These missing data are due to breakage of individual artifacts.

\section{Artifact Classes}

Ten classes of unifacial and edge-modified flake artifacts are identified in this study. Within the unifacial tool classes are thick unifaces, thin unifaces, edge-modified flakes, retouched blades, denticulates, microspurs, microdenticulates, notches, spurs, and "multiple tools" (i.e., those with more than one morphologically unique working surface). In more functionally defined taxonomies such as those developed by Frison and Bradley (1980) for Folsom stone tools, or Goodyear (1995) for Dalton tools, or the morphofunctional typology developed by Bordes (1961) for Middle Paleolithic flake tools, many of the artifact categories defined in this chapter would be called scrapers, planes, knives, gravers, burins, or spokeshaves. Microwear analysis has suggested that such functional classification systems may not accurately represent the possible tasks to which a particular stone tool may be applied. For the purposes of this study, these terms were avoided in the 
classification systematics. Only where microwear analysis allows a functional assignment with clear linkage between microwear traces and inferred prehistoric use should such terms be employed. This is of particular importance when dealing with assemblages such as this that contain large numbers of apparently multifunctional stone tools.

Nonfunctional typologies (indeed typologies of these artifact forms in general) are rare in the literature. Therefore, a framework based in part on previous work by Collins (1974, 1979), as well as those studies prepared by Black (1986), Robinson and Turpin (1993), Fields (1984), and Tomka and Fields (1990), among others, was adopted for use on the Wilson-Leonard assemblage. The majority of flake type definitions are adapted from Collins (1974) and supplemented by analyses contained in Boisvert et al. (1979). Artifact classes were established after inspection of the assemblage and were morphologically grouped as discussed above. Generic artifact class definitions are provided below.

\section{Flake Type}

Unifacial and edge-modified flake tools were classified according to the flake type of the tool blank. Flake type is defined by the combination of commonly identified platform types and flake characteristics. Four flake types were used: biface thinning, normal, sequent, and indeterminate. Typical biface thinning flakes are thin and curved, with dispersed bulbs of percussion, and they may or may not have lipped platforms. Platforms on biface thinning flakes are usually well prepared, exhibit multiple facets, may be lipped, and may retain traces of edge grinding, although occasional specimens will retain platform cortex on a generally unprepared striking platform. Sequent flakes (Collins 1974) retain a negative flake scar on the dorsal surface indicative of previous flake removal from the same position on the core. Sequent flake platforms may be simple or faceted and usually have a distinctive "winged" shape. These flakes usually have a salient bulb of percussion. Normal flake platforms are generally single faceted with a broad platform, although some also have moderately prepared multiple facet platforms. Normal flake bulbs of percussion range from dispersed to salient. All flake types exhibit a variety of flake terminations, i.e., feather, stepped, hinge, or overshot (Crabtree 1972). Indeterminate flake types have missing or crushed platforms.

\section{Dorsal Surface Cortex}

Five distinctive dorsal cortical states were noted. Flakes with remnant cortex covering the dorsal surface and lacking invasive dorsal flake scars were classified as having primary cortex. Flakes having at least one invasive dorsal flake scar prior to edge modification were categorized as having secondary cortex. Platform cortex was noted on those flakes exhibiting a natural unprepared platform. Those flakes with cortex along a lateral margin were categorized as edge cortex flakes. Those flake types lacking cortex or those flakes where cortex was a trivial factor were classified as noncortex flakes.

\section{Location and Number of Modified Edges}

The location and number of modified edges observed on tool surfaces were recorded on a sample of tools analyzed (see Sample Selection). Location was recorded in terms of left, right, distal, or proximal, with the tool oriented dorsal surface up and the platform positioned proximal to the analyst. Areas of ventral edge modification were noted separately in the same fashion, except with the ventral face up.

\section{Retouch Morphology}

Several categories of edge morphology observed below require brief elaboration. Figure 17-2 illustrates these categories. Regular retouch is defined as edge flaking that has similar macroscopic size, shape, and distribution across a flake margin. Irregular retouch lacks demonstrable regularity in flake scar size, shape, or distribution. Continuous retouch is defined as contiguous serial flaking along flake margins with overlapping regular negative flake scars. Continuous modification may reflect intentional human modification (either sharpening or dulling) of the flake margin, or accretional edge modification acquired through tool utilization, or unidentified postdepositional damage. Discontinuous retouch is defined as noncontiguous areas of edge flaking that do not significantly overlap and may be random single flake detachments or trivial areas of edge modification not longer than $4 \mathrm{~mm}$. Discontinuous retouch clusters are assumed to represent incipient accretional retouch. Single flake random detachments are assumed to represent edge damage not clearly related to human agency (Tringham et al. 1974), although it is possible this damage may also represent accretional chipping acquired during use. Further microscopic examination of these modified areas is necessary to confirm this latter pattern. Invasive retouch is intentional modification that intrudes more than $1 \mathrm{~cm}$ into the flake body. Noninvasive retouch intrudes less than $1 \mathrm{~cm}$ into the flake body and may or may not be intentional.

\section{Edge Morphology}

Simple and complex edge morphologies were noted in this study. Simple edge-morphology categories are concave, convex, and straight areas of retouch and are found on edge-modified flakes, unifaces, and "multiple tools" (see below). These morphological categories represent regular continuous units of edge modification unless otherwise noted. Complex morphologies involve a combination of distinctive tool edge profiles such as denticulate, notch, spur, or burin with another simple or complex morphologic 


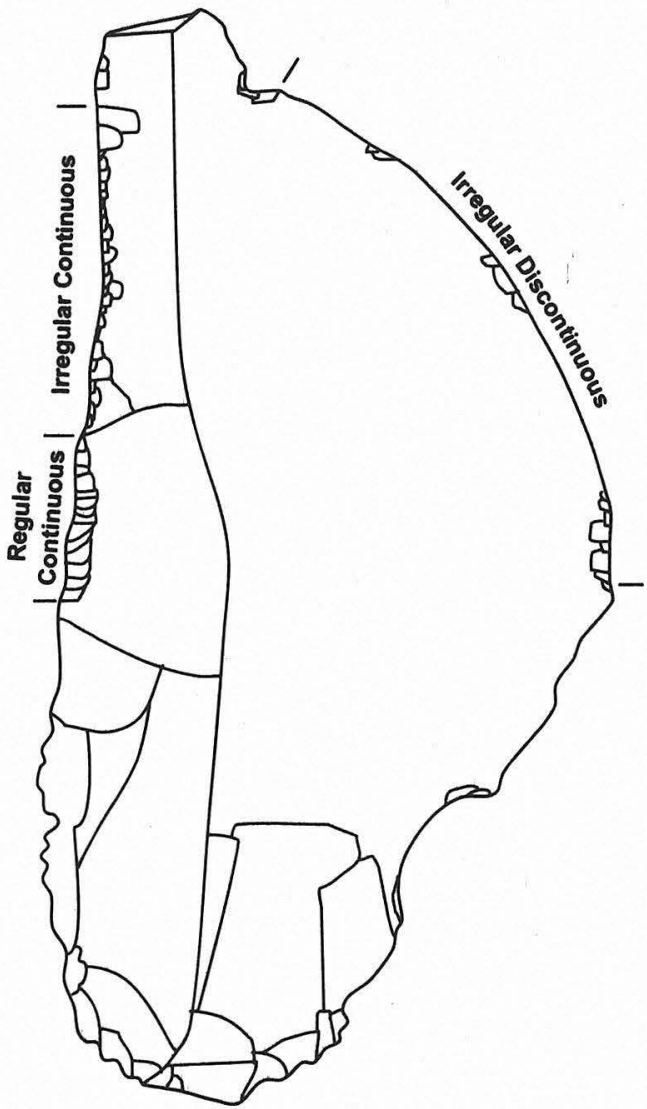

FIGURE 17-2. Examples of edge retouch on unifaces and edgemodified flakes.

unit. Multiple notches and multiple spurs, or concave/convex invasive modification are examples of complex morphologies. Multiple tools, or those artifacts where multiple tool morphologies are present, represent the typical example of complex edge morphologies. Where modified edges differ morphologically along the same flake margin, each separate morphology is recorded.

\section{Edge Angle}

Edge angle was recorded as either steep (greater than $45^{\circ}$ ) or shallow ( $45^{\circ}$ or less). This angle corresponds to the spine plane angle defined by Tringham et al. (1974). Due to difficulty in obtaining accurate edge angle measurements along the extent of edge modification, this distinction is an approximation. Where tools were observed with multiple modified edge segments, the predominant edge angle estimate was recorded unless significant variation between modified edges was noted. In these cases, separate edge angle estimates were recorded.

\section{Metric Variables}

Length, width, and thickness were collected on the subset selected for complete analysis (see Sample Selec- tion). Maximum length and width to the nearest $0.1 \mathrm{~mm}$ were measured with dial calipers on the unifacial tools in this sample by orienting the tool platform down, dorsal side facing the analyst. Thickness was obtained on the point of maximum thickness perpendicular to the long and intermediate axes of the tool. Additional measurements (see Figure 17-1) were made on modified edges of 458 specimens selected nonrandomly (see Sample Selection). All sampled modified edges were measured to the nearest $1 \mathrm{~mm}$ using a cloth tape measure. The first variable, length of modified edge, is the sum of the length of all modified edges for each tool. The second variable, potential edge length available for modification, is defined as the total flake margin available for retouch or utilization. When broken flake margins were encountered, fracture length was excluded from these measurements. Finally, the total flake circumference was obtained on each of these artifacts.

\section{RESULTS}

\section{Artifact Descriptions and Descriptive Statistics}

\section{Thick Unifaces $(N=74)$}

Thick unifaces are defined for the purposes of this study as those flaked stone tools that exhibit extensive, continuous, invasive (greater than $1-\mathrm{cm}$ intrusion into body), and regular retouch over one surface of the tool and have a maximum thickness of $15 \mathrm{~mm}$ or more. Artifacts in this class have three or more rows of overlapping intentional marginal flaking, which often completely alters the original flake outline. It is this substantial amount of invasive modification and alteration of flake margins that distinguishes the uniface category from simple edge-modified flakes. Most thick unifaces have convex retouch, although a small number have straight, concave, or somewhat denticulated edges. The great majority have modification on their dorsal surfaces, but a few have flake removals on the ventral surface. The retouch angle in a majority of cases is steep. Seven thick uniface fragments were identified. Descriptive statistics are given in Table 17-1 and examples are shown in Figures 17-3 and 17-4.

Thick unifaces are almost exclusively made on large cortical flakes. These tools tend to be more simply made, exhibiting fewer, though often times much larger, dorsal flake scars forming the employable unit. In Figure 17-3a, the specimen shows total edge modification with steep proximal and distal edge angles. More representative of this artifact category is the specimen shown in Figure 17-3b. This specimen shows more-random flaking and possibly was discarded as unsuitable for further modification. Convergent-edged specimens like that illustrated in Figure 17-3c are also found. Note the very steep convergent edge angles and extensive invasive flaking on the ventral surface (see Figure 17-3c). Figures 17-4a and 17-4b illustrate typical steep- and convex-edged thick unifaces. Figure $17-4 \mathrm{c}$ illustrates a thick uniface with a heavily 
TABLE 17-1

Thick Uniface Descriptive Statistics ( $\mathrm{n}=16$; in $\mathrm{mm}$ )

\begin{tabular}{l|c|c|c|c|c}
\hline & Length & Width & Thickness & $\begin{array}{c}\text { Total } \\
\text { Modified Edge }\end{array}$ & $\begin{array}{c}\text { Potential } \\
\text { Modified Edge }\end{array}$ \\
\hline Minimum & 42 & 33 & 16 & 30 & 70 \\
Maximum & 116 & 115 & 34 & 300 & 366 \\
Range & 74 & 82 & 18 & 270 & 296 \\
Mean & $70 \pm 21$ & $51 \pm 21$ & $20 \pm 5$ & $131 \pm 65$ & $187 \pm 76$ \\
\hline \hline
\end{tabular}

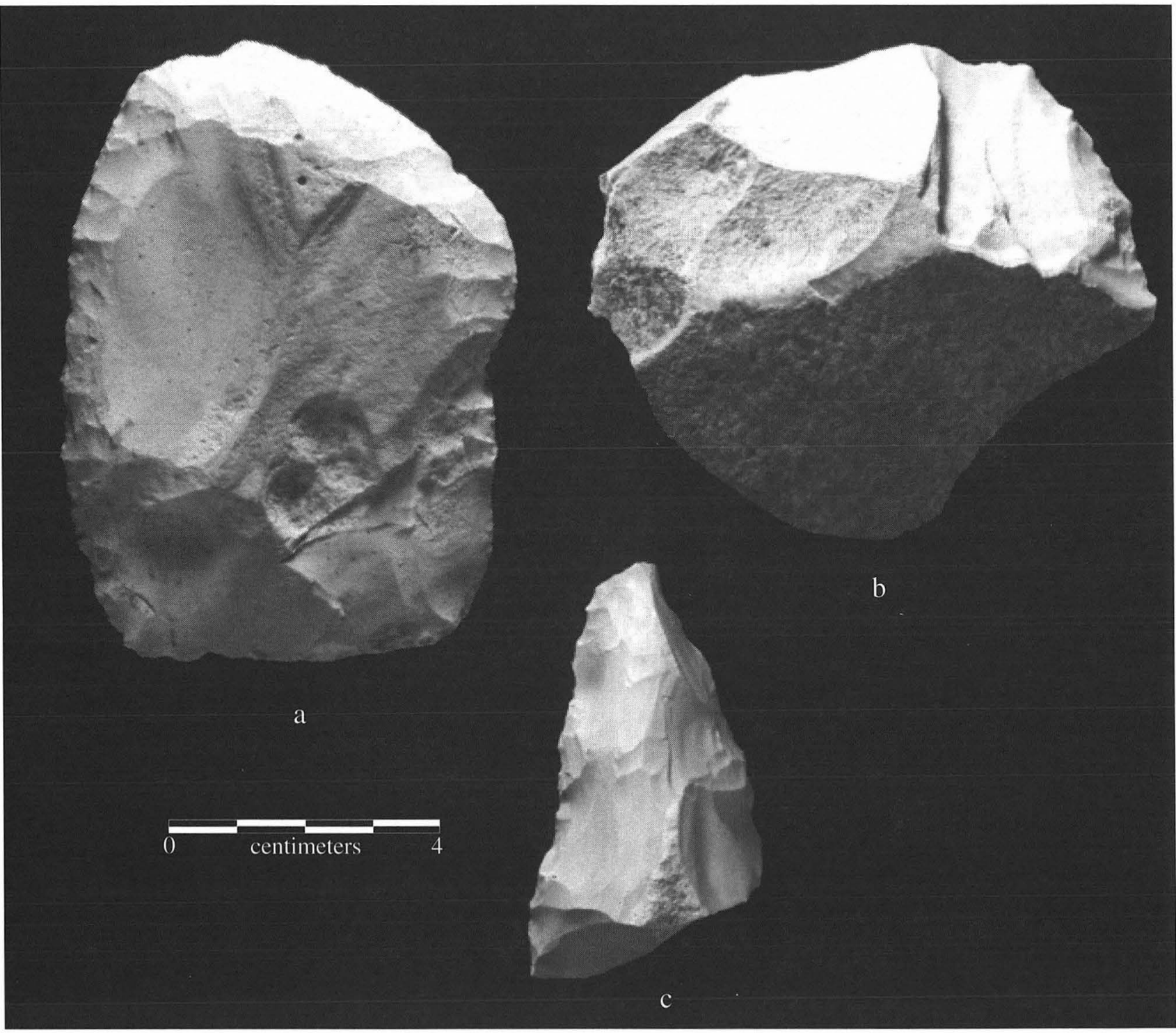

FIGURE 17-3. Thick unifaces. (a) Specimen 17J2-3, note total edge modification with steep distal retouch; (b) 16Q2-9; (c) 30R2-1, dorsal, note convergent steep retouch.

battered proximal margin. Figure 17-4d illustrates an atypical large specimen.

\section{Thin Unifaces $(N=386)$}

Thin unifaces exhibit the same characteristics as thick unifaces except they measure less than $15 \mathrm{~mm}$ in maximum thickness (Table 17-2). Examples are shown in Figures 17-5 through 17-8 (see also Figure 22-5a). Also identified were 229 thin uniface fragments, but these were not subjected to detailed analyses. These are lateral or distal fragments that contain all of the characteristics of the thin unifaces plus show evidence of transverse, lateral, or radial fractures that are usually snap or bend breaks. 

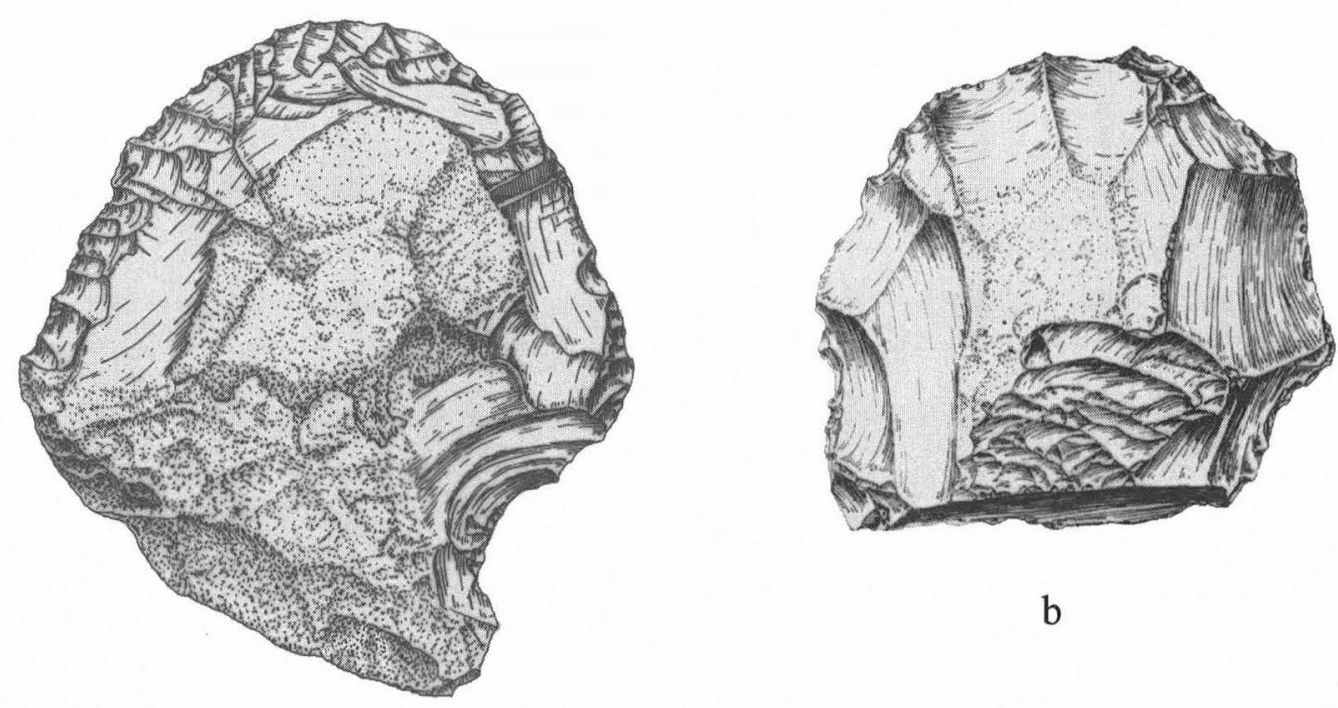

$\mathrm{b}$

a

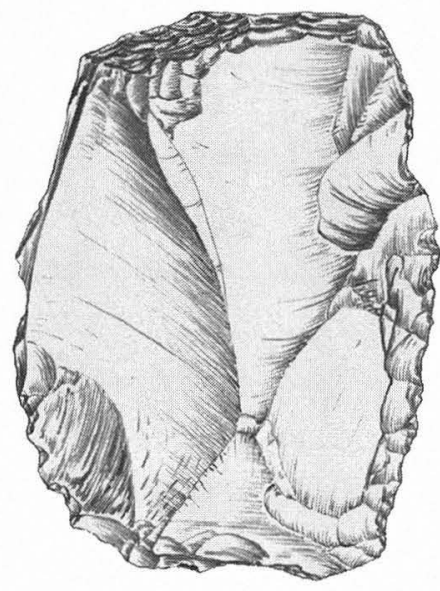

C
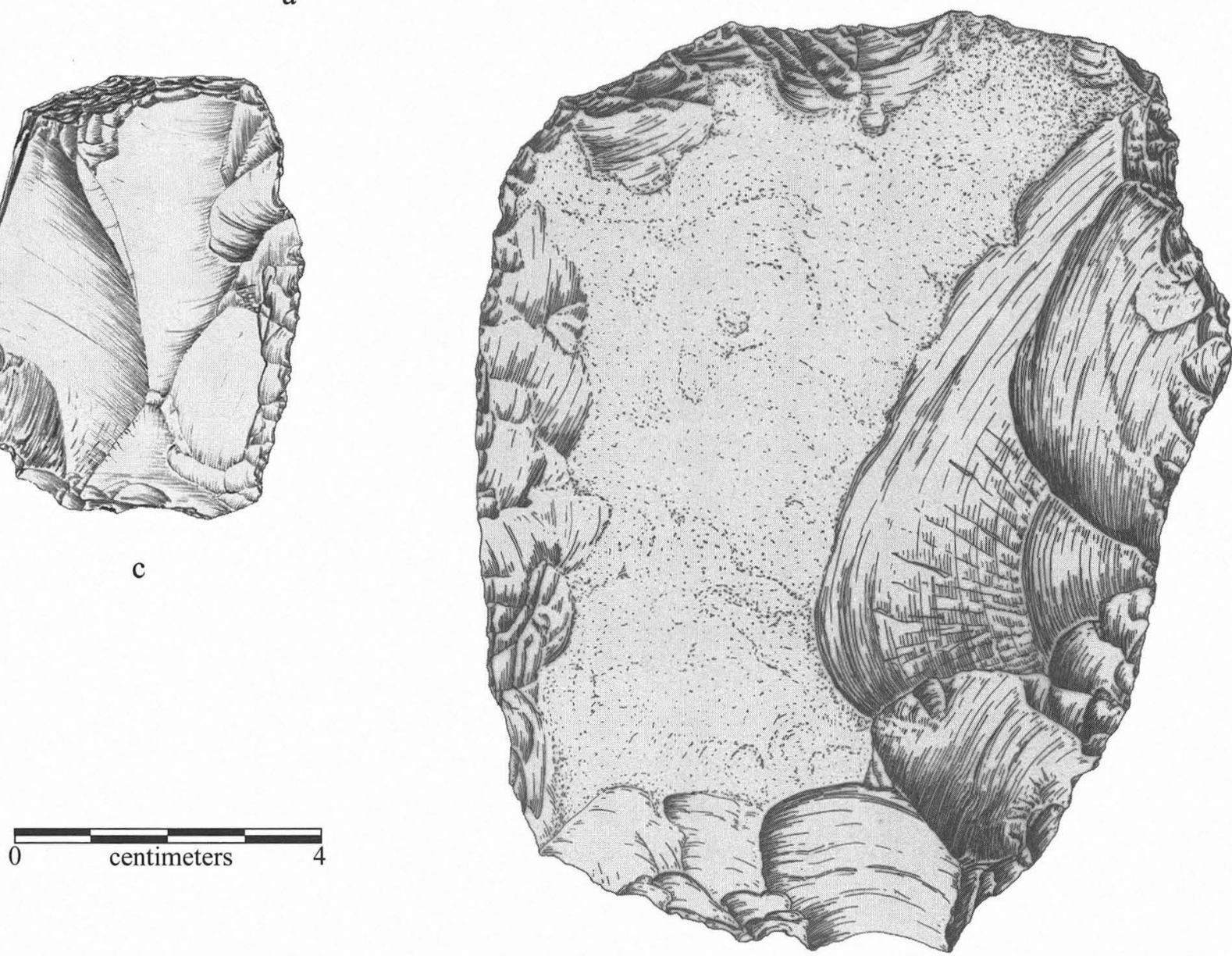

d

FIGURE 17-4. Examples of thick unifaces with modifications (a, c-d) and a multiple tool (b). (a) 22P-28; (b) 17CC2-2; (c) 21R-11; (d) 33S16.

Slightly over half of the thin unifaces have convex edge profiles, approximately a third have straight edges, an eighth have cortex edges, and the remainder have complex edge pro- files. Most have alteration on their dorsal surface, but about $15 \%$ have ventral modification. Most have steep edge angles, but approximately $20 \%$ have more-acute edge angles. 
TABLE $17-2$

Thin Uniface Descriptive Statistics ( $\mathrm{n}=79$; in $\mathrm{mm}$ )

\begin{tabular}{l|c|c|c|c|c}
\hline & Length & Width & Thickness & $\begin{array}{c}\text { Total } \\
\text { Modified Edge }\end{array}$ & $\begin{array}{c}\text { Potential } \\
\text { Modified Edge }\end{array}$ \\
\hline Minimum & 17 & 16 & 4 & 10 & 34 \\
Maximum & 97 & 102 & 14 & 187 & 243 \\
Range & 80 & 86 & 10 & 177 & 209 \\
Mean & $48 \pm 17$ & $38 \pm 15$ & $10 \pm 2$ & $77 \pm 45$ & $119 \pm 49$ \\
\hline \hline
\end{tabular}

\section{Edge-modified Flakes $(N=1,817)$}

Edge-modified flakes are those flakes or flake fragments possessing regular, moderately invasive $(2-9 \mathrm{~mm})$ discontinuous or continuous edge flaking. Edge modification exhibits a range of variables. These artifacts have either irregular, or regular concave, convex, or straight-edge modified margins. The modification does not consist of more than two overlapping rows of edge flaking. Flake outlines may or may not be significantly altered from the original flake shape and exhibit margins that show regularly retouched outlines. Even in those specimens that appear to have significantly altered the edge margins, these are generally much less altered than the thin or thick uniface categories.

Typical edge-modified flake specimens exhibit continuous to discontinuous, irregular, noninvasive areas of edge modification longer than $5 \mathrm{~mm}$, without alteration of flake margins. These artifacts may represent the earlystage accumulation of utilization-related edge damage that has not accreted sufficiently to significantly modify the flake margin. Alternatively, this category may result from random edge damage unrelated to prehistoric utilization. Low-power scanning of these artifacts at $10 \mathrm{X}$ was performed in an attempt to identify postdepositional and/or modern excavation-related edge damage, such as metal streaking or apparently fresh flake scars or fracture surfaces. When possible, artifacts possessing edge damage attributable to these factors and having no other edge modification clearly identifiable as intentional prehistoric modifications were classified as debitage and eliminated from the tool sample.

An additional form recognized during preliminary analysis exhibits edge modification that, without microwear study, is not clearly attributable to either prehistoric human agency or to modern excavation related activities. However, no postdepositional and/or modern excavation-related edge damage was noted on these. These flakes have single or multiple, irregular-shaped, randomly dispersed flake detachments with few unequivocal modified edges noted. Without microwear analysis of these artifacts, their classification as debitage would be premature, however. Metric variables are provided in Table 17-3, and examples of all of these forms are shown in Figures 17-9 through 17-11.

Most edge-modified flakes have straight modifications, while convex and concave are also common. A few complex edge profiles were noted. Most edge-modified flakes also exhibit dorsal modification, but ventral surface modifications were clearly present. Acute angles are common on modified edges.

The artifact shown in Figure 17-10g is noteworthy among the large collection of edge-modified flakes analyzed in this study. It is a noncortex flake with heavy edge grinding present on all lateral margins. Minimal retouch is visible along the distal flake margins; however, the obvious extensive grinding modification clearly sets this specimen aside from the other artifacts. Future use-wear analysis may provide more insight into the function of this and similar modified flakes. Figure 17-9d also shows another unique artifact. Specimen 33R2-10 is a flat, extremely smooth, bipolar-flaked edge-modified tool (possibly a pièce esquillée) made on unidentified nonsiliceous raw material. The surface appears smoothed, with extremely rounded though not faceted lateral margins. Low-power magnification did not reveal microwear traces on this tool (Dale Hudler, personal communication 1996).

At the Peerless Bottoms site, 41HP175, a small sample of edge-modified flakes was analyzed and the authors of that study associated these implements with cutting and light scraping tasks (Klement et al. 1993:188). Fields (1984) also associated cutting and scraping activities with edgemodified flakes at 41LN134 based on observed edge angles. In other studies (Koldehoff 1987; Parry and Kelly 1987; Robinson and Turpin 1993), artifacts such as these have been classified variously as "expedient tools" or labeled as either "retouched" or "utilized" flakes. Microwear analyses conducted by Kay and Driskill (see Chapter 22) provide evidence that, while in specific instances these terms are more or less accurate, they cannot always be applied to an entire morphological class. Some modified edges were found to be areas of intentional backing or dulling, while macroscopically, the actual working surface appeared unmodified. In other instances, macroscopically unmodified debitage yielded positive wear traces consistent with cutting or light scraping activities, while other unmodified flakes were shown to bear traces of hafting wear (see Chapter 22). While it may be questioned that the production of these tools is truly ad hoc, their importance and the apparent reliance on them by the peoples who made and used them is without question. 


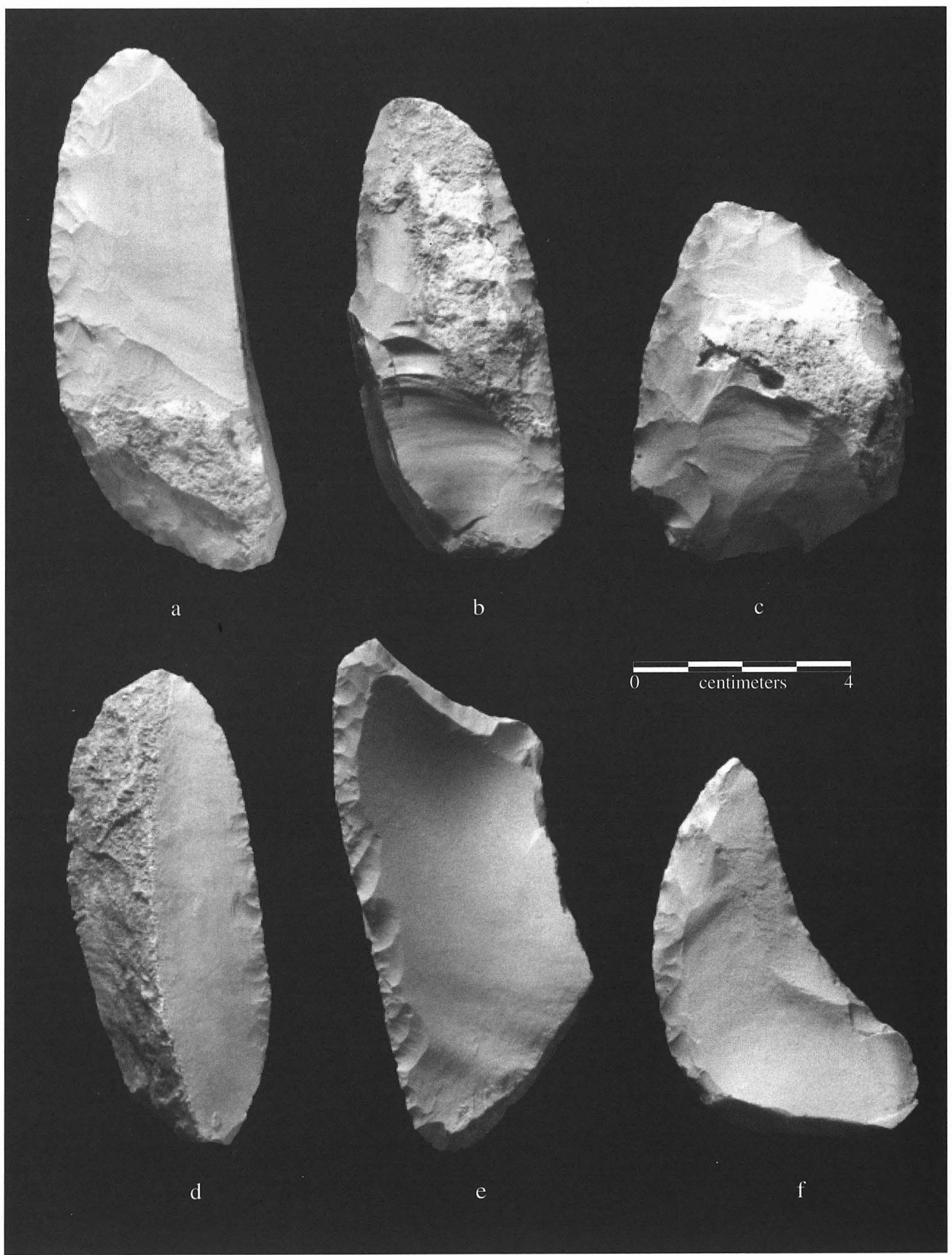

FIGURE 17-5. Thin unifaces. (a) 10K-6; (b) 30Y2A-1; (c) 34R2-2, note total edge modification; (d) 20G-19; (e) 22L-17; (f) 31S1-7. 


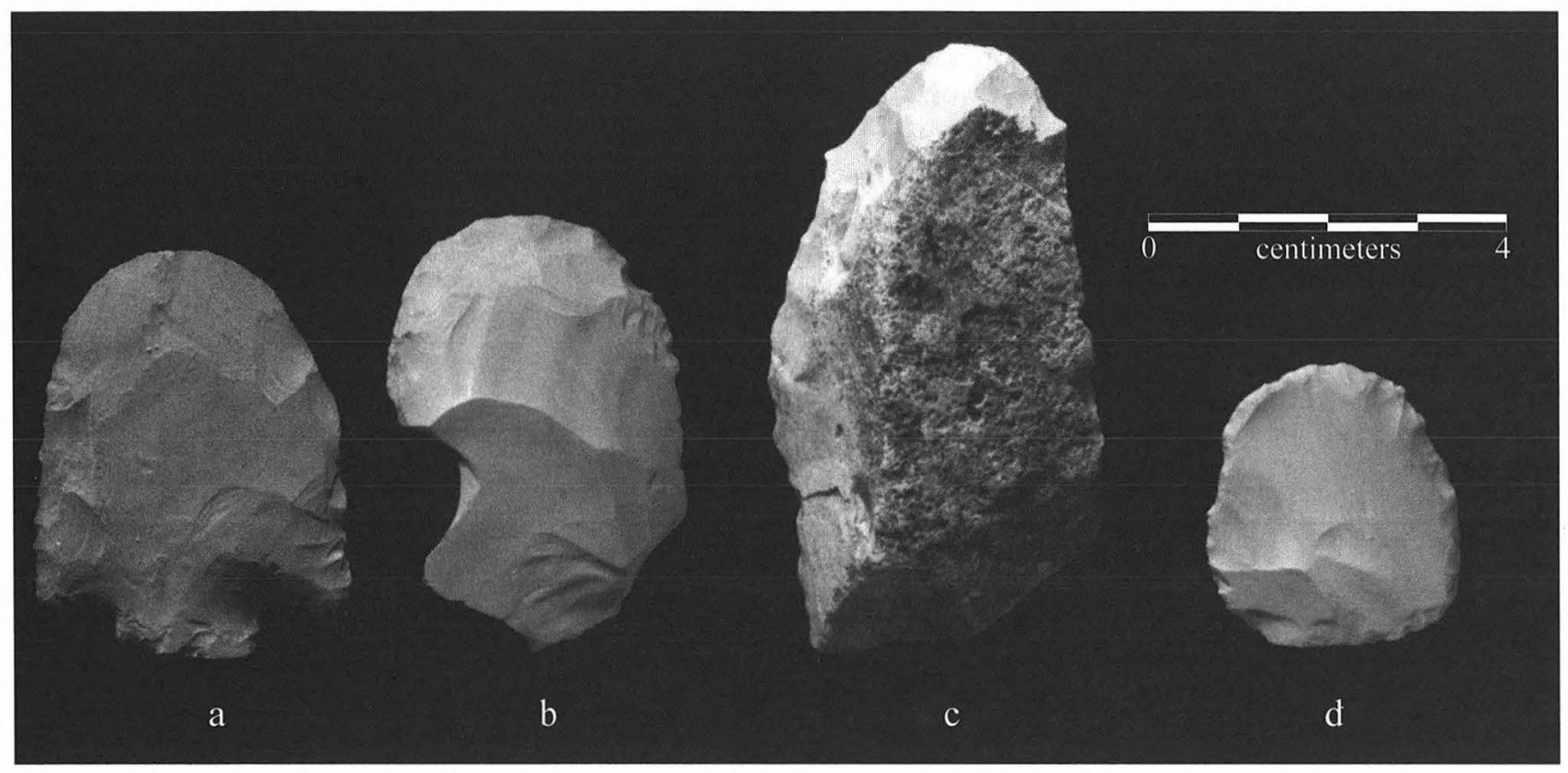

FIGURE 17-6. Thin (a, b) and thick (c) unifaces. (a) 24Z1D-3, formalized hafted uniface); (b) 23O-10; (c) 21F-16; (d) 29U1C-11.

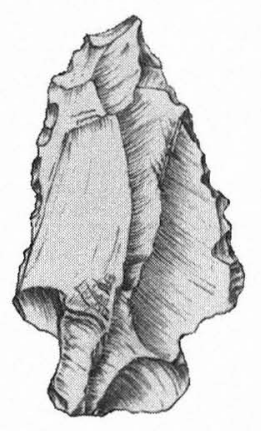

a

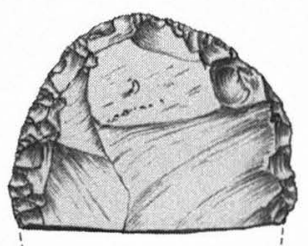

d

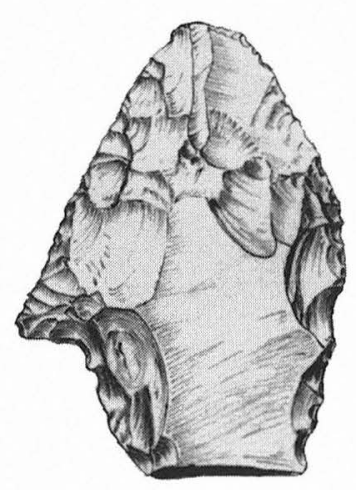

$\mathrm{b}$

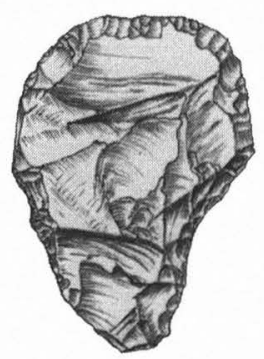

$\mathrm{e}$

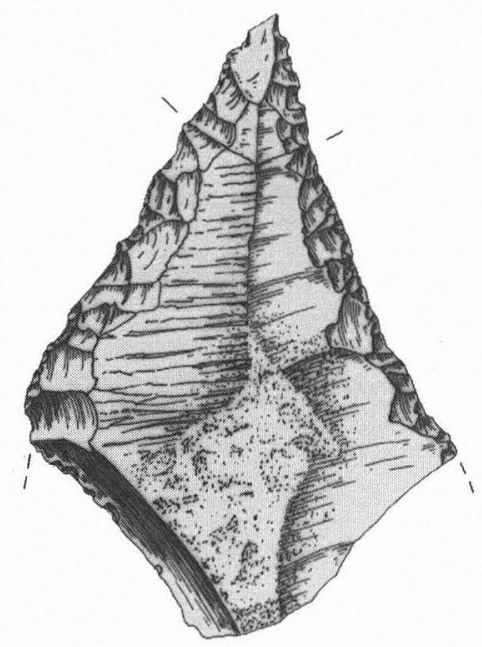

c

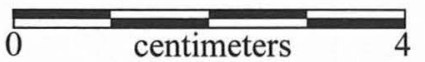

FIGURE 17-7. Distally convergent modification edge-modified flake (a-c), thin unifaces (b-c), and distally convex modified specimens (d-e). (a) 13AA-1; (b) 26KK2A-1; (c) 27GG2A-1; (d) 15L2-5; (e) 17Y1-2. 


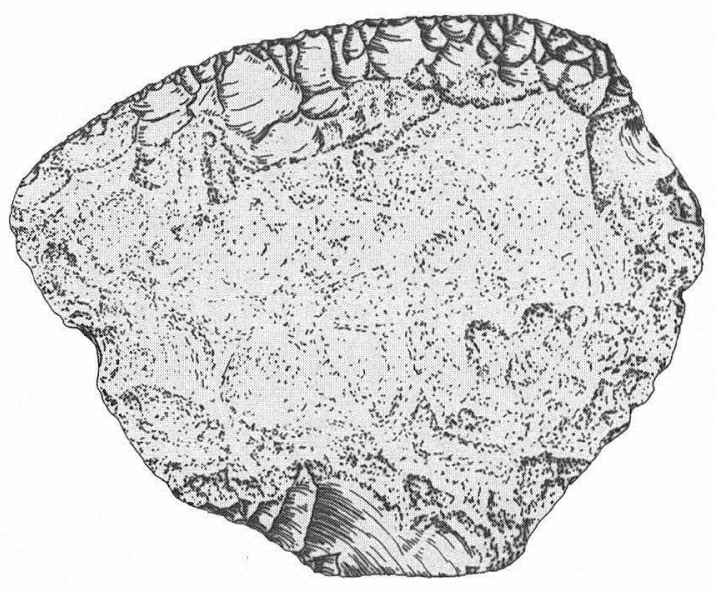

a

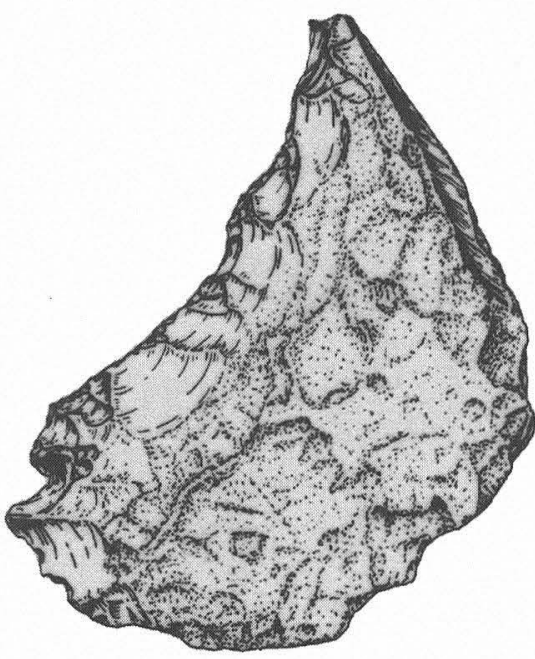

c
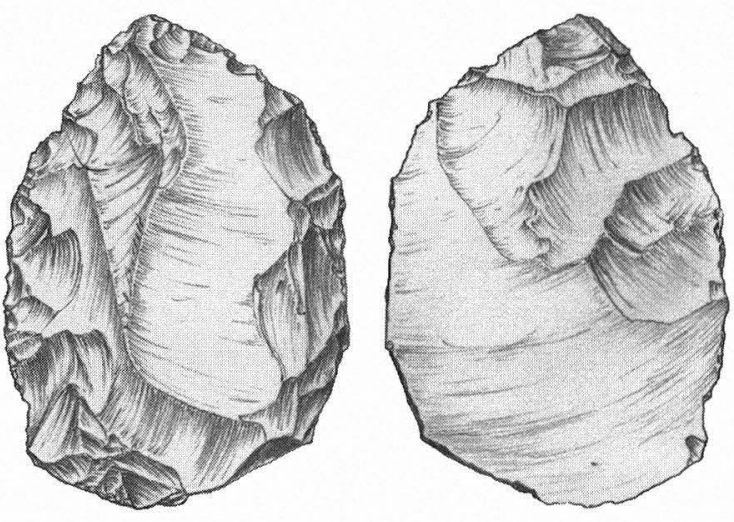

e

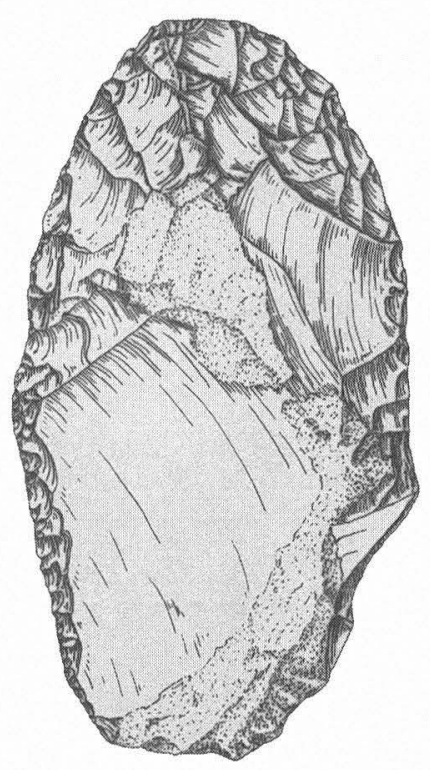

b
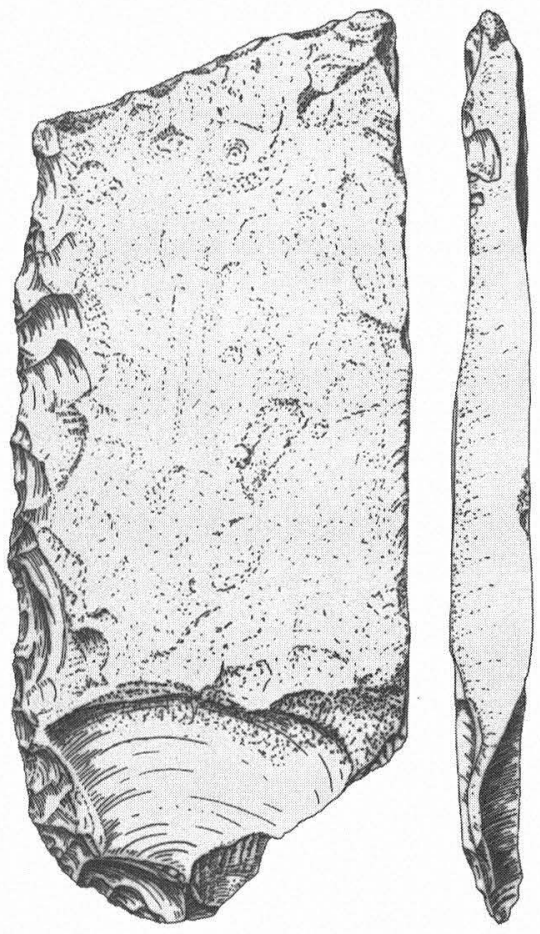

d

FIGURE 17-8. Thin unifaces. (a) 19F-11; (b) 33Q2-20; (c) 17V1-3; (d) 25AA2A-2; (e) 32R1-13, note extensive bulb modification on ventral view. 
TABLE $17-3$

Edge-modified Flakes Descriptive Statistics ( $\mathrm{n}=620$; in $\mathrm{mm}$ )

\begin{tabular}{l|c|c|c|c|c}
\hline & & & & Total & $\begin{array}{c}\text { Potential } \\
\text { Modified Edge }\end{array}$ \\
\hline Minimum & Length & Width & Thickness & 5 & 5 \\
Maximum & 4 & 5 & 2 & 190 & 325 \\
Range & 112 & 117 & 37 & 186 & 320 \\
Mean & 108 & 112 & 35 & $34 \pm 30$ & $95 \pm 52$ \\
\hline \hline
\end{tabular}

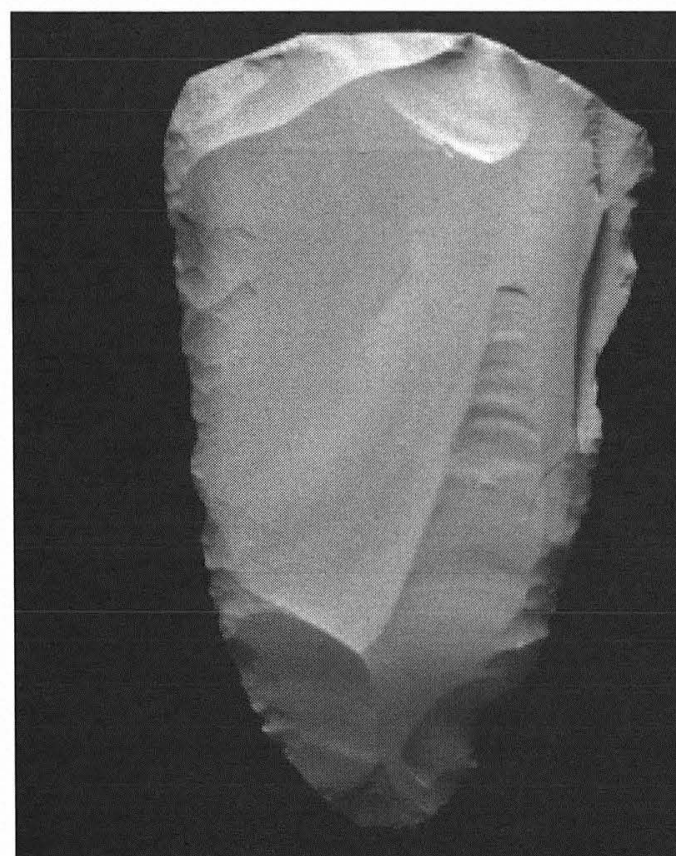

a

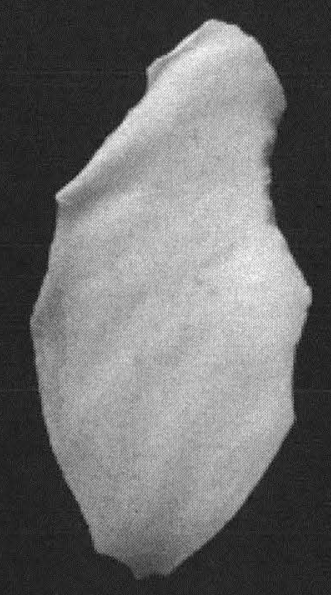

C

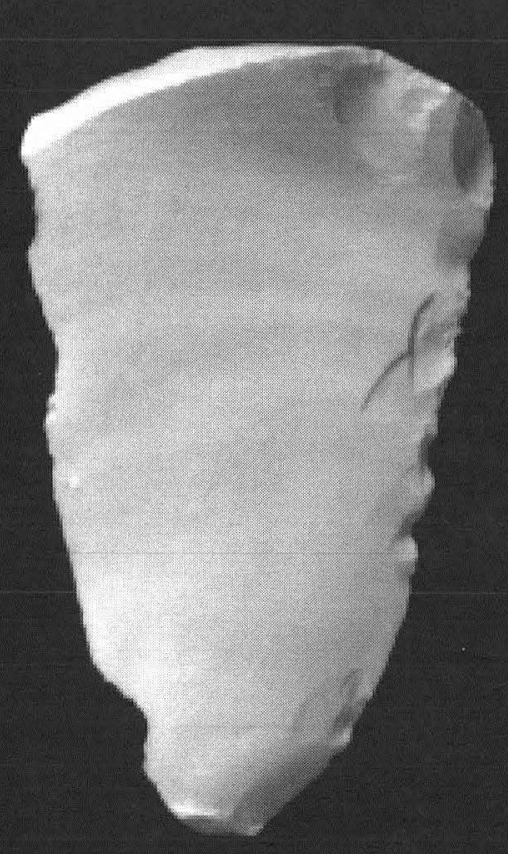

$a^{9}$

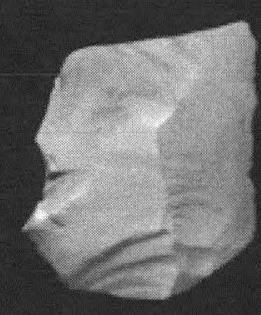

b
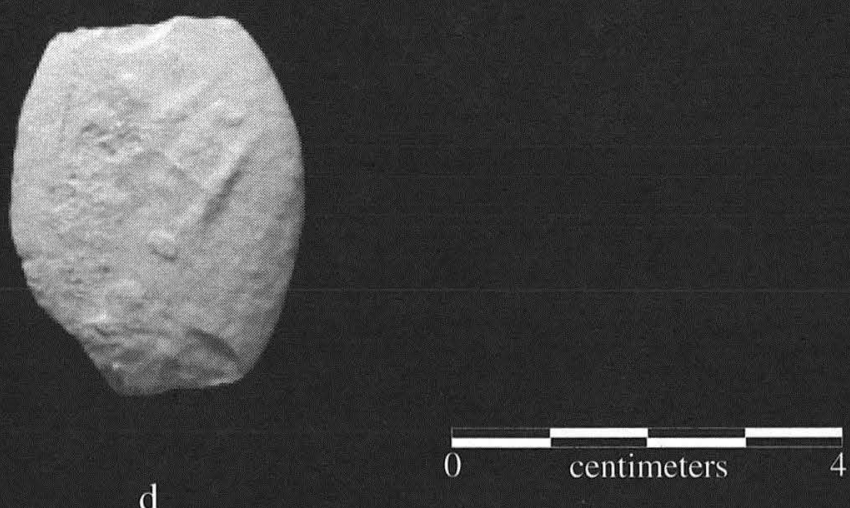

d

centimeters

FIGURE 17-9. Edge-modified flakes. (a) 37QQ1C-2, dorsal, showing continuous irregular retouch along right and left lateral margins; $\left(\mathrm{a}^{1}\right)$ 37QQ1C-2, ventral, showing noninvasive bifacial retouch; (b) 1949-1, note continuous regular straight retouch along right lateral margin; (c) $21 \mathrm{H}-18$, note continuous regular concave retouch along distal right margin; (d) 33R2-10, possible pièce esquillée; note bipolar flaking. 


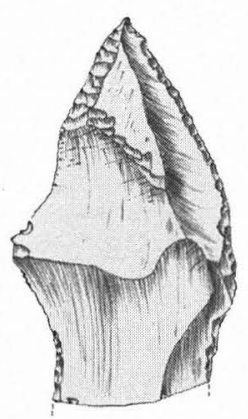

a

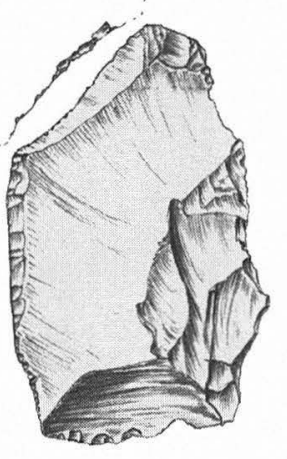

d

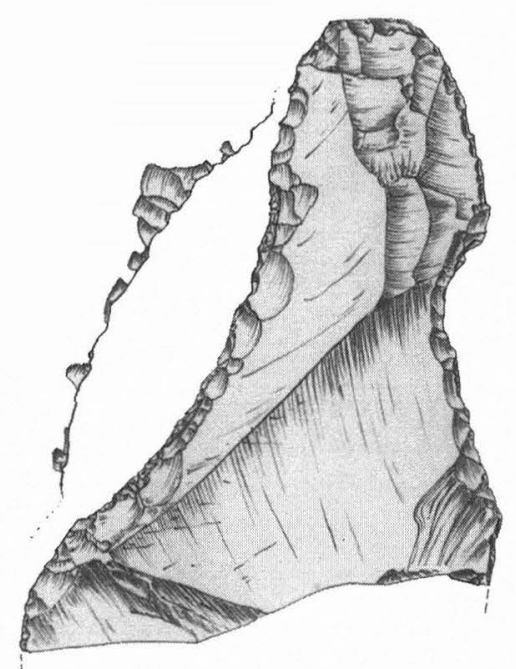

b

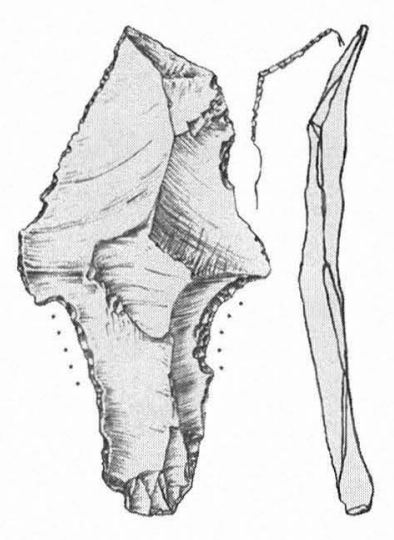

e

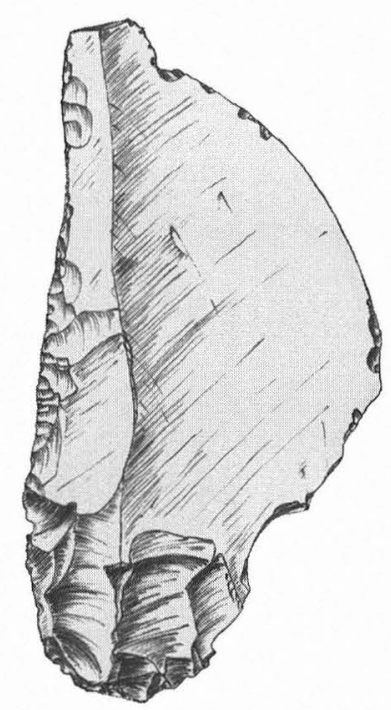

c

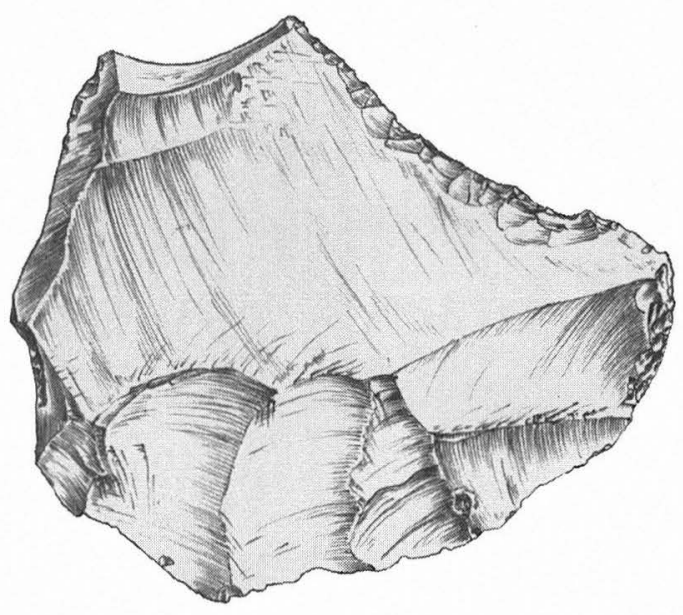

f
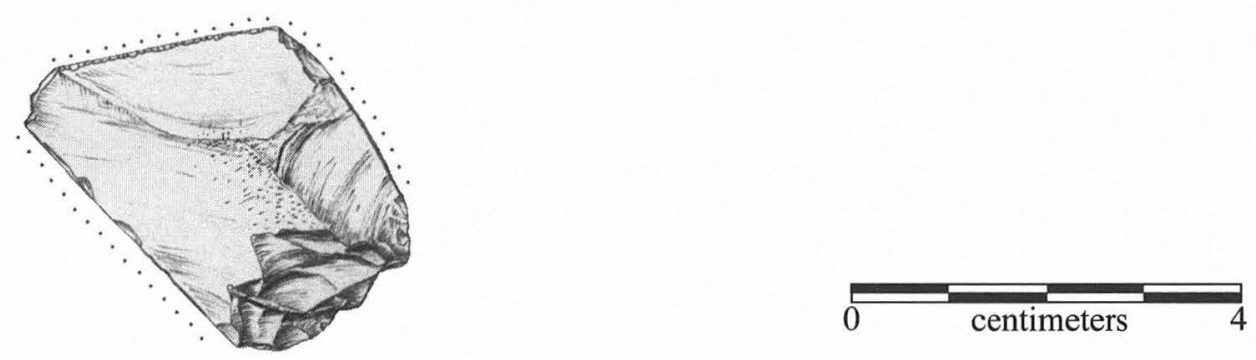

g

FiguRE 17-10. Edge-modified flakes. (a) 572-3; (b) 10D-11; (c) 23R-9; (d) 17CC2-1; (e) 37HC-17, possible hafted flake with continuous regular straight retouch along left lateral margin and minor dulling along proximal lateral margins; (f) $942-1$, note both dorsal and noninvasive ventral modification; $(\mathrm{g})$ 19I-8, note extensive grinding along lateral margins and minimal distal microflaking. 

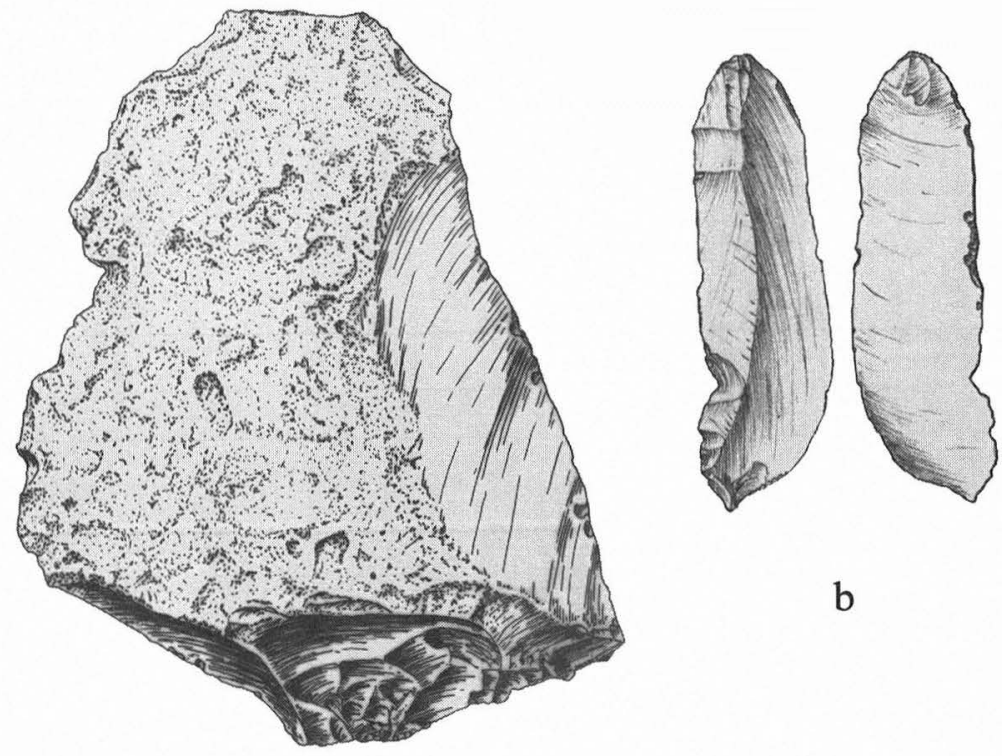

b

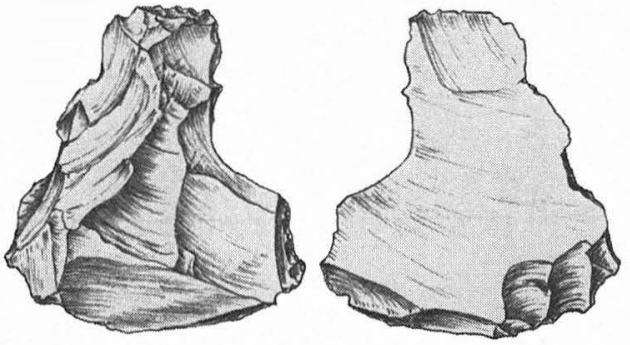

c

a

FIGURE 17-11. Edge-modified flakes. (a) 23J-17, note continuous, regular, straight microretouch; (b) 1786-1, bladelike flake with discontinuous, irregular, ventral retouch; (c) 682-2, continuous, irregular, ventral retouch.

\section{Retouched Blades $(N=30)$}

In this study blade definition follows Crabtree (1972), with integration of Johnson's (1983) more specific criteria. Blade length is at least twice the maximum width, with clear platform preparation, long, straight parallel lateral edges, and generally at least one central arris indicative of previous parallel-sided blade removal. Platform preparation ranges from simple single-facet platforms to complex multiple-faceted prepared platforms. Blades can have naturally backed (i.e., cortex) lateral edges or exhibit intentional flaked backing. Only blades exhibiting lateral (including flaked backing) or distal edge modification are included in this category. Examples are shown in Figures 17-12 and 1713 (see also Figures 7-7c, 7-8a, b, and 22-5c).

In an assemblage this large, many blade-like flakes are often noted, though few appear to belong to any formal blade manufacturing process. Rather, through the normal course of lithic reduction, a number of these blade-like flakes occur (Parry 1994). These may be unaltered or have natural backing and generally fit Crabtree's definition, although they do not have classic parallel dorsal flake scars and trapezoidal cross section indicative of previous blade removals. Although some of these blade-like artifacts might be classified by other analysts as blades, in this study, blade-like flakes with unifacial alteration are included in the other appropriate categories and excluded from this group.

Thirty retouched blades were analyzed in this study. Artifacts made on Clovis-like blades at the site include one laterally edge-modified artifact, 15V2-2 (see Figure 17-12a), and one thin uniface that is a distally modified blade (16I1C2, Figure 17-12b).

Another specimen (14EE2B-2) here initially classified as a retouched Clovis-like blade, is somewhat problematical. Although in every aspect it seems to be a tool made on a blade, it is possible that it was trimmed from a wider flake as discussed and illustrated by Collins (1996:16 and Figure 2.3). Assuming that this piece is in fact a retouched blade, it is retouched on its entire perimeter with a convergence at the bulbar end, one convex edge, and one convex edge with a broad notch that produces a shouldered appearance and a more-pointed convergence at the distal end (see Figures 7-8a and 22$5 \mathrm{c})$. The overall specimen is remarkable for its resemblance to atypical (in that flaking does not cover the entire face) single-shouldered points of Solutrean style (pointe a cran solutreene atypique a retouche non couvrante) in the Upper Paleolithic of Western Europe (Brezillon 1968:293-296). It would also be atypical of Solutrean points in that the shoulder is at the distal rather than the bulbar end of the parent piece. This artifact was found in the early Paleoindian Bison Bone Bed of Unit I but is patinated more than most other chert objects from that context.

Artifacts made on Toyah or Late Prehistoric blades include those shown in Figures 17-12c-g and 17-13. All illustrated examples exhibit moderate to extensive lateral edge modification. No other distal convex edge-modified blades were noted. Five additional blades were observed, 


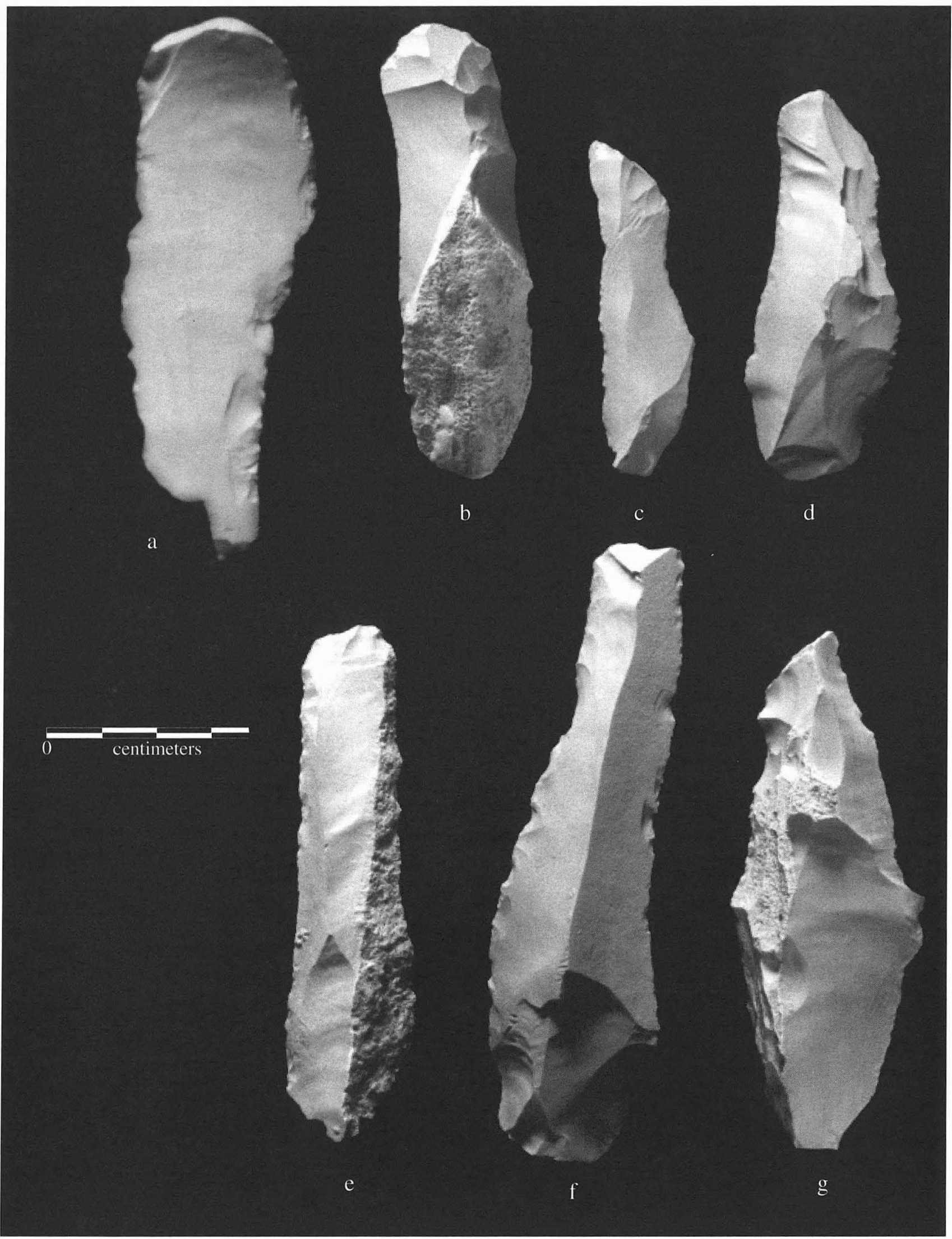

FIGURE 17-12. Retouched blades. (a) 15V2-2; (b) 16II1C-2, note extensive distal modification; (c) 503-8; (d) 6F-5; (e) 6G-7; (f) 6C-12; (g) 21I-11. Note extensive lateral modification in all but $b$. 


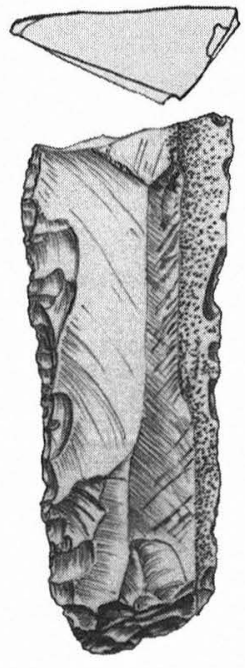

a

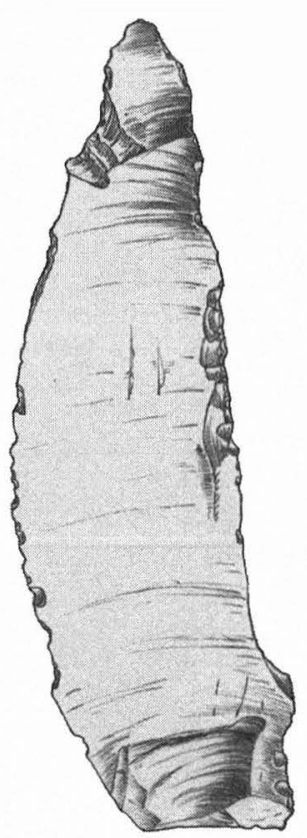

b

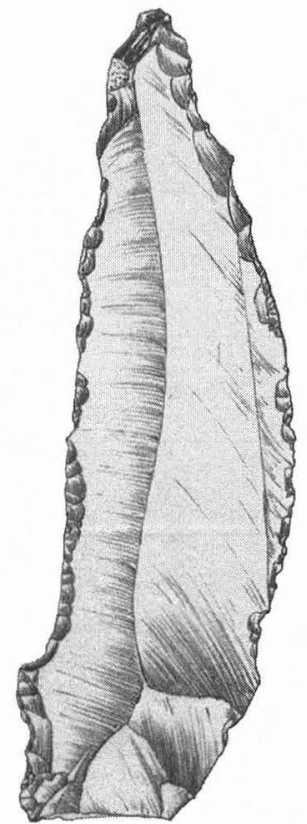

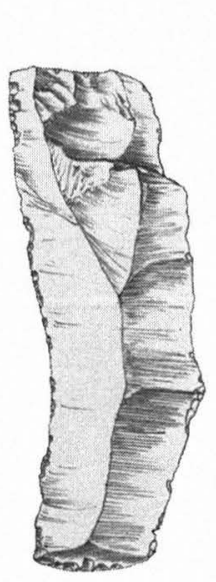

c
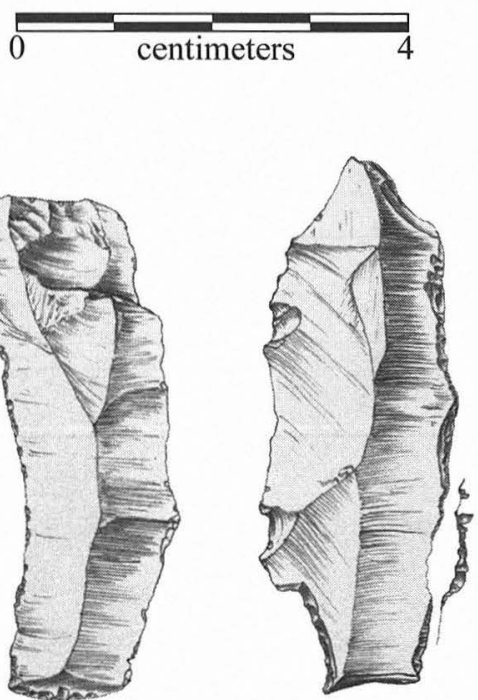

d

FIGURE 17-13. Retouched blades and multiple tool. (a) 108-12; (b) 286-2; (c) 37BC-14; (d) 37EB-20.

TABLE $17-4$

Retouched Blade Descriptive Statistics ( $\mathrm{n}=13$; in $\mathrm{mm}$ )

\begin{tabular}{l|c|c|c|c|c}
\hline & & & & Total & $\begin{array}{c}\text { Potential } \\
\text { Modified Edge }\end{array}$ \\
\hline Minimum & Length & Width & Thickness & Modified Edge & 97 \\
Maximum & 36 & 14 & 3 & 16 & 174 \\
Range & 111 & 37 & 14 & 135 & 77 \\
Mean & 74 & 23 & $7 \pm 3$ & $68 \pm 45$ & $126 \pm 26$ \\
\hline \hline
\end{tabular}

but these lack alteration and are described elsewhere. Descriptive statistics are given in Table 17-4.

\section{Denticulates $(N=94)$}

Denticulates exhibit multiple areas with coarse, regular to semiregular coalescent or dispersed multiple notch edge modification. Coalescent notches form groups of contiguous notch and spur groups producing a coarse sawtoothed pattern along the edge. Dispersed multiple-notched retouch consists of several areas of notched flaking separated by unretouched flake margins, with notched areas not generally found in combination with central spurs. Central spurs are generally broad and may be intact or attenuated. Three star-shaped specimens exhibit coarse coalescent regular dorsal edge modification. Descriptive statistics are given in Table 17-5 below. Examples are shown in Figures $17-14$ and $17-15 \mathrm{a}$.

Coarse denticulated retouch flakes are usually convex (Figure 17-14a, c), though they occasionally exhibit con- cave or straight retouched margins, as shown in Figure 17$14 \mathrm{~b}$. Kay analyzed one artifact in this category, Specimen 17D-9, which bore traces of use wear, though the contact material was indeterminate (see Chapter 22).

\section{Notched Flakes $(N=171)$}

Notched flakes, or notches, have invasive single or multiple nonadjoining concave modified edges. Notches differ from simple concave edge modification by exhibiting a significantly abrupt concave retouched depression into the flake body which tangibly alters the flake outline. Notches are produced either by intentional edge modification or by accretional edge damage acquired through repeated use, although without microwear analysis of specific artifacts, this discrimination is not made here. Usually notched modified edges are not combined with further retouch but rather exist as an area of isolated retouch. On the other hand, concave retouched modified edges generally extend over a longer flake segment and appear to function 


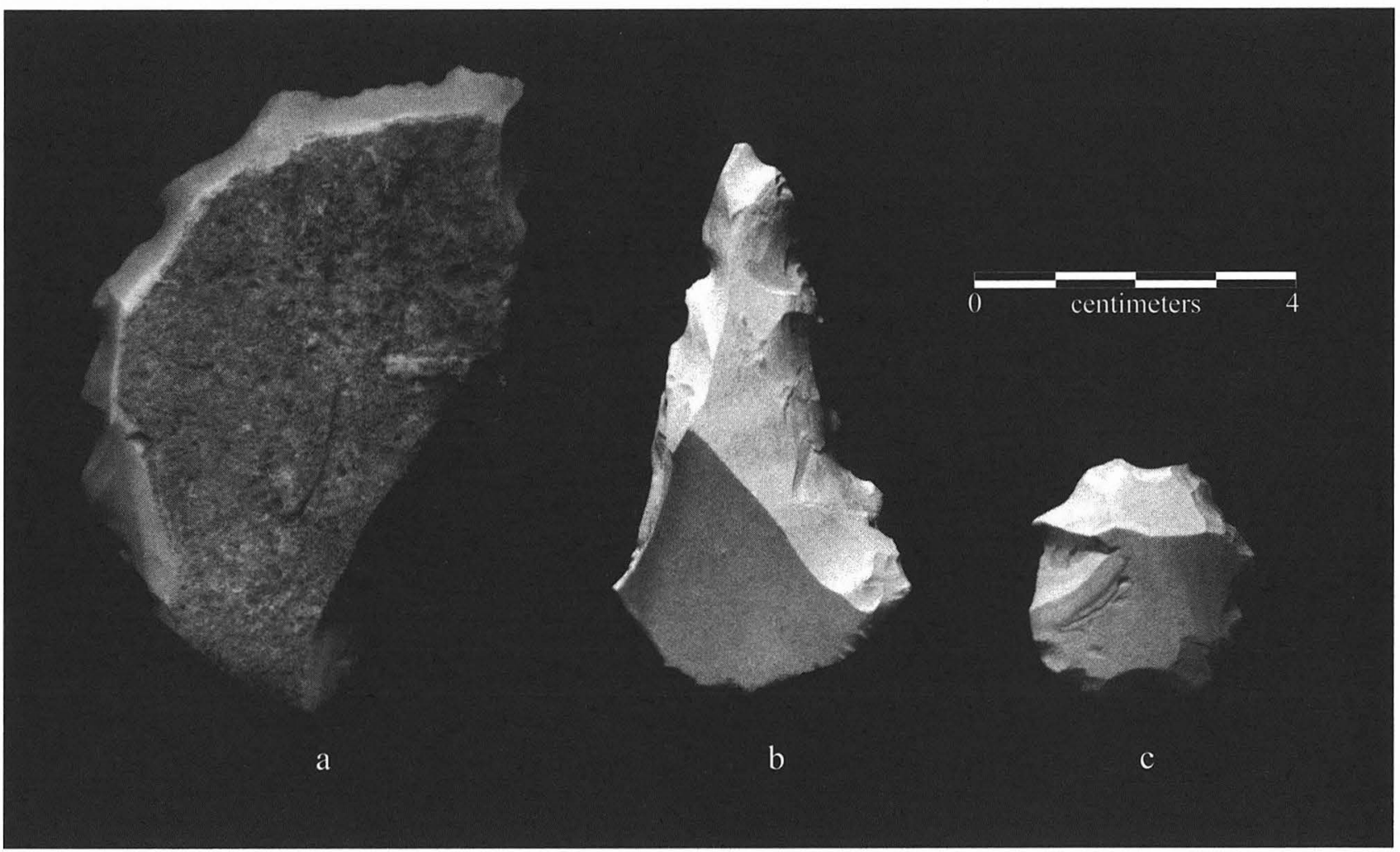

FigURE 17-14. Denticulates. (a) 21F-14; (b) 37HC-23; (c) 16I1-7.

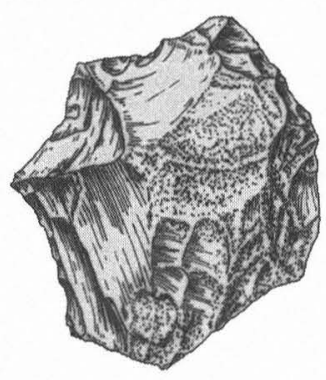

a
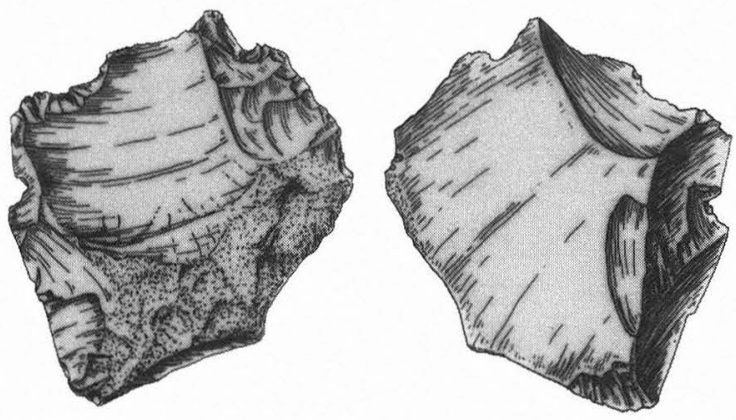

b

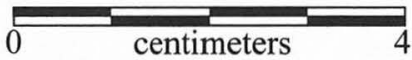

FIGURE 17-15. Miscellaneous small flake tools. (a) 27S1-6, denticulate; (b) 14J2-3, multiple tool with burin and edge-modified flake employable units.

as a larger modified edge than notches. In instances where a notch is paired with a spur or acutely retouched modified edge, it may prove difficult to isolate the intended functional unit. Therefore, these distinctive combination employable units are included in the multiple tool category defined below. Frison and Stanford (1982) report many notches from the Agate Basin site, though they are generally combined with other functional units and form what they term "composite tools." Notched flake mor- phology ranges from examples with broad, noninvasive to invasive, semicircular concave retouch along the flake margin and moderate to extensive secondary microflaking noted on modified edges, to those artifacts exhibiting narrow noninvasive to invasive concave retouch along the flake margin with minimal secondary microflaking in the notch. Finally, a small number of artifacts exhibiting a single V-shaped invasive flake scar without secondary microflaking in the notch are included in the notched flake 
TABLE $17-5$

Denticulate Descriptive Statistics ( $\mathrm{n}=21$; in $\mathrm{mm}$ )

\begin{tabular}{l|c|c|c|c|c}
\hline & & & & Total & $\begin{array}{c}\text { Potential } \\
\text { Modified Edge }\end{array}$ \\
\hline Minimum & Length & Width & Thickness & Modified Edge & 56 \\
Maximum & 13 & 21 & 3 & 25 & 100 \\
Range & 69 & 56 & 19 & 75 & 195 \\
Mean & 56 & 35 & 15 & $65 \pm 29$ & $117 \pm 48$ \\
\hline \hline
\end{tabular}

sample. Descriptive statistics are given in Table 17-6. Notched flake examples are shown in Figure 17-16.

\section{Spurred Flakes $(N=66)$}

Spurred flakes, or spurs, have one or more areas of concave or straight retouch that converge to form an acute angle relative to the flake margin. Spurs have single or multiple areas of continuous convergent regular concave or straight retouch present that brackets a central spur. A spurred edge may be formed either along a natural acute angle flake margin, by retouch along a broken flake margin, or entirely by retouching an acute angle into the unbroken flake margin. Total retouched area is usually greater than $5 \mathrm{~mm}$. Distal spur morphology may be sharp or attenuated.

Spurred flakes (Figure 17-17) are usually categorized as gravers or perforators, depending on spur morphology. Without microwear study, these tools should not be classed as such. Lynott (1975) examined the microwear traces found on replicated experimentally used "graver" tools. Though high-power observations were not made during this early study, enough doubt exists based on the lowpower observations to preclude functional classification of these tools on morphological criteria. Goodyear (1995) also speculates that tools similar to these may have functioned as microdrills used in the production of bone needles. Some of these tools (e.g., Figure 17-17a) exhibit modified edge morphology similar to those analyzed as perforators in Chapter 16. Descriptive statistics are given in Table 17-7.

\section{Microspurs and Microdenticulates $(N=191)$}

Microspurs and microdenticulates refer to the scale of retouch rather than the overall size of the artifact. Microspurs have a single area of continuous regular concave retouch forming a central microspur and exhibit a more needle-like morphology than the spurs described above. Microspurs often do not significantly disrupt the original flake outline. Total retouched area is usually less than $5 \mathrm{~mm}$. The utilization of an arris as a strengthening point for the microspur is common, though not a requisite. Descriptive statistics are given in Table 17-8. Examples are shown in Figure 17$18 \mathrm{a}-\mathrm{c}$.

Microdenticulates ("serrated flakes") exhibit fine to very fine coalescent sawtooth to serrated regular microretouch (Figure 17-18d-h). Central spur groups may exhibit sharp needle-like distal tips or may be attenuated (Figure $17-18 \mathrm{~g}, \mathrm{~h})$, presumably through use. These artifacts may be made either along natural flake margins or along broken flake margins. These distinctions notwithstanding, the boundary between the multiple microspur and microdenticulate categories is vague at best. Therefore it may be easier to view these types of artifacts as a single group possibly existing along a retouch continuum. Thus, microspurs may exist as a single area of fine spurred retouch, whereas the tool form may broaden into a microdenticulate with subsequent retouch. This includes forms intentionally produced to have only a single microspur and which would be functionally classified by others as gravers, as well as those forms that exhibit fine saw-toothed retouch. For these reasons, these forms are lumped in a separate category rather than included with the more coarsely retouched spurs or denticulates.

Multiple Tools $(N=332)$

Multiple tools have two or more morphologically unique working surfaces (e.g., notch and spur, thin uniface and burin, denticulate and spur, and so on). By its nature, this class of tool allows an almost infinite typology based on

TABLE 17-6

Notched Flake Descriptive Statistics ( $\mathrm{n}=35$; in $\mathrm{mm}$ )

\begin{tabular}{l|c|c|c|c|c}
\hline & Length & Width & Thickness & $\begin{array}{c}\text { Total } \\
\text { Modified Edge }\end{array}$ & $\begin{array}{c}\text { Potential } \\
\text { Modified Edge }\end{array}$ \\
\hline Minimum & 8 & 12 & 2 & 6 & 44 \\
Maximum & 66 & 55 & 29 & 45 & 180 \\
Range & 58 & 43 & 27 & 39 & 136 \\
Mean & $32 \pm 14$ & $29 \pm 12$ & $7 \pm 5$ & $20 \pm 17$ & $111 \pm 53$ \\
\hline \hline
\end{tabular}




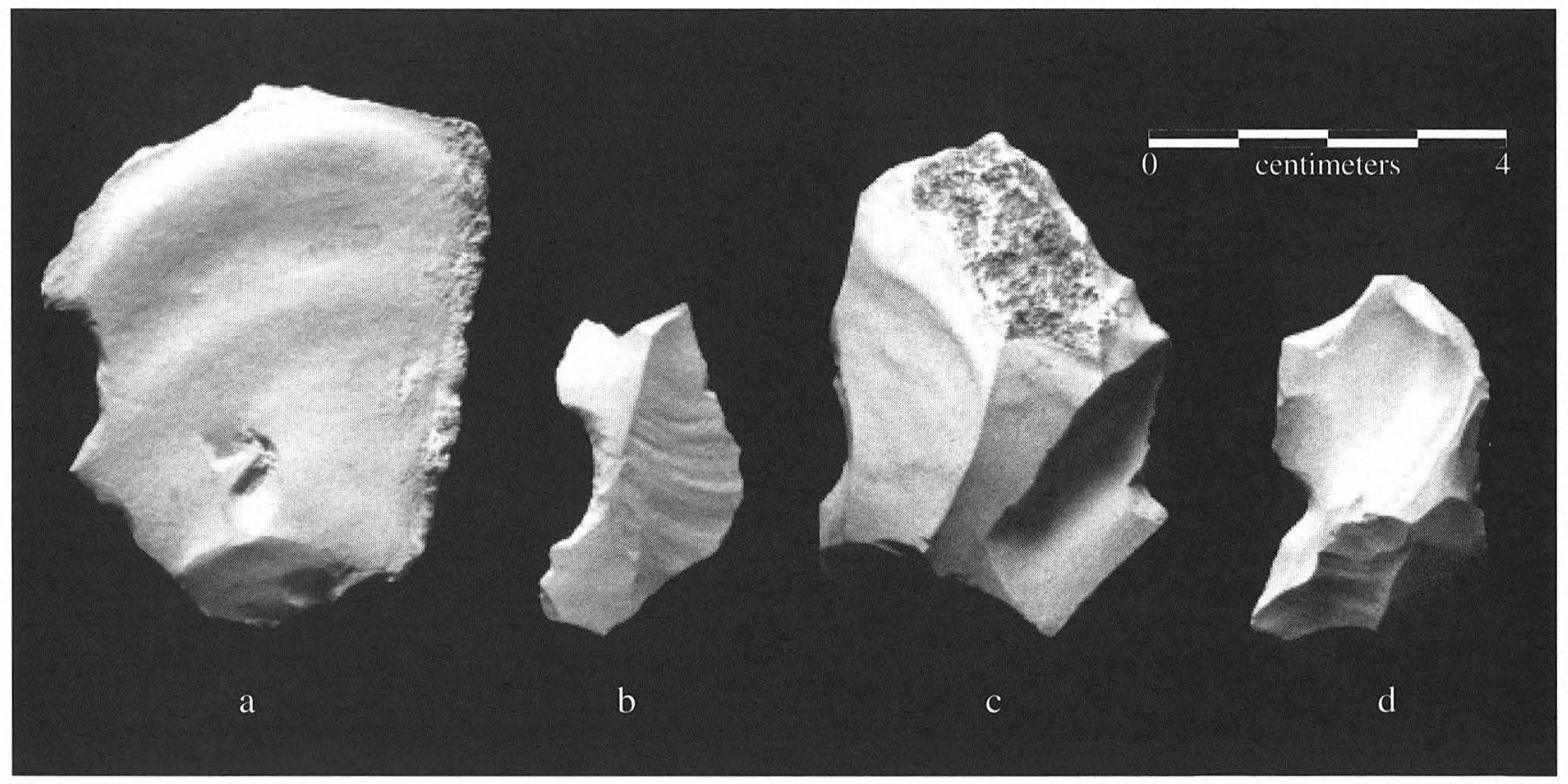

FIGURE 17-16. Notched flakes. (a) 635-3; (b) 15B-5; (c) 37ED-46; (d) $12 \mathrm{P}-7$.

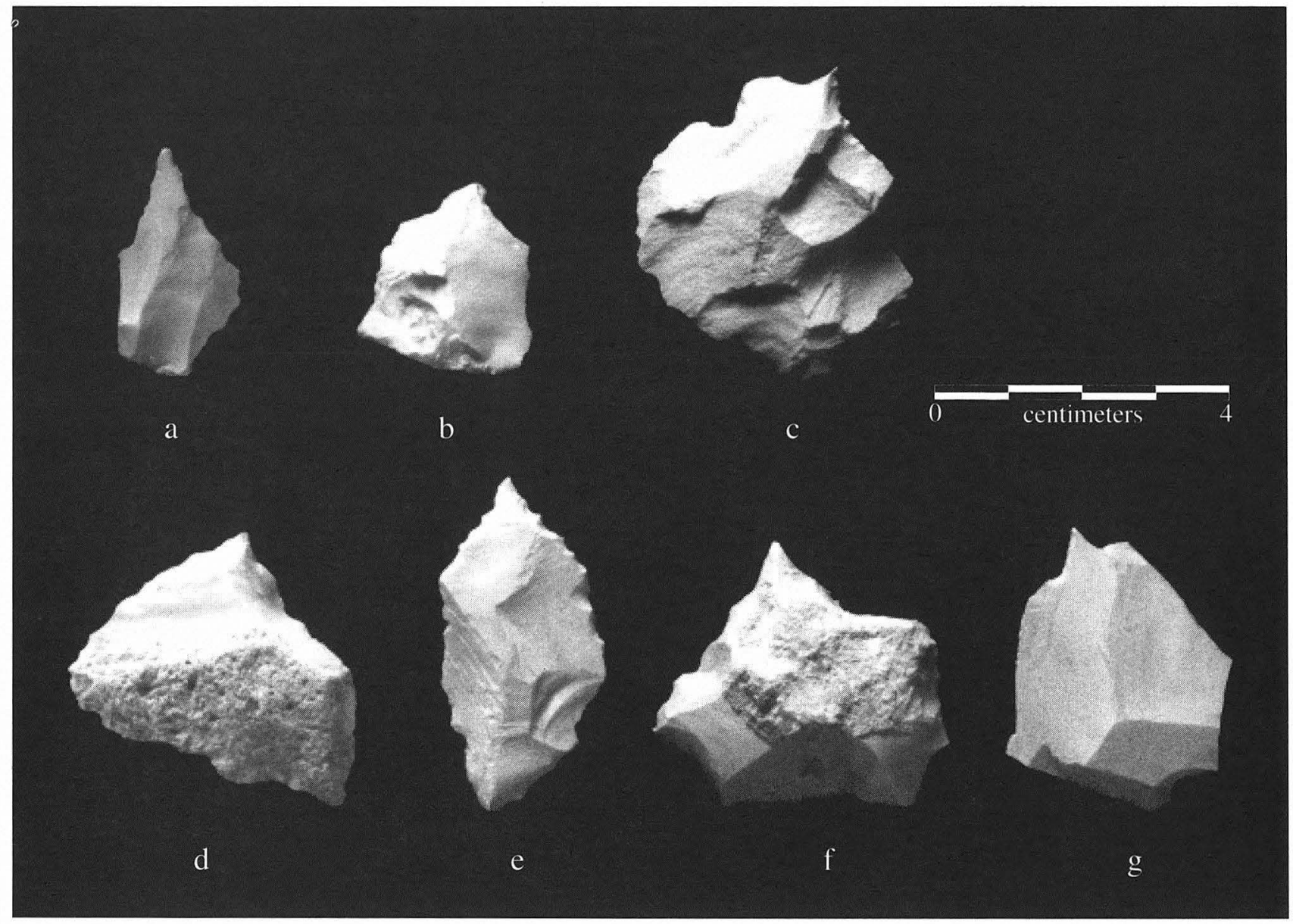

FIGURE 17-17. Spurred flakes. (a) 16S1-3a; (b) 1685-2; (c) 872-4; (d) 556-2; (e) 29R1-2, bipointed with serrated lateral margins; (f) $1832-$ $1 ;(\mathrm{g}) 34 \mathrm{~S} 2 \mathrm{C}-2$. 
TABLE $17-7$

Spurred Flake Descriptive Statistics ( $\mathrm{n}=33$; in $\mathrm{mm})$

\begin{tabular}{l|c|c|c|c|c}
\hline & Length & Width & Thickness & $\begin{array}{c}\text { Total } \\
\text { Modified Edge }\end{array}$ & $\begin{array}{c}\text { Potential } \\
\text { Modified Edge }\end{array}$ \\
\hline Minimum & 7 & 7 & 3 & 20 & 30 \\
Maximum & 63 & 75 & 22 & 40 & 160 \\
Range & 56 & 69 & 19 & 20 & 130 \\
Mean & $33 \pm 13$ & $30 \pm 13$ & $8 \pm 4$ & $27 \pm 7$ & $97 \pm 45$ \\
\hline \hline
\end{tabular}

TABLE $17-8$

Microspur/Microdenticulate Descriptive Statistics ( $\mathrm{n}=34$; in $\mathrm{mm}$ )

\begin{tabular}{l|c|c|c|c|c}
\hline & & & & Total & $\begin{array}{c}\text { Potential } \\
\text { Modified Edge }\end{array}$ \\
\hline Minimum & Length & Width & Thickness & Modified Edge & 9 \\
Maximum & 13 & 9 & 2 & 9 & 140 \\
Range & 48 & 39 & 13 & 131 & 131 \\
Mean & 35 & 30 & $5 \pm 2$ & $37 \pm 29$ & $70 \pm 33$ \\
\hline \hline
\end{tabular}

modified edge form. Of the 332 tools for which employable unit data is recorded, 10 distinct edge-modification morphologies were identified for the first employable unit alone. These are notches, spurs, convex edges, straight edges, concave edges, burins, denticulated edges, microspurs/ micro-denticulates, and ground or battered edges. To list these data individually would be cumbersome at best, and many combinations would be represented by a single example. Stratigraphic representation of these tools is easier to visualize, though only when these tools are viewed as a class. A problem with an assemblage as complex as this category is therefore readily apparent. As these data are parsed into numerous analytic units, they become nearly impossible to grasp. Therefore, these tools are addressed as an aggregate class, and specific modified-edge morphological combinations will be only commented upon. Employable unit frequency is given in Table 17-9 below, but assignment to group (e.g., employable unit 2 ) is arbitrary. The most common form is a notched edge (see Table 17-9). Some typical examples exhibiting multiple employable units in various combinations are shown in Figures 17-19 and 17-20. Descriptive statistics are given in Table 17-10.

Multiple tools have been noted in several Paleoindian assemblages (e.g., Frison and Stanford 1982). These tools may be the result of a simple ad hoc use-life history and these employable units accumulated accretionally as needed within a single occupation. Alternatively, they may have been intentionally manufactured to perform specific multiple functions, thus reducing the weight of transportable personal gear (Binford 1978, 1979). Another possibility is that these tools functioned as site furniture and as such were subject to recycling by subsequent inhabitants of the site, during which process they acquired new employable units functionally specific to the new users' task requirements. Regardless of their gen- esis, these tools generally appear to have been called upon to perform multiple roles during their effective use lives. Further study of these artifacts is recommended, with emphasis on microwear studies that may identify task-specific tool function relative to employable unit morphologies. One multiple tool examined by Kay exhibited positive use-wear traces (see Chapter 22). Specimen 37DA-7, has two modified edges, a dihedral burin and straight unifacial retouched margin. Kay found mediumhard contact-material wear on the burin and attributed the modified edge to intentional backing, possibly as a prehension aid. In fact, burins are present on 15 tools identified in the fully analyzed sample of this class. These may be functional burins as Kay has identified, or alternatively, they may represent rejuvenation of modified edges that are masking the sample by their distinctive burin tip morphology (see Uniface Rejuvenation section below).

\section{Other Modifications of Unifacial Flake Tools}

\section{Etching, Scoring, or Engraving}

Four artifacts illustrated in Figure 17-21 fall within previously defined tool classes but stand out due to dorsal cortex modification. Specimen 100-6 (Figure 17-21a) is a thin uniface with shallow etching in a cross-hatched pattern on the dorsal cortical surface. Specimen 20R-6 (Figure 17-21b) is a thin uniface with total edge modification and ventral noninvasive to invasive bifacial retouch and bulb modification. The dorsal cortical surface exhibits what appears to be random etching across the surface. Specimen 37HA-1 (Figure 17-21c) is a small edge-modified flake with deep subparallel incisions or grooves trending obliquely across the cortical surface. The incisions or grooves are deeper adjacent to the proximal flake margin and get progressively more shallow and narrow distally. 


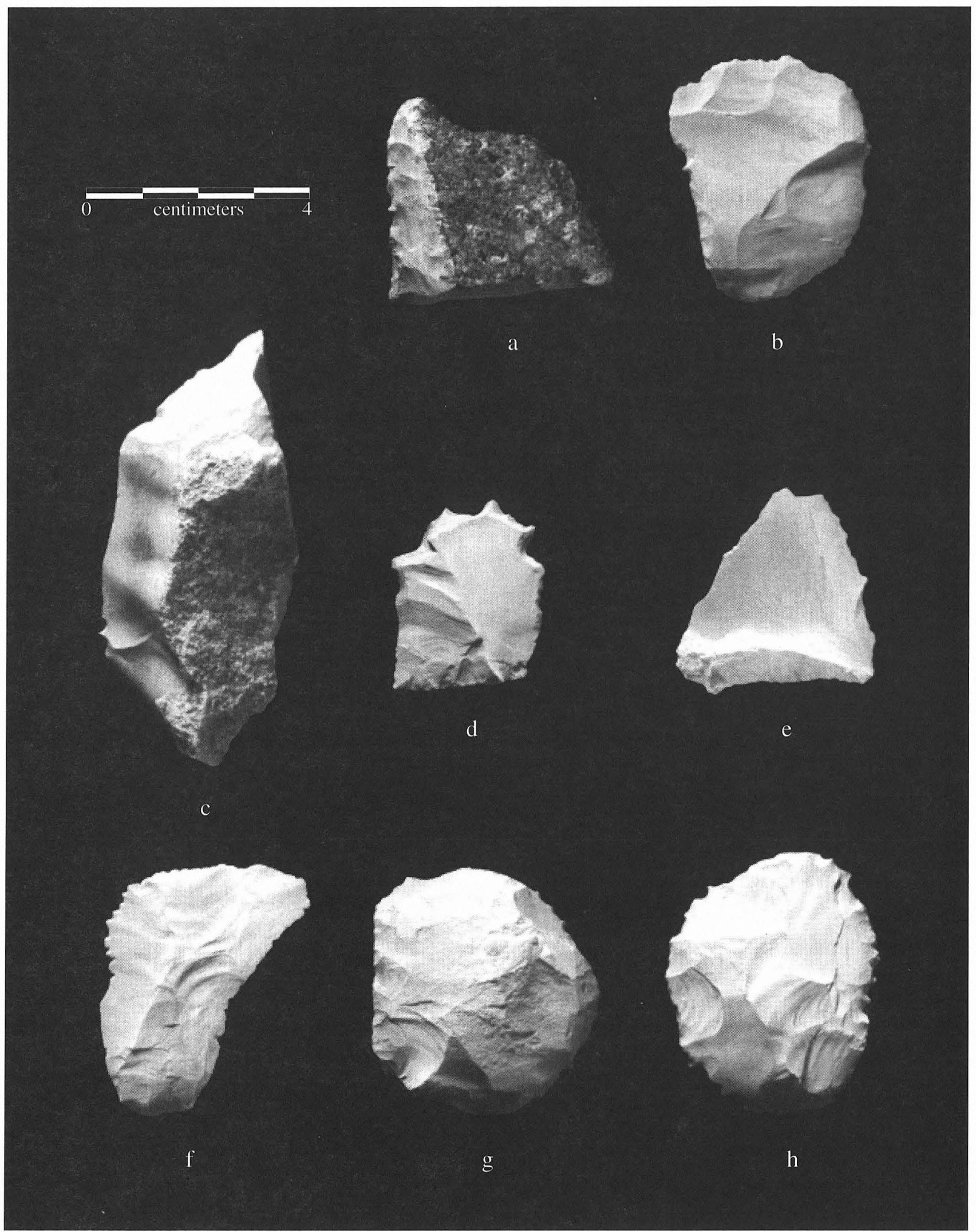

FIGURE 17-18. Microspurs/microdenticulates. (a) thin uniface fragment with attenuated microdenticulation, note single remaining microspur; (b-c) microspurs; (d-f) microdenticulates; $(\mathrm{g}, \mathrm{h}$ ) microdenticulates with attenuated microspurs. Specimen numbers: (a) 15Z2-4; (b) 166-8; (c) 31Q2-6; (d) 3A-17; (e) 532-4; (f) 16Q1-10; (g) 27T1-6; (h) 31R1-12. 
TABLE 17-9

Multiple Tool Employable Unit Frequencies

\begin{tabular}{|c|c|c|c|c|c|c|}
\hline Edge Morphology & $\begin{array}{l}\text { Employable } \\
\text { Unit } 1\end{array}$ & $\begin{array}{c}\text { Employable } \\
\text { Unit } 2\end{array}$ & $\begin{array}{c}\text { Employable } \\
\text { Unit } 3\end{array}$ & $\begin{array}{l}\text { Employable } \\
\text { Unit } 4\end{array}$ & Total & $\%$ \\
\hline Notch & 121 & 45 & 15 & 2 & 183 & 23.7 \\
\hline Spur & 22 & 100 & 22 & 4 & 148 & 19.1 \\
\hline Convex & 63 & 67 & 15 & 2 & 147 & 19.0 \\
\hline Straight & 43 & 45 & 26 & 1 & 115 & 14.9 \\
\hline Concave & 24 & 19 & 11 & 2 & 56 & 7.2 \\
\hline Burin & 26 & 22 & 8 & 1 & 57 & 7.4 \\
\hline Denticulate & 29 & 17 & 2 & - & 48 & 6.2 \\
\hline $\begin{array}{l}\text { Microspur/ } \\
\text { Microdenticulate }\end{array}$ & 11 & 5 & - & - & 16 & 2.1 \\
\hline Ground or Battered & - & 3 & - & - & 3 & 0.0 \\
\hline
\end{tabular}

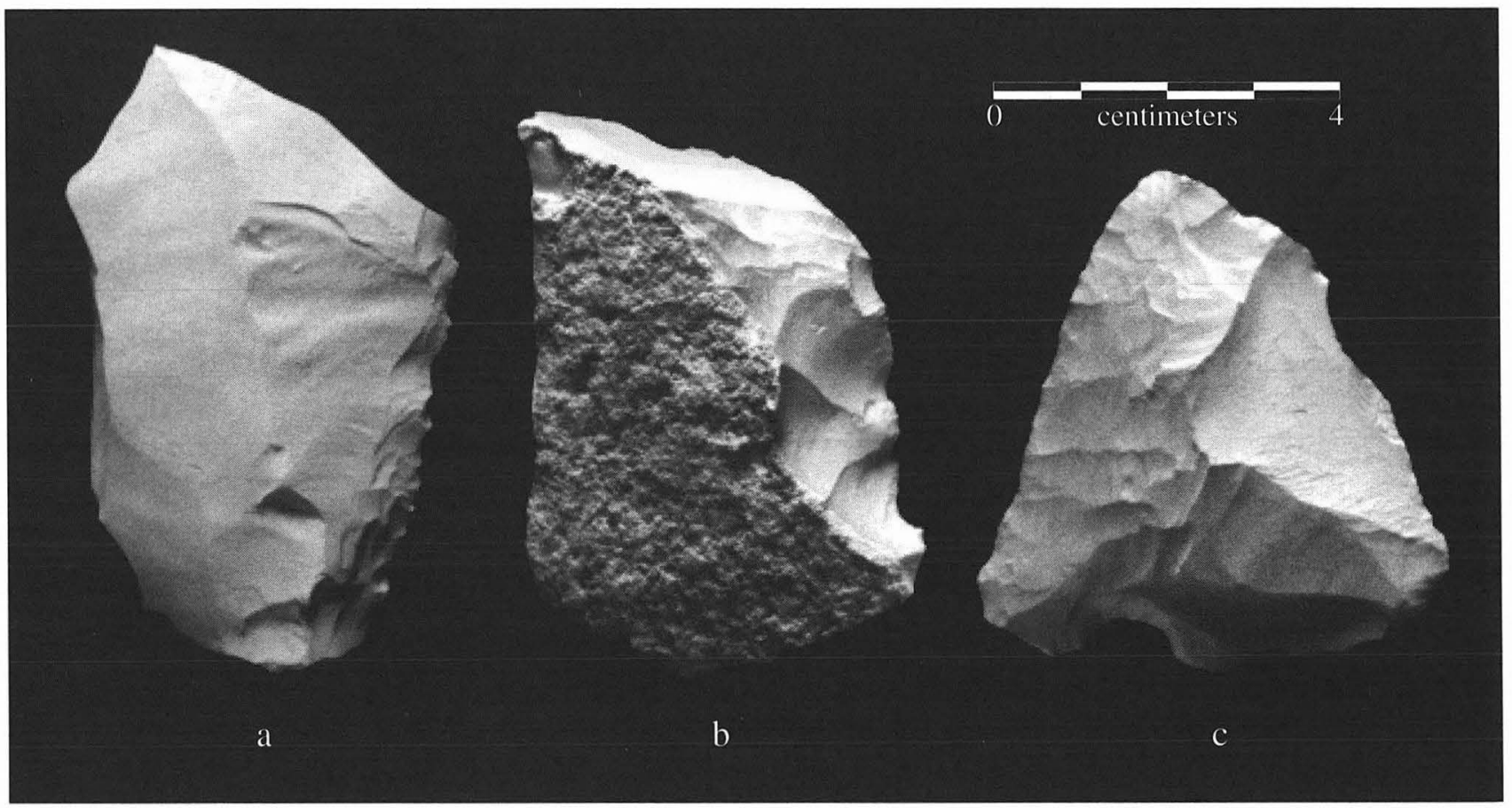

FIGURE 17-19. Multiple tools. (a) 22Q-28, thin uniface with distal dihedral burin; (b) 24S2-1, thin uniface with distal monohedral burin; (c) 51BB-10, thin uniface with broad notch.

Specimen 30LL1C-1 (Figure 17-21d) is an thin uniface with a hachured engraving pattern prominent across the cortical surface. Microwear analysis by Driskell (see Chapter 22) revealed polish attributed to hide working. Engraving on this specimen resembles that on several from very early contexts at the nearby Gault site (see Collins et al. 1991). Data collected on the Wilson-Leonard specimens is contained in their respective class summary statistics.

\section{Edge Rejuvenation}

Edge rejuvenation of unifacial tools is necessary when the working edge of the tool becomes dull from repeated use or the edge angle becomes too steep to perform the required task (Dibble 1987; Jelinek 1976; Shafer 1970, 1971). Artifacts or edge-rejuvenation flakes (Figure 17-22b, d-g) possessing features similar to those in rejuvenation methods noted by Shafer $(1970,1971)$ and Jelinek (1966) were found at the Wilson-Leonard site. Artifact 20I-11(Figure 17-22a) shows rejuvenation utilizing the burin blow method (Vaughn 1985) with remnants of a notch possibly used to set up the rejuvenation burin blow (Method A; Shafer 1970, 1971). Artifact 19F-13 (Figure 17-22c) also illustrates this method of side-struck unifacial rejuvenation. Artifact 4K-5 (Figure 17-22d) shows the ventral blow rejuvenation Method C described by Shafer $(1970,1971)$. Using this 


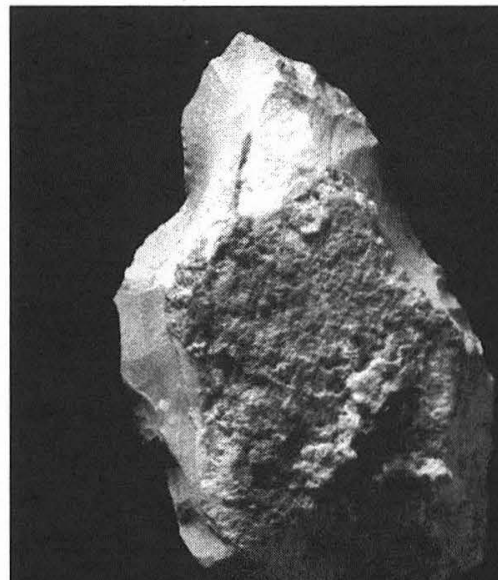

a

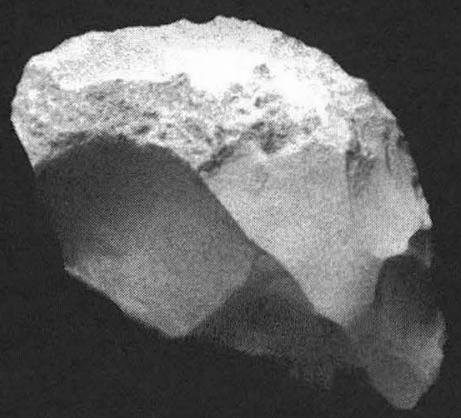

d
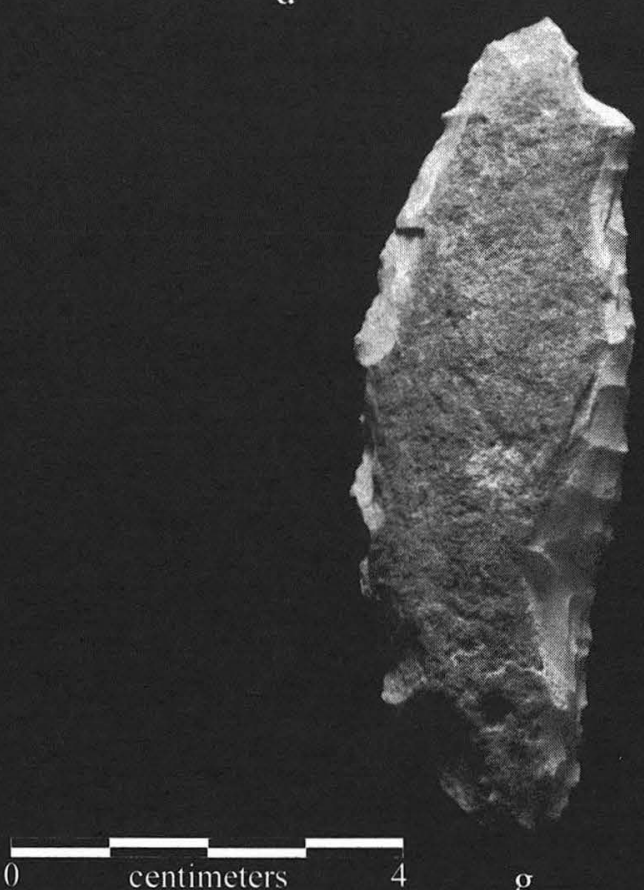

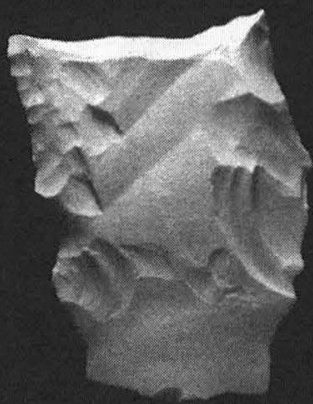

b

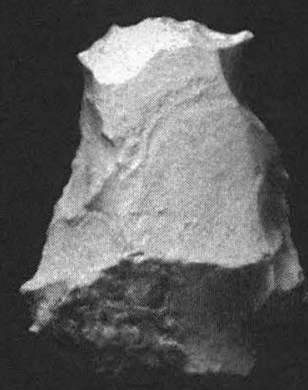

e

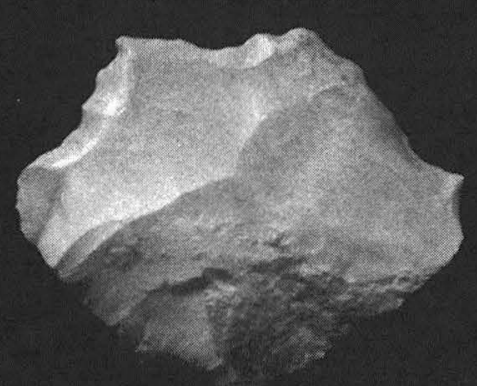

c

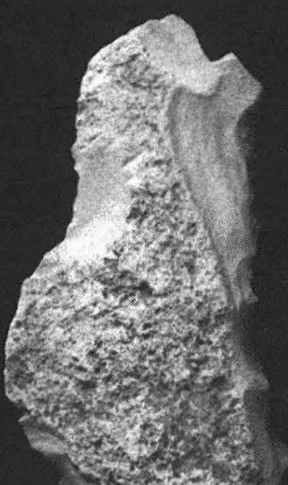

(a)

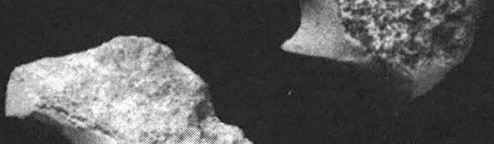

f

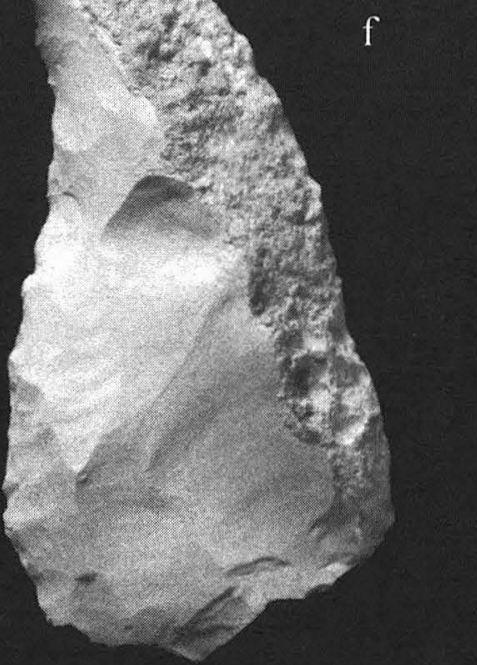

h

FigURE 17-20. Multiple tools. (a) 36R-12, thick uniface with spur; (b) 26T2B-2, multiple spur; (c) 29T2D-10, microdenticulate with multiple spurs; (d) 27T2B-2, thin uniface with microdenticulate; (e) 23O-11, thin uniface with spur; (f) 37BD-31, thin uniface with multiple spurs; (g) 36D-30, thin uniface with spur and group 2 notch; (h) 33Q2-14, thin uniface with spur. 
TABLE $17-10$

Multiple Tool Descriptive Statistics ( $\mathrm{n}=74$; in mm)

\begin{tabular}{l|c|c|c|c|c}
\hline & Length & Width & Thickness & $\begin{array}{c}\text { Total } \\
\text { Modified Edge }\end{array}$ & $\begin{array}{c}\text { Potential } \\
\text { Modified Edge }\end{array}$ \\
\hline Minimum & 6 & 11 & 2 & 7 & 22 \\
Maximum & 85 & 84 & 26 & 182 & 207 \\
Range & 79 & 73 & 24 & 175 & 185 \\
Mean & $43 \pm 16$ & $35 \pm 13$ & $9 \pm 4$ & $74 \pm 43$ & $117 \pm 48$ \\
\hline \hline
\end{tabular}

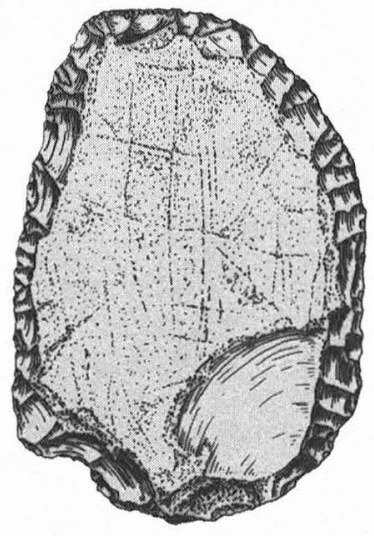

a
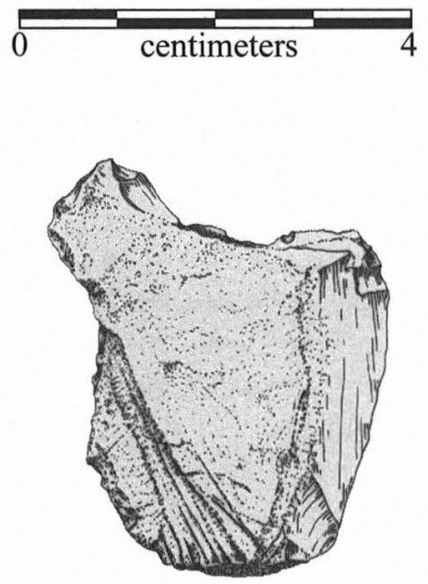

c
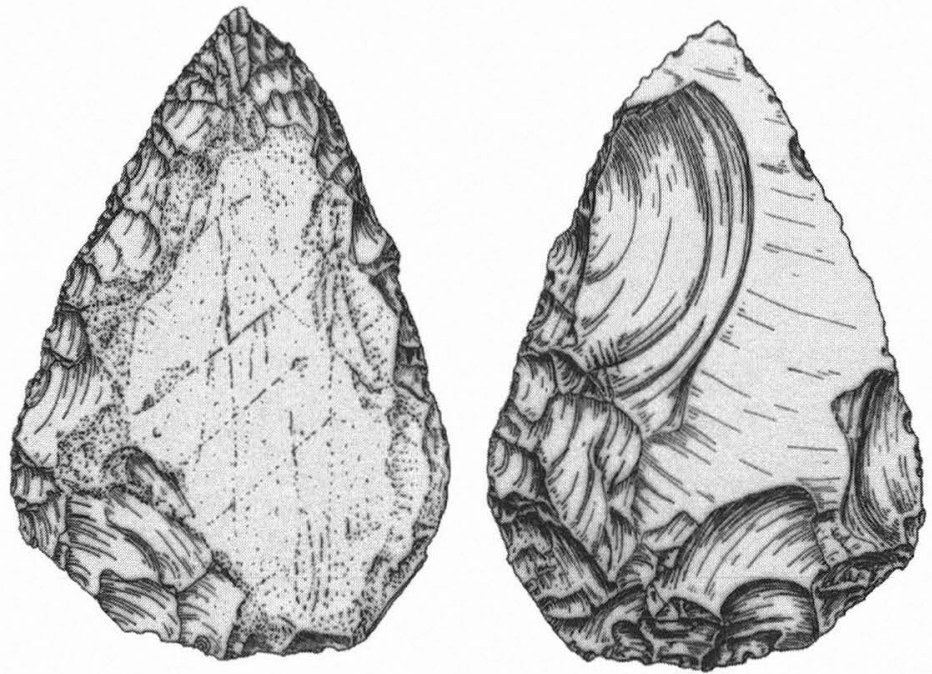

b
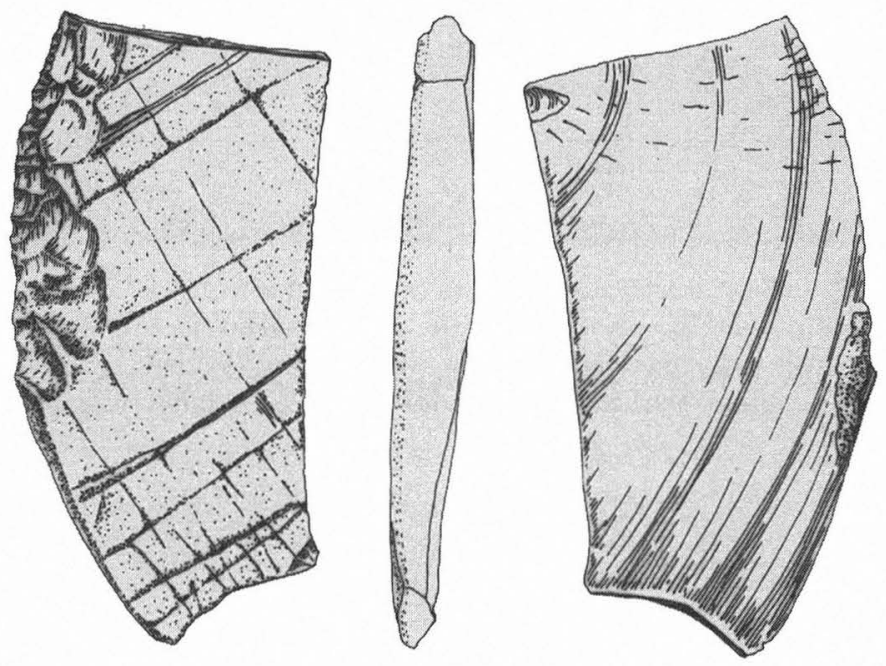

$\mathrm{d}$

FIGURE 17-21. Etched, scored, or engraved artifacts. (a, b) thin unifaces, etched dorsal cortex; (c) edge-modified flake, grooved cortex; (d) thin uniface, patterned engraving. Specimen numbers: (a) 10O-6; (b) 20R-6; (c) 37HA-1; (d) 30LL1C-1.

technique, the uniface is struck on the ventral distal margin, removing the unifacially flaked surface and rejuvenating the tool.

Vaughn (1985:488-496) argues that burin blow technology (traditionally seen as creating a specialized functional form, the burin used for engraving wood, bone, or antler) is equally adapted to removing unwanted or unusable worn, chipped-flake, blade, or uniface edges. In his study of Cassegros flints, the microwear traces on $75 \%$ of the so-called burins were on a remnant edge-modified surface. Barton et al. (1996) have also addressed the question of "burin" function and posit that the presence of burins on multiple tools may 


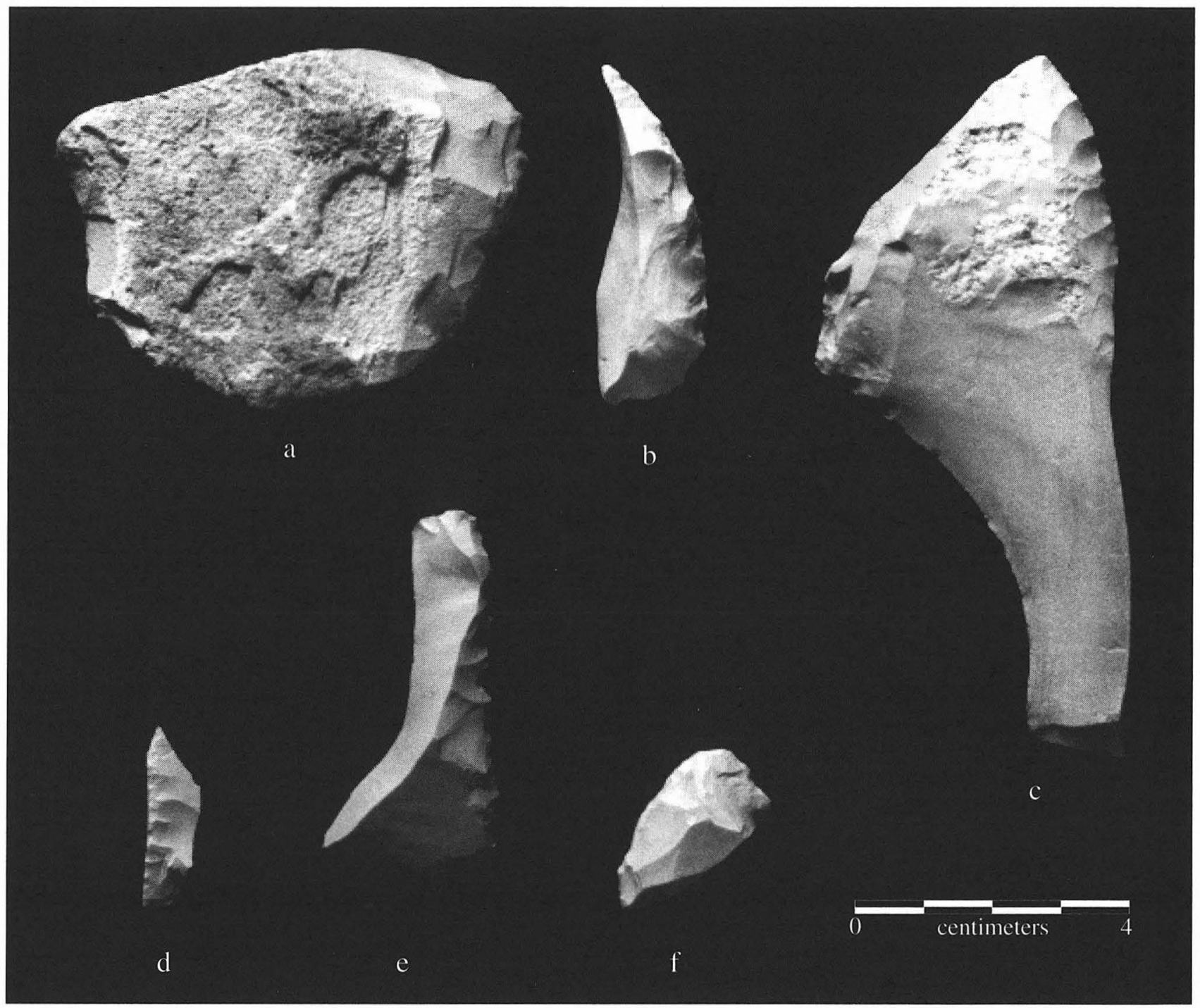

FIGURE 17-22. Rejuvenated unifaces and uniface rejuvenation spalls. (a) 20I-11, rejuvenated uniface, note notch remnant to set up burin blow distally; (b) 12M-8, uniface rejuvenation spall; (c) 19F-13, rejuvenated uniface, side struck with retouch remnant distally; (d) 4K-5, uniface rejuvenation spall, dorsal- to ventral-struck rejuvenation; (e) $7 \mathrm{H}-6$, uniface rejuvenation spall, side-struck rejuvenation that plunged through tool; (f) $13 \mathrm{U}-3$, uniface rejuvenation spall, side struck with resulting rejuvenated surface near $90^{\circ}$.

represent tangs and may point to haft elements previously unrecognized in tool assemblages. Thus, with respect to unifacial rejuvenation, the use of the term burin should be avoided unless microwear analysis reveals use-wear traces on the beveled tip created by the burin blow.

\section{Assessing the Possibility of Microlithic Tool Strategies}

At one point in this study, primarily based on the results of the analysis of cores, it was suggested that perhaps a microlithic tool strategy or tradition might be embedded in the unifacial tool classes. This was based on the occurrence of very small cores that did not appear exhausted and from which multiple flakes less than $3 \mathrm{~cm}$ in length had been detached. Unifacial tools equal to or smaller than $3 \mathrm{~cm}$ were inspected. However, these small tools oc- cur in most unifacial tool classes. To further assess the possibility of microlithic tool strategies, histograms of length for all tool classes were constructed. The histograms demonstrate that all unifacial tool classes have unimodal distributions and in all cases the modes are larger than $3 \mathrm{~cm}$, the traditional cutoff point for defining microlithic tools (Figure 17-23). No tool classes have significant modes below $3 \mathrm{~cm}$. These data suggest that a wide range of flake sizes were used for many of the tool forms and that slightly more than 300 of these happened to be shorter than $3 \mathrm{~cm}$ (examples shown in Figure 17-24). Only thick uniface and retouched blade categories do not have specimens smaller than $3 \mathrm{~cm}$.

If microlithic strategies were employed one would expect a specific tool form to be associated with small flakes, and the size mode of this tool class would be distinctly 
shorter (smaller) than other tool forms. Alternatively, if evidence of hafting could be clearly associated with the smaller forms of a specific tool class and this evidence was lacking on the larger specimens, then a case could be constructed for at least a hafting strategy for smaller tool forms. An inspection of the modes (see Figure 17-23) shows that edgemodified flakes, microspurs/microdenticulates, and spurs have the smallest modes at $3.1,3.4$, and $3.1 \mathrm{~cm}$, respectively. Notches and multiple tools are next in modal size (3.8 and $4.1 \mathrm{~cm}$ ), and these are followed thin unifaces, retouched blades, and thick unifaces $(4.7,6.5$, and 7.9 , respectively). Clearly, size was a criterion for selecting blanks for specific unifacial tools, but the histogram demonstrates that a wide range in size was acceptable for all tool classes. In summary these data fail to support a hypothesis for a microlithic strategy for the unifacial tools, thus all unifacial tools are analyzed by the morphological classes described above.

\section{STATISTICALANALYSIS OF UNIFACIAL TOOLS ANDATTRIBUTES}

This brief analysis looks at flake and tool attributes by unifacial tool classes. It attempts to further characterize the unifacial tool classes by inspecting the pattern of flake and

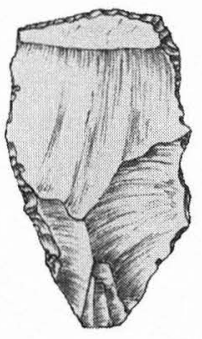

a

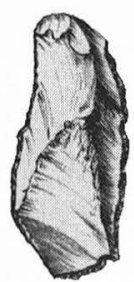

b

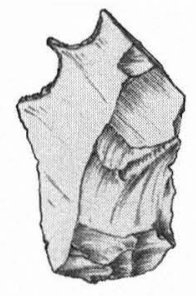

C

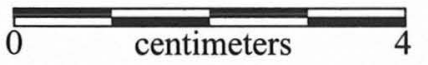

Figure 17-24. Examples of tools less than $3 \mathrm{~cm}$ in maximum dimension. (a, b) flake tools; (c) microspur. Specimen numbers: (a) 748-4; (b) 685-8; (c) 28T2D-7.

material attributes among the unifacial tools and identifying significant deviations from the overall patterns. This analysis demonstrates the nature of the flake blank used for specific tool classes and for which tools material selection was important. A diachronic analysis is presented in Chapter 11. Adjusted residuals as well as row and column percentages are used to identify significant patterns. Adjusted residuals

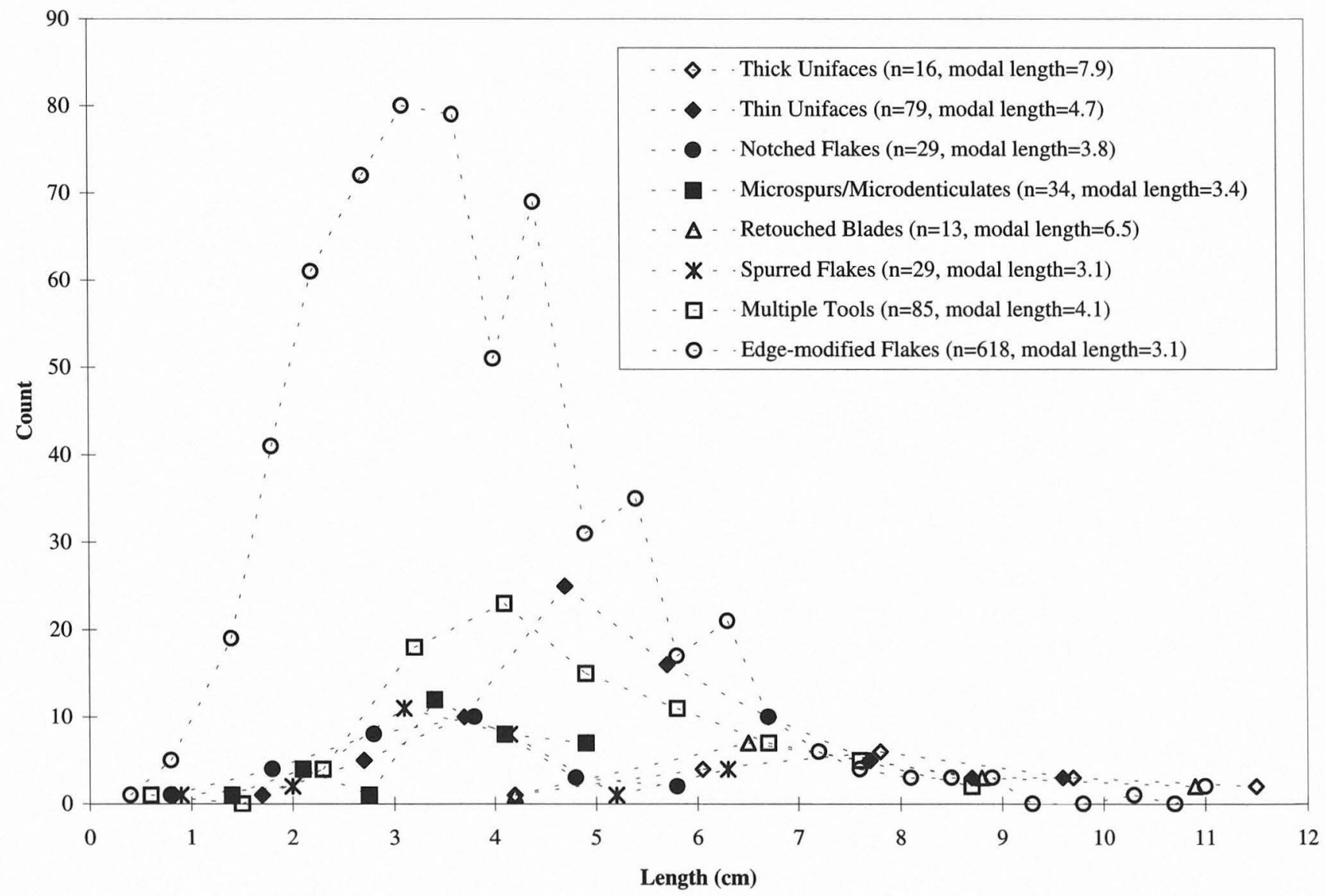

FIGURE 17-23. Distribution of lengths of unifacial tool categories. 
measure the difference between observed and expected values in contingency table cells, and converts this difference to a z-score (Haberman 1978; Norusis 1993). Adjusted residuals with the value of either +2 or greater or -2 or less are considered significant; a more complete explanation is presented below.

\section{Distribution of Flake Type by Unifacial Tool Class}

The distribution of flake type by unifacial tool class demonstrates that the most significant patterning occurs with the use of biface thinning flakes and normal flakes (Table 17-11). At this point it is worthwhile to discuss in more detail the mechanics behind contingency table analysis.

The first step in contingency table analysis and analysis of adjusted residuals is to divide the table into row and column variables, tabulate the individual cell values, and sum the row and column totals. Then expected values (not shown) are calculated. In the case of Table 17-11, the expected values can best be understood by looking at the overall percentages of different flake types (biface thinning, normal, and sequent). These are $37.3 \%, 0.3 \%, 60.2 \%$, and $2.4 \%$, respectively. Calculating the expected values of these flake types for each tool class assumes that the percentage of the flake types will remain the same as the overall percentages for each tool class (see column total percentages). This is achieved by multiplying the total number of each tool (e.g., 76 denticulates) by the given frequency of each flake type. For example, the expected number of denticulates made on biface thinning flakes is approximately 30 ( $76 \times 0.39$ ), and the expected number of edgemodified flakes made on biface thinning flakes is about 701 $(1,798 \times 0.39)$. The obvious next step is to look at the difference between the observed value and the expected value for each cell. However, these numbers are not very useful because large samples will have large differences, and small samples will have small differences. It is difficult to determine which is significant. To control for the sample size affect, the difference between the observed and expected values in each cell is divided by the square root of that cell's expected value. The result is called the standardized residual. Unfortunately, standardized residuals are not easy to interpret in statistical terms. However, if the standardized residual is divided by their estimated variance then the interpretation is much more straightforward. The result of this calculation is called the adjusted residual. Adjusted residuals have the same distribution as a z-score, and thus can be easily interpreted in terms of significance. If an adjusted residual equals +2.0 or -2.0 , then the chance that the observed value is equal to the expected value is $5 \%$. If the adjusted residual is greater than +2.0 or less than -2.0 , then the chances are even smaller than $5 \%$ that the observed and expected values are statistically equal. A brief look at the adjusted residuals presented in this chapter will show that the largest percentages and the largest samples are not necessarily significant. For example, in Table 17-11 normal flakes were used for $55.8 \%$ of all notches, but the adjusted residual is -1.0 and clearly not significant.

Returning to the analysis of flake types, over $97 \%$ of the unifacial tools were made on normal and biface thinning flakes. Biface thinning flakes occur in less than expected numbers as denticulates, multiple tools, thick unifaces, and thin unifaces but in higher than expected numbers as edgemodified flakes, microdenticulates, and microspurs. Denticulates, multiple tools, thick unifaces, and thin unifaces were made on normal flakes in greater than expected numbers, but normal flakes are used for edge-modified flakes and microspurs-microdenticulates in significantly less than expected numbers. Notches, retouched blades, and spurs show no significant deviation from the overall frequency distribution shown by the column total percentages.

One interpretation of these results is that normal flakes are produced, in part, for blanks for specific tool classes (thick and thin unifaces and denticulates) and that biface thinning flakes are produced during the course of biface tool production. The timing of tool use may play a role in creating the patterns observed here as well. For example, if the production of normal flakes takes place at a different time than the production of bifaces, then the lack of patterning among notches, retouched blades, and spurs suggests that these tools may have been manufactured at any time. Blades may be a special case. However, the favoring of biface thinning flakes for edge-modified flakes, microdenticulates, and microspurs suggests that their manufacture and perhaps use coincides with the manufacturing of bifaces. Also it is possible that the more intense use of biface thinning flakes represents a more expedient technological strategy since presumably these are byproducts of biface tool manufacture and not a specific product (i.e., tool blank) by themselves. Thus, edge-modified flakes, microdenticulates, and microspurs could be part of an expedient strategy. It is unlikely that biface thinning flakes were large enough for the manufacture of thick and thin unifaces.

\section{Distribution of Dorsal Surface Cortex by Unifacial Tool Class}

The distribution of dorsal surface cortex categories by unifacial tool class demonstrates that the most significant patterning occurs among denticulates, edge-modified flakes, microdenticulates, thick unifaces, and thin unifaces (Table 17-12). Primary flakes occur in higher than expected frequencies on denticulates, thick unifaces, and thin unifaces, while primary flakes occur in lower than expected numbers on edge-modified flakes and microspurs-microdenticulates. Secondary flakes occur in greater than expected frequencies on denticulates, thick unifaces, and thin unifaces but in signifi- 
TABLE 17-11

Distribution of Flake Types by Unifacial Tool Class

\begin{tabular}{|c|c|c|c|c|}
\hline \multirow[b]{2}{*}{ Tool Class } & \multicolumn{3}{|c|}{ Flake Types } & \multirow[b]{2}{*}{ Row Total and Percent } \\
\hline & Biface Thinning & Normal & Sequent & \\
\hline $\begin{array}{l}\text { Denticulate } \\
\text { Count } \\
\text { Row } \% \\
\text { Adjusted residual } \\
\end{array}$ & $\begin{array}{r}7 \\
9.2 \\
-5.1 \\
\end{array}$ & $\begin{array}{r}67 \\
88.2 \\
5.0 \\
\end{array}$ & $\begin{array}{r}2 \\
2.6 \\
0.1 \\
\end{array}$ & $\begin{array}{l}76 \\
2.5\end{array}$ \\
\hline $\begin{array}{l}\text { Edge Modified Flake } \\
\text { Count } \\
\text { Row } \% \\
\text { Adjusted residual } \\
\end{array}$ & $\begin{array}{r}815 \\
46.4 \\
12.1 \\
\end{array}$ & $\begin{array}{r}901 \\
51.3 \\
-11.8 \\
\end{array}$ & $\begin{array}{r}41 \\
2.3 \\
-0.5 \\
\end{array}$ & $\begin{array}{c}1,757 \\
58.1\end{array}$ \\
\hline $\begin{array}{l}\text { Microspur-Microdenticulate } \\
\text { Count } \\
\text { Row } \% \\
\text { Adjusted residual } \\
\end{array}$ & $\begin{array}{r}102 \\
53.7 \\
4.8 \\
\end{array}$ & $\begin{array}{r}86 \\
45.3 \\
-4.3 \\
\end{array}$ & $\begin{array}{r}2 \\
1.1 \\
-1.3 \\
\end{array}$ & $\begin{array}{c}190 \\
6.3\end{array}$ \\
\hline $\begin{array}{l}\text { Multiple Tools } \\
\text { Count } \\
\text { Row \% } \\
\text { Adjusted residual } \\
\end{array}$ & $\begin{array}{r}74 \\
28.7 \\
-3.0 \\
\end{array}$ & $\begin{array}{r}178 \\
69.0 \\
3.0 \\
\end{array}$ & $\begin{array}{r}6 \\
2.3 \\
-0.1 \\
\end{array}$ & $\begin{array}{r}258 \\
8.5\end{array}$ \\
\hline $\begin{array}{l}\text { Notch } \\
\qquad \begin{array}{l}\text { Count } \\
\text { Row } \% \\
\text { Adjusted residual }\end{array}\end{array}$ & $\begin{array}{r}50 \\
41.7 \\
1.0 \\
\end{array}$ & $\begin{array}{r}67 \\
55.8 \\
-1.0 \\
\end{array}$ & $\begin{array}{r}3 \\
2.5 \\
0.0 \\
\end{array}$ & $\begin{array}{l}120 \\
4.0\end{array}$ \\
\hline $\begin{array}{l}\text { Retouched Blade } \\
\text { Count } \\
\text { Row \% } \\
\text { Adjusted residual } \\
\end{array}$ & $\begin{array}{r}8 \\
30.8 \\
-0.7 \\
\end{array}$ & $\begin{array}{r}18 \\
69.2 \\
0.9 \\
\end{array}$ & $\begin{array}{r}0 \\
0.0 \\
-0.8 \\
\end{array}$ & $\begin{array}{l}26 \\
0.9\end{array}$ \\
\hline $\begin{array}{l}\text { Spur } \\
\qquad \text { Count } \\
\text { Row } \% \\
\quad \text { Adjusted residual } \\
\end{array}$ & $\begin{array}{r}23 \\
35.4 \\
-0.3 \\
\end{array}$ & $\begin{array}{r}40 \\
61.5 \\
0.2 \\
\end{array}$ & $\begin{array}{r}2 \\
3.1 \\
0.3 \\
\end{array}$ & $\begin{array}{l}65 \\
2.2\end{array}$ \\
\hline $\begin{array}{l}\text { Thick Uniface Fragment } \\
\text { Count } \\
\text { Row } \% \\
\text { Adjusted residual } \\
\end{array}$ & $\begin{array}{r}0 \\
0.0 \\
-2.0 \\
\end{array}$ & $\begin{array}{r}7 \\
100.0 \\
2.2 \\
\end{array}$ & $\begin{array}{r}0 \\
0.0 \\
-0.4 \\
\end{array}$ & $\begin{array}{c}7 \\
0.2\end{array}$ \\
\hline $\begin{array}{l}\text { Thick Uniface } \\
\text { Count } \\
\text { Row \% } \\
\text { Adjusted residual }\end{array}$ & $\begin{array}{r}1 \\
1.7 \\
-5.7 \\
\end{array}$ & $\begin{array}{r}58 \\
98.3 \\
6.0 \\
\end{array}$ & $\begin{array}{r}0 \\
0.0 \\
-1.2 \\
\end{array}$ & $\begin{array}{l}59 \\
2.0\end{array}$ \\
\hline $\begin{array}{l}\text { Thin Uniface Fragment } \\
\text { Count } \\
\text { Row } \% \\
\text { Adjusted residual }\end{array}$ & $\begin{array}{r}10 \\
5.3 \\
-9.4 \\
\end{array}$ & $\begin{array}{r}176 \\
93.6 \\
9.7 \\
\end{array}$ & $\begin{array}{r}2 \\
1.1 \\
-1.3 \\
\end{array}$ & $\begin{array}{c}188 \\
6.2\end{array}$ \\
\hline $\begin{array}{l}\text { Thin Uniface } \\
\text { Count } \\
\text { Row \% } \\
\text { Adjusted residual }\end{array}$ & $\begin{array}{r}39 \\
14.1 \\
-8.4 \\
\end{array}$ & $\begin{array}{r}222 \\
80.1 \\
7.1 \\
\end{array}$ & $\begin{array}{r}16 \\
5.8 \\
3.8 \\
\end{array}$ & $\begin{array}{c}277 \\
9.2\end{array}$ \\
\hline $\begin{array}{l}\text { Column Total: } \\
\text { Column Percent: }\end{array}$ & $\begin{array}{r}1,129 \\
37.3 \\
\end{array}$ & $\begin{array}{r}1,820 \\
60.2 \\
\end{array}$ & $\begin{array}{r}74 \\
2.4 \\
\end{array}$ & $\begin{array}{l}3,023 \\
100.0 \\
\end{array}$ \\
\hline
\end{tabular}

cantly less than expected frequencies on edge-modified flakes and microspur-microdenticulates. Interior flakes occur in greater than expected numbers on edge-modified flakes and microspur-microdenticulates, but in less than expected frequencies on denticulates, thick unifaces, and thin unifaces. Multiple tools, notches, retouched blades, and spurs are statistically similar to the overall pattern.
The result of this analysis suggests that denticulates, thick unifaces, and thin unifaces are being manufactured on flakes removed at an earlier stage of reduction than edgemodified flakes and microspur-microdenticulates. Again, the timing of tool manufacture and use as well as the timing of overall lithic reduction may play a role in the patterns found within certain tool classes. 
TABLE $17-12$

Distribution of Cortex Categories by Unifacial Tool Class

\begin{tabular}{|c|c|c|c|c|}
\hline \multirow[b]{2}{*}{ Tool Class } & \multicolumn{3}{|c|}{ Cortex } & \multirow[b]{2}{*}{ Row Total and Percent } \\
\hline & Primary & Secondary & Interior & \\
\hline $\begin{array}{l}\text { Denticulate } \\
\text { Count } \\
\text { Row } \% \\
\text { Adjusted residual }\end{array}$ & $\begin{array}{c}6 \\
7.9 \\
2.2 \\
\end{array}$ & $\begin{array}{c}38 \\
50.0 \\
2.6 \\
\end{array}$ & $\begin{array}{c}32 \\
42.1 \\
-3.4 \\
\end{array}$ & $\begin{array}{l}76 \\
2.5\end{array}$ \\
\hline $\begin{array}{l}\text { Edge Modified Flake } \\
\text { Count } \\
\text { Row \% } \\
\text { Adjusted residual }\end{array}$ & $\begin{array}{r}33 \\
1.9 \\
-5.5 \\
\end{array}$ & $\begin{array}{r}598 \\
34.0 \\
-2.3 \\
\end{array}$ & $\begin{array}{c}1,126 \\
64.1 \\
4.3 \\
\end{array}$ & $\begin{array}{c}1,757 \\
58.1\end{array}$ \\
\hline $\begin{array}{l}\text { Microspur-Microdenticulate } \\
\text { Count } \\
\text { Row } \% \\
\text { Adjusted residual } \\
\end{array}$ & $\begin{array}{c}1 \\
0.5 \\
-2.3 \\
\end{array}$ & $\begin{array}{c}50 \\
26.3 \\
-2.8 \\
\end{array}$ & $\begin{array}{c}139 \\
73.2 \\
3.6 \\
\end{array}$ & $\begin{array}{l}190 \\
6.3\end{array}$ \\
\hline $\begin{array}{l}\text { Multiple Tools } \\
\text { Count } \\
\text { Row } \% \\
\text { Adjusted residual }\end{array}$ & $\begin{array}{c}8 \\
3.1 \\
-0.3 \\
\end{array}$ & $\begin{array}{c}93 \\
36.0 \\
0.1 \\
\end{array}$ & $\begin{array}{c}157 \\
60.9 \\
0.0 \\
\end{array}$ & $\begin{array}{l}258 \\
8.5\end{array}$ \\
\hline $\begin{array}{l}\text { Notch } \\
\text { Count } \\
\text { Row } \% \\
\text { Adjusted residual }\end{array}$ & $\begin{array}{c}1 \\
0.8 \\
-1.6 \\
\end{array}$ & $\begin{array}{c}41 \\
34.2 \\
-0.4 \\
\end{array}$ & $\begin{array}{c}78 \\
65.0 \\
1.0 \\
\end{array}$ & $\begin{array}{l}120 \\
4.0\end{array}$ \\
\hline $\begin{array}{l}\text { Retouched Blade } \\
\text { Count } \\
\text { Row } \% \\
\text { Adjusted residual }\end{array}$ & $\begin{array}{c}1 \\
3.8 \\
0.1 \\
\end{array}$ & $\begin{array}{c}12 \\
46.2 \\
1.1 \\
\end{array}$ & $\begin{array}{c}13 \\
50.0 \\
-1.1 \\
\end{array}$ & $\begin{array}{l}26 \\
0.9\end{array}$ \\
\hline $\begin{array}{l}\text { Spur } \\
\text { Count } \\
\text { Row } \% \\
\text { Adjusted residual }\end{array}$ & $\begin{array}{c}0 \\
0.0 \\
-1.5 \\
\end{array}$ & $\begin{array}{c}26 \\
40.0 \\
-0.7 \\
\end{array}$ & $\begin{array}{c}39 \\
60.0 \\
-0.1 \\
\end{array}$ & $\begin{array}{l}65 \\
2.2\end{array}$ \\
\hline $\begin{array}{l}\text { Thick Uniface Fragment } \\
\text { Count } \\
\text { Row \% } \\
\text { Adjusted residual }\end{array}$ & $\begin{array}{c}1 \\
14.3 \\
1.6 \\
\end{array}$ & $\begin{array}{c}4 \\
57.1 \\
1.2 \\
\end{array}$ & $\begin{array}{c}2 \\
28.6 \\
-1.8 \\
\end{array}$ & $\begin{array}{c}7 \\
0.2\end{array}$ \\
\hline $\begin{array}{l}\text { Thick Uniface } \\
\text { Count } \\
\text { Row \% } \\
\text { Adjusted residual }\end{array}$ & $\begin{array}{c}5 \\
8.5 \\
2.2 \\
\end{array}$ & $\begin{array}{c}40 \\
67.8 \\
5.2 \\
\end{array}$ & $\begin{array}{c}14 \\
23.7 \\
-5.9 \\
\end{array}$ & $\begin{array}{l}59 \\
0.2\end{array}$ \\
\hline $\begin{array}{l}\text { Thin Uniface Fragment } \\
\text { Count } \\
\text { Row \% } \\
\text { Adjusted residual }\end{array}$ & $\begin{array}{l}11 \\
5.9 \\
1.9 \\
\end{array}$ & $\begin{array}{c}50 \\
26.6 \\
-2.7 \\
\end{array}$ & $\begin{array}{c}127 \\
67.6 \\
1.9 \\
\end{array}$ & $\begin{array}{l}188 \\
6.2\end{array}$ \\
\hline $\begin{array}{l}\text { Thin Uniface } \\
\text { Count } \\
\text { Row } \% \\
\text { Adjusted residual } \\
\end{array}$ & $\begin{array}{c}36 \\
13.0 \\
9.2 \\
\end{array}$ & $\begin{array}{c}129 \\
46.6 \\
3.9 \\
\end{array}$ & $\begin{array}{l}112 \\
40.4 \\
-7.3 \\
\end{array}$ & $\begin{array}{c}277 \\
9.2\end{array}$ \\
\hline $\begin{array}{l}\text { Column Total: } \\
\text { Column Percent: }\end{array}$ & $\begin{array}{l}103 \\
3.0 \\
\end{array}$ & $\begin{array}{l}1,081 \\
36.0 \\
\end{array}$ & $\begin{array}{c}1,839 \\
61.0 \\
\end{array}$ & $\begin{array}{c}3,023 \\
100 \\
\end{array}$ \\
\hline
\end{tabular}

\section{Distribution of Lithic Raw Material by Unifacial Tool Class}

The distribution of lithic raw material types by unifacial tool classes suggests little selectivity (Table 17-13). The only significant deviations are higher than expected numbers of non-Edwards cherts used for denticulates, nonlocal Edwards cherts occurring in greater than expected frequencies on microspurs-microdenticulates, and local Edward cherts occurring in less than expected frequencies on thick unifaces. Almost $88 \%$ of all unifacial tools were made on local Edwards cherts. Nonlocal cherts were the next most common material, but only $8.8 \%$ of the unifacial tools were made on materials in this category. 
TABLE $17-13$

Distribution of Raw Materials by Unifacial Tool Class

\begin{tabular}{|c|c|c|c|c|c|}
\hline \multirow[b]{2}{*}{ Tool Class } & \multicolumn{4}{|c|}{ Raw Material } & \multirow[b]{2}{*}{$\begin{array}{c}\text { Row Total and } \\
\text { Percent }\end{array}$} \\
\hline & $\begin{array}{l}\text { Local Edwards } \\
\text { Chert }\end{array}$ & $\begin{array}{c}\text { Nonlocal Edwards } \\
\text { Chert }\end{array}$ & $\begin{array}{c}\text { Non-Edwards } \\
\text { Materials }\end{array}$ & Indeterminate & \\
\hline $\begin{array}{l}\text { Denticulate } \\
\text { Count } \\
\text { Row } \% \\
\text { Adjusted residual } \\
\end{array}$ & $\begin{array}{c}61 \\
85.9 \\
-0.3 \\
\end{array}$ & $\begin{array}{c}8 \\
11.3 \\
0.7 \\
\end{array}$ & $\begin{array}{c}2 \\
2.8 \\
3.0 \\
\end{array}$ & $\begin{array}{c}0 \\
0.0 \\
-1.6 \\
\end{array}$ & $\begin{array}{c}71 \\
2.9\end{array}$ \\
\hline $\begin{array}{l}\text { Edge-modified Flake } \\
\text { Count } \\
\text { Row } \% \\
\text { Adjusted residual } \\
\end{array}$ & $\begin{array}{c}1,194 \\
87.8 \\
1.0 \\
\end{array}$ & $\begin{array}{l}121 \\
8.9 \\
0.2 \\
\end{array}$ & $\begin{array}{c}2 \\
0.1 \\
-2.5 \\
\end{array}$ & $\begin{array}{r}43 \\
3.2 \\
-1.1 \\
\end{array}$ & $\begin{array}{c}1,360 \\
55.2\end{array}$ \\
\hline $\begin{array}{l}\text { Microspur-Microdenticulate } \\
\text { Count } \\
\text { Row } \% \\
\text { Adjusted residual } \\
\end{array}$ & $\begin{array}{r}156 \\
82.1 \\
-2.2 \\
\end{array}$ & $\begin{array}{c}29 \\
15.3 \\
3.3 \\
\end{array}$ & $\begin{array}{c}0 \\
0.0 \\
-1.0 \\
\end{array}$ & $\begin{array}{c}5 \\
2.6 \\
-0.7 \\
\end{array}$ & $\begin{array}{l}190 \\
7.7\end{array}$ \\
\hline $\begin{array}{l}\text { Multiple Tools } \\
\text { Count } \\
\text { Row \% } \\
\text { Adjusted residual }\end{array}$ & $\begin{array}{c}188 \\
90.4 \\
1.4 \\
\end{array}$ & $\begin{array}{r}14 \\
6.7 \\
-1.1 \\
\end{array}$ & $\begin{array}{c}2 \\
1.0 \\
1.2 \\
\end{array}$ & $\begin{array}{c}4 \\
1.9 \\
-1.3 \\
\end{array}$ & $\begin{array}{l}208 \\
8.4\end{array}$ \\
\hline $\begin{array}{l}\text { Notch } \\
\text { Count } \\
\text { Row \% } \\
\text { Adjusted residual } \\
\end{array}$ & $\begin{array}{c}84 \\
85.7 \\
-0.5 \\
\end{array}$ & $\begin{array}{c}10 \\
10.2 \\
0.5 \\
\end{array}$ & $\begin{array}{c}0 \\
0.0 \\
-0.7 \\
\end{array}$ & $\begin{array}{c}4 \\
4.1 \\
0.3 \\
\end{array}$ & $\begin{array}{l}98 \\
4.0\end{array}$ \\
\hline $\begin{array}{l}\text { Retouched Blade } \\
\text { Count } \\
\text { Row \% } \\
\text { Adjusted residual }\end{array}$ & $\begin{array}{c}24 \\
92.3 \\
0.8 \\
\end{array}$ & $\begin{array}{c}2 \\
7.7 \\
-0.2 \\
\end{array}$ & $\begin{array}{c}0 \\
0.0 \\
-0.3 \\
\end{array}$ & $\begin{array}{c}0 \\
0.0 \\
-1.0 \\
\end{array}$ & $\begin{array}{l}26 \\
1.1\end{array}$ \\
\hline $\begin{array}{l}\text { Spur } \\
\text { Count } \\
\text { Row \% } \\
\text { Adjusted residual } \\
\end{array}$ & $\begin{array}{c}58 \\
89.2 \\
0.5 \\
\end{array}$ & $\begin{array}{c}4 \\
6.2 \\
-0.8 \\
\end{array}$ & $\begin{array}{c}0 \\
0.0 \\
-0.5 \\
\end{array}$ & $\begin{array}{c}3 \\
4.6 \\
0.5 \\
\end{array}$ & $\begin{array}{c}65 \\
2.6\end{array}$ \\
\hline $\begin{array}{l}\text { Thick Uniface Fragment } \\
\text { Count } \\
\text { Row \% } \\
\text { Adjusted residual } \\
\end{array}$ & $\begin{array}{c}6 \\
85.7 \\
-0.1 \\
\end{array}$ & $\begin{array}{c}1 \\
14.3 \\
0.5 \\
\end{array}$ & $\begin{array}{c}0 \\
0.0 \\
-0.2 \\
\end{array}$ & $\begin{array}{c}0 \\
0.0 \\
-0.5 \\
\end{array}$ & $\begin{array}{c}7 \\
0.3\end{array}$ \\
\hline $\begin{array}{l}\text { Thick Uniface } \\
\text { Count } \\
\text { Row } \% \\
\text { Adjusted residual }\end{array}$ & $\begin{array}{c}51 \\
96.2 \\
2.0 \\
\end{array}$ & $\begin{array}{c}2 \\
3.8 \\
-1.3 \\
\end{array}$ & $\begin{array}{c}0 \\
0.0 \\
-0.5 \\
\end{array}$ & $\begin{array}{c}0 \\
0.0 \\
-1.4 \\
\end{array}$ & $\begin{array}{l}53 \\
2.2\end{array}$ \\
\hline $\begin{array}{l}\text { Thin Uniface Fragment } \\
\text { Count } \\
\text { Row } \% \\
\text { Adjusted residual }\end{array}$ & $\begin{array}{l}122 \\
77.2 \\
-3.9 \\
\end{array}$ & $\begin{array}{r}10 \\
6.3 \\
-1.1 \\
\end{array}$ & $\begin{array}{c}3 \\
1.9 \\
2.8 \\
\end{array}$ & $\begin{array}{c}23 \\
14.6 \\
7.8 \\
\end{array}$ & $\begin{array}{c}158 \\
6.4\end{array}$ \\
\hline $\begin{array}{l}\text { Thin Uniface } \\
\text { Count } \\
\text { Row } \% \\
\text { Adjusted residual }\end{array}$ & $\begin{array}{c}203 \\
89.8 \\
1.2 \\
\end{array}$ & $\begin{array}{r}16 \\
7.1 \\
-1.0 \\
\end{array}$ & $\begin{array}{c}2 \\
0.9 \\
1.0 \\
\end{array}$ & $\begin{array}{c}5 \\
2.2 \\
-1.1 \\
\end{array}$ & $\begin{array}{l}226 \\
9.2\end{array}$ \\
\hline $\begin{array}{l}\text { Column Total: } \\
\text { Column Percent: }\end{array}$ & $\begin{array}{l}2,147 \\
87.2\end{array}$ & $\begin{array}{l}217 \\
8.8\end{array}$ & $\begin{array}{l}11 \\
0.4\end{array}$ & $\begin{array}{l}87 \\
3.5\end{array}$ & $\begin{array}{l}2,462 \\
100.0\end{array}$ \\
\hline
\end{tabular}

\section{Distribution of Raw Material Textures by Unifacial Tool Class}

The distribution of fine-grained cherts and medium/ coarse-grained cherts by unifacial tool classes is very homogeneous and shows little selectivity (Table 17-14). Finegrained cherts were used for over $77 \%$ of all unifacial tool classes. The only significant variation away from this pattern is in thick unifaces which occur on medium/coarsegrained cherts in greater than expected numbers and on fine-grained cherts in less than expected numbers. Even though thin unifaces have significant adjusted residual values for chalcedony and petrified wood, the sample size is low. 
TABLE 17-14

Distribution of Raw Material Textures by Unifacial Tool Class

\begin{tabular}{|c|c|c|c|c|c|}
\hline \multirow[b]{2}{*}{ Tool Class } & \multicolumn{4}{|c|}{ Raw Material } & \multirow[b]{2}{*}{$\begin{array}{c}\text { Row Total and } \\
\text { Percent }\end{array}$} \\
\hline & Chalcedony & Fine Chert & $\begin{array}{c}\text { Medium/Coarse } \\
\text { Chert }\end{array}$ & $\begin{array}{c}\text { Petrified } \\
\text { Wood }\end{array}$ & \\
\hline $\begin{array}{l}\text { Denticulate } \\
\text { Count } \\
\text { Row } \% \\
\text { Adjusted residual } \\
\end{array}$ & $\begin{array}{c}0 \\
0.0 \\
-0.2 \\
\end{array}$ & $\begin{array}{c}53 \\
74.6 \\
-0.2 \\
\end{array}$ & $\begin{array}{c}18 \\
25.4 \\
-0.2 \\
\end{array}$ & $\begin{array}{c}0 \\
0.0 \\
-0.2 \\
\end{array}$ & $\begin{array}{l}71 \\
3.0\end{array}$ \\
\hline $\begin{array}{l}\text { Edge-modified Flake } \\
\text { Count } \\
\text { Row } \% \\
\text { Adjusted residual }\end{array}$ & $\begin{array}{c}0 \\
0.0 \\
-1.1 \\
\end{array}$ & $\begin{array}{c}999 \\
75.9 \\
0.4 \\
\end{array}$ & $\begin{array}{r}318 \\
24.1 \\
-0.3 \\
\end{array}$ & $\begin{array}{c}0 \\
0.0 \\
-1.1 \\
\end{array}$ & $\begin{array}{c}1,317 \\
55.4\end{array}$ \\
\hline $\begin{array}{l}\text { Microspur-Microdenticulate } \\
\text { Count } \\
\text { Row } \% \\
\text { Adjusted residual } \\
\end{array}$ & $\begin{array}{c}0 \\
0.0 \\
-0.3 \\
\end{array}$ & $\begin{array}{r}138 \\
74.6 \\
-0.3 \\
\end{array}$ & $\begin{array}{c}47 \\
25.4 \\
0.3 \\
\end{array}$ & $\begin{array}{c}0 \\
0.0 \\
-0.3 \\
\end{array}$ & $\begin{array}{l}185 \\
7.8\end{array}$ \\
\hline $\begin{array}{l}\text { Multiple Tools } \\
\text { Count } \\
\text { Row } \% \\
\text { Adjusted residual } \\
\end{array}$ & $\begin{array}{c}0 \\
0.0 \\
-0.3 \\
\end{array}$ & $\begin{array}{c}163 \\
79.9 \\
1.5 \\
\end{array}$ & $\begin{array}{c}41 \\
20.1 \\
-1.5 \\
\end{array}$ & $\begin{array}{c}0 \\
0.0 \\
-0.3 \\
\end{array}$ & $\begin{array}{l}204 \\
8.6\end{array}$ \\
\hline $\begin{array}{l}\text { Notch } \\
\text { Count } \\
\text { Row \% } \\
\text { Adjusted residual }\end{array}$ & $\begin{array}{c}0 \\
0.0 \\
-0.2 \\
\end{array}$ & $\begin{array}{c}72 \\
76.6 \\
0.2 \\
\end{array}$ & $\begin{array}{c}22 \\
23.4 \\
-0.2 \\
\end{array}$ & $\begin{array}{c}0 \\
0.0 \\
-0.3 \\
\end{array}$ & $\begin{array}{l}94 \\
4.0\end{array}$ \\
\hline $\begin{array}{l}\text { Retouched Blade } \\
\text { Count } \\
\text { Row } \% \\
\text { Adjusted residual }\end{array}$ & $\begin{array}{c}0 \\
0.0 \\
-0.1 \\
\end{array}$ & $\begin{array}{c}19 \\
73.1 \\
-0.3 \\
\end{array}$ & $\begin{array}{c}7 \\
26.9 \\
0.3 \\
\end{array}$ & $\begin{array}{c}0 \\
0.0 \\
-0.1 \\
\end{array}$ & $\begin{array}{l}26 \\
1.1\end{array}$ \\
\hline $\begin{array}{l}\text { Spur } \\
\qquad \text { Count } \\
\quad \text { Row } \% \\
\text { Adjusted residual }\end{array}$ & $\begin{array}{c}0 \\
0.0 \\
-0.2 \\
\end{array}$ & $\begin{array}{c}46 \\
74.2 \\
-0.3 \\
\end{array}$ & $\begin{array}{c}16 \\
25.8 \\
0.3 \\
\end{array}$ & $\begin{array}{c}0 \\
0.0 \\
-0.2 \\
\end{array}$ & $\begin{array}{c}62 \\
2.6\end{array}$ \\
\hline $\begin{array}{l}\text { Thick Uniface Fragment } \\
\text { Count } \\
\text { Row } \% \\
\text { Adjusted residual }\end{array}$ & $\begin{array}{c}0 \\
0.0 \\
-0.1 \\
\end{array}$ & $\begin{array}{c}7 \\
100 \\
1.5 \\
\end{array}$ & $\begin{array}{c}0 \\
0.0 \\
-1.5 \\
\end{array}$ & $\begin{array}{c}0 \\
0.0 \\
-0.1 \\
\end{array}$ & $\begin{array}{c}7 \\
0.3\end{array}$ \\
\hline $\begin{array}{l}\text { Thick Uniface } \\
\text { Count } \\
\text { Row \% } \\
\text { Adjusted residual }\end{array}$ & $\begin{array}{c}0 \\
0.0 \\
-0.2 \\
\end{array}$ & $\begin{array}{c}28 \\
52.8 \\
-3.9 \\
\end{array}$ & $\begin{array}{c}25 \\
47.2 \\
3.9 \\
\end{array}$ & $\begin{array}{c}0 \\
0.0 \\
-0.2 \\
\end{array}$ & $\begin{array}{l}53 \\
2.2\end{array}$ \\
\hline $\begin{array}{l}\text { Thin Uniface Fragment } \\
\text { Count } \\
\text { Row } \% \\
\text { Adjusted residual }\end{array}$ & $\begin{array}{c}0 \\
0.0 \\
-0.2 \\
\end{array}$ & $\begin{array}{c}114 \\
84.4 \\
2.5 \\
\end{array}$ & $\begin{array}{c}21 \\
15.6 \\
-2.5 \\
\end{array}$ & $\begin{array}{c}0 \\
0.0 \\
-0.2 \\
\end{array}$ & $\begin{array}{l}135 \\
5.7\end{array}$ \\
\hline $\begin{array}{l}\text { Thin Uniface } \\
\text { Count } \\
\text { Row \% } \\
\text { Adjusted residual }\end{array}$ & $\begin{array}{c}1 \\
0.4 \\
3.1 \\
\end{array}$ & $\begin{array}{r}157 \\
70.4 \\
-1.9 \\
\end{array}$ & $\begin{array}{c}64 \\
28.7 \\
1.6 \\
\end{array}$ & $\begin{array}{c}1 \\
0.4 \\
3.1 \\
\end{array}$ & $\begin{array}{l}223 \\
9.4\end{array}$ \\
\hline $\begin{array}{l}\text { Column Total: } \\
\text { Column Percent: }\end{array}$ & $\begin{array}{c}1 \\
0.0\end{array}$ & $\begin{array}{c}1,796 \\
75.6\end{array}$ & $\begin{array}{l}579 \\
24.4\end{array}$ & $\begin{array}{c}1 \\
0.0\end{array}$ & $\begin{array}{l}2,377 \\
100.0\end{array}$ \\
\hline
\end{tabular}

\section{Distribution of Unifacial Tool Classes in the Valley Floor and Valley Margin Units and Stratigraphic Units}

The distribution of unifacial tool classes in all valley floor and valley margin units indicates that few significant differences exist between these two site areas (Table 1715). Approximately two-thirds of all unifacial tools were recovered in the valley floor units. Inspection of adjusted residuals indicates that multiple tools, thin uniface fragments, and spurs display patterns that significantly deviate from the general distributions. The valley floor units have significantly more and the valley margins have significantly fewer of these tools than would be expected. The detailed distribution of unifacial tool classes within the stratigraphic 
TABLE $17-15$

Distribution of Unifacial Tool Classes in the Valley Floor and Valley Margin

\begin{tabular}{|c|c|c|c|}
\hline Tool Class & Floor & Margin & Row Total and Percent \\
\hline $\begin{array}{l}\text { Denticulate } \\
\text { Count } \\
\text { Row } \% \\
\text { Adjusted residual } \\
\end{array}$ & $\begin{array}{c}54 \\
59.3 \\
-1.6 \\
\end{array}$ & $\begin{array}{c}37 \\
40.7 \\
1.6 \\
\end{array}$ & $\begin{array}{l}91 \\
2.7\end{array}$ \\
\hline $\begin{array}{l}\text { Edge Modified Flake } \\
\text { Count } \\
\text { Row } \% \\
\text { Adjusted residual }\end{array}$ & $\begin{array}{l}1,176 \\
65.9 \\
-1.9 \\
\end{array}$ & $\begin{array}{c}607 \\
34.1 \\
1.9 \\
\end{array}$ & $\begin{array}{c}1,780 \\
53.6\end{array}$ \\
\hline $\begin{array}{l}\text { Microspur-Microdenticulate } \\
\text { Count } \\
\text { Row } \% \\
\text { Adjusted residual }\end{array}$ & $\begin{array}{l}125 \\
66.8 \\
-0.1 \\
\end{array}$ & $\begin{array}{c}62 \\
33.2 \\
0.1 \\
\end{array}$ & $\begin{array}{c}187 \\
5.6\end{array}$ \\
\hline $\begin{array}{l}\text { Multiple Tools } \\
\text { Count } \\
\text { Row \% } \\
\text { Adjusted residual }\end{array}$ & $\begin{array}{c}233 \\
72.1 \\
2.0 \\
\end{array}$ & $\begin{array}{c}90 \\
27.9 \\
-2.0 \\
\end{array}$ & $\begin{array}{l}323 \\
9.7\end{array}$ \\
\hline $\begin{array}{l}\text { Notch } \\
\text { Count } \\
\text { Row \% } \\
\text { Adjusted residual }\end{array}$ & $\begin{array}{c}113 \\
68.1 \\
0.2 \\
\end{array}$ & $\begin{array}{c}53 \\
31.9 \\
-0.2 \\
\end{array}$ & $\begin{array}{c}166 \\
5.0\end{array}$ \\
\hline $\begin{array}{l}\text { Retouched Blade } \\
\text { Count } \\
\text { Row } \% \\
\text { Adjusted residual }\end{array}$ & $\begin{array}{c}19 \\
65.5 \\
-0.2 \\
\end{array}$ & $\begin{array}{c}10 \\
34.5 \\
0.2 \\
\end{array}$ & $\begin{array}{l}29 \\
0.9\end{array}$ \\
\hline $\begin{array}{l}\text { Spur } \\
\text { Count } \\
\text { Row } \% \\
\text { Adjusted residual }\end{array}$ & $\begin{array}{c}59 \\
89.4 \\
3.9 \\
\end{array}$ & $\begin{array}{c}7 \\
10.6 \\
-3.9 \\
\end{array}$ & $\begin{array}{l}66 \\
2.0\end{array}$ \\
\hline $\begin{array}{l}\text { Thick Uniface Fragment } \\
\text { Count } \\
\text { Row \% } \\
\text { Adjusted residual } \\
\end{array}$ & $\begin{array}{c}6 \\
85.7 \\
1.0 \\
\end{array}$ & $\begin{array}{c}1 \\
14.3 \\
-1.0 \\
\end{array}$ & $\begin{array}{c}7 \\
0.2\end{array}$ \\
\hline $\begin{array}{l}\text { Thick Uniface } \\
\text { Count } \\
\text { Row } \% \\
\text { Adjusted residual }\end{array}$ & $\begin{array}{c}46 \\
65.7 \\
-0.3 \\
\end{array}$ & $\begin{array}{c}24 \\
34.3 \\
0.3 \\
\end{array}$ & $\begin{array}{l}70 \\
2.1\end{array}$ \\
\hline $\begin{array}{l}\text { Thin Uniface Fragment } \\
\text { Count } \\
\text { Row \% } \\
\text { Adjusted residual } \\
\end{array}$ & $\begin{array}{c}167 \\
75.2 \\
2.6 \\
\end{array}$ & $\begin{array}{c}55 \\
24.8 \\
-2.6 \\
\end{array}$ & $\begin{array}{c}222 \\
6.7\end{array}$ \\
\hline $\begin{array}{l}\text { Thin Uniface } \\
\text { Count } \\
\text { Row } \% \\
\text { Adjusted residual }\end{array}$ & $\begin{array}{l}238 \\
63.1 \\
-1.8 \\
\end{array}$ & $\begin{array}{c}139 \\
36.9 \\
1.8\end{array}$ & $\begin{array}{c}377 \\
11.4\end{array}$ \\
\hline $\begin{array}{l}\text { Column Total: } \\
\text { Column Percent: }\end{array}$ & $\begin{array}{c}2,233 \\
67.3\end{array}$ & $\begin{array}{l}1,085 \\
32.7\end{array}$ & $\begin{array}{l}3,318 \\
100.0\end{array}$ \\
\hline
\end{tabular}

subdivisions within the valley margins and valley floor settings are presented in Tables 17-16 and 17-17.

\section{Distribution by Amount and Degree of Modification}

Two metric variables, total length of modified edge and potential edge length available for modification, can be used to further document and analyze the unifacial tool classes. The measurements are not intended to serve purely descrip- tive purposes, but rather are used to assess the way unifacial tool classes were used, the intensity of their use, and any changes in these patterns over time. For our purposes here, we will inspect the mean values by unifacial tool classes. These are presented in the tables above and are not duplicated here. First, if we look at a bivariate plot of mean potential edge length and mean total modification length for each unifacial tool class (Figure 17-25) it appears that a weak linear correlation exists, and it does $\left(\mathrm{s}^{2}=0.624\right.$; $\left.\mathrm{p}=0.113\right)$; how- 
TABLE $17-16$

Unifacial Tool Classes by Stratigraphic Unit (Valley Floor)

\begin{tabular}{|c|c|c|c|c|c|c|c|c|c|c|c|c|}
\hline Unit & $\begin{array}{l}\text { Retouched } \\
\text { Blade }\end{array}$ & Denticulate & \begin{tabular}{|l|} 
Edge- \\
modified \\
Flake
\end{tabular} & \begin{tabular}{|l|} 
Microspur/ \\
Micro- \\
denticulate \\
\end{tabular} & $\begin{array}{l}\text { Multiple } \\
\text { Tool }\end{array}$ & Notch & Spur & \begin{tabular}{|l} 
Thick \\
Uniface
\end{tabular} & \begin{tabular}{|l|} 
Thick \\
Uniface \\
Fragment \\
\end{tabular} & $\begin{array}{l}\text { Thin } \\
\text { Uniface } \\
\end{array}$ & \begin{tabular}{|l|} 
Thin \\
Uniface \\
Fragment \\
\end{tabular} & Totals \\
\hline Fill & & & 4 & & 1 & & & 5 & & 2 & & 7 \\
\hline IIIc/Fill & 1 & & 13 & & 2 & 2 & & 8 & & & 1 & 19 \\
\hline IIIb/c/Fill & 1 & & 3 & & 1 & & & 6 & 3 & 1 & & 6 \\
\hline IIIc/Pot & 1 & 1 & 39 & 2 & 8 & 1 & 1 & 1 & & 20 & 2 & 75 \\
\hline IIIc & 6 & 23 & 424 & 13 & 70 & 55 & 11 & 7 & 1 & 51 & 36 & 694 \\
\hline $\mathrm{IIIb} / \mathrm{c}$ & 3 & 10 & 167 & 5 & 22 & 15 & 3 & & & 10 & 9 & 252 \\
\hline IIIb & 4 & 11 & 222 & 20 & 33 & 16 & 8 & 6 & & 37 & 26 & 386 \\
\hline $\mathrm{IIIa} / \mathrm{b}$ & 4 & 6 & 116 & 18 & 21 & 6 & 9 & 16 & 2 & 14 & 22 & 217 \\
\hline IIIa & 2 & 16 & 219 & 65 & 61 & 23 & 13 & 3 & & 55 & 33 & 495 \\
\hline III & & 1 & 2 & 6 & 5 & 2 & & & & 2 & 1 & 19 \\
\hline II/IIIa & 1 & 9 & 102 & 12 & 14 & 17 & 6 & & & 27 & 14 & 208 \\
\hline II & & 1 & 105 & 12 & 25 & 10 & 7 & & & 34 & 21 & 233 \\
\hline $\mathrm{Id} / \mathrm{II}$ & 1 & 1 & 18 & 1 & 2 & 1 & & 2 & & 7 & 6 & 40 \\
\hline Isi-c/II & & & 7 & & & & & 3 & 1 & 1 & 2 & 10 \\
\hline I/II & & & & & 1 & & & & & 1 & & 2 \\
\hline Id & & & 1 & & & & & & & 1 & 2 & 4 \\
\hline Isi-c/Id & & 2 & 18 & & 6 & 1 & & & & 9 & 1 & 39 \\
\hline Isi-c & & & 17 & & 3 & 3 & 1 & 1 & & 13 & 11 & 52 \\
\hline Isi/Isi-c & & & 13 & & & & & 1 & & 2 & 2 & 17 \\
\hline Icl/Isi-c & & & 4 & & 1 & & & 3 & & 1 & 1 & 7 \\
\hline Isi/Icl/Isi-c & & & 8 & & 2 & & 1 & & & 3 & & 14 \\
\hline I & & & & & 2 & & & & & 1 & & 4 \\
\hline Icl & & 1 & 6 & & & & 1 & & & 4 & 2 & 15 \\
\hline Isi/Icl & & & 13 & 1 & 2 & 1 & 3 & & & 7 & 3 & 33 \\
\hline Isi & 2 & & 12 & & & & & & & 4 & & 18 \\
\hline Isi/D & & & & & 1 & & & & & & & 1 \\
\hline Isi/D? & & & 1 & & 1 & & & & & 1 & & 3 \\
\hline Igl/Isi & & & 8 & & 1 & & & & & 3 & & 12 \\
\hline Igl?/I & & & 1 & & & & & & & & & 1 \\
\hline Igl/Isi/Icl & & & 1 & & & & & & & & & 1 \\
\hline Igl & & & 2 & 1 & 1 & & & & & & & 4 \\
\hline Totals: & 26 & 82 & 1,550 & 126 & 287 & 153 & 64 & 62 & 7 & 312 & 196 & 2,888 \\
\hline
\end{tabular}

TABLE 17-17

Unifacial Tool Classes by Stratigraphic Unit (Valley Margin)

\begin{tabular}{|c|c|c|c|c|c|c|c|c|c|c|c|c|}
\hline Unit & $\begin{array}{l}\text { Retouched } \\
\text { Blade }\end{array}$ & Denticulate & \begin{tabular}{|l|} 
Edge- \\
modified \\
Flake
\end{tabular} & $\begin{array}{l}\text { Microspur/ } \\
\text { Micro- } \\
\text { denticulate } \\
\end{array}$ & \begin{tabular}{|l} 
Multiple \\
Tool
\end{tabular} & Notch & Spur & \begin{tabular}{|l|} 
Thick \\
Uniface \\
\end{tabular} & \begin{tabular}{|l|} 
Thick \\
Uniface \\
Fragment
\end{tabular} & $\begin{array}{l}\text { Thin } \\
\text { Uniface } \\
\end{array}$ & \begin{tabular}{|l} 
Thin \\
Uniface \\
Fragment
\end{tabular} & Totals \\
\hline Surface & 1 & 2 & 30 & 4 & 8 & 5 & & 4 & & 9 & 3 & 66 \\
\hline Unknown & 1 & 1 & 28 & 1 & 4 & 1 & 1 & & & 4 & 4 & 45 \\
\hline Y/IIIc & & 3 & 37 & 2 & 5 & 2 & & 1 & & 12 & 5 & 67 \\
\hline Y/IIIb/c & & & 1 & & & 1 & & & & 2 & & 4 \\
\hline Y/IIIb & & & 9 & & 1 & & & & & 2 & & 12 \\
\hline$Y$ & & 1 & 26 & 4 & 1 & 2 & & & & 12 & 2 & 48 \\
\hline $\mathrm{X} / \mathrm{Y} / \mathrm{IIIb}$ & 1 & & 6 & 2 & 2 & & & & & & 1 & 11 \\
\hline X/IIIb & & 1 & 7 & & 4 & 1 & & & & 3 & 2 & 19 \\
\hline $\mathrm{X} / \mathrm{Y}$ & & & 22 & 6 & 5 & 1 & 1 & 1 & & 3 & 2 & 41 \\
\hline $\mathrm{X} / \mathrm{IIIa} / \mathrm{b}$ & & & 4 & & & & & & & 1 & 1 & 6 \\
\hline X/IIIa & & & 10 & & & 1 & & & & 3 & & 14 \\
\hline X/IV/IIIa & 1 & & 8 & 3 & 5 & & & 1 & & 4 & 1 & 22 \\
\hline $\mathrm{X}$ & & 3 & 62 & 10 & 9 & 4 & 1 & 2 & & 15 & 8 & 115 \\
\hline $\mathrm{X} / \mathrm{II}$ & & 1 & 17 & 3 & 1 & & & 3 & & 5 & 5 & 35 \\
\hline $\mathrm{X} / \mathrm{Id} / \mathrm{II}$ & & & & & 1 & & & & & & & 1 \\
\hline X/Isi-c/Id/II & & & 3 & & & & & & & & & 3 \\
\hline Totals: & 4 & 12 & 270 & 35 & 46 & 18 & 3 & 12 & 0 & 75 & 34 & 509 \\
\hline
\end{tabular}




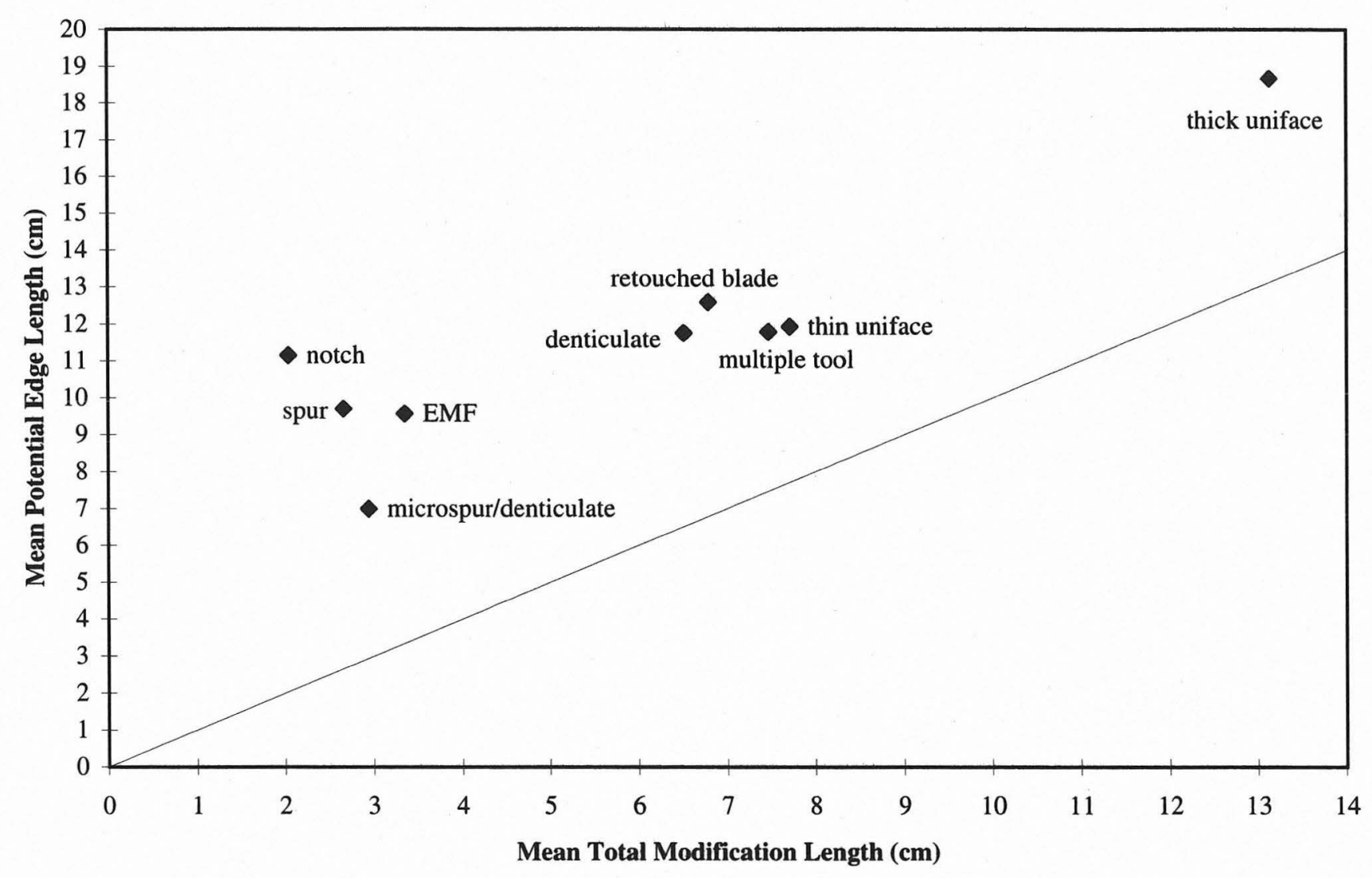

FIGURE $1 \%-25$. Distribution of mean potential edge length and mean total modification length by tool class.

ever the strength of this correlation, weak as it is, depends on the extreme outlying status of thick unifaces. However, a fair amount of variability does exist in the mean modification length. This variability can be more accurately assessed by measuring the vertical distance of each point from the diagonal line which represents $100 \%$ modification. Not surprisingly, notches and spurs have some of the least amount of edge modification, but so do thin unifaces. Also surprising is the high amount of edge modification exhibited by edge-modified flakes. Nevertheless, it appears that as the potential edge increases in length so does the mean length of modification, in other words that the total amount of modification is controlled in part by the size of the artifact class.

Another way to look at these data is to calculate the mean percent of modified edge. This is achieved by dividing the total modification length by the potential edge length and calculating means. These are plotted in Figure 17-26 against the mean potential edge length. This shows that no significant correlation exists between the size of the artifact and the percentage of its edge that is altered by unifacial flake removals $\left(r^{2}=0.497 ; p=0.503\right)$. This bivariate plot also shows that edge-modified flakes have the highest percentage of their edges modified. However, do not be misled into thinking that this excludes them from functioning within an expedient technological strategy.
The temporal changes of these variables are inspected in Figure 17-27, and they are not stable nor do they change drastically. The mean percent of modified edge was calculated for edge-modified flakes and thin unifaces in terms of simplified stratigraphic units. Figure 17-27 plots the means and standard errors for edge-modified flakes. This shows that significant variation exists. The amount of edge modification on edge-modified flakes generally declines through time. While it fluctuates in the Paleoindian levels (Unit I-lower through Unit II), it is fairly high. In the Archaic and Late Prehistoric stratigraphic units it consistently drops (Unit IIIa through Unit IIIc). This suggests that edge-modified flakes become increasingly more expedient through time and especially during the Holocene.

\section{SUMMARY AND DISCUSSION}

At the Wilson Leonard site, unifacial chipped stone tools generally are variable in form, unlike more-formalized unifaces seen in some assemblages. They tend to be made on quite variable flake blanks and seem to be further modified to fulfill a wide variety of activities. The amount of modification necessary to produce a workable unifacial edge is both a function of the shape of the initial blank and the specific task requirements (Frison and Stanford 1982; Kuhn 1994). The description and analysis of unifacial tools demonstrates 


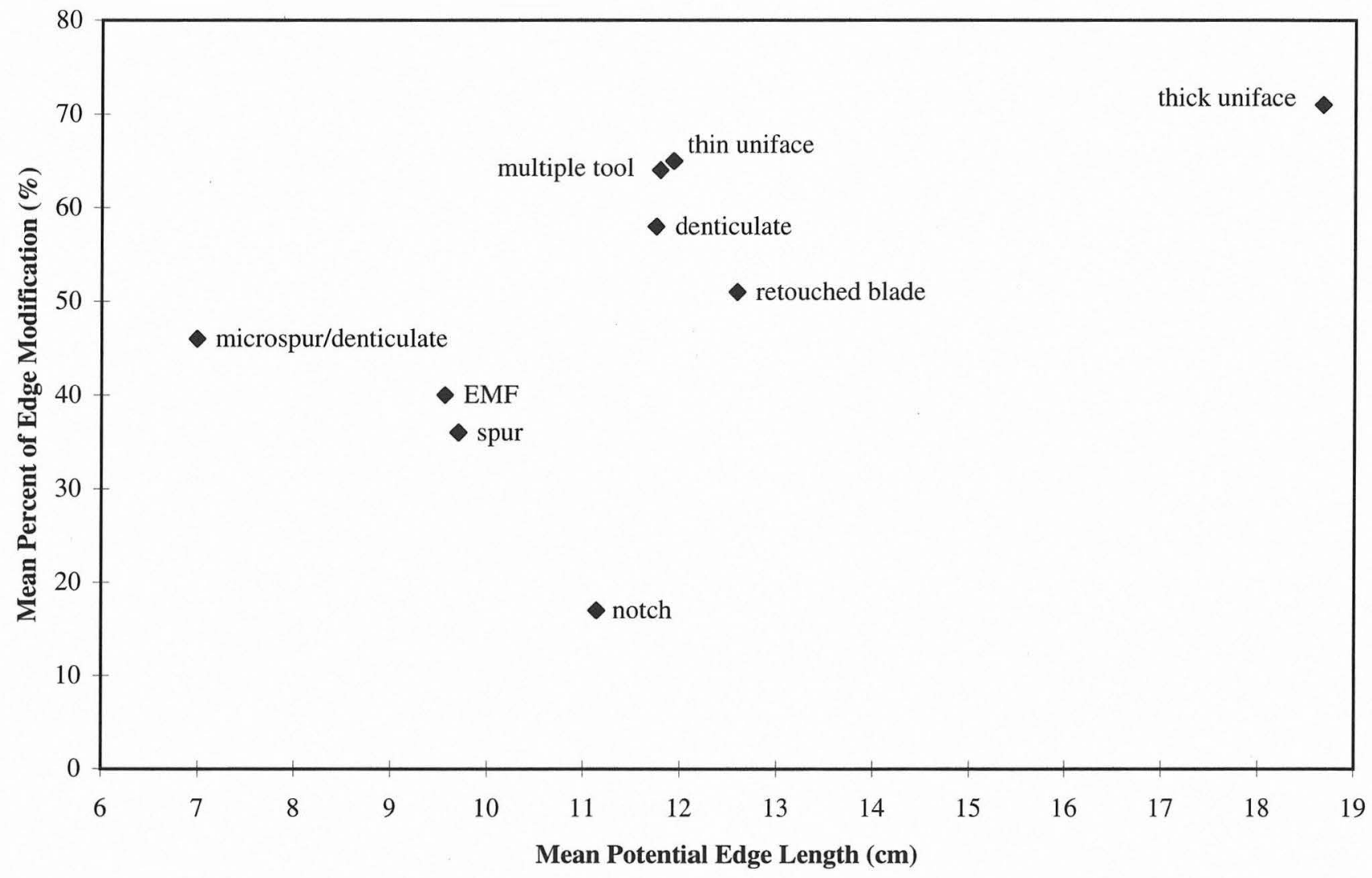

FIGURE 17-26. Distribution of unifacial tool class by mean percent of edge modification and mean potential edge length.

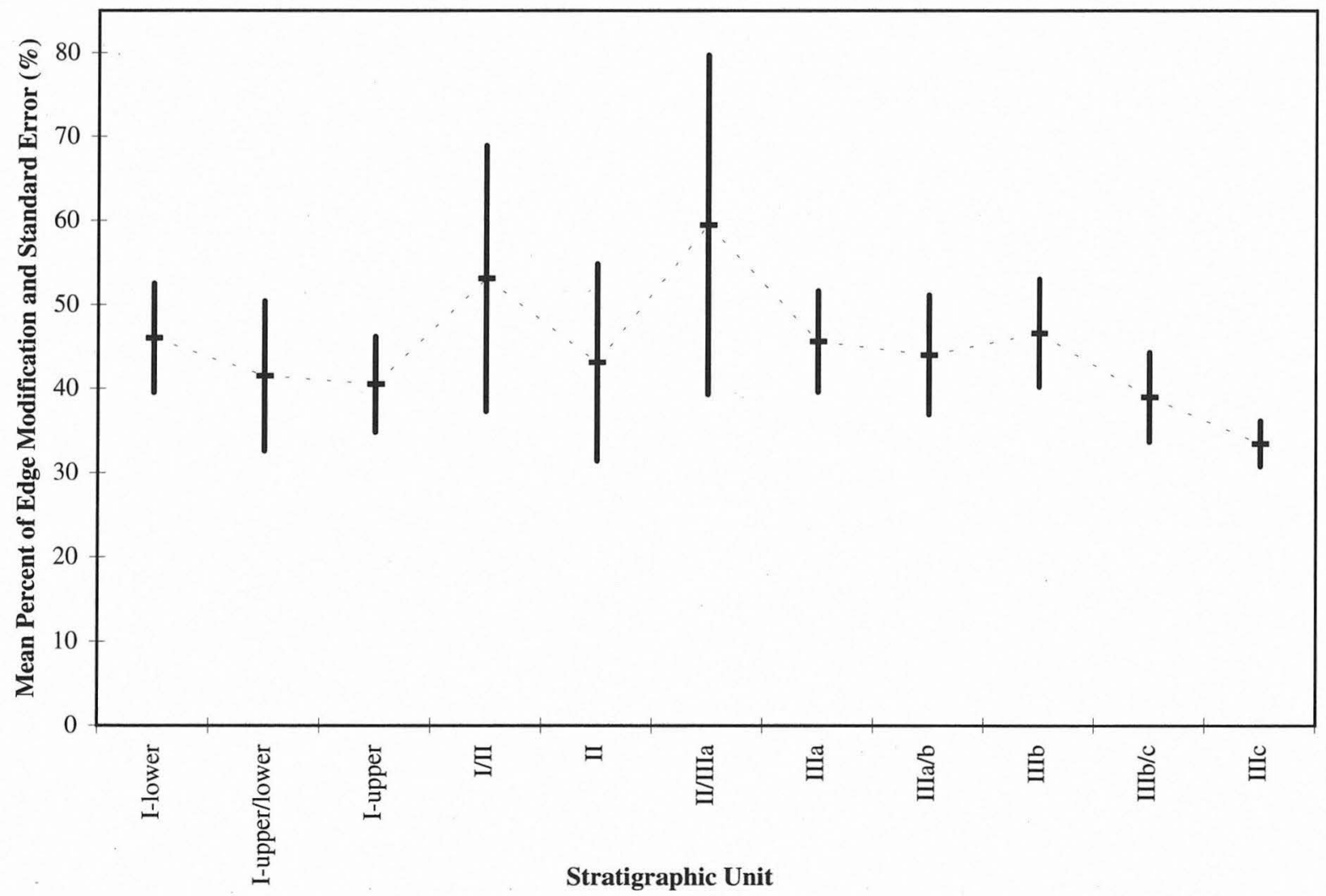

FIGURE 17-27. Changes in modified edge length on edge-modified flakes over time (by stratigraphic unit). 
that significant patterning exists in some tool classes and among some attributes but not in all. It is likely that the patterns observed in terms of unifacial tool forms are a reflection of tool design choices as well as the absence of selectivity. The timing of tool manufacture or use in relation to the timing of core reduction and biface manufacture may play a role as well. It is entirely possible that seasonal mobility patterns and the distribution of raw materials also influence the nature of these uniface assemblages.

In summary, most denticulates are made on secondary and interior normal flakes of local fine-grained Edwards chert. Edge-modified flakes are made on approximately equal numbers of biface thinning flakes and normal flakes. Most are interior secondary flakes of local fine-grained Edwards chert. Microdenticulates are made on approximately equal numbers of biface thinning and normal flakes. These are usually interior or secondary flakes of finegrained Edwards chert. Approximately two-thirds of the microspurs are made on biface thinning flakes and most of the remainder are made on normal flakes, although a significant but small number are made on edge-collapse flakes as well. Most are interior or secondary flakes made of finegrained local Edwards chert. Multiple tools are made on normal and biface thinning flakes. Two-thirds are interior and most of the remainder are secondary flakes. Most multiple tools are made of fine-grained local Edwards chert. Notches are made in approximately equal numbers of normal flakes and biface thinning flakes, and most are interior and secondary flakes. Most notches are made of fine-grained local Edwards chert. Retouched blades are both normal and biface thinning flakes made on equal numbers of secondary and interior flakes. Most retouched blades are made of local Edwards cherts. Of these, approximately two-thirds are fine grained and one-third medium-coarse grained. Spurs are made on normal and biface thinning flakes and mostly of interior and secondary flakes. Most spurs are made of fine-grained local Edwards cherts, but approximately onethird are made on medium-coarse-grained cherts. Thick unifaces are made almost exclusively on normal flakes. Approximately two-thirds are on secondary flakes and slightly less than one-fourth are interior flakes. Approximately three-fifths are fine-grained cherts and two-fifths are medium-coarse cherts. Virtually all are local Edwards cherts. The majority of thick unifaces have steep convex modified edges. These data suggest, although do not prove, that a generic interpretation of these thicker tools as scrapers is probably correct. Thin unifaces are mostly made on normal flakes, but a significant although small number are also made on sequent flakes. Thin unifaces occur in roughly equal numbers of secondary and interior flakes made on finegrained local Edwards chert. For thin unifaces, steep convex modified edges are the majority, though straight and concave modified edges are much better represented than for the thick unifaces. For shallow angle thin unifaces, as well as more-formalized steep angle thin unifaces such as those shown in Figure 17-7b and c, functional assignment should remain dependent on microwear traces.

Several analytical units in this study (e.g., edge angle, edge-modification morphology, total edge-modification length, as well as initial microwear studies performed separately and reported in Chapter 22) enable us to make general statements concerning possible functions of these tools. Many of the unifacial tools shown in Figures 17-5 through 17-8 most likely functioned as cutting or scraping tools, however, evidence of microscopic use-wear or organic residue is needed to more confidently infer function. Unifaces such as 24Z1D-3 (see Figure 17-6a) are commonly identified as "scrapers" based on their distinctive, more-formalized morphology. Use-wear analysis of that artifact by Driskill (see Chapter 22) supports such an inference; his findings suggest use on hides. Kay examined eight selected tools from this class and found evidence for butchering use on artifacts 33Q2-15 and 34U2C-3 (see Chapter 22). Soft to medium contact material and cutting wear were noted on 37LA-5. Indeterminate wear was noted on 33Q2-20 (see Figure 17-8b). Kay identified wear on 14FF1B-1 as burin/ radial break tool use. The three remaining tools (36E-23, 29U1C-11, and 37EE1C-1) yielded negative or indeterminate results. Based on these results, if conventional categorization as "scrapers" were made for all these tools, we would have made gross errors in inferring function based on form. Wilmsen (1970) presumed artifact function based on observed edge angles. By his criteria, most of the unifacial tools from the site would be categorized as heavy cutting or scraping tools. In his experimental studies, Solls (1985) demonstrated the most likely function for heavy, steepangled unifaces traditionally labeled "scraper planes" was as a tool for the pulping of yucca leaves, probably for fiber production. Obviously more investigation of the relationship between unifacial working edge morphology and function is necessary.

Spurred flakes were also analyzed for use-wear (see Chapter 22). On one specimen, Driskell found polish suggestive of bone or antler which may have derived from either working that material with the flake and/or from socketing it in a bone or antler haft.

Kay has also identified an artifact (16S2-9, not shown here) from the microdenticulate class as a graving tool, although wear results were negative (see Chapter 22). Attenuation of central spurs is speculated by Kay as the probable result of graving. Obviously, with respect to these fine microspurs, any significant utilization against a hard contact material will attenuate the microspur. Artifacts 28T2D7 (see Figure 17-24c), 37O2A-2, and 24Q1-3 belonging to the spurred retouch classes have been analyzed by Kay (see Chapter 22) and identified as graving tools. Generally, these artifacts are presumed to be used to engrave or groove bone and other hard materials. This is hard to imagine given the delicate needle-like quality of the fine microspur shown in Figure 17-24c. It seems logical that these were used on 
some much softer material. Ethnographic reports of aboriginal tattooing (Newcomb 1961) or scarification (Griffen 1983) practices may provide an additional explanation for these artifact classes.

Future microwear study of the sizable small-tool assemblage from the Wilson-Leonard site should provide further insight into uniface and small flake tool function. Recent issues raised about bifacial chipped stone tool-life histories, specifically first use/last use questions, are particularly applicable to this site assemblage (see Chapter 22). Resolution of the questions raised in this study about multiple modified edge relationships to tool multifunctionality should be possible. With the recent advances in microwear analysis of stone tools, such as those made by Kay and others (see Chapter 22), this issue of serial acquisition of functional modified edges should be addressed by future analysts. 


\section{Chapter 18}

\section{CORE TOOLS, BATTERED STONES, CORES, AND TESTED AND UNMODIFIED CHERT MATERIALS}

by Susan W. Dial and Michael B. Collins

\section{INTRODUCTION}

Materials produced by the initial stage of toolmaking as well as certain tools likely derived from this stage are reported in this chapter. As the starting point in the lithic tool manufacturing process, selection of raw material would necessarily consider type, quality, size, and availability. Modifications to the materials, both by natural and human processes, provide additional information. These criteria are a focal point for this study along with technological evidence manifest in the cores and tools themselves.

Although the group described here is relatively small, impressions of more-specialized tool industries and activities at various times in the site's history can be traced. Of particular interest are small exhausted cores bearing evidence of microflake removal and, concomitantly, important implications for the manufacture of composite microlithic tools, particularly in Early Archaic times. Battered stones, some of which may have been recycled from exhausted cores for use as hammerstones, were present throughout the site's history but in markedly high frequencies during Early Paleoindian times. A small number of possible blade core fragments also were recovered in early contexts. These items, along with core tools bearing an inordinately high frequency of mineral deposits, raise intriguing questions about use and function of certain poorly understood tool categories.

Sections below are organized into three categories as follows: core tools; battered stones; cores, core fragments, and tested and untested raw materials. For each group, metric data and raw material characteristics are presented following guidelines and terminology set forth in Chapter 12.

\section{CORE TOOLS}

A group of 64 large pebble implements are characterized herein as core tools. Known variously as choppers, pebble tools, and hand axes, among other appellations, core tools vary widely in size and shape but are bound by the common attributes of generally simple manufacture by percussion flaking and at least one working edge (Epstein 1969:53-62; Suhm 1955; Turner and Hester 1993:245). On most specimens, more than half of the original cortex covering remains, with the working edge or edges formed by the bifacial removal of several large flakes.

Four subcategories based on morphological differences were identified: flat to wedge-shaped with convex end; rounded with squared-off proximal end; small triangular; and flat, straight-edged (Figures 18-1, 18-2). The 45 specimens comprising these groups are complete or nearly so. An additional 19 specimens are either aberrant forms or are too fragmentary to be categorized, although they exhibit sections of a worked or battered edge or other morphological attributes suggestive of the core tool group as a whole. Likely, however, there is some overlap between the core tool fragments group and other lithic categories, namely, cores, Stage I bifaces, and battered stones.

For the core tool analysis, particular attention was paid to mineral precipitates, which were present in relatively high frequencies and in a variety of locational patterns on the tools. These data were recorded, albeit somewhat subjectively, according to the relative degree of heaviness of accumulations as well as their location on the tool.

\section{Descriptions}

\section{Group 1: Flat to Wedge-shaped, Convex End $(N=10)$}

The 10 tools in this group are distinguished by an overall large size, a flat to wedge-shaped longitudinal profile, and an elongate convex to straight-edged working end (see Figure 18-1a). Although unmodified, proximal ends are generally rounded to blocky in shape, affording good prehension.

As shown in Table 18-1, Group 1 tools range from 85 to $154 \mathrm{~mm}$ in maximum length (mean $=130.6 \mathrm{~mm}$ ), 73 to $122 \mathrm{~mm}$ in 

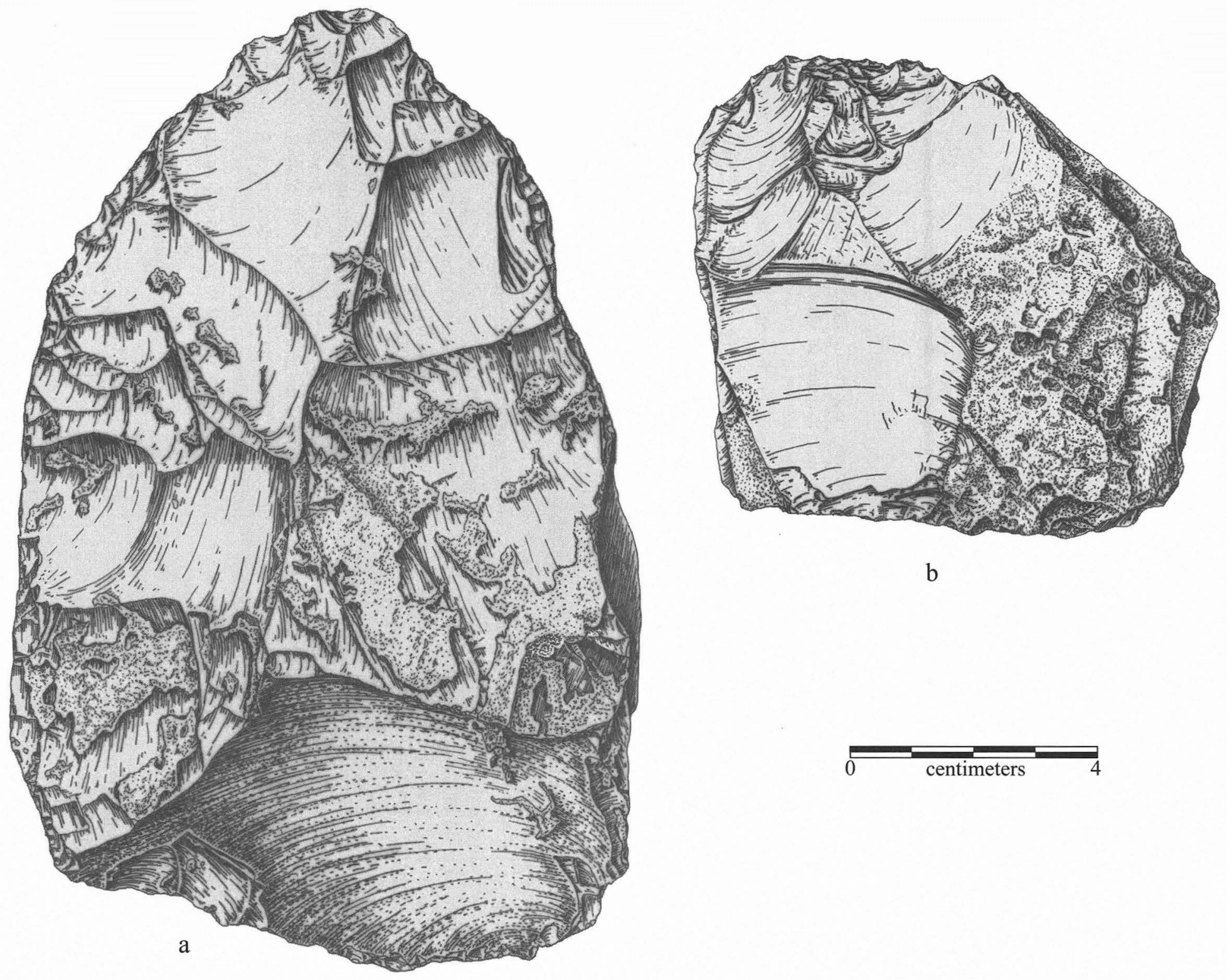

b 


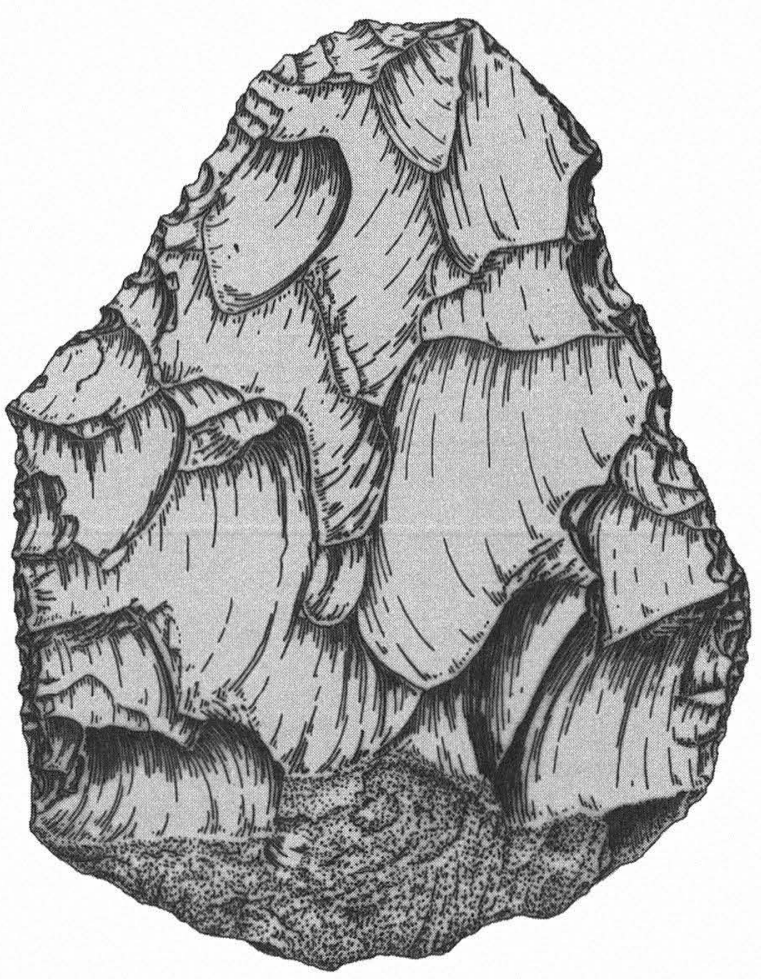

a
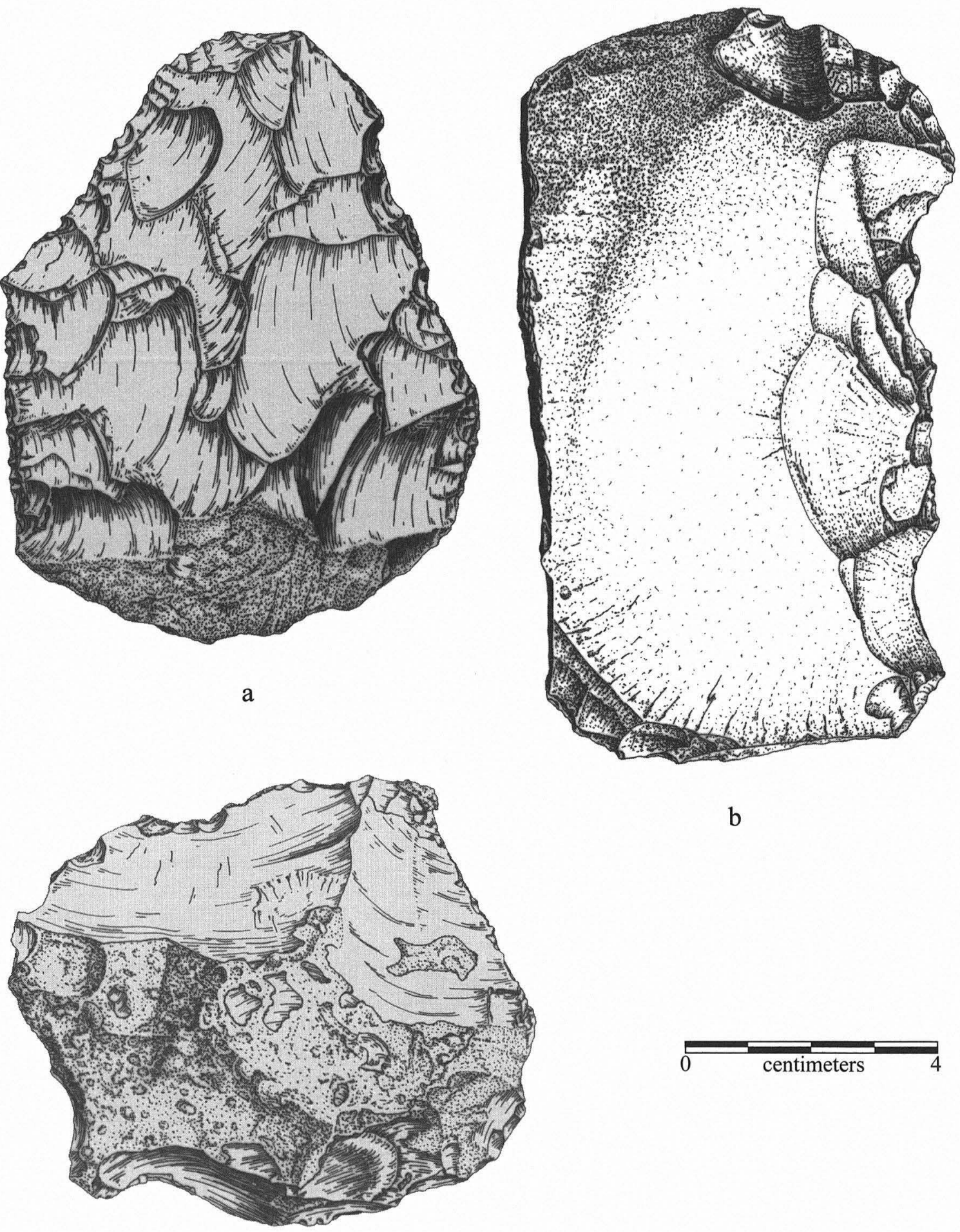

b

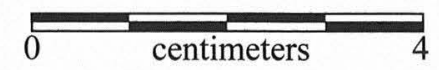

c

FIGURE 18-2. Examples of core-tool forms. (a) Group 3, small triangular (Specimen 11F-2); (b) Group 4, flat, straight edged (Specimen 0153); (c) Group 5, miscellaneous (Specimen 50TA-2). 
TABLE 18-1

Group 1 Core Tool Attributes $(\mathrm{N}=10)$

\begin{tabular}{|c|c|c|c|c|c|c|c|c|c|c|}
\hline \multirow[b]{2}{*}{ tem } & \multirow[b]{2}{*}{ Unit } & \multirow[b]{2}{*}{ Cultural Period } & \multicolumn{3}{|c|}{ Measurements (mm) } & \multicolumn{3}{|c|}{ Cortex } & \multicolumn{2}{|c|}{ Mineral Precipitates } \\
\hline & & & Length & Width & Thickness & $\begin{array}{l}\text { Stream } \\
\text { Rolled }\end{array}$ & $\begin{array}{c}\text { Upland } \\
\text { Residual }\end{array}$ & Nodular & $\begin{array}{l}\text { Heavy } \\
\text { Overall }\end{array}$ & On Cortex \\
\hline \multicolumn{11}{|c|}{ Valley Floor A: } \\
\hline 306-1 & |IIIc & Late Prehistoric/Late Archaic & 85 & 73 & 28 & \multirow{6}{*}{$\mathrm{x}$} & $\mathrm{X}$ & & \multirow{6}{*}{$\mathrm{X}$} & \\
\hline $87-1$ & IIIIc & Late Prehistoric/Late Archaic & 125 & 114 & 37 & & $\mathrm{x}$ & & & \\
\hline $3 \mathrm{~J}-6$ & IIIb & Middle/Early Archaic & 132 & 92 & 47 & & $\mathrm{X}$ & & & $\mathrm{X}$ \\
\hline $.3 \mathrm{~J}-8$ & IIIb & Middle/Early Archaic & 138 & 102 & 28 & & $\mathrm{X}$ & & & $\mathrm{X}$ \\
\hline $4 \mathrm{~K} 2-13$ & IIIIb & Early Archaic & 133 & 116 & 63 & & & & & \\
\hline $360-10$ & IIIIa & Early Archaic & 146 & 81 & 38 & & & $\mathrm{X}$ & & $\mathrm{X}$ \\
\hline \multicolumn{11}{|c|}{ Valley Margin: } \\
\hline $.0 \mathrm{D}-7$ & |IIIc & Late Archaic & 104 & 122 & 53 & $\mathrm{x}$ & & & \multirow{3}{*}{$\mathrm{X}$} & \multirow{4}{*}{$\mathrm{X}$} \\
\hline JJ-8 & & Early Archaic & 154 & 103 & 50 & $\mathrm{x}$ & & & & \\
\hline $9 \mathrm{~L}-10$ & $\mathrm{X} / \mathrm{Y}$ IIIb & Early Archaic & 150 & 85 & 60 & $\mathrm{x}$ & & & & \\
\hline iN-1 & $\mathrm{X} / \mathrm{Y}$ & Early Archaic & 139 & 80 & 35 & $\mathrm{x}$ & & & $\mathrm{x}$ & \\
\hline
\end{tabular}

width (mean $=96.8 \mathrm{~mm}$ ), and 28 to $63 \mathrm{~mm}$ in thickness $(m e a n=43.9 \mathrm{~mm})$. Working edges on all specimens display step and hinge flaking. Three specimens exhibit additional modification including "nibbling" flake removal (13J-8), battering (10D-7), and slight beveling (306-1).

All 10 of the Group 1 tools are made of coarse, local chert with large sections of cortex (see Table 18-1). On 5 specimens, cortex appears stream rolled, and cortex on 4 bears evidence of weathering on an upland surface (upland residual). A single specimen exhibits nodular cortex. Mineral precipitates are present on 7, or 70\%; on these, accumulations are chiefly on cortex-covered areas on 4 , and are heavy overall on 3 (see Table 18-1). Patina is absent on all Group 1 specimens.

Of particular interest is a flat, convex-edged tool with crosshatching of numerous fine striations over much of the cortex surface of one face (Figure 18-3). To a large extent, the striae are centered over a small, ca. 12-mm-diameter, 2-mm-deep oval concavity which appears to have been worn down around the edges by repeated cutting action. The presence of mineral deposits within the striae rules out the possibility that these small lines may have been caused by heavy brushing during laboratory processing. On the opposing face, a few scratches overlying mineral encrustations suggest excavation damage.

In many respects, this specimen resembles a lap tool, an expedient device used as a flat cutting surface. What is unusual about this specimen is its size and its possible dual function as a chopper-like core tool as well as a cutting surface. As a cutting surface, the tool would seem relatively small, constrained on two sides by natural tabular facets and on the distal end, by the chipped working edge of the tool. In several areas, striations appear to extend slightly into the flaked, decorticate surface. It is unclear whether the concavity served a purpose-such as helping in some way to stabilize the material while it was cut—or whether it was coincidental—either present in the original rock piece or enlarged through repeated cutting and wear. Equally puzzling is what the worked material might have been. Clearly, it must have been relatively small, as well as soft or pliable enough to cut with a stone tool. Possible choices include berries, bulbs, or immature nuts (Ken Brown, personal communication 1996). Other substances might be herbs, mushrooms, or roots requiring fine processing or mincing for use as medicines or hallucinogens. As a chopper-like core tool, the specimen would have afforded good prehension. The wide, convex working edge appears acute and fresh, except for one small slightly dulled section. From Unit IIIa, this specimen is attributed to the Early Archaic.

\section{Group 2: Rounded (N=16)}

Specimens in this category are generally smaller and more rounded than angular in outline and longitudinal section than other types (see Figure 18-1b). Working edges are acute and convex to pointed in shape; proximal, or butt, ends are squared-off or blocky, in some cases as a result of a tabular facet. Sizes are highly variable, ranging from 58 to $93 \mathrm{~mm}$ in length (mean=76.2 $\mathrm{mm}$ ); 49 to $106 \mathrm{~mm}$ in width (mean $=78.7 \mathrm{~mm}$ ); and 28 to $84 \mathrm{~mm}$ in thickness (mean $=46.8$ $\mathrm{mm}$ ) (Table 18-2). Working edges on all Group 2 specimens display step and hinge fracturing, seven $(43.8 \%$; Specimens 36D-28, 15R1-4, 23Q-2, 30BB2A-1, 34NN1\&2A, 50PC-5, and 3A-11) also exhibit evidence of crushing or battering, and three (18.8\%; Specimens 28T1A-1, 50PC-5, and $9 \mathrm{~F}-5$ ) have nibbling.

Group 2 tools were made chiefly on coarse cherts (14, or $87.5 \%$ ). Specimens 15R1-4 and 50PC-5 are made of fine-grained chert. Cortex indicates that 7 specimens were made on stream-rolled pebbles, 6 on upland residual cherts, and 1 bears nodular attributes. Mineral precipitates are present on 10 , or $62.5 \%$ of these tools. Patina is absent on all but specimens 23Q-2 and 28T1A-1. 


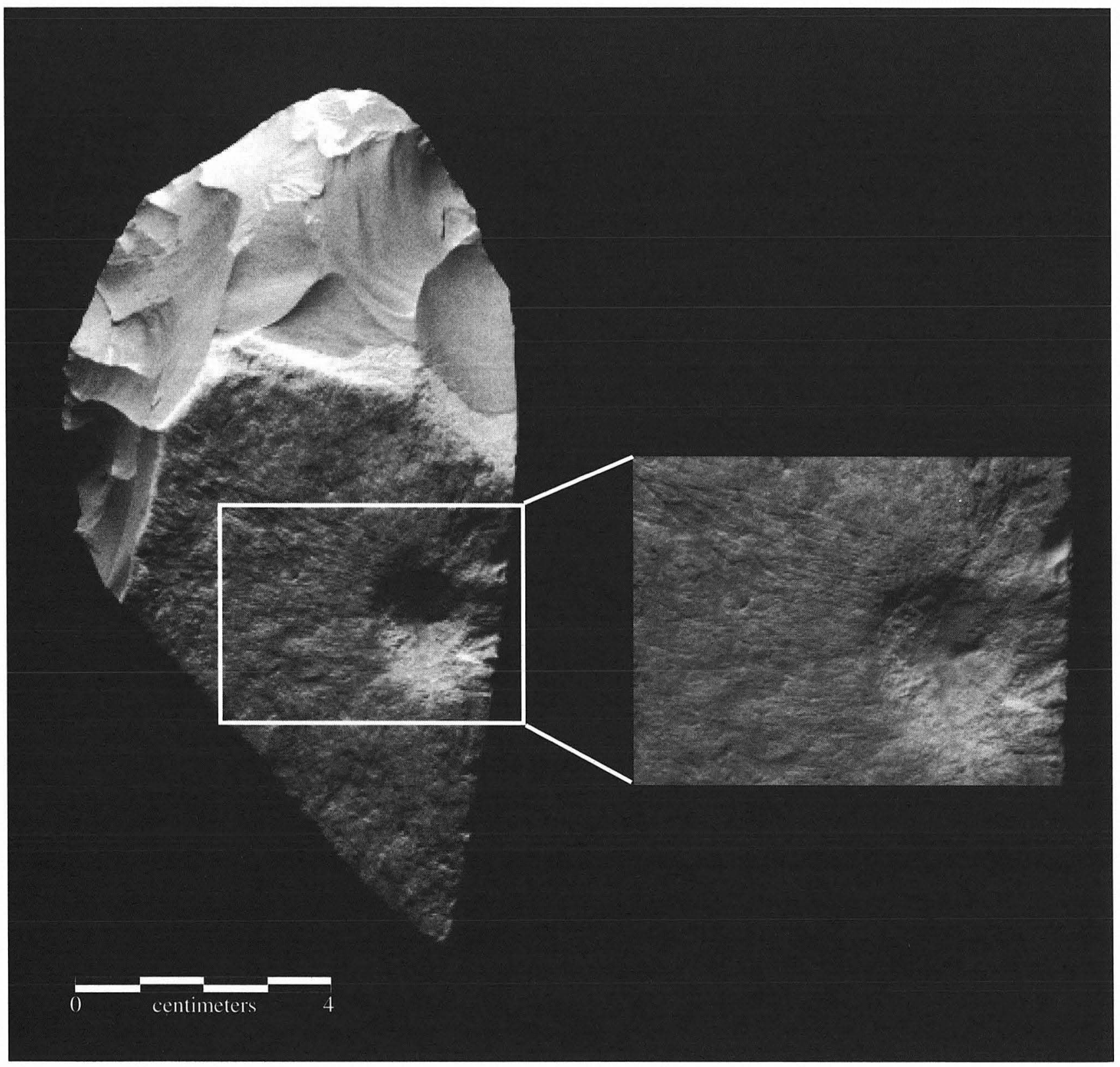

FIGURE 18-3. Early Archaic Form 1 core tool (Specimen 360-10) with linear striations centered over circular indentation in cortex area; inset shows enlargement of incised area.

\section{Group 3: Small, Triangular $(N=5)$}

Group 3 specimens are generally smaller and thinner than other categories (see Figure 18-2a). With triangular to subtriangular outlines, some are a smaller version of those in Group 1, ranging from 92 to $107 \mathrm{~mm}$ in length (mean=99.4 mm); 67 to $82 \mathrm{~mm}$ in width (mean $=73.2 \mathrm{~mm}$ ); and 22 to $27 \mathrm{~mm}$ in thickness (mean $=25.0 \mathrm{~mm})($ Table 18-3). All of these specimens show hinge and step flaking and nibbling is also evident on three specimens (37R1B-1, 11F-2, and 18N-6).

Four of the tools were made on coarse, local cherts, and one (18N-6) on fine-grained chert. Cortex, present on all speci- mens, varies from stream modified to upland residual and was unclassified on two. Mineral precipitates are evident on three specimens, and patina is present on two (37R1B-1 and $11 \mathrm{~F}-2)$.

\section{Group 4: Flat, Straight-edged $(N=14)$}

The chief identifier of this group is a long, straight to slightly concave working edge formed on roughly flat, angular pebbles with naturally squared-off sides and proximal ends (see Figure 18-2b). Most afford good prehension. A wide range of sizes is encompassed in this category (Table 
TABLE $18-2$

Group 2 Core Tool Attributes $(\mathrm{N}=16)$

\begin{tabular}{|c|c|c|c|c|c|c|c|c|c|c|c|c|c|c|}
\hline \multirow[b]{2}{*}{ Item } & \multirow[b]{2}{*}{ Unit } & \multirow[b]{2}{*}{ Cultural Period } & \multicolumn{3}{|c|}{ Measurements (mm) } & \multicolumn{4}{|c|}{ Cortex } & \multicolumn{5}{|c|}{ Mineral Precipitates } \\
\hline & & & Length & Width & Thickness & $\begin{array}{l}\text { Stream } \\
\text { Rolled } \\
\end{array}$ & $\begin{array}{c}\text { Upland } \\
\text { Residual } \\
\end{array}$ & Nodular & $\begin{array}{c}\text { Indeter- } \\
\text { minate }\end{array}$ & $\begin{array}{l}\text { Heavy } \\
\text { Overall } \\
\end{array}$ & \begin{tabular}{|c|} 
Set Back \\
$10-30 \mathrm{~mm}$ \\
\end{tabular} & Unpatterned & $\begin{array}{c}\text { On } \\
\text { Cortex } \\
\end{array}$ & $\begin{array}{c}\text { In Fractures, } \\
\text { On Edges }\end{array}$ \\
\hline \multicolumn{2}{|c|}{ Valley Floor A: } & & & & & & & & & & & & & \\
\hline $36 \mathrm{D}-28$ & IIIIc & Late Archaic & 74 & 81 & 47 & & $\mathrm{X}$ & & & & & & $\mathrm{X}$ & \\
\hline $389-1$ & IIIlb & Middle Archaic & 79 & 96 & 66 & & $\mathrm{X}$ & & & & $\mathrm{X}$ & & $\mathrm{X}$ & \\
\hline $23 \mathrm{~L}-3$ & IIIlb & Early Archaic & 66 & 102 & 54 & & $\mathrm{X}$ & & & & & $\mathrm{X}$ & & \\
\hline $655-5$ & IIIa/b & Early Archaic & 93 & 78 & 84 & $\mathrm{X}$ & & & & & & & $\mathrm{X}$ & \\
\hline $15 \mathrm{R} 1-4$ & IIIIa & Early Archaic & 58 & 49 & 28 & & & & & & & & & \\
\hline $35 \mathrm{R} 2-30$ & IIIIa & Early Archaic & 90 & 87 & 52 & $\mathrm{X}$ & & & & & & & & \\
\hline $2432-1$ & II//II & Early Archaic/Late Paleoindian & 67 & 106 & 48 & & & & $\mathrm{X}$ & $\mathrm{X}$ & & & & \\
\hline 23Q-2 & II/IIIa & Early Archaic/Late Paleoindian & 82 & 78 & 38 & & $\mathrm{X}$ & & & & & & $X$ & \\
\hline $35 \mathrm{~T} 1 \mathrm{~B}-3$ & II/IIIa & Early Archaic/Late Paleoindian & 62 & 71 & 35 & $\mathrm{X}$ & & & & & & & $\mathrm{X}$ & \\
\hline 28T1A-1 & II & Late Paleoindian & 81 & 91 & 31 & & $\mathrm{X}$ & & & & $\mathrm{X}$ & & $\mathrm{X}$ & \\
\hline $30 \mathrm{BB} 2 \mathrm{~A}-1$ & Isi-c & Late Paleoindian & 63 & 58 & 36 & & $\mathrm{X}$ & & & & & & & \\
\hline $34 \mathrm{NN} 1 \& 2 \mathrm{~A}$ & Isi//cl & Early Paleoindian & 68 & 74 & 33 & $\mathrm{X}$ & & & & & & & & \\
\hline \multicolumn{2}{|c|}{ Valley Floor B: } & & & & & & & & & & & & & \\
\hline $50 \mathrm{PC}-5$ & III & Early Archaic & 88 & 72 & 36 & & & $\mathrm{X}$ & & & & & & $\mathrm{X}$ \\
\hline \multicolumn{2}{|c|}{ Valley Margin: } & & & & & & & & & & & & & \\
\hline $3 \mathrm{~A}-11$ & IIIC & Late Prehistoric & 77 & 83 & 52 & $\mathrm{X}$ & & & & & & & & \\
\hline $9 \mathrm{~F}-5$ & IIIIc & Late/Middle Archaic & 91 & 63 & 53 & $\mathrm{X}$ & & & & & & & & \\
\hline $11 \mathrm{~N}-2$ & IIIa/b & Early Archaic & 80 & 70 & 55 & $\mathrm{X}$ & & & & & & & $\mathrm{X}$ & \\
\hline
\end{tabular}


TABLE $18-3$

Group 3 Core Tool Attributes (N=5)

\begin{tabular}{|c|c|c|c|c|c|c|c|c|c|c|c|}
\hline \multirow[b]{2}{*}{ Item } & \multirow[b]{2}{*}{ Unit } & \multirow[b]{2}{*}{ Cultural Period } & \multicolumn{3}{|c|}{ Measurements (mm) } & \multicolumn{3}{|c|}{ Cortex } & \multicolumn{3}{|c|}{ Mineral Precipitates } \\
\hline & & & Length & Width & Thickness & \begin{tabular}{|l|} 
Stream \\
Rolled
\end{tabular} & \begin{tabular}{|l|} 
Upland \\
Residual
\end{tabular} & Indeterminate & \begin{tabular}{|l|} 
Set Back \\
$10-30 \mathrm{~mm}$
\end{tabular} & $\begin{array}{l}\text { On } \\
\text { Cortex }\end{array}$ & $\begin{array}{l}\text { In Fractures, } \\
\text { On Edge }\end{array}$ \\
\hline \multicolumn{2}{|c|}{ Valley Floor A: } & & & & & & \multirow[b]{2}{*}{$\mathrm{X}$} & & \multirow[b]{2}{*}{$X$} & \multirow[b]{2}{*}{$\mathrm{X}$} & \multirow[b]{2}{*}{$X$} \\
\hline $\begin{array}{l}\text { 13M-1 } \\
\text { 37R1B-1 }\end{array}$ & \begin{tabular}{|l} 
IIIlb \\
IIIIa
\end{tabular} & $\begin{array}{l}\text { Early Archaic } \\
\text { Early Archaic }\end{array}$ & $\begin{array}{l}101 \\
100\end{array}$ & $\begin{array}{l}71 \\
82\end{array}$ & $\begin{array}{l}27 \\
25\end{array}$ & & & $\mathrm{X}$ & & & \\
\hline \multicolumn{2}{|c|}{ Valley Margin: } & & & & & & & \multirow[b]{2}{*}{$\mathrm{X}$} & & \multirow[b]{2}{*}{$X$} & \\
\hline $\begin{array}{l}11 \mathrm{~F}-2 \\
20 \mathrm{G}-8 \\
18 \mathrm{~N}-6\end{array}$ & $\mid \begin{array}{l}\text { IIIIc } \\
\text { IIIIc } \\
X\end{array}$ & $\begin{array}{l}\text { Late/Middle Archaic } \\
\text { Middle/Early Archaic } \\
\text { Early Archaic }\end{array}$ & $\begin{array}{r}97 \\
92 \\
107\end{array}$ & $\begin{array}{l}76 \\
67 \\
70\end{array}$ & $\begin{array}{l}22 \\
25 \\
26\end{array}$ & $\mathrm{X}$ & & & & & \\
\hline
\end{tabular}

18-4). Lengths range from 51 to $120 \mathrm{~mm}$ (mean $=86.6 \mathrm{~mm})$; widths from 74 to $132 \mathrm{~mm}$ (mean $=96.4 \mathrm{~mm}$ ); and thicknesses from 25 to $44 \mathrm{~mm}$ (mean=35.1 $\mathrm{mm}$ ). Edges on two tools (24Z2D-1 and 35NN1\&2D-4) appear crushed or battered, and two others (21T-3 and 22X-4) are slightly beveled. All of the tools in this group show step and hinge flaking.

Local coarse cherts predominate as raw material choice (10 or $71.4 \%$ ), while one specimen (17V2-5) is made of local fine-grained chert, one (22X-4) is made of nonlocal fine chert, and two (13D-7 and 35NN1\&2D-4) are made of nonlocal coarse-grained chert. Cortex evidencing either upland weathering $(5$, or $35.7 \%)$ or stream modification (4, or $28.6 \%$ ) is most common in this group. Mineral precipitates are present on a majority of the specimens $(10$, or $71.4 \%$ ), and patina was noted on only one specimen (8R-2).

Figure 18-4 illustrates two Group 1 core tools from Paleoindian and Late Archaic contexts. Specimen 35NN1\&2D-4 (Figure 18-4a), of coarse, honey-colored chert, is unusual in its two potential working edges, háving been flaked bifacially along the short axis as well as unifacially along one lateral edge. The other specimen, 24D, is more typical in its straight, acute working edge.

\section{Group 5: Fragments and Miscellaneous $(N=19)$}

A total of 19 specimens comprises Group 5 (Table 18-5), a combination of miscellaneous specimens and likely core tool fragments based on form and edge modifications (see Figure 18-2c). Small size and fragmented condition prevents assignment to a more-definitive morphological category, but at least one edge on each appears modified for use as a tool. Fine-grained cherts predominate (12, or $63.2 \%)$ among which are 2 (37U1A-4 and 32KK2A-1) of nonlocal materials, and cortex type is chiefly upland residual ( 10 or $52.6 \%)$. Mineral precipitates were recorded on 14 , or $73.7 \%$ of the fragments; on 5, deposits were chiefly set back from the working edge. Patina was present on 3 specimens (OR460, 35X1A-1, and 50TA-2).

Organic residue analysis was performed by Jeffrey Hurst on a single core tool fragment recovered from mixed Late Paleoindian/Early Archaic contexts (OR460, Figure 18-5). As discussed in Chapter 23, fatty acids of both animal and vegetal derivation were identified on that specimen.

\section{Distribution and Comments}

Core tools were present in all major stratigraphic units at the Wilson-Leonard site. Although the sample of 64 is not large by some site standards, it is sufficient to trace, at least impressionistically, certain diachronic distributional patterns.

Of the four morphological groups (excluding fragments), the rounded, fist-sized Group 2 tools were the most frequently encountered, with a total of 16 recovered. As shown in Table 18-2, these tools are consistently present in relatively small numbers in each of the major stratigraphic units. Tools of this form also exhibit one of the highest relative frequencies of edge battering or crushing.

In contrast, the straight-edged Group 4 tools were limited chiefly to lower and upper deposits and are notably absent in contexts attributable to the Early Archaic. Displaying a markedly different distributional pattern, the large, flat to wedge-shaped Group 1 tools are not present in lower stratigraphic units, but are confined to Archaic-age deposits in Unit III. The few Group 3 tools (small, triangular) also were recovered only in Archaic contexts.

Although each of these patterns likely is drawn over many centuries - if not several millennia, as in the Early and Late Archaic samples-they may point to potentially significant morphological differences in what has long been a poorly understood tool category. The findings produced through organic residue analysis of one core tool fragment suggest use on more than one material, in this case, vegetable and faunal matter (see Chapter 23). The heavily striated Group 1 specimen described above, with its hints of dual use as both a chopper-like core tool and a lap tool for cutting, is a prime candidate for further study and may inform as to what material(s) were being worked using this and others of the rather distinctive, flat, convex-end tools during Archaic times. 
TABLE 18-4

Group 4 Core Tool Attributes ( $N=14)$

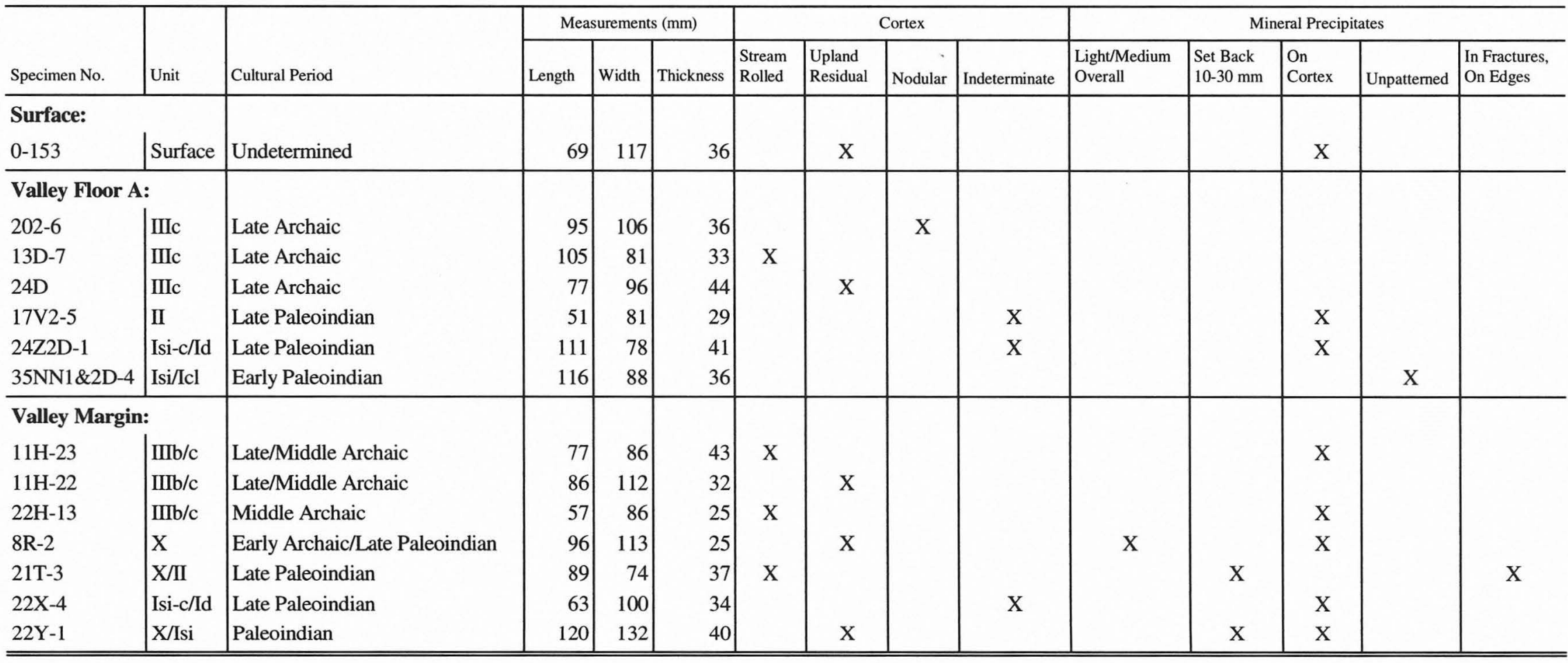




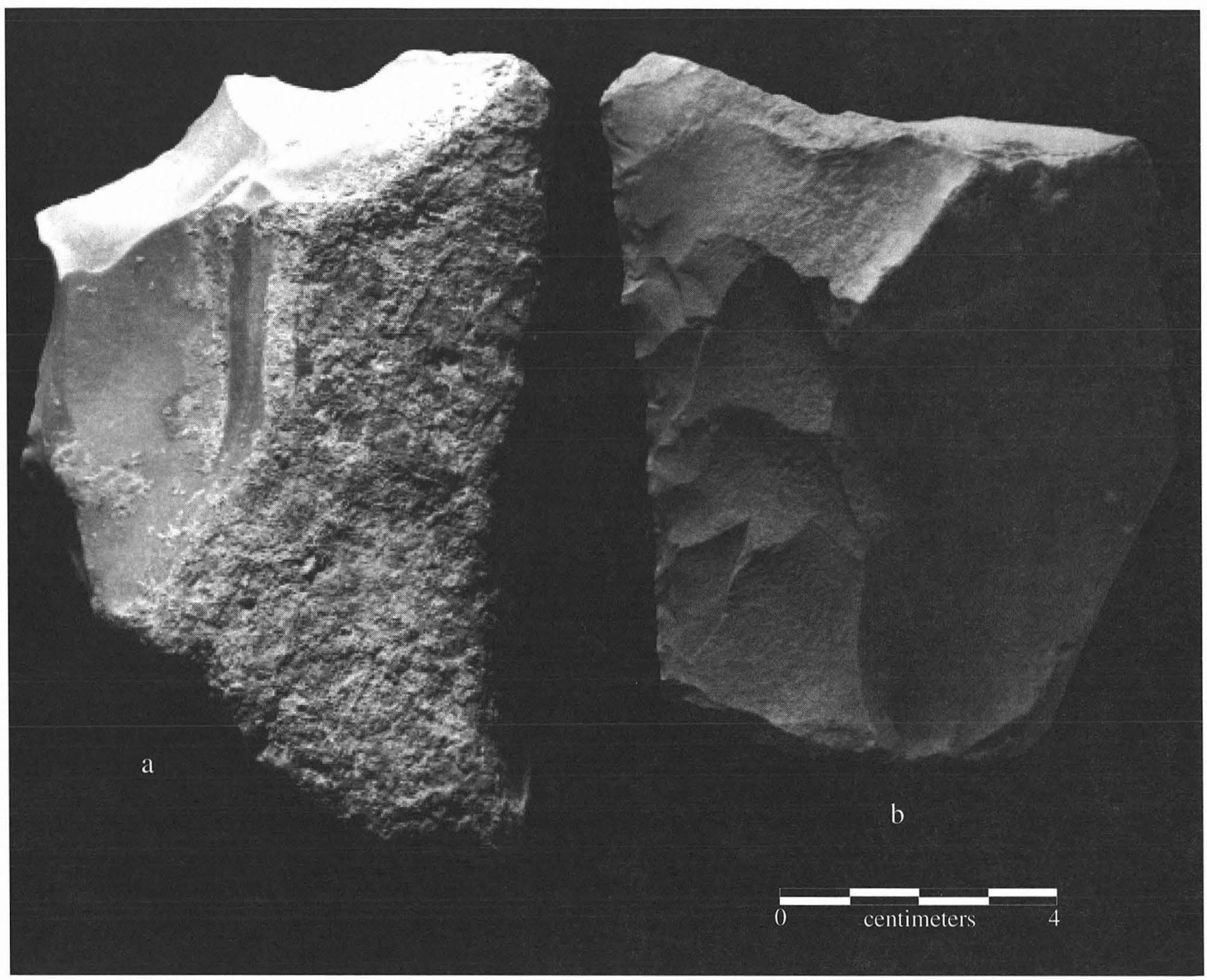

FIGURE 18-4. Group 4 tools from Early Paleoindian and Late Archaic contexts. (a) 35NN1\&2D-4; (b) $24 \mathrm{D}$.

A clear selection for coarse chert is shown in all types of core tools, which likely indicates that these were specifically made as heavy-duty tools. Of the total 64 specimens, the majority (43, or $67.2 \%$ ) were made on local Edwards tan to cream-colored coarse chert, and an additional $3(4.7 \%)$ implements are on coarse cherts from other locales. Only $17(26.6 \%)$ specimens were made on fine-grained cherts, and of these, 12 (70.6\%) fall into the miscellaneous/fragments category, an aspect that would suggest that tools of this material are less durable and more easily fractured. Alternatively, these particular specimens may be byproducts of the biface reduction process - either exhausted cores or core fragments-which may have been recycled as core tools. Their small size also may have led to misclassification, in some cases.

As has been discussed in previous sections, fine-grained cherts predominate in most other lithic categories, with the exception of heavy, bifacial Clear Fork tools, for which coarser cherts also appear to have been preferred (see Chapter 15). Perhaps significantly, the few core tools made on finer cherts are most frequent in deposits attributed to the Early and Late Paleoindian and Early Archaic (14 of 17, or 82.4\%), a distributional pattern also exhibited among Clear Fork tools and Brushy Creek bifaces (see Chapter 15).

Cortex, present on almost all specimens ( $\mathrm{n}=51,79.7 \%)$, suggests that cherts used for manufacturing core tools were chosen chiefly from upland locales $(44.0 \%)$ or, less prevalently, from stream areas $(30.5 \%)$ or embedded chert outcroppings (11.9\%). Eight (13.6\%) tools bear cortex that could not be classified.

Of particular note is the consistent presence of encrustations of mineral precipitates on core tools from all contexts (43 or $67.2 \%$ ). As shown in Tables 18-1 through 18-5, there appears to be no correlation between its presence and raw material, cortex type, stratigraphic placement in the site, or tool shape. Mineral precipitates appear to form with nearly equal frequency on coarse as well as fine-grained cherts, in early as well as late contexts, and on all of the core tool morphological groups. Although not easily quantifiable, 
TABLE $18-5$

Group 5 Core Tool Attributes (N=19)

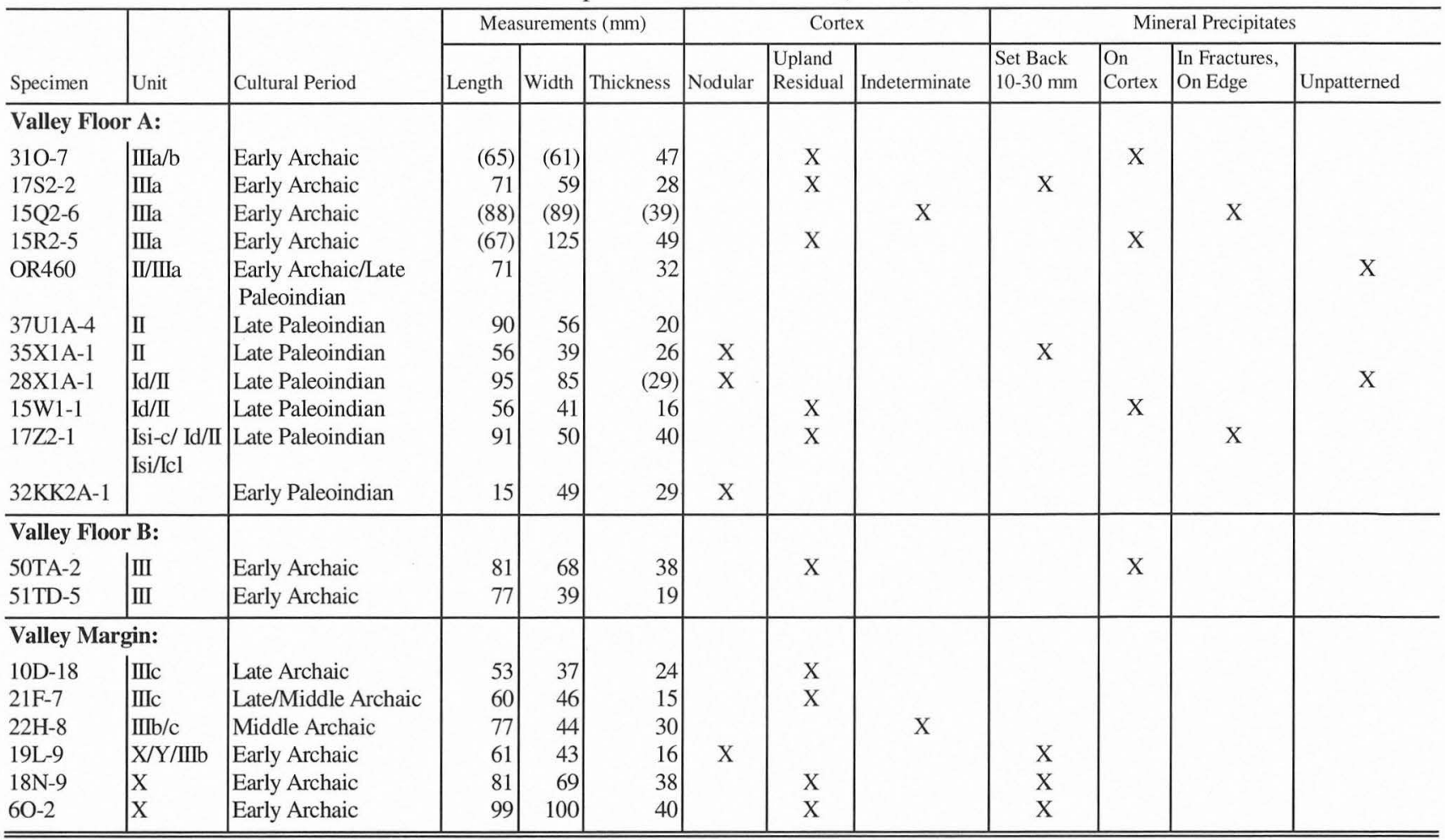

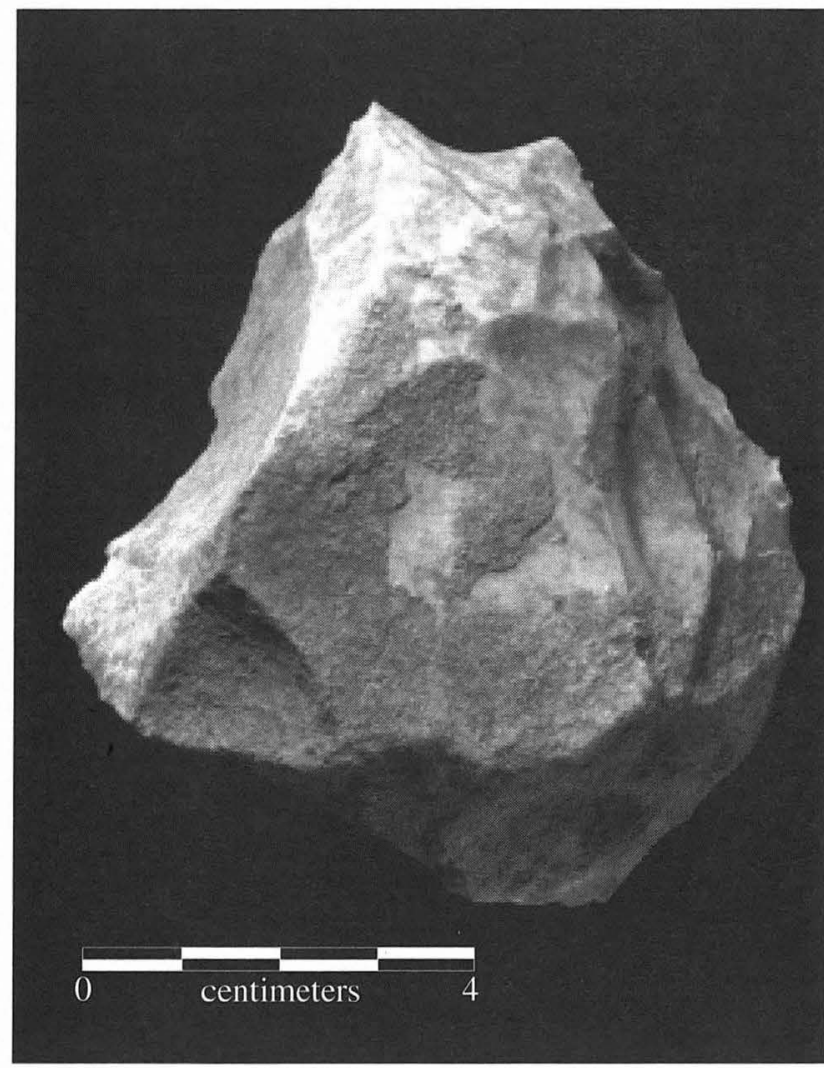

FIGURE 18-5. Core tool fragment (OR460) found to have evidence of both faunal and vegetal residues (see Chapter 23). accumulations appear somewhat heavier on the flat Group 1 tools.

Location of mineral precipitates on individual specimens is varied, perhaps significantly (Figure 18-6a-c). On 10, the deposits form an almost arc-like pattern, set back some 10-30 $\mathrm{mm}$ from the tools' lateral margins and distal ends (e.g., see Figure $18-6 a)$. On the majority (25, or $58.1 \%$ of those with mineral precipitates), deposits are concentrated mainly on corticate areas, which typically are in the proximal area (see Figure 18-6b). Although these locational patterns were of necessity recorded somewhat impressionistically, they suggest that mineral precipitates most frequently accumulated away from the working edge and often on the butt end. If, as Collins (1994:143) has suggested, these residues are related to use, these locational patterns may indicate that certain worked materials (meat, hide, vegetable matter) tended to build up on the tool as it was used and accumulate in greatest amounts back from the working edge or where the tool was gripped.

Barring further chemical analyses, however, explanations and implications are difficult to explore. Significantly, a similar distributional pattern was observed on core tools or "choppers" from the Mustang Branch site (Collins 1994:142-143). In that group, drawn chiefly from a Late Archaic burned rock midden where no other tool classes had mineral encrustations, a total of $13(39 \%)$ bore patchy residues resembling calcium carbonates for which a possible use-related derivation was ascribed. 


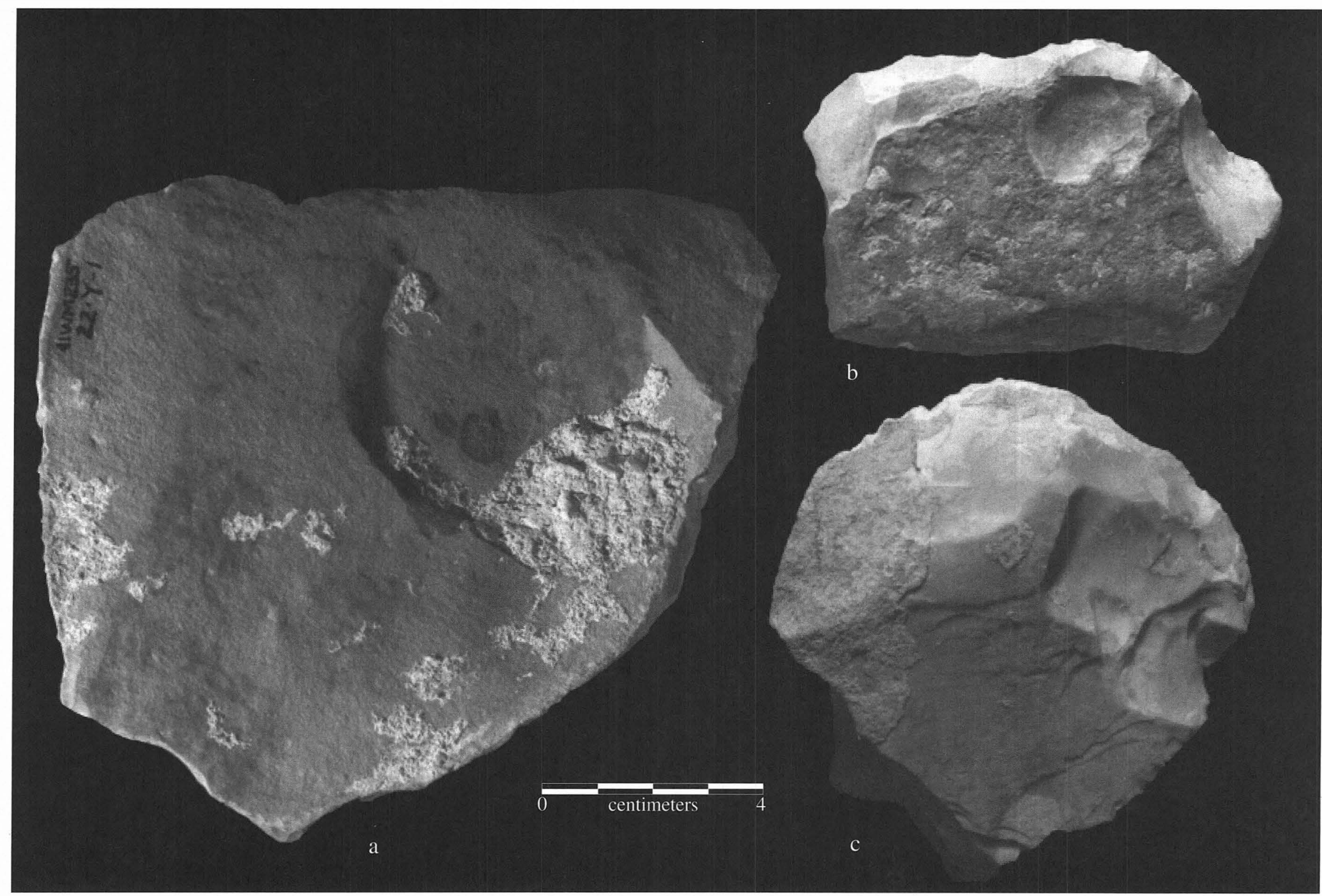

FIGURE 18-6. Mineral precipitates on core tools. (a) Specimen 22Y-1, showing deposits generally set well back from working edge (top of tool; flake removal to form working edge is primarily on opposite face, not shown); (b) Specimen 22H-13, with light deposits on one face; (c) Specimen 28T1A-1 with heavy deposits chiefly on cortex-covered proximal area. 


\section{BATTEREDSTONE ARTIFACTS}

Fifty stones with evidence of percussion damage form this group which is divided into four descriptive subgroups. Three of these subgroups are inferred to represent ordinary hammerstones and one subgroup, from Early Paleoindian context, consists of stones with unusual patterns of batter marks that do not seem to be hammerstones in the common usage of that term. Following the descriptions is a discussion of the occurrences, associations, and interpretations seen in the battered stone group with particular attention given to this unusual subgroup.

Several of the attributes (weight, raw material, cortex type, cortex distribution, and mineral precipitates) reported for battered stones are shared with other categories of lithic artifacts and have been defined elsewhere (primarily in Chapter 12, above). Dimensions reported are maximum length, width, and thickness as measured on the three principal, perpendicular axes of the stone without regard to form or location of the cultural modifications. Evidence for battering, as the identifying characteristic of this group, has two primary expressions, crushing and incomplete conical fracturing. Crushing of the stone fabric is visible to some degree in all of the lithologies (chert, quartzite, sandstone, limestone, and basalt). Circular to oval scars demarcating the apexes of embedded Hertzian fracture cones are also present on some of the chert artifacts in this group. These scars are visible on the surfaces of the chert as isolated, complete circles or as clusters of intersecting circles. In the latter cases, there is generally also crushing and irregular pitting where bits of material have broken away around and between the cones. Generally the diameters of the cone apexes, which indicate the contact area of impact, are between 1.5 and $3.5 \mathrm{~mm}$. Two attributes, degree and areal extent, are associated with these expressions of crushing and conical fracturing (collectively called "battering"). Degree of battering is subjective and is expressed as light, moderate, or heavy, depending on how much the surface topography of the stone has been altered. Light battering has not changed the shape of the surface, moderate battering has slightly modified the contour of the stone's surface (creating slight pitting of faces or rounding of edges), and heavy battering has imparted an artificial contour to the stone (deep pitting of surfaces or extensive rounding of edges). Battering is noted as being either limited to one or more small areas ("patchy") or as covering much of the surface of the stone ("extensive"); these are also subjective categories and are judged relative to the size of the stone.

\section{Descriptions}

\section{Battered Cobbles (N=10)}

Ten cobble-sized stones- 3 stream cobbles, 4 upland pieces of chert, 1 nodule of limestone and 2 without corti- cal evidence for origin, exhibiting one or more areas of battering-comprise this category (Table 18-6). These are inferred to have been used as hammerstones, probably in chert knapping and possibly in additional ways. The defining characteristic of this subgroup is that each piece retains much of the natural form of the parent stone with human alteration occurring almost exclusively as battering; that is to say, these stones evidently had served no prior or subsequent functions.

The four chert residuum pieces range from one small flattish piece weighing $70 \mathrm{~g}$ to three larger globular pieces weighing between 255 and $287 \mathrm{~g}$; the former has limited battering primarily on two edges, and two of the latter have concentrated patches of heavy battering (Table 18-7). One of the larger pieces of residuum chert is extensively battered on the full perimeter of its greatest circumference and also has batter marks on its other surfaces. There are some sizable fracture scars on all of these, but they seem to be either incidental to use as hammerstones or the result of weathering (thermal spalling) of the stone prior to use.

The elongate, flattish nodule of battered limestone, from the earliest Paleoindian component, is probably of Comanche Peak limestone; it shows battering and flaking along its edges and especially at both ends (Figure 18-7a). The three stream cobbles include one each of chert, dense limestone, and dense sandstone. One of basalt lacks cortex and one of quartzite has indeterminate cortex. All but the quartzite piece-which is heavily battered and fractured from use - have limited patches of battering. The basalt and sandstone pieces are broken.

\section{Large Core Hammerstones ( $N=10)$}

Ten flaked chert pieces (mostly cores) recycled as hammerstones constitute this group (Table 18-8). Except for one fragment, these stones all weigh more than $80 \mathrm{~g}$. Seven are angular to globular flaked chert pieces, evidently having been depleted or broken as cores. Each has one or more acute edges exhibiting heavy battering (Table 18-9). On two of these, the battered, crushed edge is concave, evidently from concentrated attrition (1A-11, 7A-19; Figure 18-8). One specimen is a heat-damaged core (33MM2D-1). Another (Figure 18-9a) is a small, patinated chert piece from Early Paleoindian context; it is here interpreted as a core because there are a few very sharp points and edges, but the battering is in two stages of patination (fresh and moderately patinated) and is widely dispersed over both edges and surfaces. These latter characteristics resemble those of stream-battered stones, but in a stream, it is unlikely that any sharp edges or points would survive. The battering occurred in two episodes with enough of a time lapse for patination to occur after this stone was evidently depleted as a core; it is possible that the earlier battering (and possibly even some of the flaking) occurred in a stream gravel. 
TABLE $18-6$

Attributes of Battered Cobbles (N=10)

\begin{tabular}{|c|c|c|c|c|c|c|c|c|c|c|c|c|c|c|c|}
\hline \multirow[b]{2}{*}{ Specimen } & \multirow[b]{2}{*}{ Unit } & \multirow[b]{2}{*}{ Cultural Period } & \multicolumn{4}{|c|}{ Measurements (mm) } & \multicolumn{5}{|c|}{ Cortex } & \multicolumn{4}{|c|}{ Mineral Precipitates } \\
\hline & & & Length & Width & Thickness & $\begin{array}{l}\text { Weight } \\
\text { (g) }\end{array}$ & Nodular & \begin{tabular}{|l} 
Upland \\
Residual
\end{tabular} & $\begin{array}{l}\text { Stream } \\
\text { Rolled }\end{array}$ & Indeterminate & Amount & Heavy* & $\begin{array}{l}\text { Medium } \\
\text { Overall }\end{array}$ & $\begin{array}{l}\text { Light } \\
\text { Overall }\end{array}$ & Light* \\
\hline \multicolumn{16}{|c|}{ Valley Floor A: } \\
\hline $736-1$ & IIIa & Early Archaic & 41 & 34 & 30 & 71 & & & & & & & & & \\
\hline 33T1B-8 & II/IIIa & Early Archaic/Late Paleoindian & 94 & 83 & 42 & 407 & & & $\mathrm{X}$ & & $3 / 4$ or more & $\mathrm{X}$ & & & \\
\hline $34 \mathrm{~W} 1 \mathrm{~B}-3$ & III & Late Paleoindian & 62 & 46 & 19 & 70 & & $\mathrm{X}$ & & & $3 / 4$ or more & & $\mathrm{X}$ & & \\
\hline 37RR1A-2 & Isi & Early Paleoindian & 99 & 71 & 37 & 358 & $X$ & & & & $3 / 4$ or more & & & $\mathrm{x}$ & \\
\hline \multicolumn{16}{|c|}{ Valley Margin: } \\
\hline 1D-3 & IIIIc & Late Archaic & 78 & 57 & 56 & 275 & & $\mathrm{X}$ & & & $1 / 2-3 / 4$ & & & & \\
\hline $20 \mathrm{~F}-1$ & IIII & Late/Middle Archaic & 62 & 67 & 41 & 255 & & $\mathrm{X}$ & & & $3 / 4$ or more & & & & \\
\hline 4G-1 & $\mathrm{Y} / \mathrm{III} \mathrm{c}$ & Archaic & 80 & 63 & 27 & 195 & & & $\mathrm{x}$ & & $3 / 4$ or more & & & & $\mathrm{x}$ \\
\hline $4 \mathrm{G}-2$ & $\mathrm{Y} / \mathrm{IIIC}$ & Archaic & 87 & 75 & 45 & 414 & & & & $\mathrm{X}$ & & $\mathrm{X}$ & & & \\
\hline 9P-1 & $\mathrm{x}$ & Early Archaic & 80 & 62 & 38 & 261 & & & $\mathrm{X}$ & & $3 / 4$ or more & & & $\mathrm{X}$ & \\
\hline $190-2$ & $\mathrm{x}$ & Early Archaic & 75 & 56 & 51 & 287 & & $\mathrm{X}$ & & & $3 / 4$ or more & & & & \\
\hline
\end{tabular}


TABLE $18-7$

Battering Attributes of Battered Cobbles $(\mathrm{N}=10)$

\begin{tabular}{|c|c|c|c|c|c|c|c|c|}
\hline \multirow[b]{2}{*}{ Specimen } & \multirow[b]{2}{*}{ Unit } & \multirow[b]{2}{*}{ Cultural Period } & \multicolumn{6}{|c|}{ Battering } \\
\hline & & & \begin{tabular}{|l|} 
Light/ \\
Patchy
\end{tabular} & $\begin{array}{l}\text { Moderate/ } \\
\text { Patchy }\end{array}$ & \begin{tabular}{|l|} 
Moderate/ \\
Extensive
\end{tabular} & $\begin{array}{l}\text { Heavy/ } \\
\text { Patchy }\end{array}$ & \begin{tabular}{|l|} 
Heavy/ \\
Extensive
\end{tabular} & Distribution \\
\hline $\begin{array}{l}\text { Valley Floo } \\
736-1 \\
33 \text { T1B-8 } \\
\text { 34W1B-3 } \\
\text { 37RR1A-2 }\end{array}$ & \begin{tabular}{|l} 
IIIIa \\
II/IIIa \\
II \\
Isi
\end{tabular} & $\begin{array}{l}\text { Early Archaic } \\
\text { Early Archaic/Late Paleoindian } \\
\text { Late Paleoindian } \\
\text { Early Paleoindian }\end{array}$ & & $\begin{array}{l}X \\
X\end{array}$ & & $\mathrm{X}$ & $\mathrm{X}$ & $\begin{array}{l}\text { edge } \\
\text { edges, face } \\
\text { edges } \\
\text { edges, faces }\end{array}$ \\
\hline $\begin{array}{l}\text { Valley Mar } \\
1 \mathrm{D}-3 \\
20 \mathrm{~F}-1 \\
4 \mathrm{G}-1 \\
4 \mathrm{G}-2 \\
9 \mathrm{P}-1 \\
19 \mathrm{O}-2\end{array}$ & $\mid \begin{array}{l}\text { IIIl } \\
\text { IIIc } \\
\text { Y/IIIc } \\
\text { Y/IIIc } \\
\mathrm{X} \\
\mathrm{X}\end{array}$ & $\begin{array}{l}\text { Late Archaic } \\
\text { Late/Middle Archaic } \\
\text { Archaic } \\
\text { Archaic } \\
\text { Early Archaic } \\
\text { Early Archaic }\end{array}$ & $\mathrm{X}$ & $X$ & $\mathrm{X}$ & $\mathrm{X}$ & $\begin{array}{l}X \\
X\end{array}$ & $\begin{array}{l}\text { edges, face } \\
\text { edges, face } \\
\text { edge } \\
\text { edge reworked break } \\
\text { edge } \\
\text { edges }\end{array}$ \\
\hline
\end{tabular}

The most interesting single specimen (Figure 18-9b) is also from Late Paleoindian context. This is an elongate core fragment with two broad bands of extreme battering and crushing; none of the original contour of the stone survived in the rounded, slightly concave areas produced by this battering. The extent of battering and crushing on this stone indicates many hundreds (if not thousands) of impacts.

Three pieces in this group are fragments of moderately thin bifaces with heavy battering along one of the formerly sharp bifacial edges (Figure 18-9c-e); the battering is advanced in degree and includes much crushing. In two instances the resulting battered edge is concave (see Figure 18-9d, e).

Some of the battered stones in this subgroup resemble knappers' hammerstones, but the concave-edged pieces and the one (28BB2A-1) with the broad bands of battering are not typical of such implements where promontories are more often the point of impact. Other possible uses might include crushing bones or shaping other stones by pecking.

\section{Small Core Hammerstones $(N=24)$}

Twenty-four small, apparently depleted cores with multiple flake scars and with one or more battered edges comprise this subgroup (Tables 18-10 and 18-11; Figure 18-10a-h). They weigh $80 \mathrm{~g}$ or less. One (33T1B-9) is of quartzite and 23 are of chert. Of the chert pieces, 5 (OR690, 31V1D-2, 11C-9, $3 \mathrm{~J}-5$, and 20O-4) are made of nonlocal fine-grained Edwards material, 1 (24S1-5) is of local Edwards medium- to coarse-grained chert, and the remainder are made of local Edwards fine-grained chert. These are extensively flaked pieces, either exhausted cores or fragments of other kinds of chipped stone objects that have subsequently been employed as hammerstones. In most cases, the battering is moderate to heavy and tends to be along acute edges and promontories, sometimes with additional battering on lim- ited areas of faces. The majority of these closely resemble knappers' hammerstones.

\section{Face-battered Chert Pieces $(N=6)$}

A chunk, a core, a core fragment, two flakes, and a flake fragment make up the face-battered chert pieces. All are from Paleoindian contexts, and all are made of nonlocal fine-grained Edwards chert (Table 18-12). Each specimen has dispersed or concentrated scars at points of impact (Figure 18-11). Embedded, small partial cones of percussion indicate sharp blows to or from hard objects. What sets this group apart is that the impact points occur primarily on faces rather than on edges or on promontories (Table 18-13). The number of blows ranges from one (see Figure 18-11e) to scores (see Figure 18-11c). The extent of damage on some of these suggests prolonged usage whereas others seem to have been used comparatively little. These could represent anvils as easily as hammers. The position of the battering does not resemble that imparted to flintknappers' hammerstones. Also enigmatic is that, except for one specimen (35KK2A-2) which weighs $196 \mathrm{~g}$, these are light-weight objects (75 $\mathrm{g}$ or less), yet they received (or delivered) very sharp blows from (or to) very hard objects. The points of impact were also quite small, less than $3 \mathrm{~mm}$ across in almost all cases. At least two seem to have broken as a result of this usage, which is not surprising given the evidence of concentrated forces.

In addition to the six pieces described here, a tested cobble from the same context (17JJ2/KK1-1; described below) has a small number of Hertzian cone scars on the flat distal end of the core. These indicate limited usage of that cobble in a manner similar to that of these battered objects.

\section{Discussion}

Some general observations can be made in regard to the battered stones comprising this group. First, it seems un- 


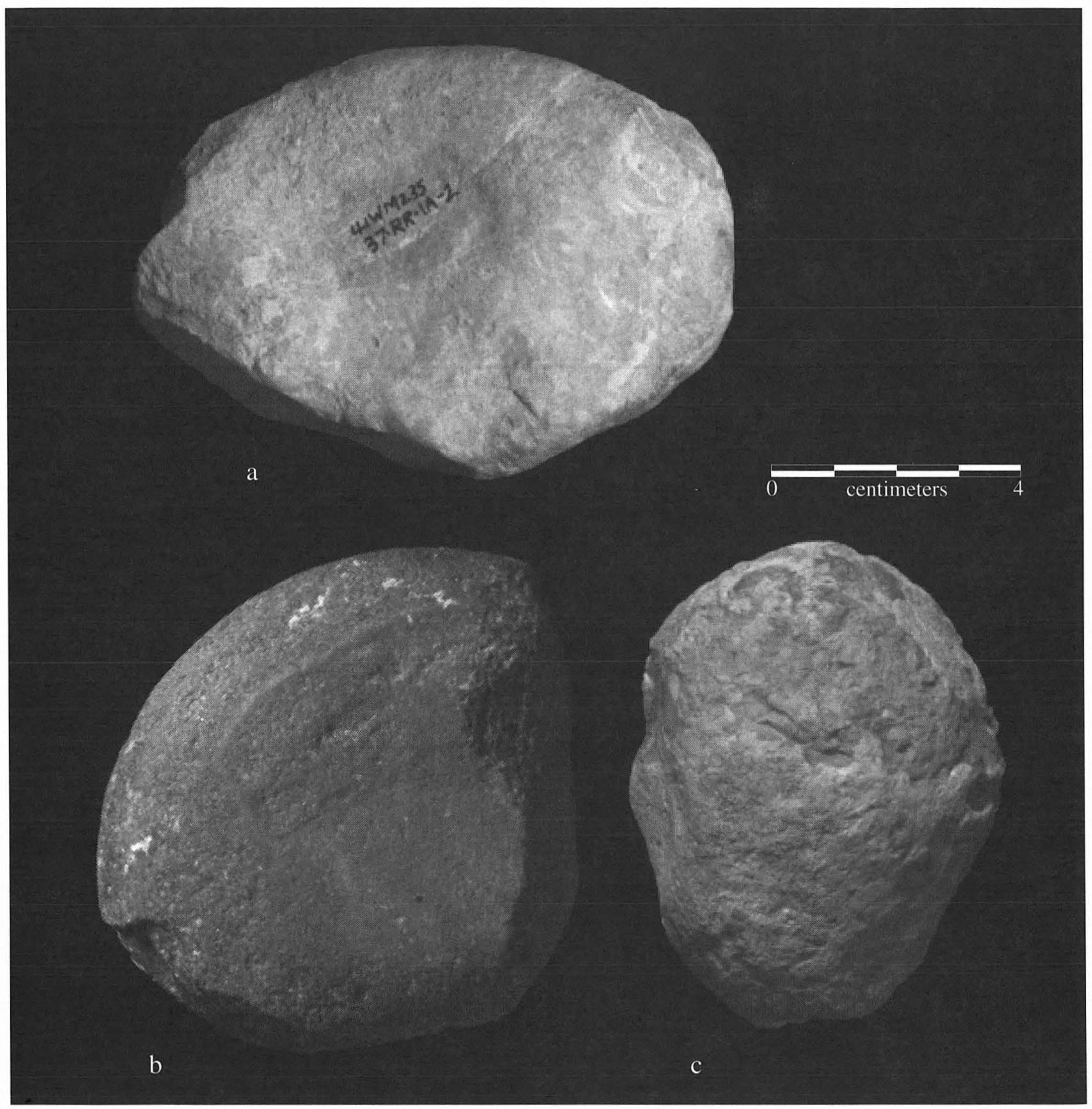

FIGURE 18-7. Representative battered cobbles. (a) 37RR1A-2; (b) 4G-2; (c) 1D-3.

usual that the large lithic collection (some 10,000 stone artifacts and 500,000 pieces of flaking debris) from this site (where considerable knapping occurred and which is near major chert outcrops) includes just 50 hammerstones that appear to have been used in knapping (ca. $0.5 \%$ of the implements). Evidently hammerstones were kept in service for extended periods, much of the knapping was accomplished using antler or other perishable tools, and possibly the excavated sample is deficient in hammerstones for some reason. Alternatively, perhaps 50 hammerstones is representative of their usage at this site; if so, the average rate of loss in the sampled part of the site is less than 1 hammerstone every 210 years (ca. 50 battered stones in roughly 10,500 years). It is noteworthy that 12 of the 50 battered stone artifacts occur in the Paleoindian contexts of Unit I.

The forms of the recovered and identified hammerstones are dominated by chert, either as cobbles or as pieces of chipped stone. Natural cobbles used as hammers are distributed sparsely throughout the occupation of the site, with no discernible times of abundance or scarcity. Large and small core hammerstones are also fairly evenly distributed through the stratigraphic sequence except that small core 
TABLE $18-8$

Attributes of Large Core Hammerstones ( $\mathrm{N}=10)$

\begin{tabular}{|c|c|c|c|c|c|c|c|c|c|c|c|}
\hline \multirow[b]{2}{*}{ Specimen } & \multirow[b]{2}{*}{ Unit } & \multirow[b]{2}{*}{ Cultural Period } & \multicolumn{4}{|c|}{ Measurements (mm) } & \multicolumn{3}{|c|}{ Cortex } & \multicolumn{2}{|c|}{ Mineral Precipitates } \\
\hline & & & Length & Width & Thickness & \begin{tabular}{|l|} 
Weight \\
(g)
\end{tabular} & Nodular & \begin{tabular}{|l|} 
Upland \\
Residual
\end{tabular} & Amount & Light* & Medium* \\
\hline \multicolumn{2}{|c|}{ Valley Floor A: } & & & & & & \multirow{7}{*}{$\mathrm{X}$} & & & \multirow{7}{*}{$\mathrm{X}$} & \\
\hline $13 \mathrm{D}-8$ & |IIc & Late Archaic & 60 & 76 & 37 & 166 & & & & & \\
\hline $30 \mathrm{R} 2-20$ & IIIIa & Early Archaic & 79 & 49 & 18 & 85 & & $\mathrm{X}$ & $1 / 4$ or less & & \\
\hline 17AA1-2 & Isi-c/Id/II & Late Paleoindian & 77 & 54 & 40 & 152 & & $\mathrm{X}$ & $1 / 4-1 / 2$ & & \\
\hline 28BB2A-1 & Isi-c & Late Paleoindian & 76 & 48 & 33 & 164 & & & $1 / 4$ or less & & \\
\hline 24FF2B-1 & |Isi/Icl/Isi-c & Paleoindian & 66 & 42 & 35 & 124 & & $\mathrm{X}$ & $1 / 4$ or less & & \\
\hline 33MM2D-1 & Isi/Icl & Early Paleoindian & 82 & 75 & 60 & 409 & & $\mathrm{X}$ & $1 / 4$ or less & & \\
\hline \multicolumn{2}{|c|}{ Valley Margin: } & & & & & & & & & \multirow{5}{*}{$\mathrm{X}$} & \multirow[b]{5}{*}{$\mathrm{X}$} \\
\hline $1 \mathrm{~A}-11$ & IIIc & Late Prehistoric & 91 & 69 & 35 & 236 & & $\mathrm{X}$ & $1 / 4-1 / 2$ & & \\
\hline $7 A-19$ & |IIc/POT & Late Prehistoric/Archaic & 107 & 54 & 35 & 277 & & $\mathrm{X}$ & $1 / 4$ or less & & \\
\hline $21 \mathrm{R}-4$ & III & Late Paleoindian & 64 & 54 & 21 & 81 & & & & & \\
\hline $22 Q-15$ & III & Late Paleoindian & 72 & 36 & 22 & 57 & & $\mathrm{X}$ & $1 / 4$ or less & & \\
\hline
\end{tabular}

*Concentrated.

TABLE 18-9

Battering Attributes of Large Core Hammerstones $(\mathrm{N}=10)$

\begin{tabular}{|c|c|c|c|c|c|}
\hline \multirow[b]{2}{*}{ Specimen } & \multirow[b]{2}{*}{ Unit } & \multirow[b]{2}{*}{ Cultural Period } & \multicolumn{3}{|c|}{ Battering } \\
\hline & & & Heavy/Patchy & Heavy/Extensive & Distribution \\
\hline \multicolumn{6}{|c|}{ Valley Floor A: } \\
\hline $13 \mathrm{D}-8$ & IIIIc & Late Archaic & $\mathrm{X}$ & & edges \\
\hline $30 \mathrm{R} 2-20$ & IIIIa & Early Archaic & & $\mathrm{X}$ & edges \\
\hline 17AA1-2 & Isi-c/Id/II & Late Paleoindian & $\mathrm{X}$ & & edges \\
\hline 28BB2A-1 & Isi-c & Late Paleoindian & & $\mathrm{X}$ & edges, faces \\
\hline 24FF2B-1 & Isi/Icl/Isi-c & Paleoindian & & $\mathrm{X}$ & edges, faces, ridges \\
\hline 33MM2D-1 & Isi//cl & Early Paleoindian & $\mathrm{X}$ & & edges \\
\hline \multicolumn{6}{|c|}{ Valley Margin: } \\
\hline $1 \mathrm{~A}-11$ & |IIIc & Late Prehistoric & & $\mathrm{X}$ & edges \\
\hline 7A-19 & IIIc/POT & Late Prehistoric/Archaic & & $\mathrm{X}$ & edges \\
\hline $21 \mathrm{R}-4$ & II & Late Paleoindian & & $\mathrm{X}$ & edge \\
\hline 22Q-15 & II & Late Paleoindian & & $\mathrm{X}$ & edge \\
\hline
\end{tabular}

hammerstones are almost absent from Unit I. Only the six face-battered stones ( 7 counting the core described elsewhere) occur in a single interval (see Table 18-12). These are found in the lowest cultural zone (Early Paleoindian).

Battered stones are predominantly of chert (44 of 50, or $88.0 \%$ ). The battered cobbles are the most variable lithologically with only 5 of the 10 being chert; the other 5 are of limestone $(\mathrm{n}=2)$, basalt, quartzite, and sandstone. It is also of interest to note the lithological characteristics among the 44. Thirty-one (70.5\%) are of fine-grained chert, and 13 $(29.5 \%)$ are of medium-coarse grained chert. Among the battered cobbles, all of the 5 chert specimens are of medium-coarse texture. The large core hammerstones are all of chert, 3 being fine- and 7 being medium-coarsegrained. The 24 small core hammerstones are of particular interest, there being 1 of quartzite and 1 of mediumcoarse-grained chert. The 22 fine-grained pieces include 8 of the distinctive black chert that occurs infrequently in most artifact categories except for Paleoindian projectile points and Nolan dart points of Archaic age.
Along with inferring that the majority of these $50 \mathrm{ob}-$ jects were, or could have been, used as knappers' hammerstones among other possible uses, the question has been raised regarding the uses that would have produced the concave-edged pieces of various ages and the face-battered pieces of Early Paleoindian age. If the concave-edged configuration did not result from knapping, other possibilities include bone-breaking for marrow extraction or the shaping and maintenance of pecked stone tools. The small sample of 5 does not provide a robust data set for addressing these questions, but some hints may reside in the distributional evidence. These pieces occur in four stratigraphic contexts (1 each in Units Isi-c, II, and IIIa and 2 in Unit IIIc). Manos, some of which are shaped and maintained by pecking, occur in all of these units (see Chapter 20). Alternatively, if bone breaking accounts for these tools, only bison among the central Texas Holocene faunal succession have bones of the size and toughness that might cause this degree of damage. Bison occurrences through time in central Texas (Collins 1995:Table 2) correlate in 

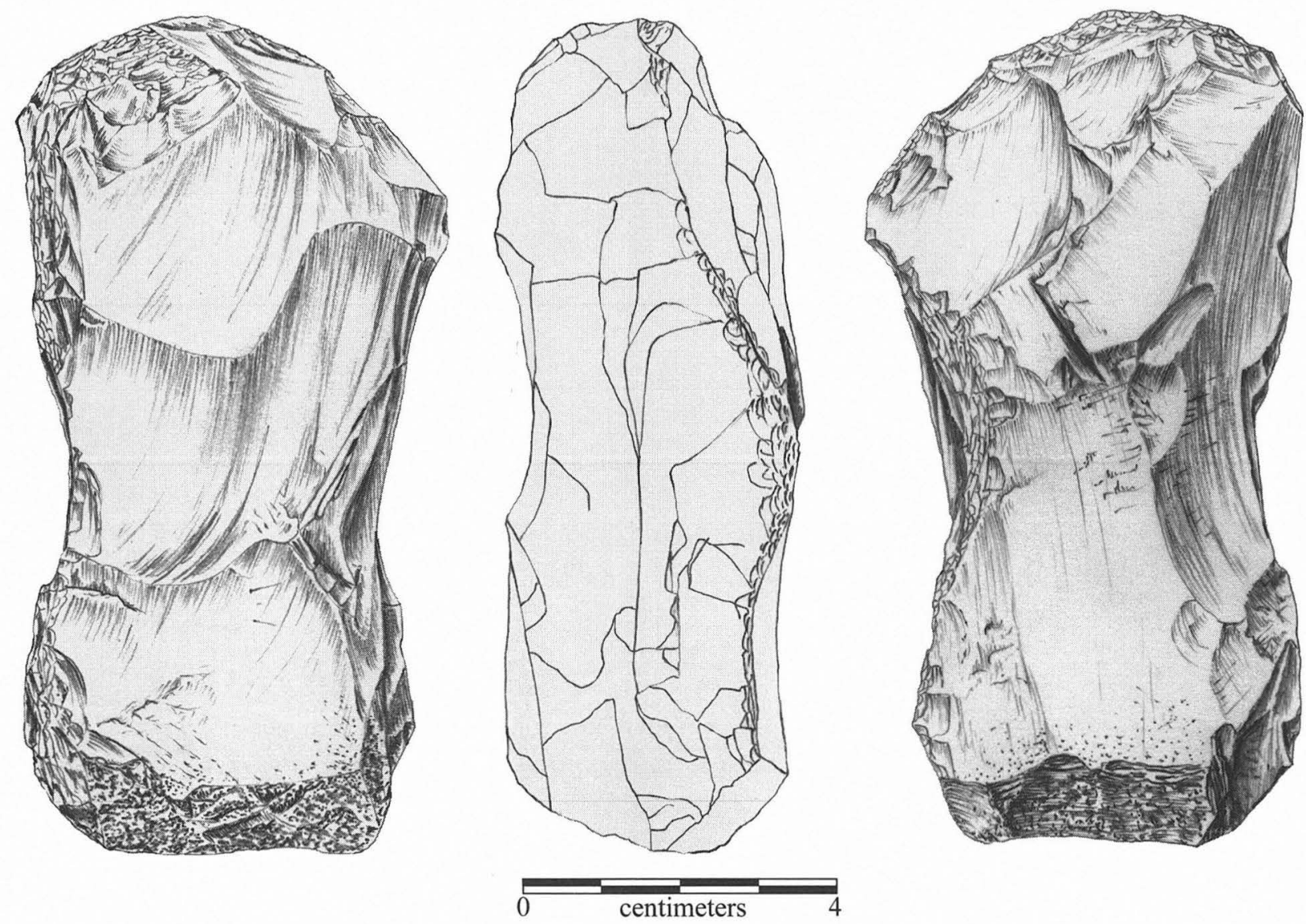

FIGURE 18-8. Large core hammerstone (three views) with concave edges from concentrated attrition of heavy battering (Specimen 7A-19).

general with the distribution of concave-edged hammerstones with the possible exception of Unit Isi-c. Unit Isi-c represents a previously unknown prehistoric cultural interval and the presence of bison in this unit is indicated by only a single specimen. Identifiable bison bone at Wilson-Leonard (see Chapter 33) do not correlate closely to the distribution of these hammerstones, but bone preservation may be a factor.

Although it is not identified, the activity that produced the face-battered stones appears to have occurred only in early Paleoindian times. One possibility to consider is the driving of wedges to split bone or wood. However, there are no pièce esquillées (interpreted as probable wedges) in the early Paleoindian components associated with these face-battered pieces.

\section{CORES ANDTESTED AND UNMODIFIED CHERTMATERIALS}

The final category is referred to here as the core assemblage. This broad group encompasses cores and core fragments as well as tested and unmodified raw materials of sufficient size to have been intended as cores. Cores and fragments are classified into six groups based on morphology, flake removal, degree of completeness, and alteration of raw material. These are multidirectional cores, unidirectional cores, microcores (in three size ranges), core fragments, chunks and indeterminate fragments, and thermally or cryogenically altered fragments. Tested and unmodified materials constitute a seventh group. Metric attributes for cores and microcores are summarized in tabular form. For all other categories, size ranges based on maximum diameter, rather than specific measurements, are given.

\section{Descriptions}

\section{Multidirectional Cores $(N=25)$}

This category consists of 25 generally complete, unbroken cores, the majority of which exhibit multidirectional flaking as well as both prepared and natural platforms (Figures 18-12 and 18-13). As distinguished from the various microcore and core fragment groups, below, specimens in this category appear to have been deposited while still potentially serviceable. Although there is substantial variability in size, most cores in this category are fist-sized (Table 18-14). The weights of these specimens range from 60 to $640 \mathrm{~g}$.

Raw material is predominately local coarse-grained chert (16, or $64.0 \%)$. An additional 7 , or $28.0 \%$ were made 


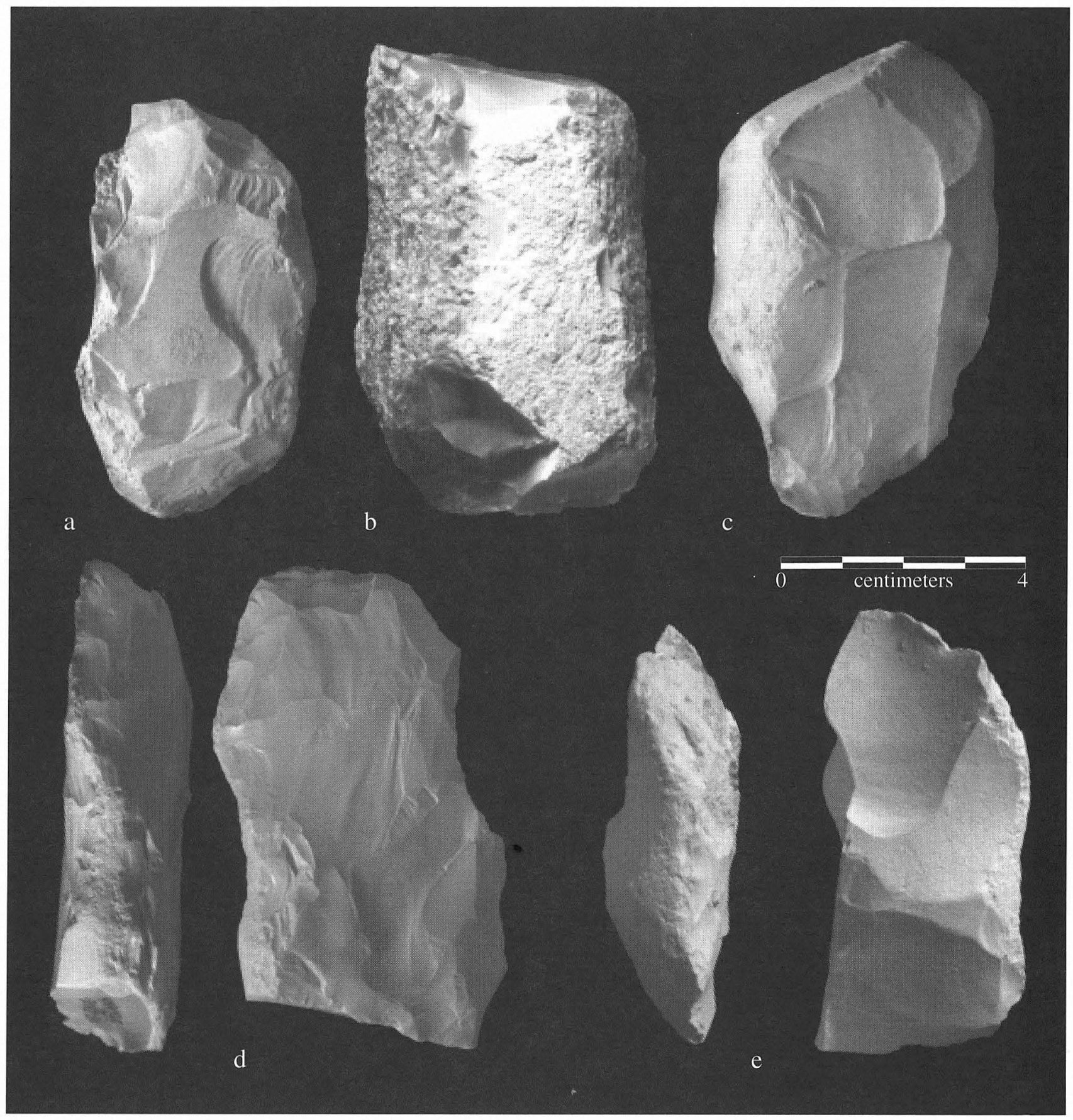

FIGURE 18-9. Large core hammerstones (a, b) and core hammerstones made on moderately thin biface fragments (c-e). (a) 24FF2B-1; (b) 28BB2A-1; (c) 17AA1-2; (d) 30R2-20; (e) 22Q-15.

of fine-grained cherts, and $1(4.0 \%)$ is of quartz (10E-4, discussed below). Only 1 specimen is of non-Edwards chert. It is a stream-rolled, cylindrical pebble of coarse olive green chert, from which five to six flakes have been removed.

Cortex, present on all but 2 specimens, indicates most (12 or $52.2 \%$ ) were derived from upland settings; 5 others (21.7\%) likely were collected as stream cobbles; 4 (17.4\%) bear nodular cortex; and $2(8.7 \%)$ are indeterminate. Only 9 specimens $(36.0 \%)$ exhibit mineral precipitates, and these are sparsely deposited for the most part in fracture scars or on cortex. Patina is present on $2(8.0 \%)$ specimens.

One unusual core bears further comment. A large, tabular block of quartz, Specimen 10E-4, shows some evidence of bipolar, blade-like flake removal as well as scars of overlying, multidirectional flake extractions that are generally more glossy than the others and perhaps more recent (Figure 18-14). Its overall dimensions are $130 \mathrm{~mm}$ in length; $79 \mathrm{~mm}$ in width; and $58 \mathrm{~mm}$ in thickness; and it weighs $230 \mathrm{~g}$. This 
TABLE 18-10

Attributes of Small Core Hammerstones ( $N=24)$

\begin{tabular}{|c|c|c|c|c|c|c|c|c|c|c|c|c|c|c|c|}
\hline \multirow[b]{2}{*}{ Specimen } & \multirow[b]{2}{*}{ Unit } & \multirow[b]{2}{*}{ Cultural Period } & \multicolumn{4}{|c|}{ Measurements (mm) } & \multicolumn{5}{|c|}{ Cortex } & \multicolumn{4}{|c|}{ Mineral Precipitates } \\
\hline & & & Length & Width & Thickness & \begin{tabular}{|l|} 
Weight \\
$(\mathrm{g})$
\end{tabular} & Nodular & \begin{tabular}{|l|} 
Upland \\
Residual
\end{tabular} & $\begin{array}{l}\text { Stream } \\
\text { Rolled }\end{array}$ & Indeterminate & Amount & $\begin{array}{l}\text { Light } \\
\text { Overall }\end{array}$ & Light* & Medium* & Heavy* \\
\hline \multicolumn{2}{|c|}{ Valley Floor A: } & & & & & & & & & & & & & & \\
\hline $13 \mathrm{~A}-1$ & IIIIc & Late Prehistoric & 55 & 40 & 28 & 40 & & & $\mathrm{x}$ & & $1 / 2-3 / 4$ & & & & \\
\hline $15 \mathrm{~L} 1-2$ & IIIlb & Early Archaic & 48 & 41 & 24 & 43 & & & & & & & & & \\
\hline $15 \mathrm{~L} 1-3$ & IIIlb & Early Archaic & 53 & 45 & 28 & 60 & & & & & & & & & \\
\hline $16 \mathrm{~L} 2-2$ & IIIb & Early Archaic & 48 & 36 & 21 & 32 & & & & & & & & & \\
\hline 29Q1-1 & IIIа & Early Archaic & 55 & 41 & 23 & 56 & $\mathrm{x}$ & & & & $1 / 4$ or less & & & & \\
\hline $29 R 2-4$ & IIIIa & Early Archaic & 36 & 45 & 34 & 46 & $\mathrm{x}$ & & & & $1 / 4$ or less & & & & \\
\hline 33R1-3 & IIIIa & Early Archaic & 39 & 32 & 16 & 19 & & $\mathrm{X}$ & & & $1 / 4$ or less & & & & \\
\hline $34 Q 2-5$ & IIIа & Early Archaic & 39 & 32 & 18 & 23 & $\mathrm{X}$ & & & & $1 / 4$ or less & & & & \\
\hline 35R1-1 & IIIIa & Early Archaic & 36 & 39 & 13 & 21 & & & & & & & & & \\
\hline $12 \mathrm{R}-7$ & II/IIIa & Early Archaic/Late Paleoindian & 41 & 51 & 19 & 45 & & & & & & & & & \\
\hline $17 \mathrm{~T} 1-6$ & II/IIIa & Early Archaic/Late Paleoindian & 40 & 38 & 20 & 20 & $\mathrm{x}$ & & & & $1 / 2-3 / 4$ & & & & \\
\hline $24 S 1-5$ & II/IIIa & Early Archaic/Late Paleoindian & 44 & 52 & 27 & 75 & $\mathrm{X}$ & & & & $1 / 2-3 / 4$ & & & & \\
\hline 33Т1B-9 & II/IIIa & Early Archaic/Late Paleoindian & 37 & 38 & 21 & 35 & & & & & & & $\mathrm{X}$ & & \\
\hline OR690 & II/IIIa & Early Archaic/Late Paleoindian & 47 & 45 & 32 & 80 & & & $\mathrm{X}$ & & $1 / 4$ or less & & & & $\mathrm{x}$ \\
\hline $12 S-3$ & III & Late Paleoindian & 47 & 42 & 28 & 49 & $\mathrm{X}$ & & & & $1 / 4$ or less & & & & \\
\hline 16U1-1 & II & Late Paleoindian & 65 & 34 & 19 & 57 & & & & $\mathrm{X}$ & $1 / 4$ or less & & & & \\
\hline 31V1D-2 & II & Late Paleoindian & 43 & 50 & 18 & 52 & & & & $\mathrm{X}$ & $1 / 2-3 / 4$ & & & & \\
\hline $32 \mathrm{~V} 1 \mathrm{~A}-3$ & II & Late Paleoindian & 45 & 35 & 26 & 56 & & & & & & $\mathrm{X}$ & & & \\
\hline $1428-2$ & Isi-c & Late Paleoindian & 39 & 41 & 29 & 55 & & & & $\mathrm{X}$ & $1 / 4-1 / 2$ & & & $\mathrm{X}$ & \\
\hline \multicolumn{2}{|c|}{ Valley Margin: } & & & & & & & & & & & & & & \\
\hline $11 \mathrm{C}-9$ & |IIIc & Late Archaic & 46 & 38 & 29 & 52 & & & & $\mathrm{x}$ & $1 / 4$ or less & & & & \\
\hline $8 \mathrm{I}-17$ & $\mathrm{Y} / \mathrm{IIIC}$ & Archaic & 45 & 48 & 30 & 61 & & & & & & & & & $\mathrm{X}$ \\
\hline $3 J-5$ & $\mathrm{Y}$ & Early Archaic & 52 & 40 & 20 & 37 & & & & & & & & $\mathrm{X}$ & \\
\hline $20 \mathrm{O}-4$ & $\mathrm{X} / \mathrm{III}$ & Early Archaic & 45 & 35 & 19 & 30 & $\mathrm{X}$ & & & & $1 / 4$ or less & $\mathrm{X}$ & & & \\
\hline $18 \mathrm{~N}-3$ & $\mathrm{x}$ & Early Archaic & 48 & 34 & 16 & 30 & $\mathrm{X}$ & & & & $1 / 4-1 / 2$ & & $\mathrm{X}$ & & \\
\hline
\end{tabular}


TABLE 18-11

Battering Attributes of Small Core Hammerstones ( $\mathrm{N}=24)$

\begin{tabular}{|c|c|c|c|c|c|c|}
\hline \multirow[b]{2}{*}{ Specimen } & \multirow[b]{2}{*}{ Unit } & \multirow[b]{2}{*}{ Cultural Period } & \multicolumn{4}{|c|}{ Battering } \\
\hline & & & Moderate/Patchy & Heavy/Patchy & Heavy/Extensive & Distribution \\
\hline \multicolumn{7}{|c|}{ Valley Floor A: } \\
\hline $13 \mathrm{~A}-1$ & |IIIc & Late Prehistoric & \multirow{7}{*}{$\mathrm{X}$} & $\mathrm{X}$ & \multirow{19}{*}{$X$} & edges \\
\hline 15L1-2 & IIIb & Early Archaic & & $\mathrm{X}$ & & edge \\
\hline $15 \mathrm{~L} 1-3$ & IIIlb & Early Archaic & & $\mathrm{X}$ & & edge \\
\hline $16 \mathrm{~L} 2-2$ & IIlb & Early Archaic & & $X$ & & edges \\
\hline 29Q1-1 & IIIIa & Early Archaic & & $\mathrm{X}$ & & edges, faces \\
\hline 29R2-4 & ШІа & Early Archaic & & & & edge \\
\hline 33R1-3 & IIIIa & Early Archaic & & $\mathrm{X}$ & & edges \\
\hline $34 Q 2-5$ & IIIIa & Early Archaic & \multirow[t]{7}{*}{$\mathrm{X}$} & & & edge \\
\hline $35 \mathrm{R} 1-1$ & IIIIa & Early Archaic & & $\mathrm{X}$ & & edge \\
\hline $12 \mathrm{R}-7$ & II//IIIa & Early Archaic/Late Paleoindian & & $\mathrm{X}$ & & edges \\
\hline $17 \mathrm{~T} 1-6$ & |ІІ/ІІІа & Early Archaic/Late Paleoindian & & $\mathrm{X}$ & & edges \\
\hline $24 \mathrm{~S} 1-5$ & II/IIIIa & Early Archaic/Late Paleoindian & & $\mathrm{X}$ & & edges \\
\hline 33Т1B-9 & |ІІ/ШІа & Early Archaic/Late Paleoindian & & & & edges \\
\hline OR690 & |Ш/Ша & Early Archaic/Late Paleoindian & & $\mathrm{X}$ & & edges \\
\hline $12 S-3$ & & Late Paleoindian & \multirow[t]{3}{*}{$X$} & & & face \\
\hline $16 U 1-1$ & III & Late Paleoindian & & $\mathrm{X}$ & & edges \\
\hline $31 \mathrm{~V} 1 \mathrm{D}-2$ & II & Late Paleoindian & & $\mathrm{X}$ & & edges \\
\hline $32 \mathrm{~V} 1 \mathrm{~A}-3$ & II & Late Paleoindian & \multirow[t]{2}{*}{$\mathrm{X}$} & & & edges \\
\hline $1428-2$ & Isi-c & Late Paleoindian & & $\mathrm{X}$ & & edges \\
\hline \multicolumn{7}{|c|}{ Valley Margin: } \\
\hline $11 \mathrm{C}-9$ & IIIIc & Late Archaic & \multirow{5}{*}{$\mathrm{X}$} & & \multirow[t]{5}{*}{$X$} & \multirow{5}{*}{$\begin{array}{l}\text { edges } \\
\text { edges } \\
\text { edge } \\
\text { edges, face } \\
\text { edges }\end{array}$} \\
\hline $8 \mathrm{I}-17$ & IIII & Archaic & & $\mathrm{X}$ & & \\
\hline $3 \mathrm{~J}-5$ & & Early Archaic & & $\mathrm{X}$ & & \\
\hline $200-4$ & $\mathrm{X} / \mathrm{III} \mathrm{a}$ & Early Archaic & & & & \\
\hline $18 \mathrm{~N}-3$ & $\mathrm{X}$ & Early Archaic & & $\mathrm{X}$ & & \\
\hline
\end{tabular}

TABLE 18-12

Attributes of Face-battered Stones $(\mathrm{N}=6)$

\begin{tabular}{|c|c|c|c|c|c|c|c|c|c|c|}
\hline \multirow[b]{2}{*}{ Specimen } & \multirow[b]{2}{*}{ Unit } & \multirow[b]{2}{*}{ Cultural Period } & \multicolumn{4}{|c|}{ Measurements (mm) } & \multicolumn{3}{|c|}{ Cortex } & \multirow{2}{*}{\begin{tabular}{|l} 
Mineral Precipitates \\
Light Concentrated
\end{tabular}} \\
\hline & & & Length & Width & Thickness & $\begin{array}{l}\text { Weight } \\
(\mathrm{g})\end{array}$ & Nodular & $\begin{array}{l}\text { Upland } \\
\text { Residual }\end{array}$ & Amount & \\
\hline \multicolumn{11}{|c|}{ Valley Floor A: } \\
\hline 27II1D-1 & Isi/Icl & Early Paleoindian & 41 & 36 & 27 & 41 & $\mathrm{X}$ & & $1 / 4$ or less & $\mathrm{X}$ \\
\hline 29LL1C-1 & Isi/Icl & Early Paleoindian & 57 & 51 & 21 & 57 & $\mathrm{X}$ & & $1 / 4-1 / 2$ & $\mathrm{X}$ \\
\hline $31 \mathrm{KK} 1 \mathrm{~A}-1$ & Isi/Icl & Early Paleoindian & 50 & 51 & 26 & 65 & $\mathrm{X}$ & & $1 / 4$ or less & \\
\hline $34 N N 1 B$ & Isi/Icl & Early Paleoindian & 81 & 73 & 16 & 74 & & $\mathrm{X}$ & $1 / 2-3 / 4$ & \\
\hline $35 \mathrm{KK} 2 \mathrm{~A}-2$ & Isi/Icl & Early Paleoindian & 75 & 51 & 36 & 196 & & $\mathrm{X}$ & $1 / 4-1 / 2$ & $\mathrm{X}$ \\
\hline $37001 \mathrm{~A}-4$ & Isi/Icl & Early Paleoindian & 61 & 50 & 30 & 75 & & & & \\
\hline
\end{tabular}

specimen also exhibits areas of reddish brown residue resembling hematite, although these likely were deposited through natural processes (C. Gene Mear, personal communication 1995). This core is one of four items recovered as a possible Late Archaic "cache;" the other specimens are Stage I bifaces of fine-grained local chert (see Feature 11, Chapter 26).

\section{Unidirectional Cores $(N=2)$}

Two early specimens are aberrant morphologically within the larger group of cores, and both were submitted for special technical studies. The first, recovered from mixed Late Paleoindian/Early Archaic deposits (Unit II/IIIa transi- tion zone), is a complete conical core from which flakes were struck unidirectionally from a flat prepared platform (Figure 18-15a). Flake scars originating from this platform extend around the full circumference of the core. With an opposing planar surface of much smaller diameter, the overall aspect of the specimen is almost pyramidal. Its measurements are $58 \mathrm{~mm}$ in length; $49 \mathrm{~mm}$ in width; and $28 \mathrm{~mm}$ in thickness; and $296 \mathrm{~g}$ in weight.

Several aspects of this core suggest its use as a tool. Platform edges are dulled and slightly battered on at least one area, and in the adjacent flaked platform surface, a series of parallel linear striations are present. Like the core tools described above, this specimen is made of coarse material and displays encrustations of mineral precipitates 


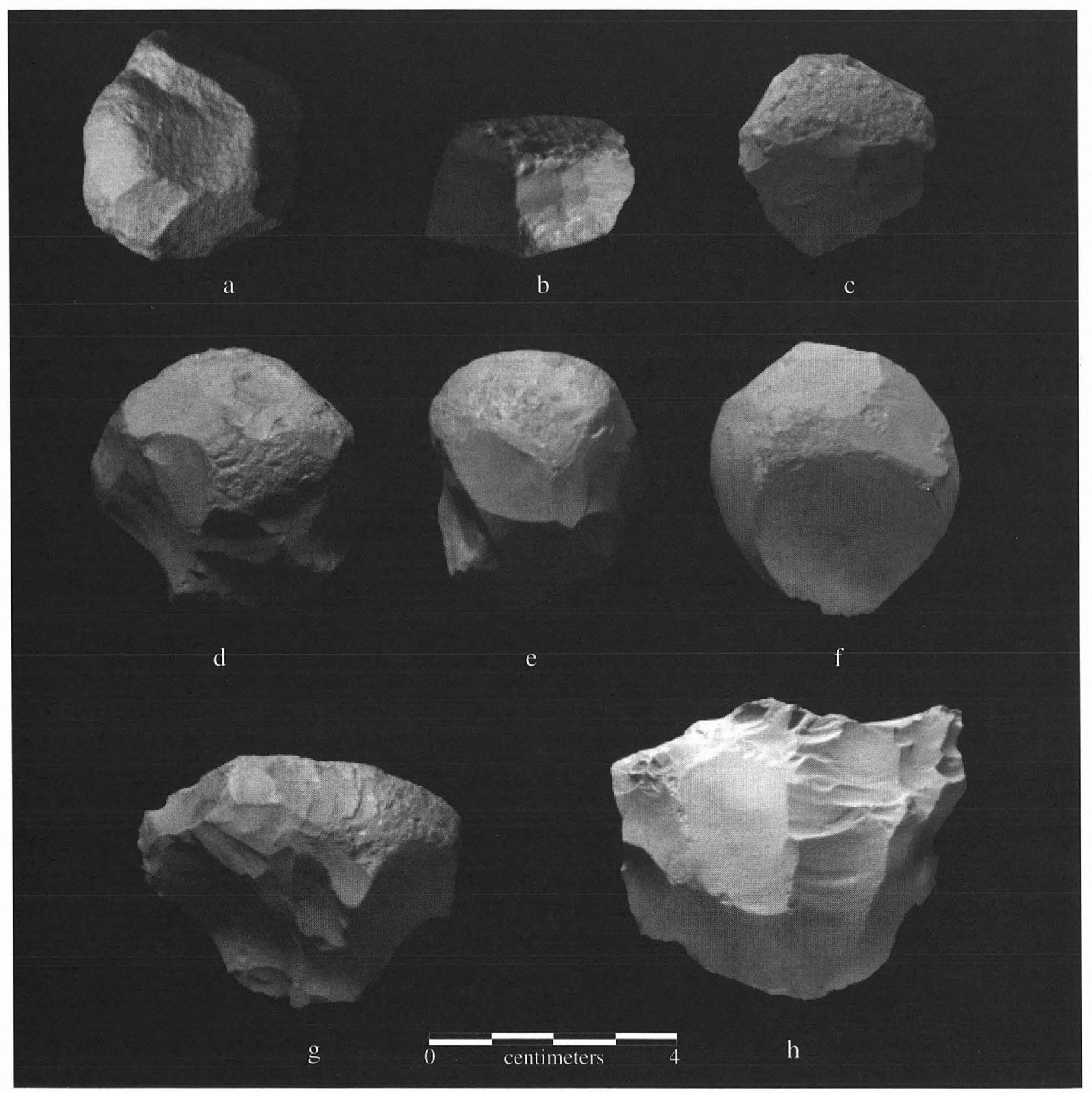

FIGURE 18-10. Small core hammerstones (a-g) and face-battered stone (h). (a) 33T1B-9; (b) 17T1-6; (c) 33R1-3; (d) 8I-17; (e) 11C-9; (f) 1428-2; (g) 15L1-3; (h) 37OO1A-1.

which, in this case, are particularly concentrated along and overlapping the platform edge, although not on the platform surface. In form, this specimen is somewhat reminiscent of domed scrapers or "push" planes from the Valley of Oaxaca, Mexico. Experimental use-wear studies conducted on this tool type by Hester and Heizer (1972) suggest their possible use as pulping planes to extract cordage fibers from agave. Of particular relevance are their findings of battered and dulled planar edges with associated linear striations, a complex of wear that may be attributable to contact of the stone tool against other stone on which the plant material was placed for processing (Hester and Heizer 1972:111).

The second specimen (Figure 18-15b) appears to be a large flake detached from the face of a conical blade core. Made on waxy greenish brown, fine-grained Edwards chert, its dimensions are $88 \mathrm{~mm}$ in length, $50 \mathrm{~mm}$ in width, and 25 $\mathrm{mm}$ in thickness, and $77 \mathrm{~g}$ in weight. Long, narrow blade-like flake scars and ridges resemble the face of a conical blade core, and the flake platform is a remnant of a larger, prepared platform, the edges of which have been scarred and crushed by multiple hinge flakes. However, the flake scars are not all 


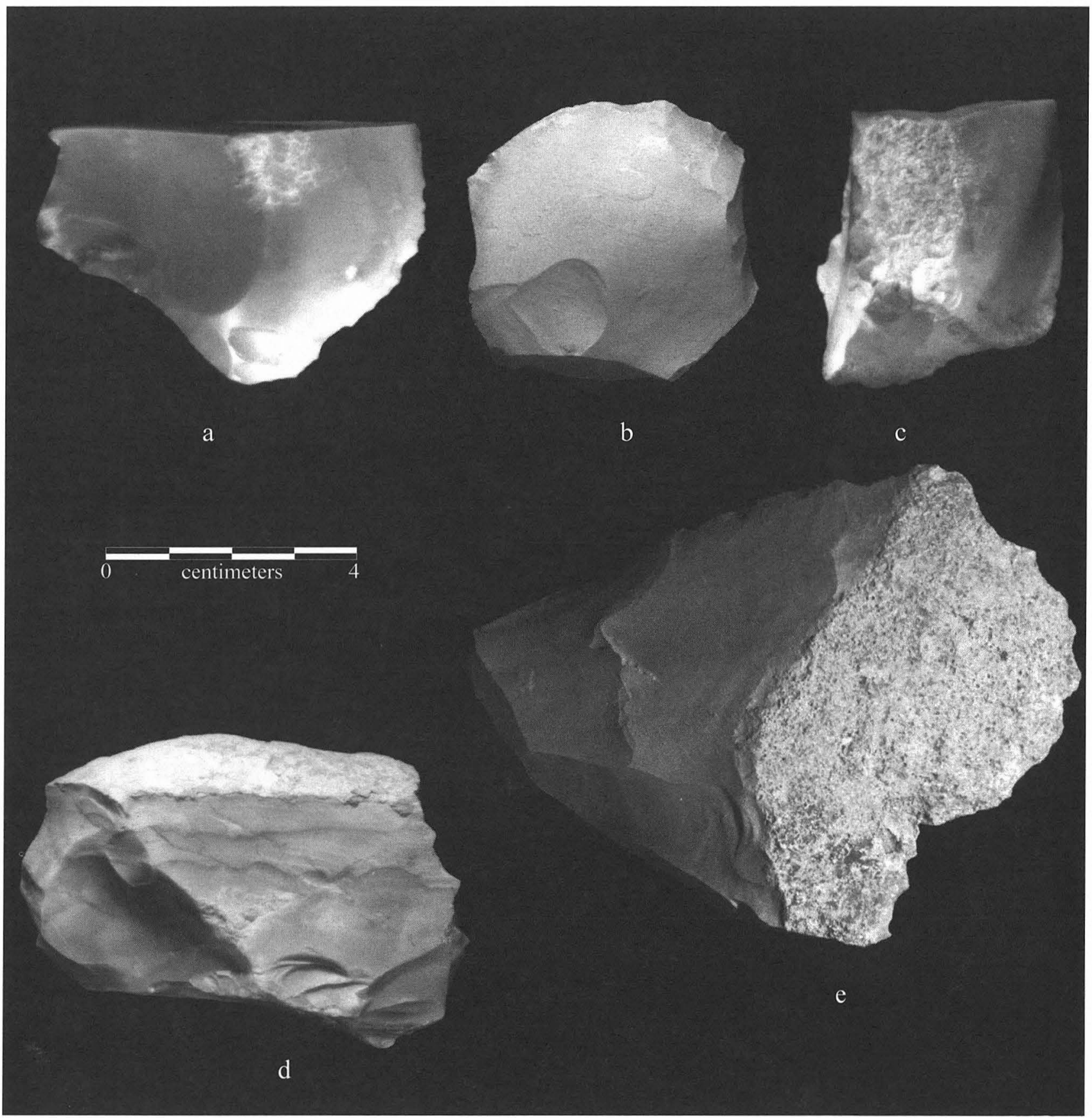

FigURE 18-11. Face-battered chert pieces. (a) 29LL1C-1; (b) 31KK1A-1; (c) 27II1D-1; (d) 35KK2A-2; (e) 34NN1B-2.

TABLE 18-13

Battering Attributes of Face-battered Stones $(\mathrm{N}=6)$

\begin{tabular}{|c|c|c|c|c|c|c|}
\hline \multirow[b]{2}{*}{ Specimen } & \multirow[b]{2}{*}{ Unit } & \multirow[b]{2}{*}{ Cultural Period } & \multicolumn{4}{|c|}{ Battering } \\
\hline & & & Light/Patchy & Moderate/Patchy & Heavy/Patchy & Distribution \\
\hline \multicolumn{7}{|c|}{ Valley Floor A: } \\
\hline $\begin{array}{l}\text { 27II1D-1 } \\
\text { 29LL1C-1 } \\
\text { 31KK1A-1 } \\
\text { 34NN1B-2 } \\
\text { 35KK2A-2 } \\
\text { 37O01A-1 }\end{array}$ & \begin{tabular}{|l} 
Isi/Icl \\
Isi//cl \\
Isi/Icl \\
Isi/Icl \\
Isi/Icl \\
Isi//cl
\end{tabular} & $\begin{array}{l}\text { Early Paleoindian } \\
\text { Early Paleoindian } \\
\text { Early Paleoindian } \\
\text { Early Paleoindian } \\
\text { Early Paleoindian } \\
\text { Early Paleoindian }\end{array}$ & $\mathrm{X}$ & $\begin{array}{l}X \\
X \\
X \\
X\end{array}$ & $\mathrm{X}$ & $\begin{array}{l}\text { faces } \\
\text { face } \\
\text { face } \\
\text { face } \\
\text { faces } \\
\text { edges, face }\end{array}$ \\
\hline
\end{tabular}




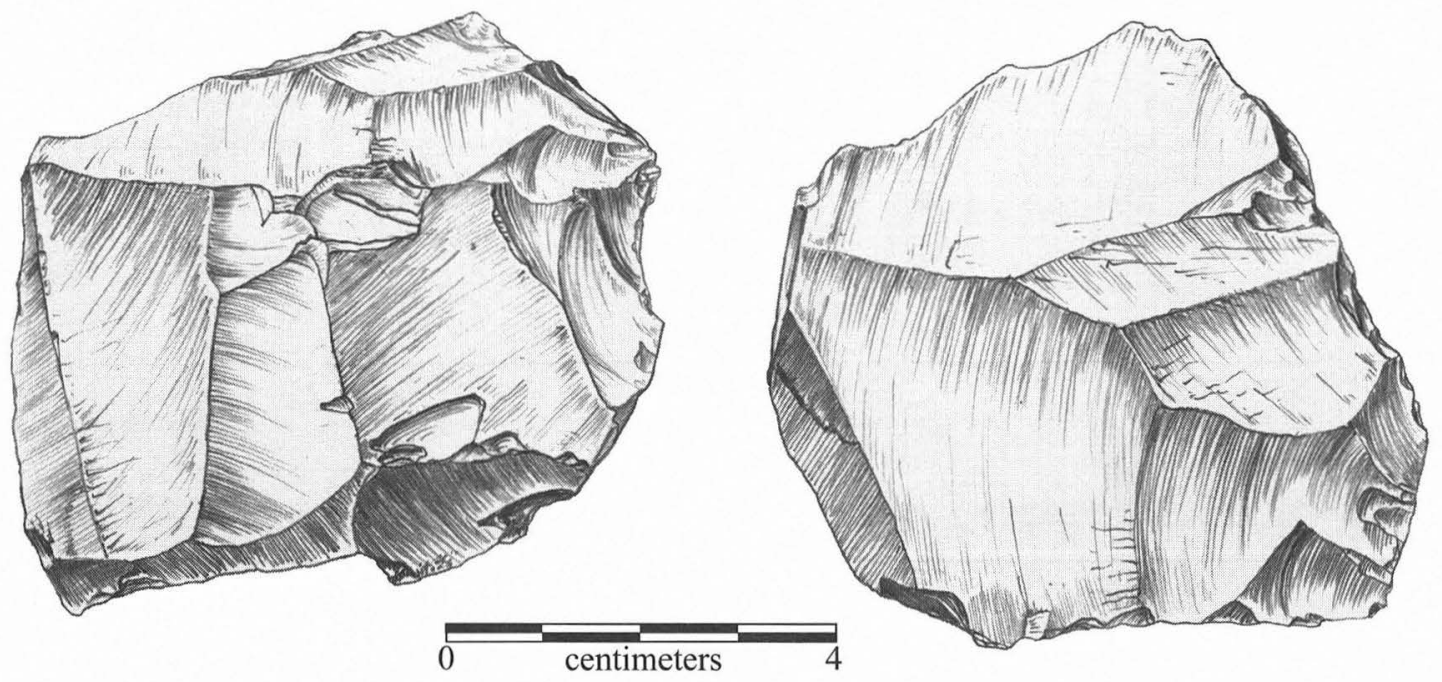

FIGURE 18-12. Representative core (two faces) showing multidirectional flake removal (37LA-1).

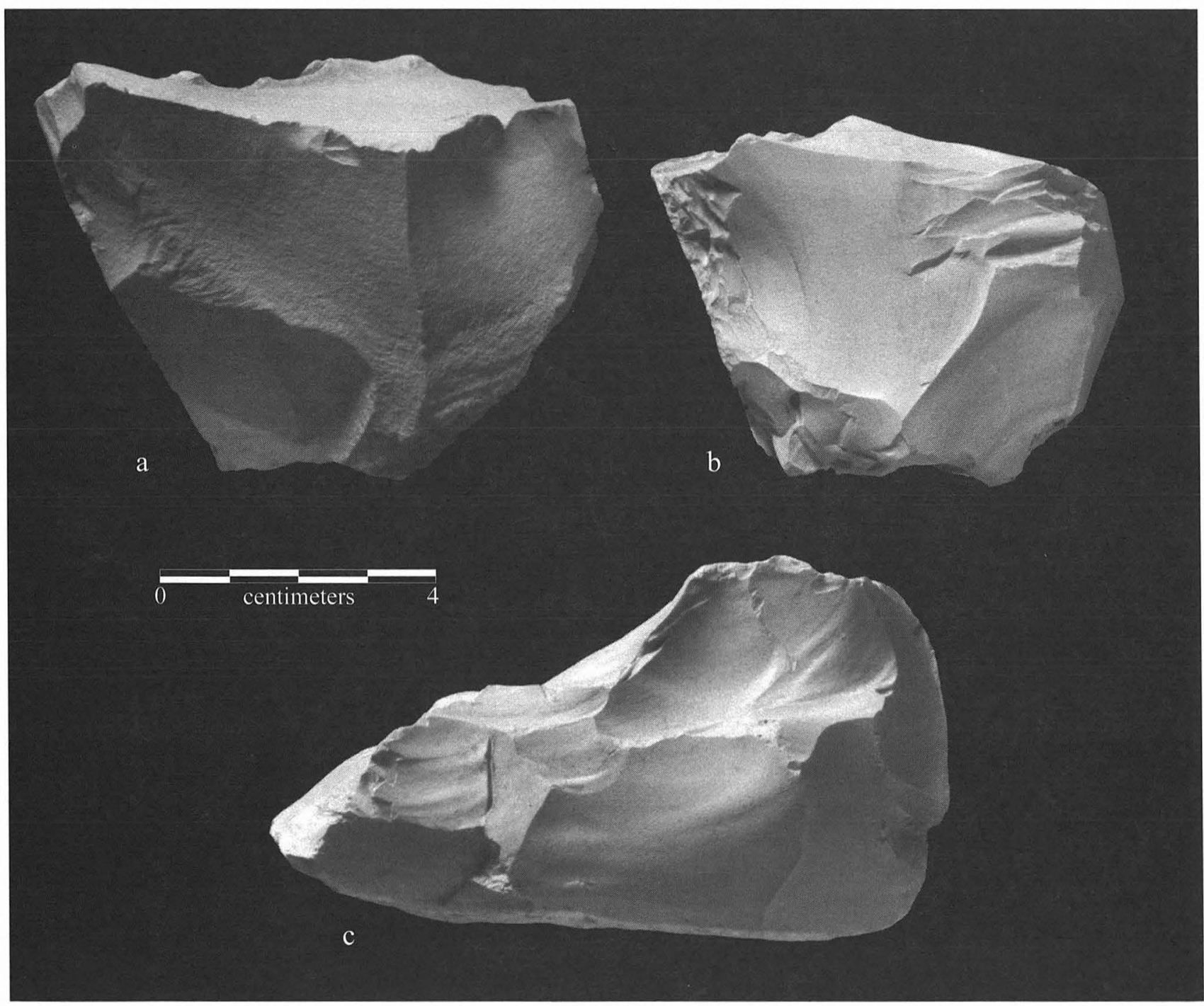

FIGURE 18-13. Examples of multidirectional cores from various contexts. (a) 3A-10 (Unit IIIc); (b) 15Q2-7 (Unit IIIa); (c) 31NN1A-1 (Unit Igl/Isi). 
TABLE 18-14

Metric Attributes of Multidirectional Cores

\begin{tabular}{l|c|c|c|c}
\hline & Length $(\mathrm{mm})$ & Width $(\mathrm{mm})$ & Thickness $(\mathrm{mm})$ & Weight $(\mathrm{g})$ \\
\hline Mean & 79.9 & 64.5 & 44.9 & 266.4 \\
Standard deviation & 15.5 & 11.1 & 8.6 & 127.2 \\
Range & 75 & 43 & 34 & 580 \\
Minimum & 55 & 46 & 25 & 60 \\
Maximum & 130 & 89 & 59 & 640 \\
Count & 25 & 25 & 25 & 25 \\
\hline \hline
\end{tabular}

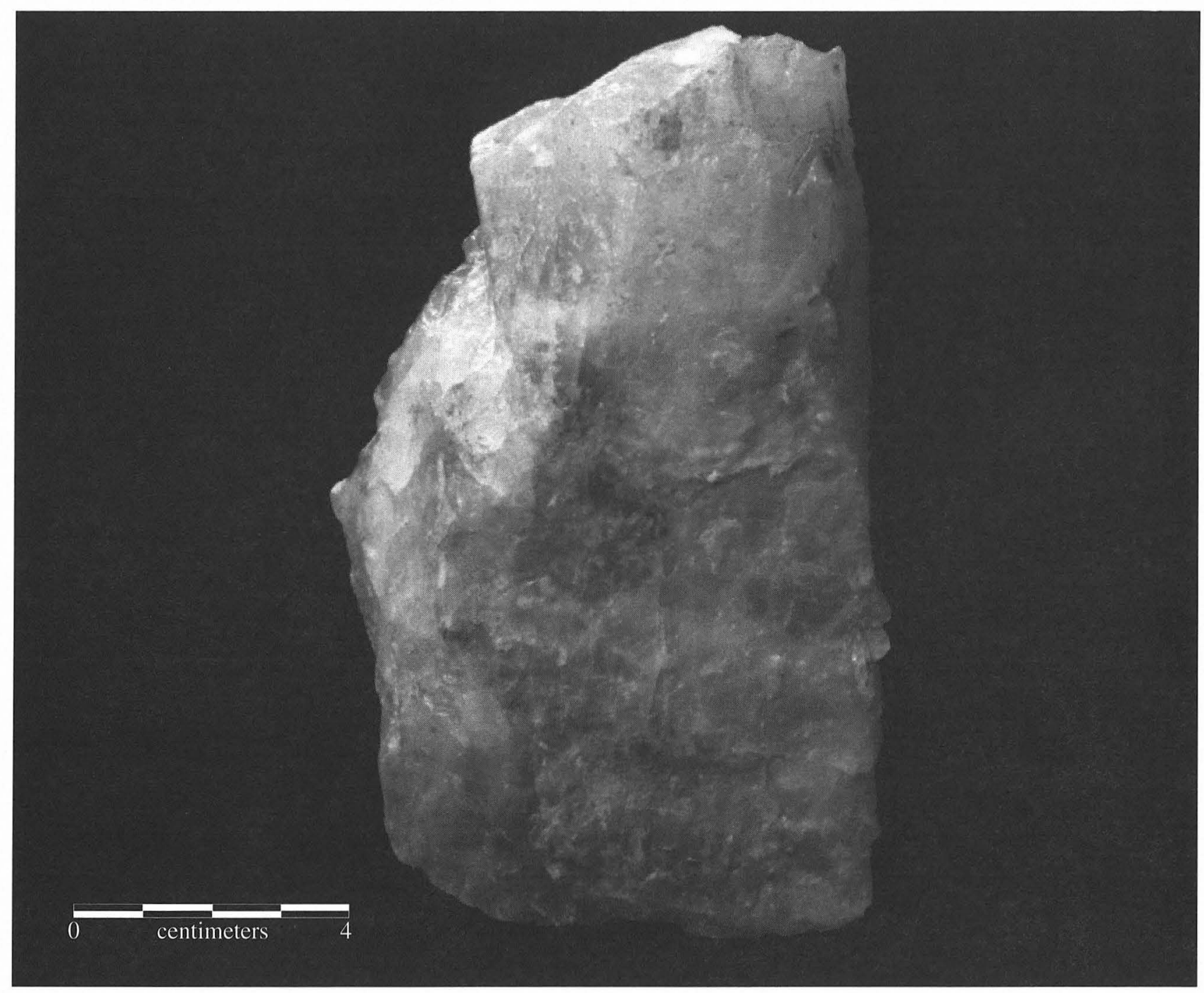

FIGURE 18-14. Quartz core (10E-4) from Late Archaic “cache," showing multidirectional and blade-like flake scars.

unidirectional, and if this is a piece of a blade core, the knapper was not successful in detaching blades. Although microflaking is present along one lateral section, use-wear analysis revealed no evidence of use (see Driskell, Chapter 22). Recovered from a stratigraphically undifferentiated deposit in Unit I, the possible blade core fragment likely is related to an Early Paleoindian occupation.

\section{Microcores $(N=50)$}

In a previous chapter, microlithic flake tools (arbitrarily defined as $30 \mathrm{~mm}$ or less in maximum dimension) are reported (see Chapter 17). In the following section, a group of 50 microcores from which such tools may have derived are discussed. The microcores are defined on the basis of hav- 


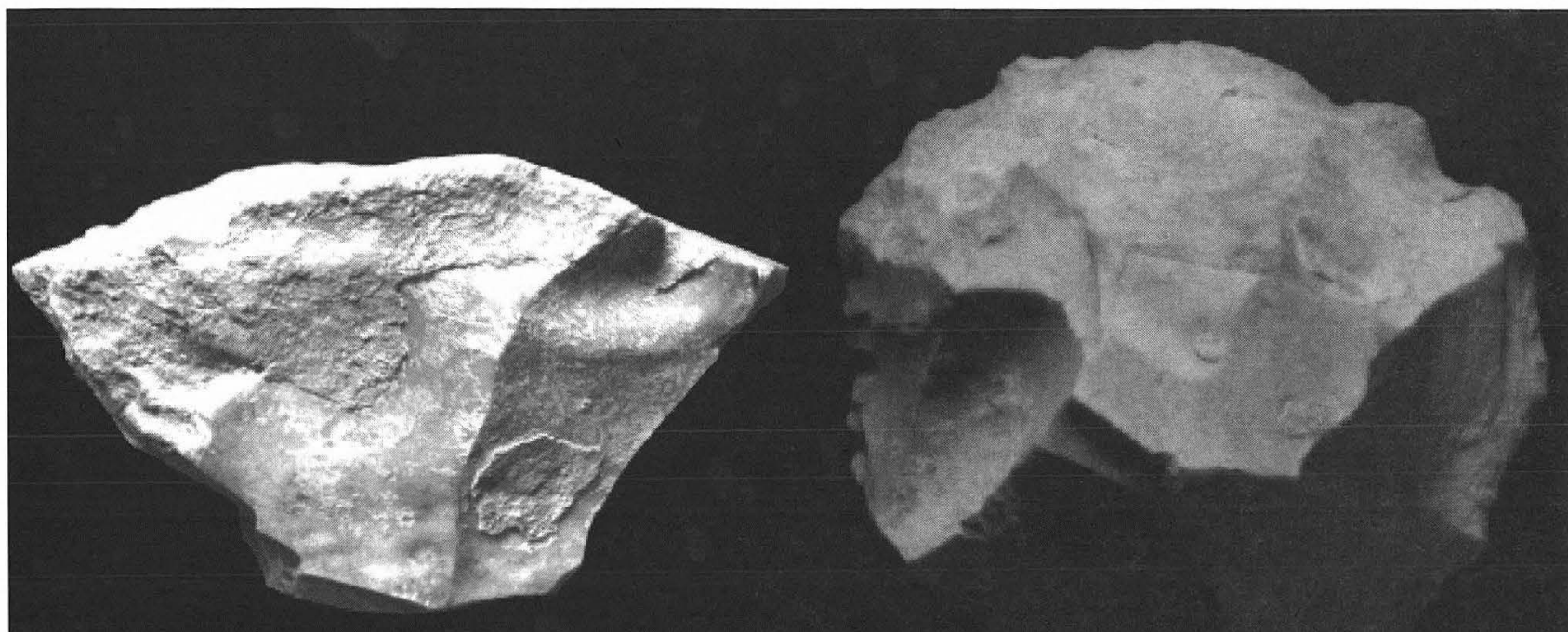

a
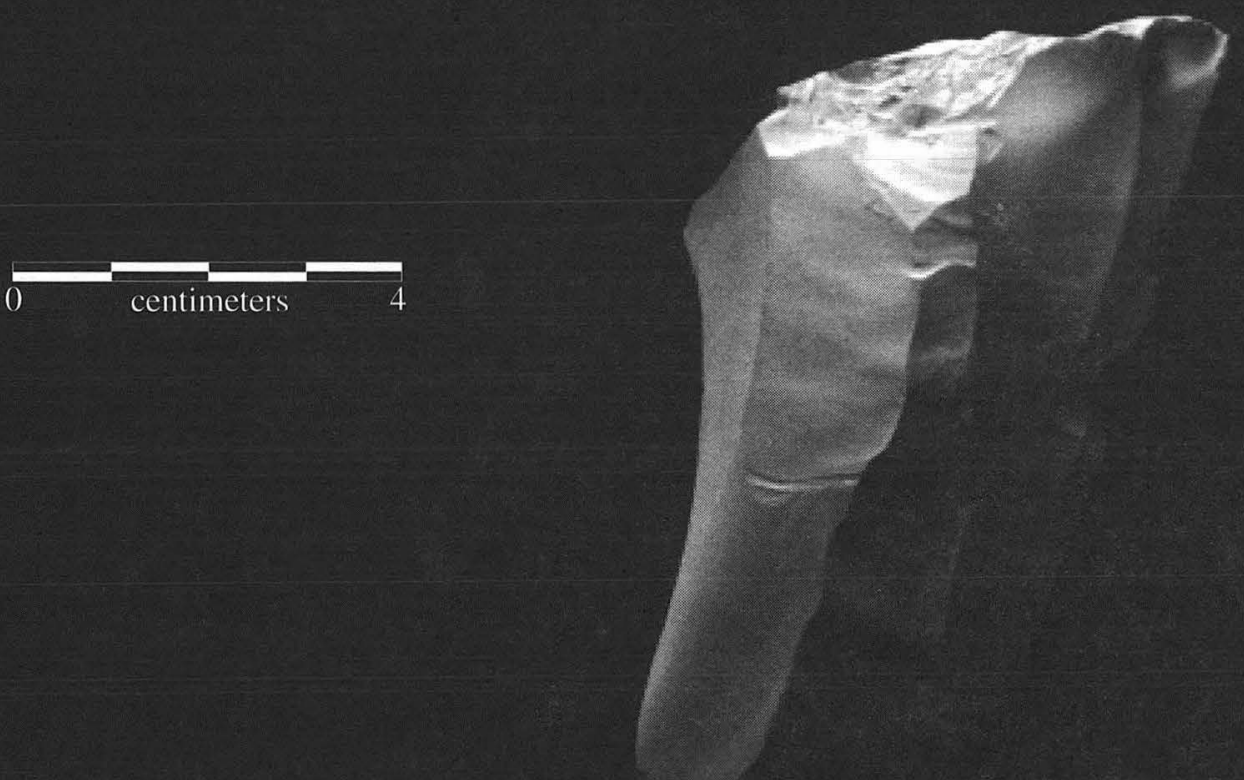

FIGURE 18-15. Unidirectional cores. (a) cylindrical core OR481 (two views); (b) blade core fragment 50KK1\&2A-1.

ing multiple flake scars of less than $40 \mathrm{~mm}$ in length, clearly indicating the intentional production of small flakes (Figures 18-16 and 18-17). Most of these cores appear to have been reduced by direct percussion and are multidirectional with multiple platforms. The scars do not generally reach microblade proportions.

In some cases, these microcores may be depleted nuclei of larger cores discarded when small size prevented further flaking or because the platforms or core faces became inadequate. Some, however, clearly were made on small pebbles. Ranging in shape from rounded to wedge-like to near planoconvex, they are classified into three subgroups based on length of flake scars. These are large (30-40-mm flake scars); intermediate (20-30-mm flake scars); and small (10-20-mm flake scars).

\section{LARGE $(\mathrm{N}=12)$}

In addition to displaying relatively large, ca. 30-40-mm flake scars, specimens in this group are larger overall than the other two categories (see Figures 18-16a and 18-17a, b; Table 18-15). Most (eight, or $66.7 \%$ ) are made of fine-grained cherts; four $(33.3 \%$ ) are of coarse-grained cherts. All but two retain cortex, although chiefly in small 

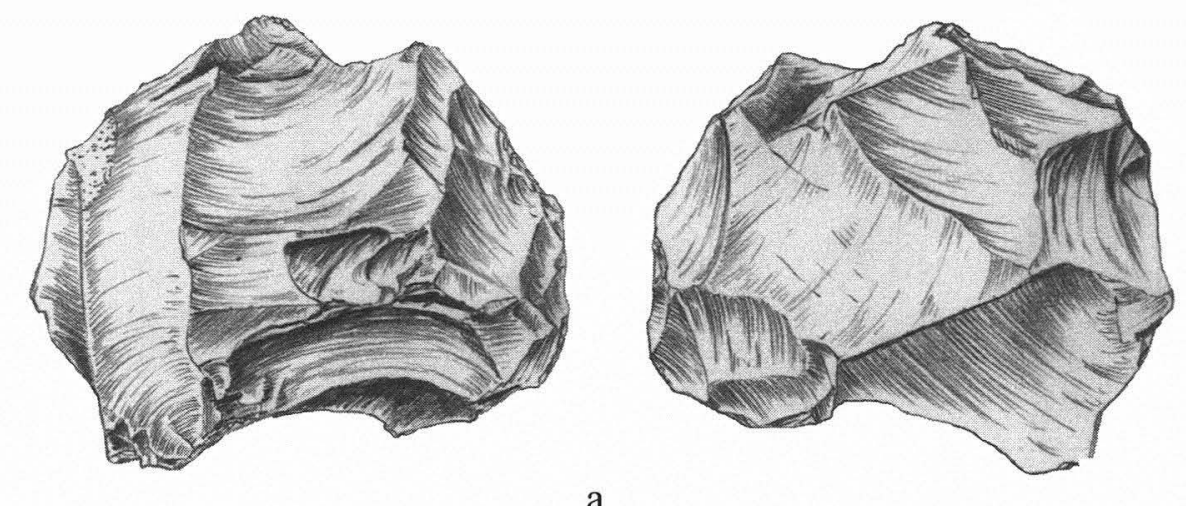

a

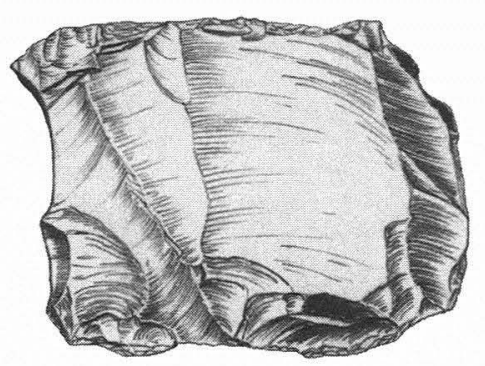

b
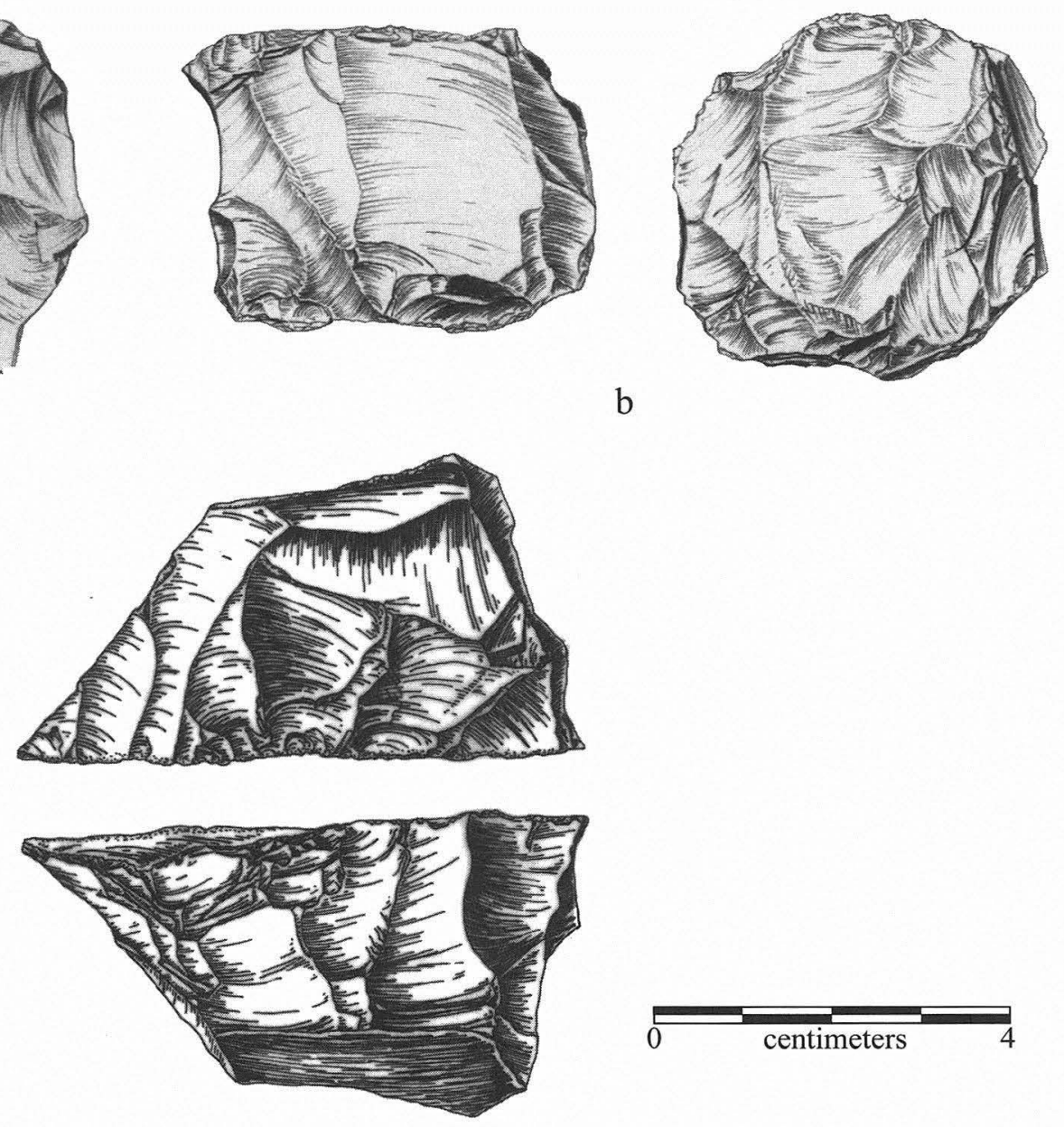

d

FIGURE 18-16. Representative microcores, showing varying flake scar sizes. (a) large flake scars (17U1-2); (b) intermediate size scars (21Q-5); (c, d) small flake scars (c, 12P-11; d, 37CB-15) 


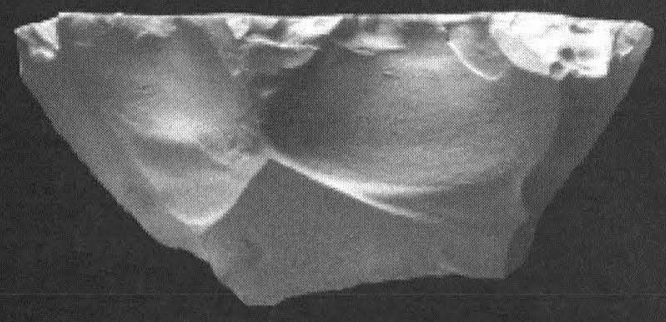

a

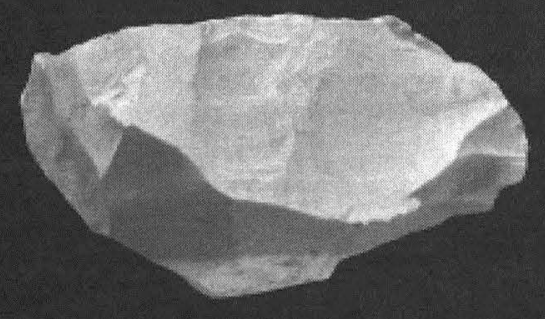

c

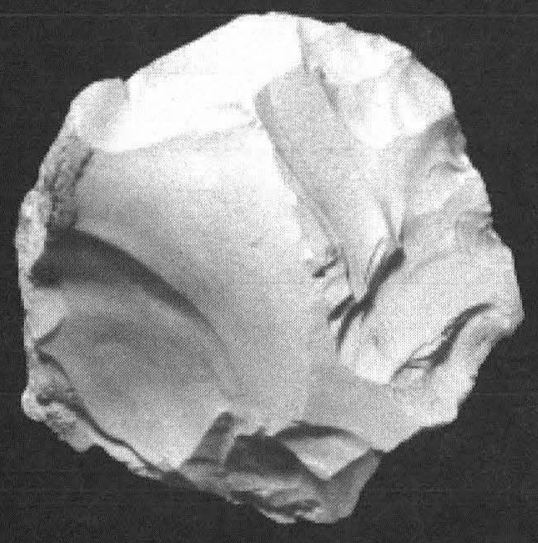

e

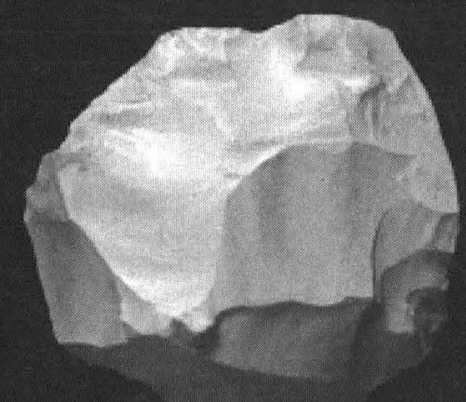

g

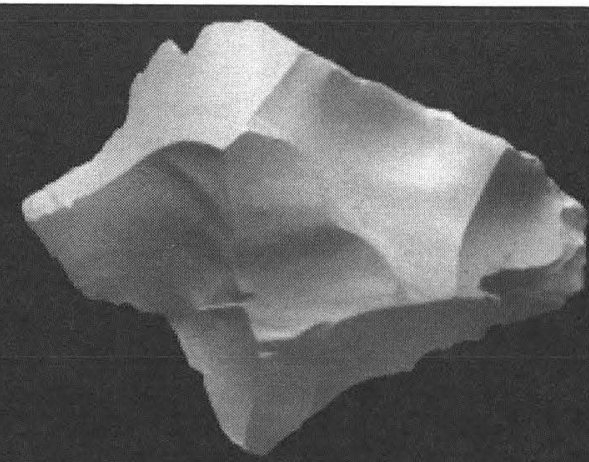

b
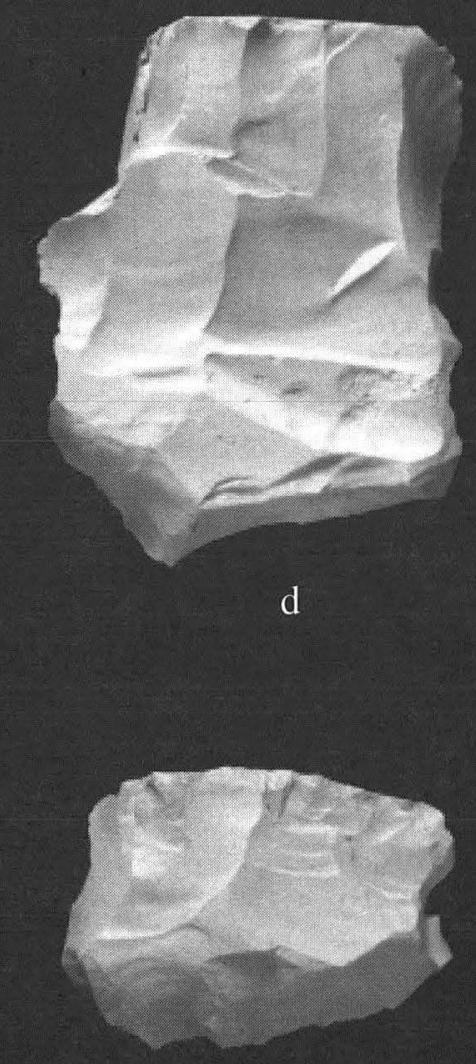

f

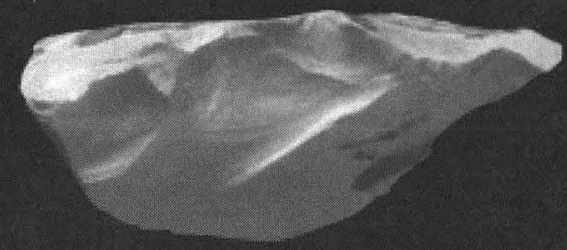

h

FIGURE 18-17. Representative microcores. (a) 30DD1A-2; (b) 15Z1-1; (c) 32O-12; (d) 17C-11; (e) 1429-1; (f) 9C-25; (g) 25S1-5; (h) 18J5. 
amounts (less than a quarter of the surface), and of these, most evidence upland origins (five, or 50.0\%), with a lesser number bearing nodular remnants (three, or $30.0 \%$ ) or stream modification (two, or $20.0 \%$ ). Small amounts of mineral precipitates are present on three $(25.0 \%)$, and patina is present on one $(8.3 \%)$.

Within this group, one specimen from a very early context (28LL1A-1; Unit Isi) is notable for what appears to be a small patch of incised nodular cortex (Figure 18-18). Of dark gray fine-grained chert, it is a split pebble fragment with a large bulb of percussion and multiple step and hinge flakes where attempts at further flaking failed. Its dimensions are $61 \mathrm{~mm}$ in length; $49 \mathrm{~mm}$ in width; and $31 \mathrm{~mm}$ in thickness; and it weighs $84 \mathrm{~g}$. Although incising of nodular cortex has been observed in other tool categories (see Chapter 17), reasons for this modification are unknown.

\section{INTERMEDIATE $(\mathrm{N}=21)$}

This subgroup includes microcores with both smalland medium-sized flake scars (20-33 mm in length), as exemplified in Figures 18-16b and 18-17c-e). See Table 18-15 for mean dimensions. The large majority (18 of 21 or $85.7 \%$ ) are of fine-grained local gray cherts, with the 2 being coarse-grained and 1 indeterminate. Cortex, present on 15 $(71.4 \%)$, is chiefly in small patches and predominately beto-

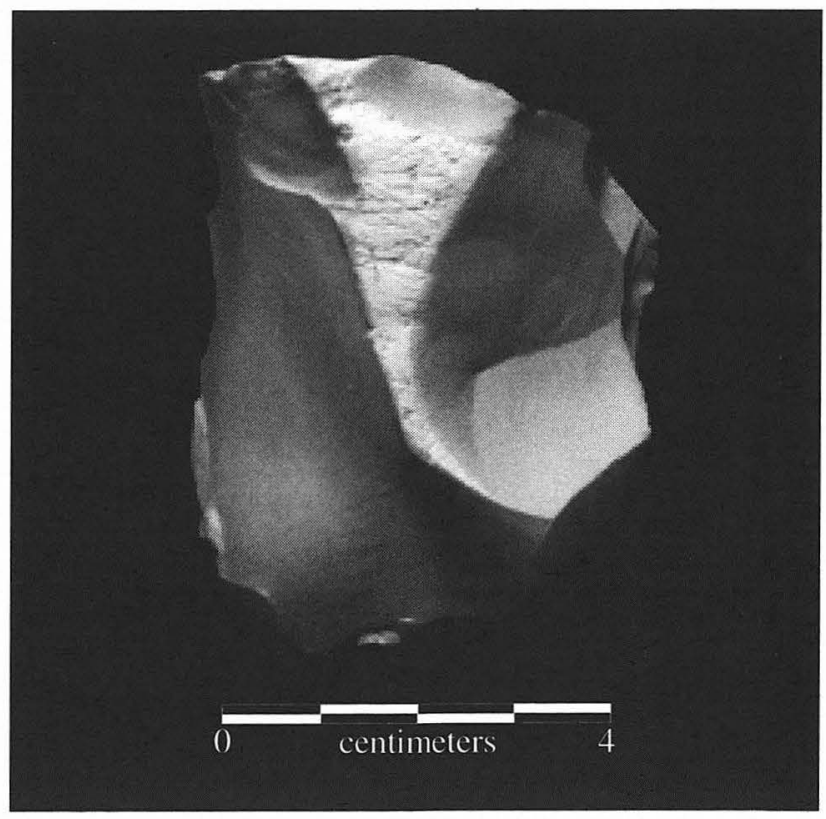

FIGURE 18-18. Large flake microcore 28LL1A-1 showing incised nodular cortex.

kens upland weathering (7 or 46.7\%); 4 (26.7\%) others display nodular cortex, and 4 (26.7\%) are indeterminate. Mineral precipitates are present in small amounts on 4 (19.0\%) and are heavy on 1 (4.8\%). Patina is evident on 5 (23.8\%).

TABLE $18-15$

Metric Attributes of Microcores

\begin{tabular}{|c|c|c|c|c|}
\hline & Length (mm) & Width (mm) & Thickness (mm) & Weight (g) \\
\hline \multicolumn{5}{|l|}{ Large: } \\
\hline Mean & 61.8 & 46.9 & 25.8 & 74.8 \\
\hline Standard deviation & 7.6 & 6 & 7.2 & 33.2 \\
\hline Range & 25 & 17 & 24 & 112 \\
\hline Minimum & 49 & 38 & 16 & 45 \\
\hline Maximum & 74 & 55 & 40 & 157 \\
\hline Count & 12 & 12 & 12 & 12 \\
\hline \multicolumn{5}{|l|}{ Intermediate: } \\
\hline Mean & 49.1 & 41.7 & 26.3 & 58.8 \\
\hline Standard deviation & 7.7 & 7.7 & 10.3 & 29.6 \\
\hline Range & 25 & 32 & 37 & 94 \\
\hline Minimum & 35 & 29 & 12 & 24 \\
\hline Maximum & 60 & 61 & 49 & 118 \\
\hline Count & 21 & 21 & 21 & 21 \\
\hline \multicolumn{5}{|l|}{ Small: } \\
\hline Mean & 47.4 & 34.2 & 22.6 & 38.1 \\
\hline Standard deviation & 8.1 & 6 & 6.8 & 17.6 \\
\hline Range & 27 & 20 & 20 & 73 \\
\hline Minimum & 37 & 24 & 13 & 15 \\
\hline Maximum & 64 & 44 & 33 & 88 \\
\hline Count & 17 & 17 & 17 & 17 \\
\hline
\end{tabular}


Although these specimens exhibit a range of flake scar sizes, it is clear that many were made on small pebbles and were not reduced from larger cores. This is well-illustrated in Figure 18-19, on which cortex remnants on one face and tabular end delimit the original size of the parent pebble. That these cores were reused and recycled is also apparent. On one specimen (see Figure 18-16b) the patinated surface has been partially flaked off, indicating use on at least two temporally distant occasions. Similar differential patination is visible on 17C-1.1 (see Figure 18-17d). Small patches of battering on the ridge scars of the pebble core 22O-9 (see Figure 18-19) suggest that some exhausted microcores were recycled as hammerstones, similar to those described above (see Battered Stones).

\section{$\operatorname{SMALL}(\mathrm{N}=17)$}

Small microcores vary from rounded with generally multidirectional, small flake scars to markedly planoconvex with more unidirectional flake removals (as illustrated in Figures 18-16c, d, and 18-17f-h). Dimensions for this group are generally diminutive (see Table 18-15). Flake scars generally range from 10 to $20 \mathrm{~mm}$ in length. With the exception of one indeterminate specimen, all small microcores are of fine-grained cherts. A little over one-half (58.8\%) retain small cortex patches, and these primarily indicate upland weathering ( 6 of 10 , or $60.0 \%$ ), with a few showing nodular remnants (3, or $30.0 \%)$, and 1 indeterminate. Mineral precipitates are present on $7(41.2 \%)$, and patina is displayed on $1(5.9 \%)$.

\section{Core Fragments $(N=71)$}

Specimens in this category are broken sections of cores with evidence of flake removal (Figure 18-20a). As shown in Table 18-16, core fragments predominately fall in the intermediate size category $(46-75 \mathrm{~mm})$. Fine-grained cherts dominate in this group with $52(73.2 \%)$, followed by 19 (26.8\%) coarse-grained chert specimens. Cortex, present on 56 specimens $(78.9 \%)$, indicates upland weathering on $31(55.4 \%)$. Cortex on $12(21.4 \%)$ is nodular, on $4(7.1 \%)$ is stream modified, and on $9(16.1 \%)$ is indeterminate. Patina is present on $23(32.4 \%)$ and mineral precipitates on 27 $(38.0 \%)$.

\section{Chunks and Indeterminate Fragments $(N=23)$}

Best characterized as indeterminate "chunks," specimens in this category are generally small and angular. They may be fragments of cores, tested pebbles, or crude bifaces, but generally they are too small or indistinct to categorize further. Predominate size range is small (less than $45 \mathrm{~mm}$ ) (see Table 18-16). The large majority of chunks are of fine-grained chert ( 20 of 23 , or $87.0 \%$ ), with the remainder being common coarse-grained chert. Almost all cherts are deemed to be of local origin ( 20 of 23 , or $87.0 \%$ ), and based on cortex (present on 17 , or $73.9 \%$ ), most appear to derive from upland settings ( 10 of 17 , or $58.8 \%$ ), with a smaller number evidencing stream modification (5 of 17 , or $29.4 \%$ ), and 4 indeterminate. Mineral precipitates were observed on $9(39.1 \%)$ and patina on $6(26.1 \%)$.

\section{Thermally/Cryogenically Altered Fragments $(N=89)$}

Fragments in this category appear to have been thermally or cryogenically altered, variously displaying potlidding, crazing, and/or pockmarks (Figure 18-20b). Although cryogenic alterations occurred as a result of natural processes, thermal alterations may have occurred through human means (e.g., as a result of discard into a hearth area) or by natural processes (e.g., grass fire). In either case, resultant color and texture changes have served to hinder material classification on many. A total of 51 specimens (57.3\%) are of fine-grained cherts; 23 (25.8\%) are of coarse

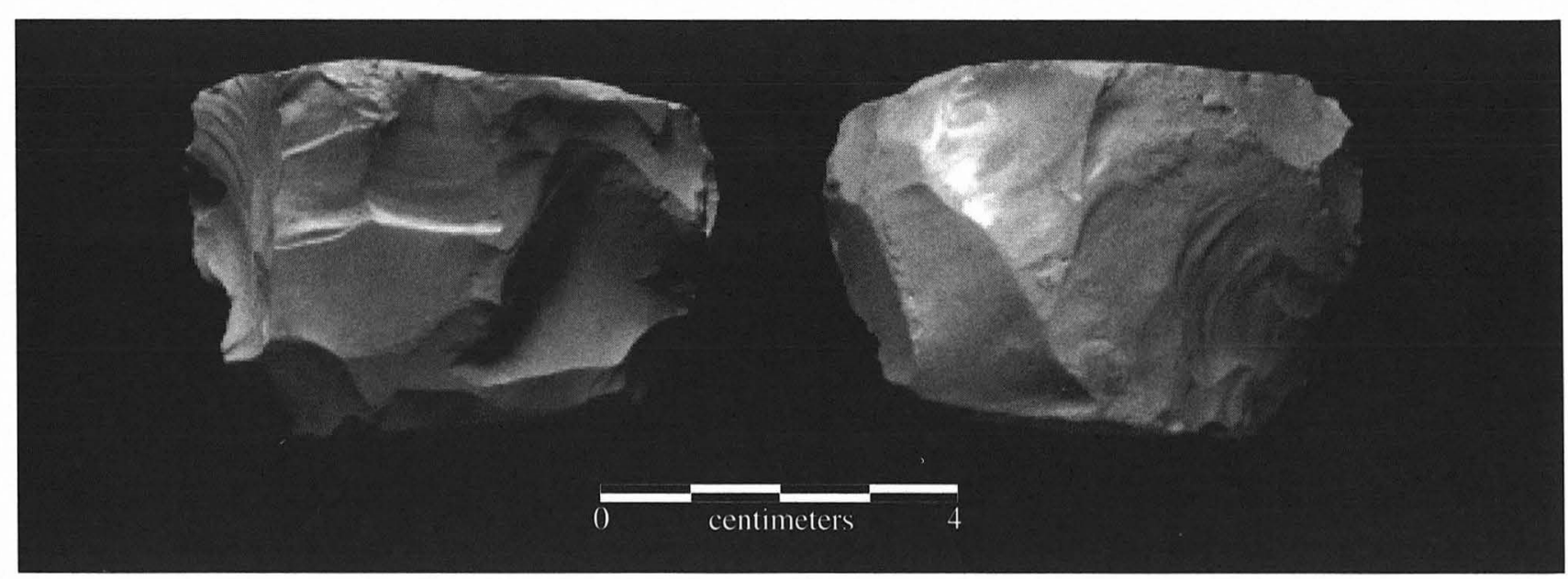

FIGURE 18-19. Microcore 22O-9 with intermediate (medium and small) flake scars made on a small pebble and likely used as hammerstone (note light battering on ridges). 


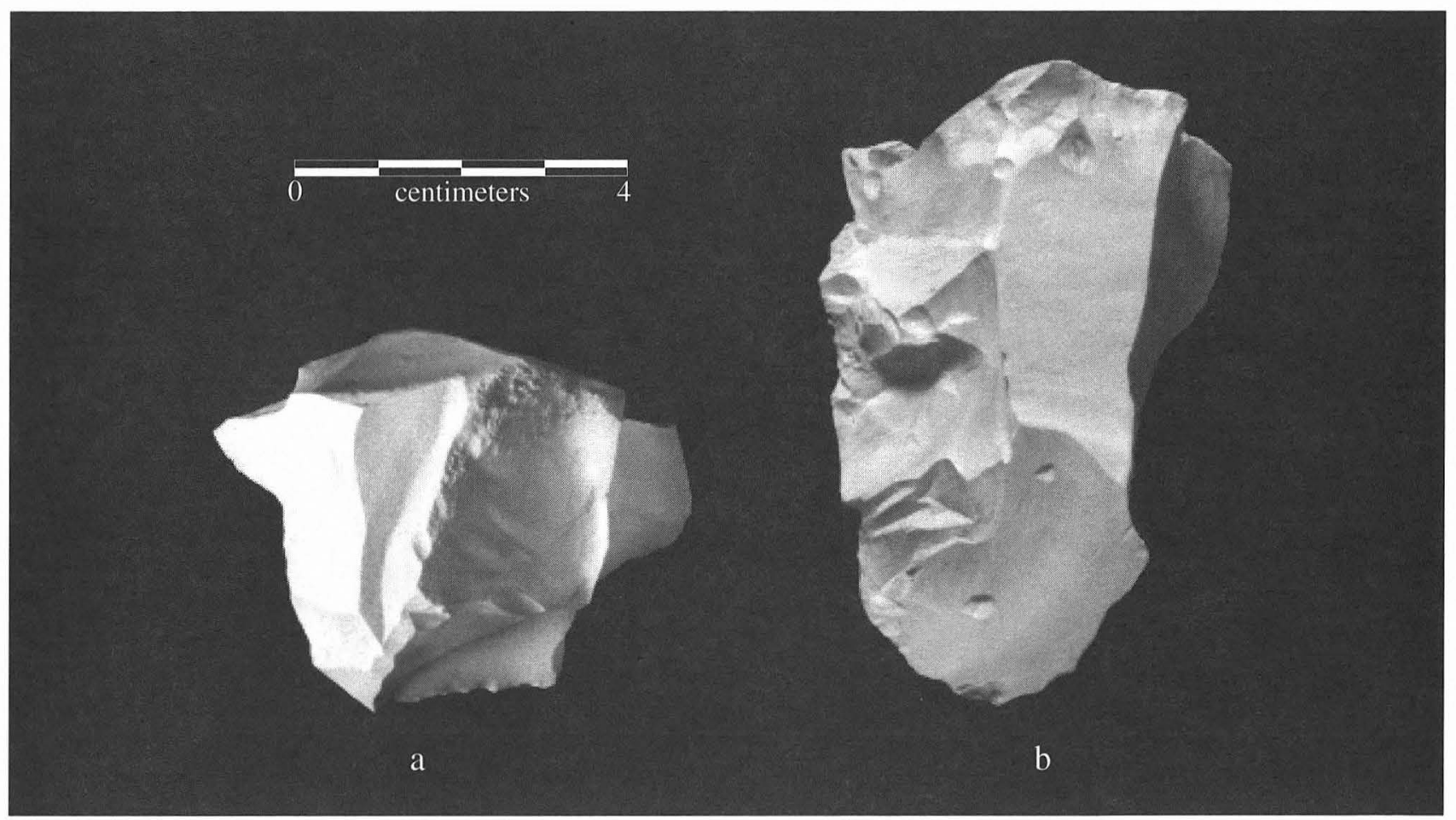

FIGURE 18-20. Examples of core fragments. (a) miscellaneous fragment (24AA2B-2); (b) thermally altered fragment (28DD2D-1).

TABLE 18-16

Size Attributes of Core Fragments, Chunks, and Unmodified and Tested Material

\begin{tabular}{|c|c|c|c|c|c|c|}
\hline & \multicolumn{3}{|c|}{ Size Range (mm) } & \multicolumn{3}{|c|}{ Weight $(\mathrm{g})$} \\
\hline Group & Small & Medium & Large & Mean & Range & $\begin{array}{l}\text { Standard } \\
\text { Deviation }\end{array}$ \\
\hline Core fragments & 20 & 32 & 19 & 78.9 & 10 to 260 & 56.4 \\
\hline Indeterminate/chunks & 14 & 8 & 1 & 39.6 & 6 to 239 & 52.2 \\
\hline $\begin{array}{l}\text { Thermal/cryogenically altered } \\
\text { fragments }\end{array}$ & 42 & 34 & 13 & 61.8 & 1 to 587 & 90 \\
\hline $\begin{array}{l}\text { Unmodified and tested pebbles } \\
\text { and cobbles }\end{array}$ & 0 & 3 & 18 & 324.9 & 81 to 1123 & 276.9 \\
\hline
\end{tabular}

cherts; and $14(15.7 \%)$ are indeterminate. Among the total, the classification of $11(12.4 \%)$ as non-Edwards cherts may be incorrect, inasmuch as heat alteration may have caused "false negatives" when specimens were scanned with ultraviolet light for fluorescent properties. Cortex was observed on well over half of the specimens ( 51 of 89 , or $57.3 \%$ ) but is not characterized further due to thermal changes on some. A total of $33(37.1 \%)$ shows mineral precipitates, and 9 (10.1\%) display patina. As shown in Table 18-16, most specimens tend to fall in the small size range (less than $45 \mathrm{~mm}$ ).

\section{Tested and Unmodified Pebbles and Cobbles $(N=21)$}

Specimens in this category represent raw materials likely procured as potential cores. Many of the tested pieces can be characterized as "opportunistic cores" from which sev- eral usable flakes were struck, following no specific sequence (Frison and Bradley 1980:22), whereas others were minimally flaked to determine the quality of the material. Unmodified materials in this category include rounded stream pebbles and cobbles as well as tabular cherts and other raw materials of sufficient size to have had potential as cores. Most (18, or $85.7 \%$ ) fall in the large size range $(>75 \mathrm{~mm})$, with the remaining 3 observed ranging from 46 to $75 \mathrm{~mm}$ (see Table 18-16).

Fine- and coarse-grained cherts are evenly represented, with nine each for a total of $85.7 \%$. Two others appear to be non-Edwards. Of these, one (9F-3), resembles Jasper (C. Gene Mear, personal communication 1995). The other is a coarse-grained, indeterminate dark gray material. All specimens are corticate, $10(47.6 \%)$ evidencing upland weathering; 7 (33.3\%) are stream modified; 2 (9.5\%) nodular; and 2 indeterminate. A large number (17 of 21 or $81.0 \%$ ) exhibit 
mineral precipitates, and on 6 , deposits are heavy. There are no specimens with patina in this group.

One specimen (Figure 18-21) is interesting both for its early context (Unit Isi/Icl, attributed to Early Paleoindian times) as well as the technological evidence it bears. An oblong tabular cobble of waxy, reddish brown banded chert, this specimen appears to be a failed blade core. A single, large blade-like flake has been removed from one tabular facet, and thick enamel-like cortex covers the remainder of the specimen. Heavy battering, present on both long ends, may have been related to platform preparation, or the piece may have been used at some point as a hammerstone. It measures $121 \mathrm{~mm}$ in length; $68 \mathrm{~mm}$ in width; and $52 \mathrm{~mm}$ in thickness; and it weighs $526 \mathrm{~g}$.

\section{Summary and Interpretations: Variation Over Time}

With a few notable exceptions, a largely unpatterned core technology is evinced throughout much of the site's history (Table 18-17). Complete, multidirectional, amorphous cores are present in very low frequencies in all major stratigraphic units. Peak proportions, as relative percentages of the core collection within stratigraphic units, are seen in Unit IIIa (4 specimens; $8.9 \%$ of assemblage) and IIIb (3 specimens, $13.3 \%$ of the assemblage).

More-formalized core technology is suggested only in a few specimens. Evidence of blade removal was observed on two specimens from Early Paleoindian deposits. One is a possible blade core fragment, the other a tested and battered tabular chert cobble. From a much later context, an unusual tabular quartz core bearing both multidirectional and blade-like flake scars was recovered in a possible Late Archaic biface cache.

In addition to the possible blade core fragment, there are three specimens with some attributes of blade removal; all are from deep in the site. These cannot definitely be classified as parts of blade cores, and each is placed in another category elsewhere in these descriptive chapters, but mention is made of them here. When viewed in light of the small number of blades and blade fragments also documented at the site, the presence of some blade core debris is expectable.

The first (37001A-1) is a large fragment of an apparent unidirectional core with prepared platform. This specimen shows subsequent face battering (see above). The fragment is too small to be identified with certainty, but its face with multiple elongate scars, its prepared platform, and its platform angle resemble those of Clovis-age, wedge-shaped cores (Collins 1996).

Another aberrant specimen (350O2C) possibly represents an unsuccessful attempt to remove a blade core tablet flake. This is a large flake (categorized as unmodified flaking debris) with a prominent bulb of percussion. Its exterior face is almost entirely a single, deep negative flake scar. Its perimeter is partly a thin edge, partly irregular flake scars, and partly, of particular interest, three flake scar remnants that resemble the proximal portion of a polyhedral blade core. One of these scars was the platform struck in the detachment of the flake being described; it and another scar are of flakes (blades?) struck from the concave flake scar that is now the exterior of the present flake. If, in fact, this were struck to remove the platform of a blade core, it detached in an orientation that would have produced an acute angle between the face of the core and the rejuvenated platform, rendering the core unusable.

Finally, a large uniface (16HH2D-3) described in Chapter 17 also has attributes that resemble those of a blade core. It is a large flake with two long, prominent scars on the exterior that suggest the face of a blade core. Modification of this flake has invaded both faces and removed any attributes that could definitively identify this as part of a blade core.

Blade core debris is highly distinctive and easily recognized for the most part, but, as with any knapping, some

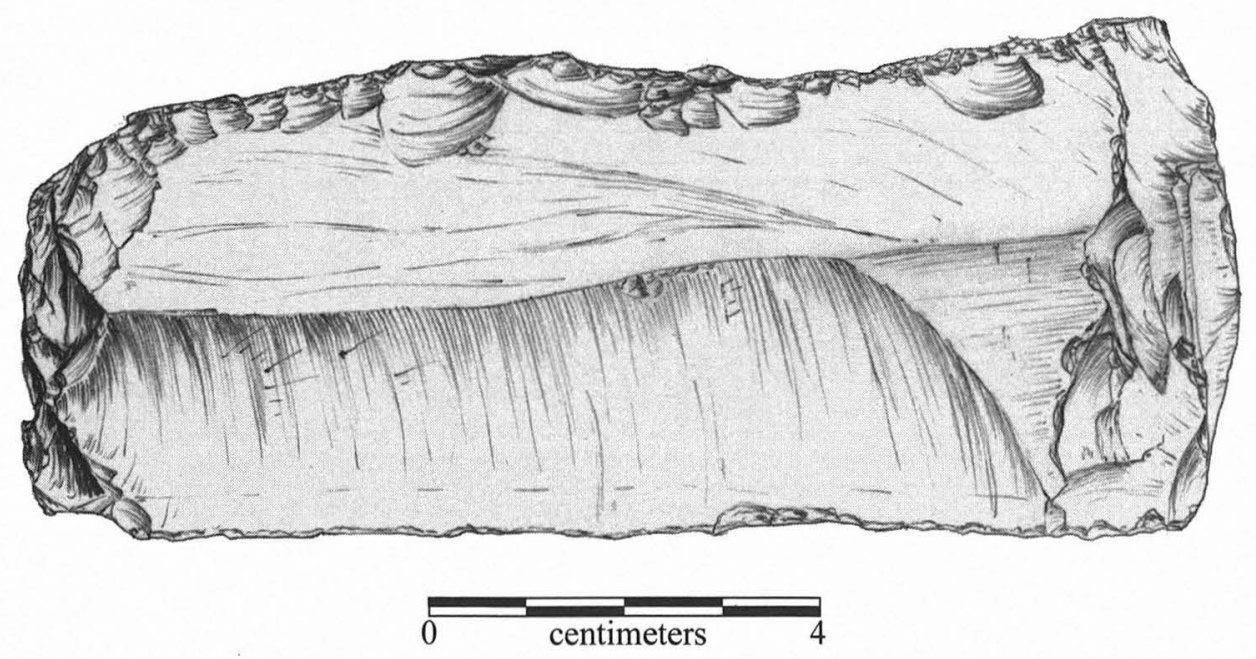

FIGURE 18-21. Tested cobble showing failed attempt at blade extraction (17JJ2KK1-1). 
TABLE 18-17

Core Assemblage by Group and Geologic Strata

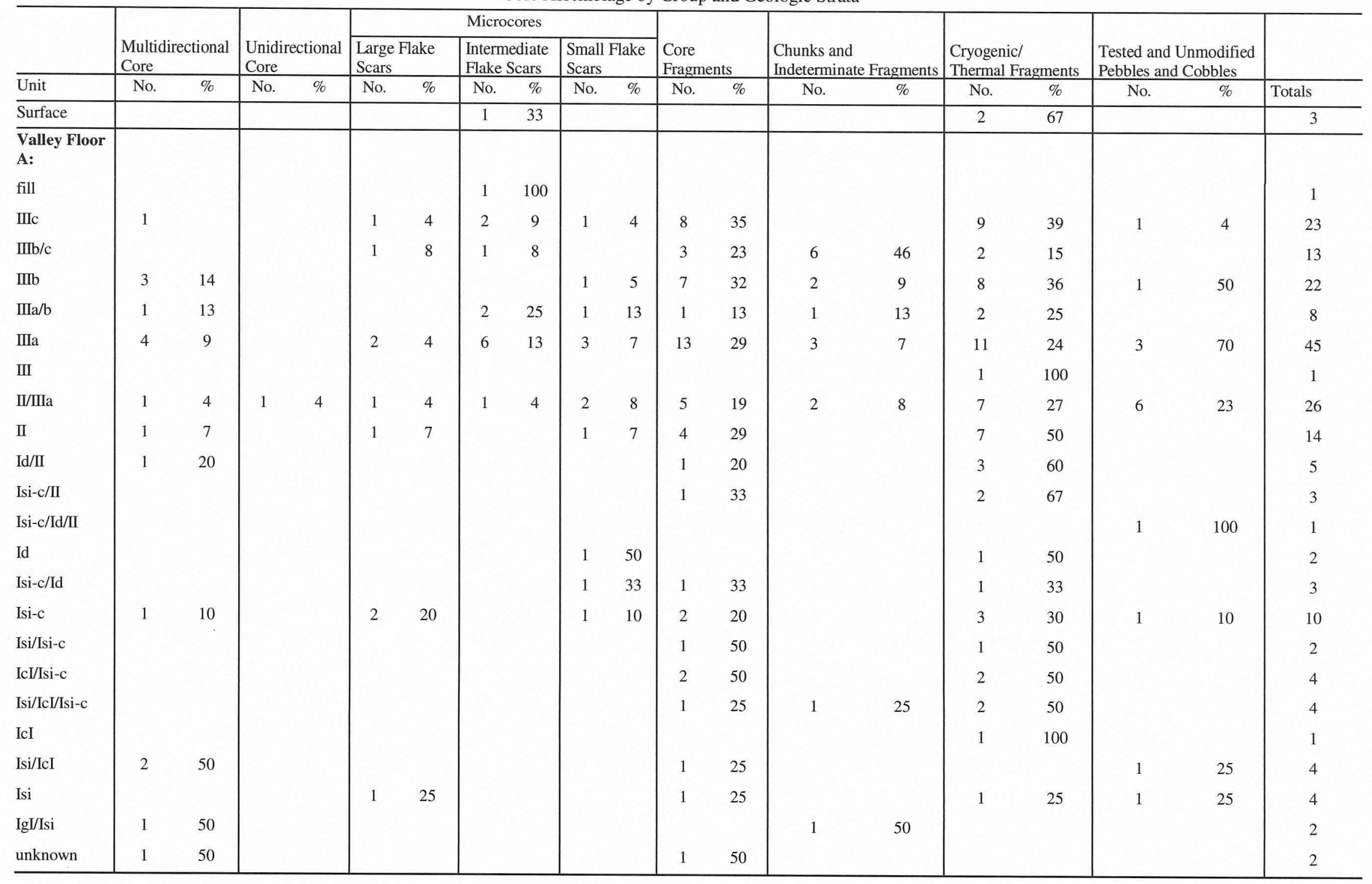


Table 18-17, (continued)

\begin{tabular}{|c|c|c|c|c|c|c|c|c|c|c|c|c|c|c|c|c|c|c|c|}
\hline \multirow[b]{3}{*}{ Unit } & \multirow{2}{*}{\multicolumn{2}{|c|}{$\begin{array}{l}\text { Multidirectional } \\
\text { Core }\end{array}$}} & \multirow{2}{*}{\multicolumn{2}{|c|}{$\begin{array}{l}\text { Unidirectional } \\
\text { Core }\end{array}$}} & \multicolumn{6}{|c|}{ Microcores } & \multirow{2}{*}{\multicolumn{2}{|c|}{\begin{tabular}{|l} 
Core \\
Fragments
\end{tabular}}} & \multirow{2}{*}{\multicolumn{2}{|c|}{$\begin{array}{l}\text { Chunks and } \\
\text { Indeterminate Fragments }\end{array}$}} & \multirow{2}{*}{\multicolumn{2}{|c|}{\begin{tabular}{|l} 
Cryogenic/ \\
Thermal Fragments
\end{tabular}}} & \multirow{2}{*}{\multicolumn{2}{|c|}{$\begin{array}{l}\text { Tested and Unmodified } \\
\text { Pebbles and Cobbles } \\
\end{array}$}} & \multirow{4}{*}{ Totals } \\
\hline & & & & & \multicolumn{2}{|c|}{\begin{tabular}{|l|} 
Large Flake \\
Scars \\
\end{tabular}} & \multicolumn{2}{|c|}{\begin{tabular}{|l|} 
Intermediate \\
Flake Scars
\end{tabular}} & \multicolumn{2}{|c|}{\begin{tabular}{|l} 
Small Flake \\
Scars
\end{tabular}} & & & & & & & & & \\
\hline & No. & $\%$ & No. & $\%$ & No. & $\%$ & No. & $\%$ & No. & $\%$ & No. & $\%$ & No. & $\%$ & No. & $\%$ & No. & $\%$ & \\
\hline \multicolumn{19}{|c|}{$\begin{array}{l}\text { Valley Floor } \\
\text { B: } \\
\end{array}$} & \\
\hline III & & & 1 & 50 & & & 1 & 9 & 1 & 9 & 2 & 18 & 1 & 50 & 6 & 55 & 1 & 9 & $\begin{array}{l}1 \\
2\end{array}$ \\
\hline \multicolumn{20}{|l|}{$\begin{array}{l}\text { Valley } \\
\text { Margin: }\end{array}$} \\
\hline$\overline{\text { IIIc }}$ & 3 & 19 & & & 2 & 13 & & & 2 & 13 & 1 & 6 & 2 & 13 & 3 & 19 & 3 & 19 & 16 \\
\hline IIIc POT & & & & & & & & & & & 1 & 50 & & & 1 & 50 & & & 2 \\
\hline $\mathrm{IIIb} / \mathrm{c}$ & 1 & 33 & & & & & & & & & 2 & 67 & & & & & & & 3 \\
\hline Y/IIIc & & & & & & & & & 1 & 25 & 1 & 25 & & & 2 & 50 & & & 4 \\
\hline $\mathrm{Y} / \mathrm{IIl} \mathrm{b} / \mathrm{c}$ & & & & & & & & & & & & & & & 1 & 100 & & & 1 \\
\hline IIIb & & & & & & & & & & & 1 & 50 & & & 1 & 50 & & & 2 \\
\hline Y & & & & & & & 1 & 25 & & & & & 2 & 50 & & & 1 & 25 & 4 \\
\hline $\mathrm{X} / \mathrm{Y}$ & & & & & & & & & & & 1 & 50 & & & 1 & 50 & & & 2 \\
\hline III $/ \mathrm{b}$ & & & & & & & & & & & 1 & 50 & 1 & 50 & & & & & 2 \\
\hline X/IIIa/b & & & & & & & 1 & 100 & & & & & & & & & & & 1 \\
\hline IIIa & & & & & & & 1 & 100 & & & & & & & & & & & 1 \\
\hline IIIIIIa & & & & & & & 1 & 100 & & & & & & & & & & & 1 \\
\hline X/II/IIIa & 1 & 50 & & & & & & & & & 1 & 50 & & & & & & & 2 \\
\hline II & 1 & 25 & & & & & & & & & 1 & 25 & & & 2 & 50 & & & 4 \\
\hline $\mathrm{X}$ & & & & & 1 & 7 & 1 & 7 & 1 & 7 & 5 & 33 & 1 & 7 & 6 & 40 & & & 15 \\
\hline $\mathrm{X} / \mathrm{II}$ & 2 & 50 & & & & & 1 & 25 & & & 1 & 25 & & & & & & & 4 \\
\hline $\mathrm{X} / \mathrm{Isi}$ & & & & & & & & & & & & & & & & & 1 & 100 & 1 \\
\hline Totals: & 25 & 9 & 2 & 1 & 12 & 4 & 21 & 7 & 17 & 6 & 71 & 25 & 23 & 8 & 89 & 32 & 21 & 8 & 281 \\
\hline
\end{tabular}


of the pieces produced are less definitive. These three pieces cannot be used alone to argue for blade production at this site, but neither should they be totally disregarded when evaluating the few blades and blade fragments present.

Microcores make up 18\% of the total core assemblage. They have multiple platforms and multidirectional flake scar attributes indicative of direct percussion with a comparatively hard percussor. They occur predominately in Early Archaic contexts (Unit IIIa, $\mathrm{n}=11$; Unit III $\mathrm{a} / \mathrm{b}$ transition zone, $n=3$ ). The occurrence of microlithic tools with traces of use wear in conjunction with these microcores is evidence that some form of composite tools were being made. This interpretation is discussed further in Chapter 17. It appears, too, that some small cores of this type later were used as hammerstones, based on the markedly similar suite of attributes seen in both tool forms, that is, size, shape, and generally fine-grained raw material marked by varying degrees of battering.

Core fragments and chunks account for the largest proportion of the core assemblage by stratigraphic unit. Thermally or cryogenically altered fragments, although distributed in small amounts throughout (see Table 18-17), show high frequency in Unit II (7 or 50.0\% of the core assemblage for that unit in the Valley Floor, as well as 2 or $50.0 \%$ in Valley Margin).

Within the full assemblage, fine-grained Edwards cherts clearly predominate (219 or $77.9 \%$ ) (Tables 18-18, 18-19). Of those, the large majority are identified as local cherts. However, significant variance from this pattern can be seen within specific core categories and according to stratigraphic provenience in the site. A large proportion of "complete" cores were made of coarse-grained cherts, in contrast to the predominately fine-grained exhausted microcores. This difference may be due chiefly to the poorer flaking quality of the coarse-grained cherts which may have hindered degree of reduction. When viewed over time and in stratigraphic units with a sample of sufficient size, a marked increase in frequency of total coarse cherts is seen in contexts related to Early Archaic and possibly Middle Archaic times; these include Valley Floor Units IIIa $(n=16,34.8 \%)$, and IIIb $(n=9,40.9 \%)$. This pattern is of interest, also, when considered with the large numbers of coarse chert Clear Fork tools discarded at the site during roughly the same time periods (see Chapter 15), although the implications of such a correlation are unclear. The coarse chert core materials described here are not of sufficient size to produce Clear Fork tools.

The few, relatively large (i.e., $>300$ g) chert cores and fragments of fine-grained cherts are chiefly present in Paleoindian contexts (see Table 18-19). Although raw material groups have been collapsed into larger categories for lithic studies in this volume, it is worth mentioning several within the fine-grained group that show particular patterning. Within Unit I are almost all specimens of the fine-grained black cherts ( 7 of 10 , or $70.0 \%$ ). Of exceptional quality, these glossy cherts are believed to derive from sources not far to the northeast, near Georgetown (see Chapter 19). The other three specimens are from predominately Early Archaic contexts (the Unit II/IIIa transition zone, IIIa, and IIIa/b). Another fine-grained material predominately limited to early contexts is a fine-grained waxy greenish gray-brown chert. A total of 11 of the 16 specimens $(68.8 \%)$ of this material was recovered in Unit I, with 3 others in Late Paleoindian and Early Archaic contexts and 2 in higher Archaic deposits. Although cherts of this sort have been found in Paleoindian deposits at nearby sites, it may be that material identification has been skewed by groundwater staining which may have caused the greenish coloration (see Chapter 19 for a more complete discussion of these cherts). Seeing a similar pattern in the debitage sample from the site, Masson (see Chapter 19) has noted that the temporally limited exploitation of these distinctive fine cherts maybe due either to greater mobility of the occupants during Paleoindian times or better accessibility of the cherts during early times.

Of interest also in relation to raw material acquisition strategies are probable procurement locales, suggested by cortex "type." Based on this factor, upland cherts appear to have been exploited throughout much of the site's history ( $n=97$, or $34.5 \%$ of the total core assemblage), followed by nodular cherts ( $\mathrm{n}=45$, or $16.0 \%$ of total assemblage), and stream-altered materials ( $\mathrm{n}=23$, or $8.2 \%$ of total assemblage). Significantly high proportions were taken from upland areas during late occupations at the site (Valley Floor Unit IIIc: $n=12$, or $52.2 \%$; Valley Margin IIIc: $n=7$, or $43.8 \%$ ) (see Table 18-18).

These indications of the chert sources being exploited by knappers at the Wilson-Leonard locality are consistent with the occurrence of chert in the area. Chert is virtually absent in the gravels of Brushy Creek upstream from the mouth of South Brushy Creek. The upland surface east, northwest, and southwest of the site at distances mostly $1.5 \mathrm{~km}$ or less is capped by chert-bearing Edwards limestone. At a few points of active erosion, nodular Edwards chert could have been accessible, but the most abundant occurrences are residual cherts in the thin upland soils.

Mineral precipitates are present in significantly high frequencies (e.g., $48 \%$ or greater) in almost all lower stratigraphic units, beginning with the Unit II/IIIa transition zone. This pattern is roughly repeated in the Valley Margin units, although somewhat larger percentages also are seen in higher units.

Patina is sparsely present within the assemblage as a whole ( $\mathrm{n}=45$ or $16.4 \%$ ). From a depositional perspective, it is perhaps important to note the somewhat higher incidence of patina among specimens in the major (i.e., nontransitional) stratigraphic units, including Unit IIIb $(n=5$, or $23.8 \%)$; Unit IIIa $(\mathrm{n}=7$, or $15.5 \%)$; and Unit II $(\mathrm{n}=4$, or $28.6 \%)$. 
TABLE 18-18

Core Assemblage Raw Material Characteristics by Geologic Strata

\begin{tabular}{|c|c|c|c|c|c|c|c|c|c|c|c|c|c|c|c|c|c|}
\hline \multirow[b]{2}{*}{ Unit } & \multicolumn{8}{|c|}{ Raw Material } & \multicolumn{5}{|c|}{ Cortex Type } & \multicolumn{2}{|c|}{ Patina } & \multicolumn{2}{|c|}{$\begin{array}{c}\text { Mineral } \\
\text { Precipitates }\end{array}$} \\
\hline & $\begin{array}{l}\text { Local } \\
\text { Edwards } \\
\text { Fine } \\
\text { Chert }\end{array}$ & \begin{tabular}{|l|} 
Local \\
Edwards \\
Medium/ \\
Coarse \\
Chert
\end{tabular} & $\begin{array}{l}\text { Nonlocal } \\
\text { Edwards } \\
\text { Fine } \\
\text { Chert }\end{array}$ & \begin{tabular}{|l|} 
Nonlocal \\
Edwards \\
Medium/ \\
Coarse \\
Chert \\
\end{tabular} & $\begin{array}{l}\text { Indeterminate } \\
\text { Non-Edwards } \\
\text { Fine Chert }\end{array}$ & $\begin{array}{l}\text { Indeter- } \\
\text { minate Non- } \\
\text { Edwards } \\
\text { Medium/ } \\
\text { Coarse Chert }\end{array}$ & Quartz & $\begin{array}{l}\text { Indeter- } \\
\text { minate } \\
\text { Heat } \\
\text { Altered }\end{array}$ & Absent & $\begin{array}{l}\text { Upland } \\
\text { Residual }\end{array}$ & Nodular & $\begin{array}{l}\text { Stream } \\
\text { Modified }\end{array}$ & $\begin{array}{l}\text { Indeter- } \\
\text { minate }\end{array}$ & Absent & Present & Absent & Present \\
\hline Surface & 5 & & & & & & & & 1 & & & & 2 & 2 & 1 & 1 & 2 \\
\hline \multicolumn{18}{|c|}{ Valley Floor A } \\
\hline fill & 1 & & & & & & & & 1 & & & & & & 1 & 1 & \\
\hline IIIc & 18 & 3 & & 1 & & & & 1 & 6 & 12 & 3 & & 2 & 19 & 4 & 23 & \\
\hline $\mathrm{IIIb} / \mathrm{c}$ & 11 & 2 & & & & & & & 3 & 4 & 4 & 1 & 1 & 13 & & 12 & 1 \\
\hline IIIb & 11 & 7 & & 2 & 1 & 1 & & & 9 & 7 & 2 & 3 & 1 & 17 & 5 & 20 & 2 \\
\hline IIIa/b & 3 & 3 & & 1 & & & & & 1 & 3 & 1 & 1 & 2 & 8 & & 8 & \\
\hline ШІа & 25 & 15 & 3 & 1 & & 2 & & & 13 & 15 & 6 & 5 & 6 & 38 & 7 & 33 & 13 \\
\hline III & 1 & & & & & & & & 1 & & & & & 1 & & 1 & \\
\hline ш/யа & 18 & 5 & 1 & 1 & & 2 & & & 3 & 13 & 3 & 5 & 1 & 22 & 3 & 13 & 12 \\
\hline II & 6 & 1 & 2 & & & 2 & & & 7 & 3 & 1 & & 3 & 10 & 4 & 5 & 9 \\
\hline $\mathrm{Id} / \mathrm{II}$ & 2 & 1 & & 1 & & & & 1 & 3 & 1 & 2 & & & 5 & & & 5 \\
\hline Isi(c)/II & 2 & & & & & & & & 1 & & 1 & & 1 & 2 & 1 & 1 & 2 \\
\hline Isi(c)/Id/II & & 1 & & & & & & & & & & & 1 & 1 & & & 1 \\
\hline Id & 1 & 1 & & & & & & & 1 & & & & 1 & 2 & & & 2 \\
\hline Isi(c)/Id & 1 & 1 & & & & & & 1 & 1 & & & 1 & 1 & 2 & 1 & 1 & 2 \\
\hline Isi-c & 2 & 4 & 2 & & & & & 2 & 3 & 4 & 1 & & 2 & 9 & & 4 & 6 \\
\hline Isi/Isi-c & & & & & & & & 1 & 1 & 1 & & & & 2 & & 1 & 1 \\
\hline IcI/Isi-c & 2 & & 2 & & & & & & 1 & 2 & 1 & & & 3 & 1 & 3 & 1 \\
\hline Isi/IcL/Isi-c & 2 & & 2 & & & & & & & 3 & & & 1 & 3 & 1 & 1 & 3 \\
\hline IcI & & & 1 & & & & & & & & 1 & & & 1 & & 1 & \\
\hline Isi/IcI & & & 4 & & & & & & & 2 & 2 & & & 3 & 1 & 2 & 2 \\
\hline Isi & 2 & 1 & 1 & & & & & & 2 & 1 & 1 & & & 1 & 2 & 2 & 2 \\
\hline $\mathrm{Ig} / / \mathrm{si}$ & & & 2 & & & & & & 1 & 1 & & & & 2 & & 1 & \\
\hline unknown & 1 & 1 & & & & & & & & 1 & & 1 & & 2 & & 2 & \\
\hline
\end{tabular}


Table 18-18, (continued)

\begin{tabular}{|c|c|c|c|c|c|c|c|c|c|c|c|c|c|c|c|c|c|}
\hline \multirow[b]{2}{*}{ Unit } & \multicolumn{8}{|c|}{ Raw Material } & \multicolumn{5}{|c|}{ Cortex Type } & \multicolumn{2}{|c|}{ Patina } & \multicolumn{2}{|c|}{$\begin{array}{c}\text { Mineral } \\
\text { Precipitates }\end{array}$} \\
\hline & \begin{tabular}{|l|} 
Local \\
Edwards \\
Fine \\
Chert \\
\end{tabular} & \begin{tabular}{|l|} 
Local \\
Edwards \\
Medium/ \\
Coarse \\
Chert \\
\end{tabular} & \begin{tabular}{|l|} 
Nonlocal \\
Edwards \\
Fine \\
Chert \\
\end{tabular} & \begin{tabular}{|l|} 
Nonlocal \\
Edwards \\
Medium/ \\
Coarse \\
Chert \\
\end{tabular} & $\begin{array}{l}\text { Indeterminate } \\
\text { Non-Edwards } \\
\text { Fine Chert }\end{array}$ & \begin{tabular}{|l|} 
Indeter- \\
minate Non- \\
Edwards \\
Medium/ \\
Coarse Chert \\
\end{tabular} & Quartz & \begin{tabular}{|l} 
Indeter- \\
minate \\
Heat \\
Altered \\
\end{tabular} & Absent & $\begin{array}{l}\text { Upland } \\
\text { Residual }\end{array}$ & Nodular & $\begin{array}{l}\text { Stream } \\
\text { Modified }\end{array}$ & $\begin{array}{l}\text { Indeter- } \\
\text { minate }\end{array}$ & Absent & Present & Absent & Present \\
\hline \multicolumn{18}{|c|}{ Valley Floor B } \\
\hline $\begin{array}{l}\text { III } \\
\text { I }\end{array}$ & 4 & 2 & $\begin{array}{l}1 \\
2\end{array}$ & & & 1 & & 2 & 3 & $\begin{array}{l}2 \\
1\end{array}$ & 3 & 1 & 2 & $\begin{array}{c}10 \\
1\end{array}$ & 1 & 3 & $\begin{array}{l}8 \\
1\end{array}$ \\
\hline \multicolumn{18}{|c|}{ Valley Margin } \\
\hline$\overline{\text { IIIc }}$ & 6 & 4 & 2 & 1 & & 1 & 1 & & 2 & 7 & 5 & 1 & 1 & 13 & 2 & 13 & 3 \\
\hline IIIc POT & 2 & & & & & & & & 2 & & & & & 1 & 1 & 2 & \\
\hline $\mathrm{IIIb} / \mathrm{c}$ & & 3 & & & & & & & 1 & 1 & & & 1 & 3 & & 2 & 1 \\
\hline Y/IIIc & 2 & & 1 & & & 1 & & & 1 & 1 & 1 & & 1 & 3 & 1 & 2 & 2 \\
\hline $\mathrm{Y} / \mathrm{mll} \mathrm{b} / \mathrm{c}$ & 1 & & & & & & & & & 1 & & & & 1 & & & 1 \\
\hline IIIb & 1 & 1 & & & & & & & & 1 & & 1 & & 1 & 1 & 2 & \\
\hline $\mathrm{Y}$ & 4 & & & & & & & & 2 & 1 & & 1 & & 4 & & & 4 \\
\hline $\mathrm{X} / \mathrm{Y}$ & 1 & 1 & & & & & & & & 1 & & & 1 & 1 & 1 & & 2 \\
\hline $\mathrm{III} / \mathrm{b}$ & 2 & & & & & & & & & 1 & 1 & & & 1 & 1 & & 2 \\
\hline $\mathrm{X} / \mathrm{II} \mathrm{a} / \mathrm{b}$ & & 1 & & & & & & & & 1 & & & & 1 & & & 1 \\
\hline IIIa & 1 & & & & & & & & & 1 & & & & 1 & & & 1 \\
\hline II/IIIa & 1 & & & & & & & & & & & & 1 & & 1 & & \\
\hline $\mathrm{X} / \mathrm{II} / \mathrm{III}$ & 1 & 1 & & & & & & & & 1 & & & 1 & 2 & & 1 & 1 \\
\hline II & 3 & & & & & 1 & & & 2 & 1 & & & & 3 & 1 & 1 & 3 \\
\hline$x$ & 10 & 3 & 1 & 1 & & & & 1 & 4 & 2 & 4 & 1 & 4 & 11 & 3 & 3 & 11 \\
\hline $\mathrm{X} / \mathrm{II}$ & 3 & & & & & 1 & & & 1 & & 2 & 1 & & 4 & & 2 & 2 \\
\hline $\mathrm{X} / \mathrm{si}$ & & 1 & & & & & & & & 1 & & & & 1 & & & 1 \\
\hline Total & 156 & 63 & 27 & 9 & 1 & 12 & 1 & 9 & 77 & 97 & 45 & 23 & 37 & 230 & 45 & 167 & 110 \\
\hline
\end{tabular}


TABLE $18-19$

Distribution of Core Assemblage by Count, Weight, and Chert Type in Key Archeological Zones

\begin{tabular}{l|l|r|r|r|r|r}
\hline \multirow{2}{*}{ Archeological Chronology } & \multirow{2}{*}{ Chert Type } & \multicolumn{3}{|c|}{ Weight $(\mathrm{g})$} & \multirow{2}{*}{ Totals } \\
\cline { 3 - 6 } & coarse & $<100$ & $100-199$ & $200-299$ & $>300$ & \multicolumn{1}{c}{$2(100 \%)$} \\
& fine & $2(100 \%)$ & $0(0 \%)$ & $0(0 \%)$ & $0(0 \%)$ & $0(0 \%)$ \\
\hline Late Prehistoric & coarse & $0(0 \%)$ & $0(0 \%)$ & $0(0 \%)$ & $0(0 \%)$ & $0(0 \%)$ \\
& fine & $1(100 \%)$ & $0(0 \%)$ & $0(0 \%)$ & $0(0 \%)$ & $1(100 \%)$ \\
& coarse & $0(0 \%)$ & $0(0 \%)$ & $0(0 \%)$ & $0(0 \%)$ & $0(0 \%)$ \\
\hline Middle Archaic & fine & $4(57 \%)$ & $0(0 \%)$ & $0(0 \%)$ & $0(0 \%)$ & $4(57 \%)$ \\
& coarse & $3(43 \%)$ & $0(0 \%)$ & $0(0 \%)$ & $0(0 \%)$ & $3(43 \%)$ \\
\hline Early Archaic & fine & $15(21 \%)$ & $0(0 \%)$ & $2(3 \%)$ & $6(9 \%)$ & $23(33 \%)$ \\
& foarse & $47(67 \%)$ & $0(0 \%)$ & $0(0 \%)$ & $0(0 \%)$ & $47(67 \%)$ \\
\hline Early Archaic/Late Paleoindian & fine & $19(79 \%)$ & $0(0 \%)$ & $2(8 \%)$ & $1(4 \%)$ & $4(17 \%)$ \\
& foarse & $9(26 \%)$ & $1(3 \%)$ & $0(0 \%)$ & $1(4 \%)$ & $20(83 \%)$ \\
\hline Late Paleoindian & fine & $17(49 \%)$ & $6(17 \%)$ & $2(6 \%)$ & $3(9 \%)$ & $10(29 \%)$ \\
& coarse & $0(0 \%)$ & $0(0 \%)$ & $0(0 \%)$ & $0(0 \%)$ & $25(71 \%)$ \\
\hline Early Paleoindian & fine & $3(27 \%)$ & $3(27 \%)$ & $0(0 \%)$ & $5(45 \%)$ & $11(100 \%)$ \\
& & & & &
\end{tabular}

\section{CONCLUSIONS}

Cores, simple core tools, battered stones, and tested chert cobbles together embody the various primary lithic technologies and raw material procurement strategies employed over time at the Wilson-Leonard site. Given that use of the site spans a ca. 11,000-year period, the overall size of the core and cobble collection is surprisingly small.

With several faintly evident but notable exceptions, core technology appears to have been largely unpatterned during much of the site's history. A more-formalized blade technology during early Paleoindian times is hinted at with a few core fragments. By far, however, the most significant finding among this suite of artifacts is the presence of microcores, indicating the production of small flakes. Particularly prevalent in Early Archaic times, these microcores, and the corresponding microlithic tools (see Chapter 17), constitute a previously overlooked aspect of technology in the Archaic of Central Texas. Their implications for toolmaking in the Archaic is a priority for future inquiry.

For much of the site's history, lithic tool makers took advantage of fine-grained local cherts chiefly from upland settings as well as stream cobbles and pebbles. These cherts were used exhaustively, for the most part, as evidenced in the small, fine-grained core nuclei left behind. Significantly, battered stones-some of which may have been recycled from exhausted cores-are predominately of fine-grained cherts. Likely employed as hammerstones, these tools are particularly common in Early Paleoindian deposits.

Coarse-grained local cherts were selected throughout much of the site's history for core tools, suggesting use in heavy tasks requiring more durable materials. Surprisingly, the majority of larger, blocky, multidirectional cores were of coarse-grained chert, suggesting that flakes of this material type were needed for use in other tools or implements.

A particularly striking pattern is seen in the high frequencies of mineral precipitates on core tools as opposed to other tool types (see Tables 18-1 through 18-5). Mineral precipitates were recorded on almost $75 \%$ of core tools, as opposed to only $35 \%$ of battered stone and $40 \%$ of the core assemblage specimens. In most other tool types (e.g., projectile points, bifaces, Clear Fork tools, unifaces), comparatively low frequencies (ca. 30-35\%) overall were observed, although higher frequencies are present within certain stratigraphic units. In those cases, the highest incidence was seen in Unit II and older deposits.

While explanations for the presence or absence of mineral precipitates on stone artifacts are unknown, the patterned distribution seen here and at other sites (Collins 1994) clearly points to the need for future study. Pedogenic precipitates, especially those containing calcium, tend to accumulate slowly over time. Were the precipitates observed on the core tools strictly pedogenic, they should be more frequent and more developed in a single temporal cline. This is clearly not the case, as core tools bearing precipitates derive from all stratigraphic sections of the site.

As noted in Chapter 12, the broad term "mineral precipitates" was deliberately chosen during analysis to encompass calcium carbonates, calcium phosphates, and other salts or residues that may be difficult to distinguish visually. Our interpretation is that the precipitates on these core tools occur directly or indirectly as a result of the buildup of tenacious organic materials during their use. This may prove to be of great benefit in future studies of the function of the tools. 
"This page intentionally left blank" 


\section{REFERENCES CITED IN VOLUME II}

Aikens, C. M.

1970 Hogup Cave. Anthropological Papers 98. University of Utah, Salt Lake City.

Alexander, H. L., Jr.

1963 The Levi Site: A Paleo-Indian Campsite in Central Texas. American Antiquity 28:510-528.

Amick, D. S.

1995 Patterns of Technological Variation Among Folsom and Midland Projectile Points in the American Southwest. Plains Anthropologist 40:23-38.

Banks, K. M., L. A. Hannus, and R. P. Winham

1995 Foreword: Three Reservoirs, 40 Years Later. In Archeological Investigations in Three Reservoir Areas in South Dakota and Wyoming, Part I: Angostura Reservoir, by R. P. Wheeler. Smithsonian Institution, Bureau of American Ethnology, River Basin Surveys Papers, Inter-Agency Archeological Salvage Program. Reprints in Anthropology, Vol. 46, J\&L Reprint Company, Lincoln.

Barton, C. M., K. I. Olszewski, and N. R. Coinman

1996 Beyond the Graver: Reconsidering Burin Function. Journal of Field Archaeology 23(1):111-125.

Beck, C., and G. T. Jones

1993 The Multipurpose Function of Great Basin Stemmed Series Points. Current Research in the Pleistocene 10:52-53.

Bell, R.

1957 Clear Fork Gouges Found in Oklahoma. Bulletin of the Texas Archeological Society 28:285-288.

Binford, L. R.

1978 Nunamiut Ethnoarchaeology. Academic Press, New York.

1979 Organization and Formation Processes: Looking at Curated Technologies. Journal of Anthropological Research 35(3):255-273.

Bement, L. C.

1986 Excavation of the Late Pleistocene Deposits of Bonfire Shelter, Val Verde County, Texas. Archeology Series 1. Texas Archeological Survey, The University of Texas at Austin.

Black, S. L.

1986 The Clemente and Herminia Hinojosa Site, 41JW8: A Toyah Horizon Campsite in Southern Texas. Special Report 18. Center for Archaeological Research, The University of Texas at San Antonio.

Black, S. L., and A. J. McGraw

1985 The Panther Springs Creek Site: Cultural Change and Continuity within the Upper Salado Creek Watershed, South Central Texas. Archaeological Survey Report 100. Center for Archaeological Research, The University of Texas at San Antonio.
Blaine, J. C.

1968 A Preliminary Report of an Early Man Site in West Texas. Transactions of the Third Regional Archeological Symposium for Southeastern New Mexico and Western Texas 3:1-11. South Plains Archeological Society, Lubbock.

Bleed, P.

1986 The Optimal Design of Hunting Weapons: Maintainability or Reliability. American Antiquity 51(4):737747.

Black, S. L.

1986 The Clemente and Herminia Hinojosa Site, 41JW8: A Toyah Horizon Campsite in Southern Texas. Special Report 18. Center for Archaeological Research, The University of Texas at San Antonio.

Bordes, F.

1961 Typologic du Paléolithique luférieur et Moyen. Delmus, Bordeaux.

Bousman, C. B.

1993 Hunter-Gatherer Adaptations, Economic Risk, and Tool Design. Lithic Technology 18(1 and 2):59-86.

Bradley, B.

1993 Paleo-Indian Flaked Stone Technology in the North American High Plains. In From Kostenki to Clovis: Upper Paleolithic-Paleo-Indian Adaptations, edited by O. Soffer, pp. 251-262. Plenum Press, New York.

Brezillon, M. N.

1968 La Denomination de objets de peirre taillee. IV supplement a Gallia Prehistoire. Centre National de la Recherche Scientifique, Paris.

Broecker, W. W., and J. L. Kulp

1957 Lamont Natural Radiocarbon Measurements IV. Science 126(3287):1324-1334.

Brown, K. M.

1985 Three Caches of Guadalupe Tools from South Texas. Bulletin of the Texas Archeological Society 56:75-125.

Brown, K. M., D. R. Potter, G. D. Hall, and S. L. Black

1982 Excavations at 41LK67: A Prehistoric Site in the Choke Canyon Reservoir, South Texas. Choke Canyon Series 5. Center for Archaeological Research, The University of Texas at San Antonio.

Campbell, T. N.

1948 The Merrell Site: Archaeological Remains Associated with Alluvial Terrace Deposits. Bulletin of the Texas Archeological and Paleontological Society 19:7-35.

1959 A List of Radiocarbon Dates from Archeological Sites in Texas. Bulletin of the Texas Archeological Society 30:311320. 
Chadderdon, M. F.

1983 Baker Cave, Val Verde County, Texas: The 1976 Excavations. Special Report 13. Center for Archaeological Research, The University of Texas at San Antonio.

Chandler, C. K.

1974 Use Wear Analysis of "Clear Fork" Tools from the Falcon Reservoir Area, Southern Texas. La Tierra 1(4):1521.

Chandler, C. K., F. Knolle, and M. M. Knolle

1983 Paleo-Indian Projectile Points from Jim Wells and Nueces County, Texas. La Tierra 10(2):23-27.

Christenson, A. L.

1986 Projectile Point Size and Projectile Aerodynamics: An Exploratory Study. Plains Anthropologist 31(112):109128.

Clark, J. D.

1969 Kalambo Falls Prehistoric Site, Volume I. Cambridge University Press, Cambridge.

1974 Kalambo Falls Prehistoric Site, Volume II. Cambridge University Press, Cambridge.

Coffin, E. F.

1932 Archaeological Exploration of a Rock Shelter in Brewster County, Texas. Indian Notes and Monographs 48. Museum of the American Indian, Heye Foundation, New York.

Collins, M. B.

1969 Test Excavations at Amistad International Reservoir, Fall 1967. Papers of the Texas Archeological Salvage Project 16. The University of Texas at Austin.

1972 The Devil's Hollow Site, A Stratified Archaic Campsite in Central Texas. Bulletin of the Texas Archeological Society 43:77-100.

1974 A Functional Analysis of Lithic Technology among Prehistoric Hunter-Gatherers of Southwestern France and Western Texas. Unpublished Ph.D. dissertation, University of Arizona, Tuscon.

1975 Lithic Technology as a Means of Processual Inference. In Lithic Technology, Making and Using Stone Tools, edited by E. Swanson, pp. 15-34. Mouton Publishers, The Hague.

1994 Late Archaic Evidence. In Human Ecology in the Middle Onion Creek Valley, Hays County, Texas, by R. Ricklis and M. B. Collins. Studies in Archeology 19. Texas Archeological Research Laboratory, The University of Texas at Austin.

1995 Forty Years of Archeology in Central Texas. Bulletin of the Texas Archeological Society 66:361-400.

1996 The Kevin Davis Cache (41NV659) and Clovis Blade Technology in the South Central United States. Report submitted to the Office of the State Archeologist, Texas Historical Commission, Austin.

n.d. Clovis and Folsom Lithic Technology on and near the Southern Plains: Similar Ends, Different Means. Manuscript on file with author.

Collins, M. B. (editor)

1979 Excavations at Four Archaic Site in the Lower Ohio Valley, Jefferson County, Kentucky. Occasional Papers in Anthropology 1. Department of Anthropology, University of Kentucky, Lexington.

Collins, M. B., and A. C. Kerr

1993 Archeology of the Earliest Texans. Paper presented at the 64th Annual Meeting of the Texas Archeological Society, Laredo.

Collins, M. B., B. Ellis, and C. D. Ellis

1990 Excavations at the Camp Pearl Wheat Site (41KR243), An Early Archaic Campsite on Town Creek, Kerr County, Texas. Studies in Archeology 6. Texas Archeological Research Laboratory, The University of Texas at Austin.

Collins, M. B., and T. R. Hester

1968 A Wooden Mortar and Pestle from Val Verde County, Texas. Bulletin of the Texas Archeological Society 39:18.

Collins, M. B., T. R. Hester, D. Olmstead, and P. J. Headrick

1991 Engraved Pebbles from Early Archeological Contexts in Central Texas. Current Research in the Pleistocene 8:1315.

Cotter, J. L.

1937 The Occurrence of Flints and Extinct Animals in Pluvial Deposits near Clovis, New Mexico. Part IV, Report on Excavation at the Gravel Pit, 1936. Proceedings of the Academy of Natural Sciences of Philadelphia 89:1-16.

1938 The Occurrence of Flints and Extinct Animals in Pluvial Deposits near Clovis, New Mexico, Part VI, Report on Field Season of 1937. Proceedings of the Academy of Natural Sciences of Philadelphia 90:113-117.

Crabtree, D. E.

1972 An Introduction to Flintworking. Occasional Paper No. 28. Idaho State University, Pocatello.

1982 Introduction to Flintworking, $2^{\text {nd }}$ ed. Occasional Papers of the Idaho Museum of Natural History 28. Idaho Museum of Natural History, Pocatello.

Crawford, D. D.

1965 The Granite Beach Site, Llano County, Texas. Bulletin of the Texas Archeological Society 36:71-98.

Crook, W. W., and R. K. Harris

1952 The Trinity Aspect of the Archaic Horizon: The Carrollton and Elam Foci. Bulletin of the Texas Archeological Society 23:7-38. 
Daugherty, R. D.

1988 Ozette Village Site. In Historical Dictionary of North American Archaeology, edited by E. B. Jelks and J. C. Jelks, pp. 354-355. Greenwood Press, New York.

Davis, E. M.

1962 The Archeology of the Lime Creek Site in Southwestern Nebraska. Special Publication No. 3. University of Nebraska State Museum, University of Nebraska, Lincoln.

Dellinger, S. C.

1936 Baby Cradles of the Ozark Bluff Dwellers. American Antiquity 1:197-311.

Demars, P. Y., and P. Laurent

1989 Types D'Outils Lithiques du Paleolithique Superieur en Europe. Cahiers du Quaternaire 14. Centre National de la Rechorche Scientifique, Paris.

Dial, S. W.

1993 Prehistoric and Historic Archeology of the Upper Barton Creek Watershed. Unpublished master's thesis, Department of Anthropology, The University of Texas at Austin.

Dibble, D. S.

1970 On the Significance of Additional Radiocarbon Dates from Bonfire Shelter, Texas. Plains Anthropologist 15(50, Part 1):251-254.

Dibble, D. S., and D. Lorrain

1968 Bonfire Shelter: A Stratified Bison Kill Site, Val Verde County, Texas. Miscellaneous Papers 1. Texas Memorial Museum, Austin.

Dibble, H. L.

1987 The Interpretation of Middle Paleolithic Scraper Morphology. American Antiquity 52(1):109-117.

Driskell, B. N.

1986 The Chipped Stone Tool Production/Use Cycle: Its Potential in Activity Analysis of Disturbed Sites. International Series 305. British Archaeological Reports, Oxford.

1994 Stratigraphy and Chronology at Dust Cave. Journal of Alabama Archaeology 4(1\&2):17-34.

Early, A. M., and G. Sabo, III

1988 Previous Archeological Investigations. In Human Adaptation in the Ozark and Ouachita Mountains, by G. Sabo III, A. M. Early, J. C. Rose, B. A. Burnett, L. Voegele, Jr., and J. P. Harcourt, pp. 15-33. Final Report, Study Unit 1, Ozark-Arkansas-Ouachita, Archeological Research, Synthesis, and Overview Report. U. S. Army Corps of Engineers, Southwestern Division, Dallas.

Epstein, J. F.

1969 The San Isidro Site: An Early Man Campsite in Nuevo Leon Mexico. Anthropology Series 7. Department of Anthropology, The University of Texas at Austin.
Fenenga, F.

1953 The Weights of Chipped Stone Points: A Clue to Their Functions. Southwestern Journal of Anthropology 9:309323.

Fields, R. C.

1984 Archeological Investigations at 41LN134 and 41 LN144, Jewett Mine Project, Leon County, Texas. Reports of Investigations 35. Prewitt and Associates, Inc., Austin.

Fish, P. R.

1979 The Interpretive Potential of Mousterian Debitage. Anthropological Research Papers No. 16. Arizona State University, Tempe.

Flenniken, J. J., and A. W. Raymond

1986 Morphological Projectile Point Typology: Replication Experimentation and Technological Analysis. American Antiquity 52(3):603-614.

Flinn, R., and J. Flinn

1968 The High Bluff Site on the Clear Fork of the Brazos River. Bulletin of the Texas Archeological Society 38:93125.

Folsom, F., and M. E. Folsom

1993 America's Ancient Treasures. University of New Mexico Press, Albuquerque.

Forrester, R. E.

1985 Horn Shelter Number 2: The North End, A Preliminary Report. Central Texas Archeologist 10:21-35.

1996 Horn Shelter Number 2: The North End. A Stratified Rock Shelter in Bosque County, Texas. Occasional Papers of the Strecker Museum No. 3. Baylor University, Waco.

Friis-Hansen, J.

1990 Mesolithic Cutting Arrows: Functional Analysis of Arrows Used in the Hunting of Large Game. American Antiquity 64:494-504.

Frison, G. C.

1978 Prehistoric Hunters of the High Plains. Academic Press, San Diego.

Frison, G. C., and B. A. Bradley

1980 Folsom Tools and Technology at the Hanson Site, Wyoming. University of New Mexico Press, Albuquerque.

Frison, G. C., and D. C. Grey

1980 Pryor Stemmed: A Specialized Late Paleoindian Ecological Adaptation. Plains Anthropologist 25(87):2746.

Frison, G. C., and D. J. Stanford

1982 The Agate Basin Site: A Record of the Paleoindian Occupation of the Northwestern High Plains. Academic Press, New York. 
Goode, G. T.

1989 Private Collections from the Kennedy Bluffs Site. In $E x$ cavations at 41BP19, the Kennedy Bluffs Site, Bastrop County, Texas, edited by L. C. Bement, pp. 67-154. Contract Reports in Archeology 5. Highway Design Division, Texas State Department of Highways and Public Transportation, Austin.

Goodyear, A. C.

1974 The Brand Site: A Techno-Functional Study of a Dalton Site in Northeast Arkansas. Publications in Archeology Research Series 7. Arkansas Archeological Survey, Fayetteville.

1982 The Chronological Position of the Dalton Horizon in the Southeastern United States. American Antiquity 47(2):382395 .

1995 The Brand Site: A Techo-Functional Study of a Dalton Site in Northeast Arkansas. Arkansas Archeological Survey Research Series No. 7. Arkansas Archeological Survey, Fayetteville.

Gould, R. A.

1980 Living Archaeology. Cambridge University Press, Cambridge.

Gregg, J.

1958 Commerce of the Prairies. University of Oklahoma Press, Norman.

Greiser, S. T.

1985 Predictive Models of Hunter-Gatherer Subsistence and Settlement Strategies on the Central High Plains. Plains Anthropologist Memoir 20.

Griffen, W. B.

1983 Southern Periphery: East. In Southwest, edited by A. F. Ortiz, pp. 329-342. Handbook of North American Indians, vol. 10, W. C. Sturtevant, general editor. Smithsonian Institution, Washington, D.C.

Haas, H., V. Holliday, and R. Stuckenrath

1986 Dating of Holocene Stratigraphy with Soluble and Insoluble Organic Fractions at the Lubbock Lake Archaeological Site, Texas: An Ideal Case Study. Radiocarbon 28(2A):473-485.

Haberman, S. J.

1978 Analysis of Quantitative Data, Vol. 1. Academic Press, New York.

Hall, G. D.

1981 Allens Creek: A Study in Cultural Prehistory of the Lower Brazos River Valley, Texas. Report 61. Texas Archeological Survey, The University of Texas at Austin.

Hall, G. D., S. L. Black, and C. Graves

1982 Archaeological Investigations at Choke Canyon Reservoir, South Texas: The Phase I Findings. Choke Canyon Series 5. Center for Archaeological Research, The University of Texas at San Antonio.
Hall, G. D., T. R. Hester, and S. L. Black

1986 The Prehistoric Sites at Choke Canyon Reservoir, Southern Texas: Results of Phase II Archaeological Investigations. Choke Canyon Series 10. Center for Archaeological Research, The University of Texas at San Antonio.

Harriott, T.

1893 Narrative of the First English Plantation of Virginia. Bernard Quaritch, London.

Hartwell, W. T.

1995 The Ryan's Site Cache: Comparison to Plainview. Plains Anthropologist 40(2A):165-184.

Hayden, B., and J. Kamminga

1979 An Introduction to Use-Wear: The First Conference on Lithic Use-Wear. In Lithic Use-Wear Analysis, edited by B. Hayden, pp. 1-13. Academic Press, New York.

Hayden, B., N. Franco, and J. Spafford

1996 Evaluating Lithic Strategies and Design Criteria. In Stone Tools: Theoretical Insights into Human Prehistory, edited by G. H. Odell, pp. 9-45. Plenum Press, New York.

Hays, T. R.

1982 Archaeological Investigations at the San Gabriel Reservoir Districts, Central Texas. 4 volumes. Report submitted to the U.S. Army Corps of Engineers, Fort Worth District, by the Archaeological Program, Institute of Applied Sciences, North Texas State University, Denton.

Heizer, R. F., and A. D. Krieger

1956 The Archaeology of Humboldt Cave, Churchill County, Nevada. University of California Publications in American Archaeology and Ethnology 47(1):1-190. Berkeley.

Hester, J. J.

1972 Blackwater Locality No. 1: A Stratified, Early Man Site in Eastern New Mexico. Publication of the Fort Burgwin Research Center No. 8. Southern Methodist University, Dallas.

Hester, T. R.

1969 Archeological Investigations in Kenedy and Kleburg Counties, Texas, August, 1967. Report 15. Archeological Program, State Building Commission, Austin.

1971 Archeological Investigations at the La Jita Site, Uvalde County, Texas. Bulletin of the Texas Archeological Society 42:51-148.

1977 The Current Status of Paleoindian Studies in Southern Texas and Northeastern Mexico. In Paleoindian Lifeways, edited by E. Johnson, pp. 169-186. The Museum Journal 17. West Texas Museum Association, Texas Tech University, Lubbock.

1979a Early Populations in Prehistoric Texas. Archaeology 32(6):26-33.

1979b Notes on Gower, Jetta, and Other Projectile Points of the Pre-Archaic Period. La Tierra 6(3):5-8. 
1980 Digging into South Texas Prehistory. Corona, San Antonio.

1983 Late Paleoindian Occupations at Baker Cave, Southwestern Texas. Bulletin of the Texas Archeological Society 53:101-119.

1989 The Archaic of the Texas-Mexico Borderlands. Paper presented for the Southern North American Archaic Symposium, La Jitas, Texas, Oct. 7-13, 1989, Manuscript on file, Texas Archeological Research Laboratory, The University of Texas at Austin.

1990 Early Archaic "Eccentric" Lithic Artifacts in Southern and Central Texas. La Tierra 17(3):1-5.

1991 Notes on South Texas Archaeology: 1991-2, The Plainview Points from the St. Mary's Hall Site, South Central Texas. La Tierra 18(2):1-4.

1993 Lithic Typology: Background, Goals, and a Personal Perspective. Lithic Technology 18(1\&2):1-4.

1995a The Prehistory of South Texas. Bulletin of the Texas Archeological Society 66:427-459.

1995b Notes on South Texas Archaeology 1995-1: Early Archaic Chronology in South Texas. La Tierra 22(1):4-5.

Hester, T. R., M. B. Collins, and P. J. Headrick

1992 Paleo-Indian Engraved Stones from the Gault Site. La Tierra 19(4):3-5.

Hester, T. R., D. Gilbow, and A. D. Albee

1973 A Functional Analysis of "Clear Fork" Artifacts from the Rio Grande Plain, Texas. American Antiquity 38(1):9096.

Hester, T. R., and R. F. Heizer

1972 Problems in the Functional Interpretation of Artifacts: Scraper Planes from Mitla and Yazul, Oaxaca. Papers on Archaeology 14. Contributions of the University of California Research Facility, University of California, Berkeley.

Hester, T. R., and D. Knepper

1991 Further Notes on the Lithics of the Plainview Occupation at the St. Mary's Hall Site, South Central Texas. $\mathrm{La}$ Tierra 18(3):1-5.

Hester, T. R., and C. M. Whatley

1992 Chipped Stone Artifacts from Site 41DM59, Dimmit County, Southern Texas. La Tierra 19(3):1-7.

Highley, C. L.

1995 Chipped Stones. In Archeological Excavations at the Loma Sandia Site (41LK28), A Prehistoric Cemetery and Campsite in Live Oak County, Texas, by A. J. Taylor and C. L. Highley, pp. 405-484. Studies in Archeology 20. Texas Archeological Research Laboratory, The University of Texas at Austin.
Hoffman, C. M.

1985 Projectile Point Maintenance and Typology: Assessment with Factor Analysis and Canonical Correlation. In Fo: Concordance in Archaeological Analysis: Bridging Data Structure, Quantitative Technique, and Theory, edited by C. Carr, pp. 566-612. Westport Publishers, Kansas City.

Hofman, J. L.

1977 A Technological Analysis of Clear Fork Tool Production. Bulletin of the Oklahoma Anthropological Society 26:105122.

1989 Prehistoric Culture History: Hunters and Gatherers in the Southern Great Plains. In From Clovis to Comanchero: Archeological Overview of the Southern Great Plains, edited by J. L. Hofman, R. L. Brooks, J. S. Hays, D. W. Owsley, R. L. Jantz, M. K. Marks, and M. H. Manhein, pp. 25-60. Arkansas Archeological Survey Research Series 35. Arkansas Archeological Survey, Fayetteville.

Hofman, J. L., L. C. Todd, and M. B. Collins

1991 Identification of Central Texas Edwards Chert at the Folsom and Lindenmeier Sites. Plains Anthropologist 36(137):297-308.

Holder, P., and J. Wilke

1949 The Frontier Complex: A Preliminary Report on a Prehistoric Hunter's Camp in Southwestern Nebraska. American Antiquity 14(4, Part 1):260-266.

Holliday, V. T.

1985 New Data on the Stratigraphy and Pedology of the Clovis and Plainview Sites, Southern High Plains. Quaternary Research 23:388-402.

1990 Investigations of the Plainview Site and Middle Running Water Draw. In Fifty Years of Discovery: The Lubbock Lake Landmark, Guidebook to the Quaternary History of the Llano Estacado, edited by V. T. Holliday and E. Johnson, pp. 93-104. Lubbock Lake Landmark Quaternary Research Center Series No. 2. Museum of Texas Tech University, Lubbock.

Holliday, V. T., and E. Johnson

1990 An Overview of the Cultural Chronology of the Lubbock Lake Landmark. In Fifty Years of Discovery: The Lubbock Lake Landmark, Guidebook to the Quaternary History of the Llano Estacado, edited by V. T. Holliday, and E. Johnson, pp. 19-54. Lubbock Lake Landmark Quaternary Research Series No. 2. Museum of Texas Tech University, Lubbock.

Holliday, V. T., E. Johnson, H. Haas, and R. Stuckenrath

1983 Radiocarbon Ages from the Lubbock Lake Site, 19501980: Framework for Cultural and Ecological Change on the Southern High Plains. Plains Anthropologist 28(101):165-182.

1985 Radiocarbon Ages for the Lubbock Lake Site: 1981-1984. Plains Anthropologist 30(110), Pt. 1:227-291. 
Holliday, V. T., and K. A. Grombacher

1974 An Assessment of the Archeological and Historical Resources to be Affected by the Proposed Chiltipin Creek Flood Control Project, San Patricio County, Texas. Research Report 29. Texas Archeological Survey, The University of Texas at Austin.

Howard, C.

1975 A Study of the Clear Fork Gouge. Bulletin of the Texas Archeological Society 44:51-60.

Howard, E. B.

1935 Evidence of Early Man in North America. The Museum Journal 24. University of Pennsylvannia, Philadelphia.

1943 The Finley Site: Discovery of Yuma Points, in situ, near Eden, Wyoming. American Antiquity 8(3):224-234.

Hudler, D. B.

1997 Determining Clear Fork Tool Function through Use-Wear Analysis: A Discussion of Use-Wear Methods and Clear Fork Tools. Studies in Archeology 25. Texas Archeological Research Laboratory, The University of Texas at Austin.

Hughes, J. T.

1949 Investigations in Western South Dakota and Northeastern Wyoming. American Antiquity 14:266-277.

1980 Some Early and Northerly Occurrences of the Clear Fork Gouge. Papers on the Prehistory of Northeastern Mexico and Adjacent Texas, edited by J. F. Epstein, T. R. Hester, and C. Graves, pp. 143-146. Special Report 9. Center for Archaeological Research, The University of Texas at San Antonio.

Irwin-Williams, C., H. Irwin, G. Agogino, and C. V. Haynes

1973 Hell Gap: Paleo-Indian Occupation on the High Plains. Plains Anthropologist 18:40-53.

Jelinek, A. J.

1966 Some Distinctive Flakes and Flake Tools from the Llano Estacado. Papers of the Michigan Academy of Science, Arts and Letters 51:399-405.

1976 Form, Function, and Style in Lithic Analysis. In Cultural Change and Continuity, edited by C. B. Cleland, pp. 1934. Academic Press, New York.

Jelks, E. B.

1962 The Kyle Site: A Stratified Central Texas Aspect Site in Hill County, Texas. Archaeology Series 5. The University of Texas at Austin.

Jennings, J. D.

1957 Danger Cave. Anthropological Papers 27. University of Utah, Salt Lake City.

Johnson, E. (editor)

1987 Lubbock Lake: Late Quaternary Studies on the Southern High Plains. Texas A\&M University Press, College Station.
Johnson, E., and V. T. Holliday

1980 A Plainview Kill/Butchering Locale on the Llano Estacado-The Lubbock Lake Site. Plains Anthropologist 25(Part 1):89-111.

Johnson, J. K.

1979 Archaic Biface Manufacture: Production Failures, A Chronicle of the Misbegotten. Lithic Technology 8(2):2535 .

1981 Yellow Creek Archaeological Project, Vol. 2. Papers of the Center for Archaeological Research 2. Center for Archaeological Research, University of Mississippi, Oxford.

1983 Poverty Point Period Blade Technology in the Yazoo Basin, Mississippi. Lithic Technology 12(3):49-56.

Johnson, L., Jr.

1962 The Yarbrough and Miller Sites of Northeastern Texas, with a Preliminary Definition of the La Harpe Aspect. Bulletin of the Texas Archeological Society 32:141-284.

1964 The Devil's Mouth Site, A Stratified Campsite at Amistad Reservoir, Val Verde County, Texas. Archeology Series 6. Department of Anthropology, The University of Texas at Austin.

1967 Toward a Statistical Overview of the Archaic Cultures of Central and Southwestern Texas. Bulletin 12. Texas Memorial Museum, The University of Texas at Austin.

1989 Great Plains Interlopers in the Eastern Woodlands during Late Paleo-Indian Times. Report 36. Office of the State Archeologist, Texas Historical Commission, Austin.

1991 Early Archaic Life at the Sleeper Archaeological Site, 41BC65 of the Texas Hill Country, Blanco County, Texas. Publications in Archaeology Report 39. Texas State Department of Highways and Public Transportation, Austin.

Johnson, L., Jr., and G. Goode

1994 A New Try at Dating and Characterizing Holocene Climates, as well as Archeological Periods, on the Eastern Edwards Plateau. Bulletin of the Texas Archeological Society $65: 1-54$.

Johnson, R., and G. Bhattacharyya

1985 Statistics: Principles and Methods. John Wiley \& Sons, New York.

Judge, W. J.

1973 Paleoindian Occupation of the Central Rio Grande Valley in New Mexico. University of New Mexico Press, Albuquerque.

Justice, N. D.

1995 Stone Age Spear and Arrow Points of the Midcontinental and Eastern United States. Indiana University Press, Blommington. 
Karbula, J.

1993 The Ekols Site: Continuing Studies. Newsletter of the Friends of TARL 1(2):29.

Kay, M.

n.d. Microwear Examination of Lake Ilo Project Folsom Artifacts. Manuscript on file with author.

Keeley, L. H.

1982 Hafting and Retooling: Effects on the Archaeological Record. American Antiquity 47(4):798-809.

Kelley, J. C.

1947 The Lehman Rock Shelter: A Stratified Site of the Toyah, Uvalde, and Round Rock Foci. Bulletin of the Texas Archeological Society 18:115-128.

Kelly, R. L.

1988 The Three Sides of a Biface. American Antiquity 53(4):717-734.

Kelly, T. C.

1962 The Crumley Site: A Stratified Burnt Rock Midden, Travis County, Texas. Bulletin of the Texas Archeological Society 31:239-272.

1982 Criteria for Classification of Plainview and Golondrina Projectile Points. La Tierra 9(3):2-25.

1983a The Barber Paleo-Indian Point. La Tierra 10(4):1025.

1983b The Brom Cooper Paleo-Indian Collection from McMullen County, Texas. La Tierra 10(3):17-40.

1987 Archaeology of the Gamenthaler Valley, Gillespie County, Central Texas: A Preliminary Report. La Tierra 14(1):527.

Klement, L. W., R. C. Fields, E. F. Gadus, J. B. McLerran, and C. B. Bousman

1993 The Peerless Bottoms Site, 41HP175. In Excavations at the Tick, Spike, Johns Creek, and Peerless Bottoms Sites, Cooper Lake Project, Delta and Hopkins Counties, Texas, by R. C. Fields, E. F. Gadus, L. W. Klement, C. B. Bousman, and J. B. McLerran, pp. 165-226. Reports of Investigations 91. Prewitt and Associates, Inc., Austin.

Knecht, H.

1988 Upper Paleolithic Burins. BAR International Series 434. British Archaeological Reports, Oxford.

Knudson, R.

1973 Organizational Variability in Late Paleo-Indian Assemblages. Unpublished Ph.D. dissertation, Department of Anthropology, Washington State University, Pullman.

1983 Organizational Variability in Late Paleoindian Assemblages. Reports of Investigations 60. Laboratory of Anthropology, Washington State University, Pullman.
Koldehoff, B.

1987 The Cahokia Flake Tool Industry: Socioeconomic Implications for Late Prehistory in the Central Mississippi Valley. In The Organization of Core Technology, edited by J. K. Johnson and C. A. Morrow, pp. 187-206. Westview Press, Boulder.

Krieger, A. D.

1944 The Typological Concept. American Antiquity 3:271288.

1947a Artifacts from the Plainview Bison Bed. In Fossil Bison and Associated Artifacts from Plainview, Texas. Bulletin of the Geological Society of America 58:927-954.

1947b Certain Projectile Points of Early American Hunters. Bulletin of the Texas Archeolgical and Paleontological Society 18:7-27.

Kuhn, S.

1990 A Geometric Index of Reduction for Unifacial Stone Tools. Journal of Archaeological Science 17:583-593.

1994 A Formal Approach to the Design and Assembly of Mobile Toolkits. American Antiquity 59(3):426-442.

Le Blanc, $\mathrm{R}$.

1992 Wedges, Pièces Esquillées, Bipolar Cores, and Other Things: An Alternative to Shott's View of Bipolar Industries. North American Archaeologist 13:1-14.

Lewenstein, S.

1987 Stone Tool Use at Cerros. The University of Texas Press, Austin.

Lynott, M. J.

1975 Explanation of Microwear Pattern on Gravers. Plains Anthropologist 20(68):121-128.

Mallouf, R. J.

1981 A Case Study of Plow Damage to Chert Artifacts: The Brookeen Creek Cache, Hill County, Texas. Office of the State Archeologist Report 33. Texas Historical Commission, Austin.

Marchbanks, M. L., and M. B. Collins

1993 Trace Organic Residue Analysis from Early Archaic Artifacts. Current Research in the Pleistocene 10:8486.

Marcy, R. B.

1963 Thirty Years of Army Life on the Border. Harper, New York.

McDermott, J. F. (editor)

1940 Tixier's Travels on the Osage Prairies. University of Oklahoma Press, Norman.

McDonald, G.

1968 Debert: A Palaeo-Indian Site in Central Nova Scotia. Anthropology Papers 16. National Museum of Canada, Ottawa. 
McKinney, W. W.

1981 Early Holocene Adaptations in Central and Southern Texas: The Problem of the Paleo-Indian Archaic Transition. Bulletin of the Texas Archeological Society 52:91120 .

McReynolds, R.

1984 Two-Tanged Knife Forms from Val Verde County, Texas La Tierra 11(2):6-11.

1993 Some Examples of Bandy Points. La Tierra 20(3):9-16.

Miller, E. O., and E. B. Jelks

1952 Archeological Excavations at the Belton Reservoir, Coryell Country, Texas. Bulletin of the Texas Archeological Society 23:168-217.

Morse, D. F

1971 The Hawkins Cache: A Significant Dalton Find in Northeast Arkansas. Arkansas Archeologist 12(1):9-20.

Morse, D. F., and A. C. Goodyear

1973 The Significance of the Dalton Adze in Northeast Arkansas. Plains Anthropologist 18(62):316-322.

Morse, D. F., and P. A. Morse

1983 Archaeology of the Central Mississippi Valley. Academic Press, New York.

Mosteller, F., S. F. Fienberg, and R. E. K. Rourke

1983 Beginning Statistics with Data Analysis. Addison-Wesley, Reading, Massachusetts.

Nelson, M. C.

1991 The Study of Technological Organization. In Archaeological Method and Theory, Vol. 3, edited by M. B. Schiffer, pp. 57-100. University of Arizona Press, Tucson.

Newcomb, W. W., Jr.

1961 The Indians of Texas: From Prehistoric to Modern Times. The University of Texas Press, Austin.

Nichols, P. W.

1959 Archaeological Report of Site on Gus B. Mauermann Ranch, Travis County, Texas. Unpublished master's thesis, Department of Anthropology, The University of Texas at Austin.

Norusis, M. J.

1993 SPSS for Windows, Base System User's Guide, Release 6.0. SPSS, Inc., Chicago.

Parry, W. J.

1994 Prismatic Blade Technologies in North America. In The Organization of North American Prehistoric Chipped Stone Tool Technologies, edited by P. J. Carr, pp. 87-98. Archaeological Series No. 7. International Monographs in Prehistory, Ann Arbor.

Parry, W. J., and R. L. Kelly

1987 Expedient Core Technology and Sedentism. In The Or- ganization of Core Technology, edited by J. K. Johnson and C. A. Morrow, 285-304. Westview Press, Boulder.

Patterson, J. T.

1936 The Corner Tang Flint Artifact of Texas. University of Texas Bulletin 3618, Anthropological Papers 1(4). The University of Texas at Austin.

Patterson, L. W.

1985 Distinguishing Between Arrow and Spear Points on the Upper Texas Coast. Lithic Technology 14:81-89.

Patterson, L. W., and J. Hudgins

1985 Paleo-Indian Occupations in Wharton County, Texas. Bulletin of the Texas Archeological Society 56:155-170.

Perino, G.

1968 Guide to the Identification of Certain American Indian Projectile Points. Special Bulletin 3. Oklahoma Anthropological Society, Norman.

Perttula, T.

1984 Interim Report on the Quince Site (34A+-134) CK Atoka, Oklahoma. Oklahoma Archeological Society Newsletter $32(3): 2-6$.

Peter, D. E

1982 Projectile Point Classification. In Archaeological Investigations at San Gabriel Reservoir Districts, Central Texas, edited by T. R. Hays, pp. 14.3-14.35. Institute of Applied Sciences, North Texas State University.

Pimentel, R. A., and J. D. Smith

1985 BIOSTAT II: A Multivariate Statistical Toolbox. Second edition. Sigma Soft, Placentia, California.

Prewitt, E. R

1981a Archeological Excavations at the Loeve-Fox, Loeve, and Tombstone Bluff Sites in the Granger Lake District of Central Texas. Archaeological Investigations at the San Gabriel Reservoir Districts, Vol. 4. North Texas State University, Denton

1981b Cultural Chronology in Central Texas. Bulletin of the Texas Archeological Society 52:65-89.

1981c A Wooden Mortar from the Stockton Plateau of Texas. Journal of Field Archaeology 8:111-117.

1983 Andice: An Early Archaic Dart Point Type. La Tierra 10(3):17-40.

1984 Appendix 2: Artifact Descriptions. In Settlement in the Upper Bull Creek Basin: An Inventory and Assessment of Cultural Resources at the Canyon Creek Development, Travis County, Texas, edited by M. A. Howard and M. D. Freeman, pp. 175-204. Reports of Investigations 32. Prewitt and Associates, Inc., Austin.

1985 From Circleville to Toyah: Comments on Central Texas Chronology. Bulletin of the Texas Archeological Society 54:201-238. 
1995 Distribution of Typed Projectile Points in Texas. Bulletin of the Texas Archeological Society 66:83-174.

n.d. The Rogers Spring Site 41TV39. Unpublished manuscript on file at the Texas Archeological Research Laboratory.

Prikryl, D. J.

1990 Lower Elm Fork Prehistory. A Redefinition of Cultural Concepts and Chronologies along the Trinity River, NorthCentral Texas. Office of the State Archeologist Report 37. Texas Historical Commission, Austin.

Purdy, B.

1981 Florida's Prehistoric Stone Technology. University Presses of Florida, Gainesville.

Ray, C. N.

1938 The Clear Fork Culture Complex. Bulletin of the Texas Archeological and Paleontological Society 10:193-207.

1941 The Various Types of the Clear Fork Gouge. Bulletin of the Texas Archeological and Paleontology Society 13:152162.

1961 Deductions Concerning the Clear Fork Gouge. Bulletin of the Texas Archeological Society 30:199-207.

Redder, A. J.

1985 Horn Shelter Number 2: The South End, A Preliminary Report. Central Texas Archeologist 10:37-65.

Renaud, E. B.

1931 Prehistoric Flaked Points from Colorado and Neighboring Districts. Proceedings of the Colorado Museum of Natural History 10(2):6-17.

1932 Yuma and Folsom Artifacts (New Material). Proceedings of the Colorado Museum of Natural History 11(2):518.

Robinson, D. L., and S. Turpin

1993 Hunter-Gatherer Mobility and Settlement in the Brazos Uplands: Archeology in the Calvert Mine, Robertson County, East-Central Texas. Studies in Archeology 14. Texas Archeological Research Laboratory, The University of Texas at Austin.

Root, M. J.

1994 Archaeological Field and Laboratory Techniques. In Archeology of the Bobtail Wolf Site (32DU955A): 19931994 Progress Report, edited by M. J. Root and A. M. Emerson, pp. 23-48. Project Report 26. Center for Northwest Anthropology, Washington State University, Pullman.

Runnels, C.

1985 Trade and the Demand for Millstones in Southern Greece in the Neolithic and Early Bronze Age. In Prehistoric Production and Exchange: The Aegean and Eastern Mediterranean, edited by B. Knapp and T. Stech, pp. 30-43. Institute of Archaeology Monograph 25, University of California at Los Angeles.
Schiffer, M. B.

1972 Archaeological Context and Systemic Context.American Antiquity 37:156-165.

Sellards, E. H.

1955 Fossil Bison and Associated Artifacts from Milnesand, New Mexico. American Antiquity 20(336-344).

Sellards, E. H., G. L. Evans, and G. E. Meade

1947 Fossil Bison and Associated Artifacts from Plainview Texas. Bulletin of the Geological Society of America 58:927954.

Shafer, H. J.

1963 Test Excavations at the Youngsport Site: A Stratified Terrace Site in Bell County, Texas. Bulletin of the Texas Archeological Society 24:57-81.

1970 Notes on Uniface Retouch Technology. American Antiquity 35(4):480-487.

1971 Investigations into South Plains Prehistory, West Central Texas: Salvage Archeology in the Robert Lee Reservoir District. Papers of the Texas Archeological Salvage Project No. 20. Texas Archeological Salvage Project, The University of Texas at Austin.

1973 Lithic Technology at the George C. Davis Site, Cherokee County, Texas. Ph.D. dissertation, Department of Anthropology, The University of Texas at Austin.

1983 The Lithic Artifacts of the Pulltrouser Swamp Area: Settlements and Fields. In Pulltrouser Swamp: Ancient Maya Habitat, Agriculture, and Settlement in Northern Belize, edited by B. L. Turner and P. D. Harrison, pp. 212-245. The University of Texas Press, Austin.

Shafer, H. J., and V. M. Bryant, Jr.

1977 Archeological and Botanical Studies at Hinds Cave, Val Verde County, Texas. Special Series No. 1. Anthropology Laboratory, Texas A\&M University, College Station.

Shennan, S.

1988 Quantifying Archaeology. Academic Press, San Diego.

Shiner, J.

1975 The Clear Fork Gouge Revisited. Bulletin of the Texas Archeological Society 46:179-188.

Shott, M. J.

1993 Spears, Darts, and Arrows: Late Woodland Hunting Techniques in the Upper Ohio Valley. American Antiquity 58:425-443.

Sollberger, J. B.

1971 A Technological Study of Beveled Knives. Plains Anthropologist 16(53):209-218.

Sollberger, J. B., and T. R. Hester

1972 The Strohacker Site: A Review of Pre-Archaic Manifestations in Texas. Plains Anthropologist 17(58):326-344. 
Solls, R. A.

1985 The Scraper Plane: A Functional Interpretation. Journal of Field Archaeology 12:99-106.

Sorrow, W. M.

1968 The Devil's Mouth Site: The Third Season 1967. Papers of the Texas Archeological Salvage Project 14. Texas Archeological Salvage Project, The University of Texas at Austin.

1969 The John Ischy Site: A Burnt Rock Midden in Williamson County, Texas. Papers of the Texas Archeological Salvage Project 18, The University of Texas at Austin.

Sorrow, W. M., H. J. Shafer, and R. E. Ross

1967 Excavations at Stillhouse Hollow Reservoir. Papers of the Texas Archeological Salvage Project 12. Texas Archeological Salvage Project, The University of Texas at Austin.

Speer, R. D.

1990 History of the Plainview Site. In Fifty Years of Discovery: The Lubbock Lake Landmark, Guidebook to the Quaternary History of the Llano Estacado, edited by V. T. Holliday and E. Johnson, pp. 79-92. Lubbock Lake Landmark Quaternary Research Center Series Number 2. Museum of Texas Tech University, Lubbock.

Story, D. A.

1990 Cultural History of the Native Americans. In The Archeology and Bioarcheology of the Gulf Coastal Plain, Volume 1, by D. A. Story, J. A. Guy, B. A. Burnett, M. D. Freeman, J. C. Rose, D. G. Steele, B. W. Olive, and K. J. Reinhard, pp. 163-366. Arkansas Archeological Survey Research Series 38. Arkansas Archeological Survey, Fayetteville.

Story, D. A., J. A. Guy, B. A. Burnett, M. D. Freeman, J. C. Rose, D. G. Steele, B. W. Olive, and K. J. Reinhard

1990 The Archeology and Bioarcheology of the Gulf Coastal Plain, Volume 1. Arkansas Archeological Survey Research Series 38. Arkansas Archeological Survey, Fayetteville.

Suhm, D. A.

1961 The Beidleman Ranch Site: An Early Man Kill Site in Stonewall County, Texas. Bulletin of the Texas Archeological Society 30(for 1960):207-212.

1955 Excavations at the Collins Site, Travis County, Texas. Bulletin of the Texas Archeological Society 26:7-54.

Suhm D. A., and E. B. Jelks

1962 Handbook of Texas Archeology: Type Descriptions. Special Publications 1, Texas Archeological Society, and Bulletin 4, Texas Memorial Museum, Austin.

Suhm, D. A., A. D. Krieger, and E. B. Jelks

1954 An Introductory Handbook of Texas Archeology. Bulletin of the Texas Archeological Society 25.

Taylor, A. J., and C. L. Highley

1995 Archeological Investigations at the Loma Sandia Site (41LK28), A Prehistoric Cemetery and Campsite in Live
Oak County, Texas. Studies in Archeology 20. Texas Archeological Research Laboratory, The University of Texas at Austin.

Tennis, C., M. Renner, and R. J. Hard (editors)

1996 Archaeological Survey of Laughlin Air Force Base, Val Verde County, Texas. Survey Report 239. Center for Archaeological Research, The University of Texas at San Antonio.

Thomas, D. H.

1978 Arrowheads and Atlatl Darts: How the Stones Got the Shaft. American Antiquity 43:461-472.

Thomas, P. M., Jr., and L. J. Campbell

1978 A Multicomponent Site on the Happyville Bend of Little River: 16LA37-The Whatley Site. Report of Investigations 11. New World Research, Baton Rouge.

Thoms, A. V.

1992 Late Pleistocene and Early Holocene Regional Land Use Patterns: A Perspective from the Preliminary Results of Archaeological Studies at the Richard Beene Site, 41BX831, Lower Medina River, South Texas. In Late Cenozoic Alluvial Stratigraphy and Prehistory of the Inner Gulf Coastal Plain, edited by R. Mandel, and S. C. Caran. Series 4. South Central Friends of the Pleistocene, Lubbock Lake Quaternary Research Center, Lubbock.

1993 Knocking Sense from Old Rocks: Typologies and the Narrow Perspective of the Angostura Point Type. Lithic Technology 18(1\&2):16-27.

1995 Investigations at the Richard Beene Site, South Central Texas. Cultural Research Management News and Views 8(1):10-12,17.

Titmus, G. L., and J. C. Woods

1991 A Closer Look at Margin "Grinding" on Folsom and Clovis Points. Journal of California and Great Basin Anthropology 13(2):194-203.

Tomka, S.

n.d. Biface Manufacture Failures at 41BP19. Unpublished manuscript on file at the Texas Archeological Research Laboratory, The University of Texas at Austin.

Tomka, S. A., and R. C. Fields

1990 Chipped Stone Artifacts, 41LN29A and 41LN106. In Excavations at the Charles Cox, Lambs Creek Knoll, and Buffalo Branch Sites, Jewett Mine Project, Leon and Freestone Counties, Texas, Reports of Investigations 70. Prewitt and Associates, Inc, Austin.

Tomka, S., and E. R. Prewitt

1993 What Do I Call Thee? Projectile Point Types and Archaeological Interpretations: Perspective from Texas. Lithic Technology 18(1\&2):49-58.

Trigham, R., G. Cooper, G. Odell, B. Voytek, and A. Whitman 1974 Experimentation in the Formation of Edge Damage: A 
New Approach to Lithic Analysis. Journal of Field Archaeology 1:171-195.

Turner, S. E., and T. R. Hester

1985 A Field Guide to Stone Artifacts of Texas Indians. Texas Monthly Press, Austin.

1993 A Field Guide to Stone Artifacts of Texas Indians. $2^{\text {nd }}$ ed. Gulf Publishing Co., Houston.

Vaughn, P.

1985 The Burin Blow Technique: Creator or Eliminator? Journal of Field Archaeology 14(4):488-496.

Webb, C. H., J. L. Shiner, and E. W. Roberts

1971 The John Pearce Site (16CD56): A San Patrice Site in Caddo Parish, Louisiana. Bulletin of the Texas Archeological Society 42:1-49.

Weber, C.

1994 A Replication Technique for Andice/Bell Points. In Archaic and Late Prehistoric Human Ecology in the Middle Onion Creek Valley, Hays County, Texas, Volume 2, edited by R. A. Ricklis and M. B. Collins, pp. 629-651. Studies in Archeology 19. Texas Archeological Research Laboratory, The University of Texas at Austin.

Weir, F. A.

1956 Surface Artifacts from La Perdida, Starr County, Texas. Bulletin of the Texas Archeological Society 27:59-78.

1976 The Central Texas Archaic. Unpublished Ph.D. dissertation, Washington State University, Pullman.

1985 An Early Holocene Burial at the Wilson-Leonard Site in Central Texas. Mammoth Trumpet 2(1):3.

Wesolowsky, A. B., T. R. Hester, and D. R. Brown

1976 Archeological Excavations at the Jetta Court Site (41TV151) Travis County, Texas. Bulletin of the Texas Archeological Society 47:25-87.

Wheat, J. B.

1972 The Olsen-Chubbuck Site: A Paleo-Indian Bison Kill. Memoirs of the Society for American Archaeology 26.

Wheeler, R. P.

1954 Selected Projectile Point Types of the United States: II. Bulletin of the Oklahoma Anthropological Society 2:1-6.
1995 Archeological Investigations in Three Reservoir Areas in South Dakota and Wyoming, Part I: Angostura Reservoir. Smithsonian Institution, Bureau of American Ethnology, River Basin Surveys Papers, Inter-Agency Archeological Salvage Program Vol. 46. J\&L Reprint Company, Lincoln, Nebraska.

Whittaker, J. C.

1994 Flintknapping: Making and Understanding Stone Tools. The University of Texas Press, Austin.

Willey, P. S., B. R. Harrison, and J. T. Hughes

1978 The Rex Rodgers Site. In Archeology at Mackenzie Reservoir, by J. T. Hughes and P. S. Willey, pp. 51-114. Survey Report 24. Office of the State Archeologist, Texas Historical Commission, Austin.

Wilmsen, E. N.

1970 Lithic Analysis and Cultural Inference: A Paleo-Indian Case. Anthropological Papers of the University of Arizona Number 16. University of Arizona Press, Tucson.

Wilmsen, E. N., and F. H. H. Roberts, Jr.

1978 Lindenmeier, 1934-1974. Smithsonian Contributions to Anthropology, Number 24. Smithsonian Institution Press, Washington, D.C.

Winship, G. P.

1896 The Coronado Expedition, 1540-1542. 14th Annual Report. Bureau of American Ethnology, Washington, D.C.

Wood, W. R., and R. B. McMillan (editors)

1976 Prehistoric Man and His Environments: A Case Study in the Ozark Highland. Academic Press, New York.

Word, J. H., and C. L. Douglas

1970 Excavations at Baker Cave, Val Verde County, Texas. Bulletin 16. Texas Memorial Museum, The University of Texas at Austin.

Wormington, H. M.

1957 Ancient Man in North America. Popular Series 4. Denver Museum of Natural History, Denver.

Wyckoff, D. J.

1994 Introduction to the 1991 Bulletin. In Bulletin of the Oklahoma Antropological Society, Vol. XL, edited by D. G. Wyckoff and D. Shockey, pp. 1-8. Transcript Press, Norman, Oklahoma. 
"This page intentionally left blank" 
"This page intentionally left blank" 
"This page intentionally left blank" 
"This page intentionally left blank" 
"This page intentionally left blank" 
"This page intentionally left blank" 
"This page intentionally left blank" 
"This page intentionally left blank" 
ISBN 1-887072-26-8 (Volume II)

ISBN 1-887072-24-1 (5 Volume Set) 